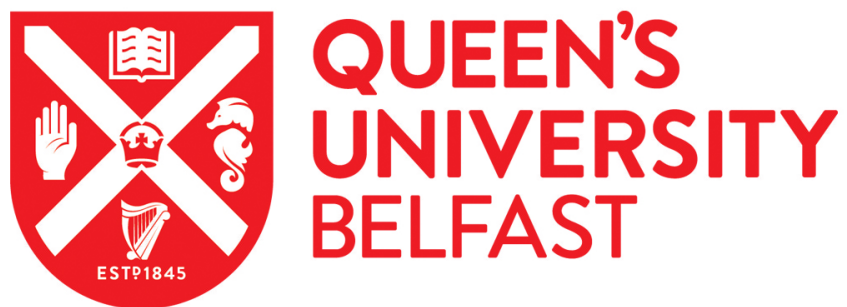

\section{Provenance and depositional variability of the Thin Mud Facies in the lower Ganges-Brahmaputra delta, West Bengal Sundarbans, India}

Flood, R. P., Barr, I. D., Weltje, G. J., Roberson, S., Russell, M. I., Meneely, J., \& Orford, J. D. (2018).

Provenance and depositional variability of the Thin Mud Facies in the lower Ganges-Brahmaputra delta, West Bengal Sundarbans, India. Marine Geology, 395, 198-218. https://doi.org/10.1016/j.margeo.2017.09.001

Published in:

Marine Geology

Document Version:

Peer reviewed version

Queen's University Belfast - Research Portal:

Link to publication record in Queen's University Belfast Research Portal

Publisher rights

(C) 2017 Elsevier B.V.

This manuscript version is made available under the CC-BY-NC-ND 4.0 license http://creativecommons.org/licenses/by-nc-nd/4.0/,which permits distribution and reproduction for noncommercial purposes, provided the author and source are cited

\section{General rights}

Copyright for the publications made accessible via the Queen's University Belfast Research Portal is retained by the author(s) and / or other copyright owners and it is a condition of accessing these publications that users recognise and abide by the legal requirements associated with these rights.

Take down policy

The Research Portal is Queen's institutional repository that provides access to Queen's research output. Every effort has been made to ensure that content in the Research Portal does not infringe any person's rights, or applicable UK laws. If you discover content in the Research Portal that you believe breaches copyright or violates any law, please contact openaccess@qub.ac.uk. 


\section{Highlights}

- Holocene TMF in the West Bengal Sundarbans determined to show intensively weathered, terrestrial sediment derived from the Ganges Alluvial Plain (GAP)

- Lithofacies is indicative of muddy tidal flat with aggradation and fining-up in grain size.

- Sediment provenance indicates a continuing G-B sediment source from the active delta front, with sediment reworked over the far-western abandoned delta by tidal-estuarine forcing. 
1 Provenance and depositional variability of the Thin Mud Facies in the lower

2 Ganges-Brahmaputra delta, West Bengal Sundarbans, India

3

4 R.P., Flood ${ }^{1, *}$, I.D., Barr ${ }^{1}$, G.J., Weltje ${ }^{2}$, S., Roberson ${ }^{3}$, M.I., Russel1 ${ }^{1}$, J. Meneely ${ }^{1}$, $5 \quad$ J.D., Orford ${ }^{1}$

$7 \quad{ }^{1}$ School of Natural and Built Environment, Queen's University Belfast, Belfast, BT7

$8 \quad 1 N N$, Northern Ireland, UK

$9 \quad{ }^{2}$ Department of Earth and Environmental Sciences, Geology Division, University of

10 Leuven, Celestijnenlaan 200E, 3001 Leuven-Heverlee, Belgium

$11{ }^{3}$ Geological Survey of Northern Ireland, Dundonald House, Upper Newtownards

12 Road, Belfast BT4 3SB, Northern Ireland, UK

$14{ }^{*}$ Corresponding author. Present address: Department of Petroleum and Energy

15 Engineering, The American University in Cairo, New Cairo 11835, Arab Republic of

16 Egypt. Email address: rory.flood@aucegypt.edu

18 Keywords: Ganges-Brahmaputra delta; West Bengal Sundarbans; grain-size; XRD;

19 XRF; mineralogy; geochemistry; weathering; provenance.

21 ABSTRACT

22 The Sundarbans is one of the largest coastal wetland sites in the world and covers an 23 area of approximately one million hectares of the western delta of the Ganges and

24 Brahmaputra (G-B) rivers (located across Bangladesh and India). Since the late 25 Holocene, the western delta has not been directly fluvially sourced, due to the 26 Ganges shift to the east (present-day Bangladesh). The depositional facies (Thin Mud 
27 Facies) of the late-Holocene abandoned western region (The Sundarbans) is derived

28 from dominant estuary-tidal dynamics, however the provenance of the associated

29 TMF sedimentation in this far western zone (Indian Sundarbans per se) is as yet

30 equivocal. In this study, sediment cores from the Indian Sundarbans (Saptamukhi-

31 Thakuran estuary) were closely examined for grain-size distributions (GSDs),

32 mineralogy through X-ray diffraction (XRD), and geochemistry with X-ray

33 fluorescence (XRF). The TMF in the West Bengal Sundarbans has been determined

34 to show intensively weathered, terrestrial sediment, derived principally from the

35 Ganges Alluvial Plain (GAP). There is a predominance of quartz, mica and clay

36 minerals, with quartz interpreted as a product of low-relief tropical weathering

37 sourced via the G-B Rivers draining the Himalayas. Lithofacies interpreted through

38 GSD analysis of the TMF is indicative of a muddy tidal flat environment with

39 aggradation and a general fining-up trend between the adjacent estuaries. The

40 sediment provenance indicates a continuing G-B sediment source, which moves

41 westward along the Bay of Bengal, from the active delta front and is then reworked

42 over the far-western abandoned delta by tidal-estuarine forcing. 


\section{INTRODUCTION}

44 The Indian Sundarbans comprises just over 400,000 hectares of mangrove land cover

45 in the western sector of the Ganges-Brahmaputra (G-B) delta, and is cross-cut by a

46 number of approximately north-south estuarine channels; the Mooriganga,

47 Saptamukhi, Thakuran, Matla, Bidya, Gosaba, and Haribhanga (Fig. 1). The overall

48 morphology of the far-western G-B delta reflects that of a tide-dominated system.

49 Depositionally it falls on the extreme of a west-east continuum of tidal, mixed tidal-

50 fluvial, and purely fluvial in the contemporary G-B river mouth estuary (Rogers et

51 al., 2013). The eastern sector of the G-B delta comprises the fluvially dominated

52 system, where fluvially-driven shoreline progradation occurred following the joining

53 of the Ganges and Brahmaputra Rivers (Allison, 1998a), while the older abandoned

54 part of the delta, in the west, comprises the non-fluvially dominated system (no

55 longer directly linked fluvially to the G-B River sources) (Rogers et al., 2013). This

56 western part of the delta, which underlies the present day Indian Sundarbans, was

57 fluvially abandoned prior to c. 5000 cal yr BP, as the Ganges River migrated

58 eastward towards its present position (Goodbred and Kuehl, 2000a; Sarkar et al.,

59 2009). Over the last 4,000 yrs, the West Bengal Sundarbans area is thought to be

60 estuary-tidal in terms of process domination, leading to the deposition of what is

61 termed the "Thin Mud Facies" (TMF: Goodbred and Kuehl, 2000a). The origin of

62 TMF sediments in what is now the Bangladesh Sundarbans, is considered to be

63 related to reworking of G-B sourced muds from the delta front (Allison et al., 2003;

64 Rogers et al., 2013), but a similar provenance for the Indian Sundarbans TMF has

65 still to be substantiated. The western extent of the G-B delta is now considered to be

66 undergoing net delta front erosion as a result of eustatic sea-level rise and tectonic

67 subsidence (Allison, 1998b; Allison et al., 2003) and, by inference, is seen as one 
68 possible source for the extreme western TMF. This paper aims to establish

69 sedimentary depositional processes and provenance in the West Bengal Sundarbans

70 during the last 4-5,000 yrs, and addresses the potential sources of sediment in the far

71 "abandoned" western sector of the G-B delta system, by analysing sedimentary cores

72 from three deltaic islands in the far-western Sundarbans; Lothian, Dhanchi, and

73 Gplot, shown in Fig. 1(ii).

\section{LATE-PLEISTOCENE AND HOLOCENE SEDIMENTARY FACIES OF}

\section{THE LOWER GANGES-BRAHMAPUTRA DELTA}

\section{2.1. Background}

78 The majority of studies on the Quaternary stratigraphy and sedimentary facies of the

79 Ganges-Brahmaputra (G-B) system have determined the appropriate broad-scale

80 sedimentary facies models (e.g., Morgan and McIntire, 1959; Coleman, 1969;

81 Umitsu, 1987, 1993; Kuehl et al., 1989; Johnson and Alam, 1991; Lindsay et al., 82 1991; Curray, 1991; Johnson, 1994; Hait et al., 1996; Kuehl et al., 1997; Hübscher et 83 al., 1998; Stanley and Hait, 2000; Goodbred and Kuehl, 2000a, 2000b; Goodbred et 84 al., 2003; Allison et al., 2003; Sarkar et al., 2009). These studies show variability in 85 the use of facies descriptions for the G-B delta and these tend to follow the broader 86 scale late Quaternary evolution of the delta (e.g., Goodbred and Kuehl, 2000a), or

87 Holocene evolution of the lower delta plain (e.g., Allison et al., 2003). In terms of the 88 entire G-B delta, the oldest stratigraphic units are dated from 18,000 cal yr BP and 89 consist of coarse channel sands, indicative of lowstand alluvial valleys and oxidized 90 laterites associated with subaerial weather exposure (Goodbred et al., 2003). Fining-

91 up of the channel sands and a silty mud unit with wood and marine fossils is 92 understood to have taken place from 10,000-11,000 cal yr BP, and has been 
93 interpreted as a mangrove-colonized coastal plain (Goodbred et al., 2003). From c.

$9411,000-3,000 \mathrm{cal}$ yr BP, muddy fluvial sands of the mid-Holocene prograding river

95 channels overlay these coastal facies, with the upper delta stratigraphy consisting of

96 single or multiple fining-up sand sequences that are locally interspersed with silty

97 floodplain deposits. The late Quaternary stratigraphic facies of the G-B delta have

98 been broken down into six principal groups by Goodbred and Kuehl (2000a):

99 Oxidised Facies (OF), Sand Facies (SF), Lower Delta Mud Facies (LDMF), Muddy

100 Sand Facies (MSF), Sylhet Mud Facies (SMF), and Thin Mud Facies (TMF). This

101 description of stratigraphic facies succession has been further developed by Allison

102 et al. (2003) to look specifically at the lower delta plain stratigraphy. The lower delta

103 plain stratigraphy has been further divided into five units: Muddy Sand (MS),

104 Mottled Mud (MM), Interbedded Mud (IM), Laminated Sand (LS), and Peaty Mud

105 (PM) (Allison et al., 2003). The fining up in grain size from the MS to IM/MM,

106 located west of the active G-B river mouths is attributed to either older phases or

107 multiple phases of progradation of the lower delta plain (Allison et al., 2003).

108 Subaqueous shoal sedimentation has been linked to the MSF as a result of the

109 reduced mud content and cross-stratification associated with bedload transport

110 (Allison et al., 2003). Current energies have been found to diminish in the preserved

111 upward section of the core sections examined by Allison et al. (2003) which allow

112 for enhanced deposition of mud, illustrating this upward fining of the sequences.

114 2.2. Thin Mud Facies of the Ganges-Brahmaputra delta

115 Goodbred and Kuehl (2000a) describe the TMF as representing the cap unit of 116 coarser underlying facies units throughout the Bengal Basin, consisting of overbank 117 deposits of the modern and recent floodplain system. Deposition of this facies unit 
118 took place from approximately $5000 \mathrm{cal} \mathrm{yr}$ BP to present, and is present within a

119 depth of 5-7 $\mathrm{m}$ to the surface of the G-B delta. This unit is generally poorly preserved

120 in the deeper stratigraphy indicative of rapid channel migration and frequent avulsion

121 and subsequent removal. As outlined by Allison et al. (2003) the TMF and surface

122 sediments of the lower delta plain tend to be very homogenous silts to clayey silts

123 with the Sundarbans in the west presenting slightly finer material on average in

124 comparison to that of the eastern delta. The lithostratigraphy of the near surface

125 sediments appears to be homogenous with a network of mangrove roots at a depth of

$126 \quad 3-4 \mathrm{~m}$ below the surface. Bed thicknesses tend to range from 3-10 $\mathrm{cm}$, with a series

127 of alternating layers of clay dominant and clay-deficient silts.

128 The TMF is understood to be found in floodplain environments and absent

129 near active fluvial channels and is interpreted as abandoned floodplain overbank

130 deposits (Goodbred and Kuehl, 2000a). In contrast to Goodbred and Kuehl's

131 observations of the TMF as a product of abandoned floodplain and overbank

132 deposits, Allison et al. (2003) regard the source of TMF sediments as reworked

133 Bengal Bay nearshore muds, transported into the delta plain by a mixture of cyclones

134 and constant tidal activity up the abandoned river channels, which post 5,000 yrs ago

135 have been transformed into tidal-dominant estuaries.

136 The central criticism with these interpretations of lithofacies is that grain-size

137 distributions (GSDs) represent a mixture of sediment populations, corresponding to

138 different production and/or transport mechanisms (cf., Weltje and Prins, 2003; 2007).

139 In the conceptual model of spatio-temporal grain-size variation developed by Weltje

140 and Prins $(2003 ; 2007)$, each size fraction corresponds to a characteristic process,

141 termed a dynamic population (DP). DPs may be defined in probabilistic terms as an

142 assemblage of grains that are likely to occur together, as they respond to dynamics of 
143 sediment production and transport in a similar manner (Weltje and Prins, 2003). DPs

144 provide a link between GSD variation and palaeoenvironmental reconstructions, as 145 they can be coupled with the physical laws that govern sediment production and

146 transport (Weltje and Prins, 2003). Tying this understanding of GSD and physical

147 laws of production and transport of sediments, means that when a sediment is being

148 eroded, the probability of any grain going into transport increases with diminishing

149 grain-size (McLaren and Bowles, 1985). Here we present the first discussion of the

150 production and transport of sediment within the G-B Delta that more fully

151 encompasses the various processes contributing to grain-size variability in TMF

152 lithofacies. For the purposes of this study, analysis is focussed primarily on the TMF

153 unit as described by Goodbred and Kuehl (2000a).

155 2.3. Mineralogy and geochemistry of the Ganges-Brahmaputra Rivers

156 Both the Ganges and Brahmaputra rivers drain lithologically complex basins and 157 lack a dominant lithology (Small et al., 2009). The drainage basin of the Ganges 158 River is composed of highly weathered sediments and volcanics with clay dominance 159 (Coleman, 1969; Lupker et al., 2012; 2013; Goodbred et al., 2014). Detrital grains of 160 higher quartz and lower feldspars contents in surface sediments of the Bengal Basin 161 (BB) are indicative of a source zone of low-relief tropical weathering (Potter, 1978; 162 Datta and Subramanian, 1997). The lack of BB carbonate minerals is attributed to 163 both weathering and the settling effects of detrital carbonates during transport in the 164 upper reaches of the G-B Rivers (Datta and Subramanian, 1997).

165 The Ganges catchment streams draining the Himalaya have been well studied 166 (e.g., Galy and France-Lanord, 1999; Dalai et al., 2002; Bickle et al., 2003, 167 Chakrapani, 2005; Garzanti et al., 2010, 2011; Fontorbe et al., 2013; Frings et al. 
168 2015) and are characterised by sediment supply from rapid physical and chemical

169 weathering. Carbonate weathering is dominant with minor contributions from silicate

170 weathering and hot springs (Frings et al., 2015). Differences in chemistries of

171 headwaters in the southern tributaries of the Ganges compared to other streams

172 draining the Himalaya have found cation compositions dominated by $\mathrm{Na}^{+}$

173 (Rengarajan et al., 2009) instead of $\mathrm{Ca}^{2+}$ (Frings et al., 2015).

174 Detrital micas dominate the clay fraction $(\sim 80 \%)$ in Himalayan streams of

175 the Ganges with authigenic clays more dominant in the alluvial plain (Chakrapani et

176 al., 1995). The alluvial plain is the dominant zone of weathering and clay formation

177 in the G-B Rivers with predominance of illite and kaolinite (Sarin et al., 1989;

178 Chakrapani et al., 1995; Datta and Subramanian, 1997; Heroy et al., 2003; Frings et 179 al., 2015).

180 The key distinction between the Ganges and Brahmaputra is silica enrichment

181 in the sand fraction of Ganges sediment-attributable to chemical weathering of

182 feldspar (Singh et al., 2005a, b; Bhuiyan et al., 2011). Fluvial transport is considered

183 a major source of mineral sorting, controlling the geochemistry of weathering

184 products (Singh et al., 2005a). Sediments in the upper catchment of the Ganges, the

185 Ganges Alluvial Plain (GAP), have undergone chemical weathering of incipient to

186 moderate intensity, with a first-stage of weathering in the Himalayas, followed by a

187 second stage of weathering in the GAP (Singh et al. (2005a). The clay mineralogy

188 and chemical index of alteration (CIA) (Nesbitt and Young, 1982) of sediments in

189 the Brahmaputra show that weathering is generally less intense than in the Ganges

190 and GAP (Singh et al., 2005b; Bhuiyan et al., 2011). This lower weathering intensity

191 is attributed to higher runoff rates with greater physical erosion within the

192 Brahmaputra. Heavy rainfall occurs during the SW and NE monsoons in the eastern 
193 Himalaya and, as a result, there is increased runoff, which limits the potential for

194 alteration of sediments through chemical weathering (Singh et al., 2005b).

Rogers et al. (2013) examined present-day sediment composition through sediment traps and radioisotope geochemistry, particularly through inventories of ${ }^{7} \mathrm{Be}\left(\mathrm{t}_{1 / 2}=53.3\right.$ days; $\left.477.7 \mathrm{KeV}\right),{ }^{210} \mathrm{~Pb}\left(\mathrm{t}_{1 / 2}=22.3\right.$ years; $\left.46.5 \mathrm{KeV}\right),{ }^{234} \mathrm{Th}\left(\mathrm{t}_{1 / 2}=\right.$

19824.1 days; $63.3 \mathrm{KeV})$ and ${ }^{137} \mathrm{Cs}\left(\mathrm{t}_{1 / 2}=30.1\right.$ years; $\left.661 \mathrm{KeV}\right)$. The results from the 199 inventory of ${ }^{7} \mathrm{Be}$ have shown that atmospheric deposition cannot alone be responsible 200 for accumulation of ${ }^{7} \mathrm{Be}$ in sediments (Rogers et al., 2013). It has been proposed that $201{ }^{7}$ Be can only be added with the input of new sediment (Rogers et al., 2013). These 202 findings from Rogers et al. (2013) indicate that there is rapid transport of G-B 203 sediments to the inner BB shelf, sourced from sediment eroded from the surface of 204 the G-B fluvial catchment. While on the coastal shelf, the river sediment plume is 205 dispersed westward by prevailing currents. This sediment plume remains in 206 suspension and available for transport onshore through a series of tidal creeks and 207 onto the subaerial delta plain (Kuehl et al., 1989; Barua et al., 1994; Rogers et al., 208 2013).

209 In a previous study, Flood et al. (2016), the joint geochemical and grain size 210 composition from the West Bengal Sundarbans was modelled using compositional 211 data analysis (CoDa) and partial least squares (PLS) regression. It was found that 212 there was a strong relationship between zirconium $(\mathrm{Zr})$ and coarse grained sediment, 213 with coarse clay and medium/coarse-silt found to be related to rubidium ( $\mathrm{Rb}$ ). Fine214 grained sediment provenance was found to relate to $\mathrm{Rb}$ and $\mathrm{K}$, with coarse grained 215 sediment provenance linked to Zr. Calcium was interpreted to relate to external 216 environmental controls (e.g., sea-level/ tidal inundation) as $\mathrm{Ca}$ is generally only 217 present in liquid form in the marine environment. There was a negative covariance of 
$218 \mathrm{Ca}$ with $\mathrm{Zr}$, Ti, $\mathrm{K}$, and $\mathrm{Rb}$ with decline in $\mathrm{Ca}$ and concomitant increases in $\mathrm{Zr}$, $\mathrm{Ti}, \mathrm{K}$,

219 and $\mathrm{Rb}$ interpreted as terrestrial derived sediment flux with diminished marine or

220 carbonate deposition (Flood et al., 2016).

\section{3. METHODOLOGY}

222 3.1. Fieldwork

223 Coring was carried out using a motor driven percussion coring device with latitude,

224 longitude and elevation recorded with a differential GPS and reported as above mean 225 sea-level (amsl). Coring was carried out at Lothian $\left(21^{\circ} 42^{\prime} 0.9252^{\prime \prime} \mathrm{N}, 88^{\circ} 18^{\prime}\right.$

$22646.0188^{\prime \prime}$ E; 4.539 amsl), Gplot (21 41' 24.3456" N, 88²4' 9.4788" E; 2.532 amsl),

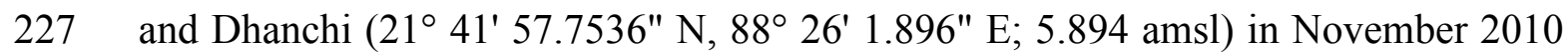
228 (sites shown in Fig. 1(ii)). These cores were all taken through the TMF sequence, 229 varying between 5-7 m overall depth, before moving into the underlying sand facies 230 of the fluviatile dominated delta. In total, 202 samples $(8 \mathrm{~cm}$ apart $)$ were collected 231 from the three cores (Lothian, $n=83$, Gplot, $n=46$, and Dhanchi, $n=73$ ). Samples were 232 divided into three groups for laboratory analysis (i.e., laser granulometry, XRD, and $233 \mathrm{XRF})$.

235 3.2. Analysis of grain-size distributions

236 GSDs were analysed following Flood et al. (2015; 2016) using a MalvernMastersizer 2372000 instrument. Data were aggregated into quarter phi intervals ( $\varphi$ scale) over the 238 range of $0.02-2000 \mu \mathrm{m}$. Analysis of GSD composition followed the methodology 239 developed recently by Flood et al. (2015) through compositional data analysis $240(\mathrm{CoDa})$ and multivariate statistics through principal components analysis (PCA) and 241 cluster analyses (CA). In order to assess the textural characteristics of sediments 242 from the Sundarbans, this study has adopted the approaches advocated by Flood et al. 
243 (2015), whereby the GSD data are log-ratio transformed. A description of these

244 statistical procedures are outlined in Flood et al. $(2015 ; 2016)$. PCA and CA results

245 from Lothian Island GSDs have been published previously (Flood et al., 2015) and

246 will not be presented again in this study.

247 Centred log-ratio (clr) transformation was carried out on the GSDs prior to

248 multivariate statistical analysis using the 'compositions' package for the $\mathrm{R}$ statistical

249 environment (R Development Core Team, 2011). R was downloaded freely at

250 http://www.r-project.org for Windows (current version 3.4.1, 62 MB). PCA,

251 hierarchical cluster and k-means cluster analyses were carried out on the log-

252 transformed GSDs using the statistical package IBM® ${ }^{\circledR}$ SPSS ${ }^{\circledR}$ Statistics version

25319.0.

255 3.3. X-ray diffraction and mineralogical analysis

256 Selected core samples were lightly homogenised to an equal grain size fraction $(<75$

$257 \mu \mathrm{m}$ fraction) using an agate mortar and pestle in order to determine sediment

258 mineralogy. X-ray powder diffraction (XRPD) was performed using a $3 \mathrm{~kW}$

259 PANalytical X'pert Pro Powder Diffractometer (Almelo, The Netherlands) $\theta / \theta$

260 goniometer with a $\mathrm{CuK}_{\alpha 1}$ electrode producing monochromatic radiation $(\lambda=1.54060$

$261 \AA, 40 \mathrm{kV}, 40 \mathrm{~mA}$ ) between 3 and $63^{\circ} 2 \theta$ with a step size of $0.02^{\circ}$ using an

262 X'Celerator multichannel detector.

263 Qualitative and quantitative analyses of XRD data was performed using 264 X'Pert HighScore Plus software, Version 2.2b (2006) with mineral identification 265 performed with the JCPDS PDF-2 database from the International Centre for 266 Diffraction Data ${ }^{\circledR}(\mathrm{ICDD}, 2002)$. Multivariate statistical methods were employed to 267 detect, describe, and classify patterns within the mineralogical data. PCA and 
268 hierarchical cluster analysis (HCA) was carried out to allow samples to be 269 agglomerated into representative mineralogical groups.

270 The Rietveld refinement method (Rietveld, 1967; 1969) of quantitative

271 analysis was performed on diffraction samples originating from the cluster centroids

272 most representative of the cluster groups. The Rietveld refinement method involves

273 fitting an observed diffraction pattern with a synthetic pattern, which is a sum of 274 patterns calculated for each phase in the sample (Snyder and Bish, 1989; Bish, 1994;

275 Hillier, 2000). XRD was repeated on the cluster representative samples (between 3

276 and $65^{\circ} 2 \theta$ with a step size of $0.02^{\circ}$ ) with further qualitative analysis. The output 277 parameters from the fitting procedure are presented as a set of agreement indices 278 following the refinement.

280 3.4. X-ray fluorescence and geochemistry

281 Data acquisition using ED-XRF was undertaken following the approach outlined by 282 Flood et al. (2016), using a Bruker S1 TURBO SD portable X-ray fluorescence 283 (PXRF) spectrometer (Bruker Corporation, Massachusetts, USA) consisting of a 10 $284 \mathrm{~mm}$ X-Flash ${ }^{\circledR}$ SDD Peltier-cooled detector with a 4-W X-ray tube with an Ag target 285 and a maximum voltage of $40 \mathrm{kV}$. Analysis was performed on discrete samples 286 collected from the Lothian, Gplot, and Dhanchi Island cores. These samples were 287 each measured for 30 seconds, with a set of 22 international geochemical reference 288 standards (see supplementary appendix) measured for $120 \mathrm{~s}$, this was performed for 289 instrument calibration purposes. The 10 elements that are generally listed as oxides 290 in the major element chemical analysis, $\mathrm{Al}, \mathrm{Si}, \mathrm{Ti}, \mathrm{Fe}, \mathrm{Mn}, \mathrm{Mg}, \mathrm{Ca}, \mathrm{Na}, \mathrm{K}$, and $\mathrm{P}$ 291 were determined in all samples. Trace elements $\mathrm{Ba}, \mathrm{V}, \mathrm{Cr}, \mathrm{Co}, \mathrm{Ni}, \mathrm{Cu}, \mathrm{Zn}, \mathrm{Rb}$, and $292 \mathrm{Zr}$ were also analysed. The precision and accuracy of the preparation and the 
293 instrumental performance of the PXRF was checked using the international reference

294 samples and a summary of these is provided by Flood et al. (2016). No discrepancies

295 were found between the analytical data obtained and the consensus data with the

296 international reference samples.

298 3.5. Major and trace element interpretation

299 Sundarban sediment maturity classification was obtained using, the $\mathrm{Na}_{2} \mathrm{O} / \mathrm{K}_{2} \mathrm{O}$ ratio

300 index of chemical maturity (Pettijohn et al., 1972), and the $\mathrm{Fe}_{2} \mathrm{O}_{3} / \mathrm{Al}_{2} \mathrm{O}_{3}$ ratio for

301 mineral stability (Herron, 1988). These were plotted against $\mathrm{SiO}_{2} / \mathrm{Al}_{2} \mathrm{O}_{3}$ ratio as an 302 assessment of quartz and clay mineral abundance (Potter, 1978). A bivariate plot of 303 major element geochemistry has also been used to infer tectonic provenance, using $304 \mathrm{~K}_{2} \mathrm{O} / \mathrm{Na}_{2} \mathrm{O}$ plotted against $\mathrm{SiO}_{2}$ (Roser and Korsch, 1986).

305 In order to quantify weathering intensity the use of the CIA as proposed by 306 Nesbitt and Young (1982) was applied in this study, where:

$308 \mathrm{CIA}=\left[\mathrm{Al}_{2} \mathrm{O}_{3} /\left(\mathrm{Al}_{2} \mathrm{O}_{3}+\mathrm{CaO}^{*}+\mathrm{Na}_{2} \mathrm{O}+\mathrm{K}_{2} \mathrm{O}\right)\right] * 100$ Eq. 1.

310 Taking the molecular proportions, and with $\mathrm{CaO}^{*}$ representing $\mathrm{CaO}$ in silicates only 311 (Nesbitt and Young, 1989; Nesbitt et al., 1996). In this approach, a CIA value of 100 312 would suggest intense chemical weathering along with complete removal of all the 313 alkali and alkaline earth elements (Singh et al., 2005a). CIA values of 45-55 indicate 314 almost no weathering (Singh et al., 2005a). CIA values for average upper continental 315 crust (UCC) and unaltered granite rocks are at 47 and 50, respectively (Singh et al., 316 2005a). A correction for $\mathrm{CaO}$ is required in order to account for the presence of $\mathrm{Ca}$ in 317 carbonates and phosphates and this is generally conducted by calculating corrections 
318 from measured $\mathrm{CO}_{2}$ and $\mathrm{P}_{2} \mathrm{O}_{5}$ contents (Singh et al., 2005a). Where these data are

319 unavailable, approximate corrections can be made by assuming reasonable $\mathrm{Ca} / \mathrm{Na}$ 320 ratios in silicate material (Singh et al., 2005a). In this study, $\mathrm{CaO}$ content was

321 corrected for phosphate using available $\mathrm{P}_{2} \mathrm{O}_{5}$, where if the remaining number of 322 moles is less than that of $\mathrm{Na}_{2} \mathrm{O}$, this $\mathrm{CaO}$ value was adopted (cf., Singh et al., 2005a).

323 The rationale for using CIA is that it offers a quantitative measure of feldspar 324 weathering by relating $\mathrm{Al}$, which is enriched in the weathering residues, to $\mathrm{Na}, \mathrm{Ca}$, 325 and $\mathrm{K}$, which in principal should be removed during plagioclase and K-feldspar 326 weathering (Nesbitt and Young, 1982; Sheldon and Tabor, 2009; Buggle et al., 327 2011). As clay content increases there should also be an increase in $\mathrm{Al}$, whereas $\mathrm{Ca}$, $328 \mathrm{~K}$, and Na should decrease, leading to higher CIA values (Sheldon and Tabor, 2009). 329 The identification and evaluation of major element mobility within the 330 Sundarbans during weathering was carried out using elemental ratios calculated with 331 respect to the least mobile element $\mathrm{Al}$, which is believed to stay in the weathered 332 material (cf., Singh et al., 2005a). The ratio of the content of element $\boldsymbol{D}$ and $\mathrm{Al}_{2} \mathrm{O}_{3}$ in 333 the Sundarbans sediments was divided by the ratio of the same element content in $334 \mathrm{UCC}$ in the following element ratio:

335 Element ratio $(\boldsymbol{D})=\frac{\boldsymbol{D} / \mathrm{Al}_{2} \mathrm{O}_{3}(\text { Sundarbans sediments })}{\boldsymbol{D} / \mathrm{Al}_{2} \mathrm{O}_{3}(\mathrm{UCC})}$ Eq. 2.

337 This ratio refers to the relative enrichment or depletion of the element with a value of $338>1$ indicating enrichment, $<1$ indicating depletion, and $=1$, indicating no change in 339 the relative abundance.

340 As weathering proceeds, the mobilisation, fractionation, and redistribution of 341 major and trace elements takes place, and as a result, weathering is impacted by 
342 dissolution and formation of primary minerals and secondary minerals, respectively

343 (Chesworth et al., 1981; Nesbitt et al., 1980; Fritz and Mohr, 1984). Along with

344 these, redox processes, transportation, co-precipitation and ion exchange with

345 various minerals may take place (Chesworth et al., 1981; Nesbitt et al., 1980; Fritz

346 and Mohr, 1984). Since titanium is a relatively immobile element during weathering

347 (Nesbitt, 1979), it has been used previously by Singh et al. (2005a) for the

348 calculation of chemical mobility of major and trace elements. Following this

349 approach, the percentage increase and decrease of a selected element, element $\boldsymbol{D}$, was

350 calculated in the following manner:

352 Percentage change $(\%)=\left\{\left(\boldsymbol{D} / \mathrm{TiO}_{2}\right)_{\text {sample }} /\left(\boldsymbol{D} / \mathrm{TiO}_{2}\right)_{U C \mathrm{C}}-1\right\}$

Eq. 3.

354 Relative to $\mathrm{TiO}_{2}$, percentage change of elements were plotted against the CIA and

355 provide a basis for assessing the increase or decrease in chemical mobility during

356 chemical weathering (cf., Singh et al., 2005a).

357 These data are examined with reference to published data from Singh et al.

358 (2005a) and Bhuiyan et al. (2011), for the GAP in India, and Brahmaputra-Jamuna

359 River in Bangladesh, respectively. Data for UCC from Taylor and McLennan (1985)

360 and World Sediments from McLennan (1995) are discussed to facilitate

361 interpretation of the data from this study, alongside Singh et al. (2005a) and Bhuiyan

362 et al. (2011). 


\section{RESULTS}

365 4.1. Multivariate statistical analysis results from Gplot and Dhanchi grain-size

366 distributions: PCA and CA results

368 Figure 2 (a-c) shows the percentage (\%) sand, silt, and clay composition from the

369 Lothian, Gplot, and Dhanchi Island cores, respectively. The overarching grain size is 370 silt which comprises $\sim 70-80 \%$ of the sediment, with Gplot showing a 371 proportionally higher sand content.

372 The results from the multivariate statistical analysis of GSDs from the Gplot 373 and Dhanchi Island cores are shown in Fig. 3(a-j). Coefficients from the first and 374 second principal components (PCs) from Gplot are shown in Fig. 3(a, b) with PC1 375 (Fig. 3a) illustrating very coarse silt to fine-medium sand (4.00 $\varphi-1.25 \varphi)$ and 376 negative coefficients corresponding to fine clay to coarse silt fractions (12.02 $\varphi-4.24$

$377 \varphi$ ). PC2 coefficients (Fig. 3b) show highest positive values for coarse silt and very 378 coarse silt, with highest negative values for fine and medium sand. Table 1 379 summarises the PCA carried out from the first four PCs ( $95 \%$ of variance), with $38071 \%$ of the cumulative variance of $\mathrm{PC} 1$ and $14 \%$ by $\mathrm{PC} 2$.

381 Vertical distribution of PC scores for Gplot are shown in Fig. 3(c, d) with 382 PC1 (Fig. 3c) scores showing positive scores from the base of the core, at $\sim 386 \mathrm{~cm}$ 383 to a depth of $\sim 230 \mathrm{~cm}$. Positive scores from $\sim 230 \mathrm{~cm}$ progress into negative scores 384 up to the core surface. This succession is interpreted as dominant fining-up in the 385 core, with coarse material (sand and coarse silt) dominant at the core base, then, at $386200 \mathrm{~cm}$, the position is reversed with the predominance of medium - fine silt and 387 clay and the absence of sand and coarse silt. 
PC2 scores (Fig. 3d) reflect that within this fining-up of the sequence,

389 varying degrees of silt composition make up the core with some sandy and coarse

390 material interspersed throughout with negative scores in PC2 reflecting very-coarse

391 silt and sand.

392 Cluster analysis (CA) through Ward's (1963) hierarchical cluster analysis

393 (HCA) and k-means cluster analysis indicate that three groups of sedimentary facies

394 effectively explain the GSD variation, with their vertical disposition depicted in Fig.

395 4(a). The grain size association with each of the sedimentary facies follows:

396 - Facies 1a (F1a): medium clay to medium silt;

397 - Facies 2a (F2a): medium to very coarse silt with some sand, and;

398 - Facies 3a (F3a): composed of very fine to very coarse sand.

399 From the base of the core to a depth of $250 \mathrm{~cm}$ there are fluctuating trend 400 between F3a and F2a. From $250 \mathrm{~cm}$ to $200 \mathrm{~cm}$ the cluster membership is mainly 401 composed of F2a, with a fluctuating membership of F2a to F1a from $200 \mathrm{~cm}$ to 40 $402 \mathrm{~cm}$. From $40 \mathrm{~cm}$ to the core surface the sequence is primarily composed of fine-to403 medium silt and clay. The fluctuating variability in cluster group association appear 404 indicative of the PCA results, with F3a, and F2a composed of varying levels of sand 405 and silt (coarse-medium) while F1a appears to be characteristic of fine and medium 406 silt with clay composition. The vertical trend of these groups through the Gplot 407 Island core indicates a fining-up sequence (Fig. 4(a)).

408 Association of these sedimentary facies to PCs extracted is illustrated in Fig. 409 3(e), with a biplot of PC1 and PC2. PC1 is interpreted as fining-up trend (horizontal 410 axis) with PC2 of an oscillating trend between coarse and fine material. Sample 411 points located in the positive quadrant for PC1 and 2 reflect very-coarse silt and fine 412 sand composition that comprise F2a samples. Samples in the positive PC1 and 
413 negative PC2 quadrant reflect very-coarse silt with fine-medium-coarse sand with

414 F3a samples in this quadrant indicative of sand near the core base. Samples in the 415 negative PC1 and PC2 quadrant are composed of F1a, indicative of medium clay to

416 fine silt in the upper parts of the core. A facies transition order is present, with

417 coarser sands and coarser silts at the core base, overlain by a greater oscillating

418 pattern of cluster groups from $\sim 200 \mathrm{~cm}$ to core surface (Fig. 4(a)). The ordering and 419 appearance of sedimentary facies suggests there are two distinctive broad 420 stratigraphical Facies; (i) a lower sandy-silt; and (ii) an upper oscillating sequence of 421 fine/medium-silt and clay, as shown in Fig. 4(a).

422 Dhanchi grain-size coefficients from PC1 and PC2 are shown in Fig. 3(f,g) 423 with positive coefficients from PC1 (Fig. 3f) corresponding to medium clay and very 424 coarse silt $(12.02 \varphi-6.00 \varphi)$ and negative coefficients for very coarse silt to sand 425 fractions $(5.01 \varphi--1.00 \varphi)$. PC2 coefficients (Fig. 3g) indicate highest positive 426 values are very coarse silt $(5.00 \varphi-4.00 \varphi)$ and coarse silt $(6.00 \varphi-5.00 \varphi)$, with high 427 negative coefficients indicative of clay $(7.00 \varphi-6.00 \varphi)$ and sand $(4.00 \varphi--1.00 \varphi)$. 428 Table 2 is a summary of the PCA with the first four components ( $~ 99 \%$ of variance), 429 with $\mathrm{PC} 1$ comprising $\sim 86 \%$ of the cumulative variance and $\mathrm{PC} 2$ of $8 \%$ of 430 cumulative variance.

431 Fig. 3(h, i) shows the score distributions throughout the Dhanchi core for PC1 432 and PC2. PC1 scores (Fig. 3h) show that from core base to $\sim 300 \mathrm{~cm}$ depth, sediment 433 is composed of very coarse silt and sand with some finer material (clay and fine silts) 434 and overlain by coarser sediment from $\sim 300 \mathrm{~cm}$ to the core surface. PC2 scores (Fig. 435 3i) show fluctuating coarse sediment throughout the core, with highest positive 436 scores for coarse silt and sand with finer-grained sediment between $\sim 450 \mathrm{~cm}$ to 0 $437 \mathrm{~cm}$, denoted by slightly negative to low scores. Results indicate that the sequence is 
438 composed almost entirely of various grades of silt with clay, and sand. These

439 characteristics would suggest a mixture of size fractions throughout the Dhanchi

440 sequence but with dominant fining-up.

441 HCA and k-means cluster analysis show three groups of sedimentary facies

442 that explain GSD trend in the Dhanchi core (Fig. 4b). Grain size association with

443 each of the sedimentary facies is:

444 - Facies 1b (F1b): two samples, considered to be an outlier;

445 - Facies 2b (F2b): two samples, considered to be an outlier;

446 - Facies 3b (F3b): medium and coarse silt with clay;

447 - Facies 4b (F4b): coarse silt with some sand, and;

448 - Facies 5b (F5b): sand (very fine sand to very coarse sand) with silt.

449 From core base to $320 \mathrm{~cm}$ a fluctuating trend between F5b and F4b is found,

450 and from $320 \mathrm{~cm}$ to $60 \mathrm{~cm}$ cluster membership varies between F4b and F3b, with the

451 top $60 \mathrm{~cm}$ composed of F3b and some F2b. F5b, F4b. F3b indicate varying degrees

452 of silt (coarse-medium-fine) and sand, F4b and F3b comprising fine and medium silt

453 with clay, with F1b of coarse silt, and F2b clay. Sand and silt fluctuations are shown

454 by F5b and F4b, with F4b and F3b for coarse silt to medium and fine silt.

455 Biplot analysis of sedimentary facies and PCs from the Dhanchi are shown in

456 Fig. 3j. Biplot quadrants reveal that in positive PC1 and PC2, there is an overall

457 coarse-silt composition. Positive PC1 and negative PC2 reflect predominantly F4b

458 samples with F4b samples most negatively represented along the second principal

459 component, indicative of very-coarse silt to sand, with F5b showing the transition

460 from very-coarse silt and sand to very-coarse clay and medium clay. The negative

461 PC1 and PC2 quadrant is composed of some F4b and F5b samples, with negative

462 PC1 and positive PC2 quadrant composed mainly of F5b and F1b. 
PC1 is interpreted as a fining-up trend (horizontal axis) with PC2 composed

464 of coarse-silt and sand fractions. Positions of some outliers in the F1b and F2b

465 samples show the latter indicative of coarse- to very coarse-silt and former of sand

466 and clay. Cluster groups of F4b and F3b represent coarse sediment (coarse silt and

467 sand), with F3b of the progression into fine material and fining-up. Facies order or

468 stacking pattern is present, coarser silts and sand dominating the sequence from core

469 base at $558 \mathrm{~cm}$ to $\sim 250 \mathrm{~cm}$, overlain by an oscillating trend of cluster groups $\sim 250$

$470 \mathrm{~cm}$ to $\sim 60 \mathrm{~cm}$. The oscillating trend is overlain by homogeneous, medium- and

471 finer-silts with clays from $\sim 60 \mathrm{~cm}$ to core surface (Fig. $4 \mathrm{~b}$ ). Ordering of sedimentary

472 facies and appearance in core suggests there are three distinctive broad

473 stratigraphical facies; (i) a lower coarse-silt and sand; (ii) a middle medium-fine

474 silty-clay; and (iii) an upper medium- to very fine-silt and clay.

476 4.2. X-ray powder diffraction: results from the Sundarbans

477 Table 3 shows the most representative cluster samples with their site and depth in 478 each core with data analysis outlining mineral groups within the cores. Following

479 Rietveld refinement, observed and calculated spectra for cluster representative 480 samples are given in supplementary data. Agreement indices for each diffractogram 481 are shown in Table 4 with 'goodness-of-fit' best interpreted from the residual $\left(\mathrm{R}_{\mathrm{p}}\right)$ 482 and weighted residual $\left(\mathrm{R}_{\mathrm{wp}}\right)$ profiles. Optimum fit based on these parameters is 483 generally less than $5 \%$ and $10 \%$ for $\mathrm{R}_{\mathrm{p}}$ and $\mathrm{R}_{\mathrm{wp}}$, respectively. These are expected to 484 be a little higher in geological material (O'Meara 2013, pers. comm.; Speakman 485 2013, pers. comm.; Pecharsky and Zavalij, 2009). 
488 clusters $1,3,5$, and 6 having a series of residual and weighted residual values 489 between $13.1-9.3 \%$ and 9.1-6.2\%, respectively. Rietveld Refinement carried out on 490 the six cluster representative samples appears to show well-fitted models for the 491 observed spectra. Mineralogical composition is shown in Table 5 with a data 492 summary in Table 6.

493 High abundance of quartz is found in all clusters with cluster 4 and 2 having 494 the highest (78.6\%) and lowest (34.8\%), respectively. Clinochlore was present in all 495 but two clusters (2 and 5) with highest (5.5\%) and lowest (0.9\%) in 6 and 4 , 496 respectively. Muscovite was found in nearly all clusters, except cluster 4, with 497 highest (50.9\%) and lowest (18.8\%) in cluster 2 and 1. Albite (plagioclase feldspar) 498 present in all clusters with highest (13\%) and lowest (4.9\%) contents in cluster 5 and 4994 , respectively. Microcline (alkali feldspar) was present in cluster 4 at $8.2 \%$. Clay 500 minerals consisted of kaolinite, dickite, and possibly vermiculite at only trace 501 amounts $(<1.0 \%)$ detected in clusters 2 and 5 at $0.7 \%$ and $0.4 \%$, respectively. 502 Dickite (polymorph of kaolinite) was present in cluster 4 (4.6\%), with kaolinite in 503 all cluster representative samples, with the exception of cluster 4 . The highest $504(13.6 \%)$ and lowest values $(6.7 \%)$ of kaolinite were in cluster 2 and 5, respectively.

505 A plot of the first three principal components is shown in Fig. 5, with $\sim 95 \%$ 506 of the variance accounted by these components. PC1 is composed mainly of quartz in 507 all of the samples examined (Table 5) with phyllosilicates, particularly mica (i.e., 508 muscovite) and clays (i.e., clinochlore and kaolinite) illustrated by PC2. Feldspars 509 (i.e., albite, microcline and Ca plagioclase) comprise PC3 with Fig. 5a showing 510 cluster 5 in the negative PC1 and PC2 quadrant, potentially attributed to albite. 511 Progression of scores along PC2 show predominantly clusters 3, 6, and 2, and reflect 512 increasing muscovite and kaolinite composition (i.e., muscovite: $22.1 \% \rightarrow 33.6 \% \rightarrow$ 
$51350.9 \%$; kaolinite: $6.9 \% \rightarrow 11.9 \% \rightarrow 13.6 \%$ ). This trend in PC2 appears to continue

514 with cluster 4, with this cluster varying more positively in PC3, interpreted as high

515 microcline content. Cluster 1 appears distinct as it tends to vary negatively along

516 PC2, reflecting lower quantities of phyllosilicates and having higher albite and

517 clinochlore contents, with the latter absent from cluster 5.

518 The distribution of the mineral clusters from each of the Sundarbans cores is

519 shown in Fig. 5(e), Lothian (i) displaying a sporadic and fluctuating trend in clusters

5202,4 and 6 at core base to c. $500 \mathrm{~cm}$, and an oscillating trend between clusters 4 and 2

521 from c. $500 \mathrm{~cm}$ to c. $170 \mathrm{~cm}$, overlain by another oscillating trend between cluster 5

522 and 3. Cluster 1 is dominant in Gplot core (ii) from the core base to c. $225 \mathrm{~cm}$,

523 followed by an oscillating trend between 6 and clusters 2 and 3 from c. $225 \mathrm{~cm}$ to

524 core surface. Two oscillating trends are found in the Dhanchi core (iii), at the core

525 base to c. $220 \mathrm{~cm}$ composed of clusters 2 and 4, followed by clusters 6 and 2 from c.

$526220 \mathrm{~cm}$ to c. $40 \mathrm{~cm}$ depth with a final oscillating trend from c. $110 \mathrm{~cm}$ to $50 \mathrm{~cm}$ with

527 clusters 3 and 6.

529 4.3. X-ray fluorescence: results from major and trace element geochemistry of the

530 Sundarbans

532 The bulk chemistry of the Lothian, Gplot, and Dhanchi island cores consists mainly

533 of the oxides of three elements: Al, Si and, Fe. The total composition of these

534 elements in Lothian, Gplot, and Dhanchi island cores is approximately $84 \%, 83 \%$

535 and $86 \%$. The average $\mathrm{SiO}_{2} / \mathrm{Al}_{2} \mathrm{O}_{3}$ ratios of Lothian, Gplot, and Dhanchi are 4.1, 4.5,

536 and 4.2, respectively. This is interpreted to reflect mineral composition and grain-

537 size variability, in which GSD is mainly silt-sized with quartz dominant in coarse- 
538 size fractions, and clay minerals in fine grain-size fractions. Aluminosilicate minerals

539 are preferentially transported as suspended load with quartz tending to be found as

540 bedload (cf., Singh et al., 2005a).

\section{4.3.1. Rock classification and tectonic provenance of Sundarbans sediments}

543 Ratios of the major elements of $\mathrm{Al}_{2} \mathrm{O}_{3} / \mathrm{SiO}_{2}$ and $\mathrm{Fe}_{2} \mathrm{O}_{3} / \mathrm{SiO}_{2}$ of the Sundarbans, along 544 with data from Singh et al. (2005a) and Bhuiyan et al. (2011), were plotted with 545 respect to the Himalaya and the Siwalik, following Singh et al. (2005a) (Fig. 6). The 546 Himalayas tend to possess a mean chemical composition that is similar to the average 547 UCC (Galy and France-Lanord, 2001). The linear relationship found with the

548 Sundarbans sediments indicates a grain-size component, with the lower parts of each 549 core being enriched with quartz. This is seen in Fig. 6 with the position of the 550 Sundarbans samples from Lothian and Gplot being clustered in close proximity to 551 the channel sediments samples from Singh et al. (2005a), with these samples 552 understood to be enriched in quartz. This sort of mineral sorting response is further 553 evidenced by the trend towards phyllosilicates that is substantiated by the presence of 554 suspended sediments from Singh et al. (2005a). The $\mathrm{Al}_{2} \mathrm{O}_{3} / \mathrm{SiO}_{2}$ and $\mathrm{Fe}_{2} \mathrm{O}_{3} / \mathrm{SiO}_{2}$ 555 ratios found in this study appear to reflect the compositional maturity of sediments, 556 originating from the Himalayas and Siwaliks, and moving towards the delta, with the 557 Sundarbans evidence of the highest weathering maturity.

558 The Pettijohn and Herron diagrams (Fig. 7a \& b) indicate that clastic 559 weathering products of the Sundarbans are primarily litharenite and 560 greywacke/wacke, with Lothian and Dhanchi representing the most mature sediment.

561 The presence of Gplot samples within arkose, with all Sundarbans samples plotting 562 towards litharenite and greywacke/wacke, may be interpreted as grain-size variation 
563 with increasing clay content. The tectonic provenance discrimination plots show that

564 the majority of the Sundarbans samples fall into the active continental margin field

565 (Fig. 8), with some scatter of samples into the island arc field.

\section{4.3.2. Element enrichment ratios for the Sundarbans}

568 Element ratios in the Lothian, Gplot, Dhanchi, Singh et al. (2005a) and Bhuiyan et al. 569 (2011) datasets are shown in Fig. 9. $\mathrm{SiO}_{2}$ mobility is considered important for 570 interpreting chemical weathering, soil formation and distribution of elements in 571 natural waters (Singh et al., 2005a). There is a depletion in $\mathrm{SiO}_{2}$ relative to Singh et 572 al. (2005) data points, but enriched compared to Bhuiyan et al. (2011). Titanium is 573 understood to be a relatively immobile element and appears to be most enriched in 574 the Lothian and Dhanchi cores, but most depleted in the Gplot Island core. There is a 575 progressive depletion in $\mathrm{CaO}$ and $\mathrm{Na}_{2} \mathrm{O}$ throughout the Sundarbans cores, which is 576 reflective of these being highly mobile elements. Potassium shows some depletion in 577 the Sundarbans cores, with Gplot being more enriched in $\mathrm{K}_{2} \mathrm{O}$ relative to Lothian and 578 Dhanchi. As discussed by Singh et al. (2005a), potassium tends to be preferentially 579 adsorbed to clay minerals, as found by Flood et al. (2016) for the Sundarbans. 580 Therefore, the enrichment of $\mathrm{K}_{2} \mathrm{O}$ may be due to silicate minerals formed during 581 weathering or alteration of existing clay minerals such as montmorillonite, which 582 tend to incorporate potassium relative to $\mathrm{Na}_{2} \mathrm{O}$ (Singh et al., 2005a). Clay minerals 583 also have a tendency to incorporate magnesium as well as $\mathrm{K}_{2} \mathrm{O}$, and lose $\mathrm{Na}_{2} \mathrm{O}$, 584 which can be see with the pronounced enrichment of $\mathrm{MgO}$ in the Dhanchi and 585 Lothian cores. Due to the highly mobile nature of $\mathrm{Na}_{2} \mathrm{O}$ and $\mathrm{CaO}$ during weathering, 586 these may be readily dissolved and taken up into and enriched in solution (i.e., in 587 aqueous phase) as opposed to being deposited as part of the sediment load. This has 
588 been found by Singh et al. (2005a) and Singh et al. (2005b), who show a

589 concentration of these elements in the dissolved fraction of fluvial sediments. $\mathrm{MgO}$

590 shows some mobility between sites in the Sundarbans with Lothian and Dhanchi

591 slightly enriched compared to Gplot. Although Mg may dissolve during weathering

592 and enter the aqueous phase, it has been found to be depleted in channel sediments,

593 but mobile within suspended and floodplain sediments (Singh et al., 2005a). This

594 may explain the $\mathrm{Mg}$ variability between sites in the Sundarbans, with Gplot

595 composed of coarse-grained, channel-type sediment, relative to Lothian and

596 Dhanchi.

597 4.3.3. Inter-element variability from the Sundarbans

598 Fig. 10 displays inter-element relationships plotted on variation diagrams using

$599 \mathrm{Al}_{2} \mathrm{O}_{3}, \mathrm{SiO}_{2}$ and $\mathrm{TiO}_{2}$ along the x-axis. In the majority of the variation diagrams,

600 some linear trend from Lothian, Gplot, and Dhanchi are observed with positive or

601 negative correlations. Relatively strong negative correlation of $\mathrm{SiO}_{2}$ with $\mathrm{Al}_{2} \mathrm{O}_{3}$ and

$602 \mathrm{Fe}_{2} \mathrm{O}_{3}$ indicates grainsize control on the geochemistry of the Sundarbans' weathering

603 products, with little correlation $\left(\mathrm{R}^{2}=0.04\right)$ with $\mathrm{K}_{2} \mathrm{O}$. The increasing trend of $\mathrm{TiO}_{2}$

604 with $\mathrm{Fe}_{2} \mathrm{O}_{3}$ is attributed to the enrichment of heavy minerals in Sundarbans

605 sediments. However, $\mathrm{MgO}$ and $\mathrm{K}_{2} \mathrm{O}$ have different patterns with $\mathrm{Al}_{2} \mathrm{O}_{3}$ and $\mathrm{TiO}_{2}$,

606 respectively. Plotting the chemically immobile $\mathrm{TiO}_{2}$ against $\mathrm{Al}_{2} \mathrm{O}_{3}$ provides further

607 insight into the hydrodynamic and chemical behaviour of the major mineral phases

608 and indicates that they were concentrated into the fine-grained sediment fraction (i.e.,

609 monotonic relationship reflects decreasing grain-size). High concentrations of $\mathrm{TiO}_{2}$

610 in Sundarbans sediments and a strong correlation with $\mathrm{Al}_{2} \mathrm{O}_{3}$ indicate that $\mathrm{TiO}_{2}$ could

611 potentially be derived from mica, although there is a poor correlation with $\mathrm{K}_{2} \mathrm{O}$ and

$612 \mathrm{TiO}_{2} . \mathrm{TiO}_{2}$ plotted against $\mathrm{Fe}_{2} \mathrm{O}_{3}$ displays a good correlation $\left(\mathrm{R}^{2}=0.68\right)$ indicating 
613 their common sources from ferromagnesium minerals such as biotite, amphibole, and

614 pyroxene (cf., Singh et al., 2005a). A poor correlation of $\mathrm{K}_{2} \mathrm{O}$ with $\mathrm{MgO}$ and $\mathrm{Al}_{2} \mathrm{O}_{3}$

615 indicates an absence (or failure to detect) of illite in these sediments (cf., Singh et al.,

616 2005a). The strong correlation found for $\mathrm{Fe}_{2} \mathrm{O}_{3}$ with $\mathrm{Al}_{2} \mathrm{O}_{3}$ may be associated with

617 alteration of biotite into aluminosilicates and Fe(III) oxides (cf., Singh et al., 2005a).

618 The distribution of major elements in the Sundarbans sediments suggests that

619 weathering products have strong inter-elemental linkage.

621 4.3.4. A-CN-K diagram and chemical index of alteration (CIA) for Sundarbans

622 Fig. 11 (a) shows a ternary A-CN-K diagram along with the location of important 623 rock-forming minerals, UCC (Taylor and McLennan, 1985) and natural waters (cf., 624 Singh et al., 2005a). The sediments from the Sundarbans tend to plot towards the $625 \mathrm{Al}_{2} \mathrm{O}_{3}$ apex, indicating loss of $\mathrm{Na}$ and $\mathrm{Ca}$ during weathering compared to the UCC, 626 with a tendency for samples to plot towards A-CN, with a slightly larger proportion 627 of samples towards the A of the apex. CIA values for Lothian, Gplot, and Dhanchi 628 are 46-67, 53-76, and 52-65, respectively. The average CIA of the Sundarbans is 629 compared with Singh et al. (2005a) and Bhuiyan et al. (2011) (Fig. 11b). There 630 appears to be a fair degree of weathering found in the Sundarbans sediments, with 631 Gplot Island showing the highest mean CIA. The Sundarbans data show a higher 632 degree of weathering, relative to Bhuiyan et al. (2011) from the Brahmaputra-Jamuna 633 River. With the exception of the flood and suspended sediment CIA values of Singh 634 et al. (2005a), the Sundarbans CIA values show an increasing degree of weathering 635 compared with channel sediments. 
638 Shown in Fig. (12 c,d) are A-CNK-FM and S/10-CM-NK ternary plots (Nesbitt and

639 Young, 1989; Singh et al., 2005a). Sundarbans samples tend to be positioned in the

640 centre of the ternary diagram (Fig. 11c), with Gplot closer to feldspar composition

641 (cf., Singh et al., 2005a). Increased weathering of fine-grained material, tends to have

642 a winnowing effect of Fe-oxyhydroxides and biotite, which may explain the

643 enrichment towards the FM apex with Lothian and Dhanchi (cf., Singh et al., 2005a).

644 The presence of suspended sediments from Singh et al. (2005a) closest to the FM

645 apex may indicate secondary sedimentary processes concentrating heavy minerals in

646 sediment (Singh et al., 2005a). The suspended sediments from Singh et al. (2005a)

647 may be considered to be one end-member in this ternary diagram, with Gplot being

648 the opposing end-member in the A-CNK apex. The arrows depicted show the

649 predicted weathering trends that have been found in the GAP, in which Sundarbans

650 samples appear to following this predicted weathering trend.

651 In the S/10-CM-NK diagram (Fig. 11d) all of the Sundarbans samples plot 652 away from the S/10-CM apex and towards NK, with Gplot samples plotting more 653 strongly towards the S/10-NK apex and, Lothian and Dhanchi plotting towards the 654 CM-NK apex. Compared with Singh et al. (2005a) and Bhuiyan et al. (2011), there is 655 some similarity present, with the exception of suspended sediments of Singh et al. 656 (2005a) which plot towards the CM apex. The position of the Gplot samples at the $657 \mathrm{~S} / 10-\mathrm{NK}$ apex may be attributed to greater quartz presence in these samples, relative 658 to Lothian and Dhanchi, which show a decrease in quartz, as these plot towards the 659 CM-NK apex. 
662 The chemical mobility is shown in Fig. 12 for the Sundarbans sediments during 663 weathering processes, and is calculated in terms of $\%$ change normalised to $\mathrm{TiO}_{2}$ for 664 individual major/trace elements against progressive chemical alteration, using the 665 CIA values. A decreasing trend was found in chemical mobility of $\mathrm{Na}_{2} \mathrm{O}_{3}(\mathrm{a}), \mathrm{MgO}$ 666 (b), $\mathrm{Al}_{2} \mathrm{O}_{3}(\mathrm{c}), \mathrm{SiO}_{2}(\mathrm{~d}), \mathrm{K}_{2} \mathrm{O}$ (f) in all cores, with $\mathrm{CaO}$ (g) and $\mathrm{Zr}$ (q) showing the 667 greatest decreasing trend. An increase was found in $\mathrm{P}_{2} \mathrm{O}_{5}$ (e) and $\mathrm{Fe}_{2} \mathrm{O}_{3}$ (i) mobility, 668 with an unvarying trend in $\mathrm{Ba}(\mathrm{j})$, and $\mathrm{V}(\mathrm{k}) . \mathrm{Mn}(\mathrm{h}), \mathrm{Cr}(\mathrm{l}), \mathrm{Ni}(\mathrm{m})$, and $\mathrm{Cu}(\mathrm{n})$ 669 appear to have no discernible trend with CIA. A slight increase was found in $\mathrm{Zn}$ (o) 670 with a slightly greater increase in $\mathrm{Rb}(\mathrm{p})$ with increased CIA.

\section{5. DISCUSSION}

673 Deposition of the TMF in the far western extent of the Sundarbans is shown in Fig.

674 13. There is a gradual shift in the dominance of fluvial processes in deposition from

675 c. 5,000 cal yr BP to a more mixed fluvio-tidal depositional system from c. 3,000 cal

$676 \mathrm{yr}$ BP, to its current state as a tidal dominant delta. Sedimentation rates for the

677 present-day eastern Sundarbans have been reported as being c. $1.0 \pm 0.9 \mathrm{~cm} \mathrm{yr}^{-1}$

678 (Rogers et al., 2013) whereas Flood (2014) and Flood et al. (2015) determine a

679 sedimentation rate of c. $1.4 \mathrm{~mm} \mathrm{yr}^{-1}$ for the far western Sundarbans over the late

680 Holocene (c. last 4,000 cal yrs). The model proposed in this study is that there is a

681 gradual (or rapid) loss of the westerly tidal transported sediment plume along the Bay

682 of Bengal coastline. This primarily suspended load then moves up the flood

683 dominant estuaries of the Sundarban blind rivers (Bhattacharyya et al., 2013), to be

684 deposited in the distributive system between estuaries, as the sediment plume moves

685 westward (cf., Rogers et al., 2013). The TMF in the most western extent of the G-B 
686 delta is proposed as being a diachronous facies unit, with fluvial dominance

687 prevalent c. 5,000 yrs BP that gradually developed into a mixed fluvio-tidal

688 dominant delta from c. 3,000 yrs BP, to being a tidal dominant delta at present.

689 Sediment is sourced primarily from the Ganges river and delivered to the coastal

690 shelf as a plume that is re-worked onto the delta plain with the emerging tidal

691 supplied sediment plume thinning in volume and sediment size as it is carried further

692 west to the tidal dominant delta (Fig. 13). The depositional model proposed in this

693 study is a series of incremental developments from previous studies, particularly

694 from Goodbred and Kuehl (2000a), Allison et al. (2003), and Rogers et al. (2013).

695 The TMF in the far western extent of the Sundarbans is considered to reflect

696 abandoned floodplain overbank deposits (cf., Goodbred and Kuehl, 2000a). The

697 processes of deposition reflecting tidal/marine processes (cf. Allison et al., 2003),

698 with the source of the TMF sediments being the Ganges River associated with

699 monsoon flood discharge and sediment being dispersed westwards by currents

700 followed by onshore transport (Rogers et al., 2013).

702 5.1. Lithofacies variability and dynamics in the TMF: evidence from the West

703 Bengal Sundarbans

704

705 The Lothian and Dhanchi Island GSDs may be interpreted as reflecting a muddy tidal

706 flat environment with the overarching presence of silt and clay (e.g., Fig. 2, Fig. 4b),

707 although it is difficult to determine sedimentary structures from the cores (cf.,

708 Allison et al., 2003; Goodbred and Saito, 2011; Flood, 2014; Flood et al., 2015).

709 The dominant upward-fining in GSDs from these cores may be attributed to

710 slack water deposits as a result of tidal deposition (cf. Bass et al., 2002). In terms of 
711 silt and clay deposition through tides, suspension of sediments lasts longer than sands

712 and may therefore be transported over long distances and may be advected into or

713 away from a depositional site (Bass et al., 2002). These fining-up sequences,

714 comprising muddy tidal flats may cap subtidal sand ridges (Wells, 1995). A similar

715 model of facies succession has been proposed by Goodbred and Saito (2011), where

716 the migration of tidal channels and creeks across tidal flats, contribute to fining up

717 facies succession. The clay fraction indicated by the first and second principal

718 components may represent the mud-drapes and fluid-muds attributed to slack water

719 or poor water flows (cf., Wells, 1995). However, this is difficult to judge as a limited

720 sample population was used in the GSD analysis, thus only the general trend of

721 fining-up (e.g., Dhanchi PCA score plots and GSD facies shown in Fig. 3(h, i)).

722 Gplot GSDs may be interpreted as sub- to intertidal-tidal flat environment

723 (cf., Goodbred and Saito, 2011). Transition between the two broader sedimentary

724 sub-units indicates the lower sub-unit was within closer proximity to fluvial sources

725 of sediment given coarse GSDs (Fig. 2 and Fig. 3(a-d)) (Hughes, 2011). GSD

726 interpretation indicates a correlation with effective transport energy as opposed to

727 any direct correlation with proximity to marine or fluvial sources. With a relatively

728 coarser sediment load, the probability of sediment transport processes that maintain

729 such a sediment load decreases (McLaren and Bowles, 1985). As sediment deposits

730 become coarser in a sequence, depositional processes take on the attributes of low-

731 energy functions with sediments becoming finer (McLaren and Bowles, 1985). From

732 Gplot, although coarse sediment is present, the likelihood of transitioning into a finer

733 facies increases with time. Cross phase similarity with PC1 from Dhanchi and Gplot

734 Island is found with signs being different - but PCs measuring the same

735 phenomenon; dominant GSDs (Fig. 3 and 4). There is a similar fining-upwards drift 
736 through the core (Fig. 2 and Fig. 3(c-e)) with PC2 indicative of the presence or

737 absence of the 4.5 to $7 \varphi$ elements, and acting as a bridge to the presence of very fine

738 and slightly coarser sediment. Three broader stratigraphic facies have been identified

739 in the Lothian and Dhanchi Island core data which are similar to those developed by

740 Allison et al. (2003). It is proposed that the statistically derived facies presented in

741 this study may be considered to range over two of the principal facies that Allison et

742 al. (2003) outlined in their study (i.e., those of the 'intertidal shoal' and 'supratidal'

743 facies).

744 Lithofacies of the Sundarbans and the TMF demonstrate a high degree of 745 variability between core-sites, however application of multivariate statistics offers

746 enhanced scope for palaeoenvironment interpretation. The lithofacies models

747 developed are applied to each site separately, with this approach taken given the

748 GSDs from the Gplot Island core would skew the analysis of the GSD data from the

749 Lothian and Dhanchi Island cores. Although the sites are relatively close (within 10

$750 \mathrm{~km}$ ), sediment dynamics and variability can be understood to be highly divergent

751 when the Gplot GSD data is examined. One potential explanation for this variability

752 may be in the depositional environment of the Gplot site. Tidal dominated

753 environments are indicative of intertidal to shallow subtidal zones, which are

754 dominated by muddy tidal flats and tidal channels and, may also include channel-

755 mouth and channel-side bar features (Goodbred and Saito, 2011). These tidal ridges

756 are understood to accrete vertically and horizontally (akin to subdued flooding

757 levees, i.e., channel-side features) until forming a shallow intertidal flat with

758 vegetation succession taking place (Goodbred and Saito, 2011). 
761 Mineralogy from the West Bengal Sundarbans reveals key variability between the

762 islands. Terrigenous minerals are abundant in all cores, principally quartz, feldspars,

763 and muscovite mica. Quartz in Holocene sedimentary environments varies under a

764 number of circumstances (see Wedepohl, 1978; Kabata-Pendias, 2001; Hinman,

765 1998; Salminen et al., 2005; Garzanti et al., 2011). Quartz in these sediments may

766 reflect detrital deposition of weathering-resistant sediment in the West Bengal

767 Sundarbans. The Ganges River is understood to carry more quartz and less Ca-

768 plagioclase than the Brahmaputra river (Garzanti et al., 2011). With increased

769 erosion and sorting, the ratio of feldspar to quartz is generally diminished in sand

770 composition with higher proportion of quartz indicative of coarse grain-size

771 sediments (Nesbitt et al., 1996). This is seen in the mineral clusters 5, 4, 3, and 1

772 with mineralogy from Gplot (depth of c. $386 \mathrm{~cm}$ to c. $225 \mathrm{~cm}$ ), having high quartz,

773 muscovite, albite, and kaolinite contents.

774 There is a dominant fluctuation between high quartz and high muscovite in

775 Dhanchi, particularly in clusters 2, 4, and 6 (core base to c. $220 \mathrm{~cm}$ ). Mica may

776 illustrate winnowing and active deposition on continental shelf systems (Doyle et al.,

777 1968; Adegoke and Stanley, 1972; Doyle et al., 1979; Dias et al., 1984). Common

778 minerals like quartz, feldspars, and calcite are generally associated with significantly

779 reduced heavy mineral loads (Garzanti et al., 2008). Heavy minerals tend to be

780 enriched in the fine tail of the grain size distribution with quartz, feldspars, and

781 calcite found to comprise the coarse tail (Garzanti et al., 2008). Thus, micas make up

782 the coarsest tail of a grain size distribution for sediment due to their platy shape, with

783 the finest grain size fraction composed of zircon, monazite or magnetite because of

784 their extreme density (Garzanti et al., 2008). The predominance of mica and reduced 
785 quartz content from the base of the core to c. $220 \mathrm{~cm}$ reflect this variability in 786 hydrodynamic sorting of minerals. From $220 \mathrm{~cm}$ to the core surface, mica 787 composition is reduced with much less variability in quartz composition (see Table 5 788 and Fig. 5).

789 In the Lothian core, fluctuations between clusters 2, 4, and 6 from the base of 790 the core to c. $500 \mathrm{~cm}$ may reflect variability in sand and silt deposition. Cluster 2 has 791 higher muscovite abundance (50.9\%) relative to quartz $(34.8 \%)$ with cluster 4 made 792 up of $78.6 \%$ quartz and no muscovite, variability in these detrital minerals may be a 793 result of diverging settling velocities (cf., Dias et al., 1984). Mineralogical variability 794 over time scales of centuries of deposition in terms of sediment hydraulics may be 795 attributed to the relative height of the tidal frame and the upward development of an 796 intertidal mudflat (cf., Allen, 1990; 2000), with fluctuations in tidal frame that 797 contribute fluctuations in settling of finer (i.e., muscovite) with coarser sediments 798 (i.e., quartz grains). Cluster 6 is composed of nearly equal quantities of quartz and 799 muscovite at $38.8 \%$ and $33.6 \%$, with kaolinite at $11.9 \%$, indicative of reduced 800 energy.

801 Chemical weathering in lowland rivers may control the formation of kaolinite 802 from feldspars and mica mineral assemblages, demonstrated in Fig. 14(a) where a 803 strong correlation is found between muscovite and kaolinite. Smectite, attributed to 804 be an indicator mineral for Ganges sediment provenance (Heroy et al., 2003) was not 805 found in this study or by Sarin et al. (1989), where kaolinite content ranged between $806 \quad 6.7 \%$ and $13.6 \%$. The lack of smectite is attributed to the requirement of dehydration 807 experiments needed for positive smectite identification. Illite was not found either 808 but may be attributed to late stage weathering within the GAP (cf., Singh et al., 809 2005a). 
811 kaolinite formation possibly a function of weathering in mica (cf., Stoch and Sikora,

812 1976). Muscovite mica to kaolinite formation is the result of transformation of:

813 muscovite $\rightarrow$ mixed layer muscovite/montmorillonite $\rightarrow$ montmorillonite $\rightarrow$

814 kaolinite (Stoch and Sikora, 1976). It is uncommon for montmorillonite to be found

815 and this may be due to montmorillonite existing in an unstable intermediate phase

816 during weathering (Stoch and Sikora, 1976).

817 Kaolinite and quartz shown in Fig. 14(b) being highly negatively correlated

$818\left(\mathrm{R}^{2}=-0.96\right)$. Correlation is attributed to grain-size variability and has been known to

819 occur in estuaries where quartz is dominant in sand and phyllosilicates in greater

820 abundance in finest fraction (Galán et al., 2003). Where there are greater quantities

821 of quartz there is a concomitant decrease in kaolinite.

822 Oscillating trends in mineral clusters found in the Dhanchi core are indicative

823 of silt and sand-size sediment, similar to the Lothian Island core, with the Gplot core

824 showing oscillating variability in mineral clusters associated with high mica and

825 quartz content, indicative of varying deposition in sand and silt. As the feldspar is

826 composed mainly of albite, chemical weathering tends to alter plagioclase feldspar as

827 opposed to K-feldspar and quartz, with the abundance of albite indicative of

828 weathered sediment (Grant 1963; Nesbitt and Young 1989; Nesbitt et al., 1996).

829 High quartz content compared to other minerals is indicative of low-relief

830 tropical weathering within the Bengal Basin (Potter, 1978; Mukherjee et al., 2009).

831 In gentle slopes, water easily penetrates into the substrate, dissolving the most

832 soluble constituents and accumulating the less mobile ones (Gutierrez, 2005).

833 Formation of kaolinite from feldspar and mica in soil and sediments as a result of

834 meteoric water drainage (Islam et al., 2002; Bjørlykke, 1998). This has been known 
835 to occur within warm, humid climates, particularly under tropical conditions (Islam

836 et al., 2002). Formation of kaolinite is due to greater leaching conditions with high-

837 rainfall (Ehlmann, 1968; Islam et al., 2002).

839 5.3. Geochemistry of the West Bengal Sundarbans

840 The geochemistry of the Holocene sediment from the West Bengal Sundarbans can

841 be characterised as intensively weathered, terrestrial sediment derived from the

842 Ganges River, principally the GAP. Therefore we suggest that sediments of the TMF

843 are derived from the weathering and transport of Himalayan derived sediments, with

844 geochemical data supporting the two stage weathering model proposed by Singh et

845 al. (2005a), with initial weathering in the Himalaya and subsequent weathering under

846 a humid sub-tropical climate (Singh et al., 2005a). The first and second weathering

847 cycles are related to illite, and smectite dominance, respectively (Sarin et al., 1989;

848 Singh et al., 2005a). Although illite-smectite variability was not found in this study,

849 several aspects of the geochemical data validate both the source and processes (i.e.,

850 'second cycle' weathering products) in the TMF of the West Bengal Sundarbans.

851 The maturity of sediments in the Sundarbans can be derived from Fig. 6 and

852 from Fig. 7(a) following the Pettijohn et al. (1972) index of chemical maturity with

853 mineral stability following Herron (1988) (Fig. 7b). The $\mathrm{Al}_{2} \mathrm{O}_{3} / \mathrm{SiO}_{2}$ versus

$854 \mathrm{Fe}_{2} \mathrm{O}_{3} / \mathrm{SiO}_{2}$ presented in Fig. 6 for the Sundarbans along with the Siwaliks and the

855 Himalayan sources show that the increasing ratios from low-to-high are indicative of

856 decreasing quartz proportion and enrichment in phyllosilicates, respectively (Singh et

857 al., 2005a; Garzanti et al., 2010; Garzanti et al., 2011). The linear trend shown in Fig.

8586 may correspond to mineralogical sorting of sediments during transportation (Singh

859 et al., 2005a; Garzanti et al., 2008; Garzanti et al., 2009). The lower ratios found in 
860 samples from Gplot, with the subsequent increasing trend, indicate increasing 861 compositional maturity in Sundarbans sediments (cf., Singh et al., 2005). Textural 862 maturity is further substantiated by the Pettijohn et al. (1972) classification scheme, 863 where the majority of Sundarbans samples are found to be mostly litharenite and 864 greywacke/wacke. Mineral stability in the modified classification scheme of 865 Pettijohn et al. (1972) by Herron (1988) shows a consistency in the Sundarbans being 866 mineralogical mature, with most samples found to be litharenites and wacke (Fig. 867 7b). As discussed by Herron (1988), the $\mathrm{Fe}_{2} \mathrm{O}_{3} / \mathrm{K}_{2} \mathrm{O}$ ratio can be considered an 868 indicator of mineralogical stability. The most stable rock-forming minerals found at 869 low temperature and pressures in sedimentary environments are K-feldspar, 870 muscovite mica, and quartz (Herron, 1988). K-feldspar and muscovite mica tend to 871 have high quantities of $\mathrm{K}$, and in all three there is a low Fe content (Herron, 1988). 872 There tends to be a higher Fe and $\mathrm{Mg}$ content in less stable rock-forming minerals 873 (Herron, 1988). Stable mineral assemblages therefore possess low $\mathrm{Fe}_{2} \mathrm{O}_{3} / \mathrm{K}_{2} \mathrm{O}$ ratios, 874 while less stable mineral assemblages that are found close to sediment source have 875 high $\mathrm{Fe}_{2} \mathrm{O}_{3} / \mathrm{K}_{2} \mathrm{O}$ ratios (Herron, 1988). The $\mathrm{SiO}_{2} / \mathrm{Al}_{2} \mathrm{O}_{3}$ ratio allows for a distinction 876 between high-ratio sandstones and quartz-rich sands, and low-ratio shales (Herron, 877 1988). With the exception of some of the samples from Gplot, the majority of 878 Sundarbans sediments plot within a mineralogically-stable, silica-depleted litharenite 879 classification. Furthermore, these Sundarbans samples appear to be more mature 880 relative to those of Singh et al. (2005a) and Bhuiyan et al. (2011), indicating that 881 these samples are located further from their source. The presence of the Sundarbans 882 samples in these plots shows that these are also undergoing stronger degrees of 883 weathering, relative to the upper reaches of the GAP and Brahmaputra-Jamuna 884 rivers. A complex relationship exists between tectonic setting and having a unique 
885 geochemical signatures, with source and depositional sites having divergent tectonic 886 settings (McLennan et al., 1990; Bahlburg, 1998; Armstrong-Altrin and Verma, 887 2005).

888 The enrichment ratios found in the Sundarbans sediments illustrate

889 dissolution during chemical weathering, whereby $\mathrm{SiO}_{2}, \mathrm{Na}_{2} \mathrm{O}$, and $\mathrm{K}_{2} \mathrm{O}$ depletion 890 may be attributed to the dissolution of feldspar minerals (Nesbitt and Young, 1984;

891 Singh et al., 2005). Furthermore, increased distance from sediment source, coupled 892 with increased weathering, indicate a depletion of $\mathrm{SiO}_{2}$ and $\mathrm{K}_{2} \mathrm{O}$ in the Sundarbans 893 sediments, relative to what was found by Singh et al. (2005a). The weathering of 894 biotite and ferromagnesium minerals tends to release $\mathrm{Mg}$ and $\mathrm{K}$ (Clow and Drever, 895 1996; Singh et al., 2005a; Garzanti et al., 2010; Lupker et al., 2012; Bouchez et al., 896 2012). The depletion of $\mathrm{Na}$ and $\mathrm{Ca}$ may be attributed to their highly mobile nature 897 during chemical weathering (Nesbitt and Young, 1984; Singh et al., 2005a; Bouchez 898 et al., 2012). This then results in these elements being concentrated in dissolved river 899 water loads, hence the subsequent depleted nature of these in the Sundarbans 900 samples. The immobility of Ti means that it is enriched in all Sundarbans samples, 901 mainly Lothian and Dhanchi, with a lower enrichment ratio in Gplot, which was 902 found to have a ratio similar to the UCC. Potassium has been found to be less mobile 903 than $\mathrm{Ti}$, and may be associated with clay mineral formation within the GAP whereby $904 \mathrm{~K}$ is preferentially adsorbed by clay minerals (cf., Singh et al., 2005a). The 905 enrichment of $\mathrm{Fe}_{2} \mathrm{O}_{3}$ in the Sundarbans samples may be attributed to weathering and 906 depositional maturity of the Sundarbans, relative to the Gomati River and 907 Brahmaputra-Jamuna sites examined by Singh et al. (2005a) and Bhuiyan et al. 908 (2011), respectively. 
The inter-element relationships found in the major element geochemistry

910 show a generally negative trend with $\mathrm{SiO}_{2}$, indicating a grain-size control on the

911 geochemistry of weathering products (Singh et al., 2005a; Garzanti et al., 2010,

912 2011; von Eynatten et al., 2012; Lupker et al., 2013). Fe, Al, and Si are considered

913 immobile elements during Himalayan erosion since they are found to be resistant to

914 chemical weathering (Galy and France-Lanord, 2001; Lupker et al., 2013). The

915 relationship between $\mathrm{TiO}_{2}$ and $\mathrm{Fe}_{2} \mathrm{O}_{3}$ was attributed to both enrichment of heavy

916 minerals in Sundarbans sediment and a common source of ferromagnesium minerals.

917 A strong correlation for $\mathrm{TiO}_{2}$ with $\mathrm{Al}_{2} \mathrm{O}_{3}$ and $\mathrm{K}_{2} \mathrm{O}$ may indicate that Ti was derived

918 from mica in fluvial sediments (Galy and France-Lanord, 2001; Singh et al., 2005a;

919 Lupker et al., 2013). Although the correlation for $\mathrm{K}_{2} \mathrm{O}$ was poor $\left(\mathrm{R}^{2}=0.06\right)$, the 920 correlation with $\mathrm{Al}_{2} \mathrm{O}_{3}$ was relatively strong $\left(\mathrm{R}^{2}=0.66\right)$. The poor correlation with

$921 \mathrm{~K}_{2} \mathrm{O}$ may be attributed to the substitution effect of $\mathrm{K}$ with $\mathrm{Rb}$ (El-Makky and Sediek,

922 2012). Singh et al. (2005a) consider correlation between $\mathrm{K}_{2} \mathrm{O}$ with $\mathrm{MgO}$ and $\mathrm{Al}_{2} \mathrm{O}_{3}$

923 (Fig. 10d,f) to reflect illite in suspended sediments. However, this was not found in

924 this study, indicating increased weathering and illite removal within the Sundarbans.

925 The lack of smectite may also be linked to the lack of correlation found between

$926 \mathrm{MgO}$ and $\mathrm{Al}_{2} \mathrm{O}_{3}$, where $\mathrm{MgO}$ has weathered out of sediment and dissolved into

927 solution (Fig. 10f). Dissolution of $\mathrm{MgO}$ has been found by Singh et al. (2005b) to

928 contribute heavily to the cation budget of the Brahmaputra, where on average $75 \%$ of

929 the cations are $\mathrm{Ca}$ and $\mathrm{Mg}$.

930 The A-CN-K ternary diagram and CIA (Fig. 11a,b) reveal a trend of 931 increasing weathering throughout the Sundarbans sediments. With progressive 932 chemical weathering, there is an increase in clay mineral composition with a 933 concomitant decrease in feldspars and other minerals (Singh et al., 2005a; Garzanti et 
934 al., 2010, 2011; Bouchez et al., 2012; Lupker et al., 2012, 2013). This is further

935 evidenced by Sundarbans sediments following a predicted weathering trend along the

936 A-CN apex of the ternary diagram. The location of both Lothian and Dhanchi

937 samples, along with Gplot samples at the highest point on the $\mathrm{A}-\mathrm{CN}-\mathrm{K}$ ternary

938 diagram is indicative of higher intensity chemical weathering. The $\mathrm{A}-\mathrm{CNK}-\mathrm{FM}$

939 ternary diagram (Fig. 11c) illustrates the predominant trend of Sundarbans samples

940 to follow the predicted weathering trend in the GAP as proposed by Singh et al.

941 (2005a). The S/10-CM-NK ternary diagram (Fig. 11d) shows that data plot parallel

942 to the S/10-CM apex, similarly to those Singh et al. (2005a) and Bhuiyan et al.

943 (2011) data. This trend indicates the control of $\mathrm{Na}$ and $\mathrm{K}$ mobility during weathering

944 (Nesbitt and Young, 1984; Singh et al., 2005a; Garzanti et al., 2011; Lupker et al.,

945 2012; Bouchez et al., 2012). The proximity of the Sundarbans samples to Na and K

946 illustrates the higher degree of weathering in the Sundarbans, compared with Singh

947 et al. (2005a) and Bhuiyan et al. (2011). The primary distinction between Ganges and

948 Brahmaputra sources in the G-B Delta is the degree of weathering in sediments, with

949 Brahmaputra derived sediments being considerably less weathered than those for the

950 Ganges. What this study has shown is that not only are sediments from the

951 Sundarbans more weathered than those found in the Brahmaputra, ruling out a

952 Brahmaputra source of sediment; but that sediments are more intensively weathered

953 than those found by Singh et al. (2005a), with the exception of floodplain and

954 suspended sediments. At the Sundarbans, sediments are considered to be channel

955 sediments, and, given the lithofacies found in this study, may be considered to be 956 intensively weathered.

957 Chemical mobility within the Sundarbans sediments backs up the inference 958 regarding intense weathering, since increasing weathering intensity is associated with 
959 decreasing variability in $\mathrm{Si}, \mathrm{Na}$, and $\mathrm{K}$. The rapid decrease in $\mathrm{CaO}$ throughout the

960 Sundarbans sediments shows the predominance of $\mathrm{Ca}$ to be released during the

961 weathering of feldspars, but not retained in the clay fraction and being dissolved into

962 solution (Singh et al., 2005a). This is supported by Singh et al. (2005b) whereby the

963 cation budget of the Brahmaputra is dominated by $\mathrm{Ca}$ and $\mathrm{Mg}$, with dissolution of

$964 \mathrm{CaO}$ increasing as a result of weathering (Fig. 12g). Ca from the Ganges River

965 sediment has been found to be reduced by half from the initial Ca composition

966 (Bouchez et al., 2012).

967 Zirconium, and its mineral constituent zircon may be related to grain-size

968 variability, whereby $\mathrm{Zr}$ has been shown to be correlated with coarse-size fractions

969 (e.g., fine to very-fine sands) (Flood et al., 2016). With increased weathering, there

970 may be a concomitant increase in clay minerals, leading to fining-up in lithofacies.

971 Sediments in the sites examined in this study are composed of the TMF

972 which are derived from the Ganges River through the GAP, having undergone two

973 cycles of weathering. The provenance and depositional model outlined in this study

974 is both enhanced and adds to the model of Rogers et al. (2013), whereby

975 sedimentation is locally heterogeneous in the Sundarbans with seasonal delivery of

976 sediment distributed throughout all parts. Although Rogers et al. (2013) analysed

977 present-day sedimentation in the Bangladesh Sundarbans, the present study has

978 demonstrated that sediments from the West Bengal Sundarbans are sourced from the

979 GAP, through the Ganges River. As Rogers et al. (2013) have found the majority of

980 sediment deposited on the tidal delta plain during monsoonal activity was derived

981 from flooding. The catchment and upper floodplain may be considered the principal

982 source (i.e., GAP) of sediments accreting on the lower delta plain surface (Rogers et

983 al., 2013). 
985 5.4. Summary of the mineralogy, grain size, and chemical index of alteration from

986 the Sundarbans

988 The relationship between mineral clusters, GSD facies, and CIA is shown in Fig. 15

989 from the Lothian, Gplot, and Dhanchi cores. In each core there is a distinct trend,

990 with increasing weathering intensity moving from the base of the core to the core

991 surface shown by the CIA values along with a fining-up in GSD facies. Mineral

992 clusters demonstrate this relationship between mineralogy and GSD facies, with

993 higher quartz, muscovite, and albite indicative of coarse grained sediment, and

994 kaolinite of fine grained sediment (e.g., mineral clusters 6, 4, 2). It has been found

995 that quartz, feldspar, and heavy minerals steadily increase with depth relative to

996 micas, such as muscovite and clay-rich aggregates in the G-B delta (Garzanti et al.,

997 2011). The fluctuations between muscovite mica and quartz reflect most strongly the

998 differentiating settling velocities of sediment, whereby size shifts for micas, which

999 settle slower than quartz in spite of their higher density (Garzanti et al., 2008). In this

1000 respect, grain size facies illustrate the continuous fluctuations between slightly

1001 coarser, quartz dominated silts, and finer, muscovite mica dominated clays. This is

1002 seen with the increase in kaolinite content moving up through each of the cores.

1003 Chemical weathering indices have been found to be similar in both the Ganges and

1004 Brahmaputra sediments, indicative of significant weathering in monsoonal climates

1005 (Garzanti et al., 2011). The CIA for each of the cores shown in Fig. 15 has illustrated

1006 the variability in suspension sorting, the weathering indices decrease with depth

1007 through the cores from the Sundarbans (cf., Garzanti et al., 2011). This trend in

1008 decreasing weathering intensity has been found to be higher in the Ganges plains 
1009 than in the Brahmaputra (Garzanti et al., 2011). This increase in CIA moving up-

1010 core, is understood to be a hydraulic-sorting effect, as a result of decreasing $\mathrm{Al}$,

1011 related to phyllosilicates, and increasing $\mathrm{Na}$ and $\mathrm{Ca}$, associated with plagioclase,

1012 offset by a decrease in $\mathrm{K}$ and Mg (Garzanti et al., 2011).

1013 The mineralogy of sediments from the Himalayas is composed primarily of 1014 quartz, micas, and feldspars, with the finer fraction composed mainly of 1015 phyllosilicates, clay assemblages and hydroxides (Garzanti et al., 2010, 2011; Lupker 1016 et al., 2013). During sediment transport these minerals are segregated with coarse1017 grained quartz enriched in bedload at the bottom of the water column and 1018 phyllosilicates and clays found to be enriched in shallow surface waters (Lupker et 1019 al., 2013). The sediments of the TMF presented in this study reflect this partitioning 1020 of mineralogy and geochemistry as a result of sediment transport.

\section{6. CONCLUSION}

1023 The TMF of the Ganges-Brahmaputra Delta have been examined and found to reveal 1024 intensively weathered, terrestrial sediment derived from the Ganges River, 1025 principally the GAP. The TMF in the West Bengal Sundarbans are composed of 1026 sediments sourced from the Ganges that went through initial in-situ weathering, prior 1027 to being eroded and chemically weathered in the GAP, and finally being transported 1028 to the lower delta plain during the monsoon. The TMF is proposed as being 1029 diachronous in terms of the role played by fluvial and marine processes in 1030 deposition. The depositional model for the TMF in the far western extent of the G-B 1031 delta is that the sediment plume is then transported westwards by prevailing currents, 1032 and through tides these sediments are deposited onto the delta plain. This study 
1033 presents a first-order approximation of lithofacies and geochemistry of the TMF on

1034 the western abandoned lower Ganges-Brahmaputra delta and demonstrates that:

1035 1. Geochemical data enhances the weathering model proposed by Singh et al.

1036 (2005a), with sediments of the TMF having undergone at least two cycles of

1037 weathering.

1038 2. Mineralogy of the cores collected shows a predominance of quartz and mica

1039 with clay minerals. Quartz supply is interpreted as indicative of terrestrial

$1040 \quad$ sources of sediment, draining the Himalayas.

1041 3. Kaolinite formation is derived from feldspar and muscovite mica with 1042 kaolinite the product of intense chemical weathering.

1043 4. Fining upward trend in grain size distributions in the West Bengal $1044 \quad$ Sundarbans.

1045 5. Dhanchi and Lothian Island lithofacies are considered to be muddy tidal flats

1046 with Gplot Island indicating an intertidal to shallow subtidal environment

1047 with possible channel-mouth and channel-side bar deposits.

1048 6. Geochemical, mineralogical and lithofacies composition of the TMF suggest 1049 it is locally heterogeneous with sediment derived from the Ganges and 1050 deposited tidally in a low-energy system following the model of Rogers et al. $1051 \quad$ (2013).

1052

1053 ACKNOWLEDGEMENTS

1054 RPF acknowledges the support provided by a Department for Employment and 1055 Learning (Northern Ireland) research studentship and the Department of Education 1056 and Science's Higher Education Grant Scheme (ROI) via Laois County Council 1057 (ROI). RPF also acknowledges the School of Natural and Built Environment 
1058 (formerly the School of Geography, Archaeology and Palaeoecology), Queen's 1059 University, Belfast (QUB) for the fieldwork support provided by their Soulby 1060 Research Fund. RPF acknowledges the assistance and technical support provided by 1061 Paul O’Meara (PANanalytical B.V.), Mike Dobby (MikeDobby Analytical 1062 Consultant), and Pat McBride (QUB), for their help and assistance with XRD, XRF, 1063 and laser granulometry, respectively. RPF and JDO would like to acknowledge the 1064 support and assistance of Vincent van Walt and Van Walt Environmental Ltd., in the 1065 core extraction and research support, while logistical support in the Sundarbans 1066 provided through the Institute of Environmental Studies \& Wetland Management 1067 (IESWM), Kolkata, West Bengal, India (S. Bhattacharyya and K. Sen Sarma) is 1068 gratefully acknowledged. RPF would also like to acknowledge the help and support 1069 provided by Mary Bourke of Trinity College Dublin (TCD), Irene Delgado1070 Fernandez and Breandán Anraoi MacGabhann from Edge Hill University (EHU) for 1071 their help and support of this research. The authors would like to acknowledge the 1072 helpful comments from the editor of Marine Geology, an anonymous reviewer, and 1073 Laura Stutenbecker (Institute of Geological Sciences, University of Bern). 
REFERENCES

1075 Adegoke, O.S., Stanley, D.J., 1972. Mica and shell as indicators of energy level and depositional regime on the Nigerian Shelf. Marine Geology, 13, M61-M66.

Aitchison, J., 1986. The Statistical Analysis of Compositional Data: Monographs on Statistics and Applied Probability. Chapman \& Hall Ltd., London (436 pp.).

1079 Allen, G.P., Castaing, P., Klingebiel, A., 1972. Distinction of elementary sand 1080 populations in the Gironde estuary (France) by R-mode factor analysis of 1081 grain-size data. Sedimentology 19, 21-35.

Allen, J.R.L., 1990. Salt-marsh growth and stratification: a numerical model with special reference to the Severn Estuary, southwest Britain. Marine Geology, $95,77-96$.

Allen, J.R.L., 2000. Morphodynamics of Holocene salt marshes: a review sketch from the Atlantic and Southern North Sea coasts of Europe. Quaternary

Allison, M.A. 1998a. Geologic framework and environmental status of the GangesBrahmaputra delta. Journal of Coastal Research 14, 826-836.

1090 Allison, M.A., 1998b. Historical changes in the Ganges-Brahmaputra delta. Journal 1091 of Coastal Research 14, 1269-1275.

1092

Allison, M.A., Kepple, E.B., 2001. Modern sediment supply to the lower delta plain of the Ganges-Brahmaputra River in Bangladesh. Geo-Marine Letters 21, 55-

1095 Allison, M.A., Khan, S.R., Goodbred Jr., S.L., Kuehl, S.A., 2003. Stratigraphic 1096 evolution of the late Holocene Ganges-Brahmaputra lower delta plain. Sedimentary Geology 155, 317-342. 
1098 Andrews, J.T., Vogt, C., 2014. Source to sink: statistical identification of regional variations in the mineralogy of surface sediments in the western Nordic Seas $\left(58^{\circ} \mathrm{N}-75^{\circ} \mathrm{N} ; 10^{\circ} \mathrm{W}-40^{\circ} \mathrm{W}\right)$. Marine Geology $357,151-162$.

1101 Anthony, E.J., 2015. Wave influence in the construction, shaping and destruction of 1102 river deltas: A review. Marine Geology 361, 53-78.

1103 Armstrong-Altrin, J.S., Verma, S.P., 2005. Critical evaluation of six tectonic setting 1104 discrimination diagrams using geochemical data of Neogene sediments from 1105 known tectonic settings. Sedimentary Geology 177, 115-129.

1106 Auerbach, L.W., Goodbred, Jr., S. L., Mondal, D.R., Wilson, C.A., Ahmed, K.R., 1107 Roy, K., Steckler, M.S., Small, C., Gilligan, J.M., Ackerly, B.A., 2015. Flood 1108 risk of natural and embanked landscapes on the Ganges-Brahmaputra tidal 1109 delta plain. Nature Climate Change 5, 153-157.

1110 Bahlburg H., 1998. The geochemistry and provenance of Ordovician turbidites in the 1111 Argentine Puna. In: Panhhurst, R.J., Rapela, C.W, (Eds.). The proto-andean 1112 margin of Gondwana. Geological Society of London, Special Paper 142, 127 111342.

1114 Barua, D.K., Kuehl, S.A., Miller, R.L., Moore, R.S., 1994. Suspended sediment 1115 distribution and residual transport in the coastal ocean off the Ganges 1116 Brahmaputra River mouth. Marine Geology 120, 41-61.

1117 Bass, S.J., Aldridge, J.N., McCave, I.N., Vincent, C.E., 2002. Phase relationships 1118 between fine sediment suspensions and tidal currents in coastal seas. Journal of 1119 Geophysical Research 107(C10), 3146, doi:10.1029/2001JC001269

1120 Bates, C.C., 1953. Rational theory of delta formation. Bulletin of the American $1121 \quad$ Association of Petroleum Geologists 37, 2119-2162. 
1122 Berner, E.K., Berner, R.A., 1996. Global Environment: Water, Air and Geochemical 1123 Cycles. Prentice Hall, Inc., Upper Saddle River, NJ.

1124 Bhatia, M.R., 1983. Plate tectonics and geochemical composition of sandstones. The Journal of Geology, 611-627.

1126 Bhattacharyya, S., Pethick, J., Sensarma, K. 2013. Managerial response to sea level

1130 Bhuiyan, M.A.H., Rahman, M.J.J., Dampare, S.B., Suzuki, S., 2011. Provenance, 1131 tectonics and source weathering of modern fluvial sediments of the 1132 Brahmaputra-Jamuna River, Bangladesh: inference from geochemistry. $1133 \quad$ Journal of Geochemical Exploration 111, 113-137.

1134 Bickle, M.J., Bunbury, J., Chapman, H.J., Harris, N.B., Fairchild, I.J., Ahmad, T., 1135 2003. Fluxes of $\mathrm{Sr}$ into the headwaters of the Ganges. Geochimica et $1136 \quad$ Cosmochimica Acta 67, 2567-2584.

1137 Bish, D.L., 1994. Quantitative X-ray diffraction analysis of soils. In: Amonette, J.E., 1138 Zelazny, L.W. (Eds.), Quantitative Methods in Soil Mineralogy. Soil Science 1139 Society of America, Madison, USA, pp. 267-295 (Chapter 9).

1140 Bjørlykke, K., 1998. Clay mineral diagenesis in sedimentary basins - a key to the 1141 prediction of rock properties. Examples from the North Sea Basin. Clay 1142 minerals 33, 15-34.

1143 Boto, K.G., Wellington, J.T., 1984. Soil characteristics and nutrient status in a 1144 northern Australian mangrove forest. Estuaries 7, 61-69. 
1145 Bouchez, J., Gaillardet, J., Lupker, M., Louvat, P., France-Lanord, C., Maurice, L., 1146 Armijos, E., Moquet, J.S., 2012. Floodplains of large rivers: Weathering 1147 reactors or simple silos?. Chemical Geology 332, 166-184.

1148 Buggle, B., Glaser, B., Hambach, U., Gerasimenko, N., Marković, S., 2011. An 1149 evaluation of geochemical weathering indices in loess-paleosol studies. $1150 \quad$ Quaternary International 240, 12-21.

1151 Canuel, E.A., Martens, C.S., 1993. Seasonal variability in the sources and alteration 1152 of organic matter associated with recently deposited sediments. Organic $1153 \quad$ Geochemistry 20, 563-577.

1154 Chakrapani, G.J., 2005. Major and trace element geochemistry in upper Ganga River 1155 in the Himalayas, India. Environmental Geology 48, 189-201.

1156 Chakrapani, G.J., Subramanian, V., Gibbs, R.J., Jha, P.K., 1995. Size characteristics 1157 and mineralogy of suspended sediments of the Ganges River, India. $1158 \quad$ Environmental Geology 25, 192-196.

1159 Chayes, F., 1960. On correlation between variables of constant sum. Journal of $1160 \quad$ Geophysical Research 6, 4185-4193.

1161 Chayes, F., 1971. Ratio Correlation: A Manual for Students of Petrology and 1162 Geochemistry. University of Chicago Press, Chicago (99 pp.).

1163 Chesworth, W., Dejou, J., Larroque, P., 1981. The weathering of basalt and relative 1164 mobilities of the major elements at Belbex, France. Geochimica et $1165 \quad$ Cosmochimica Acta 45, 1235-1243.

1166 Clow, D.W., Drever, J.I., 1996. Weathering rates as a function of flow through an $1167 \quad$ alpine soil. Chemical Geology 132, 131-141.

1168 Coleman, J.M., 1969. Brahmaputra River: channel processes and sedimentation. 1169 Sedimentary Geology 3, 129-239. 
1170 Curray, J.R., 1991. Possible greenschist metamorphism at the base of a 22-km

1171 sedimentary section, Bay of Bengal. Geology 19, 1097-1100.

1172 Dalai, T.K., Krishnaswami, S., Sarin, M.M., 2002. Major ion chemistry in the

1173 headwaters of the Yamuna river system: Chemical weathering, its temperature

1174 dependence and $\mathrm{CO}_{2}$ consumption in the Himalaya. Geochimica et

$1175 \quad$ Cosmochimica Acta 66, 3397-3416.

1176 Dalrymple, R.W., Zaitlin, B.A., Boyd, R.A., 1992. A conceptual model of estuarine 1177 sedimentation. Journal of Sedimentary Petrology 62, 1130-1146.

1178 Datta, D.K., Subramanian, V., 1997. Texture and mineralogy of sediments from the 1179 Ganges-Brahmaputra-Meghna river system in the Bengal Basin, Bangladesh 1180 and their environmental implications. Environmental Geology 30, 181-188.

1181 Dias, J.A., Pilkey, O.H., Heilweil, V.M., 1984. Detrital mica: Environmental 1182 significance in North Portugal continental shelf sediments. Comunicações dos 1183 Serviços Geológicos de Portugal 70, 93-101.

1184 Doyle, L.J., Cleary, W.J., Pilkey, O.H., 1968. Mica: its use in determining shelf1185 depositional regimes. Marine Geology 6, 381-389.

1186 Drever, J.I., 1988. The Geochemistry of Natural Waters. Prentice-Hall, Englewood Cliff, NJ.

1188 Dronkers, J., 1986. Tidal asymmetry and estuarine morphology. Netherlands Journal 1189 of Sea Research 20, 117-131

1190 Dutta, M.K., Chowdhury, C., Jana, T.K., Mukhopadhyay, S.K., 2013. Dynamics and 1191 exchange fluxes of methane in the estuarine mangrove environment of the 1192 Sundarbans, NE coast of India. Atmospheric Environment 77, 631-639.

1193 Ehlmann, A.J., 1968. Clay mineralogy of weathered products and of river sediments, 1194 Puerto Rico. Journal of Sedimentary Petrology 38, 885-894. 
1195 El-Makky, A.M., Sediek, K.N., 2012. Stream sediments geochemical exploration in 1196 the northwestern part of Wadi Allaqi Area, South Eastern Desert, Egypt. $1197 \quad$ Natural resources research 21, 95-115.

1198 Fan, D., Tu, J., Shang, S., Cai, G., 2014. Characteristics of tidal-bore deposits and 1199 facies associations in the Qiantang Estuary, China. Marine Geology 348, 1-14.

1200 Flood, R.P., 2014. Post Mid-Holocene Sedimentation of the West Bengal

1201 Sundarbans. Unpublished Ph.D. Thesis, Queen's University, Belfast, 646 pp.

1202 Flood, R.P., Bloemsma, M.R., Weltje, G.J., Barr, I.D., O’Rourke, S.M., Turner, J.N.

1203 and Orford, J.D., 2016. Compositional data analysis of Holocene sediments 1204 from the West Bengal Sundarbans, India: Geochemical proxies for grain-size variability in a delta environment. Applied Geochemistry 75, 222-235.

1206 Flood, R.P., Orford, J.D., McKinley, J.M., Roberson, S., 2015. Effective grain size 1207 distribution analysis for interpretation of tidal-deltaic facies: West Bengal

1209 Fontorbe, G., Christina, L., Chapman, H.J. Bickle, M.J., 2013. The silicon isotopic 1210 composition of the Ganges and its tributaries. Earth and Planetary Science $1211 \quad$ Letters $381,21-30$.

1212 Frings, P.J., Clymans, W., Fontorbe, G., Gray, W., Chakrapani, G., Conley, D.J., De La Rocha, C., 2015. Silicate weathering in the Ganges alluvial plain. Earth and Planetary Science Letters 427, 136-148.

1215 Fritz, S.J., Mohr, D.W., 1984. Chemical alteration in the micro weathering environment within a spheroidally-weathered anorthosite boulder. Geochimica et Cosmochimica Acta 48, 2527-2535.

1218 Galán, E., Gómez-Ariza, J.L., González, I., Fernández-Caliani, J.C., Morales, E., 1219 Giráldez, I., 2003. Heavy metal partitioning in river sediments severely 
polluted by acid mine drainage in the Iberian Pyrite Belt. Applied $1221 \quad$ Geochemistry 18, 409-421.

1222 Galy, A., France-Lanord, C., 1999. Weathering processes in the Ganges1223 Brahmaputra basin and the riverine alkalinity budget. Chemical Geology 159, $1224 \quad 31-60$.

1225 Galy, A., France-Lanord, C., 2001. Higher erosion rates in the Himalaya: 1226 Geochemical constraints on riverine fluxes. Geology 29, 23-26.

1227 Garzanti, E., Andò, S. and Vezzoli, G., 2008. Settling equivalence of detrital 1228 minerals and grain-size dependence of sediment composition. Earth and $1229 \quad$ Planetary Science Letters 273, 138-151.

1230 Garzanti, E., Andó, S., France-Lanord, C., Censi, P., Vignola, P., Galy, V., Lupker, 1231 M., 2011. Mineralogical and chemical variability of fluvial sediments 2. 1232 Suspended-load silt (Ganga-Brahmaputra, Bangladesh). Earth and Planetary $1233 \quad$ Science Letters 302, 107-120.

1234 Garzanti, E., Andò, S., France-Lanord, C., Vezzoli, G., Censi, P., Galy, V., Najman, 1235 Y., 2010. Mineralogical and chemical variability of fluvial sediments: 1.

1236 Bedload sand (Ganga-Brahmaputra, Bangladesh). Earth and Planetary Science $1237 \quad$ Letters 299, 368-381.

1238 Garzanti, E., Andò, S., Vezzoli, G., 2009. Grain-size dependence of sediment 1239 composition and environmental bias in provenance studies. Earth and Planetary $1240 \quad$ Science Letters 277, 422-432.

1241 Giri, C., Pengra, B., Zhu, Z., Singh, A., Tieszen, L.L., 2007. Monitoring mangrove 1242 forest dynamics of the Sundarbans in Bangladesh and India using multi1243 temporal satellite data from 1973 to 2000. Estuarine, Coastal and Shelf Science $1244 \quad 73,91-100$. 
1245 Goodbred Jr., S.L. 2003. Response of the Ganges dispersal system to climate change:

1246 a source-to-sink view since the last interstade. Sedimentary Geology 162, 83$1247 \quad 104$.

1248 Goodbred Jr., S.L., Kuehl, S.A., 2000a. The significance of large sediment supply, 1249 active tectonism and eustasy on margin sequence development: Late 1250 Quaternary stratigraphy and evolution of the Ganges-Brahmaputra 1251 delta. Sedimentary Geology 133, 227-248.

1252 Goodbred Jr., S.L., Kuehl, S.A., 2000b. Enormous Ganges-Brahmaputra sediment 1253 discharge during strengthened early Holocene monsoon. Geology 28, 1083$1254 \quad 1086$.

1255 Goodbred Jr., S.L., Kuehl, S.A., Steckler, M.S., Sarker, M.H. 2003. Controls on 1256 facies distribution and stratigraphic preservation in the Ganges-Brahmaputra 1257 delta sequence. Sedimentary Geology 155, 301-316.

1258 Goodbred, S.L., Paolo, P.M., Ullah, M.S., Pate, R.D., Khan, S.R., Kuehl, S.A., 1259 Singh, S.K., Rahaman, W., 2014. Piecing together the Ganges-Brahmaputra1260 Meghna River delta: Use of sediment provenance to reconstruct the history and 1261 interaction of multiple fluvial systems during Holocene delta evolution.

1262 Geological Society of America Bulletin 126, 1495-1510.

1263 Goodbred, S.L., Saito, Y., 2011. Tide-dominated Deltas. In: Davis, R. Dalyrymple, 1264 R.W. (Eds.) Principles of Tidal Sedimentology. Springer, New York, pp. 1291265149.

1266 Grant, W.H., 1963. Weathering of Stone Mountain Granite. In Ingersoll, E.C. ed. 1267 Clays and Clay Minerals, v. 11, pp. 65-73.

1268 Gutierrez, M., 2005. Climatic Geomorphology. Elsevier, Amsterdam. 
1269 Hait, A.K., Das, J.K., Ghosh, S., Ray, A.K., Saha, A.K., Chanda, S., 1996. New dates 1270 of Pleisto-Holocene subcrop samples from south Bengal, India. Indian Journal $1271 \quad$ of Earth Sciences 23, 79-82.

1272 Hart, M.G.R., 1959. Sulphur oxidation in tidal mangrove soils of Sierra Leone. Plant 1273 and Soil 11, 215-236.

1274 Helsel, D.R., 2005. Nondetects and Data Analysis: Statistics for Censored 1275 Environmental Data. John Wiley and Sons, New York (250 pp.).

1276 Heroy, D.C., Kuehl, S.A. Goodbred, S.L., 2003. Mineralogy of the Ganges and 1277 Brahmaputra Rivers: implications for river switching and Late Quaternary $1278 \quad$ climate change. Sedimentary Geology 155, 343-359.

1279 Herron, M.M., 1988. Geochemical classification of terrigeneous sands and shales 1280 from core or log data. Journal of Sedimentary Petrology 58, 820-829.

1281 Hesse, P.R., 1961. Some differences between the soils of Rhizophora and Avicennia 1282 mangrove swamps in Sierra Leone. Plant and Soil 14, 335-346.

1283 Hillier, S., 2000. Accurate quantitative analysis of clay and other minerals in 1284 sandstones by XRD: comparison of a Rietveld and a reference intensity ratio 1285 (RIR) method and the importance of sample preparation. Clay Minerals 35, $1286 \quad 291-302$

1287 Hinman, N.W., 1998. Sequences of silica phase transitions: effects of Na, Mg, K, Al, $1288 \quad$ and Fe ions. Marine Geology 147, 13-24.

1289 Hübscher, C., Breitzke, M., Michels, K., Kudrass, H.R., Spiess, V., Wiedicke, M., 1290 1998. Late Quaternary seismic stratigraphy of the eastern Bengal Shelf. Marine $1291 \quad$ Geophysical Researches 20, 57-71. 
1292 Hughes, Z.J., 2011. Tidal Channels on Tidal Flats and Marshes. In Davis, R.A., 1293 Dalrymple, R.W. (Eds.) Principles of tidal sedimentology. Springer, New $1294 \quad$ York, pp. 269-300.

1295 ICDD, 2002. International Centre for Diffraction Data. Mineral Powder diffraction 1296 file data book. (941 p.).

1297 Islam, M.R., Stuart, R., Risto, A., Vesa, P., 2002. Mineralogical changes during 1298 intense chemical weathering of sedimentary rocks in Bangladesh. Journal of

1300 Johnson, M.R.W., 1994. Volume balance of erosional loss and sediment deposition 1301 related to Himalayan uplifts. Journal of the Geological Society 151, 217-220.

1302 Johnson, S.Y., Alam, A.M.N., 1991. Sedimentation and tectonics of the Sylhet 1303 trough, Bangladesh. Geological Society of America Bulletin 103, 1513-1527.

1304 Kabata-Pendias, A., Pendias, H., 2000. Trace elements in soils and plants. 3rd ed. 1305 Boca Raton, Florida; London: CRC Press, 413 p.

1306 Kuehl, S.A., Hariu, T.M. and Moore, W.S., 1989. Shelf sedimentation off the 1307 Ganges-Brahmaputra river system: Evidence for sediment bypassing to the $1308 \quad$ Bengal fan. Geology 17, 1132-1135.

1309 Kuehl, S.A., Levy, B.M., Moore, W.S. and Allison, M.A., 1997. Subaqueous delta of 1310 the Ganges-Brahmaputra river system. Marine Geology 144, 81-96.

1311 Lindsay, J.F., Holliday, D.W., Hulbert, A.G., 1991. Sequence Stratigraphy and the 1312 Evolution of the Ganges-Brahmaputra Delta Complex (1). AAPG Bulletin 75, $1313 \quad 1233-1254$.

1314 Lupker, M., France-Lanord, C., Galy, V., Lavé, J., Gaillardet, J., Gajurel, A.P., 1315 Guilmette, C., Rahman, M., Singh, S.K. and Sinha, R., 2012. Predominant 
floodplain over mountain weathering of Himalayan sediments (Ganga basin).

$1317 \quad$ Geochimica et Cosmochimica Acta 84, 410-432.

1318 Lupker, M., France-Lanord, C., Galy, V., Lavé, J., Kudrass, H., 2013. Increasing

1319 chemical weathering in the Himalayan system since the Last Glacial

1320 Maximum. Earth and Planetary Science Letters 365, 243-252.

1321 McLaren, P., Bowles, D., 1985. The effects of sediment transport on grain-size 1322 distributions. Journal of Sedimentary Research 55, 0457-0470.

1323 McLennan, S.M., 1995. Sediments and soils: chemistry and abundances. In: Ahrens, 1324 T.J. (Ed.), Rock Physics and Phase Relations: A Handbook of Physical 1325 Constants, vol. 3. American Geophysical Union Reference Shelf, 8-19.

1326 McLennan, S.M., Taylor, S.R., McCulloch, M.T., Maynard, J.B., 1990. Geochemical 1327 and $\mathrm{Nd}-\mathrm{Sr}$ isotopic composition of deep-sea turbidites: crustal evolution and 1328 plate tectonic associations. Geochimica et Cosmochimica Acta 54, 2015-2050.

1329 Middelburg, J.J., Nieuwenhuize, J., Slim, F.J., Ohowa, B., 1996. Sediment 1330 biogeochemistry in an East African mangrove forest (Gazi bay, $1331 \quad$ Kenya). Biogeochemistry 34, 133-155.

1332 Morgan, J.P. and McIntire, W.G., 1959. Quaternary geology of the Bengal basin, 1333 East Pakistan and India. Geological Society of America Bulletin 70, 319-342.

1334 Mukherjee, A., Fryar, A.E., Thomas, W.A., 2009. Geologic, geomorphic and 1335 hydrologic framework and evolution of the Bengal basin, India and 1336 Bangladesh. Journal of Asian Earth Sciences 34, 227-244.

1337 Nesbitt, H.W., 1979. Mobility and fractionation of rare earth elements during 1338 weathering of a granodiorite. Science $279,206-210$. 
1339 Nesbitt, H.W., Markovics, G., 1980. Chemical processes affecting alkalis and 1340 alkaline earths during continental weathering. Geochimica et Cosmochimica $1341 \quad$ Acta 44, 1659-1666.

1342 Nesbitt, H.W., Young, G.M., 1982. Early Proterozoic climates and plate motions 1343 inferred from major element chemistry of lutites. Nature 299, 715-717.

1344 Nesbitt, H.W., Young, G.M., 1989. Formation and diagenesis of weathering profiles. 1345 The Journal of Geology 129-147.

1346 Nesbitt, H.W., Young, G.M., McLennan, S.M., Keays, R.R., 1996. Effects of 1347 chemical weathering and sorting on the petrogenesis of siliciclastic sediments, 1348 with implications for provenance studies. The Journal of Geology 104, 525$1349 \quad 542$.

1350 Pearson, K., 1896. On the form of spurious correlations which may arise when 1351 indices are used in the measurement of organs. Proceedings of the Royal $1352 \quad$ Society of London 60, 489-502.

1353 Pecharsky, V.K., Zavalij, P.Y., 2009. Fundamentals of powder diffraction and 1354 structural characterization of materials, 2nd edn., New York: Springer, 768 p.

1355 Pethick, J.S., 1980. Velocities, surges and asymmetry in tidal channels. Estuarine, $1356 \quad$ Coastal and Marine Science 11, 331-345.

1357 Pethick, J.S., Orford, J.D., 2013. Rapid rise in effective sea-level in southwest 1358 Bangladesh: Its causes and contemporary rates. Global and Planetary Change $1359 \quad 111,237-245$.

1360 Pettijohn, F., Potter, P., Siever, R., 1972. Sand and Sandstone: New York, Springer$1361 \quad$ Verlag, $618 \mathrm{p}$. 
1362 Pomerancblum, M., 1966. The distribution of heavy minerals and their hydraulic 1363 equivalents in sediments of the Mediterranean continental shelf of Israel. 1364 Journal of Sedimentary Petrology 36, 162-174.

1365 Potter, P.E., 1978. Petrology and chemistry of modern big river sands. The Journal of 1366 Geology 86, 423-449.

1367 Prins, M.A., Postma, G. 2000. Effects of climate, sea level, and tectonics unraveled 1368 for last deglaciation turbidite records of the Arabian Sea. Geology 28, 3751369378.

1370 Purkait, B., Majumdar, D.D., 2014. Distinguishing different sedimentary facies in a 1371 deltaic system. Sedimentary Geology 308, 53-62.

1372 R Development Core Team, 2011. R: A Language and Environment for Statistical 1373 Computing. Compositional Data Analysis in the Geosciences - From Theory 1374 to Practice. R Foundation for Statistical Computing, Vienna Available online at $1375 \quad \mathrm{http}: / / \mathrm{www}$. Rproject.org/. (last accessed 13.12.2014.).

1376 Rengarajan, R., Singh, S.K., Sarin, M.M. and Krishnaswami, S., 2009. Strontium

1377 isotopes and major ion chemistry in the Chambal River system, India:

1378 implications to silicate erosion rates of the Ganga. Chemical Geology 260, 87$1379 \quad 101$.

1380 Rengarajan, R., Singh, S.K., Sarin, M.M. Krishnaswami, S., 2009. Strontium 1381 isotopes and major ion chemistry in the Chambal River system, India: 1382 implications to silicate erosion rates of the Ganga. Chemical Geology 260, 87$1383 \quad 101$.

1384 Rietveld, H., 1969. A profile refinement method for nuclear and magnetic structures. 1385 Journal of applied Crystallography 2, 65-71. 
1386 Rietveld, H.M., 1967. Line profiles of neutron powder-diffraction peaks for structure refinement. Acta Crystallographica 22, 151-152.

1388 Roberson, S., Weltje, G.J., 2014. Inter-instrument comparison of particle-size analysers. Sedimentology 61, 1157-1174.

1390 Rogers, K.G., Goodbred Jr., S.L., Mondal, D.R., 2013. Monsoon sedimentation on

Roser, B.P., Korsch, R.J., 1986. Determination of tectonic setting of sandstonemudstone suites using content and ratio. The Journal of Geology 94, 635-650.

1395 Salminen, R., Batista, M.J., Bidovec, M., Demetriades, A., De Vivo, B., De Vos, W., Duris, M., Gilucis, A., Gregorauskiene, V., Halamic, J., Heitzmann, P., Lima, A., Jordan, G., Klaver, G., Klein, P., Lis, J., Locutura, J., Marsina, K., Mazreku, A., O’Connor, P.J., Olsson, S.Å., Ottesen, R.-T., Petersell, V., Plant, J.A., Reeder, S., Salpeteur, I., Sandström, H., Siewers, U., Steenfelt, A., Tarvainen, T., 2005. Geochemical Atlas of Europe. Part 1: Background Information, Methodology and Maps. Geological Survey of Finland, Espoo downloaded at 01.11.2016 http://www.gtk.fi/publ/foregsatlas.

Sarin, M.M., Krishnaswami, S., Dilli, K., Somayajulu, B.L.K. and Moore, W.S., 1989. Major ion chemistry of the Ganga-Brahmaputra river system: Weathering processes and fluxes to the Bay of Bengal. Geochimica et Cosmochimica Acta 53, 997-1009.

1410 isotopes. Quaternary Science Reviews 28, 2564-2581. 
1411 Sarkar, S.K., Frančišković-Bilinksi, S., Bhattacharya, A., Saha, M., Bilinski, H., 1412 2004. Levels of elements in the surficial estuarine sediments of the Hugli 1413 River, northeast India and their environmental implications. Environment $1414 \quad$ International 30, 1089-1098.

1415 Schimanski, A., Stattegger, K., 2005. Deglacial and Holocene evolution of the 1416 Vietnam shelf: stratigraphy, sediments and sea-level change. Marine Geology $1417 \quad 214,365-387$.

1418 Sheldon, N.D., Tabor, N.J., 2009. Quantitative paleoenvironmental and paleoclimatic 1419 reconstruction using paleosols. Earth-Science Reviews 95, 1-52.

1420 Singh, M., Sharma, M. and Tobschall, H.J., 2005a. Weathering of the Ganga alluvial 1421 plain, northern India: implications from fluvial geochemistry of the Gomati $1422 \quad$ River. Applied Geochemistry 20, 1-21.

1423 Singh, S.K., Sarin, M.M. and France-Lanord, C., 2005b. Chemical erosion in the 1424 eastern Himalaya: major ion composition of the Brahmaputra and $\delta^{13} \mathrm{C}$ of 1425 dissolved inorganic carbon. Geochimica et Cosmochimica Acta 69, 3573$1426 \quad 3588$.

1427 Small, C., Steckler, M., Seeber, L., Akhter, S.H., Goodbred, S., Mia, B. and Imam, 1428 B., 2009. Spectroscopy of sediments in the Ganges-Brahmaputra delta: 1429 Spectral effects of moisture, grain size and lithology. Remote Sensing of $1430 \quad$ Environment 113, 342-361

1431 Snyder, R.L., Bish, D.L., 1989. Quantitative analysis. In: Bish, D.L., Post, J.E. (Eds), 1432 Modern Powder Diffraction, Reviews in Mineralogy, Volume 20, 1433 Mineralogical Society of America, USA, 101-144 (Chapter 5). 
1434 Stallard, R.F., 1988. Weathering and erosion in the Humid tropics. In: Lerman, A., 1435 Meybeck, M. (Eds.), Physical and Chemical Weathering in Geochemical $1436 \quad$ Cycles. Kluwer, Dodretch, 225-246.

1437 Stanley, D.J., Hait, A.K., 2000. Deltas, radiocarbon dating, and measurements of 1438 sediment storage and subsidence. Geology 28, 295-298.

1439 Stoch, L., Sikora, W., 1976. Transformations of micas in the process of 1440 kaolinitization of granites and gneisses. Clays and Clay Minerals 24, 156-162.

1441 Taylor, S.R. and McLennan, S.M., 1985. The continental crust: its composition and 1442 evolution. Oxford, Blackwell, London, $312+\mathrm{XV}$ p.

1443 Thomas, M.F., 2003. Late Quaternary sediment fluxes from tropical watersheds. $1444 \quad$ Sedimentary Geology 162, 63-81.

1445 Twilley, R.R., 1988. Coupling of mangroves to the productivity of estuarine and 1446 coastal waters. In: Jansson, B.O. (Ed.) Coastal offshore Ecosystem: 1447 Interactions. Lecture Notes on Coastal and Estuarine Studies, vol. 22. Springer$1448 \quad$ Verlag, Berlin, pp. 155-180.

1449 Umitsu, M., 1987. Late Quaternary sedimentary environment and landform evolution 1450 in the Bengal lowland: Geographical Review of Japan 60, 164-178.

1451 Umitsu, M., 1993. Late Quaternary sedimentary environments and landforms in the $1452 \quad$ Ganges delta: Sedimentary Geology 83, 177-186.

1453 von Eynatten, H., Tolosana-Delgado, R., Karius, V., 2012. Sediment generation in 1454 modern glacial settings: grain-size and source-rock control on sediment 1455 composition. Sedimentary Geology 280, 80-92.

1456 Ward, J.H., 1963. Hierarchical grouping to optimize an objective function. Journal of 1457 the American Statistical Association 58, 236-244. 
1458 Wedepohl K.H., 1978. Handbook of Geochemistry 11, Sections 73, B-G. Springer$1459 \quad$ Verlag.

1460 Wells, J.T., 1995. Tide-Dominated Estuaries and Tidal Rivers. In: Perillo, G.M. 1461 (Ed.) Geomorphology and sedimentology of estuaries - Developments in 1462 Sedimentology, vol. 53. Elsevier, Amsterdam, 179-206.

1463 Weltje, G.J., Prins, M.A., 2003. Muddled or mixed? Inferring palaeoclimate from 1464 size distributions of deep-sea clastics. Sedimentary Geology 162, 39-62.

1465 Weltje, G.J., Prins, M.A., 2007. Genetically meaningful decomposition of grain-size 1466 distributions. Sedimentary Geology 202, 409-424.

1468 List of tables

1469 Table 1 Total variance explained by the PCA on the clr-transformed data from Gplot $1470 \quad$ Island core

1471 Table 2 Total variance explained by the PCA on the clr-transformed data from 1472 Dhanchi Island core

1473 Table 3 Cluster representative samples: sites and depths

1474 Table 4 Agreement indices from the Rietveld refinement carried out on the XRD 1475 cluster representative samples from the Lothian, Gplot, and Dhanchi Island $1476 \quad$ core samples

1477 Table 5 Percentage (\%) relative abundance of minerals detected with XRD and $1478 \quad$ Rietveld refinement

1479 Table 6 Summary of the distinguishing mineralogical compositions from cluster $1480 \quad$ representative samples

1481 Supplementary Table 1 XRD counts data for cluster representative samples and 1482 Rietveld refinement 
1483 Supplementary Table 2 Tally of reported values, minimum values, and maximum 1484 values of elemental concentrations $(\%, \mathrm{ppm})$ for the suite of calibration 1485 international geochemical reference standards used in the Bruker AXS $1486 \quad$ S1CalProcess calibration software

1488 List of figures

1489 Fig. 1 (i) Extent of the G-B tidal delta complex, (a) West Bengal Sundarbans (India),

(b) East Bengal Sundarbans (Bangladesh), and (c) Kuakata Peninsula

Fig. 3 First (a) and second (b) principal component loadings for each grain size class (Bangladesh) (adapted from Rogers et al., 2013), and (ii) sites cored in the West Bengal Sundarbans, India (November 2010) (after Flood et al., 2015)

Fig. 2 Percentage (\%) sand/silt/clay composition of the Lothian, Gplot, and Dhanchi Island cores of the clr-transformed GSD data (Gplot Island), score plots for first (c) and second (d) principal components (Gplot Island), biplot of first and second principal components (Gplot Island) (e), first (f) and second (g) principal component loadings (Dhanchi Island), first component (h) and second

1502 Fig. 4 Vertical transition of the broad stratigraphical facies of the TMF for Gplot Island (a) and Dhanchi Island (b) cores

1504 Fig. 5 (a) Biplot of the first and second principal components; (b) biplot of the 1505 second and third principal components; (c) biplot of the first and third principal components; (d) triplot of the first, second, and third principal components; (e) 
distribution of XRD cluster groups throughout the cores, with Lothian Island

1509 Fig. $6 \mathrm{Al}_{2} \mathrm{O}_{3} / \mathrm{SiO}_{2}$ versus $\mathrm{Fe}_{2} \mathrm{O}_{3} / \mathrm{SiO}_{2}$ for river sediments of the West Bengal 1510 Sundarbans, Ganga alluvial plain, Siwaliks and the Himalayan sources, and 1511 data from Singh et al. (2005a) and Bhuiyan et al. (2011). Lower and higher ratios are indicative of the quartz dominance moving towards enrichment of phyllosilicates, respectively. Linear trend in mineralogical sorting is indicative of transportation. Gray ellipses indicate composition of source area: the Himalaya and the Siwaliks (Galy and France-Lanord, 2001). Star corresponds to average UCC (Taylor and McLennan, 1985). CS: channel sediments, FS: flood sediments, and SS: suspended sediments. *Data points from Singh et al.

Fig. 7 Geochemical classification diagrams of the West Bengal Sundarbans (2005a), **average data points from Singh et al. (2005a), ***average data points from Bhuiyan et al. (2011)

1526 Fig. 8 Tectonic discrimination diagram for the West Bengal Sundarbans sediments. 1527 Boundaries of fields are from Roser and Korsch (1986). CS: channel 1528 sediments, FS: flood sediments, and SS: suspended sediments. *Data points 1529 from Singh et al. (2005a), **average data points from Singh et al. (2005a), $1530 \quad * * *$ average data points from Bhuiyan et al. (2011) 
1531 Fig. 9 Element ratio of West Bengal Sundarbans sediments calculated from average major element concentrations normalised to UCC (Taylor and McLennan, 1985) with respect to $\mathrm{Al}_{2} \mathrm{O}_{3}$ (Eq. 2). CS: channel sediments, FS: flood sediments, and SS: suspended sediments. ${ }^{* * A v e r a g e ~ d a t a ~ p o i n t s ~ f r o m ~ S i n g h ~ e t ~}$

Fig. 10 Variation diagrams of major elements in West Bengal Sundarbans sediments, data are plotted against $\mathrm{Al}_{2} \mathrm{O}_{3}, \mathrm{SiO}_{2}$ and $\mathrm{TiO}_{2}$. For reference, UCC and World

Fig. 11 (a) A-CN-K ternary diagram of molecular proportions showing sediment suites from the West Bengal Sundarbans. $\mathrm{A}=\mathrm{Al}_{2} \mathrm{O}_{3} ; \mathrm{C}=\mathrm{CaO} ; \mathrm{N}=\mathrm{Na}_{2} \mathrm{O}$ and $\mathrm{K}=\mathrm{K}_{2} \mathrm{O}$. Also plotted is the UCC (Taylor and McLennan, 1985) in a grey cross, along with idealised mineral compositions. Shown are the predicted weathering trends exhibited by the Ganga alluvial plain with arrows, which had experienced incipient to moderate chemical weathering. (b) Bar plot of average

1553 Fig. 12 Chemical mobility in the West Bengal Sundarbans sediments during weathering processes, calculated in terms of percentage change (normalised 
with respect to $\mathrm{TiO}_{2}$ ) of individual major/trace elements vs. progressive degree of chemical alteration (CIA values) (Eq. 3)

1557 Fig. 13 Cartoon example of depositional model for the far western extent of the 1558 Ganges-Brahmaputra delta in the West Bengal Sundarbans. The TMF is 1559 proposed as being a diachronous facies unit whereby fluvial dominance was 1560 prevalent c. 5,000 yrs BP that gradually developed into a mixed fluvio-tidal 1561 dominant delta from c. 3,000 yrs BP, to finally its current state as being a tidal 1562 dominant delta. Sediment is sourced primarily from the Ganges river and 1563 delivered to the coastal shelf as a dominantly monsoonal plume that is re1564 worked onto the delta plain, with the emerging tidal supplied sediment plume 1565 thinning in volume and sediment size as it is carried further west to the tidal dominant delta (figure not to scale)

1567 Fig. 14 (a) kaolinite and muscovite regression of XRD cluster representative samples. A strong positive correlation $\left(\mathrm{R}^{2}=0.90\right)$ is found between these clay and mica mineral compositions, indicative of weathering of mica. (b) kaolinite and quartz regression of cluster representative samples. A strong negative correlation $\left(\mathrm{R}^{2}=-0.96\right)$ is found between kaolinite and quartz, indicative of

1573 Fig. 15 Mineralogy, grain size distribution (GSD) facies, and chemical index of 1574 alteration (CIA) from the Lothian, Gplot, and Dhanchi Island cores, with $1575 \quad$ Lothian Island GSD adapted from Flood et al. (2015)

1577 List of supplementary information

1578 Supplementary Table 1: XRD and Rietveld refinement output for cluster 1579 representative samples 
1580 Supplementary Table 2: XRF: Tally of reported values, minimum values, and 1581 maximum values of elemental concentrations $(\%, \mathrm{ppm})$ for the suite of 1582 calibration international geochemical reference standards used in the Bruker 1583 AXS S1CalProcess calibration software for PXRF analysis 

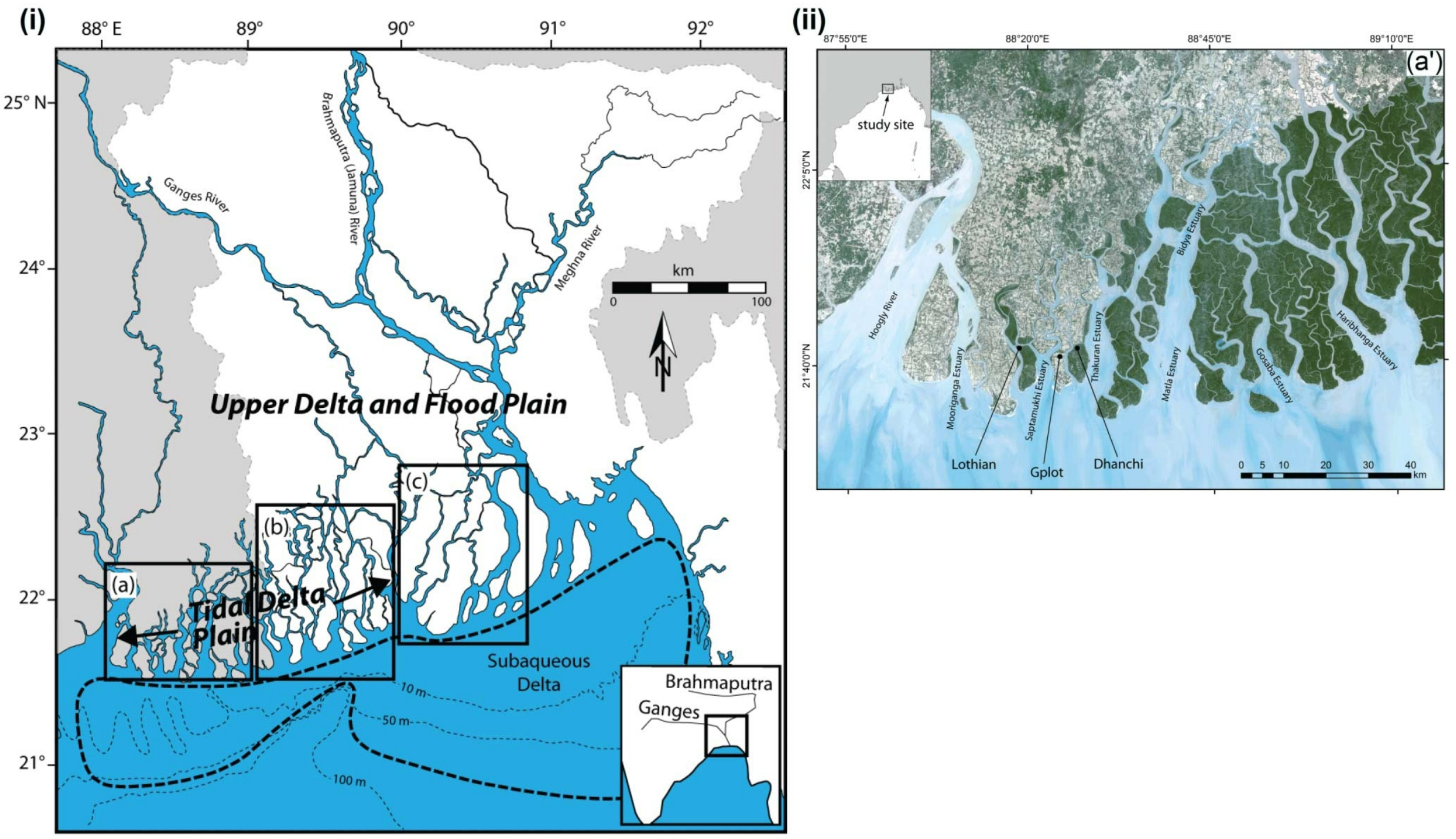


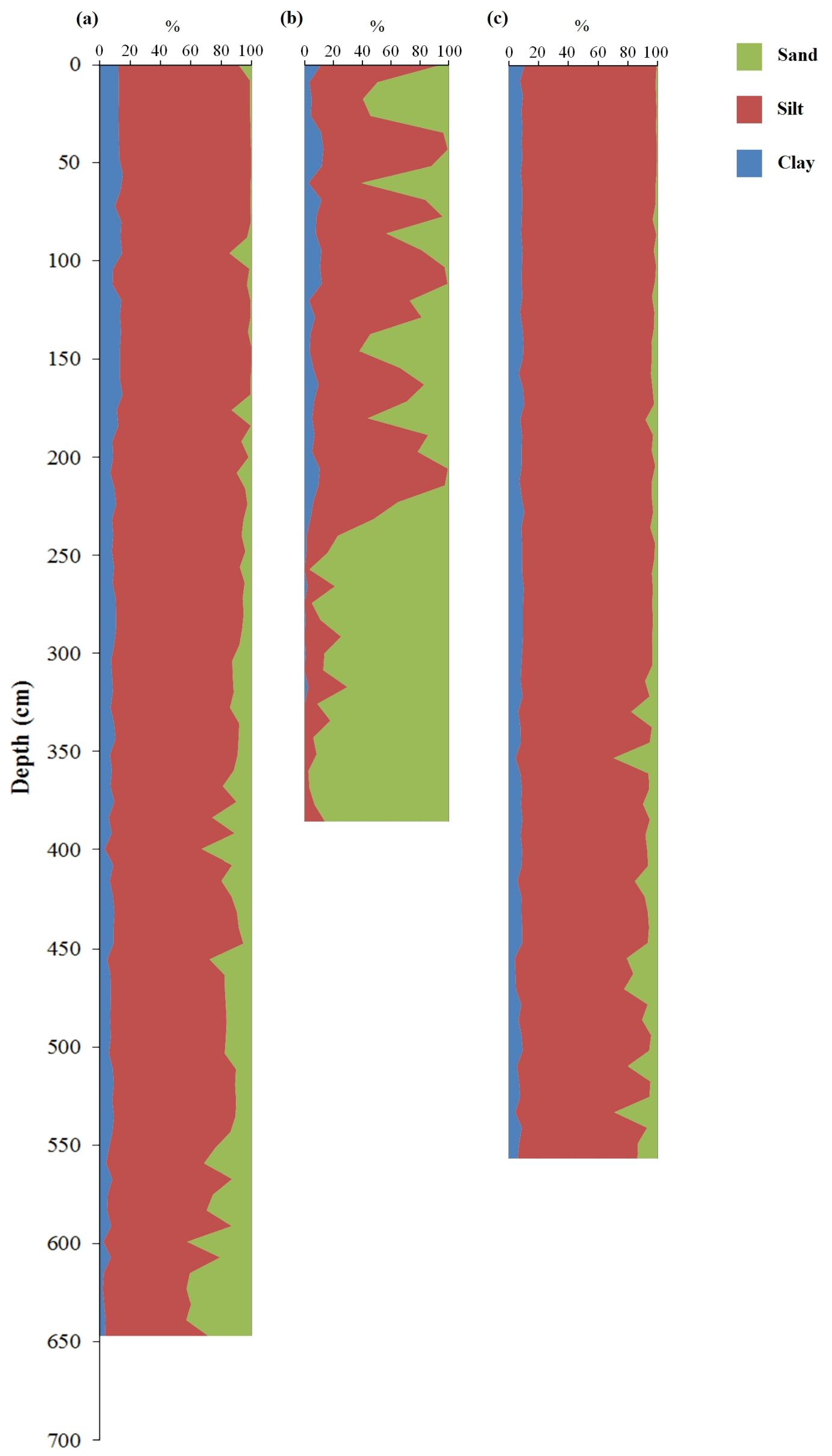




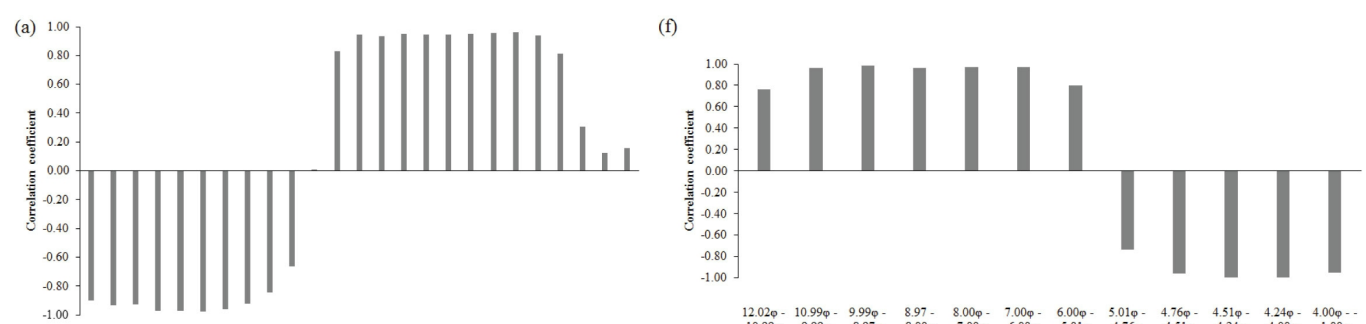

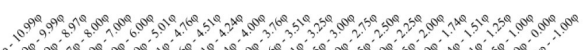

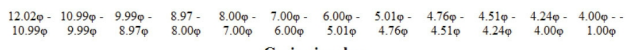

Grain size class

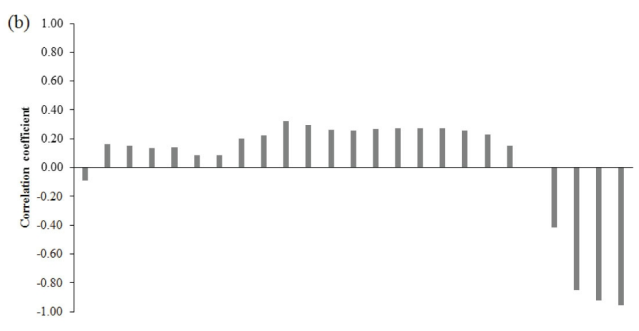

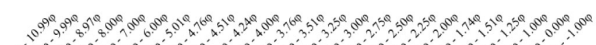

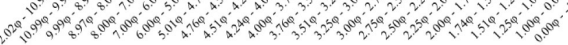

\section{Grain size class}

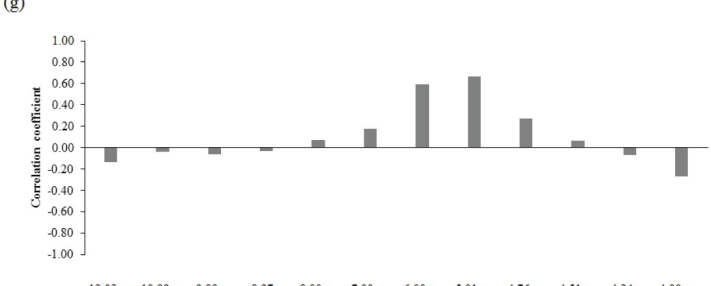

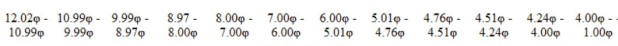

(c)

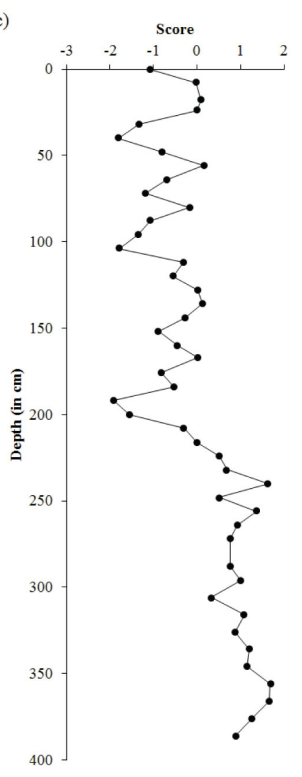

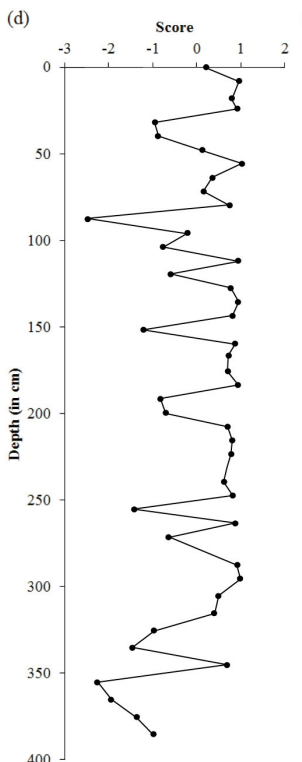

(h)

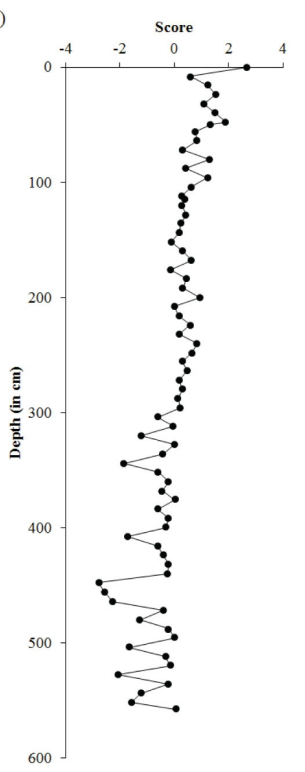

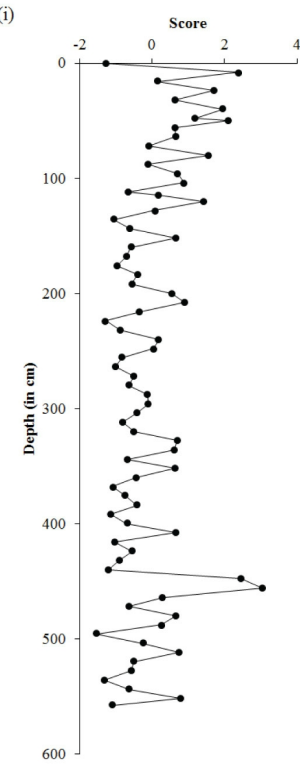

(e)

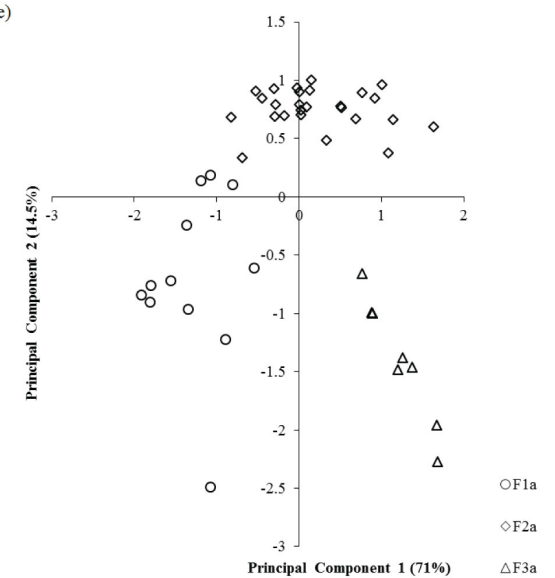

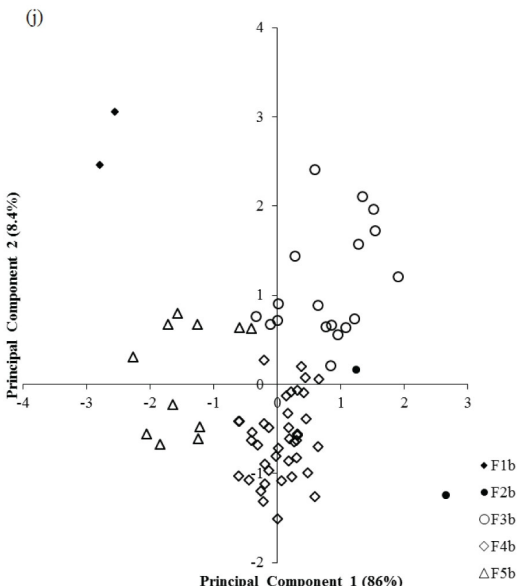


(a)

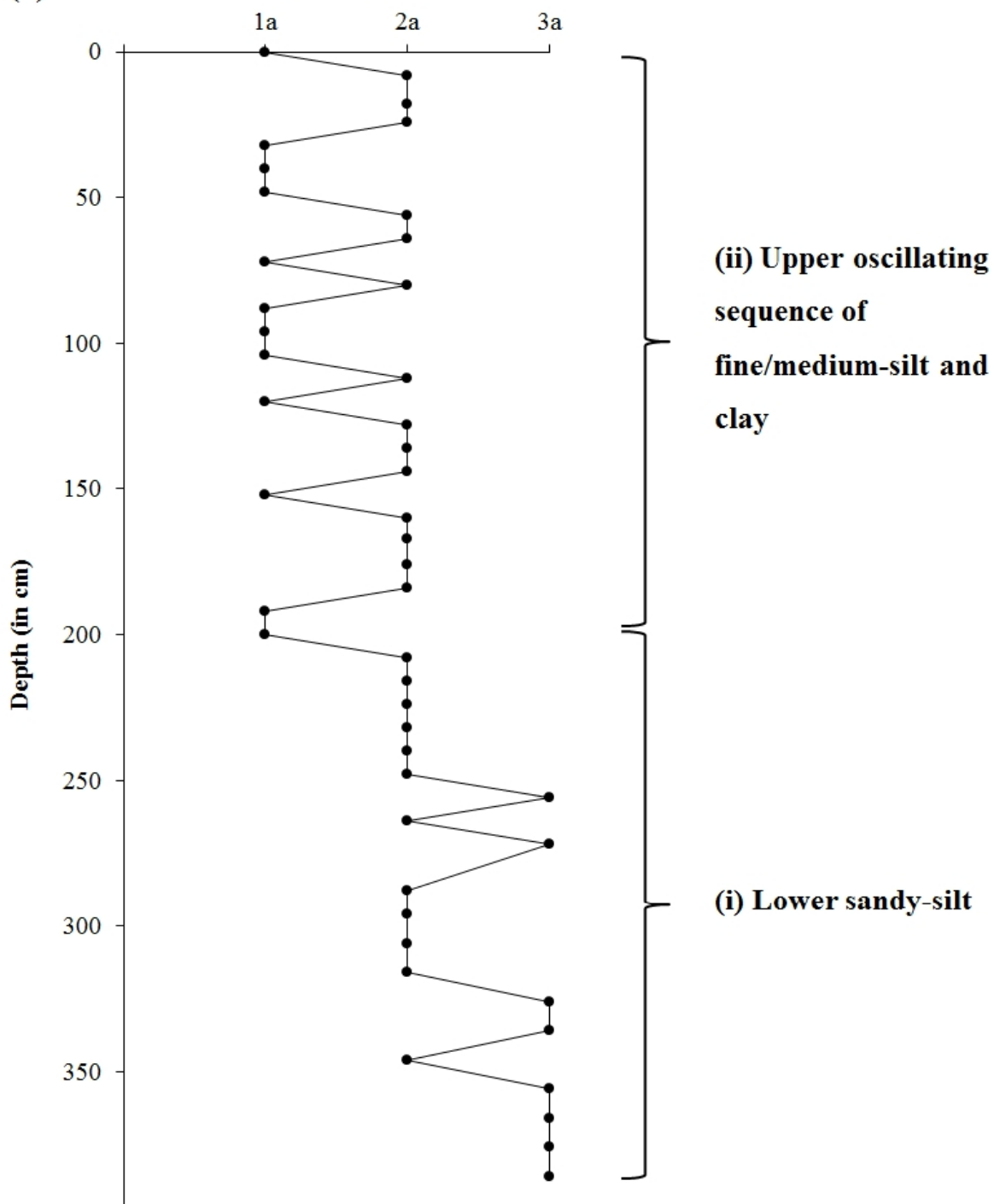

(b)

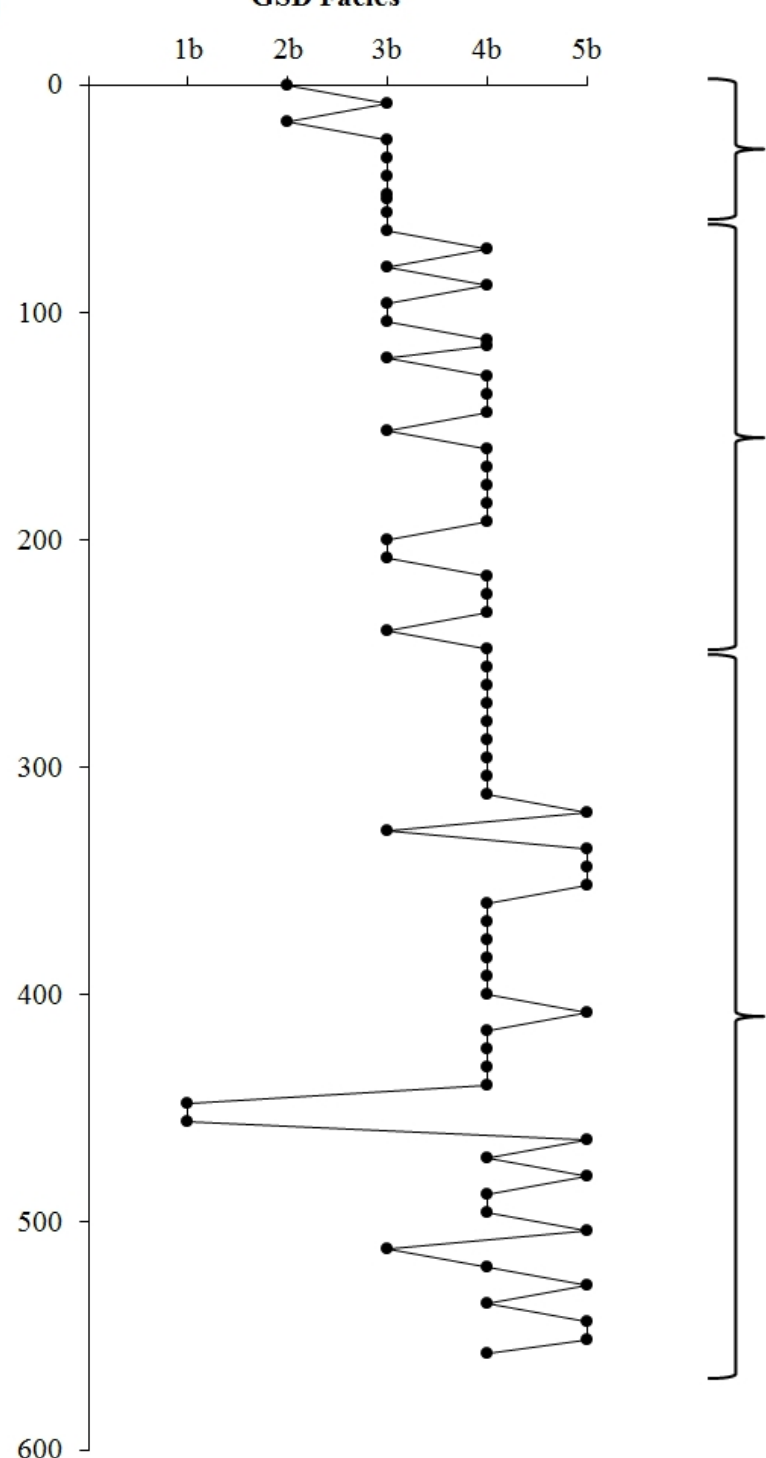

(iii) Upper medium-

to very fine-silt and clay

(ii) Middle mediumfine silty-clay

(i) Lower coarse-silt and sand 

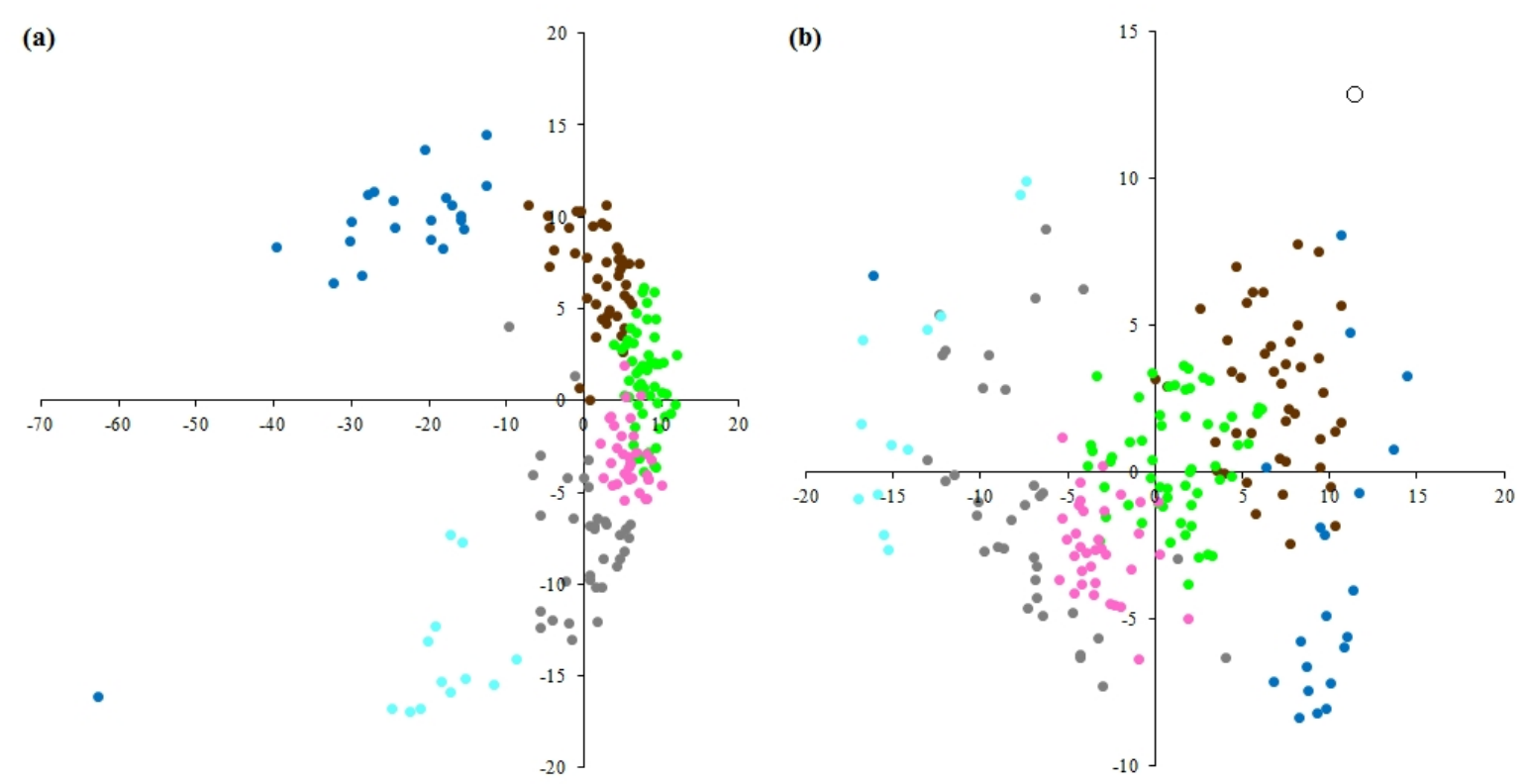

\begin{tabular}{|l|}
\hline Legend \\
Cluster 1 \\
Cluster 2 \\
Cluster 3 \\
Cluster 4 \\
Cluster 5 \\
Cluster 6 \\
\hline
\end{tabular}
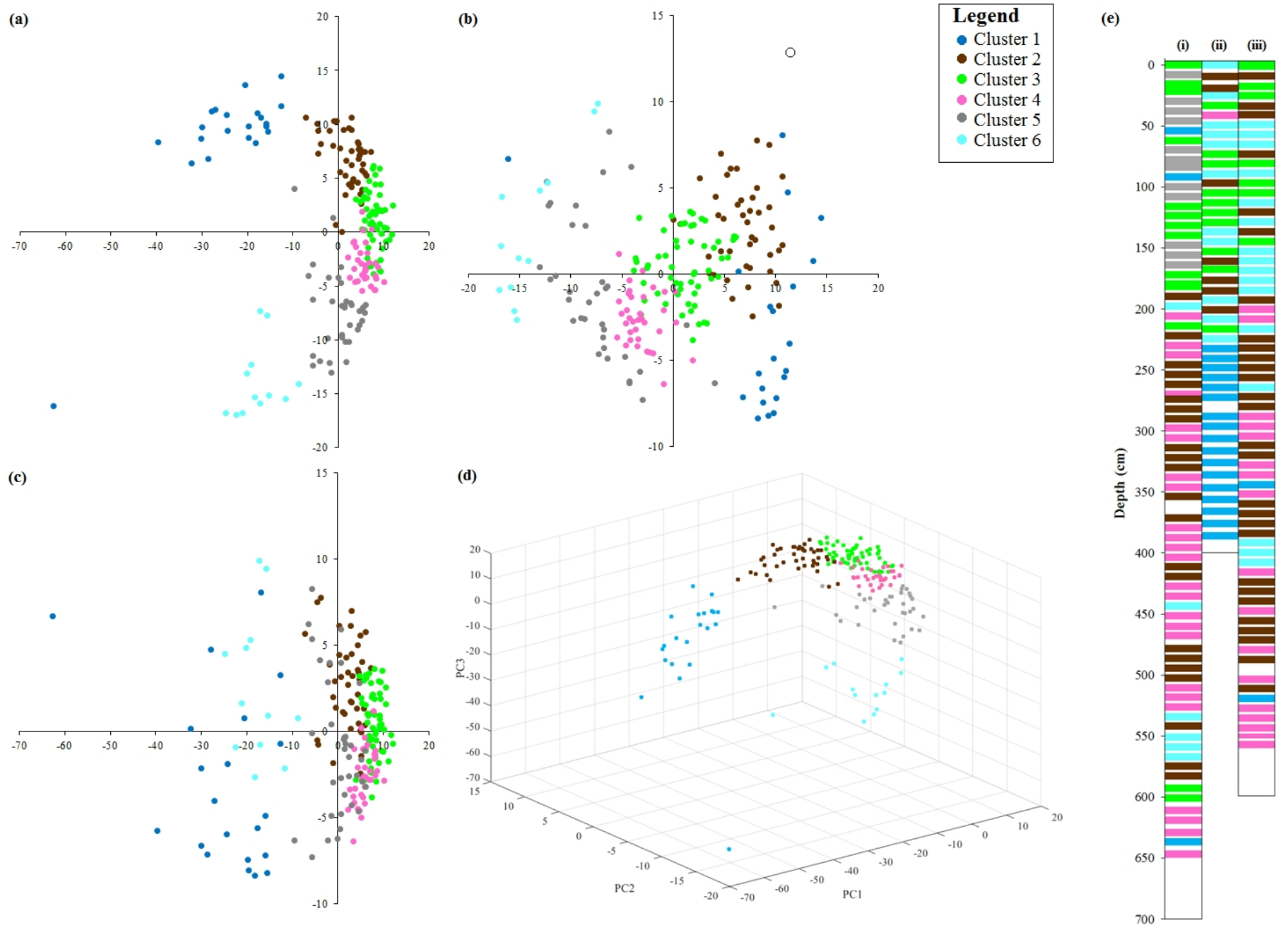


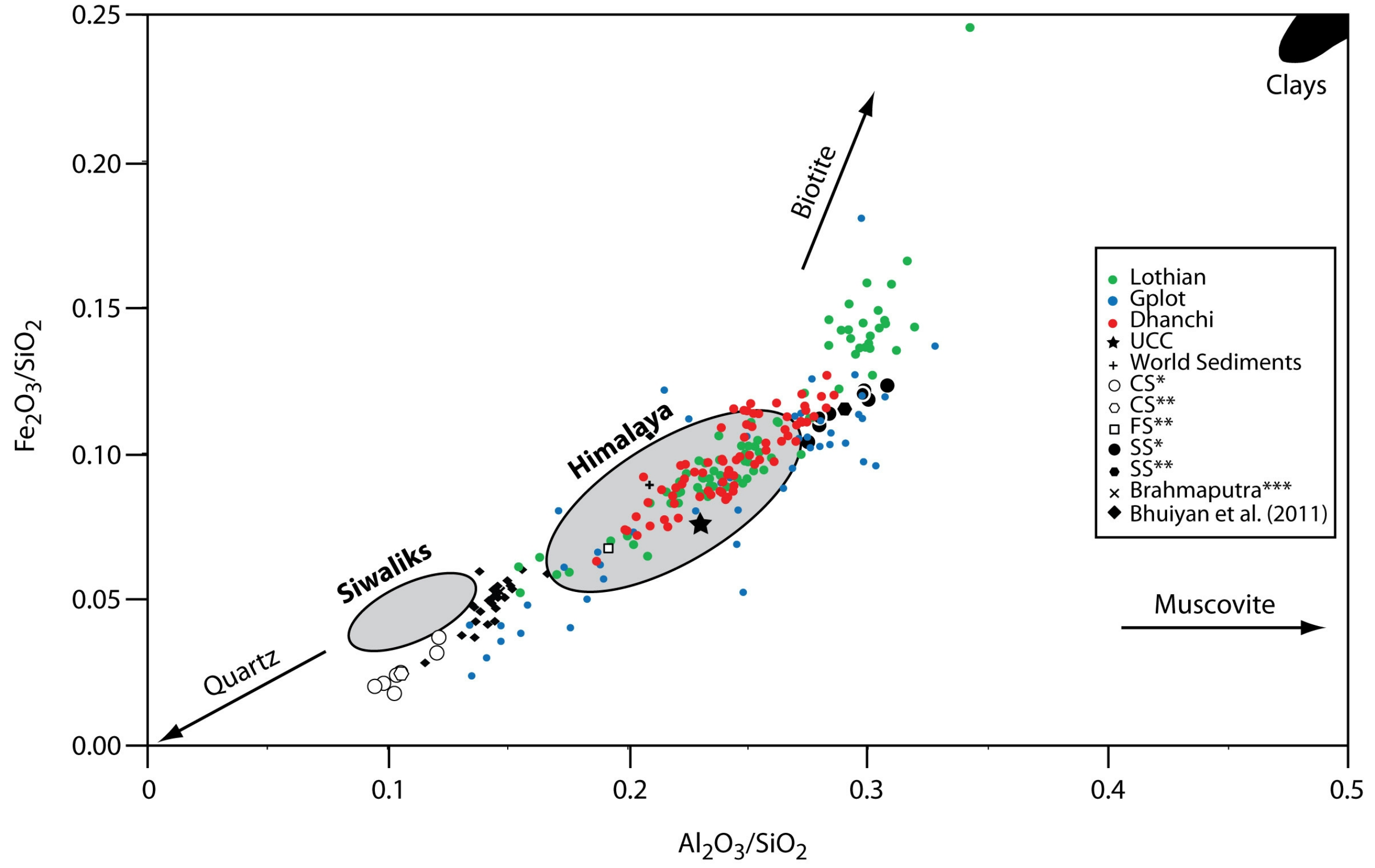



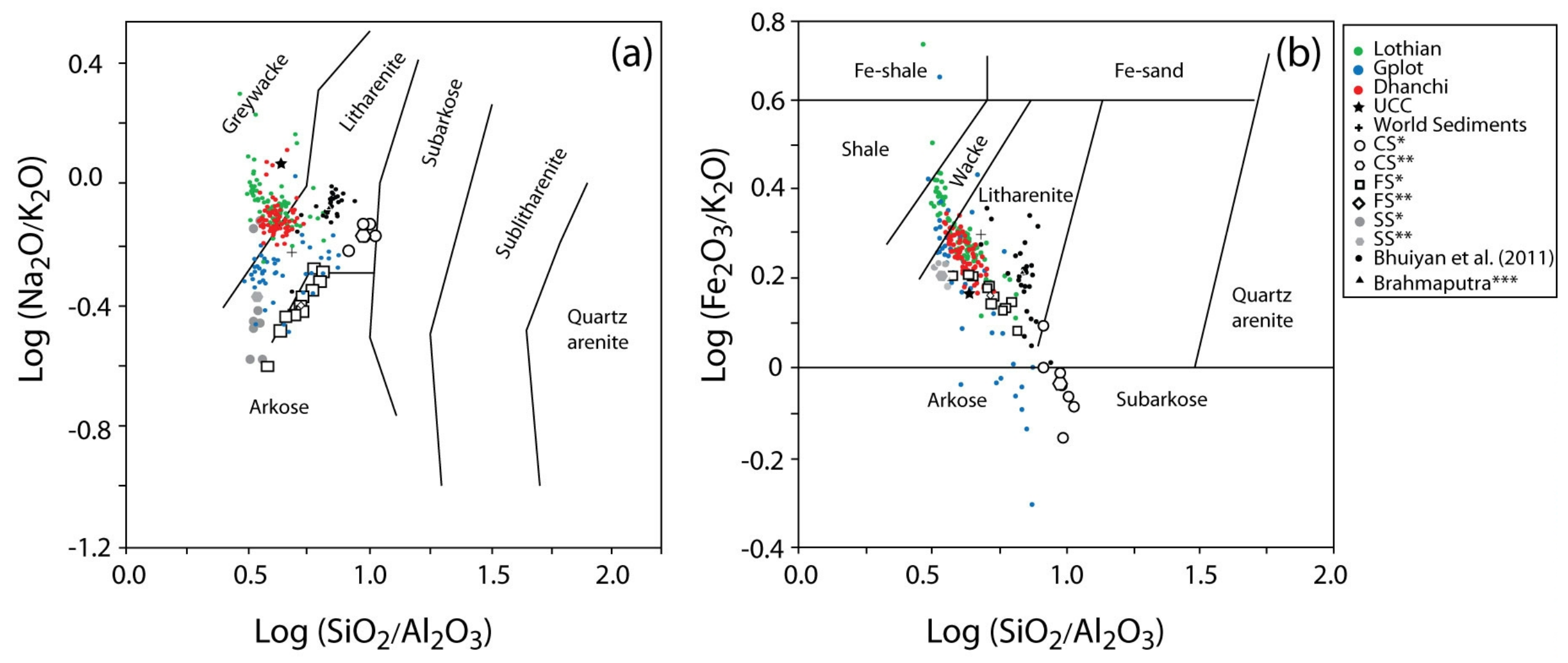


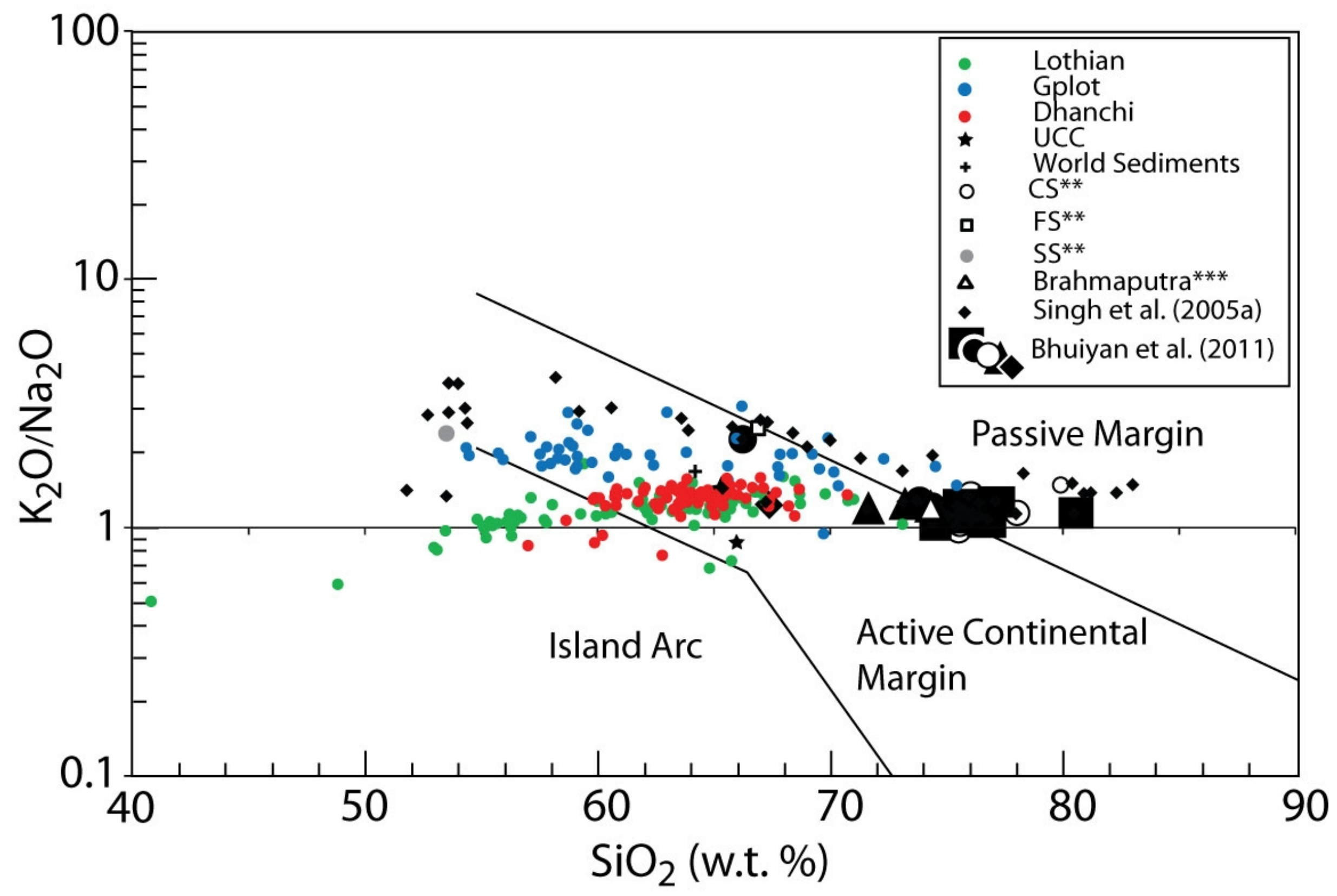




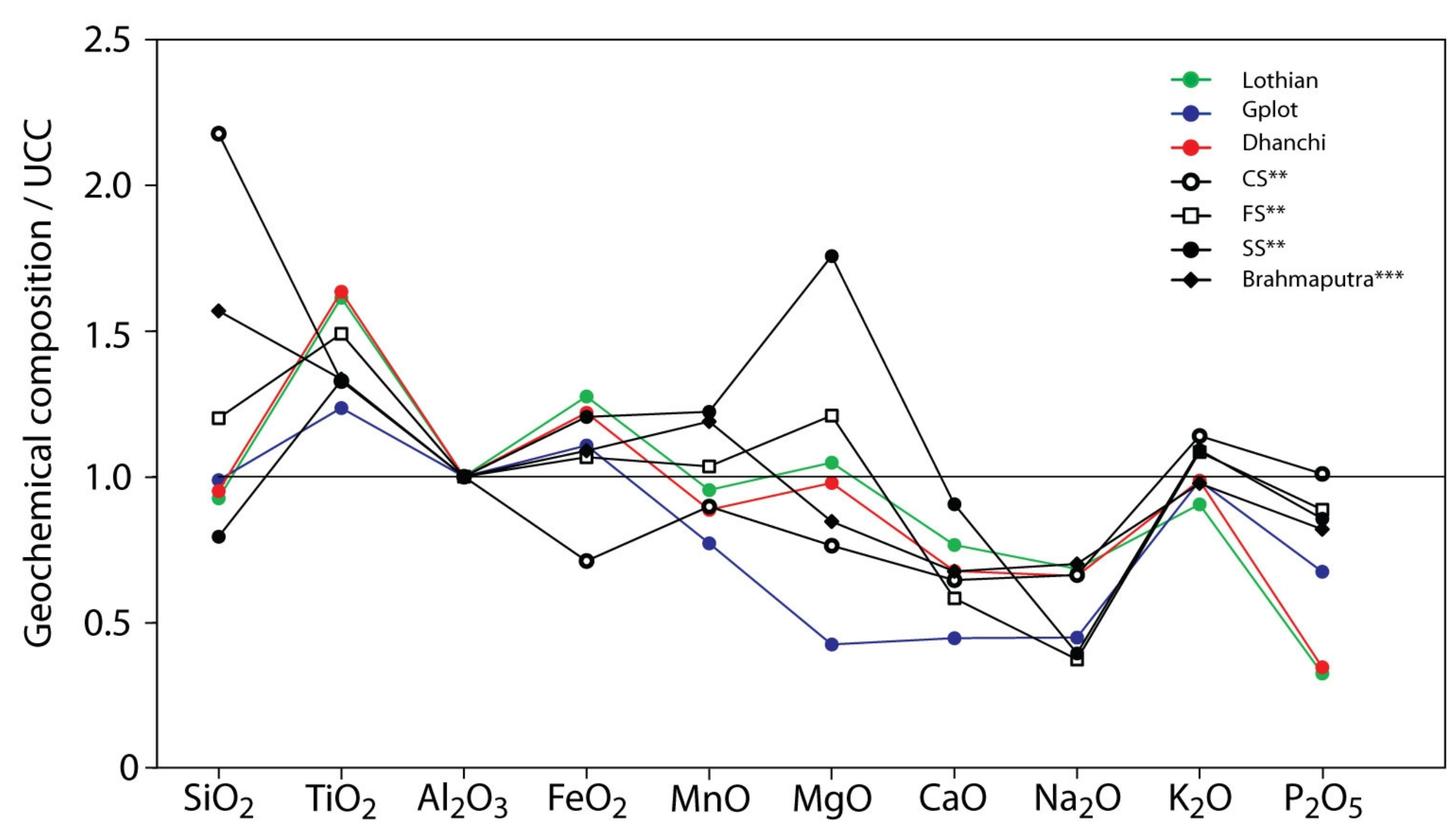



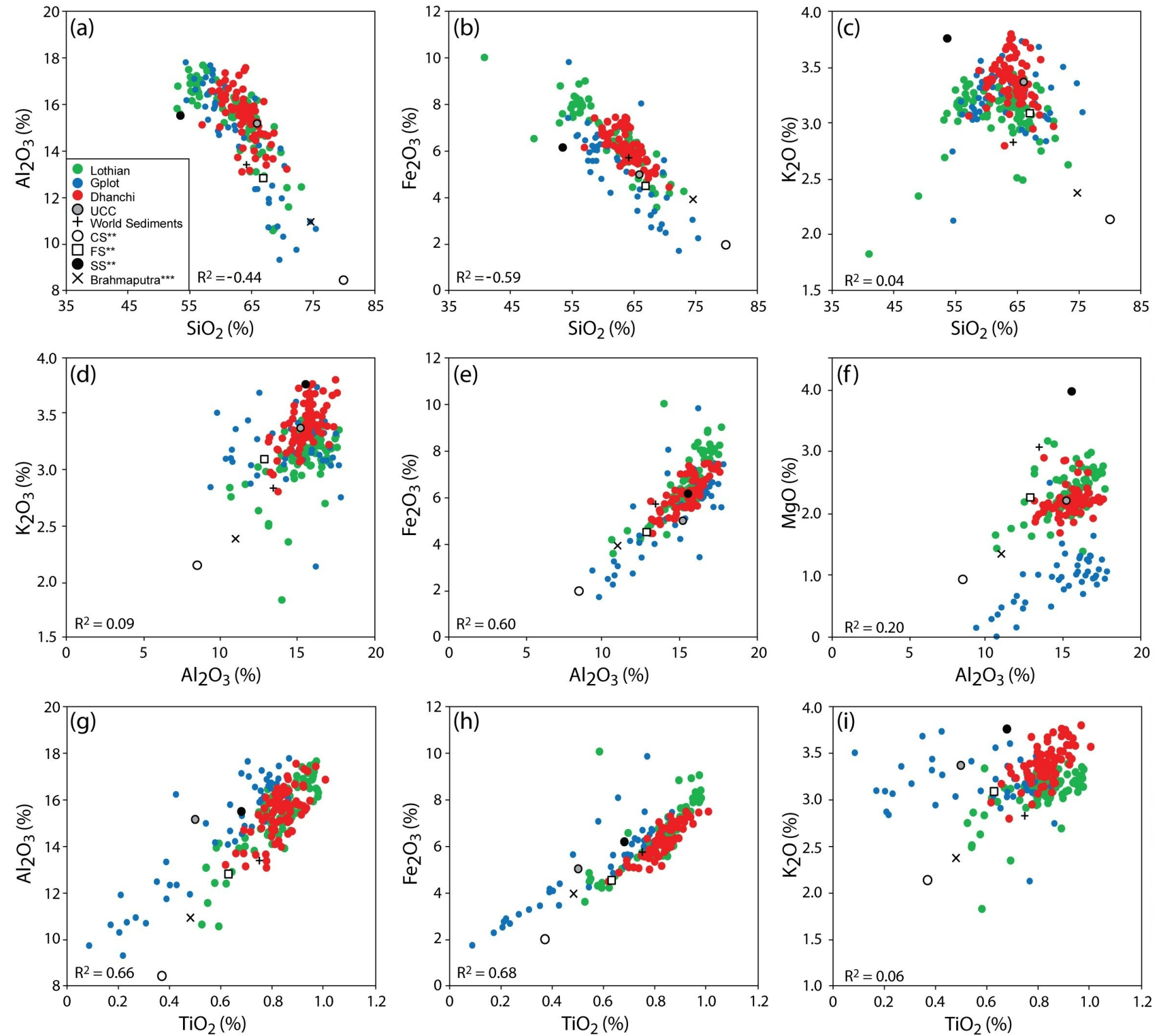

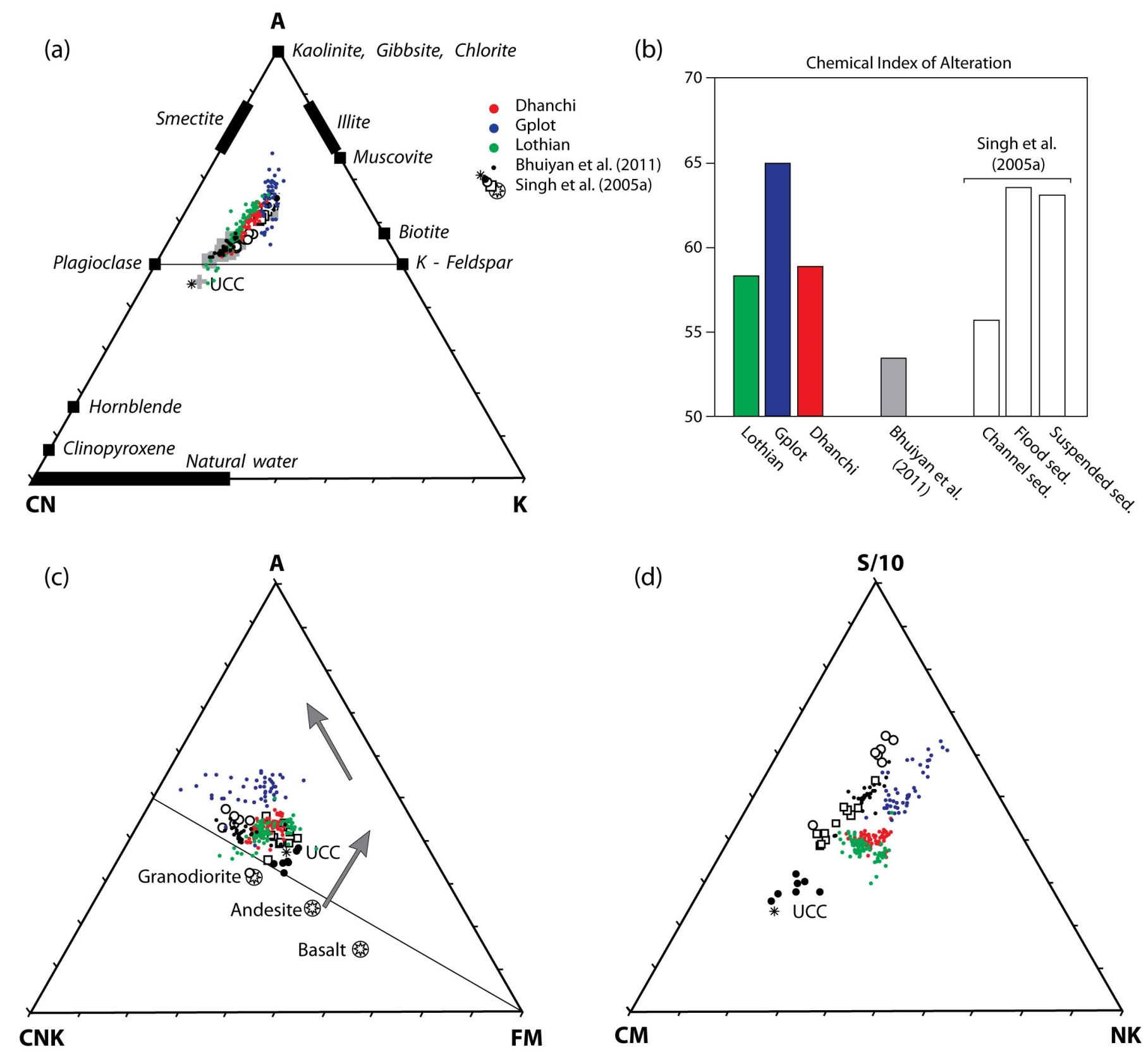

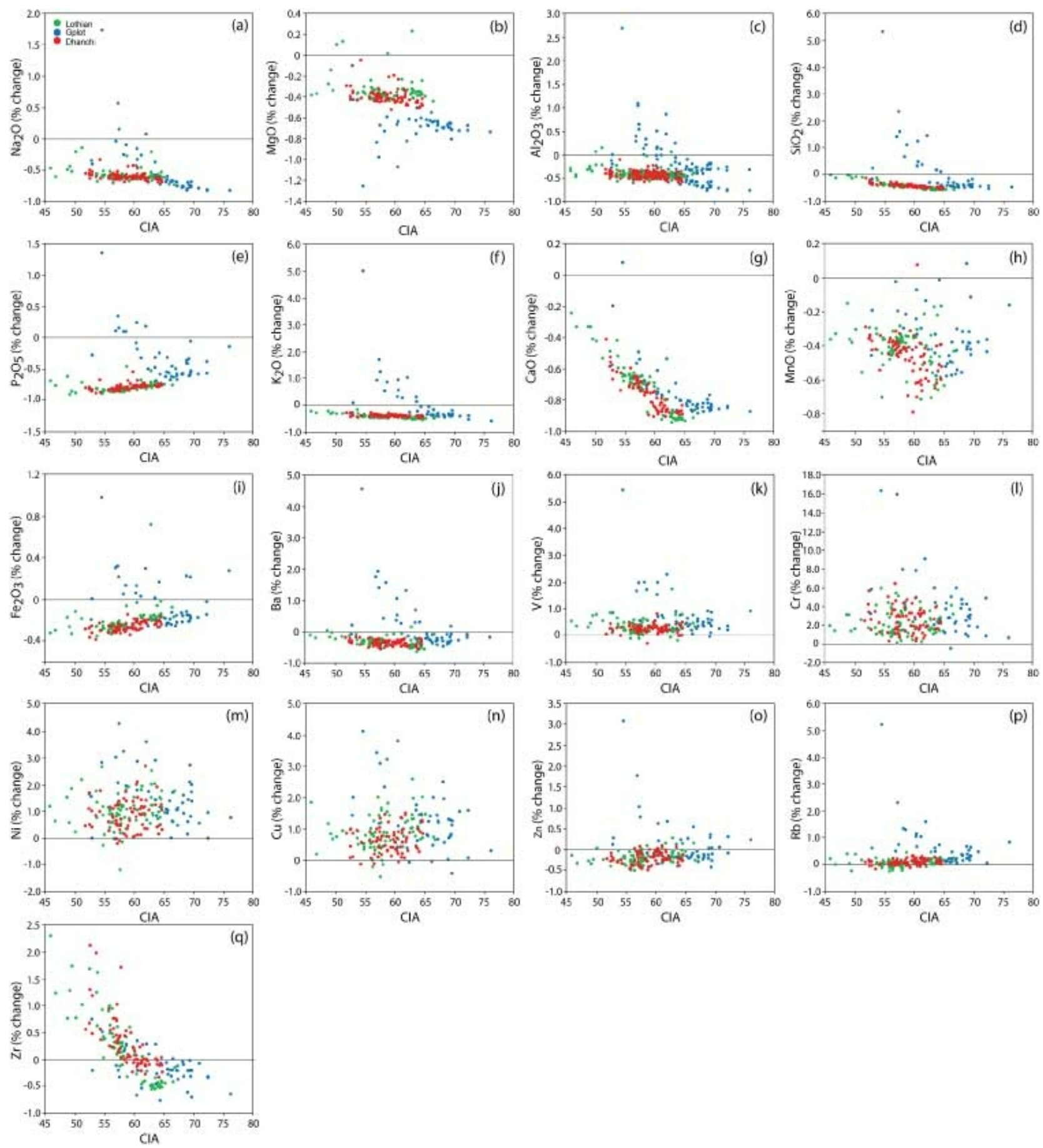
Active delta

$\nabla$ Semi active delta

Abandoned delta
Sediment plume

Fluvial sediment source

† Re-worked fluvial sediment source
(F) Fluvial dominant delta

(M) Mixed fluvio-tidal delta

(T) Tidal dominant delta
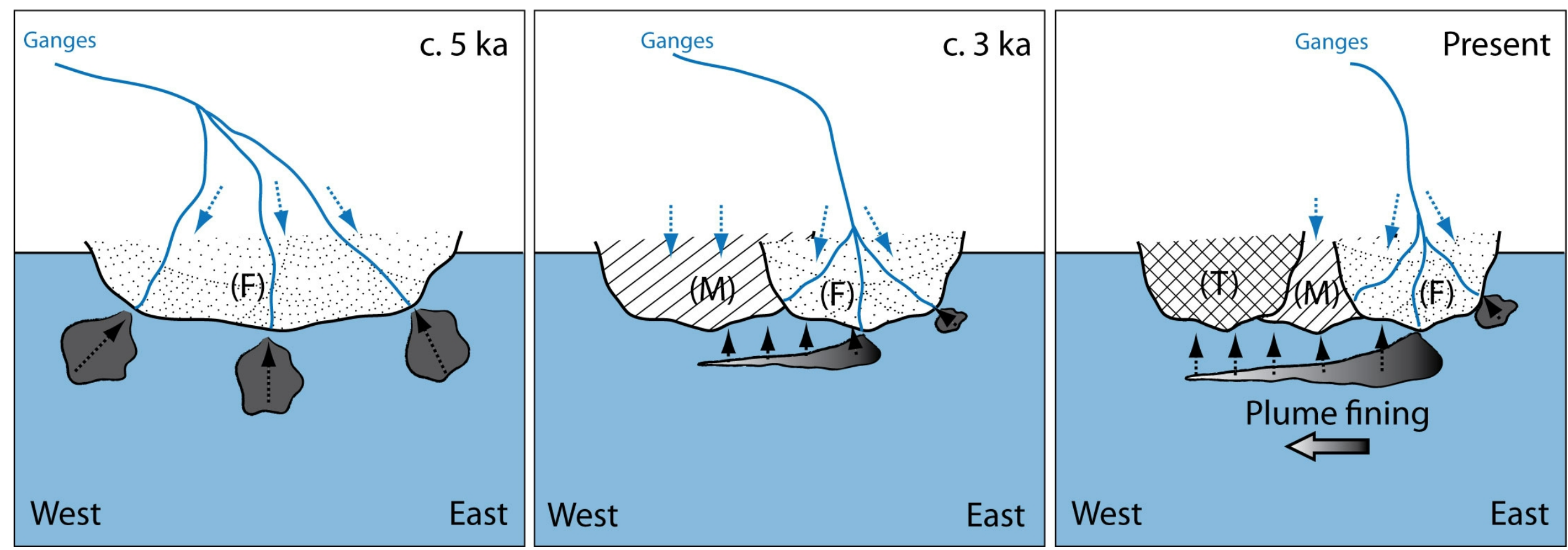
(a)

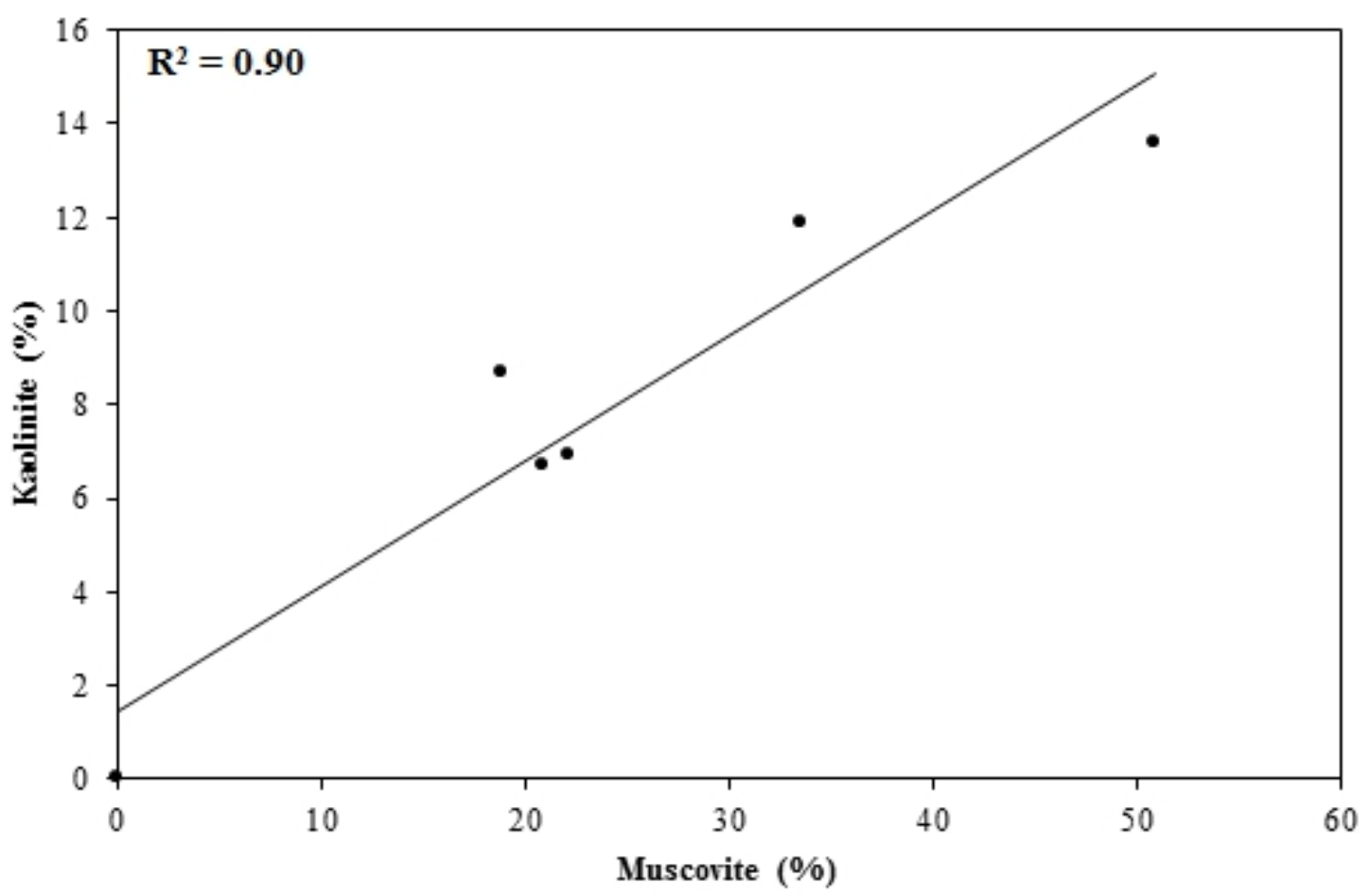

(b)

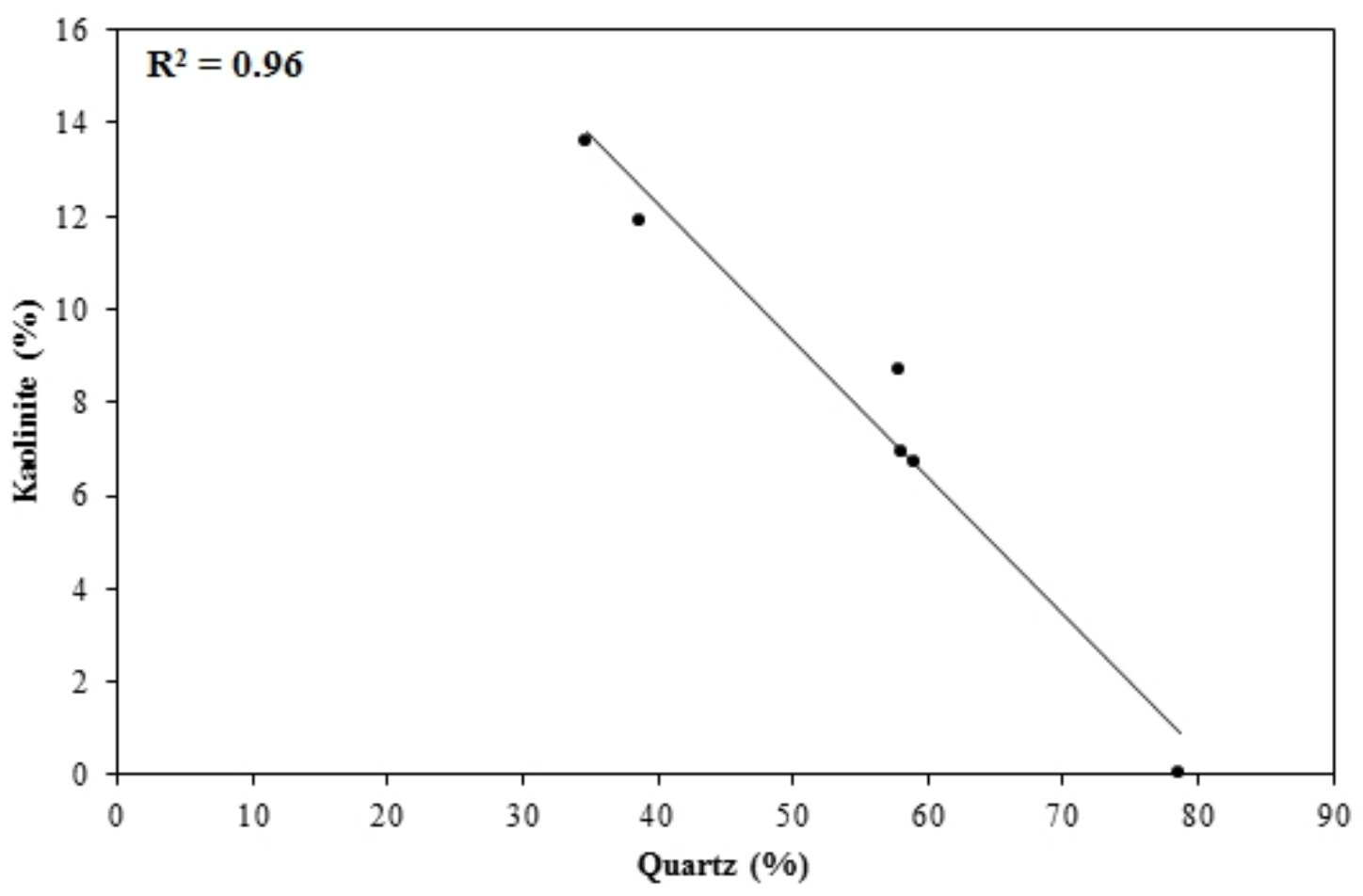




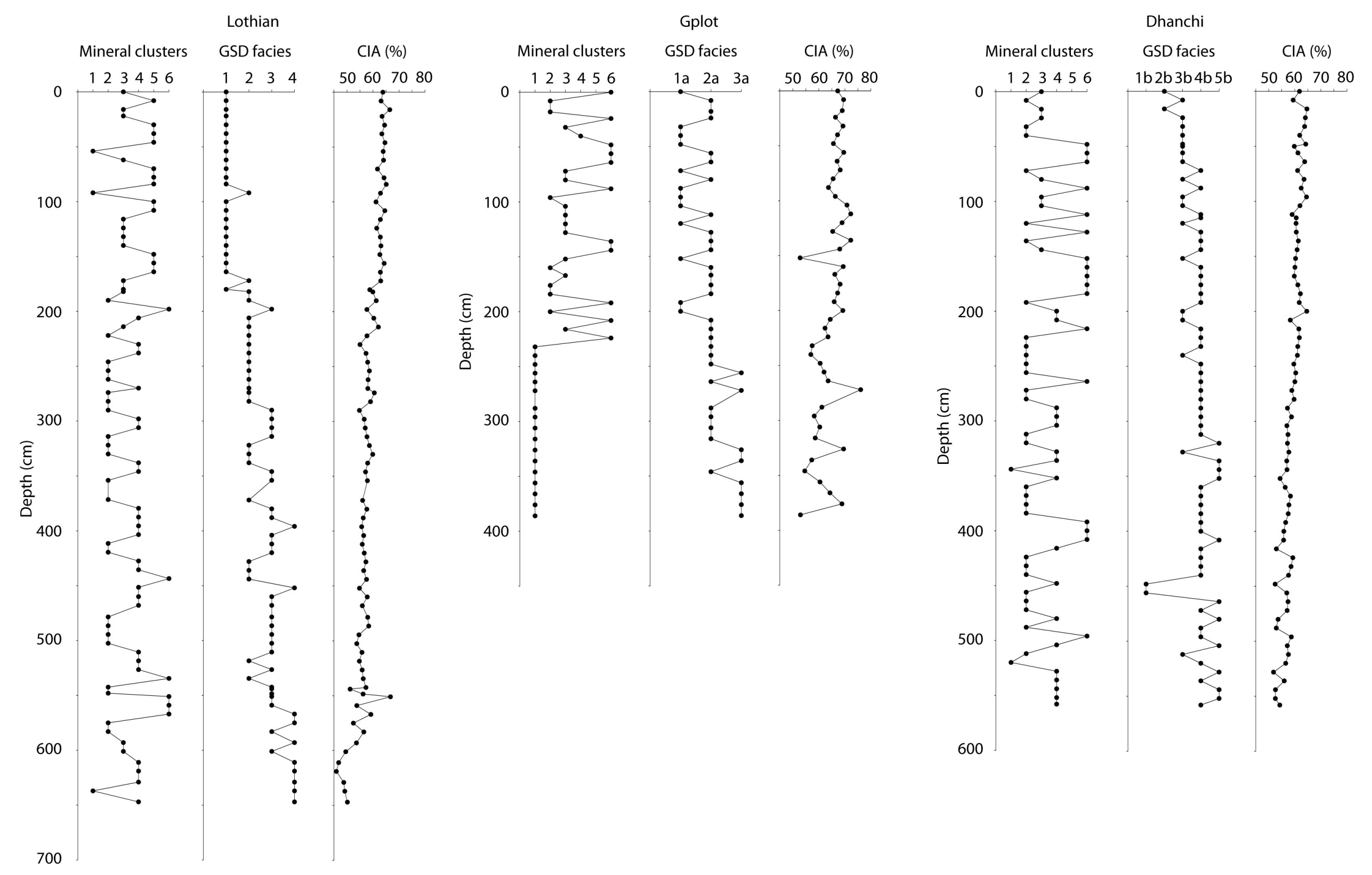




\begin{tabular}{llccrr}
\hline $\begin{array}{l}\text { Principal } \\
\text { component }\end{array}$ & Total & $\begin{array}{c}\text { Initial Eigenvalues } \\
\text { \% of Variance }\end{array}$ & Cumulative \% & To1 \\
\hline \hline & 1 & 17.859 & 71.436 & 71.436 & 17.859 \\
& 2 & 3.633 & 14.531 & 85.966 & 3.633 \\
& 3 & 1.840 & 7.360 & 93.327 & 1.840 \\
& 4 & 0.523 & 2.090 & 95.417 & 0.523 \\
\hline
\end{tabular}


Extraction Sums of Squared Loadings

\begin{tabular}{|c|c|c|}
\hline tal & $\%$ of Variance & Cumulative \% \\
\hline & 71.436 & 71.436 \\
\hline & 14.531 & 85.966 \\
\hline & 7.360 & 93.327 \\
\hline & 2.090 & 95.417 \\
\hline
\end{tabular}




\begin{tabular}{lcccrr}
\hline $\begin{array}{l}\text { Principal } \\
\text { component }\end{array}$ & Total & $\begin{array}{c}\text { Initial Eigenvalues } \\
\text { \% of Variance }\end{array}$ & Cumulative \% & To1 \\
\hline \hline 1 & 10.294 & 85.787 & 85.787 & 10.294 \\
& 2 & 1.008 & 8.399 & 94.186 & 1.008 \\
& 3 & 0.622 & 5.181 & 99.367 & 0.622 \\
& 4 & 0.048 & 0.4 & 99.767 & 0.048 \\
\hline
\end{tabular}


Extraction Sums of Squared Loadings

\begin{tabular}{lll} 
tal & $\%$ of Variance & Cumulative \% \\
\hline \hline
\end{tabular}

$\begin{array}{ll}85.787 & 85.787\end{array}$

$8.399 \quad 94.186$

$5.181 \quad 99.367$

0.4

99.767 


\begin{tabular}{lrc}
\hline Site & Depth (cm) & Cluster group \\
\hline \hline Gplot Island & 356 & 1 \\
Dhanchi Island & 456 & 2 \\
Lothian Island & 22 & 3 \\
Dhanchi Island & 304 & 4 \\
Lothian Island & 78 & 5 \\
Dhanchi Island & 184 & 6 \\
\hline
\end{tabular}




\begin{tabular}{lrrrrr}
\hline Statistic (\%) & Cluster 1 & Cluster 2 & Cluster 3 & Cluster 4 & \multicolumn{2}{c}{ Cluster 5 } \\
\hline \hline $\mathrm{R}_{\mathrm{p}}$ & 8.728 & 5.551 & 7.126 & 15.295 & 9.134 \\
Weighted $\mathrm{R}_{\mathrm{p}}$ & 12.765 & 7.631 & 9.901 & 24.955 & 13.055 \\
$\mathrm{R}_{\exp }$ & 4.271 & 4.038 & 4.251 & 4.569 & 4.418 \\
$\mathrm{X}^{2}$ & 8.932 & 3.571 & 5.425 & 29.831 & 8.731 \\
D-statistic & 0.491 & 0.438 & 0.594 & 0.229 & 0.432 \\
Weighted D-statistic & 0.318 & 0.421 & 0.375 & 0.176 & 0.338 \\
\hline
\end{tabular}




$\begin{array}{r}\hline \multicolumn{1}{c}{\text { Cluster 6 }} \\ \hline \hline 6.168 \\ 9.253 \\ 4.043 \\ 5.237 \\ 0.355 \\ 0.342 \\ \hline\end{array}$




\begin{tabular}{lrrrrrrr}
\hline & Quartz & \multicolumn{1}{c}{ Muscovite } & Albite & \multicolumn{1}{c}{ Microcline } & Clinochlore & Kaolinite & Dickite \\
\hline \hline Cluster 1 & 58 & 18.8 & 10.4 & 0 & 4.2 & 8.7 & 0 \\
Cluster 2 & 34.8 & 50.9 & 0 & 0 & 0 & 13.6 & 0 \\
Cluster 3 & 58.2 & 22.1 & 10.2 & 0 & 2.7 & 6.9 & 0 \\
Cluster 4 & 78.6 & 0 & 7.7 & 8.2 & 0.9 & 0 & 4.6 \\
Cluster 5 & 59 & 20.9 & 13 & 0 & 0 & 6.7 & 0 \\
Cluster 6 & 38.8 & 33.6 & 10.1 & 0 & 5.5 & 11.9 & 0 \\
\hline
\end{tabular}


Vermiculite

0.7

0

0

0.4

0 


\section{Cluster Mineralogical characteristics}

Cluster 1 Relatively high quartz, muscovite, albite and clinochlore; moderate kaolinite abundance; absence of vermiculite, dickite, microcline.

Cluster 2

Relatively high muscovite and kaolinite; low quartz and possibly low vermiculite, absent of clinochlore, albite, dickite, microcline.

Cluster 3 Relatively high quartz, muscovite, albite; relatively high clinochlore, kaolinite and absent of vermiculite, dickite, microcline.

Cluster 4 High quartz content; relatively high microcline, and dickite; low albite and clinochlore; absence of muscovite and kaolinite.

Cluster 5 Relatively high quartz, muscovite, and albite content; low kaolinite content; absence of clinochlore, vermiculite, microcline, dickite.

Cluster 6 Relatively high muscovite, kaolinite, clinochlore and albite content; relatively low quartz content; complete absence of vermiculite, dickite, microcline. 


\begin{tabular}{llr}
\hline Lab Code (XRD) & Sample Code & Depth (in cm) \\
\hline \hline RF322 & LTCS1 - 117 & 78 \\
RF329 & LTCS1 - 24 & 22 \\
RF336 & GPCS3-342 & 356 \\
RF408 & DHCS6 - 456 & 456 \\
RF427 & DHCS4 - 304 & 304 \\
RF442 & DHCS3 - 184 & 184 \\
\hline
\end{tabular}


No. Pos. [ ${ }^{\circ} 2$ Th. lobs [cts] Icalc [cts] lback [cts] CT [s] ESD D spacings

\begin{tabular}{|c|c|c|c|c|c|c|}
\hline 1 & 3.0003 & 2313.603 & 2313.603 & 2313.603 & 122.5994 & 29.42347 \\
\hline 2 & 3.0173 & 2283.218 & 2285.62 & 2285.62 & 122.5994 & 29.25773 \\
\hline 3 & 3.0343 & 2213.511 & 2257.636 & 2257.636 & 122.5994 & 29.09385 \\
\hline 4 & 3.0513 & 2301.458 & 2229.653 & 2229.653 & 122.5994 & 28.9318 \\
\hline 5 & 3.0683 & 2125.983 & 2201.669 & 2201.669 & 122.5994 & 28.77154 \\
\hline 6 & 3.0853 & 2124.616 & 2173.686 & 2173.686 & 122.5994 & 28.61305 \\
\hline 7 & 3.1023 & 2030.367 & 2145.702 & 2145.702 & 122.5994 & 28.45629 \\
\hline 8 & 3.1193 & 1948.789 & 2117.719 & 2117.719 & 122.5994 & 28.30124 \\
\hline 9 & 3.1363 & 1972.899 & 2089.736 & 2089.735 & 122.5994 & 28.14788 \\
\hline 10 & 3.1533 & 1966.595 & 2061.752 & 2061.752 & 122.5994 & 27.99617 \\
\hline 11 & 3.1703 & 1894.765 & 2033.769 & 2033.769 & 122.5994 & 27.84608 \\
\hline 12 & 3.1873 & 1842.451 & 2005.785 & 2005.785 & 122.5994 & 27.6976 \\
\hline 13 & 3.2043 & 1846.79 & 1977.802 & 1977.802 & 122.5994 & 27.55069 \\
\hline 14 & 3.2213 & 1835.629 & 1949.818 & 1949.818 & 122.5994 & 27.40534 \\
\hline 15 & 3.2383 & 1813.03 & 1921.835 & 1921.835 & 122.5994 & 27.26151 \\
\hline 16 & 3.2553 & 1783.85 & 1893.851 & 1893.851 & 122.5994 & 27.11918 \\
\hline 17 & 3.2723 & 1743.775 & 1865.868 & 1865.868 & 122.5994 & 26.97833 \\
\hline 18 & 3.2893 & 1735.116 & 1837.884 & 1837.884 & 122.5994 & 26.83894 \\
\hline 19 & 3.3063 & 1778.939 & 1809.901 & 1809.901 & 122.5994 & 26.70098 \\
\hline 20 & 3.3233 & 1708.75 & 1781.917 & 1781.917 & 122.5994 & 26.56443 \\
\hline 21 & 3.3403 & 1668.125 & 1753.934 & 1753.934 & 122.5994 & 26.42928 \\
\hline 22 & 3.3573 & 1669.826 & 1725.95 & 1725.95 & 122.5994 & 26.29549 \\
\hline 23 & 3.3743 & 1667.071 & 1697.967 & 1697.967 & 122.5994 & 26.16305 \\
\hline 24 & 3.3913 & 1644.165 & 1669.984 & 1669.983 & 122.5994 & 26.03194 \\
\hline 25 & 3.4083 & 1643.684 & 1642 & 1642 & 122.5994 & 25.90213 \\
\hline 26 & 3.4253 & 1619.767 & 1632.5 & 1632.5 & 122.5994 & 25.77362 \\
\hline 27 & 3.4423 & 1652.388 & 1623 & 1623 & 122.5994 & 25.64637 \\
\hline 28 & 3.4593 & 1601.68 & 1613.5 & 1613.5 & 122.5994 & 25.52038 \\
\hline 29 & 3.4763 & 1558.757 & 1604 & 1604 & 122.5994 & 25.39561 \\
\hline 30 & 3.4933 & 1613.149 & 1594.5 & 1594.5 & 122.5994 & 25.27207 \\
\hline 31 & 3.5103 & 1578.422 & 1585 & 1585 & 122.5994 & 25.14971 \\
\hline 32 & 3.5273 & 1608.369 & 1575.5 & 1575.5 & 122.5994 & 25.02854 \\
\hline 33 & 3.5443 & 1590.621 & 1566 & 1566 & 122.5994 & 24.90853 \\
\hline 34 & 3.5613 & 1590.752 & 1556.5 & 1556.5 & 122.5994 & 24.78967 \\
\hline 35 & 3.5783 & 1553.656 & 1547 & 1547 & 122.5994 & 24.67194 \\
\hline 36 & 3.5953 & 1530.75 & 1537.5 & 1537.5 & 122.5994 & 24.55532 \\
\hline 37 & 3.6123 & 1486.795 & 1528 & 1528 & 122.5994 & 24.4398 \\
\hline 38 & 3.6293 & 1536.482 & 1518.5 & 1518.5 & 122.5994 & 24.32536 \\
\hline 39 & 3.6463 & 1501.347 & 1509 & 1509 & 122.5994 & 24.21198 \\
\hline 40 & 3.6633 & 1477.044 & 1499.5 & 1499.5 & 122.5994 & 24.09966 \\
\hline 41 & 3.6803 & 1454.793 & 1490 & 1490 & 122.5994 & 23.98838 \\
\hline 42 & 3.6973 & 1471.862 & 1480.5 & 1480.5 & 122.5994 & 23.87812 \\
\hline 43 & 3.7143 & 1440.091 & 1471 & 1471 & 122.5994 & 23.76887 \\
\hline 44 & 3.7313 & 1471.692 & 1461.5 & 1461.5 & 122.5994 & 23.66062 \\
\hline 45 & 3.7483 & 1445.635 & 1452 & 1452 & 122.5994 & 23.55335 \\
\hline 46 & 3.7653 & 1400.203 & 1442.5 & 1442.5 & 122.5994 & 23.44704 \\
\hline 47 & 3.7823 & 1473.79 & 1433 & 1433 & 122.5994 & 23.3417 \\
\hline
\end{tabular}




\begin{tabular}{|c|c|c|c|c|c|c|}
\hline 48 & 3.7993 & 1450.853 & 1423.5 & 1423.5 & 122.5994 & 23.23729 \\
\hline 49 & 3.8163 & 1384.827 & 1414 & 1414 & 122.5994 & 23.13382 \\
\hline 50 & 3.8333 & 1411.316 & 1407.458 & 1407.458 & 122.5994 & 23.03126 \\
\hline 51 & 3.8503 & 1370.131 & 1400.917 & 1400.917 & 122.5994 & 22.92961 \\
\hline 52 & 3.8673 & 1384.571 & 1394.375 & 1394.375 & 122.5994 & 22.82886 \\
\hline 53 & 3.8843 & 1392.164 & 1387.833 & 1387.833 & 122.5994 & 22.72898 \\
\hline 54 & 3.9013 & 1395.629 & 1381.292 & 1381.292 & 122.5994 & 22.62998 \\
\hline 55 & 3.9183 & 1378.825 & 1374.75 & 1374.75 & 122.5994 & 22.53184 \\
\hline 56 & 3.9353 & 1387.188 & 1368.208 & 1368.208 & 122.5994 & 22.43454 \\
\hline 57 & 3.9523 & 1367.492 & 1361.667 & 1361.667 & 122.5994 & 22.33808 \\
\hline 58 & 3.9693 & 1341.889 & 1355.125 & 1355.125 & 122.5994 & 22.24245 \\
\hline 59 & 3.9863 & 1294.27 & 1348.583 & 1348.583 & 122.5994 & 22.14763 \\
\hline 60 & 4.0033 & 1325.085 & 1342.042 & 1342.042 & 122.5994 & 22.05362 \\
\hline 61 & 4.0203 & 1256.421 & 1335.5 & 1335.5 & 122.5994 & 21.9604 \\
\hline 62 & 4.0373 & 1288.405 & 1328.958 & 1328.958 & 122.5994 & 21.86797 \\
\hline 63 & 4.0543 & 1322.703 & 1322.417 & 1322.417 & 122.5994 & 21.77632 \\
\hline 64 & 4.0713 & 1325.598 & 1315.875 & 1315.875 & 122.5994 & 21.68543 \\
\hline 65 & 4.0883 & 1273.717 & 1309.333 & 1309.333 & 122.5994 & 21.59529 \\
\hline 66 & 4.1053 & 1307.529 & 1302.792 & 1302.792 & 122.5994 & 21.50591 \\
\hline 67 & 4.1223 & 1315.848 & 1296.25 & 1296.25 & 122.5994 & 21.41726 \\
\hline 68 & 4.1393 & 1296.838 & 1289.708 & 1289.708 & 122.5994 & 21.32933 \\
\hline 69 & 4.1563 & 1249.563 & 1283.167 & 1283.167 & 122.5994 & 21.24213 \\
\hline 70 & 4.1733 & 1234.775 & 1276.625 & 1276.625 & 122.5994 & 21.15564 \\
\hline 71 & 4.1903 & 1242.369 & 1270.083 & 1270.083 & 122.5994 & 21.06985 \\
\hline 72 & 4.2073 & 1266.768 & 1263.542 & 1263.542 & 122.5994 & 20.98475 \\
\hline 73 & 4.2243 & 1263.542 & 1257 & 1257 & 122.5994 & 20.90034 \\
\hline 74 & 4.2413 & 1261.403 & 1253.063 & 1253.063 & 122.5994 & 20.81661 \\
\hline 75 & 4.2583 & 1230.43 & 1249.125 & 1249.125 & 122.5994 & 20.73354 \\
\hline 76 & 4.2753 & 1259.599 & 1245.188 & 1245.188 & 122.5994 & 20.65114 \\
\hline 77 & 4.2923 & 1262.434 & 1241.25 & 1241.25 & 122.5994 & 20.56939 \\
\hline 78 & 4.3093 & 1220.639 & 1237.313 & 1237.313 & 122.5994 & 20.48828 \\
\hline 79 & 4.3263 & 1220.531 & 1233.375 & 1233.375 & 122.5994 & 20.40781 \\
\hline 80 & 4.3433 & 1215.219 & 1229.438 & 1229.438 & 122.5994 & 20.32797 \\
\hline 81 & 4.3603 & 1181.167 & 1225.5 & 1225.5 & 122.5994 & 20.24875 \\
\hline 82 & 4.3773 & 1154.486 & 1221.563 & 1221.563 & 122.5994 & 20.17015 \\
\hline 83 & 4.3943 & 1154.388 & 1217.625 & 1217.625 & 122.5994 & 20.09216 \\
\hline 84 & 4.4113 & 1193.779 & 1213.688 & 1213.688 & 122.5994 & 20.01477 \\
\hline 85 & 4.4283 & 1181.829 & 1209.75 & 1209.75 & 122.5994 & 19.93797 \\
\hline 86 & 4.4453 & 1210.893 & 1205.813 & 1205.813 & 122.5994 & 19.86176 \\
\hline 87 & 4.4623 & 1142.303 & 1201.875 & 1201.875 & 122.5994 & 19.78613 \\
\hline 88 & 4.4793 & 1212.688 & 1197.938 & 1197.938 & 122.5994 & 19.71108 \\
\hline 89 & 4.4963 & 1218.743 & 1194 & 1194 & 122.5994 & 19.63659 \\
\hline 90 & 4.5133 & 1198.956 & 1190.063 & 1190.063 & 122.5994 & 19.56267 \\
\hline 91 & 4.5303 & 1158.603 & 1186.125 & 1186.125 & 122.5994 & 19.48929 \\
\hline 92 & 4.5473 & 1175.617 & 1182.188 & 1182.188 & 122.5994 & 19.41647 \\
\hline 93 & 4.5643 & 1123.383 & 1178.25 & 1178.25 & 122.5994 & 19.34419 \\
\hline 94 & 4.5813 & 1169.691 & 1174.313 & 1174.313 & 122.5994 & 19.27245 \\
\hline 95 & 4.5983 & 1229.862 & 1170.375 & 1170.375 & 122.5994 & 19.20124 \\
\hline 96 & 4.6153 & 1138.691 & 1166.438 & 1166.438 & 122.5994 & 19.13055 \\
\hline
\end{tabular}




\begin{tabular}{|c|c|c|c|c|c|c|}
\hline 97 & 4.6323 & 1201.372 & 1162.5 & 1162.5 & 122.5994 & 19.06038 \\
\hline 98 & 4.6493 & 1162.239 & 1158.563 & 1158.563 & 122.5994 & 18.99073 \\
\hline 99 & 4.6663 & 1144.452 & 1154.625 & 1154.625 & 122.5994 & 18.92158 \\
\hline 100 & 4.6833 & 1059.18 & 1150.688 & 1150.688 & 122.5994 & 18.85293 \\
\hline 101 & 4.7003 & 1128.421 & 1146.75 & 1146.75 & 122.5994 & 18.78479 \\
\hline 102 & 4.7173 & 1154.617 & 1142.813 & 1142.813 & 122.5994 & 18.71713 \\
\hline 103 & 4.7343 & 1119.467 & 1138.875 & 1138.875 & 122.5994 & 18.64996 \\
\hline 104 & 4.7513 & 1128.48 & 1134.938 & 1134.938 & 122.5994 & 18.58327 \\
\hline 105 & 4.7683 & 1099.082 & 1131 & 1131 & 122.5994 & 18.51705 \\
\hline 106 & 4.7853 & 1077.171 & 1127.063 & 1127.063 & 122.5994 & 18.45131 \\
\hline 107 & 4.8023 & 1056.112 & 1123.125 & 1123.125 & 122.5994 & 18.38603 \\
\hline 108 & 4.8193 & 1107.187 & 1119.188 & 1119.188 & 122.5994 & 18.32121 \\
\hline 109 & 4.8363 & 1125.476 & 1115.25 & 1115.25 & 122.5994 & 18.25685 \\
\hline 110 & 4.8533 & 1087.241 & 1111.313 & 1111.313 & 122.5994 & 18.19294 \\
\hline 111 & 4.8703 & 1096.757 & 1107.375 & 1107.375 & 122.5994 & 18.12947 \\
\hline 112 & 4.8873 & 1083.537 & 1103.438 & 1103.438 & 122.5994 & 18.06645 \\
\hline 113 & 4.9043 & 1101.042 & 1099.5 & 1099.5 & 122.5994 & 18.00386 \\
\hline 114 & 4.9213 & 1103.151 & 1095.563 & 1095.563 & 122.5994 & 17.94171 \\
\hline 115 & 4.9383 & 1077.806 & 1091.625 & 1091.625 & 122.5994 & 17.87998 \\
\hline 116 & 4.9553 & 1116.537 & 1087.688 & 1087.688 & 122.5994 & 17.81868 \\
\hline 117 & 4.9723 & 1136.557 & 1083.75 & 1083.75 & 122.5994 & 17.7578 \\
\hline 118 & 4.9893 & 1113.178 & 1079.813 & 1079.813 & 122.5994 & 17.69733 \\
\hline 119 & 5.0063 & 1139.316 & 1075.875 & 1075.875 & 122.5994 & 17.63727 \\
\hline 120 & 5.0233 & 1083.248 & 1071.938 & 1071.938 & 122.5994 & 17.57762 \\
\hline 121 & 5.0403 & 1044.378 & 1068 & 1068 & 122.5994 & 17.51837 \\
\hline 122 & 5.0573 & 1061.535 & 1066.875 & 1066.875 & 122.5994 & 17.45953 \\
\hline 123 & 5.0743 & 1040.974 & 1065.75 & 1065.75 & 122.5994 & 17.40107 \\
\hline 124 & 5.0913 & 1053.3 & 1064.625 & 1064.625 & 122.5994 & 17.34301 \\
\hline 125 & 5.1083 & 1022.031 & 1063.5 & 1063.5 & 122.5994 & 17.28533 \\
\hline 126 & 5.1253 & 1056.366 & 1062.375 & 1062.375 & 122.5994 & 17.22803 \\
\hline 127 & 5.1423 & 1077.759 & 1061.25 & 1061.25 & 122.5994 & 17.17112 \\
\hline 128 & 5.1593 & 1095.169 & 1061.272 & 1060.125 & 122.5994 & 17.11458 \\
\hline 129 & 5.1763 & 1077.413 & 1060.755 & 1059 & 122.5994 & 17.05841 \\
\hline 130 & 5.1933 & 1004.648 & 1059.686 & 1057.875 & 122.5994 & 17.00261 \\
\hline 131 & 5.2103 & 1001.039 & 1058.619 & 1056.75 & 122.5994 & 16.94717 \\
\hline 132 & 5.2273 & 1041.641 & 1057.556 & 1055.625 & 122.5994 & 16.89209 \\
\hline 133 & 5.2443 & 1088.27 & 1056.495 & 1054.5 & 122.5994 & 16.83737 \\
\hline 134 & 5.2613 & 1028.39 & 1055.438 & 1053.375 & 122.5994 & 16.78301 \\
\hline 135 & 5.2783 & 1034.613 & 1054.384 & 1052.25 & 122.5994 & 16.72899 \\
\hline 136 & 5.2953 & 1048.518 & 1053.334 & 1051.125 & 122.5994 & 16.67532 \\
\hline 137 & 5.3123 & 1067.838 & 1052.288 & 1050 & 122.5994 & 16.622 \\
\hline 138 & 5.3293 & 1056.951 & 1051.246 & 1048.875 & 122.5994 & 16.56901 \\
\hline 139 & 5.3463 & 1031.194 & 1050.209 & 1047.75 & 122.5994 & 16.51637 \\
\hline 140 & 5.3633 & 1024.049 & 1049.177 & 1046.625 & 122.5994 & 16.46405 \\
\hline 141 & 5.3803 & 999.0201 & 1048.15 & 1045.5 & 122.5994 & 16.41207 \\
\hline 142 & 5.3973 & 1037.705 & 1047.128 & 1044.375 & 122.5994 & 16.36041 \\
\hline 143 & 5.4143 & 1062.42 & 1046.113 & 1043.25 & 122.5994 & 16.30908 \\
\hline 144 & 5.4313 & 1053.762 & 1045.105 & 1042.125 & 122.5994 & 16.25807 \\
\hline 145 & 5.4483 & 1025.252 & 1044.104 & 1041 & 122.5994 & 16.20738 \\
\hline
\end{tabular}




\begin{tabular}{|c|c|c|c|c|c|c|}
\hline 146 & 5.4653 & 1036.859 & 1043.495 & 1040.26 & 122.5994 & 16.15701 \\
\hline 147 & 5.4823 & 1030.526 & 1042.896 & 1039.521 & 122.5994 & 16.10695 \\
\hline 148 & 5.4993 & 1001.607 & 1042.306 & 1038.781 & 122.5994 & 16.05719 \\
\hline 149 & 5.5163 & 1047.397 & 1041.725 & 1038.042 & 122.5994 & 16.00775 \\
\hline 150 & 5.5333 & 1025.061 & 1041.156 & 1037.302 & 122.5994 & 15.9586 \\
\hline 151 & 5.5503 & 986.8845 & 1040.598 & 1036.563 & 122.5994 & 15.90976 \\
\hline 152 & 5.5673 & 1011.23 & 1040.053 & 1035.823 & 122.5994 & 15.86122 \\
\hline 153 & 5.5843 & 1012.953 & 1039.523 & 1035.083 & 122.5994 & 15.81297 \\
\hline 154 & 5.6013 & 995.6937 & 1039.009 & 1034.344 & 122.5994 & 15.76502 \\
\hline 155 & 5.6183 & 960.0591 & 1038.511 & 1033.604 & 122.5994 & 15.71735 \\
\hline 156 & 5.6353 & 998.5501 & 1038.033 & 1032.865 & 122.5994 & 15.66998 \\
\hline 157 & 5.6523 & 1007.649 & 1037.576 & 1032.125 & 122.5994 & 15.62289 \\
\hline 158 & 5.6693 & 1009.609 & 1037.142 & 1031.385 & 122.5994 & 15.57608 \\
\hline 159 & 5.6863 & 983.4449 & 1036.735 & 1030.646 & 122.5994 & 15.52955 \\
\hline 160 & 5.7033 & 1006.49 & 1036.356 & 1029.906 & 122.5994 & 15.4833 \\
\hline 161 & 5.7203 & 1040.425 & 1036.011 & 1029.167 & 122.5994 & 15.43732 \\
\hline 162 & 5.7373 & 1016.135 & 1035.703 & 1028.427 & 122.5994 & 15.39162 \\
\hline 163 & 5.7543 & 1007.688 & 1035.437 & 1027.688 & 122.5994 & 15.34618 \\
\hline 164 & 5.7713 & 1017.519 & 1035.221 & 1026.948 & 122.5994 & 15.30102 \\
\hline 165 & 5.7883 & 1001.191 & 1035.063 & 1026.208 & 122.5994 & 15.25612 \\
\hline 166 & 5.8053 & 1009.737 & 1034.975 & 1025.469 & 122.5994 & 15.21148 \\
\hline 167 & 5.8223 & 1016.695 & 1034.971 & 1024.729 & 122.5994 & 15.16711 \\
\hline 168 & 5.8393 & 992.5143 & 1035.072 & 1023.99 & 122.5994 & 15.12299 \\
\hline 169 & 5.8563 & 1042.855 & 1035.305 & 1023.25 & 122.5994 & 15.07913 \\
\hline 170 & 5.8733 & 1069.897 & 1035.511 & 1022.313 & 122.5994 & 15.03552 \\
\hline 171 & 5.8903 & 1049.943 & 1035.937 & 1021.375 & 122.5994 & 14.99216 \\
\hline 172 & 5.9073 & 1060.971 & 1036.647 & 1020.438 & 122.5994 & 14.94906 \\
\hline 173 & 5.9243 & 1015.29 & 1037.728 & 1019.5 & 122.5994 & 14.9062 \\
\hline 174 & 5.9413 & 1015.203 & 1039.283 & 1018.563 & 122.5994 & 14.86358 \\
\hline 175 & 5.9583 & 1043.151 & 1041.444 & 1017.625 & 122.5994 & 14.82121 \\
\hline 176 & 5.9753 & 1044.798 & 1044.361 & 1016.688 & 122.5994 & 14.77909 \\
\hline 177 & 5.9923 & 1003.147 & 1048.208 & 1015.75 & 122.5994 & 14.7372 \\
\hline 178 & 6.0093 & 997.341 & 1053.168 & 1014.813 & 122.5994 & 14.69554 \\
\hline 179 & 6.0263 & 1019.662 & 1059.432 & 1013.875 & 122.5994 & 14.65413 \\
\hline 180 & 6.0433 & 1057.02 & 1067.176 & 1012.938 & 122.5994 & 14.61294 \\
\hline 181 & 6.0603 & 1079.903 & 1076.549 & 1012 & 122.5994 & 14.57199 \\
\hline 182 & 6.0773 & 1094.206 & 1087.646 & 1011.063 & 122.5994 & 14.53126 \\
\hline 183 & 6.0943 & 1089.576 & 1100.49 & 1010.125 & 122.5994 & 14.49077 \\
\hline 184 & 6.1113 & 1088.989 & 1115.002 & 1009.188 & 122.5994 & 14.4505 \\
\hline 185 & 6.1283 & 1133.764 & 1130.974 & 1008.25 & 122.5994 & 14.41045 \\
\hline 186 & 6.1453 & 1064.202 & 1148.036 & 1007.313 & 122.5994 & 14.37062 \\
\hline 187 & 6.1623 & 1102.269 & 1165.615 & 1006.375 & 122.5994 & 14.33102 \\
\hline 188 & 6.1793 & 1122.588 & 1182.897 & 1005.438 & 122.5994 & 14.29163 \\
\hline 189 & 6.1963 & 1136.594 & 1198.803 & 1004.5 & 122.5994 & 14.25246 \\
\hline 190 & 6.2133 & 1161.985 & 1212.034 & 1003.563 & 122.5994 & 14.2135 \\
\hline 191 & 6.2303 & 1243.025 & 1221.217 & 1002.625 & 122.5994 & 14.17475 \\
\hline 192 & 6.2473 & 1209.507 & 1225.193 & 1001.688 & 122.5994 & 14.13622 \\
\hline 193 & 6.2643 & 1202.639 & 1223.339 & 1000.75 & 122.5994 & 14.0979 \\
\hline 194 & 6.2813 & 1237.99 & 1215.777 & 999.8125 & 122.5994 & 14.05978 \\
\hline
\end{tabular}




\begin{tabular}{|c|c|c|c|c|c|c|}
\hline 195 & 6.2983 & 1229.56 & 1203.329 & 998.875 & 122.5994 & 14.02187 \\
\hline 196 & 6.3153 & 1236.12 & 1187.254 & 997.9375 & 122.5994 & 13.98416 \\
\hline 197 & 6.3323 & 1301.07 & 1168.924 & 997 & 122.5994 & 13.94666 \\
\hline 198 & 6.3493 & 1275.905 & 1149.578 & 996.0625 & 122.5994 & 13.90935 \\
\hline 199 & 6.3663 & 1264.925 & 1130.213 & 995.125 & 122.5994 & 13.87225 \\
\hline 200 & 6.3833 & 1131.559 & 1111.564 & 994.1875 & 122.5994 & 13.83534 \\
\hline 201 & 6.4003 & 1061.395 & 1094.139 & 993.25 & 122.5994 & 13.79863 \\
\hline 202 & 6.4173 & 1058.155 & 1078.252 & 992.3125 & 122.5994 & 13.76212 \\
\hline 203 & 6.4343 & 1077.648 & 1064.069 & 991.375 & 122.5994 & 13.72579 \\
\hline 204 & 6.4513 & 1049.101 & 1051.635 & 990.4375 & 122.5994 & 13.68966 \\
\hline 205 & 6.4683 & 1034.356 & 1040.903 & 989.5 & 122.5994 & 13.65372 \\
\hline 206 & 6.4853 & 1038.349 & 1031.758 & 988.5625 & 122.5994 & 13.61797 \\
\hline 207 & 6.5023 & 1035.85 & 1024.04 & 987.625 & 122.5994 & 13.5824 \\
\hline 208 & 6.5193 & 1029.285 & 1017.567 & 986.6875 & 122.5994 & 13.54702 \\
\hline 209 & 6.5363 & 1034.109 & 1012.151 & 985.75 & 122.5994 & 13.51183 \\
\hline 210 & 6.5533 & 1053.013 & 1007.608 & 984.8125 & 122.5994 & 13.47682 \\
\hline 211 & 6.5703 & 1072.243 & 1003.773 & 983.875 & 122.5994 & 13.44198 \\
\hline 212 & 6.5873 & 1031.504 & 1000.501 & 982.9375 & 122.5994 & 13.40733 \\
\hline 213 & 6.6043 & 1030.072 & 997.6693 & 982 & 122.5994 & 13.37286 \\
\hline 214 & 6.6213 & 1072.655 & 995.179 & 981.0625 & 122.5994 & 13.33856 \\
\hline 215 & 6.6383 & 1011.616 & 992.9521 & 980.125 & 122.5994 & 13.30444 \\
\hline 216 & 6.6553 & 976.6433 & 990.9281 & 979.1875 & 122.5994 & 13.2705 \\
\hline 217 & 6.6723 & 1006.444 & 989.0615 & 978.25 & 122.5994 & 13.23672 \\
\hline 218 & 6.6893 & 979.7262 & 987.1201 & 977.1146 & 122.5994 & 13.20312 \\
\hline 219 & 6.7063 & 995.7118 & 985.277 & 975.9792 & 122.5994 & 13.16969 \\
\hline 220 & 6.7233 & 1038.659 & 983.5132 & 974.8438 & 122.5994 & 13.13643 \\
\hline 221 & 6.7403 & 1002.947 & 981.815 & 973.7083 & 122.5994 & 13.10334 \\
\hline 222 & 6.7573 & 1005.068 & 980.1721 & 972.5729 & 122.5994 & 13.07041 \\
\hline 223 & 6.7743 & 967.4055 & 978.5763 & 971.4375 & 122.5994 & 13.03765 \\
\hline 224 & 6.7913 & 959.7409 & 977.0214 & 970.3021 & 122.5994 & 13.00505 \\
\hline 225 & 6.8083 & 996.9927 & 975.5024 & 969.1667 & 122.5994 & 12.97261 \\
\hline 226 & 6.8253 & 978.4254 & 974.0151 & 968.0313 & 122.5994 & 12.94034 \\
\hline 227 & 6.8423 & 952.0361 & 972.556 & 966.8958 & 122.5994 & 12.90823 \\
\hline 228 & 6.8593 & 1026.645 & 971.1221 & 965.7604 & 122.5994 & 12.87627 \\
\hline 229 & 6.8763 & 1013.643 & 969.7109 & 964.625 & 122.5994 & 12.84448 \\
\hline 230 & 6.8933 & 1005.342 & 968.3203 & 963.4896 & 122.5994 & 12.81284 \\
\hline 231 & 6.9103 & 998.9032 & 966.9481 & 962.3542 & 122.5994 & 12.78136 \\
\hline 232 & 6.9273 & 974.1772 & 965.5926 & 961.2188 & 122.5994 & 12.75003 \\
\hline 233 & 6.9443 & 1023.574 & 964.2524 & 960.0833 & 122.5994 & 12.71886 \\
\hline 234 & 6.9613 & 973.6803 & 962.9261 & 958.9479 & 122.5994 & 12.68783 \\
\hline 235 & 6.9783 & 992.0212 & 961.6125 & 957.8125 & 122.5994 & 12.65696 \\
\hline 236 & 6.9953 & 988.1213 & 960.3105 & 956.6771 & 122.5994 & 12.62624 \\
\hline 237 & 7.0123 & 1022.997 & 959.0191 & 955.5417 & 122.5994 & 12.59567 \\
\hline 238 & 7.0293 & 1008.747 & 957.7374 & 954.4063 & 122.5994 & 12.56525 \\
\hline 239 & 7.0463 & 981.439 & 956.4646 & 953.2708 & 122.5994 & 12.53497 \\
\hline 240 & 7.0633 & 947.7549 & 955.2002 & 952.1354 & 122.5994 & 12.50484 \\
\hline 241 & 7.0803 & 944.5271 & 953.9432 & 951 & 122.5994 & 12.47485 \\
\hline 242 & 7.0973 & 951.3794 & 952.7549 & 949.9261 & 122.5994 & 12.44501 \\
\hline 243 & 7.1143 & 951.8458 & 951.5731 & 948.8522 & 122.5994 & 12.41531 \\
\hline
\end{tabular}




\begin{tabular}{|c|c|c|c|c|c|c|}
\hline 244 & 7.1313 & 1009.808 & 950.3973 & 947.7783 & 122.5994 & 12.38575 \\
\hline 245 & 7.1483 & 995.461 & 949.227 & 946.7044 & 122.5994 & 12.35633 \\
\hline 246 & 7.1653 & 957.1665 & 948.062 & 945.6305 & 122.5994 & 12.32706 \\
\hline 247 & 7.1823 & 946.5913 & 946.9017 & 944.5566 & 122.5994 & 12.29792 \\
\hline 248 & 7.1993 & 964.6036 & 945.7459 & 943.4827 & 122.5994 & 12.26892 \\
\hline 249 & 7.2163 & 922.167 & 944.5944 & 942.4089 & 122.5994 & 12.24005 \\
\hline 250 & 7.2333 & 952.4363 & 943.4467 & 941.335 & 122.5994 & 12.21132 \\
\hline 251 & 7.2503 & 989.8558 & 942.3027 & 940.2611 & 122.5994 & 12.18273 \\
\hline 252 & 7.2673 & 961.1946 & 941.1621 & 939.1872 & 122.5994 & 12.15427 \\
\hline 253 & 7.2843 & 931.9146 & 940.0247 & 938.1133 & 122.5994 & 12.12594 \\
\hline 254 & 7.3013 & 956.2105 & 938.8903 & 937.0394 & 122.5994 & 12.09774 \\
\hline 255 & 7.3183 & 988.0584 & 937.7588 & 935.9655 & 122.5994 & 12.06968 \\
\hline 256 & 7.3353 & 965.6209 & 936.6298 & 934.8916 & 122.5994 & 12.04175 \\
\hline 257 & 7.3523 & 942.8716 & 934.3882 & 933.8177 & 122.5994 & 12.01394 \\
\hline 258 & 7.3693 & 951.1862 & 932.7438 & 932.7438 & 122.5994 & 11.98627 \\
\hline 259 & 7.3863 & 945.8975 & 931.6699 & 931.6699 & 122.5994 & 11.95872 \\
\hline 260 & 7.4033 & 934.6106 & 930.596 & 930.596 & 122.5994 & 11.93129 \\
\hline 261 & 7.4203 & 942.3909 & 929.5222 & 929.5221 & 122.5994 & 11.904 \\
\hline 262 & 7.4373 & 966.272 & 928.4482 & 928.4482 & 122.5994 & 11.87683 \\
\hline 263 & 7.4543 & 942.0262 & 927.3743 & 927.3743 & 122.5994 & 11.84978 \\
\hline 264 & 7.4713 & 952.5384 & 926.3005 & 926.3005 & 122.5994 & 11.82285 \\
\hline 265 & 7.4883 & 984.2351 & 925.2266 & 925.2266 & 122.5994 & 11.79605 \\
\hline 266 & 7.5053 & 996.6685 & 923.958 & 923.958 & 122.5994 & 11.76937 \\
\hline 267 & 7.5223 & 994.0144 & 922.6895 & 922.6895 & 122.5994 & 11.74281 \\
\hline 268 & 7.5393 & 1011.129 & 921.4209 & 921.4209 & 122.5994 & 11.71637 \\
\hline 269 & 7.5563 & 909.199 & 920.1523 & 920.1523 & 122.5994 & 11.69005 \\
\hline 270 & 7.5733 & 957.7466 & 918.8838 & 918.8838 & 122.5994 & 11.66385 \\
\hline 271 & 7.5903 & 934.7345 & 917.6152 & 917.6152 & 122.5994 & 11.63776 \\
\hline 272 & 7.6073 & 944.4913 & 916.3467 & 916.3467 & 122.5994 & 11.61179 \\
\hline 273 & 7.6243 & 959.1939 & 915.0781 & 915.0781 & 122.5994 & 11.58594 \\
\hline 274 & 7.6413 & 907.6799 & 913.8096 & 913.8096 & 122.5994 & 11.5602 \\
\hline 275 & 7.6583 & 868.0772 & 912.541 & 912.541 & 122.5994 & 11.53458 \\
\hline 276 & 7.6753 & 924.7963 & 911.2725 & 911.2725 & 122.5994 & 11.50907 \\
\hline 277 & 7.6923 & 953.2901 & 910.0039 & 910.0039 & 122.5994 & 11.48367 \\
\hline 278 & 7.7093 & 948.8773 & 908.7354 & 908.7354 & 122.5994 & 11.45839 \\
\hline 279 & 7.7263 & 942.6213 & 907.4668 & 907.4668 & 122.5994 & 11.43321 \\
\hline 280 & 7.7433 & 918.0079 & 906.1982 & 906.1982 & 122.5994 & 11.40815 \\
\hline 281 & 7.7603 & 894.9169 & 909.442 & 904.9297 & 122.5994 & 11.3832 \\
\hline 282 & 7.7773 & 931.6574 & 908.3126 & 903.6611 & 122.5994 & 11.35835 \\
\hline 283 & 7.7943 & 960.5995 & 909.4829 & 902.3926 & 122.5994 & 11.33362 \\
\hline 284 & 7.8113 & 950.4519 & 908.4386 & 901.124 & 122.5994 & 11.30899 \\
\hline 285 & 7.8283 & 910.7166 & 907.405 & 899.8555 & 122.5994 & 11.28447 \\
\hline 286 & 7.8453 & 948.6577 & 906.3828 & 898.5869 & 122.5994 & 11.26006 \\
\hline 287 & 7.8623 & 938.4046 & 905.3727 & 897.3184 & 122.5994 & 11.23575 \\
\hline 288 & 7.8793 & 887.3274 & 904.3757 & 896.0498 & 122.5994 & 11.21154 \\
\hline 289 & 7.8963 & 909.6888 & 903.3926 & 894.7813 & 122.5994 & 11.18745 \\
\hline 290 & 7.9133 & 907.925 & 902.2112 & 893.2996 & 122.5994 & 11.16345 \\
\hline 291 & 7.9303 & 917.6843 & 901.0458 & 891.818 & 122.5994 & 11.13956 \\
\hline 292 & 7.9473 & 864.6715 & 899.8973 & 890.3364 & 122.5994 & 11.11577 \\
\hline
\end{tabular}




\begin{tabular}{|c|c|c|c|c|c|c|}
\hline 293 & 7.9643 & 890.5497 & 898.767 & 888.8548 & 122.5994 & 11.09208 \\
\hline 294 & 7.9813 & 925.0441 & 897.6563 & 887.3732 & 122.5994 & 11.06849 \\
\hline 295 & 7.9983 & 950.7871 & 896.5667 & 885.8916 & 122.5994 & 11.045 \\
\hline 296 & 8.0153 & 931.9323 & 895.4996 & 884.41 & 122.5994 & 11.02162 \\
\hline 297 & 8.0323 & 909.833 & 894.457 & 882.9284 & 122.5994 & 10.99833 \\
\hline 298 & 8.0493 & 920.2137 & 893.4408 & 881.4468 & 122.5994 & 10.97514 \\
\hline 299 & 8.0663 & 934.9584 & 892.453 & 879.9652 & 122.5994 & 10.95204 \\
\hline 300 & 8.0833 & 913.7067 & 891.4961 & 878.4836 & 122.5994 & 10.92905 \\
\hline 301 & 8.1003 & 936.2131 & 890.5725 & 877.002 & 122.5994 & 10.90615 \\
\hline 302 & 8.1173 & 938.8907 & 889.6852 & 875.5203 & 122.5994 & 10.88335 \\
\hline 303 & 8.1343 & 909.0393 & 888.8375 & 874.0387 & 122.5994 & 10.86064 \\
\hline 304 & 8.1513 & 930.4611 & 888.0327 & 872.5571 & 122.5994 & 10.83803 \\
\hline 305 & 8.1683 & 926.4945 & 887.2748 & 871.0755 & 122.5994 & 10.81551 \\
\hline 306 & 8.1853 & 942.2431 & 886.5681 & 869.5939 & 122.5994 & 10.79309 \\
\hline 307 & 8.2023 & 935.9053 & 885.9177 & 868.1123 & 122.5994 & 10.77075 \\
\hline 308 & 8.2193 & 925.4699 & 885.3289 & 866.6307 & 122.5994 & 10.74852 \\
\hline 309 & 8.2363 & 911.2998 & 884.808 & 865.1491 & 122.5994 & 10.72637 \\
\hline 310 & 8.2533 & 919.5825 & 884.3619 & 863.6675 & 122.5994 & 10.70431 \\
\hline 311 & 8.2703 & 926.1869 & 883.9985 & 862.1859 & 122.5994 & 10.68235 \\
\hline 312 & 8.2873 & 949.6549 & 883.7269 & 860.7043 & 122.5994 & 10.66047 \\
\hline 313 & 8.3043 & 960.9629 & 883.5575 & 859.2227 & 122.5994 & 10.63869 \\
\hline 314 & 8.3213 & 979.3532 & 883.5023 & 857.741 & 122.5994 & 10.61699 \\
\hline 315 & 8.3383 & 957.76 & 883.5758 & 856.2594 & 122.5994 & 10.59538 \\
\hline 316 & 8.3553 & 972.804 & 883.7952 & 854.7778 & 122.5994 & 10.57386 \\
\hline 317 & 8.3723 & 974.6576 & 884.1815 & 853.2962 & 122.5994 & 10.55243 \\
\hline 318 & 8.3893 & 963.2491 & 884.7617 & 851.8146 & 122.5994 & 10.53109 \\
\hline 319 & 8.4063 & 987.3368 & 885.5695 & 850.333 & 122.5994 & 10.50983 \\
\hline 320 & 8.4233 & 1008.613 & 886.6499 & 848.8514 & 122.5994 & 10.48865 \\
\hline 321 & 8.4403 & 1011.259 & 888.0626 & 847.3698 & 122.5994 & 10.46757 \\
\hline 322 & 8.4573 & 960.0909 & 889.8875 & 845.8882 & 122.5994 & 10.44656 \\
\hline 323 & 8.4743 & 1079.332 & 892.2317 & 844.4066 & 122.5994 & 10.42565 \\
\hline 324 & 8.4913 & 1033.374 & 895.2391 & 842.925 & 122.5994 & 10.40481 \\
\hline 325 & 8.5083 & 1005.642 & 899.0995 & 841.4434 & 122.5994 & 10.38406 \\
\hline 326 & 8.5253 & 1055.84 & 904.0613 & 839.9618 & 122.5994 & 10.36339 \\
\hline 327 & 8.5423 & 1055.992 & 910.4409 & 838.4801 & 122.5994 & 10.34281 \\
\hline 328 & 8.5593 & 1037.962 & 918.6322 & 836.9985 & 122.5994 & 10.3223 \\
\hline 329 & 8.5763 & 1070.062 & 929.1125 & 835.5169 & 122.5994 & 10.30188 \\
\hline 330 & 8.5933 & 1081.395 & 942.4396 & 834.0353 & 122.5994 & 10.28154 \\
\hline 331 & 8.6103 & 1076.59 & 959.2441 & 832.5537 & 122.5994 & 10.26128 \\
\hline 332 & 8.6273 & 1143.287 & 980.2025 & 831.0721 & 122.5994 & 10.24109 \\
\hline 333 & 8.6443 & 1153.144 & 1006.004 & 829.5905 & 122.5994 & 10.22099 \\
\hline 334 & 8.6613 & 1174.189 & 1037.298 & 828.1089 & 122.5994 & 10.20097 \\
\hline 335 & 8.6783 & 1175.733 & 1074.632 & 826.6273 & 122.5994 & 10.18102 \\
\hline 336 & 8.6953 & 1220.486 & 1118.384 & 825.1457 & 122.5994 & 10.16116 \\
\hline 337 & 8.7123 & 1179.602 & 1168.665 & 823.6641 & 122.5994 & 10.14137 \\
\hline 338 & 8.7293 & 1204.287 & 1225.031 & 821.9694 & 122.5994 & 10.12166 \\
\hline 339 & 8.7463 & 1240.366 & 1287.012 & 820.2747 & 122.5994 & 10.10202 \\
\hline 340 & 8.7633 & 1258.633 & 1353.373 & 818.5801 & 122.5994 & 10.08246 \\
\hline 341 & 8.7803 & 1346.543 & 1422.175 & 816.8854 & 122.5994 & 10.06298 \\
\hline
\end{tabular}




\begin{tabular}{|c|c|c|c|c|c|c|}
\hline 342 & 8.7973 & 1364.126 & 1490.643 & 815.1908 & 122.5994 & 10.04357 \\
\hline 343 & 8.8143 & 1409.552 & 1555.076 & 813.4961 & 122.5994 & 10.02424 \\
\hline 344 & 8.8313 & 1514.93 & 1610.919 & 811.8014 & 122.5994 & 10.00498 \\
\hline 345 & 8.8483 & 1590.652 & 1653.205 & 810.1068 & 122.5994 & 9.9858 \\
\hline 346 & 8.8653 & 1630.864 & 1677.385 & 808.4121 & 122.5994 & 9.96669 \\
\hline 347 & 8.8823 & 1748.463 & 1680.461 & 806.7174 & 122.5994 & 9.94765 \\
\hline 348 & 8.8993 & 1887.646 & 1661.866 & 805.0228 & 122.5994 & 9.92869 \\
\hline 349 & 8.9163 & 1965.708 & 1623.643 & 803.3281 & 122.5994 & 9.90979 \\
\hline 350 & 8.9333 & 2180.846 & 1569.792 & 801.6335 & 122.5994 & 9.89097 \\
\hline 351 & 8.9503 & 2303.464 & 1505.208 & 799.9388 & 122.5994 & 9.87222 \\
\hline 352 & 8.9673 & 2160.515 & 1434.678 & 798.2441 & 122.5994 & 9.85355 \\
\hline 353 & 8.9843 & 1858.135 & 1362.291 & 796.5495 & 122.5994 & 9.83494 \\
\hline 354 & 9.0013 & 1499.866 & 1291.222 & 794.8548 & 122.5994 & 9.8164 \\
\hline 355 & 9.0183 & 1182.488 & 1223.754 & 793.1602 & 122.5994 & 9.79794 \\
\hline 356 & 9.0353 & 1059.214 & 1161.423 & 791.4655 & 122.5994 & 9.77954 \\
\hline 357 & 9.0523 & 999.4232 & 1105.145 & 789.7708 & 122.5994 & 9.76121 \\
\hline 358 & 9.0693 & 943.9778 & 1055.337 & 788.0762 & 122.5994 & 9.74295 \\
\hline 359 & 9.0863 & 934.5616 & 1012.029 & 786.3815 & 122.5994 & 9.72476 \\
\hline 360 & 9.1033 & 918.7612 & 974.9479 & 784.6868 & 122.5994 & 9.70664 \\
\hline 361 & 9.1203 & 894.1557 & 943.6162 & 782.9922 & 122.5994 & 9.68859 \\
\hline 362 & 9.1373 & 816.7941 & 917.189 & 781.0661 & 122.5994 & 9.6706 \\
\hline 363 & 9.1543 & 790.8134 & 895.2195 & 779.14 & 122.5994 & 9.65268 \\
\hline 364 & 9.1713 & 807.973 & 877.0218 & 777.2139 & 122.5994 & 9.63482 \\
\hline 365 & 9.1883 & 798.0678 & 861.9408 & 775.2878 & 122.5994 & 9.61704 \\
\hline 366 & 9.2053 & 814.6452 & 849.3862 & 773.3617 & 122.5994 & 9.59931 \\
\hline 367 & 9.2223 & 793.1695 & 838.8447 & 771.4355 & 122.5994 & 9.58166 \\
\hline 368 & 9.2393 & 804.7745 & 829.8863 & 769.5094 & 122.5994 & 9.56407 \\
\hline 369 & 9.2563 & 796.1834 & 822.1603 & 767.5833 & 122.5994 & 9.54654 \\
\hline 370 & 9.2733 & 784.6945 & 815.3905 & 765.6572 & 122.5994 & 9.52908 \\
\hline 371 & 9.2903 & 800.2012 & 809.3623 & 763.7311 & 122.5994 & 9.51168 \\
\hline 372 & 9.3073 & 778.8174 & 803.9127 & 761.805 & 122.5994 & 9.49434 \\
\hline 373 & 9.3243 & 783.9924 & 798.9197 & 759.8789 & 122.5994 & 9.47707 \\
\hline 374 & 9.3413 & 789.1959 & 794.2915 & 757.9528 & 122.5994 & 9.45986 \\
\hline 375 & 9.3583 & 771.2998 & 789.9605 & 756.0267 & 122.5994 & 9.44272 \\
\hline 376 & 9.3753 & 740.5604 & 785.876 & 754.1006 & 122.5994 & 9.42563 \\
\hline 377 & 9.3923 & 749.3005 & 781.9993 & 752.1745 & 122.5994 & 9.40861 \\
\hline 378 & 9.4093 & 737.4776 & 778.3008 & 750.2484 & 122.5994 & 9.39165 \\
\hline 379 & 9.4263 & 759.5378 & 774.7571 & 748.3223 & 122.5994 & 9.37475 \\
\hline 380 & 9.4433 & 746.15 & 771.3492 & 746.3962 & 122.5994 & 9.35791 \\
\hline 381 & 9.4603 & 750.774 & 768.0618 & 744.4701 & 122.5994 & 9.34113 \\
\hline 382 & 9.4773 & 753.9987 & 764.8817 & 742.5439 & 122.5994 & 9.32441 \\
\hline 383 & 9.4943 & 772.1973 & 761.7979 & 740.6178 & 122.5994 & 9.30776 \\
\hline 384 & 9.5113 & 775.0327 & 758.8005 & 738.6917 & 122.5994 & 9.29116 \\
\hline 385 & 9.5283 & 731.8144 & 755.8813 & 736.7656 & 122.5994 & 9.27462 \\
\hline 386 & 9.5453 & 726.1905 & 752.8022 & 734.6087 & 122.5994 & 9.25814 \\
\hline 387 & 9.5623 & 709.737 & 749.7876 & 732.4518 & 122.5994 & 9.24172 \\
\hline 388 & 9.5793 & 743.6203 & 746.8314 & 730.2949 & 122.5994 & 9.22536 \\
\hline 389 & 9.5963 & 730.898 & 743.9286 & 728.138 & 122.5994 & 9.20905 \\
\hline 390 & 9.6133 & 718.6238 & 741.0746 & 725.9811 & 122.5994 & 9.19281 \\
\hline
\end{tabular}




\begin{tabular}{|c|c|c|c|c|c|c|}
\hline 391 & 9.6303 & 677.4464 & 738.2653 & 723.8242 & 122.5994 & 9.17662 \\
\hline 392 & 9.6473 & 681.3329 & 735.4969 & 721.6673 & 122.5994 & 9.16048 \\
\hline 393 & 9.6643 & 699.9046 & 732.7662 & 719.5104 & 122.5994 & 9.14441 \\
\hline 394 & 9.6813 & 668.9033 & 730.0701 & 717.3535 & 122.5994 & 9.12839 \\
\hline 395 & 9.6983 & 672.8911 & 727.406 & 715.1966 & 122.5994 & 9.11243 \\
\hline 396 & 9.7153 & 699.6499 & 724.7714 & 713.0397 & 122.5994 & 9.09652 \\
\hline 397 & 9.7323 & 726.8878 & 722.1641 & 710.8828 & 122.5994 & 9.08067 \\
\hline 398 & 9.7493 & 728.1476 & 719.5819 & 708.7259 & 122.5994 & 9.06487 \\
\hline 399 & 9.7663 & 727.3651 & 717.0233 & 706.569 & 122.5994 & 9.04913 \\
\hline 400 & 9.7833 & 697.1777 & 714.4863 & 704.4121 & 122.5994 & 9.03344 \\
\hline 401 & 9.8003 & 673.9703 & 711.9695 & 702.2552 & 122.5994 & 9.01781 \\
\hline 402 & 9.8173 & 705.9135 & 709.4716 & 700.0983 & 122.5994 & 9.00224 \\
\hline 403 & 9.8343 & 706.3124 & 706.9912 & 697.9414 & 122.5994 & 8.98671 \\
\hline 404 & 9.8513 & 689.8736 & 704.5271 & 695.7845 & 122.5994 & 8.97124 \\
\hline 405 & 9.8683 & 697.4026 & 702.0784 & 693.6276 & 122.5994 & 8.95583 \\
\hline 406 & 9.8853 & 679.8112 & 699.6439 & 691.4707 & 122.5994 & 8.94046 \\
\hline 407 & 9.9023 & 682.2815 & 697.2227 & 689.3138 & 122.5994 & 8.92515 \\
\hline 408 & 9.9193 & 674.6188 & 694.8142 & 687.1569 & 122.5994 & 8.90989 \\
\hline 409 & 9.9363 & 682.9931 & 692.4174 & 685 & 122.5994 & 8.89469 \\
\hline 410 & 9.9533 & 699.0638 & 691.376 & 684.1875 & 122.5994 & 8.87953 \\
\hline 411 & 9.9703 & 676.4295 & 690.345 & 683.375 & 122.5994 & 8.86443 \\
\hline 412 & 9.9873 & 675.1988 & 684.8681 & 682.5625 & 122.5994 & 8.84938 \\
\hline 413 & 10.0043 & 644.9228 & 683.9867 & 681.75 & 122.5994 & 8.83438 \\
\hline 414 & 10.0213 & 626.7288 & 680.9375 & 680.9375 & 122.5994 & 8.81943 \\
\hline 415 & 10.0383 & 676.0371 & 680.125 & 680.125 & 122.5994 & 8.80454 \\
\hline 416 & 10.0553 & 671.5196 & 679.3125 & 679.3125 & 122.5994 & 8.78969 \\
\hline 417 & 10.0723 & 685.6832 & 678.5 & 678.5 & 122.5994 & 8.77489 \\
\hline 418 & 10.0893 & 676.3019 & 677.6875 & 677.6875 & 122.5994 & 8.76014 \\
\hline 419 & 10.1063 & 625.2907 & 676.875 & 676.875 & 122.5994 & 8.74545 \\
\hline 420 & 10.1233 & 635.5213 & 676.0625 & 676.0625 & 122.5994 & 8.7308 \\
\hline 421 & 10.1403 & 664.4876 & 675.25 & 675.25 & 122.5994 & 8.7162 \\
\hline 422 & 10.1573 & 635.0519 & 674.4375 & 674.4375 & 122.5994 & 8.70165 \\
\hline 423 & 10.1743 & 635.4553 & 673.625 & 673.625 & 122.5994 & 8.68715 \\
\hline 424 & 10.1913 & 643.1793 & 672.8125 & 672.8125 & 122.5994 & 8.6727 \\
\hline 425 & 10.2083 & 670.1871 & 672 & 672 & 122.5994 & 8.65829 \\
\hline 426 & 10.2253 & 664.9086 & 671.1875 & 671.1875 & 122.5994 & 8.64394 \\
\hline 427 & 10.2423 & 657.921 & 670.375 & 670.375 & 122.5994 & 8.62963 \\
\hline 428 & 10.2593 & 647.084 & 669.5625 & 669.5625 & 122.5994 & 8.61537 \\
\hline 429 & 10.2763 & 661.8675 & 668.75 & 668.75 & 122.5994 & 8.60115 \\
\hline 430 & 10.2933 & 687.6234 & 667.9375 & 667.9375 & 122.5994 & 8.58698 \\
\hline 431 & 10.3103 & 689.062 & 667.125 & 667.125 & 122.5994 & 8.57286 \\
\hline 432 & 10.3273 & 689.8882 & 666.3125 & 666.3125 & 122.5994 & 8.55879 \\
\hline 433 & 10.3443 & 695.8905 & 665.5 & 665.5 & 122.5994 & 8.54476 \\
\hline 434 & 10.3613 & 666.8723 & 664.6875 & 664.6875 & 122.5994 & 8.53078 \\
\hline 435 & 10.3783 & 672.5507 & 663.875 & 663.875 & 122.5994 & 8.51685 \\
\hline 436 & 10.3953 & 642.3217 & 663.0625 & 663.0625 & 122.5994 & 8.50296 \\
\hline 437 & 10.4123 & 645.7116 & 662.25 & 662.25 & 122.5994 & 8.48911 \\
\hline 438 & 10.4293 & 622.0014 & 661.4375 & 661.4375 & 122.5994 & 8.47531 \\
\hline 439 & 10.4463 & 614.2736 & 660.625 & 660.625 & 122.5994 & 8.46156 \\
\hline
\end{tabular}




\begin{tabular}{|c|c|c|c|c|c|c|}
\hline 440 & 10.4633 & 635.775 & 659.8125 & 659.8125 & 122.5994 & 8.44785 \\
\hline 441 & 10.4803 & 657.7689 & 659 & 659 & 122.5994 & 8.43418 \\
\hline 442 & 10.4973 & 643.1222 & 658.1875 & 658.1875 & 122.5994 & 8.42056 \\
\hline 443 & 10.5143 & 656.3576 & 657.375 & 657.375 & 122.5994 & 8.40699 \\
\hline 444 & 10.5313 & 669.9269 & 656.5625 & 656.5625 & 122.5994 & 8.39345 \\
\hline 445 & 10.5483 & 669.6349 & 655.75 & 655.75 & 122.5994 & 8.37996 \\
\hline 446 & 10.5653 & 696.367 & 654.9375 & 654.9375 & 122.5994 & 8.36652 \\
\hline 447 & 10.5823 & 685.6603 & 654.125 & 654.125 & 122.5994 & 8.35312 \\
\hline 448 & 10.5993 & 683.7223 & 653.3125 & 653.3125 & 122.5994 & 8.33976 \\
\hline 449 & 10.6163 & 715.5149 & 652.5 & 652.5 & 122.5994 & 8.32644 \\
\hline 450 & 10.6333 & 700.8892 & 651.6875 & 651.6875 & 122.5994 & 8.31317 \\
\hline 451 & 10.6503 & 686.4528 & 650.875 & 650.875 & 122.5994 & 8.29994 \\
\hline 452 & 10.6673 & 671.7738 & 650.0625 & 650.0625 & 122.5994 & 8.28675 \\
\hline 453 & 10.6843 & 650.797 & 649.25 & 649.25 & 122.5994 & 8.2736 \\
\hline 454 & 10.7013 & 652.7904 & 648.4375 & 648.4375 & 122.5994 & 8.26049 \\
\hline 455 & 10.7183 & 643.6016 & 647.625 & 647.625 & 122.5994 & 8.24743 \\
\hline 456 & 10.7353 & 640.102 & 646.8125 & 646.8125 & 122.5994 & 8.23441 \\
\hline 457 & 10.7523 & 631.9226 & 646 & 646 & 122.5994 & 8.22143 \\
\hline 458 & 10.7693 & 663.969 & 644.9583 & 644.9583 & 122.5994 & 8.20849 \\
\hline 459 & 10.7863 & 662.0045 & 643.9167 & 643.9167 & 122.5994 & 8.19559 \\
\hline 460 & 10.8033 & 656.0085 & 642.875 & 642.875 & 122.5994 & 8.18273 \\
\hline 461 & 10.8203 & 669.2007 & 641.8333 & 641.8333 & 122.5994 & 8.16991 \\
\hline 462 & 10.8373 & 597.0078 & 640.7917 & 640.7917 & 122.5994 & 8.15713 \\
\hline 463 & 10.8543 & 670.9269 & 639.75 & 639.75 & 122.5994 & 8.1444 \\
\hline 464 & 10.8713 & 631.7306 & 638.7083 & 638.7083 & 122.5994 & 8.1317 \\
\hline 465 & 10.8883 & 639.936 & 637.6667 & 637.6667 & 122.5994 & 8.11904 \\
\hline 466 & 10.9053 & 614.9912 & 636.625 & 636.625 & 122.5994 & 8.10642 \\
\hline 467 & 10.9223 & 611.305 & 635.5833 & 635.5833 & 122.5994 & 8.09384 \\
\hline 468 & 10.9393 & 652.9565 & 634.5417 & 634.5417 & 122.5994 & 8.0813 \\
\hline 469 & 10.9563 & 607.2163 & 633.5 & 633.5 & 122.5994 & 8.0688 \\
\hline 470 & 10.9733 & 634.1369 & 632.4583 & 632.4583 & 122.5994 & 8.05634 \\
\hline 471 & 10.9903 & 627.8566 & 631.4167 & 631.4167 & 122.5994 & 8.04392 \\
\hline 472 & 11.0073 & 648.1845 & 630.375 & 630.375 & 122.5994 & 8.03153 \\
\hline 473 & 11.0243 & 631.9382 & 629.3333 & 629.3333 & 122.5994 & 8.01919 \\
\hline 474 & 11.0413 & 614.1743 & 628.2917 & 628.2917 & 122.5994 & 8.00688 \\
\hline 475 & 11.0583 & 610.0664 & 627.25 & 627.25 & 122.5994 & 7.99461 \\
\hline 476 & 11.0753 & 605.7849 & 626.2083 & 626.2083 & 122.5994 & 7.98237 \\
\hline 477 & 11.0923 & 602.4926 & 625.1667 & 625.1667 & 122.5994 & 7.97018 \\
\hline 478 & 11.1093 & 632.168 & 624.125 & 624.125 & 122.5994 & 7.95802 \\
\hline 479 & 11.1263 & 605.9066 & 623.0833 & 623.0833 & 122.5994 & 7.9459 \\
\hline 480 & 11.1433 & 599.8831 & 622.0417 & 622.0417 & 122.5994 & 7.93381 \\
\hline 481 & 11.1603 & 621.562 & 621 & 621 & 122.5994 & 7.92177 \\
\hline 482 & 11.1773 & 639.8798 & 620.7424 & 620.7424 & 122.5994 & 7.90976 \\
\hline 483 & 11.1943 & 630.0218 & 620.4847 & 620.4847 & 122.5994 & 7.89778 \\
\hline 484 & 11.2113 & 626.0623 & 620.2271 & 620.2271 & 122.5994 & 7.88585 \\
\hline 485 & 11.2283 & 609.8196 & 619.9694 & 619.9694 & 122.5994 & 7.87394 \\
\hline 486 & 11.2453 & 606.1039 & 619.7117 & 619.7118 & 122.5994 & 7.86208 \\
\hline 487 & 11.2623 & 617.7153 & 619.4541 & 619.4541 & 122.5994 & 7.85025 \\
\hline 488 & 11.2793 & 619.8166 & 619.1965 & 619.1965 & 122.5994 & 7.83846 \\
\hline
\end{tabular}




\begin{tabular}{|c|c|c|c|c|c|c|}
\hline 489 & 11.2963 & 637.6355 & 618.9388 & 618.9388 & 122.5994 & 7.8267 \\
\hline 490 & 11.3133 & 639.7072 & 618.6812 & 618.6812 & 122.5994 & 7.81498 \\
\hline 491 & 11.3303 & 616.4929 & 618.4235 & 618.4235 & 122.5994 & 7.80329 \\
\hline 492 & 11.3473 & 616.371 & 618.1658 & 618.1659 & 122.5994 & 7.79164 \\
\hline 493 & 11.3643 & 616.2739 & 619.8354 & 617.9082 & 122.5994 & 7.78002 \\
\hline 494 & 11.3813 & 616.093 & 619.6348 & 617.6506 & 122.5994 & 7.76844 \\
\hline 495 & 11.3983 & 623.1605 & 620.407 & 617.3929 & 122.5994 & 7.75689 \\
\hline 496 & 11.4153 & 638.1275 & 620.2403 & 617.1353 & 122.5994 & 7.74537 \\
\hline 497 & 11.4323 & 628.2327 & 620.0936 & 616.8776 & 122.5994 & 7.73389 \\
\hline 498 & 11.4493 & 596.1826 & 619.936 & 616.62 & 122.5994 & 7.72245 \\
\hline 499 & 11.4663 & 575.3316 & 619.7831 & 616.3623 & 122.5994 & 7.71104 \\
\hline 500 & 11.4833 & 607.1345 & 619.6352 & 616.1047 & 122.5994 & 7.69966 \\
\hline 501 & 11.5003 & 601.3333 & 619.4926 & 615.847 & 122.5994 & 7.68832 \\
\hline 502 & 11.5173 & 614.5902 & 619.3557 & 615.5894 & 122.5994 & 7.67701 \\
\hline 503 & 11.5343 & 623.9838 & 619.2249 & 615.3317 & 122.5994 & 7.66573 \\
\hline 504 & 11.5513 & 633.2715 & 619.1005 & 615.0741 & 122.5994 & 7.65449 \\
\hline 505 & 11.5683 & 656.5375 & 618.9831 & 614.8164 & 122.5994 & 7.64328 \\
\hline 506 & 11.5853 & 678.4019 & 618.6826 & 614.3683 & 122.5994 & 7.6321 \\
\hline 507 & 11.6023 & 662.8391 & 618.39 & 613.9202 & 122.5994 & 7.62095 \\
\hline 508 & 11.6193 & 628.2499 & 618.1058 & 613.472 & 122.5994 & 7.60984 \\
\hline 509 & 11.6363 & 682.1306 & 617.8309 & 613.0239 & 122.5994 & 7.59876 \\
\hline 510 & 11.6533 & 642.3375 & 617.5657 & 612.5758 & 122.5994 & 7.58772 \\
\hline 511 & 11.6703 & 582.4034 & 617.311 & 612.1277 & 122.5994 & 7.5767 \\
\hline 512 & 11.6873 & 654.9499 & 617.0677 & 611.6796 & 122.5994 & 7.56572 \\
\hline 513 & 11.7043 & 640.3517 & 616.8367 & 611.2314 & 122.5994 & 7.55477 \\
\hline 514 & 11.7213 & 653.8093 & 616.6189 & 610.7833 & 122.5994 & 7.54385 \\
\hline 515 & 11.7383 & 617.7497 & 616.4154 & 610.3352 & 122.5994 & 7.53296 \\
\hline 516 & 11.7553 & 658.852 & 616.2275 & 609.8871 & 122.5994 & 7.52211 \\
\hline 517 & 11.7723 & 639.5483 & 616.0565 & 609.439 & 122.5994 & 7.51128 \\
\hline 518 & 11.7893 & 631.9602 & 615.9037 & 608.9908 & 122.5994 & 7.50049 \\
\hline 519 & 11.8063 & 612.9987 & 615.7709 & 608.5427 & 122.5994 & 7.48973 \\
\hline 520 & 11.8233 & 629.1494 & 615.6599 & 608.0946 & 122.5994 & 7.479 \\
\hline 521 & 11.8403 & 624.0713 & 615.5728 & 607.6465 & 122.5994 & 7.4683 \\
\hline 522 & 11.8573 & 628.5223 & 615.5117 & 607.1984 & 122.5994 & 7.45763 \\
\hline 523 & 11.8743 & 651.0944 & 615.4791 & 606.7502 & 122.5994 & 7.44699 \\
\hline 524 & 11.8913 & 632.8745 & 615.4781 & 606.3021 & 122.5994 & 7.43638 \\
\hline 525 & 11.9083 & 662.3885 & 615.5118 & 605.854 & 122.5994 & 7.4258 \\
\hline 526 & 11.9253 & 629.7252 & 615.5839 & 605.4059 & 122.5994 & 7.41525 \\
\hline 527 & 11.9423 & 629.3215 & 615.6989 & 604.9578 & 122.5994 & 7.40474 \\
\hline 528 & 11.9593 & 675.9431 & 615.8615 & 604.5096 & 122.5994 & 7.39425 \\
\hline 529 & 11.9763 & 661.9076 & 616.0779 & 604.0615 & 122.5994 & 7.38379 \\
\hline 530 & 11.9933 & 647.6079 & 616.3553 & 603.6134 & 122.5994 & 7.37336 \\
\hline 531 & 12.0103 & 647.4032 & 616.7025 & 603.1653 & 122.5994 & 7.36297 \\
\hline 532 & 12.0273 & 652.6057 & 617.1307 & 602.7172 & 122.5994 & 7.3526 \\
\hline 533 & 12.0443 & 655.6738 & 617.6542 & 602.269 & 122.5994 & 7.34226 \\
\hline 534 & 12.0613 & 669.1201 & 618.2918 & 601.8209 & 122.5994 & 7.33195 \\
\hline 535 & 12.0783 & 655.3722 & 619.0681 & 601.3728 & 122.5994 & 7.32166 \\
\hline 536 & 12.0953 & 644.9471 & 620.0157 & 600.9247 & 122.5994 & 7.31141 \\
\hline 537 & 12.1123 & 643.1548 & 621.1777 & 600.4766 & 122.5994 & 7.30119 \\
\hline
\end{tabular}




\begin{tabular}{|c|c|c|c|c|c|c|}
\hline 538 & 12.1293 & 682.0273 & 622.6109 & 600.0284 & 122.5994 & 7.29099 \\
\hline 539 & 12.1463 & 683.3974 & 624.3892 & 599.5803 & 122.5994 & 7.28083 \\
\hline 540 & 12.1633 & 653.4201 & 626.6068 & 599.1322 & 122.5994 & 7.27069 \\
\hline 541 & 12.1803 & 707.1342 & 629.3828 & 598.6841 & 122.5994 & 7.26058 \\
\hline 542 & 12.1973 & 700.8952 & 632.8625 & 598.236 & 122.5994 & 7.2505 \\
\hline 543 & 12.2143 & 703.9683 & 637.2212 & 597.7878 & 122.5994 & 7.24045 \\
\hline 544 & 12.2313 & 759.3715 & 642.6611 & 597.3397 & 122.5994 & 7.23042 \\
\hline 545 & 12.2483 & 782.32 & 649.4092 & 596.8916 & 122.5994 & 7.22042 \\
\hline 546 & 12.2653 & 755.8966 & 657.7101 & 596.4435 & 122.5994 & 7.21045 \\
\hline 547 & 12.2823 & 741.2278 & 667.8137 & 595.9954 & 122.5994 & 7.20051 \\
\hline 548 & 12.2993 & 744.0046 & 679.9617 & 595.5472 & 122.5994 & 7.1906 \\
\hline 549 & 12.3163 & 715.4623 & 694.3647 & 595.0991 & 122.5994 & 7.18071 \\
\hline 550 & 12.3333 & 783.4001 & 711.1808 & 594.651 & 122.5994 & 7.17085 \\
\hline 551 & 12.3503 & 745.4189 & 730.4884 & 594.2029 & 122.5994 & 7.16102 \\
\hline 552 & 12.3673 & 796.146 & 752.2563 & 593.7548 & 122.5994 & 7.15121 \\
\hline 553 & 12.3843 & 806.5751 & 776.3177 & 593.3066 & 122.5994 & 7.14144 \\
\hline 554 & 12.4013 & 847.931 & 802.1357 & 592.6681 & 122.5994 & 7.13168 \\
\hline 555 & 12.4183 & 896.2281 & 829.3392 & 592.0295 & 122.5994 & 7.12196 \\
\hline 556 & 12.4353 & 855.4248 & 857.1034 & 591.3909 & 122.5994 & 7.11226 \\
\hline 557 & 12.4523 & 863.8938 & 884.2974 & 590.7523 & 122.5994 & 7.10259 \\
\hline 558 & 12.4693 & 870.1753 & 909.4783 & 590.1137 & 122.5994 & 7.09294 \\
\hline 559 & 12.4863 & 911.8593 & 930.9424 & 589.4751 & 122.5994 & 7.08333 \\
\hline 560 & 12.5033 & 888.5525 & 946.9113 & 588.8365 & 122.5994 & 7.07373 \\
\hline 561 & 12.5203 & 909.34 & 955.8327 & 588.1979 & 122.5994 & 7.06417 \\
\hline 562 & 12.5373 & 976.0715 & 956.7302 & 587.5593 & 122.5994 & 7.05463 \\
\hline 563 & 12.5543 & 1016.006 & 949.4553 & 586.9207 & 122.5994 & 7.04511 \\
\hline 564 & 12.5713 & 1065.014 & 934.72 & 586.2821 & 122.5994 & 7.03562 \\
\hline 565 & 12.5883 & 1080.595 & 913.9006 & 585.6436 & 122.5994 & 7.02616 \\
\hline 566 & 12.6053 & 1060.214 & 888.7131 & 585.005 & 122.5994 & 7.01672 \\
\hline 567 & 12.6223 & 992.5039 & 860.891 & 584.3664 & 122.5994 & 7.00731 \\
\hline 568 & 12.6393 & 882.7292 & 831.9754 & 583.7278 & 122.5994 & 6.99792 \\
\hline 569 & 12.6563 & 781.0295 & 803.2117 & 583.0892 & 122.5994 & 6.98856 \\
\hline 570 & 12.6733 & 735.1587 & 775.5367 & 582.4506 & 122.5994 & 6.97923 \\
\hline 571 & 12.6903 & 690.5913 & 749.611 & 581.812 & 122.5994 & 6.96991 \\
\hline 572 & 12.7073 & 690.3286 & 725.8572 & 581.1734 & 122.5994 & 6.96063 \\
\hline 573 & 12.7243 & 658.9258 & 704.5114 & 580.5348 & 122.5994 & 6.95137 \\
\hline 574 & 12.7413 & 650.3139 & 685.6545 & 579.8962 & 122.5994 & 6.94213 \\
\hline 575 & 12.7583 & 631.326 & 669.2473 & 579.2576 & 122.5994 & 6.93292 \\
\hline 576 & 12.7753 & 626.2523 & 655.1608 & 578.6191 & 122.5994 & 6.92373 \\
\hline 577 & 12.7923 & 645.1721 & 643.2005 & 577.9805 & 122.5994 & 6.91457 \\
\hline 578 & 12.8093 & 634.7008 & 632.901 & 577.1063 & 122.5994 & 6.90543 \\
\hline 579 & 12.8263 & 613.5408 & 624.2498 & 576.2321 & 122.5994 & 6.89631 \\
\hline 580 & 12.8433 & 605.1713 & 616.9993 & 575.3579 & 122.5994 & 6.88722 \\
\hline 581 & 12.8603 & 584.2957 & 610.9155 & 574.4837 & 122.5994 & 6.87816 \\
\hline 582 & 12.8773 & 571.279 & 605.7858 & 573.6095 & 122.5994 & 6.86912 \\
\hline 583 & 12.8943 & 619.7733 & 601.4252 & 572.7354 & 122.5994 & 6.8601 \\
\hline 584 & 12.9113 & 614.7811 & 597.6771 & 571.8612 & 122.5994 & 6.8511 \\
\hline 585 & 12.9283 & 575.3919 & 594.4126 & 570.987 & 122.5994 & 6.84213 \\
\hline 586 & 12.9453 & 574.0326 & 591.5282 & 570.1128 & 122.5994 & 6.83319 \\
\hline
\end{tabular}




\begin{tabular}{|c|c|c|c|c|c|c|}
\hline 587 & 12.9623 & 561.5252 & 588.9422 & 569.2386 & 122.5994 & 6.82426 \\
\hline 588 & 12.9793 & 585.5969 & 586.5918 & 568.3644 & 122.5994 & 6.81536 \\
\hline 589 & 12.9963 & 576.8464 & 584.4286 & 567.4902 & 122.5994 & 6.80649 \\
\hline 590 & 13.0133 & 564.8654 & 582.4163 & 566.616 & 122.5994 & 6.79763 \\
\hline 591 & 13.0303 & 540.4444 & 580.5273 & 565.7419 & 122.5994 & 6.7888 \\
\hline 592 & 13.0473 & 566.1133 & 578.7407 & 564.8677 & 122.5994 & 6.78 \\
\hline 593 & 13.0643 & 580.7876 & 577.0403 & 563.9935 & 122.5994 & 6.77121 \\
\hline 594 & 13.0813 & 595.5789 & 575.4142 & 563.1193 & 122.5994 & 6.76245 \\
\hline 595 & 13.0983 & 588.8553 & 573.8522 & 562.2451 & 122.5994 & 6.75371 \\
\hline 596 & 13.1153 & 577.8845 & 572.3467 & 561.3709 & 122.5994 & 6.74499 \\
\hline 597 & 13.1323 & 526.0037 & 570.8913 & 560.4967 & 122.5994 & 6.7363 \\
\hline 598 & 13.1493 & 546.5397 & 569.4805 & 559.6226 & 122.5994 & 6.72763 \\
\hline 599 & 13.1663 & 560.4105 & 568.1097 & 558.7484 & 122.5994 & 6.71898 \\
\hline 600 & 13.1833 & 565.9197 & 566.7752 & 557.8742 & 122.5994 & 6.71036 \\
\hline 601 & 13.2003 & 549.2738 & 565.4733 & 557 & 122.5994 & 6.70175 \\
\hline 602 & 13.2173 & 560.6976 & 564.6171 & 556.5417 & 122.5994 & 6.69317 \\
\hline 603 & 13.2343 & 548.2374 & 563.7877 & 556.0833 & 122.5994 & 6.68461 \\
\hline 604 & 13.2513 & 529.4866 & 562.9832 & 555.625 & 122.5994 & 6.67607 \\
\hline 605 & 13.2683 & 552.1322 & 562.2012 & 555.1667 & 122.5994 & 6.66756 \\
\hline 606 & 13.2853 & 556.5649 & 561.4398 & 554.7083 & 122.5994 & 6.65907 \\
\hline 607 & 13.3023 & 533.9499 & 560.6973 & 554.25 & 122.5994 & 6.65059 \\
\hline 608 & 13.3193 & 546.9576 & 559.9724 & 553.7917 & 122.5994 & 6.64214 \\
\hline 609 & 13.3363 & 606.1368 & 559.2633 & 553.3333 & 122.5994 & 6.63371 \\
\hline 610 & 13.3533 & 561.6683 & 558.5692 & 552.875 & 122.5994 & 6.62531 \\
\hline 611 & 13.3703 & 555.338 & 557.8887 & 552.4167 & 122.5994 & 6.61692 \\
\hline 612 & 13.3873 & 518.9245 & 557.2208 & 551.9583 & 122.5994 & 6.60856 \\
\hline 613 & 13.4043 & 568.8314 & 556.5646 & 551.5 & 122.5994 & 6.60021 \\
\hline 614 & 13.4213 & 576.3019 & 555.9193 & 551.0417 & 122.5994 & 6.59189 \\
\hline 615 & 13.4383 & 556.9704 & 555.2841 & 550.5833 & 122.5994 & 6.58359 \\
\hline 616 & 13.4553 & 562.4833 & 554.6583 & 550.125 & 122.5994 & 6.57531 \\
\hline 617 & 13.4723 & 578.3823 & 554.0411 & 549.6667 & 122.5994 & 6.56705 \\
\hline 618 & 13.4893 & 559.5895 & 553.4321 & 549.2083 & 122.5994 & 6.55881 \\
\hline 619 & 13.5063 & 538.6896 & 552.8307 & 548.75 & 122.5994 & 6.5506 \\
\hline 620 & 13.5233 & 555.7114 & 552.2365 & 548.2917 & 122.5994 & 6.5424 \\
\hline 621 & 13.5403 & 557.4396 & 551.6488 & 547.8333 & 122.5994 & 6.53423 \\
\hline 622 & 13.5573 & 565.1677 & 551.0674 & 547.375 & 122.5994 & 6.52607 \\
\hline 623 & 13.5743 & 539.6915 & 550.4918 & 546.9167 & 122.5994 & 6.51793 \\
\hline 624 & 13.5913 & 549.3953 & 549.9216 & 546.4583 & 122.5994 & 6.50982 \\
\hline 625 & 13.6083 & 538.2501 & 549.3411 & 546 & 122.5994 & 6.50173 \\
\hline 626 & 13.6253 & 550.8433 & 549.0574 & 545.8177 & 122.5994 & 6.49365 \\
\hline 627 & 13.6423 & 553.2192 & 548.7783 & 545.6354 & 122.5994 & 6.4856 \\
\hline 628 & 13.6593 & 533.7157 & 548.5034 & 545.4531 & 122.5994 & 6.47757 \\
\hline 629 & 13.6763 & 538.4862 & 548.2325 & 545.2708 & 122.5994 & 6.46955 \\
\hline 630 & 13.6933 & 552.5874 & 546.0761 & 545.0885 & 122.5994 & 6.46156 \\
\hline 631 & 13.7103 & 562.2345 & 545.8655 & 544.9063 & 122.5994 & 6.45358 \\
\hline 632 & 13.7273 & 536.9169 & 544.7239 & 544.724 & 122.5994 & 6.44563 \\
\hline 633 & 13.7443 & 551.622 & 544.5417 & 544.5417 & 122.5994 & 6.4377 \\
\hline 634 & 13.7613 & 567.141 & 544.3594 & 544.3594 & 122.5994 & 6.42978 \\
\hline 635 & 13.7783 & 558.6349 & 544.1771 & 544.1771 & 122.5994 & 6.42189 \\
\hline
\end{tabular}




\begin{tabular}{|c|c|c|c|c|c|c|}
\hline 636 & 13.7953 & 553.8484 & 543.9948 & 543.9948 & 122.5994 & 6.41401 \\
\hline 637 & 13.8123 & 561.9716 & 543.8125 & 543.8125 & 122.5994 & 6.40615 \\
\hline 638 & 13.8293 & 559.7266 & 543.6302 & 543.6302 & 122.5994 & 6.39832 \\
\hline 639 & 13.8463 & 570.8199 & 543.4479 & 543.4479 & 122.5994 & 6.3905 \\
\hline 640 & 13.8633 & 568.8511 & 543.2656 & 543.2656 & 122.5994 & 6.3827 \\
\hline 641 & 13.8803 & 566.4181 & 543.0833 & 543.0833 & 122.5994 & 6.37492 \\
\hline 642 & 13.8973 & 574.0215 & 542.9011 & 542.901 & 122.5994 & 6.36716 \\
\hline 643 & 13.9143 & 603.742 & 542.7188 & 542.7188 & 122.5994 & 6.35942 \\
\hline 644 & 13.9313 & 565.7056 & 542.5364 & 542.5365 & 122.5994 & 6.3517 \\
\hline 645 & 13.9483 & 577.3976 & 542.3542 & 542.3542 & 122.5994 & 6.344 \\
\hline 646 & 13.9653 & 580.8209 & 542.1719 & 542.1719 & 122.5994 & 6.33631 \\
\hline 647 & 13.9823 & 585.1321 & 541.9896 & 541.9896 & 122.5994 & 6.32865 \\
\hline 648 & 13.9993 & 563.5922 & 541.8073 & 541.8073 & 122.5994 & 6.321 \\
\hline 649 & 14.0163 & 561.7005 & 541.625 & 541.625 & 122.5994 & 6.31337 \\
\hline 650 & 14.0333 & 543.5443 & 541.4427 & 541.4427 & 122.5994 & 6.30576 \\
\hline 651 & 14.0503 & 529.2105 & 541.2604 & 541.2604 & 122.5994 & 6.29817 \\
\hline 652 & 14.0673 & 538.3851 & 541.0781 & 541.0781 & 122.5994 & 6.2906 \\
\hline 653 & 14.0843 & 561.8364 & 540.8958 & 540.8958 & 122.5994 & 6.28304 \\
\hline 654 & 14.1013 & 565.0365 & 540.7136 & 540.7135 & 122.5994 & 6.27551 \\
\hline 655 & 14.1183 & 537.8876 & 540.5313 & 540.5313 & 122.5994 & 6.26799 \\
\hline 656 & 14.1353 & 534.0513 & 540.3489 & 540.349 & 122.5994 & 6.26049 \\
\hline 657 & 14.1523 & 538.2501 & 540.1667 & 540.1667 & 122.5994 & 6.25301 \\
\hline 658 & 14.1693 & 540.7683 & 539.9844 & 539.9844 & 122.5994 & 6.24554 \\
\hline 659 & 14.1863 & 552.561 & 539.8021 & 539.8021 & 122.5994 & 6.2381 \\
\hline 660 & 14.2033 & 533.1772 & 539.6198 & 539.6198 & 122.5994 & 6.23067 \\
\hline 661 & 14.2203 & 552.2287 & 539.4375 & 539.4375 & 122.5994 & 6.22326 \\
\hline 662 & 14.2373 & 541.0388 & 539.2552 & 539.2552 & 122.5994 & 6.21587 \\
\hline 663 & 14.2543 & 508.4102 & 539.0729 & 539.0729 & 122.5994 & 6.20849 \\
\hline 664 & 14.2713 & 525.3428 & 538.8906 & 538.8906 & 122.5994 & 6.20113 \\
\hline 665 & 14.2883 & 532.7113 & 538.7083 & 538.7083 & 122.5994 & 6.19379 \\
\hline 666 & 14.3053 & 555.895 & 538.5261 & 538.526 & 122.5994 & 6.18647 \\
\hline 667 & 14.3223 & 524.4559 & 538.3438 & 538.3438 & 122.5994 & 6.17917 \\
\hline 668 & 14.3393 & 547.3063 & 538.1614 & 538.1615 & 122.5994 & 6.17188 \\
\hline 669 & 14.3563 & 533.0931 & 537.9792 & 537.9792 & 122.5994 & 6.16461 \\
\hline 670 & 14.3733 & 539.1237 & 537.7969 & 537.7969 & 122.5994 & 6.15736 \\
\hline 671 & 14.3903 & 557.7423 & 537.6146 & 537.6146 & 122.5994 & 6.15012 \\
\hline 672 & 14.4073 & 546.1319 & 537.4323 & 537.4323 & 122.5994 & 6.1429 \\
\hline 673 & 14.4243 & 553.3822 & 537.25 & 537.25 & 122.5994 & 6.1357 \\
\hline 674 & 14.4413 & 548.7032 & 536.8646 & 536.8646 & 122.5994 & 6.12851 \\
\hline 675 & 14.4583 & 517.5678 & 536.4792 & 536.4792 & 122.5994 & 6.12135 \\
\hline 676 & 14.4753 & 520.3184 & 536.0938 & 536.0938 & 122.5994 & 6.1142 \\
\hline 677 & 14.4923 & 528.9796 & 535.7083 & 535.7083 & 122.5994 & 6.10706 \\
\hline 678 & 14.5093 & 555.6366 & 535.3229 & 535.3229 & 122.5994 & 6.09995 \\
\hline 679 & 14.5263 & 551.0829 & 534.9375 & 534.9375 & 122.5994 & 6.09284 \\
\hline 680 & 14.5433 & 539.2459 & 534.5521 & 534.5521 & 122.5994 & 6.08576 \\
\hline 681 & 14.5603 & 545.8473 & 534.1667 & 534.1667 & 122.5994 & 6.07869 \\
\hline 682 & 14.5773 & 529.4799 & 533.7813 & 533.7813 & 122.5994 & 6.07164 \\
\hline 683 & 14.5943 & 504.6193 & 533.3958 & 533.3958 & 122.5994 & 6.06461 \\
\hline 684 & 14.6113 & 503.5321 & 533.0104 & 533.0104 & 122.5994 & 6.05759 \\
\hline
\end{tabular}




\begin{tabular}{|c|c|c|c|c|c|c|}
\hline 685 & 14.6283 & 542.6264 & 532.625 & 532.625 & 122.5994 & 6.05059 \\
\hline 686 & 14.6453 & 529.5204 & 532.2396 & 532.2396 & 122.5994 & 6.0436 \\
\hline 687 & 14.6623 & 516.3909 & 531.8542 & 531.8542 & 122.5994 & 6.03664 \\
\hline 688 & 14.6793 & 518.8564 & 531.4688 & 531.4688 & 122.5994 & 6.02968 \\
\hline 689 & 14.6963 & 505.2984 & 531.0833 & 531.0833 & 122.5994 & 6.02275 \\
\hline 690 & 14.7133 & 531.855 & 530.6979 & 530.6979 & 122.5994 & 6.01583 \\
\hline 691 & 14.7303 & 496.0814 & 530.3125 & 530.3125 & 122.5994 & 6.00892 \\
\hline 692 & 14.7473 & 515.6639 & 529.9271 & 529.9271 & 122.5994 & 6.00203 \\
\hline 693 & 14.7643 & 532.0949 & 529.5417 & 529.5417 & 122.5994 & 5.99516 \\
\hline 694 & 14.7813 & 521.0783 & 529.1563 & 529.1563 & 122.5994 & 5.9883 \\
\hline 695 & 14.7983 & 490.5185 & 528.7708 & 528.7708 & 122.5994 & 5.98146 \\
\hline 696 & 14.8153 & 544.8371 & 528.3854 & 528.3854 & 122.5994 & 5.97464 \\
\hline 697 & 14.8323 & 529.4678 & 528 & 528 & 122.5994 & 5.96783 \\
\hline 698 & 14.8493 & 533.5749 & 527.5833 & 527.5833 & 122.5994 & 5.96103 \\
\hline 699 & 14.8663 & 529.0946 & 527.1667 & 527.1667 & 122.5994 & 5.95425 \\
\hline 700 & 14.8833 & 535.2789 & 526.75 & 526.75 & 122.5994 & 5.94749 \\
\hline 701 & 14.9003 & 576.3061 & 526.3333 & 526.3333 & 122.5994 & 5.94074 \\
\hline 702 & 14.9173 & 575.7649 & 525.9167 & 525.9167 & 122.5994 & 5.93401 \\
\hline 703 & 14.9343 & 517.9323 & 525.5 & 525.5 & 122.5994 & 5.9273 \\
\hline 704 & 14.9513 & 504.0463 & 525.0833 & 525.0833 & 122.5994 & 5.92059 \\
\hline 705 & 14.9683 & 523.9377 & 524.6667 & 524.6667 & 122.5994 & 5.91391 \\
\hline 706 & 14.9853 & 539.8009 & 524.25 & 524.25 & 122.5994 & 5.90724 \\
\hline 707 & 15.0023 & 508.7183 & 523.8333 & 523.8333 & 122.5994 & 5.90058 \\
\hline 708 & 15.0193 & 540.2261 & 523.4167 & 523.4167 & 122.5994 & 5.89394 \\
\hline 709 & 15.0363 & 559.8763 & 523 & 523 & 122.5994 & 5.88732 \\
\hline 710 & 15.0533 & 583.2314 & 522.5833 & 522.5833 & 122.5994 & 5.88071 \\
\hline 711 & 15.0703 & 529.8116 & 522.1667 & 522.1667 & 122.5994 & 5.87411 \\
\hline 712 & 15.0873 & 517.1429 & 521.75 & 521.75 & 122.5994 & 5.86753 \\
\hline 713 & 15.1043 & 512.188 & 521.3333 & 521.3333 & 122.5994 & 5.86096 \\
\hline 714 & 15.1213 & 510.6843 & 520.9167 & 520.9167 & 122.5994 & 5.85441 \\
\hline 715 & 15.1383 & 525.2256 & 520.5 & 520.5 & 122.5994 & 5.84788 \\
\hline 716 & 15.1553 & 575.6564 & 520.0833 & 520.0833 & 122.5994 & 5.84136 \\
\hline 717 & 15.1723 & 563.6948 & 519.6667 & 519.6667 & 122.5994 & 5.83485 \\
\hline 718 & 15.1893 & 522.4675 & 519.25 & 519.25 & 122.5994 & 5.82836 \\
\hline 719 & 15.2063 & 532.2572 & 518.8333 & 518.8333 & 122.5994 & 5.82188 \\
\hline 720 & 15.2233 & 547.5702 & 518.4167 & 518.4167 & 122.5994 & 5.81542 \\
\hline 721 & 15.2403 & 518.4835 & 518 & 518 & 122.5994 & 5.80897 \\
\hline 722 & 15.2573 & 495.2427 & 517.8646 & 517.8646 & 122.5994 & 5.80253 \\
\hline 723 & 15.2743 & 509.0047 & 517.7292 & 517.7292 & 122.5994 & 5.79611 \\
\hline 724 & 15.2913 & 522.9264 & 517.5938 & 517.5938 & 122.5994 & 5.78971 \\
\hline 725 & 15.3083 & 521.0583 & 517.4583 & 517.4583 & 122.5994 & 5.78332 \\
\hline 726 & 15.3253 & 540.2727 & 517.3229 & 517.3229 & 122.5994 & 5.77694 \\
\hline 727 & 15.3423 & 545.3483 & 517.1875 & 517.1875 & 122.5994 & 5.77058 \\
\hline 728 & 15.3593 & 511.9163 & 517.0521 & 517.0521 & 122.5994 & 5.76423 \\
\hline 729 & 15.3763 & 506.2414 & 516.9167 & 516.9167 & 122.5994 & 5.75789 \\
\hline 730 & 15.3933 & 516.9929 & 516.7813 & 516.7813 & 122.5994 & 5.75157 \\
\hline 731 & 15.4103 & 539.4142 & 516.6458 & 516.6458 & 122.5994 & 5.74527 \\
\hline 732 & 15.4273 & 527.379 & 516.5104 & 516.5104 & 122.5994 & 5.73897 \\
\hline 733 & 15.4443 & 523.2769 & 516.375 & 516.375 & 122.5994 & 5.73269 \\
\hline
\end{tabular}




\begin{tabular}{|c|c|c|c|c|c|c|}
\hline 734 & 15.4613 & 545.8659 & 516.2396 & 516.2396 & 122.5994 & 5.72643 \\
\hline 735 & 15.4783 & 515.7847 & 516.1042 & 516.1042 & 122.5994 & 5.72018 \\
\hline 736 & 15.4953 & 521.0505 & 515.9688 & 515.9688 & 122.5994 & 5.71394 \\
\hline 737 & 15.5123 & 509.2087 & 515.8333 & 515.8333 & 122.5994 & 5.70772 \\
\hline 738 & 15.5293 & 494.7604 & 515.6979 & 515.6979 & 122.5994 & 5.70151 \\
\hline 739 & 15.5463 & 512.5835 & 515.5625 & 515.5625 & 122.5994 & 5.69531 \\
\hline 740 & 15.5633 & 511.1908 & 515.4271 & 515.4271 & 122.5994 & 5.68913 \\
\hline 741 & 15.5803 & 540.3948 & 515.2917 & 515.2917 & 122.5994 & 5.68296 \\
\hline 742 & 15.5973 & 547.3067 & 515.1563 & 515.1563 & 122.5994 & 5.6768 \\
\hline 743 & 15.6143 & 556.6104 & 515.0208 & 515.0208 & 122.5994 & 5.67066 \\
\hline 744 & 15.6313 & 459.2979 & 514.8854 & 514.8854 & 122.5994 & 5.66453 \\
\hline 745 & 15.6483 & 528.9425 & 514.75 & 514.75 & 122.5994 & 5.65842 \\
\hline 746 & 15.6653 & 525.2445 & 514.6146 & 514.6146 & 122.5994 & 5.65231 \\
\hline 747 & 15.6823 & 551.5436 & 514.4792 & 514.4792 & 122.5994 & 5.64622 \\
\hline 748 & 15.6993 & 536.8827 & 514.3438 & 514.3438 & 122.5994 & 5.64015 \\
\hline 749 & 15.7163 & 519.8497 & 514.2083 & 514.2083 & 122.5994 & 5.63409 \\
\hline 750 & 15.7333 & 522.5167 & 514.0729 & 514.0729 & 122.5994 & 5.62804 \\
\hline 751 & 15.7503 & 534.7904 & 513.9375 & 513.9375 & 122.5994 & 5.622 \\
\hline 752 & 15.7673 & 484.709 & 513.8021 & 513.8021 & 122.5994 & 5.61598 \\
\hline 753 & 15.7843 & 499.9264 & 513.6667 & 513.6667 & 122.5994 & 5.60997 \\
\hline 754 & 15.8013 & 550.3716 & 513.5313 & 513.5313 & 122.5994 & 5.60397 \\
\hline 755 & 15.8183 & 537.7228 & 513.3958 & 513.3958 & 122.5994 & 5.59798 \\
\hline 756 & 15.8353 & 518.7359 & 513.2604 & 513.2604 & 122.5994 & 5.59201 \\
\hline 757 & 15.8523 & 512.892 & 513.125 & 513.125 & 122.5994 & 5.58605 \\
\hline 758 & 15.8693 & 530.4758 & 512.9896 & 512.9896 & 122.5994 & 5.58011 \\
\hline 759 & 15.8863 & 571.4793 & 512.8542 & 512.8542 & 122.5994 & 5.57418 \\
\hline 760 & 15.9033 & 519.9967 & 512.7188 & 512.7188 & 122.5994 & 5.56826 \\
\hline 761 & 15.9203 & 521.1909 & 512.5833 & 512.5833 & 122.5994 & 5.56235 \\
\hline 762 & 15.9373 & 543.0914 & 512.4479 & 512.4479 & 122.5994 & 5.55645 \\
\hline 763 & 15.9543 & 521.6568 & 512.3125 & 512.3125 & 122.5994 & 5.55057 \\
\hline 764 & 15.9713 & 524.3898 & 512.1771 & 512.1771 & 122.5994 & 5.5447 \\
\hline 765 & 15.9883 & 518.4351 & 512.0417 & 512.0417 & 122.5994 & 5.53884 \\
\hline 766 & 16.0053 & 510.7403 & 511.9063 & 511.9063 & 122.5994 & 5.533 \\
\hline 767 & 16.0223 & 502.1419 & 511.7708 & 511.7708 & 122.5994 & 5.52717 \\
\hline 768 & 16.0393 & 515.685 & 511.6354 & 511.6354 & 122.5994 & 5.52135 \\
\hline 769 & 16.0563 & 528.5988 & 511.5 & 511.5 & 122.5994 & 5.51554 \\
\hline 770 & 16.0733 & 532.4364 & 511.2292 & 511.2292 & 122.5994 & 5.50974 \\
\hline 771 & 16.0903 & 529.7575 & 510.9583 & 510.9583 & 122.5994 & 5.50396 \\
\hline 772 & 16.1073 & 527.9605 & 510.6875 & 510.6875 & 122.5994 & 5.49819 \\
\hline 773 & 16.1243 & 519.6689 & 510.4167 & 510.4167 & 122.5994 & 5.49243 \\
\hline 774 & 16.1413 & 480.1071 & 510.1458 & 510.1458 & 122.5994 & 5.48668 \\
\hline 775 & 16.1583 & 488.0832 & 509.875 & 509.875 & 122.5994 & 5.48095 \\
\hline 776 & 16.1753 & 504.8008 & 509.6042 & 509.6042 & 122.5994 & 5.47523 \\
\hline 777 & 16.1923 & 506.2595 & 509.3333 & 509.3333 & 122.5994 & 5.46952 \\
\hline 778 & 16.2093 & 535.8666 & 509.0625 & 509.0625 & 122.5994 & 5.46382 \\
\hline 779 & 16.2263 & 547.5702 & 508.7917 & 508.7917 & 122.5994 & 5.45813 \\
\hline 780 & 16.2433 & 558.3257 & 508.5208 & 508.5208 & 122.5994 & 5.45246 \\
\hline 781 & 16.2603 & 525.3825 & 508.25 & 508.25 & 122.5994 & 5.4468 \\
\hline 782 & 16.2773 & 504.4181 & 507.9792 & 507.9792 & 122.5994 & 5.44115 \\
\hline
\end{tabular}




\begin{tabular}{|c|c|c|c|c|c|c|}
\hline 783 & 16.2943 & 512.9423 & 507.7083 & 507.7083 & 122.5994 & 5.43551 \\
\hline 784 & 16.3113 & 511.4027 & 507.4375 & 507.4375 & 122.5994 & 5.42988 \\
\hline 785 & 16.3283 & 499.0676 & 507.1667 & 507.1667 & 122.5994 & 5.42427 \\
\hline 786 & 16.3453 & 493.3376 & 506.8958 & 506.8958 & 122.5994 & 5.41866 \\
\hline 787 & 16.3623 & 521.6351 & 506.625 & 506.625 & 122.5994 & 5.41307 \\
\hline 788 & 16.3793 & 494.9541 & 506.3542 & 506.3542 & 122.5994 & 5.40749 \\
\hline 789 & 16.3963 & 522.9948 & 506.0833 & 506.0833 & 122.5994 & 5.40192 \\
\hline 790 & 16.4133 & 472.1081 & 505.8125 & 505.8125 & 122.5994 & 5.39637 \\
\hline 791 & 16.4303 & 506.1823 & 505.5417 & 505.5417 & 122.5994 & 5.39082 \\
\hline 792 & 16.4473 & 501.9439 & 505.2708 & 505.2708 & 122.5994 & 5.38529 \\
\hline 793 & 16.4643 & 510.4012 & 505 & 505 & 122.5994 & 5.37977 \\
\hline 794 & 16.4813 & 502.3315 & 505.25 & 505.25 & 122.5994 & 5.37426 \\
\hline 795 & 16.4983 & 509.9561 & 505.5 & 505.5 & 122.5994 & 5.36876 \\
\hline 796 & 16.5153 & 508.0869 & 505.75 & 505.75 & 122.5994 & 5.36327 \\
\hline 797 & 16.5323 & 549.6238 & 506 & 506 & 122.5994 & 5.35779 \\
\hline 798 & 16.5493 & 472.4621 & 506.25 & 506.25 & 122.5994 & 5.35233 \\
\hline 799 & 16.5663 & 505.4961 & 506.5 & 506.5 & 122.5994 & 5.34687 \\
\hline 800 & 16.5833 & 515.0728 & 506.75 & 506.75 & 122.5994 & 5.34143 \\
\hline 801 & 16.6003 & 562.1655 & 507 & 507 & 122.5994 & 5.336 \\
\hline 802 & 16.6173 & 522.6218 & 507.25 & 507.25 & 122.5994 & 5.33058 \\
\hline 803 & 16.6343 & 499.4855 & 507.5 & 507.5 & 122.5994 & 5.32517 \\
\hline 804 & 16.6513 & 500.3439 & 507.75 & 507.75 & 122.5994 & 5.31977 \\
\hline 805 & 16.6683 & 514.4417 & 508 & 508 & 122.5994 & 5.31438 \\
\hline 806 & 16.6853 & 509.0321 & 509.5799 & 508.25 & 122.5994 & 5.309 \\
\hline 807 & 16.7023 & 527.5067 & 509.871 & 508.5 & 122.5994 & 5.30364 \\
\hline 808 & 16.7193 & 533.62 & 510.1639 & 508.75 & 122.5994 & 5.29828 \\
\hline 809 & 16.7363 & 526.5642 & 511.1281 & 509 & 122.5994 & 5.29294 \\
\hline 810 & 16.7533 & 515.2996 & 511.4461 & 509.25 & 122.5994 & 5.28761 \\
\hline 811 & 16.7703 & 527.1182 & 511.7674 & 509.5 & 122.5994 & 5.28229 \\
\hline 812 & 16.7873 & 526.4215 & 512.0922 & 509.75 & 122.5994 & 5.27698 \\
\hline 813 & 16.8043 & 508.3264 & 512.4208 & 510 & 122.5994 & 5.27168 \\
\hline 814 & 16.8213 & 497.5849 & 512.7533 & 510.25 & 122.5994 & 5.26639 \\
\hline 815 & 16.8383 & 502.4392 & 513.0901 & 510.5 & 122.5994 & 5.26111 \\
\hline 816 & 16.8553 & 510.163 & 513.4315 & 510.75 & 122.5994 & 5.25584 \\
\hline 817 & 16.8723 & 509.3097 & 513.7778 & 511 & 122.5994 & 5.25058 \\
\hline 818 & 16.8893 & 515.2996 & 514.0042 & 511.125 & 122.5994 & 5.24534 \\
\hline 819 & 16.9063 & 526.2985 & 514.2364 & 511.25 & 122.5994 & 5.2401 \\
\hline 820 & 16.9233 & 502.7116 & 514.4745 & 511.375 & 122.5994 & 5.23487 \\
\hline 821 & 16.9403 & 512.9589 & 514.7192 & 511.5 & 122.5994 & 5.22966 \\
\hline 822 & 16.9573 & 510.899 & 514.9708 & 511.625 & 122.5994 & 5.22445 \\
\hline 823 & 16.9743 & 523.6312 & 515.23 & 511.75 & 122.5994 & 5.21926 \\
\hline 824 & 16.9913 & 533.62 & 515.4974 & 511.875 & 122.5994 & 5.21408 \\
\hline 825 & 17.0083 & 533.62 & 515.7737 & 512 & 122.5994 & 5.2089 \\
\hline 826 & 17.0253 & 544.0337 & 516.0595 & 512.125 & 122.5994 & 5.20374 \\
\hline 827 & 17.0423 & 533.234 & 516.3557 & 512.25 & 122.5994 & 5.19859 \\
\hline 828 & 17.0593 & 510.9473 & 516.6632 & 512.375 & 122.5994 & 5.19345 \\
\hline 829 & 17.0763 & 509.9373 & 516.983 & 512.5 & 122.5994 & 5.18831 \\
\hline 830 & 17.0933 & 533.7711 & 517.3162 & 512.625 & 122.5994 & 5.18319 \\
\hline 831 & 17.1103 & 530.3169 & 517.6641 & 512.75 & 122.5994 & 5.17808 \\
\hline
\end{tabular}




\begin{tabular}{|c|c|c|c|c|c|c|}
\hline 832 & 17.1273 & 528.9493 & 518.028 & 512.875 & 122.5994 & 5.17298 \\
\hline 833 & 17.1443 & 541.532 & 518.4095 & 513 & 122.5994 & 5.16789 \\
\hline 834 & 17.1613 & 555.8875 & 518.8104 & 513.125 & 122.5994 & 5.16281 \\
\hline 835 & 17.1783 & 471.5795 & 519.2327 & 513.25 & 122.5994 & 5.15774 \\
\hline 836 & 17.1953 & 567.6737 & 519.6785 & 513.375 & 122.5994 & 5.15268 \\
\hline 837 & 17.2123 & 498.2128 & 520.1505 & 513.5 & 122.5994 & 5.14762 \\
\hline 838 & 17.2293 & 524.3782 & 520.6516 & 513.625 & 122.5994 & 5.14258 \\
\hline 839 & 17.2463 & 518.6173 & 521.1851 & 513.75 & 122.5994 & 5.13755 \\
\hline 840 & 17.2633 & 483.4085 & 521.7552 & 513.875 & 122.5994 & 5.13253 \\
\hline 841 & 17.2803 & 548.539 & 522.3667 & 514 & 122.5994 & 5.12752 \\
\hline 842 & 17.2973 & 502.6265 & 523.3641 & 514.4635 & 122.5994 & 5.12252 \\
\hline 843 & 17.3143 & 510.711 & 524.4163 & 514.9271 & 122.5994 & 5.11753 \\
\hline 844 & 17.3313 & 513.2902 & 525.5328 & 515.3906 & 122.5994 & 5.11255 \\
\hline 845 & 17.3483 & 527.5468 & 526.7261 & 515.8542 & 122.5994 & 5.10758 \\
\hline 846 & 17.3653 & 515.7622 & 528.0125 & 516.3177 & 122.5994 & 5.10261 \\
\hline 847 & 17.3823 & 503.7301 & 529.4141 & 516.7813 & 122.5994 & 5.09766 \\
\hline 848 & 17.3993 & 530.0441 & 530.9605 & 517.2448 & 122.5994 & 5.09272 \\
\hline 849 & 17.4163 & 535.8394 & 532.6909 & 517.7083 & 122.5994 & 5.08779 \\
\hline 850 & 17.4333 & 539.1875 & 534.658 & 518.1719 & 122.5994 & 5.08286 \\
\hline 851 & 17.4503 & 526.3643 & 536.9294 & 518.6354 & 122.5994 & 5.07795 \\
\hline 852 & 17.4673 & 528.4529 & 539.592 & 519.099 & 122.5994 & 5.07305 \\
\hline 853 & 17.4843 & 533.8597 & 542.7546 & 519.5625 & 122.5994 & 5.06815 \\
\hline 854 & 17.5013 & 509.4545 & 546.5486 & 520.026 & 122.5994 & 5.06327 \\
\hline 855 & 17.5183 & 520.3798 & 551.129 & 520.4896 & 122.5994 & 5.05839 \\
\hline 856 & 17.5353 & 547.64 & 556.6712 & 520.9531 & 122.5994 & 5.05353 \\
\hline 857 & 17.5523 & 566.0852 & 563.3657 & 521.4167 & 122.5994 & 5.04867 \\
\hline 858 & 17.5693 & 552.2603 & 571.4086 & 521.8802 & 122.5994 & 5.04382 \\
\hline 859 & 17.5863 & 550.0983 & 580.9873 & 522.3438 & 122.5994 & 5.03899 \\
\hline 860 & 17.6033 & 543.0731 & 592.2676 & 522.8073 & 122.5994 & 5.03416 \\
\hline 861 & 17.6203 & 559.6413 & 605.3676 & 523.2708 & 122.5994 & 5.02934 \\
\hline 862 & 17.6373 & 563.3487 & 620.3387 & 523.7344 & 122.5994 & 5.02453 \\
\hline 863 & 17.6543 & 576.3924 & 637.139 & 524.1979 & 122.5994 & 5.01973 \\
\hline 864 & 17.6713 & 589.2086 & 655.6047 & 524.6615 & 122.5994 & 5.01494 \\
\hline 865 & 17.6883 & 583.671 & 675.4174 & 525.125 & 122.5994 & 5.01016 \\
\hline 866 & 17.7053 & 603.6326 & 696.0661 & 525.5885 & 122.5994 & 5.00539 \\
\hline 867 & 17.7223 & 624.5298 & 716.8091 & 526.0521 & 122.5994 & 5.00062 \\
\hline 868 & 17.7393 & 629.9496 & 736.6553 & 526.5156 & 122.5994 & 4.99587 \\
\hline 869 & 17.7563 & 657.607 & 754.3659 & 526.9792 & 122.5994 & 4.99112 \\
\hline 870 & 17.7733 & 681.7267 & 768.5969 & 527.4427 & 122.5994 & 4.98639 \\
\hline 871 & 17.7903 & 752.5958 & 778.0956 & 527.9063 & 122.5994 & 4.98166 \\
\hline 872 & 17.8073 & 737.8334 & 781.9574 & 528.3698 & 122.5994 & 4.97694 \\
\hline 873 & 17.8243 & 740.1491 & 779.8611 & 528.8333 & 122.5994 & 4.97224 \\
\hline 874 & 17.8413 & 815.6861 & 772.1177 & 529.2969 & 122.5994 & 4.96754 \\
\hline 875 & 17.8583 & 844.566 & 759.5749 & 529.7604 & 122.5994 & 4.96285 \\
\hline 876 & 17.8753 & 818.2537 & 743.4153 & 530.224 & 122.5994 & 4.95816 \\
\hline 877 & 17.8923 & 802.6288 & 724.9307 & 530.6875 & 122.5994 & 4.95349 \\
\hline 878 & 17.9093 & 747.8031 & 705.3358 & 531.151 & 122.5994 & 4.94883 \\
\hline 879 & 17.9263 & 673.7161 & 685.6441 & 531.6146 & 122.5994 & 4.94417 \\
\hline 880 & 17.9433 & 642.255 & 666.6407 & 532.0781 & 122.5994 & 4.93953 \\
\hline
\end{tabular}




\begin{tabular}{|c|c|c|c|c|c|c|}
\hline 881 & 17.9603 & 566.8529 & 648.8801 & 532.5417 & 122.5994 & 4.93489 \\
\hline 882 & 17.9773 & 603.2506 & 632.7187 & 533.0052 & 122.5994 & 4.93026 \\
\hline 883 & 17.9943 & 595.5931 & 618.3508 & 533.4688 & 122.5994 & 4.92564 \\
\hline 884 & 18.0113 & 595.3711 & 605.8419 & 533.9323 & 122.5994 & 4.92103 \\
\hline 885 & 18.0283 & 589.2762 & 595.1581 & 534.3958 & 122.5994 & 4.91643 \\
\hline 886 & 18.0453 & 532.8528 & 586.1932 & 534.8594 & 122.5994 & 4.91184 \\
\hline 887 & 18.0623 & 519.4545 & 578.7918 & 535.3229 & 122.5994 & 4.90725 \\
\hline 888 & 18.0793 & 575.6415 & 572.7712 & 535.7865 & 122.5994 & 4.90267 \\
\hline 889 & 18.0963 & 524.8119 & 567.9361 & 536.25 & 122.5994 & 4.89811 \\
\hline 890 & 18.1133 & 560.4849 & 563.9561 & 536.5729 & 122.5994 & 4.89355 \\
\hline 891 & 18.1303 & 526.754 & 560.7944 & 536.8958 & 122.5994 & 4.889 \\
\hline 892 & 18.1473 & 558.4832 & 558.2931 & 537.2188 & 122.5994 & 4.88446 \\
\hline 893 & 18.1643 & 525.1861 & 556.3167 & 537.5417 & 122.5994 & 4.87992 \\
\hline 894 & 18.1813 & 545.5753 & 554.7527 & 537.8646 & 122.5994 & 4.8754 \\
\hline 895 & 18.1983 & 541.5505 & 553.5107 & 538.1875 & 122.5994 & 4.87088 \\
\hline 896 & 18.2153 & 543.9273 & 552.5189 & 538.5104 & 122.5994 & 4.86638 \\
\hline 897 & 18.2323 & 521.063 & 551.7223 & 538.8333 & 122.5994 & 4.86188 \\
\hline 898 & 18.2493 & 537.0317 & 551.0788 & 539.1563 & 122.5994 & 4.85739 \\
\hline 899 & 18.2663 & 555.8923 & 550.5569 & 539.4792 & 122.5994 & 4.8529 \\
\hline 900 & 18.2833 & 535.2087 & 550.1332 & 539.8021 & 122.5994 & 4.84843 \\
\hline 901 & 18.3003 & 538.2501 & 549.7899 & 540.125 & 122.5994 & 4.84396 \\
\hline 902 & 18.3173 & 541.8495 & 549.5139 & 540.4479 & 122.5994 & 4.83951 \\
\hline 903 & 18.3343 & 543.7084 & 549.2949 & 540.7708 & 122.5994 & 4.83506 \\
\hline 904 & 18.3513 & 546.284 & 549.1252 & 541.0938 & 122.5994 & 4.83062 \\
\hline 905 & 18.3683 & 561.243 & 548.9985 & 541.4167 & 122.5994 & 4.82618 \\
\hline 906 & 18.3853 & 564.2075 & 548.9099 & 541.7396 & 122.5994 & 4.82176 \\
\hline 907 & 18.4023 & 543.2928 & 548.8552 & 542.0625 & 122.5994 & 4.81734 \\
\hline 908 & 18.4193 & 536.3491 & 548.831 & 542.3854 & 122.5994 & 4.81294 \\
\hline 909 & 18.4363 & 550.088 & 548.8344 & 542.7083 & 122.5994 & 4.80854 \\
\hline 910 & 18.4533 & 552.197 & 548.863 & 543.0313 & 122.5994 & 4.80414 \\
\hline 911 & 18.4703 & 552.2603 & 548.9149 & 543.3542 & 122.5994 & 4.79976 \\
\hline 912 & 18.4873 & 557.9369 & 548.9885 & 543.6771 & 122.5994 & 4.79539 \\
\hline 913 & 18.5043 & 552.8994 & 549.0826 & 544 & 122.5994 & 4.79102 \\
\hline 914 & 18.5213 & 523.8564 & 549.8315 & 544.9583 & 122.5994 & 4.78666 \\
\hline 915 & 18.5383 & 522.9162 & 550.5996 & 545.9167 & 122.5994 & 4.78231 \\
\hline 916 & 18.5553 & 561.2549 & 551.3862 & 546.875 & 122.5994 & 4.77797 \\
\hline 917 & 18.5723 & 562.5135 & 552.1915 & 547.8333 & 122.5994 & 4.77363 \\
\hline 918 & 18.5893 & 529.8571 & 553.0156 & 548.7917 & 122.5994 & 4.7693 \\
\hline 919 & 18.6063 & 551.3192 & 553.8978 & 549.75 & 122.5994 & 4.76498 \\
\hline 920 & 18.6233 & 554.3987 & 554.7614 & 550.7083 & 122.5994 & 4.76067 \\
\hline 921 & 18.6403 & 571.1423 & 555.6446 & 551.6667 & 122.5994 & 4.75637 \\
\hline 922 & 18.6573 & 583.8153 & 556.5667 & 552.625 & 122.5994 & 4.75207 \\
\hline 923 & 18.6743 & 565.5812 & 557.4886 & 553.5833 & 122.5994 & 4.74779 \\
\hline 924 & 18.6913 & 620.4229 & 558.6955 & 554.5417 & 122.5994 & 4.74351 \\
\hline 925 & 18.7083 & 590.9294 & 559.8843 & 555.5 & 122.5994 & 4.73923 \\
\hline 926 & 18.7253 & 555.5135 & 560.868 & 556.4583 & 122.5994 & 4.73497 \\
\hline 927 & 18.7423 & 574.2083 & 561.8612 & 557.4167 & 122.5994 & 4.73071 \\
\hline 928 & 18.7593 & 575.9448 & 563.5275 & 558.375 & 122.5994 & 4.72646 \\
\hline 929 & 18.7763 & 576.0108 & 564.9143 & 559.3333 & 122.5994 & 4.72222 \\
\hline
\end{tabular}




\begin{tabular}{|c|c|c|c|c|c|c|}
\hline 930 & 18.7933 & 629.4058 & 565.9274 & 560.2917 & 122.5994 & 4.71799 \\
\hline 931 & 18.8103 & 557.164 & 566.9218 & 561.25 & 122.5994 & 4.71376 \\
\hline 932 & 18.8273 & 577.5642 & 568.3956 & 562.2083 & 122.5994 & 4.70955 \\
\hline 933 & 18.8443 & 627.099 & 569.3519 & 563.1667 & 122.5994 & 4.70534 \\
\hline 934 & 18.8613 & 662.2458 & 570.281 & 564.125 & 122.5994 & 4.70113 \\
\hline 935 & 18.8783 & 622.9305 & 571.1873 & 565.0833 & 122.5994 & 4.69694 \\
\hline 936 & 18.8953 & 647.8793 & 572.0773 & 566.0417 & 122.5994 & 4.69275 \\
\hline 937 & 18.9123 & 629.3241 & 571.6504 & 567 & 122.5994 & 4.68857 \\
\hline 938 & 18.9293 & 615.5953 & 572.4322 & 567.8214 & 122.5994 & 4.6844 \\
\hline 939 & 18.9463 & 565.5201 & 573.2183 & 568.6427 & 122.5994 & 4.68023 \\
\hline 940 & 18.9633 & 568.5609 & 573.3702 & 569.4641 & 122.5994 & 4.67608 \\
\hline 941 & 18.9803 & 583.5502 & 574.198 & 570.2855 & 122.5994 & 4.67193 \\
\hline 942 & 18.9973 & 548.7128 & 575.0415 & 571.1069 & 122.5994 & 4.66778 \\
\hline 943 & 19.0143 & 555.1715 & 575.9024 & 571.9282 & 122.5994 & 4.66365 \\
\hline 944 & 19.0313 & 517.8537 & 576.7818 & 572.7496 & 122.5994 & 4.65952 \\
\hline 945 & 19.0483 & 529.3347 & 577.6802 & 573.571 & 122.5994 & 4.6554 \\
\hline 946 & 19.0653 & 574.6574 & 578.5975 & 574.3923 & 122.5994 & 4.65129 \\
\hline 947 & 19.0823 & 544.5551 & 579.5337 & 575.2137 & 122.5994 & 4.64718 \\
\hline 948 & 19.0993 & 558.2614 & 580.5786 & 576.0351 & 122.5994 & 4.64309 \\
\hline 949 & 19.1163 & 568.4433 & 581.5542 & 576.8564 & 122.5994 & 4.63899 \\
\hline 950 & 19.1333 & 541.4176 & 582.548 & 577.6778 & 122.5994 & 4.63491 \\
\hline 951 & 19.1503 & 527.9553 & 583.6048 & 578.4992 & 122.5994 & 4.63084 \\
\hline 952 & 19.1673 & 529.6886 & 584.6364 & 579.3206 & 122.5994 & 4.62677 \\
\hline 953 & 19.1843 & 537.7964 & 585.6871 & 580.1419 & 122.5994 & 4.6227 \\
\hline 954 & 19.2013 & 576.3629 & 586.7576 & 580.9633 & 122.5994 & 4.61865 \\
\hline 955 & 19.2183 & 565.2947 & 587.8491 & 581.7847 & 122.5994 & 4.6146 \\
\hline 956 & 19.2353 & 558.9457 & 588.9633 & 582.606 & 122.5994 & 4.61056 \\
\hline 957 & 19.2523 & 557.1507 & 590.1021 & 583.4274 & 122.5994 & 4.60653 \\
\hline 958 & 19.2693 & 576.7399 & 591.2678 & 584.2488 & 122.5994 & 4.6025 \\
\hline 959 & 19.2863 & 603.269 & 592.4634 & 585.0701 & 122.5994 & 4.59849 \\
\hline 960 & 19.3033 & 589.7308 & 593.6927 & 585.8915 & 122.5994 & 4.59447 \\
\hline 961 & 19.3203 & 583.9876 & 594.9601 & 586.7129 & 122.5994 & 4.59047 \\
\hline 962 & 19.3373 & 588.9353 & 596.0669 & 587.3301 & 122.5994 & 4.58647 \\
\hline 963 & 19.3543 & 597.3205 & 597.2244 & 587.9473 & 122.5994 & 4.58248 \\
\hline 964 & 19.3713 & 593.1639 & 598.4415 & 588.5645 & 122.5994 & 4.5785 \\
\hline 965 & 19.3883 & 608.709 & 599.7294 & 589.1816 & 122.5994 & 4.57452 \\
\hline 966 & 19.4053 & 645.8239 & 601.1027 & 589.7988 & 122.5994 & 4.57055 \\
\hline 967 & 19.4223 & 627.6317 & 602.5798 & 590.416 & 122.5994 & 4.56659 \\
\hline 968 & 19.4393 & 624.0449 & 604.184 & 591.0332 & 122.5994 & 4.56264 \\
\hline 969 & 19.4563 & 629.159 & 605.9449 & 591.6504 & 122.5994 & 4.55869 \\
\hline 970 & 19.4733 & 629.9496 & 607.8997 & 592.2676 & 122.5994 & 4.55475 \\
\hline 971 & 19.4903 & 625.6592 & 610.0938 & 592.8848 & 122.5994 & 4.55081 \\
\hline 972 & 19.5073 & 616.1244 & 612.5829 & 593.502 & 122.5994 & 4.54688 \\
\hline 973 & 19.5243 & 656.5853 & 615.4341 & 594.1191 & 122.5994 & 4.54296 \\
\hline 974 & 19.5413 & 665.3195 & 618.7259 & 594.7363 & 122.5994 & 4.53905 \\
\hline 975 & 19.5583 & 655.8155 & 622.5488 & 595.3535 & 122.5994 & 4.53514 \\
\hline 976 & 19.5753 & 660.3159 & 627.4424 & 595.9707 & 122.5994 & 4.53124 \\
\hline 977 & 19.5923 & 707.5657 & 632.6599 & 596.5879 & 122.5994 & 4.52735 \\
\hline 978 & 19.6093 & 660.6126 & 638.7442 & 597.2051 & 122.5994 & 4.52346 \\
\hline
\end{tabular}




\begin{tabular}{|c|c|c|c|c|c|c|}
\hline 979 & 19.6263 & 667.2248 & 645.9102 & 597.8223 & 122.5994 & 4.51958 \\
\hline 980 & 19.6433 & 700.2358 & 654.0897 & 598.4395 & 122.5994 & 4.51571 \\
\hline 981 & 19.6603 & 676.6755 & 663.4747 & 599.0566 & 122.5994 & 4.51184 \\
\hline 982 & 19.6773 & 740.4394 & 674.1415 & 599.6738 & 122.5994 & 4.50798 \\
\hline 983 & 19.6943 & 744.601 & 686.1285 & 600.291 & 122.5994 & 4.50413 \\
\hline 984 & 19.7113 & 753.3393 & 699.423 & 600.9082 & 122.5994 & 4.50029 \\
\hline 985 & 19.7283 & 826.4981 & 714.3073 & 601.5254 & 122.5994 & 4.49645 \\
\hline 986 & 19.7453 & 818.9552 & 729.7148 & 601.9307 & 122.5994 & 4.49261 \\
\hline 987 & 19.7623 & 846.7288 & 745.9607 & 602.3361 & 122.5994 & 4.48879 \\
\hline 988 & 19.7793 & 856.5781 & 763.5242 & 602.7414 & 122.5994 & 4.48497 \\
\hline 989 & 19.7963 & 892.6941 & 780.4243 & 603.1467 & 122.5994 & 4.48115 \\
\hline 990 & 19.8133 & 914.5129 & 796.9982 & 603.5521 & 122.5994 & 4.47735 \\
\hline 991 & 19.8303 & 932.7036 & 812.7517 & 603.9574 & 122.5994 & 4.47355 \\
\hline 992 & 19.8473 & 902.9873 & 827.1232 & 604.3627 & 122.5994 & 4.46975 \\
\hline 993 & 19.8643 & 876.8339 & 839.4794 & 604.7681 & 122.5994 & 4.46597 \\
\hline 994 & 19.8813 & 929.9582 & 849.4291 & 605.1734 & 122.5994 & 4.46219 \\
\hline 995 & 19.8983 & 946.7549 & 855.6082 & 605.5787 & 122.5994 & 4.45841 \\
\hline 996 & 19.9153 & 936.7039 & 857.7933 & 605.9841 & 122.5994 & 4.45465 \\
\hline 997 & 19.9323 & 957.491 & 855.7022 & 606.3894 & 122.5994 & 4.45088 \\
\hline 998 & 19.9493 & 972.2941 & 849.3986 & 606.7947 & 122.5994 & 4.44713 \\
\hline 999 & 19.9663 & 962.5026 & 839.3033 & 607.2001 & 122.5994 & 4.44338 \\
\hline 1000 & 19.9833 & 933.2474 & 826.1364 & 607.6054 & 122.5994 & 4.43964 \\
\hline 1001 & 20.0003 & 930.2674 & 810.7813 & 608.0107 & 122.5994 & 4.4359 \\
\hline 1002 & 20.0173 & 866.8078 & 794.1718 & 608.4161 & 122.5994 & 4.43218 \\
\hline 1003 & 20.0343 & 825.1074 & 777.1797 & 608.8214 & 122.5994 & 4.42845 \\
\hline 1004 & 20.0513 & 831.874 & 760.5441 & 609.2267 & 122.5994 & 4.42474 \\
\hline 1005 & 20.0683 & 808.8535 & 744.8308 & 609.6321 & 122.5994 & 4.42103 \\
\hline 1006 & 20.0853 & 802.2253 & 730.4344 & 610.0374 & 122.5994 & 4.41732 \\
\hline 1007 & 20.1023 & 763.5171 & 717.5949 & 610.4427 & 122.5994 & 4.41363 \\
\hline 1008 & 20.1193 & 747.1363 & 706.4147 & 610.8481 & 122.5994 & 4.40994 \\
\hline 1009 & 20.1363 & 800.9808 & 696.8846 & 611.2534 & 122.5994 & 4.40625 \\
\hline 1010 & 20.1533 & 771.9281 & 688.6964 & 611.453 & 122.5994 & 4.40257 \\
\hline 1011 & 20.1703 & 750.774 & 681.8865 & 611.6526 & 122.5994 & 4.3989 \\
\hline 1012 & 20.1873 & 702.2606 & 676.2393 & 611.8522 & 122.5994 & 4.39523 \\
\hline 1013 & 20.2043 & 732.0291 & 671.5213 & 612.0518 & 122.5994 & 4.39157 \\
\hline 1014 & 20.2213 & 745.3039 & 667.5088 & 612.2514 & 122.5994 & 4.38792 \\
\hline 1015 & 20.2383 & 710.1179 & 664.0151 & 612.451 & 122.5994 & 4.38427 \\
\hline 1016 & 20.2553 & 702.2631 & 660.9037 & 612.6507 & 122.5994 & 4.38063 \\
\hline 1017 & 20.2723 & 702.2631 & 658.0898 & 612.8503 & 122.5994 & 4.377 \\
\hline 1018 & 20.2893 & 716.286 & 655.5334 & 613.0499 & 122.5994 & 4.37337 \\
\hline 1019 & 20.3063 & 708.6036 & 653.2288 & 613.2495 & 122.5994 & 4.36974 \\
\hline 1020 & 20.3233 & 737.4348 & 651.1914 & 613.4491 & 122.5994 & 4.36613 \\
\hline 1021 & 20.3403 & 679.422 & 649.4479 & 613.6487 & 122.5994 & 4.36252 \\
\hline 1022 & 20.3573 & 670.9879 & 648.0295 & 613.8483 & 122.5994 & 4.35891 \\
\hline 1023 & 20.3743 & 650.4746 & 646.9688 & 614.0479 & 122.5994 & 4.35531 \\
\hline 1024 & 20.3913 & 691.2988 & 646.2991 & 614.2475 & 122.5994 & 4.35172 \\
\hline 1025 & 20.4083 & 658.1312 & 646.0554 & 614.4471 & 122.5994 & 4.34813 \\
\hline 1026 & 20.4253 & 738.1045 & 646.2745 & 614.6467 & 122.5994 & 4.34455 \\
\hline 1027 & 20.4423 & 705.1833 & 646.9954 & 614.8463 & 122.5994 & 4.34098 \\
\hline
\end{tabular}




\begin{tabular}{|c|c|c|c|c|c|c|}
\hline 1028 & 20.4593 & 680.121 & 648.2589 & 615.0459 & 122.5994 & 4.33741 \\
\hline 1029 & 20.4763 & 711.3172 & 650.1116 & 615.2455 & 122.5994 & 4.33385 \\
\hline 1030 & 20.4933 & 654.8263 & 652.5856 & 615.4451 & 122.5994 & 4.33029 \\
\hline 1031 & 20.5103 & 631.616 & 655.7236 & 615.6447 & 122.5994 & 4.32674 \\
\hline 1032 & 20.5273 & 689.7821 & 659.5605 & 615.8443 & 122.5994 & 4.3232 \\
\hline 1033 & 20.5443 & 672.8004 & 664.3038 & 616.0439 & 122.5994 & 4.31966 \\
\hline 1034 & 20.5613 & 652.9749 & 669.5915 & 616.2436 & 122.5994 & 4.31612 \\
\hline 1035 & 20.5783 & 680.3044 & 675.5967 & 616.4432 & 122.5994 & 4.3126 \\
\hline 1036 & 20.5953 & 641.0097 & 682.3762 & 616.6428 & 122.5994 & 4.30907 \\
\hline 1037 & 20.6123 & 676.8954 & 689.6759 & 616.8424 & 122.5994 & 4.30556 \\
\hline 1038 & 20.6293 & 699.9967 & 697.4814 & 617.042 & 122.5994 & 4.30205 \\
\hline 1039 & 20.6463 & 707.6348 & 705.6548 & 617.2416 & 122.5994 & 4.29855 \\
\hline 1040 & 20.6633 & 717.1639 & 714.0431 & 617.4412 & 122.5994 & 4.29505 \\
\hline 1041 & 20.6803 & 744.2486 & 722.5137 & 617.6408 & 122.5994 & 4.29156 \\
\hline 1042 & 20.6973 & 705.3713 & 731.0031 & 617.8404 & 122.5994 & 4.28807 \\
\hline 1043 & 20.7143 & 727.3242 & 739.5917 & 618.04 & 122.5994 & 4.28459 \\
\hline 1044 & 20.7313 & 742.2599 & 748.6184 & 618.2396 & 122.5994 & 4.28111 \\
\hline 1045 & 20.7483 & 745.3039 & 758.9197 & 618.4392 & 122.5994 & 4.27764 \\
\hline 1046 & 20.7653 & 742.6631 & 772.2701 & 618.6388 & 122.5994 & 4.27418 \\
\hline 1047 & 20.7823 & 767.8908 & 792.0287 & 618.8384 & 122.5994 & 4.27072 \\
\hline 1048 & 20.7993 & 822.3671 & 823.6888 & 619.038 & 122.5994 & 4.26727 \\
\hline 1049 & 20.8163 & 866.1959 & 874.5989 & 619.2376 & 122.5994 & 4.26382 \\
\hline 1050 & 20.8333 & 865.3031 & 951.9438 & 619.4372 & 122.5994 & 4.26038 \\
\hline 1051 & 20.8503 & 946.5643 & 1058.908 & 619.6368 & 122.5994 & 4.25695 \\
\hline 1052 & 20.8673 & 1034.896 & 1188.431 & 619.8364 & 122.5994 & 4.25352 \\
\hline 1053 & 20.8843 & 1121.586 & 1314.984 & 620.0361 & 122.5994 & 4.25009 \\
\hline 1054 & 20.9013 & 1352.324 & 1394.131 & 620.2357 & 122.5994 & 4.24667 \\
\hline 1055 & 20.9183 & 1585.06 & 1397.737 & 620.4353 & 122.5994 & 4.24326 \\
\hline 1056 & 20.9353 & 1794.796 & 1342.821 & 620.6349 & 122.5994 & 4.23985 \\
\hline 1057 & 20.9523 & 1730.273 & 1251.141 & 620.8345 & 122.5994 & 4.23645 \\
\hline 1058 & 20.9693 & 1499.365 & 1134.285 & 620.8283 & 122.5994 & 4.23306 \\
\hline 1059 & 20.9863 & 1285.592 & 1013.906 & 620.8222 & 122.5994 & 4.22967 \\
\hline 1060 & 21.0033 & 1152.432 & 910.6007 & 620.8161 & 122.5994 & 4.22628 \\
\hline 1061 & 21.0203 & 988.4023 & 832.0905 & 620.81 & 122.5994 & 4.2229 \\
\hline 1062 & 21.0373 & 822.5821 & 776.8356 & 620.8039 & 122.5994 & 4.21953 \\
\hline 1063 & 21.0543 & 780.9443 & 738.9467 & 620.7977 & 122.5994 & 4.21616 \\
\hline 1064 & 21.0713 & 730.6481 & 714.2275 & 620.7916 & 122.5994 & 4.2128 \\
\hline 1065 & 21.0883 & 709.285 & 697.3257 & 620.7855 & 122.5994 & 4.20944 \\
\hline 1066 & 21.1053 & 692.9744 & 685.0756 & 620.7794 & 122.5994 & 4.20609 \\
\hline 1067 & 21.1223 & 691.7029 & 675.0925 & 620.7732 & 122.5994 & 4.20274 \\
\hline 1068 & 21.1393 & 676.6741 & 667.6832 & 620.7671 & 122.5994 & 4.1994 \\
\hline 1069 & 21.1563 & 660.8222 & 661.6456 & 620.761 & 122.5994 & 4.19606 \\
\hline 1070 & 21.1733 & 610.1589 & 656.4929 & 620.7549 & 122.5994 & 4.19273 \\
\hline 1071 & 21.1903 & 670.2858 & 652.384 & 620.7487 & 122.5994 & 4.18941 \\
\hline 1072 & 21.2073 & 651.851 & 648.9783 & 620.7426 & 122.5994 & 4.18609 \\
\hline 1073 & 21.2243 & 684.0847 & 646.1539 & 620.7365 & 122.5994 & 4.18277 \\
\hline 1074 & 21.2413 & 647.1999 & 643.812 & 620.7304 & 122.5994 & 4.17946 \\
\hline 1075 & 21.2583 & 617.7696 & 641.8734 & 620.7242 & 122.5994 & 4.17616 \\
\hline 1076 & 21.2753 & 636.8444 & 640.2751 & 620.7181 & 122.5994 & 4.17286 \\
\hline
\end{tabular}




\begin{tabular}{|c|c|c|c|c|c|c|}
\hline 1077 & 21.2923 & 611.2626 & 638.9686 & 620.712 & 122.5994 & 4.16957 \\
\hline 1078 & 21.3093 & 595.6079 & 637.9185 & 620.7059 & 122.5994 & 4.16628 \\
\hline 1079 & 21.3263 & 620.0216 & 637.1005 & 620.6997 & 122.5994 & 4.163 \\
\hline 1080 & 21.3433 & 623.2691 & 636.491 & 620.6936 & 122.5994 & 4.15972 \\
\hline 1081 & 21.3603 & 631.4807 & 636.1096 & 620.6875 & 122.5994 & 4.15645 \\
\hline 1082 & 21.3773 & 620.0216 & 635.7451 & 620.4818 & 122.5994 & 4.15318 \\
\hline 1083 & 21.3943 & 628.5091 & 635.6434 & 620.276 & 122.5994 & 4.14992 \\
\hline 1084 & 21.4113 & 651.7313 & 635.7465 & 620.0703 & 122.5994 & 4.14666 \\
\hline 1085 & 21.4283 & 637.5371 & 636.1069 & 619.8646 & 122.5994 & 4.14341 \\
\hline 1086 & 21.4453 & 614.0125 & 636.7002 & 619.6589 & 122.5994 & 4.14016 \\
\hline 1087 & 21.4623 & 630.9162 & 637.6252 & 619.4531 & 122.5994 & 4.13692 \\
\hline 1088 & 21.4793 & 628.7212 & 638.8478 & 619.2474 & 122.5994 & 4.13369 \\
\hline 1089 & 21.4963 & 639.9155 & 640.3654 & 619.0417 & 122.5994 & 4.13046 \\
\hline 1090 & 21.5133 & 627.1003 & 642.1719 & 618.8359 & 122.5994 & 4.12723 \\
\hline 1091 & 21.5303 & 641.4983 & 644.2249 & 618.6302 & 122.5994 & 4.12401 \\
\hline 1092 & 21.5473 & 626.9168 & 646.4772 & 618.4245 & 122.5994 & 4.12079 \\
\hline 1093 & 21.5643 & 610.0664 & 648.8528 & 618.2188 & 122.5994 & 4.11758 \\
\hline 1094 & 21.5813 & 625.0117 & 651.2386 & 618.013 & 122.5994 & 4.11438 \\
\hline 1095 & 21.5983 & 633.1431 & 653.4961 & 617.8073 & 122.5994 & 4.11118 \\
\hline 1096 & 21.6153 & 642.8692 & 655.4553 & 617.6016 & 122.5994 & 4.10798 \\
\hline 1097 & 21.6323 & 645.1721 & 656.934 & 617.3958 & 122.5994 & 4.10479 \\
\hline 1098 & 21.6493 & 612.1868 & 657.7673 & 617.1901 & 122.5994 & 4.10161 \\
\hline 1099 & 21.6663 & 602.7488 & 657.8416 & 616.9844 & 122.5994 & 4.09843 \\
\hline 1100 & 21.6833 & 594.2964 & 657.119 & 616.7786 & 122.5994 & 4.09525 \\
\hline 1101 & 21.7003 & 622.0212 & 655.6407 & 616.5729 & 122.5994 & 4.09209 \\
\hline 1102 & 21.7173 & 650.5554 & 653.514 & 616.3672 & 122.5994 & 4.08892 \\
\hline 1103 & 21.7343 & 630.2415 & 650.8895 & 616.1615 & 122.5994 & 4.08576 \\
\hline 1104 & 21.7513 & 605.5484 & 647.9366 & 615.9557 & 122.5994 & 4.08261 \\
\hline 1105 & 21.7683 & 618.9223 & 644.8207 & 615.75 & 122.5994 & 4.07946 \\
\hline 1106 & 21.7853 & 632.7979 & 641.5063 & 615.3646 & 122.5994 & 4.07631 \\
\hline 1107 & 21.8023 & 603.4248 & 638.1232 & 614.9792 & 122.5994 & 4.07317 \\
\hline 1108 & 21.8193 & 591.3169 & 635.6214 & 614.5938 & 122.5994 & 4.07004 \\
\hline 1109 & 21.8363 & 586.427 & 632.8331 & 614.2083 & 122.5994 & 4.06691 \\
\hline 1110 & 21.8533 & 615.2622 & 630.2247 & 613.8229 & 122.5994 & 4.06378 \\
\hline 1111 & 21.8703 & 611.2615 & 628.2341 & 613.4375 & 122.5994 & 4.06066 \\
\hline 1112 & 21.8873 & 628.1213 & 626.2543 & 613.0521 & 122.5994 & 4.05754 \\
\hline 1113 & 21.9043 & 580.0005 & 624.3407 & 612.6667 & 122.5994 & 4.05443 \\
\hline 1114 & 21.9213 & 585.0793 & 622.8383 & 612.2813 & 122.5994 & 4.05133 \\
\hline 1115 & 21.9383 & 623.7948 & 621.5299 & 611.8958 & 122.5994 & 4.04823 \\
\hline 1116 & 21.9553 & 590.8248 & 620.2899 & 611.5104 & 122.5994 & 4.04513 \\
\hline 1117 & 21.9723 & 591.4172 & 619.2805 & 611.125 & 122.5994 & 4.04204 \\
\hline 1118 & 21.9893 & 665.3057 & 618.3838 & 610.7396 & 122.5994 & 4.03895 \\
\hline 1119 & 22.0063 & 654.1766 & 617.577 & 610.3542 & 122.5994 & 4.03587 \\
\hline 1120 & 22.0233 & 636.2182 & 616.8442 & 609.9688 & 122.5994 & 4.03279 \\
\hline 1121 & 22.0403 & 654.8499 & 616.1744 & 609.5833 & 122.5994 & 4.02972 \\
\hline 1122 & 22.0573 & 696.3419 & 615.5601 & 609.1979 & 122.5994 & 4.02665 \\
\hline 1123 & 22.0743 & 695.4541 & 614.0924 & 608.8125 & 122.5994 & 4.02359 \\
\hline 1124 & 22.0913 & 721.7704 & 613.6046 & 608.4271 & 122.5994 & 4.02053 \\
\hline 1125 & 22.1083 & 732.9239 & 613.1654 & 608.0417 & 122.5994 & 4.01748 \\
\hline
\end{tabular}




\begin{tabular}{|c|c|c|c|c|c|c|}
\hline 1126 & 22.1253 & 701.0941 & 612.3065 & 607.6563 & 122.5994 & 4.01443 \\
\hline 1127 & 22.1423 & 688.683 & 611.9871 & 607.2708 & 122.5994 & 4.01139 \\
\hline 1128 & 22.1593 & 660.5023 & 611.7273 & 606.8854 & 122.5994 & 4.00835 \\
\hline 1129 & 22.1763 & 653.6211 & 611.5363 & 606.5 & 122.5994 & 4.00532 \\
\hline 1130 & 22.1933 & 627.7685 & 611.2446 & 605.9375 & 122.5994 & 4.00229 \\
\hline 1131 & 22.2103 & 621.0942 & 611.0388 & 605.375 & 122.5994 & 3.99926 \\
\hline 1132 & 22.2273 & 628.4215 & 610.9283 & 604.8125 & 122.5994 & 3.99624 \\
\hline 1133 & 22.2443 & 611.3132 & 610.9219 & 604.25 & 122.5994 & 3.99322 \\
\hline 1134 & 22.2613 & 625.0448 & 611.2911 & 603.6875 & 122.5994 & 3.99021 \\
\hline 1135 & 22.2783 & 590.5739 & 611.5201 & 603.125 & 122.5994 & 3.98721 \\
\hline 1136 & 22.2953 & 594.5395 & 611.8662 & 602.5625 & 122.5994 & 3.98421 \\
\hline 1137 & 22.3123 & 622.2299 & 612.3235 & 602 & 122.5994 & 3.98121 \\
\hline 1138 & 22.3293 & 608.9285 & 613.0159 & 601.4375 & 122.5994 & 3.97822 \\
\hline 1139 & 22.3463 & 595.2395 & 613.6571 & 600.875 & 122.5994 & 3.97523 \\
\hline 1140 & 22.3633 & 610.2803 & 614.3453 & 600.3125 & 122.5994 & 3.97224 \\
\hline 1141 & 22.3803 & 627.966 & 615.0354 & 599.75 & 122.5994 & 3.96927 \\
\hline 1142 & 22.3973 & 624.0497 & 615.6691 & 599.1875 & 122.5994 & 3.96629 \\
\hline 1143 & 22.4143 & 603.8016 & 616.1796 & 598.625 & 122.5994 & 3.96332 \\
\hline 1144 & 22.4313 & 609.9219 & 616.5001 & 598.0625 & 122.5994 & 3.96036 \\
\hline 1145 & 22.4483 & 595.9294 & 616.5776 & 597.5 & 122.5994 & 3.9574 \\
\hline 1146 & 22.4653 & 602.5957 & 616.3843 & 596.9375 & 122.5994 & 3.95444 \\
\hline 1147 & 22.4823 & 602.1086 & 615.9238 & 596.375 & 122.5994 & 3.95149 \\
\hline 1148 & 22.4993 & 608.7378 & 615.2289 & 595.8125 & 122.5994 & 3.94854 \\
\hline 1149 & 22.5163 & 615.0515 & 614.3549 & 595.25 & 122.5994 & 3.9456 \\
\hline 1150 & 22.5333 & 597.252 & 613.5056 & 594.6875 & 122.5994 & 3.94266 \\
\hline 1151 & 22.5503 & 627.3882 & 612.4923 & 594.125 & 122.5994 & 3.93973 \\
\hline 1152 & 22.5673 & 602.1467 & 611.5225 & 593.5625 & 122.5994 & 3.9368 \\
\hline 1153 & 22.5843 & 564.1209 & 610.7357 & 593 & 122.5994 & 3.93387 \\
\hline 1154 & 22.6013 & 603.9163 & 610.6977 & 593.0625 & 122.5994 & 3.93095 \\
\hline 1155 & 22.6183 & 606.8528 & 610.9172 & 593.125 & 122.5994 & 3.92803 \\
\hline 1156 & 22.6353 & 564.0716 & 611.4645 & 593.1875 & 122.5994 & 3.92512 \\
\hline 1157 & 22.6523 & 603.3416 & 612.4081 & 593.25 & 122.5994 & 3.92222 \\
\hline 1158 & 22.6693 & 623.4358 & 613.8319 & 593.3125 & 122.5994 & 3.91931 \\
\hline 1159 & 22.6863 & 629.6001 & 615.8077 & 593.375 & 122.5994 & 3.91641 \\
\hline 1160 & 22.7033 & 588.4297 & 618.4075 & 593.4375 & 122.5994 & 3.91352 \\
\hline 1161 & 22.7203 & 590.3017 & 621.7032 & 593.5 & 122.5994 & 3.91063 \\
\hline 1162 & 22.7373 & 600.0016 & 625.7479 & 593.5625 & 122.5994 & 3.90774 \\
\hline 1163 & 22.7543 & 590.5383 & 631.1442 & 593.625 & 122.5994 & 3.90486 \\
\hline 1164 & 22.7713 & 610.3339 & 636.5981 & 593.6875 & 122.5994 & 3.90199 \\
\hline 1165 & 22.7883 & 620.0216 & 643.0073 & 593.75 & 122.5994 & 3.89911 \\
\hline 1166 & 22.8053 & 622.7138 & 650.1028 & 593.8125 & 122.5994 & 3.89625 \\
\hline 1167 & 22.8223 & 657.7466 & 657.9387 & 593.875 & 122.5994 & 3.89338 \\
\hline 1168 & 22.8393 & 628.3831 & 665.924 & 593.9375 & 122.5994 & 3.89052 \\
\hline 1169 & 22.8563 & 620.3333 & 673.9363 & 594 & 122.5994 & 3.88767 \\
\hline 1170 & 22.8733 & 649.2222 & 681.5721 & 594.0625 & 122.5994 & 3.88482 \\
\hline 1171 & 22.8903 & 620.2944 & 688.3494 & 594.125 & 122.5994 & 3.88197 \\
\hline 1172 & 22.9073 & 647.1672 & 693.7584 & 594.1875 & 122.5994 & 3.87913 \\
\hline 1173 & 22.9243 & 656.4862 & 697.3532 & 594.25 & 122.5994 & 3.87629 \\
\hline 1174 & 22.9413 & 653.1554 & 698.8314 & 594.3125 & 122.5994 & 3.87346 \\
\hline
\end{tabular}




\begin{tabular}{|c|c|c|c|c|c|c|}
\hline 1175 & 22.9583 & 637.9494 & 698.1126 & 594.375 & 122.5994 & 3.87063 \\
\hline 1176 & 22.9753 & 664.8704 & 695.3276 & 594.4375 & 122.5994 & 3.8678 \\
\hline 1177 & 22.9923 & 659.0386 & 690.7743 & 594.5 & 122.5994 & 3.86498 \\
\hline 1178 & 23.0093 & 621.6418 & 684.8658 & 594.5625 & 122.5994 & 3.86216 \\
\hline 1179 & 23.0263 & 623.284 & 678.0663 & 594.625 & 122.5994 & 3.85935 \\
\hline 1180 & 23.0433 & 634.8331 & 670.8273 & 594.6875 & 122.5994 & 3.85654 \\
\hline 1181 & 23.0603 & 640.8873 & 663.5599 & 594.75 & 122.5994 & 3.85374 \\
\hline 1182 & 23.0773 & 630.8018 & 656.5836 & 594.8125 & 122.5994 & 3.85093 \\
\hline 1183 & 23.0943 & 651.6843 & 650.1381 & 594.875 & 122.5994 & 3.84814 \\
\hline 1184 & 23.1113 & 647.0314 & 644.3842 & 594.9375 & 122.5994 & 3.84535 \\
\hline 1185 & 23.1283 & 650.5239 & 639.4255 & 595 & 122.5994 & 3.84256 \\
\hline 1186 & 23.1453 & 627.6772 & 635.3108 & 595.0625 & 122.5994 & 3.83977 \\
\hline 1187 & 23.1623 & 633.564 & 632.0558 & 595.125 & 122.5994 & 3.83699 \\
\hline 1188 & 23.1793 & 638.9834 & 629.6517 & 595.1875 & 122.5994 & 3.83422 \\
\hline 1189 & 23.1963 & 636.3285 & 628.0763 & 595.25 & 122.5994 & 3.83145 \\
\hline 1190 & 23.2133 & 624.7302 & 627.2979 & 595.3125 & 122.5994 & 3.82868 \\
\hline 1191 & 23.2303 & 601.1983 & 627.2791 & 595.375 & 122.5994 & 3.82592 \\
\hline 1192 & 23.2473 & 610.7299 & 627.9786 & 595.4375 & 122.5994 & 3.82316 \\
\hline 1193 & 23.2643 & 591.5305 & 629.3497 & 595.5 & 122.5994 & 3.8204 \\
\hline 1194 & 23.2813 & 579.2282 & 631.3391 & 595.5625 & 122.5994 & 3.81765 \\
\hline 1195 & 23.2983 & 561.1017 & 633.8823 & 595.625 & 122.5994 & 3.8149 \\
\hline 1196 & 23.3153 & 596.9657 & 636.8997 & 595.6875 & 122.5994 & 3.81216 \\
\hline 1197 & 23.3323 & 593.4861 & 640.2903 & 595.75 & 122.5994 & 3.80942 \\
\hline 1198 & 23.3493 & 604.042 & 643.9265 & 595.8125 & 122.5994 & 3.80669 \\
\hline 1199 & 23.3663 & 602.4609 & 647.6469 & 595.875 & 122.5994 & 3.80396 \\
\hline 1200 & 23.3833 & 588.6023 & 651.2581 & 595.9375 & 122.5994 & 3.80123 \\
\hline 1201 & 23.4003 & 573.825 & 654.5448 & 596 & 122.5994 & 3.79851 \\
\hline 1202 & 23.4173 & 608.0851 & 657.4984 & 596.2656 & 122.5994 & 3.79579 \\
\hline 1203 & 23.4343 & 619.3387 & 659.7444 & 596.5313 & 122.5994 & 3.79307 \\
\hline 1204 & 23.4513 & 598.1591 & 661.1869 & 596.7969 & 122.5994 & 3.79036 \\
\hline 1205 & 23.4683 & 564.8807 & 661.8248 & 597.0625 & 122.5994 & 3.78765 \\
\hline 1206 & 23.4853 & 548.6005 & 661.7491 & 597.3281 & 122.5994 & 3.78495 \\
\hline 1207 & 23.5023 & 602.2117 & 661.1238 & 597.5938 & 122.5994 & 3.78225 \\
\hline 1208 & 23.5193 & 619.5784 & 660.1616 & 597.8594 & 122.5994 & 3.77955 \\
\hline 1209 & 23.5363 & 624.9702 & 659.0969 & 598.125 & 122.5994 & 3.77686 \\
\hline 1210 & 23.5533 & 610.0366 & 658.1613 & 598.3906 & 122.5994 & 3.77418 \\
\hline 1211 & 23.5703 & 607.0902 & 657.4919 & 598.6563 & 122.5994 & 3.77149 \\
\hline 1212 & 23.5873 & 660.5023 & 657.4034 & 598.9219 & 122.5994 & 3.76881 \\
\hline 1213 & 23.6043 & 660.1035 & 657.951 & 599.1875 & 122.5994 & 3.76614 \\
\hline 1214 & 23.6213 & 658.4581 & 659.1797 & 599.4531 & 122.5994 & 3.76346 \\
\hline 1215 & 23.6383 & 690.2949 & 661.2018 & 599.7188 & 122.5994 & 3.7608 \\
\hline 1216 & 23.6553 & 713.6478 & 663.9701 & 599.9844 & 122.5994 & 3.75813 \\
\hline 1217 & 23.6723 & 686.4528 & 667.4507 & 600.25 & 122.5994 & 3.75547 \\
\hline 1218 & 23.6893 & 683.5564 & 671.5856 & 600.5156 & 122.5994 & 3.75281 \\
\hline 1219 & 23.7063 & 674.0555 & 676.3214 & 600.7813 & 122.5994 & 3.75016 \\
\hline 1220 & 23.7233 & 675.8674 & 681.5961 & 601.0469 & 122.5994 & 3.74751 \\
\hline 1221 & 23.7403 & 650.3785 & 687.3433 & 601.3125 & 122.5994 & 3.74487 \\
\hline 1222 & 23.7573 & 679.731 & 693.4798 & 601.5781 & 122.5994 & 3.74223 \\
\hline 1223 & 23.7743 & 662.5435 & 699.8665 & 601.8438 & 122.5994 & 3.73959 \\
\hline
\end{tabular}




\begin{tabular}{|c|c|c|c|c|c|c|}
\hline 1224 & 23.7913 & 631.6032 & 706.2908 & 602.1094 & 122.5994 & 3.73696 \\
\hline 1225 & 23.8083 & 647.0685 & 712.4799 & 602.375 & 122.5994 & 3.73433 \\
\hline 1226 & 23.8253 & 658.0356 & 717.7549 & 602.4505 & 122.5994 & 3.7317 \\
\hline 1227 & 23.8423 & 656.5229 & 721.8629 & 602.526 & 122.5994 & 3.72908 \\
\hline 1228 & 23.8593 & 685.3409 & 724.3497 & 602.6016 & 122.5994 & 3.72646 \\
\hline 1229 & 23.8763 & 673.8226 & 724.8178 & 602.6771 & 122.5994 & 3.72384 \\
\hline 1230 & 23.8933 & 670.8225 & 723.1175 & 602.7526 & 122.5994 & 3.72123 \\
\hline 1231 & 23.9103 & 654.1882 & 719.2981 & 602.8281 & 122.5994 & 3.71863 \\
\hline 1232 & 23.9273 & 672.9315 & 713.5975 & 602.9036 & 122.5994 & 3.71602 \\
\hline 1233 & 23.9443 & 688.8953 & 706.3873 & 602.9792 & 122.5994 & 3.71342 \\
\hline 1234 & 23.9613 & 684.9621 & 698.1149 & 603.0547 & 122.5994 & 3.71083 \\
\hline 1235 & 23.9783 & 682.0888 & 689.2458 & 603.1302 & 122.5994 & 3.70823 \\
\hline 1236 & 23.9953 & 656.8402 & 680.2072 & 603.2057 & 122.5994 & 3.70565 \\
\hline 1237 & 24.0123 & 632.2956 & 671.3572 & 603.2813 & 122.5994 & 3.70306 \\
\hline 1238 & 24.0293 & 623.0938 & 664.0428 & 603.3568 & 122.5994 & 3.70048 \\
\hline 1239 & 24.0463 & 650.9075 & 655.8075 & 603.4323 & 122.5994 & 3.6979 \\
\hline 1240 & 24.0633 & 652.0876 & 648.86 & 603.5078 & 122.5994 & 3.69533 \\
\hline 1241 & 24.0803 & 638.2889 & 642.7166 & 603.5833 & 122.5994 & 3.69276 \\
\hline 1242 & 24.0973 & 642.153 & 637.6595 & 603.6589 & 122.5994 & 3.69019 \\
\hline 1243 & 24.1143 & 667.4764 & 633.1229 & 603.7344 & 122.5994 & 3.68763 \\
\hline 1244 & 24.1313 & 638.0298 & 629.3065 & 603.8099 & 122.5994 & 3.68507 \\
\hline 1245 & 24.1483 & 664.0928 & 626.139 & 603.8854 & 122.5994 & 3.68251 \\
\hline 1246 & 24.1653 & 667.7744 & 623.5399 & 603.9609 & 122.5994 & 3.67996 \\
\hline 1247 & 24.1823 & 624.7043 & 621.4285 & 604.0365 & 122.5994 & 3.67741 \\
\hline 1248 & 24.1993 & 619.1805 & 619.7265 & 604.112 & 122.5994 & 3.67487 \\
\hline 1249 & 24.2163 & 611.5666 & 618.3623 & 604.1875 & 122.5994 & 3.67233 \\
\hline 1250 & 24.2333 & 645.1955 & 617.2728 & 604.263 & 122.5994 & 3.66979 \\
\hline 1251 & 24.2503 & 659.431 & 616.3943 & 604.3385 & 122.5994 & 3.66725 \\
\hline 1252 & 24.2673 & 660.4266 & 615.7026 & 604.4141 & 122.5994 & 3.66472 \\
\hline 1253 & 24.2843 & 627.2004 & 615.1515 & 604.4896 & 122.5994 & 3.6622 \\
\hline 1254 & 24.3013 & 719.6656 & 614.7128 & 604.5651 & 122.5994 & 3.65967 \\
\hline 1255 & 24.3183 & 671.8047 & 614.3597 & 604.6406 & 122.5994 & 3.65715 \\
\hline 1256 & 24.3353 & 718.1746 & 614.0854 & 604.7161 & 122.5994 & 3.65464 \\
\hline 1257 & 24.3523 & 692.0918 & 613.8729 & 604.7917 & 122.5994 & 3.65212 \\
\hline 1258 & 24.3693 & 705.8044 & 613.7126 & 604.8672 & 122.5994 & 3.64961 \\
\hline 1259 & 24.3863 & 673.3017 & 613.598 & 604.9427 & 122.5994 & 3.64711 \\
\hline 1260 & 24.4033 & 704.7645 & 613.5325 & 605.0182 & 122.5994 & 3.64461 \\
\hline 1261 & 24.4203 & 676.5885 & 614.4409 & 605.0938 & 122.5994 & 3.64211 \\
\hline 1262 & 24.4373 & 684.0014 & 614.4698 & 605.1693 & 122.5994 & 3.63961 \\
\hline 1263 & 24.4543 & 701.4506 & 614.5342 & 605.2448 & 122.5994 & 3.63712 \\
\hline 1264 & 24.4713 & 686.9397 & 615.0961 & 605.3203 & 122.5994 & 3.63463 \\
\hline 1265 & 24.4883 & 641.0985 & 615.2446 & 605.3958 & 122.5994 & 3.63215 \\
\hline 1266 & 24.5053 & 645.1721 & 615.4296 & 605.4714 & 122.5994 & 3.62967 \\
\hline 1267 & 24.5223 & 632.0717 & 615.6521 & 605.5469 & 122.5994 & 3.62719 \\
\hline 1268 & 24.5393 & 596.9125 & 615.914 & 605.6224 & 122.5994 & 3.62471 \\
\hline 1269 & 24.5563 & 613.6522 & 616.2178 & 605.6979 & 122.5994 & 3.62224 \\
\hline 1270 & 24.5733 & 620.6968 & 616.5662 & 605.7734 & 122.5994 & 3.61978 \\
\hline 1271 & 24.5903 & 643.521 & 616.9626 & 605.849 & 122.5994 & 3.61731 \\
\hline 1272 & 24.6073 & 626.2701 & 617.1486 & 605.9245 & 122.5994 & 3.61485 \\
\hline
\end{tabular}




\begin{tabular}{|c|c|c|c|c|c|c|}
\hline 1273 & 24.6243 & 594.8742 & 617.6612 & 606 & 122.5994 & 3.6124 \\
\hline 1274 & 24.6413 & 587.5437 & 618.9916 & 606.8318 & 122.5994 & 3.60994 \\
\hline 1275 & 24.6583 & 598.5721 & 620.3928 & 607.6637 & 122.5994 & 3.60749 \\
\hline 1276 & 24.6753 & 620.8401 & 621.735 & 608.4955 & 122.5994 & 3.60504 \\
\hline 1277 & 24.6923 & 628.217 & 623.2899 & 609.3274 & 122.5994 & 3.6026 \\
\hline 1278 & 24.7093 & 627.1801 & 624.9336 & 610.1592 & 122.5994 & 3.60016 \\
\hline 1279 & 24.7263 & 632.5479 & 626.6783 & 610.9911 & 122.5994 & 3.59772 \\
\hline 1280 & 24.7433 & 643.1873 & 628.5334 & 611.8229 & 122.5994 & 3.59529 \\
\hline 1281 & 24.7603 & 633.5637 & 630.5163 & 612.6548 & 122.5994 & 3.59286 \\
\hline 1282 & 24.7773 & 603.9148 & 632.6453 & 613.4866 & 122.5994 & 3.59043 \\
\hline 1283 & 24.7943 & 636.4035 & 634.9421 & 614.3185 & 122.5994 & 3.58801 \\
\hline 1284 & 24.8113 & 678.2443 & 637.4311 & 615.1503 & 122.5994 & 3.58559 \\
\hline 1285 & 24.8283 & 670.1845 & 640.1413 & 615.9822 & 122.5994 & 3.58317 \\
\hline 1286 & 24.8453 & 684.1512 & 643.1057 & 616.814 & 122.5994 & 3.58076 \\
\hline 1287 & 24.8623 & 669.1018 & 646.2321 & 617.6459 & 122.5994 & 3.57835 \\
\hline 1288 & 24.8793 & 676.7859 & 649.8365 & 618.4777 & 122.5994 & 3.57594 \\
\hline 1289 & 24.8963 & 712.9527 & 653.8475 & 619.3096 & 122.5994 & 3.57354 \\
\hline 1290 & 24.9133 & 669.7427 & 658.3496 & 620.1414 & 122.5994 & 3.57114 \\
\hline 1291 & 24.9303 & 705.7228 & 663.3875 & 620.9733 & 122.5994 & 3.56874 \\
\hline 1292 & 24.9473 & 757.5865 & 669.2235 & 621.8051 & 122.5994 & 3.56635 \\
\hline 1293 & 24.9643 & 749.7247 & 675.9377 & 622.637 & 122.5994 & 3.56396 \\
\hline 1294 & 24.9813 & 714.2612 & 683.1191 & 623.4688 & 122.5994 & 3.56157 \\
\hline 1295 & 24.9983 & 734.754 & 692.0495 & 624.3007 & 122.5994 & 3.55919 \\
\hline 1296 & 25.0153 & 698.2731 & 702.2626 & 625.1325 & 122.5994 & 3.55681 \\
\hline 1297 & 25.0323 & 715.2024 & 713.8453 & 625.9644 & 122.5994 & 3.55443 \\
\hline 1298 & 25.0493 & 723.1221 & 726.3057 & 626.6056 & 122.5994 & 3.55206 \\
\hline 1299 & 25.0663 & 713.6735 & 740.3379 & 627.2468 & 122.5994 & 3.54969 \\
\hline 1300 & 25.0833 & 738.4131 & 755.4825 & 627.888 & 122.5994 & 3.54732 \\
\hline 1301 & 25.1003 & 739.8538 & 771.4462 & 628.5292 & 122.5994 & 3.54496 \\
\hline 1302 & 25.1173 & 692.0572 & 787.774 & 629.1704 & 122.5994 & 3.5426 \\
\hline 1303 & 25.1343 & 697.5054 & 803.8644 & 629.8116 & 122.5994 & 3.54024 \\
\hline 1304 & 25.1513 & 736.3971 & 818.9681 & 630.4528 & 122.5994 & 3.53789 \\
\hline 1305 & 25.1683 & 789.9473 & 832.2391 & 631.094 & 122.5994 & 3.53553 \\
\hline 1306 & 25.1853 & 799.2226 & 842.8658 & 631.7352 & 122.5994 & 3.53319 \\
\hline 1307 & 25.2023 & 848.585 & 850.2136 & 632.3764 & 122.5994 & 3.53084 \\
\hline 1308 & 25.2193 & 861.5315 & 853.9651 & 633.0176 & 122.5994 & 3.5285 \\
\hline 1309 & 25.2363 & 850.3323 & 854.1747 & 633.6588 & 122.5994 & 3.52616 \\
\hline 1310 & 25.2533 & 896.2681 & 851.2411 & 634.3 & 122.5994 & 3.52383 \\
\hline 1311 & 25.2703 & 843.5884 & 845.8281 & 634.9412 & 122.5994 & 3.52149 \\
\hline 1312 & 25.2873 & 836.6168 & 838.7723 & 635.5824 & 122.5994 & 3.51917 \\
\hline 1313 & 25.3043 & 855.0491 & 830.9889 & 636.2236 & 122.5994 & 3.51684 \\
\hline 1314 & 25.3213 & 809.5563 & 823.3711 & 636.8648 & 122.5994 & 3.51452 \\
\hline 1315 & 25.3383 & 776.4572 & 816.7123 & 637.506 & 122.5994 & 3.5122 \\
\hline 1316 & 25.3553 & 799.2442 & 811.6429 & 638.1472 & 122.5994 & 3.50988 \\
\hline 1317 & 25.3723 & 788.1882 & 808.6037 & 638.7884 & 122.5994 & 3.50757 \\
\hline 1318 & 25.3893 & 778.4156 & 807.8241 & 639.4296 & 122.5994 & 3.50526 \\
\hline 1319 & 25.4063 & 775.6997 & 809.5287 & 640.0707 & 122.5994 & 3.50295 \\
\hline 1320 & 25.4233 & 767.6749 & 813.1552 & 640.7119 & 122.5994 & 3.50065 \\
\hline 1321 & 25.4403 & 770.8316 & 818.4944 & 641.3531 & 122.5994 & 3.49835 \\
\hline
\end{tabular}




\begin{tabular}{|c|c|c|c|c|c|c|}
\hline 1322 & 25.4573 & 743.6628 & 824.7217 & 641.7872 & 122.5994 & 3.49605 \\
\hline 1323 & 25.4743 & 773.5979 & 831.3231 & 642.2212 & 122.5994 & 3.49375 \\
\hline 1324 & 25.4913 & 795.1637 & 837.1407 & 642.6552 & 122.5994 & 3.49146 \\
\hline 1325 & 25.5083 & 792.5101 & 841.2873 & 643.0892 & 122.5994 & 3.48917 \\
\hline 1326 & 25.5253 & 786.7689 & 842.9302 & 643.5232 & 122.5994 & 3.48689 \\
\hline 1327 & 25.5423 & 823.5387 & 841.5397 & 643.9572 & 122.5994 & 3.48461 \\
\hline 1328 & 25.5593 & 848.8493 & 836.9559 & 644.3912 & 122.5994 & 3.48233 \\
\hline 1329 & 25.5763 & 871.4257 & 830.0907 & 644.8252 & 122.5994 & 3.48005 \\
\hline 1330 & 25.5933 & 825.1323 & 819.9252 & 645.2592 & 122.5994 & 3.47778 \\
\hline 1331 & 25.6103 & 795.7824 & 807.7792 & 645.6932 & 122.5994 & 3.47551 \\
\hline 1332 & 25.6273 & 839.7145 & 794.3459 & 646.1272 & 122.5994 & 3.47324 \\
\hline 1333 & 25.6443 & 783.829 & 785.2885 & 646.5612 & 122.5994 & 3.47098 \\
\hline 1334 & 25.6613 & 767.8661 & 771.451 & 646.9953 & 122.5994 & 3.46872 \\
\hline 1335 & 25.6783 & 818.3414 & 758.1493 & 647.4293 & 122.5994 & 3.46646 \\
\hline 1336 & 25.6953 & 777.9485 & 745.7466 & 647.8633 & 122.5994 & 3.4642 \\
\hline 1337 & 25.7123 & 772.7892 & 734.4776 & 648.2973 & 122.5994 & 3.46195 \\
\hline 1338 & 25.7293 & 757.2101 & 729.8562 & 648.7313 & 122.5994 & 3.4597 \\
\hline 1339 & 25.7463 & 716.6985 & 721.3212 & 649.1653 & 122.5994 & 3.45746 \\
\hline 1340 & 25.7633 & 760.471 & 714.0703 & 649.5993 & 122.5994 & 3.45521 \\
\hline 1341 & 25.7803 & 722.9847 & 708.0319 & 650.0333 & 122.5994 & 3.45297 \\
\hline 1342 & 25.7973 & 731.1841 & 704.6086 & 650.4673 & 122.5994 & 3.45074 \\
\hline 1343 & 25.8143 & 706.2898 & 700.7192 & 650.9013 & 122.5994 & 3.4485 \\
\hline 1344 & 25.8313 & 726.2404 & 697.7026 & 651.3353 & 122.5994 & 3.44627 \\
\hline 1345 & 25.8483 & 652.0585 & 695.4329 & 651.7693 & 122.5994 & 3.44404 \\
\hline 1346 & 25.8653 & 693.8338 & 693.5687 & 651.9758 & 122.5994 & 3.44182 \\
\hline 1347 & 25.8823 & 652.087 & 692.2341 & 652.1823 & 122.5994 & 3.4396 \\
\hline 1348 & 25.8993 & 675.5612 & 691.3448 & 652.3888 & 122.5994 & 3.43738 \\
\hline 1349 & 25.9163 & 655.5981 & 690.827 & 652.5953 & 122.5994 & 3.43516 \\
\hline 1350 & 25.9333 & 712.0968 & 690.6232 & 652.8017 & 122.5994 & 3.43295 \\
\hline 1351 & 25.9503 & 682.461 & 690.6887 & 653.0082 & 122.5994 & 3.43074 \\
\hline 1352 & 25.9673 & 700.8936 & 690.9905 & 653.2147 & 122.5994 & 3.42853 \\
\hline 1353 & 25.9843 & 618.4018 & 691.5019 & 653.4212 & 122.5994 & 3.42633 \\
\hline 1354 & 26.0013 & 700.4201 & 692.213 & 653.6277 & 122.5994 & 3.42413 \\
\hline 1355 & 26.0183 & 685.7571 & 693.1104 & 653.8341 & 122.5994 & 3.42193 \\
\hline 1356 & 26.0353 & 680.4175 & 694.1551 & 654.0406 & 122.5994 & 3.41973 \\
\hline 1357 & 26.0523 & 689.1771 & 695.4116 & 654.2471 & 122.5994 & 3.41754 \\
\hline 1358 & 26.0693 & 741.1872 & 696.8484 & 654.4536 & 122.5994 & 3.41535 \\
\hline 1359 & 26.0863 & 706.9444 & 698.449 & 654.6601 & 122.5994 & 3.41316 \\
\hline 1360 & 26.1033 & 704.6671 & 700.2515 & 654.8665 & 122.5994 & 3.41098 \\
\hline 1361 & 26.1203 & 654.6213 & 702.242 & 655.073 & 122.5994 & 3.40879 \\
\hline 1362 & 26.1373 & 673.8904 & 704.4264 & 655.2795 & 122.5994 & 3.40662 \\
\hline 1363 & 26.1543 & 656.9378 & 706.8113 & 655.486 & 122.5994 & 3.40444 \\
\hline 1364 & 26.1713 & 675.8811 & 709.4069 & 655.6925 & 122.5994 & 3.40227 \\
\hline 1365 & 26.1883 & 707.5732 & 712.2289 & 655.8989 & 122.5994 & 3.4001 \\
\hline 1366 & 26.2053 & 709.4625 & 715.3028 & 656.1054 & 122.5994 & 3.39793 \\
\hline 1367 & 26.2223 & 712.9033 & 718.6664 & 656.3119 & 122.5994 & 3.39577 \\
\hline 1368 & 26.2393 & 704.1531 & 722.3723 & 656.5184 & 122.5994 & 3.3936 \\
\hline 1369 & 26.2563 & 675.9255 & 726.4894 & 656.7249 & 122.5994 & 3.39145 \\
\hline 1370 & 26.2733 & 660.058 & 730.8522 & 656.6953 & 122.5994 & 3.38929 \\
\hline
\end{tabular}




\begin{tabular}{|c|c|c|c|c|c|c|}
\hline 1371 & 26.2903 & 678.0874 & 735.8324 & 656.6657 & 122.5994 & 3.38714 \\
\hline 1372 & 26.3073 & 712.9311 & 741.5397 & 656.6361 & 122.5994 & 3.38499 \\
\hline 1373 & 26.3243 & 698.6423 & 748.1214 & 656.6065 & 122.5994 & 3.38284 \\
\hline 1374 & 26.3413 & 668.4538 & 755.751 & 656.577 & 122.5994 & 3.38069 \\
\hline 1375 & 26.3583 & 718.9573 & 764.6328 & 656.5474 & 122.5994 & 3.37855 \\
\hline 1376 & 26.3753 & 762.7215 & 773.9587 & 656.5178 & 122.5994 & 3.37641 \\
\hline 1377 & 26.3923 & 784.2545 & 786.144 & 656.4882 & 122.5994 & 3.37428 \\
\hline 1378 & 26.4093 & 788.1065 & 800.4536 & 656.4587 & 122.5994 & 3.37214 \\
\hline 1379 & 26.4263 & 806.0683 & 817.3051 & 656.4291 & 122.5994 & 3.37001 \\
\hline 1380 & 26.4433 & 798.9543 & 836.6955 & 656.3995 & 122.5994 & 3.36789 \\
\hline 1381 & 26.4603 & 853.6644 & 860.3146 & 656.3699 & 122.5994 & 3.36576 \\
\hline 1382 & 26.4773 & 886.3548 & 888.4604 & 656.3404 & 122.5994 & 3.36364 \\
\hline 1383 & 26.4943 & 960.0807 & 922.2709 & 656.3108 & 122.5994 & 3.36152 \\
\hline 1384 & 26.5113 & 1039.072 & 963.4941 & 656.2812 & 122.5994 & 3.3594 \\
\hline 1385 & 26.5283 & 1076.635 & 1015.244 & 656.2516 & 122.5994 & 3.35729 \\
\hline 1386 & 26.5453 & 1157.697 & 1083.714 & 656.2221 & 122.5994 & 3.35517 \\
\hline 1387 & 26.5623 & 1304.479 & 1181.352 & 656.1925 & 122.5994 & 3.35307 \\
\hline 1388 & 26.5793 & 1413.71 & 1331.104 & 656.1629 & 122.5994 & 3.35096 \\
\hline 1389 & 26.5963 & 1576.858 & 1569.107 & 656.1333 & 122.5994 & 3.34886 \\
\hline 1390 & 26.6133 & 1851.312 & 1940.39 & 656.1037 & 122.5994 & 3.34676 \\
\hline 1391 & 26.6303 & 2204.421 & 2483.275 & 656.0742 & 122.5994 & 3.34466 \\
\hline 1392 & 26.6473 & 2622.882 & 3200.84 & 656.0446 & 122.5994 & 3.34256 \\
\hline 1393 & 26.6643 & 3373.195 & 4026.044 & 656.015 & 122.5994 & 3.34047 \\
\hline 1394 & 26.6813 & 4164.591 & 4767.527 & 655.7519 & 122.5994 & 3.33838 \\
\hline 1395 & 26.6983 & 5409.322 & 5154.772 & 655.4888 & 122.5994 & 3.33629 \\
\hline 1396 & 26.7153 & 6491.624 & 5107.624 & 655.2257 & 122.5994 & 3.33421 \\
\hline 1397 & 26.7323 & 6180.3 & 4822.046 & 654.9626 & 122.5994 & 3.33213 \\
\hline 1398 & 26.7493 & 4946.023 & 4451.899 & 654.6995 & 122.5994 & 3.33005 \\
\hline 1399 & 26.7663 & 4084.941 & 3987.406 & 654.4364 & 122.5994 & 3.32797 \\
\hline 1400 & 26.7833 & 3916.738 & 3428.349 & 654.1733 & 122.5994 & 3.3259 \\
\hline 1401 & 26.8003 & 3442.098 & 2874.994 & 653.9102 & 122.5994 & 3.32383 \\
\hline 1402 & 26.8173 & 2479.98 & 2410.987 & 653.6471 & 122.5994 & 3.32176 \\
\hline 1403 & 26.8343 & 1899.749 & 2061.272 & 653.384 & 122.5994 & 3.31969 \\
\hline 1404 & 26.8513 & 1654.722 & 1814.292 & 653.1209 & 122.5994 & 3.31763 \\
\hline 1405 & 26.8683 & 1561.363 & 1642.727 & 652.8578 & 122.5994 & 3.31557 \\
\hline 1406 & 26.8853 & 1498.353 & 1519.826 & 652.5947 & 122.5994 & 3.31351 \\
\hline 1407 & 26.9023 & 1447.993 & 1424.143 & 652.3316 & 122.5994 & 3.31146 \\
\hline 1408 & 26.9193 & 1346.948 & 1342.416 & 652.0685 & 122.5994 & 3.3094 \\
\hline 1409 & 26.9363 & 1280.412 & 1268.105 & 651.8054 & 122.5994 & 3.30735 \\
\hline 1410 & 26.9533 & 1235.943 & 1198.858 & 651.5423 & 122.5994 & 3.30531 \\
\hline 1411 & 26.9703 & 1144.83 & 1134.368 & 651.2792 & 122.5994 & 3.30326 \\
\hline 1412 & 26.9873 & 1037.099 & 1074.957 & 651.0161 & 122.5994 & 3.30122 \\
\hline 1413 & 27.0043 & 982.0974 & 1020.987 & 650.753 & 122.5994 & 3.29918 \\
\hline 1414 & 27.0213 & 944.0624 & 972.6575 & 650.4899 & 122.5994 & 3.29714 \\
\hline 1415 & 27.0383 & 869.62 & 929.9612 & 650.2268 & 122.5994 & 3.29511 \\
\hline 1416 & 27.0553 & 824.5745 & 892.7113 & 649.9637 & 122.5994 & 3.29308 \\
\hline 1417 & 27.0723 & 776.6171 & 860.5811 & 649.7006 & 122.5994 & 3.29105 \\
\hline 1418 & 27.0893 & 767.1879 & 833.1448 & 649.4375 & 122.5994 & 3.28902 \\
\hline 1419 & 27.1063 & 767.2022 & 809.9183 & 649.1744 & 122.5994 & 3.287 \\
\hline
\end{tabular}




\begin{tabular}{|c|c|c|c|c|c|c|}
\hline 1420 & 27.1233 & 758.7581 & 790.3829 & 648.9113 & 122.5994 & 3.28497 \\
\hline 1421 & 27.1403 & 774.7151 & 774.0298 & 648.6482 & 122.5994 & 3.28295 \\
\hline 1422 & 27.1573 & 748.6141 & 760.3711 & 648.3851 & 122.5994 & 3.28094 \\
\hline 1423 & 27.1743 & 747.4948 & 748.9594 & 648.1219 & 122.5994 & 3.27892 \\
\hline 1424 & 27.1913 & 730.4097 & 739.3979 & 647.8588 & 122.5994 & 3.27691 \\
\hline 1425 & 27.2083 & 690.7296 & 731.3448 & 647.5957 & 122.5994 & 3.2749 \\
\hline 1426 & 27.2253 & 688.0682 & 724.5131 & 647.3326 & 122.5994 & 3.2729 \\
\hline 1427 & 27.2423 & 720.8457 & 718.6679 & 647.0695 & 122.5994 & 3.27089 \\
\hline 1428 & 27.2593 & 682.4051 & 713.6203 & 646.8064 & 122.5994 & 3.26889 \\
\hline 1429 & 27.2763 & 714.1128 & 709.2213 & 646.5433 & 122.5994 & 3.26689 \\
\hline 1430 & 27.2933 & 702.5211 & 705.3539 & 646.2802 & 122.5994 & 3.2649 \\
\hline 1431 & 27.3103 & 699.8164 & 701.9289 & 646.0171 & 122.5994 & 3.2629 \\
\hline 1432 & 27.3273 & 689.6968 & 698.8773 & 645.754 & 122.5994 & 3.26091 \\
\hline 1433 & 27.3443 & 648.8502 & 696.1467 & 645.4909 & 122.5994 & 3.25892 \\
\hline 1434 & 27.3613 & 683.075 & 693.6968 & 645.2278 & 122.5994 & 3.25694 \\
\hline 1435 & 27.3783 & 728.2006 & 691.4805 & 644.9647 & 122.5994 & 3.25495 \\
\hline 1436 & 27.3953 & 687.8269 & 689.5079 & 644.7016 & 122.5994 & 3.25297 \\
\hline 1437 & 27.4123 & 681.2227 & 687.7449 & 644.4385 & 122.5994 & 3.25099 \\
\hline 1438 & 27.4293 & 695.6962 & 686.1791 & 644.1754 & 122.5994 & 3.24902 \\
\hline 1439 & 27.4463 & 737.2162 & 684.7946 & 643.9123 & 122.5994 & 3.24704 \\
\hline 1440 & 27.4633 & 698.6073 & 683.6053 & 643.6492 & 122.5994 & 3.24507 \\
\hline 1441 & 27.4803 & 717.9006 & 682.6047 & 643.3861 & 122.5994 & 3.2431 \\
\hline 1442 & 27.4973 & 723.9813 & 681.5674 & 642.8895 & 122.5994 & 3.24114 \\
\hline 1443 & 27.5143 & 797.0886 & 680.8488 & 642.3929 & 122.5994 & 3.23917 \\
\hline 1444 & 27.5313 & 862.327 & 680.2633 & 641.8962 & 122.5994 & 3.23721 \\
\hline 1445 & 27.5483 & 822.9105 & 679.9467 & 641.3996 & 122.5994 & 3.23525 \\
\hline 1446 & 27.5653 & 804.1972 & 679.952 & 640.903 & 122.5994 & 3.2333 \\
\hline 1447 & 27.5823 & 729.7915 & 680.3942 & 640.4063 & 122.5994 & 3.23134 \\
\hline 1448 & 27.5993 & 774.717 & 681.2466 & 639.9097 & 122.5994 & 3.22939 \\
\hline 1449 & 27.6163 & 751.6284 & 682.6599 & 639.4131 & 122.5994 & 3.22744 \\
\hline 1450 & 27.6333 & 755.2055 & 684.7532 & 638.9165 & 122.5994 & 3.22549 \\
\hline 1451 & 27.6503 & 744.3162 & 687.6596 & 638.4198 & 122.5994 & 3.22355 \\
\hline 1452 & 27.6673 & 721.8127 & 691.523 & 637.9232 & 122.5994 & 3.22161 \\
\hline 1453 & 27.6843 & 739.7014 & 696.4951 & 637.4266 & 122.5994 & 3.21967 \\
\hline 1454 & 27.7013 & 756.2641 & 702.7225 & 636.9299 & 122.5994 & 3.21773 \\
\hline 1455 & 27.7183 & 742.4012 & 710.3354 & 636.4333 & 122.5994 & 3.2158 \\
\hline 1456 & 27.7353 & 704.2471 & 719.2441 & 635.9367 & 122.5994 & 3.21386 \\
\hline 1457 & 27.7523 & 723.6621 & 729.8825 & 635.4401 & 122.5994 & 3.21193 \\
\hline 1458 & 27.7693 & 780.3766 & 742.0391 & 634.9434 & 122.5994 & 3.21001 \\
\hline 1459 & 27.7863 & 805.1471 & 755.6111 & 634.4468 & 122.5994 & 3.20808 \\
\hline 1460 & 27.8033 & 843.2949 & 765.4662 & 633.9502 & 122.5994 & 3.20616 \\
\hline 1461 & 27.8203 & 854.0883 & 781.1951 & 633.4536 & 122.5994 & 3.20424 \\
\hline 1462 & 27.8373 & 864.2771 & 797.1866 & 632.9569 & 122.5994 & 3.20232 \\
\hline 1463 & 27.8543 & 864.3762 & 812.6953 & 632.4603 & 122.5994 & 3.2004 \\
\hline 1464 & 27.8713 & 895.8917 & 824.4128 & 631.9637 & 122.5994 & 3.19849 \\
\hline 1465 & 27.8883 & 915.1472 & 836.2036 & 631.467 & 122.5994 & 3.19658 \\
\hline 1466 & 27.9053 & 900.0168 & 844.4664 & 630.7394 & 122.5994 & 3.19467 \\
\hline 1467 & 27.9223 & 906.5535 & 848.7945 & 630.0118 & 122.5994 & 3.19276 \\
\hline 1468 & 27.9393 & 939.5821 & 848.9221 & 629.2842 & 122.5994 & 3.19086 \\
\hline
\end{tabular}




\begin{tabular}{|c|c|c|c|c|c|c|}
\hline 1469 & 27.9563 & 983.023 & 842.0679 & 628.5566 & 122.5994 & 3.18896 \\
\hline 1470 & 27.9733 & 1017.924 & 834.4215 & 627.829 & 122.5994 & 3.18706 \\
\hline 1471 & 27.9903 & 1026.398 & 823.5735 & 627.1013 & 122.5994 & 3.18516 \\
\hline 1472 & 28.0073 & 1044.997 & 810.2046 & 626.3737 & 122.5994 & 3.18327 \\
\hline 1473 & 28.0243 & 1004.821 & 793.6443 & 625.6461 & 122.5994 & 3.18137 \\
\hline 1474 & 28.0413 & 1001.474 & 777.5536 & 624.9185 & 122.5994 & 3.17948 \\
\hline 1475 & 28.0583 & 970.9137 & 761.1569 & 624.1909 & 122.5994 & 3.1776 \\
\hline 1476 & 28.0753 & 896.9025 & 745.0307 & 623.4633 & 122.5994 & 3.17571 \\
\hline 1477 & 28.0923 & 903.3745 & 729.6168 & 622.7357 & 122.5994 & 3.17383 \\
\hline 1478 & 28.1093 & 794.904 & 715.2216 & 622.008 & 122.5994 & 3.17195 \\
\hline 1479 & 28.1263 & 811.3864 & 702.0446 & 621.2804 & 122.5994 & 3.17007 \\
\hline 1480 & 28.1433 & 759.2108 & 690.19 & 620.5528 & 122.5994 & 3.16819 \\
\hline 1481 & 28.1603 & 713.3723 & 679.6854 & 619.8252 & 122.5994 & 3.16632 \\
\hline 1482 & 28.1773 & 759.2749 & 670.4979 & 619.0976 & 122.5994 & 3.16445 \\
\hline 1483 & 28.1943 & 738.1357 & 662.5496 & 618.37 & 122.5994 & 3.16258 \\
\hline 1484 & 28.2113 & 681.3489 & 655.7329 & 617.6423 & 122.5994 & 3.16071 \\
\hline 1485 & 28.2283 & 710.3275 & 649.9244 & 616.9147 & 122.5994 & 3.15884 \\
\hline 1486 & 28.2453 & 663.0327 & 644.9963 & 616.1871 & 122.5994 & 3.15698 \\
\hline 1487 & 28.2623 & 660.4266 & 640.8248 & 615.4595 & 122.5994 & 3.15512 \\
\hline 1488 & 28.2793 & 645.2538 & 637.2975 & 614.7319 & 122.5994 & 3.15326 \\
\hline 1489 & 28.2963 & 680.555 & 634.3115 & 614.0043 & 122.5994 & 3.15141 \\
\hline 1490 & 28.3133 & 641.8528 & 631.5666 & 613.0593 & 122.5994 & 3.14955 \\
\hline 1491 & 28.3303 & 682.5129 & 629.212 & 612.1142 & 122.5994 & 3.1477 \\
\hline 1492 & 28.3473 & 621.5716 & 627.194 & 611.1692 & 122.5994 & 3.14585 \\
\hline 1493 & 28.3643 & 638.5588 & 625.4736 & 610.2242 & 122.5994 & 3.14401 \\
\hline 1494 & 28.3813 & 629.4053 & 624.0204 & 609.2792 & 122.5994 & 3.14216 \\
\hline 1495 & 28.3983 & 662.0022 & 622.8156 & 608.3342 & 122.5994 & 3.14032 \\
\hline 1496 & 28.4153 & 669.1005 & 621.8454 & 607.3892 & 122.5994 & 3.13848 \\
\hline 1497 & 28.4323 & 649.1529 & 621.1002 & 606.4442 & 122.5994 & 3.13664 \\
\hline 1498 & 28.4493 & 603.2957 & 620.5711 & 605.4992 & 122.5994 & 3.13481 \\
\hline 1499 & 28.4663 & 620.847 & 620.247 & 604.5541 & 122.5994 & 3.13297 \\
\hline 1500 & 28.4833 & 647.8709 & 620.1122 & 603.6091 & 122.5994 & 3.13114 \\
\hline 1501 & 28.5003 & 666.5826 & 620.1434 & 602.6641 & 122.5994 & 3.12931 \\
\hline 1502 & 28.5173 & 641.974 & 620.306 & 601.7191 & 122.5994 & 3.12749 \\
\hline 1503 & 28.5343 & 622.8522 & 620.5513 & 600.7741 & 122.5994 & 3.12566 \\
\hline 1504 & 28.5513 & 648.4625 & 620.8131 & 599.8291 & 122.5994 & 3.12384 \\
\hline 1505 & 28.5683 & 637.9053 & 621.008 & 598.8841 & 122.5994 & 3.12202 \\
\hline 1506 & 28.5853 & 656.5487 & 621.0398 & 597.9391 & 122.5994 & 3.1202 \\
\hline 1507 & 28.6023 & 692.7437 & 620.8124 & 596.994 & 122.5994 & 3.11838 \\
\hline 1508 & 28.6193 & 647.117 & 620.2467 & 596.049 & 122.5994 & 3.11657 \\
\hline 1509 & 28.6363 & 601.9216 & 619.2964 & 595.104 & 122.5994 & 3.11476 \\
\hline 1510 & 28.6533 & 604.0049 & 617.9535 & 594.159 & 122.5994 & 3.11295 \\
\hline 1511 & 28.6703 & 612.8953 & 616.2427 & 593.214 & 122.5994 & 3.11114 \\
\hline 1512 & 28.6873 & 592.7943 & 614.2124 & 592.269 & 122.5994 & 3.10934 \\
\hline 1513 & 28.7043 & 611.9623 & 611.9268 & 591.324 & 122.5994 & 3.10753 \\
\hline 1514 & 28.7213 & 610.3643 & 609.2668 & 590.1855 & 122.5994 & 3.10573 \\
\hline 1515 & 28.7383 & 582.1593 & 606.504 & 589.047 & 122.5994 & 3.10394 \\
\hline 1516 & 28.7553 & 587.5048 & 603.7117 & 587.9085 & 122.5994 & 3.10214 \\
\hline 1517 & 28.7723 & 601.4338 & 600.9523 & 586.77 & 122.5994 & 3.10034 \\
\hline
\end{tabular}




\begin{tabular}{|c|c|c|c|c|c|c|}
\hline 1518 & 28.7893 & 608.3274 & 598.2737 & 585.6315 & 122.5994 & 3.09855 \\
\hline 1519 & 28.8063 & 606.8392 & 595.7089 & 584.493 & 122.5994 & 3.09676 \\
\hline 1520 & 28.8233 & 588.5342 & 594.5456 & 583.3545 & 122.5994 & 3.09497 \\
\hline 1521 & 28.8403 & 574.0549 & 592.2938 & 582.216 & 122.5994 & 3.09319 \\
\hline 1522 & 28.8573 & 610.2894 & 590.1862 & 581.0775 & 122.5994 & 3.09141 \\
\hline 1523 & 28.8743 & 574.0638 & 588.2239 & 579.939 & 122.5994 & 3.08962 \\
\hline 1524 & 28.8913 & 571.6174 & 587.0081 & 578.8005 & 122.5994 & 3.08784 \\
\hline 1525 & 28.9083 & 560.9538 & 585.305 & 577.662 & 122.5994 & 3.08607 \\
\hline 1526 & 28.9253 & 556.9704 & 583.7048 & 576.5235 & 122.5994 & 3.08429 \\
\hline 1527 & 28.9423 & 564.8756 & 582.1971 & 575.385 & 122.5994 & 3.08252 \\
\hline 1528 & 28.9593 & 582.8745 & 580.7632 & 574.2465 & 122.5994 & 3.08075 \\
\hline 1529 & 28.9763 & 552.194 & 579.393 & 573.108 & 122.5994 & 3.07898 \\
\hline 1530 & 28.9933 & 635.2511 & 578.0757 & 571.9695 & 122.5994 & 3.07721 \\
\hline 1531 & 29.0103 & 590.4193 & 576.8024 & 570.831 & 122.5994 & 3.07545 \\
\hline 1532 & 29.0273 & 554.2163 & 574.5103 & 569.6925 & 122.5994 & 3.07369 \\
\hline 1533 & 29.0443 & 570.5183 & 573.3353 & 568.554 & 122.5994 & 3.07193 \\
\hline 1534 & 29.0613 & 599.8108 & 572.1851 & 567.4155 & 122.5994 & 3.07017 \\
\hline 1535 & 29.0783 & 585.4803 & 571.0568 & 566.277 & 122.5994 & 3.06841 \\
\hline 1536 & 29.0953 & 556.841 & 569.4206 & 565.1385 & 122.5994 & 3.06666 \\
\hline 1537 & 29.1123 & 552.2603 & 568.3452 & 564 & 122.5994 & 3.0649 \\
\hline 1538 & 29.1293 & 555.3019 & 568.0589 & 563.6341 & 122.5994 & 3.06315 \\
\hline 1539 & 29.1463 & 595.3711 & 567.7888 & 563.2682 & 122.5994 & 3.06141 \\
\hline 1540 & 29.1633 & 590.9442 & 567.5346 & 562.9023 & 122.5994 & 3.05966 \\
\hline 1541 & 29.1803 & 559.5416 & 567.2339 & 562.5365 & 122.5994 & 3.05792 \\
\hline 1542 & 29.1973 & 573.7701 & 566.9669 & 562.1706 & 122.5994 & 3.05618 \\
\hline 1543 & 29.2143 & 560.0191 & 566.766 & 561.8047 & 122.5994 & 3.05444 \\
\hline 1544 & 29.2313 & 557.002 & 566.5828 & 561.4388 & 122.5994 & 3.0527 \\
\hline 1545 & 29.2483 & 568.6874 & 566.3867 & 561.0729 & 122.5994 & 3.05096 \\
\hline 1546 & 29.2653 & 550.8705 & 566.2179 & 560.707 & 122.5994 & 3.04923 \\
\hline 1547 & 29.2823 & 568.4987 & 566.093 & 560.3411 & 122.5994 & 3.0475 \\
\hline 1548 & 29.2993 & 569.6928 & 565.988 & 559.9753 & 122.5994 & 3.04577 \\
\hline 1549 & 29.3163 & 599.7868 & 565.9031 & 559.6094 & 122.5994 & 3.04404 \\
\hline 1550 & 29.3333 & 564.9917 & 565.8386 & 559.2435 & 122.5994 & 3.04231 \\
\hline 1551 & 29.3503 & 598.1604 & 565.7953 & 558.8776 & 122.5994 & 3.04059 \\
\hline 1552 & 29.3673 & 565.3736 & 565.7742 & 558.5117 & 122.5994 & 3.03887 \\
\hline 1553 & 29.3843 & 558.7538 & 565.7776 & 558.1458 & 122.5994 & 3.03715 \\
\hline 1554 & 29.4013 & 580.0091 & 565.8092 & 557.7799 & 122.5994 & 3.03543 \\
\hline 1555 & 29.4183 & 569.0365 & 565.8738 & 557.4141 & 122.5994 & 3.03372 \\
\hline 1556 & 29.4353 & 534.8789 & 565.978 & 557.0482 & 122.5994 & 3.032 \\
\hline 1557 & 29.4523 & 519.8497 & 566.1297 & 556.6823 & 122.5994 & 3.03029 \\
\hline 1558 & 29.4693 & 526.6861 & 566.3391 & 556.3164 & 122.5994 & 3.02858 \\
\hline 1559 & 29.4863 & 538.4672 & 566.6195 & 555.9505 & 122.5994 & 3.02688 \\
\hline 1560 & 29.5033 & 560.9075 & 566.9879 & 555.5846 & 122.5994 & 3.02517 \\
\hline 1561 & 29.5203 & 581.4612 & 567.4659 & 555.2188 & 122.5994 & 3.02347 \\
\hline 1562 & 29.5373 & 571.5939 & 567.959 & 554.7292 & 122.5994 & 3.02177 \\
\hline 1563 & 29.5543 & 533.4675 & 568.6284 & 554.2396 & 122.5994 & 3.02007 \\
\hline 1564 & 29.5713 & 529.7582 & 569.5232 & 553.75 & 122.5994 & 3.01837 \\
\hline 1565 & 29.5883 & 544.2596 & 570.707 & 553.2604 & 122.5994 & 3.01667 \\
\hline 1566 & 29.6053 & 547.5702 & 572.2597 & 552.7708 & 122.5994 & 3.01498 \\
\hline
\end{tabular}




\begin{tabular}{|c|c|c|c|c|c|c|}
\hline 1567 & 29.6223 & 577.3849 & 574.2806 & 552.2813 & 122.5994 & 3.01329 \\
\hline 1568 & 29.6393 & 569.8794 & 576.8889 & 551.7917 & 122.5994 & 3.0116 \\
\hline 1569 & 29.6563 & 554.7379 & 580.2238 & 551.3021 & 122.5994 & 3.00991 \\
\hline 1570 & 29.6733 & 555.8357 & 584.4418 & 550.8125 & 122.5994 & 3.00822 \\
\hline 1571 & 29.6903 & 583.0774 & 589.7115 & 550.3229 & 122.5994 & 3.00654 \\
\hline 1572 & 29.7073 & 578.8793 & 596.2048 & 549.8333 & 122.5994 & 3.00486 \\
\hline 1573 & 29.7243 & 560.3296 & 604.0864 & 549.3438 & 122.5994 & 3.00318 \\
\hline 1574 & 29.7413 & 601.8719 & 613.3954 & 548.8542 & 122.5994 & 3.0015 \\
\hline 1575 & 29.7583 & 576.4057 & 624.4397 & 548.3646 & 122.5994 & 2.99983 \\
\hline 1576 & 29.7753 & 641.4235 & 637.1591 & 547.875 & 122.5994 & 2.99815 \\
\hline 1577 & 29.7923 & 587.024 & 651.5103 & 547.3854 & 122.5994 & 2.99648 \\
\hline 1578 & 29.8093 & 631.3171 & 667.2919 & 546.8958 & 122.5994 & 2.99481 \\
\hline 1579 & 29.8263 & 630.2599 & 684.3154 & 546.4063 & 122.5994 & 2.99314 \\
\hline 1580 & 29.8433 & 674.8261 & 702.0602 & 545.9167 & 122.5994 & 2.99147 \\
\hline 1581 & 29.8603 & 655.4603 & 719.8516 & 545.4271 & 122.5994 & 2.98981 \\
\hline 1582 & 29.8773 & 672.2147 & 736.7949 & 544.9375 & 122.5994 & 2.98815 \\
\hline 1583 & 29.8943 & 753.7638 & 751.8118 & 544.4479 & 122.5994 & 2.98649 \\
\hline 1584 & 29.9113 & 693.9514 & 763.7861 & 543.9583 & 122.5994 & 2.98483 \\
\hline 1585 & 29.9283 & 731.2426 & 771.7469 & 543.4688 & 122.5994 & 2.98317 \\
\hline 1586 & 29.9453 & 751.7836 & 775.0793 & 542.9792 & 122.5994 & 2.98152 \\
\hline 1587 & 29.9623 & 716.2685 & 773.6184 & 542.4896 & 122.5994 & 2.97986 \\
\hline 1588 & 29.9793 & 741.3665 & 767.5989 & 542 & 122.5994 & 2.97821 \\
\hline 1589 & 29.9963 & 743.5241 & 757.54 & 541.5104 & 122.5994 & 2.97656 \\
\hline 1590 & 30.0133 & 710.6124 & 744.1422 & 541.0208 & 122.5994 & 2.97492 \\
\hline 1591 & 30.0303 & 712.4884 & 728.2313 & 540.5313 & 122.5994 & 2.97327 \\
\hline 1592 & 30.0473 & 673.2398 & 710.7041 & 540.0417 & 122.5994 & 2.97163 \\
\hline 1593 & 30.0643 & 669.4973 & 692.4496 & 539.5521 & 122.5994 & 2.96999 \\
\hline 1594 & 30.0813 & 640.5749 & 674.2529 & 539.0625 & 122.5994 & 2.96835 \\
\hline 1595 & 30.0983 & 637.5571 & 656.7469 & 538.5729 & 122.5994 & 2.96671 \\
\hline 1596 & 30.1153 & 608.0074 & 640.3851 & 538.0833 & 122.5994 & 2.96507 \\
\hline 1597 & 30.1323 & 621.5611 & 625.4565 & 537.5938 & 122.5994 & 2.96344 \\
\hline 1598 & 30.1493 & 618.9171 & 612.1052 & 537.1042 & 122.5994 & 2.96181 \\
\hline 1599 & 30.1663 & 619.0401 & 600.3713 & 536.6146 & 122.5994 & 2.96018 \\
\hline 1600 & 30.1833 & 573.598 & 590.212 & 536.125 & 122.5994 & 2.95855 \\
\hline 1601 & 30.2003 & 600.9964 & 582.4208 & 535.6354 & 122.5994 & 2.95692 \\
\hline 1602 & 30.2173 & 573.9783 & 575.0994 & 535.1458 & 122.5994 & 2.9553 \\
\hline 1603 & 30.2343 & 583.8961 & 568.9587 & 534.6563 & 122.5994 & 2.95367 \\
\hline 1604 & 30.2513 & 620.1251 & 563.8331 & 534.1667 & 122.5994 & 2.95205 \\
\hline 1605 & 30.2683 & 604.9723 & 559.9982 & 533.6771 & 122.5994 & 2.95043 \\
\hline 1606 & 30.2853 & 587.5394 & 556.4495 & 533.1875 & 122.5994 & 2.94881 \\
\hline 1607 & 30.3023 & 564.9669 & 553.4766 & 532.6979 & 122.5994 & 2.9472 \\
\hline 1608 & 30.3193 & 552.2603 & 550.9656 & 532.2083 & 122.5994 & 2.94558 \\
\hline 1609 & 30.3363 & 555.0777 & 548.8213 & 531.7188 & 122.5994 & 2.94397 \\
\hline 1610 & 30.3533 & 577.7494 & 546.8424 & 531.1055 & 122.5994 & 2.94236 \\
\hline 1611 & 30.3703 & 603.0695 & 545.0911 & 530.4922 & 122.5994 & 2.94075 \\
\hline 1612 & 30.3873 & 581.1709 & 543.5193 & 529.8789 & 122.5994 & 2.93915 \\
\hline 1613 & 30.4043 & 572.9267 & 542.09 & 529.2656 & 122.5994 & 2.93754 \\
\hline 1614 & 30.4213 & 572.6303 & 540.7751 & 528.6523 & 122.5994 & 2.93594 \\
\hline 1615 & 30.4383 & 588.7843 & 539.5539 & 528.0391 & 122.5994 & 2.93434 \\
\hline
\end{tabular}




\begin{tabular}{|c|c|c|c|c|c|c|}
\hline 1616 & 30.4553 & 538.8211 & 538.4087 & 527.4258 & 122.5994 & 2.93274 \\
\hline 1617 & 30.4723 & 566.8176 & 537.3312 & 526.8125 & 122.5994 & 2.93114 \\
\hline 1618 & 30.4893 & 580.8209 & 536.3105 & 526.1992 & 122.5994 & 2.92955 \\
\hline 1619 & 30.5063 & 553.7121 & 535.34 & 525.5859 & 122.5994 & 2.92795 \\
\hline 1620 & 30.5233 & 620.6015 & 534.4142 & 524.9727 & 122.5994 & 2.92636 \\
\hline 1621 & 30.5403 & 609.4636 & 533.5416 & 524.3594 & 122.5994 & 2.92477 \\
\hline 1622 & 30.5573 & 618.2082 & 532.6942 & 523.7461 & 122.5994 & 2.92318 \\
\hline 1623 & 30.5743 & 615.5514 & 531.8815 & 523.1328 & 122.5994 & 2.9216 \\
\hline 1624 & 30.5913 & 610.4927 & 531.1015 & 522.5195 & 122.5994 & 2.92001 \\
\hline 1625 & 30.6083 & 600.7583 & 530.3527 & 521.9063 & 122.5994 & 2.91843 \\
\hline 1626 & 30.6253 & 576.8859 & 529.6404 & 521.293 & 122.5994 & 2.91685 \\
\hline 1627 & 30.6423 & 585.5106 & 528.9509 & 520.6797 & 122.5994 & 2.91527 \\
\hline 1628 & 30.6593 & 561.5034 & 528.2902 & 520.0664 & 122.5994 & 2.91369 \\
\hline 1629 & 30.6763 & 534.4217 & 527.6581 & 519.4531 & 122.5994 & 2.91211 \\
\hline 1630 & 30.6933 & 560.0025 & 527.0549 & 518.8398 & 122.5994 & 2.91054 \\
\hline 1631 & 30.7103 & 526.4242 & 526.4814 & 518.2266 & 122.5994 & 2.90897 \\
\hline 1632 & 30.7273 & 494.5165 & 525.9384 & 517.6133 & 122.5994 & 2.9074 \\
\hline 1633 & 30.7443 & 512.8594 & 525.4272 & 517 & 122.5994 & 2.90583 \\
\hline 1634 & 30.7613 & 530.2676 & 525.6046 & 517.0416 & 122.5994 & 2.90426 \\
\hline 1635 & 30.7783 & 540.4587 & 525.8181 & 517.0832 & 122.5994 & 2.9027 \\
\hline 1636 & 30.7953 & 548.8592 & 526.0703 & 517.1248 & 122.5994 & 2.90113 \\
\hline 1637 & 30.8123 & 532.6952 & 526.3651 & 517.1663 & 122.5994 & 2.89957 \\
\hline 1638 & 30.8293 & 533.1328 & 526.707 & 517.2079 & 122.5994 & 2.89801 \\
\hline 1639 & 30.8463 & 555.6724 & 527.1021 & 517.2495 & 122.5994 & 2.89645 \\
\hline 1640 & 30.8633 & 584.6796 & 527.5583 & 517.2911 & 122.5994 & 2.89489 \\
\hline 1641 & 30.8803 & 581.6648 & 528.0864 & 517.3327 & 122.5994 & 2.89334 \\
\hline 1642 & 30.8973 & 562.2377 & 528.7005 & 517.3743 & 122.5994 & 2.89179 \\
\hline 1643 & 30.9143 & 608.4536 & 529.4197 & 517.4159 & 122.5994 & 2.89023 \\
\hline 1644 & 30.9313 & 626.2886 & 530.2698 & 517.4574 & 122.5994 & 2.88868 \\
\hline 1645 & 30.9483 & 617.5269 & 531.9689 & 517.499 & 122.5994 & 2.88714 \\
\hline 1646 & 30.9653 & 635.9955 & 533.2145 & 517.5406 & 122.5994 & 2.88559 \\
\hline 1647 & 30.9823 & 655.8383 & 534.7285 & 517.5822 & 122.5994 & 2.88405 \\
\hline 1648 & 30.9993 & 679.8756 & 536.5828 & 517.6238 & 122.5994 & 2.8825 \\
\hline 1649 & 31.0163 & 677.3886 & 539.1963 & 517.6654 & 122.5994 & 2.88096 \\
\hline 1650 & 31.0333 & 658.0383 & 542.0153 & 517.7069 & 122.5994 & 2.87942 \\
\hline 1651 & 31.0503 & 645.1744 & 544.2419 & 517.7485 & 122.5994 & 2.87788 \\
\hline 1652 & 31.0673 & 653.7738 & 548.4958 & 517.7901 & 122.5994 & 2.87635 \\
\hline 1653 & 31.0843 & 624.4106 & 553.6271 & 517.8317 & 122.5994 & 2.87481 \\
\hline 1654 & 31.1013 & 605.1205 & 559.7477 & 517.8733 & 122.5994 & 2.87328 \\
\hline 1655 & 31.1183 & 608.3339 & 566.3298 & 517.9149 & 122.5994 & 2.87175 \\
\hline 1656 & 31.1353 & 588.414 & 574.6962 & 517.9565 & 122.5994 & 2.87022 \\
\hline 1657 & 31.1523 & 621.423 & 584.2224 & 517.998 & 122.5994 & 2.86869 \\
\hline 1658 & 31.1693 & 628.7619 & 594.6844 & 517.8612 & 122.5994 & 2.86717 \\
\hline 1659 & 31.1863 & 596.8552 & 606.1337 & 517.7243 & 122.5994 & 2.86564 \\
\hline 1660 & 31.2033 & 606.1005 & 618.3389 & 517.5874 & 122.5994 & 2.86412 \\
\hline 1661 & 31.2203 & 614.981 & 630.9449 & 517.4505 & 122.5994 & 2.8626 \\
\hline 1662 & 31.2373 & 629.8219 & 643.4456 & 517.3136 & 122.5994 & 2.86108 \\
\hline 1663 & 31.2543 & 627.4209 & 655.1878 & 517.1768 & 122.5994 & 2.85956 \\
\hline 1664 & 31.2713 & 625.0117 & 665.4161 & 517.0399 & 122.5994 & 2.85805 \\
\hline
\end{tabular}




\begin{tabular}{|c|c|c|c|c|c|c|}
\hline 1665 & 31.2883 & 641.2026 & 673.3722 & 516.903 & 122.5994 & 2.85653 \\
\hline 1666 & 31.3053 & 666.3245 & 678.4487 & 516.7661 & 122.5994 & 2.85502 \\
\hline 1667 & 31.3223 & 636.2256 & 680.311 & 516.6292 & 122.5994 & 2.85351 \\
\hline 1668 & 31.3393 & 643.1661 & 679.0317 & 516.4924 & 122.5994 & 2.852 \\
\hline 1669 & 31.3563 & 645.1721 & 674.6401 & 516.3555 & 122.5994 & 2.85049 \\
\hline 1670 & 31.3733 & 659.1328 & 667.6147 & 516.2186 & 122.5994 & 2.84899 \\
\hline 1671 & 31.3903 & 599.787 & 658.4354 & 516.0817 & 122.5994 & 2.84748 \\
\hline 1672 & 31.4073 & 600.035 & 647.7061 & 515.9448 & 122.5994 & 2.84598 \\
\hline 1673 & 31.4243 & 619.4619 & 635.9348 & 515.8079 & 122.5994 & 2.84448 \\
\hline 1674 & 31.4413 & 660.3684 & 623.7633 & 515.6711 & 122.5994 & 2.84298 \\
\hline 1675 & 31.4583 & 596.0958 & 611.72 & 515.5342 & 122.5994 & 2.84148 \\
\hline 1676 & 31.4753 & 632.8937 & 600.2253 & 515.3973 & 122.5994 & 2.83999 \\
\hline 1677 & 31.4923 & 580.5258 & 589.5781 & 515.2604 & 122.5994 & 2.83849 \\
\hline 1678 & 31.5093 & 576.7425 & 579.9636 & 515.1235 & 122.5994 & 2.837 \\
\hline 1679 & 31.5263 & 584.6456 & 571.4722 & 514.9867 & 122.5994 & 2.83551 \\
\hline 1680 & 31.5433 & 571.2597 & 564.1201 & 514.8498 & 122.5994 & 2.83402 \\
\hline 1681 & 31.5603 & 543.8143 & 557.8651 & 514.7129 & 122.5994 & 2.83253 \\
\hline 1682 & 31.5773 & 574.3962 & 552.6279 & 514.576 & 122.5994 & 2.83105 \\
\hline 1683 & 31.5943 & 573.8239 & 548.3037 & 514.4391 & 122.5994 & 2.82956 \\
\hline 1684 & 31.6113 & 595.8929 & 544.7753 & 514.3022 & 122.5994 & 2.82808 \\
\hline 1685 & 31.6283 & 557.3129 & 541.9258 & 514.1654 & 122.5994 & 2.8266 \\
\hline 1686 & 31.6453 & 540.5345 & 539.6481 & 514.0285 & 122.5994 & 2.82512 \\
\hline 1687 & 31.6623 & 536.1855 & 537.8506 & 513.8916 & 122.5994 & 2.82364 \\
\hline 1688 & 31.6793 & 540.1348 & 536.4604 & 513.7547 & 122.5994 & 2.82216 \\
\hline 1689 & 31.6963 & 535.0994 & 535.4244 & 513.6178 & 122.5994 & 2.82069 \\
\hline 1690 & 31.7133 & 544.5708 & 534.7086 & 513.481 & 122.5994 & 2.81922 \\
\hline 1691 & 31.7303 & 536.9616 & 534.2999 & 513.3441 & 122.5994 & 2.81774 \\
\hline 1692 & 31.7473 & 515.7782 & 534.2054 & 513.2072 & 122.5994 & 2.81627 \\
\hline 1693 & 31.7643 & 532.6413 & 534.4509 & 513.0703 & 122.5994 & 2.81481 \\
\hline 1694 & 31.7813 & 538.3213 & 535.08 & 512.9334 & 122.5994 & 2.81334 \\
\hline 1695 & 31.7983 & 526.2177 & 536.1515 & 512.7965 & 122.5994 & 2.81187 \\
\hline 1696 & 31.8153 & 529.5998 & 537.7376 & 512.6597 & 122.5994 & 2.81041 \\
\hline 1697 & 31.8323 & 555.107 & 539.9206 & 512.5228 & 122.5994 & 2.80895 \\
\hline 1698 & 31.8493 & 526.5545 & 542.7887 & 512.3859 & 122.5994 & 2.80749 \\
\hline 1699 & 31.8663 & 527.2117 & 546.4299 & 512.249 & 122.5994 & 2.80603 \\
\hline 1700 & 31.8833 & 546.6067 & 550.9265 & 512.1121 & 122.5994 & 2.80457 \\
\hline 1701 & 31.9003 & 543.4808 & 556.3433 & 511.9753 & 122.5994 & 2.80311 \\
\hline 1702 & 31.9173 & 511.8553 & 562.7198 & 511.8384 & 122.5994 & 2.80166 \\
\hline 1703 & 31.9343 & 537.2133 & 570.0572 & 511.7015 & 122.5994 & 2.80021 \\
\hline 1704 & 31.9513 & 540.7069 & 578.3055 & 511.5646 & 122.5994 & 2.79876 \\
\hline 1705 & 31.9683 & 553.8602 & 587.3478 & 511.4277 & 122.5994 & 2.79731 \\
\hline 1706 & 31.9853 & 589.6197 & 596.8032 & 511.1124 & 122.5994 & 2.79586 \\
\hline 1707 & 32.0023 & 596.7367 & 606.542 & 510.797 & 122.5994 & 2.79441 \\
\hline 1708 & 32.0193 & 591.8923 & 616.1354 & 510.4817 & 122.5994 & 2.79297 \\
\hline 1709 & 32.0363 & 590.4334 & 625.0432 & 510.1663 & 122.5994 & 2.79152 \\
\hline 1710 & 32.0533 & 586.8274 & 632.6467 & 509.851 & 122.5994 & 2.79008 \\
\hline 1711 & 32.0703 & 574.1432 & 638.3546 & 509.5356 & 122.5994 & 2.78864 \\
\hline 1712 & 32.0873 & 559.5521 & 641.716 & 509.2203 & 122.5994 & 2.7872 \\
\hline 1713 & 32.1043 & 622.3016 & 642.5101 & 508.9049 & 122.5994 & 2.78577 \\
\hline
\end{tabular}




\begin{tabular}{|c|c|c|c|c|c|c|}
\hline 1714 & 32.1213 & 564.0013 & 640.759 & 508.5896 & 122.5994 & 2.78433 \\
\hline 1715 & 32.1383 & 594.5143 & 636.6681 & 508.2743 & 122.5994 & 2.7829 \\
\hline 1716 & 32.1553 & 636.0641 & 630.554 & 507.9589 & 122.5994 & 2.78147 \\
\hline 1717 & 32.1723 & 598.9323 & 622.805 & 507.6436 & 122.5994 & 2.78003 \\
\hline 1718 & 32.1893 & 532.6947 & 613.8615 & 507.3282 & 122.5994 & 2.7786 \\
\hline 1719 & 32.2063 & 583.6003 & 604.2089 & 507.0129 & 122.5994 & 2.77718 \\
\hline 1720 & 32.2233 & 520.8042 & 594.3162 & 506.6975 & 122.5994 & 2.77575 \\
\hline 1721 & 32.2403 & 520.1335 & 584.6116 & 506.3822 & 122.5994 & 2.77433 \\
\hline 1722 & 32.2573 & 547.3697 & 575.4274 & 506.0668 & 122.5994 & 2.7729 \\
\hline 1723 & 32.2743 & 543.7954 & 567.0085 & 505.7515 & 122.5994 & 2.77148 \\
\hline 1724 & 32.2913 & 561.0797 & 559.5037 & 505.4361 & 122.5994 & 2.77006 \\
\hline 1725 & 32.3083 & 547.0309 & 552.9855 & 505.1208 & 122.5994 & 2.76864 \\
\hline 1726 & 32.3253 & 499.4379 & 547.4693 & 504.8054 & 122.5994 & 2.76722 \\
\hline 1727 & 32.3423 & 517.7516 & 542.9258 & 504.4901 & 122.5994 & 2.76581 \\
\hline 1728 & 32.3593 & 529.3386 & 539.2859 & 504.1747 & 122.5994 & 2.76439 \\
\hline 1729 & 32.3763 & 511.13 & 536.4567 & 503.8594 & 122.5994 & 2.76298 \\
\hline 1730 & 32.3933 & 533.5588 & 534.1068 & 503.3236 & 122.5994 & 2.76157 \\
\hline 1731 & 32.4103 & 525.5742 & 532.3381 & 502.7878 & 122.5994 & 2.76016 \\
\hline 1732 & 32.4273 & 500.5374 & 530.1537 & 502.252 & 122.5994 & 2.75875 \\
\hline 1733 & 32.4443 & 506.0564 & 529.1441 & 501.7161 & 122.5994 & 2.75735 \\
\hline 1734 & 32.4613 & 500.5606 & 528.2902 & 501.1803 & 122.5994 & 2.75594 \\
\hline 1735 & 32.4783 & 510.0746 & 527.4601 & 500.6445 & 122.5994 & 2.75454 \\
\hline 1736 & 32.4953 & 495.8678 & 526.1144 & 500.1087 & 122.5994 & 2.75313 \\
\hline 1737 & 32.5123 & 499.7404 & 525.0488 & 499.5729 & 122.5994 & 2.75173 \\
\hline 1738 & 32.5293 & 487.9544 & 523.7795 & 499.0371 & 122.5994 & 2.75033 \\
\hline 1739 & 32.5463 & 498.1862 & 522.2977 & 498.5013 & 122.5994 & 2.74894 \\
\hline 1740 & 32.5633 & 502.2653 & 520.6158 & 497.9655 & 122.5994 & 2.74754 \\
\hline 1741 & 32.5803 & 511.2781 & 518.7624 & 497.4297 & 122.5994 & 2.74615 \\
\hline 1742 & 32.5973 & 497.9995 & 516.7758 & 496.8939 & 122.5994 & 2.74475 \\
\hline 1743 & 32.6143 & 480.041 & 514.7036 & 496.3581 & 122.5994 & 2.74336 \\
\hline 1744 & 32.6313 & 470.8448 & 512.6427 & 495.8223 & 122.5994 & 2.74197 \\
\hline 1745 & 32.6483 & 508.7144 & 510.5497 & 495.2865 & 122.5994 & 2.74058 \\
\hline 1746 & 32.6653 & 537.7721 & 508.5118 & 494.7507 & 122.5994 & 2.73919 \\
\hline 1747 & 32.6823 & 498.0852 & 506.5598 & 494.2148 & 122.5994 & 2.73781 \\
\hline 1748 & 32.6993 & 496.9945 & 504.716 & 493.679 & 122.5994 & 2.73642 \\
\hline 1749 & 32.7163 & 477.6413 & 503.0038 & 493.1432 & 122.5994 & 2.73504 \\
\hline 1750 & 32.7333 & 461.8847 & 501.4059 & 492.6074 & 122.5994 & 2.73366 \\
\hline 1751 & 32.7503 & 486.5957 & 499.9317 & 492.0716 & 122.5994 & 2.73228 \\
\hline 1752 & 32.7673 & 509.508 & 498.5761 & 491.5358 & 122.5994 & 2.7309 \\
\hline 1753 & 32.7843 & 505.8167 & 497.3305 & 491 & 122.5994 & 2.72952 \\
\hline 1754 & 32.8013 & 464.1494 & 496.9846 & 491.2708 & 122.5994 & 2.72815 \\
\hline 1755 & 32.8183 & 484.8892 & 496.7342 & 491.5417 & 122.5994 & 2.72677 \\
\hline 1756 & 32.8353 & 483.7877 & 496.5608 & 491.8125 & 122.5994 & 2.7254 \\
\hline 1757 & 32.8523 & 511.1602 & 496.4536 & 492.0833 & 122.5994 & 2.72403 \\
\hline 1758 & 32.8693 & 480.4305 & 496.4022 & 492.3542 & 122.5994 & 2.72266 \\
\hline 1759 & 32.8863 & 496.443 & 496.3988 & 492.625 & 122.5994 & 2.72129 \\
\hline 1760 & 32.9033 & 475.5604 & 496.4329 & 492.8958 & 122.5994 & 2.71992 \\
\hline 1761 & 32.9203 & 521.3173 & 496.4988 & 493.1667 & 122.5994 & 2.71856 \\
\hline 1762 & 32.9373 & 496.8802 & 496.591 & 493.4375 & 122.5994 & 2.71719 \\
\hline
\end{tabular}




\begin{tabular}{|c|c|c|c|c|c|c|}
\hline 1763 & 32.9543 & 472.7823 & 496.7049 & 493.7083 & 122.5994 & 2.71583 \\
\hline 1764 & 32.9713 & 508.1629 & 496.8368 & 493.9792 & 122.5994 & 2.71447 \\
\hline 1765 & 32.9883 & 480.9719 & 496.984 & 494.25 & 122.5994 & 2.71311 \\
\hline 1766 & 33.0053 & 454.2628 & 497.1442 & 494.5208 & 122.5994 & 2.71175 \\
\hline 1767 & 33.0223 & 524.3133 & 497.3157 & 494.7917 & 122.5994 & 2.71039 \\
\hline 1768 & 33.0393 & 565.703 & 497.4974 & 495.0625 & 122.5994 & 2.70904 \\
\hline 1769 & 33.0563 & 525.8789 & 497.6884 & 495.3333 & 122.5994 & 2.70768 \\
\hline 1770 & 33.0733 & 563.2594 & 497.8879 & 495.6042 & 122.5994 & 2.70633 \\
\hline 1771 & 33.0903 & 583.1798 & 498.0956 & 495.875 & 122.5994 & 2.70498 \\
\hline 1772 & 33.1073 & 559.4166 & 498.3112 & 496.1458 & 122.5994 & 2.70363 \\
\hline 1773 & 33.1243 & 585.8616 & 498.5345 & 496.4167 & 122.5994 & 2.70228 \\
\hline 1774 & 33.1413 & 614.1367 & 498.7664 & 496.6875 & 122.5994 & 2.70093 \\
\hline 1775 & 33.1583 & 570.5249 & 499.005 & 496.9583 & 122.5994 & 2.69958 \\
\hline 1776 & 33.1753 & 528.7085 & 499.2511 & 497.2292 & 122.5994 & 2.69824 \\
\hline 1777 & 33.1923 & 560.3846 & 499.5049 & 497.5 & 122.5994 & 2.6969 \\
\hline 1778 & 33.2093 & 541.5964 & 499.0893 & 497.7708 & 122.5994 & 2.69556 \\
\hline 1779 & 33.2263 & 545.0735 & 499.378 & 498.0417 & 122.5994 & 2.69422 \\
\hline 1780 & 33.2433 & 523.2116 & 499.6726 & 498.3125 & 122.5994 & 2.69288 \\
\hline 1781 & 33.2603 & 521.1563 & 499.9733 & 498.5833 & 122.5994 & 2.69154 \\
\hline 1782 & 33.2773 & 525.9514 & 499.927 & 498.8542 & 122.5994 & 2.6902 \\
\hline 1783 & 33.2943 & 533.6998 & 500.2495 & 499.125 & 122.5994 & 2.68887 \\
\hline 1784 & 33.3113 & 537.7899 & 500.5775 & 499.3958 & 122.5994 & 2.68753 \\
\hline 1785 & 33.3283 & 522.0466 & 500.9115 & 499.6667 & 122.5994 & 2.6862 \\
\hline 1786 & 33.3453 & 505.2354 & 501.2523 & 499.9375 & 122.5994 & 2.68487 \\
\hline 1787 & 33.3623 & 505.063 & 501.5967 & 500.2083 & 122.5994 & 2.68354 \\
\hline 1788 & 33.3793 & 515.2406 & 501.9529 & 500.4792 & 122.5994 & 2.68221 \\
\hline 1789 & 33.3963 & 517.5027 & 502.3172 & 500.75 & 122.5994 & 2.68089 \\
\hline 1790 & 33.4133 & 485.1081 & 502.6897 & 501.0208 & 122.5994 & 2.67956 \\
\hline 1791 & 33.4303 & 471.7072 & 503.1062 & 501.2917 & 122.5994 & 2.67824 \\
\hline 1792 & 33.4473 & 495.2896 & 503.4949 & 501.5625 & 122.5994 & 2.67692 \\
\hline 1793 & 33.4643 & 504.7469 & 504.2275 & 501.8333 & 122.5994 & 2.6756 \\
\hline 1794 & 33.4813 & 505.619 & 504.6433 & 502.1042 & 122.5994 & 2.67428 \\
\hline 1795 & 33.4983 & 507.1137 & 505.0681 & 502.375 & 122.5994 & 2.67296 \\
\hline 1796 & 33.5153 & 505.1164 & 505.5039 & 502.6458 & 122.5994 & 2.67164 \\
\hline 1797 & 33.5323 & 516.397 & 505.9538 & 502.9167 & 122.5994 & 2.67033 \\
\hline 1798 & 33.5493 & 474.5277 & 506.4249 & 503.1875 & 122.5994 & 2.66901 \\
\hline 1799 & 33.5663 & 485.4093 & 507.2198 & 503.4583 & 122.5994 & 2.6677 \\
\hline 1800 & 33.5833 & 501.7119 & 507.7628 & 503.7292 & 122.5994 & 2.66639 \\
\hline 1801 & 33.6003 & 525.0587 & 508.3486 & 504 & 122.5994 & 2.66508 \\
\hline 1802 & 33.6173 & 506.2886 & 509.5638 & 504.8516 & 122.5994 & 2.66377 \\
\hline 1803 & 33.6343 & 476.8548 & 510.8324 & 505.7032 & 122.5994 & 2.66246 \\
\hline 1804 & 33.6513 & 474.4062 & 512.1698 & 506.5548 & 122.5994 & 2.66115 \\
\hline 1805 & 33.6683 & 493.1723 & 513.5209 & 507.4064 & 122.5994 & 2.65985 \\
\hline 1806 & 33.6853 & 446.7362 & 514.9496 & 508.258 & 122.5994 & 2.65855 \\
\hline 1807 & 33.7023 & 481.0612 & 516.4127 & 509.1096 & 122.5994 & 2.65724 \\
\hline 1808 & 33.7193 & 520.0364 & 517.8905 & 509.9612 & 122.5994 & 2.65594 \\
\hline 1809 & 33.7363 & 492.0273 & 519.3569 & 510.8128 & 122.5994 & 2.65464 \\
\hline 1810 & 33.7533 & 525.823 & 520.7393 & 511.6644 & 122.5994 & 2.65335 \\
\hline 1811 & 33.7703 & 503.3415 & 522.0835 & 512.516 & 122.5994 & 2.65205 \\
\hline
\end{tabular}




\begin{tabular}{|c|c|c|c|c|c|c|}
\hline 1812 & 33.7873 & 501.7119 & 523.316 & 513.3676 & 122.5994 & 2.65075 \\
\hline 1813 & 33.8043 & 467.0633 & 524.4136 & 514.2192 & 122.5994 & 2.64946 \\
\hline 1814 & 33.8213 & 519.7495 & 525.3658 & 515.0708 & 122.5994 & 2.64817 \\
\hline 1815 & 33.8383 & 515.0823 & 526.5809 & 515.9224 & 122.5994 & 2.64687 \\
\hline 1816 & 33.8553 & 508.3494 & 527.2665 & 516.774 & 122.5994 & 2.64558 \\
\hline 1817 & 33.8723 & 542.952 & 527.8339 & 517.6257 & 122.5994 & 2.6443 \\
\hline 1818 & 33.8893 & 488.2316 & 528.5192 & 518.4773 & 122.5994 & 2.64301 \\
\hline 1819 & 33.9063 & 531.6576 & 528.9205 & 519.3289 & 122.5994 & 2.64172 \\
\hline 1820 & 33.9233 & 546.5923 & 529.2745 & 520.1805 & 122.5994 & 2.64044 \\
\hline 1821 & 33.9403 & 518.8372 & 529.8155 & 521.0321 & 122.5994 & 2.63915 \\
\hline 1822 & 33.9573 & 558.3582 & 530.1609 & 521.8837 & 122.5994 & 2.63787 \\
\hline 1823 & 33.9743 & 541.8957 & 531.3377 & 522.7353 & 122.5994 & 2.63659 \\
\hline 1824 & 33.9913 & 542.5102 & 531.7773 & 523.5869 & 122.5994 & 2.63531 \\
\hline 1825 & 34.0083 & 555.7493 & 532.2725 & 524.4385 & 122.5994 & 2.63403 \\
\hline 1826 & 34.0253 & 545.9866 & 532.64 & 525.099 & 122.5994 & 2.63275 \\
\hline 1827 & 34.0423 & 531.7387 & 533.5361 & 525.7595 & 122.5994 & 2.63148 \\
\hline 1828 & 34.0593 & 529.6644 & 534.0546 & 526.42 & 122.5994 & 2.6302 \\
\hline 1829 & 34.0763 & 519.9469 & 534.6425 & 527.0805 & 122.5994 & 2.62893 \\
\hline 1830 & 34.0933 & 536.5528 & 535.2984 & 527.741 & 122.5994 & 2.62766 \\
\hline 1831 & 34.1103 & 555.0058 & 536.02 & 528.4015 & 122.5994 & 2.62639 \\
\hline 1832 & 34.1273 & 577.1445 & 536.8459 & 529.062 & 122.5994 & 2.62512 \\
\hline 1833 & 34.1443 & 568.8876 & 537.7167 & 529.7225 & 122.5994 & 2.62385 \\
\hline 1834 & 34.1613 & 575.1945 & 538.713 & 530.383 & 122.5994 & 2.62258 \\
\hline 1835 & 34.1783 & 557.286 & 539.6852 & 531.0435 & 122.5994 & 2.62132 \\
\hline 1836 & 34.1953 & 580.2295 & 540.7213 & 531.704 & 122.5994 & 2.62005 \\
\hline 1837 & 34.2123 & 588.0368 & 541.8268 & 532.3645 & 122.5994 & 2.61879 \\
\hline 1838 & 34.2293 & 563.8905 & 543.0109 & 533.025 & 122.5994 & 2.61753 \\
\hline 1839 & 34.2463 & 556.6016 & 544.3307 & 533.6855 & 122.5994 & 2.61627 \\
\hline 1840 & 34.2633 & 573.9318 & 545.7196 & 534.346 & 122.5994 & 2.61501 \\
\hline 1841 & 34.2803 & 602.1332 & 547.2449 & 535.0065 & 122.5994 & 2.61375 \\
\hline 1842 & 34.2973 & 607.017 & 548.9374 & 535.667 & 122.5994 & 2.61249 \\
\hline 1843 & 34.3143 & 595.9531 & 550.8353 & 536.3275 & 122.5994 & 2.61124 \\
\hline 1844 & 34.3313 & 596.8214 & 552.9825 & 536.988 & 122.5994 & 2.60998 \\
\hline 1845 & 34.3483 & 583.8009 & 555.4998 & 537.6485 & 122.5994 & 2.60873 \\
\hline 1846 & 34.3653 & 586.1848 & 558.3036 & 538.309 & 122.5994 & 2.60748 \\
\hline 1847 & 34.3823 & 583.4734 & 561.5196 & 538.9695 & 122.5994 & 2.60623 \\
\hline 1848 & 34.3993 & 595.2858 & 565.2057 & 539.63 & 122.5994 & 2.60498 \\
\hline 1849 & 34.4163 & 603.9342 & 569.4144 & 540.2905 & 122.5994 & 2.60373 \\
\hline 1850 & 34.4333 & 612.8429 & 574.0216 & 540.7464 & 122.5994 & 2.60249 \\
\hline 1851 & 34.4503 & 631.2249 & 579.1862 & 541.2023 & 122.5994 & 2.60124 \\
\hline 1852 & 34.4673 & 626.6804 & 584.9507 & 541.6582 & 122.5994 & 2.6 \\
\hline 1853 & 34.4843 & 659.5548 & 591.2893 & 542.1141 & 122.5994 & 2.59875 \\
\hline 1854 & 34.5013 & 638.9653 & 598.1413 & 542.57 & 122.5994 & 2.59751 \\
\hline 1855 & 34.5183 & 695.028 & 605.3956 & 543.0259 & 122.5994 & 2.59627 \\
\hline 1856 & 34.5353 & 650.0762 & 612.8956 & 543.4818 & 122.5994 & 2.59503 \\
\hline 1857 & 34.5523 & 703.3877 & 620.4312 & 543.9377 & 122.5994 & 2.5938 \\
\hline 1858 & 34.5693 & 662.6659 & 627.7479 & 544.3936 & 122.5994 & 2.59256 \\
\hline 1859 & 34.5863 & 680.6742 & 634.5671 & 544.8494 & 122.5994 & 2.59132 \\
\hline 1860 & 34.6033 & 756.2543 & 640.6291 & 545.3053 & 122.5994 & 2.59009 \\
\hline
\end{tabular}




\begin{tabular}{|c|c|c|c|c|c|c|}
\hline 1861 & 34.6203 & 681.6762 & 645.7338 & 545.7612 & 122.5994 & 2.58886 \\
\hline 1862 & 34.6373 & 707.4323 & 649.764 & 546.2171 & 122.5994 & 2.58762 \\
\hline 1863 & 34.6543 & 701.8481 & 652.7136 & 546.673 & 122.5994 & 2.58639 \\
\hline 1864 & 34.6713 & 697.0529 & 654.6345 & 547.1289 & 122.5994 & 2.58516 \\
\hline 1865 & 34.6883 & 700.8745 & 655.6391 & 547.5848 & 122.5994 & 2.58394 \\
\hline 1866 & 34.7053 & 735.2027 & 655.9166 & 548.0407 & 122.5994 & 2.58271 \\
\hline 1867 & 34.7223 & 731.7921 & 655.8713 & 548.4966 & 122.5994 & 2.58148 \\
\hline 1868 & 34.7393 & 694.5675 & 655.5621 & 548.9525 & 122.5994 & 2.58026 \\
\hline 1869 & 34.7563 & 733.4667 & 655.4966 & 549.4084 & 122.5994 & 2.57904 \\
\hline 1870 & 34.7733 & 724.3447 & 656.042 & 549.8643 & 122.5994 & 2.57781 \\
\hline 1871 & 34.7903 & 703.9166 & 657.5416 & 550.3201 & 122.5994 & 2.57659 \\
\hline 1872 & 34.8073 & 697.0708 & 660.3621 & 550.776 & 122.5994 & 2.57537 \\
\hline 1873 & 34.8243 & 728.1404 & 664.6028 & 551.2319 & 122.5994 & 2.57416 \\
\hline 1874 & 34.8413 & 760.6162 & 670.2987 & 551.4907 & 122.5994 & 2.57294 \\
\hline 1875 & 34.8583 & 750.5125 & 677.7117 & 551.7494 & 122.5994 & 2.57172 \\
\hline 1876 & 34.8753 & 767.5738 & 686.795 & 552.0081 & 122.5994 & 2.57051 \\
\hline 1877 & 34.8923 & 796.9358 & 697.359 & 552.2669 & 122.5994 & 2.56929 \\
\hline 1878 & 34.9093 & 820.7154 & 709.0795 & 552.5256 & 122.5994 & 2.56808 \\
\hline 1879 & 34.9263 & 799.5743 & 721.8622 & 552.7844 & 122.5994 & 2.56687 \\
\hline 1880 & 34.9433 & 799.2466 & 734.4775 & 553.0431 & 122.5994 & 2.56566 \\
\hline 1881 & 34.9603 & 794.5985 & 746.7386 & 553.3018 & 122.5994 & 2.56445 \\
\hline 1882 & 34.9773 & 786.0072 & 758.1099 & 553.5606 & 122.5994 & 2.56325 \\
\hline 1883 & 34.9943 & 826.5463 & 768.2782 & 553.8193 & 122.5994 & 2.56204 \\
\hline 1884 & 35.0113 & 840.6072 & 776.8239 & 554.0781 & 122.5994 & 2.56083 \\
\hline 1885 & 35.0283 & 832.6478 & 783.5699 & 554.3368 & 122.5994 & 2.55963 \\
\hline 1886 & 35.0453 & 838.3619 & 787.8737 & 554.5955 & 122.5994 & 2.55843 \\
\hline 1887 & 35.0623 & 854.4593 & 789.4362 & 554.8543 & 122.5994 & 2.55723 \\
\hline 1888 & 35.0793 & 868.0167 & 788.2116 & 555.113 & 122.5994 & 2.55603 \\
\hline 1889 & 35.0963 & 855.5706 & 784.239 & 555.3717 & 122.5994 & 2.55483 \\
\hline 1890 & 35.1133 & 854.4488 & 777.8063 & 555.6305 & 122.5994 & 2.55363 \\
\hline 1891 & 35.1303 & 820.0598 & 769.3823 & 555.8892 & 122.5994 & 2.55243 \\
\hline 1892 & 35.1473 & 793.0618 & 759.3521 & 556.148 & 122.5994 & 2.55124 \\
\hline 1893 & 35.1643 & 781.7763 & 748.1737 & 556.4067 & 122.5994 & 2.55004 \\
\hline 1894 & 35.1813 & 790.6255 & 736.2191 & 556.6654 & 122.5994 & 2.54885 \\
\hline 1895 & 35.1983 & 778.5034 & 723.8563 & 556.9242 & 122.5994 & 2.54766 \\
\hline 1896 & 35.2153 & 726.4052 & 711.4399 & 557.1829 & 122.5994 & 2.54647 \\
\hline 1897 & 35.2323 & 704.5265 & 699.3134 & 557.4417 & 122.5994 & 2.54528 \\
\hline 1898 & 35.2493 & 663.902 & 687.5718 & 557.5178 & 122.5994 & 2.54409 \\
\hline 1899 & 35.2663 & 681.3363 & 676.5906 & 557.594 & 122.5994 & 2.5429 \\
\hline 1900 & 35.2833 & 646.6955 & 666.4784 & 557.6701 & 122.5994 & 2.54171 \\
\hline 1901 & 35.3003 & 644.4947 & 657.259 & 557.7463 & 122.5994 & 2.54053 \\
\hline 1902 & 35.3173 & 659.0818 & 648.918 & 557.8224 & 122.5994 & 2.53934 \\
\hline 1903 & 35.3343 & 665.185 & 641.4172 & 557.8986 & 122.5994 & 2.53816 \\
\hline 1904 & 35.3513 & 617.7403 & 634.7203 & 557.9748 & 122.5994 & 2.53698 \\
\hline 1905 & 35.3683 & 591.3816 & 628.7841 & 558.0509 & 122.5994 & 2.5358 \\
\hline 1906 & 35.3853 & 609.8068 & 623.5649 & 558.1271 & 122.5994 & 2.53462 \\
\hline 1907 & 35.4023 & 600.0949 & 619.2754 & 558.2032 & 122.5994 & 2.53344 \\
\hline 1908 & 35.4193 & 592.7839 & 615.3149 & 558.2794 & 122.5994 & 2.53226 \\
\hline 1909 & 35.4363 & 656.4547 & 611.8763 & 558.3556 & 122.5994 & 2.53109 \\
\hline
\end{tabular}




\begin{tabular}{|c|c|c|c|c|c|c|}
\hline 1910 & 35.4533 & 586.3078 & 608.8837 & 558.4317 & 122.5994 & 2.52991 \\
\hline 1911 & 35.4703 & 594.5049 & 606.2639 & 558.5079 & 122.5994 & 2.52874 \\
\hline 1912 & 35.4873 & 582.7684 & 604.0593 & 558.584 & 122.5994 & 2.52757 \\
\hline 1913 & 35.5043 & 561.1064 & 602.3053 & 558.6602 & 122.5994 & 2.5264 \\
\hline 1914 & 35.5213 & 564.6487 & 600.4332 & 558.7364 & 122.5994 & 2.52523 \\
\hline 1915 & 35.5383 & 605.2407 & 598.7338 & 558.8125 & 122.5994 & 2.52406 \\
\hline 1916 & 35.5553 & 602.1137 & 597.2021 & 558.8887 & 122.5994 & 2.52289 \\
\hline 1917 & 35.5723 & 587.1714 & 595.8472 & 558.9648 & 122.5994 & 2.52172 \\
\hline 1918 & 35.5893 & 576.0768 & 594.6881 & 559.041 & 122.5994 & 2.52056 \\
\hline 1919 & 35.6063 & 575.9448 & 593.7496 & 559.1172 & 122.5994 & 2.51939 \\
\hline 1920 & 35.6233 & 581.688 & 593.0641 & 559.1933 & 122.5994 & 2.51823 \\
\hline 1921 & 35.6403 & 584.8229 & 592.6673 & 559.2695 & 122.5994 & 2.51707 \\
\hline 1922 & 35.6573 & 556.9354 & 592.7428 & 559.3456 & 122.5994 & 2.51591 \\
\hline 1923 & 35.6743 & 564.5698 & 593.1534 & 559.4218 & 122.5994 & 2.51475 \\
\hline 1924 & 35.6913 & 591.6506 & 593.8288 & 559.4979 & 122.5994 & 2.51359 \\
\hline 1925 & 35.7083 & 581.8872 & 594.8934 & 559.5741 & 122.5994 & 2.51243 \\
\hline 1926 & 35.7253 & 594.3768 & 596.0654 & 559.6503 & 122.5994 & 2.51127 \\
\hline 1927 & 35.7423 & 590.2467 & 597.8818 & 559.7264 & 122.5994 & 2.51012 \\
\hline 1928 & 35.7593 & 572.1089 & 600.0067 & 559.8026 & 122.5994 & 2.50896 \\
\hline 1929 & 35.7763 & 590.6052 & 602.4581 & 559.8787 & 122.5994 & 2.50781 \\
\hline 1930 & 35.7933 & 590.6262 & 605.032 & 559.9549 & 122.5994 & 2.50666 \\
\hline 1931 & 35.8103 & 590.1865 & 607.3788 & 560.0311 & 122.5994 & 2.50551 \\
\hline 1932 & 35.8273 & 583.0711 & 610.1005 & 560.1072 & 122.5994 & 2.50436 \\
\hline 1933 & 35.8443 & 559.1609 & 613.0189 & 560.1834 & 122.5994 & 2.50321 \\
\hline 1934 & 35.8613 & 565.2436 & 615.7856 & 560.2595 & 122.5994 & 2.50206 \\
\hline 1935 & 35.8783 & 575.0073 & 618.6238 & 560.3357 & 122.5994 & 2.50091 \\
\hline 1936 & 35.8953 & 547.3192 & 621.476 & 560.4119 & 122.5994 & 2.49977 \\
\hline 1937 & 35.9123 & 558.3312 & 624.592 & 560.488 & 122.5994 & 2.49862 \\
\hline 1938 & 35.9293 & 555.144 & 627.8593 & 560.5642 & 122.5994 & 2.49748 \\
\hline 1939 & 35.9463 & 574.2378 & 631.3583 & 560.6403 & 122.5994 & 2.49634 \\
\hline 1940 & 35.9633 & 610.3328 & 634.8237 & 560.7165 & 122.5994 & 2.4952 \\
\hline 1941 & 35.9803 & 580.2286 & 638.2172 & 560.7927 & 122.5994 & 2.49406 \\
\hline 1942 & 35.9973 & 581.1679 & 641.3444 & 560.8688 & 122.5994 & 2.49292 \\
\hline 1943 & 36.0143 & 589.389 & 643.973 & 560.945 & 122.5994 & 2.49178 \\
\hline 1944 & 36.0313 & 610.3613 & 645.8738 & 561.0211 & 122.5994 & 2.49064 \\
\hline 1945 & 36.0483 & 578.7057 & 646.886 & 561.0973 & 122.5994 & 2.48951 \\
\hline 1946 & 36.0653 & 567.1052 & 646.7693 & 560.9909 & 122.5994 & 2.48837 \\
\hline 1947 & 36.0823 & 604.5218 & 645.3511 & 560.8845 & 122.5994 & 2.48724 \\
\hline 1948 & 36.0993 & 575.3456 & 643.5317 & 560.778 & 122.5994 & 2.48611 \\
\hline 1949 & 36.1163 & 615.8784 & 640.8098 & 560.6716 & 122.5994 & 2.48498 \\
\hline 1950 & 36.1333 & 634.0065 & 637.7253 & 560.5652 & 122.5994 & 2.48385 \\
\hline 1951 & 36.1503 & 583.2349 & 634.1927 & 560.4588 & 122.5994 & 2.48272 \\
\hline 1952 & 36.1673 & 530.0703 & 630.1584 & 560.3524 & 122.5994 & 2.48159 \\
\hline 1953 & 36.1843 & 585.5715 & 626.1864 & 560.2459 & 122.5994 & 2.48046 \\
\hline 1954 & 36.2013 & 609.3074 & 621.4556 & 560.1395 & 122.5994 & 2.47934 \\
\hline 1955 & 36.2183 & 575.463 & 617.6891 & 560.0331 & 122.5994 & 2.47821 \\
\hline 1956 & 36.2353 & 561.3319 & 614.2198 & 559.9267 & 122.5994 & 2.47709 \\
\hline 1957 & 36.2523 & 554.5095 & 611.2207 & 559.8203 & 122.5994 & 2.47597 \\
\hline 1958 & 36.2693 & 534.6339 & 608.6663 & 559.7138 & 122.5994 & 2.47485 \\
\hline
\end{tabular}




\begin{tabular}{|c|c|c|c|c|c|c|}
\hline 1959 & 36.2863 & 579.5178 & 606.351 & 559.6074 & 122.5994 & 2.47372 \\
\hline 1960 & 36.3033 & 570.5685 & 605.0141 & 559.501 & 122.5994 & 2.47261 \\
\hline 1961 & 36.3203 & 565.5693 & 604.3535 & 559.3946 & 122.5994 & 2.47149 \\
\hline 1962 & 36.3373 & 556.3308 & 604.4348 & 559.2882 & 122.5994 & 2.47037 \\
\hline 1963 & 36.3543 & 529.6211 & 605.2261 & 559.1817 & 122.5994 & 2.46925 \\
\hline 1964 & 36.3713 & 520.6651 & 606.7687 & 559.0753 & 122.5994 & 2.46814 \\
\hline 1965 & 36.3883 & 544.3621 & 608.936 & 558.9689 & 122.5994 & 2.46702 \\
\hline 1966 & 36.4053 & 571.0446 & 611.9613 & 558.8625 & 122.5994 & 2.46591 \\
\hline 1967 & 36.4223 & 585.302 & 615.8141 & 558.7561 & 122.5994 & 2.4648 \\
\hline 1968 & 36.4393 & 557.0792 & 620.2151 & 558.6496 & 122.5994 & 2.46369 \\
\hline 1969 & 36.4563 & 552.4958 & 625.3345 & 558.5432 & 122.5994 & 2.46258 \\
\hline 1970 & 36.4733 & 568.6258 & 631.2074 & 558.2688 & 122.5994 & 2.46147 \\
\hline 1971 & 36.4903 & 594.001 & 638.7894 & 557.9944 & 122.5994 & 2.46036 \\
\hline 1972 & 36.5073 & 625.3584 & 649.8433 & 557.7199 & 122.5994 & 2.45926 \\
\hline 1973 & 36.5243 & 644.398 & 666.7568 & 557.4455 & 122.5994 & 2.45815 \\
\hline 1974 & 36.5413 & 641.1941 & 692.8 & 557.1711 & 122.5994 & 2.45705 \\
\hline 1975 & 36.5583 & 702.807 & 730.4351 & 556.8967 & 122.5994 & 2.45594 \\
\hline 1976 & 36.5753 & 809.2508 & 779.1409 & 556.6222 & 122.5994 & 2.45484 \\
\hline 1977 & 36.5923 & 850.3686 & 832.5121 & 556.3478 & 122.5994 & 2.45374 \\
\hline 1978 & 36.6093 & 919.5714 & 875.1759 & 556.0734 & 122.5994 & 2.45264 \\
\hline 1979 & 36.6263 & 977.8253 & 887.6929 & 555.799 & 122.5994 & 2.45154 \\
\hline 1980 & 36.6433 & 915.2534 & 868.4095 & 555.5245 & 122.5994 & 2.45044 \\
\hline 1981 & 36.6603 & 857.4158 & 837.059 & 555.2501 & 122.5994 & 2.44934 \\
\hline 1982 & 36.6773 & 778.811 & 810.8959 & 554.9757 & 122.5994 & 2.44825 \\
\hline 1983 & 36.6943 & 748.4303 & 793.7201 & 554.7013 & 122.5994 & 2.44715 \\
\hline 1984 & 36.7113 & 797.5009 & 777.4106 & 554.4268 & 122.5994 & 2.44606 \\
\hline 1985 & 36.7283 & 771.6311 & 752.6469 & 554.1524 & 122.5994 & 2.44496 \\
\hline 1986 & 36.7453 & 694.1035 & 720.0731 & 553.878 & 122.5994 & 2.44387 \\
\hline 1987 & 36.7623 & 640.9078 & 687.7963 & 553.6036 & 122.5994 & 2.44278 \\
\hline 1988 & 36.7793 & 637.5372 & 660.9197 & 553.3292 & 122.5994 & 2.44169 \\
\hline 1989 & 36.7963 & 636.2647 & 641.0805 & 553.0547 & 122.5994 & 2.4406 \\
\hline 1990 & 36.8133 & 603.4013 & 627.6583 & 552.7803 & 122.5994 & 2.43951 \\
\hline 1991 & 36.8303 & 607.4806 & 619.2606 & 552.5059 & 122.5994 & 2.43843 \\
\hline 1992 & 36.8473 & 597.6748 & 613.9004 & 552.2315 & 122.5994 & 2.43734 \\
\hline 1993 & 36.8643 & 574.6575 & 610.4528 & 551.957 & 122.5994 & 2.43626 \\
\hline 1994 & 36.8813 & 583.8818 & 607.9647 & 551.5363 & 122.5994 & 2.43517 \\
\hline 1995 & 36.8983 & 616.6327 & 606.1444 & 551.1156 & 122.5994 & 2.43409 \\
\hline 1996 & 36.9153 & 624.7085 & 604.7643 & 550.6948 & 122.5994 & 2.43301 \\
\hline 1997 & 36.9323 & 620.2173 & 603.6835 & 550.2741 & 122.5994 & 2.43193 \\
\hline 1998 & 36.9493 & 586.8194 & 602.6438 & 549.8534 & 122.5994 & 2.43085 \\
\hline 1999 & 36.9663 & 590.383 & 601.8024 & 549.4326 & 122.5994 & 2.42977 \\
\hline 2000 & 36.9833 & 610.0624 & 600.884 & 549.0119 & 122.5994 & 2.42869 \\
\hline 2001 & 37.0003 & 600.5121 & 599.7667 & 548.5911 & 122.5994 & 2.42761 \\
\hline 2002 & 37.0173 & 610.9785 & 598.3651 & 548.1704 & 122.5994 & 2.42654 \\
\hline 2003 & 37.0343 & 623.8135 & 596.7561 & 547.7497 & 122.5994 & 2.42546 \\
\hline 2004 & 37.0513 & 611.1356 & 594.7234 & 547.3289 & 122.5994 & 2.42439 \\
\hline 2005 & 37.0683 & 616.4487 & 592.412 & 546.9082 & 122.5994 & 2.42331 \\
\hline 2006 & 37.0853 & 599.5181 & 589.8719 & 546.4875 & 122.5994 & 2.42224 \\
\hline 2007 & 37.1023 & 656.2769 & 587.163 & 546.0667 & 122.5994 & 2.42117 \\
\hline
\end{tabular}




\begin{tabular}{|c|c|c|c|c|c|c|}
\hline 2008 & 37.1193 & 657.6195 & 584.3545 & 545.646 & 122.5994 & 2.4201 \\
\hline 2009 & 37.1363 & 646.2883 & 581.6236 & 545.2253 & 122.5994 & 2.41903 \\
\hline 2010 & 37.1533 & 635.5738 & 578.5248 & 544.8045 & 122.5994 & 2.41797 \\
\hline 2011 & 37.1703 & 589.3389 & 575.946 & 544.3838 & 122.5994 & 2.4169 \\
\hline 2012 & 37.1873 & 606.8565 & 573.6039 & 543.9631 & 122.5994 & 2.41583 \\
\hline 2013 & 37.2043 & 610.697 & 571.5586 & 543.5423 & 122.5994 & 2.41477 \\
\hline 2014 & 37.2213 & 600.8629 & 569.8496 & 543.1216 & 122.5994 & 2.4137 \\
\hline 2015 & 37.2383 & 635.2724 & 568.5281 & 542.7008 & 122.5994 & 2.41264 \\
\hline 2016 & 37.2553 & 605.9733 & 567.374 & 542.2801 & 122.5994 & 2.41158 \\
\hline 2017 & 37.2723 & 601.0839 & 566.7488 & 541.8594 & 122.5994 & 2.41052 \\
\hline 2018 & 37.2893 & 567.3349 & 566.3195 & 541.3073 & 122.5994 & 2.40946 \\
\hline 2019 & 37.3063 & 566.1045 & 566.2114 & 540.7552 & 122.5994 & 2.4084 \\
\hline 2020 & 37.3233 & 558.4592 & 566.3052 & 540.2031 & 122.5994 & 2.40734 \\
\hline 2021 & 37.3403 & 557.7749 & 566.5922 & 539.651 & 122.5994 & 2.40628 \\
\hline 2022 & 37.3573 & 554.3517 & 566.9968 & 539.099 & 122.5994 & 2.40523 \\
\hline 2023 & 37.3743 & 566.5822 & 567.4263 & 538.5469 & 122.5994 & 2.40417 \\
\hline 2024 & 37.3913 & 563.6391 & 567.9978 & 537.9948 & 122.5994 & 2.40312 \\
\hline 2025 & 37.4083 & 564.7279 & 568.6423 & 537.4427 & 122.5994 & 2.40207 \\
\hline 2026 & 37.4253 & 585.0641 & 569.3075 & 536.8906 & 122.5994 & 2.40101 \\
\hline 2027 & 37.4423 & 569.7846 & 569.9784 & 536.3385 & 122.5994 & 2.39996 \\
\hline 2028 & 37.4593 & 566.8963 & 570.6091 & 535.7865 & 122.5994 & 2.39891 \\
\hline 2029 & 37.4763 & 594.4615 & 571.1063 & 535.2344 & 122.5994 & 2.39786 \\
\hline 2030 & 37.4933 & 601.8789 & 571.6445 & 534.6823 & 122.5994 & 2.39682 \\
\hline 2031 & 37.5103 & 603.6594 & 571.7961 & 534.1302 & 122.5994 & 2.39577 \\
\hline 2032 & 37.5273 & 601.6674 & 571.6967 & 533.5781 & 122.5994 & 2.39472 \\
\hline 2033 & 37.5443 & 593.2916 & 571.3555 & 533.026 & 122.5994 & 2.39368 \\
\hline 2034 & 37.5613 & 638.6036 & 570.824 & 532.474 & 122.5994 & 2.39263 \\
\hline 2035 & 37.5783 & 624.6342 & 570.1832 & 531.9219 & 122.5994 & 2.39159 \\
\hline 2036 & 37.5953 & 650.6881 & 569.6096 & 531.3698 & 122.5994 & 2.39055 \\
\hline 2037 & 37.6123 & 619.751 & 569.0278 & 530.8177 & 122.5994 & 2.38951 \\
\hline 2038 & 37.6293 & 622.5273 & 568.3872 & 530.2656 & 122.5994 & 2.38847 \\
\hline 2039 & 37.6463 & 571.1186 & 568.2348 & 529.7135 & 122.5994 & 2.38743 \\
\hline 2040 & 37.6633 & 586.872 & 568.4293 & 529.1615 & 122.5994 & 2.38639 \\
\hline 2041 & 37.6803 & 612.3613 & 569.0493 & 528.6094 & 122.5994 & 2.38535 \\
\hline 2042 & 37.6973 & 619.5266 & 570.0055 & 527.9173 & 122.5994 & 2.38431 \\
\hline 2043 & 37.7143 & 597.0137 & 571.4532 & 527.2253 & 122.5994 & 2.38328 \\
\hline 2044 & 37.7313 & 628.4867 & 573.3608 & 526.5332 & 122.5994 & 2.38224 \\
\hline 2045 & 37.7483 & 639.9657 & 575.2018 & 525.8411 & 122.5994 & 2.38121 \\
\hline 2046 & 37.7653 & 625.0967 & 577.7224 & 525.1491 & 122.5994 & 2.38018 \\
\hline 2047 & 37.7823 & 638.7061 & 580.2637 & 524.457 & 122.5994 & 2.37914 \\
\hline 2048 & 37.7993 & 582.9058 & 582.5737 & 523.765 & 122.5994 & 2.37811 \\
\hline 2049 & 37.8163 & 630.094 & 584.3864 & 523.0729 & 122.5994 & 2.37708 \\
\hline 2050 & 37.8333 & 628.2205 & 585.2856 & 522.3809 & 122.5994 & 2.37605 \\
\hline 2051 & 37.8503 & 611.2471 & 585.499 & 521.6888 & 122.5994 & 2.37503 \\
\hline 2052 & 37.8673 & 607.535 & 584.8094 & 520.9967 & 122.5994 & 2.374 \\
\hline 2053 & 37.8843 & 533.0256 & 583.2794 & 520.3047 & 122.5994 & 2.37297 \\
\hline 2054 & 37.9013 & 573.3922 & 580.9066 & 519.6126 & 122.5994 & 2.37195 \\
\hline 2055 & 37.9183 & 541.8268 & 578.0569 & 518.9206 & 122.5994 & 2.37092 \\
\hline 2056 & 37.9353 & 567.4459 & 574.7396 & 518.2285 & 122.5994 & 2.3699 \\
\hline
\end{tabular}




\begin{tabular}{|c|c|c|c|c|c|c|}
\hline 2057 & 37.9523 & 589.2131 & 571.1221 & 517.5365 & 122.5994 & 2.36888 \\
\hline 2058 & 37.9693 & 550.5921 & 567.3765 & 516.8444 & 122.5994 & 2.36786 \\
\hline 2059 & 37.9863 & 577.5391 & 563.691 & 516.1523 & 122.5994 & 2.36684 \\
\hline 2060 & 38.0033 & 548.4649 & 560.1903 & 515.4603 & 122.5994 & 2.36582 \\
\hline 2061 & 38.0203 & 532.0603 & 557.1383 & 514.7682 & 122.5994 & 2.3648 \\
\hline 2062 & 38.0373 & 524.061 & 554.5816 & 514.0762 & 122.5994 & 2.36378 \\
\hline 2063 & 38.0543 & 510.6726 & 552.5807 & 513.3841 & 122.5994 & 2.36276 \\
\hline 2064 & 38.0713 & 531.7699 & 551.1509 & 512.6921 & 122.5994 & 2.36175 \\
\hline 2065 & 38.0883 & 514.2554 & 550.2756 & 512 & 122.5994 & 2.36073 \\
\hline 2066 & 38.1053 & 496.6384 & 549.6926 & 511.125 & 122.5994 & 2.35972 \\
\hline 2067 & 38.1223 & 505.7848 & 549.4939 & 510.25 & 122.5994 & 2.3587 \\
\hline 2068 & 38.1393 & 530.5026 & 549.5485 & 509.375 & 122.5994 & 2.35769 \\
\hline 2069 & 38.1563 & 522.7522 & 549.7002 & 508.5 & 122.5994 & 2.35668 \\
\hline 2070 & 38.1733 & 509.2359 & 549.7861 & 507.625 & 122.5994 & 2.35567 \\
\hline 2071 & 38.1903 & 526.8323 & 549.4858 & 506.75 & 122.5994 & 2.35466 \\
\hline 2072 & 38.2073 & 544.6099 & 549.0458 & 505.875 & 122.5994 & 2.35365 \\
\hline 2073 & 38.2243 & 543.4501 & 548.2375 & 505 & 122.5994 & 2.35264 \\
\hline 2074 & 38.2413 & 487.9282 & 547.0609 & 504.125 & 122.5994 & 2.35164 \\
\hline 2075 & 38.2583 & 485.3886 & 545.7678 & 503.25 & 122.5994 & 2.35063 \\
\hline 2076 & 38.2753 & 504.6135 & 543.8848 & 502.375 & 122.5994 & 2.34963 \\
\hline 2077 & 38.2923 & 512.3553 & 541.8624 & 501.5 & 122.5994 & 2.34862 \\
\hline 2078 & 38.3093 & 512.118 & 539.6729 & 500.625 & 122.5994 & 2.34762 \\
\hline 2079 & 38.3263 & 533.5471 & 537.3899 & 499.75 & 122.5994 & 2.34662 \\
\hline 2080 & 38.3433 & 536.1658 & 535.0936 & 498.875 & 122.5994 & 2.34562 \\
\hline 2081 & 38.3603 & 537.049 & 532.9692 & 498 & 122.5994 & 2.34462 \\
\hline 2082 & 38.3773 & 510.023 & 530.8664 & 497.125 & 122.5994 & 2.34362 \\
\hline 2083 & 38.3943 & 516.1195 & 528.9407 & 496.25 & 122.5994 & 2.34262 \\
\hline 2084 & 38.4113 & 494.0709 & 527.2245 & 495.375 & 122.5994 & 2.34162 \\
\hline 2085 & 38.4283 & 484.7709 & 525.7371 & 494.5 & 122.5994 & 2.34062 \\
\hline 2086 & 38.4453 & 550.8735 & 524.4857 & 493.625 & 122.5994 & 2.33963 \\
\hline 2087 & 38.4623 & 512.8102 & 523.4695 & 492.75 & 122.5994 & 2.33863 \\
\hline 2088 & 38.4793 & 539.3401 & 522.6249 & 491.875 & 122.5994 & 2.33764 \\
\hline 2089 & 38.4963 & 488.4249 & 522.063 & 491 & 122.5994 & 2.33664 \\
\hline 2090 & 38.5133 & 475.6534 & 521.715 & 490.125 & 122.5994 & 2.33565 \\
\hline 2091 & 38.5303 & 514.0001 & 521.5664 & 489.25 & 122.5994 & 2.33466 \\
\hline 2092 & 38.5473 & 484.3634 & 521.5901 & 488.375 & 122.5994 & 2.33367 \\
\hline 2093 & 38.5643 & 551.1277 & 521.7423 & 487.5 & 122.5994 & 2.33268 \\
\hline 2094 & 38.5813 & 455.8661 & 521.9283 & 486.625 & 122.5994 & 2.33169 \\
\hline 2095 & 38.5983 & 518.9112 & 522.119 & 485.75 & 122.5994 & 2.3307 \\
\hline 2096 & 38.6153 & 503.0972 & 522.7871 & 484.875 & 122.5994 & 2.32972 \\
\hline 2097 & 38.6323 & 520.6882 & 522.6257 & 484 & 122.5994 & 2.32873 \\
\hline 2098 & 38.6493 & 525.275 & 521.9963 & 483.125 & 122.5994 & 2.32775 \\
\hline 2099 & 38.6663 & 495.3669 & 521.0828 & 482.25 & 122.5994 & 2.32676 \\
\hline 2100 & 38.6833 & 487.686 & 519.7345 & 481.375 & 122.5994 & 2.32578 \\
\hline 2101 & 38.7003 & 508.7045 & 517.9765 & 480.5 & 122.5994 & 2.3248 \\
\hline 2102 & 38.7173 & 511.5187 & 515.8371 & 479.625 & 122.5994 & 2.32381 \\
\hline 2103 & 38.7343 & 482.6805 & 513.3098 & 478.75 & 122.5994 & 2.32283 \\
\hline 2104 & 38.7513 & 490.5944 & 510.5789 & 477.875 & 122.5994 & 2.32185 \\
\hline 2105 & 38.7683 & 482.8778 & 507.6392 & 477 & 122.5994 & 2.32087 \\
\hline
\end{tabular}




\begin{tabular}{|c|c|c|c|c|c|c|}
\hline 2106 & 38.7853 & 483.4238 & 504.5593 & 476.125 & 122.5994 & 2.3199 \\
\hline 2107 & 38.8023 & 488.7595 & 501.4202 & 475.25 & 122.5994 & 2.31892 \\
\hline 2108 & 38.8193 & 478.1009 & 498.29 & 474.375 & 122.5994 & 2.31794 \\
\hline 2109 & 38.8363 & 469.0387 & 495.2421 & 473.5 & 122.5994 & 2.31697 \\
\hline 2110 & 38.8533 & 464.5509 & 492.3309 & 472.625 & 122.5994 & 2.31599 \\
\hline 2111 & 38.8703 & 478.6923 & 489.5914 & 471.75 & 122.5994 & 2.31502 \\
\hline 2112 & 38.8873 & 473.1029 & 487.0469 & 470.875 & 122.5994 & 2.31405 \\
\hline 2113 & 38.9043 & 456.0271 & 484.7058 & 470 & 122.5994 & 2.31307 \\
\hline 2114 & 38.9213 & 471.8041 & 484.1519 & 470.7083 & 122.5994 & 2.3121 \\
\hline 2115 & 38.9383 & 490.7552 & 483.8027 & 471.4167 & 122.5994 & 2.31113 \\
\hline 2116 & 38.9553 & 492.8641 & 483.3974 & 472.125 & 122.5994 & 2.31016 \\
\hline 2117 & 38.9723 & 490.3323 & 483.4149 & 472.8333 & 122.5994 & 2.30919 \\
\hline 2118 & 38.9893 & 493.7204 & 483.5955 & 473.5417 & 122.5994 & 2.30823 \\
\hline 2119 & 39.0063 & 489.1663 & 483.9275 & 474.25 & 122.5994 & 2.30726 \\
\hline 2120 & 39.0233 & 498.0361 & 484.4009 & 474.9583 & 122.5994 & 2.30629 \\
\hline 2121 & 39.0403 & 505.1812 & 485.0665 & 475.6667 & 122.5994 & 2.30533 \\
\hline 2122 & 39.0573 & 507.5516 & 485.702 & 476.375 & 122.5994 & 2.30436 \\
\hline 2123 & 39.0743 & 513.6895 & 486.5795 & 477.0833 & 122.5994 & 2.3034 \\
\hline 2124 & 39.0913 & 497.1033 & 487.5929 & 477.7917 & 122.5994 & 2.30244 \\
\hline 2125 & 39.1083 & 504.0208 & 488.7511 & 478.5 & 122.5994 & 2.30148 \\
\hline 2126 & 39.1253 & 491.3907 & 490.0671 & 479.2083 & 122.5994 & 2.30052 \\
\hline 2127 & 39.1423 & 480.8 & 491.5671 & 479.9167 & 122.5994 & 2.29956 \\
\hline 2128 & 39.1593 & 510.3422 & 493.3012 & 480.625 & 122.5994 & 2.2986 \\
\hline 2129 & 39.1763 & 505.6857 & 495.2022 & 481.3333 & 122.5994 & 2.29764 \\
\hline 2130 & 39.1933 & 480.2804 & 497.3409 & 482.0417 & 122.5994 & 2.29668 \\
\hline 2131 & 39.2103 & 457.4217 & 499.7399 & 482.75 & 122.5994 & 2.29572 \\
\hline 2132 & 39.2273 & 486.9708 & 502.5147 & 483.4583 & 122.5994 & 2.29477 \\
\hline 2133 & 39.2443 & 516.3533 & 505.4975 & 484.1667 & 122.5994 & 2.29381 \\
\hline 2134 & 39.2613 & 536.3638 & 508.7962 & 484.875 & 122.5994 & 2.29286 \\
\hline 2135 & 39.2783 & 484.5017 & 512.4245 & 485.5833 & 122.5994 & 2.29191 \\
\hline 2136 & 39.2953 & 483.7888 & 516.4016 & 486.2917 & 122.5994 & 2.29095 \\
\hline 2137 & 39.3123 & 500.9811 & 520.7002 & 487 & 122.5994 & 2.29 \\
\hline 2138 & 39.3293 & 467.3166 & 525.2185 & 487.4792 & 122.5994 & 2.28905 \\
\hline 2139 & 39.3463 & 501.7041 & 529.9181 & 487.9583 & 122.5994 & 2.2881 \\
\hline 2140 & 39.3633 & 497.2523 & 534.918 & 488.4375 & 122.5994 & 2.28715 \\
\hline 2141 & 39.3803 & 493.1923 & 540.0767 & 488.9167 & 122.5994 & 2.2862 \\
\hline 2142 & 39.3973 & 500.6468 & 545.9943 & 489.3958 & 122.5994 & 2.28526 \\
\hline 2143 & 39.4143 & 487.9077 & 553.1221 & 489.875 & 122.5994 & 2.28431 \\
\hline 2144 & 39.4313 & 527.9482 & 562.8436 & 490.3542 & 122.5994 & 2.28336 \\
\hline 2145 & 39.4483 & 524.153 & 577.5255 & 490.8333 & 122.5994 & 2.28242 \\
\hline 2146 & 39.4653 & 561.7637 & 600.2702 & 491.3125 & 122.5994 & 2.28148 \\
\hline 2147 & 39.4823 & 626.6361 & 633.9938 & 491.7917 & 122.5994 & 2.28053 \\
\hline 2148 & 39.4993 & 688.3814 & 679.1819 & 492.2708 & 122.5994 & 2.27959 \\
\hline 2149 & 39.5163 & 731.3974 & 731.3791 & 492.75 & 122.5994 & 2.27865 \\
\hline 2150 & 39.5333 & 790.5059 & 777.7545 & 493.2292 & 122.5994 & 2.27771 \\
\hline 2151 & 39.5503 & 816.0968 & 798.3046 & 493.7083 & 122.5994 & 2.27677 \\
\hline 2152 & 39.5673 & 749.6783 & 784.1412 & 494.1875 & 122.5994 & 2.27583 \\
\hline 2153 & 39.5843 & 662.0007 & 751.2145 & 494.6667 & 122.5994 & 2.27489 \\
\hline 2154 & 39.6013 & 620.4327 & 720.4385 & 495.1458 & 122.5994 & 2.27395 \\
\hline
\end{tabular}




\begin{tabular}{|c|c|c|c|c|c|c|}
\hline 2155 & 39.6183 & 626.4064 & 701.27 & 495.625 & 122.5994 & 2.27302 \\
\hline 2156 & 39.6353 & 664.6021 & 690.8259 & 496.1042 & 122.5994 & 2.27208 \\
\hline 2157 & 39.6523 & 639.3507 & 677.63 & 496.5833 & 122.5994 & 2.27115 \\
\hline 2158 & 39.6693 & 611.7335 & 653.4128 & 497.0625 & 122.5994 & 2.27021 \\
\hline 2159 & 39.6863 & 581.5517 & 622.126 & 497.5417 & 122.5994 & 2.26928 \\
\hline 2160 & 39.7033 & 558.8161 & 591.85 & 498.0208 & 122.5994 & 2.26835 \\
\hline 2161 & 39.7203 & 529.5668 & 567.2255 & 498.5 & 122.5994 & 2.26741 \\
\hline 2162 & 39.7373 & 515.2802 & 549.2502 & 498.7708 & 122.5994 & 2.26648 \\
\hline 2163 & 39.7543 & 487.8106 & 537.3632 & 499.0417 & 122.5994 & 2.26555 \\
\hline 2164 & 39.7713 & 485.6217 & 529.9899 & 499.3125 & 122.5994 & 2.26462 \\
\hline 2165 & 39.7883 & 524.7932 & 525.5774 & 499.5833 & 122.5994 & 2.2637 \\
\hline 2166 & 39.8053 & 498.475 & 522.8477 & 499.8542 & 122.5994 & 2.26277 \\
\hline 2167 & 39.8223 & 490.4979 & 521.0944 & 500.125 & 122.5994 & 2.26184 \\
\hline 2168 & 39.8393 & 540.8886 & 519.814 & 500.3958 & 122.5994 & 2.26092 \\
\hline 2169 & 39.8563 & 514.3293 & 519.1566 & 500.6667 & 122.5994 & 2.25999 \\
\hline 2170 & 39.8733 & 506.3389 & 518.8632 & 500.9375 & 122.5994 & 2.25907 \\
\hline 2171 & 39.8903 & 493.7564 & 518.9153 & 501.2083 & 122.5994 & 2.25814 \\
\hline 2172 & 39.9073 & 503.0016 & 519.2172 & 501.4792 & 122.5994 & 2.25722 \\
\hline 2173 & 39.9243 & 517.4879 & 519.7097 & 501.75 & 122.5994 & 2.2563 \\
\hline 2174 & 39.9413 & 489.4213 & 520.51 & 502.0208 & 122.5994 & 2.25538 \\
\hline 2175 & 39.9583 & 503.7457 & 521.5911 & 502.2917 & 122.5994 & 2.25446 \\
\hline 2176 & 39.9753 & 547.6933 & 522.8012 & 502.5625 & 122.5994 & 2.25354 \\
\hline 2177 & 39.9923 & 515.3563 & 524.1682 & 502.8333 & 122.5994 & 2.25262 \\
\hline 2178 & 40.0093 & 510.1105 & 525.6473 & 503.1042 & 122.5994 & 2.2517 \\
\hline 2179 & 40.0263 & 530.4178 & 527.1867 & 503.375 & 122.5994 & 2.25078 \\
\hline 2180 & 40.0433 & 529.6055 & 528.7223 & 503.6458 & 122.5994 & 2.24987 \\
\hline 2181 & 40.0603 & 537.3162 & 530.2119 & 503.9167 & 122.5994 & 2.24895 \\
\hline 2182 & 40.0773 & 538.1884 & 531.6563 & 504.1875 & 122.5994 & 2.24804 \\
\hline 2183 & 40.0943 & 538.3117 & 532.9842 & 504.4583 & 122.5994 & 2.24712 \\
\hline 2184 & 40.1113 & 520.3404 & 534.2387 & 504.7292 & 122.5994 & 2.24621 \\
\hline 2185 & 40.1283 & 501.9803 & 535.4371 & 505 & 122.5994 & 2.2453 \\
\hline 2186 & 40.1453 & 501.6539 & 536.4116 & 505.0942 & 122.5994 & 2.24439 \\
\hline 2187 & 40.1623 & 498.2953 & 537.3477 & 505.1885 & 122.5994 & 2.24347 \\
\hline 2188 & 40.1793 & 522.8398 & 538.2529 & 505.2827 & 122.5994 & 2.24256 \\
\hline 2189 & 40.1963 & 497.3563 & 539.157 & 505.377 & 122.5994 & 2.24166 \\
\hline 2190 & 40.2133 & 499.0263 & 540.1403 & 505.4712 & 122.5994 & 2.24075 \\
\hline 2191 & 40.2303 & 514.8116 & 541.4604 & 505.5654 & 122.5994 & 2.23984 \\
\hline 2192 & 40.2473 & 512.002 & 543.3413 & 505.6597 & 122.5994 & 2.23893 \\
\hline 2193 & 40.2643 & 517.5668 & 546.4965 & 505.7539 & 122.5994 & 2.23803 \\
\hline 2194 & 40.2813 & 515.7117 & 551.8599 & 505.8481 & 122.5994 & 2.23712 \\
\hline 2195 & 40.2983 & 561.8357 & 560.4801 & 505.9424 & 122.5994 & 2.23622 \\
\hline 2196 & 40.3153 & 575.4419 & 573.0209 & 506.0366 & 122.5994 & 2.23531 \\
\hline 2197 & 40.3323 & 576.4066 & 589.0464 & 506.1309 & 122.5994 & 2.23441 \\
\hline 2198 & 40.3493 & 637.7233 & 606.0496 & 506.2251 & 122.5994 & 2.23351 \\
\hline 2199 & 40.3663 & 655.8019 & 618.4194 & 506.3193 & 122.5994 & 2.23261 \\
\hline 2200 & 40.3833 & 654.1677 & 620.2404 & 506.4136 & 122.5994 & 2.23171 \\
\hline 2201 & 40.4003 & 587.3278 & 612.3594 & 506.5078 & 122.5994 & 2.23081 \\
\hline 2202 & 40.4173 & 559.3749 & 601.7127 & 506.6021 & 122.5994 & 2.22991 \\
\hline 2203 & 40.4343 & 559.9576 & 593.7057 & 506.6963 & 122.5994 & 2.22901 \\
\hline
\end{tabular}




\begin{tabular}{|c|c|c|c|c|c|c|}
\hline 2204 & 40.4513 & 589.4775 & 589.6613 & 506.7905 & 122.5994 & 2.22811 \\
\hline 2205 & 40.4683 & 580.7543 & 587.2698 & 506.8848 & 122.5994 & 2.22721 \\
\hline 2206 & 40.4853 & 568.2227 & 582.4584 & 506.979 & 122.5994 & 2.22632 \\
\hline 2207 & 40.5023 & 543.532 & 573.6794 & 507.0732 & 122.5994 & 2.22542 \\
\hline 2208 & 40.5193 & 529.2052 & 563.1125 & 507.1675 & 122.5994 & 2.22453 \\
\hline 2209 & 40.5363 & 524.3597 & 553.2073 & 507.2617 & 122.5994 & 2.22363 \\
\hline 2210 & 40.5533 & 510.0036 & 544.9752 & 507.182 & 122.5994 & 2.22274 \\
\hline 2211 & 40.5703 & 494.9043 & 538.7858 & 507.1024 & 122.5994 & 2.22185 \\
\hline 2212 & 40.5873 & 492.7927 & 534.3187 & 507.0227 & 122.5994 & 2.22096 \\
\hline 2213 & 40.6043 & 508.9457 & 530.8582 & 506.943 & 122.5994 & 2.22007 \\
\hline 2214 & 40.6213 & 508.662 & 528.4033 & 506.8634 & 122.5994 & 2.21918 \\
\hline 2215 & 40.6383 & 507.5612 & 526.4529 & 506.7837 & 122.5994 & 2.21829 \\
\hline 2216 & 40.6553 & 510.711 & 524.8679 & 506.704 & 122.5994 & 2.2174 \\
\hline 2217 & 40.6723 & 510.711 & 523.5936 & 506.6243 & 122.5994 & 2.21651 \\
\hline 2218 & 40.6893 & 498.2548 & 522.6146 & 506.5447 & 122.5994 & 2.21563 \\
\hline 2219 & 40.7063 & 509.0253 & 521.3749 & 506.465 & 122.5994 & 2.21474 \\
\hline 2220 & 40.7233 & 518.7471 & 520.9984 & 506.3853 & 122.5994 & 2.21385 \\
\hline 2221 & 40.7403 & 537.4493 & 520.905 & 506.3057 & 122.5994 & 2.21297 \\
\hline 2222 & 40.7573 & 532.0202 & 521.0853 & 506.226 & 122.5994 & 2.21209 \\
\hline 2223 & 40.7743 & 521.537 & 521.5211 & 506.1463 & 122.5994 & 2.2112 \\
\hline 2224 & 40.7913 & 508.5021 & 522.1859 & 506.0667 & 122.5994 & 2.21032 \\
\hline 2225 & 40.8083 & 525.7065 & 523.0424 & 505.987 & 122.5994 & 2.20944 \\
\hline 2226 & 40.8253 & 541.0847 & 524.0385 & 505.9073 & 122.5994 & 2.20856 \\
\hline 2227 & 40.8423 & 547.1436 & 525.1183 & 505.8276 & 122.5994 & 2.20768 \\
\hline 2228 & 40.8593 & 501.4222 & 526.2048 & 505.748 & 122.5994 & 2.2068 \\
\hline 2229 & 40.8763 & 518.3981 & 527.2219 & 505.6683 & 122.5994 & 2.20592 \\
\hline 2230 & 40.8933 & 533.1261 & 528.1017 & 505.5886 & 122.5994 & 2.20504 \\
\hline 2231 & 40.9103 & 515.5824 & 528.8004 & 505.509 & 122.5994 & 2.20417 \\
\hline 2232 & 40.9273 & 501.7497 & 529.5167 & 505.4293 & 122.5994 & 2.20329 \\
\hline 2233 & 40.9443 & 515.6939 & 529.8428 & 505.3496 & 122.5994 & 2.20241 \\
\hline 2234 & 40.9613 & 527.4948 & 530.0265 & 505.2699 & 122.5994 & 2.20154 \\
\hline 2235 & 40.9783 & 496.348 & 530.0668 & 505.1903 & 122.5994 & 2.20066 \\
\hline 2236 & 40.9953 & 488.0667 & 529.9995 & 505.1106 & 122.5994 & 2.19979 \\
\hline 2237 & 41.0123 & 524.9587 & 529.9639 & 505.0309 & 122.5994 & 2.19892 \\
\hline 2238 & 41.0293 & 498.8989 & 529.7278 & 504.9513 & 122.5994 & 2.19805 \\
\hline 2239 & 41.0463 & 537.478 & 529.421 & 504.8716 & 122.5994 & 2.19718 \\
\hline 2240 & 41.0633 & 539.2991 & 529.0455 & 504.7919 & 122.5994 & 2.19631 \\
\hline 2241 & 41.0803 & 558.2567 & 528.6112 & 504.7122 & 122.5994 & 2.19544 \\
\hline 2242 & 41.0973 & 519.4238 & 528.1204 & 504.6326 & 122.5994 & 2.19457 \\
\hline 2243 & 41.1143 & 501.8269 & 527.5835 & 504.5529 & 122.5994 & 2.1937 \\
\hline 2244 & 41.1313 & 504.0608 & 527.0369 & 504.4732 & 122.5994 & 2.19283 \\
\hline 2245 & 41.1483 & 510.8281 & 526.475 & 504.3936 & 122.5994 & 2.19196 \\
\hline 2246 & 41.1653 & 527.641 & 525.9321 & 504.3139 & 122.5994 & 2.1911 \\
\hline 2247 & 41.1823 & 549.7374 & 525.3735 & 504.2342 & 122.5994 & 2.19023 \\
\hline 2248 & 41.1993 & 501.4562 & 524.9488 & 504.1545 & 122.5994 & 2.18937 \\
\hline 2249 & 41.2163 & 510.564 & 524.5948 & 504.0749 & 122.5994 & 2.18851 \\
\hline 2250 & 41.2333 & 544.1869 & 524.318 & 503.9952 & 122.5994 & 2.18764 \\
\hline 2251 & 41.2503 & 507.2811 & 524.1579 & 503.9155 & 122.5994 & 2.18678 \\
\hline 2252 & 41.2673 & 499.7897 & 524.0264 & 503.8359 & 122.5994 & 2.18592 \\
\hline
\end{tabular}




\begin{tabular}{|c|c|c|c|c|c|c|}
\hline 2253 & 41.2843 & 497.966 & 523.9111 & 503.7562 & 122.5994 & 2.18506 \\
\hline 2254 & 41.3013 & 530.7644 & 523.8405 & 503.6765 & 122.5994 & 2.1842 \\
\hline 2255 & 41.3183 & 545.2869 & 523.7288 & 503.5968 & 122.5994 & 2.18334 \\
\hline 2256 & 41.3353 & 547.5075 & 523.5248 & 503.5172 & 122.5994 & 2.18248 \\
\hline 2257 & 41.3523 & 530.1805 & 523.189 & 503.4375 & 122.5994 & 2.18162 \\
\hline 2258 & 41.3693 & 515.2406 & 522.9326 & 503.1839 & 122.5994 & 2.18076 \\
\hline 2259 & 41.3863 & 531.3498 & 522.1401 & 502.9303 & 122.5994 & 2.17991 \\
\hline 2260 & 41.4033 & 523.5135 & 521.1962 & 502.6768 & 122.5994 & 2.17905 \\
\hline 2261 & 41.4203 & 539.6663 & 520.1588 & 502.4232 & 122.5994 & 2.1782 \\
\hline 2262 & 41.4373 & 524.1545 & 519.2097 & 502.1696 & 122.5994 & 2.17734 \\
\hline 2263 & 41.4543 & 515.3041 & 518.0014 & 501.916 & 122.5994 & 2.17649 \\
\hline 2264 & 41.4713 & 516.645 & 516.9481 & 501.6624 & 122.5994 & 2.17564 \\
\hline 2265 & 41.4883 & 508.9143 & 515.6123 & 501.4089 & 122.5994 & 2.17478 \\
\hline 2266 & 41.5053 & 499.6221 & 514.224 & 501.1553 & 122.5994 & 2.17393 \\
\hline 2267 & 41.5223 & 487.3097 & 512.9818 & 500.9017 & 122.5994 & 2.17308 \\
\hline 2268 & 41.5393 & 483.9536 & 511.8043 & 500.6481 & 122.5994 & 2.17223 \\
\hline 2269 & 41.5563 & 501.8427 & 510.8059 & 500.3945 & 122.5994 & 2.17138 \\
\hline 2270 & 41.5733 & 543.9416 & 509.8016 & 500.141 & 122.5994 & 2.17053 \\
\hline 2271 & 41.5903 & 502.1822 & 508.7883 & 499.8874 & 122.5994 & 2.16969 \\
\hline 2272 & 41.6073 & 489.0804 & 508.0241 & 499.6338 & 122.5994 & 2.16884 \\
\hline 2273 & 41.6243 & 492.2834 & 507.3728 & 499.3802 & 122.5994 & 2.16799 \\
\hline 2274 & 41.6413 & 509.1516 & 506.8335 & 499.1266 & 122.5994 & 2.16715 \\
\hline 2275 & 41.6583 & 506.5257 & 506.4047 & 498.873 & 122.5994 & 2.1663 \\
\hline 2276 & 41.6753 & 510.6004 & 506.0853 & 498.6195 & 122.5994 & 2.16546 \\
\hline 2277 & 41.6923 & 480.4622 & 505.8729 & 498.3659 & 122.5994 & 2.16461 \\
\hline 2278 & 41.7093 & 483.921 & 505.7393 & 498.1123 & 122.5994 & 2.16377 \\
\hline 2279 & 41.7263 & 488.9332 & 505.7692 & 497.8587 & 122.5994 & 2.16293 \\
\hline 2280 & 41.7433 & 529.4003 & 505.9322 & 497.6051 & 122.5994 & 2.16209 \\
\hline 2281 & 41.7603 & 537.3985 & 506.2431 & 497.3516 & 122.5994 & 2.16124 \\
\hline 2282 & 41.7773 & 522.2299 & 506.5504 & 496.9268 & 122.5994 & 2.1604 \\
\hline 2283 & 41.7943 & 499.1596 & 507.0459 & 496.502 & 122.5994 & 2.15957 \\
\hline 2284 & 41.8113 & 514.1413 & 507.7597 & 496.0771 & 122.5994 & 2.15873 \\
\hline 2285 & 41.8283 & 503.7619 & 508.7173 & 495.6523 & 122.5994 & 2.15789 \\
\hline 2286 & 41.8453 & 498.1036 & 509.9445 & 495.2275 & 122.5994 & 2.15705 \\
\hline 2287 & 41.8623 & 547.633 & 511.4644 & 494.8027 & 122.5994 & 2.15621 \\
\hline 2288 & 41.8793 & 548.4818 & 513.2913 & 494.3779 & 122.5994 & 2.15538 \\
\hline 2289 & 41.8963 & 539.6022 & 515.4304 & 493.9531 & 122.5994 & 2.15454 \\
\hline 2290 & 41.9133 & 498.4774 & 517.8699 & 493.5283 & 122.5994 & 2.15371 \\
\hline 2291 & 41.9303 & 512.9088 & 520.5804 & 493.1035 & 122.5994 & 2.15287 \\
\hline 2292 & 41.9473 & 530.875 & 523.5059 & 492.6787 & 122.5994 & 2.15204 \\
\hline 2293 & 41.9643 & 497.5026 & 526.5553 & 492.2539 & 122.5994 & 2.15121 \\
\hline 2294 & 41.9813 & 516.2438 & 529.6057 & 491.8291 & 122.5994 & 2.15038 \\
\hline 2295 & 41.9983 & 556.1592 & 532.5038 & 491.4043 & 122.5994 & 2.14955 \\
\hline 2296 & 42.0153 & 518.6037 & 534.9911 & 490.9795 & 122.5994 & 2.14872 \\
\hline 2297 & 42.0323 & 507.2584 & 537.0291 & 490.5547 & 122.5994 & 2.14789 \\
\hline 2298 & 42.0493 & 522.9874 & 538.4384 & 490.1299 & 122.5994 & 2.14706 \\
\hline 2299 & 42.0663 & 533.6811 & 539.1682 & 489.7051 & 122.5994 & 2.14623 \\
\hline 2300 & 42.0833 & 518.7188 & 539.2498 & 489.2803 & 122.5994 & 2.1454 \\
\hline 2301 & 42.1003 & 501.7119 & 538.7631 & 488.8555 & 122.5994 & 2.14457 \\
\hline
\end{tabular}




\begin{tabular}{|c|c|c|c|c|c|c|}
\hline 2302 & 42.1173 & 499.5516 & 537.7772 & 488.4307 & 122.5994 & 2.14375 \\
\hline 2303 & 42.1343 & 483.2391 & 536.4387 & 488.0059 & 122.5994 & 2.14292 \\
\hline 2304 & 42.1513 & 508.1349 & 534.8069 & 487.5811 & 122.5994 & 2.1421 \\
\hline 2305 & 42.1683 & 514.7038 & 532.9835 & 487.1562 & 122.5994 & 2.14127 \\
\hline 2306 & 42.1853 & 499.9016 & 530.8925 & 486.5339 & 122.5994 & 2.14045 \\
\hline 2307 & 42.2023 & 488.17 & 528.8763 & 485.9115 & 122.5994 & 2.13963 \\
\hline 2308 & 42.2193 & 502.3272 & 527.0445 & 485.2891 & 122.5994 & 2.1388 \\
\hline 2309 & 42.2363 & 497.6278 & 525.6457 & 484.6667 & 122.5994 & 2.13798 \\
\hline 2310 & 42.2533 & 479.3937 & 524.7691 & 484.0443 & 122.5994 & 2.13716 \\
\hline 2311 & 42.2703 & 506.6439 & 524.5319 & 483.4219 & 122.5994 & 2.13634 \\
\hline 2312 & 42.2873 & 533.114 & 525.0275 & 482.7995 & 122.5994 & 2.13552 \\
\hline 2313 & 42.3043 & 524.4704 & 526.3105 & 482.1771 & 122.5994 & 2.1347 \\
\hline 2314 & 42.3213 & 492.4131 & 528.4658 & 481.5547 & 122.5994 & 2.13388 \\
\hline 2315 & 42.3383 & 523.0767 & 531.5086 & 480.9323 & 122.5994 & 2.13307 \\
\hline 2316 & 42.3553 & 548.7844 & 535.4686 & 480.3099 & 122.5994 & 2.13225 \\
\hline 2317 & 42.3723 & 532.8604 & 540.37 & 479.6875 & 122.5994 & 2.13143 \\
\hline 2318 & 42.3893 & 548.9105 & 546.402 & 479.0651 & 122.5994 & 2.13062 \\
\hline 2319 & 42.4063 & 531.859 & 553.8725 & 478.4427 & 122.5994 & 2.1298 \\
\hline 2320 & 42.4233 & 520.7403 & 563.5164 & 477.8203 & 122.5994 & 2.12899 \\
\hline 2321 & 42.4403 & 593.7115 & 576.5104 & 477.1979 & 122.5994 & 2.12818 \\
\hline 2322 & 42.4573 & 609.4346 & 594.3574 & 476.5755 & 122.5994 & 2.12736 \\
\hline 2323 & 42.4743 & 663.5489 & 618.1547 & 475.9531 & 122.5994 & 2.12655 \\
\hline 2324 & 42.4913 & 738.4638 & 647.7429 & 475.3307 & 122.5994 & 2.12574 \\
\hline 2325 & 42.5083 & 767.6104 & 679.752 & 474.7083 & 122.5994 & 2.12493 \\
\hline 2326 & 42.5253 & 822.7492 & 705.9993 & 474.0859 & 122.5994 & 2.12412 \\
\hline 2327 & 42.5423 & 784.3827 & 714.7534 & 473.4635 & 122.5994 & 2.12331 \\
\hline 2328 & 42.5593 & 672.4489 & 702.1186 & 472.8411 & 122.5994 & 2.1225 \\
\hline 2329 & 42.5763 & 624.6342 & 678.4065 & 472.2188 & 122.5994 & 2.12169 \\
\hline 2330 & 42.5933 & 625.611 & 655.8478 & 471.3763 & 122.5994 & 2.12089 \\
\hline 2331 & 42.6103 & 660.7223 & 640.3129 & 470.5339 & 122.5994 & 2.12008 \\
\hline 2332 & 42.6273 & 602.35 & 631.1295 & 469.6914 & 122.5994 & 2.11927 \\
\hline 2333 & 42.6443 & 631.3031 & 622.3185 & 468.849 & 122.5994 & 2.11847 \\
\hline 2334 & 42.6613 & 582.0872 & 607.0696 & 468.0065 & 122.5994 & 2.11766 \\
\hline 2335 & 42.6783 & 551.642 & 585.1288 & 467.1641 & 122.5994 & 2.11686 \\
\hline 2336 & 42.6953 & 550.8361 & 561.5029 & 466.3216 & 122.5994 & 2.11605 \\
\hline 2337 & 42.7123 & 492.6025 & 540.2609 & 465.4792 & 122.5994 & 2.11525 \\
\hline 2338 & 42.7293 & 476.4937 & 523.0903 & 464.6367 & 122.5994 & 2.11445 \\
\hline 2339 & 42.7463 & 504.573 & 510.0248 & 463.7943 & 122.5994 & 2.11365 \\
\hline 2340 & 42.7633 & 497.6915 & 500.3487 & 462.9518 & 122.5994 & 2.11285 \\
\hline 2341 & 42.7803 & 484.8354 & 493.1073 & 462.1094 & 122.5994 & 2.11205 \\
\hline 2342 & 42.7973 & 488.0461 & 487.4891 & 461.2669 & 122.5994 & 2.11125 \\
\hline 2343 & 42.8143 & 469.856 & 482.9494 & 460.4245 & 122.5994 & 2.11045 \\
\hline 2344 & 42.8313 & 509.2883 & 479.1676 & 459.582 & 122.5994 & 2.10965 \\
\hline 2345 & 42.8483 & 502.326 & 475.956 & 458.7396 & 122.5994 & 2.10885 \\
\hline 2346 & 42.8653 & 475.6997 & 473.2117 & 457.8971 & 122.5994 & 2.10805 \\
\hline 2347 & 42.8823 & 472.9206 & 470.8268 & 457.0547 & 122.5994 & 2.10726 \\
\hline 2348 & 42.8993 & 481.761 & 468.8417 & 456.2122 & 122.5994 & 2.10646 \\
\hline 2349 & 42.9163 & 486.1808 & 467.0269 & 455.3698 & 122.5994 & 2.10567 \\
\hline 2350 & 42.9333 & 481.5757 & 465.4293 & 454.5273 & 122.5994 & 2.10487 \\
\hline
\end{tabular}




\begin{tabular}{|c|c|c|c|c|c|c|}
\hline 2351 & 42.9503 & 477.2122 & 464.0155 & 453.6849 & 122.5994 & 2.10408 \\
\hline 2352 & 42.9673 & 475.1944 & 462.7645 & 452.8424 & 122.5994 & 2.10329 \\
\hline 2353 & 42.9843 & 441.9581 & 461.6419 & 452 & 122.5994 & 2.10249 \\
\hline 2354 & 43.0013 & 433.2367 & 461.6846 & 452.2083 & 122.5994 & 2.1017 \\
\hline 2355 & 43.0183 & 443.691 & 461.9812 & 452.4167 & 122.5994 & 2.10091 \\
\hline 2356 & 43.0353 & 448.1072 & 462.2103 & 452.625 & 122.5994 & 2.10012 \\
\hline 2357 & 43.0523 & 437.7663 & 462.506 & 452.8333 & 122.5994 & 2.09933 \\
\hline 2358 & 43.0693 & 432.6977 & 462.8517 & 453.0417 & 122.5994 & 2.09854 \\
\hline 2359 & 43.0863 & 486.7289 & 463.2305 & 453.25 & 122.5994 & 2.09775 \\
\hline 2360 & 43.1033 & 489.4738 & 463.6248 & 453.4583 & 122.5994 & 2.09696 \\
\hline 2361 & 43.1203 & 453.758 & 464.0167 & 453.6667 & 122.5994 & 2.09618 \\
\hline 2362 & 43.1373 & 441.7999 & 464.4422 & 453.875 & 122.5994 & 2.09539 \\
\hline 2363 & 43.1543 & 434.191 & 464.5632 & 454.0833 & 122.5994 & 2.0946 \\
\hline 2364 & 43.1713 & 464.8405 & 464.8448 & 454.2917 & 122.5994 & 2.09382 \\
\hline 2365 & 43.1883 & 456.082 & 465.0286 & 454.5 & 122.5994 & 2.09303 \\
\hline 2366 & 43.2053 & 453.6465 & 465.154 & 454.7083 & 122.5994 & 2.09225 \\
\hline 2367 & 43.2223 & 423.3024 & 465.1957 & 454.9167 & 122.5994 & 2.09147 \\
\hline 2368 & 43.2393 & 455.3412 & 465.1615 & 455.125 & 122.5994 & 2.09068 \\
\hline 2369 & 43.2563 & 449.8179 & 465.0641 & 455.3333 & 122.5994 & 2.0899 \\
\hline 2370 & 43.2733 & 461.953 & 464.8136 & 455.5417 & 122.5994 & 2.08912 \\
\hline 2371 & 43.2903 & 483.8156 & 464.6337 & 455.75 & 122.5994 & 2.08834 \\
\hline 2372 & 43.3073 & 437.0232 & 464.4179 & 455.9583 & 122.5994 & 2.08756 \\
\hline 2373 & 43.3243 & 455.4026 & 464.2026 & 456.1667 & 122.5994 & 2.08678 \\
\hline 2374 & 43.3413 & 498.5367 & 463.9896 & 456.375 & 122.5994 & 2.086 \\
\hline 2375 & 43.3583 & 442.2085 & 463.7748 & 456.5833 & 122.5994 & 2.08522 \\
\hline 2376 & 43.3753 & 415.7855 & 463.6127 & 456.7917 & 122.5994 & 2.08444 \\
\hline 2377 & 43.3923 & 482.879 & 463.4974 & 457 & 122.5994 & 2.08367 \\
\hline 2378 & 43.4093 & 471.4768 & 463.2325 & 457 & 122.5994 & 2.08289 \\
\hline 2379 & 43.4263 & 450.2043 & 463.0365 & 457 & 122.5994 & 2.08211 \\
\hline 2380 & 43.4433 & 452.851 & 462.9169 & 457 & 122.5994 & 2.08134 \\
\hline 2381 & 43.4603 & 446.8626 & 462.8805 & 457 & 122.5994 & 2.08056 \\
\hline 2382 & 43.4773 & 439.6759 & 462.955 & 457 & 122.5994 & 2.07979 \\
\hline 2383 & 43.4943 & 452.7265 & 463.0678 & 457 & 122.5994 & 2.07902 \\
\hline 2384 & 43.5113 & 454.1132 & 463.3116 & 457 & 122.5994 & 2.07824 \\
\hline 2385 & 43.5283 & 428.9469 & 463.6538 & 457 & 122.5994 & 2.07747 \\
\hline 2386 & 43.5453 & 433.5937 & 464.0953 & 457 & 122.5994 & 2.0767 \\
\hline 2387 & 43.5623 & 472.5387 & 464.6329 & 457 & 122.5994 & 2.07593 \\
\hline 2388 & 43.5793 & 492.9057 & 465.2612 & 457 & 122.5994 & 2.07516 \\
\hline 2389 & 43.5963 & 482.7837 & 465.9904 & 457 & 122.5994 & 2.07439 \\
\hline 2390 & 43.6133 & 471.765 & 466.7558 & 457 & 122.5994 & 2.07362 \\
\hline 2391 & 43.6303 & 468.6856 & 467.1539 & 457 & 122.5994 & 2.07285 \\
\hline 2392 & 43.6473 & 463.2438 & 468.0242 & 457 & 122.5994 & 2.07208 \\
\hline 2393 & 43.6643 & 479.3943 & 468.8878 & 457 & 122.5994 & 2.07131 \\
\hline 2394 & 43.6813 & 477.5504 & 469.7087 & 457 & 122.5994 & 2.07055 \\
\hline 2395 & 43.6983 & 464.3575 & 470.2838 & 457 & 122.5994 & 2.06978 \\
\hline 2396 & 43.7153 & 469.4229 & 470.9156 & 457 & 122.5994 & 2.06902 \\
\hline 2397 & 43.7323 & 451.2991 & 471.4162 & 457 & 122.5994 & 2.06825 \\
\hline 2398 & 43.7493 & 450.4207 & 471.7888 & 457 & 122.5994 & 2.06749 \\
\hline 2399 & 43.7663 & 469.0959 & 472.06 & 457 & 122.5994 & 2.06672 \\
\hline
\end{tabular}




\begin{tabular}{|c|c|c|c|c|c|c|}
\hline 2400 & 43.7833 & 465.6164 & 472.0549 & 457 & 122.5994 & 2.06596 \\
\hline 2401 & 43.8003 & 456.7354 & 472.2343 & 457 & 122.5994 & 2.0652 \\
\hline 2402 & 43.8173 & 450.9425 & 472.1779 & 456.8333 & 122.5994 & 2.06444 \\
\hline 2403 & 43.8343 & 452.2959 & 472.2534 & 456.6667 & 122.5994 & 2.06367 \\
\hline 2404 & 43.8513 & 453.6465 & 472.4138 & 456.5 & 122.5994 & 2.06291 \\
\hline 2405 & 43.8683 & 466.2238 & 472.6987 & 456.3333 & 122.5994 & 2.06215 \\
\hline 2406 & 43.8853 & 480.6329 & 473.1471 & 456.1667 & 122.5994 & 2.06139 \\
\hline 2407 & 43.9023 & 470.666 & 473.7967 & 456 & 122.5994 & 2.06064 \\
\hline 2408 & 43.9193 & 463.1117 & 474.6719 & 455.8333 & 122.5994 & 2.05988 \\
\hline 2409 & 43.9363 & 497.8942 & 475.7846 & 455.6667 & 122.5994 & 2.05912 \\
\hline 2410 & 43.9533 & 469.6764 & 477.131 & 455.5 & 122.5994 & 2.05836 \\
\hline 2411 & 43.9703 & 440.4755 & 478.6896 & 455.3333 & 122.5994 & 2.05761 \\
\hline 2412 & 43.9873 & 455.3919 & 480.423 & 455.1667 & 122.5994 & 2.05685 \\
\hline 2413 & 44.0043 & 493.2046 & 482.2731 & 455 & 122.5994 & 2.0561 \\
\hline 2414 & 44.0213 & 493.2672 & 484.1617 & 454.8333 & 122.5994 & 2.05534 \\
\hline 2415 & 44.0383 & 451.9832 & 485.9889 & 454.6667 & 122.5994 & 2.05459 \\
\hline 2416 & 44.0553 & 499.7148 & 487.6436 & 454.5 & 122.5994 & 2.05383 \\
\hline 2417 & 44.0723 & 510.6773 & 489.012 & 454.3333 & 122.5994 & 2.05308 \\
\hline 2418 & 44.0893 & 492.6085 & 489.9949 & 454.1667 & 122.5994 & 2.05233 \\
\hline 2419 & 44.1063 & 434.3025 & 490.5167 & 454 & 122.5994 & 2.05158 \\
\hline 2420 & 44.1233 & 493.9552 & 490.5352 & 453.8333 & 122.5994 & 2.05083 \\
\hline 2421 & 44.1403 & 428.8352 & 490.0438 & 453.6667 & 122.5994 & 2.05008 \\
\hline 2422 & 44.1573 & 433.358 & 489.0708 & 453.5 & 122.5994 & 2.04933 \\
\hline 2423 & 44.1743 & 440.5874 & 487.668 & 453.3333 & 122.5994 & 2.04858 \\
\hline 2424 & 44.1913 & 443.6782 & 485.908 & 453.1667 & 122.5994 & 2.04783 \\
\hline 2425 & 44.2083 & 479.7992 & 483.8595 & 453 & 122.5994 & 2.04708 \\
\hline 2426 & 44.2253 & 436.3241 & 482.0132 & 453.2608 & 122.5994 & 2.04633 \\
\hline 2427 & 44.2423 & 456.2 & 480.0251 & 453.5216 & 122.5994 & 2.04559 \\
\hline 2428 & 44.2593 & 468.5323 & 477.9587 & 453.7825 & 122.5994 & 2.04484 \\
\hline 2429 & 44.2763 & 469.9906 & 475.8853 & 454.0433 & 122.5994 & 2.04409 \\
\hline 2430 & 44.2933 & 462.8862 & 473.8773 & 454.3041 & 122.5994 & 2.04335 \\
\hline 2431 & 44.3103 & 468.9684 & 471.9982 & 454.5649 & 122.5994 & 2.0426 \\
\hline 2432 & 44.3273 & 425.2765 & 470.2968 & 454.8258 & 122.5994 & 2.04186 \\
\hline 2433 & 44.3443 & 441.006 & 468.8084 & 455.0866 & 122.5994 & 2.04112 \\
\hline 2434 & 44.3613 & 444.9885 & 467.5516 & 455.3474 & 122.5994 & 2.04037 \\
\hline 2435 & 44.3783 & 446.2985 & 466.5016 & 455.6082 & 122.5994 & 2.03963 \\
\hline 2436 & 44.3953 & 462.2057 & 467.2587 & 455.8691 & 122.5994 & 2.03889 \\
\hline 2437 & 44.4123 & 451.7856 & 466.7506 & 456.1299 & 122.5994 & 2.03815 \\
\hline 2438 & 44.4293 & 442.2644 & 466.4544 & 456.3907 & 122.5994 & 2.03741 \\
\hline 2439 & 44.4463 & 459.1909 & 466.3499 & 456.6515 & 122.5994 & 2.03667 \\
\hline 2440 & 44.4633 & 462.7269 & 466.4143 & 456.9124 & 122.5994 & 2.03593 \\
\hline 2441 & 44.4803 & 466.5979 & 466.6067 & 457.1732 & 122.5994 & 2.03519 \\
\hline 2442 & 44.4973 & 455.1877 & 466.9268 & 457.434 & 122.5994 & 2.03445 \\
\hline 2443 & 44.5143 & 437.1358 & 468.0909 & 457.6948 & 122.5994 & 2.03372 \\
\hline 2444 & 44.5313 & 438.694 & 468.5834 & 457.9556 & 122.5994 & 2.03298 \\
\hline 2445 & 44.5483 & 446.6565 & 469.0585 & 458.2165 & 122.5994 & 2.03224 \\
\hline 2446 & 44.5653 & 464.0908 & 469.5056 & 458.4773 & 122.5994 & 2.03151 \\
\hline 2447 & 44.5823 & 479.1973 & 469.8977 & 458.7381 & 122.5994 & 2.03077 \\
\hline 2448 & 44.5993 & 486.8214 & 470.2197 & 458.9989 & 122.5994 & 2.03004 \\
\hline
\end{tabular}




\begin{tabular}{|c|c|c|c|c|c|c|}
\hline 2449 & 44.6163 & 470.897 & 470.4702 & 459.2598 & 122.5994 & 2.0293 \\
\hline 2450 & 44.6333 & 456.2704 & 470.4727 & 459.3371 & 122.5994 & 2.02857 \\
\hline 2451 & 44.6503 & 459.7651 & 470.4458 & 459.4145 & 122.5994 & 2.02784 \\
\hline 2452 & 44.6673 & 456.0077 & 470.3546 & 459.4918 & 122.5994 & 2.0271 \\
\hline 2453 & 44.6843 & 453.6465 & 470.2292 & 459.5692 & 122.5994 & 2.02637 \\
\hline 2454 & 44.7013 & 450.4376 & 470.0776 & 459.6465 & 122.5994 & 2.02564 \\
\hline 2455 & 44.7183 & 477.0769 & 469.9099 & 459.7239 & 122.5994 & 2.02491 \\
\hline 2456 & 44.7353 & 506.0767 & 469.739 & 459.8012 & 122.5994 & 2.02418 \\
\hline 2457 & 44.7523 & 491.8477 & 469.5793 & 459.8786 & 122.5994 & 2.02345 \\
\hline 2458 & 44.7693 & 473.201 & 469.4447 & 459.9559 & 122.5994 & 2.02272 \\
\hline 2459 & 44.7863 & 482.5822 & 469.3463 & 460.0333 & 122.5994 & 2.02199 \\
\hline 2460 & 44.8033 & 464.194 & 469.2914 & 460.1106 & 122.5994 & 2.02127 \\
\hline 2461 & 44.8203 & 511.6461 & 469.3997 & 460.188 & 122.5994 & 2.02054 \\
\hline 2462 & 44.8373 & 502.366 & 469.4449 & 460.2653 & 122.5994 & 2.01981 \\
\hline 2463 & 44.8543 & 459.0954 & 469.4845 & 460.3427 & 122.5994 & 2.01909 \\
\hline 2464 & 44.8713 & 462.288 & 469.6276 & 460.42 & 122.5994 & 2.01836 \\
\hline 2465 & 44.8883 & 467.0474 & 469.8184 & 460.4974 & 122.5994 & 2.01764 \\
\hline 2466 & 44.9053 & 491.3206 & 470.0581 & 460.5747 & 122.5994 & 2.01691 \\
\hline 2467 & 44.9223 & 457.4003 & 470.3485 & 460.6521 & 122.5994 & 2.01619 \\
\hline 2468 & 44.9393 & 514.1491 & 470.6833 & 460.7295 & 122.5994 & 2.01547 \\
\hline 2469 & 44.9563 & 503.5577 & 471.0458 & 460.8068 & 122.5994 & 2.01474 \\
\hline 2470 & 44.9733 & 513.8522 & 471.8337 & 460.8842 & 122.5994 & 2.01402 \\
\hline 2471 & 44.9903 & 474.6771 & 472.3473 & 460.9615 & 122.5994 & 2.0133 \\
\hline 2472 & 45.0073 & 500.3589 & 472.9251 & 461.0389 & 122.5994 & 2.01258 \\
\hline 2473 & 45.0243 & 516.9572 & 473.5754 & 461.1162 & 122.5994 & 2.01186 \\
\hline 2474 & 45.0413 & 524.3597 & 474.311 & 461.1936 & 122.5994 & 2.01114 \\
\hline 2475 & 45.0583 & 512.1007 & 475.1441 & 461.2709 & 122.5994 & 2.01042 \\
\hline 2476 & 45.0753 & 489.0408 & 476.0961 & 461.3483 & 122.5994 & 2.0097 \\
\hline 2477 & 45.0923 & 541.6865 & 477.4012 & 461.4256 & 122.5994 & 2.00898 \\
\hline 2478 & 45.1093 & 532.7458 & 478.6858 & 461.503 & 122.5994 & 2.00826 \\
\hline 2479 & 45.1263 & 530.1976 & 480.1981 & 461.5803 & 122.5994 & 2.00755 \\
\hline 2480 & 45.1433 & 552.197 & 481.998 & 461.6577 & 122.5994 & 2.00683 \\
\hline 2481 & 45.1603 & 538.881 & 484.1646 & 461.735 & 122.5994 & 2.00611 \\
\hline 2482 & 45.1773 & 533.0803 & 486.7938 & 461.8124 & 122.5994 & 2.0054 \\
\hline 2483 & 45.1943 & 548.4506 & 490.0088 & 461.8897 & 122.5994 & 2.00468 \\
\hline 2484 & 45.2113 & 534.9738 & 493.9815 & 461.9671 & 122.5994 & 2.00397 \\
\hline 2485 & 45.2283 & 542.8379 & 498.7976 & 462.0444 & 122.5994 & 2.00326 \\
\hline 2486 & 45.2453 & 532.1733 & 504.4684 & 462.1218 & 122.5994 & 2.00254 \\
\hline 2487 & 45.2623 & 535.6546 & 511.5863 & 462.1991 & 122.5994 & 2.00183 \\
\hline 2488 & 45.2793 & 530.9993 & 520.1277 & 462.2765 & 122.5994 & 2.00112 \\
\hline 2489 & 45.2963 & 540.4811 & 530.2852 & 462.3538 & 122.5994 & 2.00041 \\
\hline 2490 & 45.3133 & 563.7543 & 542.1363 & 462.4312 & 122.5994 & 1.9997 \\
\hline 2491 & 45.3303 & 572.7709 & 555.7879 & 462.5085 & 122.5994 & 1.99899 \\
\hline 2492 & 45.3473 & 566.5146 & 571.2561 & 462.5859 & 122.5994 & 1.99828 \\
\hline 2493 & 45.3643 & 553.9936 & 588.3497 & 462.6632 & 122.5994 & 1.99757 \\
\hline 2494 & 45.3813 & 592.5169 & 607.0566 & 462.7406 & 122.5994 & 1.99686 \\
\hline 2495 & 45.3983 & 614.981 & 626.8892 & 462.818 & 122.5994 & 1.99615 \\
\hline 2496 & 45.4153 & 632.2359 & 647.2814 & 462.8953 & 122.5994 & 1.99544 \\
\hline 2497 & 45.4323 & 618.8902 & 667.3909 & 462.9727 & 122.5994 & 1.99473 \\
\hline
\end{tabular}




\begin{tabular}{|c|c|c|c|c|c|c|}
\hline 2498 & 45.4493 & 603.0249 & 685.9965 & 462.8665 & 122.5994 & 1.99403 \\
\hline 2499 & 45.4663 & 622.4434 & 702.1191 & 462.7604 & 122.5994 & 1.99332 \\
\hline 2500 & 45.4833 & 629.9496 & 714.6528 & 462.6543 & 122.5994 & 1.99262 \\
\hline 2501 & 45.5003 & 666.4607 & 722.834 & 462.5482 & 122.5994 & 1.99191 \\
\hline 2502 & 45.5173 & 630.4616 & 726.4071 & 462.4421 & 122.5994 & 1.99121 \\
\hline 2503 & 45.5343 & 641.0633 & 725.7723 & 462.3359 & 122.5994 & 1.9905 \\
\hline 2504 & 45.5513 & 636.4431 & 721.2216 & 462.2298 & 122.5994 & 1.9898 \\
\hline 2505 & 45.5683 & 666.3373 & 713.5052 & 462.1237 & 122.5994 & 1.9891 \\
\hline 2506 & 45.5853 & 643.3033 & 703.1212 & 462.0176 & 122.5994 & 1.98839 \\
\hline 2507 & 45.6023 & 658.7911 & 690.431 & 461.9115 & 122.5994 & 1.98769 \\
\hline 2508 & 45.6193 & 627.1684 & 675.774 & 461.8053 & 122.5994 & 1.98699 \\
\hline 2509 & 45.6363 & 615.3505 & 659.603 & 461.6992 & 122.5994 & 1.98629 \\
\hline 2510 & 45.6533 & 576.689 & 642.506 & 461.5931 & 122.5994 & 1.98559 \\
\hline 2511 & 45.6703 & 561.7188 & 625.2474 & 461.487 & 122.5994 & 1.98489 \\
\hline 2512 & 45.6873 & 575.1687 & 608.3486 & 461.3809 & 122.5994 & 1.98419 \\
\hline 2513 & 45.7043 & 541.4898 & 592.4236 & 461.2747 & 122.5994 & 1.98349 \\
\hline 2514 & 45.7213 & 516.6502 & 577.9754 & 461.1686 & 122.5994 & 1.9828 \\
\hline 2515 & 45.7383 & 532.7132 & 565.3711 & 461.0625 & 122.5994 & 1.9821 \\
\hline 2516 & 45.7553 & 526.4542 & 554.9309 & 460.9564 & 122.5994 & 1.9814 \\
\hline 2517 & 45.7723 & 542.3347 & 547.2205 & 460.8503 & 122.5994 & 1.9807 \\
\hline 2518 & 45.7893 & 540.3796 & 543.0627 & 460.7441 & 122.5994 & 1.98001 \\
\hline 2519 & 45.8063 & 558.2689 & 543.4706 & 460.638 & 122.5994 & 1.97931 \\
\hline 2520 & 45.8233 & 592.4398 & 549.3345 & 460.5319 & 122.5994 & 1.97862 \\
\hline 2521 & 45.8403 & 621.1961 & 560.7577 & 460.4258 & 122.5994 & 1.97792 \\
\hline 2522 & 45.8573 & 636.0511 & 575.7678 & 460.077 & 122.5994 & 1.97723 \\
\hline 2523 & 45.8743 & 658.6534 & 589.8575 & 459.7282 & 122.5994 & 1.97654 \\
\hline 2524 & 45.8913 & 608.3576 & 595.5798 & 459.3794 & 122.5994 & 1.97585 \\
\hline 2525 & 45.9083 & 554.8902 & 589.2255 & 459.0306 & 122.5994 & 1.97515 \\
\hline 2526 & 45.9253 & 541.3765 & 576.1733 & 458.6818 & 122.5994 & 1.97446 \\
\hline 2527 & 45.9423 & 561.4177 & 564.3041 & 458.333 & 122.5994 & 1.97377 \\
\hline 2528 & 45.9593 & 559.3721 & 557.9897 & 457.9842 & 122.5994 & 1.97308 \\
\hline 2529 & 45.9763 & 569.2798 & 557.8621 & 457.6354 & 122.5994 & 1.97239 \\
\hline 2530 & 45.9933 & 572.3434 & 561.2065 & 457.2866 & 122.5994 & 1.9717 \\
\hline 2531 & 46.0103 & 566.3822 & 563.1816 & 456.9378 & 122.5994 & 1.97101 \\
\hline 2532 & 46.0273 & 562.7249 & 560.1682 & 456.589 & 122.5994 & 1.97032 \\
\hline 2533 & 46.0443 & 547.3113 & 553.2612 & 456.2402 & 122.5994 & 1.96964 \\
\hline 2534 & 46.0613 & 499.5196 & 545.5153 & 455.8914 & 122.5994 & 1.96895 \\
\hline 2535 & 46.0783 & 486.0604 & 538.7998 & 455.5426 & 122.5994 & 1.96826 \\
\hline 2536 & 46.0953 & 469.9038 & 533.6889 & 455.1938 & 122.5994 & 1.96757 \\
\hline 2537 & 46.1123 & 475.1272 & 529.9446 & 454.8451 & 122.5994 & 1.96689 \\
\hline 2538 & 46.1293 & 473.1224 & 527.123 & 454.4963 & 122.5994 & 1.9662 \\
\hline 2539 & 46.1463 & 488.9707 & 524.7535 & 454.1475 & 122.5994 & 1.96552 \\
\hline 2540 & 46.1633 & 492.3012 & 522.4976 & 453.7987 & 122.5994 & 1.96483 \\
\hline 2541 & 46.1803 & 495.5218 & 520.147 & 453.4499 & 122.5994 & 1.96415 \\
\hline 2542 & 46.1973 & 460.8333 & 517.5844 & 453.1011 & 122.5994 & 1.96347 \\
\hline 2543 & 46.2143 & 452.8364 & 514.7504 & 452.7523 & 122.5994 & 1.96278 \\
\hline 2544 & 46.2313 & 454.8594 & 511.6416 & 452.4035 & 122.5994 & 1.9621 \\
\hline 2545 & 46.2483 & 444.3915 & 508.2979 & 452.0547 & 122.5994 & 1.96142 \\
\hline 2546 & 46.2653 & 473.5915 & 504.5658 & 451.4691 & 122.5994 & 1.96074 \\
\hline
\end{tabular}




\begin{tabular}{|c|c|c|c|c|c|c|}
\hline 2547 & 46.2823 & 487.3949 & 500.7872 & 450.8835 & 122.5994 & 1.96006 \\
\hline 2548 & 46.2993 & 464.0543 & 497.0656 & 450.2979 & 122.5994 & 1.95938 \\
\hline 2549 & 46.3163 & 440.5008 & 493.4926 & 449.7122 & 122.5994 & 1.9587 \\
\hline 2550 & 46.3333 & 465.6681 & 490.1323 & 449.1266 & 122.5994 & 1.95802 \\
\hline 2551 & 46.3503 & 437.7017 & 487.0258 & 448.541 & 122.5994 & 1.95734 \\
\hline 2552 & 46.3673 & 425.3439 & 484.1919 & 447.9554 & 122.5994 & 1.95666 \\
\hline 2553 & 46.3843 & 439.4543 & 481.6367 & 447.3698 & 122.5994 & 1.95599 \\
\hline 2554 & 46.4013 & 456.7471 & 479.3622 & 446.7842 & 122.5994 & 1.95531 \\
\hline 2555 & 46.4183 & 449.9047 & 477.3696 & 446.1986 & 122.5994 & 1.95463 \\
\hline 2556 & 46.4353 & 441.1759 & 475.6622 & 445.613 & 122.5994 & 1.95396 \\
\hline 2557 & 46.4523 & 424.4904 & 474.2412 & 445.0273 & 122.5994 & 1.95328 \\
\hline 2558 & 46.4693 & 420.8515 & 473.1101 & 444.4417 & 122.5994 & 1.95261 \\
\hline 2559 & 46.4863 & 456.0906 & 472.2752 & 443.8561 & 122.5994 & 1.95193 \\
\hline 2560 & 46.5033 & 411.2584 & 471.7252 & 443.2705 & 122.5994 & 1.95126 \\
\hline 2561 & 46.5203 & 462.6639 & 471.4525 & 442.6849 & 122.5994 & 1.95058 \\
\hline 2562 & 46.5373 & 462.1619 & 471.4308 & 442.0993 & 122.5994 & 1.94991 \\
\hline 2563 & 46.5543 & 429.6836 & 471.6171 & 441.5137 & 122.5994 & 1.94924 \\
\hline 2564 & 46.5713 & 468.4236 & 471.9466 & 440.9281 & 122.5994 & 1.94857 \\
\hline 2565 & 46.5883 & 475.2185 & 472.3356 & 440.3424 & 122.5994 & 1.9479 \\
\hline 2566 & 46.6053 & 455.3709 & 472.6859 & 439.7568 & 122.5994 & 1.94722 \\
\hline 2567 & 46.6223 & 457.7278 & 471.4327 & 439.1712 & 122.5994 & 1.94655 \\
\hline 2568 & 46.6393 & 436.2371 & 471.4308 & 438.5856 & 122.5994 & 1.94588 \\
\hline 2569 & 46.6563 & 428.6965 & 471.1222 & 438 & 122.5994 & 1.94521 \\
\hline 2570 & 46.6733 & 449.0009 & 470.5901 & 437.5104 & 122.5994 & 1.94455 \\
\hline 2571 & 46.6903 & 438.1121 & 469.7697 & 437.0208 & 122.5994 & 1.94388 \\
\hline 2572 & 46.7073 & 442.2977 & 468.7144 & 436.5313 & 122.5994 & 1.94321 \\
\hline 2573 & 46.7243 & 459.9544 & 467.476 & 436.0417 & 122.5994 & 1.94254 \\
\hline 2574 & 46.7413 & 478.9102 & 465.3657 & 435.5521 & 122.5994 & 1.94188 \\
\hline 2575 & 46.7583 & 457.4054 & 463.8686 & 435.0625 & 122.5994 & 1.94121 \\
\hline 2576 & 46.7753 & 451.1157 & 462.2437 & 434.5729 & 122.5994 & 1.94054 \\
\hline 2577 & 46.7923 & 437.6647 & 460.5052 & 434.0833 & 122.5994 & 1.93988 \\
\hline 2578 & 46.8093 & 440.7066 & 458.6773 & 433.5938 & 122.5994 & 1.93921 \\
\hline 2579 & 46.8263 & 460.7867 & 456.7923 & 433.1042 & 122.5994 & 1.93855 \\
\hline 2580 & 46.8433 & 445.3596 & 454.8912 & 432.6146 & 122.5994 & 1.93788 \\
\hline 2581 & 46.8603 & 424.0828 & 453.0074 & 432.125 & 122.5994 & 1.93722 \\
\hline 2582 & 46.8773 & 425.6829 & 451.1676 & 431.6354 & 122.5994 & 1.93656 \\
\hline 2583 & 46.8943 & 429.4404 & 449.4496 & 431.1458 & 122.5994 & 1.9359 \\
\hline 2584 & 46.9113 & 413.171 & 447.8395 & 430.6563 & 122.5994 & 1.93523 \\
\hline 2585 & 46.9283 & 450.9019 & 446.3574 & 430.1667 & 122.5994 & 1.93457 \\
\hline 2586 & 46.9453 & 445.3343 & 445.0317 & 429.6771 & 122.5994 & 1.93391 \\
\hline 2587 & 46.9623 & 424.4166 & 443.8818 & 429.1875 & 122.5994 & 1.93325 \\
\hline 2588 & 46.9793 & 460.9286 & 442.8451 & 428.6979 & 122.5994 & 1.93259 \\
\hline 2589 & 46.9963 & 444.5963 & 441.8304 & 428.2083 & 122.5994 & 1.93193 \\
\hline 2590 & 47.0133 & 422.7323 & 441.0611 & 427.7188 & 122.5994 & 1.93127 \\
\hline 2591 & 47.0303 & 405.4859 & 440.4111 & 427.2292 & 122.5994 & 1.93061 \\
\hline 2592 & 47.0473 & 415.2155 & 439.8587 & 426.7396 & 122.5994 & 1.92996 \\
\hline 2593 & 47.0643 & 432.9637 & 439.3913 & 426.25 & 122.5994 & 1.9293 \\
\hline 2594 & 47.0813 & 453.6152 & 438.9815 & 425.7604 & 122.5994 & 1.92864 \\
\hline 2595 & 47.0983 & 447.5472 & 438.5031 & 425.2708 & 122.5994 & 1.92799 \\
\hline
\end{tabular}




\begin{tabular}{|c|c|c|c|c|c|c|}
\hline 2596 & 47.1153 & 455.8092 & 438.0467 & 424.7813 & 122.5994 & 1.92733 \\
\hline 2597 & 47.1323 & 458.021 & 437.5535 & 424.2917 & 122.5994 & 1.92667 \\
\hline 2598 & 47.1493 & 428.4243 & 436.9267 & 423.8021 & 122.5994 & 1.92602 \\
\hline 2599 & 47.1663 & 439.3823 & 436.1968 & 423.3125 & 122.5994 & 1.92536 \\
\hline 2600 & 47.1833 & 440.9577 & 435.3851 & 422.8229 & 122.5994 & 1.92471 \\
\hline 2601 & 47.2003 & 398.5671 & 434.2069 & 422.3333 & 122.5994 & 1.92406 \\
\hline 2602 & 47.2173 & 423.3885 & 433.2415 & 421.8438 & 122.5994 & 1.9234 \\
\hline 2603 & 47.2343 & 440.7145 & 432.2167 & 421.3542 & 122.5994 & 1.92275 \\
\hline 2604 & 47.2513 & 428.3366 & 431.1602 & 420.8646 & 122.5994 & 1.9221 \\
\hline 2605 & 47.2683 & 412.3775 & 430.0429 & 420.375 & 122.5994 & 1.92145 \\
\hline 2606 & 47.2853 & 420.621 & 428.8946 & 419.8854 & 122.5994 & 1.9208 \\
\hline 2607 & 47.3023 & 429.9434 & 427.7321 & 419.3958 & 122.5994 & 1.92014 \\
\hline 2608 & 47.3193 & 447.5519 & 426.5063 & 418.9063 & 122.5994 & 1.91949 \\
\hline 2609 & 47.3363 & 429.8801 & 425.3813 & 418.4167 & 122.5994 & 1.91885 \\
\hline 2610 & 47.3533 & 438.7948 & 424.2915 & 417.9271 & 122.5994 & 1.9182 \\
\hline 2611 & 47.3703 & 425.6232 & 423.2517 & 417.4375 & 122.5994 & 1.91755 \\
\hline 2612 & 47.3873 & 430.382 & 422.2689 & 416.9479 & 122.5994 & 1.9169 \\
\hline 2613 & 47.4043 & 424.4673 & 421.3468 & 416.4583 & 122.5994 & 1.91625 \\
\hline 2614 & 47.4213 & 442.8311 & 420.486 & 415.9688 & 122.5994 & 1.9156 \\
\hline 2615 & 47.4383 & 452.6206 & 419.6346 & 415.4792 & 122.5994 & 1.91496 \\
\hline 2616 & 47.4553 & 467.9702 & 418.8911 & 414.9896 & 122.5994 & 1.91431 \\
\hline 2617 & 47.4723 & 471.7371 & 418.197 & 414.5 & 122.5994 & 1.91366 \\
\hline 2618 & 47.4893 & 446.1832 & 417.348 & 413.8125 & 122.5994 & 1.91302 \\
\hline 2619 & 47.5063 & 453.7146 & 416.5348 & 413.125 & 122.5994 & 1.91237 \\
\hline 2620 & 47.5233 & 439.3977 & 415.7502 & 412.4375 & 122.5994 & 1.91173 \\
\hline 2621 & 47.5403 & 431.5489 & 415.0288 & 411.75 & 122.5994 & 1.91109 \\
\hline 2622 & 47.5573 & 446.8978 & 414.2424 & 411.0625 & 122.5994 & 1.91044 \\
\hline 2623 & 47.5743 & 463.9972 & 413.5092 & 410.375 & 122.5994 & 1.9098 \\
\hline 2624 & 47.5913 & 444.5815 & 412.7863 & 409.6875 & 122.5994 & 1.90916 \\
\hline 2625 & 47.6083 & 433.3364 & 412.0741 & 409 & 122.5994 & 1.90851 \\
\hline 2626 & 47.6253 & 425.9511 & 411.347 & 408.3125 & 122.5994 & 1.90787 \\
\hline 2627 & 47.6423 & 400.0772 & 410.6643 & 407.625 & 122.5994 & 1.90723 \\
\hline 2628 & 47.6593 & 416.7798 & 410.0207 & 406.9375 & 122.5994 & 1.90659 \\
\hline 2629 & 47.6763 & 421.8288 & 409.3791 & 406.25 & 122.5994 & 1.90595 \\
\hline 2630 & 47.6933 & 437.3732 & 408.8286 & 405.5625 & 122.5994 & 1.90531 \\
\hline 2631 & 47.7103 & 446.1884 & 408.2439 & 404.875 & 122.5994 & 1.90467 \\
\hline 2632 & 47.7273 & 420.9741 & 407.6936 & 404.1875 & 122.5994 & 1.90403 \\
\hline 2633 & 47.7443 & 434.1154 & 407.1692 & 403.5 & 122.5994 & 1.9034 \\
\hline 2634 & 47.7613 & 441.2597 & 406.5641 & 402.8125 & 122.5994 & 1.90276 \\
\hline 2635 & 47.7783 & 396.0335 & 406.1516 & 402.125 & 122.5994 & 1.90212 \\
\hline 2636 & 47.7953 & 415.5532 & 405.7902 & 401.4375 & 122.5994 & 1.90148 \\
\hline 2637 & 47.8123 & 444.6429 & 405.4807 & 400.75 & 122.5994 & 1.90085 \\
\hline 2638 & 47.8293 & 407.0158 & 405.2548 & 400.0625 & 122.5994 & 1.90021 \\
\hline 2639 & 47.8463 & 419.7941 & 405.0423 & 399.375 & 122.5994 & 1.89958 \\
\hline 2640 & 47.8633 & 409.3198 & 404.8676 & 398.6875 & 122.5994 & 1.89894 \\
\hline 2641 & 47.8803 & 381.8591 & 404.7198 & 398 & 122.5994 & 1.89831 \\
\hline 2642 & 47.8973 & 399.1209 & 405.6017 & 398.3958 & 122.5994 & 1.89767 \\
\hline 2643 & 47.9143 & 410.8062 & 406.5545 & 398.7917 & 122.5994 & 1.89704 \\
\hline 2644 & 47.9313 & 444.5481 & 407.4989 & 399.1875 & 122.5994 & 1.89641 \\
\hline
\end{tabular}




\begin{tabular}{|c|c|c|c|c|c|c|}
\hline 2645 & 47.9483 & 464.6162 & 408.4285 & 399.5833 & 122.5994 & 1.89577 \\
\hline 2646 & 47.9653 & 428.1938 & 409.3049 & 399.9792 & 122.5994 & 1.89514 \\
\hline 2647 & 47.9823 & 439.8285 & 410.198 & 400.375 & 122.5994 & 1.89451 \\
\hline 2648 & 47.9993 & 453.1612 & 411.065 & 400.7708 & 122.5994 & 1.89388 \\
\hline 2649 & 48.0163 & 441.2723 & 411.8954 & 401.1667 & 122.5994 & 1.89325 \\
\hline 2650 & 48.0333 & 384.3146 & 412.6769 & 401.5625 & 122.5994 & 1.89262 \\
\hline 2651 & 48.0503 & 445.0911 & 413.3987 & 401.9583 & 122.5994 & 1.89199 \\
\hline 2652 & 48.0673 & 440.5616 & 414.0593 & 402.3542 & 122.5994 & 1.89136 \\
\hline 2653 & 48.0843 & 430.7773 & 414.6494 & 402.75 & 122.5994 & 1.89073 \\
\hline 2654 & 48.1013 & 461.3499 & 415.2271 & 403.1458 & 122.5994 & 1.8901 \\
\hline 2655 & 48.1183 & 405.3302 & 415.8005 & 403.5417 & 122.5994 & 1.88947 \\
\hline 2656 & 48.1353 & 416.1201 & 416.3952 & 403.9375 & 122.5994 & 1.88884 \\
\hline 2657 & 48.1523 & 420.4945 & 417.0307 & 404.3333 & 122.5994 & 1.88822 \\
\hline 2658 & 48.1693 & 440.956 & 417.7183 & 404.7292 & 122.5994 & 1.88759 \\
\hline 2659 & 48.1863 & 432.4421 & 418.4627 & 405.125 & 122.5994 & 1.88696 \\
\hline 2660 & 48.2033 & 479.3471 & 419.2625 & 405.5208 & 122.5994 & 1.88634 \\
\hline 2661 & 48.2203 & 456.2728 & 420.1115 & 405.9167 & 122.5994 & 1.88571 \\
\hline 2662 & 48.2373 & 441.1703 & 420.9994 & 406.3125 & 122.5994 & 1.88509 \\
\hline 2663 & 48.2543 & 454.8262 & 421.9099 & 406.7083 & 122.5994 & 1.88446 \\
\hline 2664 & 48.2713 & 438.3815 & 422.8479 & 407.1042 & 122.5994 & 1.88384 \\
\hline 2665 & 48.2883 & 410.3331 & 423.7493 & 407.5 & 122.5994 & 1.88322 \\
\hline 2666 & 48.3053 & 448.0575 & 424.3337 & 407.6563 & 122.5994 & 1.88259 \\
\hline 2667 & 48.3223 & 440.6117 & 424.9523 & 407.8125 & 122.5994 & 1.88197 \\
\hline 2668 & 48.3393 & 435.8074 & 425.5577 & 407.9688 & 122.5994 & 1.88135 \\
\hline 2669 & 48.3563 & 432.6449 & 426.1658 & 408.125 & 122.5994 & 1.88073 \\
\hline 2670 & 48.3733 & 432.6712 & 426.7665 & 408.2813 & 122.5994 & 1.8801 \\
\hline 2671 & 48.3903 & 457.1793 & 427.3602 & 408.4375 & 122.5994 & 1.87948 \\
\hline 2672 & 48.4073 & 458.4692 & 427.9425 & 408.5938 & 122.5994 & 1.87886 \\
\hline 2673 & 48.4243 & 443.1905 & 428.4673 & 408.75 & 122.5994 & 1.87824 \\
\hline 2674 & 48.4413 & 449.9202 & 428.894 & 408.9063 & 122.5994 & 1.87762 \\
\hline 2675 & 48.4583 & 469.1484 & 429.2556 & 409.0625 & 122.5994 & 1.87701 \\
\hline 2676 & 48.4753 & 456.2618 & 429.5143 & 409.2188 & 122.5994 & 1.87639 \\
\hline 2677 & 48.4923 & 436.1295 & 429.675 & 409.375 & 122.5994 & 1.87577 \\
\hline 2678 & 48.5093 & 430.211 & 429.7476 & 409.5313 & 122.5994 & 1.87515 \\
\hline 2679 & 48.5263 & 441.4507 & 429.7615 & 409.6875 & 122.5994 & 1.87453 \\
\hline 2680 & 48.5433 & 452.1255 & 429.7429 & 409.8438 & 122.5994 & 1.87392 \\
\hline 2681 & 48.5603 & 437.0257 & 429.7089 & 410 & 122.5994 & 1.8733 \\
\hline 2682 & 48.5773 & 425.6194 & 429.6646 & 410.1563 & 122.5994 & 1.87269 \\
\hline 2683 & 48.5943 & 448.1634 & 429.6024 & 410.3125 & 122.5994 & 1.87207 \\
\hline 2684 & 48.6113 & 421.5658 & 429.5107 & 410.4688 & 122.5994 & 1.87145 \\
\hline 2685 & 48.6283 & 404.98 & 429.3822 & 410.625 & 122.5994 & 1.87084 \\
\hline 2686 & 48.6453 & 416.5888 & 429.2197 & 410.7813 & 122.5994 & 1.87023 \\
\hline 2687 & 48.6623 & 420.2097 & 429.036 & 410.9375 & 122.5994 & 1.86961 \\
\hline 2688 & 48.6793 & 433.791 & 428.8669 & 411.0938 & 122.5994 & 1.869 \\
\hline 2689 & 48.6963 & 458.0291 & 428.7376 & 411.25 & 122.5994 & 1.86839 \\
\hline 2690 & 48.7133 & 447.7019 & 428.6715 & 411.4063 & 122.5994 & 1.86777 \\
\hline 2691 & 48.7303 & 398.4415 & 428.6808 & 411.5625 & 122.5994 & 1.86716 \\
\hline 2692 & 48.7473 & 432.3053 & 428.7657 & 411.7188 & 122.5994 & 1.86655 \\
\hline 2693 & 48.7643 & 391.9547 & 428.9133 & 411.875 & 122.5994 & 1.86594 \\
\hline
\end{tabular}




\begin{tabular}{|c|c|c|c|c|c|c|}
\hline 2694 & 48.7813 & 414.5309 & 429.0999 & 412.0313 & 122.5994 & 1.86533 \\
\hline 2695 & 48.7983 & 452.4297 & 429.2999 & 412.1875 & 122.5994 & 1.86472 \\
\hline 2696 & 48.8153 & 420.6626 & 429.5037 & 412.3438 & 122.5994 & 1.86411 \\
\hline 2697 & 48.8323 & 404.16 & 429.6136 & 412.5 & 122.5994 & 1.8635 \\
\hline 2698 & 48.8493 & 416.0007 & 429.6208 & 412.6563 & 122.5994 & 1.86289 \\
\hline 2699 & 48.8663 & 413.6189 & 429.5002 & 412.8125 & 122.5994 & 1.86228 \\
\hline 2700 & 48.8833 & 440.3524 & 429.2458 & 412.9688 & 122.5994 & 1.86168 \\
\hline 2701 & 48.9003 & 431.9189 & 428.87 & 413.125 & 122.5994 & 1.86107 \\
\hline 2702 & 48.9173 & 422.9024 & 428.4311 & 413.2813 & 122.5994 & 1.86046 \\
\hline 2703 & 48.9343 & 415.4345 & 427.8847 & 413.4375 & 122.5994 & 1.85985 \\
\hline 2704 & 48.9513 & 440.2214 & 427.2966 & 413.5938 & 122.5994 & 1.85925 \\
\hline 2705 & 48.9683 & 444.6347 & 426.6487 & 413.75 & 122.5994 & 1.85864 \\
\hline 2706 & 48.9853 & 392.3161 & 425.9611 & 413.9063 & 122.5994 & 1.85804 \\
\hline 2707 & 49.0023 & 422.4904 & 425.2431 & 414.0625 & 122.5994 & 1.85743 \\
\hline 2708 & 49.0193 & 419.2324 & 424.5092 & 414.2188 & 122.5994 & 1.85683 \\
\hline 2709 & 49.0363 & 429.5532 & 423.7809 & 414.375 & 122.5994 & 1.85622 \\
\hline 2710 & 49.0533 & 430.2178 & 423.0994 & 414.5313 & 122.5994 & 1.85562 \\
\hline 2711 & 49.0703 & 428.2658 & 422.4521 & 414.6875 & 122.5994 & 1.85502 \\
\hline 2712 & 49.0873 & 427.2569 & 421.8741 & 414.8438 & 122.5994 & 1.85441 \\
\hline 2713 & 49.1043 & 402.4585 & 421.3561 & 415 & 122.5994 & 1.85381 \\
\hline 2714 & 49.1213 & 408.7314 & 421.2514 & 415.4583 & 122.5994 & 1.85321 \\
\hline 2715 & 49.1383 & 425.4015 & 421.2393 & 415.9167 & 122.5994 & 1.85261 \\
\hline 2716 & 49.1553 & 428.8978 & 421.3208 & 416.375 & 122.5994 & 1.85201 \\
\hline 2717 & 49.1723 & 446.822 & 421.4947 & 416.8333 & 122.5994 & 1.85141 \\
\hline 2718 & 49.1893 & 432.6977 & 421.7545 & 417.2917 & 122.5994 & 1.85081 \\
\hline 2719 & 49.2063 & 418.821 & 422.1048 & 417.75 & 122.5994 & 1.85021 \\
\hline 2720 & 49.2233 & 422.6652 & 422.4986 & 418.2083 & 122.5994 & 1.84961 \\
\hline 2721 & 49.2403 & 419.4616 & 423.0062 & 418.6667 & 122.5994 & 1.84901 \\
\hline 2722 & 49.2573 & 411.0124 & 424.7089 & 419.125 & 122.5994 & 1.84841 \\
\hline 2723 & 49.2743 & 466.5519 & 425.379 & 419.5833 & 122.5994 & 1.84781 \\
\hline 2724 & 49.2913 & 451.3119 & 426.0963 & 420.0417 & 122.5994 & 1.84722 \\
\hline 2725 & 49.3083 & 428.821 & 426.8293 & 420.5 & 122.5994 & 1.84662 \\
\hline 2726 & 49.3253 & 442.0757 & 427.5936 & 420.9583 & 122.5994 & 1.84602 \\
\hline 2727 & 49.3423 & 426.1896 & 428.3513 & 421.4167 & 122.5994 & 1.84543 \\
\hline 2728 & 49.3593 & 399.9363 & 429.0816 & 421.875 & 122.5994 & 1.84483 \\
\hline 2729 & 49.3763 & 426.0955 & 429.7665 & 422.3333 & 122.5994 & 1.84424 \\
\hline 2730 & 49.3933 & 447.1187 & 430.4055 & 422.7917 & 122.5994 & 1.84364 \\
\hline 2731 & 49.4103 & 415.8053 & 430.9737 & 423.25 & 122.5994 & 1.84305 \\
\hline 2732 & 49.4273 & 413.3108 & 431.4853 & 423.7083 & 122.5994 & 1.84245 \\
\hline 2733 & 49.4443 & 435.557 & 431.9452 & 424.1667 & 122.5994 & 1.84186 \\
\hline 2734 & 49.4613 & 407.9976 & 432.3101 & 424.625 & 122.5994 & 1.84126 \\
\hline 2735 & 49.4783 & 444.3774 & 432.6816 & 425.0833 & 122.5994 & 1.84067 \\
\hline 2736 & 49.4953 & 418.4123 & 433.0137 & 425.5417 & 122.5994 & 1.84008 \\
\hline 2737 & 49.5123 & 444.5881 & 433.3122 & 426 & 122.5994 & 1.83949 \\
\hline 2738 & 49.5293 & 408.9621 & 433.4509 & 426.3229 & 122.5994 & 1.8389 \\
\hline 2739 & 49.5463 & 407.6445 & 433.5686 & 426.6458 & 122.5994 & 1.8383 \\
\hline 2740 & 49.5633 & 434.9401 & 433.6914 & 426.9688 & 122.5994 & 1.83771 \\
\hline 2741 & 49.5803 & 379.0664 & 433.8246 & 427.2917 & 122.5994 & 1.83712 \\
\hline 2742 & 49.5973 & 443.6205 & 433.9548 & 427.6146 & 122.5994 & 1.83653 \\
\hline
\end{tabular}




\begin{tabular}{|c|c|c|c|c|c|c|}
\hline 2743 & 49.6143 & 445.1673 & 434.1391 & 427.9375 & 122.5994 & 1.83594 \\
\hline 2744 & 49.6313 & 440.249 & 434.3612 & 428.2604 & 122.5994 & 1.83536 \\
\hline 2745 & 49.6483 & 404.797 & 434.6059 & 428.5833 & 122.5994 & 1.83477 \\
\hline 2746 & 49.6653 & 424.6736 & 434.8958 & 428.9063 & 122.5994 & 1.83418 \\
\hline 2747 & 49.6823 & 427.4175 & 435.277 & 429.2292 & 122.5994 & 1.83359 \\
\hline 2748 & 49.6993 & 415.3604 & 435.7238 & 429.5521 & 122.5994 & 1.833 \\
\hline 2749 & 49.7163 & 412.1775 & 436.2426 & 429.875 & 122.5994 & 1.83242 \\
\hline 2750 & 49.7333 & 433.7946 & 436.8403 & 430.1979 & 122.5994 & 1.83183 \\
\hline 2751 & 49.7503 & 396.0977 & 437.5228 & 430.5208 & 122.5994 & 1.83124 \\
\hline 2752 & 49.7673 & 417.688 & 438.2979 & 430.8438 & 122.5994 & 1.83066 \\
\hline 2753 & 49.7843 & 428.8547 & 439.1098 & 431.1667 & 122.5994 & 1.83007 \\
\hline 2754 & 49.8013 & 438.3615 & 440.0952 & 431.4896 & 122.5994 & 1.82949 \\
\hline 2755 & 49.8183 & 422.5728 & 441.1966 & 431.8125 & 122.5994 & 1.8289 \\
\hline 2756 & 49.8353 & 435.2027 & 442.4209 & 432.1354 & 122.5994 & 1.82832 \\
\hline 2757 & 49.8523 & 423.9114 & 443.7804 & 432.4583 & 122.5994 & 1.82774 \\
\hline 2758 & 49.8693 & 439.4425 & 445.2629 & 432.7813 & 122.5994 & 1.82715 \\
\hline 2759 & 49.8863 & 443.6203 & 446.8689 & 433.1042 & 122.5994 & 1.82657 \\
\hline 2760 & 49.9033 & 442.9109 & 448.6283 & 433.4271 & 122.5994 & 1.82599 \\
\hline 2761 & 49.9203 & 447.7773 & 450.5188 & 433.75 & 122.5994 & 1.82541 \\
\hline 2762 & 49.9373 & 420.8141 & 452.3224 & 433.8607 & 122.5994 & 1.82482 \\
\hline 2763 & 49.9543 & 456.1578 & 454.2437 & 433.9714 & 122.5994 & 1.82424 \\
\hline 2764 & 49.9713 & 457.4123 & 456.2811 & 434.082 & 122.5994 & 1.82366 \\
\hline 2765 & 49.9883 & 443.0419 & 458.4424 & 434.1927 & 122.5994 & 1.82308 \\
\hline 2766 & 50.0053 & 462.1083 & 460.7333 & 434.3034 & 122.5994 & 1.8225 \\
\hline 2767 & 50.0223 & 472.9647 & 463.2691 & 434.4141 & 122.5994 & 1.82192 \\
\hline 2768 & 50.0393 & 461.9096 & 466.1015 & 434.5247 & 122.5994 & 1.82134 \\
\hline 2769 & 50.0563 & 467.5403 & 469.4977 & 434.6354 & 122.5994 & 1.82076 \\
\hline 2770 & 50.0733 & 475.3034 & 473.7641 & 434.7461 & 122.5994 & 1.82019 \\
\hline 2771 & 50.0903 & 491.1072 & 479.5738 & 434.8568 & 122.5994 & 1.81961 \\
\hline 2772 & 50.1073 & 533.2155 & 488.2744 & 434.9674 & 122.5994 & 1.81903 \\
\hline 2773 & 50.1243 & 567.4706 & 502.5505 & 435.0781 & 122.5994 & 1.81845 \\
\hline 2774 & 50.1413 & 625.4344 & 526.8837 & 435.1888 & 122.5994 & 1.81788 \\
\hline 2775 & 50.1583 & 738.1271 & 567.5063 & 435.2995 & 122.5994 & 1.8173 \\
\hline 2776 & 50.1753 & 872.8906 & 630.5978 & 435.4102 & 122.5994 & 1.81672 \\
\hline 2777 & 50.1923 & 1007.874 & 718.6907 & 435.5208 & 122.5994 & 1.81615 \\
\hline 2778 & 50.2093 & 1057.805 & 825.2278 & 435.6315 & 122.5994 & 1.81557 \\
\hline 2779 & 50.2263 & 1003.588 & 927.5662 & 435.7422 & 122.5994 & 1.815 \\
\hline 2780 & 50.2433 & 857.0669 & 984.3763 & 435.8529 & 122.5994 & 1.81442 \\
\hline 2781 & 50.2603 & 698.9908 & 964.1888 & 435.9635 & 122.5994 & 1.81385 \\
\hline 2782 & 50.2773 & 645.1929 & 887.5037 & 436.0742 & 122.5994 & 1.81328 \\
\hline 2783 & 50.2943 & 622.7955 & 801.9343 & 436.1849 & 122.5994 & 1.8127 \\
\hline 2784 & 50.3113 & 669.3566 & 740.2385 & 436.2956 & 122.5994 & 1.81213 \\
\hline 2785 & 50.3283 & 748.2637 & 714.4647 & 436.4063 & 122.5994 & 1.81156 \\
\hline 2786 & 50.3453 & 771.6624 & 720.0743 & 436.2767 & 122.5994 & 1.81099 \\
\hline 2787 & 50.3623 & 691.1732 & 739.0508 & 436.1471 & 122.5994 & 1.81042 \\
\hline 2788 & 50.3793 & 617.4174 & 743.2379 & 436.0176 & 122.5994 & 1.80985 \\
\hline 2789 & 50.3963 & 548.4902 & 714.002 & 435.888 & 122.5994 & 1.80927 \\
\hline 2790 & 50.4133 & 492.5158 & 660.6191 & 435.7585 & 122.5994 & 1.8087 \\
\hline 2791 & 50.4303 & 449.6559 & 603.222 & 435.6289 & 122.5994 & 1.80813 \\
\hline
\end{tabular}




\begin{tabular}{|c|c|c|c|c|c|c|}
\hline 2792 & 50.4473 & 457.7988 & 554.3701 & 435.4993 & 122.5994 & 1.80756 \\
\hline 2793 & 50.4643 & 452.4062 & 518.2515 & 435.3698 & 122.5994 & 1.807 \\
\hline 2794 & 50.4813 & 432.8964 & 494.1371 & 435.2402 & 122.5994 & 1.80643 \\
\hline 2795 & 50.4983 & 399.3668 & 479.005 & 435.1107 & 122.5994 & 1.80586 \\
\hline 2796 & 50.5153 & 427.1958 & 469.6664 & 434.9811 & 122.5994 & 1.80529 \\
\hline 2797 & 50.5323 & 441.2854 & 463.6346 & 434.8516 & 122.5994 & 1.80472 \\
\hline 2798 & 50.5493 & 414.3876 & 459.4127 & 434.722 & 122.5994 & 1.80416 \\
\hline 2799 & 50.5663 & 393.7357 & 456.2822 & 434.5924 & 122.5994 & 1.80359 \\
\hline 2800 & 50.5833 & 422.7257 & 453.7421 & 434.4629 & 122.5994 & 1.80302 \\
\hline 2801 & 50.6003 & 449.351 & 451.7051 & 434.3333 & 122.5994 & 1.80246 \\
\hline 2802 & 50.6173 & 462.3116 & 450.1132 & 434.2038 & 122.5994 & 1.80189 \\
\hline 2803 & 50.6343 & 471.0338 & 448.9752 & 434.0742 & 122.5994 & 1.80133 \\
\hline 2804 & 50.6513 & 483.4153 & 448.3863 & 433.9447 & 122.5994 & 1.80076 \\
\hline 2805 & 50.6683 & 469.4194 & 448.3383 & 433.8151 & 122.5994 & 1.8002 \\
\hline 2806 & 50.6853 & 470.2574 & 448.7927 & 433.6855 & 122.5994 & 1.79963 \\
\hline 2807 & 50.7023 & 451.6548 & 449.5312 & 433.556 & 122.5994 & 1.79907 \\
\hline 2808 & 50.7193 & 477.8577 & 449.9429 & 433.4264 & 122.5994 & 1.79851 \\
\hline 2809 & 50.7363 & 472.9483 & 449.3908 & 433.2969 & 122.5994 & 1.79794 \\
\hline 2810 & 50.7533 & 434.4301 & 447.6027 & 432.9801 & 122.5994 & 1.79738 \\
\hline 2811 & 50.7703 & 434.8472 & 445.4134 & 432.6634 & 122.5994 & 1.79682 \\
\hline 2812 & 50.7873 & 440.8002 & 443.4776 & 432.3467 & 122.5994 & 1.79626 \\
\hline 2813 & 50.8043 & 458.0205 & 442.096 & 432.0299 & 122.5994 & 1.7957 \\
\hline 2814 & 50.8213 & 435.8779 & 441.3177 & 431.7132 & 122.5994 & 1.79514 \\
\hline 2815 & 50.8383 & 430.6377 & 440.9583 & 431.3965 & 122.5994 & 1.79458 \\
\hline 2816 & 50.8553 & 442.3206 & 440.6385 & 431.0798 & 122.5994 & 1.79402 \\
\hline 2817 & 50.8723 & 429.4244 & 439.9336 & 430.763 & 122.5994 & 1.79346 \\
\hline 2818 & 50.8893 & 458.0922 & 438.7543 & 430.4463 & 122.5994 & 1.7929 \\
\hline 2819 & 50.9063 & 439.5149 & 437.379 & 430.1296 & 122.5994 & 1.79234 \\
\hline 2820 & 50.9233 & 421.4857 & 436.0796 & 429.8128 & 122.5994 & 1.79178 \\
\hline 2821 & 50.9403 & 421.6382 & 434.983 & 429.4961 & 122.5994 & 1.79122 \\
\hline 2822 & 50.9573 & 403.3044 & 434.1107 & 429.1794 & 122.5994 & 1.79066 \\
\hline 2823 & 50.9743 & 413.3966 & 433.4285 & 428.8626 & 122.5994 & 1.79011 \\
\hline 2824 & 50.9913 & 429.4806 & 432.8825 & 428.5459 & 122.5994 & 1.78955 \\
\hline 2825 & 51.0083 & 442.1756 & 432.4243 & 428.2292 & 122.5994 & 1.78899 \\
\hline 2826 & 51.0253 & 448.5838 & 432.0192 & 427.9124 & 122.5994 & 1.78844 \\
\hline 2827 & 51.0423 & 425.8997 & 431.6528 & 427.5957 & 122.5994 & 1.78788 \\
\hline 2828 & 51.0593 & 393.7218 & 431.2962 & 427.279 & 122.5994 & 1.78733 \\
\hline 2829 & 51.0763 & 429.3695 & 430.9463 & 426.9622 & 122.5994 & 1.78677 \\
\hline 2830 & 51.0933 & 425.9433 & 430.5951 & 426.6455 & 122.5994 & 1.78622 \\
\hline 2831 & 51.1103 & 473.6506 & 430.2373 & 426.3288 & 122.5994 & 1.78566 \\
\hline 2832 & 51.1273 & 444.331 & 429.8704 & 426.012 & 122.5994 & 1.78511 \\
\hline 2833 & 51.1443 & 433.249 & 429.4946 & 425.6953 & 122.5994 & 1.78456 \\
\hline 2834 & 51.1613 & 436.5834 & 429.0497 & 425.3786 & 122.5994 & 1.784 \\
\hline 2835 & 51.1783 & 412.616 & 428.6674 & 425.0618 & 122.5994 & 1.78345 \\
\hline 2836 & 51.1953 & 404.0756 & 428.2777 & 424.7451 & 122.5994 & 1.7829 \\
\hline 2837 & 51.2123 & 392.746 & 427.8841 & 424.4284 & 122.5994 & 1.78235 \\
\hline 2838 & 51.2293 & 423.6396 & 427.4859 & 424.1117 & 122.5994 & 1.78179 \\
\hline 2839 & 51.2463 & 408.6084 & 427.0829 & 423.7949 & 122.5994 & 1.78124 \\
\hline 2840 & 51.2633 & 417.1647 & 426.6759 & 423.4782 & 122.5994 & 1.78069 \\
\hline
\end{tabular}




\begin{tabular}{|c|c|c|c|c|c|c|}
\hline 2841 & 51.2803 & 432.0321 & 426.2678 & 423.1615 & 122.5994 & 1.78014 \\
\hline 2842 & 51.2973 & 460.6746 & 424.6646 & 422.8447 & 122.5994 & 1.77959 \\
\hline 2843 & 51.3143 & 447.5124 & 424.3069 & 422.528 & 122.5994 & 1.77904 \\
\hline 2844 & 51.3313 & 444.931 & 424.0103 & 422.2113 & 122.5994 & 1.77849 \\
\hline 2845 & 51.3483 & 476.4951 & 423.6785 & 421.8945 & 122.5994 & 1.77794 \\
\hline 2846 & 51.3653 & 440.8089 & 423.3649 & 421.5778 & 122.5994 & 1.7774 \\
\hline 2847 & 51.3823 & 424.3193 & 423.0735 & 421.2611 & 122.5994 & 1.77685 \\
\hline 2848 & 51.3993 & 432.9467 & 422.8164 & 420.9443 & 122.5994 & 1.7763 \\
\hline 2849 & 51.4163 & 447.5219 & 422.584 & 420.6276 & 122.5994 & 1.77575 \\
\hline 2850 & 51.4333 & 420.2097 & 422.3898 & 420.3109 & 122.5994 & 1.77521 \\
\hline 2851 & 51.4503 & 421.6273 & 422.2409 & 419.9941 & 122.5994 & 1.77466 \\
\hline 2852 & 51.4673 & 407.8597 & 422.17 & 419.6774 & 122.5994 & 1.77411 \\
\hline 2853 & 51.4843 & 380.5975 & 422.1341 & 419.3607 & 122.5994 & 1.77357 \\
\hline 2854 & 51.5013 & 389.6663 & 422.1679 & 419.0439 & 122.5994 & 1.77302 \\
\hline 2855 & 51.5183 & 405.1695 & 422.2773 & 418.7272 & 122.5994 & 1.77248 \\
\hline 2856 & 51.5353 & 437.0006 & 422.4724 & 418.4105 & 122.5994 & 1.77193 \\
\hline 2857 & 51.5523 & 440.4342 & 422.749 & 418.0938 & 122.5994 & 1.77139 \\
\hline 2858 & 51.5693 & 428.3257 & 422.9257 & 417.5898 & 122.5994 & 1.77084 \\
\hline 2859 & 51.5863 & 402.9121 & 423.1872 & 417.0859 & 122.5994 & 1.7703 \\
\hline 2860 & 51.6033 & 390.7611 & 423.5075 & 416.582 & 122.5994 & 1.76976 \\
\hline 2861 & 51.6203 & 430.31 & 423.9125 & 416.0781 & 122.5994 & 1.76921 \\
\hline 2862 & 51.6373 & 435.4015 & 424.3637 & 415.5742 & 122.5994 & 1.76867 \\
\hline 2863 & 51.6543 & 434.0624 & 424.8305 & 415.0703 & 122.5994 & 1.76813 \\
\hline 2864 & 51.6713 & 416.3126 & 425.313 & 414.5664 & 122.5994 & 1.76759 \\
\hline 2865 & 51.6883 & 402.4579 & 425.6869 & 414.0625 & 122.5994 & 1.76705 \\
\hline 2866 & 51.7053 & 427.8917 & 425.938 & 413.5586 & 122.5994 & 1.7665 \\
\hline 2867 & 51.7223 & 427.1467 & 426.02 & 413.0547 & 122.5994 & 1.76596 \\
\hline 2868 & 51.7393 & 414.6982 & 425.8939 & 412.5508 & 122.5994 & 1.76542 \\
\hline 2869 & 51.7563 & 420.7457 & 425.5653 & 412.0469 & 122.5994 & 1.76488 \\
\hline 2870 & 51.7733 & 418.428 & 425.0392 & 411.543 & 122.5994 & 1.76434 \\
\hline 2871 & 51.7903 & 392.0648 & 424.3448 & 411.0391 & 122.5994 & 1.7638 \\
\hline 2872 & 51.8073 & 399.4702 & 423.5132 & 410.5352 & 122.5994 & 1.76327 \\
\hline 2873 & 51.8243 & 394.5501 & 422.5902 & 410.0313 & 122.5994 & 1.76273 \\
\hline 2874 & 51.8413 & 415.0279 & 421.7861 & 409.5273 & 122.5994 & 1.76219 \\
\hline 2875 & 51.8583 & 430.6872 & 420.6662 & 409.0234 & 122.5994 & 1.76165 \\
\hline 2876 & 51.8753 & 397.7114 & 419.4848 & 408.5195 & 122.5994 & 1.76111 \\
\hline 2877 & 51.8923 & 424.1043 & 418.2488 & 408.0156 & 122.5994 & 1.76058 \\
\hline 2878 & 51.9093 & 395.6561 & 416.9766 & 407.5117 & 122.5994 & 1.76004 \\
\hline 2879 & 51.9263 & 418.0622 & 415.6981 & 407.0078 & 122.5994 & 1.7595 \\
\hline 2880 & 51.9433 & 412.5913 & 414.4397 & 406.5039 & 122.5994 & 1.75897 \\
\hline 2881 & 51.9603 & 392.6651 & 413.2209 & 406 & 122.5994 & 1.75843 \\
\hline 2882 & 51.9773 & 424.0405 & 413.1608 & 406.4688 & 122.5994 & 1.7579 \\
\hline 2883 & 51.9943 & 376.7149 & 413.0608 & 406.9375 & 122.5994 & 1.75736 \\
\hline 2884 & 52.0113 & 416.8654 & 413.0417 & 407.4063 & 122.5994 & 1.75683 \\
\hline 2885 & 52.0283 & 432.9598 & 413.1052 & 407.875 & 122.5994 & 1.75629 \\
\hline 2886 & 52.0453 & 366.8884 & 413.2495 & 408.3438 & 122.5994 & 1.75576 \\
\hline 2887 & 52.0623 & 395.1039 & 413.4709 & 408.8125 & 122.5994 & 1.75523 \\
\hline 2888 & 52.0793 & 420.6153 & 413.7693 & 409.2813 & 122.5994 & 1.75469 \\
\hline 2889 & 52.0963 & 395.3559 & 414.1243 & 409.75 & 122.5994 & 1.75416 \\
\hline
\end{tabular}




\begin{tabular}{|c|c|c|c|c|c|c|}
\hline 2890 & 52.1133 & 412.8869 & 414.5341 & 410.2188 & 122.5994 & 1.75363 \\
\hline 2891 & 52.1303 & 391.0786 & 414.991 & 410.6875 & 122.5994 & 1.7531 \\
\hline 2892 & 52.1473 & 379.945 & 415.4892 & 411.1563 & 122.5994 & 1.75257 \\
\hline 2893 & 52.1643 & 419.8343 & 416.0268 & 411.625 & 122.5994 & 1.75204 \\
\hline 2894 & 52.1813 & 411.0312 & 416.6055 & 412.0938 & 122.5994 & 1.7515 \\
\hline 2895 & 52.1983 & 428.1816 & 417.2299 & 412.5625 & 122.5994 & 1.75097 \\
\hline 2896 & 52.2153 & 417.4002 & 417.9085 & 413.0313 & 122.5994 & 1.75044 \\
\hline 2897 & 52.2323 & 416.4114 & 418.6358 & 413.5 & 122.5994 & 1.74991 \\
\hline 2898 & 52.2493 & 408.0944 & 419.4326 & 413.9688 & 122.5994 & 1.74938 \\
\hline 2899 & 52.2663 & 413.7806 & 420.2939 & 414.4375 & 122.5994 & 1.74886 \\
\hline 2900 & 52.2833 & 419.8055 & 421.2198 & 414.9063 & 122.5994 & 1.74833 \\
\hline 2901 & 52.3003 & 421.8698 & 422.2078 & 415.375 & 122.5994 & 1.7478 \\
\hline 2902 & 52.3173 & 418.0254 & 423.2528 & 415.8438 & 122.5994 & 1.74727 \\
\hline 2903 & 52.3343 & 411.8042 & 424.3458 & 416.3125 & 122.5994 & 1.74674 \\
\hline 2904 & 52.3513 & 417.9302 & 425.4715 & 416.7813 & 122.5994 & 1.74622 \\
\hline 2905 & 52.3683 & 423.9432 & 426.6331 & 417.25 & 122.5994 & 1.74569 \\
\hline 2906 & 52.3853 & 449.3659 & 427.7495 & 417.7188 & 122.5994 & 1.74516 \\
\hline 2907 & 52.4023 & 425.6083 & 428.8108 & 418.1875 & 122.5994 & 1.74464 \\
\hline 2908 & 52.4193 & 405.8865 & 429.7838 & 418.6563 & 122.5994 & 1.74411 \\
\hline 2909 & 52.4363 & 394.9084 & 430.646 & 419.125 & 122.5994 & 1.74359 \\
\hline 2910 & 52.4533 & 419.4075 & 431.3919 & 419.5938 & 122.5994 & 1.74306 \\
\hline 2911 & 52.4703 & 433.2973 & 432.0341 & 420.0625 & 122.5994 & 1.74254 \\
\hline 2912 & 52.4873 & 423.323 & 432.6899 & 420.5313 & 122.5994 & 1.74201 \\
\hline 2913 & 52.5043 & 396.5558 & 433.2168 & 421 & 122.5994 & 1.74149 \\
\hline 2914 & 52.5213 & 411.3319 & 433.6958 & 421.4688 & 122.5994 & 1.74096 \\
\hline 2915 & 52.5383 & 415.8462 & 434.156 & 421.9375 & 122.5994 & 1.74044 \\
\hline 2916 & 52.5553 & 414.9805 & 434.6071 & 422.4063 & 122.5994 & 1.73992 \\
\hline 2917 & 52.5723 & 440.8944 & 435.0604 & 422.875 & 122.5994 & 1.73939 \\
\hline 2918 & 52.5893 & 442.9574 & 435.5346 & 423.3438 & 122.5994 & 1.73887 \\
\hline 2919 & 52.6063 & 421.4733 & 436.0577 & 423.8125 & 122.5994 & 1.73835 \\
\hline 2920 & 52.6233 & 446.4663 & 436.8189 & 424.2813 & 122.5994 & 1.73783 \\
\hline 2921 & 52.6403 & 415.8727 & 437.5659 & 424.75 & 122.5994 & 1.73731 \\
\hline 2922 & 52.6573 & 421.9519 & 438.4547 & 425.2188 & 122.5994 & 1.73679 \\
\hline 2923 & 52.6743 & 446.1523 & 439.5287 & 425.6875 & 122.5994 & 1.73627 \\
\hline 2924 & 52.6913 & 457.1013 & 440.8102 & 426.1563 & 122.5994 & 1.73575 \\
\hline 2925 & 52.7083 & 438.5934 & 442.3148 & 426.625 & 122.5994 & 1.73523 \\
\hline 2926 & 52.7253 & 460.5551 & 444.0484 & 427.0938 & 122.5994 & 1.73471 \\
\hline 2927 & 52.7423 & 422.1313 & 446.052 & 427.5625 & 122.5994 & 1.73419 \\
\hline 2928 & 52.7593 & 432.3283 & 448.286 & 428.0313 & 122.5994 & 1.73367 \\
\hline 2929 & 52.7763 & 462.3001 & 450.6758 & 428.5 & 122.5994 & 1.73315 \\
\hline 2930 & 52.7933 & 416.7445 & 453.1182 & 428.8125 & 122.5994 & 1.73263 \\
\hline 2931 & 52.8103 & 439.3776 & 455.7269 & 429.125 & 122.5994 & 1.73212 \\
\hline 2932 & 52.8273 & 406.3437 & 458.4995 & 429.4375 & 122.5994 & 1.7316 \\
\hline 2933 & 52.8443 & 441.7828 & 461.4002 & 429.75 & 122.5994 & 1.73108 \\
\hline 2934 & 52.8613 & 443.2432 & 464.3666 & 430.0625 & 122.5994 & 1.73056 \\
\hline 2935 & 52.8783 & 400.5865 & 467.2951 & 430.375 & 122.5994 & 1.73005 \\
\hline 2936 & 52.8953 & 451.6345 & 470.1174 & 430.6875 & 122.5994 & 1.72953 \\
\hline 2937 & 52.9123 & 417.528 & 472.4457 & 431 & 122.5994 & 1.72902 \\
\hline 2938 & 52.9293 & 427.6339 & 474.3372 & 431.3125 & 122.5994 & 1.7285 \\
\hline
\end{tabular}




\begin{tabular}{|c|c|c|c|c|c|c|}
\hline 2939 & 52.9463 & 440.9577 & 475.5991 & 431.625 & 122.5994 & 1.72799 \\
\hline 2940 & 52.9633 & 435.1862 & 476.1828 & 431.9375 & 122.5994 & 1.72747 \\
\hline 2941 & 52.9803 & 423.6103 & 476.1403 & 432.25 & 122.5994 & 1.72696 \\
\hline 2942 & 52.9973 & 445.2693 & 475.5801 & 432.5625 & 122.5994 & 1.72644 \\
\hline 2943 & 53.0143 & 443.9005 & 474.6769 & 432.875 & 122.5994 & 1.72593 \\
\hline 2944 & 53.0313 & 438.263 & 473.435 & 433.1875 & 122.5994 & 1.72542 \\
\hline 2945 & 53.0483 & 428.4358 & 471.9358 & 433.5 & 122.5994 & 1.7249 \\
\hline 2946 & 53.0653 & 424.737 & 470.2649 & 433.8125 & 122.5994 & 1.72439 \\
\hline 2947 & 53.0823 & 442.4977 & 468.4279 & 434.125 & 122.5994 & 1.72388 \\
\hline 2948 & 53.0993 & 457.9161 & 466.4011 & 434.4375 & 122.5994 & 1.72337 \\
\hline 2949 & 53.1163 & 457.9161 & 464.2557 & 434.75 & 122.5994 & 1.72286 \\
\hline 2950 & 53.1333 & 456.093 & 462.0657 & 435.0625 & 122.5994 & 1.72234 \\
\hline 2951 & 53.1503 & 437.8308 & 459.9133 & 435.375 & 122.5994 & 1.72183 \\
\hline 2952 & 53.1673 & 445.353 & 457.8992 & 435.6875 & 122.5994 & 1.72132 \\
\hline 2953 & 53.1843 & 434.2636 & 456.0323 & 436 & 122.5994 & 1.72081 \\
\hline 2954 & 53.2013 & 422.0981 & 454.3956 & 436.3333 & 122.5994 & 1.7203 \\
\hline 2955 & 53.2183 & 460.1012 & 452.992 & 436.6667 & 122.5994 & 1.71979 \\
\hline 2956 & 53.2353 & 445.6576 & 451.8285 & 437 & 122.5994 & 1.71928 \\
\hline 2957 & 53.2523 & 447.2327 & 450.9096 & 437.3333 & 122.5994 & 1.71878 \\
\hline 2958 & 53.2693 & 467.5935 & 450.2261 & 437.6667 & 122.5994 & 1.71827 \\
\hline 2959 & 53.2863 & 448.9631 & 449.8027 & 438 & 122.5994 & 1.71776 \\
\hline 2960 & 53.3033 & 450.8679 & 449.6113 & 438.3333 & 122.5994 & 1.71725 \\
\hline 2961 & 53.3203 & 431.9686 & 449.6381 & 438.6667 & 122.5994 & 1.71674 \\
\hline 2962 & 53.3373 & 429.0996 & 449.8878 & 439 & 122.5994 & 1.71624 \\
\hline 2963 & 53.3543 & 444.8818 & 450.3551 & 439.3333 & 122.5994 & 1.71573 \\
\hline 2964 & 53.3713 & 446.3156 & 451.0352 & 439.6667 & 122.5994 & 1.71522 \\
\hline 2965 & 53.3883 & 465.0492 & 451.9245 & 440 & 122.5994 & 1.71472 \\
\hline 2966 & 53.4053 & 450.5149 & 453.0295 & 440.3333 & 122.5994 & 1.71421 \\
\hline 2967 & 53.4223 & 462.6164 & 454.3225 & 440.6667 & 122.5994 & 1.71371 \\
\hline 2968 & 53.4393 & 448.6088 & 455.8056 & 441 & 122.5994 & 1.7132 \\
\hline 2969 & 53.4563 & 452.5652 & 457.4652 & 441.3333 & 122.5994 & 1.7127 \\
\hline 2970 & 53.4733 & 424.1598 & 459.3212 & 441.6667 & 122.5994 & 1.71219 \\
\hline 2971 & 53.4903 & 473.9335 & 461.2669 & 442 & 122.5994 & 1.71169 \\
\hline 2972 & 53.5073 & 432.2857 & 463.308 & 442.3333 & 122.5994 & 1.71118 \\
\hline 2973 & 53.5243 & 440.3878 & 465.4011 & 442.6667 & 122.5994 & 1.71068 \\
\hline 2974 & 53.5413 & 445.0326 & 467.5045 & 443 & 122.5994 & 1.71018 \\
\hline 2975 & 53.5583 & 457.7814 & 469.5172 & 443.3333 & 122.5994 & 1.70968 \\
\hline 2976 & 53.5753 & 440.9121 & 471.486 & 443.6667 & 122.5994 & 1.70917 \\
\hline 2977 & 53.5923 & 437.5565 & 473.3228 & 444 & 122.5994 & 1.70867 \\
\hline 2978 & 53.6093 & 456.1029 & 475.1679 & 444.4583 & 122.5994 & 1.70817 \\
\hline 2979 & 53.6263 & 426.5303 & 476.832 & 444.9167 & 122.5994 & 1.70767 \\
\hline 2980 & 53.6433 & 454.1648 & 478.3802 & 445.375 & 122.5994 & 1.70717 \\
\hline 2981 & 53.6603 & 455.9071 & 479.8532 & 445.8333 & 122.5994 & 1.70667 \\
\hline 2982 & 53.6773 & 438.7275 & 481.2835 & 446.2917 & 122.5994 & 1.70617 \\
\hline 2983 & 53.6943 & 433.2434 & 482.6828 & 446.75 & 122.5994 & 1.70567 \\
\hline 2984 & 53.7113 & 479.6739 & 484.0336 & 447.2083 & 122.5994 & 1.70517 \\
\hline 2985 & 53.7283 & 468.2603 & 485.2635 & 447.6667 & 122.5994 & 1.70467 \\
\hline 2986 & 53.7453 & 424.3193 & 486.3781 & 448.125 & 122.5994 & 1.70417 \\
\hline 2987 & 53.7623 & 433.8005 & 487.334 & 448.5833 & 122.5994 & 1.70367 \\
\hline
\end{tabular}




\begin{tabular}{|c|c|c|c|c|c|c|}
\hline 2988 & 53.7793 & 464.5305 & 488.0257 & 449.0417 & 122.5994 & 1.70317 \\
\hline 2989 & 53.7963 & 459.8323 & 488.4957 & 449.5 & 122.5994 & 1.70267 \\
\hline 2990 & 53.8133 & 447.2603 & 488.794 & 449.9583 & 122.5994 & 1.70217 \\
\hline 2991 & 53.8303 & 486.9526 & 488.9424 & 450.4167 & 122.5994 & 1.70168 \\
\hline 2992 & 53.8473 & 487.792 & 489.0029 & 450.875 & 122.5994 & 1.70118 \\
\hline 2993 & 53.8643 & 462.2057 & 489.0052 & 451.3333 & 122.5994 & 1.70068 \\
\hline 2994 & 53.8813 & 460.6029 & 488.9512 & 451.7917 & 122.5994 & 1.70019 \\
\hline 2995 & 53.8983 & 472.5557 & 488.9682 & 452.25 & 122.5994 & 1.69969 \\
\hline 2996 & 53.9153 & 470.0255 & 488.7778 & 452.7083 & 122.5994 & 1.69919 \\
\hline 2997 & 53.9323 & 450.7918 & 488.4948 & 453.1667 & 122.5994 & 1.6987 \\
\hline 2998 & 53.9493 & 466.3892 & 488.1217 & 453.625 & 122.5994 & 1.6982 \\
\hline 2999 & 53.9663 & 455.8328 & 487.6815 & 454.0833 & 122.5994 & 1.69771 \\
\hline 3000 & 53.9833 & 440.9577 & 487.1815 & 454.5417 & 122.5994 & 1.69721 \\
\hline 3001 & 54.0003 & 469.5149 & 486.6509 & 455 & 122.5994 & 1.69672 \\
\hline 3002 & 54.0173 & 487.7509 & 485.9764 & 455.3233 & 122.5994 & 1.69623 \\
\hline 3003 & 54.0343 & 473.6332 & 485.3125 & 455.6466 & 122.5994 & 1.69573 \\
\hline 3004 & 54.0513 & 469.2783 & 484.6794 & 455.97 & 122.5994 & 1.69524 \\
\hline 3005 & 54.0683 & 501.9199 & 484.0958 & 456.2933 & 122.5994 & 1.69475 \\
\hline 3006 & 54.0853 & 470.916 & 483.6909 & 456.6166 & 122.5994 & 1.69425 \\
\hline 3007 & 54.1023 & 459.6405 & 483.2638 & 456.9399 & 122.5994 & 1.69376 \\
\hline 3008 & 54.1193 & 469.6513 & 482.9095 & 457.2633 & 122.5994 & 1.69327 \\
\hline 3009 & 54.1363 & 483.4763 & 482.6148 & 457.5866 & 122.5994 & 1.69278 \\
\hline 3010 & 54.1533 & 485.0892 & 482.3587 & 457.9099 & 122.5994 & 1.69229 \\
\hline 3011 & 54.1703 & 497.6339 & 482.1171 & 458.2332 & 122.5994 & 1.6918 \\
\hline 3012 & 54.1873 & 453.9871 & 481.8823 & 458.5566 & 122.5994 & 1.69131 \\
\hline 3013 & 54.2043 & 458.2099 & 481.6096 & 458.8799 & 122.5994 & 1.69082 \\
\hline 3014 & 54.2213 & 441.1278 & 481.1944 & 459.2032 & 122.5994 & 1.69033 \\
\hline 3015 & 54.2383 & 433.323 & 480.9898 & 459.5265 & 122.5994 & 1.68984 \\
\hline 3016 & 54.2553 & 462.1633 & 480.6238 & 459.8499 & 122.5994 & 1.68935 \\
\hline 3017 & 54.2723 & 474.2667 & 480.237 & 460.1732 & 122.5994 & 1.68886 \\
\hline 3018 & 54.2893 & 483.3159 & 479.8429 & 460.4965 & 122.5994 & 1.68837 \\
\hline 3019 & 54.3063 & 451.6022 & 479.4642 & 460.8198 & 122.5994 & 1.68788 \\
\hline 3020 & 54.3233 & 499.609 & 479.1171 & 461.1431 & 122.5994 & 1.68739 \\
\hline 3021 & 54.3403 & 467.2453 & 478.8217 & 461.4665 & 122.5994 & 1.6869 \\
\hline 3022 & 54.3573 & 488.4564 & 478.5756 & 461.7898 & 122.5994 & 1.68642 \\
\hline 3023 & 54.3743 & 462.0162 & 478.5174 & 462.1131 & 122.5994 & 1.68593 \\
\hline 3024 & 54.3913 & 454.8854 & 478.4091 & 462.4364 & 122.5994 & 1.68544 \\
\hline 3025 & 54.4083 & 478.432 & 478.3734 & 462.7598 & 122.5994 & 1.68496 \\
\hline 3026 & 54.4253 & 463.8195 & 478.2532 & 462.909 & 122.5994 & 1.68447 \\
\hline 3027 & 54.4423 & 476.2973 & 478.233 & 463.0583 & 122.5994 & 1.68398 \\
\hline 3028 & 54.4593 & 449.3969 & 478.3093 & 463.2076 & 122.5994 & 1.6835 \\
\hline 3029 & 54.4763 & 460.8464 & 478.4888 & 463.3569 & 122.5994 & 1.68301 \\
\hline 3030 & 54.4933 & 520.4465 & 478.8319 & 463.5061 & 122.5994 & 1.68253 \\
\hline 3031 & 54.5103 & 498.374 & 479.1842 & 463.6554 & 122.5994 & 1.68204 \\
\hline 3032 & 54.5273 & 480.1894 & 479.5848 & 463.8047 & 122.5994 & 1.68156 \\
\hline 3033 & 54.5443 & 480.2406 & 480.0208 & 463.9539 & 122.5994 & 1.68108 \\
\hline 3034 & 54.5613 & 516.3062 & 480.4142 & 464.1032 & 122.5994 & 1.68059 \\
\hline 3035 & 54.5783 & 480.6265 & 480.7659 & 464.2525 & 122.5994 & 1.68011 \\
\hline 3036 & 54.5953 & 448.2225 & 481.0663 & 464.4018 & 122.5994 & 1.67963 \\
\hline
\end{tabular}




\begin{tabular}{|c|c|c|c|c|c|c|}
\hline 3037 & 54.6123 & 453.4721 & 481.3183 & 464.551 & 122.5994 & 1.67914 \\
\hline 3038 & 54.6293 & 472.4537 & 481.5522 & 464.7003 & 122.5994 & 1.67866 \\
\hline 3039 & 54.6463 & 475.1944 & 481.7846 & 464.8496 & 122.5994 & 1.67818 \\
\hline 3040 & 54.6633 & 480.3029 & 482.0461 & 464.9988 & 122.5994 & 1.6777 \\
\hline 3041 & 54.6803 & 488.4751 & 482.3542 & 465.1481 & 122.5994 & 1.67722 \\
\hline 3042 & 54.6973 & 486.4626 & 482.6993 & 465.2974 & 122.5994 & 1.67674 \\
\hline 3043 & 54.7143 & 478.1308 & 483.0317 & 465.4467 & 122.5994 & 1.67625 \\
\hline 3044 & 54.7313 & 468.7923 & 483.5278 & 465.5959 & 122.5994 & 1.67577 \\
\hline 3045 & 54.7483 & 470.8157 & 484.1021 & 465.7452 & 122.5994 & 1.67529 \\
\hline 3046 & 54.7653 & 484.4043 & 484.7762 & 465.8945 & 122.5994 & 1.67481 \\
\hline 3047 & 54.7823 & 490.4071 & 485.5846 & 466.0437 & 122.5994 & 1.67433 \\
\hline 3048 & 54.7993 & 488.4751 & 487.1741 & 466.193 & 122.5994 & 1.67386 \\
\hline 3049 & 54.8163 & 493.5276 & 488.4487 & 466.3423 & 122.5994 & 1.67338 \\
\hline 3050 & 54.8333 & 486.6911 & 489.932 & 466.296 & 122.5994 & 1.6729 \\
\hline 3051 & 54.8503 & 501.8518 & 492.083 & 466.2498 & 122.5994 & 1.67242 \\
\hline 3052 & 54.8673 & 521.1328 & 495.4401 & 466.2036 & 122.5994 & 1.67194 \\
\hline 3053 & 54.8843 & 565.3649 & 501.0981 & 466.1573 & 122.5994 & 1.67146 \\
\hline 3054 & 54.9013 & 616.1613 & 510.3253 & 466.1111 & 122.5994 & 1.67099 \\
\hline 3055 & 54.9183 & 633.6693 & 524.7068 & 466.0648 & 122.5994 & 1.67051 \\
\hline 3056 & 54.9353 & 664.9707 & 544.9611 & 466.0186 & 122.5994 & 1.67003 \\
\hline 3057 & 54.9523 & 637.6429 & 570.2094 & 465.9723 & 122.5994 & 1.66956 \\
\hline 3058 & 54.9693 & 594.4247 & 595.4285 & 465.9261 & 122.5994 & 1.66908 \\
\hline 3059 & 54.9863 & 616.9422 & 611.519 & 465.8798 & 122.5994 & 1.6686 \\
\hline 3060 & 55.0033 & 557.888 & 609.8128 & 465.8336 & 122.5994 & 1.66813 \\
\hline 3061 & 55.0203 & 535.0448 & 593.0334 & 465.7874 & 122.5994 & 1.66765 \\
\hline 3062 & 55.0373 & 557.87 & 572.2053 & 465.7411 & 122.5994 & 1.66718 \\
\hline 3063 & 55.0543 & 600.0135 & 555.767 & 465.6949 & 122.5994 & 1.6667 \\
\hline 3064 & 55.0713 & 570.0887 & 547.7393 & 465.6486 & 122.5994 & 1.66623 \\
\hline 3065 & 55.0883 & 579.8209 & 548.6064 & 465.6024 & 122.5994 & 1.66576 \\
\hline 3066 & 55.1053 & 585.3299 & 556.2396 & 465.5561 & 122.5994 & 1.66528 \\
\hline 3067 & 55.1223 & 539.7881 & 566.1591 & 465.5099 & 122.5994 & 1.66481 \\
\hline 3068 & 55.1393 & 533.6028 & 571.9712 & 465.4636 & 122.5994 & 1.66434 \\
\hline 3069 & 55.1563 & 561.0811 & 569.6147 & 465.4174 & 122.5994 & 1.66386 \\
\hline 3070 & 55.1733 & 515.8521 & 561.1504 & 465.3712 & 122.5994 & 1.66339 \\
\hline 3071 & 55.1903 & 531.1937 & 551.4974 & 465.3249 & 122.5994 & 1.66292 \\
\hline 3072 & 55.2073 & 493.2438 & 543.5305 & 465.2787 & 122.5994 & 1.66245 \\
\hline 3073 & 55.2243 & 550.495 & 538.1033 & 465.2324 & 122.5994 & 1.66197 \\
\hline 3074 & 55.2413 & 530.2887 & 534.9044 & 465.1862 & 122.5994 & 1.6615 \\
\hline 3075 & 55.2583 & 507.4756 & 533.2023 & 465.1399 & 122.5994 & 1.66103 \\
\hline 3076 & 55.2753 & 515.3587 & 532.1792 & 465.0937 & 122.5994 & 1.66056 \\
\hline 3077 & 55.2923 & 520.3193 & 531.481 & 465.0474 & 122.5994 & 1.66009 \\
\hline 3078 & 55.3093 & 540.1057 & 531.1077 & 465.0012 & 122.5994 & 1.65962 \\
\hline 3079 & 55.3263 & 532.1034 & 531.2391 & 464.955 & 122.5994 & 1.65915 \\
\hline 3080 & 55.3433 & 557.2296 & 532.4653 & 464.9087 & 122.5994 & 1.65868 \\
\hline 3081 & 55.3603 & 600.1924 & 535.4694 & 464.8625 & 122.5994 & 1.65821 \\
\hline 3082 & 55.3773 & 586.197 & 540.9592 & 464.8162 & 122.5994 & 1.65774 \\
\hline 3083 & 55.3943 & 559.7626 & 549.0291 & 464.77 & 122.5994 & 1.65728 \\
\hline 3084 & 55.4113 & 569.5732 & 558.8973 & 464.7237 & 122.5994 & 1.65681 \\
\hline 3085 & 55.4283 & 553.6851 & 567.5575 & 464.6775 & 122.5994 & 1.65634 \\
\hline
\end{tabular}




\begin{tabular}{|c|c|c|c|c|c|c|}
\hline 3086 & 55.4453 & 533.6077 & 570.3809 & 464.6312 & 122.5994 & 1.65587 \\
\hline 3087 & 55.4623 & 516.1592 & 564.4246 & 464.585 & 122.5994 & 1.65541 \\
\hline 3088 & 55.4793 & 526.4113 & 552.635 & 464.5388 & 122.5994 & 1.65494 \\
\hline 3089 & 55.4963 & 511.0003 & 540.2368 & 464.4925 & 122.5994 & 1.65447 \\
\hline 3090 & 55.5133 & 486.4295 & 530.6743 & 464.4463 & 122.5994 & 1.65401 \\
\hline 3091 & 55.5303 & 512.5313 & 525.3506 & 464.4 & 122.5994 & 1.65354 \\
\hline 3092 & 55.5473 & 562.1848 & 524.2124 & 464.3538 & 122.5994 & 1.65307 \\
\hline 3093 & 55.5643 & 548.5413 & 526.0366 & 464.3075 & 122.5994 & 1.65261 \\
\hline 3094 & 55.5813 & 519.7901 & 528.511 & 464.2613 & 122.5994 & 1.65214 \\
\hline 3095 & 55.5983 & 530.4233 & 528.6643 & 464.215 & 122.5994 & 1.65168 \\
\hline 3096 & 55.6153 & 524.5829 & 525.1978 & 464.1688 & 122.5994 & 1.65121 \\
\hline 3097 & 55.6323 & 518.1546 & 519.6669 & 464.1226 & 122.5994 & 1.65075 \\
\hline 3098 & 55.6493 & 540.3997 & 514.2067 & 463.8808 & 122.5994 & 1.65028 \\
\hline 3099 & 55.6663 & 513.2585 & 510.2625 & 463.639 & 122.5994 & 1.64982 \\
\hline 3100 & 55.6833 & 486.7906 & 508.2717 & 463.3973 & 122.5994 & 1.64936 \\
\hline 3101 & 55.7003 & 474.7714 & 508.1436 & 463.1555 & 122.5994 & 1.64889 \\
\hline 3102 & 55.7173 & 486.8016 & 509.5946 & 462.9138 & 122.5994 & 1.64843 \\
\hline 3103 & 55.7343 & 512.8662 & 512.3529 & 462.672 & 122.5994 & 1.64797 \\
\hline 3104 & 55.7513 & 526.9177 & 516.1298 & 462.4302 & 122.5994 & 1.64751 \\
\hline 3105 & 55.7683 & 507.5241 & 520.8939 & 462.1885 & 122.5994 & 1.64704 \\
\hline 3106 & 55.7853 & 512.6435 & 526.5966 & 461.9467 & 122.5994 & 1.64658 \\
\hline 3107 & 55.8023 & 504.1342 & 532.9149 & 461.705 & 122.5994 & 1.64612 \\
\hline 3108 & 55.8193 & 528.8169 & 539.7849 & 461.4632 & 122.5994 & 1.64566 \\
\hline 3109 & 55.8363 & 509.8048 & 546.9573 & 461.2214 & 122.5994 & 1.6452 \\
\hline 3110 & 55.8533 & 490.2508 & 554.106 & 460.9797 & 122.5994 & 1.64474 \\
\hline 3111 & 55.8703 & 529.8666 & 560.8173 & 460.7379 & 122.5994 & 1.64428 \\
\hline 3112 & 55.8873 & 512.1447 & 566.6302 & 460.4962 & 122.5994 & 1.64382 \\
\hline 3113 & 55.9043 & 532.6146 & 571.1591 & 460.2544 & 122.5994 & 1.64336 \\
\hline 3114 & 55.9213 & 520.1833 & 574.1217 & 460.0126 & 122.5994 & 1.6429 \\
\hline 3115 & 55.9383 & 566.4734 & 575.5056 & 459.7709 & 122.5994 & 1.64244 \\
\hline 3116 & 55.9553 & 542.303 & 575.3428 & 459.5291 & 122.5994 & 1.64198 \\
\hline 3117 & 55.9723 & 518.3875 & 573.9698 & 459.2874 & 122.5994 & 1.64152 \\
\hline 3118 & 55.9893 & 491.1653 & 571.6452 & 459.0456 & 122.5994 & 1.64107 \\
\hline 3119 & 56.0063 & 525.7782 & 568.5833 & 458.8038 & 122.5994 & 1.64061 \\
\hline 3120 & 56.0233 & 492.9057 & 564.864 & 458.5621 & 122.5994 & 1.64015 \\
\hline 3121 & 56.0403 & 493.9592 & 560.5897 & 458.3203 & 122.5994 & 1.63969 \\
\hline 3122 & 56.0573 & 503.8891 & 555.5401 & 457.8616 & 122.5994 & 1.63924 \\
\hline 3123 & 56.0743 & 506.7695 & 549.9871 & 457.4028 & 122.5994 & 1.63878 \\
\hline 3124 & 56.0913 & 467.3607 & 544.0421 & 456.9441 & 122.5994 & 1.63832 \\
\hline 3125 & 56.1083 & 473.7964 & 538.0225 & 456.4854 & 122.5994 & 1.63787 \\
\hline 3126 & 56.1253 & 504.738 & 531.9408 & 456.0266 & 122.5994 & 1.63741 \\
\hline 3127 & 56.1423 & 552.0687 & 526.0889 & 455.5679 & 122.5994 & 1.63696 \\
\hline 3128 & 56.1593 & 488.595 & 520.4708 & 455.1091 & 122.5994 & 1.6365 \\
\hline 3129 & 56.1763 & 453.7364 & 515.4717 & 454.6504 & 122.5994 & 1.63605 \\
\hline 3130 & 56.1933 & 435.3186 & 511.0115 & 454.1917 & 122.5994 & 1.63559 \\
\hline 3131 & 56.2103 & 471.5198 & 507.0962 & 453.7329 & 122.5994 & 1.63514 \\
\hline 3132 & 56.2273 & 498.9992 & 503.7103 & 453.2742 & 122.5994 & 1.63468 \\
\hline 3133 & 56.2443 & 505.2125 & 500.8132 & 452.8154 & 122.5994 & 1.63423 \\
\hline 3134 & 56.2613 & 512.6881 & 498.3563 & 452.3567 & 122.5994 & 1.63378 \\
\hline
\end{tabular}




\begin{tabular}{|c|c|c|c|c|c|c|}
\hline 3135 & 56.2783 & 505.5416 & 496.3407 & 451.8979 & 122.5994 & 1.63332 \\
\hline 3136 & 56.2953 & 502.9726 & 494.5276 & 451.4392 & 122.5994 & 1.63287 \\
\hline 3137 & 56.3123 & 535.156 & 492.8397 & 450.9805 & 122.5994 & 1.63242 \\
\hline 3138 & 56.3293 & 536.2167 & 491.3525 & 450.5217 & 122.5994 & 1.63196 \\
\hline 3139 & 56.3463 & 517.7163 & 489.9433 & 450.063 & 122.5994 & 1.63151 \\
\hline 3140 & 56.3633 & 500.4513 & 488.3099 & 449.6042 & 122.5994 & 1.63106 \\
\hline 3141 & 56.3803 & 491.4094 & 487.0514 & 449.1455 & 122.5994 & 1.63061 \\
\hline 3142 & 56.3973 & 487.0549 & 485.812 & 448.6868 & 122.5994 & 1.63016 \\
\hline 3143 & 56.4143 & 487.5559 & 484.6338 & 448.228 & 122.5994 & 1.62971 \\
\hline 3144 & 56.4313 & 512.6599 & 483.4958 & 447.7693 & 122.5994 & 1.62926 \\
\hline 3145 & 56.4483 & 492.5395 & 482.5119 & 447.3105 & 122.5994 & 1.62881 \\
\hline 3146 & 56.4653 & 499.059 & 481.2871 & 446.6309 & 122.5994 & 1.62836 \\
\hline 3147 & 56.4823 & 486.1945 & 480.0879 & 445.9513 & 122.5994 & 1.62791 \\
\hline 3148 & 56.4993 & 478.3043 & 478.9022 & 445.2717 & 122.5994 & 1.62746 \\
\hline 3149 & 56.5163 & 492.6384 & 477.7074 & 444.5921 & 122.5994 & 1.62701 \\
\hline 3150 & 56.5333 & 483.8991 & 476.4734 & 443.9125 & 122.5994 & 1.62656 \\
\hline 3151 & 56.5503 & 472.9041 & 475.0519 & 443.2329 & 122.5994 & 1.62611 \\
\hline 3152 & 56.5673 & 446.9993 & 473.7006 & 442.5533 & 122.5994 & 1.62566 \\
\hline 3153 & 56.5843 & 444.5086 & 472.1089 & 441.8737 & 122.5994 & 1.62521 \\
\hline 3154 & 56.6013 & 459.4953 & 470.5397 & 441.1941 & 122.5994 & 1.62477 \\
\hline 3155 & 56.6183 & 451.16 & 468.8439 & 440.5145 & 122.5994 & 1.62432 \\
\hline 3156 & 56.6353 & 457.0309 & 467.2068 & 439.8349 & 122.5994 & 1.62387 \\
\hline 3157 & 56.6523 & 457.9161 & 465.6371 & 439.1553 & 122.5994 & 1.62342 \\
\hline 3158 & 56.6693 & 470.007 & 464.1754 & 438.4757 & 122.5994 & 1.62298 \\
\hline 3159 & 56.6863 & 479.1149 & 462.8412 & 437.7961 & 122.5994 & 1.62253 \\
\hline 3160 & 56.7033 & 466.9077 & 461.5941 & 437.1165 & 122.5994 & 1.62209 \\
\hline 3161 & 56.7203 & 449.6481 & 460.6175 & 436.4368 & 122.5994 & 1.62164 \\
\hline 3162 & 56.7373 & 436.1822 & 459.6538 & 435.7572 & 122.5994 & 1.62119 \\
\hline 3163 & 56.7543 & 386.8134 & 458.8027 & 435.0776 & 122.5994 & 1.62075 \\
\hline 3164 & 56.7713 & 462.5534 & 458.0435 & 434.398 & 122.5994 & 1.6203 \\
\hline 3165 & 56.7883 & 466.5152 & 457.4339 & 433.7184 & 122.5994 & 1.61986 \\
\hline 3166 & 56.8053 & 463.9863 & 456.9667 & 433.0388 & 122.5994 & 1.61941 \\
\hline 3167 & 56.8223 & 440.0506 & 456.585 & 432.3592 & 122.5994 & 1.61897 \\
\hline 3168 & 56.8393 & 434.3836 & 456.3275 & 431.6796 & 122.5994 & 1.61853 \\
\hline 3169 & 56.8563 & 440.515 & 456.1306 & 431 & 122.5994 & 1.61808 \\
\hline 3170 & 56.8733 & 419.942 & 456.5396 & 430.9167 & 122.5994 & 1.61764 \\
\hline 3171 & 56.8903 & 429.1668 & 456.9271 & 430.8333 & 122.5994 & 1.6172 \\
\hline 3172 & 56.9073 & 420.2241 & 457.1742 & 430.75 & 122.5994 & 1.61675 \\
\hline 3173 & 56.9243 & 475.4853 & 457.2542 & 430.6667 & 122.5994 & 1.61631 \\
\hline 3174 & 56.9413 & 430.1636 & 457.1427 & 430.5833 & 122.5994 & 1.61587 \\
\hline 3175 & 56.9583 & 426.7294 & 456.8529 & 430.5 & 122.5994 & 1.61543 \\
\hline 3176 & 56.9753 & 454.3161 & 456.3973 & 430.4167 & 122.5994 & 1.61499 \\
\hline 3177 & 56.9923 & 446.7555 & 455.8598 & 430.3333 & 122.5994 & 1.61454 \\
\hline 3178 & 57.0093 & 454.4013 & 455.2819 & 430.25 & 122.5994 & 1.6141 \\
\hline 3179 & 57.0263 & 443.9134 & 454.1085 & 430.1667 & 122.5994 & 1.61366 \\
\hline 3180 & 57.0433 & 462.2057 & 453.5836 & 430.0833 & 122.5994 & 1.61322 \\
\hline 3181 & 57.0603 & 444.5939 & 453.1054 & 430 & 122.5994 & 1.61278 \\
\hline 3182 & 57.0773 & 435.0327 & 452.6826 & 429.9167 & 122.5994 & 1.61234 \\
\hline 3183 & 57.0943 & 445.8025 & 452.3248 & 429.8333 & 122.5994 & 1.6119 \\
\hline
\end{tabular}




\begin{tabular}{|c|c|c|c|c|c|c|}
\hline 3184 & 57.1113 & 449.2407 & 452.004 & 429.75 & 122.5994 & 1.61146 \\
\hline 3185 & 57.1283 & 434.6799 & 451.8435 & 429.6667 & 122.5994 & 1.61102 \\
\hline 3186 & 57.1453 & 425.5438 & 451.7671 & 429.5833 & 122.5994 & 1.61058 \\
\hline 3187 & 57.1623 & 420.5824 & 451.8907 & 429.5 & 122.5994 & 1.61015 \\
\hline 3188 & 57.1793 & 401.7682 & 451.8823 & 429.4167 & 122.5994 & 1.60971 \\
\hline 3189 & 57.1963 & 447.2421 & 452.3515 & 429.3333 & 122.5994 & 1.60927 \\
\hline 3190 & 57.2133 & 484.9788 & 453.0101 & 429.25 & 122.5994 & 1.60883 \\
\hline 3191 & 57.2303 & 452.9512 & 453.8746 & 429.1667 & 122.5994 & 1.60839 \\
\hline 3192 & 57.2473 & 421.7482 & 455.0213 & 429.0833 & 122.5994 & 1.60796 \\
\hline 3193 & 57.2643 & 427.4226 & 456.5529 & 429 & 122.5994 & 1.60752 \\
\hline 3194 & 57.2813 & 421.0384 & 458.5454 & 428.7917 & 122.5994 & 1.60708 \\
\hline 3195 & 57.2983 & 434.1776 & 461.1654 & 428.5833 & 122.5994 & 1.60665 \\
\hline 3196 & 57.3153 & 434.7334 & 464.3459 & 428.375 & 122.5994 & 1.60621 \\
\hline 3197 & 57.3323 & 445.4665 & 467.5407 & 428.1667 & 122.5994 & 1.60578 \\
\hline 3198 & 57.3493 & 449.4999 & 469.6535 & 427.9583 & 122.5994 & 1.60534 \\
\hline 3199 & 57.3663 & 449.3969 & 469.5992 & 427.75 & 122.5994 & 1.6049 \\
\hline 3200 & 57.3833 & 439.0085 & 467.6003 & 427.5417 & 122.5994 & 1.60447 \\
\hline 3201 & 57.4003 & 462.1178 & 465.0659 & 427.3333 & 122.5994 & 1.60403 \\
\hline 3202 & 57.4173 & 477.9146 & 463.0068 & 427.125 & 122.5994 & 1.6036 \\
\hline 3203 & 57.4343 & 452.6669 & 461.9109 & 426.9167 & 122.5994 & 1.60317 \\
\hline 3204 & 57.4513 & 422.6419 & 461.863 & 426.7083 & 122.5994 & 1.60273 \\
\hline 3205 & 57.4683 & 435.8431 & 462.6627 & 426.5 & 122.5994 & 1.6023 \\
\hline 3206 & 57.4853 & 440.789 & 463.8692 & 426.2917 & 122.5994 & 1.60186 \\
\hline 3207 & 57.5023 & 396.91 & 464.6832 & 426.0833 & 122.5994 & 1.60143 \\
\hline 3208 & 57.5193 & 420.2205 & 464.6432 & 425.875 & 122.5994 & 1.601 \\
\hline 3209 & 57.5363 & 425.1286 & 463.3858 & 425.6667 & 122.5994 & 1.60057 \\
\hline 3210 & 57.5533 & 452.495 & 461.6093 & 425.4583 & 122.5994 & 1.60013 \\
\hline 3211 & 57.5703 & 429.3896 & 459.8187 & 425.25 & 122.5994 & 1.5997 \\
\hline 3212 & 57.5873 & 440.2156 & 458.2733 & 425.0417 & 122.5994 & 1.59927 \\
\hline 3213 & 57.6043 & 435.8611 & 457.0271 & 424.8333 & 122.5994 & 1.59884 \\
\hline 3214 & 57.6213 & 461.746 & 456.0549 & 424.625 & 122.5994 & 1.59841 \\
\hline 3215 & 57.6383 & 452.7667 & 455.2518 & 424.4167 & 122.5994 & 1.59798 \\
\hline 3216 & 57.6553 & 424.4166 & 454.4803 & 424.2083 & 122.5994 & 1.59755 \\
\hline 3217 & 57.6723 & 424.3193 & 453.7603 & 424 & 122.5994 & 1.59711 \\
\hline 3218 & 57.6893 & 424.4166 & 452.9754 & 423.7917 & 122.5994 & 1.59668 \\
\hline 3219 & 57.7063 & 433.39 & 452.2216 & 423.5833 & 122.5994 & 1.59625 \\
\hline 3220 & 57.7233 & 465.5937 & 451.4701 & 423.375 & 122.5994 & 1.59582 \\
\hline 3221 & 57.7403 & 475.3034 & 450.7525 & 423.1667 & 122.5994 & 1.5954 \\
\hline 3222 & 57.7573 & 456.004 & 450.0911 & 422.9583 & 122.5994 & 1.59497 \\
\hline 3223 & 57.7743 & 416.1393 & 449.5091 & 422.75 & 122.5994 & 1.59454 \\
\hline 3224 & 57.7913 & 425.7522 & 448.9924 & 422.5417 & 122.5994 & 1.59411 \\
\hline 3225 & 57.8083 & 431.5138 & 448.5111 & 422.3333 & 122.5994 & 1.59368 \\
\hline 3226 & 57.8253 & 430.4094 & 448.0211 & 422.125 & 122.5994 & 1.59325 \\
\hline 3227 & 57.8423 & 415.1859 & 447.4296 & 421.9167 & 122.5994 & 1.59282 \\
\hline 3228 & 57.8593 & 411.6111 & 446.7855 & 421.7083 & 122.5994 & 1.5924 \\
\hline 3229 & 57.8763 & 414.2702 & 446.0501 & 421.5 & 122.5994 & 1.59197 \\
\hline 3230 & 57.8933 & 416.0304 & 445.1657 & 421.2917 & 122.5994 & 1.59154 \\
\hline 3231 & 57.9103 & 430.5346 & 444.1733 & 421.0833 & 122.5994 & 1.59112 \\
\hline 3232 & 57.9273 & 447.5439 & 443.0972 & 420.875 & 122.5994 & 1.59069 \\
\hline
\end{tabular}




\begin{tabular}{|c|c|c|c|c|c|c|}
\hline 3233 & 57.9443 & 449.1657 & 441.9627 & 420.6667 & 122.5994 & 1.59026 \\
\hline 3234 & 57.9613 & 422.0098 & 440.7748 & 420.4583 & 122.5994 & 1.58984 \\
\hline 3235 & 57.9783 & 418.3535 & 439.5802 & 420.25 & 122.5994 & 1.58941 \\
\hline 3236 & 57.9953 & 443.6986 & 438.368 & 420.0417 & 122.5994 & 1.58899 \\
\hline 3237 & 58.0123 & 432.7904 & 437.1411 & 419.8333 & 122.5994 & 1.58856 \\
\hline 3238 & 58.0293 & 414.9202 & 435.9166 & 419.625 & 122.5994 & 1.58814 \\
\hline 3239 & 58.0463 & 403.9546 & 434.5975 & 419.4167 & 122.5994 & 1.58771 \\
\hline 3240 & 58.0633 & 434.2305 & 433.3662 & 419.2083 & 122.5994 & 1.58729 \\
\hline 3241 & 58.0803 & 443.3816 & 432.1592 & 419 & 122.5994 & 1.58686 \\
\hline 3242 & 58.0973 & 431.872 & 430.8278 & 418.625 & 122.5994 & 1.58644 \\
\hline 3243 & 58.1143 & 406.9168 & 429.5555 & 418.25 & 122.5994 & 1.58602 \\
\hline 3244 & 58.1313 & 412.5211 & 428.3726 & 417.875 & 122.5994 & 1.58559 \\
\hline 3245 & 58.1483 & 419.4828 & 427.2583 & 417.5 & 122.5994 & 1.58517 \\
\hline 3246 & 58.1653 & 420.2097 & 426.2328 & 417.125 & 122.5994 & 1.58475 \\
\hline 3247 & 58.1823 & 423.7663 & 425.3307 & 416.75 & 122.5994 & 1.58432 \\
\hline 3248 & 58.1993 & 435.0678 & 424.4482 & 416.375 & 122.5994 & 1.5839 \\
\hline 3249 & 58.2163 & 404.2919 & 423.6886 & 416 & 122.5994 & 1.58348 \\
\hline 3250 & 58.2333 & 433.3264 & 423.0039 & 415.625 & 122.5994 & 1.58306 \\
\hline 3251 & 58.2503 & 429.0486 & 422.3837 & 415.25 & 122.5994 & 1.58264 \\
\hline 3252 & 58.2673 & 409.1303 & 421.8915 & 414.875 & 122.5994 & 1.58221 \\
\hline 3253 & 58.2843 & 400.1949 & 421.3618 & 414.5 & 122.5994 & 1.58179 \\
\hline 3254 & 58.3013 & 432.45 & 420.9054 & 414.125 & 122.5994 & 1.58137 \\
\hline 3255 & 58.3183 & 420.2097 & 420.4519 & 413.75 & 122.5994 & 1.58095 \\
\hline 3256 & 58.3353 & 420.8942 & 420.0387 & 413.375 & 122.5994 & 1.58053 \\
\hline 3257 & 58.3523 & 435.8252 & 419.673 & 413 & 122.5994 & 1.58011 \\
\hline 3258 & 58.3693 & 419.6647 & 419.2909 & 412.625 & 122.5994 & 1.57969 \\
\hline 3259 & 58.3863 & 412.8666 & 418.9351 & 412.25 & 122.5994 & 1.57927 \\
\hline 3260 & 58.4033 & 419.3133 & 418.6116 & 411.875 & 122.5994 & 1.57885 \\
\hline 3261 & 58.4203 & 416.7893 & 418.3287 & 411.5 & 122.5994 & 1.57843 \\
\hline 3262 & 58.4373 & 442.4168 & 418.1331 & 411.125 & 122.5994 & 1.57802 \\
\hline 3263 & 58.4543 & 429.3196 & 417.87 & 410.75 & 122.5994 & 1.5776 \\
\hline 3264 & 58.4713 & 425.8234 & 417.7996 & 410.375 & 122.5994 & 1.57718 \\
\hline 3265 & 58.4883 & 400.0533 & 417.7773 & 410 & 122.5994 & 1.57676 \\
\hline 3266 & 58.5053 & 401.9768 & 418.6504 & 410.4583 & 122.5994 & 1.57634 \\
\hline 3267 & 58.5223 & 406.9045 & 419.6291 & 410.9167 & 122.5994 & 1.57593 \\
\hline 3268 & 58.5393 & 405.2475 & 420.679 & 411.375 & 122.5994 & 1.57551 \\
\hline 3269 & 58.5563 & 421.0473 & 421.82 & 411.8333 & 122.5994 & 1.57509 \\
\hline 3270 & 58.5733 & 443.842 & 423.0279 & 412.2917 & 122.5994 & 1.57468 \\
\hline 3271 & 58.5903 & 435.5931 & 424.2845 & 412.75 & 122.5994 & 1.57426 \\
\hline 3272 & 58.6073 & 441.0588 & 425.4689 & 413.2083 & 122.5994 & 1.57384 \\
\hline 3273 & 58.6243 & 437.6031 & 426.7347 & 413.6667 & 122.5994 & 1.57343 \\
\hline 3274 & 58.6413 & 420.4969 & 427.944 & 414.125 & 122.5994 & 1.57301 \\
\hline 3275 & 58.6583 & 426.1387 & 429.0404 & 414.5833 & 122.5994 & 1.5726 \\
\hline 3276 & 58.6753 & 445.7844 & 429.921 & 415.0417 & 122.5994 & 1.57218 \\
\hline 3277 & 58.6923 & 430.8 & 430.6805 & 415.5 & 122.5994 & 1.57177 \\
\hline 3278 & 58.7093 & 428.5324 & 431.2877 & 415.9583 & 122.5994 & 1.57135 \\
\hline 3279 & 58.7263 & 430.4962 & 431.7129 & 416.4167 & 122.5994 & 1.57094 \\
\hline 3280 & 58.7433 & 442.3568 & 432.0157 & 416.875 & 122.5994 & 1.57052 \\
\hline 3281 & 58.7603 & 425.1639 & 432.2369 & 417.3333 & 122.5994 & 1.57011 \\
\hline
\end{tabular}




\begin{tabular}{|c|c|c|c|c|c|c|}
\hline 3282 & 58.7773 & 416.1715 & 432.393 & 417.7917 & 122.5994 & 1.5697 \\
\hline 3283 & 58.7943 & 426.865 & 432.4933 & 418.25 & 122.5994 & 1.56928 \\
\hline 3284 & 58.8113 & 425.912 & 432.6411 & 418.7083 & 122.5994 & 1.56887 \\
\hline 3285 & 58.8283 & 421.6089 & 432.7372 & 419.1667 & 122.5994 & 1.56846 \\
\hline 3286 & 58.8453 & 460.6268 & 432.8563 & 419.625 & 122.5994 & 1.56804 \\
\hline 3287 & 58.8623 & 437.9717 & 432.9839 & 420.0833 & 122.5994 & 1.56763 \\
\hline 3288 & 58.8793 & 450.6156 & 433.1127 & 420.5417 & 122.5994 & 1.56722 \\
\hline 3289 & 58.8963 & 434.0608 & 433.2742 & 421 & 122.5994 & 1.56681 \\
\hline 3290 & 58.9133 & 446.816 & 433.4904 & 421.4583 & 122.5994 & 1.5664 \\
\hline 3291 & 58.9303 & 440.4377 & 433.7797 & 421.9167 & 122.5994 & 1.56599 \\
\hline 3292 & 58.9473 & 430.0281 & 434.1265 & 422.375 & 122.5994 & 1.56557 \\
\hline 3293 & 58.9643 & 449.403 & 434.5564 & 422.8333 & 122.5994 & 1.56516 \\
\hline 3294 & 58.9813 & 461.0535 & 435.1053 & 423.2917 & 122.5994 & 1.56475 \\
\hline 3295 & 58.9983 & 443.6208 & 435.7289 & 423.75 & 122.5994 & 1.56434 \\
\hline 3296 & 59.0153 & 441.0588 & 436.4162 & 424.2083 & 122.5994 & 1.56393 \\
\hline 3297 & 59.0323 & 468.3863 & 437.1599 & 424.6667 & 122.5994 & 1.56352 \\
\hline 3298 & 59.0493 & 442.776 & 437.9613 & 425.125 & 122.5994 & 1.56311 \\
\hline 3299 & 59.0663 & 461.4741 & 438.7902 & 425.5833 & 122.5994 & 1.5627 \\
\hline 3300 & 59.0833 & 466.4387 & 439.7484 & 426.0417 & 122.5994 & 1.56229 \\
\hline 3301 & 59.1003 & 492.5397 & 440.8151 & 426.5 & 122.5994 & 1.56189 \\
\hline 3302 & 59.1173 & 424.5212 & 442.0127 & 426.9583 & 122.5994 & 1.56148 \\
\hline 3303 & 59.1343 & 429.4286 & 443.3469 & 427.4167 & 122.5994 & 1.56107 \\
\hline 3304 & 59.1513 & 449.6867 & 444.7858 & 427.875 & 122.5994 & 1.56066 \\
\hline 3305 & 59.1683 & 455.6864 & 447.2686 & 428.3333 & 122.5994 & 1.56025 \\
\hline 3306 & 59.1853 & 470.9189 & 448.9815 & 428.7917 & 122.5994 & 1.55984 \\
\hline 3307 & 59.2023 & 440.8221 & 450.7508 & 429.25 & 122.5994 & 1.55944 \\
\hline 3308 & 59.2193 & 468.5292 & 452.5145 & 429.7083 & 122.5994 & 1.55903 \\
\hline 3309 & 59.2363 & 467.6977 & 454.2742 & 430.1667 & 122.5994 & 1.55862 \\
\hline 3310 & 59.2533 & 416.2014 & 456.0382 & 430.625 & 122.5994 & 1.55822 \\
\hline 3311 & 59.2703 & 428.1018 & 457.6685 & 431.0833 & 122.5994 & 1.55781 \\
\hline 3312 & 59.2873 & 418.0892 & 459.2145 & 431.5417 & 122.5994 & 1.5574 \\
\hline 3313 & 59.3043 & 437.8654 & 460.6665 & 432 & 122.5994 & 1.557 \\
\hline 3314 & 59.3213 & 441.0588 & 462.1381 & 432.5703 & 122.5994 & 1.55659 \\
\hline 3315 & 59.3383 & 450.0728 & 463.5074 & 433.1406 & 122.5994 & 1.55619 \\
\hline 3316 & 59.3553 & 470.8448 & 464.7068 & 433.7109 & 122.5994 & 1.55578 \\
\hline 3317 & 59.3723 & 460.5503 & 465.6859 & 434.2813 & 122.5994 & 1.55538 \\
\hline 3318 & 59.3893 & 442.5131 & 466.4245 & 434.8516 & 122.5994 & 1.55497 \\
\hline 3319 & 59.4063 & 460.4399 & 466.8969 & 435.4219 & 122.5994 & 1.55457 \\
\hline 3320 & 59.4233 & 464.487 & 467.1346 & 435.9922 & 122.5994 & 1.55416 \\
\hline 3321 & 59.4403 & 433.256 & 467.0982 & 436.5625 & 122.5994 & 1.55376 \\
\hline 3322 & 59.4573 & 441.875 & 466.8525 & 437.1328 & 122.5994 & 1.55336 \\
\hline 3323 & 59.4743 & 447.8274 & 466.4401 & 437.7031 & 122.5994 & 1.55295 \\
\hline 3324 & 59.4913 & 447.4148 & 465.9153 & 438.2734 & 122.5994 & 1.55255 \\
\hline 3325 & 59.5083 & 462.3887 & 465.3145 & 438.8438 & 122.5994 & 1.55215 \\
\hline 3326 & 59.5253 & 439.3193 & 464.7071 & 439.4141 & 122.5994 & 1.55174 \\
\hline 3327 & 59.5423 & 427.749 & 464.0584 & 439.9844 & 122.5994 & 1.55134 \\
\hline 3328 & 59.5593 & 444.1512 & 463.43 & 440.5547 & 122.5994 & 1.55094 \\
\hline 3329 & 59.5763 & 457.2655 & 462.8346 & 441.125 & 122.5994 & 1.55054 \\
\hline 3330 & 59.5933 & 473.1014 & 462.2905 & 441.6953 & 122.5994 & 1.55014 \\
\hline
\end{tabular}




\begin{tabular}{|c|c|c|c|c|c|c|}
\hline 3331 & 59.6103 & 465.6169 & 461.8141 & 442.2656 & 122.5994 & 1.54973 \\
\hline 3332 & 59.6273 & 446.5409 & 461.4208 & 442.8359 & 122.5994 & 1.54933 \\
\hline 3333 & 59.6443 & 452.8011 & 461.1313 & 443.4063 & 122.5994 & 1.54893 \\
\hline 3334 & 59.6613 & 439.8433 & 460.9611 & 443.9766 & 122.5994 & 1.54853 \\
\hline 3335 & 59.6783 & 444.9498 & 460.9338 & 444.5469 & 122.5994 & 1.54813 \\
\hline 3336 & 59.6953 & 489.0981 & 461.0623 & 445.1172 & 122.5994 & 1.54773 \\
\hline 3337 & 59.7123 & 451.1635 & 461.7193 & 445.6875 & 122.5994 & 1.54733 \\
\hline 3338 & 59.7293 & 464.1293 & 462.0378 & 446.1035 & 122.5994 & 1.54693 \\
\hline 3339 & 59.7463 & 478.0069 & 462.5313 & 446.5195 & 122.5994 & 1.54653 \\
\hline 3340 & 59.7633 & 498.231 & 463.1694 & 446.9355 & 122.5994 & 1.54613 \\
\hline 3341 & 59.7803 & 505.7081 & 463.9551 & 447.3516 & 122.5994 & 1.54573 \\
\hline 3342 & 59.7973 & 433.6612 & 464.8486 & 447.7676 & 122.5994 & 1.54533 \\
\hline 3343 & 59.8143 & 435.7146 & 465.9519 & 448.1836 & 122.5994 & 1.54494 \\
\hline 3344 & 59.8313 & 492.9477 & 467.168 & 448.5996 & 122.5994 & 1.54454 \\
\hline 3345 & 59.8483 & 493.1264 & 468.5753 & 449.0156 & 122.5994 & 1.54414 \\
\hline 3346 & 59.8653 & 463.0603 & 470.3561 & 449.4316 & 122.5994 & 1.54374 \\
\hline 3347 & 59.8823 & 466.4872 & 472.3463 & 449.8477 & 122.5994 & 1.54334 \\
\hline 3348 & 59.8993 & 537.1175 & 474.7881 & 450.2637 & 122.5994 & 1.54295 \\
\hline 3349 & 59.9163 & 470.4513 & 477.9008 & 450.6797 & 122.5994 & 1.54255 \\
\hline 3350 & 59.9333 & 571.7121 & 482.1099 & 451.0957 & 122.5994 & 1.54215 \\
\hline 3351 & 59.9503 & 587.0833 & 488.3214 & 451.5117 & 122.5994 & 1.54176 \\
\hline 3352 & 59.9673 & 667.503 & 498.288 & 451.9277 & 122.5994 & 1.54136 \\
\hline 3353 & 59.9843 & 730.258 & 515.1004 & 452.3438 & 122.5994 & 1.54096 \\
\hline 3354 & 60.0013 & 767.3861 & 542.934 & 452.7598 & 122.5994 & 1.54057 \\
\hline 3355 & 60.0183 & 905.0207 & 586.0479 & 453.1758 & 122.5994 & 1.54017 \\
\hline 3356 & 60.0353 & 788.0676 & 646.2603 & 453.5918 & 122.5994 & 1.53978 \\
\hline 3357 & 60.0523 & 803.9314 & 719.1927 & 454.0078 & 122.5994 & 1.53938 \\
\hline 3358 & 60.0693 & 696.6632 & 789.6174 & 454.4238 & 122.5994 & 1.53899 \\
\hline 3359 & 60.0863 & 646.651 & 828.8979 & 454.8398 & 122.5994 & 1.53859 \\
\hline 3360 & 60.1033 & 598.4512 & 814.228 & 455.2559 & 122.5994 & 1.5382 \\
\hline 3361 & 60.1203 & 586.6179 & 757.6981 & 455.6719 & 122.5994 & 1.5378 \\
\hline 3362 & 60.1373 & 612.7898 & 690.63 & 455.9245 & 122.5994 & 1.53741 \\
\hline 3363 & 60.1543 & 662.97 & 635.7714 & 456.1771 & 122.5994 & 1.53701 \\
\hline 3364 & 60.1713 & 690.5813 & 603.3168 & 456.4297 & 122.5994 & 1.53662 \\
\hline 3365 & 60.1883 & 670.3056 & 595.3068 & 456.6823 & 122.5994 & 1.53623 \\
\hline 3366 & 60.2053 & 690.7641 & 608.3008 & 456.9349 & 122.5994 & 1.53583 \\
\hline 3367 & 60.2223 & 635.5708 & 634.1254 & 457.1875 & 122.5994 & 1.53544 \\
\hline 3368 & 60.2393 & 548.1546 & 659.4841 & 457.4401 & 122.5994 & 1.53505 \\
\hline 3369 & 60.2563 & 542.8379 & 667.0379 & 457.6927 & 122.5994 & 1.53466 \\
\hline 3370 & 60.2733 & 516.2381 & 648.5132 & 457.9453 & 122.5994 & 1.53426 \\
\hline 3371 & 60.2903 & 493.003 & 613.8608 & 458.1979 & 122.5994 & 1.53387 \\
\hline 3372 & 60.3073 & 499.6173 & 577.3306 & 458.4505 & 122.5994 & 1.53348 \\
\hline 3373 & 60.3243 & 499.3551 & 546.9578 & 458.7031 & 122.5994 & 1.53309 \\
\hline 3374 & 60.3413 & 479.5913 & 525.1952 & 458.9557 & 122.5994 & 1.5327 \\
\hline 3375 & 60.3583 & 477.0171 & 511.2764 & 459.2083 & 122.5994 & 1.53231 \\
\hline 3376 & 60.3753 & 483.9536 & 503.0616 & 459.4609 & 122.5994 & 1.53192 \\
\hline 3377 & 60.3923 & 489.6013 & 498.422 & 459.7135 & 122.5994 & 1.53153 \\
\hline 3378 & 60.4093 & 489.9795 & 495.6973 & 459.9661 & 122.5994 & 1.53113 \\
\hline 3379 & 60.4263 & 479.3819 & 493.9961 & 460.2188 & 122.5994 & 1.53074 \\
\hline
\end{tabular}




\begin{tabular}{|c|c|c|c|c|c|c|}
\hline 3380 & 60.4433 & 487.6867 & 492.9568 & 460.4714 & 122.5994 & 1.53035 \\
\hline 3381 & 60.4603 & 461.3269 & 492.0738 & 460.724 & 122.5994 & 1.52997 \\
\hline 3382 & 60.4773 & 462.176 & 491.4007 & 460.9766 & 122.5994 & 1.52958 \\
\hline 3383 & 60.4943 & 473.2892 & 490.8618 & 461.2292 & 122.5994 & 1.52919 \\
\hline 3384 & 60.5113 & 475.1944 & 490.6242 & 461.4818 & 122.5994 & 1.5288 \\
\hline 3385 & 60.5283 & 464.7664 & 490.6154 & 461.7344 & 122.5994 & 1.52841 \\
\hline 3386 & 60.5453 & 465.7941 & 490.7298 & 461.8089 & 122.5994 & 1.52802 \\
\hline 3387 & 60.5623 & 470.321 & 491.1361 & 461.8835 & 122.5994 & 1.52763 \\
\hline 3388 & 60.5793 & 433.7173 & 491.8494 & 461.958 & 122.5994 & 1.52724 \\
\hline 3389 & 60.5963 & 466.4847 & 492.9755 & 462.0326 & 122.5994 & 1.52686 \\
\hline 3390 & 60.6133 & 455.1508 & 494.5658 & 462.1071 & 122.5994 & 1.52647 \\
\hline 3391 & 60.6303 & 426.0722 & 496.4966 & 462.1816 & 122.5994 & 1.52608 \\
\hline 3392 & 60.6473 & 469.1687 & 498.8532 & 462.2562 & 122.5994 & 1.52569 \\
\hline 3393 & 60.6643 & 458.1319 & 501.5975 & 462.3307 & 122.5994 & 1.52531 \\
\hline 3394 & 60.6813 & 462.4573 & 504.9341 & 462.4053 & 122.5994 & 1.52492 \\
\hline 3395 & 60.6983 & 491.4353 & 508.4718 & 462.4798 & 122.5994 & 1.52453 \\
\hline 3396 & 60.7153 & 441.2602 & 512.6273 & 462.5544 & 122.5994 & 1.52415 \\
\hline 3397 & 60.7323 & 445.1673 & 516.5966 & 462.6289 & 122.5994 & 1.52376 \\
\hline 3398 & 60.7493 & 446.8183 & 520.5904 & 462.7035 & 122.5994 & 1.52338 \\
\hline 3399 & 60.7663 & 461.7238 & 524.3687 & 462.778 & 122.5994 & 1.52299 \\
\hline 3400 & 60.7833 & 461.3988 & 527.6787 & 462.8525 & 122.5994 & 1.52261 \\
\hline 3401 & 60.8003 & 476.0835 & 530.2801 & 462.9271 & 122.5994 & 1.52222 \\
\hline 3402 & 60.8173 & 444.8199 & 531.9989 & 463.0016 & 122.5994 & 1.52184 \\
\hline 3403 & 60.8343 & 497.4309 & 532.8095 & 463.0762 & 122.5994 & 1.52145 \\
\hline 3404 & 60.8513 & 478.6031 & 532.8808 & 463.1507 & 122.5994 & 1.52107 \\
\hline 3405 & 60.8683 & 462.0214 & 532.2352 & 463.2253 & 122.5994 & 1.52068 \\
\hline 3406 & 60.8853 & 429.0029 & 531.3472 & 463.2998 & 122.5994 & 1.5203 \\
\hline 3407 & 60.9023 & 457.3064 & 530.0259 & 463.3743 & 122.5994 & 1.51992 \\
\hline 3408 & 60.9193 & 466.6222 & 528.5507 & 463.4489 & 122.5994 & 1.51953 \\
\hline 3409 & 60.9363 & 485.4579 & 526.9427 & 463.5234 & 122.5994 & 1.51915 \\
\hline 3410 & 60.9533 & 508.8405 & 525.1663 & 463.598 & 122.5994 & 1.51877 \\
\hline 3411 & 60.9703 & 491.1055 & 523.1837 & 463.6725 & 122.5994 & 1.51838 \\
\hline 3412 & 60.9873 & 507.1836 & 520.9288 & 463.7471 & 122.5994 & 1.518 \\
\hline 3413 & 61.0043 & 485.4862 & 518.4454 & 463.8216 & 122.5994 & 1.51762 \\
\hline 3414 & 61.0213 & 452.9618 & 515.8228 & 463.8962 & 122.5994 & 1.51724 \\
\hline 3415 & 61.0383 & 452.7309 & 513.1749 & 463.9707 & 122.5994 & 1.51685 \\
\hline 3416 & 61.0553 & 472.7541 & 510.652 & 464.0452 & 122.5994 & 1.51647 \\
\hline 3417 & 61.0723 & 468.8549 & 508.3479 & 464.1198 & 122.5994 & 1.51609 \\
\hline 3418 & 61.0893 & 460.1518 & 506.2719 & 464.1943 & 122.5994 & 1.51571 \\
\hline 3419 & 61.1063 & 481.2171 & 504.4837 & 464.2689 & 122.5994 & 1.51533 \\
\hline 3420 & 61.1233 & 480.018 & 502.9713 & 464.3434 & 122.5994 & 1.51495 \\
\hline 3421 & 61.1403 & 469.051 & 501.6893 & 464.418 & 122.5994 & 1.51457 \\
\hline 3422 & 61.1573 & 468.3232 & 500.5737 & 464.4925 & 122.5994 & 1.51419 \\
\hline 3423 & 61.1743 & 463.8055 & 499.6225 & 464.5671 & 122.5994 & 1.51381 \\
\hline 3424 & 61.1913 & 448.3905 & 498.0867 & 464.6416 & 122.5994 & 1.51343 \\
\hline 3425 & 61.2083 & 455.334 & 497.573 & 464.7161 & 122.5994 & 1.51305 \\
\hline 3426 & 61.2253 & 489.5938 & 497.2114 & 464.7907 & 122.5994 & 1.51267 \\
\hline 3427 & 61.2423 & 498.6315 & 497.0933 & 464.8652 & 122.5994 & 1.51229 \\
\hline 3428 & 61.2593 & 481.0077 & 497.2335 & 464.9398 & 122.5994 & 1.51191 \\
\hline
\end{tabular}




\begin{tabular}{|c|c|c|c|c|c|c|}
\hline 3429 & 61.2763 & 449.3524 & 497.6266 & 465.0143 & 122.5994 & 1.51153 \\
\hline 3430 & 61.2933 & 454.1752 & 498.2545 & 465.0889 & 122.5994 & 1.51115 \\
\hline 3431 & 61.3103 & 482.2991 & 499.0576 & 465.1634 & 122.5994 & 1.51078 \\
\hline 3432 & 61.3273 & 481.2379 & 499.989 & 465.238 & 122.5994 & 1.5104 \\
\hline 3433 & 61.3443 & 468.5521 & 500.9958 & 465.3125 & 122.5994 & 1.51002 \\
\hline 3434 & 61.3613 & 493.2182 & 501.8719 & 465.209 & 122.5994 & 1.50964 \\
\hline 3435 & 61.3783 & 485.4555 & 502.7876 & 465.1055 & 122.5994 & 1.50926 \\
\hline 3436 & 61.3953 & 504.1636 & 503.67 & 465.002 & 122.5994 & 1.50889 \\
\hline 3437 & 61.4123 & 469.4072 & 504.653 & 464.8984 & 122.5994 & 1.50851 \\
\hline 3438 & 61.4293 & 508.5665 & 505.7535 & 464.7949 & 122.5994 & 1.50813 \\
\hline 3439 & 61.4463 & 506.4397 & 506.9459 & 464.6914 & 122.5994 & 1.50776 \\
\hline 3440 & 61.4633 & 475.3634 & 508.2569 & 464.5879 & 122.5994 & 1.50738 \\
\hline 3441 & 61.4803 & 454.4879 & 509.6623 & 464.4844 & 122.5994 & 1.50701 \\
\hline 3442 & 61.4973 & 505.5337 & 511.1124 & 464.3809 & 122.5994 & 1.50663 \\
\hline 3443 & 61.5143 & 487.697 & 512.5374 & 464.2773 & 122.5994 & 1.50625 \\
\hline 3444 & 61.5313 & 479.522 & 513.8749 & 464.1738 & 122.5994 & 1.50588 \\
\hline 3445 & 61.5483 & 526.288 & 515.0783 & 464.0703 & 122.5994 & 1.5055 \\
\hline 3446 & 61.5653 & 502.824 & 516.174 & 463.9668 & 122.5994 & 1.50513 \\
\hline 3447 & 61.5823 & 516.0466 & 517.2494 & 463.8633 & 122.5994 & 1.50475 \\
\hline 3448 & 61.5993 & 542.2556 & 518.3958 & 463.7598 & 122.5994 & 1.50438 \\
\hline 3449 & 61.6163 & 520.431 & 519.8609 & 463.6563 & 122.5994 & 1.504 \\
\hline 3450 & 61.6333 & 544.9252 & 521.7284 & 463.5527 & 122.5994 & 1.50363 \\
\hline 3451 & 61.6503 & 550.0337 & 524.0897 & 463.4492 & 122.5994 & 1.50326 \\
\hline 3452 & 61.6673 & 544.1428 & 526.9684 & 463.3457 & 122.5994 & 1.50288 \\
\hline 3453 & 61.6843 & 556.6246 & 530.3268 & 463.2422 & 122.5994 & 1.50251 \\
\hline 3454 & 61.7013 & 566.8011 & 534.0938 & 463.1387 & 122.5994 & 1.50214 \\
\hline 3455 & 61.7183 & 536.6254 & 538.1146 & 463.0352 & 122.5994 & 1.50176 \\
\hline 3456 & 61.7353 & 546.0081 & 542.2017 & 462.9316 & 122.5994 & 1.50139 \\
\hline 3457 & 61.7523 & 555.7252 & 546.1191 & 462.8281 & 122.5994 & 1.50102 \\
\hline 3458 & 61.7693 & 554.4591 & 549.3746 & 462.5319 & 122.5994 & 1.50065 \\
\hline 3459 & 61.7863 & 537.359 & 551.9319 & 462.2357 & 122.5994 & 1.50027 \\
\hline 3460 & 61.8033 & 534.8501 & 553.4966 & 461.9395 & 122.5994 & 1.4999 \\
\hline 3461 & 61.8203 & 551.2274 & 553.9365 & 461.6432 & 122.5994 & 1.49953 \\
\hline 3462 & 61.8373 & 557.3479 & 553.2316 & 461.347 & 122.5994 & 1.49916 \\
\hline 3463 & 61.8543 & 547.617 & 551.5272 & 461.0508 & 122.5994 & 1.49879 \\
\hline 3464 & 61.8713 & 559.8805 & 549.0859 & 460.7546 & 122.5994 & 1.49842 \\
\hline 3465 & 61.8883 & 583.6591 & 546.179 & 460.4583 & 122.5994 & 1.49805 \\
\hline 3466 & 61.9053 & 538.8183 & 543.0364 & 460.1621 & 122.5994 & 1.49768 \\
\hline 3467 & 61.9223 & 528.9835 & 539.7961 & 459.8659 & 122.5994 & 1.49731 \\
\hline 3468 & 61.9393 & 516.7364 & 536.1685 & 459.5697 & 122.5994 & 1.49694 \\
\hline 3469 & 61.9563 & 526.7516 & 532.7916 & 459.2734 & 122.5994 & 1.49657 \\
\hline 3470 & 61.9733 & 538.5802 & 529.2449 & 458.9772 & 122.5994 & 1.4962 \\
\hline 3471 & 61.9903 & 521.5606 & 525.4963 & 458.681 & 122.5994 & 1.49583 \\
\hline 3472 & 62.0073 & 518.3483 & 521.5754 & 458.3848 & 122.5994 & 1.49546 \\
\hline 3473 & 62.0243 & 510.2168 & 517.4771 & 458.0885 & 122.5994 & 1.49509 \\
\hline 3474 & 62.0413 & 509.5921 & 513.3499 & 457.7923 & 122.5994 & 1.49472 \\
\hline 3475 & 62.0583 & 500.6421 & 509.3187 & 457.4961 & 122.5994 & 1.49435 \\
\hline 3476 & 62.0753 & 487.1342 & 505.4841 & 457.1999 & 122.5994 & 1.49398 \\
\hline 3477 & 62.0923 & 494.4604 & 501.9129 & 456.9036 & 122.5994 & 1.49361 \\
\hline
\end{tabular}




\begin{tabular}{|c|c|c|c|c|c|c|}
\hline 3478 & 62.1093 & 486.4508 & 498.6425 & 456.6074 & 122.5994 & 1.49325 \\
\hline 3479 & 62.1263 & 476.7618 & 495.6573 & 456.3112 & 122.5994 & 1.49288 \\
\hline 3480 & 62.1433 & 501.7101 & 492.9678 & 456.015 & 122.5994 & 1.49251 \\
\hline 3481 & 62.1603 & 506.3175 & 490.3469 & 455.7187 & 122.5994 & 1.49214 \\
\hline 3482 & 62.1773 & 466.6833 & 487.9799 & 455.2096 & 122.5994 & 1.49178 \\
\hline 3483 & 62.1943 & 470.2586 & 485.8761 & 454.7005 & 122.5994 & 1.49141 \\
\hline 3484 & 62.2113 & 427.0504 & 483.9834 & 454.1914 & 122.5994 & 1.49104 \\
\hline 3485 & 62.2283 & 503.1491 & 482.3033 & 453.6823 & 122.5994 & 1.49068 \\
\hline 3486 & 62.2453 & 467.2514 & 480.8187 & 453.1732 & 122.5994 & 1.49031 \\
\hline 3487 & 62.2623 & 462.5683 & 479.5044 & 452.6641 & 122.5994 & 1.48994 \\
\hline 3488 & 62.2793 & 505.9573 & 478.3387 & 452.1549 & 122.5994 & 1.48958 \\
\hline 3489 & 62.2963 & 496.3817 & 477.3297 & 451.6458 & 122.5994 & 1.48921 \\
\hline 3490 & 62.3133 & 479.3666 & 476.3365 & 451.1367 & 122.5994 & 1.48885 \\
\hline 3491 & 62.3303 & 472.8274 & 475.3356 & 450.6276 & 122.5994 & 1.48848 \\
\hline 3492 & 62.3473 & 448.0678 & 474.3947 & 450.1185 & 122.5994 & 1.48812 \\
\hline 3493 & 62.3643 & 436.2059 & 473.4156 & 449.6094 & 122.5994 & 1.48775 \\
\hline 3494 & 62.3813 & 438.6824 & 472.3788 & 449.1003 & 122.5994 & 1.48739 \\
\hline 3495 & 62.3983 & 469.3079 & 471.2831 & 448.5911 & 122.5994 & 1.48702 \\
\hline 3496 & 62.4153 & 466.5405 & 470.1438 & 448.082 & 122.5994 & 1.48666 \\
\hline 3497 & 62.4323 & 487.001 & 468.9862 & 447.5729 & 122.5994 & 1.48629 \\
\hline 3498 & 62.4493 & 490.8436 & 467.8342 & 447.0638 & 122.5994 & 1.48593 \\
\hline 3499 & 62.4663 & 466.4116 & 466.7296 & 446.5547 & 122.5994 & 1.48557 \\
\hline 3500 & 62.4833 & 450.0242 & 465.6385 & 446.0456 & 122.5994 & 1.4852 \\
\hline 3501 & 62.5003 & 447.5845 & 464.5473 & 445.5365 & 122.5994 & 1.48484 \\
\hline 3502 & 62.5173 & 444.3969 & 463.5163 & 445.0273 & 122.5994 & 1.48448 \\
\hline 3503 & 62.5343 & 416.6092 & 462.4876 & 444.5182 & 122.5994 & 1.48411 \\
\hline 3504 & 62.5513 & 446.9107 & 461.4032 & 444.0091 & 122.5994 & 1.48375 \\
\hline 3505 & 62.5683 & 469.1431 & 460.3655 & 443.5 & 122.5994 & 1.48339 \\
\hline 3506 & 62.5853 & 461.2856 & 459.0828 & 442.7708 & 122.5994 & 1.48303 \\
\hline 3507 & 62.6023 & 446.2856 & 457.7893 & 442.0417 & 122.5994 & 1.48267 \\
\hline 3508 & 62.6193 & 429.192 & 456.4828 & 441.3125 & 122.5994 & 1.4823 \\
\hline 3509 & 62.6363 & 438.3745 & 455.2124 & 440.5833 & 122.5994 & 1.48194 \\
\hline 3510 & 62.6533 & 462.2209 & 453.9702 & 439.8542 & 122.5994 & 1.48158 \\
\hline 3511 & 62.6703 & 446.7415 & 452.7632 & 439.125 & 122.5994 & 1.48122 \\
\hline 3512 & 62.6873 & 415.1407 & 451.5854 & 438.3958 & 122.5994 & 1.48086 \\
\hline 3513 & 62.7043 & 424.9737 & 450.4226 & 437.6667 & 122.5994 & 1.4805 \\
\hline 3514 & 62.7213 & 445.9095 & 449.1026 & 436.9375 & 122.5994 & 1.48014 \\
\hline 3515 & 62.7383 & 457.187 & 447.9485 & 436.2083 & 122.5994 & 1.47978 \\
\hline 3516 & 62.7553 & 462.3116 & 446.7803 & 435.4792 & 122.5994 & 1.47942 \\
\hline 3517 & 62.7723 & 459.412 & 445.6075 & 434.75 & 122.5994 & 1.47906 \\
\hline 3518 & 62.7893 & 460.9075 & 444.4363 & 434.0208 & 122.5994 & 1.4787 \\
\hline 3519 & 62.8063 & 418.9877 & 443.3017 & 433.2917 & 122.5994 & 1.47834 \\
\hline 3520 & 62.8233 & 414.4542 & 442.2094 & 432.5625 & 122.5994 & 1.47798 \\
\hline 3521 & 62.8403 & 441.8288 & 441.1171 & 431.8333 & 122.5994 & 1.47762 \\
\hline 3522 & 62.8573 & 452.6216 & 440.1566 & 431.1042 & 122.5994 & 1.47726 \\
\hline 3523 & 62.8743 & 457.0828 & 439.2679 & 430.375 & 122.5994 & 1.4769 \\
\hline 3524 & 62.8913 & 430.9748 & 438.4519 & 429.6458 & 122.5994 & 1.47655 \\
\hline 3525 & 62.9083 & 378.5326 & 437.5648 & 428.9167 & 122.5994 & 1.47619 \\
\hline 3526 & 62.9253 & 398.4885 & 436.8967 & 428.1875 & 122.5994 & 1.47583 \\
\hline
\end{tabular}




\begin{tabular}{|c|c|c|c|c|c|c|}
\hline 3527 & 62.9423 & 421.6007 & 435.9686 & 427.4583 & 122.5994 & 1.47547 \\
\hline 3528 & 62.9593 & 435.4717 & 435.448 & 426.7292 & 122.5994 & 1.47512 \\
\hline 3529 & 62.9763 & 406.9403 & 434.9895 & 426 & 122.5994 & 1.47476 \\
\hline 3530 & 62.9933 & 446.3607 & 434.7191 & 425.4094 & 122.5994 & 1.4744 \\
\hline 3531 & 63.0103 & 417.6992 & 434.4862 & 424.8188 & 122.5994 & 1.47404 \\
\hline 3532 & 63.0273 & 420.1032 & 434.5552 & 424.2281 & 122.5994 & 1.47369 \\
\hline 3533 & 63.0443 & 420.306 & 434.3414 & 423.6375 & 122.5994 & 1.47333 \\
\hline 3534 & 63.0613 & 432.6977 & 434.1021 & 423.0469 & 122.5994 & 1.47297 \\
\hline 3535 & 63.0783 & 432.759 & 433.7372 & 422.4563 & 122.5994 & 1.47262 \\
\hline 3536 & 63.0953 & 435.3814 & 433.4046 & 421.8657 & 122.5994 & 1.47226 \\
\hline 3537 & 63.1123 & 412.0505 & 432.8169 & 421.2751 & 122.5994 & 1.47191 \\
\hline 3538 & 63.1293 & 414.5963 & 432.3441 & 420.6844 & 122.5994 & 1.47155 \\
\hline 3539 & 63.1463 & 434.2945 & 431.8096 & 420.0938 & 122.5994 & 1.4712 \\
\hline 3540 & 63.1633 & 422.2253 & 431.232 & 419.5032 & 122.5994 & 1.47084 \\
\hline 3541 & 63.1803 & 446.9657 & 430.6336 & 418.9126 & 122.5994 & 1.47049 \\
\hline 3542 & 63.1973 & 391.2553 & 430.0295 & 418.322 & 122.5994 & 1.47013 \\
\hline 3543 & 63.2143 & 400.1814 & 429.5469 & 417.7313 & 122.5994 & 1.46978 \\
\hline 3544 & 63.2313 & 448.1278 & 429.0092 & 417.1407 & 122.5994 & 1.46942 \\
\hline 3545 & 63.2483 & 449.3945 & 428.4672 & 416.5501 & 122.5994 & 1.46907 \\
\hline 3546 & 63.2653 & 419.9242 & 427.9455 & 415.9595 & 122.5994 & 1.46872 \\
\hline 3547 & 63.2823 & 406.8052 & 427.4356 & 415.3689 & 122.5994 & 1.46836 \\
\hline 3548 & 63.2993 & 413.1779 & 426.8905 & 414.7783 & 122.5994 & 1.46801 \\
\hline 3549 & 63.3163 & 431.1885 & 426.3792 & 414.1876 & 122.5994 & 1.46765 \\
\hline 3550 & 63.3333 & 421.0628 & 425.8501 & 413.597 & 122.5994 & 1.4673 \\
\hline 3551 & 63.3503 & 414.3421 & 425.2719 & 413.0064 & 122.5994 & 1.46695 \\
\hline 3552 & 63.3673 & 411.254 & 424.6588 & 412.4158 & 122.5994 & 1.4666 \\
\hline 3553 & 63.3843 & 420.1675 & 423.9748 & 411.8252 & 122.5994 & 1.46624 \\
\hline 3554 & 63.4013 & 419.6838 & 423.2133 & 411.2345 & 122.5994 & 1.46589 \\
\hline 3555 & 63.4183 & 402.7037 & 422.3697 & 410.6439 & 122.5994 & 1.46554 \\
\hline 3556 & 63.4353 & 420.6834 & 421.4737 & 410.0533 & 122.5994 & 1.46519 \\
\hline 3557 & 63.4523 & 422.9031 & 420.5457 & 409.4627 & 122.5994 & 1.46484 \\
\hline 3558 & 63.4693 & 419.187 & 419.614 & 408.8721 & 122.5994 & 1.46449 \\
\hline 3559 & 63.4863 & 402.1867 & 418.6861 & 408.2815 & 122.5994 & 1.46413 \\
\hline 3560 & 63.5033 & 415.7962 & 417.8111 & 407.6908 & 122.5994 & 1.46378 \\
\hline 3561 & 63.5203 & 452.1418 & 416.9671 & 407.1002 & 122.5994 & 1.46343 \\
\hline 3562 & 63.5373 & 434.4259 & 416.154 & 406.5096 & 122.5994 & 1.46308 \\
\hline 3563 & 63.5543 & 425.3179 & 415.3017 & 405.919 & 122.5994 & 1.46273 \\
\hline 3564 & 63.5713 & 427.1135 & 414.524 & 405.3284 & 122.5994 & 1.46238 \\
\hline 3565 & 63.5883 & 456.2472 & 413.7428 & 404.7377 & 122.5994 & 1.46203 \\
\hline 3566 & 63.6053 & 458.7101 & 412.9853 & 404.1471 & 122.5994 & 1.46168 \\
\hline 3567 & 63.6223 & 432.4229 & 412.2347 & 403.5565 & 122.5994 & 1.46133 \\
\hline 3568 & 63.6393 & 433.6391 & 411.5021 & 402.9659 & 122.5994 & 1.46098 \\
\hline 3569 & 63.6563 & 424.0169 & 410.8042 & 402.3753 & 122.5994 & 1.46063 \\
\hline 3570 & 63.6733 & 401.1165 & 410.1139 & 401.7847 & 122.5994 & 1.46028 \\
\hline 3571 & 63.6903 & 386.3371 & 409.4464 & 401.194 & 122.5994 & 1.45994 \\
\hline 3572 & 63.7073 & 410.9222 & 408.8065 & 400.6034 & 122.5994 & 1.45959 \\
\hline 3573 & 63.7243 & 419.6217 & 408.1408 & 400.0128 & 122.5994 & 1.45924 \\
\hline 3574 & 63.7413 & 438.419 & 407.5501 & 399.4222 & 122.5994 & 1.45889 \\
\hline 3575 & 63.7583 & 386.4319 & 406.9946 & 398.8316 & 122.5994 & 1.45854 \\
\hline
\end{tabular}




\begin{tabular}{|c|c|c|c|c|c|c|}
\hline 3576 & 63.7753 & 432.2843 & 406.508 & 398.2409 & 122.5994 & 1.45819 \\
\hline 3577 & 63.7923 & 417.4102 & 406.0949 & 397.6503 & 122.5994 & 1.45785 \\
\hline 3578 & 63.8093 & 444.1155 & 406.1146 & 397.4025 & 122.5994 & 1.4575 \\
\hline 3579 & 63.8263 & 400.7217 & 406.2482 & 397.1546 & 122.5994 & 1.45715 \\
\hline 3580 & 63.8433 & 457.7194 & 406.4907 & 396.9068 & 122.5994 & 1.4568 \\
\hline 3581 & 63.8603 & 428.6647 & 406.8402 & 396.6589 & 122.5994 & 1.45646 \\
\hline 3582 & 63.8773 & 434.7175 & 407.3759 & 396.4111 & 122.5994 & 1.45611 \\
\hline 3583 & 63.8943 & 473.7648 & 408.0824 & 396.1632 & 122.5994 & 1.45576 \\
\hline 3584 & 63.9113 & 452.0798 & 408.9888 & 395.9154 & 122.5994 & 1.45542 \\
\hline 3585 & 63.9283 & 438.8004 & 410.1172 & 395.6675 & 122.5994 & 1.45507 \\
\hline 3586 & 63.9453 & 451.9221 & 411.5073 & 395.4197 & 122.5994 & 1.45473 \\
\hline 3587 & 63.9623 & 432.6348 & 413.1638 & 395.1718 & 122.5994 & 1.45438 \\
\hline 3588 & 63.9793 & 390.9907 & 415.1094 & 394.924 & 122.5994 & 1.45404 \\
\hline 3589 & 63.9963 & 421.7704 & 417.3586 & 394.6761 & 122.5994 & 1.45369 \\
\hline 3590 & 64.0133 & 485.1616 & 419.9496 & 394.4283 & 122.5994 & 1.45335 \\
\hline 3591 & 64.0303 & 470.8869 & 422.942 & 394.1804 & 122.5994 & 1.453 \\
\hline 3592 & 64.0473 & 496.6092 & 426.5325 & 393.9325 & 122.5994 & 1.45266 \\
\hline 3593 & 64.0643 & 475.7505 & 431.1067 & 393.6847 & 122.5994 & 1.45231 \\
\hline 3594 & 64.0813 & 522.7321 & 437.1441 & 393.4368 & 122.5994 & 1.45197 \\
\hline 3595 & 64.0983 & 521.3352 & 445.3544 & 393.189 & 122.5994 & 1.45162 \\
\hline 3596 & 64.1153 & 511.0876 & 456.1676 & 392.9411 & 122.5994 & 1.45128 \\
\hline 3597 & 64.1323 & 509.8477 & 469.2576 & 392.6933 & 122.5994 & 1.45094 \\
\hline 3598 & 64.1493 & 482.3295 & 482.803 & 392.4454 & 122.5994 & 1.45059 \\
\hline 3599 & 64.1663 & 457.7514 & 492.7514 & 392.1976 & 122.5994 & 1.45025 \\
\hline 3600 & 64.1833 & 430.7912 & 493.755 & 391.9497 & 122.5994 & 1.44991 \\
\hline 3601 & 64.2003 & 427.3401 & 485.1051 & 391.7019 & 122.5994 & 1.44956 \\
\hline 3602 & 64.2173 & 445.0652 & 471.7466 & 391.454 & 122.5994 & 1.44922 \\
\hline 3603 & 64.2343 & 458.1513 & 458.6807 & 391.2062 & 122.5994 & 1.44888 \\
\hline 3604 & 64.2513 & 442.3135 & 448.6909 & 390.9583 & 122.5994 & 1.44854 \\
\hline 3605 & 64.2683 & 424.9976 & 442.6585 & 390.7105 & 122.5994 & 1.44819 \\
\hline 3606 & 64.2853 & 454.3519 & 440.4997 & 390.4626 & 122.5994 & 1.44785 \\
\hline 3607 & 64.3023 & 465.8744 & 441.5619 & 390.2148 & 122.5994 & 1.44751 \\
\hline 3608 & 64.3193 & 459.6972 & 444.6641 & 389.9669 & 122.5994 & 1.44717 \\
\hline 3609 & 64.3363 & 465.9649 & 447.8678 & 389.7191 & 122.5994 & 1.44683 \\
\hline 3610 & 64.3533 & 468.1285 & 448.3976 & 389.4712 & 122.5994 & 1.44649 \\
\hline 3611 & 64.3703 & 440.9663 & 444.1149 & 389.2234 & 122.5994 & 1.44614 \\
\hline 3612 & 64.3873 & 439.9728 & 436.225 & 388.9755 & 122.5994 & 1.4458 \\
\hline 3613 & 64.4043 & 407.1596 & 427.4261 & 388.7277 & 122.5994 & 1.44546 \\
\hline 3614 & 64.4213 & 412.9226 & 419.5152 & 388.4798 & 122.5994 & 1.44512 \\
\hline 3615 & 64.4383 & 411.0258 & 413.2153 & 388.232 & 122.5994 & 1.44478 \\
\hline 3616 & 64.4553 & 404.7589 & 408.5174 & 387.9841 & 122.5994 & 1.44444 \\
\hline 3617 & 64.4723 & 430.3988 & 405.1039 & 387.7362 & 122.5994 & 1.4441 \\
\hline 3618 & 64.4893 & 440.3231 & 402.5904 & 387.4884 & 122.5994 & 1.44376 \\
\hline 3619 & 64.5063 & 409.5729 & 400.6696 & 387.2405 & 122.5994 & 1.44342 \\
\hline 3620 & 64.5233 & 418.1502 & 399.0996 & 386.9927 & 122.5994 & 1.44308 \\
\hline 3621 & 64.5403 & 409.4509 & 397.8501 & 386.7448 & 122.5994 & 1.44275 \\
\hline 3622 & 64.5573 & 389.7484 & 396.8169 & 386.497 & 122.5994 & 1.44241 \\
\hline 3623 & 64.5743 & 384.4795 & 395.9569 & 386.2491 & 122.5994 & 1.44207 \\
\hline 3624 & 64.5913 & 403.1386 & 395.2392 & 386.0013 & 122.5994 & 1.44173 \\
\hline
\end{tabular}




$\begin{array}{rrrrrrr}3625 & 64.6083 & 407.9938 & 394.6401 & 385.7534 & 122.5994 & 1.44139 \\ 3626 & 64.6253 & 420.0729 & 394.1391 & 385.5056 & 122.5994 & 1.44105 \\ 3627 & 64.6423 & 420.1579 & 393.7177 & 385.2577 & 122.5994 & 1.44071 \\ 3628 & 64.6593 & 412.1161 & 393.3572 & 385.0099 & 122.5994 & 1.44038 \\ 3629 & 64.6763 & 416.4649 & 393.0446 & 384.762 & 122.5994 & 1.44004 \\ 3630 & 64.6933 & 421.6745 & 392.7422 & 384.5142 & 122.5994 & 1.4397 \\ 3631 & 64.7103 & 435.5699 & 392.4804 & 384.2663 & 122.5994 & 1.43936 \\ 3632 & 64.7273 & 421.1654 & 392.2265 & 384.0185 & 122.5994 & 1.43903 \\ 3633 & 64.7443 & 396.0058 & 391.9722 & 383.7706 & 122.5994 & 1.43869 \\ 3634 & 64.7613 & 376.5093 & 391.7121 & 383.5228 & 122.5994 & 1.43835 \\ 3635 & 64.7783 & 398.1666 & 391.4439 & 383.2749 & 122.5994 & 1.43802 \\ 3636 & 64.7953 & 407.2416 & 391.1689 & 383.0271 & 122.5994 & 1.43768 \\ 3637 & 64.8123 & 402.1977 & 390.8894 & 382.7792 & 122.5994 & 1.43735 \\ 3638 & 64.8293 & 402.242 & 390.6076 & 382.5314 & 122.5994 & 1.43701 \\ 3639 & 64.8463 & 420.1134 & 390.3246 & 382.2835 & 122.5994 & 1.43667 \\ 3640 & 64.8633 & 423.9952 & 390.0307 & 382.0357 & 122.5994 & 1.43634 \\ 3641 & 64.8803 & 420.9978 & 389.7478 & 381.7878 & 122.5994 & 1.436 \\ 3642 & 64.8973 & 401.3581 & 389.4643 & 381.5399 & 122.5994 & 1.43567 \\ 3643 & 64.9143 & 406.32 & 389.1984 & 381.2921 & 122.5994 & 1.43533 \\ 3644 & 64.9313 & 410.1249 & 388.9515 & 381.0442 & 122.5994 & 1.435 \\ 3645 & 64.9483 & 432.6977 & 388.733 & 380.7964 & 122.5994 & 1.43466 \\ 3646 & 64.9653 & 410.1425 & 388.5542 & 380.5485 & 122.5994 & 1.43433 \\ 3647 & 64.9823 & 380.3007 & 388.6751 & 380.5485 & 122.5994 & 1.434\end{array}$


No. Pos. $\left[{ }^{\circ} 2\right.$ Th. lobs [cts] Icalc [cts] lback [cts] CT [s] ESD D spacings

\begin{tabular}{|c|c|c|c|c|c|c|}
\hline 1 & 3.0003 & 2352.243 & 2352 & 2352 & 122.5994 & 29.42347 \\
\hline 2 & 3.0173 & 2208.004 & 2321 & 2321 & 122.5994 & 29.25773 \\
\hline 3 & 3.0343 & 2161.063 & 2291 & 2291 & 122.5994 & 29.09385 \\
\hline 4 & 3.0513 & 2133.259 & 2260 & 2260 & 122.5994 & 28.9318 \\
\hline 5 & 3.0683 & 2112.826 & 2229 & 2229 & 122.5994 & 28.77154 \\
\hline 6 & 3.0853 & 2067.75 & 2198 & 2198 & 122.5994 & 28.61305 \\
\hline 7 & 3.1023 & 1967.941 & 2168 & 2168 & 122.5994 & 28.45629 \\
\hline 8 & 3.1193 & 1948.789 & 2137 & 2137 & 122.5994 & 28.30124 \\
\hline 9 & 3.1363 & 1956.256 & 2106 & 2106 & 122.5994 & 28.14788 \\
\hline 10 & 3.1533 & 1873.553 & 2075 & 2075 & 122.5994 & 27.99617 \\
\hline 11 & 3.1703 & 1837.366 & 2045 & 2045 & 122.5994 & 27.84608 \\
\hline 12 & 3.1873 & 1837.453 & 2014 & 2014 & 122.5994 & 27.6976 \\
\hline 13 & 3.2043 & 1807.863 & 1983 & 1983 & 122.5994 & 27.55069 \\
\hline 14 & 3.2213 & 1864.258 & 1952 & 1952 & 122.5994 & 27.40534 \\
\hline 15 & 3.2383 & 1747.223 & 1922 & 1922 & 122.5994 & 27.26151 \\
\hline 16 & 3.2553 & 1741.864 & 1891 & 1891 & 122.5994 & 27.11918 \\
\hline 17 & 3.2723 & 1724.853 & 1860 & 1860 & 122.5994 & 26.97833 \\
\hline 18 & 3.2893 & 1732.246 & 1829 & 1829 & 122.5994 & 26.83894 \\
\hline 19 & 3.3063 & 1728.69 & 1799 & 1799 & 122.5994 & 26.70098 \\
\hline 20 & 3.3233 & 1659.506 & 1768 & 1768 & 122.5994 & 26.56443 \\
\hline 21 & 3.3403 & 1664.844 & 1737 & 1737 & 122.5994 & 26.42928 \\
\hline 22 & 3.3573 & 1657.039 & 1706 & 1706 & 122.5994 & 26.29549 \\
\hline 23 & 3.3743 & 1630.966 & 1676 & 1676 & 122.5994 & 26.16305 \\
\hline 24 & 3.3913 & 1644.457 & 1645 & 1645 & 122.5994 & 26.03194 \\
\hline 25 & 3.4083 & 1619.673 & 1614 & 1614 & 122.5994 & 25.90213 \\
\hline 26 & 3.4253 & 1586.581 & 1604 & 1604 & 122.5994 & 25.77362 \\
\hline 27 & 3.4423 & 1581.509 & 1594 & 1594 & 122.5994 & 25.64637 \\
\hline 28 & 3.4593 & 1648.461 & 1585 & 1585 & 122.5994 & 25.52038 \\
\hline 29 & 3.4763 & 1621.068 & 1575 & 1575 & 122.5994 & 25.39561 \\
\hline 30 & 3.4933 & 1591.016 & 1565 & 1565 & 122.5994 & 25.27207 \\
\hline 31 & 3.5103 & 1528.955 & 1556 & 1556 & 122.5994 & 25.14971 \\
\hline 32 & 3.5273 & 1542.126 & 1546 & 1546 & 122.5994 & 25.02854 \\
\hline 33 & 3.5443 & 1595.605 & 1536 & 1536 & 122.5994 & 24.90853 \\
\hline 34 & 3.5613 & 1562.893 & 1526 & 1526 & 122.5994 & 24.78967 \\
\hline 35 & 3.5783 & 1521.614 & 1516 & 1516 & 122.5994 & 24.67194 \\
\hline 36 & 3.5953 & 1542.699 & 1507 & 1507 & 122.5994 & 24.55532 \\
\hline 37 & 3.6123 & 1516.023 & 1497 & 1497 & 122.5994 & 24.4398 \\
\hline 38 & 3.6293 & 1438.433 & 1487 & 1487 & 122.5994 & 24.32536 \\
\hline 39 & 3.6463 & 1458.58 & 1478 & 1478 & 122.5994 & 24.21198 \\
\hline 40 & 3.6633 & 1447.688 & 1468 & 1468 & 122.5994 & 24.09966 \\
\hline 41 & 3.6803 & 1463.686 & 1458 & 1458 & 122.5994 & 23.98838 \\
\hline 42 & 3.6973 & 1473.663 & 1448 & 1448 & 122.5994 & 23.87812 \\
\hline 43 & 3.7143 & 1406.067 & 1438 & 1438 & 122.5994 & 23.76887 \\
\hline 44 & 3.7313 & 1433.58 & 1429 & 1429 & 122.5994 & 23.66062 \\
\hline 45 & 3.7483 & 1379.613 & 1419 & 1419 & 122.5994 & 23.55335 \\
\hline 46 & 3.7653 & 1456.615 & 1409 & 1409 & 122.5994 & 23.44704 \\
\hline 47 & 3.7823 & 1421.685 & 1400 & 1400 & 122.5994 & 23.3417 \\
\hline
\end{tabular}




\begin{tabular}{|c|c|c|c|c|c|c|}
\hline 48 & 3.7993 & 1304.716 & 1390 & 1390 & 122.5994 & 23.23729 \\
\hline 49 & 3.8163 & 1433.722 & 1380 & 1380 & 122.5994 & 23.13382 \\
\hline 50 & 3.8333 & 1356.238 & 1375 & 1375 & 122.5994 & 23.03126 \\
\hline 51 & 3.8503 & 1393.57 & 1369 & 1369 & 122.5994 & 22.92961 \\
\hline 52 & 3.8673 & 1419.103 & 1364 & 1364 & 122.5994 & 22.82886 \\
\hline 53 & 3.8843 & 1390.842 & 1359 & 1359 & 122.5994 & 22.72898 \\
\hline 54 & 3.9013 & 1336.377 & 1354 & 1354 & 122.5994 & 22.62998 \\
\hline 55 & 3.9183 & 1348.67 & 1348 & 1348 & 122.5994 & 22.53184 \\
\hline 56 & 3.9353 & 1282.942 & 1343 & 1343 & 122.5994 & 22.43454 \\
\hline 57 & 3.9523 & 1282.963 & 1338 & 1338 & 122.5994 & 22.33808 \\
\hline 58 & 3.9693 & 1278.214 & 1332 & 1332 & 122.5994 & 22.24245 \\
\hline 59 & 3.9863 & 1339.566 & 1327 & 1327 & 122.5994 & 22.14763 \\
\hline 60 & 4.0033 & 1322.013 & 1322 & 1322 & 122.5994 & 22.05362 \\
\hline 61 & 4.0203 & 1297.682 & 1316 & 1316 & 122.5994 & 21.9604 \\
\hline 62 & 4.0373 & 1331.578 & 1311 & 1311 & 122.5994 & 21.86797 \\
\hline 63 & 4.0543 & 1318.251 & 1306 & 1306 & 122.5994 & 21.77632 \\
\hline 64 & 4.0713 & 1362.571 & 1301 & 1301 & 122.5994 & 21.68543 \\
\hline 65 & 4.0883 & 1304.982 & 1295 & 1295 & 122.5994 & 21.59529 \\
\hline 66 & 4.1053 & 1281.739 & 1290 & 1290 & 122.5994 & 21.50591 \\
\hline 67 & 4.1223 & 1272.271 & 1285 & 1285 & 122.5994 & 21.41726 \\
\hline 68 & 4.1393 & 1269.932 & 1279 & 1279 & 122.5994 & 21.32933 \\
\hline 69 & 4.1563 & 1269.191 & 1274 & 1274 & 122.5994 & 21.24213 \\
\hline 70 & 4.1733 & 1275.262 & 1269 & 1269 & 122.5994 & 21.15564 \\
\hline 71 & 4.1903 & 1221.306 & 1264 & 1264 & 122.5994 & 21.06985 \\
\hline 72 & 4.2073 & 1242.243 & 1258 & 1258 & 122.5994 & 20.98475 \\
\hline 73 & 4.2243 & 1273.149 & 1253 & 1253 & 122.5994 & 20.90034 \\
\hline 74 & 4.2413 & 1256.191 & 1248 & 1248 & 122.5994 & 20.81661 \\
\hline 75 & 4.2583 & 1236.471 & 1243 & 1243 & 122.5994 & 20.73354 \\
\hline 76 & 4.2753 & 1237.966 & 1237 & 1237 & 122.5994 & 20.65114 \\
\hline 77 & 4.2923 & 1214.996 & 1232 & 1232 & 122.5994 & 20.56939 \\
\hline 78 & 4.3093 & 1201.699 & 1227 & 1227 & 122.5994 & 20.48828 \\
\hline 79 & 4.3263 & 1199.412 & 1222 & 1222 & 122.5994 & 20.40781 \\
\hline 80 & 4.3433 & 1201.998 & 1217 & 1217 & 122.5994 & 20.32797 \\
\hline 81 & 4.3603 & 1219.16 & 1211 & 1211 & 122.5994 & 20.24875 \\
\hline 82 & 4.3773 & 1225.023 & 1206 & 1206 & 122.5994 & 20.17015 \\
\hline 83 & 4.3943 & 1203.709 & 1201 & 1201 & 122.5994 & 20.09216 \\
\hline 84 & 4.4113 & 1159.467 & 1196 & 1196 & 122.5994 & 20.01477 \\
\hline 85 & 4.4283 & 1149.231 & 1190 & 1190 & 122.5994 & 19.93797 \\
\hline 86 & 4.4453 & 1194.687 & 1185 & 1185 & 122.5994 & 19.86176 \\
\hline 87 & 4.4623 & 1169.007 & 1180 & 1180 & 122.5994 & 19.78613 \\
\hline 88 & 4.4793 & 1180.716 & 1175 & 1175 & 122.5994 & 19.71108 \\
\hline 89 & 4.4963 & 1183.348 & 1170 & 1170 & 122.5994 & 19.63659 \\
\hline 90 & 4.5133 & 1121.348 & 1164 & 1164 & 122.5994 & 19.56267 \\
\hline 91 & 4.5303 & 1172.608 & 1159 & 1159 & 122.5994 & 19.48929 \\
\hline 92 & 4.5473 & 1236.4 & 1154 & 1154 & 122.5994 & 19.41647 \\
\hline 93 & 4.5643 & 1124.666 & 1149 & 1149 & 122.5994 & 19.34419 \\
\hline 94 & 4.5813 & 1135.645 & 1144 & 1144 & 122.5994 & 19.27245 \\
\hline 95 & 4.5983 & 1102.41 & 1138 & 1138 & 122.5994 & 19.20124 \\
\hline 96 & 4.6153 & 1111.254 & 1133 & 1133 & 122.5994 & 19.13055 \\
\hline
\end{tabular}




\begin{tabular}{|c|c|c|c|c|c|c|}
\hline 97 & 4.6323 & 1159.701 & 1128 & 1128 & 122.5994 & 19.06038 \\
\hline 98 & 4.6493 & 1117.334 & 1125 & 1125 & 122.5994 & 18.99073 \\
\hline 99 & 4.6663 & 1137.147 & 1123 & 1123 & 122.5994 & 18.92158 \\
\hline 100 & 4.6833 & 1146.564 & 1120 & 1120 & 122.5994 & 18.85293 \\
\hline 101 & 4.7003 & 1125.938 & 1118 & 1118 & 122.5994 & 18.78479 \\
\hline 102 & 4.7173 & 1110.034 & 1115 & 1115 & 122.5994 & 18.71713 \\
\hline 103 & 4.7343 & 1124.746 & 1112 & 1112 & 122.5994 & 18.64996 \\
\hline 104 & 4.7513 & 1158.428 & 1110 & 1110 & 122.5994 & 18.58327 \\
\hline 105 & 4.7683 & 1132.874 & 1107 & 1107 & 122.5994 & 18.51705 \\
\hline 106 & 4.7853 & 1095.417 & 1105 & 1105 & 122.5994 & 18.45131 \\
\hline 107 & 4.8023 & 1088.858 & 1102 & 1102 & 122.5994 & 18.38603 \\
\hline 108 & 4.8193 & 1108.212 & 1100 & 1100 & 122.5994 & 18.32121 \\
\hline 109 & 4.8363 & 1086.561 & 1097 & 1097 & 122.5994 & 18.25685 \\
\hline 110 & 4.8533 & 1108.879 & 1094 & 1094 & 122.5994 & 18.19294 \\
\hline 111 & 4.8703 & 1101.584 & 1092 & 1092 & 122.5994 & 18.12947 \\
\hline 112 & 4.8873 & 1078.831 & 1089 & 1089 & 122.5994 & 18.06645 \\
\hline 113 & 4.9043 & 1078.929 & 1087 & 1087 & 122.5994 & 18.00386 \\
\hline 114 & 4.9213 & 1082.392 & 1084 & 1084 & 122.5994 & 17.94171 \\
\hline 115 & 4.9383 & 1059.218 & 1082 & 1082 & 122.5994 & 17.87998 \\
\hline 116 & 4.9553 & 1087.796 & 1079 & 1079 & 122.5994 & 17.81868 \\
\hline 117 & 4.9723 & 1118.866 & 1076 & 1076 & 122.5994 & 17.7578 \\
\hline 118 & 4.9893 & 1051.753 & 1074 & 1074 & 122.5994 & 17.69733 \\
\hline 119 & 5.0063 & 1009.905 & 1071 & 1071 & 122.5994 & 17.63727 \\
\hline 120 & 5.0233 & 1070.655 & 1069 & 1069 & 122.5994 & 17.57762 \\
\hline 121 & 5.0403 & 1053.009 & 1066 & 1066 & 122.5994 & 17.51837 \\
\hline 122 & 5.0573 & 1088.857 & 1065 & 1065 & 122.5994 & 17.45953 \\
\hline 123 & 5.0743 & 1110.63 & 1064 & 1064 & 122.5994 & 17.40107 \\
\hline 124 & 5.0913 & 1049.334 & 1063 & 1063 & 122.5994 & 17.34301 \\
\hline 125 & 5.1083 & 1049.882 & 1062 & 1062 & 122.5994 & 17.28533 \\
\hline 126 & 5.1253 & 1058.261 & 1061 & 1061 & 122.5994 & 17.22803 \\
\hline 127 & 5.1423 & 1037.896 & 1060 & 1060 & 122.5994 & 17.17112 \\
\hline 128 & 5.1593 & 1054.098 & 1060 & 1060 & 122.5994 & 17.11458 \\
\hline 129 & 5.1763 & 1067.704 & 1059 & 1059 & 122.5994 & 17.05841 \\
\hline 130 & 5.1933 & 1054.132 & 1058 & 1058 & 122.5994 & 17.00261 \\
\hline 131 & 5.2103 & 1003.908 & 1057 & 1057 & 122.5994 & 16.94717 \\
\hline 132 & 5.2273 & 1039.349 & 1056 & 1056 & 122.5994 & 16.89209 \\
\hline 133 & 5.2443 & 1033.493 & 1055 & 1055 & 122.5994 & 16.83737 \\
\hline 134 & 5.2613 & 1086.58 & 1054 & 1054 & 122.5994 & 16.78301 \\
\hline 135 & 5.2783 & 1032.385 & 1053 & 1053 & 122.5994 & 16.72899 \\
\hline 136 & 5.2953 & 1035.606 & 1052 & 1052 & 122.5994 & 16.67532 \\
\hline 137 & 5.3123 & 1007.237 & 1051 & 1051 & 122.5994 & 16.622 \\
\hline 138 & 5.3293 & 1023.02 & 1050 & 1050 & 122.5994 & 16.56901 \\
\hline 139 & 5.3463 & 999.3322 & 1050 & 1050 & 122.5994 & 16.51637 \\
\hline 140 & 5.3633 & 1055.707 & 1049 & 1049 & 122.5994 & 16.46405 \\
\hline 141 & 5.3803 & 1050.07 & 1048 & 1048 & 122.5994 & 16.41207 \\
\hline 142 & 5.3973 & 1073.647 & 1047 & 1047 & 122.5994 & 16.36041 \\
\hline 143 & 5.4143 & 1015.561 & 1046 & 1046 & 122.5994 & 16.30908 \\
\hline 144 & 5.4313 & 1084.982 & 1045 & 1045 & 122.5994 & 16.25807 \\
\hline 145 & 5.4483 & 1034.939 & 1044 & 1044 & 122.5994 & 16.20738 \\
\hline
\end{tabular}




\begin{tabular}{|c|c|c|c|c|c|c|}
\hline 146 & 5.4653 & 1022.261 & 1043 & 1043 & 122.5994 & 16.15701 \\
\hline 147 & 5.4823 & 1047.192 & 1043 & 1043 & 122.5994 & 16.10695 \\
\hline 148 & 5.4993 & 994.2989 & 1042 & 1042 & 122.5994 & 16.05719 \\
\hline 149 & 5.5163 & 1006.08 & 1042 & 1042 & 122.5994 & 16.00775 \\
\hline 150 & 5.5333 & 1011.23 & 1041 & 1041 & 122.5994 & 15.9586 \\
\hline 151 & 5.5503 & 1005.509 & 1041 & 1041 & 122.5994 & 15.90976 \\
\hline 152 & 5.5673 & 993.8298 & 1041.941 & 1040 & 122.5994 & 15.86122 \\
\hline 153 & 5.5843 & 1026.295 & 1043 & 1040 & 122.5994 & 15.81297 \\
\hline 154 & 5.6013 & 1032.987 & 1042.142 & 1039 & 122.5994 & 15.76502 \\
\hline 155 & 5.6183 & 1006.22 & 1042.294 & 1039 & 122.5994 & 15.71735 \\
\hline 156 & 5.6353 & 1036.83 & 1041.458 & 1038 & 122.5994 & 15.66998 \\
\hline 157 & 5.6523 & 1027.732 & 1041.634 & 1038 & 122.5994 & 15.62289 \\
\hline 158 & 5.6693 & 1028.246 & 1040.823 & 1037 & 122.5994 & 15.57608 \\
\hline 159 & 5.6863 & 1028.154 & 1040.028 & 1036 & 122.5994 & 15.52955 \\
\hline 160 & 5.7033 & 1021.069 & 1040.25 & 1036 & 122.5994 & 15.4833 \\
\hline 161 & 5.7203 & 1037.974 & 1039.49 & 1035 & 122.5994 & 15.43732 \\
\hline 162 & 5.7373 & 1028.346 & 1039.751 & 1035 & 122.5994 & 15.39162 \\
\hline 163 & 5.7543 & 1065.951 & 1039.035 & 1034 & 122.5994 & 15.34618 \\
\hline 164 & 5.7713 & 1056.093 & 1039.345 & 1034 & 122.5994 & 15.30102 \\
\hline 165 & 5.7883 & 1033.082 & 1038.684 & 1033 & 122.5994 & 15.25612 \\
\hline 166 & 5.8053 & 1059.782 & 1039.057 & 1033 & 122.5994 & 15.21148 \\
\hline 167 & 5.8223 & 1077.721 & 1038.466 & 1032 & 122.5994 & 15.16711 \\
\hline 168 & 5.8393 & 1061.544 & 1038.919 & 1032 & 122.5994 & 15.12299 \\
\hline 169 & 5.8563 & 1032.516 & 1038.42 & 1031 & 122.5994 & 15.07913 \\
\hline 170 & 5.8733 & 1009.507 & 1037.976 & 1030 & 122.5994 & 15.03552 \\
\hline 171 & 5.8903 & 1004.88 & 1038.596 & 1030 & 122.5994 & 14.99216 \\
\hline 172 & 5.9073 & 1035.388 & 1038.291 & 1029 & 122.5994 & 14.94906 \\
\hline 173 & 5.9243 & 1018.305 & 1039.072 & 1029 & 122.5994 & 14.9062 \\
\hline 174 & 5.9413 & 1056.158 & 1038.954 & 1028 & 122.5994 & 14.86358 \\
\hline 175 & 5.9583 & 1075.829 & 1039.956 & 1028 & 122.5994 & 14.82121 \\
\hline 176 & 5.9753 & 1075.829 & 1040.105 & 1027 & 122.5994 & 14.77909 \\
\hline 177 & 5.9923 & 1015.05 & 1041.433 & 1027 & 122.5994 & 14.7372 \\
\hline 178 & 6.0093 & 998.5501 & 1041.99 & 1026 & 122.5994 & 14.69554 \\
\hline 179 & 6.0263 & 1068.136 & 1043.852 & 1026 & 122.5994 & 14.65413 \\
\hline 180 & 6.0433 & 1076.836 & 1045.133 & 1025 & 122.5994 & 14.61294 \\
\hline 181 & 6.0603 & 1104.68 & 1047.014 & 1024 & 122.5994 & 14.57199 \\
\hline 182 & 6.0773 & 1055.991 & 1050.767 & 1024 & 122.5994 & 14.53126 \\
\hline 183 & 6.0943 & 1110.051 & 1054.794 & 1023 & 122.5994 & 14.49077 \\
\hline 184 & 6.1113 & 1109.296 & 1061.659 & 1023 & 122.5994 & 14.4505 \\
\hline 185 & 6.1283 & 1173.706 & 1070.099 & 1022 & 122.5994 & 14.41045 \\
\hline 186 & 6.1453 & 1163.098 & 1083.011 & 1022 & 122.5994 & 14.37062 \\
\hline 187 & 6.1623 & 1069.359 & 1099.377 & 1021 & 122.5994 & 14.33102 \\
\hline 188 & 6.1793 & 1109.86 & 1122.126 & 1021 & 122.5994 & 14.29163 \\
\hline 189 & 6.1963 & 1150.737 & 1149.941 & 1020 & 122.5994 & 14.25246 \\
\hline 190 & 6.2133 & 1188.466 & 1185.007 & 1020 & 122.5994 & 14.2135 \\
\hline 191 & 6.2303 & 1265.974 & 1224.721 & 1019 & 122.5994 & 14.17475 \\
\hline 192 & 6.2473 & 1324.947 & 1269.352 & 1019 & 122.5994 & 14.13622 \\
\hline 193 & 6.2643 & 1316.463 & 1313.621 & 1018 & 122.5994 & 14.0979 \\
\hline 194 & 6.2813 & 1263.539 & 1353.351 & 1017 & 122.5994 & 14.05978 \\
\hline
\end{tabular}




\begin{tabular}{|c|c|c|c|c|c|c|}
\hline 195 & 6.2983 & 1304.636 & 1381.782 & 1016 & 122.5994 & 14.02187 \\
\hline 196 & 6.3153 & 1407.931 & 1393.478 & 1016 & 122.5994 & 13.98416 \\
\hline 197 & 6.3323 & 1390.325 & 1383.486 & 1015 & 122.5994 & 13.94666 \\
\hline 198 & 6.3493 & 1411.953 & 1355.138 & 1014 & 122.5994 & 13.90935 \\
\hline 199 & 6.3663 & 1396.583 & 1315.573 & 1014 & 122.5994 & 13.87225 \\
\hline 200 & 6.3833 & 1340.35 & 1269.621 & 1013 & 122.5994 & 13.83534 \\
\hline 201 & 6.4003 & 1221.314 & 1223.701 & 1012 & 122.5994 & 13.79863 \\
\hline 202 & 6.4173 & 1126.51 & 1181.334 & 1011 & 122.5994 & 13.76212 \\
\hline 203 & 6.4343 & 1139.863 & 1144.445 & 1010 & 122.5994 & 13.72579 \\
\hline 204 & 6.4513 & 1100.359 & 1114.769 & 1010 & 122.5994 & 13.68966 \\
\hline 205 & 6.4683 & 1080.74 & 1090.216 & 1009 & 122.5994 & 13.65372 \\
\hline 206 & 6.4853 & 1084.586 & 1071.157 & 1008 & 122.5994 & 13.61797 \\
\hline 207 & 6.5023 & 1022.166 & 1057.685 & 1008 & 122.5994 & 13.5824 \\
\hline 208 & 6.5193 & 1011.999 & 1046.816 & 1007 & 122.5994 & 13.54702 \\
\hline 209 & 6.5363 & 1031.072 & 1038.637 & 1006 & 122.5994 & 13.51183 \\
\hline 210 & 6.5533 & 1027.155 & 1032.389 & 1005 & 122.5994 & 13.47682 \\
\hline 211 & 6.5703 & 1067.327 & 1027.483 & 1004 & 122.5994 & 13.44198 \\
\hline 212 & 6.5873 & 1050.045 & 1024.498 & 1004 & 122.5994 & 13.40733 \\
\hline 213 & 6.6043 & 1043.821 & 1021.144 & 1003 & 122.5994 & 13.37286 \\
\hline 214 & 6.6213 & 1035.468 & 1018.231 & 1002 & 122.5994 & 13.33856 \\
\hline 215 & 6.6383 & 999.7509 & 1016.635 & 1002 & 122.5994 & 13.30444 \\
\hline 216 & 6.6553 & 992.2685 & 1014.278 & 1001 & 122.5994 & 13.2705 \\
\hline 217 & 6.6723 & 1004.537 & 1012.107 & 1000 & 122.5994 & 13.23672 \\
\hline 218 & 6.6893 & 1002.747 & 1010.086 & 999 & 122.5994 & 13.20312 \\
\hline 219 & 6.7063 & 1002.95 & 1008.188 & 998 & 122.5994 & 13.16969 \\
\hline 220 & 6.7233 & 1004.88 & 1006.394 & 997 & 122.5994 & 13.13643 \\
\hline 221 & 6.7403 & 990.9934 & 1004.688 & 996 & 122.5994 & 13.10334 \\
\hline 222 & 6.7573 & 976.5245 & 1003.058 & 995 & 122.5994 & 13.07041 \\
\hline 223 & 6.7743 & 1007.733 & 1001.494 & 994 & 122.5994 & 13.03765 \\
\hline 224 & 6.7913 & 977.3745 & 999.9855 & 993 & 122.5994 & 13.00505 \\
\hline 225 & 6.8083 & 1024.301 & 998.5268 & 992 & 122.5994 & 12.97261 \\
\hline 226 & 6.8253 & 967.829 & 997.1114 & 991 & 122.5994 & 12.94034 \\
\hline 227 & 6.8423 & 1042.31 & 995.7341 & 990 & 122.5994 & 12.90823 \\
\hline 228 & 6.8593 & 1022.104 & 995.3903 & 990 & 122.5994 & 12.87627 \\
\hline 229 & 6.8763 & 1000.346 & 994.0763 & 989 & 122.5994 & 12.84448 \\
\hline 230 & 6.8933 & 975.2584 & 992.7888 & 988 & 122.5994 & 12.81284 \\
\hline 231 & 6.9103 & 997.2909 & 991.5248 & 987 & 122.5994 & 12.78136 \\
\hline 232 & 6.9273 & 962.1071 & 990.2819 & 986 & 122.5994 & 12.75003 \\
\hline 233 & 6.9443 & 973.328 & 989.0579 & 985 & 122.5994 & 12.71886 \\
\hline 234 & 6.9613 & 919.4884 & 987.8509 & 984 & 122.5994 & 12.68783 \\
\hline 235 & 6.9783 & 1020.728 & 986.6592 & 983 & 122.5994 & 12.65696 \\
\hline 236 & 6.9953 & 933.7599 & 985.4815 & 982 & 122.5994 & 12.62624 \\
\hline 237 & 7.0123 & 988.3669 & 984.3163 & 981 & 122.5994 & 12.59567 \\
\hline 238 & 7.0293 & 947.4524 & 983.1626 & 980 & 122.5994 & 12.56525 \\
\hline 239 & 7.0463 & 992.2401 & 982.0192 & 979 & 122.5994 & 12.53497 \\
\hline 240 & 7.0633 & 992.2401 & 978.9854 & 978 & 122.5994 & 12.50484 \\
\hline 241 & 7.0803 & 991.2375 & 977 & 977 & 122.5994 & 12.47485 \\
\hline 242 & 7.0973 & 982.4734 & 976 & 976 & 122.5994 & 12.44501 \\
\hline 243 & 7.1143 & 964.6344 & 975 & 975 & 122.5994 & 12.41531 \\
\hline
\end{tabular}




\begin{tabular}{|c|c|c|c|c|c|c|}
\hline 244 & 7.1313 & 959.0842 & 974 & 974 & 122.5994 & 12.38575 \\
\hline 245 & 7.1483 & 992.5083 & 972 & 972 & 122.5994 & 12.35633 \\
\hline 246 & 7.1653 & 1034.137 & 971 & 971 & 122.5994 & 12.32706 \\
\hline 247 & 7.1823 & 979.4902 & 970 & 970 & 122.5994 & 12.29792 \\
\hline 248 & 7.1993 & 956.4307 & 969 & 969 & 122.5994 & 12.26892 \\
\hline 249 & 7.2163 & 979.751 & 968 & 968 & 122.5994 & 12.24005 \\
\hline 250 & 7.2333 & 983.3152 & 967 & 967 & 122.5994 & 12.21132 \\
\hline 251 & 7.2503 & 993.5949 & 966 & 966 & 122.5994 & 12.18273 \\
\hline 252 & 7.2673 & 1006.45 & 964 & 964 & 122.5994 & 12.15427 \\
\hline 253 & 7.2843 & 955.8007 & 963 & 963 & 122.5994 & 12.12594 \\
\hline 254 & 7.3013 & 956.8685 & 962 & 962 & 122.5994 & 12.09774 \\
\hline 255 & 7.3183 & 944.3535 & 961 & 961 & 122.5994 & 12.06968 \\
\hline 256 & 7.3353 & 942.0118 & 960 & 960 & 122.5994 & 12.04175 \\
\hline 257 & 7.3523 & 970.3662 & 959 & 959 & 122.5994 & 12.01394 \\
\hline 258 & 7.3693 & 983.3934 & 957 & 957 & 122.5994 & 11.98627 \\
\hline 259 & 7.3863 & 954.737 & 956 & 956 & 122.5994 & 11.95872 \\
\hline 260 & 7.4033 & 927.6386 & 955 & 955 & 122.5994 & 11.93129 \\
\hline 261 & 7.4203 & 944.8621 & 954 & 954 & 122.5994 & 11.904 \\
\hline 262 & 7.4373 & 958.612 & 953 & 953 & 122.5994 & 11.87683 \\
\hline 263 & 7.4543 & 964.9478 & 952 & 952 & 122.5994 & 11.84978 \\
\hline 264 & 7.4713 & 959.059 & 951 & 951 & 122.5994 & 11.82285 \\
\hline 265 & 7.4883 & 963.2362 & 949 & 949 & 122.5994 & 11.79605 \\
\hline 266 & 7.5053 & 949.8517 & 948 & 948 & 122.5994 & 11.76937 \\
\hline 267 & 7.5223 & 973.9513 & 947 & 947 & 122.5994 & 11.74281 \\
\hline 268 & 7.5393 & 967.3588 & 945 & 945 & 122.5994 & 11.71637 \\
\hline 269 & 7.5563 & 946.8052 & 944 & 944 & 122.5994 & 11.69005 \\
\hline 270 & 7.5733 & 923.2831 & 942 & 942 & 122.5994 & 11.66385 \\
\hline 271 & 7.5903 & 952.812 & 941 & 941 & 122.5994 & 11.63776 \\
\hline 272 & 7.6073 & 945.6104 & 940 & 940 & 122.5994 & 11.61179 \\
\hline 273 & 7.6243 & 937.2822 & 938 & 938 & 122.5994 & 11.58594 \\
\hline 274 & 7.6413 & 968.7565 & 937 & 937 & 122.5994 & 11.5602 \\
\hline 275 & 7.6583 & 934.7558 & 936 & 936 & 122.5994 & 11.53458 \\
\hline 276 & 7.6753 & 924.683 & 934 & 934 & 122.5994 & 11.50907 \\
\hline 277 & 7.6923 & 918.4542 & 933 & 933 & 122.5994 & 11.48367 \\
\hline 278 & 7.7093 & 900.7145 & 931 & 931 & 122.5994 & 11.45839 \\
\hline 279 & 7.7263 & 953.8146 & 930 & 930 & 122.5994 & 11.43321 \\
\hline 280 & 7.7433 & 906.1114 & 929 & 929 & 122.5994 & 11.40815 \\
\hline 281 & 7.7603 & 943.1148 & 927 & 927 & 122.5994 & 11.3832 \\
\hline 282 & 7.7773 & 963.8281 & 926 & 926 & 122.5994 & 11.35835 \\
\hline 283 & 7.7943 & 899.3965 & 925 & 925 & 122.5994 & 11.33362 \\
\hline 284 & 7.8113 & 965.29 & 923 & 923 & 122.5994 & 11.30899 \\
\hline 285 & 7.8283 & 880.7715 & 922 & 922 & 122.5994 & 11.28447 \\
\hline 286 & 7.8453 & 879.0028 & 920 & 920 & 122.5994 & 11.26006 \\
\hline 287 & 7.8623 & 933.5433 & 919 & 919 & 122.5994 & 11.23575 \\
\hline 288 & 7.8793 & 920.2109 & 923.0435 & 918 & 122.5994 & 11.21154 \\
\hline 289 & 7.8963 & 932.675 & 923.7014 & 916 & 122.5994 & 11.18745 \\
\hline 290 & 7.9133 & 950.5979 & 922.9694 & 915 & 122.5994 & 11.16345 \\
\hline 291 & 7.9303 & 967.8289 & 922.2516 & 914 & 122.5994 & 11.13956 \\
\hline 292 & 7.9473 & 946.4647 & 920.549 & 912 & 122.5994 & 11.11577 \\
\hline
\end{tabular}




\begin{tabular}{|c|c|c|c|c|c|c|}
\hline 293 & 7.9643 & 966.1561 & 919.8626 & 911 & 122.5994 & 11.09208 \\
\hline 294 & 7.9813 & 905.4951 & 918.1937 & 909 & 122.5994 & 11.06849 \\
\hline 295 & 7.9983 & 920.0583 & 917.5436 & 908 & 122.5994 & 11.045 \\
\hline 296 & 8.0153 & 934.8221 & 916.9137 & 907 & 122.5994 & 11.02162 \\
\hline 297 & 8.0323 & 954.8278 & 915.3057 & 905 & 122.5994 & 10.99833 \\
\hline 298 & 8.0493 & 945.187 & 914.7211 & 904 & 122.5994 & 10.97514 \\
\hline 299 & 8.0663 & 920.2842 & 914.162 & 903 & 122.5994 & 10.95204 \\
\hline 300 & 8.0833 & 887.9765 & 912.6304 & 901 & 122.5994 & 10.92905 \\
\hline 301 & 8.1003 & 891.5273 & 912.1287 & 900 & 122.5994 & 10.90615 \\
\hline 302 & 8.1173 & 974.7944 & 910.6594 & 898 & 122.5994 & 10.88335 \\
\hline 303 & 8.1343 & 1020.884 & 910.2254 & 897 & 122.5994 & 10.86064 \\
\hline 304 & 8.1513 & 988.3314 & 909.8298 & 896 & 122.5994 & 10.83803 \\
\hline 305 & 8.1683 & 973.9791 & 908.4763 & 894 & 122.5994 & 10.81551 \\
\hline 306 & 8.1853 & 948.4739 & 908.1686 & 893 & 122.5994 & 10.79309 \\
\hline 307 & 8.2023 & 949.2636 & 907.9112 & 892 & 122.5994 & 10.77075 \\
\hline 308 & 8.2193 & 963.468 & 906.7091 & 890 & 122.5994 & 10.74852 \\
\hline 309 & 8.2363 & 953.2679 & 906.5677 & 889 & 122.5994 & 10.72637 \\
\hline 310 & 8.2533 & 952.6046 & 905.4934 & 887 & 122.5994 & 10.70431 \\
\hline 311 & 8.2703 & 946.573 & 905.4932 & 886 & 122.5994 & 10.68235 \\
\hline 312 & 8.2873 & 946.7608 & 905.5751 & 885 & 122.5994 & 10.66047 \\
\hline 313 & 8.3043 & 953.0029 & 904.7482 & 883 & 122.5994 & 10.63869 \\
\hline 314 & 8.3213 & 963.9367 & 905.0231 & 882 & 122.5994 & 10.61699 \\
\hline 315 & 8.3383 & 1000.52 & 904.4115 & 880 & 122.5994 & 10.59538 \\
\hline 316 & 8.3553 & 962.5776 & 903.9277 & 878 & 122.5994 & 10.57386 \\
\hline 317 & 8.3723 & 993.5547 & 904.5878 & 877 & 122.5994 & 10.55243 \\
\hline 318 & 8.3893 & 1025.852 & 904.4114 & 875 & 122.5994 & 10.53109 \\
\hline 319 & 8.4063 & 987.7127 & 905.4221 & 874 & 122.5994 & 10.50983 \\
\hline 320 & 8.4233 & 958.1638 & 905.6492 & 872 & 122.5994 & 10.48865 \\
\hline 321 & 8.4403 & 987.9704 & 906.1307 & 870 & 122.5994 & 10.46757 \\
\hline 322 & 8.4573 & 1021.058 & 907.9158 & 869 & 122.5994 & 10.44656 \\
\hline 323 & 8.4743 & 1047.872 & 909.0713 & 867 & 122.5994 & 10.42565 \\
\hline 324 & 8.4913 & 1049.78 & 911.6884 & 866 & 122.5994 & 10.40481 \\
\hline 325 & 8.5083 & 1081.493 & 913.8932 & 864 & 122.5994 & 10.38406 \\
\hline 326 & 8.5253 & 1024.38 & 916.8616 & 862 & 122.5994 & 10.36339 \\
\hline 327 & 8.5423 & 1175.831 & 921.8339 & 861 & 122.5994 & 10.34281 \\
\hline 328 & 8.5593 & 1131.856 & 927.1362 & 859 & 122.5994 & 10.3223 \\
\hline 329 & 8.5763 & 1211.063 & 935.1994 & 858 & 122.5994 & 10.30188 \\
\hline 330 & 8.5933 & 1206.721 & 944.5777 & 856 & 122.5994 & 10.28154 \\
\hline 331 & 8.6103 & 1162.88 & 956.9622 & 854 & 122.5994 & 10.26128 \\
\hline 332 & 8.6273 & 1227.594 & 974.1769 & 853 & 122.5994 & 10.24109 \\
\hline 333 & 8.6443 & 1249.164 & 995.1625 & 851 & 122.5994 & 10.22099 \\
\hline 334 & 8.6613 & 1278.012 & 1022.933 & 850 & 122.5994 & 10.20097 \\
\hline 335 & 8.6783 & 1332.275 & 1056.506 & 848 & 122.5994 & 10.18102 \\
\hline 336 & 8.6953 & 1332.275 & 1097.808 & 846 & 122.5994 & 10.16116 \\
\hline 337 & 8.7123 & 1352.629 & 1148.546 & 845 & 122.5994 & 10.14137 \\
\hline 338 & 8.7293 & 1408.193 & 1207.07 & 843 & 122.5994 & 10.12166 \\
\hline 339 & 8.7463 & 1387.396 & 1274.213 & 841 & 122.5994 & 10.10202 \\
\hline 340 & 8.7633 & 1463.52 & 1349.108 & 839 & 122.5994 & 10.08246 \\
\hline 341 & 8.7803 & 1556.363 & 1430.009 & 837 & 122.5994 & 10.06298 \\
\hline
\end{tabular}




\begin{tabular}{|c|c|c|c|c|c|c|}
\hline 342 & 8.7973 & 1649.076 & 1515.019 & 836 & 122.5994 & 10.04357 \\
\hline 343 & 8.8143 & 1699.14 & 1597.856 & 834 & 122.5994 & 10.02424 \\
\hline 344 & 8.8313 & 1730.036 & 1673.716 & 832 & 122.5994 & 10.00498 \\
\hline 345 & 8.8483 & 1893.527 & 1735.566 & 830 & 122.5994 & 9.9858 \\
\hline 346 & 8.8653 & 2044.012 & 1776.144 & 828 & 122.5994 & 9.96669 \\
\hline 347 & 8.8823 & 2190.262 & 1789.768 & 826 & 122.5994 & 9.94765 \\
\hline 348 & 8.8993 & 2312.391 & 1774.195 & 824 & 122.5994 & 9.92869 \\
\hline 349 & 8.9163 & 2468.52 & 1732.44 & 823 & 122.5994 & 9.90979 \\
\hline 350 & 8.9333 & 2739.082 & 1667.998 & 821 & 122.5994 & 9.89097 \\
\hline 351 & 8.9503 & 2995.719 & 1589.036 & 819 & 122.5994 & 9.87222 \\
\hline 352 & 8.9673 & 3052.448 & 1502.609 & 817 & 122.5994 & 9.85355 \\
\hline 353 & 8.9843 & 2813.681 & 1414.6 & 815 & 122.5994 & 9.83494 \\
\hline 354 & 9.0013 & 2271.26 & 1329.403 & 813 & 122.5994 & 9.8164 \\
\hline 355 & 9.0183 & 1675.133 & 1251.002 & 812 & 122.5994 & 9.79794 \\
\hline 356 & 9.0353 & 1288.723 & 1179.243 & 810 & 122.5994 & 9.77954 \\
\hline 357 & 9.0523 & 1095.232 & 1116.052 & 808 & 122.5994 & 9.76121 \\
\hline 358 & 9.0693 & 1036.537 & 1061.645 & 806 & 122.5994 & 9.74295 \\
\hline 359 & 9.0863 & 994.7823 & 1015.711 & 804 & 122.5994 & 9.72476 \\
\hline 360 & 9.1033 & 966.1701 & 977.5606 & 802 & 122.5994 & 9.70664 \\
\hline 361 & 9.1203 & 902.1599 & 946.2866 & 800 & 122.5994 & 9.68859 \\
\hline 362 & 9.1373 & 928.4049 & 920.8752 & 798 & 122.5994 & 9.6706 \\
\hline 363 & 9.1543 & 932.8515 & 900.3106 & 796 & 122.5994 & 9.65268 \\
\hline 364 & 9.1713 & 862.6446 & 883.6464 & 794 & 122.5994 & 9.63482 \\
\hline 365 & 9.1883 & 846.7773 & 870.0474 & 792 & 122.5994 & 9.61704 \\
\hline 366 & 9.2053 & 841.8673 & 858.8143 & 790 & 122.5994 & 9.59931 \\
\hline 367 & 9.2223 & 815.8599 & 849.3828 & 788 & 122.5994 & 9.58166 \\
\hline 368 & 9.2393 & 802.0348 & 841.3124 & 786 & 122.5994 & 9.56407 \\
\hline 369 & 9.2563 & 821.8353 & 833.27 & 783 & 122.5994 & 9.54654 \\
\hline 370 & 9.2733 & 856.4011 & 827.0081 & 781 & 122.5994 & 9.52908 \\
\hline 371 & 9.2903 & 825.0137 & 821.347 & 779 & 122.5994 & 9.51168 \\
\hline 372 & 9.3073 & 840.7132 & 816.1569 & 777 & 122.5994 & 9.49434 \\
\hline 373 & 9.3243 & 772.9235 & 811.344 & 775 & 122.5994 & 9.47707 \\
\hline 374 & 9.3413 & 800.0456 & 806.8395 & 773 & 122.5994 & 9.45986 \\
\hline 375 & 9.3583 & 768.0916 & 802.593 & 771 & 122.5994 & 9.44272 \\
\hline 376 & 9.3753 & 782.8236 & 798.5658 & 769 & 122.5994 & 9.42563 \\
\hline 377 & 9.3923 & 763.3678 & 793.728 & 766 & 122.5994 & 9.40861 \\
\hline 378 & 9.4093 & 732.2524 & 790.0555 & 764 & 122.5994 & 9.39165 \\
\hline 379 & 9.4263 & 794.9159 & 786.5284 & 762 & 122.5994 & 9.37475 \\
\hline 380 & 9.4433 & 755.415 & 783.1301 & 760 & 122.5994 & 9.35791 \\
\hline 381 & 9.4603 & 752.7123 & 779.8466 & 758 & 122.5994 & 9.34113 \\
\hline 382 & 9.4773 & 767.2361 & 776.6656 & 756 & 122.5994 & 9.32441 \\
\hline 383 & 9.4943 & 785.9485 & 773.5768 & 754 & 122.5994 & 9.30776 \\
\hline 384 & 9.5113 & 759.7093 & 770.5707 & 752 & 122.5994 & 9.29116 \\
\hline 385 & 9.5283 & 725.3056 & 766.6393 & 749 & 122.5994 & 9.27462 \\
\hline 386 & 9.5453 & 754.7022 & 763.7755 & 747 & 122.5994 & 9.25814 \\
\hline 387 & 9.5623 & 775.0296 & 760.973 & 745 & 122.5994 & 9.24172 \\
\hline 388 & 9.5793 & 754.748 & 757.2261 & 742 & 122.5994 & 9.22536 \\
\hline 389 & 9.5963 & 719.6963 & 754.5299 & 740 & 122.5994 & 9.20905 \\
\hline 390 & 9.6133 & 732.4982 & 750.88 & 737 & 122.5994 & 9.19281 \\
\hline
\end{tabular}




\begin{tabular}{|c|c|c|c|c|c|c|}
\hline 391 & 9.6303 & 770.9621 & 748.2723 & 735 & 122.5994 & 9.17662 \\
\hline 392 & 9.6473 & 753.0406 & 745.7033 & 733 & 122.5994 & 9.16048 \\
\hline 393 & 9.6643 & 746.7769 & 742.1699 & 730 & 122.5994 & 9.14441 \\
\hline 394 & 9.6813 & 722.1415 & 739.6691 & 728 & 122.5994 & 9.12839 \\
\hline 395 & 9.6983 & 712.6719 & 736.1984 & 725 & 122.5994 & 9.11243 \\
\hline 396 & 9.7153 & 729.0306 & 733.7554 & 723 & 122.5994 & 9.09652 \\
\hline 397 & 9.7323 & 734.1701 & 731.338 & 721 & 122.5994 & 9.08067 \\
\hline 398 & 9.7493 & 723.1822 & 727.9442 & 718 & 122.5994 & 9.06487 \\
\hline 399 & 9.7663 & 671.371 & 725.5724 & 716 & 122.5994 & 9.04913 \\
\hline 400 & 9.7833 & 652.9155 & 723.2209 & 714 & 122.5994 & 9.03344 \\
\hline 401 & 9.8003 & 704.0805 & 719.8884 & 711 & 122.5994 & 9.01781 \\
\hline 402 & 9.8173 & 715.5485 & 717.5734 & 709 & 122.5994 & 9.00224 \\
\hline 403 & 9.8343 & 725.0143 & 714.2748 & 706 & 122.5994 & 8.98671 \\
\hline 404 & 9.8513 & 702.1913 & 711.9914 & 704 & 122.5994 & 8.97124 \\
\hline 405 & 9.8683 & 704.7717 & 709.7222 & 702 & 122.5994 & 8.95583 \\
\hline 406 & 9.8853 & 694.0148 & 701.5532 & 699 & 122.5994 & 8.94046 \\
\hline 407 & 9.9023 & 690.2754 & 699.4688 & 697 & 122.5994 & 8.92515 \\
\hline 408 & 9.9193 & 691.7029 & 694 & 694 & 122.5994 & 8.90989 \\
\hline 409 & 9.9363 & 683.5425 & 692 & 692 & 122.5994 & 8.89469 \\
\hline 410 & 9.9533 & 698.0188 & 690 & 690 & 122.5994 & 8.87953 \\
\hline 411 & 9.9703 & 706.6263 & 688 & 688 & 122.5994 & 8.86443 \\
\hline 412 & 9.9873 & 680.9613 & 686 & 686 & 122.5994 & 8.84938 \\
\hline 413 & 10.0043 & 671.4861 & 684 & 684 & 122.5994 & 8.83438 \\
\hline 414 & 10.0213 & 680.0393 & 682 & 682 & 122.5994 & 8.81943 \\
\hline 415 & 10.0383 & 671.782 & 680 & 680 & 122.5994 & 8.80454 \\
\hline 416 & 10.0553 & 734.9296 & 679 & 679 & 122.5994 & 8.78969 \\
\hline 417 & 10.0723 & 664.4098 & 677 & 677 & 122.5994 & 8.77489 \\
\hline 418 & 10.0893 & 630.8663 & 675 & 675 & 122.5994 & 8.76014 \\
\hline 419 & 10.1063 & 625.0068 & 673 & 673 & 122.5994 & 8.74545 \\
\hline 420 & 10.1233 & 660.6607 & 671 & 671 & 122.5994 & 8.7308 \\
\hline 421 & 10.1403 & 672.5305 & 669 & 669 & 122.5994 & 8.7162 \\
\hline 422 & 10.1573 & 713.9889 & 667 & 667 & 122.5994 & 8.70165 \\
\hline 423 & 10.1743 & 646.8082 & 665 & 665 & 122.5994 & 8.68715 \\
\hline 424 & 10.1913 & 664.5722 & 663 & 663 & 122.5994 & 8.6727 \\
\hline 425 & 10.2083 & 654.1186 & 661 & 661 & 122.5994 & 8.65829 \\
\hline 426 & 10.2253 & 648.8769 & 659 & 659 & 122.5994 & 8.64394 \\
\hline 427 & 10.2423 & 669.0719 & 658 & 658 & 122.5994 & 8.62963 \\
\hline 428 & 10.2593 & 663.4097 & 656 & 656 & 122.5994 & 8.61537 \\
\hline 429 & 10.2763 & 669.5675 & 654 & 654 & 122.5994 & 8.60115 \\
\hline 430 & 10.2933 & 637.0528 & 652 & 652 & 122.5994 & 8.58698 \\
\hline 431 & 10.3103 & 616.3887 & 650 & 650 & 122.5994 & 8.57286 \\
\hline 432 & 10.3273 & 637.82 & 648 & 648 & 122.5994 & 8.55879 \\
\hline 433 & 10.3443 & 646.6367 & 646 & 646 & 122.5994 & 8.54476 \\
\hline 434 & 10.3613 & 663.3798 & 645 & 645 & 122.5994 & 8.53078 \\
\hline 435 & 10.3783 & 674.258 & 645 & 645 & 122.5994 & 8.51685 \\
\hline 436 & 10.3953 & 652.977 & 644 & 644 & 122.5994 & 8.50296 \\
\hline 437 & 10.4123 & 681.992 & 643 & 643 & 122.5994 & 8.48911 \\
\hline 438 & 10.4293 & 679.141 & 642 & 642 & 122.5994 & 8.47531 \\
\hline 439 & 10.4463 & 671.47 & 642 & 642 & 122.5994 & 8.46156 \\
\hline
\end{tabular}




\begin{tabular}{|c|c|c|c|c|c|c|}
\hline 440 & 10.4633 & 670.3251 & 641 & 641 & 122.5994 & 8.44785 \\
\hline 441 & 10.4803 & 659.8673 & 640 & 640 & 122.5994 & 8.43418 \\
\hline 442 & 10.4973 & 663.2344 & 640 & 640 & 122.5994 & 8.42056 \\
\hline 443 & 10.5143 & 673.9074 & 639 & 639 & 122.5994 & 8.40699 \\
\hline 444 & 10.5313 & 720.2033 & 638 & 638 & 122.5994 & 8.39345 \\
\hline 445 & 10.5483 & 721.4515 & 638 & 638 & 122.5994 & 8.37996 \\
\hline 446 & 10.5653 & 702.2631 & 637 & 637 & 122.5994 & 8.36652 \\
\hline 447 & 10.5823 & 724.6769 & 636 & 636 & 122.5994 & 8.35312 \\
\hline 448 & 10.5993 & 710.1065 & 635 & 635 & 122.5994 & 8.33976 \\
\hline 449 & 10.6163 & 712.0692 & 635 & 635 & 122.5994 & 8.32644 \\
\hline 450 & 10.6333 & 713.0174 & 634 & 634 & 122.5994 & 8.31317 \\
\hline 451 & 10.6503 & 675.4549 & 633 & 633 & 122.5994 & 8.29994 \\
\hline 452 & 10.6673 & 649.6748 & 633 & 633 & 122.5994 & 8.28675 \\
\hline 453 & 10.6843 & 623.3241 & 632 & 632 & 122.5994 & 8.2736 \\
\hline 454 & 10.7013 & 615.0515 & 631 & 631 & 122.5994 & 8.26049 \\
\hline 455 & 10.7183 & 649.939 & 630 & 630 & 122.5994 & 8.24743 \\
\hline 456 & 10.7353 & 636.4258 & 630 & 630 & 122.5994 & 8.23441 \\
\hline 457 & 10.7523 & 621.9073 & 629 & 629 & 122.5994 & 8.22143 \\
\hline 458 & 10.7693 & 632.5049 & 629 & 629 & 122.5994 & 8.20849 \\
\hline 459 & 10.7863 & 613.7331 & 629 & 629 & 122.5994 & 8.19559 \\
\hline 460 & 10.8033 & 640.8226 & 629 & 629 & 122.5994 & 8.18273 \\
\hline 461 & 10.8203 & 658.8933 & 629 & 629 & 122.5994 & 8.16991 \\
\hline 462 & 10.8373 & 669.9865 & 629 & 629 & 122.5994 & 8.15713 \\
\hline 463 & 10.8543 & 618.3685 & 629 & 629 & 122.5994 & 8.1444 \\
\hline 464 & 10.8713 & 648.889 & 629 & 629 & 122.5994 & 8.1317 \\
\hline 465 & 10.8883 & 615.6312 & 629 & 629 & 122.5994 & 8.11904 \\
\hline 466 & 10.9053 & 600.3519 & 629 & 629 & 122.5994 & 8.10642 \\
\hline 467 & 10.9223 & 626.7848 & 629 & 629 & 122.5994 & 8.09384 \\
\hline 468 & 10.9393 & 688.7348 & 629 & 629 & 122.5994 & 8.0813 \\
\hline 469 & 10.9563 & 628.9438 & 629 & 629 & 122.5994 & 8.0688 \\
\hline 470 & 10.9733 & 616.8742 & 629 & 629 & 122.5994 & 8.05634 \\
\hline 471 & 10.9903 & 632.2408 & 629 & 629 & 122.5994 & 8.04392 \\
\hline 472 & 11.0073 & 610.7305 & 629 & 629 & 122.5994 & 8.03153 \\
\hline 473 & 11.0243 & 615.8252 & 629 & 629 & 122.5994 & 8.01919 \\
\hline 474 & 11.0413 & 621.7938 & 629 & 629 & 122.5994 & 8.00688 \\
\hline 475 & 11.0583 & 638.0924 & 629 & 629 & 122.5994 & 7.99461 \\
\hline 476 & 11.0753 & 656.3645 & 629 & 629 & 122.5994 & 7.98237 \\
\hline 477 & 11.0923 & 624.8219 & 629 & 629 & 122.5994 & 7.97018 \\
\hline 478 & 11.1093 & 668.9263 & 629 & 629 & 122.5994 & 7.95802 \\
\hline 479 & 11.1263 & 627.1837 & 629 & 629 & 122.5994 & 7.9459 \\
\hline 480 & 11.1433 & 648.2964 & 629 & 629 & 122.5994 & 7.93381 \\
\hline 481 & 11.1603 & 643.7473 & 629 & 629 & 122.5994 & 7.92177 \\
\hline 482 & 11.1773 & 608.0153 & 629 & 629 & 122.5994 & 7.90976 \\
\hline 483 & 11.1943 & 596.5542 & 629 & 629 & 122.5994 & 7.89778 \\
\hline 484 & 11.2113 & 624.8443 & 629 & 629 & 122.5994 & 7.88585 \\
\hline 485 & 11.2283 & 631.7914 & 629 & 629 & 122.5994 & 7.87394 \\
\hline 486 & 11.2453 & 637.6469 & 629 & 629 & 122.5994 & 7.86208 \\
\hline 487 & 11.2623 & 639.6991 & 629 & 629 & 122.5994 & 7.85025 \\
\hline 488 & 11.2793 & 619.8166 & 628 & 628 & 122.5994 & 7.83846 \\
\hline
\end{tabular}




\begin{tabular}{|c|c|c|c|c|c|c|}
\hline 489 & 11.2963 & 617.5574 & 628 & 628 & 122.5994 & 7.8267 \\
\hline 490 & 11.3133 & 615.2746 & 628 & 628 & 122.5994 & 7.81498 \\
\hline 491 & 11.3303 & 609.257 & 628 & 628 & 122.5994 & 7.80329 \\
\hline 492 & 11.3473 & 603.0329 & 628 & 628 & 122.5994 & 7.79164 \\
\hline 493 & 11.3643 & 613.874 & 628 & 628 & 122.5994 & 7.78002 \\
\hline 494 & 11.3813 & 650.8478 & 628 & 628 & 122.5994 & 7.76844 \\
\hline 495 & 11.3983 & 645.1573 & 628 & 628 & 122.5994 & 7.75689 \\
\hline 496 & 11.4153 & 630.0218 & 628 & 628 & 122.5994 & 7.74537 \\
\hline 497 & 11.4323 & 629.9857 & 628 & 628 & 122.5994 & 7.73389 \\
\hline 498 & 11.4493 & 599.2469 & 628 & 628 & 122.5994 & 7.72245 \\
\hline 499 & 11.4663 & 613.7986 & 628 & 628 & 122.5994 & 7.71104 \\
\hline 500 & 11.4833 & 617.6392 & 628 & 628 & 122.5994 & 7.69966 \\
\hline 501 & 11.5003 & 622.6026 & 628 & 628 & 122.5994 & 7.68832 \\
\hline 502 & 11.5173 & 625.0117 & 627 & 627 & 122.5994 & 7.67701 \\
\hline 503 & 11.5343 & 637.0653 & 627 & 627 & 122.5994 & 7.66573 \\
\hline 504 & 11.5513 & 652.6645 & 627 & 627 & 122.5994 & 7.65449 \\
\hline 505 & 11.5683 & 617.1541 & 627 & 627 & 122.5994 & 7.64328 \\
\hline 506 & 11.5853 & 640.4267 & 627 & 627 & 122.5994 & 7.6321 \\
\hline 507 & 11.6023 & 622.858 & 627 & 627 & 122.5994 & 7.62095 \\
\hline 508 & 11.6193 & 642.8773 & 626 & 626 & 122.5994 & 7.60984 \\
\hline 509 & 11.6363 & 598.8085 & 626 & 626 & 122.5994 & 7.59876 \\
\hline 510 & 11.6533 & 554.3396 & 626 & 626 & 122.5994 & 7.58772 \\
\hline 511 & 11.6703 & 603.7588 & 625 & 625 & 122.5994 & 7.5767 \\
\hline 512 & 11.6873 & 629.8574 & 625 & 625 & 122.5994 & 7.56572 \\
\hline 513 & 11.7043 & 586.6243 & 624 & 624 & 122.5994 & 7.55477 \\
\hline 514 & 11.7213 & 643.9956 & 626.8804 & 624 & 122.5994 & 7.54385 \\
\hline 515 & 11.7383 & 616.2388 & 628.3857 & 624 & 122.5994 & 7.53296 \\
\hline 516 & 11.7553 & 635.8706 & 627.5699 & 623 & 122.5994 & 7.52211 \\
\hline 517 & 11.7723 & 642.0514 & 627.7659 & 623 & 122.5994 & 7.51128 \\
\hline 518 & 11.7893 & 613.2188 & 627.9747 & 623 & 122.5994 & 7.50049 \\
\hline 519 & 11.8063 & 608.2769 & 627.1974 & 622 & 122.5994 & 7.48973 \\
\hline 520 & 11.8233 & 655.3347 & 627.4354 & 622 & 122.5994 & 7.479 \\
\hline 521 & 11.8403 & 649.6506 & 627.6899 & 622 & 122.5994 & 7.4683 \\
\hline 522 & 11.8573 & 615.7319 & 626.9627 & 621 & 122.5994 & 7.45763 \\
\hline 523 & 11.8743 & 598.044 & 627.2553 & 621 & 122.5994 & 7.44699 \\
\hline 524 & 11.8913 & 652.7916 & 627.5699 & 621 & 122.5994 & 7.43638 \\
\hline 525 & 11.9083 & 641.0127 & 626.9086 & 620 & 122.5994 & 7.4258 \\
\hline 526 & 11.9253 & 619.7285 & 627.274 & 620 & 122.5994 & 7.41525 \\
\hline 527 & 11.9423 & 596.924 & 627.6689 & 620 & 122.5994 & 7.40474 \\
\hline 528 & 11.9593 & 618.9486 & 627.0964 & 619 & 122.5994 & 7.39425 \\
\hline 529 & 11.9763 & 664.6554 & 627.5604 & 619 & 122.5994 & 7.38379 \\
\hline 530 & 11.9933 & 663.581 & 627.065 & 618 & 122.5994 & 7.37336 \\
\hline 531 & 12.0103 & 645.3245 & 627.615 & 618 & 122.5994 & 7.36297 \\
\hline 532 & 12.0273 & 634.8555 & 627.2159 & 617 & 122.5994 & 7.3526 \\
\hline 533 & 12.0443 & 620.3204 & 627.8742 & 617 & 122.5994 & 7.34226 \\
\hline 534 & 12.0613 & 674.3869 & 627.5974 & 616 & 122.5994 & 7.33195 \\
\hline 535 & 12.0783 & 672.0586 & 628.394 & 616 & 122.5994 & 7.32166 \\
\hline 536 & 12.0953 & 637.597 & 628.2743 & 615 & 122.5994 & 7.31141 \\
\hline 537 & 12.1123 & 650.9697 & 628.2505 & 614 & 122.5994 & 7.30119 \\
\hline
\end{tabular}




\begin{tabular}{|c|c|c|c|c|c|c|}
\hline 538 & 12.1293 & 689.5461 & 629.337 & 614 & 122.5994 & 7.29099 \\
\hline 539 & 12.1463 & 682.6933 & 629.5518 & 613 & 122.5994 & 7.28083 \\
\hline 540 & 12.1633 & 660.5023 & 630.9174 & 613 & 122.5994 & 7.27069 \\
\hline 541 & 12.1803 & 683.0706 & 631.4633 & 612 & 122.5994 & 7.26058 \\
\hline 542 & 12.1973 & 713.9969 & 633.2291 & 612 & 122.5994 & 7.2505 \\
\hline 543 & 12.2143 & 709.4868 & 634.2711 & 611 & 122.5994 & 7.24045 \\
\hline 544 & 12.2313 & 684.3248 & 636.6702 & 611 & 122.5994 & 7.23042 \\
\hline 545 & 12.2483 & 709.5314 & 638.5455 & 610 & 122.5994 & 7.22042 \\
\hline 546 & 12.2653 & 739.3714 & 641.0724 & 609 & 122.5994 & 7.21045 \\
\hline 547 & 12.2823 & 737.1666 & 645.5043 & 609 & 122.5994 & 7.20051 \\
\hline 548 & 12.2993 & 751.1851 & 650.2005 & 608 & 122.5994 & 7.1906 \\
\hline 549 & 12.3163 & 769.3856 & 657.6497 & 608 & 122.5994 & 7.18071 \\
\hline 550 & 12.3333 & 728.4942 & 666.4882 & 607 & 122.5994 & 7.17085 \\
\hline 551 & 12.3503 & 749.9102 & 679.4995 & 607 & 122.5994 & 7.16102 \\
\hline 552 & 12.3673 & 770.4564 & 695.5847 & 606 & 122.5994 & 7.15121 \\
\hline 553 & 12.3843 & 767.9871 & 716.6998 & 605 & 122.5994 & 7.14144 \\
\hline 554 & 12.4013 & 802.5439 & 744.7404 & 605 & 122.5994 & 7.13168 \\
\hline 555 & 12.4183 & 859.7522 & 778.3937 & 604 & 122.5994 & 7.12196 \\
\hline 556 & 12.4353 & 926.4152 & 819.9531 & 604 & 122.5994 & 7.11226 \\
\hline 557 & 12.4523 & 872.4321 & 867.1 & 603 & 122.5994 & 7.10259 \\
\hline 558 & 12.4693 & 953.5213 & 920.665 & 603 & 122.5994 & 7.09294 \\
\hline 559 & 12.4863 & 942.4536 & 976.3124 & 602 & 122.5994 & 7.08333 \\
\hline 560 & 12.5033 & 944.5808 & 1031.231 & 601 & 122.5994 & 7.07373 \\
\hline 561 & 12.5203 & 1017.943 & 1081 & 601 & 122.5994 & 7.06417 \\
\hline 562 & 12.5373 & 1028.55 & 1117.121 & 600 & 122.5994 & 7.05463 \\
\hline 563 & 12.5543 & 1090.853 & 1135.624 & 600 & 122.5994 & 7.04511 \\
\hline 564 & 12.5713 & 1126.118 & 1131.242 & 599 & 122.5994 & 7.03562 \\
\hline 565 & 12.5883 & 1268.947 & 1106.755 & 599 & 122.5994 & 7.02616 \\
\hline 566 & 12.6053 & 1311.522 & 1064.517 & 598 & 122.5994 & 7.01672 \\
\hline 567 & 12.6223 & 1229.139 & 1012.698 & 598 & 122.5994 & 7.00731 \\
\hline 568 & 12.6393 & 1142.64 & 955.3961 & 597 & 122.5994 & 6.99792 \\
\hline 569 & 12.6563 & 958.1152 & 898.5109 & 596 & 122.5994 & 6.98856 \\
\hline 570 & 12.6733 & 847.5716 & 846.4092 & 596 & 122.5994 & 6.97923 \\
\hline 571 & 12.6903 & 732.6898 & 799.0925 & 595 & 122.5994 & 6.96991 \\
\hline 572 & 12.7073 & 699.3998 & 759.4818 & 595 & 122.5994 & 6.96063 \\
\hline 573 & 12.7243 & 656.2483 & 725.7147 & 594 & 122.5994 & 6.95137 \\
\hline 574 & 12.7413 & 641.6967 & 699.3763 & 594 & 122.5994 & 6.94213 \\
\hline 575 & 12.7583 & 655.3722 & 677.7114 & 593 & 122.5994 & 6.93292 \\
\hline 576 & 12.7753 & 642.2266 & 660.8026 & 592 & 122.5994 & 6.92373 \\
\hline 577 & 12.7923 & 610.3241 & 648.7061 & 592 & 122.5994 & 6.91457 \\
\hline 578 & 12.8093 & 605.5639 & 638.5541 & 591 & 122.5994 & 6.90543 \\
\hline 579 & 12.8263 & 635.449 & 630.606 & 590 & 122.5994 & 6.89631 \\
\hline 580 & 12.8433 & 636.9075 & 625.2689 & 590 & 122.5994 & 6.88722 \\
\hline 581 & 12.8603 & 612.6431 & 620.0926 & 589 & 122.5994 & 6.87816 \\
\hline 582 & 12.8773 & 610.1014 & 615.7485 & 588 & 122.5994 & 6.86912 \\
\hline 583 & 12.8943 & 589.7865 & 612.006 & 587 & 122.5994 & 6.8601 \\
\hline 584 & 12.9113 & 579.0376 & 608.7061 & 586 & 122.5994 & 6.8511 \\
\hline 585 & 12.9283 & 591.1477 & 606.7408 & 586 & 122.5994 & 6.84213 \\
\hline 586 & 12.9453 & 595.3711 & 604.036 & 585 & 122.5994 & 6.83319 \\
\hline
\end{tabular}




\begin{tabular}{|c|c|c|c|c|c|c|}
\hline 587 & 12.9623 & 580.6411 & 601.5402 & 584 & 122.5994 & 6.82426 \\
\hline 588 & 12.9793 & 565.2225 & 599.2164 & 583 & 122.5994 & 6.81536 \\
\hline 589 & 12.9963 & 583.6398 & 597.0371 & 582 & 122.5994 & 6.80649 \\
\hline 590 & 13.0133 & 585.5438 & 595.9812 & 582 & 122.5994 & 6.79763 \\
\hline 591 & 13.0303 & 590.9274 & 594.0317 & 581 & 122.5994 & 6.7888 \\
\hline 592 & 13.0473 & 584.9512 & 592.1746 & 580 & 122.5994 & 6.78 \\
\hline 593 & 13.0643 & 563.1953 & 590.3983 & 579 & 122.5994 & 6.77121 \\
\hline 594 & 13.0813 & 575.0763 & 589.6931 & 579 & 122.5994 & 6.76245 \\
\hline 595 & 13.0983 & 573.3262 & 588.0507 & 578 & 122.5994 & 6.75371 \\
\hline 596 & 13.1153 & 586.6538 & 586.4638 & 577 & 122.5994 & 6.74499 \\
\hline 597 & 13.1323 & 543.6487 & 584.9264 & 576 & 122.5994 & 6.7363 \\
\hline 598 & 13.1493 & 573.5153 & 583.433 & 575 & 122.5994 & 6.72763 \\
\hline 599 & 13.1663 & 564.2159 & 582.9791 & 575 & 122.5994 & 6.71898 \\
\hline 600 & 13.1833 & 574.4114 & 581.5606 & 574 & 122.5994 & 6.71036 \\
\hline 601 & 13.2003 & 584.7811 & 580.1739 & 573 & 122.5994 & 6.70175 \\
\hline 602 & 13.2173 & 581.1194 & 578.8159 & 572 & 122.5994 & 6.69317 \\
\hline 603 & 13.2343 & 535.8487 & 577.4838 & 571 & 122.5994 & 6.68461 \\
\hline 604 & 13.2513 & 547.2119 & 576.1753 & 570 & 122.5994 & 6.67607 \\
\hline 605 & 13.2683 & 542.8884 & 574.8881 & 569 & 122.5994 & 6.66756 \\
\hline 606 & 13.2853 & 590.7558 & 573.6204 & 568 & 122.5994 & 6.65907 \\
\hline 607 & 13.3023 & 602.7179 & 573.3704 & 568 & 122.5994 & 6.65059 \\
\hline 608 & 13.3193 & 540.9602 & 572.1366 & 567 & 122.5994 & 6.64214 \\
\hline 609 & 13.3363 & 512.6413 & 570.9177 & 566 & 122.5994 & 6.63371 \\
\hline 610 & 13.3533 & 542.0085 & 569.7125 & 565 & 122.5994 & 6.62531 \\
\hline 611 & 13.3703 & 606.6215 & 568.5197 & 564 & 122.5994 & 6.61692 \\
\hline 612 & 13.3873 & 544.1797 & 567.3384 & 563 & 122.5994 & 6.60856 \\
\hline 613 & 13.4043 & 528.2775 & 563.4534 & 562 & 122.5994 & 6.60021 \\
\hline 614 & 13.4213 & 546.6854 & 562.3961 & 561 & 122.5994 & 6.59189 \\
\hline 615 & 13.4383 & 552.9441 & 560 & 560 & 122.5994 & 6.58359 \\
\hline 616 & 13.4553 & 579.4009 & 559 & 559 & 122.5994 & 6.57531 \\
\hline 617 & 13.4723 & 554.9915 & 558.0599 & 558 & 122.5994 & 6.56705 \\
\hline 618 & 13.4893 & 577.3846 & 557.1809 & 557 & 122.5994 & 6.55881 \\
\hline 619 & 13.5063 & 540.1126 & 556.249 & 556 & 122.5994 & 6.5506 \\
\hline 620 & 13.5233 & 536.5763 & 556.3286 & 556 & 122.5994 & 6.5424 \\
\hline 621 & 13.5403 & 539.744 & 555.3699 & 555 & 122.5994 & 6.53423 \\
\hline 622 & 13.5573 & 543.7748 & 554.4051 & 554 & 122.5994 & 6.52607 \\
\hline 623 & 13.5743 & 558.0517 & 553.4456 & 553 & 122.5994 & 6.51793 \\
\hline 624 & 13.5913 & 542.8522 & 552.4924 & 552 & 122.5994 & 6.50982 \\
\hline 625 & 13.6083 & 549.0406 & 551.5471 & 551 & 122.5994 & 6.50173 \\
\hline 626 & 13.6253 & 559.074 & 551.6112 & 551 & 122.5994 & 6.49365 \\
\hline 627 & 13.6423 & 542.623 & 551.7226 & 551 & 122.5994 & 6.4856 \\
\hline 628 & 13.6593 & 549.8718 & 551.8165 & 551 & 122.5994 & 6.47757 \\
\hline 629 & 13.6763 & 549.8264 & 550.9475 & 550 & 122.5994 & 6.46955 \\
\hline 630 & 13.6933 & 550.0735 & 551.0879 & 550 & 122.5994 & 6.46156 \\
\hline 631 & 13.7103 & 576.1985 & 551.2628 & 550 & 122.5994 & 6.45358 \\
\hline 632 & 13.7273 & 600.9892 & 551.4869 & 550 & 122.5994 & 6.44563 \\
\hline 633 & 13.7443 & 587.7251 & 551.7885 & 550 & 122.5994 & 6.4377 \\
\hline 634 & 13.7613 & 567.141 & 552.2241 & 550 & 122.5994 & 6.42978 \\
\hline 635 & 13.7783 & 555.6144 & 552.9075 & 550 & 122.5994 & 6.42189 \\
\hline
\end{tabular}




\begin{tabular}{|c|c|c|c|c|c|c|}
\hline 636 & 13.7953 & 593.8801 & 554.043 & 550 & 122.5994 & 6.41401 \\
\hline 637 & 13.8123 & 578.54 & 554.9431 & 549 & 122.5994 & 6.40615 \\
\hline 638 & 13.8293 & 545.116 & 557.9863 & 549 & 122.5994 & 6.39832 \\
\hline 639 & 13.8463 & 551.8668 & 562.4724 & 549 & 122.5994 & 6.3905 \\
\hline 640 & 13.8633 & 607.9272 & 568.3561 & 549 & 122.5994 & 6.3827 \\
\hline 641 & 13.8803 & 576.2017 & 574.8578 & 549 & 122.5994 & 6.37492 \\
\hline 642 & 13.8973 & 535.8121 & 580.2451 & 549 & 122.5994 & 6.36716 \\
\hline 643 & 13.9143 & 631.8001 & 582.6031 & 549 & 122.5994 & 6.35942 \\
\hline 644 & 13.9313 & 589.0731 & 581.2558 & 549 & 122.5994 & 6.3517 \\
\hline 645 & 13.9483 & 584.2673 & 575.9583 & 548 & 122.5994 & 6.344 \\
\hline 646 & 13.9653 & 620.2098 & 570.2497 & 548 & 122.5994 & 6.33631 \\
\hline 647 & 13.9823 & 572.3013 & 564.7841 & 548 & 122.5994 & 6.32865 \\
\hline 648 & 13.9993 & 553.3811 & 560.6146 & 548 & 122.5994 & 6.321 \\
\hline 649 & 14.0163 & 579.18 & 558.0794 & 548 & 122.5994 & 6.31337 \\
\hline 650 & 14.0333 & 544.231 & 556.9737 & 548 & 122.5994 & 6.30576 \\
\hline 651 & 14.0503 & 594.6135 & 555.73 & 547 & 122.5994 & 6.29817 \\
\hline 652 & 14.0673 & 524.7888 & 555.5912 & 547 & 122.5994 & 6.2906 \\
\hline 653 & 14.0843 & 580.2738 & 555.0153 & 547 & 122.5994 & 6.28304 \\
\hline 654 & 14.1013 & 560.5246 & 552.9448 & 546 & 122.5994 & 6.27551 \\
\hline 655 & 14.1183 & 535.928 & 551.5753 & 546 & 122.5994 & 6.26799 \\
\hline 656 & 14.1353 & 507.9512 & 549.2036 & 545 & 122.5994 & 6.26049 \\
\hline 657 & 14.1523 & 525.475 & 548.0608 & 545 & 122.5994 & 6.25301 \\
\hline 658 & 14.1693 & 538.7094 & 547.22 & 545 & 122.5994 & 6.24554 \\
\hline 659 & 14.1863 & 568.2306 & 545.6494 & 544 & 122.5994 & 6.2381 \\
\hline 660 & 14.2033 & 550.5826 & 545.2787 & 544 & 122.5994 & 6.23067 \\
\hline 661 & 14.2203 & 537.3292 & 545.0378 & 544 & 122.5994 & 6.22326 \\
\hline 662 & 14.2373 & 523.5141 & 543.8745 & 543 & 122.5994 & 6.21587 \\
\hline 663 & 14.2543 & 546.5923 & 543.756 & 543 & 122.5994 & 6.20849 \\
\hline 664 & 14.2713 & 531.4306 & 543.6646 & 543 & 122.5994 & 6.20113 \\
\hline 665 & 14.2883 & 526.5056 & 542.5908 & 542 & 122.5994 & 6.19379 \\
\hline 666 & 14.3053 & 508.498 & 542.5295 & 542 & 122.5994 & 6.18647 \\
\hline 667 & 14.3223 & 532.0993 & 542.4778 & 542 & 122.5994 & 6.17917 \\
\hline 668 & 14.3393 & 520.9864 & 541.3813 & 541 & 122.5994 & 6.17188 \\
\hline 669 & 14.3563 & 511.3269 & 541.2455 & 541 & 122.5994 & 6.16461 \\
\hline 670 & 14.3733 & 525.2809 & 541.1747 & 541 & 122.5994 & 6.15736 \\
\hline 671 & 14.3903 & 545.2677 & 540.1079 & 540 & 122.5994 & 6.15012 \\
\hline 672 & 14.4073 & 549.4174 & 540.0865 & 540 & 122.5994 & 6.1429 \\
\hline 673 & 14.4243 & 557.5 & 539.0784 & 539 & 122.5994 & 6.1357 \\
\hline 674 & 14.4413 & 554.0042 & 539.0714 & 539 & 122.5994 & 6.12851 \\
\hline 675 & 14.4583 & 536.1188 & 538.0652 & 538 & 122.5994 & 6.12135 \\
\hline 676 & 14.4753 & 544.9259 & 538.0599 & 538 & 122.5994 & 6.1142 \\
\hline 677 & 14.4923 & 545.8545 & 537.0551 & 537 & 122.5994 & 6.10706 \\
\hline 678 & 14.5093 & 523.6564 & 537.0188 & 537 & 122.5994 & 6.09995 \\
\hline 679 & 14.5263 & 507.0619 & 536.0173 & 536 & 122.5994 & 6.09284 \\
\hline 680 & 14.5433 & 519.0605 & 536 & 536 & 122.5994 & 6.08576 \\
\hline 681 & 14.5603 & 510.126 & 535 & 535 & 122.5994 & 6.07869 \\
\hline 682 & 14.5773 & 507.6429 & 535 & 535 & 122.5994 & 6.07164 \\
\hline 683 & 14.5943 & 535.5466 & 534.088 & 534 & 122.5994 & 6.06461 \\
\hline 684 & 14.6113 & 550.8327 & 534.0954 & 534 & 122.5994 & 6.05759 \\
\hline
\end{tabular}




\begin{tabular}{|c|c|c|c|c|c|c|}
\hline 685 & 14.6283 & 516.0511 & 533.1469 & 533 & 122.5994 & 6.05059 \\
\hline 686 & 14.6453 & 539.0342 & 532.16 & 532 & 122.5994 & 6.0436 \\
\hline 687 & 14.6623 & 527.6233 & 532.1749 & 532 & 122.5994 & 6.03664 \\
\hline 688 & 14.6793 & 541.5185 & 531.192 & 531 & 122.5994 & 6.02968 \\
\hline 689 & 14.6963 & 540.0532 & 531.2118 & 531 & 122.5994 & 6.02275 \\
\hline 690 & 14.7133 & 542.085 & 530.2348 & 530 & 122.5994 & 6.01583 \\
\hline 691 & 14.7303 & 542.9001 & 530.2617 & 530 & 122.5994 & 6.00892 \\
\hline 692 & 14.7473 & 555.078 & 529.2934 & 529 & 122.5994 & 6.00203 \\
\hline 693 & 14.7643 & 511.8758 & 529.3312 & 529 & 122.5994 & 5.99516 \\
\hline 694 & 14.7813 & 531.1789 & 528.3766 & 528 & 122.5994 & 5.9883 \\
\hline 695 & 14.7983 & 537.9058 & 528.4321 & 528 & 122.5994 & 5.98146 \\
\hline 696 & 14.8153 & 533.8339 & 527.5007 & 527 & 122.5994 & 5.97464 \\
\hline 697 & 14.8323 & 584.3672 & 526.5873 & 526 & 122.5994 & 5.96783 \\
\hline 698 & 14.8493 & 552.3334 & 526.7009 & 526 & 122.5994 & 5.96103 \\
\hline 699 & 14.8663 & 532.4673 & 525.8588 & 525 & 122.5994 & 5.95425 \\
\hline 700 & 14.8833 & 472.8227 & 526.0975 & 525 & 122.5994 & 5.94749 \\
\hline 701 & 14.9003 & 519.8497 & 525.4888 & 524 & 122.5994 & 5.94074 \\
\hline 702 & 14.9173 & 517.9798 & 526.1554 & 524 & 122.5994 & 5.93401 \\
\hline 703 & 14.9343 & 501.0627 & 526.2698 & 523 & 122.5994 & 5.9273 \\
\hline 704 & 14.9513 & 526.8193 & 528.0099 & 523 & 122.5994 & 5.92059 \\
\hline 705 & 14.9683 & 491.026 & 529.4603 & 522 & 122.5994 & 5.91391 \\
\hline 706 & 14.9853 & 546.1054 & 532.4559 & 522 & 122.5994 & 5.90724 \\
\hline 707 & 15.0023 & 501.4972 & 534.3672 & 521 & 122.5994 & 5.90058 \\
\hline 708 & 15.0193 & 549.3281 & 536.1104 & 521 & 122.5994 & 5.89394 \\
\hline 709 & 15.0363 & 572.9377 & 534.996 & 520 & 122.5994 & 5.88732 \\
\hline 710 & 15.0533 & 527.1036 & 532.2896 & 519 & 122.5994 & 5.88071 \\
\hline 711 & 15.0703 & 564.3334 & 529.6149 & 519 & 122.5994 & 5.87411 \\
\hline 712 & 15.0873 & 565.5686 & 525.7437 & 518 & 122.5994 & 5.86753 \\
\hline 713 & 15.1043 & 552.2287 & 523.2994 & 518 & 122.5994 & 5.86096 \\
\hline 714 & 15.1213 & 552.2603 & 520.5094 & 517 & 122.5994 & 5.85441 \\
\hline 715 & 15.1383 & 545.5005 & 519.3267 & 517 & 122.5994 & 5.84788 \\
\hline 716 & 15.1553 & 533.5894 & 517.5978 & 516 & 122.5994 & 5.84136 \\
\hline 717 & 15.1723 & 539.279 & 517.1619 & 516 & 122.5994 & 5.83485 \\
\hline 718 & 15.1893 & 543.5888 & 515.8963 & 515 & 122.5994 & 5.82836 \\
\hline 719 & 15.2063 & 559.4864 & 515.7241 & 515 & 122.5994 & 5.82188 \\
\hline 720 & 15.2233 & 561.1859 & 514.6031 & 514 & 122.5994 & 5.81542 \\
\hline 721 & 15.2403 & 524.6112 & 514.5123 & 514 & 122.5994 & 5.80897 \\
\hline 722 & 15.2573 & 543.9695 & 513.4412 & 513 & 122.5994 & 5.80253 \\
\hline 723 & 15.2743 & 563.6004 & 512.3839 & 512 & 122.5994 & 5.79611 \\
\hline 724 & 15.2913 & 521.3009 & 511.3371 & 511 & 122.5994 & 5.78971 \\
\hline 725 & 15.3083 & 516.3767 & 511.2982 & 511 & 122.5994 & 5.78332 \\
\hline 726 & 15.3253 & 528.1064 & 510.2657 & 510 & 122.5994 & 5.77694 \\
\hline 727 & 15.3423 & 521.5826 & 509.2382 & 509 & 122.5994 & 5.77058 \\
\hline 728 & 15.3593 & 552.8158 & 508.2148 & 508 & 122.5994 & 5.76423 \\
\hline 729 & 15.3763 & 537.1185 & 508.1946 & 508 & 122.5994 & 5.75789 \\
\hline 730 & 15.3933 & 535.6479 & 507.1771 & 507 & 122.5994 & 5.75157 \\
\hline 731 & 15.4103 & 507.4904 & 506.1619 & 506 & 122.5994 & 5.74527 \\
\hline 732 & 15.4273 & 486.4102 & 505.1485 & 505 & 122.5994 & 5.73897 \\
\hline 733 & 15.4443 & 504.0773 & 505.1367 & 505 & 122.5994 & 5.73269 \\
\hline
\end{tabular}




\begin{tabular}{|c|c|c|c|c|c|c|}
\hline 734 & 15.4613 & 476.6438 & 504.0469 & 504 & 122.5994 & 5.72643 \\
\hline 735 & 15.4783 & 476.9167 & 503.0433 & 503 & 122.5994 & 5.72018 \\
\hline 736 & 15.4953 & 512.3856 & 503 & 503 & 122.5994 & 5.71394 \\
\hline 737 & 15.5123 & 486.1892 & 502.0432 & 502 & 122.5994 & 5.70772 \\
\hline 738 & 15.5293 & 541.9075 & 501.0468 & 501 & 122.5994 & 5.70151 \\
\hline 739 & 15.5463 & 531.8229 & 500.0717 & 500 & 122.5994 & 5.69531 \\
\hline 740 & 15.5633 & 508.493 & 500.0781 & 500 & 122.5994 & 5.68913 \\
\hline 741 & 15.5803 & 518.79 & 499.0854 & 499 & 122.5994 & 5.68296 \\
\hline 742 & 15.5973 & 515.5564 & 498.0936 & 498 & 122.5994 & 5.6768 \\
\hline 743 & 15.6143 & 515.2701 & 497.1032 & 497 & 122.5994 & 5.67066 \\
\hline 744 & 15.6313 & 484.4292 & 497.1143 & 497 & 122.5994 & 5.66453 \\
\hline 745 & 15.6483 & 519.4241 & 496.1272 & 496 & 122.5994 & 5.65842 \\
\hline 746 & 15.6653 & 471.9462 & 496.1425 & 496 & 122.5994 & 5.65231 \\
\hline 747 & 15.6823 & 503.3532 & 496.1606 & 496 & 122.5994 & 5.64622 \\
\hline 748 & 15.6993 & 455.2015 & 497.1824 & 497 & 122.5994 & 5.64015 \\
\hline 749 & 15.7163 & 524.7722 & 497.2089 & 497 & 122.5994 & 5.63409 \\
\hline 750 & 15.7333 & 487.1053 & 497.2416 & 497 & 122.5994 & 5.62804 \\
\hline 751 & 15.7503 & 509.5056 & 497.2827 & 497 & 122.5994 & 5.622 \\
\hline 752 & 15.7673 & 509.783 & 497.3362 & 497 & 122.5994 & 5.61598 \\
\hline 753 & 15.7843 & 557.4083 & 498.4097 & 498 & 122.5994 & 5.60997 \\
\hline 754 & 15.8013 & 570.3663 & 498.5193 & 498 & 122.5994 & 5.60397 \\
\hline 755 & 15.8183 & 526.9345 & 498.6968 & 498 & 122.5994 & 5.59798 \\
\hline 756 & 15.8353 & 518.7359 & 498.9984 & 498 & 122.5994 & 5.59201 \\
\hline 757 & 15.8523 & 518.9511 & 499.5053 & 498 & 122.5994 & 5.58605 \\
\hline 758 & 15.8693 & 525.0337 & 501.3066 & 499 & 122.5994 & 5.58011 \\
\hline 759 & 15.8863 & 518.1209 & 502.455 & 499 & 122.5994 & 5.57418 \\
\hline 760 & 15.9033 & 524.4198 & 503.8955 & 499 & 122.5994 & 5.56826 \\
\hline 761 & 15.9203 & 514.8337 & 505.3634 & 499 & 122.5994 & 5.56235 \\
\hline 762 & 15.9373 & 512.6103 & 507.3481 & 500 & 122.5994 & 5.55645 \\
\hline 763 & 15.9543 & 543.79 & 507.4534 & 500 & 122.5994 & 5.55057 \\
\hline 764 & 15.9713 & 538.7167 & 506.7624 & 500 & 122.5994 & 5.5447 \\
\hline 765 & 15.9883 & 515.8687 & 505.5477 & 500 & 122.5994 & 5.53884 \\
\hline 766 & 16.0053 & 504.5452 & 504.1436 & 500 & 122.5994 & 5.533 \\
\hline 767 & 16.0223 & 505.9655 & 503.8831 & 501 & 122.5994 & 5.52717 \\
\hline 768 & 16.0393 & 492.9669 & 502.9258 & 501 & 122.5994 & 5.52135 \\
\hline 769 & 16.0563 & 498.9714 & 502.2755 & 501 & 122.5994 & 5.51554 \\
\hline 770 & 16.0733 & 509.6107 & 502.8668 & 502 & 122.5994 & 5.50974 \\
\hline 771 & 16.0903 & 504.8863 & 502.6205 & 502 & 122.5994 & 5.50396 \\
\hline 772 & 16.1073 & 537.9821 & 502.4716 & 502 & 122.5994 & 5.49819 \\
\hline 773 & 16.1243 & 512.4247 & 503.3768 & 503 & 122.5994 & 5.49243 \\
\hline 774 & 16.1413 & 506.663 & 504.3117 & 504 & 122.5994 & 5.48668 \\
\hline 775 & 16.1583 & 482.2077 & 504.2636 & 504 & 122.5994 & 5.48095 \\
\hline 776 & 16.1753 & 495.0104 & 504.2263 & 504 & 122.5994 & 5.47523 \\
\hline 777 & 16.1923 & 507.6275 & 505.1965 & 505 & 122.5994 & 5.46952 \\
\hline 778 & 16.2093 & 522.5385 & 506.1722 & 506 & 122.5994 & 5.46382 \\
\hline 779 & 16.2263 & 463.9211 & 506.1521 & 506 & 122.5994 & 5.45813 \\
\hline 780 & 16.2433 & 487.2576 & 506.1353 & 506 & 122.5994 & 5.45246 \\
\hline 781 & 16.2603 & 515.9209 & 507.1212 & 507 & 122.5994 & 5.4468 \\
\hline 782 & 16.2773 & 505.3089 & 508.1091 & 508 & 122.5994 & 5.44115 \\
\hline
\end{tabular}




\begin{tabular}{|c|c|c|c|c|c|c|}
\hline 783 & 16.2943 & 543.4633 & 508.0987 & 508 & 122.5994 & 5.43551 \\
\hline 784 & 16.3113 & 552.197 & 508.0898 & 508 & 122.5994 & 5.42988 \\
\hline 785 & 16.3283 & 589.7117 & 509.082 & 509 & 122.5994 & 5.42427 \\
\hline 786 & 16.3453 & 540.3966 & 510.0751 & 510 & 122.5994 & 5.41866 \\
\hline 787 & 16.3623 & 508.6728 & 510.0691 & 510 & 122.5994 & 5.41307 \\
\hline 788 & 16.3793 & 506.2595 & 510.0239 & 510 & 122.5994 & 5.40749 \\
\hline 789 & 16.3963 & 510.566 & 511.022 & 511 & 122.5994 & 5.40192 \\
\hline 790 & 16.4133 & 574.4049 & 512 & 512 & 122.5994 & 5.39637 \\
\hline 791 & 16.4303 & 501.9314 & 512 & 512 & 122.5994 & 5.39082 \\
\hline 792 & 16.4473 & 484.8816 & 512 & 512 & 122.5994 & 5.38529 \\
\hline 793 & 16.4643 & 529.5783 & 513 & 513 & 122.5994 & 5.37977 \\
\hline 794 & 16.4813 & 540.6116 & 514 & 514 & 122.5994 & 5.37426 \\
\hline 795 & 16.4983 & 504.705 & 514 & 514 & 122.5994 & 5.36876 \\
\hline 796 & 16.5153 & 489.8264 & 514 & 514 & 122.5994 & 5.36327 \\
\hline 797 & 16.5323 & 501.2019 & 515 & 515 & 122.5994 & 5.35779 \\
\hline 798 & 16.5493 & 504.7636 & 516 & 516 & 122.5994 & 5.35233 \\
\hline 799 & 16.5663 & 536.0174 & 516 & 516 & 122.5994 & 5.34687 \\
\hline 800 & 16.5833 & 489.9629 & 516 & 516 & 122.5994 & 5.34143 \\
\hline 801 & 16.6003 & 523.9324 & 517 & 517 & 122.5994 & 5.336 \\
\hline 802 & 16.6173 & 494.7227 & 518 & 518 & 122.5994 & 5.33058 \\
\hline 803 & 16.6343 & 528.3963 & 518 & 518 & 122.5994 & 5.32517 \\
\hline 804 & 16.6513 & 553.5154 & 518 & 518 & 122.5994 & 5.31977 \\
\hline 805 & 16.6683 & 518.4887 & 519 & 519 & 122.5994 & 5.31438 \\
\hline 806 & 16.6853 & 525.647 & 520 & 520 & 122.5994 & 5.309 \\
\hline 807 & 16.7023 & 545.91 & 520 & 520 & 122.5994 & 5.30364 \\
\hline 808 & 16.7193 & 531.2175 & 520 & 520 & 122.5994 & 5.29828 \\
\hline 809 & 16.7363 & 510.7695 & 521 & 521 & 122.5994 & 5.29294 \\
\hline 810 & 16.7533 & 518.1873 & 522 & 522 & 122.5994 & 5.28761 \\
\hline 811 & 16.7703 & 519.2681 & 522 & 522 & 122.5994 & 5.28229 \\
\hline 812 & 16.7873 & 516.4912 & 522 & 522 & 122.5994 & 5.27698 \\
\hline 813 & 16.8043 & 528.9493 & 524.9749 & 523 & 122.5994 & 5.27168 \\
\hline 814 & 16.8213 & 535.8535 & 526.0427 & 524 & 122.5994 & 5.26639 \\
\hline 815 & 16.8383 & 526.3778 & 526.114 & 524 & 122.5994 & 5.26111 \\
\hline 816 & 16.8553 & 513.1966 & 527.1839 & 524 & 122.5994 & 5.25584 \\
\hline 817 & 16.8723 & 530.6812 & 528.2974 & 525 & 122.5994 & 5.25058 \\
\hline 818 & 16.8893 & 534.8601 & 528.4169 & 525 & 122.5994 & 5.24534 \\
\hline 819 & 16.9063 & 507.3003 & 529.5431 & 526 & 122.5994 & 5.2401 \\
\hline 820 & 16.9233 & 479.1443 & 529.6763 & 526 & 122.5994 & 5.23487 \\
\hline 821 & 16.9403 & 490.7584 & 529.8172 & 526 & 122.5994 & 5.22966 \\
\hline 822 & 16.9573 & 501.7694 & 530.9662 & 527 & 122.5994 & 5.22445 \\
\hline 823 & 16.9743 & 520.4669 & 531.1241 & 527 & 122.5994 & 5.21926 \\
\hline 824 & 16.9913 & 545.4694 & 531.2916 & 527 & 122.5994 & 5.21408 \\
\hline 825 & 17.0083 & 499.2716 & 532.4694 & 528 & 122.5994 & 5.2089 \\
\hline 826 & 17.0253 & 509.7723 & 532.6584 & 528 & 122.5994 & 5.20374 \\
\hline 827 & 17.0423 & 485.3996 & 532.8595 & 528 & 122.5994 & 5.19859 \\
\hline 828 & 17.0593 & 496.3047 & 534.0738 & 529 & 122.5994 & 5.19345 \\
\hline 829 & 17.0763 & 523.9835 & 534.3025 & 529 & 122.5994 & 5.18831 \\
\hline 830 & 17.0933 & 521.3427 & 534.5468 & 529 & 122.5994 & 5.18319 \\
\hline 831 & 17.1103 & 496.6685 & 535.8083 & 530 & 122.5994 & 5.17808 \\
\hline
\end{tabular}




\begin{tabular}{|c|c|c|c|c|c|c|}
\hline 832 & 17.1273 & 524.613 & 536.0885 & 530 & 122.5994 & 5.17298 \\
\hline 833 & 17.1443 & 537.3399 & 536.3892 & 530 & 122.5994 & 5.16789 \\
\hline 834 & 17.1613 & 496.3434 & 537.7126 & 531 & 122.5994 & 5.16281 \\
\hline 835 & 17.1783 & 527.0056 & 538.0608 & 531 & 122.5994 & 5.15774 \\
\hline 836 & 17.1953 & 533.4637 & 538.4365 & 531 & 122.5994 & 5.15268 \\
\hline 837 & 17.2123 & 511.0519 & 539.8425 & 532 & 122.5994 & 5.14762 \\
\hline 838 & 17.2293 & 528.9681 & 540.2823 & 532 & 122.5994 & 5.14258 \\
\hline 839 & 17.2463 & 524.8435 & 540.7596 & 532 & 122.5994 & 5.13755 \\
\hline 840 & 17.2633 & 538.0085 & 542.2786 & 533 & 122.5994 & 5.13253 \\
\hline 841 & 17.2803 & 533.9612 & 542.8447 & 533 & 122.5994 & 5.12752 \\
\hline 842 & 17.2973 & 535.6329 & 543.4634 & 533 & 122.5994 & 5.12252 \\
\hline 843 & 17.3143 & 515.0041 & 545.142 & 534 & 122.5994 & 5.11753 \\
\hline 844 & 17.3313 & 544.371 & 545.8885 & 534 & 122.5994 & 5.11255 \\
\hline 845 & 17.3483 & 523.6898 & 546.7135 & 534 & 122.5994 & 5.10758 \\
\hline 846 & 17.3653 & 523.185 & 548.6299 & 535 & 122.5994 & 5.10261 \\
\hline 847 & 17.3823 & 535.4453 & 549.6545 & 535 & 122.5994 & 5.09766 \\
\hline 848 & 17.3993 & 538.1558 & 550.8099 & 535 & 122.5994 & 5.09272 \\
\hline 849 & 17.4163 & 567.1817 & 553.1265 & 536 & 122.5994 & 5.08779 \\
\hline 850 & 17.4333 & 525.6522 & 554.6465 & 536 & 122.5994 & 5.08286 \\
\hline 851 & 17.4503 & 559.6488 & 557.428 & 537 & 122.5994 & 5.07795 \\
\hline 852 & 17.4673 & 532.9283 & 559.551 & 537 & 122.5994 & 5.07305 \\
\hline 853 & 17.4843 & 515.2999 & 562.124 & 537 & 122.5994 & 5.06815 \\
\hline 854 & 17.5013 & 530.9512 & 566.2921 & 538 & 122.5994 & 5.06327 \\
\hline 855 & 17.5183 & 555.2492 & 570.2437 & 538 & 122.5994 & 5.05839 \\
\hline 856 & 17.5353 & 570.3385 & 575.2164 & 538 & 122.5994 & 5.05353 \\
\hline 857 & 17.5523 & 596.5842 & 582.4989 & 539 & 122.5994 & 5.04867 \\
\hline 858 & 17.5693 & 614.7794 & 590.4279 & 539 & 122.5994 & 5.04382 \\
\hline 859 & 17.5863 & 589.4532 & 600.3754 & 539 & 122.5994 & 5.03899 \\
\hline 860 & 17.6033 & 579.641 & 613.7325 & 540 & 122.5994 & 5.03416 \\
\hline 861 & 17.6203 & 545.1368 & 628.8719 & 540 & 122.5994 & 5.02934 \\
\hline 862 & 17.6373 & 563.1578 & 647.1415 & 540 & 122.5994 & 5.02453 \\
\hline 863 & 17.6543 & 564.8206 & 669.6937 & 541 & 122.5994 & 5.01973 \\
\hline 864 & 17.6713 & 563.7398 & 694.62 & 541 & 122.5994 & 5.01494 \\
\hline 865 & 17.6883 & 587.4348 & 723.7126 & 542 & 122.5994 & 5.01016 \\
\hline 866 & 17.7053 & 619.6402 & 754.5037 & 542 & 122.5994 & 5.00539 \\
\hline 867 & 17.7223 & 635.0519 & 787.1222 & 542 & 122.5994 & 5.00062 \\
\hline 868 & 17.7393 & 638.2847 & 821.2128 & 543 & 122.5994 & 4.99587 \\
\hline 869 & 17.7563 & 664.3811 & 852.8667 & 543 & 122.5994 & 4.99112 \\
\hline 870 & 17.7733 & 698.0303 & 880.6804 & 543 & 122.5994 & 4.98639 \\
\hline 871 & 17.7903 & 715.2541 & 903.0043 & 544 & 122.5994 & 4.98166 \\
\hline 872 & 17.8073 & 779.4735 & 915.4836 & 544 & 122.5994 & 4.97694 \\
\hline 873 & 17.8243 & 800.905 & 917.665 & 544 & 122.5994 & 4.97224 \\
\hline 874 & 17.8413 & 864.4472 & 910.3811 & 545 & 122.5994 & 4.96754 \\
\hline 875 & 17.8583 & 891.7045 & 892.7292 & 545 & 122.5994 & 4.96285 \\
\hline 876 & 17.8753 & 918.9529 & 867.7245 & 545 & 122.5994 & 4.95816 \\
\hline 877 & 17.8923 & 992.6585 & 838.817 & 546 & 122.5994 & 4.95349 \\
\hline 878 & 17.9093 & 924.147 & 806.4364 & 546 & 122.5994 & 4.94883 \\
\hline 879 & 17.9263 & 880.0756 & 773.6549 & 546 & 122.5994 & 4.94417 \\
\hline 880 & 17.9433 & 802.215 & 743.0656 & 547 & 122.5994 & 4.93953 \\
\hline
\end{tabular}




\begin{tabular}{|c|c|c|c|c|c|c|}
\hline 881 & 17.9603 & 683.849 & 713.7632 & 547 & 122.5994 & 4.93489 \\
\hline 882 & 17.9773 & 680.9925 & 688.4136 & 548 & 122.5994 & 4.93026 \\
\hline 883 & 17.9943 & 587.8155 & 665.3386 & 548 & 122.5994 & 4.92564 \\
\hline 884 & 18.0113 & 595.3603 & 645.5952 & 548 & 122.5994 & 4.92103 \\
\hline 885 & 18.0283 & 553.074 & 630.0466 & 549 & 122.5994 & 4.91643 \\
\hline 886 & 18.0453 & 594.3598 & 616.4238 & 549 & 122.5994 & 4.91184 \\
\hline 887 & 18.0623 & 570.0184 & 605.3792 & 549 & 122.5994 & 4.90725 \\
\hline 888 & 18.0793 & 550.6769 & 597.5333 & 550 & 122.5994 & 4.90267 \\
\hline 889 & 18.0963 & 539.1603 & 590.5067 & 550 & 122.5994 & 4.89811 \\
\hline 890 & 18.1133 & 583.8036 & 584.9502 & 550 & 122.5994 & 4.89355 \\
\hline 891 & 18.1303 & 566.2282 & 580.5577 & 550 & 122.5994 & 4.889 \\
\hline 892 & 18.1473 & 539.0451 & 577.1669 & 550 & 122.5994 & 4.88446 \\
\hline 893 & 18.1643 & 546.5193 & 575.449 & 551 & 122.5994 & 4.87992 \\
\hline 894 & 18.1813 & 558.6761 & 573.225 & 551 & 122.5994 & 4.8754 \\
\hline 895 & 18.1983 & 543.1979 & 571.4826 & 551 & 122.5994 & 4.87088 \\
\hline 896 & 18.2153 & 534.9878 & 570.0787 & 551 & 122.5994 & 4.86638 \\
\hline 897 & 18.2323 & 561.4973 & 568.9471 & 551 & 122.5994 & 4.86188 \\
\hline 898 & 18.2493 & 581.321 & 569.0777 & 552 & 122.5994 & 4.85739 \\
\hline 899 & 18.2663 & 551.7797 & 568.4521 & 552 & 122.5994 & 4.8529 \\
\hline 900 & 18.2833 & 523.3971 & 568.0255 & 552 & 122.5994 & 4.84843 \\
\hline 901 & 18.3003 & 539.128 & 567.7754 & 552 & 122.5994 & 4.84396 \\
\hline 902 & 18.3173 & 529.7611 & 567.6618 & 552 & 122.5994 & 4.83951 \\
\hline 903 & 18.3343 & 530.688 & 567.6107 & 552 & 122.5994 & 4.83506 \\
\hline 904 & 18.3513 & 535.7803 & 567.5394 & 552 & 122.5994 & 4.83062 \\
\hline 905 & 18.3683 & 556.9704 & 568.3522 & 553 & 122.5994 & 4.82618 \\
\hline 906 & 18.3853 & 563.6997 & 567.976 & 553 & 122.5994 & 4.82176 \\
\hline 907 & 18.4023 & 554.9411 & 567.3875 & 553 & 122.5994 & 4.81734 \\
\hline 908 & 18.4193 & 547.8965 & 566.6119 & 553 & 122.5994 & 4.81294 \\
\hline 909 & 18.4363 & 567.1918 & 565.7062 & 553 & 122.5994 & 4.80854 \\
\hline 910 & 18.4533 & 547.1442 & 565.7421 & 554 & 122.5994 & 4.80414 \\
\hline 911 & 18.4703 & 540.5613 & 564.789 & 554 & 122.5994 & 4.79976 \\
\hline 912 & 18.4873 & 552.2603 & 563.9007 & 554 & 122.5994 & 4.79539 \\
\hline 913 & 18.5043 & 564.0698 & 563.1099 & 554 & 122.5994 & 4.79102 \\
\hline 914 & 18.5213 & 555.9806 & 562.4315 & 554 & 122.5994 & 4.78666 \\
\hline 915 & 18.5383 & 547.5702 & 562.8669 & 555 & 122.5994 & 4.78231 \\
\hline 916 & 18.5553 & 544.2975 & 562.4099 & 555 & 122.5994 & 4.77797 \\
\hline 917 & 18.5723 & 546.2107 & 563.0497 & 556 & 122.5994 & 4.77363 \\
\hline 918 & 18.5893 & 544.1592 & 562.7749 & 556 & 122.5994 & 4.7693 \\
\hline 919 & 18.6063 & 557.037 & 562.5757 & 556 & 122.5994 & 4.76498 \\
\hline 920 & 18.6233 & 525.8104 & 563.4466 & 557 & 122.5994 & 4.76067 \\
\hline 921 & 18.6403 & 544.6839 & 563.3915 & 557 & 122.5994 & 4.75637 \\
\hline 922 & 18.6573 & 563.7824 & 564.4531 & 558 & 122.5994 & 4.75207 \\
\hline 923 & 18.6743 & 570.4478 & 564.5693 & 558 & 122.5994 & 4.74779 \\
\hline 924 & 18.6913 & 534.9404 & 565.923 & 559 & 122.5994 & 4.74351 \\
\hline 925 & 18.7083 & 537.1508 & 566.3336 & 559 & 122.5994 & 4.73923 \\
\hline 926 & 18.7253 & 555.1465 & 566.9649 & 559 & 122.5994 & 4.73497 \\
\hline 927 & 18.7423 & 565.7311 & 568.9288 & 560 & 122.5994 & 4.73071 \\
\hline 928 & 18.7593 & 598.3545 & 570.2021 & 560 & 122.5994 & 4.72646 \\
\hline 929 & 18.7763 & 590.8225 & 572.8926 & 561 & 122.5994 & 4.72222 \\
\hline
\end{tabular}




\begin{tabular}{|c|c|c|c|c|c|c|}
\hline 930 & 18.7933 & 624.6181 & 575.0414 & 561 & 122.5994 & 4.71799 \\
\hline 931 & 18.8103 & 595.8259 & 578.6505 & 562 & 122.5994 & 4.71376 \\
\hline 932 & 18.8273 & 640.2627 & 580.18 & 562 & 122.5994 & 4.70955 \\
\hline 933 & 18.8443 & 644.0271 & 583.516 & 562 & 122.5994 & 4.70534 \\
\hline 934 & 18.8613 & 626.5168 & 586.8716 & 563 & 122.5994 & 4.70113 \\
\hline 935 & 18.8783 & 645.1721 & 590.0273 & 563 & 122.5994 & 4.69694 \\
\hline 936 & 18.8953 & 651.2704 & 593.1316 & 564 & 122.5994 & 4.69275 \\
\hline 937 & 18.9123 & 696.2391 & 594.0618 & 564 & 122.5994 & 4.68857 \\
\hline 938 & 18.9293 & 684.221 & 593.7234 & 564 & 122.5994 & 4.6844 \\
\hline 939 & 18.9463 & 644.1743 & 593.63 & 565 & 122.5994 & 4.68023 \\
\hline 940 & 18.9633 & 634.9979 & 591.3326 & 565 & 122.5994 & 4.67608 \\
\hline 941 & 18.9803 & 611.7195 & 589.5496 & 566 & 122.5994 & 4.67193 \\
\hline 942 & 18.9973 & 597.8522 & 586.8256 & 566 & 122.5994 & 4.66778 \\
\hline 943 & 19.0143 & 587.9033 & 584.0279 & 566 & 122.5994 & 4.66365 \\
\hline 944 & 19.0313 & 576.6551 & 582.5089 & 567 & 122.5994 & 4.65952 \\
\hline 945 & 19.0483 & 560.5081 & 580.3263 & 567 & 122.5994 & 4.6554 \\
\hline 946 & 19.0653 & 542.927 & 579.4656 & 568 & 122.5994 & 4.65129 \\
\hline 947 & 19.0823 & 531.9863 & 577.9022 & 568 & 122.5994 & 4.64718 \\
\hline 948 & 19.0993 & 522.1531 & 577.6274 & 569 & 122.5994 & 4.64309 \\
\hline 949 & 19.1163 & 534.7956 & 576.6183 & 569 & 122.5994 & 4.63899 \\
\hline 950 & 19.1333 & 553.5352 & 575.8205 & 569 & 122.5994 & 4.63491 \\
\hline 951 & 19.1503 & 539.2521 & 576.2733 & 570 & 122.5994 & 4.63084 \\
\hline 952 & 19.1673 & 554.3728 & 575.9107 & 570 & 122.5994 & 4.62677 \\
\hline 953 & 19.1843 & 554.6932 & 576.7371 & 571 & 122.5994 & 4.6227 \\
\hline 954 & 19.2013 & 564.2544 & 576.8003 & 571 & 122.5994 & 4.61865 \\
\hline 955 & 19.2183 & 565.9853 & 577.9257 & 572 & 122.5994 & 4.6146 \\
\hline 956 & 19.2353 & 544.1727 & 578.1909 & 572 & 122.5994 & 4.61056 \\
\hline 957 & 19.2523 & 555.0376 & 578.6254 & 572 & 122.5994 & 4.60653 \\
\hline 958 & 19.2693 & 565.5285 & 580.188 & 573 & 122.5994 & 4.6025 \\
\hline 959 & 19.2863 & 553.0448 & 580.9085 & 573 & 122.5994 & 4.59849 \\
\hline 960 & 19.3033 & 562.5669 & 582.7852 & 574 & 122.5994 & 4.59447 \\
\hline 961 & 19.3203 & 580.6816 & 583.8019 & 574 & 122.5994 & 4.59047 \\
\hline 962 & 19.3373 & 579.1229 & 585.9208 & 575 & 122.5994 & 4.58647 \\
\hline 963 & 19.3543 & 599.8299 & 587.0748 & 575 & 122.5994 & 4.58248 \\
\hline 964 & 19.3713 & 578.5976 & 589.1663 & 576 & 122.5994 & 4.5785 \\
\hline 965 & 19.3883 & 613.5985 & 591.0873 & 577 & 122.5994 & 4.57452 \\
\hline 966 & 19.4053 & 603.5768 & 591.7637 & 577 & 122.5994 & 4.57055 \\
\hline 967 & 19.4223 & 614.7149 & 593.1944 & 578 & 122.5994 & 4.56659 \\
\hline 968 & 19.4393 & 600.1469 & 593.4357 & 578 & 122.5994 & 4.56264 \\
\hline 969 & 19.4563 & 575.3795 & 594.6002 & 579 & 122.5994 & 4.55869 \\
\hline 970 & 19.4733 & 604.1732 & 595.8271 & 580 & 122.5994 & 4.55475 \\
\hline 971 & 19.4903 & 653.9879 & 596.2612 & 580 & 122.5994 & 4.55081 \\
\hline 972 & 19.5073 & 642.2994 & 598.0438 & 581 & 122.5994 & 4.54688 \\
\hline 973 & 19.5243 & 635.4871 & 600.2982 & 582 & 122.5994 & 4.54296 \\
\hline 974 & 19.5413 & 658.857 & 602.1321 & 582 & 122.5994 & 4.53905 \\
\hline 975 & 19.5583 & 617.0111 & 605.642 & 583 & 122.5994 & 4.53514 \\
\hline 976 & 19.5753 & 664.4842 & 608.9171 & 583 & 122.5994 & 4.53124 \\
\hline 977 & 19.5923 & 635.0568 & 614.0364 & 584 & 122.5994 & 4.52735 \\
\hline 978 & 19.6093 & 650.1532 & 620.0639 & 585 & 122.5994 & 4.52346 \\
\hline
\end{tabular}




\begin{tabular}{|c|c|c|c|c|c|c|}
\hline 979 & 19.6263 & 644.7374 & 626.0338 & 585 & 122.5994 & 4.51958 \\
\hline 980 & 19.6433 & 637.025 & 633.8421 & 586 & 122.5994 & 4.51571 \\
\hline 981 & 19.6603 & 666.4661 & 642.5605 & 587 & 122.5994 & 4.51184 \\
\hline 982 & 19.6773 & 679.976 & 651.1163 & 587 & 122.5994 & 4.50798 \\
\hline 983 & 19.6943 & 714.9811 & 661.4114 & 588 & 122.5994 & 4.50413 \\
\hline 984 & 19.7113 & 730.6451 & 672.3214 & 589 & 122.5994 & 4.50029 \\
\hline 985 & 19.7283 & 735.2173 & 682.8924 & 589 & 122.5994 & 4.49645 \\
\hline 986 & 19.7453 & 722.3635 & 694.9951 & 590 & 122.5994 & 4.49261 \\
\hline 987 & 19.7623 & 767.6746 & 706.2221 & 590 & 122.5994 & 4.48879 \\
\hline 988 & 19.7793 & 803.1545 & 717.0524 & 590 & 122.5994 & 4.48497 \\
\hline 989 & 19.7963 & 852.8749 & 727.7086 & 591 & 122.5994 & 4.48115 \\
\hline 990 & 19.8133 & 875.2404 & 735.4319 & 591 & 122.5994 & 4.47735 \\
\hline 991 & 19.8303 & 873.8911 & 741.7403 & 592 & 122.5994 & 4.47355 \\
\hline 992 & 19.8473 & 835.4302 & 744.479 & 592 & 122.5994 & 4.46975 \\
\hline 993 & 19.8643 & 841.6656 & 744.7787 & 592 & 122.5994 & 4.46597 \\
\hline 994 & 19.8813 & 884.893 & 743.9471 & 593 & 122.5994 & 4.46219 \\
\hline 995 & 19.8983 & 928.4501 & 740.3713 & 593 & 122.5994 & 4.45841 \\
\hline 996 & 19.9153 & 924.0875 & 736.3933 & 594 & 122.5994 & 4.45465 \\
\hline 997 & 19.9323 & 847.648 & 730.205 & 594 & 122.5994 & 4.45088 \\
\hline 998 & 19.9493 & 856.8999 & 723.861 & 595 & 122.5994 & 4.44713 \\
\hline 999 & 19.9663 & 890.3045 & 715.395 & 595 & 122.5994 & 4.44338 \\
\hline 1000 & 19.9833 & 885.3179 & 705.9442 & 595 & 122.5994 & 4.43964 \\
\hline 1001 & 20.0003 & 855.3158 & 696.7974 & 596 & 122.5994 & 4.4359 \\
\hline 1002 & 20.0173 & 828.2397 & 686.3484 & 596 & 122.5994 & 4.43218 \\
\hline 1003 & 20.0343 & 787.1178 & 679.1385 & 597 & 122.5994 & 4.42845 \\
\hline 1004 & 20.0513 & 783.5395 & 669.4186 & 597 & 122.5994 & 4.42474 \\
\hline 1005 & 20.0683 & 781.7611 & 660.5477 & 597 & 122.5994 & 4.42103 \\
\hline 1006 & 20.0853 & 762.4167 & 653.8755 & 598 & 122.5994 & 4.41732 \\
\hline 1007 & 20.1023 & 774.1727 & 647.2128 & 598 & 122.5994 & 4.41363 \\
\hline 1008 & 20.1193 & 754.5649 & 642.684 & 599 & 122.5994 & 4.40994 \\
\hline 1009 & 20.1363 & 724.9839 & 638.2031 & 599 & 122.5994 & 4.40625 \\
\hline 1010 & 20.1533 & 729.7335 & 634.5934 & 599 & 122.5994 & 4.40257 \\
\hline 1011 & 20.1703 & 672.8612 & 632.76 & 600 & 122.5994 & 4.3989 \\
\hline 1012 & 20.1873 & 705.4214 & 630.4788 & 600 & 122.5994 & 4.39523 \\
\hline 1013 & 20.2043 & 748.9015 & 629.5574 & 601 & 122.5994 & 4.39157 \\
\hline 1014 & 20.2213 & 717.5077 & 627.8912 & 601 & 122.5994 & 4.38792 \\
\hline 1015 & 20.2383 & 720.6944 & 627.3614 & 602 & 122.5994 & 4.38427 \\
\hline 1016 & 20.2553 & 710.1567 & 625.9122 & 602 & 122.5994 & 4.38063 \\
\hline 1017 & 20.2723 & 703.0035 & 624.5252 & 602 & 122.5994 & 4.377 \\
\hline 1018 & 20.2893 & 688.2381 & 624.4016 & 603 & 122.5994 & 4.37337 \\
\hline 1019 & 20.3063 & 695.9581 & 623.1829 & 603 & 122.5994 & 4.36974 \\
\hline 1020 & 20.3233 & 658.4898 & 623.0756 & 604 & 122.5994 & 4.36613 \\
\hline 1021 & 20.3403 & 674.9104 & 622.1915 & 604 & 122.5994 & 4.36252 \\
\hline 1022 & 20.3573 & 665.9826 & 621.3631 & 604 & 122.5994 & 4.35891 \\
\hline 1023 & 20.3743 & 650.4212 & 621.7049 & 605 & 122.5994 & 4.35531 \\
\hline 1024 & 20.3913 & 660.5596 & 621.2308 & 605 & 122.5994 & 4.35172 \\
\hline 1025 & 20.4083 & 667.1955 & 621.9498 & 606 & 122.5994 & 4.34813 \\
\hline 1026 & 20.4253 & 709.7856 & 621.8681 & 606 & 122.5994 & 4.34455 \\
\hline 1027 & 20.4423 & 657.3298 & 622.9869 & 607 & 122.5994 & 4.34098 \\
\hline
\end{tabular}




\begin{tabular}{|c|c|c|c|c|c|c|}
\hline 1028 & 20.4593 & 673.8259 & 623.3027 & 607 & 122.5994 & 4.33741 \\
\hline 1029 & 20.4763 & 609.2475 & 623.8129 & 607 & 122.5994 & 4.33385 \\
\hline 1030 & 20.4933 & 638.6886 & 625.5215 & 608 & 122.5994 & 4.33029 \\
\hline 1031 & 20.5103 & 635.785 & 626.4412 & 608 & 122.5994 & 4.32674 \\
\hline 1032 & 20.5273 & 666.6 & 628.4871 & 609 & 122.5994 & 4.3232 \\
\hline 1033 & 20.5443 & 672.9527 & 629.8947 & 609 & 122.5994 & 4.31966 \\
\hline 1034 & 20.5613 & 674.2147 & 631.5805 & 609 & 122.5994 & 4.31612 \\
\hline 1035 & 20.5783 & 661.7613 & 633.5676 & 609 & 122.5994 & 4.3126 \\
\hline 1036 & 20.5953 & 678.9425 & 636.8746 & 610 & 122.5994 & 4.30907 \\
\hline 1037 & 20.6123 & 635.9085 & 639.5142 & 610 & 122.5994 & 4.30556 \\
\hline 1038 & 20.6293 & 663.2073 & 642.4922 & 610 & 122.5994 & 4.30205 \\
\hline 1039 & 20.6463 & 698.782 & 645.9517 & 610 & 122.5994 & 4.29855 \\
\hline 1040 & 20.6633 & 705.3677 & 649.6185 & 610 & 122.5994 & 4.29505 \\
\hline 1041 & 20.6803 & 687.7521 & 654.6539 & 611 & 122.5994 & 4.29156 \\
\hline 1042 & 20.6973 & 683.2283 & 659.1815 & 611 & 122.5994 & 4.28807 \\
\hline 1043 & 20.7143 & 686.4528 & 664.2827 & 611 & 122.5994 & 4.28459 \\
\hline 1044 & 20.7313 & 702.8626 & 670.5684 & 611 & 122.5994 & 4.28111 \\
\hline 1045 & 20.7483 & 711.2842 & 678.9176 & 611 & 122.5994 & 4.27764 \\
\hline 1046 & 20.7653 & 715.1193 & 691.8221 & 612 & 122.5994 & 4.27418 \\
\hline 1047 & 20.7823 & 753.8725 & 711.099 & 612 & 122.5994 & 4.27072 \\
\hline 1048 & 20.7993 & 775.7041 & 742.4662 & 612 & 122.5994 & 4.26727 \\
\hline 1049 & 20.8163 & 809.6099 & 792.5996 & 612 & 122.5994 & 4.26382 \\
\hline 1050 & 20.8333 & 858.5906 & 868.0896 & 612 & 122.5994 & 4.26038 \\
\hline 1051 & 20.8503 & 911.8213 & 974.5643 & 613 & 122.5994 & 4.25695 \\
\hline 1052 & 20.8673 & 1030.044 & 1106.55 & 613 & 122.5994 & 4.25352 \\
\hline 1053 & 20.8843 & 1166.537 & 1248.115 & 613 & 122.5994 & 4.25009 \\
\hline 1054 & 20.9013 & 1280.95 & 1363.932 & 613 & 122.5994 & 4.24667 \\
\hline 1055 & 20.9183 & 1439.779 & 1415.801 & 613 & 122.5994 & 4.24326 \\
\hline 1056 & 20.9353 & 1689.931 & 1400.082 & 614 & 122.5994 & 4.23985 \\
\hline 1057 & 20.9523 & 1903.147 & 1334.963 & 614 & 122.5994 & 4.23645 \\
\hline 1058 & 20.9693 & 1757.274 & 1236.653 & 614 & 122.5994 & 4.23306 \\
\hline 1059 & 20.9863 & 1507.65 & 1120.786 & 614 & 122.5994 & 4.22967 \\
\hline 1060 & 21.0033 & 1380.758 & 1009.541 & 614 & 122.5994 & 4.22628 \\
\hline 1061 & 21.0203 & 1168.417 & 916.983 & 613 & 122.5994 & 4.2229 \\
\hline 1062 & 21.0373 & 953.0707 & 848.3583 & 613 & 122.5994 & 4.21953 \\
\hline 1063 & 21.0543 & 780.4949 & 800.0206 & 613 & 122.5994 & 4.21616 \\
\hline 1064 & 21.0713 & 751.4778 & 767.1001 & 613 & 122.5994 & 4.2128 \\
\hline 1065 & 21.0883 & 755.6786 & 744.567 & 613 & 122.5994 & 4.20944 \\
\hline 1066 & 21.1053 & 701.9397 & 728.342 & 613 & 122.5994 & 4.20609 \\
\hline 1067 & 21.1223 & 716.898 & 715.6551 & 613 & 122.5994 & 4.20274 \\
\hline 1068 & 21.1393 & 687.7935 & 704.7534 & 613 & 122.5994 & 4.1994 \\
\hline 1069 & 21.1563 & 712.3578 & 694.8208 & 613 & 122.5994 & 4.19606 \\
\hline 1070 & 21.1733 & 675.9979 & 685.5649 & 613 & 122.5994 & 4.19273 \\
\hline 1071 & 21.1903 & 659.4101 & 676.8942 & 613 & 122.5994 & 4.18941 \\
\hline 1072 & 21.2073 & 614.6177 & 668.8518 & 613 & 122.5994 & 4.18609 \\
\hline 1073 & 21.2243 & 606.1431 & 661.655 & 613 & 122.5994 & 4.18277 \\
\hline 1074 & 21.2413 & 619.5893 & 655.3507 & 613 & 122.5994 & 4.17946 \\
\hline 1075 & 21.2583 & 618.3197 & 648.9575 & 612 & 122.5994 & 4.17616 \\
\hline 1076 & 21.2753 & 649.1826 & 644.5396 & 612 & 122.5994 & 4.17286 \\
\hline
\end{tabular}




\begin{tabular}{|c|c|c|c|c|c|c|}
\hline 1077 & 21.2923 & 662.9836 & 641.0319 & 612 & 122.5994 & 4.16957 \\
\hline 1078 & 21.3093 & 587.1656 & 638.3695 & 612 & 122.5994 & 4.16628 \\
\hline 1079 & 21.3263 & 585.6063 & 636.4686 & 612 & 122.5994 & 4.163 \\
\hline 1080 & 21.3433 & 638.6641 & 635.2274 & 612 & 122.5994 & 4.15972 \\
\hline 1081 & 21.3603 & 612.704 & 634.5244 & 612 & 122.5994 & 4.15645 \\
\hline 1082 & 21.3773 & 610.3126 & 634.2143 & 612 & 122.5994 & 4.15318 \\
\hline 1083 & 21.3943 & 611.2587 & 634.1268 & 612 & 122.5994 & 4.14992 \\
\hline 1084 & 21.4113 & 584.7538 & 634.0848 & 612 & 122.5994 & 4.14666 \\
\hline 1085 & 21.4283 & 623.0776 & 632.9506 & 611 & 122.5994 & 4.14341 \\
\hline 1086 & 21.4453 & 636.341 & 632.6675 & 611 & 122.5994 & 4.14016 \\
\hline 1087 & 21.4623 & 603.1558 & 633.0001 & 611 & 122.5994 & 4.13692 \\
\hline 1088 & 21.4793 & 621.1449 & 632.5691 & 611 & 122.5994 & 4.13369 \\
\hline 1089 & 21.4963 & 611.003 & 632.1715 & 611 & 122.5994 & 4.13046 \\
\hline 1090 & 21.5133 & 611.5051 & 631.9284 & 611 & 122.5994 & 4.12723 \\
\hline 1091 & 21.5303 & 633.7119 & 630.8822 & 610 & 122.5994 & 4.12401 \\
\hline 1092 & 21.5473 & 640.6632 & 631.1044 & 610 & 122.5994 & 4.12079 \\
\hline 1093 & 21.5643 & 637.533 & 631.6053 & 610 & 122.5994 & 4.11758 \\
\hline 1094 & 21.5813 & 637.3345 & 632.3578 & 610 & 122.5994 & 4.11438 \\
\hline 1095 & 21.5983 & 643.6819 & 633.303 & 610 & 122.5994 & 4.11118 \\
\hline 1096 & 21.6153 & 627.1709 & 634.3604 & 610 & 122.5994 & 4.10798 \\
\hline 1097 & 21.6323 & 605.1713 & 635.4448 & 610 & 122.5994 & 4.10479 \\
\hline 1098 & 21.6493 & 599.2585 & 635.4874 & 609 & 122.5994 & 4.10161 \\
\hline 1099 & 21.6663 & 598.4268 & 636.4515 & 609 & 122.5994 & 4.09843 \\
\hline 1100 & 21.6833 & 626.0147 & 637.3319 & 609 & 122.5994 & 4.09525 \\
\hline 1101 & 21.7003 & 640.102 & 638.1363 & 609 & 122.5994 & 4.09209 \\
\hline 1102 & 21.7173 & 609.1927 & 638.7866 & 609 & 122.5994 & 4.08892 \\
\hline 1103 & 21.7343 & 609.3619 & 639.3865 & 609 & 122.5994 & 4.08576 \\
\hline 1104 & 21.7513 & 648.3412 & 638.7927 & 608 & 122.5994 & 4.08261 \\
\hline 1105 & 21.7683 & 611.3954 & 636.866 & 608 & 122.5994 & 4.07946 \\
\hline 1106 & 21.7853 & 580.3571 & 636.8487 & 608 & 122.5994 & 4.07631 \\
\hline 1107 & 21.8023 & 619.0596 & 636.593 & 608 & 122.5994 & 4.07317 \\
\hline 1108 & 21.8193 & 577.9192 & 636.1126 & 608 & 122.5994 & 4.07004 \\
\hline 1109 & 21.8363 & 562.4605 & 635.0606 & 608 & 122.5994 & 4.06691 \\
\hline 1110 & 21.8533 & 607.6638 & 633.1633 & 607 & 122.5994 & 4.06378 \\
\hline 1111 & 21.8703 & 610.6803 & 632.0988 & 607 & 122.5994 & 4.06066 \\
\hline 1112 & 21.8873 & 628.1213 & 630.9529 & 607 & 122.5994 & 4.05754 \\
\hline 1113 & 21.9043 & 644.0529 & 629.8599 & 607 & 122.5994 & 4.05443 \\
\hline 1114 & 21.9213 & 625.9951 & 628.9993 & 607 & 122.5994 & 4.05133 \\
\hline 1115 & 21.9383 & 615.3594 & 628.6251 & 607 & 122.5994 & 4.04823 \\
\hline 1116 & 21.9553 & 576.377 & 629.153 & 607 & 122.5994 & 4.04513 \\
\hline 1117 & 21.9723 & 595.7325 & 630.9858 & 606 & 122.5994 & 4.04204 \\
\hline 1118 & 21.9893 & 624.1769 & 636.0384 & 606 & 122.5994 & 4.03895 \\
\hline 1119 & 22.0063 & 603.2316 & 645.7578 & 606 & 122.5994 & 4.03587 \\
\hline 1120 & 22.0233 & 655.7656 & 662.3245 & 606 & 122.5994 & 4.03279 \\
\hline 1121 & 22.0403 & 655.068 & 686.8132 & 606 & 122.5994 & 4.02972 \\
\hline 1122 & 22.0573 & 618.0722 & 718.4816 & 606 & 122.5994 & 4.02665 \\
\hline 1123 & 22.0743 & 706.2275 & 751.5364 & 605 & 122.5994 & 4.02359 \\
\hline 1124 & 22.0913 & 669.8939 & 776.9333 & 605 & 122.5994 & 4.02053 \\
\hline 1125 & 22.1083 & 698.5813 & 783.3484 & 605 & 122.5994 & 4.01748 \\
\hline
\end{tabular}




\begin{tabular}{|c|c|c|c|c|c|c|}
\hline 1126 & 22.1253 & 728.9301 & 773.4777 & 605 & 122.5994 & 4.01443 \\
\hline 1127 & 22.1423 & 730.2434 & 754.4745 & 605 & 122.5994 & 4.01139 \\
\hline 1128 & 22.1593 & 730.3999 & 728.9078 & 605 & 122.5994 & 4.00835 \\
\hline 1129 & 22.1763 & 698.4939 & 698.9702 & 604 & 122.5994 & 4.00532 \\
\hline 1130 & 22.1933 & 640.7495 & 672.5461 & 604 & 122.5994 & 4.00229 \\
\hline 1131 & 22.2103 & 625.3294 & 651.992 & 604 & 122.5994 & 3.99926 \\
\hline 1132 & 22.2273 & 598.7062 & 636.6378 & 603 & 122.5994 & 3.99624 \\
\hline 1133 & 22.2443 & 587.3218 & 627.4434 & 603 & 122.5994 & 3.99322 \\
\hline 1134 & 22.2613 & 610.1359 & 621.9097 & 603 & 122.5994 & 3.99021 \\
\hline 1135 & 22.2783 & 595.4182 & 617.709 & 602 & 122.5994 & 3.98721 \\
\hline 1136 & 22.2953 & 608.0258 & 615.8888 & 602 & 122.5994 & 3.98421 \\
\hline 1137 & 22.3123 & 620.6295 & 614.8733 & 602 & 122.5994 & 3.98121 \\
\hline 1138 & 22.3293 & 633.0273 & 613.3533 & 601 & 122.5994 & 3.97822 \\
\hline 1139 & 22.3463 & 615.3793 & 613.168 & 601 & 122.5994 & 3.97523 \\
\hline 1140 & 22.3633 & 602.6818 & 613.225 & 601 & 122.5994 & 3.97224 \\
\hline 1141 & 22.3803 & 592.5388 & 612.457 & 600 & 122.5994 & 3.96927 \\
\hline 1142 & 22.3973 & 610.0592 & 612.8026 & 600 & 122.5994 & 3.96629 \\
\hline 1143 & 22.4143 & 599.1474 & 613.1978 & 600 & 122.5994 & 3.96332 \\
\hline 1144 & 22.4313 & 578.7974 & 612.5753 & 599 & 122.5994 & 3.96036 \\
\hline 1145 & 22.4483 & 589.7632 & 612.8708 & 599 & 122.5994 & 3.9574 \\
\hline 1146 & 22.4653 & 601.6052 & 612.0337 & 598 & 122.5994 & 3.95444 \\
\hline 1147 & 22.4823 & 611.6691 & 612.0376 & 598 & 122.5994 & 3.95149 \\
\hline 1148 & 22.4993 & 553.4954 & 611.8832 & 598 & 122.5994 & 3.94854 \\
\hline 1149 & 22.5163 & 601.9424 & 610.5951 & 597 & 122.5994 & 3.9456 \\
\hline 1150 & 22.5333 & 595.8165 & 610.3549 & 597 & 122.5994 & 3.94266 \\
\hline 1151 & 22.5503 & 580.8209 & 609.9418 & 597 & 122.5994 & 3.93973 \\
\hline 1152 & 22.5673 & 603.2267 & 608.5449 & 596 & 122.5994 & 3.9368 \\
\hline 1153 & 22.5843 & 606.2227 & 608.2204 & 596 & 122.5994 & 3.93387 \\
\hline 1154 & 22.6013 & 581.6014 & 608.0222 & 596 & 122.5994 & 3.93095 \\
\hline 1155 & 22.6183 & 592.0763 & 608.0043 & 596 & 122.5994 & 3.92803 \\
\hline 1156 & 22.6353 & 603.6452 & 608.2243 & 596 & 122.5994 & 3.92512 \\
\hline 1157 & 22.6523 & 600.8859 & 608.6722 & 596 & 122.5994 & 3.92222 \\
\hline 1158 & 22.6693 & 582.8756 & 610.5847 & 597 & 122.5994 & 3.91931 \\
\hline 1159 & 22.6863 & 585.2444 & 611.9803 & 597 & 122.5994 & 3.91641 \\
\hline 1160 & 22.7033 & 585.6509 & 612.9584 & 597 & 122.5994 & 3.91352 \\
\hline 1161 & 22.7203 & 604.3692 & 615.7341 & 597 & 122.5994 & 3.91063 \\
\hline 1162 & 22.7373 & 595.4945 & 619.3699 & 597 & 122.5994 & 3.90774 \\
\hline 1163 & 22.7543 & 590.501 & 623.9943 & 597 & 122.5994 & 3.90486 \\
\hline 1164 & 22.7713 & 589.9374 & 629.7087 & 597 & 122.5994 & 3.90199 \\
\hline 1165 & 22.7883 & 569.0841 & 637.5637 & 598 & 122.5994 & 3.89911 \\
\hline 1166 & 22.8053 & 622.394 & 645.5289 & 598 & 122.5994 & 3.89625 \\
\hline 1167 & 22.8223 & 586.4385 & 654.4571 & 598 & 122.5994 & 3.89338 \\
\hline 1168 & 22.8393 & 601.3795 & 664.0406 & 598 & 122.5994 & 3.89052 \\
\hline 1169 & 22.8563 & 646.9545 & 674.4332 & 598 & 122.5994 & 3.88767 \\
\hline 1170 & 22.8733 & 623.4925 & 683.7004 & 598 & 122.5994 & 3.88482 \\
\hline 1171 & 22.8903 & 618.5328 & 691.7504 & 598 & 122.5994 & 3.88197 \\
\hline 1172 & 22.9073 & 642.0485 & 697.9969 & 598 & 122.5994 & 3.87913 \\
\hline 1173 & 22.9243 & 661.9634 & 702.3707 & 598 & 122.5994 & 3.87629 \\
\hline 1174 & 22.9413 & 644.6638 & 705.1887 & 599 & 122.5994 & 3.87346 \\
\hline
\end{tabular}




\begin{tabular}{|c|c|c|c|c|c|c|}
\hline 1175 & 22.9583 & 686.2722 & 704.7512 & 599 & 122.5994 & 3.87063 \\
\hline 1176 & 22.9753 & 678.1871 & 702.2933 & 599 & 122.5994 & 3.8678 \\
\hline 1177 & 22.9923 & 695.3754 & 698.3094 & 599 & 122.5994 & 3.86498 \\
\hline 1178 & 23.0093 & 673.3089 & 693.522 & 599 & 122.5994 & 3.86216 \\
\hline 1179 & 23.0263 & 652.8482 & 688.7381 & 599 & 122.5994 & 3.85935 \\
\hline 1180 & 23.0433 & 674.9475 & 684.637 & 599 & 122.5994 & 3.85654 \\
\hline 1181 & 23.0603 & 657.3688 & 682.4998 & 600 & 122.5994 & 3.85374 \\
\hline 1182 & 23.0773 & 669.0125 & 679.8466 & 600 & 122.5994 & 3.85093 \\
\hline 1183 & 23.0943 & 667.8401 & 676.189 & 600 & 122.5994 & 3.84814 \\
\hline 1184 & 23.1113 & 643.36 & 670.1745 & 600 & 122.5994 & 3.84535 \\
\hline 1185 & 23.1283 & 652.0543 & 662.4064 & 600 & 122.5994 & 3.84256 \\
\hline 1186 & 23.1453 & 643.0421 & 654.2334 & 600 & 122.5994 & 3.83977 \\
\hline 1187 & 23.1623 & 615.0515 & 645.8347 & 600 & 122.5994 & 3.83699 \\
\hline 1188 & 23.1793 & 604.4279 & 637.5764 & 600 & 122.5994 & 3.83422 \\
\hline 1189 & 23.1963 & 617.6456 & 630.2611 & 600 & 122.5994 & 3.83145 \\
\hline 1190 & 23.2133 & 632.1199 & 625.4026 & 601 & 122.5994 & 3.82868 \\
\hline 1191 & 23.2303 & 600.2842 & 621.0231 & 601 & 122.5994 & 3.82592 \\
\hline 1192 & 23.2473 & 559.945 & 617.8977 & 601 & 122.5994 & 3.82316 \\
\hline 1193 & 23.2643 & 600.9465 & 615.7306 & 601 & 122.5994 & 3.8204 \\
\hline 1194 & 23.2813 & 597.1113 & 614.2472 & 601 & 122.5994 & 3.81765 \\
\hline 1195 & 23.2983 & 554.6391 & 613.2388 & 601 & 122.5994 & 3.8149 \\
\hline 1196 & 23.3153 & 542.9001 & 612.5687 & 601 & 122.5994 & 3.81216 \\
\hline 1197 & 23.3323 & 556.6903 & 613.1572 & 602 & 122.5994 & 3.80942 \\
\hline 1198 & 23.3493 & 568.8231 & 612.9625 & 602 & 122.5994 & 3.80669 \\
\hline 1199 & 23.3663 & 612.9871 & 612.97 & 602 & 122.5994 & 3.80396 \\
\hline 1200 & 23.3833 & 601.3588 & 613.1897 & 602 & 122.5994 & 3.80123 \\
\hline 1201 & 23.4003 & 611.4997 & 613.6686 & 602 & 122.5994 & 3.79851 \\
\hline 1202 & 23.4173 & 598.4991 & 614.5247 & 602 & 122.5994 & 3.79579 \\
\hline 1203 & 23.4343 & 570.4244 & 616.0145 & 602 & 122.5994 & 3.79307 \\
\hline 1204 & 23.4513 & 587.6466 & 619.6204 & 603 & 122.5994 & 3.79036 \\
\hline 1205 & 23.4683 & 599.3394 & 624.0547 & 603 & 122.5994 & 3.78765 \\
\hline 1206 & 23.4853 & 609.3839 & 631.3212 & 603 & 122.5994 & 3.78495 \\
\hline 1207 & 23.5023 & 624.2456 & 642.0948 & 603 & 122.5994 & 3.78225 \\
\hline 1208 & 23.5193 & 654.4661 & 656.2969 & 603 & 122.5994 & 3.77955 \\
\hline 1209 & 23.5363 & 635.2208 & 673.142 & 604 & 122.5994 & 3.77686 \\
\hline 1210 & 23.5533 & 644.8414 & 686.3127 & 604 & 122.5994 & 3.77418 \\
\hline 1211 & 23.5703 & 621.2476 & 691.8195 & 604 & 122.5994 & 3.77149 \\
\hline 1212 & 23.5873 & 653.385 & 689.783 & 604 & 122.5994 & 3.76881 \\
\hline 1213 & 23.6043 & 625.5948 & 684.0143 & 604 & 122.5994 & 3.76614 \\
\hline 1214 & 23.6213 & 690.6624 & 677.1269 & 605 & 122.5994 & 3.76346 \\
\hline 1215 & 23.6383 & 683.1257 & 667.6072 & 605 & 122.5994 & 3.7608 \\
\hline 1216 & 23.6553 & 691.7056 & 658.6182 & 605 & 122.5994 & 3.75813 \\
\hline 1217 & 23.6723 & 665.6122 & 652.2584 & 605 & 122.5994 & 3.75547 \\
\hline 1218 & 23.6893 & 693.6598 & 650.1898 & 606 & 122.5994 & 3.75281 \\
\hline 1219 & 23.7063 & 690.4543 & 650.4284 & 606 & 122.5994 & 3.75016 \\
\hline 1220 & 23.7233 & 647.5015 & 652.7475 & 606 & 122.5994 & 3.74751 \\
\hline 1221 & 23.7403 & 659.2335 & 656.4269 & 606 & 122.5994 & 3.74487 \\
\hline 1222 & 23.7573 & 674.9866 & 661.6591 & 606 & 122.5994 & 3.74223 \\
\hline 1223 & 23.7743 & 680.3611 & 668.4797 & 607 & 122.5994 & 3.73959 \\
\hline
\end{tabular}




\begin{tabular}{|c|c|c|c|c|c|c|}
\hline 1224 & 23.7913 & 659.2134 & 674.7178 & 607 & 122.5994 & 3.73696 \\
\hline 1225 & 23.8083 & 645.1721 & 680.9179 & 607 & 122.5994 & 3.73433 \\
\hline 1226 & 23.8253 & 636.1815 & 687.005 & 607 & 122.5994 & 3.7317 \\
\hline 1227 & 23.8423 & 645.1993 & 692.572 & 607 & 122.5994 & 3.72908 \\
\hline 1228 & 23.8593 & 688.4764 & 697.8293 & 608 & 122.5994 & 3.72646 \\
\hline 1229 & 23.8763 & 676.3729 & 700.5222 & 608 & 122.5994 & 3.72384 \\
\hline 1230 & 23.8933 & 669.1563 & 701.4203 & 608 & 122.5994 & 3.72123 \\
\hline 1231 & 23.9103 & 684.0207 & 700.2039 & 608 & 122.5994 & 3.71863 \\
\hline 1232 & 23.9273 & 671.141 & 697.6165 & 608 & 122.5994 & 3.71602 \\
\hline 1233 & 23.9443 & 665.746 & 693.7064 & 609 & 122.5994 & 3.71342 \\
\hline 1234 & 23.9613 & 649.141 & 687.5045 & 609 & 122.5994 & 3.71083 \\
\hline 1235 & 23.9783 & 635.5765 & 680.5612 & 609 & 122.5994 & 3.70823 \\
\hline 1236 & 23.9953 & 666.6418 & 673.161 & 609 & 122.5994 & 3.70565 \\
\hline 1237 & 24.0123 & 680.2501 & 667.5609 & 610 & 122.5994 & 3.70306 \\
\hline 1238 & 24.0293 & 638.4816 & 662.1976 & 610 & 122.5994 & 3.70048 \\
\hline 1239 & 24.0463 & 599.4082 & 658.6519 & 610 & 122.5994 & 3.6979 \\
\hline 1240 & 24.0633 & 616.4621 & 658.0259 & 610 & 122.5994 & 3.69533 \\
\hline 1241 & 24.0803 & 616.4731 & 659.9012 & 610 & 122.5994 & 3.69276 \\
\hline 1242 & 24.0973 & 624.0791 & 665.9328 & 611 & 122.5994 & 3.69019 \\
\hline 1243 & 24.1143 & 637.3119 & 671.7479 & 611 & 122.5994 & 3.68763 \\
\hline 1244 & 24.1313 & 643.8779 & 675.1359 & 611 & 122.5994 & 3.68507 \\
\hline 1245 & 24.1483 & 656.9194 & 674.183 & 611 & 122.5994 & 3.68251 \\
\hline 1246 & 24.1653 & 597.6614 & 670.6306 & 611 & 122.5994 & 3.67996 \\
\hline 1247 & 24.1823 & 608.5215 & 667.686 & 612 & 122.5994 & 3.67741 \\
\hline 1248 & 24.1993 & 606.8433 & 663.8839 & 612 & 122.5994 & 3.67487 \\
\hline 1249 & 24.2163 & 634.9429 & 660.9057 & 612 & 122.5994 & 3.67233 \\
\hline 1250 & 24.2333 & 635.689 & 660.0728 & 612 & 122.5994 & 3.66979 \\
\hline 1251 & 24.2503 & 630.548 & 661.6668 & 612 & 122.5994 & 3.66725 \\
\hline 1252 & 24.2673 & 643.8393 & 664.6537 & 612 & 122.5994 & 3.66472 \\
\hline 1253 & 24.2843 & 645.1721 & 668.5607 & 612 & 122.5994 & 3.6622 \\
\hline 1254 & 24.3013 & 669.793 & 674.6248 & 612 & 122.5994 & 3.65967 \\
\hline 1255 & 24.3183 & 675.9161 & 683.4235 & 612 & 122.5994 & 3.65715 \\
\hline 1256 & 24.3353 & 686.3757 & 693.0018 & 612 & 122.5994 & 3.65464 \\
\hline 1257 & 24.3523 & 662.0491 & 699.2534 & 612 & 122.5994 & 3.65212 \\
\hline 1258 & 24.3693 & 721.5933 & 698.4197 & 612 & 122.5994 & 3.64961 \\
\hline 1259 & 24.3863 & 679.8249 & 691.4964 & 612 & 122.5994 & 3.64711 \\
\hline 1260 & 24.4033 & 661.8827 & 682.1735 & 612 & 122.5994 & 3.64461 \\
\hline 1261 & 24.4203 & 674.2983 & 673.1984 & 612 & 122.5994 & 3.64211 \\
\hline 1262 & 24.4373 & 656.477 & 661.3138 & 612 & 122.5994 & 3.63961 \\
\hline 1263 & 24.4543 & 633.1307 & 649.5936 & 612 & 122.5994 & 3.63712 \\
\hline 1264 & 24.4713 & 639.7284 & 639.8132 & 612 & 122.5994 & 3.63463 \\
\hline 1265 & 24.4883 & 601.2467 & 633.3181 & 612 & 122.5994 & 3.63215 \\
\hline 1266 & 24.5053 & 629.0442 & 628.4041 & 612 & 122.5994 & 3.62967 \\
\hline 1267 & 24.5223 & 580.5373 & 625.2659 & 612 & 122.5994 & 3.62719 \\
\hline 1268 & 24.5393 & 608.8139 & 623.3091 & 612 & 122.5994 & 3.62471 \\
\hline 1269 & 24.5563 & 617.9181 & 622.116 & 612 & 122.5994 & 3.62224 \\
\hline 1270 & 24.5733 & 651.7417 & 621.9783 & 612 & 122.5994 & 3.61978 \\
\hline 1271 & 24.5903 & 642.242 & 621.4932 & 612 & 122.5994 & 3.61731 \\
\hline 1272 & 24.6073 & 604.8442 & 621.1744 & 612 & 122.5994 & 3.61485 \\
\hline
\end{tabular}




\begin{tabular}{|c|c|c|c|c|c|c|}
\hline 1273 & 24.6243 & 589.2349 & 620.9769 & 612 & 122.5994 & 3.6124 \\
\hline 1274 & 24.6413 & 608.8935 & 622.1721 & 613 & 122.5994 & 3.60994 \\
\hline 1275 & 24.6583 & 644.6939 & 623.169 & 614 & 122.5994 & 3.60749 \\
\hline 1276 & 24.6753 & 639.3755 & 624.2455 & 615 & 122.5994 & 3.60504 \\
\hline 1277 & 24.6923 & 614.1935 & 624.3985 & 615 & 122.5994 & 3.6026 \\
\hline 1278 & 24.7093 & 603.9648 & 625.6278 & 616 & 122.5994 & 3.60016 \\
\hline 1279 & 24.7263 & 592.9427 & 626.935 & 617 & 122.5994 & 3.59772 \\
\hline 1280 & 24.7433 & 625.9454 & 628.3229 & 618 & 122.5994 & 3.59529 \\
\hline 1281 & 24.7603 & 636.9776 & 629.7952 & 619 & 122.5994 & 3.59286 \\
\hline 1282 & 24.7773 & 627.1324 & 631.3552 & 620 & 122.5994 & 3.59043 \\
\hline 1283 & 24.7943 & 619.1966 & 633.0062 & 621 & 122.5994 & 3.58801 \\
\hline 1284 & 24.8113 & 636.3668 & 634.75 & 622 & 122.5994 & 3.58559 \\
\hline 1285 & 24.8283 & 682.8615 & 635.5872 & 622 & 122.5994 & 3.58317 \\
\hline 1286 & 24.8453 & 695.4155 & 637.5156 & 623 & 122.5994 & 3.58076 \\
\hline 1287 & 24.8623 & 688.1855 & 639.5825 & 624 & 122.5994 & 3.57835 \\
\hline 1288 & 24.8793 & 708.3607 & 641.2389 & 625 & 122.5994 & 3.57594 \\
\hline 1289 & 24.8963 & 658.9371 & 643.427 & 626 & 122.5994 & 3.57354 \\
\hline 1290 & 24.9133 & 684.4157 & 645.6926 & 627 & 122.5994 & 3.57114 \\
\hline 1291 & 24.9303 & 706.2775 & 647.1998 & 628 & 122.5994 & 3.56874 \\
\hline 1292 & 24.9473 & 678.9986 & 649.7501 & 629 & 122.5994 & 3.56635 \\
\hline 1293 & 24.9643 & 721.6438 & 651.5237 & 629 & 122.5994 & 3.56396 \\
\hline 1294 & 24.9813 & 775.028 & 654.6479 & 630 & 122.5994 & 3.56157 \\
\hline 1295 & 24.9983 & 697.4622 & 658.3044 & 631 & 122.5994 & 3.55919 \\
\hline 1296 & 25.0153 & 685.7392 & 662.739 & 632 & 122.5994 & 3.55681 \\
\hline 1297 & 25.0323 & 748.2418 & 668.2702 & 633 & 122.5994 & 3.55443 \\
\hline 1298 & 25.0493 & 712.12 & 675.29 & 634 & 122.5994 & 3.55206 \\
\hline 1299 & 25.0663 & 722.4704 & 683.2534 & 634 & 122.5994 & 3.54969 \\
\hline 1300 & 25.0833 & 733.7962 & 694.6526 & 635 & 122.5994 & 3.54732 \\
\hline 1301 & 25.1003 & 723.9356 & 707.9736 & 635 & 122.5994 & 3.54496 \\
\hline 1302 & 25.1173 & 707.6913 & 725.6307 & 636 & 122.5994 & 3.5426 \\
\hline 1303 & 25.1343 & 777.4932 & 746.8834 & 637 & 122.5994 & 3.54024 \\
\hline 1304 & 25.1513 & 713.2866 & 770.7445 & 637 & 122.5994 & 3.53789 \\
\hline 1305 & 25.1683 & 727.7269 & 798.8467 & 638 & 122.5994 & 3.53553 \\
\hline 1306 & 25.1853 & 799.2226 & 829.3024 & 639 & 122.5994 & 3.53319 \\
\hline 1307 & 25.2023 & 845.0847 & 859.5343 & 639 & 122.5994 & 3.53084 \\
\hline 1308 & 25.2193 & 844.7225 & 889.1767 & 640 & 122.5994 & 3.5285 \\
\hline 1309 & 25.2363 & 949.5655 & 914.2541 & 641 & 122.5994 & 3.52616 \\
\hline 1310 & 25.2533 & 915.1251 & 930.8615 & 641 & 122.5994 & 3.52383 \\
\hline 1311 & 25.2703 & 914.8695 & 939.1492 & 642 & 122.5994 & 3.52149 \\
\hline 1312 & 25.2873 & 970.592 & 937.8753 & 643 & 122.5994 & 3.51917 \\
\hline 1313 & 25.3043 & 923.9315 & 927.0947 & 643 & 122.5994 & 3.51684 \\
\hline 1314 & 25.3213 & 871.7982 & 910.5347 & 644 & 122.5994 & 3.51452 \\
\hline 1315 & 25.3383 & 822.4486 & 889.3197 & 645 & 122.5994 & 3.5122 \\
\hline 1316 & 25.3553 & 776.1348 & 864.8665 & 645 & 122.5994 & 3.50988 \\
\hline 1317 & 25.3723 & 803.8297 & 841.5978 & 646 & 122.5994 & 3.50757 \\
\hline 1318 & 25.3893 & 823.6851 & 819.5746 & 646 & 122.5994 & 3.50526 \\
\hline 1319 & 25.4063 & 770.4817 & 802.3093 & 647 & 122.5994 & 3.50295 \\
\hline 1320 & 25.4233 & 764.6842 & 789.7861 & 648 & 122.5994 & 3.50065 \\
\hline 1321 & 25.4403 & 785.1818 & 781.5428 & 648 & 122.5994 & 3.49835 \\
\hline
\end{tabular}




\begin{tabular}{|c|c|c|c|c|c|c|}
\hline 1322 & 25.4573 & 778.7607 & 779.6907 & 649 & 122.5994 & 3.49605 \\
\hline 1323 & 25.4743 & 756.2641 & 781.7928 & 649 & 122.5994 & 3.49375 \\
\hline 1324 & 25.4913 & 761.322 & 788.5453 & 650 & 122.5994 & 3.49146 \\
\hline 1325 & 25.5083 & 767.3043 & 795.5128 & 650 & 122.5994 & 3.48917 \\
\hline 1326 & 25.5253 & 796.0154 & 802.2485 & 651 & 122.5994 & 3.48689 \\
\hline 1327 & 25.5423 & 826.7563 & 805.9778 & 651 & 122.5994 & 3.48461 \\
\hline 1328 & 25.5593 & 835.8381 & 807.2979 & 651 & 122.5994 & 3.48233 \\
\hline 1329 & 25.5763 & 824.5228 & 806.2523 & 652 & 122.5994 & 3.48005 \\
\hline 1330 & 25.5933 & 829.2808 & 800.7855 & 652 & 122.5994 & 3.47778 \\
\hline 1331 & 25.6103 & 812.7991 & 793.9348 & 653 & 122.5994 & 3.47551 \\
\hline 1332 & 25.6273 & 798.8604 & 784.5189 & 653 & 122.5994 & 3.47324 \\
\hline 1333 & 25.6443 & 793.4637 & 774.0316 & 653 & 122.5994 & 3.47098 \\
\hline 1334 & 25.6613 & 812.8409 & 764.0731 & 654 & 122.5994 & 3.46872 \\
\hline 1335 & 25.6783 & 815.6658 & 753.9789 & 654 & 122.5994 & 3.46646 \\
\hline 1336 & 25.6953 & 837.1998 & 744.3243 & 655 & 122.5994 & 3.4642 \\
\hline 1337 & 25.7123 & 833.8727 & 734.1105 & 655 & 122.5994 & 3.46195 \\
\hline 1338 & 25.7293 & 760.7098 & 725.4825 & 656 & 122.5994 & 3.4597 \\
\hline 1339 & 25.7463 & 752.7978 & 723.5159 & 656 & 122.5994 & 3.45746 \\
\hline 1340 & 25.7633 & 695.4577 & 715.6204 & 656 & 122.5994 & 3.45521 \\
\hline 1341 & 25.7803 & 725.4775 & 709.5861 & 657 & 122.5994 & 3.45297 \\
\hline 1342 & 25.7973 & 748.2906 & 703.4594 & 657 & 122.5994 & 3.45074 \\
\hline 1343 & 25.8143 & 676.3101 & 699.2496 & 658 & 122.5994 & 3.4485 \\
\hline 1344 & 25.8313 & 717.6136 & 694.9261 & 658 & 122.5994 & 3.44627 \\
\hline 1345 & 25.8483 & 723.6235 & 699.9898 & 658 & 122.5994 & 3.44404 \\
\hline 1346 & 25.8653 & 723.6235 & 698.5197 & 659 & 122.5994 & 3.44182 \\
\hline 1347 & 25.8823 & 677.8726 & 696.6959 & 659 & 122.5994 & 3.4396 \\
\hline 1348 & 25.8993 & 696.6055 & 695.4233 & 659 & 122.5994 & 3.43738 \\
\hline 1349 & 25.9163 & 681.3053 & 697.0901 & 659 & 122.5994 & 3.43516 \\
\hline 1350 & 25.9333 & 700.6805 & 696.7437 & 659 & 122.5994 & 3.43295 \\
\hline 1351 & 25.9503 & 642.2802 & 696.7535 & 659 & 122.5994 & 3.43074 \\
\hline 1352 & 25.9673 & 686.4528 & 698.0436 & 660 & 122.5994 & 3.42853 \\
\hline 1353 & 25.9843 & 682.2783 & 698.5554 & 660 & 122.5994 & 3.42633 \\
\hline 1354 & 26.0013 & 645.7812 & 699.209 & 660 & 122.5994 & 3.42413 \\
\hline 1355 & 26.0183 & 694.523 & 699.9924 & 660 & 122.5994 & 3.42193 \\
\hline 1356 & 26.0353 & 710.4413 & 700.9012 & 660 & 122.5994 & 3.41973 \\
\hline 1357 & 26.0523 & 693.2822 & 702.9598 & 661 & 122.5994 & 3.41754 \\
\hline 1358 & 26.0693 & 681.2003 & 704.1725 & 661 & 122.5994 & 3.41535 \\
\hline 1359 & 26.0863 & 693.111 & 705.5425 & 661 & 122.5994 & 3.41316 \\
\hline 1360 & 26.1033 & 654.3039 & 705.5516 & 661 & 122.5994 & 3.41098 \\
\hline 1361 & 26.1203 & 644.4277 & 707.3644 & 661 & 122.5994 & 3.40879 \\
\hline 1362 & 26.1373 & 675.256 & 709.3992 & 661 & 122.5994 & 3.40662 \\
\hline 1363 & 26.1543 & 699.4415 & 712.6736 & 662 & 122.5994 & 3.40444 \\
\hline 1364 & 26.1713 & 711.6975 & 714.4463 & 662 & 122.5994 & 3.40227 \\
\hline 1365 & 26.1883 & 700.1432 & 717.2912 & 662 & 122.5994 & 3.4001 \\
\hline 1366 & 26.2053 & 739.1965 & 720.4236 & 662 & 122.5994 & 3.39793 \\
\hline 1367 & 26.2223 & 735.3864 & 723.9417 & 662 & 122.5994 & 3.39577 \\
\hline 1368 & 26.2393 & 729.731 & 727.87 & 662 & 122.5994 & 3.3936 \\
\hline 1369 & 26.2563 & 733.5128 & 733.1737 & 663 & 122.5994 & 3.39145 \\
\hline 1370 & 26.2733 & 757.2764 & 738.1521 & 663 & 122.5994 & 3.38929 \\
\hline
\end{tabular}




\begin{tabular}{|c|c|c|c|c|c|c|}
\hline 1371 & 26.2903 & 737.5279 & 743.8049 & 663 & 122.5994 & 3.38714 \\
\hline 1372 & 26.3073 & 718.2364 & 749.2681 & 662 & 122.5994 & 3.38499 \\
\hline 1373 & 26.3243 & 715.9241 & 756.7045 & 662 & 122.5994 & 3.38284 \\
\hline 1374 & 26.3413 & 697.8265 & 765.3088 & 662 & 122.5994 & 3.38069 \\
\hline 1375 & 26.3583 & 733.7079 & 775.308 & 662 & 122.5994 & 3.37855 \\
\hline 1376 & 26.3753 & 771.9203 & 786.9639 & 662 & 122.5994 & 3.37641 \\
\hline 1377 & 26.3923 & 767.3043 & 800.577 & 662 & 122.5994 & 3.37428 \\
\hline 1378 & 26.4093 & 795.4452 & 816.4988 & 662 & 122.5994 & 3.37214 \\
\hline 1379 & 26.4263 & 827.1754 & 835.1561 & 662 & 122.5994 & 3.37001 \\
\hline 1380 & 26.4433 & 843.0085 & 857.0881 & 662 & 122.5994 & 3.36789 \\
\hline 1381 & 26.4603 & 891.9957 & 883.001 & 662 & 122.5994 & 3.36576 \\
\hline 1382 & 26.4773 & 942.5951 & 913.7867 & 662 & 122.5994 & 3.36364 \\
\hline 1383 & 26.4943 & 991.6637 & 950.573 & 662 & 122.5994 & 3.36152 \\
\hline 1384 & 26.5113 & 995.2256 & 994.886 & 662 & 122.5994 & 3.3594 \\
\hline 1385 & 26.5283 & 1119.747 & 1049.135 & 662 & 122.5994 & 3.35729 \\
\hline 1386 & 26.5453 & 1194.495 & 1117.668 & 662 & 122.5994 & 3.35517 \\
\hline 1387 & 26.5623 & 1291.486 & 1209.3 & 662 & 122.5994 & 3.35307 \\
\hline 1388 & 26.5793 & 1494.572 & 1341.634 & 661 & 122.5994 & 3.35096 \\
\hline 1389 & 26.5963 & 1569.719 & 1548.222 & 661 & 122.5994 & 3.34886 \\
\hline 1390 & 26.6133 & 1780.08 & 1870.479 & 661 & 122.5994 & 3.34676 \\
\hline 1391 & 26.6303 & 2214.493 & 2347.5 & 661 & 122.5994 & 3.34466 \\
\hline 1392 & 26.6473 & 2538.849 & 2999.357 & 661 & 122.5994 & 3.34256 \\
\hline 1393 & 26.6643 & 3139.674 & 3802.441 & 661 & 122.5994 & 3.34047 \\
\hline 1394 & 26.6813 & 3837.376 & 4649.384 & 661 & 122.5994 & 3.33838 \\
\hline 1395 & 26.6983 & 4865.745 & 5322.866 & 661 & 122.5994 & 3.33629 \\
\hline 1396 & 26.7153 & 6355.578 & 5604.273 & 660 & 122.5994 & 3.33421 \\
\hline 1397 & 26.7323 & 7637.453 & 5509.608 & 660 & 122.5994 & 3.33213 \\
\hline 1398 & 26.7493 & 7036.6 & 5220.094 & 660 & 122.5994 & 3.33005 \\
\hline 1399 & 26.7663 & 5283.672 & 4827.816 & 659 & 122.5994 & 3.32797 \\
\hline 1400 & 26.7833 & 4435.908 & 4326.244 & 659 & 122.5994 & 3.3259 \\
\hline 1401 & 26.8003 & 4498.225 & 3756.677 & 659 & 122.5994 & 3.32383 \\
\hline 1402 & 26.8173 & 3919.118 & 3218.51 & 658 & 122.5994 & 3.32176 \\
\hline 1403 & 26.8343 & 2821.277 & 2776.655 & 658 & 122.5994 & 3.31969 \\
\hline 1404 & 26.8513 & 2135.054 & 2440.375 & 658 & 122.5994 & 3.31763 \\
\hline 1405 & 26.8683 & 1914.28 & 2193.092 & 658 & 122.5994 & 3.31557 \\
\hline 1406 & 26.8853 & 1810.458 & 2008.414 & 657 & 122.5994 & 3.31351 \\
\hline 1407 & 26.9023 & 1745.249 & 1863.824 & 657 & 122.5994 & 3.31146 \\
\hline 1408 & 26.9193 & 1741.142 & 1740.806 & 657 & 122.5994 & 3.3094 \\
\hline 1409 & 26.9363 & 1643.096 & 1625.628 & 656 & 122.5994 & 3.30735 \\
\hline 1410 & 26.9533 & 1525.344 & 1516.751 & 656 & 122.5994 & 3.30531 \\
\hline 1411 & 26.9703 & 1418.95 & 1412.809 & 656 & 122.5994 & 3.30326 \\
\hline 1412 & 26.9873 & 1296.631 & 1314.615 & 655 & 122.5994 & 3.30122 \\
\hline 1413 & 27.0043 & 1193.835 & 1224.885 & 655 & 122.5994 & 3.29918 \\
\hline 1414 & 27.0213 & 1066.928 & 1144.208 & 655 & 122.5994 & 3.29714 \\
\hline 1415 & 27.0383 & 978.4518 & 1073.124 & 655 & 122.5994 & 3.29511 \\
\hline 1416 & 27.0553 & 905.0835 & 1010.62 & 654 & 122.5994 & 3.29308 \\
\hline 1417 & 27.0723 & 842.2524 & 958.2549 & 654 & 122.5994 & 3.29105 \\
\hline 1418 & 27.0893 & 801.5818 & 914.2897 & 654 & 122.5994 & 3.28902 \\
\hline 1419 & 27.1063 & 804.4565 & 876.8094 & 653 & 122.5994 & 3.287 \\
\hline
\end{tabular}




\begin{tabular}{|c|c|c|c|c|c|c|}
\hline 1420 & 27.1233 & 820.2231 & 846.8051 & 653 & 122.5994 & 3.28497 \\
\hline 1421 & 27.1403 & 799.7433 & 822.2769 & 653 & 122.5994 & 3.28295 \\
\hline 1422 & 27.1573 & 770.4554 & 801.2764 & 652 & 122.5994 & 3.28094 \\
\hline 1423 & 27.1743 & 766.1543 & 784.9497 & 652 & 122.5994 & 3.27892 \\
\hline 1424 & 27.1913 & 783.2131 & 771.5591 & 652 & 122.5994 & 3.27691 \\
\hline 1425 & 27.2083 & 757.8105 & 760.4891 & 652 & 122.5994 & 3.2749 \\
\hline 1426 & 27.2253 & 729.0468 & 750.2417 & 651 & 122.5994 & 3.2729 \\
\hline 1427 & 27.2423 & 749.6487 & 742.4241 & 651 & 122.5994 & 3.27089 \\
\hline 1428 & 27.2593 & 719.8961 & 735.7332 & 651 & 122.5994 & 3.26889 \\
\hline 1429 & 27.2763 & 733.4779 & 728.9396 & 650 & 122.5994 & 3.26689 \\
\hline 1430 & 27.2933 & 721.1081 & 723.8691 & 650 & 122.5994 & 3.2649 \\
\hline 1431 & 27.3103 & 704.7284 & 719.3931 & 650 & 122.5994 & 3.2629 \\
\hline 1432 & 27.3273 & 661.1161 & 714.4147 & 649 & 122.5994 & 3.26091 \\
\hline 1433 & 27.3443 & 675.3792 & 712.1914 & 649 & 122.5994 & 3.25892 \\
\hline 1434 & 27.3613 & 672.5615 & 708.8047 & 649 & 122.5994 & 3.25694 \\
\hline 1435 & 27.3783 & 711.3441 & 706.026 & 649 & 122.5994 & 3.25495 \\
\hline 1436 & 27.3953 & 722.9569 & 702.5432 & 648 & 122.5994 & 3.25297 \\
\hline 1437 & 27.4123 & 671.6004 & 700.36 & 648 & 122.5994 & 3.25099 \\
\hline 1438 & 27.4293 & 699.2952 & 698.2645 & 648 & 122.5994 & 3.24902 \\
\hline 1439 & 27.4463 & 707.176 & 695.5405 & 647 & 122.5994 & 3.24704 \\
\hline 1440 & 27.4633 & 677.2155 & 694.0396 & 647 & 122.5994 & 3.24507 \\
\hline 1441 & 27.4803 & 722.8342 & 692.7562 & 647 & 122.5994 & 3.2431 \\
\hline 1442 & 27.4973 & 745.5253 & 690.6906 & 646 & 122.5994 & 3.24114 \\
\hline 1443 & 27.5143 & 790.0744 & 689.8496 & 646 & 122.5994 & 3.23917 \\
\hline 1444 & 27.5313 & 809.2541 & 688.2477 & 645 & 122.5994 & 3.23721 \\
\hline 1445 & 27.5483 & 858.0169 & 687.9081 & 645 & 122.5994 & 3.23525 \\
\hline 1446 & 27.5653 & 784.4285 & 686.8645 & 644 & 122.5994 & 3.2333 \\
\hline 1447 & 27.5823 & 887.592 & 686.1616 & 643 & 122.5994 & 3.23134 \\
\hline 1448 & 27.5993 & 796.5156 & 686.8561 & 643 & 122.5994 & 3.22939 \\
\hline 1449 & 27.6163 & 839.2167 & 687.0178 & 642 & 122.5994 & 3.22744 \\
\hline 1450 & 27.6333 & 824.3058 & 688.729 & 642 & 122.5994 & 3.22549 \\
\hline 1451 & 27.6503 & 792.0404 & 690.0848 & 641 & 122.5994 & 3.22355 \\
\hline 1452 & 27.6673 & 755.0557 & 693.5062 & 641 & 122.5994 & 3.22161 \\
\hline 1453 & 27.6843 & 749.4522 & 689.5983 & 640 & 122.5994 & 3.21967 \\
\hline 1454 & 27.7013 & 749.6386 & 694.8638 & 640 & 122.5994 & 3.21773 \\
\hline 1455 & 27.7183 & 758.6423 & 700.434 & 639 & 122.5994 & 3.2158 \\
\hline 1456 & 27.7353 & 744.0264 & 708.84 & 639 & 122.5994 & 3.21386 \\
\hline 1457 & 27.7523 & 741.5603 & 715.6707 & 638 & 122.5994 & 3.21193 \\
\hline 1458 & 27.7693 & 784.9988 & 731.1105 & 638 & 122.5994 & 3.21001 \\
\hline 1459 & 27.7863 & 833.0016 & 751.2858 & 637 & 122.5994 & 3.20808 \\
\hline 1460 & 27.8033 & 849.3738 & 778.3677 & 636 & 122.5994 & 3.20616 \\
\hline 1461 & 27.8203 & 842.4384 & 813.0931 & 636 & 122.5994 & 3.20424 \\
\hline 1462 & 27.8373 & 891.6641 & 850.3453 & 635 & 122.5994 & 3.20232 \\
\hline 1463 & 27.8543 & 925.8447 & 879.8562 & 635 & 122.5994 & 3.2004 \\
\hline 1464 & 27.8713 & 929.3682 & 901.0992 & 634 & 122.5994 & 3.19849 \\
\hline 1465 & 27.8883 & 936.4737 & 911.9032 & 634 & 122.5994 & 3.19658 \\
\hline 1466 & 27.9053 & 927.6327 & 919.168 & 633 & 122.5994 & 3.19467 \\
\hline 1467 & 27.9223 & 959.9018 & 927.3805 & 632 & 122.5994 & 3.19276 \\
\hline 1468 & 27.9393 & 993.8171 & 942.2527 & 631 & 122.5994 & 3.19086 \\
\hline
\end{tabular}




\begin{tabular}{|c|c|c|c|c|c|c|}
\hline 1469 & 27.9563 & 968.3508 & 963.2546 & 631 & 122.5994 & 3.18896 \\
\hline 1470 & 27.9733 & 1027.495 & 989.3101 & 630 & 122.5994 & 3.18706 \\
\hline 1471 & 27.9903 & 1086.575 & 1016.278 & 629 & 122.5994 & 3.18516 \\
\hline 1472 & 28.0073 & 1123.781 & 1035.148 & 628 & 122.5994 & 3.18327 \\
\hline 1473 & 28.0243 & 1151.574 & 1040.581 & 628 & 122.5994 & 3.18137 \\
\hline 1474 & 28.0413 & 1160.611 & 1028.095 & 627 & 122.5994 & 3.17948 \\
\hline 1475 & 28.0583 & 1157.963 & 1003.474 & 626 & 122.5994 & 3.1776 \\
\hline 1476 & 28.0753 & 1151.021 & 971.5377 & 625 & 122.5994 & 3.17571 \\
\hline 1477 & 28.0923 & 1015.993 & 932.6498 & 625 & 122.5994 & 3.17383 \\
\hline 1478 & 28.1093 & 939.7888 & 885.0125 & 624 & 122.5994 & 3.17195 \\
\hline 1479 & 28.1263 & 925.3887 & 836.2111 & 623 & 122.5994 & 3.17007 \\
\hline 1480 & 28.1433 & 851.0039 & 793.2596 & 622 & 122.5994 & 3.16819 \\
\hline 1481 & 28.1603 & 773.134 & 757.6777 & 621 & 122.5994 & 3.16632 \\
\hline 1482 & 28.1773 & 701.1223 & 729.5452 & 621 & 122.5994 & 3.16445 \\
\hline 1483 & 28.1943 & 715.2561 & 706.7071 & 620 & 122.5994 & 3.16258 \\
\hline 1484 & 28.2113 & 718.2534 & 689.8585 & 619 & 122.5994 & 3.16071 \\
\hline 1485 & 28.2283 & 664.5347 & 678.1968 & 618 & 122.5994 & 3.15884 \\
\hline 1486 & 28.2453 & 710.373 & 672.0325 & 618 & 122.5994 & 3.15698 \\
\hline 1487 & 28.2623 & 723.2538 & 669.0744 & 617 & 122.5994 & 3.15512 \\
\hline 1488 & 28.2793 & 665.9616 & 670.3181 & 616 & 122.5994 & 3.15326 \\
\hline 1489 & 28.2963 & 670.9068 & 675.6302 & 615 & 122.5994 & 3.15141 \\
\hline 1490 & 28.3133 & 676.407 & 684.082 & 614 & 122.5994 & 3.14955 \\
\hline 1491 & 28.3303 & 684.0046 & 693.0254 & 613 & 122.5994 & 3.1477 \\
\hline 1492 & 28.3473 & 647.1688 & 697.7656 & 612 & 122.5994 & 3.14585 \\
\hline 1493 & 28.3643 & 665.6275 & 695.1666 & 611 & 122.5994 & 3.14401 \\
\hline 1494 & 28.3813 & 628.2456 & 687.7264 & 610 & 122.5994 & 3.14216 \\
\hline 1495 & 28.3983 & 674.6401 & 679.3256 & 609 & 122.5994 & 3.14032 \\
\hline 1496 & 28.4153 & 615.2635 & 670.6773 & 608 & 122.5994 & 3.13848 \\
\hline 1497 & 28.4323 & 659.8873 & 660.6423 & 607 & 122.5994 & 3.13664 \\
\hline 1498 & 28.4493 & 636.0387 & 649.7457 & 606 & 122.5994 & 3.13481 \\
\hline 1499 & 28.4663 & 632.0206 & 639.7944 & 605 & 122.5994 & 3.13297 \\
\hline 1500 & 28.4833 & 615.2871 & 631.9191 & 604 & 122.5994 & 3.13114 \\
\hline 1501 & 28.5003 & 635.5478 & 626.2544 & 603 & 122.5994 & 3.12931 \\
\hline 1502 & 28.5173 & 626.5422 & 622.5125 & 602 & 122.5994 & 3.12749 \\
\hline 1503 & 28.5343 & 652.7493 & 620.2399 & 601 & 122.5994 & 3.12566 \\
\hline 1504 & 28.5513 & 608.333 & 618.6652 & 600 & 122.5994 & 3.12384 \\
\hline 1505 & 28.5683 & 577.7446 & 618.1506 & 599 & 122.5994 & 3.12202 \\
\hline 1506 & 28.5853 & 601.5361 & 618.131 & 598 & 122.5994 & 3.1202 \\
\hline 1507 & 28.6023 & 603.5447 & 618.4645 & 597 & 122.5994 & 3.11838 \\
\hline 1508 & 28.6193 & 596.6242 & 619.0306 & 596 & 122.5994 & 3.11657 \\
\hline 1509 & 28.6363 & 623.5737 & 619.6994 & 595 & 122.5994 & 3.11476 \\
\hline 1510 & 28.6533 & 599.6064 & 620.319 & 594 & 122.5994 & 3.11295 \\
\hline 1511 & 28.6703 & 614.9577 & 620.7195 & 593 & 122.5994 & 3.11114 \\
\hline 1512 & 28.6873 & 625.1324 & 620.7293 & 592 & 122.5994 & 3.10934 \\
\hline 1513 & 28.7043 & 606.3895 & 620.2001 & 591 & 122.5994 & 3.10753 \\
\hline 1514 & 28.7213 & 591.0373 & 619.0416 & 590 & 122.5994 & 3.10573 \\
\hline 1515 & 28.7383 & 589.7754 & 617.2474 & 589 & 122.5994 & 3.10394 \\
\hline 1516 & 28.7553 & 627.2143 & 614.8886 & 588 & 122.5994 & 3.10214 \\
\hline 1517 & 28.7723 & 630.4285 & 611.0785 & 586 & 122.5994 & 3.10034 \\
\hline
\end{tabular}




\begin{tabular}{|c|c|c|c|c|c|c|}
\hline 1518 & 28.7893 & 608.7265 & 607.9434 & 585 & 122.5994 & 3.09855 \\
\hline 1519 & 28.8063 & 589.0586 & 604.6153 & 584 & 122.5994 & 3.09676 \\
\hline 1520 & 28.8233 & 600.8264 & 601.2288 & 583 & 122.5994 & 3.09497 \\
\hline 1521 & 28.8403 & 606.6268 & 597.9083 & 582 & 122.5994 & 3.09319 \\
\hline 1522 & 28.8573 & 587.4854 & 593.7504 & 580 & 122.5994 & 3.09141 \\
\hline 1523 & 28.8743 & 598.9617 & 590.8156 & 579 & 122.5994 & 3.08962 \\
\hline 1524 & 28.8913 & 582.7393 & 588.1317 & 578 & 122.5994 & 3.08784 \\
\hline 1525 & 28.9083 & 595.1879 & 585.7015 & 577 & 122.5994 & 3.08607 \\
\hline 1526 & 28.9253 & 592.3327 & 582.6609 & 575 & 122.5994 & 3.08429 \\
\hline 1527 & 28.9423 & 598.6397 & 580.743 & 574 & 122.5994 & 3.08252 \\
\hline 1528 & 28.9593 & 579.5174 & 579.0106 & 573 & 122.5994 & 3.08075 \\
\hline 1529 & 28.9763 & 551.0076 & 577.4329 & 572 & 122.5994 & 3.07898 \\
\hline 1530 & 28.9933 & 568.8167 & 574.9109 & 571 & 122.5994 & 3.07721 \\
\hline 1531 & 29.0103 & 562.4632 & 573.0579 & 569 & 122.5994 & 3.07545 \\
\hline 1532 & 29.0273 & 611.9263 & 571.8337 & 568 & 122.5994 & 3.07369 \\
\hline 1533 & 29.0443 & 568.2509 & 570.613 & 567 & 122.5994 & 3.07193 \\
\hline 1534 & 29.0613 & 552.4804 & 569.4952 & 566 & 122.5994 & 3.07017 \\
\hline 1535 & 29.0783 & 600.0318 & 567.4126 & 564 & 122.5994 & 3.06841 \\
\hline 1536 & 29.0953 & 560.8021 & 566.3577 & 563 & 122.5994 & 3.06666 \\
\hline 1537 & 29.1123 & 531.7863 & 565.0762 & 562 & 122.5994 & 3.0649 \\
\hline 1538 & 29.1293 & 584.2963 & 565.0739 & 562 & 122.5994 & 3.06315 \\
\hline 1539 & 29.1463 & 565.5776 & 564.0873 & 561 & 122.5994 & 3.06141 \\
\hline 1540 & 29.1633 & 555.3095 & 563.1152 & 560 & 122.5994 & 3.05966 \\
\hline 1541 & 29.1803 & 550.6463 & 563.1563 & 560 & 122.5994 & 3.05792 \\
\hline 1542 & 29.1973 & 592.0111 & 563.2103 & 560 & 122.5994 & 3.05618 \\
\hline 1543 & 29.2143 & 545.9102 & 562.2768 & 559 & 122.5994 & 3.05444 \\
\hline 1544 & 29.2313 & 547.7134 & 561.3558 & 558 & 122.5994 & 3.0527 \\
\hline 1545 & 29.2483 & 581.4042 & 561.4477 & 558 & 122.5994 & 3.05096 \\
\hline 1546 & 29.2653 & 560.6723 & 561.5527 & 558 & 122.5994 & 3.04923 \\
\hline 1547 & 29.2823 & 546.6166 & 560.6716 & 557 & 122.5994 & 3.0475 \\
\hline 1548 & 29.2993 & 566.5491 & 559.8049 & 556 & 122.5994 & 3.04577 \\
\hline 1549 & 29.3163 & 589.0111 & 559.9539 & 556 & 122.5994 & 3.04404 \\
\hline 1550 & 29.3333 & 590.4539 & 560.1196 & 556 & 122.5994 & 3.04231 \\
\hline 1551 & 29.3503 & 580.1692 & 559.3035 & 555 & 122.5994 & 3.04059 \\
\hline 1552 & 29.3673 & 537.675 & 558.5074 & 554 & 122.5994 & 3.03887 \\
\hline 1553 & 29.3843 & 547.4919 & 558.7334 & 554 & 122.5994 & 3.03715 \\
\hline 1554 & 29.4013 & 574.024 & 558.9841 & 554 & 122.5994 & 3.03543 \\
\hline 1555 & 29.4183 & 562.9484 & 558.2627 & 553 & 122.5994 & 3.03372 \\
\hline 1556 & 29.4353 & 560.9409 & 557.5735 & 552 & 122.5994 & 3.032 \\
\hline 1557 & 29.4523 & 575.8227 & 558.051 & 552 & 122.5994 & 3.03029 \\
\hline 1558 & 29.4693 & 566.4516 & 558.4509 & 552 & 122.5994 & 3.02858 \\
\hline 1559 & 29.4863 & 532.955 & 557.9061 & 551 & 122.5994 & 3.02688 \\
\hline 1560 & 29.5033 & 530.3103 & 557.4299 & 550 & 122.5994 & 3.02517 \\
\hline 1561 & 29.5203 & 572.5666 & 558.1021 & 550 & 122.5994 & 3.02347 \\
\hline 1562 & 29.5373 & 556.4222 & 558.8225 & 550 & 122.5994 & 3.02177 \\
\hline 1563 & 29.5543 & 551.791 & 559.6787 & 550 & 122.5994 & 3.02007 \\
\hline 1564 & 29.5713 & 554.5561 & 560.7044 & 550 & 122.5994 & 3.01837 \\
\hline 1565 & 29.5883 & 569.1673 & 561.9403 & 550 & 122.5994 & 3.01667 \\
\hline 1566 & 29.6053 & 587.3351 & 563.434 & 550 & 122.5994 & 3.01498 \\
\hline
\end{tabular}




\begin{tabular}{|c|c|c|c|c|c|c|}
\hline 1567 & 29.6223 & 554.2137 & 565.2407 & 550 & 122.5994 & 3.01329 \\
\hline 1568 & 29.6393 & 528.3474 & 567.4227 & 550 & 122.5994 & 3.0116 \\
\hline 1569 & 29.6563 & 555.4196 & 570.0477 & 550 & 122.5994 & 3.00991 \\
\hline 1570 & 29.6733 & 570.0715 & 573.187 & 550 & 122.5994 & 3.00822 \\
\hline 1571 & 29.6903 & 609.2446 & 576.804 & 550 & 122.5994 & 3.00654 \\
\hline 1572 & 29.7073 & 558.1672 & 580.2496 & 549 & 122.5994 & 3.00486 \\
\hline 1573 & 29.7243 & 546.7463 & 585.6874 & 549 & 122.5994 & 3.00318 \\
\hline 1574 & 29.7413 & 587.9672 & 592.0597 & 549 & 122.5994 & 3.0015 \\
\hline 1575 & 29.7583 & 603.8644 & 599.6158 & 549 & 122.5994 & 2.99983 \\
\hline 1576 & 29.7753 & 609.5501 & 608.6155 & 549 & 122.5994 & 2.99815 \\
\hline 1577 & 29.7923 & 614.5341 & 619.0704 & 549 & 122.5994 & 2.99648 \\
\hline 1578 & 29.8093 & 638.3768 & 630.9646 & 549 & 122.5994 & 2.99481 \\
\hline 1579 & 29.8263 & 649.7812 & 644.1545 & 549 & 122.5994 & 2.99314 \\
\hline 1580 & 29.8433 & 645.3034 & 658.3128 & 549 & 122.5994 & 2.99147 \\
\hline 1581 & 29.8603 & 686.2768 & 672.8779 & 549 & 122.5994 & 2.98981 \\
\hline 1582 & 29.8773 & 670.8349 & 687.0338 & 549 & 122.5994 & 2.98815 \\
\hline 1583 & 29.8943 & 676.8244 & 699.761 & 549 & 122.5994 & 2.98649 \\
\hline 1584 & 29.9113 & 693.8514 & 709.9907 & 549 & 122.5994 & 2.98483 \\
\hline 1585 & 29.9283 & 654.7097 & 716.8154 & 549 & 122.5994 & 2.98317 \\
\hline 1586 & 29.9453 & 756.1736 & 719.6996 & 549 & 122.5994 & 2.98152 \\
\hline 1587 & 29.9623 & 708.2388 & 717.5664 & 548 & 122.5994 & 2.97986 \\
\hline 1588 & 29.9793 & 720.7797 & 712.729 & 548 & 122.5994 & 2.97821 \\
\hline 1589 & 29.9963 & 755.5013 & 704.7486 & 548 & 122.5994 & 2.97656 \\
\hline 1590 & 30.0133 & 692.0213 & 693.3413 & 547 & 122.5994 & 2.97492 \\
\hline 1591 & 30.0303 & 681.9577 & 681.3649 & 547 & 122.5994 & 2.97327 \\
\hline 1592 & 30.0473 & 712.5576 & 668.826 & 547 & 122.5994 & 2.97163 \\
\hline 1593 & 30.0643 & 686.74 & 656.8506 & 547 & 122.5994 & 2.96999 \\
\hline 1594 & 30.0813 & 691.1202 & 645.5897 & 546 & 122.5994 & 2.96835 \\
\hline 1595 & 30.0983 & 609.8165 & 638.0581 & 546 & 122.5994 & 2.96671 \\
\hline 1596 & 30.1153 & 601.3825 & 634.1041 & 546 & 122.5994 & 2.96507 \\
\hline 1597 & 30.1323 & 626.5692 & 632.0372 & 545 & 122.5994 & 2.96344 \\
\hline 1598 & 30.1493 & 604.7762 & 632.7247 & 545 & 122.5994 & 2.96181 \\
\hline 1599 & 30.1663 & 577.0609 & 631.8456 & 545 & 122.5994 & 2.96018 \\
\hline 1600 & 30.1833 & 562.91 & 627.1852 & 545 & 122.5994 & 2.95855 \\
\hline 1601 & 30.2003 & 613.1757 & 619.4127 & 544 & 122.5994 & 2.95692 \\
\hline 1602 & 30.2173 & 641.2038 & 613.3544 & 544 & 122.5994 & 2.9553 \\
\hline 1603 & 30.2343 & 611.4836 & 609.2895 & 544 & 122.5994 & 2.95367 \\
\hline 1604 & 30.2513 & 571.7398 & 605.1055 & 543 & 122.5994 & 2.95205 \\
\hline 1605 & 30.2683 & 584.4184 & 601.9562 & 543 & 122.5994 & 2.95043 \\
\hline 1606 & 30.2853 & 596.7168 & 598.6313 & 543 & 122.5994 & 2.94881 \\
\hline 1607 & 30.3023 & 570.2072 & 594.9504 & 543 & 122.5994 & 2.9472 \\
\hline 1608 & 30.3193 & 558.973 & 588.3024 & 542 & 122.5994 & 2.94558 \\
\hline 1609 & 30.3363 & 597.6705 & 582.646 & 542 & 122.5994 & 2.94397 \\
\hline 1610 & 30.3533 & 568.3761 & 578.0854 & 542 & 122.5994 & 2.94236 \\
\hline 1611 & 30.3703 & 542.5654 & 573.3853 & 541 & 122.5994 & 2.94075 \\
\hline 1612 & 30.3873 & 579.2513 & 570.0459 & 541 & 122.5994 & 2.93915 \\
\hline 1613 & 30.4043 & 605.3812 & 566.3769 & 541 & 122.5994 & 2.93754 \\
\hline 1614 & 30.4213 & 596.2689 & 562.4128 & 540 & 122.5994 & 2.93594 \\
\hline 1615 & 30.4383 & 601.1701 & 560.8214 & 540 & 122.5994 & 2.93434 \\
\hline
\end{tabular}




\begin{tabular}{|c|c|c|c|c|c|c|}
\hline 1616 & 30.4553 & 576.1774 & 561.1183 & 540 & 122.5994 & 2.93274 \\
\hline 1617 & 30.4723 & 566.4506 & 563.8808 & 540 & 122.5994 & 2.93114 \\
\hline 1618 & 30.4893 & 585.4953 & 568.5161 & 539 & 122.5994 & 2.92955 \\
\hline 1619 & 30.5063 & 622.5749 & 577.0558 & 539 & 122.5994 & 2.92795 \\
\hline 1620 & 30.5233 & 609.2762 & 587.5895 & 539 & 122.5994 & 2.92636 \\
\hline 1621 & 30.5403 & 639.4261 & 596.6355 & 538 & 122.5994 & 2.92477 \\
\hline 1622 & 30.5573 & 649.6406 & 602.5533 & 538 & 122.5994 & 2.92318 \\
\hline 1623 & 30.5743 & 592.1494 & 602.6914 & 538 & 122.5994 & 2.9216 \\
\hline 1624 & 30.5913 & 631.1471 & 598.7557 & 538 & 122.5994 & 2.92001 \\
\hline 1625 & 30.6083 & 606.8204 & 592.4849 & 537 & 122.5994 & 2.91843 \\
\hline 1626 & 30.6253 & 609.9235 & 586.9094 & 537 & 122.5994 & 2.91685 \\
\hline 1627 & 30.6423 & 600.4044 & 580.4027 & 537 & 122.5994 & 2.91527 \\
\hline 1628 & 30.6593 & 566.4841 & 571.9235 & 536 & 122.5994 & 2.91369 \\
\hline 1629 & 30.6763 & 570.2793 & 564.3539 & 536 & 122.5994 & 2.91211 \\
\hline 1630 & 30.6933 & 539.836 & 557.8028 & 536 & 122.5994 & 2.91054 \\
\hline 1631 & 30.7103 & 568.2023 & 552.8068 & 536 & 122.5994 & 2.90897 \\
\hline 1632 & 30.7273 & 529.4388 & 548.3517 & 535 & 122.5994 & 2.9074 \\
\hline 1633 & 30.7443 & 530.4851 & 546.1326 & 535 & 122.5994 & 2.90583 \\
\hline 1634 & 30.7613 & 516.408 & 544.775 & 535 & 122.5994 & 2.90426 \\
\hline 1635 & 30.7783 & 576.3184 & 543.9584 & 535 & 122.5994 & 2.9027 \\
\hline 1636 & 30.7953 & 543.9599 & 543.4636 & 535 & 122.5994 & 2.90113 \\
\hline 1637 & 30.8123 & 517.3809 & 543.1636 & 535 & 122.5994 & 2.89957 \\
\hline 1638 & 30.8293 & 572.452 & 542.9926 & 535 & 122.5994 & 2.89801 \\
\hline 1639 & 30.8463 & 600.6274 & 542.9183 & 535 & 122.5994 & 2.89645 \\
\hline 1640 & 30.8633 & 557.5969 & 542.9255 & 535 & 122.5994 & 2.89489 \\
\hline 1641 & 30.8803 & 531.8499 & 543.0073 & 535 & 122.5994 & 2.89334 \\
\hline 1642 & 30.8973 & 576.5512 & 543.1624 & 535 & 122.5994 & 2.89179 \\
\hline 1643 & 30.9143 & 602.0403 & 543.3939 & 535 & 122.5994 & 2.89023 \\
\hline 1644 & 30.9313 & 570.6357 & 543.7095 & 535 & 122.5994 & 2.88868 \\
\hline 1645 & 30.9483 & 551.6788 & 543.2484 & 535 & 122.5994 & 2.88714 \\
\hline 1646 & 30.9653 & 577.8538 & 543.8078 & 535 & 122.5994 & 2.88559 \\
\hline 1647 & 30.9823 & 595.3711 & 544.5132 & 535 & 122.5994 & 2.88405 \\
\hline 1648 & 30.9993 & 597.4411 & 545.4047 & 535 & 122.5994 & 2.8825 \\
\hline 1649 & 31.0163 & 598.0177 & 545.852 & 535 & 122.5994 & 2.88096 \\
\hline 1650 & 31.0333 & 612.0145 & 547.3157 & 535 & 122.5994 & 2.87942 \\
\hline 1651 & 31.0503 & 607.7541 & 549.171 & 535 & 122.5994 & 2.87788 \\
\hline 1652 & 31.0673 & 565.7199 & 551.6145 & 535 & 122.5994 & 2.87635 \\
\hline 1653 & 31.0843 & 584.3423 & 554.5936 & 535 & 122.5994 & 2.87481 \\
\hline 1654 & 31.1013 & 637.8915 & 558.3514 & 535 & 122.5994 & 2.87328 \\
\hline 1655 & 31.1183 & 608.6423 & 563.0919 & 535 & 122.5994 & 2.87175 \\
\hline 1656 & 31.1353 & 564.4073 & 569.9184 & 535 & 122.5994 & 2.87022 \\
\hline 1657 & 31.1523 & 559.9548 & 577.556 & 535 & 122.5994 & 2.86869 \\
\hline 1658 & 31.1693 & 593.2812 & 587.1772 & 535 & 122.5994 & 2.86717 \\
\hline 1659 & 31.1863 & 613.4378 & 599.0193 & 535 & 122.5994 & 2.86564 \\
\hline 1660 & 31.2033 & 568.1149 & 611.9324 & 534 & 122.5994 & 2.86412 \\
\hline 1661 & 31.2203 & 567.8985 & 627.3485 & 534 & 122.5994 & 2.8626 \\
\hline 1662 & 31.2373 & 604.8425 & 641.6863 & 534 & 122.5994 & 2.86108 \\
\hline 1663 & 31.2543 & 626.4295 & 652.8115 & 534 & 122.5994 & 2.85956 \\
\hline 1664 & 31.2713 & 622.2059 & 659.1395 & 533 & 122.5994 & 2.85805 \\
\hline
\end{tabular}




\begin{tabular}{|c|c|c|c|c|c|c|}
\hline 1665 & 31.2883 & 636.1483 & 663.6824 & 533 & 122.5994 & 2.85653 \\
\hline 1666 & 31.3053 & 623.5428 & 666.5325 & 533 & 122.5994 & 2.85502 \\
\hline 1667 & 31.3223 & 654.1998 & 666.8074 & 533 & 122.5994 & 2.85351 \\
\hline 1668 & 31.3393 & 669.4561 & 662.6818 & 532 & 122.5994 & 2.852 \\
\hline 1669 & 31.3563 & 698.7774 & 656.5239 & 532 & 122.5994 & 2.85049 \\
\hline 1670 & 31.3733 & 613.8449 & 648.4304 & 532 & 122.5994 & 2.84899 \\
\hline 1671 & 31.3903 & 633.026 & 639.2438 & 532 & 122.5994 & 2.84748 \\
\hline 1672 & 31.4073 & 635.0519 & 629.498 & 532 & 122.5994 & 2.84598 \\
\hline 1673 & 31.4243 & 620.3222 & 618.5891 & 531 & 122.5994 & 2.84448 \\
\hline 1674 & 31.4413 & 629.9887 & 608.8638 & 531 & 122.5994 & 2.84298 \\
\hline 1675 & 31.4583 & 557.6717 & 599.6498 & 531 & 122.5994 & 2.84148 \\
\hline 1676 & 31.4753 & 594.0312 & 591.255 & 531 & 122.5994 & 2.83999 \\
\hline 1677 & 31.4923 & 562.5782 & 582.9397 & 530 & 122.5994 & 2.83849 \\
\hline 1678 & 31.5093 & 575.5484 & 576.8646 & 530 & 122.5994 & 2.837 \\
\hline 1679 & 31.5263 & 570.2279 & 572.0291 & 530 & 122.5994 & 2.83551 \\
\hline 1680 & 31.5433 & 559.9226 & 568.1967 & 530 & 122.5994 & 2.83402 \\
\hline 1681 & 31.5603 & 544.8353 & 564.8431 & 530 & 122.5994 & 2.83253 \\
\hline 1682 & 31.5773 & 529.0099 & 560.4553 & 529 & 122.5994 & 2.83105 \\
\hline 1683 & 31.5943 & 538.8759 & 557.1171 & 529 & 122.5994 & 2.82956 \\
\hline 1684 & 31.6113 & 576.7038 & 553.2671 & 528 & 122.5994 & 2.82808 \\
\hline 1685 & 31.6283 & 562.8091 & 551.0761 & 528 & 122.5994 & 2.8266 \\
\hline 1686 & 31.6453 & 567.6665 & 548.3549 & 527 & 122.5994 & 2.82512 \\
\hline 1687 & 31.6623 & 571.2207 & 546.8726 & 527 & 122.5994 & 2.82364 \\
\hline 1688 & 31.6793 & 569.7993 & 544.6787 & 526 & 122.5994 & 2.82216 \\
\hline 1689 & 31.6963 & 571.0421 & 543.8132 & 526 & 122.5994 & 2.82069 \\
\hline 1690 & 31.7133 & 574.2355 & 542.6196 & 525 & 122.5994 & 2.81922 \\
\hline 1691 & 31.7303 & 549.8511 & 542.9886 & 525 & 122.5994 & 2.81774 \\
\hline 1692 & 31.7473 & 534.3438 & 542.7975 & 524 & 122.5994 & 2.81627 \\
\hline 1693 & 31.7643 & 564.1792 & 544.0067 & 524 & 122.5994 & 2.81481 \\
\hline 1694 & 31.7813 & 591.2199 & 545.5551 & 524 & 122.5994 & 2.81334 \\
\hline 1695 & 31.7983 & 552.0585 & 546.3423 & 523 & 122.5994 & 2.81187 \\
\hline 1696 & 31.8153 & 519.3451 & 548.4014 & 523 & 122.5994 & 2.81041 \\
\hline 1697 & 31.8323 & 526.6929 & 549.7715 & 522 & 122.5994 & 2.80895 \\
\hline 1698 & 31.8493 & 522.0749 & 552.5938 & 522 & 122.5994 & 2.80749 \\
\hline 1699 & 31.8663 & 529.6871 & 554.9751 & 521 & 122.5994 & 2.80603 \\
\hline 1700 & 31.8833 & 540.8363 & 558.9636 & 521 & 122.5994 & 2.80457 \\
\hline 1701 & 31.9003 & 562.0282 & 562.5311 & 520 & 122.5994 & 2.80311 \\
\hline 1702 & 31.9173 & 553.3832 & 567.562 & 520 & 122.5994 & 2.80166 \\
\hline 1703 & 31.9343 & 564.6102 & 571.8451 & 519 & 122.5994 & 2.80021 \\
\hline 1704 & 31.9513 & 550.5089 & 577.1393 & 519 & 122.5994 & 2.79876 \\
\hline 1705 & 31.9683 & 542.8791 & 581.3307 & 518 & 122.5994 & 2.79731 \\
\hline 1706 & 31.9853 & 557.2531 & 586.5303 & 518 & 122.5994 & 2.79586 \\
\hline 1707 & 32.0023 & 554.9649 & 591.9683 & 518 & 122.5994 & 2.79441 \\
\hline 1708 & 32.0193 & 583.7225 & 596.8019 & 517 & 122.5994 & 2.79297 \\
\hline 1709 & 32.0363 & 562.9806 & 603.041 & 517 & 122.5994 & 2.79152 \\
\hline 1710 & 32.0533 & 552.3236 & 608.5906 & 516 & 122.5994 & 2.79008 \\
\hline 1711 & 32.0703 & 567.2291 & 615.2974 & 516 & 122.5994 & 2.78864 \\
\hline 1712 & 32.0873 & 594.8011 & 620.8843 & 515 & 122.5994 & 2.7872 \\
\hline 1713 & 32.1043 & 616.8475 & 626.9019 & 515 & 122.5994 & 2.78577 \\
\hline
\end{tabular}




\begin{tabular}{|c|c|c|c|c|c|c|}
\hline 1714 & 32.1213 & 594.6423 & 630.7593 & 514 & 122.5994 & 2.78433 \\
\hline 1715 & 32.1383 & 573.6221 & 634.0031 & 514 & 122.5994 & 2.7829 \\
\hline 1716 & 32.1553 & 592.8997 & 634.0161 & 513 & 122.5994 & 2.78147 \\
\hline 1717 & 32.1723 & 555.6901 & 632.7762 & 513 & 122.5994 & 2.78003 \\
\hline 1718 & 32.1893 & 606.7669 & 629.3267 & 513 & 122.5994 & 2.7786 \\
\hline 1719 & 32.2063 & 605.2746 & 622.8193 & 512 & 122.5994 & 2.77718 \\
\hline 1720 & 32.2233 & 548.4164 & 615.6266 & 512 & 122.5994 & 2.77575 \\
\hline 1721 & 32.2403 & 543.0001 & 606.134 & 511 & 122.5994 & 2.77433 \\
\hline 1722 & 32.2573 & 547.0959 & 596.955 & 511 & 122.5994 & 2.7729 \\
\hline 1723 & 32.2743 & 534.5081 & 586.6108 & 510 & 122.5994 & 2.77148 \\
\hline 1724 & 32.2913 & 551.6443 & 577.5271 & 510 & 122.5994 & 2.77006 \\
\hline 1725 & 32.3083 & 540.6713 & 568.0699 & 509 & 122.5994 & 2.76864 \\
\hline 1726 & 32.3253 & 516.2745 & 559.9199 & 509 & 122.5994 & 2.76722 \\
\hline 1727 & 32.3423 & 492.6712 & 552.5175 & 508 & 122.5994 & 2.76581 \\
\hline 1728 & 32.3593 & 514.9208 & 547.2955 & 508 & 122.5994 & 2.76439 \\
\hline 1729 & 32.3763 & 496.5054 & 543.217 & 508 & 122.5994 & 2.76298 \\
\hline 1730 & 32.3933 & 534.492 & 539.1624 & 507 & 122.5994 & 2.76157 \\
\hline 1731 & 32.4103 & 533.1891 & 535.6057 & 506 & 122.5994 & 2.76016 \\
\hline 1732 & 32.4273 & 514.1324 & 532.9595 & 505 & 122.5994 & 2.75875 \\
\hline 1733 & 32.4443 & 509.6272 & 531.6073 & 505 & 122.5994 & 2.75735 \\
\hline 1734 & 32.4613 & 503.6237 & 529.313 & 504 & 122.5994 & 2.75594 \\
\hline 1735 & 32.4783 & 512.7816 & 526.9351 & 503 & 122.5994 & 2.75454 \\
\hline 1736 & 32.4953 & 531.0053 & 525.4283 & 503 & 122.5994 & 2.75313 \\
\hline 1737 & 32.5123 & 486.4196 & 522.876 & 502 & 122.5994 & 2.75173 \\
\hline 1738 & 32.5293 & 484.8393 & 520.1656 & 501 & 122.5994 & 2.75033 \\
\hline 1739 & 32.5463 & 513.4501 & 518.3922 & 501 & 122.5994 & 2.74894 \\
\hline 1740 & 32.5633 & 512.7167 & 515.5591 & 500 & 122.5994 & 2.74754 \\
\hline 1741 & 32.5803 & 515.2406 & 512.8139 & 499 & 122.5994 & 2.74615 \\
\hline 1742 & 32.5973 & 500.8551 & 511.192 & 499 & 122.5994 & 2.74475 \\
\hline 1743 & 32.6143 & 499.1274 & 508.7438 & 498 & 122.5994 & 2.74336 \\
\hline 1744 & 32.6313 & 497.3 & 506.5039 & 497 & 122.5994 & 2.74197 \\
\hline 1745 & 32.6483 & 493.6457 & 504.4599 & 496 & 122.5994 & 2.74058 \\
\hline 1746 & 32.6653 & 503.7504 & 503.6428 & 496 & 122.5994 & 2.73919 \\
\hline 1747 & 32.6823 & 509.5057 & 502.0159 & 495 & 122.5994 & 2.73781 \\
\hline 1748 & 32.6993 & 507.272 & 500.562 & 494 & 122.5994 & 2.73642 \\
\hline 1749 & 32.7163 & 477.5958 & 500.1135 & 494 & 122.5994 & 2.73504 \\
\hline 1750 & 32.7333 & 490.1208 & 498.9746 & 493 & 122.5994 & 2.73366 \\
\hline 1751 & 32.7503 & 500.3465 & 497.9854 & 492 & 122.5994 & 2.73228 \\
\hline 1752 & 32.7673 & 506.4499 & 498.1547 & 492 & 122.5994 & 2.7309 \\
\hline 1753 & 32.7843 & 482.9408 & 497.4206 & 491 & 122.5994 & 2.72952 \\
\hline 1754 & 32.8013 & 493.0076 & 497.9661 & 491 & 122.5994 & 2.72815 \\
\hline 1755 & 32.8183 & 463.1317 & 498.7327 & 491 & 122.5994 & 2.72677 \\
\hline 1756 & 32.8353 & 462.4253 & 499.7411 & 491 & 122.5994 & 2.7254 \\
\hline 1757 & 32.8523 & 470.0111 & 501.0017 & 491 & 122.5994 & 2.72403 \\
\hline 1758 & 32.8693 & 463.8976 & 502.5444 & 491 & 122.5994 & 2.72266 \\
\hline 1759 & 32.8863 & 494.9361 & 504.271 & 491 & 122.5994 & 2.72129 \\
\hline 1760 & 32.9033 & 464.6068 & 506.1563 & 491 & 122.5994 & 2.71992 \\
\hline 1761 & 32.9203 & 476.7206 & 508.0991 & 491 & 122.5994 & 2.71856 \\
\hline 1762 & 32.9373 & 503.3893 & 509.9481 & 491 & 122.5994 & 2.71719 \\
\hline
\end{tabular}




\begin{tabular}{|c|c|c|c|c|c|c|}
\hline 1763 & 32.9543 & 535.3647 & 511.5283 & 491 & 122.5994 & 2.71583 \\
\hline 1764 & 32.9713 & 508.4559 & 512.6075 & 491 & 122.5994 & 2.71447 \\
\hline 1765 & 32.9883 & 501.9148 & 513.0872 & 491 & 122.5994 & 2.71311 \\
\hline 1766 & 33.0053 & 528.3016 & 512.9672 & 491 & 122.5994 & 2.71175 \\
\hline 1767 & 33.0223 & 529.0705 & 512.3349 & 491 & 122.5994 & 2.71039 \\
\hline 1768 & 33.0393 & 492.8201 & 511.2978 & 491 & 122.5994 & 2.70904 \\
\hline 1769 & 33.0563 & 488.3632 & 509.9482 & 491 & 122.5994 & 2.70768 \\
\hline 1770 & 33.0733 & 489.5883 & 508.3812 & 491 & 122.5994 & 2.70633 \\
\hline 1771 & 33.0903 & 503.6021 & 506.7167 & 491 & 122.5994 & 2.70498 \\
\hline 1772 & 33.1073 & 476.1163 & 505.0958 & 491 & 122.5994 & 2.70363 \\
\hline 1773 & 33.1243 & 486.8879 & 503.6482 & 491 & 122.5994 & 2.70228 \\
\hline 1774 & 33.1413 & 504.2126 & 502.4689 & 491 & 122.5994 & 2.70093 \\
\hline 1775 & 33.1583 & 503.0031 & 501.011 & 491 & 122.5994 & 2.69958 \\
\hline 1776 & 33.1753 & 539.1352 & 500.5089 & 491 & 122.5994 & 2.69824 \\
\hline 1777 & 33.1923 & 482.0894 & 501.3204 & 492 & 122.5994 & 2.6969 \\
\hline 1778 & 33.2093 & 485.7599 & 501.3895 & 492 & 122.5994 & 2.69556 \\
\hline 1779 & 33.2263 & 525.688 & 501.6248 & 492 & 122.5994 & 2.69422 \\
\hline 1780 & 33.2433 & 542.9624 & 501.5379 & 492 & 122.5994 & 2.69288 \\
\hline 1781 & 33.2603 & 530.5424 & 501.7446 & 492 & 122.5994 & 2.69154 \\
\hline 1782 & 33.2773 & 491.9302 & 501.7571 & 492 & 122.5994 & 2.6902 \\
\hline 1783 & 33.2943 & 476.8369 & 501.668 & 492 & 122.5994 & 2.68887 \\
\hline 1784 & 33.3113 & 481.9958 & 501.306 & 492 & 122.5994 & 2.68753 \\
\hline 1785 & 33.3283 & 489.8025 & 500.8174 & 492 & 122.5994 & 2.6862 \\
\hline 1786 & 33.3453 & 516.0991 & 500.2159 & 492 & 122.5994 & 2.68487 \\
\hline 1787 & 33.3623 & 497.4912 & 499.5429 & 492 & 122.5994 & 2.68354 \\
\hline 1788 & 33.3793 & 505.9224 & 498.9434 & 492 & 122.5994 & 2.68221 \\
\hline 1789 & 33.3963 & 516.9183 & 498.3538 & 492 & 122.5994 & 2.68089 \\
\hline 1790 & 33.4133 & 506.0941 & 497.8004 & 492 & 122.5994 & 2.67956 \\
\hline 1791 & 33.4303 & 522.1394 & 497.3777 & 492 & 122.5994 & 2.67824 \\
\hline 1792 & 33.4473 & 505.1943 & 497.114 & 492 & 122.5994 & 2.67692 \\
\hline 1793 & 33.4643 & 500.8151 & 497.0223 & 492 & 122.5994 & 2.6756 \\
\hline 1794 & 33.4813 & 495.3399 & 497.169 & 492 & 122.5994 & 2.67428 \\
\hline 1795 & 33.4983 & 478.6958 & 497.4144 & 492 & 122.5994 & 2.67296 \\
\hline 1796 & 33.5153 & 480.6398 & 497.8084 & 492 & 122.5994 & 2.67164 \\
\hline 1797 & 33.5323 & 515.9773 & 498.3329 & 492 & 122.5994 & 2.67033 \\
\hline 1798 & 33.5493 & 503.5147 & 499.1376 & 492 & 122.5994 & 2.66901 \\
\hline 1799 & 33.5663 & 494.5698 & 499.8333 & 492 & 122.5994 & 2.6677 \\
\hline 1800 & 33.5833 & 487.8361 & 500.5125 & 492 & 122.5994 & 2.66639 \\
\hline 1801 & 33.6003 & 503.5836 & 501.0628 & 492 & 122.5994 & 2.66508 \\
\hline 1802 & 33.6173 & 477.5242 & 502.5439 & 493 & 122.5994 & 2.66377 \\
\hline 1803 & 33.6343 & 497.166 & 503.5198 & 494 & 122.5994 & 2.66246 \\
\hline 1804 & 33.6513 & 502.7529 & 505.2096 & 496 & 122.5994 & 2.66115 \\
\hline 1805 & 33.6683 & 487.2317 & 505.7365 & 497 & 122.5994 & 2.65985 \\
\hline 1806 & 33.6853 & 510.2534 & 506.1797 & 498 & 122.5994 & 2.65855 \\
\hline 1807 & 33.7023 & 488.3815 & 506.6426 & 499 & 122.5994 & 2.65724 \\
\hline 1808 & 33.7193 & 483.9536 & 507.9546 & 501 & 122.5994 & 2.65594 \\
\hline 1809 & 33.7363 & 488.04 & 508.2384 & 502 & 122.5994 & 2.65464 \\
\hline 1810 & 33.7533 & 488.3632 & 508.5626 & 503 & 122.5994 & 2.65335 \\
\hline 1811 & 33.7703 & 509.5854 & 509.0699 & 504 & 122.5994 & 2.65205 \\
\hline
\end{tabular}




\begin{tabular}{|c|c|c|c|c|c|c|}
\hline 1812 & 33.7873 & 532.783 & 510.6114 & 506 & 122.5994 & 2.65075 \\
\hline 1813 & 33.8043 & 502.226 & 511.2787 & 507 & 122.5994 & 2.64946 \\
\hline 1814 & 33.8213 & 484.2329 & 511.9545 & 508 & 122.5994 & 2.64817 \\
\hline 1815 & 33.8383 & 515.7779 & 512.8553 & 509 & 122.5994 & 2.64687 \\
\hline 1816 & 33.8553 & 527.3004 & 513.9004 & 510 & 122.5994 & 2.64558 \\
\hline 1817 & 33.8723 & 493.8323 & 515.9984 & 512 & 122.5994 & 2.6443 \\
\hline 1818 & 33.8893 & 459.6184 & 517.1926 & 513 & 122.5994 & 2.64301 \\
\hline 1819 & 33.9063 & 550.9995 & 518.4354 & 514 & 122.5994 & 2.64172 \\
\hline 1820 & 33.9233 & 513.9758 & 519.8558 & 515 & 122.5994 & 2.64044 \\
\hline 1821 & 33.9403 & 555.6878 & 522.4243 & 517 & 122.5994 & 2.63915 \\
\hline 1822 & 33.9573 & 520.3756 & 524.185 & 518 & 122.5994 & 2.63787 \\
\hline 1823 & 33.9743 & 511.9425 & 526.21 & 519 & 122.5994 & 2.63659 \\
\hline 1824 & 33.9913 & 551.2718 & 528.8566 & 520 & 122.5994 & 2.63531 \\
\hline 1825 & 34.0083 & 538.4658 & 532.8213 & 522 & 122.5994 & 2.63403 \\
\hline 1826 & 34.0253 & 524.3153 & 535.5522 & 522 & 122.5994 & 2.63275 \\
\hline 1827 & 34.0423 & 565.8971 & 540.567 & 523 & 122.5994 & 2.63148 \\
\hline 1828 & 34.0593 & 558.5247 & 546.4773 & 524 & 122.5994 & 2.6302 \\
\hline 1829 & 34.0763 & 518.3994 & 553.6865 & 525 & 122.5994 & 2.62893 \\
\hline 1830 & 34.0933 & 515.2406 & 560.7243 & 526 & 122.5994 & 2.62766 \\
\hline 1831 & 34.1103 & 529.2152 & 566.3298 & 527 & 122.5994 & 2.62639 \\
\hline 1832 & 34.1273 & 533.966 & 569.6487 & 528 & 122.5994 & 2.62512 \\
\hline 1833 & 34.1443 & 525.4178 & 571.4298 & 529 & 122.5994 & 2.62385 \\
\hline 1834 & 34.1613 & 562.7593 & 572.9372 & 530 & 122.5994 & 2.62258 \\
\hline 1835 & 34.1783 & 569.3505 & 574.2764 & 531 & 122.5994 & 2.62132 \\
\hline 1836 & 34.1953 & 568.669 & 575.3515 & 532 & 122.5994 & 2.62005 \\
\hline 1837 & 34.2123 & 552.2621 & 575.6119 & 533 & 122.5994 & 2.61879 \\
\hline 1838 & 34.2293 & 546.1215 & 575.175 & 534 & 122.5994 & 2.61753 \\
\hline 1839 & 34.2463 & 559.1778 & 574.6122 & 535 & 122.5994 & 2.61627 \\
\hline 1840 & 34.2633 & 543.635 & 574.3564 & 536 & 122.5994 & 2.61501 \\
\hline 1841 & 34.2803 & 536.413 & 574.7563 & 537 & 122.5994 & 2.61375 \\
\hline 1842 & 34.2973 & 567.171 & 575.6342 & 538 & 122.5994 & 2.61249 \\
\hline 1843 & 34.3143 & 582.6602 & 577.0356 & 539 & 122.5994 & 2.61124 \\
\hline 1844 & 34.3313 & 580.7543 & 578.7913 & 540 & 122.5994 & 2.60998 \\
\hline 1845 & 34.3483 & 593.8945 & 580.6849 & 541 & 122.5994 & 2.60873 \\
\hline 1846 & 34.3653 & 610.6473 & 582.5247 & 542 & 122.5994 & 2.60748 \\
\hline 1847 & 34.3823 & 607.8772 & 584.0856 & 543 & 122.5994 & 2.60623 \\
\hline 1848 & 34.3993 & 608.8921 & 585.3868 & 544 & 122.5994 & 2.60498 \\
\hline 1849 & 34.4163 & 595.0948 & 586.5717 & 545 & 122.5994 & 2.60373 \\
\hline 1850 & 34.4333 & 579.0805 & 586.8224 & 545 & 122.5994 & 2.60249 \\
\hline 1851 & 34.4503 & 615.529 & 588.2418 & 546 & 122.5994 & 2.60124 \\
\hline 1852 & 34.4673 & 592.4064 & 589.8358 & 547 & 122.5994 & 2.6 \\
\hline 1853 & 34.4843 & 631.9035 & 590.5734 & 547 & 122.5994 & 2.59875 \\
\hline 1854 & 34.5013 & 631.2627 & 592.4445 & 548 & 122.5994 & 2.59751 \\
\hline 1855 & 34.5183 & 680.3893 & 594.4678 & 549 & 122.5994 & 2.59627 \\
\hline 1856 & 34.5353 & 653.3433 & 595.6613 & 549 & 122.5994 & 2.59503 \\
\hline 1857 & 34.5523 & 640.6969 & 598.116 & 550 & 122.5994 & 2.5938 \\
\hline 1858 & 34.5693 & 704.6616 & 600.5782 & 551 & 122.5994 & 2.59256 \\
\hline 1859 & 34.5863 & 702.3032 & 601.9716 & 551 & 122.5994 & 2.59132 \\
\hline 1860 & 34.6033 & 702.3436 & 604.1204 & 552 & 122.5994 & 2.59009 \\
\hline
\end{tabular}




\begin{tabular}{|c|c|c|c|c|c|c|}
\hline 1861 & 34.6203 & 606.9537 & 605.7811 & 553 & 122.5994 & 2.58886 \\
\hline 1862 & 34.6373 & 695.9593 & 606.1858 & 553 & 122.5994 & 2.58762 \\
\hline 1863 & 34.6543 & 671.4034 & 607.3187 & 554 & 122.5994 & 2.58639 \\
\hline 1864 & 34.6713 & 681.3008 & 608.1617 & 555 & 122.5994 & 2.58516 \\
\hline 1865 & 34.6883 & 678.9419 & 608.8502 & 556 & 122.5994 & 2.58394 \\
\hline 1866 & 34.7053 & 659.9216 & 608.4503 & 556 & 122.5994 & 2.58271 \\
\hline 1867 & 34.7223 & 660.7242 & 609.1181 & 557 & 122.5994 & 2.58148 \\
\hline 1868 & 34.7393 & 690.7269 & 609.7404 & 558 & 122.5994 & 2.58026 \\
\hline 1869 & 34.7563 & 689.5742 & 609.496 & 558 & 122.5994 & 2.57904 \\
\hline 1870 & 34.7733 & 711.3877 & 610.4976 & 559 & 122.5994 & 2.57781 \\
\hline 1871 & 34.7903 & 731.5289 & 611.8918 & 560 & 122.5994 & 2.57659 \\
\hline 1872 & 34.8073 & 686.3742 & 612.9046 & 560 & 122.5994 & 2.57537 \\
\hline 1873 & 34.8243 & 687.9941 & 615.6573 & 561 & 122.5994 & 2.57416 \\
\hline 1874 & 34.8413 & 693.2047 & 618.4237 & 561 & 122.5994 & 2.57294 \\
\hline 1875 & 34.8583 & 705.4593 & 623.4738 & 562 & 122.5994 & 2.57172 \\
\hline 1876 & 34.8753 & 698.538 & 629.1647 & 562 & 122.5994 & 2.57051 \\
\hline 1877 & 34.8923 & 686.8237 & 638.0944 & 563 & 122.5994 & 2.56929 \\
\hline 1878 & 34.9093 & 734.1784 & 648.5456 & 563 & 122.5994 & 2.56808 \\
\hline 1879 & 34.9263 & 734.0984 & 663.0606 & 564 & 122.5994 & 2.56687 \\
\hline 1880 & 34.9433 & 775.1554 & 679.587 & 564 & 122.5994 & 2.56566 \\
\hline 1881 & 34.9603 & 758.6855 & 698.1885 & 564 & 122.5994 & 2.56445 \\
\hline 1882 & 34.9773 & 755.1103 & 717.4496 & 565 & 122.5994 & 2.56325 \\
\hline 1883 & 34.9943 & 786.8229 & 732.0029 & 565 & 122.5994 & 2.56204 \\
\hline 1884 & 35.0113 & 836.8482 & 742.1559 & 566 & 122.5994 & 2.56083 \\
\hline 1885 & 35.0283 & 835.8733 & 747.6855 & 566 & 122.5994 & 2.55963 \\
\hline 1886 & 35.0453 & 794.5167 & 751.5643 & 566 & 122.5994 & 2.55843 \\
\hline 1887 & 35.0623 & 792.7975 & 755.4099 & 567 & 122.5994 & 2.55723 \\
\hline 1888 & 35.0793 & 802.1524 & 755.02 & 567 & 122.5994 & 2.55603 \\
\hline 1889 & 35.0963 & 824.3228 & 751.5186 & 568 & 122.5994 & 2.55483 \\
\hline 1890 & 35.1133 & 816.861 & 743.666 & 568 & 122.5994 & 2.55363 \\
\hline 1891 & 35.1303 & 784.0332 & 734.795 & 569 & 122.5994 & 2.55243 \\
\hline 1892 & 35.1473 & 803.7715 & 724.1307 & 569 & 122.5994 & 2.55124 \\
\hline 1893 & 35.1643 & 793.9971 & 712.796 & 569 & 122.5994 & 2.55004 \\
\hline 1894 & 35.1813 & 763.6603 & 702.2498 & 570 & 122.5994 & 2.54885 \\
\hline 1895 & 35.1983 & 715.7481 & 691.0806 & 570 & 122.5994 & 2.54766 \\
\hline 1896 & 35.2153 & 681.8829 & 681.3671 & 571 & 122.5994 & 2.54647 \\
\hline 1897 & 35.2323 & 726.8411 & 671.4349 & 571 & 122.5994 & 2.54528 \\
\hline 1898 & 35.2493 & 717.7673 & 662.3268 & 571 & 122.5994 & 2.54409 \\
\hline 1899 & 35.2663 & 677.5665 & 654.0306 & 571 & 122.5994 & 2.5429 \\
\hline 1900 & 35.2833 & 670.8994 & 647.6471 & 572 & 122.5994 & 2.54171 \\
\hline 1901 & 35.3003 & 612.9158 & 641.1888 & 572 & 122.5994 & 2.54053 \\
\hline 1902 & 35.3173 & 623.1469 & 635.738 & 572 & 122.5994 & 2.53934 \\
\hline 1903 & 35.3343 & 643.5669 & 631.334 & 572 & 122.5994 & 2.53816 \\
\hline 1904 & 35.3513 & 630.7757 & 627.9426 & 572 & 122.5994 & 2.53698 \\
\hline 1905 & 35.3683 & 587.1872 & 625.4418 & 572 & 122.5994 & 2.5358 \\
\hline 1906 & 35.3853 & 644.4159 & 624.644 & 573 & 122.5994 & 2.53462 \\
\hline 1907 & 35.4023 & 614.6871 & 623.2924 & 573 & 122.5994 & 2.53344 \\
\hline 1908 & 35.4193 & 586.4918 & 622.0203 & 573 & 122.5994 & 2.53226 \\
\hline 1909 & 35.4363 & 610.2942 & 620.4012 & 573 & 122.5994 & 2.53109 \\
\hline
\end{tabular}




\begin{tabular}{|c|c|c|c|c|c|c|}
\hline 1910 & 35.4533 & 612.4313 & 618.3678 & 573 & 122.5994 & 2.52991 \\
\hline 1911 & 35.4703 & 582.2862 & 616.3411 & 573 & 122.5994 & 2.52874 \\
\hline 1912 & 35.4873 & 595.3029 & 615.6785 & 574 & 122.5994 & 2.52757 \\
\hline 1913 & 35.5043 & 589.4382 & 614.3944 & 574 & 122.5994 & 2.5264 \\
\hline 1914 & 35.5213 & 554.4763 & 613.3503 & 574 & 122.5994 & 2.52523 \\
\hline 1915 & 35.5383 & 573.5732 & 611.9755 & 574 & 122.5994 & 2.52406 \\
\hline 1916 & 35.5553 & 600.142 & 610.3252 & 574 & 122.5994 & 2.52289 \\
\hline 1917 & 35.5723 & 588.46 & 608.565 & 574 & 122.5994 & 2.52172 \\
\hline 1918 & 35.5893 & 610.2656 & 607.7834 & 575 & 122.5994 & 2.52056 \\
\hline 1919 & 35.6063 & 599.2533 & 606.1357 & 575 & 122.5994 & 2.51939 \\
\hline 1920 & 35.6233 & 605.902 & 604.3875 & 575 & 122.5994 & 2.51823 \\
\hline 1921 & 35.6403 & 587.7861 & 602.9503 & 575 & 122.5994 & 2.51707 \\
\hline 1922 & 35.6573 & 579.1893 & 601.676 & 575 & 122.5994 & 2.51591 \\
\hline 1923 & 35.6743 & 593.7138 & 600.6025 & 575 & 122.5994 & 2.51475 \\
\hline 1924 & 35.6913 & 583.189 & 599.8223 & 575 & 122.5994 & 2.51359 \\
\hline 1925 & 35.7083 & 575.1011 & 599.3718 & 575 & 122.5994 & 2.51243 \\
\hline 1926 & 35.7253 & 599.2618 & 599.541 & 575 & 122.5994 & 2.51127 \\
\hline 1927 & 35.7423 & 590.9737 & 600.3337 & 575 & 122.5994 & 2.51012 \\
\hline 1928 & 35.7593 & 571.8443 & 601.7879 & 575 & 122.5994 & 2.50896 \\
\hline 1929 & 35.7763 & 597.8291 & 602.7701 & 574 & 122.5994 & 2.50781 \\
\hline 1930 & 35.7933 & 592.9802 & 605.2943 & 574 & 122.5994 & 2.50666 \\
\hline 1931 & 35.8103 & 587.8804 & 608.0372 & 574 & 122.5994 & 2.50551 \\
\hline 1932 & 35.8273 & 557.2043 & 610.9396 & 574 & 122.5994 & 2.50436 \\
\hline 1933 & 35.8443 & 533.5588 & 615.2361 & 574 & 122.5994 & 2.50321 \\
\hline 1934 & 35.8613 & 590.5183 & 618.9354 & 574 & 122.5994 & 2.50206 \\
\hline 1935 & 35.8783 & 608.8703 & 622.9523 & 574 & 122.5994 & 2.50091 \\
\hline 1936 & 35.8953 & 598.4028 & 627.4124 & 574 & 122.5994 & 2.49977 \\
\hline 1937 & 35.9123 & 590.7519 & 632.1633 & 574 & 122.5994 & 2.49862 \\
\hline 1938 & 35.9293 & 559.8034 & 637.313 & 574 & 122.5994 & 2.49748 \\
\hline 1939 & 35.9463 & 560.8236 & 643.049 & 574 & 122.5994 & 2.49634 \\
\hline 1940 & 35.9633 & 587.2103 & 649.1974 & 574 & 122.5994 & 2.4952 \\
\hline 1941 & 35.9803 & 602.5696 & 655.5076 & 574 & 122.5994 & 2.49406 \\
\hline 1942 & 35.9973 & 620.3815 & 661.38 & 574 & 122.5994 & 2.49292 \\
\hline 1943 & 36.0143 & 624.9401 & 665.0418 & 573 & 122.5994 & 2.49178 \\
\hline 1944 & 36.0313 & 605.1295 & 668.7122 & 573 & 122.5994 & 2.49064 \\
\hline 1945 & 36.0483 & 556.5843 & 671.1221 & 573 & 122.5994 & 2.48951 \\
\hline 1946 & 36.0653 & 600.599 & 671.8661 & 573 & 122.5994 & 2.48837 \\
\hline 1947 & 36.0823 & 580.4889 & 670.8353 & 573 & 122.5994 & 2.48724 \\
\hline 1948 & 36.0993 & 601.9592 & 668.369 & 573 & 122.5994 & 2.48611 \\
\hline 1949 & 36.1163 & 565.5188 & 664.4525 & 573 & 122.5994 & 2.48498 \\
\hline 1950 & 36.1333 & 644.4571 & 659.3512 & 573 & 122.5994 & 2.48385 \\
\hline 1951 & 36.1503 & 588.5149 & 653.2368 & 573 & 122.5994 & 2.48272 \\
\hline 1952 & 36.1673 & 590.3284 & 646.7313 & 573 & 122.5994 & 2.48159 \\
\hline 1953 & 36.1843 & 585.5849 & 639.6417 & 573 & 122.5994 & 2.48046 \\
\hline 1954 & 36.2013 & 570.953 & 632.8955 & 573 & 122.5994 & 2.47934 \\
\hline 1955 & 36.2183 & 561.9686 & 626.4755 & 573 & 122.5994 & 2.47821 \\
\hline 1956 & 36.2353 & 567.9323 & 620.636 & 573 & 122.5994 & 2.47709 \\
\hline 1957 & 36.2523 & 588.3715 & 614.5681 & 572 & 122.5994 & 2.47597 \\
\hline 1958 & 36.2693 & 563.4874 & 610.3909 & 572 & 122.5994 & 2.47485 \\
\hline
\end{tabular}




\begin{tabular}{|c|c|c|c|c|c|c|}
\hline 1959 & 36.2863 & 544.2935 & 607.0983 & 572 & 122.5994 & 2.47372 \\
\hline 1960 & 36.3033 & 584.9914 & 604.7022 & 572 & 122.5994 & 2.47261 \\
\hline 1961 & 36.3203 & 573.4378 & 603.1418 & 572 & 122.5994 & 2.47149 \\
\hline 1962 & 36.3373 & 535.6354 & 601.9948 & 572 & 122.5994 & 2.47037 \\
\hline 1963 & 36.3543 & 528.3318 & 601.4609 & 572 & 122.5994 & 2.46925 \\
\hline 1964 & 36.3713 & 551.8007 & 601.8788 & 572 & 122.5994 & 2.46814 \\
\hline 1965 & 36.3883 & 575.9543 & 602.8799 & 572 & 122.5994 & 2.46702 \\
\hline 1966 & 36.4053 & 557.6878 & 604.7505 & 572 & 122.5994 & 2.46591 \\
\hline 1967 & 36.4223 & 564.9752 & 607.4719 & 572 & 122.5994 & 2.4648 \\
\hline 1968 & 36.4393 & 601.9414 & 610.9336 & 572 & 122.5994 & 2.46369 \\
\hline 1969 & 36.4563 & 587.1281 & 615.2927 & 572 & 122.5994 & 2.46258 \\
\hline 1970 & 36.4733 & 594.4896 & 619.6219 & 571 & 122.5994 & 2.46147 \\
\hline 1971 & 36.4903 & 623.4158 & 626.4164 & 571 & 122.5994 & 2.46036 \\
\hline 1972 & 36.5073 & 605.2407 & 635.6503 & 571 & 122.5994 & 2.45926 \\
\hline 1973 & 36.5243 & 611.4884 & 648.3282 & 570 & 122.5994 & 2.45815 \\
\hline 1974 & 36.5413 & 640.7042 & 669.2196 & 570 & 122.5994 & 2.45705 \\
\hline 1975 & 36.5583 & 682.8975 & 700.2269 & 570 & 122.5994 & 2.45594 \\
\hline 1976 & 36.5753 & 748.1709 & 741.7524 & 569 & 122.5994 & 2.45484 \\
\hline 1977 & 36.5923 & 818.9673 & 793.7361 & 569 & 122.5994 & 2.45374 \\
\hline 1978 & 36.6093 & 942.7501 & 847.1669 & 569 & 122.5994 & 2.45264 \\
\hline 1979 & 36.6263 & 1016.275 & 886.4088 & 569 & 122.5994 & 2.45154 \\
\hline 1980 & 36.6433 & 982.9132 & 894.4041 & 568 & 122.5994 & 2.45044 \\
\hline 1981 & 36.6603 & 916.0677 & 875.2235 & 568 & 122.5994 & 2.44934 \\
\hline 1982 & 36.6773 & 783.1518 & 845.8008 & 568 & 122.5994 & 2.44825 \\
\hline 1983 & 36.6943 & 727.0181 & 819.2054 & 567 & 122.5994 & 2.44715 \\
\hline 1984 & 36.7113 & 784.6108 & 799.5744 & 567 & 122.5994 & 2.44606 \\
\hline 1985 & 36.7283 & 796.5901 & 778.8865 & 567 & 122.5994 & 2.44496 \\
\hline 1986 & 36.7453 & 754.3835 & 749.1182 & 566 & 122.5994 & 2.44387 \\
\hline 1987 & 36.7623 & 720.0599 & 715.2369 & 566 & 122.5994 & 2.44278 \\
\hline 1988 & 36.7793 & 658.0221 & 683.2922 & 566 & 122.5994 & 2.44169 \\
\hline 1989 & 36.7963 & 616.9436 & 656.8806 & 565 & 122.5994 & 2.4406 \\
\hline 1990 & 36.8133 & 621.897 & 639.5457 & 565 & 122.5994 & 2.43951 \\
\hline 1991 & 36.8303 & 641.5633 & 630.2609 & 565 & 122.5994 & 2.43843 \\
\hline 1992 & 36.8473 & 645.0981 & 626.3311 & 565 & 122.5994 & 2.43734 \\
\hline 1993 & 36.8643 & 594.0272 & 624.331 & 564 & 122.5994 & 2.43626 \\
\hline 1994 & 36.8813 & 624.716 & 623.0694 & 564 & 122.5994 & 2.43517 \\
\hline 1995 & 36.8983 & 620.9159 & 619.0225 & 563 & 122.5994 & 2.43409 \\
\hline 1996 & 36.9153 & 574.9516 & 615.774 & 563 & 122.5994 & 2.43301 \\
\hline 1997 & 36.9323 & 603.2869 & 613.0192 & 562 & 122.5994 & 2.43193 \\
\hline 1998 & 36.9493 & 581.6677 & 612.4594 & 562 & 122.5994 & 2.43085 \\
\hline 1999 & 36.9663 & 614.6253 & 610.9365 & 561 & 122.5994 & 2.42977 \\
\hline 2000 & 36.9833 & 610.4474 & 608.7577 & 561 & 122.5994 & 2.42869 \\
\hline 2001 & 37.0003 & 631.8345 & 603.9713 & 560 & 122.5994 & 2.42761 \\
\hline 2002 & 37.0173 & 539.0702 & 599.1544 & 559 & 122.5994 & 2.42654 \\
\hline 2003 & 37.0343 & 552.9103 & 596.1081 & 559 & 122.5994 & 2.42546 \\
\hline 2004 & 37.0513 & 564.0929 & 592.8119 & 558 & 122.5994 & 2.42439 \\
\hline 2005 & 37.0683 & 579.4118 & 590.2838 & 558 & 122.5994 & 2.42331 \\
\hline 2006 & 37.0853 & 536.8289 & 587.0565 & 557 & 122.5994 & 2.42224 \\
\hline 2007 & 37.1023 & 560.1129 & 584.7971 & 557 & 122.5994 & 2.42117 \\
\hline
\end{tabular}




\begin{tabular}{|c|c|c|c|c|c|c|}
\hline 2008 & 37.1193 & 575.0676 & 581.7687 & 556 & 122.5994 & 2.4201 \\
\hline 2009 & 37.1363 & 565.4872 & 580.1048 & 556 & 122.5994 & 2.41903 \\
\hline 2010 & 37.1533 & 542.8379 & 577.8381 & 555 & 122.5994 & 2.41797 \\
\hline 2011 & 37.1703 & 547.6217 & 576.7838 & 555 & 122.5994 & 2.4169 \\
\hline 2012 & 37.1873 & 561.6361 & 575.508 & 554 & 122.5994 & 2.41583 \\
\hline 2013 & 37.2043 & 560.2612 & 575.8562 & 554 & 122.5994 & 2.41477 \\
\hline 2014 & 37.2213 & 567.5065 & 576.0009 & 553 & 122.5994 & 2.4137 \\
\hline 2015 & 37.2383 & 585.5974 & 577.976 & 553 & 122.5994 & 2.41264 \\
\hline 2016 & 37.2553 & 562.6571 & 579.632 & 552 & 122.5994 & 2.41158 \\
\hline 2017 & 37.2723 & 543.4676 & 582.5248 & 552 & 122.5994 & 2.41052 \\
\hline 2018 & 37.2893 & 548.4695 & 583.9056 & 551 & 122.5994 & 2.40946 \\
\hline 2019 & 37.3063 & 552.8507 & 584.3773 & 550 & 122.5994 & 2.4084 \\
\hline 2020 & 37.3233 & 545.8208 & 584.2931 & 549 & 122.5994 & 2.40734 \\
\hline 2021 & 37.3403 & 545.9089 & 585.1141 & 549 & 122.5994 & 2.40628 \\
\hline 2022 & 37.3573 & 546.5383 & 584.8671 & 548 & 122.5994 & 2.40523 \\
\hline 2023 & 37.3743 & 550.9575 & 584.6371 & 547 & 122.5994 & 2.40417 \\
\hline 2024 & 37.3913 & 575.4569 & 583.5939 & 546 & 122.5994 & 2.40312 \\
\hline 2025 & 37.4083 & 587.6377 & 582.9899 & 546 & 122.5994 & 2.40207 \\
\hline 2026 & 37.4253 & 605.2407 & 581.2131 & 545 & 122.5994 & 2.40101 \\
\hline 2027 & 37.4423 & 593.5474 & 579.6022 & 544 & 122.5994 & 2.39996 \\
\hline 2028 & 37.4593 & 576.864 & 579.3094 & 544 & 122.5994 & 2.39891 \\
\hline 2029 & 37.4763 & 592.7136 & 578.3483 & 543 & 122.5994 & 2.39786 \\
\hline 2030 & 37.4933 & 618.1254 & 577.6677 & 542 & 122.5994 & 2.39682 \\
\hline 2031 & 37.5103 & 598.3943 & 577.3531 & 541 & 122.5994 & 2.39577 \\
\hline 2032 & 37.5273 & 582.2053 & 578.6174 & 541 & 122.5994 & 2.39472 \\
\hline 2033 & 37.5443 & 570.5246 & 580.1607 & 540 & 122.5994 & 2.39368 \\
\hline 2034 & 37.5613 & 577.0563 & 582.8016 & 539 & 122.5994 & 2.39263 \\
\hline 2035 & 37.5783 & 559.0614 & 586.6857 & 538 & 122.5994 & 2.39159 \\
\hline 2036 & 37.5953 & 552.197 & 592.6494 & 538 & 122.5994 & 2.39055 \\
\hline 2037 & 37.6123 & 594.8984 & 598.4142 & 537 & 122.5994 & 2.38951 \\
\hline 2038 & 37.6293 & 605.2407 & 603.7421 & 536 & 122.5994 & 2.38847 \\
\hline 2039 & 37.6463 & 588.5075 & 609.7646 & 535 & 122.5994 & 2.38743 \\
\hline 2040 & 37.6633 & 615.681 & 616.5198 & 535 & 122.5994 & 2.38639 \\
\hline 2041 & 37.6803 & 589.2514 & 622.0475 & 534 & 122.5994 & 2.38535 \\
\hline 2042 & 37.6973 & 559.3526 & 627.1936 & 533 & 122.5994 & 2.38431 \\
\hline 2043 & 37.7143 & 588.4272 & 631.7733 & 532 & 122.5994 & 2.38328 \\
\hline 2044 & 37.7313 & 595.0973 & 635.73 & 531 & 122.5994 & 2.38224 \\
\hline 2045 & 37.7483 & 629.3733 & 638.9355 & 530 & 122.5994 & 2.38121 \\
\hline 2046 & 37.7653 & 590.6083 & 641.0543 & 529 & 122.5994 & 2.38018 \\
\hline 2047 & 37.7823 & 634.6438 & 641.4993 & 528 & 122.5994 & 2.37914 \\
\hline 2048 & 37.7993 & 617.6079 & 640.5672 & 528 & 122.5994 & 2.37811 \\
\hline 2049 & 37.8163 & 571.0005 & 636.4201 & 527 & 122.5994 & 2.37708 \\
\hline 2050 & 37.8333 & 630.2719 & 630.6985 & 526 & 122.5994 & 2.37605 \\
\hline 2051 & 37.8503 & 578.8139 & 623.845 & 525 & 122.5994 & 2.37503 \\
\hline 2052 & 37.8673 & 563.4946 & 616.3226 & 524 & 122.5994 & 2.374 \\
\hline 2053 & 37.8843 & 576.8056 & 608.0753 & 523 & 122.5994 & 2.37297 \\
\hline 2054 & 37.9013 & 575.004 & 599.2367 & 522 & 122.5994 & 2.37195 \\
\hline 2055 & 37.9183 & 541.2355 & 590.1235 & 521 & 122.5994 & 2.37092 \\
\hline 2056 & 37.9353 & 517.3287 & 581.1572 & 520 & 122.5994 & 2.3699 \\
\hline
\end{tabular}




\begin{tabular}{|c|c|c|c|c|c|c|}
\hline 2057 & 37.9523 & 525.4495 & 572.3771 & 519 & 122.5994 & 2.36888 \\
\hline 2058 & 37.9693 & 536.3144 & 564.3502 & 518 & 122.5994 & 2.36786 \\
\hline 2059 & 37.9863 & 541.423 & 558.0836 & 518 & 122.5994 & 2.36684 \\
\hline 2060 & 38.0033 & 511.328 & 551.6711 & 517 & 122.5994 & 2.36582 \\
\hline 2061 & 38.0203 & 548.9536 & 546.1459 & 516 & 122.5994 & 2.3648 \\
\hline 2062 & 38.0373 & 559.7202 & 541.4644 & 515 & 122.5994 & 2.36378 \\
\hline 2063 & 38.0543 & 501.9623 & 537.6528 & 514 & 122.5994 & 2.36276 \\
\hline 2064 & 38.0713 & 500.5974 & 534.6632 & 513 & 122.5994 & 2.36175 \\
\hline 2065 & 38.0883 & 532.5557 & 532.4761 & 512 & 122.5994 & 2.36073 \\
\hline 2066 & 38.1053 & 510.711 & 531.0384 & 511 & 122.5994 & 2.35972 \\
\hline 2067 & 38.1223 & 492.6678 & 531.4332 & 511 & 122.5994 & 2.3587 \\
\hline 2068 & 38.1393 & 502.8901 & 531.6143 & 510 & 122.5994 & 2.35769 \\
\hline 2069 & 38.1563 & 509.1302 & 533.5708 & 510 & 122.5994 & 2.35668 \\
\hline 2070 & 38.1733 & 461.8799 & 535.2717 & 509 & 122.5994 & 2.35567 \\
\hline 2071 & 38.1903 & 482.9187 & 537.645 & 508 & 122.5994 & 2.35466 \\
\hline 2072 & 38.2073 & 520.3488 & 541.5599 & 508 & 122.5994 & 2.35365 \\
\hline 2073 & 38.2243 & 516.5389 & 544.7981 & 507 & 122.5994 & 2.35264 \\
\hline 2074 & 38.2413 & 513.4434 & 548.0247 & 506 & 122.5994 & 2.35164 \\
\hline 2075 & 38.2583 & 493.0648 & 551.7979 & 506 & 122.5994 & 2.35063 \\
\hline 2076 & 38.2753 & 511.4823 & 553.6408 & 505 & 122.5994 & 2.34963 \\
\hline 2077 & 38.2923 & 507.8914 & 554.2084 & 504 & 122.5994 & 2.34862 \\
\hline 2078 & 38.3093 & 494.1748 & 554.4254 & 504 & 122.5994 & 2.34762 \\
\hline 2079 & 38.3263 & 502.3238 & 552.472 & 503 & 122.5994 & 2.34662 \\
\hline 2080 & 38.3433 & 483.6061 & 550.6193 & 503 & 122.5994 & 2.34562 \\
\hline 2081 & 38.3603 & 488.4615 & 547.0747 & 502 & 122.5994 & 2.34462 \\
\hline 2082 & 38.3773 & 475.8844 & 542.8527 & 501 & 122.5994 & 2.34362 \\
\hline 2083 & 38.3943 & 488.3364 & 539.2783 & 501 & 122.5994 & 2.34262 \\
\hline 2084 & 38.4113 & 522.3825 & 534.4267 & 500 & 122.5994 & 2.34162 \\
\hline 2085 & 38.4283 & 513.7498 & 530.5537 & 500 & 122.5994 & 2.34062 \\
\hline 2086 & 38.4453 & 483.8793 & 525.91 & 499 & 122.5994 & 2.33963 \\
\hline 2087 & 38.4623 & 507.18 & 521.6848 & 498 & 122.5994 & 2.33863 \\
\hline 2088 & 38.4793 & 474.651 & 518.9368 & 498 & 122.5994 & 2.33764 \\
\hline 2089 & 38.4963 & 516.2849 & 515.8549 & 497 & 122.5994 & 2.33664 \\
\hline 2090 & 38.5133 & 485.8314 & 514.2784 & 497 & 122.5994 & 2.33565 \\
\hline 2091 & 38.5303 & 514.2714 & 513.1982 & 497 & 122.5994 & 2.33466 \\
\hline 2092 & 38.5473 & 493.1202 & 512.5151 & 497 & 122.5994 & 2.33367 \\
\hline 2093 & 38.5643 & 501.6649 & 512.1071 & 497 & 122.5994 & 2.33268 \\
\hline 2094 & 38.5813 & 487.63 & 511.8564 & 497 & 122.5994 & 2.33169 \\
\hline 2095 & 38.5983 & 468.073 & 512.7173 & 498 & 122.5994 & 2.3307 \\
\hline 2096 & 38.6153 & 493.4762 & 512.5765 & 498 & 122.5994 & 2.32972 \\
\hline 2097 & 38.6323 & 499.9706 & 512.471 & 498 & 122.5994 & 2.32873 \\
\hline 2098 & 38.6493 & 485.1226 & 512.4466 & 498 & 122.5994 & 2.32775 \\
\hline 2099 & 38.6663 & 491.5453 & 512.5237 & 498 & 122.5994 & 2.32676 \\
\hline 2100 & 38.6833 & 489.1574 & 512.7833 & 498 & 122.5994 & 2.32578 \\
\hline 2101 & 38.7003 & 511.1056 & 513.4207 & 498 & 122.5994 & 2.3248 \\
\hline 2102 & 38.7173 & 497.4086 & 514.6722 & 498 & 122.5994 & 2.32381 \\
\hline 2103 & 38.7343 & 501.3598 & 516.3782 & 498 & 122.5994 & 2.32283 \\
\hline 2104 & 38.7513 & 475.8283 & 519.5776 & 498 & 122.5994 & 2.32185 \\
\hline 2105 & 38.7683 & 517.7006 & 521.4643 & 498 & 122.5994 & 2.32087 \\
\hline
\end{tabular}




\begin{tabular}{|c|c|c|c|c|c|c|}
\hline 2106 & 38.7853 & 464.661 & 521.5668 & 498 & 122.5994 & 2.3199 \\
\hline 2107 & 38.8023 & 497.6431 & 519.801 & 498 & 122.5994 & 2.31892 \\
\hline 2108 & 38.8193 & 478.1681 & 518.4489 & 499 & 122.5994 & 2.31794 \\
\hline 2109 & 38.8363 & 468.7634 & 516.3475 & 499 & 122.5994 & 2.31697 \\
\hline 2110 & 38.8533 & 490.1491 & 515.4197 & 499 & 122.5994 & 2.31599 \\
\hline 2111 & 38.8703 & 482.299 & 515.3385 & 499 & 122.5994 & 2.31502 \\
\hline 2112 & 38.8873 & 483.3299 & 514.5723 & 499 & 122.5994 & 2.31405 \\
\hline 2113 & 38.9043 & 500.2692 & 513.3354 & 499 & 122.5994 & 2.31307 \\
\hline 2114 & 38.9213 & 503.2049 & 512.0689 & 499 & 122.5994 & 2.3121 \\
\hline 2115 & 38.9383 & 524.252 & 510.999 & 499 & 122.5994 & 2.31113 \\
\hline 2116 & 38.9553 & 515.1495 & 509.3269 & 498 & 122.5994 & 2.31016 \\
\hline 2117 & 38.9723 & 483.4035 & 508.6852 & 498 & 122.5994 & 2.30919 \\
\hline 2118 & 38.9893 & 480.4797 & 507.9389 & 498 & 122.5994 & 2.30823 \\
\hline 2119 & 39.0063 & 483.4452 & 507.2627 & 498 & 122.5994 & 2.30726 \\
\hline 2120 & 39.0233 & 503.4206 & 506.8008 & 498 & 122.5994 & 2.30629 \\
\hline 2121 & 39.0403 & 497.7691 & 506.589 & 498 & 122.5994 & 2.30533 \\
\hline 2122 & 39.0573 & 462.0818 & 506.5255 & 498 & 122.5994 & 2.30436 \\
\hline 2123 & 39.0743 & 495.6845 & 505.4464 & 497 & 122.5994 & 2.3034 \\
\hline 2124 & 39.0913 & 492.5873 & 505.3 & 497 & 122.5994 & 2.30244 \\
\hline 2125 & 39.1083 & 479.564 & 505.2095 & 497 & 122.5994 & 2.30148 \\
\hline 2126 & 39.1253 & 463.933 & 505.2809 & 497 & 122.5994 & 2.30052 \\
\hline 2127 & 39.1423 & 464.4163 & 505.5098 & 497 & 122.5994 & 2.29956 \\
\hline 2128 & 39.1593 & 483.2317 & 504.9649 & 496 & 122.5994 & 2.2986 \\
\hline 2129 & 39.1763 & 467.8949 & 505.6573 & 496 & 122.5994 & 2.29764 \\
\hline 2130 & 39.1933 & 483.3462 & 506.5961 & 496 & 122.5994 & 2.29668 \\
\hline 2131 & 39.2103 & 477.547 & 507.7975 & 496 & 122.5994 & 2.29572 \\
\hline 2132 & 39.2273 & 464.1374 & 509.2852 & 496 & 122.5994 & 2.29477 \\
\hline 2133 & 39.2443 & 520.4527 & 511.1333 & 496 & 122.5994 & 2.29381 \\
\hline 2134 & 39.2613 & 508.726 & 513.2862 & 496 & 122.5994 & 2.29286 \\
\hline 2135 & 39.2783 & 502.09 & 514.8474 & 495 & 122.5994 & 2.29191 \\
\hline 2136 & 39.2953 & 506.0355 & 517.5665 & 495 & 122.5994 & 2.29095 \\
\hline 2137 & 39.3123 & 489.0972 & 520.862 & 495 & 122.5994 & 2.29 \\
\hline 2138 & 39.3293 & 492.8183 & 524.5268 & 495 & 122.5994 & 2.28905 \\
\hline 2139 & 39.3463 & 483.9536 & 529.5823 & 496 & 122.5994 & 2.2881 \\
\hline 2140 & 39.3633 & 484.796 & 534.1206 & 496 & 122.5994 & 2.28715 \\
\hline 2141 & 39.3803 & 514.0364 & 539.3455 & 496 & 122.5994 & 2.2862 \\
\hline 2142 & 39.3973 & 491.1723 & 546.385 & 497 & 122.5994 & 2.28526 \\
\hline 2143 & 39.4143 & 529.7612 & 553.9293 & 497 & 122.5994 & 2.28431 \\
\hline 2144 & 39.4313 & 536.6769 & 564.6427 & 498 & 122.5994 & 2.28336 \\
\hline 2145 & 39.4483 & 530.6078 & 577.8618 & 498 & 122.5994 & 2.28242 \\
\hline 2146 & 39.4653 & 571.885 & 596.5894 & 498 & 122.5994 & 2.28148 \\
\hline 2147 & 39.4823 & 577.7542 & 624.3794 & 499 & 122.5994 & 2.28053 \\
\hline 2148 & 39.4993 & 608.3728 & 661.5186 & 499 & 122.5994 & 2.27959 \\
\hline 2149 & 39.5163 & 717.6672 & 709.4485 & 500 & 122.5994 & 2.27865 \\
\hline 2150 & 39.5333 & 793.1933 & 762.2274 & 500 & 122.5994 & 2.27771 \\
\hline 2151 & 39.5503 & 863.0004 & 809.645 & 500 & 122.5994 & 2.27677 \\
\hline 2152 & 39.5673 & 842.5139 & 835.0212 & 501 & 122.5994 & 2.27583 \\
\hline 2153 & 39.5843 & 722.5184 & 826.6243 & 501 & 122.5994 & 2.27489 \\
\hline 2154 & 39.6013 & 635.4679 & 796.7568 & 501 & 122.5994 & 2.27395 \\
\hline
\end{tabular}




\begin{tabular}{|c|c|c|c|c|c|c|}
\hline 2155 & 39.6183 & 613.268 & 765.4745 & 502 & 122.5994 & 2.27302 \\
\hline 2156 & 39.6353 & 630.445 & 741.4321 & 502 & 122.5994 & 2.27208 \\
\hline 2157 & 39.6523 & 673.8226 & 725.1415 & 502 & 122.5994 & 2.27115 \\
\hline 2158 & 39.6693 & 682.088 & 708.8574 & 503 & 122.5994 & 2.27021 \\
\hline 2159 & 39.6863 & 622.6055 & 682.8358 & 503 & 122.5994 & 2.26928 \\
\hline 2160 & 39.7033 & 547.9512 & 651.1553 & 504 & 122.5994 & 2.26835 \\
\hline 2161 & 39.7203 & 536.3609 & 618.8293 & 504 & 122.5994 & 2.26741 \\
\hline 2162 & 39.7373 & 526.6695 & 591.4621 & 504 & 122.5994 & 2.26648 \\
\hline 2163 & 39.7543 & 503.4229 & 570.4757 & 504 & 122.5994 & 2.26555 \\
\hline 2164 & 39.7713 & 498.5085 & 555.3721 & 504 & 122.5994 & 2.26462 \\
\hline 2165 & 39.7883 & 488.3368 & 545.0077 & 504 & 122.5994 & 2.2637 \\
\hline 2166 & 39.8053 & 486.3395 & 539.1564 & 505 & 122.5994 & 2.26277 \\
\hline 2167 & 39.8223 & 505.3815 & 534.697 & 505 & 122.5994 & 2.26184 \\
\hline 2168 & 39.8393 & 493.9681 & 531.7229 & 505 & 122.5994 & 2.26092 \\
\hline 2169 & 39.8563 & 484.0645 & 529.554 & 505 & 122.5994 & 2.25999 \\
\hline 2170 & 39.8733 & 492.8411 & 527.7393 & 505 & 122.5994 & 2.25907 \\
\hline 2171 & 39.8903 & 494.2841 & 526.1365 & 505 & 122.5994 & 2.25814 \\
\hline 2172 & 39.9073 & 505.5484 & 524.8106 & 505 & 122.5994 & 2.25722 \\
\hline 2173 & 39.9243 & 472.1014 & 523.8329 & 505 & 122.5994 & 2.2563 \\
\hline 2174 & 39.9413 & 490.9798 & 523.3083 & 505 & 122.5994 & 2.25538 \\
\hline 2175 & 39.9583 & 501.7119 & 523.0889 & 505 & 122.5994 & 2.25446 \\
\hline 2176 & 39.9753 & 523.563 & 524.1779 & 506 & 122.5994 & 2.25354 \\
\hline 2177 & 39.9923 & 502.967 & 524.5262 & 506 & 122.5994 & 2.25262 \\
\hline 2178 & 40.0093 & 516.3213 & 525.0656 & 506 & 122.5994 & 2.2517 \\
\hline 2179 & 40.0263 & 519.7901 & 525.754 & 506 & 122.5994 & 2.25078 \\
\hline 2180 & 40.0433 & 507.8945 & 526.5899 & 506 & 122.5994 & 2.24987 \\
\hline 2181 & 40.0603 & 510.2853 & 527.5076 & 506 & 122.5994 & 2.24895 \\
\hline 2182 & 40.0773 & 506.5306 & 528.3824 & 506 & 122.5994 & 2.24804 \\
\hline 2183 & 40.0943 & 506.3175 & 529.1745 & 506 & 122.5994 & 2.24712 \\
\hline 2184 & 40.1113 & 519.3837 & 529.866 & 506 & 122.5994 & 2.24621 \\
\hline 2185 & 40.1283 & 501.9803 & 530.5157 & 506 & 122.5994 & 2.2453 \\
\hline 2186 & 40.1453 & 510.9477 & 531.2393 & 506 & 122.5994 & 2.24439 \\
\hline 2187 & 40.1623 & 529.0705 & 532.1704 & 506 & 122.5994 & 2.24347 \\
\hline 2188 & 40.1793 & 526.9227 & 533.3326 & 506 & 122.5994 & 2.24256 \\
\hline 2189 & 40.1963 & 491.8778 & 534.6577 & 506 & 122.5994 & 2.24166 \\
\hline 2190 & 40.2133 & 483.5513 & 536.0756 & 506 & 122.5994 & 2.24075 \\
\hline 2191 & 40.2303 & 518.2681 & 537.2153 & 506 & 122.5994 & 2.23984 \\
\hline 2192 & 40.2473 & 508.7835 & 538.0422 & 506 & 122.5994 & 2.23893 \\
\hline 2193 & 40.2643 & 531.236 & 539.2905 & 506 & 122.5994 & 2.23803 \\
\hline 2194 & 40.2813 & 561.2914 & 542.1494 & 506 & 122.5994 & 2.23712 \\
\hline 2195 & 40.2983 & 544.0166 & 547.7629 & 506 & 122.5994 & 2.23622 \\
\hline 2196 & 40.3153 & 575.3562 & 557.0585 & 506 & 122.5994 & 2.23531 \\
\hline 2197 & 40.3323 & 613.8255 & 569.9299 & 506 & 122.5994 & 2.23441 \\
\hline 2198 & 40.3493 & 641.4034 & 585.8831 & 506 & 122.5994 & 2.23351 \\
\hline 2199 & 40.3663 & 658.8706 & 601.019 & 505 & 122.5994 & 2.23261 \\
\hline 2200 & 40.3833 & 675.4092 & 611.6633 & 505 & 122.5994 & 2.23171 \\
\hline 2201 & 40.4003 & 633.4308 & 613.2328 & 505 & 122.5994 & 2.23081 \\
\hline 2202 & 40.4173 & 605.9442 & 605.8912 & 505 & 122.5994 & 2.22991 \\
\hline 2203 & 40.4343 & 591.9832 & 595.4763 & 505 & 122.5994 & 2.22901 \\
\hline
\end{tabular}




\begin{tabular}{|c|c|c|c|c|c|c|}
\hline 2204 & 40.4513 & 592.5552 & 587.106 & 505 & 122.5994 & 2.22811 \\
\hline 2205 & 40.4683 & 564.3821 & 582.045 & 505 & 122.5994 & 2.22721 \\
\hline 2206 & 40.4853 & 572.3854 & 578.0592 & 505 & 122.5994 & 2.22632 \\
\hline 2207 & 40.5023 & 570.062 & 572.4702 & 505 & 122.5994 & 2.22542 \\
\hline 2208 & 40.5193 & 521.2646 & 563.6541 & 505 & 122.5994 & 2.22453 \\
\hline 2209 & 40.5363 & 499.7155 & 553.3653 & 505 & 122.5994 & 2.22363 \\
\hline 2210 & 40.5533 & 498.8799 & 543.7275 & 505 & 122.5994 & 2.22274 \\
\hline 2211 & 40.5703 & 481.2184 & 535.8092 & 505 & 122.5994 & 2.22185 \\
\hline 2212 & 40.5873 & 472.7044 & 529.7612 & 505 & 122.5994 & 2.22096 \\
\hline 2213 & 40.6043 & 486.6363 & 524.4034 & 504 & 122.5994 & 2.22007 \\
\hline 2214 & 40.6213 & 502.7571 & 521.3705 & 504 & 122.5994 & 2.21918 \\
\hline 2215 & 40.6383 & 492.141 & 519.3168 & 504 & 122.5994 & 2.21829 \\
\hline 2216 & 40.6553 & 501.5767 & 517.9471 & 504 & 122.5994 & 2.2174 \\
\hline 2217 & 40.6723 & 516.8853 & 517.0364 & 504 & 122.5994 & 2.21651 \\
\hline 2218 & 40.6893 & 507.2238 & 516.4018 & 504 & 122.5994 & 2.21563 \\
\hline 2219 & 40.7063 & 482.5365 & 515.9776 & 504 & 122.5994 & 2.21474 \\
\hline 2220 & 40.7233 & 431.3062 & 515.7398 & 504 & 122.5994 & 2.21385 \\
\hline 2221 & 40.7403 & 438.376 & 515.6948 & 504 & 122.5994 & 2.21297 \\
\hline 2222 & 40.7573 & 462.0883 & 515.8274 & 504 & 122.5994 & 2.21209 \\
\hline 2223 & 40.7743 & 442.4417 & 516.108 & 504 & 122.5994 & 2.2112 \\
\hline 2224 & 40.7913 & 483.3074 & 516.5132 & 504 & 122.5994 & 2.21032 \\
\hline 2225 & 40.8083 & 466.8349 & 517.0352 & 504 & 122.5994 & 2.20944 \\
\hline 2226 & 40.8253 & 439.9791 & 517.6744 & 504 & 122.5994 & 2.20856 \\
\hline 2227 & 40.8423 & 536.0265 & 517.4249 & 503 & 122.5994 & 2.20768 \\
\hline 2228 & 40.8593 & 517.9238 & 518.2517 & 503 & 122.5994 & 2.2068 \\
\hline 2229 & 40.8763 & 502.5471 & 519.0854 & 503 & 122.5994 & 2.20592 \\
\hline 2230 & 40.8933 & 484.7804 & 519.8897 & 503 & 122.5994 & 2.20504 \\
\hline 2231 & 40.9103 & 483.9536 & 520.5843 & 503 & 122.5994 & 2.20417 \\
\hline 2232 & 40.9273 & 519.7901 & 521.1246 & 503 & 122.5994 & 2.20329 \\
\hline 2233 & 40.9443 & 518.9258 & 521.4951 & 503 & 122.5994 & 2.20241 \\
\hline 2234 & 40.9613 & 480.9928 & 521.7006 & 503 & 122.5994 & 2.20154 \\
\hline 2235 & 40.9783 & 509.5052 & 521.7515 & 503 & 122.5994 & 2.20066 \\
\hline 2236 & 40.9953 & 496.5317 & 520.6874 & 502 & 122.5994 & 2.19979 \\
\hline 2237 & 41.0123 & 533.0501 & 520.5124 & 502 & 122.5994 & 2.19892 \\
\hline 2238 & 41.0293 & 523.7123 & 520.222 & 502 & 122.5994 & 2.19805 \\
\hline 2239 & 41.0463 & 489.0428 & 519.8301 & 502 & 122.5994 & 2.19718 \\
\hline 2240 & 41.0633 & 489.7154 & 519.3072 & 502 & 122.5994 & 2.19631 \\
\hline 2241 & 41.0803 & 471.9079 & 517.6977 & 501 & 122.5994 & 2.19544 \\
\hline 2242 & 41.0973 & 467.4609 & 516.9944 & 501 & 122.5994 & 2.19457 \\
\hline 2243 & 41.1143 & 503.983 & 516.2457 & 501 & 122.5994 & 2.1937 \\
\hline 2244 & 41.1313 & 506.0341 & 515.4356 & 501 & 122.5994 & 2.19283 \\
\hline 2245 & 41.1483 & 508.3716 & 513.5756 & 500 & 122.5994 & 2.19196 \\
\hline 2246 & 41.1653 & 547.5075 & 512.9642 & 500 & 122.5994 & 2.1911 \\
\hline 2247 & 41.1823 & 519.7409 & 512.324 & 500 & 122.5994 & 2.19023 \\
\hline 2248 & 41.1993 & 485.4824 & 511.8158 & 500 & 122.5994 & 2.18937 \\
\hline 2249 & 41.2163 & 513.9899 & 511.4998 & 500 & 122.5994 & 2.18851 \\
\hline 2250 & 41.2333 & 488.4751 & 510.5003 & 499 & 122.5994 & 2.18764 \\
\hline 2251 & 41.2503 & 493.8583 & 510.9196 & 499 & 122.5994 & 2.18678 \\
\hline 2252 & 41.2673 & 501.7119 & 512.063 & 499 & 122.5994 & 2.18592 \\
\hline
\end{tabular}




\begin{tabular}{|c|c|c|c|c|c|c|}
\hline 2253 & 41.2843 & 505.9191 & 513.8793 & 499 & 122.5994 & 2.18506 \\
\hline 2254 & 41.3013 & 512.8678 & 516.4836 & 499 & 122.5994 & 2.1842 \\
\hline 2255 & 41.3183 & 526.7822 & 518.5095 & 498 & 122.5994 & 2.18334 \\
\hline 2256 & 41.3353 & 510.0281 & 521.1945 & 498 & 122.5994 & 2.18248 \\
\hline 2257 & 41.3523 & 508.4018 & 522.6953 & 498 & 122.5994 & 2.18162 \\
\hline 2258 & 41.3693 & 506.1276 & 522.9805 & 498 & 122.5994 & 2.18076 \\
\hline 2259 & 41.3863 & 504.0522 & 522.6196 & 498 & 122.5994 & 2.17991 \\
\hline 2260 & 41.4033 & 501.7693 & 521.6212 & 497 & 122.5994 & 2.17905 \\
\hline 2261 & 41.4203 & 498.3832 & 521.6144 & 497 & 122.5994 & 2.1782 \\
\hline 2262 & 41.4373 & 519.489 & 521.2677 & 497 & 122.5994 & 2.17734 \\
\hline 2263 & 41.4543 & 516.7319 & 520.2674 & 497 & 122.5994 & 2.17649 \\
\hline 2264 & 41.4713 & 507.5936 & 518.5871 & 497 & 122.5994 & 2.17564 \\
\hline 2265 & 41.4883 & 493.5997 & 515.8266 & 496 & 122.5994 & 2.17478 \\
\hline 2266 & 41.5053 & 514.7979 & 513.8424 & 496 & 122.5994 & 2.17393 \\
\hline 2267 & 41.5223 & 514.1612 & 511.9038 & 496 & 122.5994 & 2.17308 \\
\hline 2268 & 41.5393 & 528.4739 & 510.0414 & 496 & 122.5994 & 2.17223 \\
\hline 2269 & 41.5563 & 504.6608 & 508.2726 & 496 & 122.5994 & 2.17138 \\
\hline 2270 & 41.5733 & 497.2423 & 505.6706 & 495 & 122.5994 & 2.17053 \\
\hline 2271 & 41.5903 & 486.054 & 504.2708 & 495 & 122.5994 & 2.16969 \\
\hline 2272 & 41.6073 & 457.8916 & 503.5646 & 495 & 122.5994 & 2.16884 \\
\hline 2273 & 41.6243 & 488.2915 & 502.5665 & 495 & 122.5994 & 2.16799 \\
\hline 2274 & 41.6413 & 492.9057 & 500.7389 & 494 & 122.5994 & 2.16715 \\
\hline 2275 & 41.6583 & 484.5892 & 500.0916 & 494 & 122.5994 & 2.1663 \\
\hline 2276 & 41.6753 & 484.0645 & 499.5795 & 494 & 122.5994 & 2.16546 \\
\hline 2277 & 41.6923 & 514.3383 & 499.1873 & 494 & 122.5994 & 2.16461 \\
\hline 2278 & 41.7093 & 479.8286 & 498.9023 & 494 & 122.5994 & 2.16377 \\
\hline 2279 & 41.7263 & 537.3037 & 497.7132 & 493 & 122.5994 & 2.16293 \\
\hline 2280 & 41.7433 & 475.616 & 497.6005 & 493 & 122.5994 & 2.16209 \\
\hline 2281 & 41.7603 & 484.7126 & 497.5524 & 493 & 122.5994 & 2.16124 \\
\hline 2282 & 41.7773 & 497.2423 & 497.6104 & 493 & 122.5994 & 2.1604 \\
\hline 2283 & 41.7943 & 498.2539 & 497.7086 & 493 & 122.5994 & 2.15957 \\
\hline 2284 & 41.8113 & 500.8509 & 496.8578 & 492 & 122.5994 & 2.15873 \\
\hline 2285 & 41.8283 & 467.9715 & 497.2878 & 492 & 122.5994 & 2.15789 \\
\hline 2286 & 41.8453 & 503.1939 & 497.5306 & 492 & 122.5994 & 2.15705 \\
\hline 2287 & 41.8623 & 515.9482 & 497.8189 & 492 & 122.5994 & 2.15621 \\
\hline 2288 & 41.8793 & 532.4796 & 498.1691 & 492 & 122.5994 & 2.15538 \\
\hline 2289 & 41.8963 & 500.582 & 498.6074 & 492 & 122.5994 & 2.15454 \\
\hline 2290 & 41.9133 & 481.1317 & 498.1637 & 491 & 122.5994 & 2.15371 \\
\hline 2291 & 41.9303 & 515.7653 & 498.8766 & 491 & 122.5994 & 2.15287 \\
\hline 2292 & 41.9473 & 491.6038 & 499.754 & 491 & 122.5994 & 2.15204 \\
\hline 2293 & 41.9643 & 505.5409 & 500.7874 & 491 & 122.5994 & 2.15121 \\
\hline 2294 & 41.9813 & 533.5444 & 502.0432 & 491 & 122.5994 & 2.15038 \\
\hline 2295 & 41.9983 & 504.1452 & 503.5243 & 491 & 122.5994 & 2.14955 \\
\hline 2296 & 42.0153 & 495.8373 & 504.2065 & 490 & 122.5994 & 2.14872 \\
\hline 2297 & 42.0323 & 508.1327 & 506.1181 & 490 & 122.5994 & 2.14789 \\
\hline 2298 & 42.0493 & 489.7661 & 508.3188 & 490 & 122.5994 & 2.14706 \\
\hline 2299 & 42.0663 & 491.5158 & 510.7993 & 490 & 122.5994 & 2.14623 \\
\hline 2300 & 42.0833 & 516.8014 & 513.5507 & 490 & 122.5994 & 2.1454 \\
\hline 2301 & 42.1003 & 531.196 & 516.5408 & 490 & 122.5994 & 2.14457 \\
\hline
\end{tabular}




\begin{tabular}{|c|c|c|c|c|c|c|}
\hline 2302 & 42.1173 & 506.195 & 518.5441 & 489 & 122.5994 & 2.14375 \\
\hline 2303 & 42.1343 & 513.1292 & 521.2739 & 489 & 122.5994 & 2.14292 \\
\hline 2304 & 42.1513 & 537.8391 & 523.3493 & 489 & 122.5994 & 2.1421 \\
\hline 2305 & 42.1683 & 515.2931 & 524.5821 & 489 & 122.5994 & 2.14127 \\
\hline 2306 & 42.1853 & 504.383 & 525.0718 & 489 & 122.5994 & 2.14045 \\
\hline 2307 & 42.2023 & 503.5491 & 525.2979 & 489 & 122.5994 & 2.13963 \\
\hline 2308 & 42.2193 & 496.0211 & 524.4326 & 488 & 122.5994 & 2.1388 \\
\hline 2309 & 42.2363 & 464.2776 & 524.601 & 488 & 122.5994 & 2.13798 \\
\hline 2310 & 42.2533 & 464.0055 & 524.8008 & 488 & 122.5994 & 2.13716 \\
\hline 2311 & 42.2703 & 461.8668 & 524.9772 & 488 & 122.5994 & 2.13634 \\
\hline 2312 & 42.2873 & 486.3958 & 525.2135 & 488 & 122.5994 & 2.13552 \\
\hline 2313 & 42.3043 & 503.9224 & 525.7257 & 488 & 122.5994 & 2.1347 \\
\hline 2314 & 42.3213 & 545.2219 & 525.7321 & 487 & 122.5994 & 2.13388 \\
\hline 2315 & 42.3383 & 542.1437 & 527.4066 & 487 & 122.5994 & 2.13307 \\
\hline 2316 & 42.3553 & 516.0457 & 529.884 & 487 & 122.5994 & 2.13225 \\
\hline 2317 & 42.3723 & 525.8809 & 533.2767 & 487 & 122.5994 & 2.13143 \\
\hline 2318 & 42.3893 & 544.9084 & 537.7282 & 487 & 122.5994 & 2.13062 \\
\hline 2319 & 42.4063 & 551.6101 & 543.5029 & 487 & 122.5994 & 2.1298 \\
\hline 2320 & 42.4233 & 552.197 & 550.1334 & 486 & 122.5994 & 2.12899 \\
\hline 2321 & 42.4403 & 596.3791 & 560.5024 & 486 & 122.5994 & 2.12818 \\
\hline 2322 & 42.4573 & 632.8687 & 575.1826 & 486 & 122.5994 & 2.12736 \\
\hline 2323 & 42.4743 & 639.9772 & 595.6929 & 486 & 122.5994 & 2.12655 \\
\hline 2324 & 42.4913 & 680.4213 & 622.9162 & 486 & 122.5994 & 2.12574 \\
\hline 2325 & 42.5083 & 718.6335 & 655.9042 & 486 & 122.5994 & 2.12493 \\
\hline 2326 & 42.5253 & 795.5939 & 689.3137 & 485 & 122.5994 & 2.12412 \\
\hline 2327 & 42.5423 & 810.5085 & 715.915 & 485 & 122.5994 & 2.12331 \\
\hline 2328 & 42.5593 & 768.6678 & 723.739 & 485 & 122.5994 & 2.1225 \\
\hline 2329 & 42.5763 & 708.7322 & 710.7748 & 485 & 122.5994 & 2.12169 \\
\hline 2330 & 42.5933 & 666.1522 & 686.5529 & 484 & 122.5994 & 2.12089 \\
\hline 2331 & 42.6103 & 669.3747 & 664.9399 & 484 & 122.5994 & 2.12008 \\
\hline 2332 & 42.6273 & 663.7792 & 650.6346 & 484 & 122.5994 & 2.11927 \\
\hline 2333 & 42.6443 & 671.2563 & 641.7133 & 483 & 122.5994 & 2.11847 \\
\hline 2334 & 42.6613 & 615.4025 & 634.1595 & 483 & 122.5994 & 2.11766 \\
\hline 2335 & 42.6783 & 572.811 & 619.7573 & 482 & 122.5994 & 2.11686 \\
\hline 2336 & 42.6953 & 552.6737 & 600.0712 & 482 & 122.5994 & 2.11605 \\
\hline 2337 & 42.7123 & 517.3729 & 577.9695 & 482 & 122.5994 & 2.11525 \\
\hline 2338 & 42.7293 & 504.9302 & 555.9715 & 481 & 122.5994 & 2.11445 \\
\hline 2339 & 42.7463 & 512.1497 & 538.4066 & 481 & 122.5994 & 2.11365 \\
\hline 2340 & 42.7633 & 475.4503 & 524.3857 & 480 & 122.5994 & 2.11285 \\
\hline 2341 & 42.7803 & 471.7156 & 515.4753 & 480 & 122.5994 & 2.11205 \\
\hline 2342 & 42.7973 & 479.5561 & 509.6689 & 480 & 122.5994 & 2.11125 \\
\hline 2343 & 42.8143 & 468.1081 & 504.6384 & 479 & 122.5994 & 2.11045 \\
\hline 2344 & 42.8313 & 486.4613 & 501.2843 & 479 & 122.5994 & 2.10965 \\
\hline 2345 & 42.8483 & 457.8693 & 497.1479 & 478 & 122.5994 & 2.10885 \\
\hline 2346 & 42.8653 & 470.797 & 494.3447 & 478 & 122.5994 & 2.10805 \\
\hline 2347 & 42.8823 & 487.2623 & 491.0637 & 477 & 122.5994 & 2.10726 \\
\hline 2348 & 42.8993 & 445.4744 & 489.2997 & 477 & 122.5994 & 2.10646 \\
\hline 2349 & 42.9163 & 457.7384 & 487.9958 & 477 & 122.5994 & 2.10567 \\
\hline 2350 & 42.9333 & 483.5965 & 486.05 & 476 & 122.5994 & 2.10487 \\
\hline
\end{tabular}




\begin{tabular}{|c|c|c|c|c|c|c|}
\hline 2351 & 42.9503 & 489.6334 & 485.3652 & 476 & 122.5994 & 2.10408 \\
\hline 2352 & 42.9673 & 486.9041 & 483.8577 & 475 & 122.5994 & 2.10329 \\
\hline 2353 & 42.9843 & 474.3862 & 483.4609 & 475 & 122.5994 & 2.10249 \\
\hline 2354 & 43.0013 & 457.3784 & 482.1279 & 474 & 122.5994 & 2.1017 \\
\hline 2355 & 43.0183 & 463.3228 & 481.8337 & 474 & 122.5994 & 2.10091 \\
\hline 2356 & 43.0353 & 439.7368 & 480.5869 & 473 & 122.5994 & 2.10012 \\
\hline 2357 & 43.0523 & 459.5434 & 479.3597 & 472 & 122.5994 & 2.09933 \\
\hline 2358 & 43.0693 & 450.8578 & 479.1636 & 472 & 122.5994 & 2.09854 \\
\hline 2359 & 43.0863 & 440.9577 & 477.9782 & 471 & 122.5994 & 2.09775 \\
\hline 2360 & 43.1033 & 447.448 & 476.8108 & 470 & 122.5994 & 2.09696 \\
\hline 2361 & 43.1203 & 446.1041 & 476.5713 & 470 & 122.5994 & 2.09618 \\
\hline 2362 & 43.1373 & 451.9501 & 475.3103 & 469 & 122.5994 & 2.09539 \\
\hline 2363 & 43.1543 & 443.3255 & 474.1136 & 468 & 122.5994 & 2.0946 \\
\hline 2364 & 43.1713 & 473.5032 & 473.843 & 468 & 122.5994 & 2.09382 \\
\hline 2365 & 43.1883 & 468.3912 & 472.7636 & 467 & 122.5994 & 2.09303 \\
\hline 2366 & 43.2053 & 462.6966 & 471.7271 & 466 & 122.5994 & 2.09225 \\
\hline 2367 & 43.2223 & 454.4382 & 471.7232 & 466 & 122.5994 & 2.09147 \\
\hline 2368 & 43.2393 & 465.7076 & 470.6778 & 465 & 122.5994 & 2.09068 \\
\hline 2369 & 43.2563 & 470.6309 & 469.6137 & 464 & 122.5994 & 2.0899 \\
\hline 2370 & 43.2733 & 462.5535 & 469.5049 & 464 & 122.5994 & 2.08912 \\
\hline 2371 & 43.2903 & 470.79 & 468.2876 & 463 & 122.5994 & 2.08834 \\
\hline 2372 & 43.3073 & 428.3865 & 467.1036 & 462 & 122.5994 & 2.08756 \\
\hline 2373 & 43.3243 & 426.4981 & 466.9154 & 462 & 122.5994 & 2.08678 \\
\hline 2374 & 43.3413 & 483.8091 & 465.7446 & 461 & 122.5994 & 2.086 \\
\hline 2375 & 43.3583 & 479.2161 & 464.6048 & 460 & 122.5994 & 2.08522 \\
\hline 2376 & 43.3753 & 472.2628 & 464.4834 & 460 & 122.5994 & 2.08444 \\
\hline 2377 & 43.3923 & 444.6508 & 463.4572 & 459 & 122.5994 & 2.08367 \\
\hline 2378 & 43.4093 & 443.4015 & 462.5456 & 458 & 122.5994 & 2.08289 \\
\hline 2379 & 43.4263 & 455.9078 & 462.8046 & 458 & 122.5994 & 2.08211 \\
\hline 2380 & 43.4433 & 440.4957 & 462.2807 & 457 & 122.5994 & 2.08134 \\
\hline 2381 & 43.4603 & 427.2887 & 461.9399 & 456 & 122.5994 & 2.08056 \\
\hline 2382 & 43.4773 & 448.8001 & 461.6666 & 455 & 122.5994 & 2.07979 \\
\hline 2383 & 43.4943 & 434.8034 & 461.2829 & 454 & 122.5994 & 2.07902 \\
\hline 2384 & 43.5113 & 445.5929 & 461.1186 & 454 & 122.5994 & 2.07824 \\
\hline 2385 & 43.5283 & 464.2968 & 460.7035 & 453 & 122.5994 & 2.07747 \\
\hline 2386 & 43.5453 & 475.5453 & 460.9062 & 452 & 122.5994 & 2.0767 \\
\hline 2387 & 43.5623 & 450.8721 & 463.0079 & 452 & 122.5994 & 2.07593 \\
\hline 2388 & 43.5793 & 430.7981 & 464.9051 & 451 & 122.5994 & 2.07516 \\
\hline 2389 & 43.5963 & 454.2757 & 466.9766 & 450 & 122.5994 & 2.07439 \\
\hline 2390 & 43.6133 & 465.6814 & 467.6714 & 449 & 122.5994 & 2.07362 \\
\hline 2391 & 43.6303 & 458.2814 & 466.6276 & 448 & 122.5994 & 2.07285 \\
\hline 2392 & 43.6473 & 446.6092 & 465.0315 & 448 & 122.5994 & 2.07208 \\
\hline 2393 & 43.6643 & 463.8144 & 462.2473 & 447 & 122.5994 & 2.07131 \\
\hline 2394 & 43.6813 & 456.4243 & 460.2039 & 446 & 122.5994 & 2.07055 \\
\hline 2395 & 43.6983 & 465.4476 & 460.0398 & 446 & 122.5994 & 2.06978 \\
\hline 2396 & 43.7153 & 463.0132 & 459.405 & 445 & 122.5994 & 2.06902 \\
\hline 2397 & 43.7323 & 448.4622 & 458.5229 & 444 & 122.5994 & 2.06825 \\
\hline 2398 & 43.7493 & 450.6954 & 456.8396 & 443 & 122.5994 & 2.06749 \\
\hline 2399 & 43.7663 & 455.3228 & 454.5587 & 442 & 122.5994 & 2.06672 \\
\hline
\end{tabular}




\begin{tabular}{|c|c|c|c|c|c|c|}
\hline 2400 & 43.7833 & 459.662 & 453.1624 & 442 & 122.5994 & 2.06596 \\
\hline 2401 & 43.8003 & 428.6027 & 450.959 & 441 & 122.5994 & 2.0652 \\
\hline 2402 & 43.8173 & 442.4513 & 451.0291 & 442 & 122.5994 & 2.06444 \\
\hline 2403 & 43.8343 & 429.4033 & 450.3654 & 442 & 122.5994 & 2.06367 \\
\hline 2404 & 43.8513 & 436.558 & 450.9196 & 443 & 122.5994 & 2.06291 \\
\hline 2405 & 43.8683 & 483.0374 & 450.6161 & 443 & 122.5994 & 2.06215 \\
\hline 2406 & 43.8853 & 458.3587 & 451.4092 & 444 & 122.5994 & 2.06139 \\
\hline 2407 & 43.9023 & 438.8159 & 452.2799 & 445 & 122.5994 & 2.06064 \\
\hline 2408 & 43.9193 & 477.5157 & 452.2294 & 445 & 122.5994 & 2.05988 \\
\hline 2409 & 43.9363 & 433.0784 & 453.2685 & 446 & 122.5994 & 2.05912 \\
\hline 2410 & 43.9533 & 441.0915 & 453.4008 & 446 & 122.5994 & 2.05836 \\
\hline 2411 & 43.9703 & 467.1025 & 454.6551 & 447 & 122.5994 & 2.05761 \\
\hline 2412 & 43.9873 & 470.9528 & 456.0239 & 448 & 122.5994 & 2.05685 \\
\hline 2413 & 44.0043 & 451.599 & 456.4967 & 448 & 122.5994 & 2.0561 \\
\hline 2414 & 44.0213 & 441.7445 & 458.0457 & 449 & 122.5994 & 2.05534 \\
\hline 2415 & 44.0383 & 456.9054 & 458.6185 & 449 & 122.5994 & 2.05459 \\
\hline 2416 & 44.0553 & 462.0419 & 460.1409 & 450 & 122.5994 & 2.05383 \\
\hline 2417 & 44.0723 & 441.4147 & 461.5426 & 451 & 122.5994 & 2.05308 \\
\hline 2418 & 44.0893 & 470.9742 & 461.7986 & 451 & 122.5994 & 2.05233 \\
\hline 2419 & 44.1063 & 474.852 & 462.873 & 452 & 122.5994 & 2.05158 \\
\hline 2420 & 44.1233 & 461.7967 & 462.7577 & 452 & 122.5994 & 2.05083 \\
\hline 2421 & 44.1403 & 452.8695 & 463.6088 & 453 & 122.5994 & 2.05008 \\
\hline 2422 & 44.1573 & 445.2648 & 464.2849 & 454 & 122.5994 & 2.04933 \\
\hline 2423 & 44.1743 & 484.0911 & 463.9056 & 454 & 122.5994 & 2.04858 \\
\hline 2424 & 44.1913 & 451.6895 & 464.4758 & 455 & 122.5994 & 2.04783 \\
\hline 2425 & 44.2083 & 465.9517 & 465.0938 & 456 & 122.5994 & 2.04708 \\
\hline 2426 & 44.2253 & 458.4447 & 464.5666 & 456 & 122.5994 & 2.04633 \\
\hline 2427 & 44.2423 & 447.7512 & 463.9901 & 456 & 122.5994 & 2.04559 \\
\hline 2428 & 44.2593 & 472.4448 & 464.3767 & 457 & 122.5994 & 2.04484 \\
\hline 2429 & 44.2763 & 452.0636 & 463.7939 & 457 & 122.5994 & 2.04409 \\
\hline 2430 & 44.2933 & 437.0803 & 464.2332 & 458 & 122.5994 & 2.04335 \\
\hline 2431 & 44.3103 & 470.5258 & 463.7096 & 458 & 122.5994 & 2.0426 \\
\hline 2432 & 44.3273 & 435.4922 & 464.2865 & 459 & 122.5994 & 2.04186 \\
\hline 2433 & 44.3443 & 448.0858 & 463.8851 & 459 & 122.5994 & 2.04112 \\
\hline 2434 & 44.3613 & 469.0335 & 464.5668 & 460 & 122.5994 & 2.04037 \\
\hline 2435 & 44.3783 & 451.0753 & 464.3504 & 460 & 122.5994 & 2.03963 \\
\hline 2436 & 44.3953 & 448.6706 & 464.278 & 460 & 122.5994 & 2.03889 \\
\hline 2437 & 44.4123 & 460.675 & 465.3567 & 461 & 122.5994 & 2.03815 \\
\hline 2438 & 44.4293 & 480.7389 & 465.6342 & 461 & 122.5994 & 2.03741 \\
\hline 2439 & 44.4463 & 462.9914 & 467.0936 & 462 & 122.5994 & 2.03667 \\
\hline 2440 & 44.4633 & 443.6834 & 467.7131 & 462 & 122.5994 & 2.03593 \\
\hline 2441 & 44.4803 & 451.7108 & 469.2928 & 463 & 122.5994 & 2.03519 \\
\hline 2442 & 44.4973 & 444.5533 & 469.5717 & 463 & 122.5994 & 2.03445 \\
\hline 2443 & 44.5143 & 447.6939 & 470.4777 & 464 & 122.5994 & 2.03372 \\
\hline 2444 & 44.5313 & 468.509 & 473.0407 & 464 & 122.5994 & 2.03298 \\
\hline 2445 & 44.5483 & 451.542 & 473.9722 & 465 & 122.5994 & 2.03224 \\
\hline 2446 & 44.5653 & 433.3613 & 474.1101 & 465 & 122.5994 & 2.03151 \\
\hline 2447 & 44.5823 & 455.2115 & 474.4341 & 465 & 122.5994 & 2.03077 \\
\hline 2448 & 44.5993 & 490.9195 & 475.8187 & 466 & 122.5994 & 2.03004 \\
\hline
\end{tabular}




\begin{tabular}{|c|c|c|c|c|c|c|}
\hline 2449 & 44.6163 & 475.2783 & 476.0946 & 466 & 122.5994 & 2.0293 \\
\hline 2450 & 44.6333 & 453.3236 & 476.2033 & 466 & 122.5994 & 2.02857 \\
\hline 2451 & 44.6503 & 452.4347 & 478.6646 & 467 & 122.5994 & 2.02784 \\
\hline 2452 & 44.6673 & 469.6207 & 478.7467 & 467 & 122.5994 & 2.0271 \\
\hline 2453 & 44.6843 & 442.9097 & 478.8728 & 467 & 122.5994 & 2.02637 \\
\hline 2454 & 44.7013 & 429.4627 & 479.0474 & 467 & 122.5994 & 2.02564 \\
\hline 2455 & 44.7183 & 487.1329 & 479.2684 & 467 & 122.5994 & 2.02491 \\
\hline 2456 & 44.7353 & 487.2179 & 480.543 & 468 & 122.5994 & 2.02418 \\
\hline 2457 & 44.7523 & 454.785 & 480.8919 & 468 & 122.5994 & 2.02345 \\
\hline 2458 & 44.7693 & 479.3057 & 481.3825 & 468 & 122.5994 & 2.02272 \\
\hline 2459 & 44.7863 & 472.4351 & 481.9481 & 468 & 122.5994 & 2.02199 \\
\hline 2460 & 44.8033 & 462.9955 & 482.7292 & 468 & 122.5994 & 2.02127 \\
\hline 2461 & 44.8203 & 486.644 & 483.738 & 468 & 122.5994 & 2.02054 \\
\hline 2462 & 44.8373 & 463.4704 & 486.0293 & 469 & 122.5994 & 2.01981 \\
\hline 2463 & 44.8543 & 513.8124 & 487.6349 & 469 & 122.5994 & 2.01909 \\
\hline 2464 & 44.8713 & 471.1972 & 489.6008 & 469 & 122.5994 & 2.01836 \\
\hline 2465 & 44.8883 & 476.025 & 491.9327 & 469 & 122.5994 & 2.01764 \\
\hline 2466 & 44.9053 & 517.7867 & 494.6564 & 469 & 122.5994 & 2.01691 \\
\hline 2467 & 44.9223 & 469.5095 & 497.7092 & 469 & 122.5994 & 2.01619 \\
\hline 2468 & 44.9393 & 513.0721 & 501.9981 & 470 & 122.5994 & 2.01547 \\
\hline 2469 & 44.9563 & 491.1074 & 505.5854 & 470 & 122.5994 & 2.01474 \\
\hline 2470 & 44.9733 & 517.6256 & 508.9109 & 470 & 122.5994 & 2.01402 \\
\hline 2471 & 44.9903 & 526.9975 & 512.0249 & 470 & 122.5994 & 2.0133 \\
\hline 2472 & 45.0073 & 485.5526 & 514.8323 & 470 & 122.5994 & 2.01258 \\
\hline 2473 & 45.0243 & 474.9765 & 517.2632 & 470 & 122.5994 & 2.01186 \\
\hline 2474 & 45.0413 & 502.3109 & 519.1991 & 470 & 122.5994 & 2.01114 \\
\hline 2475 & 45.0583 & 534.0558 & 520.4828 & 470 & 122.5994 & 2.01042 \\
\hline 2476 & 45.0753 & 516.3556 & 520.8977 & 470 & 122.5994 & 2.0097 \\
\hline 2477 & 45.0923 & 511.3001 & 520.5165 & 470 & 122.5994 & 2.00898 \\
\hline 2478 & 45.1093 & 520.3221 & 519.7388 & 470 & 122.5994 & 2.00826 \\
\hline 2479 & 45.1263 & 500.9398 & 519.1929 & 470 & 122.5994 & 2.00755 \\
\hline 2480 & 45.1433 & 497.8625 & 518.7026 & 470 & 122.5994 & 2.00683 \\
\hline 2481 & 45.1603 & 540.2745 & 518.5973 & 470 & 122.5994 & 2.00611 \\
\hline 2482 & 45.1773 & 545.2044 & 518.9276 & 470 & 122.5994 & 2.0054 \\
\hline 2483 & 45.1943 & 537.3019 & 519.728 & 470 & 122.5994 & 2.00468 \\
\hline 2484 & 45.2113 & 525.4821 & 521.2293 & 470 & 122.5994 & 2.00397 \\
\hline 2485 & 45.2283 & 501.7119 & 523.8494 & 470 & 122.5994 & 2.00326 \\
\hline 2486 & 45.2453 & 518.9021 & 528.189 & 470 & 122.5994 & 2.00254 \\
\hline 2487 & 45.2623 & 526.9545 & 534.4942 & 470 & 122.5994 & 2.00183 \\
\hline 2488 & 45.2793 & 536.5303 & 542.1465 & 469 & 122.5994 & 2.00112 \\
\hline 2489 & 45.2963 & 549.5411 & 553.2726 & 469 & 122.5994 & 2.00041 \\
\hline 2490 & 45.3133 & 540.2619 & 566.8685 & 469 & 122.5994 & 1.9997 \\
\hline 2491 & 45.3303 & 554.5446 & 583.0933 & 469 & 122.5994 & 1.99899 \\
\hline 2492 & 45.3473 & 546.7845 & 602.5516 & 469 & 122.5994 & 1.99828 \\
\hline 2493 & 45.3643 & 559.7759 & 625.8699 & 469 & 122.5994 & 1.99757 \\
\hline 2494 & 45.3813 & 585.3521 & 653.4045 & 469 & 122.5994 & 1.99686 \\
\hline 2495 & 45.3983 & 600.1382 & 685.043 & 469 & 122.5994 & 1.99615 \\
\hline 2496 & 45.4153 & 615.3861 & 720.1875 & 469 & 122.5994 & 1.99544 \\
\hline 2497 & 45.4323 & 609.487 & 757.9531 & 469 & 122.5994 & 1.99473 \\
\hline
\end{tabular}




\begin{tabular}{|c|c|c|c|c|c|c|}
\hline 2498 & 45.4493 & 637.9878 & 797.0121 & 469 & 122.5994 & 1.99403 \\
\hline 2499 & 45.4663 & 657.8439 & 835.6311 & 469 & 122.5994 & 1.99332 \\
\hline 2500 & 45.4833 & 672.3554 & 871.4821 & 469 & 122.5994 & 1.99262 \\
\hline 2501 & 45.5003 & 696.8473 & 901.8458 & 469 & 122.5994 & 1.99191 \\
\hline 2502 & 45.5173 & 702.1826 & 924.1227 & 469 & 122.5994 & 1.99121 \\
\hline 2503 & 45.5343 & 702.3436 & 935.7391 & 468 & 122.5994 & 1.9905 \\
\hline 2504 & 45.5513 & 675.2938 & 938.651 & 468 & 122.5994 & 1.9898 \\
\hline 2505 & 45.5683 & 698.3079 & 933.1044 & 468 & 122.5994 & 1.9891 \\
\hline 2506 & 45.5853 & 707.1136 & 920.8432 & 468 & 122.5994 & 1.98839 \\
\hline 2507 & 45.6023 & 688.0674 & 903.3558 & 468 & 122.5994 & 1.98769 \\
\hline 2508 & 45.6193 & 662.1026 & 881.5424 & 468 & 122.5994 & 1.98699 \\
\hline 2509 & 45.6363 & 616.6673 & 855.8948 & 468 & 122.5994 & 1.98629 \\
\hline 2510 & 45.6533 & 639.7825 & 826.8922 & 468 & 122.5994 & 1.98559 \\
\hline 2511 & 45.6703 & 639.9414 & 795.4165 & 468 & 122.5994 & 1.98489 \\
\hline 2512 & 45.6873 & 625.0833 & 762.6479 & 468 & 122.5994 & 1.98419 \\
\hline 2513 & 45.7043 & 622.5466 & 729.8268 & 468 & 122.5994 & 1.98349 \\
\hline 2514 & 45.7213 & 576.0768 & 698.3547 & 468 & 122.5994 & 1.9828 \\
\hline 2515 & 45.7383 & 577.7242 & 669.4515 & 468 & 122.5994 & 1.9821 \\
\hline 2516 & 45.7553 & 588.8291 & 643.9779 & 468 & 122.5994 & 1.9814 \\
\hline 2517 & 45.7723 & 540.2029 & 622.5699 & 468 & 122.5994 & 1.9807 \\
\hline 2518 & 45.7893 & 550.7331 & 605.8711 & 468 & 122.5994 & 1.98001 \\
\hline 2519 & 45.8063 & 555.8091 & 593.6484 & 467 & 122.5994 & 1.97931 \\
\hline 2520 & 45.8233 & 612.0308 & 588.5573 & 467 & 122.5994 & 1.97862 \\
\hline 2521 & 45.8403 & 609.1397 & 589.9889 & 467 & 122.5994 & 1.97792 \\
\hline 2522 & 45.8573 & 648.7384 & 597.6379 & 467 & 122.5994 & 1.97723 \\
\hline 2523 & 45.8743 & 677.3104 & 609.1083 & 467 & 122.5994 & 1.97654 \\
\hline 2524 & 45.8913 & 666.6773 & 618.7169 & 466 & 122.5994 & 1.97585 \\
\hline 2525 & 45.9083 & 633.0445 & 621.9156 & 466 & 122.5994 & 1.97515 \\
\hline 2526 & 45.9253 & 619.1098 & 614.2108 & 466 & 122.5994 & 1.97446 \\
\hline 2527 & 45.9423 & 562.7247 & 598.5345 & 465 & 122.5994 & 1.97377 \\
\hline 2528 & 45.9593 & 550.5685 & 584.022 & 465 & 122.5994 & 1.97308 \\
\hline 2529 & 45.9763 & 598.2372 & 574.2789 & 465 & 122.5994 & 1.97239 \\
\hline 2530 & 45.9933 & 619.4646 & 569.9469 & 465 & 122.5994 & 1.9717 \\
\hline 2531 & 46.0103 & 578.0048 & 567.7384 & 464 & 122.5994 & 1.97101 \\
\hline 2532 & 46.0273 & 546.675 & 565.5671 & 464 & 122.5994 & 1.97032 \\
\hline 2533 & 46.0443 & 529.0705 & 559.4312 & 464 & 122.5994 & 1.96964 \\
\hline 2534 & 46.0613 & 535.9045 & 548.91 & 463 & 122.5994 & 1.96895 \\
\hline 2535 & 46.0783 & 521.3616 & 538.8069 & 463 & 122.5994 & 1.96826 \\
\hline 2536 & 46.0953 & 501.8269 & 530.1509 & 463 & 122.5994 & 1.96757 \\
\hline 2537 & 46.1123 & 489.1921 & 522.5627 & 462 & 122.5994 & 1.96689 \\
\hline 2538 & 46.1293 & 452.1084 & 517.795 & 462 & 122.5994 & 1.9662 \\
\hline 2539 & 46.1463 & 474.644 & 514.2226 & 462 & 122.5994 & 1.96552 \\
\hline 2540 & 46.1633 & 479.6528 & 511.173 & 462 & 122.5994 & 1.96483 \\
\hline 2541 & 46.1803 & 477.8751 & 507.1957 & 461 & 122.5994 & 1.96415 \\
\hline 2542 & 46.1973 & 460.7021 & 504.2664 & 461 & 122.5994 & 1.96347 \\
\hline 2543 & 46.2143 & 464.3956 & 501.5429 & 461 & 122.5994 & 1.96278 \\
\hline 2544 & 46.2313 & 461.5888 & 498.1145 & 460 & 122.5994 & 1.9621 \\
\hline 2545 & 46.2483 & 458.021 & 495.968 & 460 & 122.5994 & 1.96142 \\
\hline 2546 & 46.2653 & 464.4428 & 494.0143 & 460 & 122.5994 & 1.96074 \\
\hline
\end{tabular}




\begin{tabular}{|c|c|c|c|c|c|c|}
\hline 2547 & 46.2823 & 456.4722 & 491.1136 & 459 & 122.5994 & 1.96006 \\
\hline 2548 & 46.2993 & 467.5448 & 489.1724 & 459 & 122.5994 & 1.95938 \\
\hline 2549 & 46.3163 & 463.7305 & 486.2499 & 458 & 122.5994 & 1.9587 \\
\hline 2550 & 46.3333 & 447.9117 & 484.4659 & 458 & 122.5994 & 1.95802 \\
\hline 2551 & 46.3503 & 478.7783 & 481.9175 & 457 & 122.5994 & 1.95734 \\
\hline 2552 & 46.3673 & 460.9374 & 480.5789 & 457 & 122.5994 & 1.95666 \\
\hline 2553 & 46.3843 & 457.9161 & 478.439 & 456 & 122.5994 & 1.95599 \\
\hline 2554 & 46.4013 & 442.1267 & 477.4535 & 456 & 122.5994 & 1.95531 \\
\hline 2555 & 46.4183 & 453.1476 & 475.5798 & 455 & 122.5994 & 1.95463 \\
\hline 2556 & 46.4353 & 470.4001 & 474.8016 & 455 & 122.5994 & 1.95396 \\
\hline 2557 & 46.4523 & 479.6352 & 473.0921 & 454 & 122.5994 & 1.95328 \\
\hline 2558 & 46.4693 & 417.2001 & 472.5131 & 454 & 122.5994 & 1.95261 \\
\hline 2559 & 46.4863 & 478.3437 & 471.9854 & 454 & 122.5994 & 1.95193 \\
\hline 2560 & 46.5033 & 441.9723 & 470.5987 & 453 & 122.5994 & 1.95126 \\
\hline 2561 & 46.5203 & 395.0717 & 470.3889 & 453 & 122.5994 & 1.95058 \\
\hline 2562 & 46.5373 & 424.6887 & 466.7093 & 452 & 122.5994 & 1.94991 \\
\hline 2563 & 46.5543 & 397.68 & 467.0548 & 452 & 122.5994 & 1.94924 \\
\hline 2564 & 46.5713 & 436.1583 & 466.6707 & 451 & 122.5994 & 1.94857 \\
\hline 2565 & 46.5883 & 434.7059 & 467.5451 & 451 & 122.5994 & 1.9479 \\
\hline 2566 & 46.6053 & 450.1306 & 467.6003 & 450 & 122.5994 & 1.94722 \\
\hline 2567 & 46.6223 & 459.3121 & 468.6491 & 450 & 122.5994 & 1.94655 \\
\hline 2568 & 46.6393 & 423.9488 & 468.4352 & 449 & 122.5994 & 1.94588 \\
\hline 2569 & 46.6563 & 454.8407 & 467.5197 & 449 & 122.5994 & 1.94521 \\
\hline 2570 & 46.6733 & 466.1112 & 466.7394 & 448 & 122.5994 & 1.94455 \\
\hline 2571 & 46.6903 & 439.0024 & 466.8985 & 448 & 122.5994 & 1.94388 \\
\hline 2572 & 46.7073 & 422.5079 & 466.0365 & 447 & 122.5994 & 1.94321 \\
\hline 2573 & 46.7243 & 455.0881 & 465.1436 & 446 & 122.5994 & 1.94254 \\
\hline 2574 & 46.7413 & 458.0894 & 465.1331 & 446 & 122.5994 & 1.94188 \\
\hline 2575 & 46.7583 & 465.5257 & 463.8603 & 445 & 122.5994 & 1.94121 \\
\hline 2576 & 46.7753 & 426.7786 & 463.2444 & 445 & 122.5994 & 1.94054 \\
\hline 2577 & 46.7923 & 417.3696 & 461.3316 & 444 & 122.5994 & 1.93988 \\
\hline 2578 & 46.8093 & 417.2456 & 459.2798 & 443 & 122.5994 & 1.93921 \\
\hline 2579 & 46.8263 & 434.315 & 458.1711 & 443 & 122.5994 & 1.93855 \\
\hline 2580 & 46.8433 & 455.7216 & 456.0718 & 442 & 122.5994 & 1.93788 \\
\hline 2581 & 46.8603 & 451.2616 & 454.8114 & 442 & 122.5994 & 1.93722 \\
\hline 2582 & 46.8773 & 423.9034 & 452.8157 & 441 & 122.5994 & 1.93656 \\
\hline 2583 & 46.8943 & 427.5819 & 450.9147 & 440 & 122.5994 & 1.9359 \\
\hline 2584 & 46.9113 & 415.5482 & 450.0118 & 440 & 122.5994 & 1.93523 \\
\hline 2585 & 46.9283 & 422.0702 & 448.1542 & 439 & 122.5994 & 1.93457 \\
\hline 2586 & 46.9453 & 436.7681 & 446.3492 & 438 & 122.5994 & 1.93391 \\
\hline 2587 & 46.9623 & 426.2501 & 445.6075 & 438 & 122.5994 & 1.93325 \\
\hline 2588 & 46.9793 & 417.4996 & 443.8368 & 437 & 122.5994 & 1.93259 \\
\hline 2589 & 46.9963 & 433.1114 & 442.252 & 436 & 122.5994 & 1.93193 \\
\hline 2590 & 47.0133 & 447.0147 & 441.7508 & 436 & 122.5994 & 1.93127 \\
\hline 2591 & 47.0303 & 455.5918 & 440.3566 & 435 & 122.5994 & 1.93061 \\
\hline 2592 & 47.0473 & 445.3222 & 440.0234 & 435 & 122.5994 & 1.92996 \\
\hline 2593 & 47.0643 & 434.5191 & 438.7803 & 434 & 122.5994 & 1.9293 \\
\hline 2594 & 47.0813 & 446.0003 & 437.6485 & 433 & 122.5994 & 1.92864 \\
\hline 2595 & 47.0983 & 445.9569 & 437.6718 & 433 & 122.5994 & 1.92799 \\
\hline
\end{tabular}




\begin{tabular}{|c|c|c|c|c|c|c|}
\hline 2596 & 47.1153 & 455.8092 & 436.9158 & 432 & 122.5994 & 1.92733 \\
\hline 2597 & 47.1323 & 447.1031 & 437.3003 & 432 & 122.5994 & 1.92667 \\
\hline 2598 & 47.1493 & 416.0968 & 437.1646 & 431 & 122.5994 & 1.92602 \\
\hline 2599 & 47.1663 & 468.9408 & 437.3 & 430 & 122.5994 & 1.92536 \\
\hline 2600 & 47.1833 & 447.5427 & 438.5734 & 430 & 122.5994 & 1.92471 \\
\hline 2601 & 47.2003 & 437.2526 & 438.5076 & 429 & 122.5994 & 1.92406 \\
\hline 2602 & 47.2173 & 457.2541 & 437.7903 & 428 & 122.5994 & 1.9234 \\
\hline 2603 & 47.2343 & 445.3536 & 437.5628 & 428 & 122.5994 & 1.92275 \\
\hline 2604 & 47.2513 & 419.8828 & 436.264 & 427 & 122.5994 & 1.9221 \\
\hline 2605 & 47.2683 & 374.1354 & 435.4129 & 426 & 122.5994 & 1.92145 \\
\hline 2606 & 47.2853 & 429.9199 & 436.039 & 426 & 122.5994 & 1.9208 \\
\hline 2607 & 47.3023 & 456.4073 & 436.054 & 425 & 122.5994 & 1.92014 \\
\hline 2608 & 47.3193 & 435.3284 & 437.1693 & 425 & 122.5994 & 1.91949 \\
\hline 2609 & 47.3363 & 426.0032 & 437.0119 & 424 & 122.5994 & 1.91885 \\
\hline 2610 & 47.3533 & 465.184 & 436.4019 & 423 & 122.5994 & 1.9182 \\
\hline 2611 & 47.3703 & 426.5072 & 436.45 & 423 & 122.5994 & 1.91755 \\
\hline 2612 & 47.3873 & 413.2291 & 435.2151 & 422 & 122.5994 & 1.9169 \\
\hline 2613 & 47.4043 & 407.4354 & 434.8105 & 422 & 122.5994 & 1.91625 \\
\hline 2614 & 47.4213 & 439.5715 & 433.2731 & 421 & 122.5994 & 1.9156 \\
\hline 2615 & 47.4383 & 453.6706 & 431.7298 & 420 & 122.5994 & 1.91496 \\
\hline 2616 & 47.4553 & 445.7179 & 431.1801 & 420 & 122.5994 & 1.91431 \\
\hline 2617 & 47.4723 & 383.6694 & 429.6292 & 419 & 122.5994 & 1.91366 \\
\hline 2618 & 47.4893 & 400.1098 & 429.081 & 419 & 122.5994 & 1.91302 \\
\hline 2619 & 47.5063 & 455.2032 & 428.4777 & 419 & 122.5994 & 1.91237 \\
\hline 2620 & 47.5233 & 441.0588 & 426.8387 & 418 & 122.5994 & 1.91173 \\
\hline 2621 & 47.5403 & 442.5263 & 426.1966 & 418 & 122.5994 & 1.91109 \\
\hline 2622 & 47.5573 & 455.3936 & 425.607 & 418 & 122.5994 & 1.91044 \\
\hline 2623 & 47.5743 & 465.4703 & 425.1269 & 418 & 122.5994 & 1.9098 \\
\hline 2624 & 47.5913 & 437.9614 & 424.7983 & 418 & 122.5994 & 1.90916 \\
\hline 2625 & 47.6083 & 413.9175 & 423.6441 & 417 & 122.5994 & 1.90851 \\
\hline 2626 & 47.6253 & 402.6421 & 423.6936 & 417 & 122.5994 & 1.90787 \\
\hline 2627 & 47.6423 & 390.1239 & 423.8831 & 417 & 122.5994 & 1.90723 \\
\hline 2628 & 47.6593 & 400.374 & 424.2095 & 417 & 122.5994 & 1.90659 \\
\hline 2629 & 47.6763 & 408.0944 & 423.6296 & 416 & 122.5994 & 1.90595 \\
\hline 2630 & 47.6933 & 441.1405 & 423.9913 & 416 & 122.5994 & 1.90531 \\
\hline 2631 & 47.7103 & 453.9675 & 424.413 & 416 & 122.5994 & 1.90467 \\
\hline 2632 & 47.7273 & 440.1507 & 424.7236 & 416 & 122.5994 & 1.90403 \\
\hline 2633 & 47.7443 & 431.52 & 424.8692 & 416 & 122.5994 & 1.9034 \\
\hline 2634 & 47.7613 & 425.8526 & 423.8494 & 415 & 122.5994 & 1.90276 \\
\hline 2635 & 47.7783 & 441.2196 & 423.6755 & 415 & 122.5994 & 1.90212 \\
\hline 2636 & 47.7953 & 417.9031 & 423.4108 & 415 & 122.5994 & 1.90148 \\
\hline 2637 & 47.8123 & 415.6141 & 423.0533 & 415 & 122.5994 & 1.90085 \\
\hline 2638 & 47.8293 & 408.2189 & 422.7191 & 415 & 122.5994 & 1.90021 \\
\hline 2639 & 47.8463 & 404.0639 & 421.3838 & 414 & 122.5994 & 1.89958 \\
\hline 2640 & 47.8633 & 407.1204 & 420.9945 & 414 & 122.5994 & 1.89894 \\
\hline 2641 & 47.8803 & 417.8998 & 420.575 & 414 & 122.5994 & 1.89831 \\
\hline 2642 & 47.8973 & 427.0035 & 420.1462 & 414 & 122.5994 & 1.89767 \\
\hline 2643 & 47.9143 & 407.7411 & 419.7452 & 414 & 122.5994 & 1.89704 \\
\hline 2644 & 47.9313 & 436.1547 & 419.4125 & 414 & 122.5994 & 1.89641 \\
\hline
\end{tabular}




\begin{tabular}{|c|c|c|c|c|c|c|}
\hline 2645 & 47.9483 & 426.1651 & 419.1744 & 414 & 122.5994 & 1.89577 \\
\hline 2646 & 47.9653 & 466.788 & 419.1315 & 414 & 122.5994 & 1.89514 \\
\hline 2647 & 47.9823 & 435.1941 & 419.2589 & 414 & 122.5994 & 1.89451 \\
\hline 2648 & 47.9993 & 409.2368 & 420.6769 & 415 & 122.5994 & 1.89388 \\
\hline 2649 & 48.0163 & 423.9148 & 421.5208 & 415 & 122.5994 & 1.89325 \\
\hline 2650 & 48.0333 & 420.2235 & 422.9777 & 415 & 122.5994 & 1.89262 \\
\hline 2651 & 48.0503 & 429.1376 & 425.2591 & 415 & 122.5994 & 1.89199 \\
\hline 2652 & 48.0673 & 464.7819 & 428.5124 & 415 & 122.5994 & 1.89136 \\
\hline 2653 & 48.0843 & 423.3068 & 432.6567 & 415 & 122.5994 & 1.89073 \\
\hline 2654 & 48.1013 & 470.4873 & 437.4146 & 415 & 122.5994 & 1.8901 \\
\hline 2655 & 48.1183 & 466.173 & 442.2506 & 415 & 122.5994 & 1.88947 \\
\hline 2656 & 48.1353 & 461.6666 & 446.1333 & 415 & 122.5994 & 1.88884 \\
\hline 2657 & 48.1523 & 457.3921 & 448.1194 & 415 & 122.5994 & 1.88822 \\
\hline 2658 & 48.1693 & 451.5054 & 448.7084 & 415 & 122.5994 & 1.88759 \\
\hline 2659 & 48.1863 & 448.409 & 448.6483 & 415 & 122.5994 & 1.88696 \\
\hline 2660 & 48.2033 & 473.1743 & 447.682 & 415 & 122.5994 & 1.88634 \\
\hline 2661 & 48.2203 & 453.3895 & 446.7355 & 416 & 122.5994 & 1.88571 \\
\hline 2662 & 48.2373 & 466.6222 & 444.663 & 416 & 122.5994 & 1.88509 \\
\hline 2663 & 48.2543 & 458.2959 & 443.0261 & 416 & 122.5994 & 1.88446 \\
\hline 2664 & 48.2713 & 431.9345 & 441.4995 & 416 & 122.5994 & 1.88384 \\
\hline 2665 & 48.2883 & 430.8888 & 439.872 & 416 & 122.5994 & 1.88322 \\
\hline 2666 & 48.3053 & 443.749 & 438.3543 & 416 & 122.5994 & 1.88259 \\
\hline 2667 & 48.3223 & 438.0514 & 436.6921 & 416 & 122.5994 & 1.88197 \\
\hline 2668 & 48.3393 & 455.665 & 434.5368 & 416 & 122.5994 & 1.88135 \\
\hline 2669 & 48.3563 & 443.5622 & 432.0243 & 416 & 122.5994 & 1.88073 \\
\hline 2670 & 48.3733 & 459.3148 & 429.7627 & 416 & 122.5994 & 1.8801 \\
\hline 2671 & 48.3903 & 480.1351 & 427.9863 & 416 & 122.5994 & 1.87948 \\
\hline 2672 & 48.4073 & 449.3502 & 426.7427 & 416 & 122.5994 & 1.87886 \\
\hline 2673 & 48.4243 & 434.5537 & 426.0103 & 416 & 122.5994 & 1.87824 \\
\hline 2674 & 48.4413 & 438.7515 & 426.5333 & 417 & 122.5994 & 1.87762 \\
\hline 2675 & 48.4583 & 430.667 & 426.2276 & 417 & 122.5994 & 1.87701 \\
\hline 2676 & 48.4753 & 437.3468 & 425.982 & 417 & 122.5994 & 1.87639 \\
\hline 2677 & 48.4923 & 426.6116 & 425.708 & 417 & 122.5994 & 1.87577 \\
\hline 2678 & 48.5093 & 438.7031 & 425.4561 & 417 & 122.5994 & 1.87515 \\
\hline 2679 & 48.5263 & 443.1706 & 425.2202 & 417 & 122.5994 & 1.87453 \\
\hline 2680 & 48.5433 & 444.8769 & 425.0902 & 417 & 122.5994 & 1.87392 \\
\hline 2681 & 48.5603 & 422.0235 & 424.9528 & 417 & 122.5994 & 1.8733 \\
\hline 2682 & 48.5773 & 460.0411 & 424.9081 & 417 & 122.5994 & 1.87269 \\
\hline 2683 & 48.5943 & 462.6429 & 424.7936 & 417 & 122.5994 & 1.87207 \\
\hline 2684 & 48.6113 & 444.406 & 424.7151 & 417 & 122.5994 & 1.87145 \\
\hline 2685 & 48.6283 & 453.6708 & 424.6105 & 417 & 122.5994 & 1.87084 \\
\hline 2686 & 48.6453 & 416.4126 & 425.4819 & 418 & 122.5994 & 1.87023 \\
\hline 2687 & 48.6623 & 433.2918 & 425.3469 & 418 & 122.5994 & 1.86961 \\
\hline 2688 & 48.6793 & 424.1618 & 425.229 & 418 & 122.5994 & 1.869 \\
\hline 2689 & 48.6963 & 448.1565 & 425.1493 & 418 & 122.5994 & 1.86839 \\
\hline 2690 & 48.7133 & 400.893 & 425.1163 & 418 & 122.5994 & 1.86777 \\
\hline 2691 & 48.7303 & 420.4052 & 425.148 & 418 & 122.5994 & 1.86716 \\
\hline 2692 & 48.7473 & 398.798 & 425.2261 & 418 & 122.5994 & 1.86655 \\
\hline 2693 & 48.7643 & 414.6385 & 424.3694 & 417 & 122.5994 & 1.86594 \\
\hline
\end{tabular}




\begin{tabular}{|c|c|c|c|c|c|c|}
\hline 2694 & 48.7813 & 416.1201 & 424.5606 & 417 & 122.5994 & 1.86533 \\
\hline 2695 & 48.7983 & 444.247 & 424.7944 & 417 & 122.5994 & 1.86472 \\
\hline 2696 & 48.8153 & 412.499 & 425.0639 & 417 & 122.5994 & 1.86411 \\
\hline 2697 & 48.8323 & 445.236 & 425.3615 & 417 & 122.5994 & 1.8635 \\
\hline 2698 & 48.8493 & 440.4696 & 425.6788 & 417 & 122.5994 & 1.86289 \\
\hline 2699 & 48.8663 & 425.6953 & 425.9588 & 417 & 122.5994 & 1.86228 \\
\hline 2700 & 48.8833 & 449.0906 & 426.2864 & 417 & 122.5994 & 1.86168 \\
\hline 2701 & 48.9003 & 444.4793 & 426.5959 & 417 & 122.5994 & 1.86107 \\
\hline 2702 & 48.9173 & 429.7877 & 426.8789 & 417 & 122.5994 & 1.86046 \\
\hline 2703 & 48.9343 & 389.2646 & 427.1192 & 417 & 122.5994 & 1.85985 \\
\hline 2704 & 48.9513 & 468.9028 & 427.288 & 417 & 122.5994 & 1.85925 \\
\hline 2705 & 48.9683 & 459.4638 & 427.3644 & 417 & 122.5994 & 1.85864 \\
\hline 2706 & 48.9853 & 458.1141 & 427.3573 & 417 & 122.5994 & 1.85804 \\
\hline 2707 & 49.0023 & 430.8609 & 426.3049 & 416 & 122.5994 & 1.85743 \\
\hline 2708 & 49.0193 & 462.2459 & 426.2551 & 416 & 122.5994 & 1.85683 \\
\hline 2709 & 49.0363 & 476.8674 & 426.2506 & 416 & 122.5994 & 1.85622 \\
\hline 2710 & 49.0533 & 437.9907 & 426.4024 & 416 & 122.5994 & 1.85562 \\
\hline 2711 & 49.0703 & 422.8536 & 426.5435 & 416 & 122.5994 & 1.85502 \\
\hline 2712 & 49.0873 & 420.3559 & 426.7268 & 416 & 122.5994 & 1.85441 \\
\hline 2713 & 49.1043 & 414.1486 & 426.907 & 416 & 122.5994 & 1.85381 \\
\hline 2714 & 49.1213 & 421.9005 & 427.0299 & 416 & 122.5994 & 1.85321 \\
\hline 2715 & 49.1383 & 408.7715 & 427.0419 & 416 & 122.5994 & 1.85261 \\
\hline 2716 & 49.1553 & 417.9199 & 426.9077 & 416 & 122.5994 & 1.85201 \\
\hline 2717 & 49.1723 & 429.3014 & 427.6452 & 417 & 122.5994 & 1.85141 \\
\hline 2718 & 49.1893 & 429.6372 & 427.4155 & 417 & 122.5994 & 1.85081 \\
\hline 2719 & 49.2063 & 428.0737 & 427.0789 & 417 & 122.5994 & 1.85021 \\
\hline 2720 & 49.2233 & 434.7678 & 426.9004 & 417 & 122.5994 & 1.84961 \\
\hline 2721 & 49.2403 & 435.0078 & 426.8609 & 417 & 122.5994 & 1.84901 \\
\hline 2722 & 49.2573 & 438.6712 & 427.9827 & 418 & 122.5994 & 1.84841 \\
\hline 2723 & 49.2743 & 420.5451 & 428.2988 & 418 & 122.5994 & 1.84781 \\
\hline 2724 & 49.2913 & 400.9178 & 428.9016 & 418 & 122.5994 & 1.84722 \\
\hline 2725 & 49.3083 & 423.5262 & 429.9954 & 418 & 122.5994 & 1.84662 \\
\hline 2726 & 49.3253 & 425.2624 & 431.875 & 418 & 122.5994 & 1.84602 \\
\hline 2727 & 49.3423 & 410.8155 & 434.9493 & 418 & 122.5994 & 1.84543 \\
\hline 2728 & 49.3593 & 424.6975 & 439.4017 & 418 & 122.5994 & 1.84483 \\
\hline 2729 & 49.3763 & 459.2337 & 446.015 & 419 & 122.5994 & 1.84424 \\
\hline 2730 & 49.3933 & 446.4835 & 451.8374 & 419 & 122.5994 & 1.84364 \\
\hline 2731 & 49.4103 & 406.6 & 455.8566 & 419 & 122.5994 & 1.84305 \\
\hline 2732 & 49.4273 & 463.0289 & 456.1712 & 419 & 122.5994 & 1.84245 \\
\hline 2733 & 49.4443 & 456.9109 & 453.2848 & 419 & 122.5994 & 1.84186 \\
\hline 2734 & 49.4613 & 429.2349 & 450.5454 & 420 & 122.5994 & 1.84126 \\
\hline 2735 & 49.4783 & 404.306 & 447.7959 & 420 & 122.5994 & 1.84067 \\
\hline 2736 & 49.4953 & 431.1388 & 446.7939 & 420 & 122.5994 & 1.84008 \\
\hline 2737 & 49.5123 & 440.5078 & 447.3593 & 420 & 122.5994 & 1.83949 \\
\hline 2738 & 49.5293 & 397.3811 & 448.5601 & 420 & 122.5994 & 1.8389 \\
\hline 2739 & 49.5463 & 417.7148 & 448.8603 & 420 & 122.5994 & 1.8383 \\
\hline 2740 & 49.5633 & 427.9949 & 447.2196 & 420 & 122.5994 & 1.83771 \\
\hline 2741 & 49.5803 & 436.3906 & 446.168 & 420 & 122.5994 & 1.83712 \\
\hline 2742 & 49.5973 & 408.7834 & 442.8148 & 420 & 122.5994 & 1.83653 \\
\hline
\end{tabular}




\begin{tabular}{|c|c|c|c|c|c|c|}
\hline 2743 & 49.6143 & 403.9805 & 439.9116 & 420 & 122.5994 & 1.83594 \\
\hline 2744 & 49.6313 & 454.0594 & 437.7885 & 420 & 122.5994 & 1.83536 \\
\hline 2745 & 49.6483 & 473.805 & 436.4533 & 420 & 122.5994 & 1.83477 \\
\hline 2746 & 49.6653 & 442.1142 & 435.7914 & 420 & 122.5994 & 1.83418 \\
\hline 2747 & 49.6823 & 456.4974 & 435.5227 & 420 & 122.5994 & 1.83359 \\
\hline 2748 & 49.6993 & 439.3066 & 435.3322 & 420 & 122.5994 & 1.833 \\
\hline 2749 & 49.7163 & 419.7232 & 434.8906 & 420 & 122.5994 & 1.83242 \\
\hline 2750 & 49.7333 & 391.7026 & 434.0729 & 420 & 122.5994 & 1.83183 \\
\hline 2751 & 49.7503 & 417.6739 & 433.1318 & 420 & 122.5994 & 1.83124 \\
\hline 2752 & 49.7673 & 442.7638 & 432.4045 & 420 & 122.5994 & 1.83066 \\
\hline 2753 & 49.7843 & 438.0873 & 432.1378 & 420 & 122.5994 & 1.83007 \\
\hline 2754 & 49.8013 & 460.9453 & 432.4464 & 420 & 122.5994 & 1.82949 \\
\hline 2755 & 49.8183 & 466.5508 & 433.3992 & 420 & 122.5994 & 1.8289 \\
\hline 2756 & 49.8353 & 452.4677 & 435.0128 & 420 & 122.5994 & 1.82832 \\
\hline 2757 & 49.8523 & 440.6758 & 438.8066 & 420 & 122.5994 & 1.82774 \\
\hline 2758 & 49.8693 & 431.0208 & 441.7334 & 420 & 122.5994 & 1.82715 \\
\hline 2759 & 49.8863 & 452.1937 & 445.1106 & 420 & 122.5994 & 1.82657 \\
\hline 2760 & 49.9033 & 443.3933 & 448.344 & 420 & 122.5994 & 1.82599 \\
\hline 2761 & 49.9203 & 434.2036 & 450.4912 & 420 & 122.5994 & 1.82541 \\
\hline 2762 & 49.9373 & 428.4728 & 451.291 & 420 & 122.5994 & 1.82482 \\
\hline 2763 & 49.9543 & 441.4051 & 451.6003 & 420 & 122.5994 & 1.82424 \\
\hline 2764 & 49.9713 & 473.2395 & 452.6795 & 420 & 122.5994 & 1.82366 \\
\hline 2765 & 49.9883 & 470.9528 & 455.7062 & 420 & 122.5994 & 1.82308 \\
\hline 2766 & 50.0053 & 479.4729 & 461.2035 & 420 & 122.5994 & 1.8225 \\
\hline 2767 & 50.0223 & 468.4399 & 468.352 & 419 & 122.5994 & 1.82192 \\
\hline 2768 & 50.0393 & 456.2572 & 478.2444 & 419 & 122.5994 & 1.82134 \\
\hline 2769 & 50.0563 & 474.3195 & 487.9714 & 419 & 122.5994 & 1.82076 \\
\hline 2770 & 50.0733 & 499.5481 & 495.261 & 419 & 122.5994 & 1.82019 \\
\hline 2771 & 50.0903 & 513.4291 & 498.8035 & 419 & 122.5994 & 1.81961 \\
\hline 2772 & 50.1073 & 553.1008 & 499.9579 & 419 & 122.5994 & 1.81903 \\
\hline 2773 & 50.1243 & 582.1442 & 502.003 & 418 & 122.5994 & 1.81845 \\
\hline 2774 & 50.1413 & 635.4746 & 512.1864 & 418 & 122.5994 & 1.81788 \\
\hline 2775 & 50.1583 & 722.3672 & 534.849 & 418 & 122.5994 & 1.8173 \\
\hline 2776 & 50.1753 & 794.1977 & 575.4665 & 418 & 122.5994 & 1.81672 \\
\hline 2777 & 50.1923 & 925.3612 & 638.4412 & 418 & 122.5994 & 1.81615 \\
\hline 2778 & 50.2093 & 1073.471 & 724.6853 & 418 & 122.5994 & 1.81557 \\
\hline 2779 & 50.2263 & 1109.038 & 828.3939 & 418 & 122.5994 & 1.815 \\
\hline 2780 & 50.2433 & 996.328 & 931.94 & 417 & 122.5994 & 1.81442 \\
\hline 2781 & 50.2603 & 790.678 & 1005.214 & 417 & 122.5994 & 1.81385 \\
\hline 2782 & 50.2773 & 701.4303 & 1012.529 & 417 & 122.5994 & 1.81328 \\
\hline 2783 & 50.2943 & 673.704 & 954.7682 & 417 & 122.5994 & 1.8127 \\
\hline 2784 & 50.3113 & 670.8994 & 870.5742 & 417 & 122.5994 & 1.81213 \\
\hline 2785 & 50.3283 & 732.9617 & 797.3574 & 417 & 122.5994 & 1.81156 \\
\hline 2786 & 50.3453 & 765.2733 & 752.8218 & 416 & 122.5994 & 1.81099 \\
\hline 2787 & 50.3623 & 767.2164 & 741.1526 & 416 & 122.5994 & 1.81042 \\
\hline 2788 & 50.3793 & 719.8449 & 749.7195 & 416 & 122.5994 & 1.80985 \\
\hline 2789 & 50.3963 & 611.5452 & 755.361 & 416 & 122.5994 & 1.80927 \\
\hline 2790 & 50.4133 & 537.984 & 735.3755 & 416 & 122.5994 & 1.8087 \\
\hline 2791 & 50.4303 & 500.3802 & 687.8667 & 416 & 122.5994 & 1.80813 \\
\hline
\end{tabular}




\begin{tabular}{|c|c|c|c|c|c|c|}
\hline 2792 & 50.4473 & 459.3765 & 629.1421 & 416 & 122.5994 & 1.80756 \\
\hline 2793 & 50.4643 & 479.875 & 572.921 & 415 & 122.5994 & 1.807 \\
\hline 2794 & 50.4813 & 434.5553 & 528.402 & 415 & 122.5994 & 1.80643 \\
\hline 2795 & 50.4983 & 481.6208 & 496.226 & 415 & 122.5994 & 1.80586 \\
\hline 2796 & 50.5153 & 401.0256 & 474.9437 & 415 & 122.5994 & 1.80529 \\
\hline 2797 & 50.5323 & 433.3633 & 461.7665 & 415 & 122.5994 & 1.80472 \\
\hline 2798 & 50.5493 & 429.3819 & 453.9264 & 415 & 122.5994 & 1.80416 \\
\hline 2799 & 50.5663 & 409.3826 & 448.2506 & 414 & 122.5994 & 1.80359 \\
\hline 2800 & 50.5833 & 461.3502 & 445.396 & 414 & 122.5994 & 1.80302 \\
\hline 2801 & 50.6003 & 460.0276 & 443.4478 & 414 & 122.5994 & 1.80246 \\
\hline 2802 & 50.6173 & 458.6738 & 441.9762 & 414 & 122.5994 & 1.80189 \\
\hline 2803 & 50.6343 & 431.989 & 441.0791 & 414 & 122.5994 & 1.80133 \\
\hline 2804 & 50.6513 & 416.5179 & 441.0804 & 414 & 122.5994 & 1.80076 \\
\hline 2805 & 50.6683 & 437.6019 & 442.2767 & 414 & 122.5994 & 1.8002 \\
\hline 2806 & 50.6853 & 477.3672 & 443.7779 & 413 & 122.5994 & 1.79963 \\
\hline 2807 & 50.7023 & 450.637 & 447.3883 & 413 & 122.5994 & 1.79907 \\
\hline 2808 & 50.7193 & 456.3895 & 450.9777 & 413 & 122.5994 & 1.79851 \\
\hline 2809 & 50.7363 & 453.5469 & 453.0452 & 413 & 122.5994 & 1.79794 \\
\hline 2810 & 50.7533 & 457.7164 & 452.0885 & 413 & 122.5994 & 1.79738 \\
\hline 2811 & 50.7703 & 431.3993 & 447.5809 & 412 & 122.5994 & 1.79682 \\
\hline 2812 & 50.7873 & 438.4933 & 443.1696 & 412 & 122.5994 & 1.79626 \\
\hline 2813 & 50.8043 & 432.7706 & 439.3899 & 412 & 122.5994 & 1.7957 \\
\hline 2814 & 50.8213 & 423.2091 & 435.932 & 411 & 122.5994 & 1.79514 \\
\hline 2815 & 50.8383 & 425.477 & 434.8482 & 411 & 122.5994 & 1.79458 \\
\hline 2816 & 50.8553 & 416.2155 & 434.609 & 411 & 122.5994 & 1.79402 \\
\hline 2817 & 50.8723 & 433.2553 & 433.2699 & 410 & 122.5994 & 1.79346 \\
\hline 2818 & 50.8893 & 432.4222 & 431.8135 & 410 & 122.5994 & 1.7929 \\
\hline 2819 & 50.9063 & 411.5366 & 429.3299 & 410 & 122.5994 & 1.79234 \\
\hline 2820 & 50.9233 & 428.1755 & 425.4535 & 409 & 122.5994 & 1.79178 \\
\hline 2821 & 50.9403 & 432.6021 & 422.7682 & 409 & 122.5994 & 1.79122 \\
\hline 2822 & 50.9573 & 400.2734 & 420.5523 & 409 & 122.5994 & 1.79066 \\
\hline 2823 & 50.9743 & 405.3048 & 417.8703 & 408 & 122.5994 & 1.79011 \\
\hline 2824 & 50.9913 & 397.2982 & 416.6649 & 408 & 122.5994 & 1.78955 \\
\hline 2825 & 51.0083 & 401.1224 & 415.8272 & 408 & 122.5994 & 1.78899 \\
\hline 2826 & 51.0253 & 388.5103 & 414.2495 & 407 & 122.5994 & 1.78844 \\
\hline 2827 & 51.0423 & 403.3459 & 413.8532 & 407 & 122.5994 & 1.78788 \\
\hline 2828 & 51.0593 & 408.0944 & 413.4994 & 407 & 122.5994 & 1.78733 \\
\hline 2829 & 51.0763 & 432.3991 & 412.3321 & 406 & 122.5994 & 1.78677 \\
\hline 2830 & 51.0933 & 397.8422 & 412.2183 & 406 & 122.5994 & 1.78622 \\
\hline 2831 & 51.1103 & 402.7589 & 412.1009 & 406 & 122.5994 & 1.78566 \\
\hline 2832 & 51.1273 & 411.3445 & 410.9336 & 405 & 122.5994 & 1.78511 \\
\hline 2833 & 51.1443 & 408.3615 & 410.7889 & 405 & 122.5994 & 1.78456 \\
\hline 2834 & 51.1613 & 408.0009 & 410.6016 & 405 & 122.5994 & 1.784 \\
\hline 2835 & 51.1783 & 400.1456 & 409.4675 & 404 & 122.5994 & 1.78345 \\
\hline 2836 & 51.1953 & 376.4447 & 407.4831 & 404 & 122.5994 & 1.7829 \\
\hline 2837 & 51.2123 & 456.7679 & 407.5633 & 404 & 122.5994 & 1.78235 \\
\hline 2838 & 51.2293 & 396.8112 & 407.6971 & 404 & 122.5994 & 1.78179 \\
\hline 2839 & 51.2463 & 417.5226 & 407.8598 & 404 & 122.5994 & 1.78124 \\
\hline 2840 & 51.2633 & 434.3308 & 407.0318 & 403 & 122.5994 & 1.78069 \\
\hline
\end{tabular}




\begin{tabular}{|c|c|c|c|c|c|c|}
\hline 2841 & 51.2803 & 410.1714 & 407.2367 & 403 & 122.5994 & 1.78014 \\
\hline 2842 & 51.2973 & 426.9715 & 407.515 & 403 & 122.5994 & 1.77959 \\
\hline 2843 & 51.3143 & 410.1816 & 406.9743 & 402 & 122.5994 & 1.77904 \\
\hline 2844 & 51.3313 & 377.7429 & 407.797 & 402 & 122.5994 & 1.77849 \\
\hline 2845 & 51.3483 & 388.7148 & 409.2859 & 402 & 122.5994 & 1.77794 \\
\hline 2846 & 51.3653 & 413.357 & 411.8524 & 402 & 122.5994 & 1.7774 \\
\hline 2847 & 51.3823 & 432.5985 & 415.8817 & 402 & 122.5994 & 1.77685 \\
\hline 2848 & 51.3993 & 429.4171 & 420.5253 & 401 & 122.5994 & 1.7763 \\
\hline 2849 & 51.4163 & 427.2553 & 427.2518 & 401 & 122.5994 & 1.77575 \\
\hline 2850 & 51.4333 & 438.9157 & 433.4175 & 401 & 122.5994 & 1.77521 \\
\hline 2851 & 51.4503 & 416.5851 & 435.2337 & 400 & 122.5994 & 1.77466 \\
\hline 2852 & 51.4673 & 404.1074 & 433.0753 & 400 & 122.5994 & 1.77411 \\
\hline 2853 & 51.4843 & 416.5208 & 427.8215 & 400 & 122.5994 & 1.77357 \\
\hline 2854 & 51.5013 & 405.8896 & 422.5139 & 400 & 122.5994 & 1.77302 \\
\hline 2855 & 51.5183 & 389.2303 & 419.0101 & 400 & 122.5994 & 1.77248 \\
\hline 2856 & 51.5353 & 404.6448 & 416.8134 & 399 & 122.5994 & 1.77193 \\
\hline 2857 & 51.5523 & 405.4078 & 417.8217 & 399 & 122.5994 & 1.77139 \\
\hline 2858 & 51.5693 & 397.1127 & 419.6204 & 399 & 122.5994 & 1.77084 \\
\hline 2859 & 51.5863 & 391.94 & 420.4278 & 399 & 122.5994 & 1.7703 \\
\hline 2860 & 51.6033 & 410.228 & 417.959 & 398 & 122.5994 & 1.76976 \\
\hline 2861 & 51.6203 & 416.2155 & 414.8297 & 398 & 122.5994 & 1.76921 \\
\hline 2862 & 51.6373 & 416.1201 & 411.466 & 398 & 122.5994 & 1.76867 \\
\hline 2863 & 51.6543 & 416.2155 & 408.7729 & 398 & 122.5994 & 1.76813 \\
\hline 2864 & 51.6713 & 389.8701 & 407.0122 & 398 & 122.5994 & 1.76759 \\
\hline 2865 & 51.6883 & 399.6397 & 405.2781 & 397 & 122.5994 & 1.76705 \\
\hline 2866 & 51.7053 & 413.5937 & 405.2471 & 397 & 122.5994 & 1.7665 \\
\hline 2867 & 51.7223 & 412.0505 & 405.7151 & 397 & 122.5994 & 1.76596 \\
\hline 2868 & 51.7393 & 414.942 & 406.4981 & 397 & 122.5994 & 1.76542 \\
\hline 2869 & 51.7563 & 433.7232 & 406.5156 & 396 & 122.5994 & 1.76488 \\
\hline 2870 & 51.7733 & 402.4182 & 407.7426 & 396 & 122.5994 & 1.76434 \\
\hline 2871 & 51.7903 & 382.1592 & 409.1691 & 396 & 122.5994 & 1.7638 \\
\hline 2872 & 51.8073 & 383.2365 & 410.7275 & 396 & 122.5994 & 1.76327 \\
\hline 2873 & 51.8243 & 393.6296 & 412.2832 & 396 & 122.5994 & 1.76273 \\
\hline 2874 & 51.8413 & 432.6937 & 412.6202 & 395 & 122.5994 & 1.76219 \\
\hline 2875 & 51.8583 & 406.7062 & 413.5344 & 395 & 122.5994 & 1.76165 \\
\hline 2876 & 51.8753 & 396.5331 & 413.611 & 395 & 122.5994 & 1.76111 \\
\hline 2877 & 51.8923 & 382.092 & 412.9545 & 395 & 122.5994 & 1.76058 \\
\hline 2878 & 51.9093 & 376.7905 & 411.9142 & 395 & 122.5994 & 1.76004 \\
\hline 2879 & 51.9263 & 405.5892 & 409.8547 & 394 & 122.5994 & 1.7595 \\
\hline 2880 & 51.9433 & 411.9234 & 408.9839 & 394 & 122.5994 & 1.75897 \\
\hline 2881 & 51.9603 & 385.0464 & 408.3709 & 394 & 122.5994 & 1.75843 \\
\hline 2882 & 51.9773 & 392.0006 & 407.9878 & 394 & 122.5994 & 1.7579 \\
\hline 2883 & 51.9943 & 376.6351 & 408.9221 & 395 & 122.5994 & 1.75736 \\
\hline 2884 & 52.0113 & 408.4787 & 408.7159 & 395 & 122.5994 & 1.75683 \\
\hline 2885 & 52.0283 & 419.3507 & 409.4912 & 396 & 122.5994 & 1.75629 \\
\hline 2886 & 52.0453 & 404.3643 & 409.2668 & 396 & 122.5994 & 1.75576 \\
\hline 2887 & 52.0623 & 408.7801 & 408.917 & 396 & 122.5994 & 1.75523 \\
\hline 2888 & 52.0793 & 417.6656 & 409.1324 & 397 & 122.5994 & 1.75469 \\
\hline 2889 & 52.0963 & 426.1688 & 407.8139 & 397 & 122.5994 & 1.75416 \\
\hline
\end{tabular}




\begin{tabular}{|c|c|c|c|c|c|c|}
\hline 2890 & 52.1133 & 410.754 & 407.2958 & 398 & 122.5994 & 1.75363 \\
\hline 2891 & 52.1303 & 406.2764 & 406.047 & 398 & 122.5994 & 1.7531 \\
\hline 2892 & 52.1473 & 412.8311 & 406.127 & 399 & 122.5994 & 1.75257 \\
\hline 2893 & 52.1643 & 398.2609 & 405.6659 & 399 & 122.5994 & 1.75204 \\
\hline 2894 & 52.1813 & 399.9723 & 405.5791 & 399 & 122.5994 & 1.7515 \\
\hline 2895 & 52.1983 & 421.0308 & 406.6774 & 400 & 122.5994 & 1.75097 \\
\hline 2896 & 52.2153 & 414.5086 & 406.6955 & 400 & 122.5994 & 1.75044 \\
\hline 2897 & 52.2323 & 421.4882 & 407.4675 & 401 & 122.5994 & 1.74991 \\
\hline 2898 & 52.2493 & 414.9836 & 407.0703 & 401 & 122.5994 & 1.74938 \\
\hline 2899 & 52.2663 & 411.9054 & 407.662 & 402 & 122.5994 & 1.74886 \\
\hline 2900 & 52.2833 & 441.0588 & 407.2798 & 402 & 122.5994 & 1.74833 \\
\hline 2901 & 52.3003 & 432.7153 & 407.187 & 402 & 122.5994 & 1.7478 \\
\hline 2902 & 52.3173 & 420.2097 & 408.4156 & 403 & 122.5994 & 1.74727 \\
\hline 2903 & 52.3343 & 425.7613 & 409.0492 & 403 & 122.5994 & 1.74674 \\
\hline 2904 & 52.3513 & 419.3796 & 411.1269 & 404 & 122.5994 & 1.74622 \\
\hline 2905 & 52.3683 & 404.0639 & 412.5642 & 404 & 122.5994 & 1.74569 \\
\hline 2906 & 52.3853 & 414.2436 & 414.0981 & 404 & 122.5994 & 1.74516 \\
\hline 2907 & 52.4023 & 415.7316 & 416.4155 & 405 & 122.5994 & 1.74464 \\
\hline 2908 & 52.4193 & 410.3132 & 417.2115 & 405 & 122.5994 & 1.74411 \\
\hline 2909 & 52.4363 & 419.0355 & 418.8207 & 406 & 122.5994 & 1.74359 \\
\hline 2910 & 52.4533 & 419.571 & 419.8126 & 406 & 122.5994 & 1.74306 \\
\hline 2911 & 52.4703 & 406.2246 & 421.5714 & 406 & 122.5994 & 1.74254 \\
\hline 2912 & 52.4873 & 375.3276 & 424.9413 & 407 & 122.5994 & 1.74201 \\
\hline 2913 & 52.5043 & 405.1992 & 427.1759 & 407 & 122.5994 & 1.74149 \\
\hline 2914 & 52.5213 & 428.4489 & 429.26 & 408 & 122.5994 & 1.74096 \\
\hline 2915 & 52.5383 & 434.3585 & 428.9103 & 408 & 122.5994 & 1.74044 \\
\hline 2916 & 52.5553 & 427.8053 & 428.6044 & 409 & 122.5994 & 1.73992 \\
\hline 2917 & 52.5723 & 424.4166 & 426.9648 & 409 & 122.5994 & 1.73939 \\
\hline 2918 & 52.5893 & 390.6645 & 425.6519 & 409 & 122.5994 & 1.73887 \\
\hline 2919 & 52.6063 & 418.0527 & 426.0225 & 410 & 122.5994 & 1.73835 \\
\hline 2920 & 52.6233 & 412.0635 & 426.2247 & 410 & 122.5994 & 1.73783 \\
\hline 2921 & 52.6403 & 391.493 & 427.6884 & 411 & 122.5994 & 1.73731 \\
\hline 2922 & 52.6573 & 398.2547 & 427.9636 & 411 & 122.5994 & 1.73679 \\
\hline 2923 & 52.6743 & 435.6535 & 428.7907 & 412 & 122.5994 & 1.73627 \\
\hline 2924 & 52.6913 & 437.3205 & 428.3961 & 412 & 122.5994 & 1.73575 \\
\hline 2925 & 52.7083 & 406.8019 & 428.0655 & 412 & 122.5994 & 1.73523 \\
\hline 2926 & 52.7253 & 411.5262 & 428.9867 & 413 & 122.5994 & 1.73471 \\
\hline 2927 & 52.7423 & 443.83 & 429.2828 & 413 & 122.5994 & 1.73419 \\
\hline 2928 & 52.7593 & 428.8136 & 430.9749 & 414 & 122.5994 & 1.73367 \\
\hline 2929 & 52.7763 & 376.4305 & 432.0565 & 414 & 122.5994 & 1.73315 \\
\hline 2930 & 52.7933 & 432.3467 & 434.338 & 415 & 122.5994 & 1.73263 \\
\hline 2931 & 52.8103 & 416.1201 & 435.6696 & 415 & 122.5994 & 1.73212 \\
\hline 2932 & 52.8273 & 415.0836 & 438.1013 & 416 & 122.5994 & 1.7316 \\
\hline 2933 & 52.8443 & 405.0256 & 439.489 & 416 & 122.5994 & 1.73108 \\
\hline 2934 & 52.8613 & 457.4583 & 441.8354 & 417 & 122.5994 & 1.73056 \\
\hline 2935 & 52.8783 & 454.3007 & 443.1161 & 417 & 122.5994 & 1.73005 \\
\hline 2936 & 52.8953 & 451.6345 & 445.3807 & 418 & 122.5994 & 1.72953 \\
\hline 2937 & 52.9123 & 419.6656 & 446.6538 & 418 & 122.5994 & 1.72902 \\
\hline 2938 & 52.9293 & 440.1319 & 448.8855 & 419 & 122.5994 & 1.7285 \\
\hline
\end{tabular}




\begin{tabular}{|c|c|c|c|c|c|c|}
\hline 2939 & 52.9463 & 452.7337 & 449.9963 & 419 & 122.5994 & 1.72799 \\
\hline 2940 & 52.9633 & 445.5281 & 451.8111 & 420 & 122.5994 & 1.72747 \\
\hline 2941 & 52.9803 & 430.4636 & 452.2959 & 420 & 122.5994 & 1.72696 \\
\hline 2942 & 52.9973 & 414.2796 & 453.3158 & 421 & 122.5994 & 1.72644 \\
\hline 2943 & 53.0143 & 402.713 & 453.8817 & 422 & 122.5994 & 1.72593 \\
\hline 2944 & 53.0313 & 436.7681 & 453.0589 & 422 & 122.5994 & 1.72542 \\
\hline 2945 & 53.0483 & 429.7651 & 452.96 & 423 & 122.5994 & 1.7249 \\
\hline 2946 & 53.0653 & 423.6656 & 451.7346 & 423 & 122.5994 & 1.72439 \\
\hline 2947 & 53.0823 & 421.0767 & 451.4704 & 424 & 122.5994 & 1.72388 \\
\hline 2948 & 53.0993 & 405.8733 & 450.2137 & 424 & 122.5994 & 1.72337 \\
\hline 2949 & 53.1163 & 441.9792 & 449.9464 & 425 & 122.5994 & 1.72286 \\
\hline 2950 & 53.1333 & 459.3573 & 448.6111 & 425 & 122.5994 & 1.72234 \\
\hline 2951 & 53.1503 & 433.3623 & 448.1851 & 426 & 122.5994 & 1.72183 \\
\hline 2952 & 53.1673 & 428.4967 & 446.847 & 426 & 122.5994 & 1.72132 \\
\hline 2953 & 53.1843 & 426.1121 & 446.8232 & 427 & 122.5994 & 1.72081 \\
\hline 2954 & 53.2013 & 418.4146 & 446.3695 & 427 & 122.5994 & 1.7203 \\
\hline 2955 & 53.2183 & 429.4684 & 447.6249 & 428 & 122.5994 & 1.71979 \\
\hline 2956 & 53.2353 & 422.4177 & 448.5083 & 428 & 122.5994 & 1.71928 \\
\hline 2957 & 53.2523 & 422.0205 & 449.5993 & 428 & 122.5994 & 1.71878 \\
\hline 2958 & 53.2693 & 428.4489 & 451.1739 & 429 & 122.5994 & 1.71827 \\
\hline 2959 & 53.2863 & 431.2249 & 450.7311 & 429 & 122.5994 & 1.71776 \\
\hline 2960 & 53.3033 & 455.2116 & 449.6761 & 429 & 122.5994 & 1.71725 \\
\hline 2961 & 53.3203 & 448.928 & 449.7625 & 430 & 122.5994 & 1.71674 \\
\hline 2962 & 53.3373 & 441.0588 & 449.1654 & 430 & 122.5994 & 1.71624 \\
\hline 2963 & 53.3543 & 444.0358 & 449.7465 & 431 & 122.5994 & 1.71573 \\
\hline 2964 & 53.3713 & 442.1001 & 449.5816 & 431 & 122.5994 & 1.71522 \\
\hline 2965 & 53.3883 & 450.7825 & 449.8333 & 431 & 122.5994 & 1.71472 \\
\hline 2966 & 53.4053 & 440.3162 & 451.4811 & 432 & 122.5994 & 1.71421 \\
\hline 2967 & 53.4223 & 475.3359 & 452.1458 & 432 & 122.5994 & 1.71371 \\
\hline 2968 & 53.4393 & 458.5323 & 452.5735 & 432 & 122.5994 & 1.7132 \\
\hline 2969 & 53.4563 & 404.125 & 453.7917 & 433 & 122.5994 & 1.7127 \\
\hline 2970 & 53.4733 & 424.6122 & 453.7779 & 433 & 122.5994 & 1.71219 \\
\hline 2971 & 53.4903 & 421.1029 & 453.2939 & 433 & 122.5994 & 1.71169 \\
\hline 2972 & 53.5073 & 463.0394 & 453.4591 & 434 & 122.5994 & 1.71118 \\
\hline 2973 & 53.5243 & 403.6233 & 452.7639 & 434 & 122.5994 & 1.71068 \\
\hline 2974 & 53.5413 & 477.0176 & 452.5877 & 434 & 122.5994 & 1.71018 \\
\hline 2975 & 53.5583 & 441.3656 & 453.8743 & 435 & 122.5994 & 1.70968 \\
\hline 2976 & 53.5753 & 475.0529 & 454.4782 & 435 & 122.5994 & 1.70917 \\
\hline 2977 & 53.5923 & 462.4519 & 456.0156 & 436 & 122.5994 & 1.70867 \\
\hline 2978 & 53.6093 & 465.3632 & 456.253 & 436 & 122.5994 & 1.70817 \\
\hline 2979 & 53.6263 & 444.8499 & 456.0255 & 436 & 122.5994 & 1.70767 \\
\hline 2980 & 53.6433 & 442.7045 & 456.3504 & 437 & 122.5994 & 1.70717 \\
\hline 2981 & 53.6603 & 455.4108 & 455.4588 & 437 & 122.5994 & 1.70667 \\
\hline 2982 & 53.6773 & 437.4553 & 454.5797 & 437 & 122.5994 & 1.70617 \\
\hline 2983 & 53.6943 & 445.0862 & 454.7721 & 438 & 122.5994 & 1.70567 \\
\hline 2984 & 53.7113 & 467.4153 & 454.185 & 438 & 122.5994 & 1.70517 \\
\hline 2985 & 53.7283 & 467.3258 & 453.7051 & 438 & 122.5994 & 1.70467 \\
\hline 2986 & 53.7453 & 446.0886 & 454.2659 & 439 & 122.5994 & 1.70417 \\
\hline 2987 & 53.7623 & 424.4073 & 453.8289 & 439 & 122.5994 & 1.70367 \\
\hline
\end{tabular}




\begin{tabular}{|c|c|c|c|c|c|c|}
\hline 2988 & 53.7793 & 445.9384 & 453.3679 & 439 & 122.5994 & 1.70317 \\
\hline 2989 & 53.7963 & 457.425 & 453.8722 & 440 & 122.5994 & 1.70267 \\
\hline 2990 & 53.8133 & 438.8438 & 453.3477 & 440 & 122.5994 & 1.70217 \\
\hline 2991 & 53.8303 & 466.5317 & 452.8214 & 440 & 122.5994 & 1.70168 \\
\hline 2992 & 53.8473 & 449.0268 & 453.3405 & 441 & 122.5994 & 1.70118 \\
\hline 2993 & 53.8643 & 440.5268 & 452.9029 & 441 & 122.5994 & 1.70068 \\
\hline 2994 & 53.8813 & 448.6961 & 453.8018 & 442 & 122.5994 & 1.70019 \\
\hline 2995 & 53.8983 & 448.076 & 453.7723 & 442 & 122.5994 & 1.69969 \\
\hline 2996 & 53.9153 & 450.5355 & 453.88 & 442 & 122.5994 & 1.69919 \\
\hline 2997 & 53.9323 & 427.5787 & 454.9841 & 443 & 122.5994 & 1.6987 \\
\hline 2998 & 53.9493 & 430.1355 & 454.9796 & 443 & 122.5994 & 1.6982 \\
\hline 2999 & 53.9663 & 449.4999 & 454.5539 & 443 & 122.5994 & 1.69771 \\
\hline 3000 & 53.9833 & 453.8893 & 454.9689 & 444 & 122.5994 & 1.69721 \\
\hline 3001 & 54.0003 & 437.3212 & 454.2825 & 444 & 122.5994 & 1.69672 \\
\hline 3002 & 54.0173 & 432.0443 & 454.8505 & 445 & 122.5994 & 1.69623 \\
\hline 3003 & 54.0343 & 458.6455 & 454.9188 & 445 & 122.5994 & 1.69573 \\
\hline 3004 & 54.0513 & 463.0213 & 455.8869 & 446 & 122.5994 & 1.69524 \\
\hline 3005 & 54.0683 & 441.8421 & 455.9794 & 446 & 122.5994 & 1.69475 \\
\hline 3006 & 54.0853 & 454.9656 & 457.1676 & 447 & 122.5994 & 1.69425 \\
\hline 3007 & 54.1023 & 469.4506 & 457.2419 & 447 & 122.5994 & 1.69376 \\
\hline 3008 & 54.1193 & 458.9205 & 458.2847 & 448 & 122.5994 & 1.69327 \\
\hline 3009 & 54.1363 & 426.5884 & 458.4193 & 448 & 122.5994 & 1.69278 \\
\hline 3010 & 54.1533 & 466.484 & 459.6174 & 449 & 122.5994 & 1.69229 \\
\hline 3011 & 54.1703 & 484.0645 & 460.1174 & 449 & 122.5994 & 1.6918 \\
\hline 3012 & 54.1873 & 463.5001 & 461.9103 & 450 & 122.5994 & 1.69131 \\
\hline 3013 & 54.2043 & 461.4836 & 462.769 & 450 & 122.5994 & 1.69082 \\
\hline 3014 & 54.2213 & 455.1134 & 464.7035 & 451 & 122.5994 & 1.69033 \\
\hline 3015 & 54.2383 & 439.3994 & 465.5889 & 451 & 122.5994 & 1.68984 \\
\hline 3016 & 54.2553 & 429.5164 & 467.3229 & 452 & 122.5994 & 1.68935 \\
\hline 3017 & 54.2723 & 470.1977 & 467.9383 & 452 & 122.5994 & 1.68886 \\
\hline 3018 & 54.2893 & 483.3159 & 469.4441 & 453 & 122.5994 & 1.68837 \\
\hline 3019 & 54.3063 & 467.6281 & 469.9395 & 453 & 122.5994 & 1.68788 \\
\hline 3020 & 54.3233 & 470.7692 & 471.4423 & 454 & 122.5994 & 1.68739 \\
\hline 3021 & 54.3403 & 470.8448 & 471.8062 & 454 & 122.5994 & 1.6869 \\
\hline 3022 & 54.3573 & 466.6069 & 473.0463 & 455 & 122.5994 & 1.68642 \\
\hline 3023 & 54.3743 & 446.026 & 473.1127 & 455 & 122.5994 & 1.68593 \\
\hline 3024 & 54.3913 & 484.6464 & 474.1759 & 456 & 122.5994 & 1.68544 \\
\hline 3025 & 54.4083 & 496.0894 & 473.803 & 456 & 122.5994 & 1.68496 \\
\hline 3026 & 54.4253 & 478.1661 & 473.2852 & 456 & 122.5994 & 1.68447 \\
\hline 3027 & 54.4423 & 462.6723 & 473.6874 & 457 & 122.5994 & 1.68398 \\
\hline 3028 & 54.4593 & 462.1159 & 473.1386 & 457 & 122.5994 & 1.6835 \\
\hline 3029 & 54.4763 & 441.8088 & 472.6565 & 457 & 122.5994 & 1.68301 \\
\hline 3030 & 54.4933 & 487.5078 & 473.1864 & 458 & 122.5994 & 1.68253 \\
\hline 3031 & 54.5103 & 467.3976 & 472.8831 & 458 & 122.5994 & 1.68204 \\
\hline 3032 & 54.5273 & 479.952 & 472.5768 & 458 & 122.5994 & 1.68156 \\
\hline 3033 & 54.5443 & 452.4362 & 473.2108 & 459 & 122.5994 & 1.68108 \\
\hline 3034 & 54.5613 & 470.2316 & 472.9562 & 459 & 122.5994 & 1.68059 \\
\hline 3035 & 54.5783 & 495.2932 & 472.9091 & 459 & 122.5994 & 1.68011 \\
\hline 3036 & 54.5953 & 501.8269 & 474.1787 & 460 & 122.5994 & 1.67963 \\
\hline
\end{tabular}




\begin{tabular}{|c|c|c|c|c|c|c|}
\hline 3037 & 54.6123 & 487.5471 & 474.8746 & 460 & 122.5994 & 1.67914 \\
\hline 3038 & 54.6293 & 462.4825 & 476.0739 & 460 & 122.5994 & 1.67866 \\
\hline 3039 & 54.6463 & 470.8448 & 478.6968 & 461 & 122.5994 & 1.67818 \\
\hline 3040 & 54.6633 & 465.8294 & 480.7928 & 461 & 122.5994 & 1.6777 \\
\hline 3041 & 54.6803 & 479.6446 & 482.8641 & 461 & 122.5994 & 1.67722 \\
\hline 3042 & 54.6973 & 473.4763 & 484.5327 & 461 & 122.5994 & 1.67674 \\
\hline 3043 & 54.7143 & 447.4452 & 486.5682 & 462 & 122.5994 & 1.67625 \\
\hline 3044 & 54.7313 & 464.9791 & 487.4042 & 462 & 122.5994 & 1.67577 \\
\hline 3045 & 54.7483 & 443.2173 & 488.7396 & 462 & 122.5994 & 1.67529 \\
\hline 3046 & 54.7653 & 452.4766 & 492.0287 & 463 & 122.5994 & 1.67481 \\
\hline 3047 & 54.7823 & 477.9048 & 495.4141 & 463 & 122.5994 & 1.67433 \\
\hline 3048 & 54.7993 & 489.0164 & 499.9267 & 463 & 122.5994 & 1.67386 \\
\hline 3049 & 54.8163 & 492.7927 & 506.2952 & 464 & 122.5994 & 1.67338 \\
\hline 3050 & 54.8333 & 500.9951 & 511.9987 & 464 & 122.5994 & 1.6729 \\
\hline 3051 & 54.8503 & 514.6905 & 517.438 & 464 & 122.5994 & 1.67242 \\
\hline 3052 & 54.8673 & 531.678 & 522.3392 & 464 & 122.5994 & 1.67194 \\
\hline 3053 & 54.8843 & 550.8116 & 527.2935 & 464 & 122.5994 & 1.67146 \\
\hline 3054 & 54.9013 & 607.2488 & 534.0206 & 464 & 122.5994 & 1.67099 \\
\hline 3055 & 54.9183 & 615.9349 & 543.0065 & 464 & 122.5994 & 1.67051 \\
\hline 3056 & 54.9353 & 650.7062 & 556.0148 & 464 & 122.5994 & 1.67003 \\
\hline 3057 & 54.9523 & 697.3308 & 573.9105 & 464 & 122.5994 & 1.66956 \\
\hline 3058 & 54.9693 & 687.0633 & 596.4392 & 464 & 122.5994 & 1.66908 \\
\hline 3059 & 54.9863 & 620.555 & 620.9224 & 464 & 122.5994 & 1.6686 \\
\hline 3060 & 55.0033 & 577.4574 & 640.67 & 464 & 122.5994 & 1.66813 \\
\hline 3061 & 55.0203 & 535.7082 & 646.5477 & 464 & 122.5994 & 1.66765 \\
\hline 3062 & 55.0373 & 545.0586 & 635.4486 & 464 & 122.5994 & 1.66718 \\
\hline 3063 & 55.0543 & 531.1218 & 614.8214 & 464 & 122.5994 & 1.6667 \\
\hline 3064 & 55.0713 & 597.8183 & 594.4269 & 465 & 122.5994 & 1.66623 \\
\hline 3065 & 55.0883 & 618.9905 & 577.9639 & 465 & 122.5994 & 1.66576 \\
\hline 3066 & 55.1053 & 658.2988 & 568.5945 & 465 & 122.5994 & 1.66528 \\
\hline 3067 & 55.1223 & 597.4679 & 566.4344 & 465 & 122.5994 & 1.66481 \\
\hline 3068 & 55.1393 & 590.4796 & 569.1081 & 465 & 122.5994 & 1.66434 \\
\hline 3069 & 55.1563 & 501.7656 & 571.7332 & 465 & 122.5994 & 1.66386 \\
\hline 3070 & 55.1733 & 506.8077 & 569.0523 & 465 & 122.5994 & 1.66339 \\
\hline 3071 & 55.1903 & 524.4799 & 560.1326 & 465 & 122.5994 & 1.66292 \\
\hline 3072 & 55.2073 & 520.6006 & 548.5906 & 465 & 122.5994 & 1.66245 \\
\hline 3073 & 55.2243 & 477.905 & 537.9266 & 465 & 122.5994 & 1.66197 \\
\hline 3074 & 55.2413 & 500.6457 & 529.6472 & 465 & 122.5994 & 1.6615 \\
\hline 3075 & 55.2583 & 494.0499 & 523.9858 & 465 & 122.5994 & 1.66103 \\
\hline 3076 & 55.2753 & 506.2559 & 520.7333 & 465 & 122.5994 & 1.66056 \\
\hline 3077 & 55.2923 & 530.6495 & 518.5853 & 464 & 122.5994 & 1.66009 \\
\hline 3078 & 55.3093 & 536.4114 & 519.1207 & 464 & 122.5994 & 1.65962 \\
\hline 3079 & 55.3263 & 527.907 & 520.9244 & 464 & 122.5994 & 1.65915 \\
\hline 3080 & 55.3433 & 538.5084 & 523.7923 & 464 & 122.5994 & 1.65868 \\
\hline 3081 & 55.3603 & 578.1608 & 527.6797 & 464 & 122.5994 & 1.65821 \\
\hline 3082 & 55.3773 & 565.627 & 531.8231 & 463 & 122.5994 & 1.65774 \\
\hline 3083 & 55.3943 & 567.3583 & 538.5133 & 463 & 122.5994 & 1.65728 \\
\hline 3084 & 55.4113 & 592.0774 & 547.0438 & 463 & 122.5994 & 1.65681 \\
\hline 3085 & 55.4283 & 557.6957 & 557.1314 & 463 & 122.5994 & 1.65634 \\
\hline
\end{tabular}




\begin{tabular}{|c|c|c|c|c|c|c|}
\hline 3086 & 55.4453 & 524.3597 & 567.4986 & 463 & 122.5994 & 1.65587 \\
\hline 3087 & 55.4623 & 520.7196 & 574.0662 & 462 & 122.5994 & 1.65541 \\
\hline 3088 & 55.4793 & 515.3587 & 574.6483 & 462 & 122.5994 & 1.65494 \\
\hline 3089 & 55.4963 & 507.2809 & 567.1615 & 462 & 122.5994 & 1.65447 \\
\hline 3090 & 55.5133 & 520.6741 & 555.2662 & 462 & 122.5994 & 1.65401 \\
\hline 3091 & 55.5303 & 545.2413 & 543.4285 & 462 & 122.5994 & 1.65354 \\
\hline 3092 & 55.5473 & 552.4925 & 533.3558 & 461 & 122.5994 & 1.65307 \\
\hline 3093 & 55.5643 & 549.2465 & 528.187 & 461 & 122.5994 & 1.65261 \\
\hline 3094 & 55.5813 & 540.7448 & 526.5518 & 461 & 122.5994 & 1.65214 \\
\hline 3095 & 55.5983 & 524.6029 & 527.1822 & 461 & 122.5994 & 1.65168 \\
\hline 3096 & 55.6153 & 481.9527 & 527.6847 & 461 & 122.5994 & 1.65121 \\
\hline 3097 & 55.6323 & 472.979 & 524.6307 & 460 & 122.5994 & 1.65075 \\
\hline 3098 & 55.6493 & 513.9027 & 519.6011 & 460 & 122.5994 & 1.65028 \\
\hline 3099 & 55.6663 & 501.06 & 513.3967 & 460 & 122.5994 & 1.64982 \\
\hline 3100 & 55.6833 & 478.0058 & 507.8499 & 460 & 122.5994 & 1.64936 \\
\hline 3101 & 55.7003 & 487.5213 & 503.902 & 460 & 122.5994 & 1.64889 \\
\hline 3102 & 55.7173 & 480.1978 & 500.9011 & 459 & 122.5994 & 1.64843 \\
\hline 3103 & 55.7343 & 488.2794 & 500.7364 & 459 & 122.5994 & 1.64797 \\
\hline 3104 & 55.7513 & 527.6418 & 502.1914 & 459 & 122.5994 & 1.64751 \\
\hline 3105 & 55.7683 & 502.4798 & 504.8898 & 459 & 122.5994 & 1.64704 \\
\hline 3106 & 55.7853 & 482.1035 & 508.8317 & 459 & 122.5994 & 1.64658 \\
\hline 3107 & 55.8023 & 483.3159 & 512.653 & 458 & 122.5994 & 1.64612 \\
\hline 3108 & 55.8193 & 534.0703 & 518.1019 & 458 & 122.5994 & 1.64566 \\
\hline 3109 & 55.8363 & 526.8354 & 523.8242 & 458 & 122.5994 & 1.6452 \\
\hline 3110 & 55.8533 & 536.725 & 529.4056 & 458 & 122.5994 & 1.64474 \\
\hline 3111 & 55.8703 & 538.3117 & 534.3497 & 458 & 122.5994 & 1.64428 \\
\hline 3112 & 55.8873 & 520.9446 & 538.1491 & 458 & 122.5994 & 1.64382 \\
\hline 3113 & 55.9043 & 498.1741 & 539.7026 & 457 & 122.5994 & 1.64336 \\
\hline 3114 & 55.9213 & 555.8468 & 540.7756 & 457 & 122.5994 & 1.6429 \\
\hline 3115 & 55.9383 & 501.803 & 540.4818 & 457 & 122.5994 & 1.64244 \\
\hline 3116 & 55.9553 & 488.5692 & 539.1261 & 457 & 122.5994 & 1.64198 \\
\hline 3117 & 55.9723 & 497.2423 & 537.0808 & 457 & 122.5994 & 1.64152 \\
\hline 3118 & 55.9893 & 497.9501 & 533.6456 & 456 & 122.5994 & 1.64107 \\
\hline 3119 & 56.0063 & 506.9942 & 530.6683 & 456 & 122.5994 & 1.64061 \\
\hline 3120 & 56.0233 & 515.8562 & 527.7607 & 456 & 122.5994 & 1.64015 \\
\hline 3121 & 56.0403 & 520.988 & 524.4444 & 456 & 122.5994 & 1.63969 \\
\hline 3122 & 56.0573 & 523.5592 & 519.8018 & 455 & 122.5994 & 1.63924 \\
\hline 3123 & 56.0743 & 495.1239 & 515.7409 & 455 & 122.5994 & 1.63878 \\
\hline 3124 & 56.0913 & 499.2972 & 510.3921 & 454 & 122.5994 & 1.63832 \\
\hline 3125 & 56.1083 & 480.5834 & 505.8486 & 454 & 122.5994 & 1.63787 \\
\hline 3126 & 56.1253 & 502.5997 & 501.3744 & 454 & 122.5994 & 1.63741 \\
\hline 3127 & 56.1423 & 464.5578 & 495.9716 & 453 & 122.5994 & 1.63696 \\
\hline 3128 & 56.1593 & 472.0689 & 491.9166 & 453 & 122.5994 & 1.6365 \\
\hline 3129 & 56.1763 & 475.1944 & 487.0815 & 452 & 122.5994 & 1.63605 \\
\hline 3130 & 56.1933 & 464.1936 & 483.5066 & 452 & 122.5994 & 1.63559 \\
\hline 3131 & 56.2103 & 451.2963 & 479.3021 & 451 & 122.5994 & 1.63514 \\
\hline 3132 & 56.2273 & 477.392 & 476.471 & 451 & 122.5994 & 1.63468 \\
\hline 3133 & 56.2443 & 474.8586 & 473.1068 & 450 & 122.5994 & 1.63423 \\
\hline 3134 & 56.2613 & 452.0054 & 471.1063 & 450 & 122.5994 & 1.63378 \\
\hline
\end{tabular}




\begin{tabular}{|c|c|c|c|c|c|c|}
\hline 3135 & 56.2783 & 458.6639 & 468.5172 & 449 & 122.5994 & 1.63332 \\
\hline 3136 & 56.2953 & 459.2003 & 467.272 & 449 & 122.5994 & 1.63287 \\
\hline 3137 & 56.3123 & 453.365 & 466.2699 & 449 & 122.5994 & 1.63242 \\
\hline 3138 & 56.3293 & 447.5038 & 464.4189 & 448 & 122.5994 & 1.63196 \\
\hline 3139 & 56.3463 & 448.0406 & 463.6374 & 448 & 122.5994 & 1.63151 \\
\hline 3140 & 56.3633 & 466.8677 & 462.0566 & 447 & 122.5994 & 1.63106 \\
\hline 3141 & 56.3803 & 468.4157 & 461.6247 & 447 & 122.5994 & 1.63061 \\
\hline 3142 & 56.3973 & 466.6222 & 460.1489 & 446 & 122.5994 & 1.63016 \\
\hline 3143 & 56.4143 & 446.025 & 460.0704 & 446 & 122.5994 & 1.62971 \\
\hline 3144 & 56.4313 & 464.835 & 459.1616 & 445 & 122.5994 & 1.62926 \\
\hline 3145 & 56.4483 & 481.1126 & 459.3937 & 445 & 122.5994 & 1.62881 \\
\hline 3146 & 56.4653 & 445.4851 & 458.7169 & 444 & 122.5994 & 1.62836 \\
\hline 3147 & 56.4823 & 463.7328 & 457.9344 & 443 & 122.5994 & 1.62791 \\
\hline 3148 & 56.4993 & 458.0939 & 458.3203 & 443 & 122.5994 & 1.62746 \\
\hline 3149 & 56.5163 & 425.0961 & 457.5431 & 442 & 122.5994 & 1.62701 \\
\hline 3150 & 56.5333 & 434.867 & 456.6227 & 441 & 122.5994 & 1.62656 \\
\hline 3151 & 56.5503 & 441.0588 & 456.4358 & 441 & 122.5994 & 1.62611 \\
\hline 3152 & 56.5673 & 471.7599 & 455.293 & 440 & 122.5994 & 1.62566 \\
\hline 3153 & 56.5843 & 458.4344 & 454.0699 & 439 & 122.5994 & 1.62521 \\
\hline 3154 & 56.6013 & 401.8083 & 452.7175 & 438 & 122.5994 & 1.62477 \\
\hline 3155 & 56.6183 & 434.219 & 452.3313 & 438 & 122.5994 & 1.62432 \\
\hline 3156 & 56.6353 & 456.05 & 450.8669 & 437 & 122.5994 & 1.62387 \\
\hline 3157 & 56.6523 & 450.2026 & 449.38 & 436 & 122.5994 & 1.62342 \\
\hline 3158 & 56.6693 & 433.9265 & 447.8601 & 435 & 122.5994 & 1.62298 \\
\hline 3159 & 56.6863 & 432.5985 & 447.4583 & 435 & 122.5994 & 1.62253 \\
\hline 3160 & 56.7033 & 478.1513 & 445.9504 & 434 & 122.5994 & 1.62209 \\
\hline 3161 & 56.7203 & 437.2387 & 444.4344 & 433 & 122.5994 & 1.62164 \\
\hline 3162 & 56.7373 & 440.842 & 443.9744 & 433 & 122.5994 & 1.62119 \\
\hline 3163 & 56.7543 & 424.4616 & 442.5428 & 432 & 122.5994 & 1.62075 \\
\hline 3164 & 56.7713 & 428.7799 & 441.1796 & 431 & 122.5994 & 1.6203 \\
\hline 3165 & 56.7883 & 432.2768 & 439.8617 & 430 & 122.5994 & 1.61986 \\
\hline 3166 & 56.8053 & 429.2721 & 439.617 & 430 & 122.5994 & 1.61941 \\
\hline 3167 & 56.8223 & 440.978 & 438.4674 & 429 & 122.5994 & 1.61897 \\
\hline 3168 & 56.8393 & 463.0726 & 437.4485 & 428 & 122.5994 & 1.61853 \\
\hline 3169 & 56.8563 & 421.6621 & 436.4643 & 427 & 122.5994 & 1.61808 \\
\hline 3170 & 56.8733 & 446.2875 & 436.5616 & 427 & 122.5994 & 1.61764 \\
\hline 3171 & 56.8903 & 430.8887 & 435.7716 & 426 & 122.5994 & 1.6172 \\
\hline 3172 & 56.9073 & 430.1386 & 435.0829 & 425 & 122.5994 & 1.61675 \\
\hline 3173 & 56.9243 & 452.5939 & 434.0424 & 424 & 122.5994 & 1.61631 \\
\hline 3174 & 56.9413 & 454.2808 & 433.5273 & 423 & 122.5994 & 1.61587 \\
\hline 3175 & 56.9583 & 436.277 & 433.0458 & 422 & 122.5994 & 1.61543 \\
\hline 3176 & 56.9753 & 450.6249 & 432.5752 & 421 & 122.5994 & 1.61499 \\
\hline 3177 & 56.9923 & 445.3664 & 432.0686 & 420 & 122.5994 & 1.61454 \\
\hline 3178 & 57.0093 & 431.5034 & 431.5269 & 419 & 122.5994 & 1.6141 \\
\hline 3179 & 57.0263 & 455.3535 & 430.9569 & 418 & 122.5994 & 1.61366 \\
\hline 3180 & 57.0433 & 428.5039 & 430.3755 & 417 & 122.5994 & 1.61322 \\
\hline 3181 & 57.0603 & 424.9267 & 429.8009 & 416 & 122.5994 & 1.61278 \\
\hline 3182 & 57.0773 & 446.1433 & 429.0231 & 415 & 122.5994 & 1.61234 \\
\hline 3183 & 57.0943 & 453.9569 & 428.4729 & 414 & 122.5994 & 1.6119 \\
\hline
\end{tabular}




\begin{tabular}{|c|c|c|c|c|c|c|}
\hline 3184 & 57.1113 & 433.3145 & 427.9281 & 413 & 122.5994 & 1.61146 \\
\hline 3185 & 57.1283 & 401.8672 & 427.4359 & 412 & 122.5994 & 1.61102 \\
\hline 3186 & 57.1453 & 416.1529 & 426.969 & 411 & 122.5994 & 1.61058 \\
\hline 3187 & 57.1623 & 434.0668 & 426.5272 & 410 & 122.5994 & 1.61015 \\
\hline 3188 & 57.1793 & 426.1828 & 426.0712 & 409 & 122.5994 & 1.60971 \\
\hline 3189 & 57.1963 & 429.2368 & 425.5402 & 408 & 122.5994 & 1.60927 \\
\hline 3190 & 57.2133 & 419.9536 & 424.8646 & 407 & 122.5994 & 1.60883 \\
\hline 3191 & 57.2303 & 414.7374 & 423.9947 & 406 & 122.5994 & 1.60839 \\
\hline 3192 & 57.2473 & 389.9161 & 423.0282 & 405 & 122.5994 & 1.60796 \\
\hline 3193 & 57.2643 & 409.0521 & 422.0217 & 404 & 122.5994 & 1.60752 \\
\hline 3194 & 57.2813 & 407.2317 & 422.3464 & 404 & 122.5994 & 1.60708 \\
\hline 3195 & 57.2983 & 405.8671 & 424.2565 & 405 & 122.5994 & 1.60665 \\
\hline 3196 & 57.3153 & 417.0916 & 425.934 & 405 & 122.5994 & 1.60621 \\
\hline 3197 & 57.3323 & 442.5678 & 428.3503 & 405 & 122.5994 & 1.60578 \\
\hline 3198 & 57.3493 & 382.8452 & 432.1135 & 406 & 122.5994 & 1.60534 \\
\hline 3199 & 57.3663 & 383.609 & 434.3244 & 406 & 122.5994 & 1.6049 \\
\hline 3200 & 57.3833 & 384.8772 & 434.8131 & 406 & 122.5994 & 1.60447 \\
\hline 3201 & 57.4003 & 432.556 & 433.2856 & 406 & 122.5994 & 1.60403 \\
\hline 3202 & 57.4173 & 412.1667 & 431.6059 & 407 & 122.5994 & 1.6036 \\
\hline 3203 & 57.4343 & 426.4004 & 429.0254 & 407 & 122.5994 & 1.60317 \\
\hline 3204 & 57.4513 & 420.8963 & 427.3654 & 407 & 122.5994 & 1.60273 \\
\hline 3205 & 57.4683 & 447.693 & 427.9329 & 408 & 122.5994 & 1.6023 \\
\hline 3206 & 57.4853 & 441.2974 & 428.7253 & 408 & 122.5994 & 1.60186 \\
\hline 3207 & 57.5023 & 424.7299 & 430.4698 & 408 & 122.5994 & 1.60143 \\
\hline 3208 & 57.5193 & 416.1964 & 433.6291 & 409 & 122.5994 & 1.601 \\
\hline 3209 & 57.5363 & 445.0654 & 435.4908 & 409 & 122.5994 & 1.60057 \\
\hline 3210 & 57.5533 & 437.7346 & 436.6526 & 409 & 122.5994 & 1.60013 \\
\hline 3211 & 57.5703 & 459.1848 & 438.3646 & 410 & 122.5994 & 1.5997 \\
\hline 3212 & 57.5873 & 481.6139 & 439.0362 & 410 & 122.5994 & 1.59927 \\
\hline 3213 & 57.6043 & 540.5041 & 439.9899 & 410 & 122.5994 & 1.59884 \\
\hline 3214 & 57.6213 & 481.7653 & 442.1786 & 411 & 122.5994 & 1.59841 \\
\hline 3215 & 57.6383 & 460.0478 & 443.448 & 411 & 122.5994 & 1.59798 \\
\hline 3216 & 57.6553 & 424.4166 & 444.5638 & 411 & 122.5994 & 1.59755 \\
\hline 3217 & 57.6723 & 425.1301 & 446.2941 & 412 & 122.5994 & 1.59711 \\
\hline 3218 & 57.6893 & 437.7893 & 446.4961 & 412 & 122.5994 & 1.59668 \\
\hline 3219 & 57.7063 & 462.6862 & 446.178 & 412 & 122.5994 & 1.59625 \\
\hline 3220 & 57.7233 & 440.0622 & 445.4832 & 412 & 122.5994 & 1.59582 \\
\hline 3221 & 57.7403 & 464.2785 & 445.5886 & 413 & 122.5994 & 1.5954 \\
\hline 3222 & 57.7573 & 494.9808 & 444.7408 & 413 & 122.5994 & 1.59497 \\
\hline 3223 & 57.7743 & 475.4257 & 444.024 & 413 & 122.5994 & 1.59454 \\
\hline 3224 & 57.7913 & 466.5152 & 444.5014 & 414 & 122.5994 & 1.59411 \\
\hline 3225 & 57.8083 & 455.5561 & 444.1255 & 414 & 122.5994 & 1.59368 \\
\hline 3226 & 57.8253 & 445.1173 & 443.724 & 414 & 122.5994 & 1.59325 \\
\hline 3227 & 57.8423 & 440.7176 & 444.083 & 415 & 122.5994 & 1.59282 \\
\hline 3228 & 57.8593 & 407.4886 & 442.7068 & 415 & 122.5994 & 1.5924 \\
\hline 3229 & 57.8763 & 401.4398 & 440.6483 & 415 & 122.5994 & 1.59197 \\
\hline 3230 & 57.8933 & 411.9609 & 439.3598 & 416 & 122.5994 & 1.59154 \\
\hline 3231 & 57.9103 & 447.6919 & 437.2504 & 416 & 122.5994 & 1.59112 \\
\hline 3232 & 57.9273 & 459.5252 & 435.4943 & 416 & 122.5994 & 1.59069 \\
\hline
\end{tabular}




\begin{tabular}{|c|c|c|c|c|c|c|}
\hline 3233 & 57.9443 & 421.0599 & 434.1011 & 416 & 122.5994 & 1.59026 \\
\hline 3234 & 57.9613 & 436.7066 & 434.0916 & 417 & 122.5994 & 1.58984 \\
\hline 3235 & 57.9783 & 449.9074 & 433.4701 & 417 & 122.5994 & 1.58941 \\
\hline 3236 & 57.9953 & 466.979 & 433.1939 & 417 & 122.5994 & 1.58899 \\
\hline 3237 & 58.0123 & 454.486 & 434.1004 & 418 & 122.5994 & 1.58856 \\
\hline 3238 & 58.0293 & 434.065 & 433.9323 & 418 & 122.5994 & 1.58814 \\
\hline 3239 & 58.0463 & 424.3017 & 433.7996 & 418 & 122.5994 & 1.58771 \\
\hline 3240 & 58.0633 & 440.4847 & 434.7695 & 419 & 122.5994 & 1.58729 \\
\hline 3241 & 58.0803 & 414.1607 & 434.7956 & 419 & 122.5994 & 1.58686 \\
\hline 3242 & 58.0973 & 427.085 & 434.6555 & 419 & 122.5994 & 1.58644 \\
\hline 3243 & 58.1143 & 388.1566 & 435.4301 & 420 & 122.5994 & 1.58602 \\
\hline 3244 & 58.1313 & 419.2026 & 435.0129 & 420 & 122.5994 & 1.58559 \\
\hline 3245 & 58.1483 & 446.1183 & 434.4599 & 420 & 122.5994 & 1.58517 \\
\hline 3246 & 58.1653 & 459.9045 & 433.8575 & 420 & 122.5994 & 1.58475 \\
\hline 3247 & 58.1823 & 409.9023 & 433.2901 & 420 & 122.5994 & 1.58432 \\
\hline 3248 & 58.1993 & 399.9616 & 433.8114 & 421 & 122.5994 & 1.5839 \\
\hline 3249 & 58.2163 & 382.5748 & 433.4357 & 421 & 122.5994 & 1.58348 \\
\hline 3250 & 58.2333 & 420.1951 & 433.145 & 421 & 122.5994 & 1.58306 \\
\hline 3251 & 58.2503 & 416.7111 & 433.9139 & 422 & 122.5994 & 1.58264 \\
\hline 3252 & 58.2673 & 468.1803 & 433.663 & 422 & 122.5994 & 1.58221 \\
\hline 3253 & 58.2843 & 445.9122 & 433.6212 & 422 & 122.5994 & 1.58179 \\
\hline 3254 & 58.3013 & 432.793 & 433.8554 & 422 & 122.5994 & 1.58137 \\
\hline 3255 & 58.3183 & 408.3999 & 434.6369 & 422 & 122.5994 & 1.58095 \\
\hline 3256 & 58.3353 & 435.7032 & 436.9038 & 423 & 122.5994 & 1.58053 \\
\hline 3257 & 58.3523 & 403.5157 & 438.6307 & 423 & 122.5994 & 1.58011 \\
\hline 3258 & 58.3693 & 396.6915 & 440.3368 & 423 & 122.5994 & 1.57969 \\
\hline 3259 & 58.3863 & 408.5147 & 442.0404 & 424 & 122.5994 & 1.57927 \\
\hline 3260 & 58.4033 & 447.456 & 441.2871 & 424 & 122.5994 & 1.57885 \\
\hline 3261 & 58.4203 & 431.4138 & 439.5986 & 424 & 122.5994 & 1.57843 \\
\hline 3262 & 58.4373 & 420.3121 & 437.6434 & 424 & 122.5994 & 1.57802 \\
\hline 3263 & 58.4543 & 404.5879 & 435.8263 & 424 & 122.5994 & 1.5776 \\
\hline 3264 & 58.4713 & 425.1766 & 435.4826 & 425 & 122.5994 & 1.57718 \\
\hline 3265 & 58.4883 & 429.4984 & 434.8349 & 425 & 122.5994 & 1.57676 \\
\hline 3266 & 58.5053 & 432.5985 & 435.9636 & 426 & 122.5994 & 1.57634 \\
\hline 3267 & 58.5223 & 423.7184 & 436.6438 & 426 & 122.5994 & 1.57593 \\
\hline 3268 & 58.5393 & 406.001 & 437.3406 & 426 & 122.5994 & 1.57551 \\
\hline 3269 & 58.5563 & 412.5709 & 438.5851 & 427 & 122.5994 & 1.57509 \\
\hline 3270 & 58.5733 & 412.6324 & 439.3725 & 428 & 122.5994 & 1.57468 \\
\hline 3271 & 58.5903 & 433.3037 & 438.999 & 428 & 122.5994 & 1.57426 \\
\hline 3272 & 58.6073 & 406.0369 & 438.6618 & 428 & 122.5994 & 1.57384 \\
\hline 3273 & 58.6243 & 419.1173 & 439.5244 & 429 & 122.5994 & 1.57343 \\
\hline 3274 & 58.6413 & 430.9522 & 440.6313 & 430 & 122.5994 & 1.57301 \\
\hline 3275 & 58.6583 & 409.0512 & 440.9193 & 430 & 122.5994 & 1.5726 \\
\hline 3276 & 58.6753 & 435.7847 & 441.3517 & 430 & 122.5994 & 1.57218 \\
\hline 3277 & 58.6923 & 449.6172 & 443.112 & 431 & 122.5994 & 1.57177 \\
\hline 3278 & 58.7093 & 447.3196 & 445.1152 & 432 & 122.5994 & 1.57135 \\
\hline 3279 & 58.7263 & 438.3864 & 446.3513 & 432 & 122.5994 & 1.57094 \\
\hline 3280 & 58.7433 & 406.1521 & 447.6721 & 432 & 122.5994 & 1.57052 \\
\hline 3281 & 58.7603 & 413.5942 & 449.9725 & 433 & 122.5994 & 1.57011 \\
\hline
\end{tabular}




\begin{tabular}{|c|c|c|c|c|c|c|}
\hline 3282 & 58.7773 & 435.1787 & 452.2429 & 434 & 122.5994 & 1.5697 \\
\hline 3283 & 58.7943 & 455.1057 & 453.3819 & 434 & 122.5994 & 1.56928 \\
\hline 3284 & 58.8113 & 461.135 & 454.3058 & 434 & 122.5994 & 1.56887 \\
\hline 3285 & 58.8283 & 435.7582 & 455.7901 & 435 & 122.5994 & 1.56846 \\
\hline 3286 & 58.8453 & 435.6492 & 457.0898 & 436 & 122.5994 & 1.56804 \\
\hline 3287 & 58.8623 & 455.9876 & 457.3438 & 436 & 122.5994 & 1.56763 \\
\hline 3288 & 58.8793 & 446.8217 & 457.5073 & 436 & 122.5994 & 1.56722 \\
\hline 3289 & 58.8963 & 466.5881 & 458.0988 & 437 & 122.5994 & 1.56681 \\
\hline 3290 & 58.9133 & 429.8788 & 457.9324 & 438 & 122.5994 & 1.5664 \\
\hline 3291 & 58.9303 & 419.4411 & 456.3889 & 438 & 122.5994 & 1.56599 \\
\hline 3292 & 58.9473 & 443.8562 & 454.9879 & 438 & 122.5994 & 1.56557 \\
\hline 3293 & 58.9643 & 449.3969 & 454.9258 & 439 & 122.5994 & 1.56516 \\
\hline 3294 & 58.9813 & 438.7245 & 455.2095 & 440 & 122.5994 & 1.56475 \\
\hline 3295 & 58.9983 & 415.1485 & 454.6917 & 440 & 122.5994 & 1.56434 \\
\hline 3296 & 59.0153 & 441.8281 & 454.5758 & 440 & 122.5994 & 1.56393 \\
\hline 3297 & 59.0323 & 468.7326 & 455.763 & 441 & 122.5994 & 1.56352 \\
\hline 3298 & 59.0493 & 423.2903 & 457.0504 & 442 & 122.5994 & 1.56311 \\
\hline 3299 & 59.0663 & 408.5756 & 457.155 & 442 & 122.5994 & 1.5627 \\
\hline 3300 & 59.0833 & 404.0428 & 457.043 & 442 & 122.5994 & 1.56229 \\
\hline 3301 & 59.1003 & 416.1964 & 457.9428 & 443 & 122.5994 & 1.56189 \\
\hline 3302 & 59.1173 & 437.0811 & 459.1077 & 444 & 122.5994 & 1.56148 \\
\hline 3303 & 59.1343 & 445.7635 & 459.4391 & 444 & 122.5994 & 1.56107 \\
\hline 3304 & 59.1513 & 457.9161 & 459.5246 & 444 & 122.5994 & 1.56066 \\
\hline 3305 & 59.1683 & 455.7464 & 460.2001 & 445 & 122.5994 & 1.56025 \\
\hline 3306 & 59.1853 & 435.4155 & 460.8113 & 446 & 122.5994 & 1.55984 \\
\hline 3307 & 59.2023 & 454.6559 & 460.7459 & 446 & 122.5994 & 1.55944 \\
\hline 3308 & 59.2193 & 430.0552 & 461.178 & 446 & 122.5994 & 1.55903 \\
\hline 3309 & 59.2363 & 413.9848 & 463.1016 & 447 & 122.5994 & 1.55862 \\
\hline 3310 & 59.2533 & 412.1965 & 465.3638 & 448 & 122.5994 & 1.55822 \\
\hline 3311 & 59.2703 & 450.7865 & 467.0138 & 448 & 122.5994 & 1.55781 \\
\hline 3312 & 59.2873 & 466.8168 & 470.1704 & 448 & 122.5994 & 1.5574 \\
\hline 3313 & 59.3043 & 448.1338 & 472.9099 & 449 & 122.5994 & 1.557 \\
\hline 3314 & 59.3213 & 435.068 & 474.0913 & 449 & 122.5994 & 1.55659 \\
\hline 3315 & 59.3383 & 440.9577 & 475.6693 & 450 & 122.5994 & 1.55619 \\
\hline 3316 & 59.3553 & 440.9577 & 475.9514 & 450 & 122.5994 & 1.55578 \\
\hline 3317 & 59.3723 & 442.524 & 476.2487 & 450 & 122.5994 & 1.55538 \\
\hline 3318 & 59.3893 & 445.1673 & 476.7175 & 450 & 122.5994 & 1.55497 \\
\hline 3319 & 59.4063 & 437.115 & 478.3738 & 451 & 122.5994 & 1.55457 \\
\hline 3320 & 59.4233 & 432.9202 & 479.1313 & 451 & 122.5994 & 1.55416 \\
\hline 3321 & 59.4403 & 456.4187 & 479.8516 & 451 & 122.5994 & 1.55376 \\
\hline 3322 & 59.4573 & 455.3185 & 481.446 & 452 & 122.5994 & 1.55336 \\
\hline 3323 & 59.4743 & 434.779 & 481.5826 & 452 & 122.5994 & 1.55295 \\
\hline 3324 & 59.4913 & 447.4628 & 481.2631 & 452 & 122.5994 & 1.55255 \\
\hline 3325 & 59.5083 & 457.7538 & 480.5467 & 452 & 122.5994 & 1.55215 \\
\hline 3326 & 59.5253 & 431.2147 & 480.5869 & 453 & 122.5994 & 1.55174 \\
\hline 3327 & 59.5423 & 433.0839 & 479.5148 & 453 & 122.5994 & 1.55134 \\
\hline 3328 & 59.5593 & 425.1959 & 478.399 & 453 & 122.5994 & 1.55094 \\
\hline 3329 & 59.5763 & 435.7681 & 478.2879 & 454 & 122.5994 & 1.55054 \\
\hline 3330 & 59.5933 & 427.5368 & 477.2216 & 454 & 122.5994 & 1.55014 \\
\hline
\end{tabular}




\begin{tabular}{|c|c|c|c|c|c|c|}
\hline 3331 & 59.6103 & 441.9276 & 476.1941 & 454 & 122.5994 & 1.54973 \\
\hline 3332 & 59.6273 & 446.5409 & 476.2377 & 455 & 122.5994 & 1.54933 \\
\hline 3333 & 59.6443 & 461.7533 & 475.2834 & 455 & 122.5994 & 1.54893 \\
\hline 3334 & 59.6613 & 449.5101 & 474.3839 & 455 & 122.5994 & 1.54853 \\
\hline 3335 & 59.6783 & 444.0529 & 473.579 & 455 & 122.5994 & 1.54813 \\
\hline 3336 & 59.6953 & 429.4891 & 474.0238 & 456 & 122.5994 & 1.54773 \\
\hline 3337 & 59.7123 & 460.532 & 473.5411 & 456 & 122.5994 & 1.54733 \\
\hline 3338 & 59.7293 & 467.4891 & 473.268 & 456 & 122.5994 & 1.54693 \\
\hline 3339 & 59.7463 & 484.4614 & 473.2418 & 456 & 122.5994 & 1.54653 \\
\hline 3340 & 59.7633 & 448.4081 & 474.4215 & 457 & 122.5994 & 1.54613 \\
\hline 3341 & 59.7803 & 474.4335 & 474.8636 & 457 & 122.5994 & 1.54573 \\
\hline 3342 & 59.7973 & 423.3583 & 475.3787 & 457 & 122.5994 & 1.54533 \\
\hline 3343 & 59.8143 & 502.0626 & 476.8145 & 458 & 122.5994 & 1.54494 \\
\hline 3344 & 59.8313 & 442.0192 & 477.1918 & 458 & 122.5994 & 1.54454 \\
\hline 3345 & 59.8483 & 469.2628 & 477.7473 & 458 & 122.5994 & 1.54414 \\
\hline 3346 & 59.8653 & 483.5873 & 478.8136 & 458 & 122.5994 & 1.54374 \\
\hline 3347 & 59.8823 & 470.9303 & 480.0603 & 458 & 122.5994 & 1.54334 \\
\hline 3348 & 59.8993 & 519.4532 & 482.8044 & 459 & 122.5994 & 1.54295 \\
\hline 3349 & 59.9163 & 500.5889 & 485.148 & 459 & 122.5994 & 1.54255 \\
\hline 3350 & 59.9333 & 586.7162 & 488.2799 & 459 & 122.5994 & 1.54215 \\
\hline 3351 & 59.9503 & 608.2791 & 493.5914 & 460 & 122.5994 & 1.54176 \\
\hline 3352 & 59.9673 & 649.4186 & 499.8773 & 460 & 122.5994 & 1.54136 \\
\hline 3353 & 59.9843 & 696.9581 & 509.6916 & 460 & 122.5994 & 1.54096 \\
\hline 3354 & 60.0013 & 785.2894 & 525.7164 & 460 & 122.5994 & 1.54057 \\
\hline 3355 & 60.0183 & 862.3151 & 551.6077 & 460 & 122.5994 & 1.54017 \\
\hline 3356 & 60.0353 & 883.3215 & 592.4712 & 461 & 122.5994 & 1.53978 \\
\hline 3357 & 60.0523 & 872.142 & 648.2352 & 461 & 122.5994 & 1.53938 \\
\hline 3358 & 60.0693 & 789.3133 & 718.0958 & 461 & 122.5994 & 1.53899 \\
\hline 3359 & 60.0863 & 707.6488 & 793.2401 & 462 & 122.5994 & 1.53859 \\
\hline 3360 & 60.1033 & 672.0457 & 850.5217 & 462 & 122.5994 & 1.5382 \\
\hline 3361 & 60.1203 & 626.0118 & 864.4155 & 462 & 122.5994 & 1.5378 \\
\hline 3362 & 60.1373 & 638.4318 & 828.5845 & 462 & 122.5994 & 1.53741 \\
\hline 3363 & 60.1543 & 645.4628 & 765.8025 & 462 & 122.5994 & 1.53701 \\
\hline 3364 & 60.1713 & 673.049 & 703.1258 & 462 & 122.5994 & 1.53662 \\
\hline 3365 & 60.1883 & 684.3831 & 657.0269 & 462 & 122.5994 & 1.53623 \\
\hline 3366 & 60.2053 & 694.0469 & 634.0285 & 462 & 122.5994 & 1.53583 \\
\hline 3367 & 60.2223 & 688.4427 & 634.0592 & 462 & 122.5994 & 1.53544 \\
\hline 3368 & 60.2393 & 637.4884 & 651.647 & 462 & 122.5994 & 1.53505 \\
\hline 3369 & 60.2563 & 581.2992 & 676.3064 & 462 & 122.5994 & 1.53466 \\
\hline 3370 & 60.2733 & 527.1504 & 692.5858 & 462 & 122.5994 & 1.53426 \\
\hline 3371 & 60.2903 & 520.1287 & 686.5264 & 462 & 122.5994 & 1.53387 \\
\hline 3372 & 60.3073 & 484.4299 & 658.4378 & 462 & 122.5994 & 1.53348 \\
\hline 3373 & 60.3243 & 461.875 & 620.5869 & 462 & 122.5994 & 1.53309 \\
\hline 3374 & 60.3413 & 472.6958 & 584.1592 & 462 & 122.5994 & 1.5327 \\
\hline 3375 & 60.3583 & 489.3525 & 554.6255 & 462 & 122.5994 & 1.53231 \\
\hline 3376 & 60.3753 & 501.7119 & 533.2498 & 462 & 122.5994 & 1.53192 \\
\hline 3377 & 60.3923 & 493.1828 & 519.1701 & 462 & 122.5994 & 1.53153 \\
\hline 3378 & 60.4093 & 476.9026 & 510.3879 & 462 & 122.5994 & 1.53113 \\
\hline 3379 & 60.4263 & 453.3324 & 504.9574 & 462 & 122.5994 & 1.53074 \\
\hline
\end{tabular}




\begin{tabular}{|c|c|c|c|c|c|c|}
\hline 3380 & 60.4433 & 491.3903 & 501.4633 & 462 & 122.5994 & 1.53035 \\
\hline 3381 & 60.4603 & 475.5403 & 499.0905 & 462 & 122.5994 & 1.52997 \\
\hline 3382 & 60.4773 & 462.2057 & 497.3773 & 462 & 122.5994 & 1.52958 \\
\hline 3383 & 60.4943 & 452.4724 & 496.2967 & 462 & 122.5994 & 1.52919 \\
\hline 3384 & 60.5113 & 459.5105 & 495.7851 & 462 & 122.5994 & 1.5288 \\
\hline 3385 & 60.5283 & 483.5316 & 495.7581 & 462 & 122.5994 & 1.52841 \\
\hline 3386 & 60.5453 & 484.6915 & 495.2309 & 461 & 122.5994 & 1.52802 \\
\hline 3387 & 60.5623 & 433.1183 & 495.9024 & 461 & 122.5994 & 1.52763 \\
\hline 3388 & 60.5793 & 453.7411 & 496.2212 & 461 & 122.5994 & 1.52724 \\
\hline 3389 & 60.5963 & 438.943 & 496.0064 & 461 & 122.5994 & 1.52686 \\
\hline 3390 & 60.6133 & 484.2742 & 495.3939 & 461 & 122.5994 & 1.52647 \\
\hline 3391 & 60.6303 & 475.9815 & 494.8378 & 461 & 122.5994 & 1.52608 \\
\hline 3392 & 60.6473 & 458.6836 & 494.7251 & 461 & 122.5994 & 1.52569 \\
\hline 3393 & 60.6643 & 527.7633 & 494.9751 & 461 & 122.5994 & 1.52531 \\
\hline 3394 & 60.6813 & 483.8397 & 495.4935 & 461 & 122.5994 & 1.52492 \\
\hline 3395 & 60.6983 & 437.3219 & 496.3123 & 461 & 122.5994 & 1.52453 \\
\hline 3396 & 60.7153 & 457.4112 & 497.7026 & 461 & 122.5994 & 1.52415 \\
\hline 3397 & 60.7323 & 450.3357 & 499.8305 & 461 & 122.5994 & 1.52376 \\
\hline 3398 & 60.7493 & 513.5097 & 502.3833 & 461 & 122.5994 & 1.52338 \\
\hline 3399 & 60.7663 & 449.8743 & 505.1329 & 461 & 122.5994 & 1.52299 \\
\hline 3400 & 60.7833 & 455.3678 & 508.1123 & 461 & 122.5994 & 1.52261 \\
\hline 3401 & 60.8003 & 463.3045 & 511.2706 & 461 & 122.5994 & 1.52222 \\
\hline 3402 & 60.8173 & 449.7079 & 514.4448 & 461 & 122.5994 & 1.52184 \\
\hline 3403 & 60.8343 & 470.9528 & 517.222 & 461 & 122.5994 & 1.52145 \\
\hline 3404 & 60.8513 & 465.1982 & 519.2269 & 461 & 122.5994 & 1.52107 \\
\hline 3405 & 60.8683 & 456.7736 & 520.1784 & 461 & 122.5994 & 1.52068 \\
\hline 3406 & 60.8853 & 485.8823 & 519.9894 & 461 & 122.5994 & 1.5203 \\
\hline 3407 & 60.9023 & 462.8553 & 518.8124 & 461 & 122.5994 & 1.51992 \\
\hline 3408 & 60.9193 & 451.1554 & 517.0766 & 461 & 122.5994 & 1.51953 \\
\hline 3409 & 60.9363 & 446.5524 & 515.2368 & 461 & 122.5994 & 1.51915 \\
\hline 3410 & 60.9533 & 467.7645 & 513.2492 & 461 & 122.5994 & 1.51877 \\
\hline 3411 & 60.9703 & 495.1315 & 511.4395 & 461 & 122.5994 & 1.51838 \\
\hline 3412 & 60.9873 & 492.6741 & 509.8156 & 461 & 122.5994 & 1.518 \\
\hline 3413 & 61.0043 & 486.3455 & 508.3215 & 461 & 122.5994 & 1.51762 \\
\hline 3414 & 61.0213 & 457.9161 & 505.8482 & 460 & 122.5994 & 1.51724 \\
\hline 3415 & 61.0383 & 459.9801 & 504.2527 & 460 & 122.5994 & 1.51685 \\
\hline 3416 & 61.0553 & 481.0944 & 502.4372 & 460 & 122.5994 & 1.51647 \\
\hline 3417 & 61.0723 & 482.0828 & 500.4673 & 460 & 122.5994 & 1.51609 \\
\hline 3418 & 61.0893 & 462.2057 & 497.2772 & 460 & 122.5994 & 1.51571 \\
\hline 3419 & 61.1063 & 450.6762 & 495.4912 & 460 & 122.5994 & 1.51533 \\
\hline 3420 & 61.1233 & 472.8184 & 493.7636 & 460 & 122.5994 & 1.51495 \\
\hline 3421 & 61.1403 & 486.8883 & 492.165 & 460 & 122.5994 & 1.51457 \\
\hline 3422 & 61.1573 & 474.3784 & 489.687 & 459 & 122.5994 & 1.51419 \\
\hline 3423 & 61.1743 & 470.8448 & 488.3635 & 459 & 122.5994 & 1.51381 \\
\hline 3424 & 61.1913 & 476.5139 & 487.2719 & 459 & 122.5994 & 1.51343 \\
\hline 3425 & 61.2083 & 470.9073 & 486.3672 & 459 & 122.5994 & 1.51305 \\
\hline 3426 & 61.2253 & 472.5322 & 485.6799 & 459 & 122.5994 & 1.51267 \\
\hline 3427 & 61.2423 & 456.8604 & 485.1296 & 459 & 122.5994 & 1.51229 \\
\hline 3428 & 61.2593 & 455.6699 & 484.5712 & 459 & 122.5994 & 1.51191 \\
\hline
\end{tabular}




\begin{tabular}{|c|c|c|c|c|c|c|}
\hline 3429 & 61.2763 & 467.8209 & 483.9602 & 459 & 122.5994 & 1.51153 \\
\hline 3430 & 61.2933 & 470.8448 & 482.3831 & 458 & 122.5994 & 1.51115 \\
\hline 3431 & 61.3103 & 470.8448 & 482.2772 & 458 & 122.5994 & 1.51078 \\
\hline 3432 & 61.3273 & 460.3191 & 482.6287 & 458 & 122.5994 & 1.5104 \\
\hline 3433 & 61.3443 & 476.1849 & 483.5592 & 458 & 122.5994 & 1.51002 \\
\hline 3434 & 61.3613 & 483.4734 & 485.1679 & 458 & 122.5994 & 1.50964 \\
\hline 3435 & 61.3783 & 476.1844 & 487.4551 & 458 & 122.5994 & 1.50926 \\
\hline 3436 & 61.3953 & 507.4578 & 489.3976 & 457 & 122.5994 & 1.50889 \\
\hline 3437 & 61.4123 & 481.8597 & 492.7784 & 457 & 122.5994 & 1.50851 \\
\hline 3438 & 61.4293 & 487.9362 & 496.1425 & 457 & 122.5994 & 1.50813 \\
\hline 3439 & 61.4463 & 471.4275 & 497.8808 & 456 & 122.5994 & 1.50776 \\
\hline 3440 & 61.4633 & 475.1707 & 499.5248 & 456 & 122.5994 & 1.50738 \\
\hline 3441 & 61.4803 & 480.3547 & 500.6261 & 456 & 122.5994 & 1.50701 \\
\hline 3442 & 61.4973 & 526.6122 & 500.5184 & 455 & 122.5994 & 1.50663 \\
\hline 3443 & 61.5143 & 468.7018 & 501.521 & 455 & 122.5994 & 1.50625 \\
\hline 3444 & 61.5313 & 499.2269 & 502.6684 & 455 & 122.5994 & 1.50588 \\
\hline 3445 & 61.5483 & 479.2636 & 503.9357 & 455 & 122.5994 & 1.5055 \\
\hline 3446 & 61.5653 & 545.958 & 504.2632 & 454 & 122.5994 & 1.50513 \\
\hline 3447 & 61.5823 & 505.6614 & 505.6226 & 454 & 122.5994 & 1.50475 \\
\hline 3448 & 61.5993 & 529.0705 & 506.882 & 454 & 122.5994 & 1.50438 \\
\hline 3449 & 61.6163 & 531.5614 & 506.8968 & 453 & 122.5994 & 1.504 \\
\hline 3450 & 61.6333 & 535.2786 & 507.5689 & 453 & 122.5994 & 1.50363 \\
\hline 3451 & 61.6503 & 513.5953 & 508.0366 & 453 & 122.5994 & 1.50326 \\
\hline 3452 & 61.6673 & 549.0725 & 508.4563 & 453 & 122.5994 & 1.50288 \\
\hline 3453 & 61.6843 & 567.8121 & 507.7738 & 452 & 122.5994 & 1.50251 \\
\hline 3454 & 61.7013 & 537.6827 & 507.751 & 452 & 122.5994 & 1.50214 \\
\hline 3455 & 61.7183 & 546.1513 & 507.4136 & 452 & 122.5994 & 1.50176 \\
\hline 3456 & 61.7353 & 539.6949 & 505.9769 & 451 & 122.5994 & 1.50139 \\
\hline 3457 & 61.7523 & 548.9559 & 505.8265 & 451 & 122.5994 & 1.50102 \\
\hline 3458 & 61.7693 & 547.603 & 506.1652 & 451 & 122.5994 & 1.50065 \\
\hline 3459 & 61.7863 & 520.303 & 505.9557 & 450 & 122.5994 & 1.50027 \\
\hline 3460 & 61.8033 & 539.6227 & 506.726 & 450 & 122.5994 & 1.4999 \\
\hline 3461 & 61.8203 & 520.8097 & 506.7576 & 450 & 122.5994 & 1.49953 \\
\hline 3462 & 61.8373 & 500.3705 & 505.8605 & 450 & 122.5994 & 1.49916 \\
\hline 3463 & 61.8543 & 490.5308 & 503.6068 & 449 & 122.5994 & 1.49879 \\
\hline 3464 & 61.8713 & 499.7578 & 502.4924 & 449 & 122.5994 & 1.49842 \\
\hline 3465 & 61.8883 & 508.6025 & 501.4105 & 449 & 122.5994 & 1.49805 \\
\hline 3466 & 61.9053 & 523.3656 & 499.9856 & 448 & 122.5994 & 1.49768 \\
\hline 3467 & 61.9223 & 523.1387 & 499.7753 & 448 & 122.5994 & 1.49731 \\
\hline 3468 & 61.9393 & 509.8448 & 499.543 & 448 & 122.5994 & 1.49694 \\
\hline 3469 & 61.9563 & 517.55 & 498.9997 & 448 & 122.5994 & 1.49657 \\
\hline 3470 & 61.9733 & 509.8648 & 496.8701 & 447 & 122.5994 & 1.4962 \\
\hline 3471 & 61.9903 & 498.8695 & 495.0216 & 447 & 122.5994 & 1.49583 \\
\hline 3472 & 62.0073 & 503.3622 & 492.6294 & 447 & 122.5994 & 1.49546 \\
\hline 3473 & 62.0243 & 481.2925 & 489.1657 & 446 & 122.5994 & 1.49509 \\
\hline 3474 & 62.0413 & 474.1113 & 486.9684 & 446 & 122.5994 & 1.49472 \\
\hline 3475 & 62.0583 & 462.2775 & 484.9755 & 446 & 122.5994 & 1.49435 \\
\hline 3476 & 62.0753 & 509.1317 & 482.3817 & 445 & 122.5994 & 1.49398 \\
\hline 3477 & 62.0923 & 458.4175 & 480.8167 & 445 & 122.5994 & 1.49361 \\
\hline
\end{tabular}




\begin{tabular}{|c|c|c|c|c|c|c|}
\hline 3478 & 62.1093 & 458.2127 & 479.1498 & 445 & 122.5994 & 1.49325 \\
\hline 3479 & 62.1263 & 458.7353 & 477.3614 & 445 & 122.5994 & 1.49288 \\
\hline 3480 & 62.1433 & 461.5843 & 474.3787 & 444 & 122.5994 & 1.49251 \\
\hline 3481 & 62.1603 & 447.3723 & 472.3571 & 444 & 122.5994 & 1.49214 \\
\hline 3482 & 62.1773 & 456.6186 & 470.4574 & 444 & 122.5994 & 1.49178 \\
\hline 3483 & 62.1943 & 458.021 & 467.6695 & 443 & 122.5994 & 1.49141 \\
\hline 3484 & 62.2113 & 470.0859 & 466.2014 & 443 & 122.5994 & 1.49104 \\
\hline 3485 & 62.2283 & 442.0718 & 463.9802 & 442 & 122.5994 & 1.49068 \\
\hline 3486 & 62.2453 & 474.7605 & 462.9811 & 442 & 122.5994 & 1.49031 \\
\hline 3487 & 62.2623 & 479.5227 & 461.1436 & 441 & 122.5994 & 1.48994 \\
\hline 3488 & 62.2793 & 455.3253 & 460.3986 & 441 & 122.5994 & 1.48958 \\
\hline 3489 & 62.2963 & 511.8295 & 458.655 & 440 & 122.5994 & 1.48921 \\
\hline 3490 & 62.3133 & 449.5619 & 457.7796 & 440 & 122.5994 & 1.48885 \\
\hline 3491 & 62.3303 & 502.9574 & 455.8329 & 439 & 122.5994 & 1.48848 \\
\hline 3492 & 62.3473 & 466.6155 & 454.9156 & 439 & 122.5994 & 1.48812 \\
\hline 3493 & 62.3643 & 436.012 & 453.0419 & 438 & 122.5994 & 1.48775 \\
\hline 3494 & 62.3813 & 456.0261 & 452.3178 & 438 & 122.5994 & 1.48739 \\
\hline 3495 & 62.3983 & 445.1673 & 451.6038 & 438 & 122.5994 & 1.48702 \\
\hline 3496 & 62.4153 & 448.5634 & 450.1267 & 437 & 122.5994 & 1.48666 \\
\hline 3497 & 62.4323 & 463.2639 & 449.7663 & 437 & 122.5994 & 1.48629 \\
\hline 3498 & 62.4493 & 464.4308 & 448.4849 & 436 & 122.5994 & 1.48593 \\
\hline 3499 & 62.4663 & 466.0606 & 448.2396 & 436 & 122.5994 & 1.48557 \\
\hline 3500 & 62.4833 & 485.8512 & 447.0051 & 435 & 122.5994 & 1.4852 \\
\hline 3501 & 62.5003 & 478.3319 & 446.743 & 435 & 122.5994 & 1.48484 \\
\hline 3502 & 62.5173 & 462.5696 & 445.4967 & 434 & 122.5994 & 1.48448 \\
\hline 3503 & 62.5343 & 442.675 & 445.277 & 434 & 122.5994 & 1.48411 \\
\hline 3504 & 62.5513 & 460.1417 & 443.9966 & 433 & 122.5994 & 1.48375 \\
\hline 3505 & 62.5683 & 450.9431 & 443.6299 & 433 & 122.5994 & 1.48339 \\
\hline 3506 & 62.5853 & 437.7215 & 442.0082 & 432 & 122.5994 & 1.48303 \\
\hline 3507 & 62.6023 & 451.1119 & 441.5209 & 432 & 122.5994 & 1.48267 \\
\hline 3508 & 62.6193 & 446.4441 & 440.0485 & 431 & 122.5994 & 1.4823 \\
\hline 3509 & 62.6363 & 458.021 & 438.6756 & 430 & 122.5994 & 1.48194 \\
\hline 3510 & 62.6533 & 455.7787 & 438.4901 & 430 & 122.5994 & 1.48158 \\
\hline 3511 & 62.6703 & 449.3221 & 437.5258 & 429 & 122.5994 & 1.48122 \\
\hline 3512 & 62.6873 & 433.8325 & 437.8115 & 429 & 122.5994 & 1.48086 \\
\hline 3513 & 62.7043 & 401.6922 & 437.2324 & 428 & 122.5994 & 1.4805 \\
\hline 3514 & 62.7213 & 407.7086 & 436.7517 & 427 & 122.5994 & 1.48014 \\
\hline 3515 & 62.7383 & 424.3193 & 437.0026 & 427 & 122.5994 & 1.47978 \\
\hline 3516 & 62.7553 & 424.4166 & 435.6469 & 426 & 122.5994 & 1.47942 \\
\hline 3517 & 62.7723 & 432.4267 & 434.0044 & 425 & 122.5994 & 1.47906 \\
\hline 3518 & 62.7893 & 414.799 & 433.1555 & 425 & 122.5994 & 1.4787 \\
\hline 3519 & 62.8063 & 390.1901 & 431.397 & 424 & 122.5994 & 1.47834 \\
\hline 3520 & 62.8233 & 424.8026 & 430.8507 & 424 & 122.5994 & 1.47798 \\
\hline 3521 & 62.8403 & 413.7848 & 429.5783 & 423 & 122.5994 & 1.47762 \\
\hline 3522 & 62.8573 & 448.1611 & 428.5552 & 422 & 122.5994 & 1.47726 \\
\hline 3523 & 62.8743 & 445.728 & 428.7241 & 422 & 122.5994 & 1.4769 \\
\hline 3524 & 62.8913 & 440.9577 & 428.0324 & 421 & 122.5994 & 1.47655 \\
\hline 3525 & 62.9083 & 430.7571 & 427.3083 & 420 & 122.5994 & 1.47619 \\
\hline 3526 & 62.9253 & 414.5926 & 427.3919 & 420 & 122.5994 & 1.47583 \\
\hline
\end{tabular}




\begin{tabular}{|c|c|c|c|c|c|c|}
\hline 3527 & 62.9423 & 391.2589 & 426.2086 & 419 & 122.5994 & 1.47547 \\
\hline 3528 & 62.9593 & 431.4033 & 426.1583 & 419 & 122.5994 & 1.47512 \\
\hline 3529 & 62.9763 & 406.9403 & 425.2466 & 418 & 122.5994 & 1.47476 \\
\hline 3530 & 62.9933 & 438.5028 & 425.4952 & 418 & 122.5994 & 1.4744 \\
\hline 3531 & 63.0103 & 409.5648 & 425.9178 & 418 & 122.5994 & 1.47404 \\
\hline 3532 & 63.0273 & 404.0761 & 426.3295 & 418 & 122.5994 & 1.47369 \\
\hline 3533 & 63.0443 & 408.0758 & 426.6961 & 418 & 122.5994 & 1.47333 \\
\hline 3534 & 63.0613 & 424.4166 & 426.9789 & 418 & 122.5994 & 1.47297 \\
\hline 3535 & 63.0783 & 424.9599 & 427.1588 & 418 & 122.5994 & 1.47262 \\
\hline 3536 & 63.0953 & 440.0579 & 427.2264 & 418 & 122.5994 & 1.47226 \\
\hline 3537 & 63.1123 & 424.9947 & 427.1617 & 418 & 122.5994 & 1.47191 \\
\hline 3538 & 63.1293 & 430.4821 & 427.1269 & 418 & 122.5994 & 1.47155 \\
\hline 3539 & 63.1463 & 411.1379 & 427.1821 & 418 & 122.5994 & 1.4712 \\
\hline 3540 & 63.1633 & 406.3432 & 427.2068 & 418 & 122.5994 & 1.47084 \\
\hline 3541 & 63.1803 & 418.9375 & 427.3758 & 418 & 122.5994 & 1.47049 \\
\hline 3542 & 63.1973 & 407.5114 & 426.4333 & 417 & 122.5994 & 1.47013 \\
\hline 3543 & 63.2143 & 394.0583 & 426.4538 & 417 & 122.5994 & 1.46978 \\
\hline 3544 & 63.2313 & 414.2346 & 426.3567 & 417 & 122.5994 & 1.46942 \\
\hline 3545 & 63.2483 & 408.0009 & 426.2853 & 417 & 122.5994 & 1.46907 \\
\hline 3546 & 63.2653 & 425.395 & 426.0834 & 417 & 122.5994 & 1.46872 \\
\hline 3547 & 63.2823 & 453.3996 & 425.9684 & 417 & 122.5994 & 1.46836 \\
\hline 3548 & 63.2993 & 408.2962 & 425.9932 & 417 & 122.5994 & 1.46801 \\
\hline 3549 & 63.3163 & 408.2381 & 426.0979 & 417 & 122.5994 & 1.46765 \\
\hline 3550 & 63.3333 & 422.8349 & 426.2006 & 417 & 122.5994 & 1.4673 \\
\hline 3551 & 63.3503 & 414.0889 & 426.4478 & 417 & 122.5994 & 1.46695 \\
\hline 3552 & 63.3673 & 422.3363 & 426.9614 & 417 & 122.5994 & 1.4666 \\
\hline 3553 & 63.3843 & 435.326 & 428.0706 & 417 & 122.5994 & 1.46624 \\
\hline 3554 & 63.4013 & 414.2995 & 429.8672 & 417 & 122.5994 & 1.46589 \\
\hline 3555 & 63.4183 & 414.0536 & 432.3252 & 417 & 122.5994 & 1.46554 \\
\hline 3556 & 63.4353 & 420.1413 & 434.929 & 417 & 122.5994 & 1.46519 \\
\hline 3557 & 63.4523 & 409.8095 & 436.8622 & 417 & 122.5994 & 1.46484 \\
\hline 3558 & 63.4693 & 411.1863 & 437.1534 & 417 & 122.5994 & 1.46449 \\
\hline 3559 & 63.4863 & 428.9137 & 435.8817 & 417 & 122.5994 & 1.46413 \\
\hline 3560 & 63.5033 & 432.6977 & 433.9907 & 417 & 122.5994 & 1.46378 \\
\hline 3561 & 63.5203 & 425.1899 & 432.2442 & 417 & 122.5994 & 1.46343 \\
\hline 3562 & 63.5373 & 420.2097 & 431.0126 & 417 & 122.5994 & 1.46308 \\
\hline 3563 & 63.5543 & 436.3058 & 430.5381 & 417 & 122.5994 & 1.46273 \\
\hline 3564 & 63.5713 & 415.2184 & 430.8262 & 417 & 122.5994 & 1.46238 \\
\hline 3565 & 63.5883 & 400.0533 & 430.6472 & 416 & 122.5994 & 1.46203 \\
\hline 3566 & 63.6053 & 425.9314 & 431.6096 & 416 & 122.5994 & 1.46168 \\
\hline 3567 & 63.6223 & 449.1427 & 432.3194 & 416 & 122.5994 & 1.46133 \\
\hline 3568 & 63.6393 & 434.7823 & 432.304 & 416 & 122.5994 & 1.46098 \\
\hline 3569 & 63.6563 & 415.8209 & 431.3972 & 416 & 122.5994 & 1.46063 \\
\hline 3570 & 63.6733 & 402.5739 & 430.0244 & 416 & 122.5994 & 1.46028 \\
\hline 3571 & 63.6903 & 393.4896 & 428.6838 & 416 & 122.5994 & 1.45994 \\
\hline 3572 & 63.7073 & 408.8284 & 427.6002 & 416 & 122.5994 & 1.45959 \\
\hline 3573 & 63.7243 & 422.5553 & 426.892 & 416 & 122.5994 & 1.45924 \\
\hline 3574 & 63.7413 & 406.461 & 426.4998 & 416 & 122.5994 & 1.45889 \\
\hline 3575 & 63.7583 & 404.0639 & 426.3355 & 416 & 122.5994 & 1.45854 \\
\hline
\end{tabular}




\begin{tabular}{|c|c|c|c|c|c|c|}
\hline 3576 & 63.7753 & 434.1679 & 426.2527 & 416 & 122.5994 & 1.45819 \\
\hline 3577 & 63.7923 & 379.9075 & 426.1956 & 416 & 122.5994 & 1.45785 \\
\hline 3578 & 63.8093 & 446.751 & 426.2154 & 416 & 122.5994 & 1.4575 \\
\hline 3579 & 63.8263 & 412.6971 & 427.3676 & 417 & 122.5994 & 1.45715 \\
\hline 3580 & 63.8433 & 432.5158 & 427.7297 & 417 & 122.5994 & 1.4568 \\
\hline 3581 & 63.8603 & 420.0749 & 428.4432 & 417 & 122.5994 & 1.45646 \\
\hline 3582 & 63.8773 & 411.2506 & 430.6337 & 418 & 122.5994 & 1.45611 \\
\hline 3583 & 63.8943 & 400.4855 & 432.3766 & 418 & 122.5994 & 1.45576 \\
\hline 3584 & 63.9113 & 455.5661 & 434.6565 & 418 & 122.5994 & 1.45542 \\
\hline 3585 & 63.9283 & 433.175 & 438.2519 & 419 & 122.5994 & 1.45507 \\
\hline 3586 & 63.9453 & 437.9095 & 440.7332 & 419 & 122.5994 & 1.45473 \\
\hline 3587 & 63.9623 & 441.241 & 442.8427 & 419 & 122.5994 & 1.45438 \\
\hline 3588 & 63.9793 & 422.5232 & 445.8542 & 420 & 122.5994 & 1.45404 \\
\hline 3589 & 63.9963 & 435.1172 & 447.9252 & 420 & 122.5994 & 1.45369 \\
\hline 3590 & 64.0133 & 455.8443 & 449.5036 & 420 & 122.5994 & 1.45335 \\
\hline 3591 & 64.0303 & 477.2254 & 451.1506 & 421 & 122.5994 & 1.453 \\
\hline 3592 & 64.0473 & 439.5809 & 451.4052 & 421 & 122.5994 & 1.45266 \\
\hline 3593 & 64.0643 & 477.2417 & 452.3514 & 421 & 122.5994 & 1.45231 \\
\hline 3594 & 64.0813 & 490.1815 & 454.7925 & 421 & 122.5994 & 1.45197 \\
\hline 3595 & 64.0983 & 486.7541 & 460.2425 & 422 & 122.5994 & 1.45162 \\
\hline 3596 & 64.1153 & 497.6126 & 467.0141 & 422 & 122.5994 & 1.45128 \\
\hline 3597 & 64.1323 & 502.9785 & 476.2484 & 422 & 122.5994 & 1.45094 \\
\hline 3598 & 64.1493 & 497.1283 & 489.0848 & 423 & 122.5994 & 1.45059 \\
\hline 3599 & 64.1663 & 495.5073 & 502.7938 & 423 & 122.5994 & 1.45025 \\
\hline 3600 & 64.1833 & 464.7756 & 516.1998 & 423 & 122.5994 & 1.44991 \\
\hline 3601 & 64.2003 & 454.0147 & 525.9293 & 424 & 122.5994 & 1.44956 \\
\hline 3602 & 64.2173 & 459.1907 & 525.4666 & 424 & 122.5994 & 1.44922 \\
\hline 3603 & 64.2343 & 447.551 & 515.9772 & 424 & 122.5994 & 1.44888 \\
\hline 3604 & 64.2513 & 455.6084 & 503.1873 & 425 & 122.5994 & 1.44854 \\
\hline 3605 & 64.2683 & 440.1467 & 489.7944 & 425 & 122.5994 & 1.44819 \\
\hline 3606 & 64.2853 & 461.7738 & 479.4348 & 425 & 122.5994 & 1.44785 \\
\hline 3607 & 64.3023 & 458.7903 & 474.1627 & 426 & 122.5994 & 1.44751 \\
\hline 3608 & 64.3193 & 441.0588 & 472.0352 & 426 & 122.5994 & 1.44717 \\
\hline 3609 & 64.3363 & 432.9676 & 473.2117 & 426 & 122.5994 & 1.44683 \\
\hline 3610 & 64.3533 & 442.212 & 477.4284 & 427 & 122.5994 & 1.44649 \\
\hline 3611 & 64.3703 & 443.8121 & 480.6745 & 427 & 122.5994 & 1.44614 \\
\hline 3612 & 64.3873 & 398.6291 & 481.2724 & 427 & 122.5994 & 1.4458 \\
\hline 3613 & 64.4043 & 403.1221 & 478.5339 & 428 & 122.5994 & 1.44546 \\
\hline 3614 & 64.4213 & 394.4233 & 471.5781 & 428 & 122.5994 & 1.44512 \\
\hline 3615 & 64.4383 & 394.1613 & 463.7435 & 428 & 122.5994 & 1.44478 \\
\hline 3616 & 64.4553 & 389.6938 & 457.7259 & 429 & 122.5994 & 1.44444 \\
\hline 3617 & 64.4723 & 410.6789 & 452.173 & 429 & 122.5994 & 1.4441 \\
\hline 3618 & 64.4893 & 440.1684 & 448.0504 & 429 & 122.5994 & 1.44376 \\
\hline 3619 & 64.5063 & 420.4967 & 445.9097 & 430 & 122.5994 & 1.44342 \\
\hline 3620 & 64.5233 & 402.1986 & 443.3562 & 430 & 122.5994 & 1.44308 \\
\hline 3621 & 64.5403 & 382.4949 & 441.2856 & 430 & 122.5994 & 1.44275 \\
\hline 3622 & 64.5573 & 416.5353 & 440.6797 & 431 & 122.5994 & 1.44241 \\
\hline 3623 & 64.5743 & 427.9847 & 439.5798 & 431 & 122.5994 & 1.44207 \\
\hline 3624 & 64.5913 & 393.7912 & 438.9596 & 431 & 122.5994 & 1.44173 \\
\hline
\end{tabular}




$\begin{array}{rrrrrrr}3625 & 64.6083 & 431.6843 & 438.8209 & 431 & 122.5994 & 1.44139 \\ 3626 & 64.6253 & 399.978 & 440.1311 & 432 & 122.5994 & 1.44105 \\ 3627 & 64.6423 & 419.3695 & 440.8444 & 432 & 122.5994 & 1.44071 \\ 3628 & 64.6593 & 370.2294 & 441.7547 & 432 & 122.5994 & 1.44038 \\ 3629 & 64.6763 & 410.4415 & 443.4675 & 433 & 122.5994 & 1.44004 \\ 3630 & 64.6933 & 387.1936 & 443.5643 & 433 & 122.5994 & 1.4397 \\ 3631 & 64.7103 & 422.033 & 443.0013 & 433 & 122.5994 & 1.43936 \\ 3632 & 64.7273 & 432.5523 & 443.1276 & 434 & 122.5994 & 1.43903 \\ 3633 & 64.7443 & 402.3027 & 442.3385 & 434 & 122.5994 & 1.43869 \\ 3634 & 64.7613 & 394.7927 & 441.7788 & 434 & 122.5994 & 1.43835 \\ 3635 & 64.7783 & 404.8678 & 442.4497 & 435 & 122.5994 & 1.43802 \\ 3636 & 64.7953 & 387.9551 & 442.2701 & 435 & 122.5994 & 1.43768 \\ 3637 & 64.8123 & 385.6026 & 442.1947 & 435 & 122.5994 & 1.43735 \\ 3638 & 64.8293 & 422.3068 & 443.2435 & 436 & 122.5994 & 1.43701 \\ 3639 & 64.8463 & 403.8786 & 443.3976 & 436 & 122.5994 & 1.43667 \\ 3640 & 64.8633 & 396.9891 & 443.4917 & 436 & 122.5994 & 1.43634 \\ 3641 & 64.8803 & 397.0344 & 444.5419 & 437 & 122.5994 & 1.436 \\ 3642 & 64.8973 & 422.748 & 444.5797 & 437 & 122.5994 & 1.43567 \\ 3643 & 64.9143 & 411.4356 & 444.8229 & 437 & 122.5994 & 1.43533 \\ 3644 & 64.9313 & 434.0447 & 446.4873 & 438 & 122.5994 & 1.435 \\ 3645 & 64.9483 & 416.7317 & 447.7196 & 438 & 122.5994 & 1.43466 \\ 3646 & 64.9653 & 410.3063 & 449.5481 & 438 & 122.5994 & 1.43433 \\ 3647 & 64.9823 & 438.5756 & 451.8264 & 438 & 122.5994 & 1.434\end{array}$


No. Pos. [ ${ }^{\circ} 2$ Th. lobs [cts] Icalc [cts] lback [cts] CT [s] ESD D spacings

\begin{tabular}{|c|c|c|c|c|c|c|}
\hline 1 & 3.0003 & 1814.755 & 1815 & 1815 & 122.5994 & 29.42347 \\
\hline 2 & 3.0173 & 1786.968 & 1788 & 1788 & 122.5994 & 29.25773 \\
\hline 3 & 3.0343 & 1679.254 & 1762 & 1762 & 122.5994 & 29.09385 \\
\hline 4 & 3.0513 & 1635.093 & 1735 & 1735 & 122.5994 & 28.9318 \\
\hline 5 & 3.0683 & 1559.555 & 1709 & 1709 & 122.5994 & 28.77154 \\
\hline 6 & 3.0853 & 1543.172 & 1682 & 1682 & 122.5994 & 28.61305 \\
\hline 7 & 3.1023 & 1467.887 & 1656 & 1656 & 122.5994 & 28.45629 \\
\hline 8 & 3.1193 & 1467.659 & 1629 & 1629 & 122.5994 & 28.30124 \\
\hline 9 & 3.1363 & 1438.04 & 1603 & 1603 & 122.5994 & 28.14788 \\
\hline 10 & 3.1533 & 1461.761 & 1576 & 1576 & 122.5994 & 27.99617 \\
\hline 11 & 3.1703 & 1377.995 & 1550 & 1550 & 122.5994 & 27.84608 \\
\hline 12 & 3.1873 & 1371.636 & 1523 & 1523 & 122.5994 & 27.6976 \\
\hline 13 & 3.2043 & 1334.143 & 1497 & 1497 & 122.5994 & 27.55069 \\
\hline 14 & 3.2213 & 1315.398 & 1470 & 1470 & 122.5994 & 27.40534 \\
\hline 15 & 3.2383 & 1263.886 & 1444 & 1444 & 122.5994 & 27.26151 \\
\hline 16 & 3.2553 & 1288.816 & 1417 & 1417 & 122.5994 & 27.11918 \\
\hline 17 & 3.2723 & 1288.816 & 1391 & 1391 & 122.5994 & 26.97833 \\
\hline 18 & 3.2893 & 1302.02 & 1364 & 1364 & 122.5994 & 26.83894 \\
\hline 19 & 3.3063 & 1278.154 & 1338 & 1338 & 122.5994 & 26.70098 \\
\hline 20 & 3.3233 & 1206.937 & 1311 & 1311 & 122.5994 & 26.56443 \\
\hline 21 & 3.3403 & 1229.153 & 1285 & 1285 & 122.5994 & 26.42928 \\
\hline 22 & 3.3573 & 1234.134 & 1258 & 1258 & 122.5994 & 26.29549 \\
\hline 23 & 3.3743 & 1214.345 & 1232 & 1232 & 122.5994 & 26.16305 \\
\hline 24 & 3.3913 & 1204.277 & 1205 & 1205 & 122.5994 & 26.03194 \\
\hline 25 & 3.4083 & 1155.124 & 1179 & 1179 & 122.5994 & 25.90213 \\
\hline 26 & 3.4253 & 1166.277 & 1169 & 1169 & 122.5994 & 25.77362 \\
\hline 27 & 3.4423 & 1173.394 & 1159 & 1159 & 122.5994 & 25.64637 \\
\hline 28 & 3.4593 & 1137.435 & 1150 & 1150 & 122.5994 & 25.52038 \\
\hline 29 & 3.4763 & 1133.019 & 1140 & 1140 & 122.5994 & 25.39561 \\
\hline 30 & 3.4933 & 1123.134 & 1130 & 1130 & 122.5994 & 25.27207 \\
\hline 31 & 3.5103 & 1094.151 & 1120 & 1120 & 122.5994 & 25.14971 \\
\hline 32 & 3.5273 & 1060.637 & 1110 & 1110 & 122.5994 & 25.02854 \\
\hline 33 & 3.5443 & 1058.715 & 1100 & 1100 & 122.5994 & 24.90853 \\
\hline 34 & 3.5613 & 1086.074 & 1090 & 1090 & 122.5994 & 24.78967 \\
\hline 35 & 3.5783 & 1043.228 & 1081 & 1081 & 122.5994 & 24.67194 \\
\hline 36 & 3.5953 & 1019.192 & 1071 & 1071 & 122.5994 & 24.55532 \\
\hline 37 & 3.6123 & 1057.645 & 1061 & 1061 & 122.5994 & 24.4398 \\
\hline 38 & 3.6293 & 1012.971 & 1051 & 1051 & 122.5994 & 24.32536 \\
\hline 39 & 3.6463 & 1040.426 & 1041 & 1041 & 122.5994 & 24.21198 \\
\hline 40 & 3.6633 & 1060.673 & 1032 & 1032 & 122.5994 & 24.09966 \\
\hline 41 & 3.6803 & 975.1701 & 1022 & 1022 & 122.5994 & 23.98838 \\
\hline 42 & 3.6973 & 1005.324 & 1012 & 1012 & 122.5994 & 23.87812 \\
\hline 43 & 3.7143 & 965.8432 & 1002 & 1002 & 122.5994 & 23.76887 \\
\hline 44 & 3.7313 & 955.2635 & 992 & 992 & 122.5994 & 23.66062 \\
\hline 45 & 3.7483 & 960.6743 & 982 & 982 & 122.5994 & 23.55335 \\
\hline 46 & 3.7653 & 1009.648 & 972 & 972 & 122.5994 & 23.44704 \\
\hline 47 & 3.7823 & 1049.37 & 963 & 963 & 122.5994 & 23.3417 \\
\hline
\end{tabular}




\begin{tabular}{|c|c|c|c|c|c|c|}
\hline 48 & 3.7993 & 942.2876 & 953 & 953 & 122.5994 & 23.23729 \\
\hline 49 & 3.8163 & 924.164 & 943 & 943 & 122.5994 & 23.13382 \\
\hline 50 & 3.8333 & 923.4928 & 937 & 937 & 122.5994 & 23.03126 \\
\hline 51 & 3.8503 & 912.9628 & 931 & 931 & 122.5994 & 22.92961 \\
\hline 52 & 3.8673 & 922.3974 & 924 & 924 & 122.5994 & 22.82886 \\
\hline 53 & 3.8843 & 910.3363 & 918 & 918 & 122.5994 & 22.72898 \\
\hline 54 & 3.9013 & 932.3544 & 912 & 912 & 122.5994 & 22.62998 \\
\hline 55 & 3.9183 & 871.2105 & 906 & 906 & 122.5994 & 22.53184 \\
\hline 56 & 3.9353 & 872.9108 & 900 & 900 & 122.5994 & 22.43454 \\
\hline 57 & 3.9523 & 859.6966 & 894 & 894 & 122.5994 & 22.33808 \\
\hline 58 & 3.9693 & 853.8574 & 888 & 888 & 122.5994 & 22.24245 \\
\hline 59 & 3.9863 & 817.9636 & 881 & 881 & 122.5994 & 22.14763 \\
\hline 60 & 4.0033 & 816.4133 & 875 & 875 & 122.5994 & 22.05362 \\
\hline 61 & 4.0203 & 829.1178 & 869 & 869 & 122.5994 & 21.9604 \\
\hline 62 & 4.0373 & 859.3163 & 863 & 863 & 122.5994 & 21.86797 \\
\hline 63 & 4.0543 & 831.354 & 857 & 857 & 122.5994 & 21.77632 \\
\hline 64 & 4.0713 & 850.8366 & 850 & 850 & 122.5994 & 21.68543 \\
\hline 65 & 4.0883 & 866.9053 & 844 & 844 & 122.5994 & 21.59529 \\
\hline 66 & 4.1053 & 800.9828 & 838 & 838 & 122.5994 & 21.50591 \\
\hline 67 & 4.1223 & 805.5273 & 832 & 832 & 122.5994 & 21.41726 \\
\hline 68 & 4.1393 & 827.5842 & 826 & 826 & 122.5994 & 21.32933 \\
\hline 69 & 4.1563 & 797.0568 & 820 & 820 & 122.5994 & 21.24213 \\
\hline 70 & 4.1733 & 772.8544 & 814 & 814 & 122.5994 & 21.15564 \\
\hline 71 & 4.1903 & 770.0232 & 807 & 807 & 122.5994 & 21.06985 \\
\hline 72 & 4.2073 & 773.1498 & 801 & 801 & 122.5994 & 20.98475 \\
\hline 73 & 4.2243 & 822.403 & 795 & 795 & 122.5994 & 20.90034 \\
\hline 74 & 4.2413 & 815.9709 & 790 & 790 & 122.5994 & 20.81661 \\
\hline 75 & 4.2583 & 764.3076 & 785 & 785 & 122.5994 & 20.73354 \\
\hline 76 & 4.2753 & 714.521 & 781 & 781 & 122.5994 & 20.65114 \\
\hline 77 & 4.2923 & 746.7707 & 776 & 776 & 122.5994 & 20.56939 \\
\hline 78 & 4.3093 & 785.7578 & 771 & 771 & 122.5994 & 20.48828 \\
\hline 79 & 4.3263 & 778.2501 & 766 & 766 & 122.5994 & 20.40781 \\
\hline 80 & 4.3433 & 753.5533 & 761 & 761 & 122.5994 & 20.32797 \\
\hline 81 & 4.3603 & 729.645 & 757 & 757 & 122.5994 & 20.24875 \\
\hline 82 & 4.3773 & 739.728 & 752 & 752 & 122.5994 & 20.17015 \\
\hline 83 & 4.3943 & 774.8698 & 747 & 747 & 122.5994 & 20.09216 \\
\hline 84 & 4.4113 & 740.7712 & 742 & 742 & 122.5994 & 20.01477 \\
\hline 85 & 4.4283 & 755.4631 & 738 & 738 & 122.5994 & 19.93797 \\
\hline 86 & 4.4453 & 766.3361 & 733 & 733 & 122.5994 & 19.86176 \\
\hline 87 & 4.4623 & 716.5092 & 728 & 728 & 122.5994 & 19.78613 \\
\hline 88 & 4.4793 & 698.3106 & 723 & 723 & 122.5994 & 19.71108 \\
\hline 89 & 4.4963 & 725.5784 & 718 & 718 & 122.5994 & 19.63659 \\
\hline 90 & 4.5133 & 685.2876 & 714 & 714 & 122.5994 & 19.56267 \\
\hline 91 & 4.5303 & 700.9065 & 709 & 709 & 122.5994 & 19.48929 \\
\hline 92 & 4.5473 & 677.0962 & 704 & 704 & 122.5994 & 19.41647 \\
\hline 93 & 4.5643 & 640.8385 & 699 & 699 & 122.5994 & 19.34419 \\
\hline 94 & 4.5813 & 686.4279 & 694 & 694 & 122.5994 & 19.27245 \\
\hline 95 & 4.5983 & 680.4102 & 690 & 690 & 122.5994 & 19.20124 \\
\hline 96 & 4.6153 & 647.4352 & 685 & 685 & 122.5994 & 19.13055 \\
\hline
\end{tabular}




\begin{tabular}{|c|c|c|c|c|c|c|}
\hline 97 & 4.6323 & 695.5791 & 680 & 680 & 122.5994 & 19.06038 \\
\hline 98 & 4.6493 & 677.838 & 676 & 676 & 122.5994 & 18.99073 \\
\hline 99 & 4.6663 & 692.9927 & 673 & 673 & 122.5994 & 18.92158 \\
\hline 100 & 4.6833 & 659.816 & 669 & 669 & 122.5994 & 18.85293 \\
\hline 101 & 4.7003 & 652.291 & 665 & 665 & 122.5994 & 18.78479 \\
\hline 102 & 4.7173 & 643.9331 & 661 & 661 & 122.5994 & 18.71713 \\
\hline 103 & 4.7343 & 677.4113 & 658 & 658 & 122.5994 & 18.64996 \\
\hline 104 & 4.7513 & 642.2984 & 654 & 654 & 122.5994 & 18.58327 \\
\hline 105 & 4.7683 & 652.6775 & 650 & 650 & 122.5994 & 18.51705 \\
\hline 106 & 4.7853 & 656.5284 & 647 & 647 & 122.5994 & 18.45131 \\
\hline 107 & 4.8023 & 648.017 & 643 & 643 & 122.5994 & 18.38603 \\
\hline 108 & 4.8193 & 655.3535 & 639 & 639 & 122.5994 & 18.32121 \\
\hline 109 & 4.8363 & 655.3722 & 636 & 636 & 122.5994 & 18.25685 \\
\hline 110 & 4.8533 & 639.7228 & 632 & 632 & 122.5994 & 18.19294 \\
\hline 111 & 4.8703 & 626.6882 & 628 & 628 & 122.5994 & 18.12947 \\
\hline 112 & 4.8873 & 653.3879 & 624 & 624 & 122.5994 & 18.06645 \\
\hline 113 & 4.9043 & 633.7849 & 621 & 621 & 122.5994 & 18.00386 \\
\hline 114 & 4.9213 & 603.7304 & 617 & 617 & 122.5994 & 17.94171 \\
\hline 115 & 4.9383 & 586.6036 & 613 & 613 & 122.5994 & 17.87998 \\
\hline 116 & 4.9553 & 599.2385 & 610 & 610 & 122.5994 & 17.81868 \\
\hline 117 & 4.9723 & 610.2959 & 606 & 606 & 122.5994 & 17.7578 \\
\hline 118 & 4.9893 & 597.9013 & 602 & 602 & 122.5994 & 17.69733 \\
\hline 119 & 5.0063 & 600.244 & 598 & 598 & 122.5994 & 17.63727 \\
\hline 120 & 5.0233 & 597.5035 & 595 & 595 & 122.5994 & 17.57762 \\
\hline 121 & 5.0403 & 595.3711 & 591 & 591 & 122.5994 & 17.51837 \\
\hline 122 & 5.0573 & 575.0353 & 590 & 590 & 122.5994 & 17.45953 \\
\hline 123 & 5.0743 & 591.9331 & 588 & 588 & 122.5994 & 17.40107 \\
\hline 124 & 5.0913 & 603.7398 & 587 & 587 & 122.5994 & 17.34301 \\
\hline 125 & 5.1083 & 590.4843 & 585 & 585 & 122.5994 & 17.28533 \\
\hline 126 & 5.1253 & 575.6844 & 584 & 584 & 122.5994 & 17.22803 \\
\hline 127 & 5.1423 & 598.8666 & 582 & 582 & 122.5994 & 17.17112 \\
\hline 128 & 5.1593 & 571.1629 & 581 & 581 & 122.5994 & 17.11458 \\
\hline 129 & 5.1763 & 556.9704 & 579 & 579 & 122.5994 & 17.05841 \\
\hline 130 & 5.1933 & 571.722 & 578 & 578 & 122.5994 & 17.00261 \\
\hline 131 & 5.2103 & 575.9943 & 576 & 576 & 122.5994 & 16.94717 \\
\hline 132 & 5.2273 & 552.7285 & 575 & 575 & 122.5994 & 16.89209 \\
\hline 133 & 5.2443 & 524.2655 & 574 & 574 & 122.5994 & 16.83737 \\
\hline 134 & 5.2613 & 572.3701 & 572 & 572 & 122.5994 & 16.78301 \\
\hline 135 & 5.2783 & 585.0867 & 571 & 571 & 122.5994 & 16.72899 \\
\hline 136 & 5.2953 & 581.2645 & 569 & 569 & 122.5994 & 16.67532 \\
\hline 137 & 5.3123 & 524.3335 & 568 & 568 & 122.5994 & 16.622 \\
\hline 138 & 5.3293 & 498.4782 & 566 & 566 & 122.5994 & 16.56901 \\
\hline 139 & 5.3463 & 541.4827 & 565 & 565 & 122.5994 & 16.51637 \\
\hline 140 & 5.3633 & 580.454 & 563 & 563 & 122.5994 & 16.46405 \\
\hline 141 & 5.3803 & 552.5361 & 562 & 562 & 122.5994 & 16.41207 \\
\hline 142 & 5.3973 & 575.0334 & 560 & 560 & 122.5994 & 16.36041 \\
\hline 143 & 5.4143 & 550.7566 & 559 & 559 & 122.5994 & 16.30908 \\
\hline 144 & 5.4313 & 597.9016 & 557 & 557 & 122.5994 & 16.25807 \\
\hline 145 & 5.4483 & 515.763 & 556 & 556 & 122.5994 & 16.20738 \\
\hline
\end{tabular}




\begin{tabular}{|c|c|c|c|c|c|c|}
\hline 146 & 5.4653 & 562.8416 & 554 & 554 & 122.5994 & 16.15701 \\
\hline 147 & 5.4823 & 563.2987 & 552 & 552 & 122.5994 & 16.10695 \\
\hline 148 & 5.4993 & 519.7803 & 550 & 550 & 122.5994 & 16.05719 \\
\hline 149 & 5.5163 & 544.6263 & 548 & 548 & 122.5994 & 16.00775 \\
\hline 150 & 5.5333 & 548.4061 & 547 & 547 & 122.5994 & 15.9586 \\
\hline 151 & 5.5503 & 533.62 & 545 & 545 & 122.5994 & 15.90976 \\
\hline 152 & 5.5673 & 551.4179 & 543 & 543 & 122.5994 & 15.86122 \\
\hline 153 & 5.5843 & 582.0776 & 541 & 541 & 122.5994 & 15.81297 \\
\hline 154 & 5.6013 & 530.6889 & 539 & 539 & 122.5994 & 15.76502 \\
\hline 155 & 5.6183 & 546.7497 & 537 & 537 & 122.5994 & 15.71735 \\
\hline 156 & 5.6353 & 515.3191 & 535 & 535 & 122.5994 & 15.66998 \\
\hline 157 & 5.6523 & 505.3395 & 534 & 534 & 122.5994 & 15.62289 \\
\hline 158 & 5.6693 & 519.8348 & 532 & 532 & 122.5994 & 15.57608 \\
\hline 159 & 5.6863 & 525.3368 & 530 & 530 & 122.5994 & 15.52955 \\
\hline 160 & 5.7033 & 529.7459 & 528 & 528 & 122.5994 & 15.4833 \\
\hline 161 & 5.7203 & 514.3798 & 526 & 526 & 122.5994 & 15.43732 \\
\hline 162 & 5.7373 & 527.3739 & 524 & 524 & 122.5994 & 15.39162 \\
\hline 163 & 5.7543 & 563.8909 & 522 & 522 & 122.5994 & 15.34618 \\
\hline 164 & 5.7713 & 556.8418 & 520 & 520 & 122.5994 & 15.30102 \\
\hline 165 & 5.7883 & 545.3534 & 518 & 518 & 122.5994 & 15.25612 \\
\hline 166 & 5.8053 & 555.3445 & 517 & 517 & 122.5994 & 15.21148 \\
\hline 167 & 5.8223 & 556.2876 & 516.2391 & 515 & 122.5994 & 15.16711 \\
\hline 168 & 5.8393 & 535.7296 & 514.3504 & 513 & 122.5994 & 15.12299 \\
\hline 169 & 5.8563 & 510.4959 & 512.4773 & 511 & 122.5994 & 15.07913 \\
\hline 170 & 5.8733 & 473.8874 & 512.6229 & 511 & 122.5994 & 15.03552 \\
\hline 171 & 5.8903 & 495.6645 & 513.7908 & 512 & 122.5994 & 14.99216 \\
\hline 172 & 5.9073 & 534.048 & 513.9861 & 512 & 122.5994 & 14.94906 \\
\hline 173 & 5.9243 & 546.1573 & 515.2148 & 513 & 122.5994 & 14.9062 \\
\hline 174 & 5.9413 & 498.4879 & 515.485 & 513 & 122.5994 & 14.86358 \\
\hline 175 & 5.9583 & 523.2163 & 516.8071 & 514 & 122.5994 & 14.82121 \\
\hline 176 & 5.9753 & 556.1819 & 517.1953 & 514 & 122.5994 & 14.77909 \\
\hline 177 & 5.9923 & 528.7697 & 518.6685 & 515 & 122.5994 & 14.7372 \\
\hline 178 & 6.0093 & 561.3615 & 519.2534 & 515 & 122.5994 & 14.69554 \\
\hline 179 & 6.0263 & 559.3784 & 520.9886 & 516 & 122.5994 & 14.65413 \\
\hline 180 & 6.0433 & 548.967 & 521.936 & 516 & 122.5994 & 14.61294 \\
\hline 181 & 6.0603 & 531.3529 & 524.2107 & 517 & 122.5994 & 14.57199 \\
\hline 182 & 6.0773 & 520.8017 & 526.0569 & 517 & 122.5994 & 14.53126 \\
\hline 183 & 6.0943 & 558.1832 & 530.013 & 518 & 122.5994 & 14.49077 \\
\hline 184 & 6.1113 & 584.137 & 535.1671 & 518 & 122.5994 & 14.4505 \\
\hline 185 & 6.1283 & 581.0023 & 545.3633 & 519 & 122.5994 & 14.41045 \\
\hline 186 & 6.1453 & 643.9173 & 560.9858 & 519 & 122.5994 & 14.37062 \\
\hline 187 & 6.1623 & 665.4526 & 585.9127 & 520 & 122.5994 & 14.33102 \\
\hline 188 & 6.1793 & 577.1648 & 617.5538 & 520 & 122.5994 & 14.29163 \\
\hline 189 & 6.1963 & 625.9021 & 652.0366 & 521 & 122.5994 & 14.25246 \\
\hline 190 & 6.2133 & 644.1451 & 673.99 & 521 & 122.5994 & 14.2135 \\
\hline 191 & 6.2303 & 626.5401 & 671.9796 & 522 & 122.5994 & 14.17475 \\
\hline 192 & 6.2473 & 594.8157 & 646.3134 & 522 & 122.5994 & 14.13622 \\
\hline 193 & 6.2643 & 627.392 & 612.5373 & 522 & 122.5994 & 14.0979 \\
\hline 194 & 6.2813 & 621.756 & 583.3238 & 523 & 122.5994 & 14.05978 \\
\hline
\end{tabular}




\begin{tabular}{|c|c|c|c|c|c|c|}
\hline 195 & 6.2983 & 673.1366 & 561.21 & 523 & 122.5994 & 14.02187 \\
\hline 196 & 6.3153 & 611.0701 & 548.1017 & 524 & 122.5994 & 13.98416 \\
\hline 197 & 6.3323 & 618.2853 & 539.9023 & 524 & 122.5994 & 13.94666 \\
\hline 198 & 6.3493 & 601.5387 & 536.3029 & 525 & 122.5994 & 13.90935 \\
\hline 199 & 6.3663 & 514.3218 & 533.6268 & 525 & 122.5994 & 13.87225 \\
\hline 200 & 6.3833 & 529.6114 & 532.9211 & 526 & 122.5994 & 13.83534 \\
\hline 201 & 6.4003 & 535.8947 & 531.7242 & 526 & 122.5994 & 13.79863 \\
\hline 202 & 6.4173 & 527.336 & 531.8256 & 527 & 122.5994 & 13.76212 \\
\hline 203 & 6.4343 & 533.327 & 531.1246 & 527 & 122.5994 & 13.72579 \\
\hline 204 & 6.4513 & 516.9583 & 531.5648 & 528 & 122.5994 & 13.68966 \\
\hline 205 & 6.4683 & 512.8106 & 531.1105 & 528 & 122.5994 & 13.65372 \\
\hline 206 & 6.4853 & 530.2876 & 531.7371 & 529 & 122.5994 & 13.61797 \\
\hline 207 & 6.5023 & 554.9482 & 531.4264 & 529 & 122.5994 & 13.5824 \\
\hline 208 & 6.5193 & 527.3701 & 532.1654 & 530 & 122.5994 & 13.54702 \\
\hline 209 & 6.5363 & 537.4811 & 531.944 & 530 & 122.5994 & 13.51183 \\
\hline 210 & 6.5533 & 536.249 & 532.7547 & 531 & 122.5994 & 13.47682 \\
\hline 211 & 6.5703 & 529.6082 & 532.5916 & 531 & 122.5994 & 13.44198 \\
\hline 212 & 6.5873 & 540.3739 & 533.4501 & 532 & 122.5994 & 13.40733 \\
\hline 213 & 6.6043 & 538.2346 & 533.3266 & 532 & 122.5994 & 13.37286 \\
\hline 214 & 6.6213 & 516.9832 & 534.2181 & 533 & 122.5994 & 13.33856 \\
\hline 215 & 6.6383 & 529.4402 & 533.3914 & 533 & 122.5994 & 13.30444 \\
\hline 216 & 6.6553 & 541.7358 & 534 & 534 & 122.5994 & 13.2705 \\
\hline 217 & 6.6723 & 535.2366 & 534 & 534 & 122.5994 & 13.23672 \\
\hline 218 & 6.6893 & 533.6047 & 534 & 534 & 122.5994 & 13.20312 \\
\hline 219 & 6.7063 & 530.4365 & 533 & 533 & 122.5994 & 13.16969 \\
\hline 220 & 6.7233 & 522.4722 & 533 & 533 & 122.5994 & 13.13643 \\
\hline 221 & 6.7403 & 557.5186 & 533 & 533 & 122.5994 & 13.10334 \\
\hline 222 & 6.7573 & 542.8286 & 533 & 533 & 122.5994 & 13.07041 \\
\hline 223 & 6.7743 & 501.9421 & 532 & 532 & 122.5994 & 13.03765 \\
\hline 224 & 6.7913 & 566.9008 & 532 & 532 & 122.5994 & 13.00505 \\
\hline 225 & 6.8083 & 535.4633 & 532 & 532 & 122.5994 & 12.97261 \\
\hline 226 & 6.8253 & 563.7712 & 531 & 531 & 122.5994 & 12.94034 \\
\hline 227 & 6.8423 & 542.7621 & 531 & 531 & 122.5994 & 12.90823 \\
\hline 228 & 6.8593 & 538.2501 & 531 & 531 & 122.5994 & 12.87627 \\
\hline 229 & 6.8763 & 550.9237 & 530 & 530 & 122.5994 & 12.84448 \\
\hline 230 & 6.8933 & 513.7743 & 530 & 530 & 122.5994 & 12.81284 \\
\hline 231 & 6.9103 & 550.1333 & 530 & 530 & 122.5994 & 12.78136 \\
\hline 232 & 6.9273 & 538.6512 & 530 & 530 & 122.5994 & 12.75003 \\
\hline 233 & 6.9443 & 524.5185 & 529 & 529 & 122.5994 & 12.71886 \\
\hline 234 & 6.9613 & 551.9355 & 529 & 529 & 122.5994 & 12.68783 \\
\hline 235 & 6.9783 & 528.835 & 529 & 529 & 122.5994 & 12.65696 \\
\hline 236 & 6.9953 & 523.6509 & 528 & 528 & 122.5994 & 12.62624 \\
\hline 237 & 7.0123 & 512.5335 & 528 & 528 & 122.5994 & 12.59567 \\
\hline 238 & 7.0293 & 536.4104 & 528 & 528 & 122.5994 & 12.56525 \\
\hline 239 & 7.0463 & 557.178 & 528 & 528 & 122.5994 & 12.53497 \\
\hline 240 & 7.0633 & 527.0361 & 527 & 527 & 122.5994 & 12.50484 \\
\hline 241 & 7.0803 & 533.9698 & 527 & 527 & 122.5994 & 12.47485 \\
\hline 242 & 7.0973 & 496.7864 & 527 & 527 & 122.5994 & 12.44501 \\
\hline 243 & 7.1143 & 535.525 & 526 & 526 & 122.5994 & 12.41531 \\
\hline
\end{tabular}




\begin{tabular}{|c|c|c|c|c|c|c|}
\hline 244 & 7.1313 & 538.6781 & 526 & 526 & 122.5994 & 12.38575 \\
\hline 245 & 7.1483 & 523.2624 & 526 & 526 & 122.5994 & 12.35633 \\
\hline 246 & 7.1653 & 524.8301 & 525 & 525 & 122.5994 & 12.32706 \\
\hline 247 & 7.1823 & 531.5254 & 525 & 525 & 122.5994 & 12.29792 \\
\hline 248 & 7.1993 & 526.9096 & 525 & 525 & 122.5994 & 12.26892 \\
\hline 249 & 7.2163 & 530.7925 & 524 & 524 & 122.5994 & 12.24005 \\
\hline 250 & 7.2333 & 501.2914 & 524 & 524 & 122.5994 & 12.21132 \\
\hline 251 & 7.2503 & 531.41 & 524 & 524 & 122.5994 & 12.18273 \\
\hline 252 & 7.2673 & 544.7553 & 523 & 523 & 122.5994 & 12.15427 \\
\hline 253 & 7.2843 & 533.9487 & 523 & 523 & 122.5994 & 12.12594 \\
\hline 254 & 7.3013 & 517.3033 & 523 & 523 & 122.5994 & 12.09774 \\
\hline 255 & 7.3183 & 526.2396 & 522 & 522 & 122.5994 & 12.06968 \\
\hline 256 & 7.3353 & 531.3765 & 522 & 522 & 122.5994 & 12.04175 \\
\hline 257 & 7.3523 & 528.9947 & 522 & 522 & 122.5994 & 12.01394 \\
\hline 258 & 7.3693 & 514.5665 & 521 & 521 & 122.5994 & 11.98627 \\
\hline 259 & 7.3863 & 521.8773 & 521 & 521 & 122.5994 & 11.95872 \\
\hline 260 & 7.4033 & 525.0909 & 521 & 521 & 122.5994 & 11.93129 \\
\hline 261 & 7.4203 & 499.3263 & 520 & 520 & 122.5994 & 11.904 \\
\hline 262 & 7.4373 & 539.3895 & 520 & 520 & 122.5994 & 11.87683 \\
\hline 263 & 7.4543 & 550.1939 & 520 & 520 & 122.5994 & 11.84978 \\
\hline 264 & 7.4713 & 514.2172 & 519 & 519 & 122.5994 & 11.82285 \\
\hline 265 & 7.4883 & 514.0548 & 519 & 519 & 122.5994 & 11.79605 \\
\hline 266 & 7.5053 & 513.4624 & 519 & 519 & 122.5994 & 11.76937 \\
\hline 267 & 7.5223 & 514.0463 & 520 & 520 & 122.5994 & 11.74281 \\
\hline 268 & 7.5393 & 525.4967 & 520 & 520 & 122.5994 & 11.71637 \\
\hline 269 & 7.5563 & 528.9947 & 520 & 520 & 122.5994 & 11.69005 \\
\hline 270 & 7.5733 & 521.7928 & 520 & 520 & 122.5994 & 11.66385 \\
\hline 271 & 7.5903 & 538.4914 & 521 & 521 & 122.5994 & 11.63776 \\
\hline 272 & 7.6073 & 539.0209 & 521 & 521 & 122.5994 & 11.61179 \\
\hline 273 & 7.6243 & 510.9806 & 521 & 521 & 122.5994 & 11.58594 \\
\hline 274 & 7.6413 & 530.156 & 522 & 522 & 122.5994 & 11.5602 \\
\hline 275 & 7.6583 & 525.3665 & 522 & 522 & 122.5994 & 11.53458 \\
\hline 276 & 7.6753 & 549.9486 & 522 & 522 & 122.5994 & 11.50907 \\
\hline 277 & 7.6923 & 556.6648 & 522 & 522 & 122.5994 & 11.48367 \\
\hline 278 & 7.7093 & 534.5385 & 523 & 523 & 122.5994 & 11.45839 \\
\hline 279 & 7.7263 & 542.7286 & 523 & 523 & 122.5994 & 11.43321 \\
\hline 280 & 7.7433 & 556.9118 & 523 & 523 & 122.5994 & 11.40815 \\
\hline 281 & 7.7603 & 542.9001 & 524 & 524 & 122.5994 & 11.3832 \\
\hline 282 & 7.7773 & 542.2447 & 524 & 524 & 122.5994 & 11.35835 \\
\hline 283 & 7.7943 & 530.2646 & 524 & 524 & 122.5994 & 11.33362 \\
\hline 284 & 7.8113 & 545.9006 & 525 & 525 & 122.5994 & 11.30899 \\
\hline 285 & 7.8283 & 527.4782 & 525 & 525 & 122.5994 & 11.28447 \\
\hline 286 & 7.8453 & 514.701 & 525 & 525 & 122.5994 & 11.26006 \\
\hline 287 & 7.8623 & 514.9774 & 525 & 525 & 122.5994 & 11.23575 \\
\hline 288 & 7.8793 & 536.6161 & 526 & 526 & 122.5994 & 11.21154 \\
\hline 289 & 7.8963 & 526.3131 & 526 & 526 & 122.5994 & 11.18745 \\
\hline 290 & 7.9133 & 521.3074 & 526 & 526 & 122.5994 & 11.16345 \\
\hline 291 & 7.9303 & 536.2685 & 527 & 527 & 122.5994 & 11.13956 \\
\hline 292 & 7.9473 & 527.4215 & 527 & 527 & 122.5994 & 11.11577 \\
\hline
\end{tabular}




\begin{tabular}{|c|c|c|c|c|c|c|}
\hline 293 & 7.9643 & 553.7447 & 528 & 528 & 122.5994 & 11.09208 \\
\hline 294 & 7.9813 & 525.2801 & 528 & 528 & 122.5994 & 11.06849 \\
\hline 295 & 7.9983 & 521.2302 & 528 & 528 & 122.5994 & 11.045 \\
\hline 296 & 8.0153 & 544.1856 & 529 & 529 & 122.5994 & 11.02162 \\
\hline 297 & 8.0323 & 551.6896 & 529 & 529 & 122.5994 & 10.99833 \\
\hline 298 & 8.0493 & 571.8883 & 529 & 529 & 122.5994 & 10.97514 \\
\hline 299 & 8.0663 & 556.5365 & 530 & 530 & 122.5994 & 10.95204 \\
\hline 300 & 8.0833 & 576.0108 & 530 & 530 & 122.5994 & 10.92905 \\
\hline 301 & 8.1003 & 587.0081 & 530 & 530 & 122.5994 & 10.90615 \\
\hline 302 & 8.1173 & 584.1543 & 531 & 531 & 122.5994 & 10.88335 \\
\hline 303 & 8.1343 & 566.4506 & 531 & 531 & 122.5994 & 10.86064 \\
\hline 304 & 8.1513 & 556.6117 & 532 & 532 & 122.5994 & 10.83803 \\
\hline 305 & 8.1683 & 562.9547 & 532 & 532 & 122.5994 & 10.81551 \\
\hline 306 & 8.1853 & 578.7163 & 532 & 532 & 122.5994 & 10.79309 \\
\hline 307 & 8.2023 & 555.9285 & 533 & 533 & 122.5994 & 10.77075 \\
\hline 308 & 8.2193 & 566.9503 & 533 & 533 & 122.5994 & 10.74852 \\
\hline 309 & 8.2363 & 579.6064 & 534 & 534 & 122.5994 & 10.72637 \\
\hline 310 & 8.2533 & 554.5058 & 534 & 534 & 122.5994 & 10.70431 \\
\hline 311 & 8.2703 & 546.0292 & 534 & 534 & 122.5994 & 10.68235 \\
\hline 312 & 8.2873 & 560.6271 & 535 & 535 & 122.5994 & 10.66047 \\
\hline 313 & 8.3043 & 559.6584 & 535 & 535 & 122.5994 & 10.63869 \\
\hline 314 & 8.3213 & 567.4383 & 535 & 535 & 122.5994 & 10.61699 \\
\hline 315 & 8.3383 & 564.0224 & 536 & 536 & 122.5994 & 10.59538 \\
\hline 316 & 8.3553 & 558.0222 & 536 & 536 & 122.5994 & 10.57386 \\
\hline 317 & 8.3723 & 587.6252 & 536 & 536 & 122.5994 & 10.55243 \\
\hline 318 & 8.3893 & 603.4137 & 537 & 537 & 122.5994 & 10.53109 \\
\hline 319 & 8.4063 & 592.8162 & 537 & 537 & 122.5994 & 10.50983 \\
\hline 320 & 8.4233 & 561.508 & 538 & 538 & 122.5994 & 10.48865 \\
\hline 321 & 8.4403 & 599.6378 & 538 & 538 & 122.5994 & 10.46757 \\
\hline 322 & 8.4573 & 627.7041 & 538 & 538 & 122.5994 & 10.44656 \\
\hline 323 & 8.4743 & 634.6547 & 546.2181 & 539 & 122.5994 & 10.42565 \\
\hline 324 & 8.4913 & 602.0096 & 550.3759 & 539 & 122.5994 & 10.40481 \\
\hline 325 & 8.5083 & 638.9578 & 552.4397 & 540 & 122.5994 & 10.38406 \\
\hline 326 & 8.5253 & 645.0987 & 553.6591 & 540 & 122.5994 & 10.36339 \\
\hline 327 & 8.5423 & 671.1218 & 555.0656 & 540 & 122.5994 & 10.34281 \\
\hline 328 & 8.5593 & 692.614 & 557.6993 & 541 & 122.5994 & 10.3223 \\
\hline 329 & 8.5763 & 714.9419 & 559.6115 & 541 & 122.5994 & 10.30188 \\
\hline 330 & 8.5933 & 659.2931 & 561.8682 & 541 & 122.5994 & 10.28154 \\
\hline 331 & 8.6103 & 651.9322 & 565.5568 & 542 & 122.5994 & 10.26128 \\
\hline 332 & 8.6273 & 718.1885 & 568.7933 & 542 & 122.5994 & 10.24109 \\
\hline 333 & 8.6443 & 759.4424 & 572.735 & 542 & 122.5994 & 10.22099 \\
\hline 334 & 8.6613 & 782.087 & 578.6008 & 543 & 122.5994 & 10.20097 \\
\hline 335 & 8.6783 & 809.4662 & 584.7086 & 543 & 122.5994 & 10.18102 \\
\hline 336 & 8.6953 & 839.6383 & 593.5668 & 544 & 122.5994 & 10.16116 \\
\hline 337 & 8.7123 & 832.3529 & 604.1122 & 544 & 122.5994 & 10.14137 \\
\hline 338 & 8.7293 & 926.3427 & 618.3251 & 543 & 122.5994 & 10.12166 \\
\hline 339 & 8.7463 & 1033.251 & 642.5419 & 543 & 122.5994 & 10.10202 \\
\hline 340 & 8.7633 & 1099.131 & 683.5137 & 542 & 122.5994 & 10.08246 \\
\hline 341 & 8.7803 & 1094.745 & 758.114 & 542 & 122.5994 & 10.06298 \\
\hline
\end{tabular}




\begin{tabular}{|c|c|c|c|c|c|c|}
\hline 342 & 8.7973 & 1184.906 & 883.7867 & 541 & 122.5994 & 10.04357 \\
\hline 343 & 8.8143 & 1356.283 & 1077.488 & 540 & 122.5994 & 10.02424 \\
\hline 344 & 8.8313 & 1481.219 & 1337.068 & 540 & 122.5994 & 10.00498 \\
\hline 345 & 8.8483 & 1530.051 & 1615.25 & 539 & 122.5994 & 9.9858 \\
\hline 346 & 8.8653 & 1564.016 & 1808.641 & 539 & 122.5994 & 9.96669 \\
\hline 347 & 8.8823 & 1527.536 & 1808.001 & 538 & 122.5994 & 9.94765 \\
\hline 348 & 8.8993 & 1614.053 & 1620.821 & 538 & 122.5994 & 9.92869 \\
\hline 349 & 8.9163 & 1668.216 & 1346.305 & 537 & 122.5994 & 9.90979 \\
\hline 350 & 8.9333 & 1511.208 & 1085.919 & 536 & 122.5994 & 9.89097 \\
\hline 351 & 8.9503 & 1172.114 & 888.7161 & 536 & 122.5994 & 9.87222 \\
\hline 352 & 8.9673 & 916.5267 & 757.981 & 535 & 122.5994 & 9.85355 \\
\hline 353 & 8.9843 & 774.9346 & 680.761 & 535 & 122.5994 & 9.83494 \\
\hline 354 & 9.0013 & 669.2965 & 635.9901 & 534 & 122.5994 & 9.8164 \\
\hline 355 & 9.0183 & 588.8598 & 610.73 & 534 & 122.5994 & 9.79794 \\
\hline 356 & 9.0353 & 584.9094 & 593.9745 & 533 & 122.5994 & 9.77954 \\
\hline 357 & 9.0523 & 614.9278 & 582.1518 & 532 & 122.5994 & 9.76121 \\
\hline 358 & 9.0693 & 559.5726 & 574.1395 & 532 & 122.5994 & 9.74295 \\
\hline 359 & 9.0863 & 555.3234 & 566.9344 & 531 & 122.5994 & 9.72476 \\
\hline 360 & 9.1033 & 519.5994 & 562.0006 & 531 & 122.5994 & 9.70664 \\
\hline 361 & 9.1203 & 515.255 & 557.0085 & 530 & 122.5994 & 9.68859 \\
\hline 362 & 9.1373 & 544.6903 & 552.7336 & 529 & 122.5994 & 9.6706 \\
\hline 363 & 9.1543 & 544.8762 & 549.0151 & 528 & 122.5994 & 9.65268 \\
\hline 364 & 9.1713 & 527.9233 & 544.7349 & 526 & 122.5994 & 9.63482 \\
\hline 365 & 9.1883 & 501.8283 & 541.8041 & 525 & 122.5994 & 9.61704 \\
\hline 366 & 9.2053 & 524.3622 & 539.1552 & 524 & 122.5994 & 9.59931 \\
\hline 367 & 9.2223 & 505.1753 & 536.7363 & 523 & 122.5994 & 9.58166 \\
\hline 368 & 9.2393 & 522.0455 & 534.5067 & 522 & 122.5994 & 9.56407 \\
\hline 369 & 9.2563 & 558.6427 & 531.4344 & 520 & 122.5994 & 9.54654 \\
\hline 370 & 9.2733 & 509.6129 & 529.4937 & 519 & 122.5994 & 9.52908 \\
\hline 371 & 9.2903 & 545.9297 & 521.4407 & 518 & 122.5994 & 9.51168 \\
\hline 372 & 9.3073 & 498.1778 & 517 & 517 & 122.5994 & 9.49434 \\
\hline 373 & 9.3243 & 542.721 & 516 & 516 & 122.5994 & 9.47707 \\
\hline 374 & 9.3413 & 506.7239 & 514 & 514 & 122.5994 & 9.45986 \\
\hline 375 & 9.3583 & 522.7238 & 513 & 513 & 122.5994 & 9.44272 \\
\hline 376 & 9.3753 & 489.9241 & 512 & 512 & 122.5994 & 9.42563 \\
\hline 377 & 9.3923 & 514.9332 & 511 & 511 & 122.5994 & 9.40861 \\
\hline 378 & 9.4093 & 553.336 & 509 & 509 & 122.5994 & 9.39165 \\
\hline 379 & 9.4263 & 520.7791 & 508 & 508 & 122.5994 & 9.37475 \\
\hline 380 & 9.4433 & 495.9258 & 507 & 507 & 122.5994 & 9.35791 \\
\hline 381 & 9.4603 & 490.7715 & 506 & 506 & 122.5994 & 9.34113 \\
\hline 382 & 9.4773 & 500.857 & 505 & 505 & 122.5994 & 9.32441 \\
\hline 383 & 9.4943 & 504.2507 & 503 & 503 & 122.5994 & 9.30776 \\
\hline 384 & 9.5113 & 525.7092 & 502 & 502 & 122.5994 & 9.29116 \\
\hline 385 & 9.5283 & 516.4633 & 501 & 501 & 122.5994 & 9.27462 \\
\hline 386 & 9.5453 & 513.4508 & 500 & 500 & 122.5994 & 9.25814 \\
\hline 387 & 9.5623 & 493.2419 & 499 & 499 & 122.5994 & 9.24172 \\
\hline 388 & 9.5793 & 481.1187 & 497 & 497 & 122.5994 & 9.22536 \\
\hline 389 & 9.5963 & 484.4861 & 496 & 496 & 122.5994 & 9.20905 \\
\hline 390 & 9.6133 & 490.3522 & 495 & 495 & 122.5994 & 9.19281 \\
\hline
\end{tabular}




\begin{tabular}{|c|c|c|c|c|c|c|}
\hline 391 & 9.6303 & 493.6117 & 494 & 494 & 122.5994 & 9.17662 \\
\hline 392 & 9.6473 & 521.4793 & 493 & 493 & 122.5994 & 9.16048 \\
\hline 393 & 9.6643 & 506.86 & 491 & 491 & 122.5994 & 9.14441 \\
\hline 394 & 9.6813 & 495.6547 & 490 & 490 & 122.5994 & 9.12839 \\
\hline 395 & 9.6983 & 501.2519 & 489 & 489 & 122.5994 & 9.11243 \\
\hline 396 & 9.7153 & 508.5489 & 488 & 488 & 122.5994 & 9.09652 \\
\hline 397 & 9.7323 & 492.498 & 487 & 487 & 122.5994 & 9.08067 \\
\hline 398 & 9.7493 & 498.5118 & 485 & 485 & 122.5994 & 9.06487 \\
\hline 399 & 9.7663 & 510.7695 & 484 & 484 & 122.5994 & 9.04913 \\
\hline 400 & 9.7833 & 508.1021 & 483 & 483 & 122.5994 & 9.03344 \\
\hline 401 & 9.8003 & 522.8534 & 482 & 482 & 122.5994 & 9.01781 \\
\hline 402 & 9.8173 & 530.7247 & 480 & 480 & 122.5994 & 9.00224 \\
\hline 403 & 9.8343 & 535.0119 & 479 & 479 & 122.5994 & 8.98671 \\
\hline 404 & 9.8513 & 498.8755 & 478 & 478 & 122.5994 & 8.97124 \\
\hline 405 & 9.8683 & 473.5796 & 477 & 477 & 122.5994 & 8.95583 \\
\hline 406 & 9.8853 & 477.1259 & 476 & 476 & 122.5994 & 8.94046 \\
\hline 407 & 9.9023 & 463.6658 & 474 & 474 & 122.5994 & 8.92515 \\
\hline 408 & 9.9193 & 454.7356 & 473 & 473 & 122.5994 & 8.90989 \\
\hline 409 & 9.9363 & 487.6484 & 472 & 472 & 122.5994 & 8.89469 \\
\hline 410 & 9.9533 & 469.2337 & 472 & 472 & 122.5994 & 8.87953 \\
\hline 411 & 9.9703 & 491.0503 & 472 & 472 & 122.5994 & 8.86443 \\
\hline 412 & 9.9873 & 497.2993 & 472 & 472 & 122.5994 & 8.84938 \\
\hline 413 & 10.0043 & 485.779 & 472 & 472 & 122.5994 & 8.83438 \\
\hline 414 & 10.0213 & 511.7391 & 472 & 472 & 122.5994 & 8.81943 \\
\hline 415 & 10.0383 & 486.8957 & 472 & 472 & 122.5994 & 8.80454 \\
\hline 416 & 10.0553 & 508.8478 & 472 & 472 & 122.5994 & 8.78969 \\
\hline 417 & 10.0723 & 506.4815 & 472 & 472 & 122.5994 & 8.77489 \\
\hline 418 & 10.0893 & 476.1081 & 472 & 472 & 122.5994 & 8.76014 \\
\hline 419 & 10.1063 & 497.1347 & 472 & 472 & 122.5994 & 8.74545 \\
\hline 420 & 10.1233 & 497.0954 & 472 & 472 & 122.5994 & 8.7308 \\
\hline 421 & 10.1403 & 483.8197 & 472 & 472 & 122.5994 & 8.7162 \\
\hline 422 & 10.1573 & 479.364 & 471 & 471 & 122.5994 & 8.70165 \\
\hline 423 & 10.1743 & 475.2489 & 471 & 471 & 122.5994 & 8.68715 \\
\hline 424 & 10.1913 & 473.47 & 471 & 471 & 122.5994 & 8.6727 \\
\hline 425 & 10.2083 & 465.5738 & 471 & 471 & 122.5994 & 8.65829 \\
\hline 426 & 10.2253 & 509.6813 & 471 & 471 & 122.5994 & 8.64394 \\
\hline 427 & 10.2423 & 450.1278 & 471 & 471 & 122.5994 & 8.62963 \\
\hline 428 & 10.2593 & 454.4989 & 471 & 471 & 122.5994 & 8.61537 \\
\hline 429 & 10.2763 & 467.1221 & 471 & 471 & 122.5994 & 8.60115 \\
\hline 430 & 10.2933 & 500.7105 & 471 & 471 & 122.5994 & 8.58698 \\
\hline 431 & 10.3103 & 486.3122 & 471 & 471 & 122.5994 & 8.57286 \\
\hline 432 & 10.3273 & 461.9892 & 471 & 471 & 122.5994 & 8.55879 \\
\hline 433 & 10.3443 & 480.1137 & 471 & 471 & 122.5994 & 8.54476 \\
\hline 434 & 10.3613 & 466.8059 & 471 & 471 & 122.5994 & 8.53078 \\
\hline 435 & 10.3783 & 455.0881 & 471 & 471 & 122.5994 & 8.51685 \\
\hline 436 & 10.3953 & 460.2705 & 472 & 472 & 122.5994 & 8.50296 \\
\hline 437 & 10.4123 & 469.741 & 472 & 472 & 122.5994 & 8.48911 \\
\hline 438 & 10.4293 & 480.3323 & 472 & 472 & 122.5994 & 8.47531 \\
\hline 439 & 10.4463 & 499.06 & 472 & 472 & 122.5994 & 8.46156 \\
\hline
\end{tabular}




\begin{tabular}{|c|c|c|c|c|c|c|}
\hline 440 & 10.4633 & 484.6557 & 472 & 472 & 122.5994 & 8.44785 \\
\hline 441 & 10.4803 & 505.974 & 473 & 473 & 122.5994 & 8.43418 \\
\hline 442 & 10.4973 & 544.9704 & 473 & 473 & 122.5994 & 8.42056 \\
\hline 443 & 10.5143 & 566.2499 & 473 & 473 & 122.5994 & 8.40699 \\
\hline 444 & 10.5313 & 544.8109 & 473 & 473 & 122.5994 & 8.39345 \\
\hline 445 & 10.5483 & 538.2192 & 474 & 474 & 122.5994 & 8.37996 \\
\hline 446 & 10.5653 & 551.7276 & 474 & 474 & 122.5994 & 8.36652 \\
\hline 447 & 10.5823 & 523.3007 & 474 & 474 & 122.5994 & 8.35312 \\
\hline 448 & 10.5993 & 486.3765 & 474 & 474 & 122.5994 & 8.33976 \\
\hline 449 & 10.6163 & 465.751 & 474 & 474 & 122.5994 & 8.32644 \\
\hline 450 & 10.6333 & 449.5431 & 475 & 475 & 122.5994 & 8.31317 \\
\hline 451 & 10.6503 & 453.9183 & 475 & 475 & 122.5994 & 8.29994 \\
\hline 452 & 10.6673 & 476.2701 & 475 & 475 & 122.5994 & 8.28675 \\
\hline 453 & 10.6843 & 484.009 & 475 & 475 & 122.5994 & 8.2736 \\
\hline 454 & 10.7013 & 470.9831 & 475 & 475 & 122.5994 & 8.26049 \\
\hline 455 & 10.7183 & 469.8691 & 476 & 476 & 122.5994 & 8.24743 \\
\hline 456 & 10.7353 & 491.7525 & 476 & 476 & 122.5994 & 8.23441 \\
\hline 457 & 10.7523 & 486.5152 & 476 & 476 & 122.5994 & 8.22143 \\
\hline 458 & 10.7693 & 455.1954 & 475 & 475 & 122.5994 & 8.20849 \\
\hline 459 & 10.7863 & 471.4935 & 475 & 475 & 122.5994 & 8.19559 \\
\hline 460 & 10.8033 & 456.3712 & 474 & 474 & 122.5994 & 8.18273 \\
\hline 461 & 10.8203 & 504.8913 & 473 & 473 & 122.5994 & 8.16991 \\
\hline 462 & 10.8373 & 462.2459 & 473 & 473 & 122.5994 & 8.15713 \\
\hline 463 & 10.8543 & 445.9766 & 472 & 472 & 122.5994 & 8.1444 \\
\hline 464 & 10.8713 & 482.5096 & 471 & 471 & 122.5994 & 8.1317 \\
\hline 465 & 10.8883 & 433.4936 & 471 & 471 & 122.5994 & 8.11904 \\
\hline 466 & 10.9053 & 437.0094 & 470 & 470 & 122.5994 & 8.10642 \\
\hline 467 & 10.9223 & 479.272 & 469 & 469 & 122.5994 & 8.09384 \\
\hline 468 & 10.9393 & 465.5413 & 469 & 469 & 122.5994 & 8.0813 \\
\hline 469 & 10.9563 & 449.7551 & 468 & 468 & 122.5994 & 8.0688 \\
\hline 470 & 10.9733 & 507.9307 & 467 & 467 & 122.5994 & 8.05634 \\
\hline 471 & 10.9903 & 474.7967 & 467 & 467 & 122.5994 & 8.04392 \\
\hline 472 & 11.0073 & 441.9484 & 466 & 466 & 122.5994 & 8.03153 \\
\hline 473 & 11.0243 & 469.9183 & 465 & 465 & 122.5994 & 8.01919 \\
\hline 474 & 11.0413 & 442.504 & 465 & 465 & 122.5994 & 8.00688 \\
\hline 475 & 11.0583 & 446.9131 & 464 & 464 & 122.5994 & 7.99461 \\
\hline 476 & 11.0753 & 439.5997 & 463 & 463 & 122.5994 & 7.98237 \\
\hline 477 & 11.0923 & 448.4257 & 463 & 463 & 122.5994 & 7.97018 \\
\hline 478 & 11.1093 & 448.5491 & 462 & 462 & 122.5994 & 7.95802 \\
\hline 479 & 11.1263 & 464.134 & 461 & 461 & 122.5994 & 7.9459 \\
\hline 480 & 11.1433 & 478.2804 & 461 & 461 & 122.5994 & 7.93381 \\
\hline 481 & 11.1603 & 466.7647 & 460 & 460 & 122.5994 & 7.92177 \\
\hline 482 & 11.1773 & 445.0364 & 459 & 459 & 122.5994 & 7.90976 \\
\hline 483 & 11.1943 & 438.3721 & 458 & 458 & 122.5994 & 7.89778 \\
\hline 484 & 11.2113 & 449.3201 & 458 & 458 & 122.5994 & 7.88585 \\
\hline 485 & 11.2283 & 469.4435 & 457 & 457 & 122.5994 & 7.87394 \\
\hline 486 & 11.2453 & 473.9369 & 456 & 456 & 122.5994 & 7.86208 \\
\hline 487 & 11.2623 & 460.6619 & 455 & 455 & 122.5994 & 7.85025 \\
\hline 488 & 11.2793 & 468.1994 & 454 & 454 & 122.5994 & 7.83846 \\
\hline
\end{tabular}




\begin{tabular}{|c|c|c|c|c|c|c|}
\hline 489 & 11.2963 & 479.6294 & 453 & 453 & 122.5994 & 7.8267 \\
\hline 490 & 11.3133 & 453.0334 & 452 & 452 & 122.5994 & 7.81498 \\
\hline 491 & 11.3303 & 446.3712 & 452 & 452 & 122.5994 & 7.80329 \\
\hline 492 & 11.3473 & 453.159 & 451 & 451 & 122.5994 & 7.79164 \\
\hline 493 & 11.3643 & 444.5022 & 450 & 450 & 122.5994 & 7.78002 \\
\hline 494 & 11.3813 & 467.1061 & 449 & 449 & 122.5994 & 7.76844 \\
\hline 495 & 11.3983 & 464.9425 & 448 & 448 & 122.5994 & 7.75689 \\
\hline 496 & 11.4153 & 462.0715 & 448 & 448 & 122.5994 & 7.74537 \\
\hline 497 & 11.4323 & 469.452 & 447 & 447 & 122.5994 & 7.73389 \\
\hline 498 & 11.4493 & 427.3062 & 446 & 446 & 122.5994 & 7.72245 \\
\hline 499 & 11.4663 & 449.8972 & 445 & 445 & 122.5994 & 7.71104 \\
\hline 500 & 11.4833 & 405.8453 & 444 & 444 & 122.5994 & 7.69966 \\
\hline 501 & 11.5003 & 427.2601 & 443 & 443 & 122.5994 & 7.68832 \\
\hline 502 & 11.5173 & 424.8299 & 442 & 442 & 122.5994 & 7.67701 \\
\hline 503 & 11.5343 & 447.0245 & 442 & 442 & 122.5994 & 7.66573 \\
\hline 504 & 11.5513 & 443.2585 & 441 & 441 & 122.5994 & 7.65449 \\
\hline 505 & 11.5683 & 416.6016 & 440 & 440 & 122.5994 & 7.64328 \\
\hline 506 & 11.5853 & 444.4082 & 440 & 440 & 122.5994 & 7.6321 \\
\hline 507 & 11.6023 & 464.638 & 440 & 440 & 122.5994 & 7.62095 \\
\hline 508 & 11.6193 & 454.8728 & 440 & 440 & 122.5994 & 7.60984 \\
\hline 509 & 11.6363 & 422.5648 & 440 & 440 & 122.5994 & 7.59876 \\
\hline 510 & 11.6533 & 428.1006 & 441 & 441 & 122.5994 & 7.58772 \\
\hline 511 & 11.6703 & 453.0257 & 441 & 441 & 122.5994 & 7.5767 \\
\hline 512 & 11.6873 & 420.4064 & 441 & 441 & 122.5994 & 7.56572 \\
\hline 513 & 11.7043 & 465.6117 & 441 & 441 & 122.5994 & 7.55477 \\
\hline 514 & 11.7213 & 407.5691 & 441 & 441 & 122.5994 & 7.54385 \\
\hline 515 & 11.7383 & 397.4564 & 441 & 441 & 122.5994 & 7.53296 \\
\hline 516 & 11.7553 & 421.2602 & 441 & 441 & 122.5994 & 7.52211 \\
\hline 517 & 11.7723 & 431.3548 & 441 & 441 & 122.5994 & 7.51128 \\
\hline 518 & 11.7893 & 422.8291 & 441 & 441 & 122.5994 & 7.50049 \\
\hline 519 & 11.8063 & 442.2358 & 442 & 442 & 122.5994 & 7.48973 \\
\hline 520 & 11.8233 & 443.1879 & 442 & 442 & 122.5994 & 7.479 \\
\hline 521 & 11.8403 & 419.1367 & 442 & 442 & 122.5994 & 7.4683 \\
\hline 522 & 11.8573 & 446.7961 & 442 & 442 & 122.5994 & 7.45763 \\
\hline 523 & 11.8743 & 440.7004 & 442 & 442 & 122.5994 & 7.44699 \\
\hline 524 & 11.8913 & 449.4277 & 442 & 442 & 122.5994 & 7.43638 \\
\hline 525 & 11.9083 & 437.9685 & 442 & 442 & 122.5994 & 7.4258 \\
\hline 526 & 11.9253 & 446.0035 & 442 & 442 & 122.5994 & 7.41525 \\
\hline 527 & 11.9423 & 453.9197 & 443 & 443 & 122.5994 & 7.40474 \\
\hline 528 & 11.9593 & 456.8831 & 443 & 443 & 122.5994 & 7.39425 \\
\hline 529 & 11.9763 & 481.1744 & 443 & 443 & 122.5994 & 7.38379 \\
\hline 530 & 11.9933 & 480.6618 & 442 & 442 & 122.5994 & 7.37336 \\
\hline 531 & 12.0103 & 473.4931 & 442 & 442 & 122.5994 & 7.36297 \\
\hline 532 & 12.0273 & 459.9548 & 441 & 441 & 122.5994 & 7.3526 \\
\hline 533 & 12.0443 & 452.8293 & 441 & 441 & 122.5994 & 7.34226 \\
\hline 534 & 12.0613 & 464.2765 & 440 & 440 & 122.5994 & 7.33195 \\
\hline 535 & 12.0783 & 443.8897 & 440 & 440 & 122.5994 & 7.32166 \\
\hline 536 & 12.0953 & 418.143 & 439 & 439 & 122.5994 & 7.31141 \\
\hline 537 & 12.1123 & 427.1687 & 439 & 439 & 122.5994 & 7.30119 \\
\hline
\end{tabular}




\begin{tabular}{|c|c|c|c|c|c|c|}
\hline 538 & 12.1293 & 443.8186 & 438 & 438 & 122.5994 & 7.29099 \\
\hline 539 & 12.1463 & 456.798 & 438 & 438 & 122.5994 & 7.28083 \\
\hline 540 & 12.1633 & 449.5664 & 437 & 437 & 122.5994 & 7.27069 \\
\hline 541 & 12.1803 & 446.2311 & 437 & 437 & 122.5994 & 7.26058 \\
\hline 542 & 12.1973 & 441.3357 & 436.0001 & 436 & 122.5994 & 7.2505 \\
\hline 543 & 12.2143 & 450.8159 & 436.0001 & 436 & 122.5994 & 7.24045 \\
\hline 544 & 12.2313 & 443.5154 & 435.0001 & 435 & 122.5994 & 7.23042 \\
\hline 545 & 12.2483 & 422.2381 & 435.0001 & 435 & 122.5994 & 7.22042 \\
\hline 546 & 12.2653 & 478.3457 & 434.0001 & 434 & 122.5994 & 7.21045 \\
\hline 547 & 12.2823 & 485.2031 & 434.0001 & 434 & 122.5994 & 7.20051 \\
\hline 548 & 12.2993 & 459.6489 & 433.0001 & 433 & 122.5994 & 7.1906 \\
\hline 549 & 12.3163 & 474.1992 & 433.0002 & 433 & 122.5994 & 7.18071 \\
\hline 550 & 12.3333 & 490.3678 & 432.0002 & 432 & 122.5994 & 7.17085 \\
\hline 551 & 12.3503 & 456.6281 & 432.0003 & 432 & 122.5994 & 7.16102 \\
\hline 552 & 12.3673 & 449.4484 & 431.0005 & 431 & 122.5994 & 7.15121 \\
\hline 553 & 12.3843 & 511.1897 & 431.0008 & 431 & 122.5994 & 7.14144 \\
\hline 554 & 12.4013 & 487.6182 & 430.0013 & 430 & 122.5994 & 7.13168 \\
\hline 555 & 12.4183 & 488.0663 & 430.0018 & 430 & 122.5994 & 7.12196 \\
\hline 556 & 12.4353 & 488.4191 & 429.0024 & 429 & 122.5994 & 7.11226 \\
\hline 557 & 12.4523 & 518.6592 & 429.0027 & 429 & 122.5994 & 7.10259 \\
\hline 558 & 12.4693 & 484.5628 & 428.0026 & 428 & 122.5994 & 7.09294 \\
\hline 559 & 12.4863 & 575.9198 & 428.0022 & 428 & 122.5994 & 7.08333 \\
\hline 560 & 12.5033 & 565.1645 & 427.0016 & 427 & 122.5994 & 7.07373 \\
\hline 561 & 12.5203 & 524.5272 & 427.0011 & 427 & 122.5994 & 7.06417 \\
\hline 562 & 12.5373 & 615.3672 & 426.0007 & 426 & 122.5994 & 7.05463 \\
\hline 563 & 12.5543 & 620.0216 & 426.0005 & 426 & 122.5994 & 7.04511 \\
\hline 564 & 12.5713 & 616.1144 & 425.0003 & 425 & 122.5994 & 7.03562 \\
\hline 565 & 12.5883 & 576.7239 & 425.0002 & 425 & 122.5994 & 7.02616 \\
\hline 566 & 12.6053 & 522.0074 & 424.0002 & 424 & 122.5994 & 7.01672 \\
\hline 567 & 12.6223 & 523.9956 & 424.0001 & 424 & 122.5994 & 7.00731 \\
\hline 568 & 12.6393 & 478.7188 & 423.0001 & 423 & 122.5994 & 6.99792 \\
\hline 569 & 12.6563 & 467.6736 & 423.0001 & 423 & 122.5994 & 6.98856 \\
\hline 570 & 12.6733 & 435.9706 & 422.0001 & 422 & 122.5994 & 6.97923 \\
\hline 571 & 12.6903 & 432.4714 & 422.0001 & 422 & 122.5994 & 6.96991 \\
\hline 572 & 12.7073 & 466.5687 & 421.0001 & 421 & 122.5994 & 6.96063 \\
\hline 573 & 12.7243 & 465.2442 & 421.0001 & 421 & 122.5994 & 6.95137 \\
\hline 574 & 12.7413 & 445.7395 & 420 & 420 & 122.5994 & 6.94213 \\
\hline 575 & 12.7583 & 417.8432 & 420 & 420 & 122.5994 & 6.93292 \\
\hline 576 & 12.7753 & 417.9034 & 419 & 419 & 122.5994 & 6.92373 \\
\hline 577 & 12.7923 & 419.4575 & 419 & 419 & 122.5994 & 6.91457 \\
\hline 578 & 12.8093 & 435.6153 & 419 & 419 & 122.5994 & 6.90543 \\
\hline 579 & 12.8263 & 400.496 & 419 & 419 & 122.5994 & 6.89631 \\
\hline 580 & 12.8433 & 383.8475 & 418 & 418 & 122.5994 & 6.88722 \\
\hline 581 & 12.8603 & 393.785 & 418.0538 & 418 & 122.5994 & 6.87816 \\
\hline 582 & 12.8773 & 396.0198 & 418.0591 & 418 & 122.5994 & 6.86912 \\
\hline 583 & 12.8943 & 402.5235 & 418.0919 & 418 & 122.5994 & 6.8601 \\
\hline 584 & 12.9113 & 425.5213 & 418.1015 & 418 & 122.5994 & 6.8511 \\
\hline 585 & 12.9283 & 436.8182 & 418.1125 & 418 & 122.5994 & 6.84213 \\
\hline 586 & 12.9453 & 436.8182 & 418.1255 & 418 & 122.5994 & 6.83319 \\
\hline
\end{tabular}




\begin{tabular}{|c|c|c|c|c|c|c|}
\hline 587 & 12.9623 & 429.217 & 417.1409 & 417 & 122.5994 & 6.82426 \\
\hline 588 & 12.9793 & 434.8522 & 417.1592 & 417 & 122.5994 & 6.81536 \\
\hline 589 & 12.9963 & 414.2928 & 417.2026 & 417 & 122.5994 & 6.80649 \\
\hline 590 & 13.0133 & 399.9846 & 417.2317 & 417 & 122.5994 & 6.79763 \\
\hline 591 & 13.0303 & 389.0049 & 417.2782 & 417 & 122.5994 & 6.7888 \\
\hline 592 & 13.0473 & 415.9139 & 416.3241 & 416 & 122.5994 & 6.78 \\
\hline 593 & 13.0643 & 398.6735 & 416.3827 & 416 & 122.5994 & 6.77121 \\
\hline 594 & 13.0813 & 382.1455 & 416.4598 & 416 & 122.5994 & 6.76245 \\
\hline 595 & 13.0983 & 395.6758 & 416.5675 & 416 & 122.5994 & 6.75371 \\
\hline 596 & 13.1153 & 416.2285 & 416.7336 & 416 & 122.5994 & 6.74499 \\
\hline 597 & 13.1323 & 406.8809 & 417.2303 & 416 & 122.5994 & 6.7363 \\
\hline 598 & 13.1493 & 407.4239 & 417.7703 & 416 & 122.5994 & 6.72763 \\
\hline 599 & 13.1663 & 422.1691 & 417.7594 & 415 & 122.5994 & 6.71898 \\
\hline 600 & 13.1833 & 431.7227 & 419.2996 & 415 & 122.5994 & 6.71036 \\
\hline 601 & 13.2003 & 392.1171 & 421.4264 & 415 & 122.5994 & 6.70175 \\
\hline 602 & 13.2173 & 421.852 & 423.7346 & 415 & 122.5994 & 6.69317 \\
\hline 603 & 13.2343 & 412.6771 & 425.2451 & 415 & 122.5994 & 6.68461 \\
\hline 604 & 13.2513 & 424.0528 & 425.5132 & 415 & 122.5994 & 6.67607 \\
\hline 605 & 13.2683 & 384.312 & 424.2477 & 415 & 122.5994 & 6.66756 \\
\hline 606 & 13.2853 & 419.9758 & 422.5787 & 415 & 122.5994 & 6.65907 \\
\hline 607 & 13.3023 & 404.6252 & 421.0255 & 415 & 122.5994 & 6.65059 \\
\hline 608 & 13.3193 & 421.2577 & 420.2234 & 415 & 122.5994 & 6.64214 \\
\hline 609 & 13.3363 & 435.7983 & 420.1907 & 415 & 122.5994 & 6.63371 \\
\hline 610 & 13.3533 & 421.002 & 420.5955 & 415 & 122.5994 & 6.62531 \\
\hline 611 & 13.3703 & 398.1637 & 420.8776 & 415 & 122.5994 & 6.61692 \\
\hline 612 & 13.3873 & 440.3946 & 420.6909 & 415 & 122.5994 & 6.60856 \\
\hline 613 & 13.4043 & 430.6113 & 420.1372 & 415 & 122.5994 & 6.60021 \\
\hline 614 & 13.4213 & 400.7673 & 419.4095 & 415 & 122.5994 & 6.59189 \\
\hline 615 & 13.4383 & 406.5813 & 418.8076 & 415 & 122.5994 & 6.58359 \\
\hline 616 & 13.4553 & 419.9753 & 418.4565 & 415 & 122.5994 & 6.57531 \\
\hline 617 & 13.4723 & 427.428 & 418.3713 & 415 & 122.5994 & 6.56705 \\
\hline 618 & 13.4893 & 413.2192 & 418.5085 & 415 & 122.5994 & 6.55881 \\
\hline 619 & 13.5063 & 405.4775 & 418.8333 & 415 & 122.5994 & 6.5506 \\
\hline 620 & 13.5233 & 389.1101 & 419.3531 & 415 & 122.5994 & 6.5424 \\
\hline 621 & 13.5403 & 409.425 & 420.1534 & 415 & 122.5994 & 6.53423 \\
\hline 622 & 13.5573 & 418.0286 & 421.4657 & 415 & 122.5994 & 6.52607 \\
\hline 623 & 13.5743 & 434.8792 & 423.7686 & 415 & 122.5994 & 6.51793 \\
\hline 624 & 13.5913 & 431.8163 & 427.8435 & 415 & 122.5994 & 6.50982 \\
\hline 625 & 13.6083 & 437.1574 & 433.5523 & 414 & 122.5994 & 6.50173 \\
\hline 626 & 13.6253 & 450.042 & 443.479 & 414 & 122.5994 & 6.49365 \\
\hline 627 & 13.6423 & 458.5866 & 454.5274 & 413 & 122.5994 & 6.4856 \\
\hline 628 & 13.6593 & 459.4932 & 465.3012 & 413 & 122.5994 & 6.47757 \\
\hline 629 & 13.6763 & 448.4003 & 469.0791 & 412 & 122.5994 & 6.46955 \\
\hline 630 & 13.6933 & 479.9245 & 466.3468 & 412 & 122.5994 & 6.46156 \\
\hline 631 & 13.7103 & 493.8708 & 457.8565 & 411 & 122.5994 & 6.45358 \\
\hline 632 & 13.7273 & 520.1104 & 448.4372 & 410 & 122.5994 & 6.44563 \\
\hline 633 & 13.7443 & 488.3591 & 442.724 & 410 & 122.5994 & 6.4377 \\
\hline 634 & 13.7613 & 465.9829 & 440.4175 & 409 & 122.5994 & 6.42978 \\
\hline 635 & 13.7783 & 459.8413 & 443.1143 & 409 & 122.5994 & 6.42189 \\
\hline
\end{tabular}




\begin{tabular}{|c|c|c|c|c|c|c|}
\hline 636 & 13.7953 & 412.8343 & 447.2377 & 408 & 122.5994 & 6.41401 \\
\hline 637 & 13.8123 & 414.1664 & 452.9534 & 408 & 122.5994 & 6.40615 \\
\hline 638 & 13.8293 & 478.9767 & 457.455 & 407 & 122.5994 & 6.39832 \\
\hline 639 & 13.8463 & 473.0302 & 461.5455 & 406 & 122.5994 & 6.3905 \\
\hline 640 & 13.8633 & 471.9872 & 465.8802 & 406 & 122.5994 & 6.3827 \\
\hline 641 & 13.8803 & 545.4823 & 467.6256 & 405 & 122.5994 & 6.37492 \\
\hline 642 & 13.8973 & 574.0215 & 467.5695 & 405 & 122.5994 & 6.36716 \\
\hline 643 & 13.9143 & 619.9464 & 463.0943 & 404 & 122.5994 & 6.35942 \\
\hline 644 & 13.9313 & 568.9926 & 456.7472 & 404 & 122.5994 & 6.3517 \\
\hline 645 & 13.9483 & 552.9364 & 447.7635 & 403 & 122.5994 & 6.344 \\
\hline 646 & 13.9653 & 519.8087 & 438.5324 & 402 & 122.5994 & 6.33631 \\
\hline 647 & 13.9823 & 507.1387 & 431.1223 & 402 & 122.5994 & 6.32865 \\
\hline 648 & 13.9993 & 419.5351 & 424.0671 & 401 & 122.5994 & 6.321 \\
\hline 649 & 14.0163 & 408.2803 & 419.4617 & 401 & 122.5994 & 6.31337 \\
\hline 650 & 14.0333 & 388.7916 & 415.0397 & 400 & 122.5994 & 6.30576 \\
\hline 651 & 14.0503 & 399.8355 & 412.3201 & 400 & 122.5994 & 6.29817 \\
\hline 652 & 14.0673 & 428.3288 & 409.2889 & 399 & 122.5994 & 6.2906 \\
\hline 653 & 14.0843 & 407.0305 & 406.458 & 398 & 122.5994 & 6.28304 \\
\hline 654 & 14.1013 & 374.6868 & 404.9569 & 398 & 122.5994 & 6.27551 \\
\hline 655 & 14.1183 & 417.3243 & 402.6725 & 397 & 122.5994 & 6.26799 \\
\hline 656 & 14.1353 & 431.1128 & 401.6282 & 397 & 122.5994 & 6.26049 \\
\hline 657 & 14.1523 & 414.3143 & 399.8129 & 396 & 122.5994 & 6.25301 \\
\hline 658 & 14.1693 & 402.742 & 399.1896 & 396 & 122.5994 & 6.24554 \\
\hline 659 & 14.1863 & 411.5147 & 397.7152 & 395 & 122.5994 & 6.2381 \\
\hline 660 & 14.2033 & 368.2851 & 396.3505 & 394 & 122.5994 & 6.23067 \\
\hline 661 & 14.2203 & 383.3604 & 396.0649 & 394 & 122.5994 & 6.22326 \\
\hline 662 & 14.2373 & 378.5324 & 394.8363 & 393 & 122.5994 & 6.21587 \\
\hline 663 & 14.2543 & 386.1491 & 394.6494 & 393 & 122.5994 & 6.20849 \\
\hline 664 & 14.2713 & 418.8045 & 393.4934 & 392 & 122.5994 & 6.20113 \\
\hline 665 & 14.2883 & 395.4803 & 393.3609 & 392 & 122.5994 & 6.19379 \\
\hline 666 & 14.3053 & 407.7638 & 392.2467 & 391 & 122.5994 & 6.18647 \\
\hline 667 & 14.3223 & 395.4783 & 391.1471 & 390 & 122.5994 & 6.17917 \\
\hline 668 & 14.3393 & 369.7851 & 391.0594 & 390 & 122.5994 & 6.17188 \\
\hline 669 & 14.3563 & 398.5197 & 389.9817 & 389 & 122.5994 & 6.16461 \\
\hline 670 & 14.3733 & 396.0174 & 389.9123 & 389 & 122.5994 & 6.15736 \\
\hline 671 & 14.3903 & 383.0188 & 388.8501 & 388 & 122.5994 & 6.15012 \\
\hline 672 & 14.4073 & 373.2811 & 388.7942 & 388 & 122.5994 & 6.1429 \\
\hline 673 & 14.4243 & 393.2776 & 387.7725 & 387 & 122.5994 & 6.1357 \\
\hline 674 & 14.4413 & 394.4576 & 387.7324 & 387 & 122.5994 & 6.12851 \\
\hline 675 & 14.4583 & 384.1672 & 387.6791 & 387 & 122.5994 & 6.12135 \\
\hline 676 & 14.4753 & 405.2124 & 387.6515 & 387 & 122.5994 & 6.1142 \\
\hline 677 & 14.4923 & 398.1841 & 386.6243 & 386 & 122.5994 & 6.10706 \\
\hline 678 & 14.5093 & 376.367 & 386.6004 & 386 & 122.5994 & 6.09995 \\
\hline 679 & 14.5263 & 369.5749 & 386.2946 & 386 & 122.5994 & 6.09284 \\
\hline 680 & 14.5433 & 383.8135 & 386.2909 & 386 & 122.5994 & 6.08576 \\
\hline 681 & 14.5603 & 383.6168 & 386.1469 & 386 & 122.5994 & 6.07869 \\
\hline 682 & 14.5773 & 378.9057 & 386.1539 & 386 & 122.5994 & 6.07164 \\
\hline 683 & 14.5943 & 385.5202 & 386.1622 & 386 & 122.5994 & 6.06461 \\
\hline 684 & 14.6113 & 396.3821 & 386.172 & 386 & 122.5994 & 6.05759 \\
\hline
\end{tabular}




\begin{tabular}{|c|c|c|c|c|c|c|}
\hline 685 & 14.6283 & 402.8833 & 386.1835 & 386 & 122.5994 & 6.05059 \\
\hline 686 & 14.6453 & 392.2354 & 385.197 & 385 & 122.5994 & 6.0436 \\
\hline 687 & 14.6623 & 391.0999 & 385.2129 & 385 & 122.5994 & 6.03664 \\
\hline 688 & 14.6793 & 404.5546 & 385.2301 & 385 & 122.5994 & 6.02968 \\
\hline 689 & 14.6963 & 388.8443 & 385.2404 & 385 & 122.5994 & 6.02275 \\
\hline 690 & 14.7133 & 393.9401 & 385.2955 & 385 & 122.5994 & 6.01583 \\
\hline 691 & 14.7303 & 392.6578 & 385.3243 & 385 & 122.5994 & 6.00892 \\
\hline 692 & 14.7473 & 385.1872 & 385.4384 & 385 & 122.5994 & 6.00203 \\
\hline 693 & 14.7643 & 380.6902 & 384.4957 & 384 & 122.5994 & 5.99516 \\
\hline 694 & 14.7813 & 401.8977 & 384.5653 & 384 & 122.5994 & 5.9883 \\
\hline 695 & 14.7983 & 438.4793 & 384.6512 & 384 & 122.5994 & 5.98146 \\
\hline 696 & 14.8153 & 375.6626 & 384.793 & 384 & 122.5994 & 5.97464 \\
\hline 697 & 14.8323 & 368.7419 & 384.9363 & 384 & 122.5994 & 5.96783 \\
\hline 698 & 14.8493 & 391.9514 & 384.1308 & 383 & 122.5994 & 5.96103 \\
\hline 699 & 14.8663 & 358.0767 & 383.4191 & 382 & 122.5994 & 5.95425 \\
\hline 700 & 14.8833 & 404.4786 & 383.896 & 382 & 122.5994 & 5.94749 \\
\hline 701 & 14.9003 & 411.8582 & 383.746 & 381 & 122.5994 & 5.94074 \\
\hline 702 & 14.9173 & 345.3528 & 384.2509 & 380 & 122.5994 & 5.93401 \\
\hline 703 & 14.9343 & 343.718 & 385.6997 & 379 & 122.5994 & 5.9273 \\
\hline 704 & 14.9513 & 384.6856 & 388.1628 & 378 & 122.5994 & 5.92059 \\
\hline 705 & 14.9683 & 366.1698 & 391.1134 & 377 & 122.5994 & 5.91391 \\
\hline 706 & 14.9853 & 400.7844 & 393.0758 & 376 & 122.5994 & 5.90724 \\
\hline 707 & 15.0023 & 409.4339 & 393.6998 & 376 & 122.5994 & 5.90058 \\
\hline 708 & 15.0193 & 358.2742 & 391.3795 & 375 & 122.5994 & 5.89394 \\
\hline 709 & 15.0363 & 404.9588 & 388.0724 & 374 & 122.5994 & 5.88732 \\
\hline 710 & 15.0533 & 405.9738 & 384.9641 & 373 & 122.5994 & 5.88071 \\
\hline 711 & 15.0703 & 401.1165 & 383.1606 & 372 & 122.5994 & 5.87411 \\
\hline 712 & 15.0873 & 415.0444 & 384.0343 & 372 & 122.5994 & 5.86753 \\
\hline 713 & 15.1043 & 435.6652 & 385.2245 & 371 & 122.5994 & 5.86096 \\
\hline 714 & 15.1213 & 416.0584 & 386.6577 & 370 & 122.5994 & 5.85441 \\
\hline 715 & 15.1383 & 411.8444 & 386.8366 & 369 & 122.5994 & 5.84788 \\
\hline 716 & 15.1553 & 412.4988 & 384.8807 & 368 & 122.5994 & 5.84136 \\
\hline 717 & 15.1723 & 385.0399 & 381.2655 & 367 & 122.5994 & 5.83485 \\
\hline 718 & 15.1893 & 373.9555 & 376.8426 & 366 & 122.5994 & 5.82836 \\
\hline 719 & 15.2063 & 354.751 & 373.516 & 366 & 122.5994 & 5.82188 \\
\hline 720 & 15.2233 & 371.8968 & 369.9403 & 365 & 122.5994 & 5.81542 \\
\hline 721 & 15.2403 & 372.7784 & 367.2254 & 364 & 122.5994 & 5.80897 \\
\hline 722 & 15.2573 & 360.9861 & 365.1875 & 363 & 122.5994 & 5.80253 \\
\hline 723 & 15.2743 & 352.964 & 364.5858 & 363 & 122.5994 & 5.79611 \\
\hline 724 & 15.2913 & 378.2593 & 363.2639 & 362 & 122.5994 & 5.78971 \\
\hline 725 & 15.3083 & 385.7831 & 363.0381 & 362 & 122.5994 & 5.78332 \\
\hline 726 & 15.3253 & 361.0802 & 361.8829 & 361 & 122.5994 & 5.77694 \\
\hline 727 & 15.3423 & 348.8027 & 361.7649 & 361 & 122.5994 & 5.77058 \\
\hline 728 & 15.3593 & 338.8362 & 360.6733 & 360 & 122.5994 & 5.76423 \\
\hline 729 & 15.3763 & 357.3013 & 360.5182 & 360 & 122.5994 & 5.75789 \\
\hline 730 & 15.3933 & 353.1023 & 359.4668 & 359 & 122.5994 & 5.75157 \\
\hline 731 & 15.4103 & 361.9784 & 359.3843 & 359 & 122.5994 & 5.74527 \\
\hline 732 & 15.4273 & 371.4512 & 358.3556 & 358 & 122.5994 & 5.73897 \\
\hline 733 & 15.4443 & 375.3992 & 358.3338 & 358 & 122.5994 & 5.73269 \\
\hline
\end{tabular}




\begin{tabular}{|c|c|c|c|c|c|c|}
\hline 734 & 15.4613 & 376.3455 & 357.318 & 357 & 122.5994 & 5.72643 \\
\hline 735 & 15.4783 & 346.3936 & 356.3077 & 356 & 122.5994 & 5.72018 \\
\hline 736 & 15.4953 & 375.7068 & 356.3029 & 356 & 122.5994 & 5.71394 \\
\hline 737 & 15.5123 & 380.8766 & 355.3037 & 355 & 122.5994 & 5.70772 \\
\hline 738 & 15.5293 & 370.3016 & 355.3108 & 355 & 122.5994 & 5.70151 \\
\hline 739 & 15.5463 & 348.7105 & 354.3255 & 354 & 122.5994 & 5.69531 \\
\hline 740 & 15.5633 & 359.4903 & 354.3508 & 354 & 122.5994 & 5.68913 \\
\hline 741 & 15.5803 & 385.9849 & 353.3935 & 353 & 122.5994 & 5.68296 \\
\hline 742 & 15.5973 & 380.6996 & 353.468 & 353 & 122.5994 & 5.6768 \\
\hline 743 & 15.6143 & 354.3622 & 352.6031 & 352 & 122.5994 & 5.67066 \\
\hline 744 & 15.6313 & 376.0593 & 352.8454 & 352 & 122.5994 & 5.66453 \\
\hline 745 & 15.6483 & 349.6463 & 352.2523 & 351 & 122.5994 & 5.65842 \\
\hline 746 & 15.6653 & 345.5304 & 352.8664 & 351 & 122.5994 & 5.65231 \\
\hline 747 & 15.6823 & 331.9992 & 354.6689 & 352 & 122.5994 & 5.64622 \\
\hline 748 & 15.6993 & 350.1631 & 355.5089 & 352 & 122.5994 & 5.64015 \\
\hline 749 & 15.7163 & 402.1483 & 356.0625 & 352 & 122.5994 & 5.63409 \\
\hline 750 & 15.7333 & 384.6219 & 357.1008 & 353 & 122.5994 & 5.62804 \\
\hline 751 & 15.7503 & 379.7782 & 356.6375 & 353 & 122.5994 & 5.622 \\
\hline 752 & 15.7673 & 336.5916 & 355.9598 & 353 & 122.5994 & 5.61598 \\
\hline 753 & 15.7843 & 382.0636 & 356.2154 & 354 & 122.5994 & 5.60997 \\
\hline 754 & 15.8013 & 365.6503 & 355.6015 & 354 & 122.5994 & 5.60397 \\
\hline 755 & 15.8183 & 343.557 & 355.1929 & 354 & 122.5994 & 5.59798 \\
\hline 756 & 15.8353 & 365.7191 & 355.9775 & 355 & 122.5994 & 5.59201 \\
\hline 757 & 15.8523 & 370.1364 & 355.9075 & 355 & 122.5994 & 5.58605 \\
\hline 758 & 15.8693 & 349.8322 & 355.9298 & 355 & 122.5994 & 5.58011 \\
\hline 759 & 15.8863 & 368.6469 & 356.9971 & 356 & 122.5994 & 5.57418 \\
\hline 760 & 15.9033 & 368.6469 & 357.0681 & 356 & 122.5994 & 5.56826 \\
\hline 761 & 15.9203 & 361.9485 & 357.1078 & 356 & 122.5994 & 5.56235 \\
\hline 762 & 15.9373 & 351.0986 & 358.0955 & 357 & 122.5994 & 5.55645 \\
\hline 763 & 15.9543 & 359.4571 & 358.0286 & 357 & 122.5994 & 5.55057 \\
\hline 764 & 15.9713 & 363.8605 & 357.9194 & 357 & 122.5994 & 5.5447 \\
\hline 765 & 15.9883 & 357.2167 & 358.7869 & 358 & 122.5994 & 5.53884 \\
\hline 766 & 16.0053 & 362.4531 & 358.6512 & 358 & 122.5994 & 5.533 \\
\hline 767 & 16.0223 & 370.5005 & 358.528 & 358 & 122.5994 & 5.52717 \\
\hline 768 & 16.0393 & 368.634 & 359.4273 & 359 & 122.5994 & 5.52135 \\
\hline 769 & 16.0563 & 351.048 & 359.355 & 359 & 122.5994 & 5.51554 \\
\hline 770 & 16.0733 & 350.8199 & 359.3155 & 359 & 122.5994 & 5.50974 \\
\hline 771 & 16.0903 & 367.4857 & 359.3119 & 359 & 122.5994 & 5.50396 \\
\hline 772 & 16.1073 & 354.6851 & 359.3448 & 359 & 122.5994 & 5.49819 \\
\hline 773 & 16.1243 & 351.3902 & 359.3854 & 359 & 122.5994 & 5.49243 \\
\hline 774 & 16.1413 & 369.3211 & 359.5002 & 359 & 122.5994 & 5.48668 \\
\hline 775 & 16.1583 & 368.8177 & 358.5469 & 358 & 122.5994 & 5.48095 \\
\hline 776 & 16.1753 & 377.8811 & 358.5294 & 358 & 122.5994 & 5.47523 \\
\hline 777 & 16.1923 & 368.0318 & 358.5013 & 358 & 122.5994 & 5.46952 \\
\hline 778 & 16.2093 & 360.9654 & 358.4264 & 358 & 122.5994 & 5.46382 \\
\hline 779 & 16.2263 & 378.1369 & 358.3464 & 358 & 122.5994 & 5.45813 \\
\hline 780 & 16.2433 & 396.2243 & 358.2813 & 358 & 122.5994 & 5.45246 \\
\hline 781 & 16.2603 & 393.7771 & 358.2386 & 358 & 122.5994 & 5.4468 \\
\hline 782 & 16.2773 & 361.0033 & 358.217 & 358 & 122.5994 & 5.44115 \\
\hline
\end{tabular}




\begin{tabular}{|c|c|c|c|c|c|c|}
\hline 783 & 16.2943 & 362.8358 & 358.2118 & 358 & 122.5994 & 5.43551 \\
\hline 784 & 16.3113 & 355.1423 & 358.2186 & 358 & 122.5994 & 5.42988 \\
\hline 785 & 16.3283 & 356.7218 & 358.2347 & 358 & 122.5994 & 5.42427 \\
\hline 786 & 16.3453 & 377.7286 & 358.2594 & 358 & 122.5994 & 5.41866 \\
\hline 787 & 16.3623 & 380.2571 & 358.2937 & 358 & 122.5994 & 5.41307 \\
\hline 788 & 16.3793 & 365.8463 & 357.3407 & 357 & 122.5994 & 5.40749 \\
\hline 789 & 16.3963 & 387.0464 & 357.4071 & 357 & 122.5994 & 5.40192 \\
\hline 790 & 16.4133 & 384.2159 & 357.5597 & 357 & 122.5994 & 5.39637 \\
\hline 791 & 16.4303 & 388.0887 & 357.7325 & 357 & 122.5994 & 5.39082 \\
\hline 792 & 16.4473 & 349.5523 & 358.0585 & 357 & 122.5994 & 5.38529 \\
\hline 793 & 16.4643 & 343.4939 & 358.5834 & 357 & 122.5994 & 5.37977 \\
\hline 794 & 16.4813 & 371.0729 & 359.4336 & 357 & 122.5994 & 5.37426 \\
\hline 795 & 16.4983 & 349.6565 & 360.6668 & 357 & 122.5994 & 5.36876 \\
\hline 796 & 16.5153 & 351.2904 & 362.1992 & 357 & 122.5994 & 5.36327 \\
\hline 797 & 16.5323 & 362.8973 & 363.6709 & 357 & 122.5994 & 5.35779 \\
\hline 798 & 16.5493 & 348.5918 & 364.4706 & 357 & 122.5994 & 5.35233 \\
\hline 799 & 16.5663 & 349.3819 & 363.3104 & 356 & 122.5994 & 5.34687 \\
\hline 800 & 16.5833 & 381.9317 & 362.4436 & 356 & 122.5994 & 5.34143 \\
\hline 801 & 16.6003 & 371.6343 & 361.146 & 356 & 122.5994 & 5.336 \\
\hline 802 & 16.6173 & 363.3282 & 359.7835 & 356 & 122.5994 & 5.33058 \\
\hline 803 & 16.6343 & 353.7502 & 358.6705 & 356 & 122.5994 & 5.32517 \\
\hline 804 & 16.6513 & 379.623 & 357.911 & 356 & 122.5994 & 5.31977 \\
\hline 805 & 16.6683 & 384.5886 & 357.4747 & 356 & 122.5994 & 5.31438 \\
\hline 806 & 16.6853 & 365.4297 & 357.2972 & 356 & 122.5994 & 5.309 \\
\hline 807 & 16.7023 & 342.2172 & 357.3416 & 356 & 122.5994 & 5.30364 \\
\hline 808 & 16.7193 & 346.3251 & 357.6308 & 356 & 122.5994 & 5.29828 \\
\hline 809 & 16.7363 & 347.7157 & 358.2477 & 356 & 122.5994 & 5.29294 \\
\hline 810 & 16.7533 & 358.7089 & 359.2948 & 356 & 122.5994 & 5.28761 \\
\hline 811 & 16.7703 & 374.8741 & 360.809 & 356 & 122.5994 & 5.28229 \\
\hline 812 & 16.7873 & 345.5368 & 361.6337 & 355 & 122.5994 & 5.27698 \\
\hline 813 & 16.8043 & 343.4444 & 363.2548 & 355 & 122.5994 & 5.27168 \\
\hline 814 & 16.8213 & 362.8482 & 363.946 & 355 & 122.5994 & 5.26639 \\
\hline 815 & 16.8383 & 370.6267 & 363.5251 & 355 & 122.5994 & 5.26111 \\
\hline 816 & 16.8553 & 376.6545 & 362.3307 & 355 & 122.5994 & 5.25584 \\
\hline 817 & 16.8723 & 361.1471 & 360.6913 & 355 & 122.5994 & 5.25058 \\
\hline 818 & 16.8893 & 363.5632 & 359.0377 & 355 & 122.5994 & 5.24534 \\
\hline 819 & 16.9063 & 381.05 & 357.7054 & 355 & 122.5994 & 5.2401 \\
\hline 820 & 16.9233 & 362.6899 & 356.7769 & 355 & 122.5994 & 5.23487 \\
\hline 821 & 16.9403 & 337.8271 & 356.1896 & 355 & 122.5994 & 5.22966 \\
\hline 822 & 16.9573 & 333.6729 & 355.7992 & 355 & 122.5994 & 5.22445 \\
\hline 823 & 16.9743 & 345.055 & 354.5951 & 354 & 122.5994 & 5.21926 \\
\hline 824 & 16.9913 & 344.4295 & 354.4695 & 354 & 122.5994 & 5.21408 \\
\hline 825 & 17.0083 & 334.2249 & 354.3665 & 354 & 122.5994 & 5.2089 \\
\hline 826 & 17.0253 & 373.8963 & 354.307 & 354 & 122.5994 & 5.20374 \\
\hline 827 & 17.0423 & 352.6893 & 354.2615 & 354 & 122.5994 & 5.19859 \\
\hline 828 & 17.0593 & 339.3267 & 354.2537 & 354 & 122.5994 & 5.19345 \\
\hline 829 & 17.0763 & 344.601 & 354.2271 & 354 & 122.5994 & 5.18831 \\
\hline 830 & 17.0933 & 342.8611 & 354.2061 & 354 & 122.5994 & 5.18319 \\
\hline 831 & 17.1103 & 371.542 & 354.2041 & 354 & 122.5994 & 5.17808 \\
\hline
\end{tabular}




\begin{tabular}{|c|c|c|c|c|c|c|}
\hline 832 & 17.1273 & 369.5304 & 354.1926 & 354 & 122.5994 & 5.17298 \\
\hline 833 & 17.1443 & 361.7593 & 354.1843 & 354 & 122.5994 & 5.16789 \\
\hline 834 & 17.1613 & 333.5366 & 354.1791 & 354 & 122.5994 & 5.16281 \\
\hline 835 & 17.1783 & 352.2161 & 354.1767 & 354 & 122.5994 & 5.15774 \\
\hline 836 & 17.1953 & 325.0039 & 353.1771 & 353 & 122.5994 & 5.15268 \\
\hline 837 & 17.2123 & 331.1567 & 353.1804 & 353 & 122.5994 & 5.14762 \\
\hline 838 & 17.2293 & 342.2228 & 353.1396 & 353 & 122.5994 & 5.14258 \\
\hline 839 & 17.2463 & 339.0222 & 353.1534 & 353 & 122.5994 & 5.13755 \\
\hline 840 & 17.2633 & 353.6433 & 353.1715 & 353 & 122.5994 & 5.13253 \\
\hline 841 & 17.2803 & 357.2167 & 353.1724 & 353 & 122.5994 & 5.12752 \\
\hline 842 & 17.2973 & 355.7919 & 354.2054 & 354 & 122.5994 & 5.12252 \\
\hline 843 & 17.3143 & 344.4045 & 354.2503 & 354 & 122.5994 & 5.11753 \\
\hline 844 & 17.3313 & 364.7504 & 355.3167 & 355 & 122.5994 & 5.11255 \\
\hline 845 & 17.3483 & 380.7753 & 355.4247 & 355 & 122.5994 & 5.10758 \\
\hline 846 & 17.3653 & 347.04 & 356.613 & 356 & 122.5994 & 5.10261 \\
\hline 847 & 17.3823 & 379.9469 & 356.9873 & 356 & 122.5994 & 5.09766 \\
\hline 848 & 17.3993 & 413.5999 & 358.5294 & 357 & 122.5994 & 5.09272 \\
\hline 849 & 17.4163 & 371.6177 & 360.3639 & 358 & 122.5994 & 5.08779 \\
\hline 850 & 17.4333 & 367.5875 & 361.3968 & 358 & 122.5994 & 5.08286 \\
\hline 851 & 17.4503 & 390.342 & 363.4018 & 359 & 122.5994 & 5.07795 \\
\hline 852 & 17.4673 & 371.5999 & 364.0195 & 359 & 122.5994 & 5.07305 \\
\hline 853 & 17.4843 & 372.6264 & 364.9888 & 360 & 122.5994 & 5.06815 \\
\hline 854 & 17.5013 & 384.9904 & 365.4836 & 361 & 122.5994 & 5.06327 \\
\hline 855 & 17.5183 & 370.5627 & 364.6879 & 361 & 122.5994 & 5.05839 \\
\hline 856 & 17.5353 & 381.807 & 364.7884 & 362 & 122.5994 & 5.05353 \\
\hline 857 & 17.5523 & 394.1471 & 364.0178 & 362 & 122.5994 & 5.04867 \\
\hline 858 & 17.5693 & 392.425 & 364.4676 & 363 & 122.5994 & 5.04382 \\
\hline 859 & 17.5863 & 365.453 & 365.1316 & 364 & 122.5994 & 5.03899 \\
\hline 860 & 17.6033 & 357.2167 & 364.9607 & 364 & 122.5994 & 5.03416 \\
\hline 861 & 17.6203 & 364.876 & 365.9046 & 365 & 122.5994 & 5.02934 \\
\hline 862 & 17.6373 & 386.9529 & 365.9301 & 365 & 122.5994 & 5.02453 \\
\hline 863 & 17.6543 & 397.8249 & 367.0295 & 366 & 122.5994 & 5.01973 \\
\hline 864 & 17.6713 & 400.5658 & 367.2268 & 366 & 122.5994 & 5.01494 \\
\hline 865 & 17.6883 & 413.6005 & 368.5888 & 367 & 122.5994 & 5.01016 \\
\hline 866 & 17.7053 & 420.2579 & 369.2344 & 367 & 122.5994 & 5.00539 \\
\hline 867 & 17.7223 & 417.6683 & 370.3247 & 367 & 122.5994 & 5.00062 \\
\hline 868 & 17.7393 & 472.1517 & 372.012 & 367 & 122.5994 & 4.99587 \\
\hline 869 & 17.7563 & 483.839 & 373.3304 & 366 & 122.5994 & 4.99112 \\
\hline 870 & 17.7733 & 496.8828 & 376.0099 & 366 & 122.5994 & 4.98639 \\
\hline 871 & 17.7903 & 516.0331 & 378.3684 & 366 & 122.5994 & 4.98166 \\
\hline 872 & 17.8073 & 561.4482 & 379.7625 & 366 & 122.5994 & 4.97694 \\
\hline 873 & 17.8243 & 601.9958 & 379.9086 & 366 & 122.5994 & 4.97224 \\
\hline 874 & 17.8413 & 602.3202 & 378.7374 & 366 & 122.5994 & 4.96754 \\
\hline 875 & 17.8583 & 596.3238 & 376.7409 & 366 & 122.5994 & 4.96285 \\
\hline 876 & 17.8753 & 544.32 & 373.5029 & 365 & 122.5994 & 4.95816 \\
\hline 877 & 17.8923 & 476.7432 & 371.4597 & 365 & 122.5994 & 4.95349 \\
\hline 878 & 17.9093 & 466.5687 & 369.8066 & 365 & 122.5994 & 4.94883 \\
\hline 879 & 17.9263 & 458.9032 & 368.5473 & 365 & 122.5994 & 4.94417 \\
\hline 880 & 17.9433 & 372.892 & 367.643 & 365 & 122.5994 & 4.93953 \\
\hline
\end{tabular}




\begin{tabular}{|c|c|c|c|c|c|c|}
\hline 881 & 17.9603 & 382.8955 & 366.9848 & 365 & 122.5994 & 4.93489 \\
\hline 882 & 17.9773 & 387.9236 & 366.4872 & 365 & 122.5994 & 4.93026 \\
\hline 883 & 17.9943 & 384.2562 & 365.1148 & 364 & 122.5994 & 4.92564 \\
\hline 884 & 18.0113 & 349.7349 & 364.847 & 364 & 122.5994 & 4.92103 \\
\hline 885 & 18.0283 & 391.922 & 364.66 & 364 & 122.5994 & 4.91643 \\
\hline 886 & 18.0453 & 387.4439 & 364.5308 & 364 & 122.5994 & 4.91184 \\
\hline 887 & 18.0623 & 373.4862 & 364.4404 & 364 & 122.5994 & 4.90725 \\
\hline 888 & 18.0793 & 385.796 & 364.3748 & 364 & 122.5994 & 4.90267 \\
\hline 889 & 18.0963 & 365.5866 & 364.325 & 364 & 122.5994 & 4.89811 \\
\hline 890 & 18.1133 & 398.9992 & 363.2854 & 363 & 122.5994 & 4.89355 \\
\hline 891 & 18.1303 & 363.6393 & 363.2531 & 363 & 122.5994 & 4.889 \\
\hline 892 & 18.1473 & 354.6589 & 363.226 & 363 & 122.5994 & 4.88446 \\
\hline 893 & 18.1643 & 340.1369 & 363.2032 & 363 & 122.5994 & 4.87992 \\
\hline 894 & 18.1813 & 337.6161 & 363.1837 & 363 & 122.5994 & 4.8754 \\
\hline 895 & 18.1983 & 361.0067 & 363.1224 & 363 & 122.5994 & 4.87088 \\
\hline 896 & 18.2153 & 360.0323 & 362.1113 & 362 & 122.5994 & 4.86638 \\
\hline 897 & 18.2323 & 351.9811 & 362.0633 & 362 & 122.5994 & 4.86188 \\
\hline 898 & 18.2493 & 352.2495 & 362.0363 & 362 & 122.5994 & 4.85739 \\
\hline 899 & 18.2663 & 370.6888 & 362.0331 & 362 & 122.5994 & 4.8529 \\
\hline 900 & 18.2833 & 351.3118 & 362.0268 & 362 & 122.5994 & 4.84843 \\
\hline 901 & 18.3003 & 364.4787 & 362.0267 & 362 & 122.5994 & 4.84396 \\
\hline 902 & 18.3173 & 363.1179 & 362.0268 & 362 & 122.5994 & 4.83951 \\
\hline 903 & 18.3343 & 360.1276 & 361.0266 & 361 & 122.5994 & 4.83506 \\
\hline 904 & 18.3513 & 355.2343 & 361.0279 & 361 & 122.5994 & 4.83062 \\
\hline 905 & 18.3683 & 370.7618 & 361.0264 & 361 & 122.5994 & 4.82618 \\
\hline 906 & 18.3853 & 370.465 & 361.0283 & 361 & 122.5994 & 4.82176 \\
\hline 907 & 18.4023 & 355.7434 & 361.0305 & 361 & 122.5994 & 4.81734 \\
\hline 908 & 18.4193 & 338.5663 & 361.0329 & 361 & 122.5994 & 4.81294 \\
\hline 909 & 18.4363 & 340.5473 & 361.0356 & 361 & 122.5994 & 4.80854 \\
\hline 910 & 18.4533 & 365.6138 & 360.0387 & 360 & 122.5994 & 4.80414 \\
\hline 911 & 18.4703 & 368.5417 & 360.0422 & 360 & 122.5994 & 4.79976 \\
\hline 912 & 18.4873 & 346.0019 & 360.0463 & 360 & 122.5994 & 4.79539 \\
\hline 913 & 18.5043 & 350.1827 & 360.0511 & 360 & 122.5994 & 4.79102 \\
\hline 914 & 18.5213 & 364.5822 & 360.0567 & 360 & 122.5994 & 4.78666 \\
\hline 915 & 18.5383 & 378.9822 & 360.0635 & 360 & 122.5994 & 4.78231 \\
\hline 916 & 18.5553 & 386.8245 & 360.0718 & 360 & 122.5994 & 4.77797 \\
\hline 917 & 18.5723 & 371.5943 & 361.1132 & 361 & 122.5994 & 4.77363 \\
\hline 918 & 18.5893 & 345.6432 & 361.1305 & 361 & 122.5994 & 4.7693 \\
\hline 919 & 18.6063 & 361.1853 & 361.1554 & 361 & 122.5994 & 4.76498 \\
\hline 920 & 18.6233 & 334.1181 & 361.2097 & 361 & 122.5994 & 4.76067 \\
\hline 921 & 18.6403 & 338.5153 & 361.2725 & 361 & 122.5994 & 4.75637 \\
\hline 922 & 18.6573 & 342.2172 & 361.368 & 361 & 122.5994 & 4.75207 \\
\hline 923 & 18.6743 & 364.3708 & 361.5014 & 361 & 122.5994 & 4.74779 \\
\hline 924 & 18.6913 & 371.956 & 362.6643 & 362 & 122.5994 & 4.74351 \\
\hline 925 & 18.7083 & 382.7789 & 362.8229 & 362 & 122.5994 & 4.73923 \\
\hline 926 & 18.7253 & 373.634 & 362.9244 & 362 & 122.5994 & 4.73497 \\
\hline 927 & 18.7423 & 361.8788 & 362.9546 & 362 & 122.5994 & 4.73071 \\
\hline 928 & 18.7593 & 413.1216 & 362.9472 & 362 & 122.5994 & 4.72646 \\
\hline 929 & 18.7763 & 404.4178 & 362.9302 & 362 & 122.5994 & 4.72222 \\
\hline
\end{tabular}




\begin{tabular}{|c|c|c|c|c|c|c|}
\hline 930 & 18.7933 & 416.0292 & 362.9353 & 362 & 122.5994 & 4.71799 \\
\hline 931 & 18.8103 & 380.6222 & 364.0012 & 363 & 122.5994 & 4.71376 \\
\hline 932 & 18.8273 & 417.493 & 364.151 & 363 & 122.5994 & 4.70955 \\
\hline 933 & 18.8443 & 456.3505 & 364.3968 & 363 & 122.5994 & 4.70534 \\
\hline 934 & 18.8613 & 425.7767 & 364.7517 & 363 & 122.5994 & 4.70113 \\
\hline 935 & 18.8783 & 393.1988 & 365.2372 & 363 & 122.5994 & 4.69694 \\
\hline 936 & 18.8953 & 406.4133 & 365.8805 & 363 & 122.5994 & 4.69275 \\
\hline 937 & 18.9123 & 425.521 & 367.6979 & 364 & 122.5994 & 4.68857 \\
\hline 938 & 18.9293 & 425.4845 & 368.6669 & 364 & 122.5994 & 4.6844 \\
\hline 939 & 18.9463 & 398.4611 & 369.6672 & 364 & 122.5994 & 4.68023 \\
\hline 940 & 18.9633 & 435.1936 & 370.3881 & 364 & 122.5994 & 4.67608 \\
\hline 941 & 18.9803 & 392.4669 & 370.4733 & 364 & 122.5994 & 4.67193 \\
\hline 942 & 18.9973 & 377.3915 & 369.9446 & 364 & 122.5994 & 4.66778 \\
\hline 943 & 19.0143 & 367.6191 & 369.0651 & 364 & 122.5994 & 4.66365 \\
\hline 944 & 19.0313 & 367.0432 & 369.027 & 365 & 122.5994 & 4.65952 \\
\hline 945 & 19.0483 & 378.5405 & 367.9446 & 365 & 122.5994 & 4.6554 \\
\hline 946 & 19.0653 & 379.1994 & 367.056 & 365 & 122.5994 & 4.65129 \\
\hline 947 & 19.0823 & 348.1734 & 366.444 & 365 & 122.5994 & 4.64718 \\
\hline 948 & 19.0993 & 339.9535 & 366.0324 & 365 & 122.5994 & 4.64309 \\
\hline 949 & 19.1163 & 342.2564 & 365.7833 & 365 & 122.5994 & 4.63899 \\
\hline 950 & 19.1333 & 334.6099 & 365.6353 & 365 & 122.5994 & 4.63491 \\
\hline 951 & 19.1503 & 332.0456 & 366.554 & 366 & 122.5994 & 4.63084 \\
\hline 952 & 19.1673 & 367.0513 & 366.526 & 366 & 122.5994 & 4.62677 \\
\hline 953 & 19.1843 & 377.2584 & 366.5065 & 366 & 122.5994 & 4.6227 \\
\hline 954 & 19.2013 & 359.1307 & 366.5093 & 366 & 122.5994 & 4.61865 \\
\hline 955 & 19.2183 & 365.0296 & 366.5408 & 366 & 122.5994 & 4.6146 \\
\hline 956 & 19.2353 & 385.8348 & 366.5919 & 366 & 122.5994 & 4.61056 \\
\hline 957 & 19.2523 & 409.2125 & 366.685 & 366 & 122.5994 & 4.60653 \\
\hline 958 & 19.2693 & 355.3199 & 367.8492 & 367 & 122.5994 & 4.6025 \\
\hline 959 & 19.2863 & 340.2001 & 368.1357 & 367 & 122.5994 & 4.59849 \\
\hline 960 & 19.3033 & 366.9059 & 368.6241 & 367 & 122.5994 & 4.59447 \\
\hline 961 & 19.3203 & 369.9727 & 369.4105 & 367 & 122.5994 & 4.59047 \\
\hline 962 & 19.3373 & 379.1567 & 371.5551 & 368 & 122.5994 & 4.58647 \\
\hline 963 & 19.3543 & 378.7172 & 374.0405 & 369 & 122.5994 & 4.58248 \\
\hline 964 & 19.3713 & 376.3239 & 375.699 & 369 & 122.5994 & 4.5785 \\
\hline 965 & 19.3883 & 364.975 & 377.9444 & 370 & 122.5994 & 4.57452 \\
\hline 966 & 19.4053 & 412.5154 & 379.1019 & 371 & 122.5994 & 4.57055 \\
\hline 967 & 19.4223 & 396.7946 & 379.383 & 372 & 122.5994 & 4.56659 \\
\hline 968 & 19.4393 & 388.0973 & 379.2589 & 373 & 122.5994 & 4.56264 \\
\hline 969 & 19.4563 & 384.844 & 378.8792 & 374 & 122.5994 & 4.55869 \\
\hline 970 & 19.4733 & 351.6826 & 377.5724 & 374 & 122.5994 & 4.55475 \\
\hline 971 & 19.4903 & 365.7154 & 377.6641 & 375 & 122.5994 & 4.55081 \\
\hline 972 & 19.5073 & 382.4954 & 378.2422 & 376 & 122.5994 & 4.54688 \\
\hline 973 & 19.5243 & 373.5027 & 379.5873 & 377 & 122.5994 & 4.54296 \\
\hline 974 & 19.5413 & 387.0887 & 380.9067 & 378 & 122.5994 & 4.53905 \\
\hline 975 & 19.5583 & 373.1696 & 381.273 & 378 & 122.5994 & 4.53514 \\
\hline 976 & 19.5753 & 357.5055 & 382.6097 & 379 & 122.5994 & 4.53124 \\
\hline 977 & 19.5923 & 396.0112 & 383.5093 & 380 & 122.5994 & 4.52735 \\
\hline 978 & 19.6093 & 365.3999 & 384.2135 & 381 & 122.5994 & 4.52346 \\
\hline
\end{tabular}




\begin{tabular}{|c|c|c|c|c|c|c|}
\hline 979 & 19.6263 & 391.541 & 384.836 & 382 & 122.5994 & 4.51958 \\
\hline 980 & 19.6433 & 379.2615 & 384.4554 & 382 & 122.5994 & 4.51571 \\
\hline 981 & 19.6603 & 369.1861 & 385.1278 & 383 & 122.5994 & 4.51184 \\
\hline 982 & 19.6773 & 414.0029 & 385.9338 & 384 & 122.5994 & 4.50798 \\
\hline 983 & 19.6943 & 397.5689 & 386.8888 & 385 & 122.5994 & 4.50413 \\
\hline 984 & 19.7113 & 410.5039 & 387.9485 & 386 & 122.5994 & 4.50029 \\
\hline 985 & 19.7283 & 422.9572 & 388.105 & 386 & 122.5994 & 4.49645 \\
\hline 986 & 19.7453 & 413.8158 & 389.3622 & 387 & 122.5994 & 4.49261 \\
\hline 987 & 19.7623 & 413.7386 & 390.7279 & 388 & 122.5994 & 4.48879 \\
\hline 988 & 19.7793 & 438.7027 & 392.2151 & 389 & 122.5994 & 4.48497 \\
\hline 989 & 19.7963 & 408.2086 & 394.0272 & 390 & 122.5994 & 4.48115 \\
\hline 990 & 19.8133 & 415.7171 & 396.3782 & 391 & 122.5994 & 4.47735 \\
\hline 991 & 19.8303 & 402.81 & 398.7303 & 391 & 122.5994 & 4.47355 \\
\hline 992 & 19.8473 & 410.5395 & 403.8474 & 392 & 122.5994 & 4.46975 \\
\hline 993 & 19.8643 & 428.3352 & 411.4885 & 393 & 122.5994 & 4.46597 \\
\hline 994 & 19.8813 & 426.3127 & 422.0405 & 394 & 122.5994 & 4.46219 \\
\hline 995 & 19.8983 & 422.5667 & 434.7734 & 395 & 122.5994 & 4.45841 \\
\hline 996 & 19.9153 & 401.6464 & 445.8156 & 395 & 122.5994 & 4.45465 \\
\hline 997 & 19.9323 & 418.1292 & 452.5045 & 396 & 122.5994 & 4.45088 \\
\hline 998 & 19.9493 & 404.2909 & 452.1766 & 397 & 122.5994 & 4.44713 \\
\hline 999 & 19.9663 & 402.8704 & 447.3701 & 398 & 122.5994 & 4.44338 \\
\hline 1000 & 19.9833 & 424.7847 & 439.7537 & 399 & 122.5994 & 4.43964 \\
\hline 1001 & 20.0003 & 420.9508 & 430.6391 & 400 & 122.5994 & 4.4359 \\
\hline 1002 & 20.0173 & 441.3356 & 421.3655 & 400 & 122.5994 & 4.43218 \\
\hline 1003 & 20.0343 & 417.6994 & 415.3094 & 401 & 122.5994 & 4.42845 \\
\hline 1004 & 20.0513 & 405.3577 & 411.7118 & 402 & 122.5994 & 4.42474 \\
\hline 1005 & 20.0683 & 381.3212 & 409.7898 & 403 & 122.5994 & 4.42103 \\
\hline 1006 & 20.0853 & 392.8363 & 409.1021 & 404 & 122.5994 & 4.41732 \\
\hline 1007 & 20.1023 & 406.8832 & 408.2413 & 404 & 122.5994 & 4.41363 \\
\hline 1008 & 20.1193 & 396.0349 & 408.7451 & 405 & 122.5994 & 4.40994 \\
\hline 1009 & 20.1363 & 403.0621 & 409.5468 & 406 & 122.5994 & 4.40625 \\
\hline 1010 & 20.1533 & 416.7162 & 409.6349 & 406 & 122.5994 & 4.40257 \\
\hline 1011 & 20.1703 & 402.5265 & 411.0898 & 407 & 122.5994 & 4.3989 \\
\hline 1012 & 20.1873 & 429.8456 & 412.0383 & 407 & 122.5994 & 4.39523 \\
\hline 1013 & 20.2043 & 424.9384 & 413.5718 & 407 & 122.5994 & 4.39157 \\
\hline 1014 & 20.2213 & 376.1285 & 415.6241 & 407 & 122.5994 & 4.38792 \\
\hline 1015 & 20.2383 & 403.4691 & 418.7946 & 408 & 122.5994 & 4.38427 \\
\hline 1016 & 20.2553 & 382.1183 & 420.2732 & 408 & 122.5994 & 4.38063 \\
\hline 1017 & 20.2723 & 359.8868 & 420.5335 & 408 & 122.5994 & 4.377 \\
\hline 1018 & 20.2893 & 384.4122 & 420.9763 & 409 & 122.5994 & 4.37337 \\
\hline 1019 & 20.3063 & 388.0973 & 420.0894 & 409 & 122.5994 & 4.36974 \\
\hline 1020 & 20.3233 & 402.8245 & 419.1804 & 409 & 122.5994 & 4.36613 \\
\hline 1021 & 20.3403 & 431.1191 & 418.5733 & 409 & 122.5994 & 4.36252 \\
\hline 1022 & 20.3573 & 401.0477 & 420.248 & 410 & 122.5994 & 4.35891 \\
\hline 1023 & 20.3743 & 380.4612 & 422.1361 & 410 & 122.5994 & 4.35531 \\
\hline 1024 & 20.3913 & 354.1897 & 425.0375 & 410 & 122.5994 & 4.35172 \\
\hline 1025 & 20.4083 & 419.8591 & 429.0648 & 410 & 122.5994 & 4.34813 \\
\hline 1026 & 20.4253 & 406.5416 & 434.9149 & 411 & 122.5994 & 4.34455 \\
\hline 1027 & 20.4423 & 380.8508 & 439.4873 & 411 & 122.5994 & 4.34098 \\
\hline
\end{tabular}




\begin{tabular}{|c|c|c|c|c|c|c|}
\hline 1028 & 20.4593 & 392.0267 & 441.6246 & 411 & 122.5994 & 4.33741 \\
\hline 1029 & 20.4763 & 424.5941 & 459.1546 & 412 & 122.5994 & 4.33385 \\
\hline 1030 & 20.4933 & 398.2721 & 457.4756 & 412 & 122.5994 & 4.33029 \\
\hline 1031 & 20.5103 & 411.4426 & 454.915 & 412 & 122.5994 & 4.32674 \\
\hline 1032 & 20.5273 & 414.0888 & 461.4331 & 412 & 122.5994 & 4.3232 \\
\hline 1033 & 20.5443 & 439.2959 & 460.9547 & 413 & 122.5994 & 4.31966 \\
\hline 1034 & 20.5613 & 430.7738 & 461.0422 & 413 & 122.5994 & 4.31612 \\
\hline 1035 & 20.5783 & 446.8497 & 463.2746 & 413 & 122.5994 & 4.3126 \\
\hline 1036 & 20.5953 & 506.8728 & 467.9211 & 414 & 122.5994 & 4.30907 \\
\hline 1037 & 20.6123 & 484.009 & 473.2358 & 414 & 122.5994 & 4.30556 \\
\hline 1038 & 20.6293 & 496.6324 & 480.281 & 414 & 122.5994 & 4.30205 \\
\hline 1039 & 20.6463 & 512.3988 & 489.345 & 414 & 122.5994 & 4.29855 \\
\hline 1040 & 20.6633 & 516.1551 & 501.9201 & 415 & 122.5994 & 4.29505 \\
\hline 1041 & 20.6803 & 609.7175 & 516.9389 & 415 & 122.5994 & 4.29156 \\
\hline 1042 & 20.6973 & 672.8896 & 536.3876 & 415 & 122.5994 & 4.28807 \\
\hline 1043 & 20.7143 & 687.0091 & 562.7073 & 416 & 122.5994 & 4.28459 \\
\hline 1044 & 20.7313 & 752.7611 & 596.4374 & 416 & 122.5994 & 4.28111 \\
\hline 1045 & 20.7483 & 854.7429 & 644.4042 & 416 & 122.5994 & 4.27764 \\
\hline 1046 & 20.7653 & 988.1757 & 721.1752 & 416 & 122.5994 & 4.27418 \\
\hline 1047 & 20.7823 & 1131.401 & 855.6978 & 417 & 122.5994 & 4.27072 \\
\hline 1048 & 20.7993 & 1337.825 & 1085.636 & 417 & 122.5994 & 4.26727 \\
\hline 1049 & 20.8163 & 1623.151 & 1452.305 & 417 & 122.5994 & 4.26382 \\
\hline 1050 & 20.8333 & 1938.771 & 1972.715 & 418 & 122.5994 & 4.26038 \\
\hline 1051 & 20.8503 & 2390.595 & 2595.585 & 418 & 122.5994 & 4.25695 \\
\hline 1052 & 20.8673 & 3101.774 & 3153.995 & 418 & 122.5994 & 4.25352 \\
\hline 1053 & 20.8843 & 3969.383 & 3407.567 & 418 & 122.5994 & 4.25009 \\
\hline 1054 & 20.9013 & 4943.18 & 3307.962 & 419 & 122.5994 & 4.24667 \\
\hline 1055 & 20.9183 & 5062.195 & 2996.197 & 419 & 122.5994 & 4.24326 \\
\hline 1056 & 20.9353 & 4024.211 & 2549.647 & 419 & 122.5994 & 4.23985 \\
\hline 1057 & 20.9523 & 3176.241 & 2028.121 & 420 & 122.5994 & 4.23645 \\
\hline 1058 & 20.9693 & 2563.224 & 1542 & 419 & 122.5994 & 4.23306 \\
\hline 1059 & 20.9863 & 1766.84 & 1167.266 & 419 & 122.5994 & 4.22967 \\
\hline 1060 & 21.0033 & 1081.257 & 910.0215 & 418 & 122.5994 & 4.22628 \\
\hline 1061 & 21.0203 & 793.3551 & 749.2904 & 418 & 122.5994 & 4.2229 \\
\hline 1062 & 21.0373 & 707.1268 & 653.602 & 418 & 122.5994 & 4.21953 \\
\hline 1063 & 21.0543 & 688.0279 & 595.7917 & 417 & 122.5994 & 4.21616 \\
\hline 1064 & 21.0713 & 686.4528 & 560.3186 & 417 & 122.5994 & 4.2128 \\
\hline 1065 & 21.0883 & 695.8511 & 536.3655 & 417 & 122.5994 & 4.20944 \\
\hline 1066 & 21.1053 & 603.9506 & 518.0108 & 416 & 122.5994 & 4.20609 \\
\hline 1067 & 21.1223 & 577.2439 & 504.7169 & 416 & 122.5994 & 4.20274 \\
\hline 1068 & 21.1393 & 548.7693 & 493.9186 & 416 & 122.5994 & 4.1994 \\
\hline 1069 & 21.1563 & 502.714 & 483.6169 & 415 & 122.5994 & 4.19606 \\
\hline 1070 & 21.1733 & 501.7401 & 475.6971 & 415 & 122.5994 & 4.19273 \\
\hline 1071 & 21.1903 & 470.6516 & 468.854 & 415 & 122.5994 & 4.18941 \\
\hline 1072 & 21.2073 & 459.5257 & 461.839 & 414 & 122.5994 & 4.18609 \\
\hline 1073 & 21.2243 & 402.4162 & 456.7058 & 414 & 122.5994 & 4.18277 \\
\hline 1074 & 21.2413 & 435.3779 & 451.3892 & 413 & 122.5994 & 4.17946 \\
\hline 1075 & 21.2583 & 420.2579 & 447.7983 & 413 & 122.5994 & 4.17616 \\
\hline 1076 & 21.2753 & 422.9562 & 444.7863 & 413 & 122.5994 & 4.17286 \\
\hline
\end{tabular}




\begin{tabular}{|c|c|c|c|c|c|c|}
\hline 1077 & 21.2923 & 439.6915 & 424.1529 & 412 & 122.5994 & 4.16957 \\
\hline 1078 & 21.3093 & 431.9301 & 423.1121 & 412 & 122.5994 & 4.16628 \\
\hline 1079 & 21.3263 & 425.3077 & 422.1577 & 412 & 122.5994 & 4.163 \\
\hline 1080 & 21.3433 & 412.9771 & 411.7578 & 411 & 122.5994 & 4.15972 \\
\hline 1081 & 21.3603 & 420.0835 & 411.6033 & 411 & 122.5994 & 4.15645 \\
\hline 1082 & 21.3773 & 416.0633 & 410.4526 & 410 & 122.5994 & 4.15318 \\
\hline 1083 & 21.3943 & 371.9055 & 409.7747 & 409 & 122.5994 & 4.14992 \\
\hline 1084 & 21.4113 & 389.8322 & 409.7258 & 409 & 122.5994 & 4.14666 \\
\hline 1085 & 21.4283 & 413.4149 & 408.7118 & 408 & 122.5994 & 4.14341 \\
\hline 1086 & 21.4453 & 409.2128 & 407.9434 & 407 & 122.5994 & 4.14016 \\
\hline 1087 & 21.4623 & 418.7343 & 406.9993 & 406 & 122.5994 & 4.13692 \\
\hline 1088 & 21.4793 & 441.7404 & 406.0762 & 405 & 122.5994 & 4.13369 \\
\hline 1089 & 21.4963 & 417.8437 & 406.1736 & 405 & 122.5994 & 4.13046 \\
\hline 1090 & 21.5133 & 418.4461 & 406.1574 & 404 & 122.5994 & 4.12723 \\
\hline 1091 & 21.5303 & 421.7498 & 405.3814 & 403 & 122.5994 & 4.12401 \\
\hline 1092 & 21.5473 & 411.2113 & 404.6248 & 402 & 122.5994 & 4.12079 \\
\hline 1093 & 21.5643 & 392.0473 & 406.0983 & 402 & 122.5994 & 4.11758 \\
\hline 1094 & 21.5813 & 387.9708 & 405.5918 & 401 & 122.5994 & 4.11438 \\
\hline 1095 & 21.5983 & 361.5835 & 405.1769 & 400 & 122.5994 & 4.11118 \\
\hline 1096 & 21.6153 & 367.9005 & 405.5174 & 399 & 122.5994 & 4.10798 \\
\hline 1097 & 21.6323 & 374.5525 & 405.5039 & 398 & 122.5994 & 4.10479 \\
\hline 1098 & 21.6493 & 378.8629 & 406.8257 & 398 & 122.5994 & 4.10161 \\
\hline 1099 & 21.6663 & 359.4524 & 407.748 & 397 & 122.5994 & 4.09843 \\
\hline 1100 & 21.6833 & 395.3342 & 409.8315 & 396 & 122.5994 & 4.09525 \\
\hline 1101 & 21.7003 & 410.775 & 413.7891 & 395 & 122.5994 & 4.09209 \\
\hline 1102 & 21.7173 & 395.7194 & 420.6901 & 394 & 122.5994 & 4.08892 \\
\hline 1103 & 21.7343 & 392.0473 & 432.298 & 394 & 122.5994 & 4.08576 \\
\hline 1104 & 21.7513 & 389.1542 & 446.4029 & 393 & 122.5994 & 4.08261 \\
\hline 1105 & 21.7683 & 412.3453 & 461.7429 & 392 & 122.5994 & 4.07946 \\
\hline 1106 & 21.7853 & 374.3084 & 474.4632 & 392 & 122.5994 & 4.07631 \\
\hline 1107 & 21.8023 & 385.7473 & 480.4674 & 392 & 122.5994 & 4.07317 \\
\hline 1108 & 21.8193 & 372.8501 & 484.6081 & 393 & 122.5994 & 4.07004 \\
\hline 1109 & 21.8363 & 394.9895 & 490.1432 & 393 & 122.5994 & 4.06691 \\
\hline 1110 & 21.8533 & 376.3064 & 500.7986 & 393 & 122.5994 & 4.06378 \\
\hline 1111 & 21.8703 & 403.8904 & 519.226 & 393 & 122.5994 & 4.06066 \\
\hline 1112 & 21.8873 & 393.5738 & 546.6941 & 394 & 122.5994 & 4.05754 \\
\hline 1113 & 21.9043 & 406.8657 & 574.5227 & 394 & 122.5994 & 4.05443 \\
\hline 1114 & 21.9213 & 423.4652 & 594.8073 & 394 & 122.5994 & 4.05133 \\
\hline 1115 & 21.9383 & 416.4213 & 608.0385 & 394 & 122.5994 & 4.04823 \\
\hline 1116 & 21.9553 & 479.0238 & 616.9468 & 395 & 122.5994 & 4.04513 \\
\hline 1117 & 21.9723 & 484.6139 & 611.2649 & 395 & 122.5994 & 4.04204 \\
\hline 1118 & 21.9893 & 533.0021 & 587.9796 & 395 & 122.5994 & 4.03895 \\
\hline 1119 & 22.0063 & 520.7415 & 557.0072 & 395 & 122.5994 & 4.03587 \\
\hline 1120 & 22.0233 & 617.7515 & 526.2745 & 395 & 122.5994 & 4.03279 \\
\hline 1121 & 22.0403 & 655.3694 & 497.8722 & 396 & 122.5994 & 4.02972 \\
\hline 1122 & 22.0573 & 702.2631 & 472.2239 & 396 & 122.5994 & 4.02665 \\
\hline 1123 & 22.0743 & 703.7664 & 454.0191 & 396 & 122.5994 & 4.02359 \\
\hline 1124 & 22.0913 & 708.5967 & 443.3442 & 396 & 122.5994 & 4.02053 \\
\hline 1125 & 22.1083 & 673.2898 & 440.5179 & 397 & 122.5994 & 4.01748 \\
\hline
\end{tabular}




\begin{tabular}{|c|c|c|c|c|c|c|}
\hline 1126 & 22.1253 & 608.9312 & 441.3764 & 397 & 122.5994 & 4.01443 \\
\hline 1127 & 22.1423 & 574.8764 & 444.1433 & 397 & 122.5994 & 4.01139 \\
\hline 1128 & 22.1593 & 522.5727 & 444.8074 & 397 & 122.5994 & 4.00835 \\
\hline 1129 & 22.1763 & 474.0727 & 444.6217 & 398 & 122.5994 & 4.00532 \\
\hline 1130 & 22.1933 & 446.7281 & 442.5818 & 398 & 122.5994 & 4.00229 \\
\hline 1131 & 22.2103 & 394.3506 & 439.5809 & 398 & 122.5994 & 3.99926 \\
\hline 1132 & 22.2273 & 398.6948 & 436.4321 & 398 & 122.5994 & 3.99624 \\
\hline 1133 & 22.2443 & 380.2571 & 433.312 & 398 & 122.5994 & 3.99322 \\
\hline 1134 & 22.2613 & 365.8953 & 431.3185 & 399 & 122.5994 & 3.99021 \\
\hline 1135 & 22.2783 & 352.7986 & 428.7994 & 399 & 122.5994 & 3.98721 \\
\hline 1136 & 22.2953 & 371.8971 & 426.4935 & 399 & 122.5994 & 3.98421 \\
\hline 1137 & 22.3123 & 386.8288 & 424.4131 & 399 & 122.5994 & 3.98121 \\
\hline 1138 & 22.3293 & 403.6919 & 423 & 400 & 122.5994 & 3.97822 \\
\hline 1139 & 22.3463 & 392.6669 & 422.4392 & 400 & 122.5994 & 3.97523 \\
\hline 1140 & 22.3633 & 392.43 & 423.9468 & 400 & 122.5994 & 3.97224 \\
\hline 1141 & 22.3803 & 381.1803 & 427.7627 & 400 & 122.5994 & 3.96927 \\
\hline 1142 & 22.3973 & 365.6223 & 437.3406 & 400 & 122.5994 & 3.96629 \\
\hline 1143 & 22.4143 & 397.4606 & 452.7643 & 401 & 122.5994 & 3.96332 \\
\hline 1144 & 22.4313 & 406.5362 & 468.9078 & 401 & 122.5994 & 3.96036 \\
\hline 1145 & 22.4483 & 383.0999 & 480.7851 & 401 & 122.5994 & 3.9574 \\
\hline 1146 & 22.4653 & 374.9588 & 486.3541 & 401 & 122.5994 & 3.95444 \\
\hline 1147 & 22.4823 & 381.4968 & 489.7216 & 402 & 122.5994 & 3.95149 \\
\hline 1148 & 22.4993 & 397.6493 & 488.1413 & 402 & 122.5994 & 3.94854 \\
\hline 1149 & 22.5163 & 424.0797 & 478.9791 & 402 & 122.5994 & 3.9456 \\
\hline 1150 & 22.5333 & 429.6015 & 465.7052 & 402 & 122.5994 & 3.94266 \\
\hline 1151 & 22.5503 & 419.3283 & 454.308 & 403 & 122.5994 & 3.93973 \\
\hline 1152 & 22.5673 & 403.8017 & 443.0223 & 403 & 122.5994 & 3.9368 \\
\hline 1153 & 22.5843 & 384.4689 & 432.918 & 403 & 122.5994 & 3.93387 \\
\hline 1154 & 22.6013 & 422.6038 & 424.7512 & 403 & 122.5994 & 3.93095 \\
\hline 1155 & 22.6183 & 389.1732 & 418.8032 & 403 & 122.5994 & 3.92803 \\
\hline 1156 & 22.6353 & 400.4393 & 414.8136 & 403 & 122.5994 & 3.92512 \\
\hline 1157 & 22.6523 & 390.1228 & 412.0949 & 403 & 122.5994 & 3.92222 \\
\hline 1158 & 22.6693 & 398.7486 & 410.2506 & 403 & 122.5994 & 3.91931 \\
\hline 1159 & 22.6863 & 371.2862 & 409.0196 & 403 & 122.5994 & 3.91641 \\
\hline 1160 & 22.7033 & 354.3985 & 408.2158 & 403 & 122.5994 & 3.91352 \\
\hline 1161 & 22.7203 & 379.1555 & 406.7179 & 402 & 122.5994 & 3.91063 \\
\hline 1162 & 22.7373 & 372.606 & 406.4489 & 402 & 122.5994 & 3.90774 \\
\hline 1163 & 22.7543 & 353.505 & 406.367 & 402 & 122.5994 & 3.90486 \\
\hline 1164 & 22.7713 & 384.9171 & 406.4703 & 402 & 122.5994 & 3.90199 \\
\hline 1165 & 22.7883 & 414.0307 & 406.8164 & 402 & 122.5994 & 3.89911 \\
\hline 1166 & 22.8053 & 370.2031 & 407.5572 & 402 & 122.5994 & 3.89625 \\
\hline 1167 & 22.8223 & 388.6084 & 409.031 & 402 & 122.5994 & 3.89338 \\
\hline 1168 & 22.8393 & 360.7597 & 411.564 & 402 & 122.5994 & 3.89052 \\
\hline 1169 & 22.8563 & 417.0825 & 415.6558 & 402 & 122.5994 & 3.88767 \\
\hline 1170 & 22.8733 & 399.0909 & 421.518 & 402 & 122.5994 & 3.88482 \\
\hline 1171 & 22.8903 & 424.1905 & 428.6717 & 402 & 122.5994 & 3.88197 \\
\hline 1172 & 22.9073 & 451.6551 & 435.2017 & 402 & 122.5994 & 3.87913 \\
\hline 1173 & 22.9243 & 416.1186 & 438.9609 & 402 & 122.5994 & 3.87629 \\
\hline 1174 & 22.9413 & 429.6706 & 439.1747 & 402 & 122.5994 & 3.87346 \\
\hline
\end{tabular}




\begin{tabular}{|c|c|c|c|c|c|c|}
\hline 1175 & 22.9583 & 408.3886 & 436.5929 & 402 & 122.5994 & 3.87063 \\
\hline 1176 & 22.9753 & 489.6667 & 433.7871 & 402 & 122.5994 & 3.8678 \\
\hline 1177 & 22.9923 & 484.8821 & 431.0243 & 402 & 122.5994 & 3.86498 \\
\hline 1178 & 23.0093 & 458.2695 & 426.7392 & 401 & 122.5994 & 3.86216 \\
\hline 1179 & 23.0263 & 442.4659 & 424.1915 & 401 & 122.5994 & 3.85935 \\
\hline 1180 & 23.0433 & 442.079 & 422.8387 & 401 & 122.5994 & 3.85654 \\
\hline 1181 & 23.0603 & 445.0495 & 421.5497 & 401 & 122.5994 & 3.85374 \\
\hline 1182 & 23.0773 & 451.0027 & 420.3058 & 401 & 122.5994 & 3.85093 \\
\hline 1183 & 23.0943 & 453.9284 & 418.926 & 401 & 122.5994 & 3.84814 \\
\hline 1184 & 23.1113 & 463.0936 & 417.38 & 401 & 122.5994 & 3.84535 \\
\hline 1185 & 23.1283 & 433.9249 & 415.7827 & 401 & 122.5994 & 3.84256 \\
\hline 1186 & 23.1453 & 418.1456 & 414.3969 & 401 & 122.5994 & 3.83977 \\
\hline 1187 & 23.1623 & 407.6171 & 413.5262 & 401 & 122.5994 & 3.83699 \\
\hline 1188 & 23.1793 & 387.7936 & 413.3657 & 401 & 122.5994 & 3.83422 \\
\hline 1189 & 23.1963 & 409.0519 & 413.8837 & 401 & 122.5994 & 3.83145 \\
\hline 1190 & 23.2133 & 409.5658 & 414.701 & 401 & 122.5994 & 3.82868 \\
\hline 1191 & 23.2303 & 413.1528 & 415.1173 & 401 & 122.5994 & 3.82592 \\
\hline 1192 & 23.2473 & 421.7471 & 414.7973 & 401 & 122.5994 & 3.82316 \\
\hline 1193 & 23.2643 & 389.3391 & 413.144 & 400 & 122.5994 & 3.8204 \\
\hline 1194 & 23.2813 & 390.8987 & 412.6198 & 400 & 122.5994 & 3.81765 \\
\hline 1195 & 23.2983 & 413.989 & 411.9915 & 400 & 122.5994 & 3.8149 \\
\hline 1196 & 23.3153 & 408.6953 & 411.6953 & 400 & 122.5994 & 3.81216 \\
\hline 1197 & 23.3323 & 383.7358 & 412.182 & 400 & 122.5994 & 3.80942 \\
\hline 1198 & 23.3493 & 394.1706 & 414.6778 & 400 & 122.5994 & 3.80669 \\
\hline 1199 & 23.3663 & 422.1292 & 420.3376 & 400 & 122.5994 & 3.80396 \\
\hline 1200 & 23.3833 & 393.1224 & 429.7568 & 400 & 122.5994 & 3.80123 \\
\hline 1201 & 23.4003 & 393.8788 & 443.5847 & 400 & 122.5994 & 3.79851 \\
\hline 1202 & 23.4173 & 402.746 & 459.6724 & 400 & 122.5994 & 3.79579 \\
\hline 1203 & 23.4343 & 421.5717 & 472.0638 & 400 & 122.5994 & 3.79307 \\
\hline 1204 & 23.4513 & 424.3193 & 474.8021 & 400 & 122.5994 & 3.79036 \\
\hline 1205 & 23.4683 & 443.249 & 471.1155 & 400 & 122.5994 & 3.78765 \\
\hline 1206 & 23.4853 & 481.1806 & 466.3384 & 400 & 122.5994 & 3.78495 \\
\hline 1207 & 23.5023 & 500.8568 & 460.5786 & 400 & 122.5994 & 3.78225 \\
\hline 1208 & 23.5193 & 523.7438 & 454.2076 & 400 & 122.5994 & 3.77955 \\
\hline 1209 & 23.5363 & 538.1351 & 450.7995 & 400 & 122.5994 & 3.77686 \\
\hline 1210 & 23.5533 & 552.6993 & 453.1267 & 400 & 122.5994 & 3.77418 \\
\hline 1211 & 23.5703 & 585.4834 & 461.9021 & 400 & 122.5994 & 3.77149 \\
\hline 1212 & 23.5873 & 585.5897 & 476.8319 & 400 & 122.5994 & 3.76881 \\
\hline 1213 & 23.6043 & 667.2697 & 496.0094 & 400 & 122.5994 & 3.76614 \\
\hline 1214 & 23.6213 & 691.2156 & 515.433 & 400 & 122.5994 & 3.76346 \\
\hline 1215 & 23.6383 & 727.3666 & 531.2252 & 400 & 122.5994 & 3.7608 \\
\hline 1216 & 23.6553 & 675.9352 & 541.3864 & 400 & 122.5994 & 3.75813 \\
\hline 1217 & 23.6723 & 673.4725 & 541.3194 & 400 & 122.5994 & 3.75547 \\
\hline 1218 & 23.6893 & 652.08 & 528.9804 & 400 & 122.5994 & 3.75281 \\
\hline 1219 & 23.7063 & 614.0276 & 510.8767 & 400 & 122.5994 & 3.75016 \\
\hline 1220 & 23.7233 & 597.9675 & 492.4124 & 400 & 122.5994 & 3.74751 \\
\hline 1221 & 23.7403 & 547.6935 & 473.884 & 400 & 122.5994 & 3.74487 \\
\hline 1222 & 23.7573 & 496.1296 & 457.0746 & 400 & 122.5994 & 3.74223 \\
\hline 1223 & 23.7743 & 451.5822 & 445.2284 & 400 & 122.5994 & 3.73959 \\
\hline
\end{tabular}




\begin{tabular}{|c|c|c|c|c|c|c|}
\hline 1224 & 23.7913 & 498.7047 & 439.5265 & 400 & 122.5994 & 3.73696 \\
\hline 1225 & 23.8083 & 481.3576 & 439.9478 & 400 & 122.5994 & 3.73433 \\
\hline 1226 & 23.8253 & 436.5362 & 444.3377 & 399 & 122.5994 & 3.7317 \\
\hline 1227 & 23.8423 & 425.375 & 452.9968 & 398 & 122.5994 & 3.72908 \\
\hline 1228 & 23.8593 & 430.2045 & 462.5245 & 397 & 122.5994 & 3.72646 \\
\hline 1229 & 23.8763 & 448.7966 & 468.3446 & 396 & 122.5994 & 3.72384 \\
\hline 1230 & 23.8933 & 467.141 & 470.6819 & 396 & 122.5994 & 3.72123 \\
\hline 1231 & 23.9103 & 500.676 & 471.5793 & 395 & 122.5994 & 3.71863 \\
\hline 1232 & 23.9273 & 489.7407 & 472.2383 & 394 & 122.5994 & 3.71602 \\
\hline 1233 & 23.9443 & 496.4068 & 471.3553 & 393 & 122.5994 & 3.71342 \\
\hline 1234 & 23.9613 & 511.97 & 467.1461 & 392 & 122.5994 & 3.71083 \\
\hline 1235 & 23.9783 & 492.8492 & 458.7246 & 391 & 122.5994 & 3.70823 \\
\hline 1236 & 23.9953 & 492.8492 & 449.8114 & 390 & 122.5994 & 3.70565 \\
\hline 1237 & 24.0123 & 506.1875 & 442.4837 & 389 & 122.5994 & 3.70306 \\
\hline 1238 & 24.0293 & 512.5107 & 436.7306 & 388 & 122.5994 & 3.70048 \\
\hline 1239 & 24.0463 & 525.3296 & 433.2657 & 387 & 122.5994 & 3.6979 \\
\hline 1240 & 24.0633 & 506.7068 & 433.8372 & 386 & 122.5994 & 3.69533 \\
\hline 1241 & 24.0803 & 514.3218 & 439.2018 & 385 & 122.5994 & 3.69276 \\
\hline 1242 & 24.0973 & 499.1589 & 448.3544 & 384 & 122.5994 & 3.69019 \\
\hline 1243 & 24.1143 & 463.3087 & 458.9902 & 384 & 122.5994 & 3.68763 \\
\hline 1244 & 24.1313 & 453.6985 & 464.0353 & 383 & 122.5994 & 3.68507 \\
\hline 1245 & 24.1483 & 431.223 & 462.424 & 382 & 122.5994 & 3.68251 \\
\hline 1246 & 24.1653 & 447.423 & 457.1079 & 381 & 122.5994 & 3.67996 \\
\hline 1247 & 24.1823 & 492.5992 & 450.4179 & 380 & 122.5994 & 3.67741 \\
\hline 1248 & 24.1993 & 479.7369 & 442.6745 & 379 & 122.5994 & 3.67487 \\
\hline 1249 & 24.2163 & 464.1772 & 435.7531 & 378 & 122.5994 & 3.67233 \\
\hline 1250 & 24.2333 & 520.5886 & 433.2054 & 377 & 122.5994 & 3.66979 \\
\hline 1251 & 24.2503 & 516.7341 & 437.9337 & 376 & 122.5994 & 3.66725 \\
\hline 1252 & 24.2673 & 519.4121 & 450.8035 & 375 & 122.5994 & 3.66472 \\
\hline 1253 & 24.2843 & 586.1439 & 471.5729 & 374 & 122.5994 & 3.6622 \\
\hline 1254 & 24.3013 & 571.9945 & 494.2773 & 373 & 122.5994 & 3.65967 \\
\hline 1255 & 24.3183 & 548.0102 & 509.3999 & 373 & 122.5994 & 3.65715 \\
\hline 1256 & 24.3353 & 605.0847 & 507.7097 & 372 & 122.5994 & 3.65464 \\
\hline 1257 & 24.3523 & 575.5442 & 497.072 & 371 & 122.5994 & 3.65212 \\
\hline 1258 & 24.3693 & 556.9066 & 484.1239 & 370 & 122.5994 & 3.64961 \\
\hline 1259 & 24.3863 & 555.0842 & 469.7342 & 369 & 122.5994 & 3.64711 \\
\hline 1260 & 24.4033 & 526.1878 & 454.2185 & 368 & 122.5994 & 3.64461 \\
\hline 1261 & 24.4203 & 491.5429 & 441.2635 & 367 & 122.5994 & 3.64211 \\
\hline 1262 & 24.4373 & 440.8443 & 431.7786 & 366 & 122.5994 & 3.63961 \\
\hline 1263 & 24.4543 & 420.4643 & 424.5758 & 365 & 122.5994 & 3.63712 \\
\hline 1264 & 24.4713 & 459.1294 & 417.6606 & 364 & 122.5994 & 3.63463 \\
\hline 1265 & 24.4883 & 417.2388 & 409.9092 & 363 & 122.5994 & 3.63215 \\
\hline 1266 & 24.5053 & 389.8245 & 401.9502 & 362 & 122.5994 & 3.62967 \\
\hline 1267 & 24.5223 & 395.0661 & 395.2798 & 362 & 122.5994 & 3.62719 \\
\hline 1268 & 24.5393 & 402.6505 & 388.0105 & 361 & 122.5994 & 3.62471 \\
\hline 1269 & 24.5563 & 413.4728 & 381.4094 & 360 & 122.5994 & 3.62224 \\
\hline 1270 & 24.5733 & 370.6597 & 376.1924 & 359 & 122.5994 & 3.61978 \\
\hline 1271 & 24.5903 & 380.3668 & 372.2776 & 358 & 122.5994 & 3.61731 \\
\hline 1272 & 24.6073 & 365.8831 & 369.2065 & 357 & 122.5994 & 3.61485 \\
\hline
\end{tabular}




\begin{tabular}{|c|c|c|c|c|c|c|}
\hline 1273 & 24.6243 & 361.8977 & 366.6522 & 356 & 122.5994 & 3.6124 \\
\hline 1274 & 24.6413 & 357.3421 & 366.5924 & 357 & 122.5994 & 3.60994 \\
\hline 1275 & 24.6583 & 321.7193 & 365.9678 & 357 & 122.5994 & 3.60749 \\
\hline 1276 & 24.6753 & 352.5609 & 366.8241 & 358 & 122.5994 & 3.60504 \\
\hline 1277 & 24.6923 & 396.778 & 368.4248 & 359 & 122.5994 & 3.6026 \\
\hline 1278 & 24.7093 & 386.2255 & 370.0173 & 359 & 122.5994 & 3.60016 \\
\hline 1279 & 24.7263 & 378.3577 & 373.4388 & 360 & 122.5994 & 3.59772 \\
\hline 1280 & 24.7433 & 366.5296 & 376.8338 & 361 & 122.5994 & 3.59529 \\
\hline 1281 & 24.7603 & 369.3148 & 377.6438 & 361 & 122.5994 & 3.59286 \\
\hline 1282 & 24.7773 & 376.3239 & 378.0358 & 362 & 122.5994 & 3.59043 \\
\hline 1283 & 24.7943 & 371.8598 & 378.3194 & 363 & 122.5994 & 3.58801 \\
\hline 1284 & 24.8113 & 370.9776 & 378.1976 & 363 & 122.5994 & 3.58559 \\
\hline 1285 & 24.8283 & 418.0859 & 379.5339 & 364 & 122.5994 & 3.58317 \\
\hline 1286 & 24.8453 & 393.0832 & 381.6414 & 365 & 122.5994 & 3.58076 \\
\hline 1287 & 24.8623 & 369.9623 & 383.1029 & 365 & 122.5994 & 3.57835 \\
\hline 1288 & 24.8793 & 343.934 & 384.6806 & 366 & 122.5994 & 3.57594 \\
\hline 1289 & 24.8963 & 374.5927 & 383.614 & 367 & 122.5994 & 3.57354 \\
\hline 1290 & 24.9133 & 360.9425 & 381.6233 & 367 & 122.5994 & 3.57114 \\
\hline 1291 & 24.9303 & 381.7134 & 380.8044 & 368 & 122.5994 & 3.56874 \\
\hline 1292 & 24.9473 & 385.9203 & 379.7872 & 369 & 122.5994 & 3.56635 \\
\hline 1293 & 24.9643 & 400.0288 & 377.4514 & 369 & 122.5994 & 3.56396 \\
\hline 1294 & 24.9813 & 374.9503 & 376.3921 & 370 & 122.5994 & 3.56157 \\
\hline 1295 & 24.9983 & 385.0244 & 376.0032 & 371 & 122.5994 & 3.55919 \\
\hline 1296 & 25.0153 & 388.0973 & 375.2977 & 371 & 122.5994 & 3.55681 \\
\hline 1297 & 25.0323 & 354.1094 & 376.0636 & 372 & 122.5994 & 3.55443 \\
\hline 1298 & 25.0493 & 373.8765 & 378.9871 & 375 & 122.5994 & 3.55206 \\
\hline 1299 & 25.0663 & 383.6068 & 380.885 & 377 & 122.5994 & 3.54969 \\
\hline 1300 & 25.0833 & 402.9725 & 383.8272 & 380 & 122.5994 & 3.54732 \\
\hline 1301 & 25.1003 & 431.8209 & 388.2174 & 383 & 122.5994 & 3.54496 \\
\hline 1302 & 25.1173 & 400.2476 & 391.5719 & 386 & 122.5994 & 3.5426 \\
\hline 1303 & 25.1343 & 428.7958 & 394.1449 & 388 & 122.5994 & 3.54024 \\
\hline 1304 & 25.1513 & 447.5383 & 397.9654 & 391 & 122.5994 & 3.53789 \\
\hline 1305 & 25.1683 & 399.812 & 402.1511 & 394 & 122.5994 & 3.53553 \\
\hline 1306 & 25.1853 & 459.5486 & 405.3128 & 396 & 122.5994 & 3.53319 \\
\hline 1307 & 25.2023 & 430.174 & 409.271 & 399 & 122.5994 & 3.53084 \\
\hline 1308 & 25.2193 & 444.7056 & 412.5151 & 402 & 122.5994 & 3.5285 \\
\hline 1309 & 25.2363 & 443.4388 & 415.0877 & 405 & 122.5994 & 3.52616 \\
\hline 1310 & 25.2533 & 459.3862 & 416.5839 & 407 & 122.5994 & 3.52383 \\
\hline 1311 & 25.2703 & 457.9374 & 419.1648 & 410 & 122.5994 & 3.52149 \\
\hline 1312 & 25.2873 & 417.6724 & 421.7472 & 413 & 122.5994 & 3.51917 \\
\hline 1313 & 25.3043 & 413.6893 & 424.3267 & 416 & 122.5994 & 3.51684 \\
\hline 1314 & 25.3213 & 429.5398 & 426.4067 & 418 & 122.5994 & 3.51452 \\
\hline 1315 & 25.3383 & 422.5025 & 430.0764 & 421 & 122.5994 & 3.5122 \\
\hline 1316 & 25.3553 & 415.4676 & 434.3663 & 424 & 122.5994 & 3.50988 \\
\hline 1317 & 25.3723 & 389.1643 & 438.3524 & 426 & 122.5994 & 3.50757 \\
\hline 1318 & 25.3893 & 412.3628 & 444.1339 & 429 & 122.5994 & 3.50526 \\
\hline 1319 & 25.4063 & 444.399 & 450.8267 & 432 & 122.5994 & 3.50295 \\
\hline 1320 & 25.4233 & 472.9126 & 458.5202 & 435 & 122.5994 & 3.50065 \\
\hline 1321 & 25.4403 & 479.7939 & 466.2147 & 437 & 122.5994 & 3.49835 \\
\hline
\end{tabular}




\begin{tabular}{|c|c|c|c|c|c|c|}
\hline 1322 & 25.4573 & 462.8149 & 474.7603 & 439 & 122.5994 & 3.49605 \\
\hline 1323 & 25.4743 & 497.1084 & 484.0027 & 441 & 122.5994 & 3.49375 \\
\hline 1324 & 25.4913 & 524.3597 & 494.4083 & 443 & 122.5994 & 3.49146 \\
\hline 1325 & 25.5083 & 528.905 & 508.0407 & 445 & 122.5994 & 3.48917 \\
\hline 1326 & 25.5253 & 545.5065 & 527.9138 & 447 & 122.5994 & 3.48689 \\
\hline 1327 & 25.5423 & 582.5451 & 555.9386 & 449 & 122.5994 & 3.48461 \\
\hline 1328 & 25.5593 & 575.9581 & 590.7673 & 451 & 122.5994 & 3.48233 \\
\hline 1329 & 25.5763 & 557.6757 & 627.6465 & 454 & 122.5994 & 3.48005 \\
\hline 1330 & 25.5933 & 590.4383 & 651.9601 & 456 & 122.5994 & 3.47778 \\
\hline 1331 & 25.6103 & 610.1014 & 654.2902 & 458 & 122.5994 & 3.47551 \\
\hline 1332 & 25.6273 & 591.4162 & 645.5627 & 460 & 122.5994 & 3.47324 \\
\hline 1333 & 25.6443 & 587.2067 & 641.8799 & 462 & 122.5994 & 3.47098 \\
\hline 1334 & 25.6613 & 600.4946 & 645.5309 & 464 & 122.5994 & 3.46872 \\
\hline 1335 & 25.6783 & 622.6349 & 651.6387 & 466 & 122.5994 & 3.46646 \\
\hline 1336 & 25.6953 & 626.3214 & 656.5472 & 468 & 122.5994 & 3.4642 \\
\hline 1337 & 25.7123 & 655.3176 & 650.7819 & 470 & 122.5994 & 3.46195 \\
\hline 1338 & 25.7293 & 665.6524 & 631.8523 & 472 & 122.5994 & 3.4597 \\
\hline 1339 & 25.7463 & 633.5962 & 611.7025 & 474 & 122.5994 & 3.45746 \\
\hline 1340 & 25.7633 & 629.0714 & 595.7916 & 476 & 122.5994 & 3.45521 \\
\hline 1341 & 25.7803 & 613.4512 & 579.0192 & 478 & 122.5994 & 3.45297 \\
\hline 1342 & 25.7973 & 622.7538 & 559.4452 & 480 & 122.5994 & 3.45074 \\
\hline 1343 & 25.8143 & 590.5791 & 541.353 & 482 & 122.5994 & 3.4485 \\
\hline 1344 & 25.8313 & 547.3321 & 527.4563 & 484 & 122.5994 & 3.44627 \\
\hline 1345 & 25.8483 & 538.632 & 518.2902 & 486 & 122.5994 & 3.44404 \\
\hline 1346 & 25.8653 & 516.6905 & 512.0999 & 487 & 122.5994 & 3.44182 \\
\hline 1347 & 25.8823 & 489.4693 & 509.2987 & 489 & 122.5994 & 3.4396 \\
\hline 1348 & 25.8993 & 551.141 & 506.9689 & 490 & 122.5994 & 3.43738 \\
\hline 1349 & 25.9163 & 547.4614 & 505.2026 & 491 & 122.5994 & 3.43516 \\
\hline 1350 & 25.9333 & 521.0362 & 504.2208 & 492 & 122.5994 & 3.43295 \\
\hline 1351 & 25.9503 & 564.0468 & 504.8805 & 494 & 122.5994 & 3.43074 \\
\hline 1352 & 25.9673 & 513.4575 & 505.0341 & 495 & 122.5994 & 3.42853 \\
\hline 1353 & 25.9843 & 489.6215 & 505.234 & 496 & 122.5994 & 3.42633 \\
\hline 1354 & 26.0013 & 500.2235 & 506.3869 & 498 & 122.5994 & 3.42413 \\
\hline 1355 & 26.0183 & 493.2079 & 506.5701 & 499 & 122.5994 & 3.42193 \\
\hline 1356 & 26.0353 & 520.2283 & 506.8587 & 500 & 122.5994 & 3.41973 \\
\hline 1357 & 26.0523 & 506.1513 & 508.2008 & 502 & 122.5994 & 3.41754 \\
\hline 1358 & 26.0693 & 547.5702 & 508.5604 & 503 & 122.5994 & 3.41535 \\
\hline 1359 & 26.0863 & 535.5778 & 508.9982 & 504 & 122.5994 & 3.41316 \\
\hline 1360 & 26.1033 & 510.4994 & 509.5724 & 505 & 122.5994 & 3.41098 \\
\hline 1361 & 26.1203 & 567.7009 & 511.3062 & 507 & 122.5994 & 3.40879 \\
\hline 1362 & 26.1373 & 557.9458 & 512.097 & 508 & 122.5994 & 3.40662 \\
\hline 1363 & 26.1543 & 540.1643 & 598.9316 & 509 & 122.5994 & 3.40444 \\
\hline 1364 & 26.1713 & 572.8873 & 606.6417 & 511 & 122.5994 & 3.40227 \\
\hline 1365 & 26.1883 & 564.7964 & 612.7656 & 512 & 122.5994 & 3.4001 \\
\hline 1366 & 26.2053 & 554.6862 & 621.0715 & 513 & 122.5994 & 3.39793 \\
\hline 1367 & 26.2223 & 550.2549 & 630.3875 & 514 & 122.5994 & 3.39577 \\
\hline 1368 & 26.2393 & 571.5858 & 640.8753 & 516 & 122.5994 & 3.3936 \\
\hline 1369 & 26.2563 & 622.6386 & 650.9148 & 517 & 122.5994 & 3.39145 \\
\hline 1370 & 26.2733 & 629.7992 & 661.9009 & 518 & 122.5994 & 3.38929 \\
\hline
\end{tabular}




\begin{tabular}{|c|c|c|c|c|c|c|}
\hline 1371 & 26.2903 & 638.7903 & 675.224 & 520 & 122.5994 & 3.38714 \\
\hline 1372 & 26.3073 & 694.2971 & 689.2074 & 521 & 122.5994 & 3.38499 \\
\hline 1373 & 26.3243 & 717.0728 & 705.369 & 522 & 122.5994 & 3.38284 \\
\hline 1374 & 26.3413 & 766.2786 & 724.5268 & 523 & 122.5994 & 3.38069 \\
\hline 1375 & 26.3583 & 796.7244 & 748.5717 & 525 & 122.5994 & 3.37855 \\
\hline 1376 & 26.3753 & 843.828 & 776.2039 & 526 & 122.5994 & 3.37641 \\
\hline 1377 & 26.3923 & 895.6096 & 808.446 & 527 & 122.5994 & 3.37428 \\
\hline 1378 & 26.4093 & 978.2363 & 845.8743 & 529 & 122.5994 & 3.37214 \\
\hline 1379 & 26.4263 & 1129.742 & 884.9525 & 530 & 122.5994 & 3.37001 \\
\hline 1380 & 26.4433 & 1246.792 & 927.4623 & 531 & 122.5994 & 3.36789 \\
\hline 1381 & 26.4603 & 1402.448 & 978.0367 & 533 & 122.5994 & 3.36576 \\
\hline 1382 & 26.4773 & 1605.924 & 1037.987 & 534 & 122.5994 & 3.36364 \\
\hline 1383 & 26.4943 & 1838.494 & 1111.053 & 535 & 122.5994 & 3.36152 \\
\hline 1384 & 26.5113 & 2162.535 & 1200.606 & 536 & 122.5994 & 3.3594 \\
\hline 1385 & 26.5283 & 2659.817 & 1316.483 & 538 & 122.5994 & 3.35729 \\
\hline 1386 & 26.5453 & 3228.199 & 1470.293 & 539 & 122.5994 & 3.35517 \\
\hline 1387 & 26.5623 & 3950.808 & 1694.327 & 540 & 122.5994 & 3.35307 \\
\hline 1388 & 26.5793 & 4890.8 & 2054.637 & 542 & 122.5994 & 3.35096 \\
\hline 1389 & 26.5963 & 6216.134 & 2674.712 & 543 & 122.5994 & 3.34886 \\
\hline 1390 & 26.6133 & 7954.543 & 3755.004 & 544 & 122.5994 & 3.34676 \\
\hline 1391 & 26.6303 & 10437.53 & 5534.568 & 545 & 122.5994 & 3.34466 \\
\hline 1392 & 26.6473 & 14158.21 & 8175.153 & 547 & 122.5994 & 3.34256 \\
\hline 1393 & 26.6643 & 19318.28 & 11573.46 & 548 & 122.5994 & 3.34047 \\
\hline 1394 & 26.6813 & 25153.56 & 15095.92 & 549 & 122.5994 & 3.33838 \\
\hline 1395 & 26.6983 & 26658.87 & 17397.95 & 549 & 122.5994 & 3.33629 \\
\hline 1396 & 26.7153 & 19571.16 & 17483.66 & 550 & 122.5994 & 3.33421 \\
\hline 1397 & 26.7323 & 14164.3 & 16117.32 & 550 & 122.5994 & 3.33213 \\
\hline 1398 & 26.7493 & 13586.86 & 14445.68 & 551 & 122.5994 & 3.33005 \\
\hline 1399 & 26.7663 & 12709.53 & 12576.3 & 551 & 122.5994 & 3.32797 \\
\hline 1400 & 26.7833 & 8209.373 & 10220.1 & 552 & 122.5994 & 3.3259 \\
\hline 1401 & 26.8003 & 4427.534 & 7703.45 & 553 & 122.5994 & 3.32383 \\
\hline 1402 & 26.8173 & 2828.422 & 5541.548 & 553 & 122.5994 & 3.32176 \\
\hline 1403 & 26.8343 & 2210.034 & 3936.2 & 554 & 122.5994 & 3.31969 \\
\hline 1404 & 26.8513 & 2008.365 & 2864.488 & 554 & 122.5994 & 3.31763 \\
\hline 1405 & 26.8683 & 1884.489 & 2210.23 & 555 & 122.5994 & 3.31557 \\
\hline 1406 & 26.8853 & 1645.114 & 1827.706 & 555 & 122.5994 & 3.31351 \\
\hline 1407 & 26.9023 & 1450.542 & 1598.421 & 556 & 122.5994 & 3.31146 \\
\hline 1408 & 26.9193 & 1316.276 & 1441.744 & 556 & 122.5994 & 3.3094 \\
\hline 1409 & 26.9363 & 1276.301 & 1321.093 & 557 & 122.5994 & 3.30735 \\
\hline 1410 & 26.9533 & 1177.496 & 1229.727 & 558 & 122.5994 & 3.30531 \\
\hline 1411 & 26.9703 & 1267.662 & 1168.605 & 558 & 122.5994 & 3.30326 \\
\hline 1412 & 26.9873 & 1314.319 & 1133.541 & 559 & 122.5994 & 3.30122 \\
\hline 1413 & 27.0043 & 1295.152 & 1109.574 & 559 & 122.5994 & 3.29918 \\
\hline 1414 & 27.0213 & 1135.649 & 1082.844 & 560 & 122.5994 & 3.29714 \\
\hline 1415 & 27.0383 & 1002.582 & 1039.6 & 560 & 122.5994 & 3.29511 \\
\hline 1416 & 27.0553 & 990.299 & 991.4255 & 561 & 122.5994 & 3.29308 \\
\hline 1417 & 27.0723 & 987.7938 & 948.894 & 561 & 122.5994 & 3.29105 \\
\hline 1418 & 27.0893 & 893.929 & 910.0455 & 561 & 122.5994 & 3.28902 \\
\hline 1419 & 27.1063 & 794.9341 & 873.1283 & 561 & 122.5994 & 3.287 \\
\hline
\end{tabular}




\begin{tabular}{|c|c|c|c|c|c|c|}
\hline 1420 & 27.1233 & 732.6444 & 829.6371 & 561 & 122.5994 & 3.28497 \\
\hline 1421 & 27.1403 & 687.7446 & 791.8474 & 561 & 122.5994 & 3.28295 \\
\hline 1422 & 27.1573 & 700.2846 & 760.5001 & 560 & 122.5994 & 3.28094 \\
\hline 1423 & 27.1743 & 711.8865 & 736.6836 & 560 & 122.5994 & 3.27892 \\
\hline 1424 & 27.1913 & 694.5276 & 719.3704 & 560 & 122.5994 & 3.27691 \\
\hline 1425 & 27.2083 & 618.9315 & 705.5807 & 560 & 122.5994 & 3.2749 \\
\hline 1426 & 27.2253 & 577.5766 & 693.8651 & 559 & 122.5994 & 3.2729 \\
\hline 1427 & 27.2423 & 534.5956 & 583.4232 & 559 & 122.5994 & 3.27089 \\
\hline 1428 & 27.2593 & 544.6234 & 583.0292 & 559 & 122.5994 & 3.26889 \\
\hline 1429 & 27.2763 & 563.8458 & 583.4323 & 559 & 122.5994 & 3.26689 \\
\hline 1430 & 27.2933 & 531.6504 & 583.5092 & 558 & 122.5994 & 3.2649 \\
\hline 1431 & 27.3103 & 570.4052 & 585.2418 & 558 & 122.5994 & 3.2629 \\
\hline 1432 & 27.3273 & 555.5105 & 588.3513 & 558 & 122.5994 & 3.26091 \\
\hline 1433 & 27.3443 & 566.7569 & 600.2173 & 558 & 122.5994 & 3.25892 \\
\hline 1434 & 27.3613 & 579.2157 & 605.2006 & 558 & 122.5994 & 3.25694 \\
\hline 1435 & 27.3783 & 560.4447 & 610.8516 & 557 & 122.5994 & 3.25495 \\
\hline 1436 & 27.3953 & 590.6042 & 620.5965 & 557 & 122.5994 & 3.25297 \\
\hline 1437 & 27.4123 & 649.216 & 638.7993 & 557 & 122.5994 & 3.25099 \\
\hline 1438 & 27.4293 & 703.3415 & 661.7715 & 557 & 122.5994 & 3.24902 \\
\hline 1439 & 27.4463 & 753.2788 & 701.399 & 556 & 122.5994 & 3.24704 \\
\hline 1440 & 27.4633 & 871.6062 & 772.2052 & 556 & 122.5994 & 3.24507 \\
\hline 1441 & 27.4803 & 996.5495 & 887.0179 & 556 & 122.5994 & 3.2431 \\
\hline 1442 & 27.4973 & 1103.014 & 1047.23 & 555 & 122.5994 & 3.24114 \\
\hline 1443 & 27.5143 & 1256.453 & 1239.966 & 554 & 122.5994 & 3.23917 \\
\hline 1444 & 27.5313 & 1382.837 & 1408.112 & 553 & 122.5994 & 3.23721 \\
\hline 1445 & 27.5483 & 1525.468 & 1464.127 & 552 & 122.5994 & 3.23525 \\
\hline 1446 & 27.5653 & 1668.31 & 1401.065 & 551 & 122.5994 & 3.2333 \\
\hline 1447 & 27.5823 & 1442.519 & 1306.433 & 550 & 122.5994 & 3.23134 \\
\hline 1448 & 27.5993 & 1290.669 & 1224.263 & 548 & 122.5994 & 3.22939 \\
\hline 1449 & 27.6163 & 1159.662 & 1132.022 & 547 & 122.5994 & 3.22744 \\
\hline 1450 & 27.6333 & 1106.93 & 1011.85 & 546 & 122.5994 & 3.22549 \\
\hline 1451 & 27.6503 & 918.9164 & 895.4912 & 545 & 122.5994 & 3.22355 \\
\hline 1452 & 27.6673 & 805.2488 & 809.1996 & 544 & 122.5994 & 3.22161 \\
\hline 1453 & 27.6843 & 724.4269 & 760.3627 & 543 & 122.5994 & 3.21967 \\
\hline 1454 & 27.7013 & 670.5805 & 745.765 & 542 & 122.5994 & 3.21773 \\
\hline 1455 & 27.7183 & 667.2166 & 758.4836 & 541 & 122.5994 & 3.2158 \\
\hline 1456 & 27.7353 & 732.188 & 790.2313 & 540 & 122.5994 & 3.21386 \\
\hline 1457 & 27.7523 & 823.5896 & 831.4585 & 539 & 122.5994 & 3.21193 \\
\hline 1458 & 27.7693 & 880.2382 & 871.6042 & 538 & 122.5994 & 3.21001 \\
\hline 1459 & 27.7863 & 967.9963 & 907.2765 & 537 & 122.5994 & 3.20808 \\
\hline 1460 & 27.8033 & 1062.726 & 939.108 & 536 & 122.5994 & 3.20616 \\
\hline 1461 & 27.8203 & 1159.395 & 966.103 & 535 & 122.5994 & 3.20424 \\
\hline 1462 & 27.8373 & 1195.294 & 994.9127 & 533 & 122.5994 & 3.20232 \\
\hline 1463 & 27.8543 & 1227.072 & 1042.18 & 532 & 122.5994 & 3.2004 \\
\hline 1464 & 27.8713 & 1256.287 & 1117.692 & 531 & 122.5994 & 3.19849 \\
\hline 1465 & 27.8883 & 1359.479 & 1224.462 & 530 & 122.5994 & 3.19658 \\
\hline 1466 & 27.9053 & 1479.703 & 1364.113 & 528 & 122.5994 & 3.19467 \\
\hline 1467 & 27.9223 & 1535.225 & 1530.893 & 526 & 122.5994 & 3.19276 \\
\hline 1468 & 27.9393 & 1651.522 & 1723.371 & 524 & 122.5994 & 3.19086 \\
\hline
\end{tabular}




\begin{tabular}{|c|c|c|c|c|c|c|}
\hline 1469 & 27.9563 & 1936.849 & 1908.706 & 522 & 122.5994 & 3.18896 \\
\hline 1470 & 27.9733 & 2213.605 & 2050.523 & 520 & 122.5994 & 3.18706 \\
\hline 1471 & 27.9903 & 2182.245 & 2115.465 & 518 & 122.5994 & 3.18516 \\
\hline 1472 & 28.0073 & 2056.847 & 2098.728 & 516 & 122.5994 & 3.18327 \\
\hline 1473 & 28.0243 & 2025.038 & 2009.788 & 514 & 122.5994 & 3.18137 \\
\hline 1474 & 28.0413 & 1963.091 & 1871.378 & 512 & 122.5994 & 3.17948 \\
\hline 1475 & 28.0583 & 1861.566 & 1700.899 & 510 & 122.5994 & 3.1776 \\
\hline 1476 & 28.0753 & 1690.769 & 1511.606 & 508 & 122.5994 & 3.17571 \\
\hline 1477 & 28.0923 & 1325.509 & 1319.376 & 506 & 122.5994 & 3.17383 \\
\hline 1478 & 28.1093 & 1098.546 & 1142.212 & 504 & 122.5994 & 3.17195 \\
\hline 1479 & 28.1263 & 942.0381 & 992.376 & 502 & 122.5994 & 3.17007 \\
\hline 1480 & 28.1433 & 897.2249 & 873.4818 & 500 & 122.5994 & 3.16819 \\
\hline 1481 & 28.1603 & 763.7963 & 783.6266 & 498 & 122.5994 & 3.16632 \\
\hline 1482 & 28.1773 & 649.14 & 718.3164 & 496 & 122.5994 & 3.16445 \\
\hline 1483 & 28.1943 & 591.2279 & 672.3534 & 494 & 122.5994 & 3.16258 \\
\hline 1484 & 28.2113 & 603.388 & 641.0394 & 492 & 122.5994 & 3.16071 \\
\hline 1485 & 28.2283 & 560.3359 & 621.7699 & 491 & 122.5994 & 3.15884 \\
\hline 1486 & 28.2453 & 548.0241 & 610.0721 & 489 & 122.5994 & 3.15698 \\
\hline 1487 & 28.2623 & 575.1839 & 605.1707 & 487 & 122.5994 & 3.15512 \\
\hline 1488 & 28.2793 & 543.0767 & 605.2585 & 485 & 122.5994 & 3.15326 \\
\hline 1489 & 28.2963 & 566.144 & 607.6826 & 483 & 122.5994 & 3.15141 \\
\hline 1490 & 28.3133 & 547.5702 & 607.1799 & 480 & 122.5994 & 3.14955 \\
\hline 1491 & 28.3303 & 549.8287 & 600.3419 & 477 & 122.5994 & 3.1477 \\
\hline 1492 & 28.3473 & 582.1327 & 587.7804 & 474 & 122.5994 & 3.14585 \\
\hline 1493 & 28.3643 & 544.339 & 573.6953 & 471 & 122.5994 & 3.14401 \\
\hline 1494 & 28.3813 & 551.8117 & 560.954 & 469 & 122.5994 & 3.14216 \\
\hline 1495 & 28.3983 & 514.6413 & 545.7968 & 466 & 122.5994 & 3.14032 \\
\hline 1496 & 28.4153 & 504.1165 & 530.4131 & 463 & 122.5994 & 3.13848 \\
\hline 1497 & 28.4323 & 473.3688 & 515.6492 & 460 & 122.5994 & 3.13664 \\
\hline 1498 & 28.4493 & 480.2942 & 503.8492 & 458 & 122.5994 & 3.13481 \\
\hline 1499 & 28.4663 & 460.6552 & 492.9493 & 455 & 122.5994 & 3.13297 \\
\hline 1500 & 28.4833 & 439.9486 & 484.4696 & 452 & 122.5994 & 3.13114 \\
\hline 1501 & 28.5003 & 433.4015 & 477.5116 & 449 & 122.5994 & 3.12931 \\
\hline 1502 & 28.5173 & 417.1582 & 472.5824 & 447 & 122.5994 & 3.12749 \\
\hline 1503 & 28.5343 & 391.2731 & 467.3111 & 444 & 122.5994 & 3.12566 \\
\hline 1504 & 28.5513 & 381.7779 & 462.4646 & 441 & 122.5994 & 3.12384 \\
\hline 1505 & 28.5683 & 395.7145 & 457.9095 & 438 & 122.5994 & 3.12202 \\
\hline 1506 & 28.5853 & 392.6467 & 453.593 & 435 & 122.5994 & 3.1202 \\
\hline 1507 & 28.6023 & 393.6157 & 450.2562 & 433 & 122.5994 & 3.11838 \\
\hline 1508 & 28.6193 & 406.5465 & 437.6712 & 430 & 122.5994 & 3.11657 \\
\hline 1509 & 28.6363 & 418.5971 & 434.2628 & 427 & 122.5994 & 3.11476 \\
\hline 1510 & 28.6533 & 403.4977 & 430.9955 & 424 & 122.5994 & 3.11295 \\
\hline 1511 & 28.6703 & 426.2506 & 428.7664 & 422 & 122.5994 & 3.11114 \\
\hline 1512 & 28.6873 & 432.5038 & 425.7451 & 419 & 122.5994 & 3.10934 \\
\hline 1513 & 28.7043 & 411.3933 & 418.669 & 416 & 122.5994 & 3.10753 \\
\hline 1514 & 28.7213 & 400.0075 & 418.7826 & 416 & 122.5994 & 3.10573 \\
\hline 1515 & 28.7383 & 409.3106 & 418.6858 & 416 & 122.5994 & 3.10394 \\
\hline 1516 & 28.7553 & 418.5623 & 417.4712 & 415 & 122.5994 & 3.10214 \\
\hline 1517 & 28.7723 & 400.3622 & 417.2583 & 415 & 122.5994 & 3.10034 \\
\hline
\end{tabular}




\begin{tabular}{|c|c|c|c|c|c|c|}
\hline 1518 & 28.7893 & 400.8688 & 417.0586 & 415 & 122.5994 & 3.09855 \\
\hline 1519 & 28.8063 & 384.5237 & 416.8246 & 415 & 122.5994 & 3.09676 \\
\hline 1520 & 28.8233 & 385.8545 & 416.5674 & 415 & 122.5994 & 3.09497 \\
\hline 1521 & 28.8403 & 386.4936 & 415.3462 & 414 & 122.5994 & 3.09319 \\
\hline 1522 & 28.8573 & 364.5929 & 415.2028 & 414 & 122.5994 & 3.09141 \\
\hline 1523 & 28.8743 & 377.3273 & 415.1636 & 414 & 122.5994 & 3.08962 \\
\hline 1524 & 28.8913 & 402.5375 & 415.2557 & 414 & 122.5994 & 3.08784 \\
\hline 1525 & 28.9083 & 404.8607 & 415.5095 & 414 & 122.5994 & 3.08607 \\
\hline 1526 & 28.9253 & 391.085 & 414.8802 & 413 & 122.5994 & 3.08429 \\
\hline 1527 & 28.9423 & 411.5043 & 415.2573 & 413 & 122.5994 & 3.08252 \\
\hline 1528 & 28.9593 & 423.1822 & 415.8695 & 413 & 122.5994 & 3.08075 \\
\hline 1529 & 28.9763 & 468.9326 & 416.2437 & 413 & 122.5994 & 3.07898 \\
\hline 1530 & 28.9933 & 507.159 & 415.207 & 412 & 122.5994 & 3.07721 \\
\hline 1531 & 29.0103 & 557.6203 & 414.8927 & 412 & 122.5994 & 3.07545 \\
\hline 1532 & 29.0273 & 488.5601 & 414.493 & 412 & 122.5994 & 3.07369 \\
\hline 1533 & 29.0443 & 450.7286 & 414.2114 & 412 & 122.5994 & 3.07193 \\
\hline 1534 & 29.0613 & 470.5661 & 413.8523 & 412 & 122.5994 & 3.07017 \\
\hline 1535 & 29.0783 & 416.2411 & 412.4198 & 411 & 122.5994 & 3.06841 \\
\hline 1536 & 29.0953 & 428.3845 & 412.0132 & 411 & 122.5994 & 3.06666 \\
\hline 1537 & 29.1123 & 422.015 & 411.6943 & 411 & 122.5994 & 3.0649 \\
\hline 1538 & 29.1293 & 378.3178 & 411.4729 & 411 & 122.5994 & 3.06315 \\
\hline 1539 & 29.1463 & 403.2808 & 410.3223 & 410 & 122.5994 & 3.06141 \\
\hline 1540 & 29.1633 & 392.8653 & 410.2376 & 410 & 122.5994 & 3.05966 \\
\hline 1541 & 29.1803 & 367.6152 & 409.1855 & 409 & 122.5994 & 3.05792 \\
\hline 1542 & 29.1973 & 353.5364 & 409.1516 & 409 & 122.5994 & 3.05618 \\
\hline 1543 & 29.2143 & 322.4723 & 408.1226 & 408 & 122.5994 & 3.05444 \\
\hline 1544 & 29.2313 & 365.5815 & 408.1044 & 408 & 122.5994 & 3.0527 \\
\hline 1545 & 29.2483 & 362.8099 & 407.0897 & 407 & 122.5994 & 3.05096 \\
\hline 1546 & 29.2653 & 341.322 & 407.0776 & 407 & 122.5994 & 3.04923 \\
\hline 1547 & 29.2823 & 338.5663 & 406.0677 & 406 & 122.5994 & 3.0475 \\
\hline 1548 & 29.2993 & 347.1594 & 407.059 & 406 & 122.5994 & 3.04577 \\
\hline 1549 & 29.3163 & 366.2998 & 407.123 & 406 & 122.5994 & 3.04404 \\
\hline 1550 & 29.3333 & 361.0067 & 406.1961 & 405 & 122.5994 & 3.04231 \\
\hline 1551 & 29.3503 & 357.0451 & 406.2791 & 405 & 122.5994 & 3.04059 \\
\hline 1552 & 29.3673 & 356.7002 & 405.3734 & 404 & 122.5994 & 3.03887 \\
\hline 1553 & 29.3843 & 380.9787 & 405.6788 & 404 & 122.5994 & 3.03715 \\
\hline 1554 & 29.4013 & 387.0003 & 404.7945 & 403 & 122.5994 & 3.03543 \\
\hline 1555 & 29.4183 & 358.7515 & 404.9488 & 403 & 122.5994 & 3.03372 \\
\hline 1556 & 29.4353 & 344.2679 & 404.1252 & 402 & 122.5994 & 3.032 \\
\hline 1557 & 29.4523 & 354.9071 & 404.3282 & 402 & 122.5994 & 3.03029 \\
\hline 1558 & 29.4693 & 388.3359 & 403.5538 & 401 & 122.5994 & 3.02858 \\
\hline 1559 & 29.4863 & 389.4788 & 403.829 & 401 & 122.5994 & 3.02688 \\
\hline 1560 & 29.5033 & 383.1295 & 403.153 & 400 & 122.5994 & 3.02517 \\
\hline 1561 & 29.5203 & 392.0473 & 403.5383 & 400 & 122.5994 & 3.02347 \\
\hline 1562 & 29.5373 & 411.017 & 404.0021 & 400 & 122.5994 & 3.02177 \\
\hline 1563 & 29.5543 & 426.8809 & 404.5683 & 400 & 122.5994 & 3.02007 \\
\hline 1564 & 29.5713 & 415.6144 & 405.2722 & 400 & 122.5994 & 3.01837 \\
\hline 1565 & 29.5883 & 421.3225 & 406.1725 & 400 & 122.5994 & 3.01667 \\
\hline 1566 & 29.6053 & 403.9686 & 407.3798 & 400 & 122.5994 & 3.01498 \\
\hline
\end{tabular}




\begin{tabular}{|c|c|c|c|c|c|c|}
\hline 1567 & 29.6223 & 431.9113 & 409.2615 & 400 & 122.5994 & 3.01329 \\
\hline 1568 & 29.6393 & 440.405 & 412.0436 & 400 & 122.5994 & 3.0116 \\
\hline 1569 & 29.6563 & 403.7974 & 416.7878 & 400 & 122.5994 & 3.00991 \\
\hline 1570 & 29.6733 & 389.0489 & 424.9747 & 400 & 122.5994 & 3.00822 \\
\hline 1571 & 29.6903 & 403.6241 & 438.3275 & 400 & 122.5994 & 3.00654 \\
\hline 1572 & 29.7073 & 434.4833 & 457.8533 & 400 & 122.5994 & 3.00486 \\
\hline 1573 & 29.7243 & 441.0082 & 481.7267 & 400 & 122.5994 & 3.00318 \\
\hline 1574 & 29.7413 & 492.358 & 503.594 & 400 & 122.5994 & 3.0015 \\
\hline 1575 & 29.7583 & 533.3851 & 512.7692 & 399 & 122.5994 & 2.99983 \\
\hline 1576 & 29.7753 & 530.0431 & 508.5915 & 399 & 122.5994 & 2.99815 \\
\hline 1577 & 29.7923 & 533.1426 & 500.5688 & 399 & 122.5994 & 2.99648 \\
\hline 1578 & 29.8093 & 516.5613 & 497.3009 & 399 & 122.5994 & 2.99481 \\
\hline 1579 & 29.8263 & 528.3609 & 499.5775 & 399 & 122.5994 & 2.99314 \\
\hline 1580 & 29.8433 & 551.6605 & 504.3155 & 399 & 122.5994 & 2.99147 \\
\hline 1581 & 29.8603 & 585.5086 & 511.0765 & 399 & 122.5994 & 2.98981 \\
\hline 1582 & 29.8773 & 516.2844 & 517.9886 & 399 & 122.5994 & 2.98815 \\
\hline 1583 & 29.8943 & 573.1356 & 517.5417 & 399 & 122.5994 & 2.98649 \\
\hline 1584 & 29.9113 & 504.248 & 508.0454 & 399 & 122.5994 & 2.98483 \\
\hline 1585 & 29.9283 & 542.9001 & 497.8673 & 399 & 122.5994 & 2.98317 \\
\hline 1586 & 29.9453 & 546.8185 & 490.3434 & 398 & 122.5994 & 2.98152 \\
\hline 1587 & 29.9623 & 574.3514 & 483.8054 & 398 & 122.5994 & 2.97986 \\
\hline 1588 & 29.9793 & 526.281 & 471.3609 & 397 & 122.5994 & 2.97821 \\
\hline 1589 & 29.9963 & 504.0784 & 455.8062 & 396 & 122.5994 & 2.97656 \\
\hline 1590 & 30.0133 & 472.55 & 441.3861 & 395 & 122.5994 & 2.97492 \\
\hline 1591 & 30.0303 & 477.78 & 430.7943 & 395 & 122.5994 & 2.97327 \\
\hline 1592 & 30.0473 & 461.0438 & 421.8804 & 394 & 122.5994 & 2.97163 \\
\hline 1593 & 30.0643 & 443.7155 & 415.1339 & 393 & 122.5994 & 2.96999 \\
\hline 1594 & 30.0813 & 481.1591 & 410.0968 & 392 & 122.5994 & 2.96835 \\
\hline 1595 & 30.0983 & 463.5384 & 407.5817 & 392 & 122.5994 & 2.96671 \\
\hline 1596 & 30.1153 & 461.1168 & 405.2569 & 391 & 122.5994 & 2.96507 \\
\hline 1597 & 30.1323 & 442.3256 & 404.1279 & 390 & 122.5994 & 2.96344 \\
\hline 1598 & 30.1493 & 421.1049 & 404.1471 & 389 & 122.5994 & 2.96181 \\
\hline 1599 & 30.1663 & 422.2859 & 405.7588 & 389 & 122.5994 & 2.96018 \\
\hline 1600 & 30.1833 & 438.4044 & 407.9368 & 388 & 122.5994 & 2.95855 \\
\hline 1601 & 30.2003 & 446.561 & 410.9451 & 387 & 122.5994 & 2.95692 \\
\hline 1602 & 30.2173 & 453.7801 & 414.7648 & 386 & 122.5994 & 2.9553 \\
\hline 1603 & 30.2343 & 430.7014 & 419.3644 & 386 & 122.5994 & 2.95367 \\
\hline 1604 & 30.2513 & 434.6356 & 422.615 & 385 & 122.5994 & 2.95205 \\
\hline 1605 & 30.2683 & 469.8374 & 425.3484 & 384 & 122.5994 & 2.95043 \\
\hline 1606 & 30.2853 & 490.1511 & 429.6631 & 383 & 122.5994 & 2.94881 \\
\hline 1607 & 30.3023 & 460.1394 & 435.9468 & 383 & 122.5994 & 2.9472 \\
\hline 1608 & 30.3193 & 460.7525 & 441.83 & 382 & 122.5994 & 2.94558 \\
\hline 1609 & 30.3363 & 454.7672 & 447.7018 & 381 & 122.5994 & 2.94397 \\
\hline 1610 & 30.3533 & 464.9354 & 451.5084 & 380 & 122.5994 & 2.94236 \\
\hline 1611 & 30.3703 & 526.1993 & 452.2377 & 380 & 122.5994 & 2.94075 \\
\hline 1612 & 30.3873 & 536.8506 & 449.4827 & 379 & 122.5994 & 2.93915 \\
\hline 1613 & 30.4043 & 548.0796 & 447.1354 & 378 & 122.5994 & 2.93754 \\
\hline 1614 & 30.4213 & 537.238 & 446.002 & 377 & 122.5994 & 2.93594 \\
\hline 1615 & 30.4383 & 522.3481 & 446.2966 & 377 & 122.5994 & 2.93434 \\
\hline
\end{tabular}




\begin{tabular}{|c|c|c|c|c|c|c|}
\hline 1616 & 30.4553 & 537.1098 & 445.5656 & 376 & 122.5994 & 2.93274 \\
\hline 1617 & 30.4723 & 564.7163 & 445.0328 & 375 & 122.5994 & 2.93114 \\
\hline 1618 & 30.4893 & 548.7947 & 443.7215 & 374 & 122.5994 & 2.92955 \\
\hline 1619 & 30.5063 & 577.6065 & 440.8737 & 374 & 122.5994 & 2.92795 \\
\hline 1620 & 30.5233 & 589.6999 & 434.4432 & 373 & 122.5994 & 2.92636 \\
\hline 1621 & 30.5403 & 561.787 & 427.3526 & 372 & 122.5994 & 2.92477 \\
\hline 1622 & 30.5573 & 466.3093 & 420.207 & 371 & 122.5994 & 2.92318 \\
\hline 1623 & 30.5743 & 438.516 & 413.4703 & 371 & 122.5994 & 2.9216 \\
\hline 1624 & 30.5913 & 484.3404 & 405.4119 & 370 & 122.5994 & 2.92001 \\
\hline 1625 & 30.6083 & 455.6432 & 397.8804 & 369 & 122.5994 & 2.91843 \\
\hline 1626 & 30.6253 & 394.2723 & 391.398 & 368 & 122.5994 & 2.91685 \\
\hline 1627 & 30.6423 & 376.5953 & 387.0162 & 368 & 122.5994 & 2.91527 \\
\hline 1628 & 30.6593 & 391.672 & 382.7986 & 367 & 122.5994 & 2.91369 \\
\hline 1629 & 30.6763 & 338.6717 & 380.1132 & 366 & 122.5994 & 2.91211 \\
\hline 1630 & 30.6933 & 342.0328 & 378.795 & 365 & 122.5994 & 2.91054 \\
\hline 1631 & 30.7103 & 340.171 & 379.9124 & 365 & 122.5994 & 2.90897 \\
\hline 1632 & 30.7273 & 363.5273 & 381.8965 & 364 & 122.5994 & 2.9074 \\
\hline 1633 & 30.7443 & 383.2701 & 386.641 & 363 & 122.5994 & 2.90583 \\
\hline 1634 & 30.7613 & 351.166 & 394.8395 & 362 & 122.5994 & 2.90426 \\
\hline 1635 & 30.7783 & 383.5508 & 406.1672 & 361 & 122.5994 & 2.9027 \\
\hline 1636 & 30.7953 & 391.0919 & 419.4166 & 361 & 122.5994 & 2.90113 \\
\hline 1637 & 30.8123 & 438.4987 & 429.9643 & 360 & 122.5994 & 2.89957 \\
\hline 1638 & 30.8293 & 433.1518 & 437.8599 & 359 & 122.5994 & 2.89801 \\
\hline 1639 & 30.8463 & 444.3435 & 444.2553 & 358 & 122.5994 & 2.89645 \\
\hline 1640 & 30.8633 & 434.0541 & 449.5181 & 357 & 122.5994 & 2.89489 \\
\hline 1641 & 30.8803 & 442.2569 & 451.4128 & 357 & 122.5994 & 2.89334 \\
\hline 1642 & 30.8973 & 421.7924 & 445.0194 & 356 & 122.5994 & 2.89179 \\
\hline 1643 & 30.9143 & 400.5005 & 432.4459 & 355 & 122.5994 & 2.89023 \\
\hline 1644 & 30.9313 & 379.6616 & 418.8357 & 354 & 122.5994 & 2.88868 \\
\hline 1645 & 30.9483 & 395.5678 & 407.9919 & 354 & 122.5994 & 2.88714 \\
\hline 1646 & 30.9653 & 383.4721 & 397.3286 & 353 & 122.5994 & 2.88559 \\
\hline 1647 & 30.9823 & 371.5641 & 386.6151 & 352 & 122.5994 & 2.88405 \\
\hline 1648 & 30.9993 & 368.1332 & 376.4547 & 351 & 122.5994 & 2.8825 \\
\hline 1649 & 31.0163 & 367.2254 & 368.0258 & 350 & 122.5994 & 2.88096 \\
\hline 1650 & 31.0333 & 361.0067 & 362.8013 & 350 & 122.5994 & 2.87942 \\
\hline 1651 & 31.0503 & 335.2913 & 358.544 & 349 & 122.5994 & 2.87788 \\
\hline 1652 & 31.0673 & 324.8397 & 355.4877 & 348 & 122.5994 & 2.87635 \\
\hline 1653 & 31.0843 & 336.5664 & 353.5118 & 347 & 122.5994 & 2.87481 \\
\hline 1654 & 31.1013 & 345.4042 & 352.0671 & 346 & 122.5994 & 2.87328 \\
\hline 1655 & 31.1183 & 338.2209 & 351.8939 & 346 & 122.5994 & 2.87175 \\
\hline 1656 & 31.1353 & 341.0722 & 351.0642 & 345 & 122.5994 & 2.87022 \\
\hline 1657 & 31.1523 & 356.8327 & 350.2104 & 344 & 122.5994 & 2.86869 \\
\hline 1658 & 31.1693 & 343.9701 & 350.8251 & 344 & 122.5994 & 2.86717 \\
\hline 1659 & 31.1863 & 334.8963 & 351.7186 & 344 & 122.5994 & 2.86564 \\
\hline 1660 & 31.2033 & 374.6477 & 352.5306 & 344 & 122.5994 & 2.86412 \\
\hline 1661 & 31.2203 & 380.7601 & 353.8571 & 344 & 122.5994 & 2.8626 \\
\hline 1662 & 31.2373 & 411.8159 & 355.1959 & 344 & 122.5994 & 2.86108 \\
\hline 1663 & 31.2543 & 384.944 & 356.1298 & 344 & 122.5994 & 2.85956 \\
\hline 1664 & 31.2713 & 409.8256 & 357.9687 & 345 & 122.5994 & 2.85805 \\
\hline
\end{tabular}




\begin{tabular}{|c|c|c|c|c|c|c|}
\hline 1665 & 31.2883 & 423.5587 & 358.783 & 345 & 122.5994 & 2.85653 \\
\hline 1666 & 31.3053 & 417.6057 & 360.0218 & 345 & 122.5994 & 2.85502 \\
\hline 1667 & 31.3223 & 412.6765 & 362.0317 & 345 & 122.5994 & 2.85351 \\
\hline 1668 & 31.3393 & 448.1904 & 365.4713 & 345 & 122.5994 & 2.852 \\
\hline 1669 & 31.3563 & 442.4152 & 370.2934 & 345 & 122.5994 & 2.85049 \\
\hline 1670 & 31.3733 & 480.1676 & 376.1508 & 345 & 122.5994 & 2.84899 \\
\hline 1671 & 31.3903 & 424.5801 & 381.3566 & 345 & 122.5994 & 2.84748 \\
\hline 1672 & 31.4073 & 444.0635 & 383.7866 & 345 & 122.5994 & 2.84598 \\
\hline 1673 & 31.4243 & 436.9758 & 383.5743 & 345 & 122.5994 & 2.84448 \\
\hline 1674 & 31.4413 & 432.6619 & 382.4201 & 345 & 122.5994 & 2.84298 \\
\hline 1675 & 31.4583 & 470.2764 & 381.6726 & 346 & 122.5994 & 2.84148 \\
\hline 1676 & 31.4753 & 437.3208 & 378.6816 & 346 & 122.5994 & 2.83999 \\
\hline 1677 & 31.4923 & 452.6573 & 374.3225 & 346 & 122.5994 & 2.83849 \\
\hline 1678 & 31.5093 & 496.3371 & 369.5193 & 346 & 122.5994 & 2.837 \\
\hline 1679 & 31.5263 & 437.2551 & 365.1286 & 346 & 122.5994 & 2.83551 \\
\hline 1680 & 31.5433 & 433.8565 & 361.2519 & 346 & 122.5994 & 2.83402 \\
\hline 1681 & 31.5603 & 381.8802 & 358.0012 & 346 & 122.5994 & 2.83253 \\
\hline 1682 & 31.5773 & 373.9434 & 355.7093 & 346 & 122.5994 & 2.83105 \\
\hline 1683 & 31.5943 & 389.7168 & 354.5897 & 346 & 122.5994 & 2.82956 \\
\hline 1684 & 31.6113 & 347.5114 & 354.7944 & 346 & 122.5994 & 2.82808 \\
\hline 1685 & 31.6283 & 356.994 & 356.0819 & 346 & 122.5994 & 2.8266 \\
\hline 1686 & 31.6453 & 364.7721 & 358.1954 & 346 & 122.5994 & 2.82512 \\
\hline 1687 & 31.6623 & 344.3154 & 360.326 & 346 & 122.5994 & 2.82364 \\
\hline 1688 & 31.6793 & 330.2585 & 362.2852 & 347 & 122.5994 & 2.82216 \\
\hline 1689 & 31.6963 & 362.013 & 361.7401 & 347 & 122.5994 & 2.82069 \\
\hline 1690 & 31.7133 & 372.3582 & 360.6514 & 347 & 122.5994 & 2.81922 \\
\hline 1691 & 31.7303 & 361.3598 & 359.7328 & 347 & 122.5994 & 2.81774 \\
\hline 1692 & 31.7473 & 359.9375 & 358.8297 & 347 & 122.5994 & 2.81627 \\
\hline 1693 & 31.7643 & 344.4619 & 357.4646 & 347 & 122.5994 & 2.81481 \\
\hline 1694 & 31.7813 & 343.8409 & 355.6485 & 347 & 122.5994 & 2.81334 \\
\hline 1695 & 31.7983 & 334.5043 & 353.8287 & 347 & 122.5994 & 2.81187 \\
\hline 1696 & 31.8153 & 322.1038 & 352.2899 & 347 & 122.5994 & 2.81041 \\
\hline 1697 & 31.8323 & 331.1262 & 351.0911 & 347 & 122.5994 & 2.80895 \\
\hline 1698 & 31.8493 & 340.4597 & 350.2276 & 347 & 122.5994 & 2.80749 \\
\hline 1699 & 31.8663 & 326.7731 & 350.6544 & 348 & 122.5994 & 2.80603 \\
\hline 1700 & 31.8833 & 321.2603 & 350.3073 & 348 & 122.5994 & 2.80457 \\
\hline 1701 & 31.9003 & 349.107 & 350.1614 & 348 & 122.5994 & 2.80311 \\
\hline 1702 & 31.9173 & 351.2978 & 350.266 & 348 & 122.5994 & 2.80166 \\
\hline 1703 & 31.9343 & 351.5024 & 350.5091 & 348 & 122.5994 & 2.80021 \\
\hline 1704 & 31.9513 & 347.6093 & 350.8694 & 348 & 122.5994 & 2.79876 \\
\hline 1705 & 31.9683 & 337.3713 & 351.1999 & 348 & 122.5994 & 2.79731 \\
\hline 1706 & 31.9853 & 355.3996 & 350.2352 & 347 & 122.5994 & 2.79586 \\
\hline 1707 & 32.0023 & 359.4041 & 350.2822 & 347 & 122.5994 & 2.79441 \\
\hline 1708 & 32.0193 & 341.001 & 349.4044 & 346 & 122.5994 & 2.79297 \\
\hline 1709 & 32.0363 & 343.1578 & 348.6525 & 345 & 122.5994 & 2.79152 \\
\hline 1710 & 32.0533 & 375.4741 & 348.9312 & 345 & 122.5994 & 2.79008 \\
\hline 1711 & 32.0703 & 377.9391 & 348.185 & 344 & 122.5994 & 2.78864 \\
\hline 1712 & 32.0873 & 351.5706 & 347.541 & 343 & 122.5994 & 2.7872 \\
\hline 1713 & 32.1043 & 368.1826 & 348.8706 & 343 & 122.5994 & 2.78577 \\
\hline
\end{tabular}




\begin{tabular}{|c|c|c|c|c|c|c|}
\hline 1714 & 32.1213 & 349.7095 & 350.1235 & 342 & 122.5994 & 2.78433 \\
\hline 1715 & 32.1383 & 369.3253 & 353.0624 & 341 & 122.5994 & 2.7829 \\
\hline 1716 & 32.1553 & 362.1088 & 358.876 & 341 & 122.5994 & 2.78147 \\
\hline 1717 & 32.1723 & 343.7598 & 364.6703 & 340 & 122.5994 & 2.78003 \\
\hline 1718 & 32.1893 & 363.5017 & 369.3462 & 339 & 122.5994 & 2.7786 \\
\hline 1719 & 32.2063 & 353.8043 & 370.1965 & 339 & 122.5994 & 2.77718 \\
\hline 1720 & 32.2233 & 387.6705 & 365.9125 & 338 & 122.5994 & 2.77575 \\
\hline 1721 & 32.2403 & 353.2782 & 361.2365 & 337 & 122.5994 & 2.77433 \\
\hline 1722 & 32.2573 & 327.5786 & 359.0746 & 337 & 122.5994 & 2.7729 \\
\hline 1723 & 32.2743 & 330.2594 & 356.5993 & 336 & 122.5994 & 2.77148 \\
\hline 1724 & 32.2913 & 376.2182 & 352.9187 & 335 & 122.5994 & 2.77006 \\
\hline 1725 & 32.3083 & 326.0331 & 349.0806 & 335 & 122.5994 & 2.76864 \\
\hline 1726 & 32.3253 & 344.7259 & 344.4962 & 334 & 122.5994 & 2.76722 \\
\hline 1727 & 32.3423 & 335.3609 & 341.0118 & 333 & 122.5994 & 2.76581 \\
\hline 1728 & 32.3593 & 336.2942 & 339.8908 & 333 & 122.5994 & 2.76439 \\
\hline 1729 & 32.3763 & 323.9689 & 339.284 & 332 & 122.5994 & 2.76298 \\
\hline 1730 & 32.3933 & 329.1469 & 340.4723 & 331 & 122.5994 & 2.76157 \\
\hline 1731 & 32.4103 & 347.2523 & 343.8886 & 330 & 122.5994 & 2.76016 \\
\hline 1732 & 32.4273 & 338.5275 & 348.6531 & 328 & 122.5994 & 2.75875 \\
\hline 1733 & 32.4443 & 339.5877 & 355.767 & 327 & 122.5994 & 2.75735 \\
\hline 1734 & 32.4613 & 333.8855 & 361.1392 & 326 & 122.5994 & 2.75594 \\
\hline 1735 & 32.4783 & 307.8658 & 359.838 & 324 & 122.5994 & 2.75454 \\
\hline 1736 & 32.4953 & 304.0064 & 354.7368 & 323 & 122.5994 & 2.75313 \\
\hline 1737 & 32.5123 & 304.5837 & 349.2964 & 322 & 122.5994 & 2.75173 \\
\hline 1738 & 32.5293 & 295.055 & 345.7052 & 321 & 122.5994 & 2.75033 \\
\hline 1739 & 32.5463 & 323.3217 & 342.9351 & 320 & 122.5994 & 2.74894 \\
\hline 1740 & 32.5633 & 311.0122 & 337.6768 & 318 & 122.5994 & 2.74754 \\
\hline 1741 & 32.5803 & 299.6977 & 331.9279 & 317 & 122.5994 & 2.74615 \\
\hline 1742 & 32.5973 & 315.452 & 326.3355 & 316 & 122.5994 & 2.74475 \\
\hline 1743 & 32.6143 & 318.0398 & 320.8378 & 314 & 122.5994 & 2.74336 \\
\hline 1744 & 32.6313 & 294.087 & 317.5664 & 313 & 122.5994 & 2.74197 \\
\hline 1745 & 32.6483 & 288.8826 & 315.2572 & 312 & 122.5994 & 2.74058 \\
\hline 1746 & 32.6653 & 298.7658 & 313.5472 & 311 & 122.5994 & 2.73919 \\
\hline 1747 & 32.6823 & 305.8285 & 312.1657 & 310 & 122.5994 & 2.73781 \\
\hline 1748 & 32.6993 & 298.6554 & 309.9301 & 308 & 122.5994 & 2.73642 \\
\hline 1749 & 32.7163 & 326.4038 & 308.7318 & 307 & 122.5994 & 2.73504 \\
\hline 1750 & 32.7333 & 325.3578 & 307.53 & 306 & 122.5994 & 2.73366 \\
\hline 1751 & 32.7503 & 304.1117 & 305.3414 & 304 & 122.5994 & 2.73228 \\
\hline 1752 & 32.7673 & 290.0946 & 304.1891 & 303 & 122.5994 & 2.7309 \\
\hline 1753 & 32.7843 & 317.3647 & 303.0666 & 302 & 122.5994 & 2.72952 \\
\hline 1754 & 32.8013 & 308.2701 & 302.9782 & 302 & 122.5994 & 2.72815 \\
\hline 1755 & 32.8183 & 284.2423 & 302.7852 & 302 & 122.5994 & 2.72677 \\
\hline 1756 & 32.8353 & 283.9772 & 301.6805 & 301 & 122.5994 & 2.7254 \\
\hline 1757 & 32.8523 & 329.9778 & 301.5918 & 301 & 122.5994 & 2.72403 \\
\hline 1758 & 32.8693 & 333.3643 & 301.5216 & 301 & 122.5994 & 2.72266 \\
\hline 1759 & 32.8863 & 334.2981 & 301.4777 & 301 & 122.5994 & 2.72129 \\
\hline 1760 & 32.9033 & 310.5956 & 301.2195 & 301 & 122.5994 & 2.71992 \\
\hline 1761 & 32.9203 & 294.1996 & 300.2038 & 300 & 122.5994 & 2.71856 \\
\hline 1762 & 32.9373 & 280.4132 & 300.0797 & 300 & 122.5994 & 2.71719 \\
\hline
\end{tabular}




\begin{tabular}{|c|c|c|c|c|c|c|}
\hline 1763 & 32.9543 & 297.4607 & 300.0815 & 300 & 122.5994 & 2.71583 \\
\hline 1764 & 32.9713 & 312.3959 & 300.0848 & 300 & 122.5994 & 2.71447 \\
\hline 1765 & 32.9883 & 283.6163 & 300.0894 & 300 & 122.5994 & 2.71311 \\
\hline 1766 & 33.0053 & 292.1402 & 300.0953 & 300 & 122.5994 & 2.71175 \\
\hline 1767 & 33.0223 & 285.6641 & 299.1026 & 299 & 122.5994 & 2.71039 \\
\hline 1768 & 33.0393 & 303.5638 & 299.1115 & 299 & 122.5994 & 2.70904 \\
\hline 1769 & 33.0563 & 339.7717 & 299.1223 & 299 & 122.5994 & 2.70768 \\
\hline 1770 & 33.0733 & 283.1263 & 299.1353 & 299 & 122.5994 & 2.70633 \\
\hline 1771 & 33.0903 & 297.8711 & 299.1513 & 299 & 122.5994 & 2.70498 \\
\hline 1772 & 33.1073 & 322.6778 & 298.246 & 298 & 122.5994 & 2.70363 \\
\hline 1773 & 33.1243 & 339.9798 & 298.2749 & 298 & 122.5994 & 2.70228 \\
\hline 1774 & 33.1413 & 327.2259 & 298.3153 & 298 & 122.5994 & 2.70093 \\
\hline 1775 & 33.1583 & 340.4535 & 298.3699 & 298 & 122.5994 & 2.69958 \\
\hline 1776 & 33.1753 & 312.5844 & 298.4482 & 298 & 122.5994 & 2.69824 \\
\hline 1777 & 33.1923 & 313.5288 & 298.6024 & 298 & 122.5994 & 2.6969 \\
\hline 1778 & 33.2093 & 334.5934 & 297.7793 & 297 & 122.5994 & 2.69556 \\
\hline 1779 & 33.2263 & 349.6019 & 298.0321 & 297 & 122.5994 & 2.69422 \\
\hline 1780 & 33.2433 & 327.6537 & 298.3663 & 297 & 122.5994 & 2.69288 \\
\hline 1781 & 33.2603 & 324.359 & 298.7722 & 297 & 122.5994 & 2.69154 \\
\hline 1782 & 33.2773 & 312.3683 & 299.2106 & 297 & 122.5994 & 2.6902 \\
\hline 1783 & 33.2943 & 305.125 & 298.8398 & 296 & 122.5994 & 2.68887 \\
\hline 1784 & 33.3113 & 313.6008 & 299.5593 & 296 & 122.5994 & 2.68753 \\
\hline 1785 & 33.3283 & 316.3516 & 300.6012 & 296 & 122.5994 & 2.6862 \\
\hline 1786 & 33.3453 & 306.8848 & 301.9067 & 296 & 122.5994 & 2.68487 \\
\hline 1787 & 33.3623 & 307.3088 & 302.9991 & 296 & 122.5994 & 2.68354 \\
\hline 1788 & 33.3793 & 308.526 & 302.3857 & 295 & 122.5994 & 2.68221 \\
\hline 1789 & 33.3963 & 314.5102 & 302.1104 & 295 & 122.5994 & 2.68089 \\
\hline 1790 & 33.4133 & 332.973 & 301.8357 & 295 & 122.5994 & 2.67956 \\
\hline 1791 & 33.4303 & 320.1241 & 302.1217 & 295 & 122.5994 & 2.67824 \\
\hline 1792 & 33.4473 & 300.9414 & 303.0674 & 295 & 122.5994 & 2.67692 \\
\hline 1793 & 33.4643 & 315.0393 & 303.4864 & 294 & 122.5994 & 2.6756 \\
\hline 1794 & 33.4813 & 317.3224 & 305.1065 & 294 & 122.5994 & 2.67428 \\
\hline 1795 & 33.4983 & 293.2127 & 306.5148 & 294 & 122.5994 & 2.67296 \\
\hline 1796 & 33.5153 & 303.7211 & 306.8912 & 294 & 122.5994 & 2.67164 \\
\hline 1797 & 33.5323 & 303.3735 & 305.927 & 294 & 122.5994 & 2.67033 \\
\hline 1798 & 33.5493 & 312.1431 & 304.4774 & 294 & 122.5994 & 2.66901 \\
\hline 1799 & 33.5663 & 310.7341 & 302.3828 & 293 & 122.5994 & 2.6677 \\
\hline 1800 & 33.5833 & 291.6124 & 301.7562 & 293 & 122.5994 & 2.66639 \\
\hline 1801 & 33.6003 & 295.6264 & 301.1359 & 293 & 122.5994 & 2.66508 \\
\hline 1802 & 33.6173 & 283.9443 & 302.1659 & 295 & 122.5994 & 2.66377 \\
\hline 1803 & 33.6343 & 289.3041 & 303.0939 & 297 & 122.5994 & 2.66246 \\
\hline 1804 & 33.6513 & 309.0257 & 305.3013 & 300 & 122.5994 & 2.66115 \\
\hline 1805 & 33.6683 & 290.6918 & 307.001 & 302 & 122.5994 & 2.65985 \\
\hline 1806 & 33.6853 & 302.9232 & 309.3611 & 304 & 122.5994 & 2.65855 \\
\hline 1807 & 33.7023 & 318.3755 & 312.5926 & 306 & 122.5994 & 2.65724 \\
\hline 1808 & 33.7193 & 333.119 & 316.99 & 308 & 122.5994 & 2.65594 \\
\hline 1809 & 33.7363 & 302.8426 & 322.2185 & 310 & 122.5994 & 2.65464 \\
\hline 1810 & 33.7533 & 335.5029 & 328.8327 & 313 & 122.5994 & 2.65335 \\
\hline 1811 & 33.7703 & 324.8527 & 333.6407 & 315 & 122.5994 & 2.65205 \\
\hline
\end{tabular}




\begin{tabular}{|c|c|c|c|c|c|c|}
\hline 1812 & 33.7873 & 320.5012 & 336.3445 & 317 & 122.5994 & 2.65075 \\
\hline 1813 & 33.8043 & 331.1484 & 337.3612 & 319 & 122.5994 & 2.64946 \\
\hline 1814 & 33.8213 & 306.6419 & 337.9465 & 321 & 122.5994 & 2.64817 \\
\hline 1815 & 33.8383 & 353.0321 & 338.9582 & 323 & 122.5994 & 2.64687 \\
\hline 1816 & 33.8553 & 340.493 & 341.1967 & 326 & 122.5994 & 2.64558 \\
\hline 1817 & 33.8723 & 373.6263 & 342.059 & 328 & 122.5994 & 2.6443 \\
\hline 1818 & 33.8893 & 343.6117 & 342.4788 & 330 & 122.5994 & 2.64301 \\
\hline 1819 & 33.9063 & 385.7727 & 342.9817 & 332 & 122.5994 & 2.64172 \\
\hline 1820 & 33.9233 & 343.2921 & 343.9176 & 334 & 122.5994 & 2.64044 \\
\hline 1821 & 33.9403 & 370.621 & 345.3019 & 336 & 122.5994 & 2.63915 \\
\hline 1822 & 33.9573 & 357.6505 & 347.9947 & 339 & 122.5994 & 2.63787 \\
\hline 1823 & 33.9743 & 342.2172 & 349.7983 & 341 & 122.5994 & 2.63659 \\
\hline 1824 & 33.9913 & 349.0777 & 351.5579 & 343 & 122.5994 & 2.63531 \\
\hline 1825 & 34.0083 & 364.1178 & 353.25 & 345 & 122.5994 & 2.63403 \\
\hline 1826 & 34.0253 & 331.7775 & 353.9459 & 346 & 122.5994 & 2.63275 \\
\hline 1827 & 34.0423 & 318.5151 & 355.7455 & 348 & 122.5994 & 2.63148 \\
\hline 1828 & 34.0593 & 324.5454 & 356.7964 & 349 & 122.5994 & 2.6302 \\
\hline 1829 & 34.0763 & 310.9727 & 358.4336 & 350 & 122.5994 & 2.62893 \\
\hline 1830 & 34.0933 & 322.1867 & 360.9144 & 351 & 122.5994 & 2.62766 \\
\hline 1831 & 34.1103 & 341.3794 & 364.5578 & 352 & 122.5994 & 2.62639 \\
\hline 1832 & 34.1273 & 337.2235 & 370.1069 & 354 & 122.5994 & 2.62512 \\
\hline 1833 & 34.1443 & 331.5928 & 374.2851 & 355 & 122.5994 & 2.62385 \\
\hline 1834 & 34.1613 & 347.2125 & 376.1501 & 356 & 122.5994 & 2.62258 \\
\hline 1835 & 34.1783 & 344.8579 & 376.1408 & 358 & 122.5994 & 2.62132 \\
\hline 1836 & 34.1953 & 343.8844 & 374.5182 & 359 & 122.5994 & 2.62005 \\
\hline 1837 & 34.2123 & 353.4061 & 374.0708 & 360 & 122.5994 & 2.61879 \\
\hline 1838 & 34.2293 & 345.5895 & 374.6927 & 361 & 122.5994 & 2.61753 \\
\hline 1839 & 34.2463 & 330.6414 & 375.4212 & 362 & 122.5994 & 2.61627 \\
\hline 1840 & 34.2633 & 323.9689 & 376.5153 & 364 & 122.5994 & 2.61501 \\
\hline 1841 & 34.2803 & 337.0002 & 377.2144 & 365 & 122.5994 & 2.61375 \\
\hline 1842 & 34.2973 & 339.1581 & 379.3782 & 366 & 122.5994 & 2.61249 \\
\hline 1843 & 34.3143 & 344.3045 & 384.3318 & 368 & 122.5994 & 2.61124 \\
\hline 1844 & 34.3313 & 339.9696 & 389.162 & 369 & 122.5994 & 2.60998 \\
\hline 1845 & 34.3483 & 360.2731 & 392.563 & 370 & 122.5994 & 2.60873 \\
\hline 1846 & 34.3653 & 361.8805 & 392.798 & 371 & 122.5994 & 2.60748 \\
\hline 1847 & 34.3823 & 359.9008 & 391.08 & 372 & 122.5994 & 2.60623 \\
\hline 1848 & 34.3993 & 384.0381 & 390.9559 & 374 & 122.5994 & 2.60498 \\
\hline 1849 & 34.4163 & 371.251 & 391.4241 & 375 & 122.5994 & 2.60373 \\
\hline 1850 & 34.4333 & 361.7899 & 391.5184 & 375 & 122.5994 & 2.60249 \\
\hline 1851 & 34.4503 & 398.9222 & 392.2944 & 376 & 122.5994 & 2.60124 \\
\hline 1852 & 34.4673 & 347.5375 & 392.3129 & 376 & 122.5994 & 2.6 \\
\hline 1853 & 34.4843 & 353.8668 & 395.1678 & 377 & 122.5994 & 2.59875 \\
\hline 1854 & 34.5013 & 377.2122 & 399.679 & 377 & 122.5994 & 2.59751 \\
\hline 1855 & 34.5183 & 380.3007 & 406.4157 & 377 & 122.5994 & 2.59627 \\
\hline 1856 & 34.5353 & 352.3212 & 412.9773 & 378 & 122.5994 & 2.59503 \\
\hline 1857 & 34.5523 & 396.6524 & 413.8033 & 378 & 122.5994 & 2.5938 \\
\hline 1858 & 34.5693 & 403.886 & 411.4126 & 379 & 122.5994 & 2.59256 \\
\hline 1859 & 34.5863 & 361.942 & 408.1451 & 379 & 122.5994 & 2.59132 \\
\hline 1860 & 34.6033 & 368.6793 & 407.7177 & 379 & 122.5994 & 2.59009 \\
\hline
\end{tabular}




\begin{tabular}{|c|c|c|c|c|c|c|}
\hline 1861 & 34.6203 & 372.5323 & 410.9571 & 380 & 122.5994 & 2.58886 \\
\hline 1862 & 34.6373 & 376.2483 & 413.7184 & 380 & 122.5994 & 2.58762 \\
\hline 1863 & 34.6543 & 371.4879 & 416.3745 & 381 & 122.5994 & 2.58639 \\
\hline 1864 & 34.6713 & 354.7448 & 416.7633 & 381 & 122.5994 & 2.58516 \\
\hline 1865 & 34.6883 & 331.5334 & 415.2512 & 381 & 122.5994 & 2.58394 \\
\hline 1866 & 34.7053 & 365.3487 & 413.1438 & 382 & 122.5994 & 2.58271 \\
\hline 1867 & 34.7223 & 363.0178 & 410.057 & 382 & 122.5994 & 2.58148 \\
\hline 1868 & 34.7393 & 337.5962 & 408.337 & 383 & 122.5994 & 2.58026 \\
\hline 1869 & 34.7563 & 376.2717 & 406.0033 & 383 & 122.5994 & 2.57904 \\
\hline 1870 & 34.7733 & 396.3235 & 403.5715 & 383 & 122.5994 & 2.57781 \\
\hline 1871 & 34.7903 & 368.259 & 401.7078 & 384 & 122.5994 & 2.57659 \\
\hline 1872 & 34.8073 & 416.1201 & 398.9536 & 384 & 122.5994 & 2.57537 \\
\hline 1873 & 34.8243 & 405.1276 & 396.3224 & 384 & 122.5994 & 2.57416 \\
\hline 1874 & 34.8413 & 385.8283 & 394.9938 & 385 & 122.5994 & 2.57294 \\
\hline 1875 & 34.8583 & 406.7055 & 393.3642 & 385 & 122.5994 & 2.57172 \\
\hline 1876 & 34.8753 & 389.3079 & 393.5336 & 386 & 122.5994 & 2.57051 \\
\hline 1877 & 34.8923 & 372.2795 & 393.3978 & 386 & 122.5994 & 2.56929 \\
\hline 1878 & 34.9093 & 414.2573 & 393.9117 & 386 & 122.5994 & 2.56808 \\
\hline 1879 & 34.9263 & 435.8329 & 396.1597 & 387 & 122.5994 & 2.56687 \\
\hline 1880 & 34.9433 & 417.8747 & 398.5641 & 387 & 122.5994 & 2.56566 \\
\hline 1881 & 34.9603 & 443.1265 & 403.7241 & 388 & 122.5994 & 2.56445 \\
\hline 1882 & 34.9773 & 446.9396 & 410.9743 & 388 & 122.5994 & 2.56325 \\
\hline 1883 & 34.9943 & 422.3352 & 421.7906 & 388 & 122.5994 & 2.56204 \\
\hline 1884 & 35.0113 & 424.3193 & 437.7214 & 389 & 122.5994 & 2.56083 \\
\hline 1885 & 35.0283 & 451.728 & 454.7935 & 389 & 122.5994 & 2.55963 \\
\hline 1886 & 35.0453 & 439.944 & 471.1183 & 390 & 122.5994 & 2.55843 \\
\hline 1887 & 35.0623 & 419.1624 & 483.0399 & 390 & 122.5994 & 2.55723 \\
\hline 1888 & 35.0793 & 417.039 & 488.6237 & 390 & 122.5994 & 2.55603 \\
\hline 1889 & 35.0963 & 427.4434 & 487.2302 & 391 & 122.5994 & 2.55483 \\
\hline 1890 & 35.1133 & 439.568 & 478.8273 & 391 & 122.5994 & 2.55363 \\
\hline 1891 & 35.1303 & 452.249 & 469.677 & 392 & 122.5994 & 2.55243 \\
\hline 1892 & 35.1473 & 420.8426 & 460.7602 & 392 & 122.5994 & 2.55124 \\
\hline 1893 & 35.1643 & 398.3932 & 453.2661 & 392 & 122.5994 & 2.55004 \\
\hline 1894 & 35.1813 & 407.7145 & 445.7788 & 393 & 122.5994 & 2.54885 \\
\hline 1895 & 35.1983 & 427.5965 & 435.7445 & 393 & 122.5994 & 2.54766 \\
\hline 1896 & 35.2153 & 416.7067 & 426.8716 & 394 & 122.5994 & 2.54647 \\
\hline 1897 & 35.2323 & 415.3805 & 419.3939 & 394 & 122.5994 & 2.54528 \\
\hline 1898 & 35.2493 & 352.7681 & 415.1614 & 394 & 122.5994 & 2.54409 \\
\hline 1899 & 35.2663 & 344.7273 & 412.9772 & 393 & 122.5994 & 2.5429 \\
\hline 1900 & 35.2833 & 355.6751 & 414.3658 & 393 & 122.5994 & 2.54171 \\
\hline 1901 & 35.3003 & 360.5143 & 418.0024 & 393 & 122.5994 & 2.54053 \\
\hline 1902 & 35.3173 & 381.9508 & 422.1716 & 392 & 122.5994 & 2.53934 \\
\hline 1903 & 35.3343 & 395.072 & 428.0417 & 392 & 122.5994 & 2.53816 \\
\hline 1904 & 35.3513 & 392.2189 & 434.3937 & 392 & 122.5994 & 2.53698 \\
\hline 1905 & 35.3683 & 411.481 & 439.1645 & 391 & 122.5994 & 2.5358 \\
\hline 1906 & 35.3853 & 372.9082 & 441.7054 & 391 & 122.5994 & 2.53462 \\
\hline 1907 & 35.4023 & 444.7555 & 440.0752 & 391 & 122.5994 & 2.53344 \\
\hline 1908 & 35.4193 & 403.4357 & 436.0274 & 390 & 122.5994 & 2.53226 \\
\hline 1909 & 35.4363 & 385.3904 & 434.9423 & 390 & 122.5994 & 2.53109 \\
\hline
\end{tabular}




\begin{tabular}{|c|c|c|c|c|c|c|}
\hline 1910 & 35.4533 & 408.3025 & 436.2144 & 390 & 122.5994 & 2.52991 \\
\hline 1911 & 35.4703 & 410.2174 & 436.5182 & 389 & 122.5994 & 2.52874 \\
\hline 1912 & 35.4873 & 396.2873 & 434.9378 & 389 & 122.5994 & 2.52757 \\
\hline 1913 & 35.5043 & 422.3761 & 431.341 & 389 & 122.5994 & 2.5264 \\
\hline 1914 & 35.5213 & 382.8703 & 427.569 & 388 & 122.5994 & 2.52523 \\
\hline 1915 & 35.5383 & 381.4501 & 426.4589 & 388 & 122.5994 & 2.52406 \\
\hline 1916 & 35.5553 & 396.7665 & 426.0158 & 388 & 122.5994 & 2.52289 \\
\hline 1917 & 35.5723 & 372.0984 & 423.996 & 387 & 122.5994 & 2.52172 \\
\hline 1918 & 35.5893 & 377.5609 & 421.7403 & 387 & 122.5994 & 2.52056 \\
\hline 1919 & 35.6063 & 371.8938 & 418.9809 & 387 & 122.5994 & 2.51939 \\
\hline 1920 & 35.6233 & 419.3097 & 415.9552 & 386 & 122.5994 & 2.51823 \\
\hline 1921 & 35.6403 & 416.7481 & 414.7858 & 386 & 122.5994 & 2.51707 \\
\hline 1922 & 35.6573 & 396.6465 & 413.9247 & 386 & 122.5994 & 2.51591 \\
\hline 1923 & 35.6743 & 451.3071 & 411.6253 & 385 & 122.5994 & 2.51475 \\
\hline 1924 & 35.6913 & 452.6467 & 410.0961 & 385 & 122.5994 & 2.51359 \\
\hline 1925 & 35.7083 & 436.8682 & 408.635 & 385 & 122.5994 & 2.51243 \\
\hline 1926 & 35.7253 & 421.3515 & 407.065 & 384 & 122.5994 & 2.51127 \\
\hline 1927 & 35.7423 & 394.8074 & 407.0092 & 384 & 122.5994 & 2.51012 \\
\hline 1928 & 35.7593 & 401.0179 & 406.9012 & 384 & 122.5994 & 2.50896 \\
\hline 1929 & 35.7763 & 422.0792 & 405.4896 & 383 & 122.5994 & 2.50781 \\
\hline 1930 & 35.7933 & 418.2208 & 404.0521 & 383 & 122.5994 & 2.50666 \\
\hline 1931 & 35.8103 & 403.7688 & 402.6736 & 383 & 122.5994 & 2.50551 \\
\hline 1932 & 35.8273 & 384.9771 & 400.7026 & 382 & 122.5994 & 2.50436 \\
\hline 1933 & 35.8443 & 379.0997 & 400.6058 & 382 & 122.5994 & 2.50321 \\
\hline 1934 & 35.8613 & 393.4825 & 401.9355 & 382 & 122.5994 & 2.50206 \\
\hline 1935 & 35.8783 & 373.8438 & 403.4138 & 381 & 122.5994 & 2.50091 \\
\hline 1936 & 35.8953 & 352.4085 & 406.4338 & 381 & 122.5994 & 2.49977 \\
\hline 1937 & 35.9123 & 364.684 & 409.9732 & 381 & 122.5994 & 2.49862 \\
\hline 1938 & 35.9293 & 385.6897 & 412.1396 & 380 & 122.5994 & 2.49748 \\
\hline 1939 & 35.9463 & 392.0923 & 413.6742 & 380 & 122.5994 & 2.49634 \\
\hline 1940 & 35.9633 & 350.85 & 417.1183 & 380 & 122.5994 & 2.4952 \\
\hline 1941 & 35.9803 & 357.0435 & 416.3091 & 379 & 122.5994 & 2.49406 \\
\hline 1942 & 35.9973 & 373.673 & 418.2755 & 379 & 122.5994 & 2.49292 \\
\hline 1943 & 36.0143 & 398.0172 & 421.9977 & 379 & 122.5994 & 2.49178 \\
\hline 1944 & 36.0313 & 366.3329 & 424.6212 & 378 & 122.5994 & 2.49064 \\
\hline 1945 & 36.0483 & 366.6356 & 427.2874 & 378 & 122.5994 & 2.48951 \\
\hline 1946 & 36.0653 & 395.2311 & 423.3533 & 377 & 122.5994 & 2.48837 \\
\hline 1947 & 36.0823 & 372.748 & 418.1071 & 377 & 122.5994 & 2.48724 \\
\hline 1948 & 36.0993 & 345.0614 & 412.254 & 376 & 122.5994 & 2.48611 \\
\hline 1949 & 36.1163 & 391.4202 & 408.0937 & 375 & 122.5994 & 2.48498 \\
\hline 1950 & 36.1333 & 373.9762 & 406.2359 & 375 & 122.5994 & 2.48385 \\
\hline 1951 & 36.1503 & 376.2426 & 403.4625 & 374 & 122.5994 & 2.48272 \\
\hline 1952 & 36.1673 & 357.5901 & 400.0106 & 373 & 122.5994 & 2.48159 \\
\hline 1953 & 36.1843 & 391.9948 & 396.4306 & 372 & 122.5994 & 2.48046 \\
\hline 1954 & 36.2013 & 325.0811 & 394.5265 & 372 & 122.5994 & 2.47934 \\
\hline 1955 & 36.2183 & 375.5888 & 392.695 & 371 & 122.5994 & 2.47821 \\
\hline 1956 & 36.2353 & 354.8091 & 392.0018 & 370 & 122.5994 & 2.47709 \\
\hline 1957 & 36.2523 & 322.5697 & 393.4027 & 370 & 122.5994 & 2.47597 \\
\hline 1958 & 36.2693 & 344.0357 & 394.8021 & 369 & 122.5994 & 2.47485 \\
\hline
\end{tabular}




\begin{tabular}{|c|c|c|c|c|c|c|}
\hline 1959 & 36.2863 & 329.9421 & 397.0945 & 368 & 122.5994 & 2.47372 \\
\hline 1960 & 36.3033 & 348.8421 & 401.1194 & 368 & 122.5994 & 2.47261 \\
\hline 1961 & 36.3203 & 366.7601 & 404.8776 & 367 & 122.5994 & 2.47149 \\
\hline 1962 & 36.3373 & 369.0951 & 410.0097 & 366 & 122.5994 & 2.47037 \\
\hline 1963 & 36.3543 & 342.5362 & 418.3246 & 366 & 122.5994 & 2.46925 \\
\hline 1964 & 36.3713 & 352.7676 & 427.8016 & 365 & 122.5994 & 2.46814 \\
\hline 1965 & 36.3883 & 352.4035 & 438.4744 & 364 & 122.5994 & 2.46702 \\
\hline 1966 & 36.4053 & 388.7079 & 448.5993 & 363 & 122.5994 & 2.46591 \\
\hline 1967 & 36.4223 & 397.3298 & 459.9991 & 363 & 122.5994 & 2.4648 \\
\hline 1968 & 36.4393 & 453.4091 & 475.1364 & 362 & 122.5994 & 2.46369 \\
\hline 1969 & 36.4563 & 489.9997 & 500.8738 & 361 & 122.5994 & 2.46258 \\
\hline 1970 & 36.4733 & 517.9623 & 545.8562 & 361 & 122.5994 & 2.46147 \\
\hline 1971 & 36.4903 & 607.8893 & 620.5103 & 360 & 122.5994 & 2.46036 \\
\hline 1972 & 36.5073 & 727.8981 & 743.0985 & 359 & 122.5994 & 2.45926 \\
\hline 1973 & 36.5243 & 893.5444 & 927.9456 & 359 & 122.5994 & 2.45815 \\
\hline 1974 & 36.5413 & 1096.121 & 1165.213 & 358 & 122.5994 & 2.45705 \\
\hline 1975 & 36.5583 & 1372.821 & 1405.043 & 357 & 122.5994 & 2.45594 \\
\hline 1976 & 36.5753 & 1710.676 & 1544.231 & 357 & 122.5994 & 2.45484 \\
\hline 1977 & 36.5923 & 1876.418 & 1506.233 & 356 & 122.5994 & 2.45374 \\
\hline 1978 & 36.6093 & 1713.168 & 1361.077 & 355 & 122.5994 & 2.45264 \\
\hline 1979 & 36.6263 & 1335.053 & 1225.046 & 354 & 122.5994 & 2.45154 \\
\hline 1980 & 36.6433 & 1092.412 & 1149.599 & 354 & 122.5994 & 2.45044 \\
\hline 1981 & 36.6603 & 1068.396 & 1109.169 & 353 & 122.5994 & 2.44934 \\
\hline 1982 & 36.6773 & 1147.754 & 1039.42 & 352 & 122.5994 & 2.44825 \\
\hline 1983 & 36.6943 & 1118.115 & 913.9213 & 352 & 122.5994 & 2.44715 \\
\hline 1984 & 36.7113 & 925.449 & 767.8867 & 351 & 122.5994 & 2.44606 \\
\hline 1985 & 36.7283 & 697.5159 & 640.1209 & 350 & 122.5994 & 2.44496 \\
\hline 1986 & 36.7453 & 561.6596 & 546.35 & 350 & 122.5994 & 2.44387 \\
\hline 1987 & 36.7623 & 506.9972 & 483.934 & 349 & 122.5994 & 2.44278 \\
\hline 1988 & 36.7793 & 464.4228 & 445.6475 & 348 & 122.5994 & 2.44169 \\
\hline 1989 & 36.7963 & 434.2358 & 423.4666 & 348 & 122.5994 & 2.4406 \\
\hline 1990 & 36.8133 & 402.601 & 408.4746 & 347 & 122.5994 & 2.43951 \\
\hline 1991 & 36.8303 & 392.8175 & 398.2383 & 346 & 122.5994 & 2.43843 \\
\hline 1992 & 36.8473 & 388.6888 & 390.5583 & 345 & 122.5994 & 2.43734 \\
\hline 1993 & 36.8643 & 371.4044 & 385.2408 & 345 & 122.5994 & 2.43626 \\
\hline 1994 & 36.8813 & 361.8665 & 378.5408 & 343 & 122.5994 & 2.43517 \\
\hline 1995 & 36.8983 & 367.8671 & 373.4154 & 342 & 122.5994 & 2.43409 \\
\hline 1996 & 36.9153 & 340.8645 & 369.0379 & 341 & 122.5994 & 2.43301 \\
\hline 1997 & 36.9323 & 370.6403 & 365.4144 & 340 & 122.5994 & 2.43193 \\
\hline 1998 & 36.9493 & 346.8562 & 361.3723 & 338 & 122.5994 & 2.43085 \\
\hline 1999 & 36.9663 & 349.6177 & 358.7046 & 337 & 122.5994 & 2.42977 \\
\hline 2000 & 36.9833 & 350.1615 & 356.4595 & 336 & 122.5994 & 2.42869 \\
\hline 2001 & 37.0003 & 386.5637 & 353.9931 & 334 & 122.5994 & 2.42761 \\
\hline 2002 & 37.0173 & 344.7772 & 353.6654 & 333 & 122.5994 & 2.42654 \\
\hline 2003 & 37.0343 & 386.2939 & 354.545 & 332 & 122.5994 & 2.42546 \\
\hline 2004 & 37.0513 & 361.5955 & 356.1208 & 331 & 122.5994 & 2.42439 \\
\hline 2005 & 37.0683 & 334.2889 & 356.025 & 329 & 122.5994 & 2.42331 \\
\hline 2006 & 37.0853 & 355.0807 & 354.7288 & 328 & 122.5994 & 2.42224 \\
\hline 2007 & 37.1023 & 346.5628 & 351.4268 & 327 & 122.5994 & 2.42117 \\
\hline
\end{tabular}




\begin{tabular}{|c|c|c|c|c|c|c|}
\hline 2008 & 37.1193 & 362.4245 & 347.6455 & 326 & 122.5994 & 2.4201 \\
\hline 2009 & 37.1363 & 334.1873 & 343.7878 & 324 & 122.5994 & 2.41903 \\
\hline 2010 & 37.1533 & 336.2295 & 341.8458 & 323 & 122.5994 & 2.41797 \\
\hline 2011 & 37.1703 & 345.3011 & 340.0063 & 322 & 122.5994 & 2.4169 \\
\hline 2012 & 37.1873 & 322.3179 & 336.4726 & 320 & 122.5994 & 2.41583 \\
\hline 2013 & 37.2043 & 313.4169 & 330.0038 & 319 & 122.5994 & 2.41477 \\
\hline 2014 & 37.2213 & 289.6712 & 327.5282 & 318 & 122.5994 & 2.4137 \\
\hline 2015 & 37.2383 & 319.2507 & 325.9031 & 317 & 122.5994 & 2.41264 \\
\hline 2016 & 37.2553 & 323.6503 & 324.5543 & 315 & 122.5994 & 2.41158 \\
\hline 2017 & 37.2723 & 312.3981 & 323.6236 & 314 & 122.5994 & 2.41052 \\
\hline 2018 & 37.2893 & 311.2025 & 325.6626 & 313 & 122.5994 & 2.40946 \\
\hline 2019 & 37.3063 & 305.7396 & 327.9432 & 312 & 122.5994 & 2.4084 \\
\hline 2020 & 37.3233 & 334.0242 & 328.7402 & 311 & 122.5994 & 2.40734 \\
\hline 2021 & 37.3403 & 347.6367 & 328.3571 & 310 & 122.5994 & 2.40628 \\
\hline 2022 & 37.3573 & 324.0911 & 327.8918 & 309 & 122.5994 & 2.40523 \\
\hline 2023 & 37.3743 & 320.4527 & 327.6746 & 308 & 122.5994 & 2.40417 \\
\hline 2024 & 37.3913 & 314.762 & 327.2553 & 307 & 122.5994 & 2.40312 \\
\hline 2025 & 37.4083 & 302.0901 & 325.6472 & 306 & 122.5994 & 2.40207 \\
\hline 2026 & 37.4253 & 291.9771 & 322.662 & 305 & 122.5994 & 2.40101 \\
\hline 2027 & 37.4423 & 320.4928 & 319.3324 & 304 & 122.5994 & 2.39996 \\
\hline 2028 & 37.4593 & 344.572 & 316.4159 & 303 & 122.5994 & 2.39891 \\
\hline 2029 & 37.4763 & 336.5795 & 313.9452 & 302 & 122.5994 & 2.39786 \\
\hline 2030 & 37.4933 & 328.8254 & 311.6164 & 301 & 122.5994 & 2.39682 \\
\hline 2031 & 37.5103 & 313.17 & 309.4484 & 300 & 122.5994 & 2.39577 \\
\hline 2032 & 37.5273 & 328.8213 & 307.6448 & 299 & 122.5994 & 2.39472 \\
\hline 2033 & 37.5443 & 335.9585 & 306.5465 & 298 & 122.5994 & 2.39368 \\
\hline 2034 & 37.5613 & 351.4319 & 306.2238 & 297 & 122.5994 & 2.39263 \\
\hline 2035 & 37.5783 & 297.4043 & 306.57 & 296 & 122.5994 & 2.39159 \\
\hline 2036 & 37.5953 & 319.9913 & 307.4314 & 295 & 122.5994 & 2.39055 \\
\hline 2037 & 37.6123 & 346.0984 & 308.5077 & 294 & 122.5994 & 2.38951 \\
\hline 2038 & 37.6293 & 325.4721 & 309.3509 & 293 & 122.5994 & 2.38847 \\
\hline 2039 & 37.6463 & 332.6891 & 309.6382 & 292 & 122.5994 & 2.38743 \\
\hline 2040 & 37.6633 & 309.967 & 309.0287 & 291 & 122.5994 & 2.38639 \\
\hline 2041 & 37.6803 & 335.0798 & 308.3886 & 291 & 122.5994 & 2.38535 \\
\hline 2042 & 37.6973 & 348.7072 & 306.4894 & 290 & 122.5994 & 2.38431 \\
\hline 2043 & 37.7143 & 346.2443 & 305.3116 & 289 & 122.5994 & 2.38328 \\
\hline 2044 & 37.7313 & 353.0903 & 305.3074 & 288 & 122.5994 & 2.38224 \\
\hline 2045 & 37.7483 & 328.1909 & 306.6347 & 287 & 122.5994 & 2.38121 \\
\hline 2046 & 37.7653 & 309.7951 & 308.898 & 286 & 122.5994 & 2.38018 \\
\hline 2047 & 37.7823 & 302.8088 & 311.1406 & 285 & 122.5994 & 2.37914 \\
\hline 2048 & 37.7993 & 306.2206 & 311.8177 & 284 & 122.5994 & 2.37811 \\
\hline 2049 & 37.8163 & 306.7511 & 309.5351 & 283 & 122.5994 & 2.37708 \\
\hline 2050 & 37.8333 & 313.2599 & 305.3544 & 282 & 122.5994 & 2.37605 \\
\hline 2051 & 37.8503 & 312.5683 & 301.6497 & 281 & 122.5994 & 2.37503 \\
\hline 2052 & 37.8673 & 306.2206 & 299.8849 & 280 & 122.5994 & 2.374 \\
\hline 2053 & 37.8843 & 306.2908 & 299.5836 & 279 & 122.5994 & 2.37297 \\
\hline 2054 & 37.9013 & 303.8271 & 299.0359 & 278 & 122.5994 & 2.37195 \\
\hline 2055 & 37.9183 & 295.7541 & 296.625 & 277 & 122.5994 & 2.37092 \\
\hline 2056 & 37.9353 & 308.2892 & 292.6493 & 276 & 122.5994 & 2.3699 \\
\hline
\end{tabular}




\begin{tabular}{|c|c|c|c|c|c|c|}
\hline 2057 & 37.9523 & 303.5596 & 288.4839 & 275 & 122.5994 & 2.36888 \\
\hline 2058 & 37.9693 & 295.7826 & 284.9423 & 274 & 122.5994 & 2.36786 \\
\hline 2059 & 37.9863 & 265.6645 & 282.2166 & 273 & 122.5994 & 2.36684 \\
\hline 2060 & 38.0033 & 272.7023 & 280.0283 & 272 & 122.5994 & 2.36582 \\
\hline 2061 & 38.0203 & 278.3607 & 277.9733 & 271 & 122.5994 & 2.3648 \\
\hline 2062 & 38.0373 & 258.1972 & 275.9106 & 270 & 122.5994 & 2.36378 \\
\hline 2063 & 38.0543 & 270.97 & 273.9167 & 269 & 122.5994 & 2.36276 \\
\hline 2064 & 38.0713 & 270.4017 & 272.0808 & 268 & 122.5994 & 2.36175 \\
\hline 2065 & 38.0883 & 250.4192 & 270.5745 & 267 & 122.5994 & 2.36073 \\
\hline 2066 & 38.1053 & 273.1517 & 270.2315 & 267 & 122.5994 & 2.35972 \\
\hline 2067 & 38.1223 & 285.136 & 271.1046 & 268 & 122.5994 & 2.3587 \\
\hline 2068 & 38.1393 & 270.7262 & 271.1069 & 268 & 122.5994 & 2.35769 \\
\hline 2069 & 38.1563 & 265.7028 & 272.113 & 269 & 122.5994 & 2.35668 \\
\hline 2070 & 38.1733 & 262.475 & 271.9318 & 269 & 122.5994 & 2.35567 \\
\hline 2071 & 38.1903 & 269.4824 & 272.9293 & 270 & 122.5994 & 2.35466 \\
\hline 2072 & 38.2073 & 279.2751 & 272.8893 & 270 & 122.5994 & 2.35365 \\
\hline 2073 & 38.2243 & 280.3287 & 273.0697 & 270 & 122.5994 & 2.35264 \\
\hline 2074 & 38.2413 & 274.8581 & 274.1508 & 271 & 122.5994 & 2.35164 \\
\hline 2075 & 38.2583 & 295.7237 & 274.043 & 271 & 122.5994 & 2.35063 \\
\hline 2076 & 38.2753 & 287.6285 & 274.87 & 272 & 122.5994 & 2.34963 \\
\hline 2077 & 38.2923 & 266.8339 & 274.8387 & 272 & 122.5994 & 2.34862 \\
\hline 2078 & 38.3093 & 283.0738 & 275.0233 & 272 & 122.5994 & 2.34762 \\
\hline 2079 & 38.3263 & 268.7854 & 276.5864 & 273 & 122.5994 & 2.34662 \\
\hline 2080 & 38.3433 & 276.1115 & 277.4785 & 273 & 122.5994 & 2.34562 \\
\hline 2081 & 38.3603 & 287.3087 & 279.8131 & 274 & 122.5994 & 2.34462 \\
\hline 2082 & 38.3773 & 281.1986 & 280.8023 & 274 & 122.5994 & 2.34362 \\
\hline 2083 & 38.3943 & 292.3704 & 282.253 & 275 & 122.5994 & 2.34262 \\
\hline 2084 & 38.4113 & 281.9414 & 281.9816 & 275 & 122.5994 & 2.34162 \\
\hline 2085 & 38.4283 & 259.7007 & 281.3792 & 275 & 122.5994 & 2.34062 \\
\hline 2086 & 38.4453 & 258.7404 & 282.0545 & 276 & 122.5994 & 2.33963 \\
\hline 2087 & 38.4623 & 300.7818 & 282.0194 & 276 & 122.5994 & 2.33863 \\
\hline 2088 & 38.4793 & 284.505 & 283.2485 & 277 & 122.5994 & 2.33764 \\
\hline 2089 & 38.4963 & 294.8724 & 283.4591 & 277 & 122.5994 & 2.33664 \\
\hline 2090 & 38.5133 & 295.8116 & 283.5859 & 277 & 122.5994 & 2.33565 \\
\hline 2091 & 38.5303 & 302.5166 & 284.929 & 278 & 122.5994 & 2.33466 \\
\hline 2092 & 38.5473 & 279.1552 & 286.0337 & 278 & 122.5994 & 2.33367 \\
\hline 2093 & 38.5643 & 262.8386 & 289.2084 & 279 & 122.5994 & 2.33268 \\
\hline 2094 & 38.5813 & 292.497 & 292.8972 & 279 & 122.5994 & 2.33169 \\
\hline 2095 & 38.5983 & 294.3956 & 299.1726 & 280 & 122.5994 & 2.3307 \\
\hline 2096 & 38.6153 & 273.1516 & 305.2773 & 280 & 122.5994 & 2.32972 \\
\hline 2097 & 38.6323 & 321.8865 & 310.3633 & 280 & 122.5994 & 2.32873 \\
\hline 2098 & 38.6493 & 301.9721 & 313.2992 & 281 & 122.5994 & 2.32775 \\
\hline 2099 & 38.6663 & 293.8925 & 313.162 & 281 & 122.5994 & 2.32676 \\
\hline 2100 & 38.6833 & 286.7569 & 315.336 & 282 & 122.5994 & 2.32578 \\
\hline 2101 & 38.7003 & 309.0838 & 319.3493 & 282 & 122.5994 & 2.3248 \\
\hline 2102 & 38.7173 & 304.0803 & 324.5697 & 282 & 122.5994 & 2.32381 \\
\hline 2103 & 38.7343 & 294.325 & 327.6628 & 283 & 122.5994 & 2.32283 \\
\hline 2104 & 38.7513 & 287.2912 & 323.6984 & 283 & 122.5994 & 2.32185 \\
\hline 2105 & 38.7683 & 284.105 & 317.4914 & 284 & 122.5994 & 2.32087 \\
\hline
\end{tabular}




\begin{tabular}{|c|c|c|c|c|c|c|}
\hline 2106 & 38.7853 & 279.3447 & 311.1758 & 284 & 122.5994 & 2.3199 \\
\hline 2107 & 38.8023 & 271.7822 & 308.3607 & 285 & 122.5994 & 2.31892 \\
\hline 2108 & 38.8193 & 302.7352 & 306.4516 & 285 & 122.5994 & 2.31794 \\
\hline 2109 & 38.8363 & 295.8116 & 304.3948 & 285 & 122.5994 & 2.31697 \\
\hline 2110 & 38.8533 & 293.2947 & 302.032 & 286 & 122.5994 & 2.31599 \\
\hline 2111 & 38.8703 & 284.9353 & 298.2428 & 286 & 122.5994 & 2.31502 \\
\hline 2112 & 38.8873 & 277.5254 & 295.9519 & 287 & 122.5994 & 2.31405 \\
\hline 2113 & 38.9043 & 284.5049 & 293.5768 & 287 & 122.5994 & 2.31307 \\
\hline 2114 & 38.9213 & 300.6397 & 293.0906 & 288 & 122.5994 & 2.3121 \\
\hline 2115 & 38.9383 & 289.7857 & 293.29 & 289 & 122.5994 & 2.31113 \\
\hline 2116 & 38.9553 & 277.2875 & 294.0014 & 290 & 122.5994 & 2.31016 \\
\hline 2117 & 38.9723 & 271.813 & 295.1941 & 291 & 122.5994 & 2.30919 \\
\hline 2118 & 38.9893 & 282.6799 & 296.6268 & 292 & 122.5994 & 2.30823 \\
\hline 2119 & 39.0063 & 289.5844 & 298.5254 & 293 & 122.5994 & 2.30726 \\
\hline 2120 & 39.0233 & 294.3814 & 301.0617 & 294 & 122.5994 & 2.30629 \\
\hline 2121 & 39.0403 & 286.0186 & 304.3667 & 295 & 122.5994 & 2.30533 \\
\hline 2122 & 39.0573 & 269.3187 & 308.3056 & 296 & 122.5994 & 2.30436 \\
\hline 2123 & 39.0743 & 299.2954 & 317.2688 & 297 & 122.5994 & 2.3034 \\
\hline 2124 & 39.0913 & 293.3838 & 320.6307 & 298 & 122.5994 & 2.30244 \\
\hline 2125 & 39.1083 & 286.9487 & 322.6014 & 299 & 122.5994 & 2.30148 \\
\hline 2126 & 39.1253 & 272.1428 & 324.1805 & 300 & 122.5994 & 2.30052 \\
\hline 2127 & 39.1423 & 301.1224 & 326.4511 & 301 & 122.5994 & 2.29956 \\
\hline 2128 & 39.1593 & 324.2155 & 329.4145 & 302 & 122.5994 & 2.2986 \\
\hline 2129 & 39.1763 & 321.9894 & 334.3483 & 303 & 122.5994 & 2.29764 \\
\hline 2130 & 39.1933 & 300.5236 & 335.29 & 304 & 122.5994 & 2.29668 \\
\hline 2131 & 39.2103 & 284.5945 & 334.7802 & 305 & 122.5994 & 2.29572 \\
\hline 2132 & 39.2273 & 311.6643 & 334.2435 & 306 & 122.5994 & 2.29477 \\
\hline 2133 & 39.2443 & 286.6171 & 334.7316 & 307 & 122.5994 & 2.29381 \\
\hline 2134 & 39.2613 & 332.6426 & 336.5056 & 308 & 122.5994 & 2.29286 \\
\hline 2135 & 39.2783 & 321.9078 & 339.3253 & 309 & 122.5994 & 2.29191 \\
\hline 2136 & 39.2953 & 323.8177 & 343.2843 & 310 & 122.5994 & 2.29095 \\
\hline 2137 & 39.3123 & 348.5993 & 348.9144 & 311 & 122.5994 & 2.29 \\
\hline 2138 & 39.3293 & 353.4131 & 355.7177 & 311 & 122.5994 & 2.28905 \\
\hline 2139 & 39.3463 & 349.7229 & 364.2008 & 311 & 122.5994 & 2.2881 \\
\hline 2140 & 39.3633 & 388.2341 & 372.5669 & 311 & 122.5994 & 2.28715 \\
\hline 2141 & 39.3803 & 394.5739 & 378.2075 & 311 & 122.5994 & 2.2862 \\
\hline 2142 & 39.3973 & 452.0894 & 380.4325 & 311 & 122.5994 & 2.28526 \\
\hline 2143 & 39.4143 & 494.8022 & 382.7034 & 312 & 122.5994 & 2.28431 \\
\hline 2144 & 39.4313 & 575.8437 & 386.4306 & 312 & 122.5994 & 2.28336 \\
\hline 2145 & 39.4483 & 719.0581 & 394.5276 & 312 & 122.5994 & 2.28242 \\
\hline 2146 & 39.4653 & 940.9958 & 408.0372 & 312 & 122.5994 & 2.28148 \\
\hline 2147 & 39.4823 & 1235.023 & 429.1439 & 312 & 122.5994 & 2.28053 \\
\hline 2148 & 39.4993 & 1552.071 & 464.6065 & 312 & 122.5994 & 2.27959 \\
\hline 2149 & 39.5163 & 1558.82 & 528.4162 & 312 & 122.5994 & 2.27865 \\
\hline 2150 & 39.5333 & 1368.812 & 638.9735 & 312 & 122.5994 & 2.27771 \\
\hline 2151 & 39.5503 & 1003.227 & 810.2753 & 312 & 122.5994 & 2.27677 \\
\hline 2152 & 39.5673 & 851.1121 & 1038.054 & 312 & 122.5994 & 2.27583 \\
\hline 2153 & 39.5843 & 857.4182 & 1281.219 & 312 & 122.5994 & 2.27489 \\
\hline 2154 & 39.6013 & 884.1392 & 1444.897 & 312 & 122.5994 & 2.27395 \\
\hline
\end{tabular}




\begin{tabular}{|c|c|c|c|c|c|c|}
\hline 2155 & 39.6183 & 879.7601 & 1437.491 & 312 & 122.5994 & 2.27302 \\
\hline 2156 & 39.6353 & 812.5544 & 1301.181 & 313 & 122.5994 & 2.27208 \\
\hline 2157 & 39.6523 & 633.9071 & 1154.299 & 313 & 122.5994 & 2.27115 \\
\hline 2158 & 39.6693 & 441.8025 & 1069.047 & 313 & 122.5994 & 2.27021 \\
\hline 2159 & 39.6863 & 363.7491 & 1046.85 & 313 & 122.5994 & 2.26928 \\
\hline 2160 & 39.7033 & 357.1757 & 1032.563 & 313 & 122.5994 & 2.26835 \\
\hline 2161 & 39.7203 & 350.1476 & 960.4042 & 313 & 122.5994 & 2.26741 \\
\hline 2162 & 39.7373 & 334.9456 & 831.5588 & 313 & 122.5994 & 2.26648 \\
\hline 2163 & 39.7543 & 335.1637 & 694.0507 & 313 & 122.5994 & 2.26555 \\
\hline 2164 & 39.7713 & 342.2956 & 581.6952 & 313 & 122.5994 & 2.26462 \\
\hline 2165 & 39.7883 & 314.248 & 501.0004 & 313 & 122.5994 & 2.2637 \\
\hline 2166 & 39.8053 & 306.6875 & 448.3801 & 313 & 122.5994 & 2.26277 \\
\hline 2167 & 39.8223 & 285.9023 & 415.6276 & 314 & 122.5994 & 2.26184 \\
\hline 2168 & 39.8393 & 286.687 & 393.8584 & 314 & 122.5994 & 2.26092 \\
\hline 2169 & 39.8563 & 306.1073 & 379.0339 & 314 & 122.5994 & 2.25999 \\
\hline 2170 & 39.8733 & 286.8594 & 368.5058 & 314 & 122.5994 & 2.25907 \\
\hline 2171 & 39.8903 & 287.2298 & 360.7788 & 314 & 122.5994 & 2.25814 \\
\hline 2172 & 39.9073 & 312.3126 & 354.6441 & 314 & 122.5994 & 2.25722 \\
\hline 2173 & 39.9243 & 320.4527 & 349.7318 & 314 & 122.5994 & 2.2563 \\
\hline 2174 & 39.9413 & 301.8319 & 345.7734 & 314 & 122.5994 & 2.25538 \\
\hline 2175 & 39.9583 & 290.5017 & 342.6149 & 314 & 122.5994 & 2.25446 \\
\hline 2176 & 39.9753 & 297.2494 & 340.1405 & 314 & 122.5994 & 2.25354 \\
\hline 2177 & 39.9923 & 302.1045 & 338.1809 & 314 & 122.5994 & 2.25262 \\
\hline 2178 & 40.0093 & 296.9805 & 336.5016 & 314 & 122.5994 & 2.2517 \\
\hline 2179 & 40.0263 & 286.9889 & 327.4956 & 314 & 122.5994 & 2.25078 \\
\hline 2180 & 40.0433 & 322.7798 & 327.9018 & 315 & 122.5994 & 2.24987 \\
\hline 2181 & 40.0603 & 314.6117 & 328.9918 & 315 & 122.5994 & 2.24895 \\
\hline 2182 & 40.0773 & 275.2187 & 329.2172 & 315 & 122.5994 & 2.24804 \\
\hline 2183 & 40.0943 & 324.7997 & 329.8549 & 315 & 122.5994 & 2.24712 \\
\hline 2184 & 40.1113 & 313.6882 & 330.7285 & 315 & 122.5994 & 2.24621 \\
\hline 2185 & 40.1283 & 306.3506 & 332.2437 & 315 & 122.5994 & 2.2453 \\
\hline 2186 & 40.1453 & 317.0914 & 334.2027 & 315 & 122.5994 & 2.24439 \\
\hline 2187 & 40.1623 & 338.4781 & 335.5451 & 314 & 122.5994 & 2.24347 \\
\hline 2188 & 40.1793 & 333.8463 & 338.1714 & 314 & 122.5994 & 2.24256 \\
\hline 2189 & 40.1963 & 319.7258 & 341.2433 & 314 & 122.5994 & 2.24166 \\
\hline 2190 & 40.2133 & 357.2319 & 344.5914 & 313 & 122.5994 & 2.24075 \\
\hline 2191 & 40.2303 & 399.0241 & 351.8402 & 313 & 122.5994 & 2.23984 \\
\hline 2192 & 40.2473 & 456.2004 & 364.8531 & 313 & 122.5994 & 2.23893 \\
\hline 2193 & 40.2643 & 512.4902 & 387.2352 & 312 & 122.5994 & 2.23803 \\
\hline 2194 & 40.2813 & 625.3815 & 426.4947 & 312 & 122.5994 & 2.23712 \\
\hline 2195 & 40.2983 & 798.5377 & 485.478 & 312 & 122.5994 & 2.23622 \\
\hline 2196 & 40.3153 & 920.3729 & 560.3954 & 311 & 122.5994 & 2.23531 \\
\hline 2197 & 40.3323 & 1074.11 & 637.8146 & 311 & 122.5994 & 2.23441 \\
\hline 2198 & 40.3493 & 1040.91 & 682.088 & 311 & 122.5994 & 2.23351 \\
\hline 2199 & 40.3663 & 849.1832 & 669.3558 & 310 & 122.5994 & 2.23261 \\
\hline 2200 & 40.3833 & 669.5956 & 619.313 & 310 & 122.5994 & 2.23171 \\
\hline 2201 & 40.4003 & 606.8662 & 570.2725 & 310 & 122.5994 & 2.23081 \\
\hline 2202 & 40.4173 & 644.2694 & 542.1638 & 309 & 122.5994 & 2.22991 \\
\hline 2203 & 40.4343 & 711.6103 & 536.3058 & 309 & 122.5994 & 2.22901 \\
\hline
\end{tabular}




\begin{tabular}{|c|c|c|c|c|c|c|}
\hline 2204 & 40.4513 & 658.9532 & 533.3489 & 309 & 122.5994 & 2.22811 \\
\hline 2205 & 40.4683 & 560.5761 & 510.671 & 308 & 122.5994 & 2.22721 \\
\hline 2206 & 40.4853 & 444.7122 & 470.1285 & 308 & 122.5994 & 2.22632 \\
\hline 2207 & 40.5023 & 361.2193 & 426.1852 & 308 & 122.5994 & 2.22542 \\
\hline 2208 & 40.5193 & 326.1531 & 388.5165 & 307 & 122.5994 & 2.22453 \\
\hline 2209 & 40.5363 & 307.8954 & 362.4026 & 307 & 122.5994 & 2.22363 \\
\hline 2210 & 40.5533 & 304.8036 & 344.7618 & 306 & 122.5994 & 2.22274 \\
\hline 2211 & 40.5703 & 300.2414 & 334.7964 & 306 & 122.5994 & 2.22185 \\
\hline 2212 & 40.5873 & 300.3043 & 327.8506 & 305 & 122.5994 & 2.22096 \\
\hline 2213 & 40.6043 & 316.8356 & 323.2453 & 304 & 122.5994 & 2.22007 \\
\hline 2214 & 40.6213 & 296.204 & 321.1114 & 304 & 122.5994 & 2.21918 \\
\hline 2215 & 40.6383 & 305.3814 & 319.0807 & 303 & 122.5994 & 2.21829 \\
\hline 2216 & 40.6553 & 286.4961 & 318.9357 & 303 & 122.5994 & 2.2174 \\
\hline 2217 & 40.6723 & 273.4221 & 318.3948 & 302 & 122.5994 & 2.21651 \\
\hline 2218 & 40.6893 & 280.2836 & 318.0607 & 301 & 122.5994 & 2.21563 \\
\hline 2219 & 40.7063 & 265.561 & 318.7006 & 301 & 122.5994 & 2.21474 \\
\hline 2220 & 40.7233 & 294.2754 & 318.1273 & 300 & 122.5994 & 2.21385 \\
\hline 2221 & 40.7403 & 270.1821 & 317.9465 & 300 & 122.5994 & 2.21297 \\
\hline 2222 & 40.7573 & 282.7123 & 316.0023 & 299 & 122.5994 & 2.21209 \\
\hline 2223 & 40.7743 & 264.8664 & 313.7496 & 298 & 122.5994 & 2.2112 \\
\hline 2224 & 40.7913 & 270.0163 & 312.5865 & 298 & 122.5994 & 2.21032 \\
\hline 2225 & 40.8083 & 310.2887 & 310.659 & 297 & 122.5994 & 2.20944 \\
\hline 2226 & 40.8253 & 304.5081 & 309.047 & 296 & 122.5994 & 2.20856 \\
\hline 2227 & 40.8423 & 293.5337 & 308.648 & 296 & 122.5994 & 2.20768 \\
\hline 2228 & 40.8593 & 295.5264 & 307.2127 & 295 & 122.5994 & 2.2068 \\
\hline 2229 & 40.8763 & 264.4766 & 305.759 & 294 & 122.5994 & 2.20592 \\
\hline 2230 & 40.8933 & 304.5249 & 305.4688 & 294 & 122.5994 & 2.20504 \\
\hline 2231 & 40.9103 & 306.2206 & 304.3169 & 293 & 122.5994 & 2.20417 \\
\hline 2232 & 40.9273 & 282.1758 & 303.9044 & 293 & 122.5994 & 2.20329 \\
\hline 2233 & 40.9443 & 270.0796 & 301.9319 & 292 & 122.5994 & 2.20241 \\
\hline 2234 & 40.9613 & 312.6178 & 300.7428 & 292 & 122.5994 & 2.20154 \\
\hline 2235 & 40.9783 & 298.7983 & 299.7737 & 292 & 122.5994 & 2.20066 \\
\hline 2236 & 40.9953 & 290.9504 & 299.1992 & 293 & 122.5994 & 2.19979 \\
\hline 2237 & 41.0123 & 275.5336 & 299.2348 & 293 & 122.5994 & 2.19892 \\
\hline 2238 & 41.0293 & 274.2938 & 299.5899 & 293 & 122.5994 & 2.19805 \\
\hline 2239 & 41.0463 & 267.6898 & 301.0042 & 294 & 122.5994 & 2.19718 \\
\hline 2240 & 41.0633 & 275.4222 & 301.4819 & 294 & 122.5994 & 2.19631 \\
\hline 2241 & 41.0803 & 267.1202 & 301.9198 & 294 & 122.5994 & 2.19544 \\
\hline 2242 & 41.0973 & 301.5429 & 301.2641 & 294 & 122.5994 & 2.19457 \\
\hline 2243 & 41.1143 & 300.8151 & 300.5833 & 294 & 122.5994 & 2.1937 \\
\hline 2244 & 41.1313 & 267.1503 & 300.6983 & 295 & 122.5994 & 2.19283 \\
\hline 2245 & 41.1483 & 252.8437 & 300.2731 & 295 & 122.5994 & 2.19196 \\
\hline 2246 & 41.1653 & 269.0346 & 300.427 & 295 & 122.5994 & 2.1911 \\
\hline 2247 & 41.1823 & 296.2317 & 302.0887 & 296 & 122.5994 & 2.19023 \\
\hline 2248 & 41.1993 & 301.3293 & 302.7245 & 296 & 122.5994 & 2.18937 \\
\hline 2249 & 41.2163 & 326.0399 & 303.0585 & 296 & 122.5994 & 2.18851 \\
\hline 2250 & 41.2333 & 299.227 & 302.6599 & 296 & 122.5994 & 2.18764 \\
\hline 2251 & 41.2503 & 310.2658 & 301.9401 & 296 & 122.5994 & 2.18678 \\
\hline 2252 & 41.2673 & 303.3682 & 302.2426 & 297 & 122.5994 & 2.18592 \\
\hline
\end{tabular}




\begin{tabular}{|c|c|c|c|c|c|c|}
\hline 2253 & 41.2843 & 303.8379 & 301.8846 & 297 & 122.5994 & 2.18506 \\
\hline 2254 & 41.3013 & 305.713 & 301.8837 & 297 & 122.5994 & 2.1842 \\
\hline 2255 & 41.3183 & 315.1516 & 303.1615 & 298 & 122.5994 & 2.18334 \\
\hline 2256 & 41.3353 & 300.7225 & 303.5595 & 298 & 122.5994 & 2.18248 \\
\hline 2257 & 41.3523 & 286.9488 & 303.8373 & 298 & 122.5994 & 2.18162 \\
\hline 2258 & 41.3693 & 286.0137 & 303.8109 & 298 & 122.5994 & 2.18076 \\
\hline 2259 & 41.3863 & 320.0526 & 303.5295 & 298 & 122.5994 & 2.17991 \\
\hline 2260 & 41.4033 & 316.2911 & 303.2957 & 298 & 122.5994 & 2.17905 \\
\hline 2261 & 41.4203 & 290.6875 & 303.3463 & 298 & 122.5994 & 2.1782 \\
\hline 2262 & 41.4373 & 283.463 & 304.7018 & 299 & 122.5994 & 2.17734 \\
\hline 2263 & 41.4543 & 277.7386 & 305.194 & 299 & 122.5994 & 2.17649 \\
\hline 2264 & 41.4713 & 255.4395 & 305.7611 & 299 & 122.5994 & 2.17564 \\
\hline 2265 & 41.4883 & 272.0083 & 305.9256 & 299 & 122.5994 & 2.17478 \\
\hline 2266 & 41.5053 & 292.1994 & 306.1682 & 299 & 122.5994 & 2.17393 \\
\hline 2267 & 41.5223 & 268.189 & 306.7421 & 299 & 122.5994 & 2.17308 \\
\hline 2268 & 41.5393 & 287.6602 & 307.437 & 299 & 122.5994 & 2.17223 \\
\hline 2269 & 41.5563 & 285.0128 & 308.9831 & 300 & 122.5994 & 2.17138 \\
\hline 2270 & 41.5733 & 260.6846 & 309.3392 & 300 & 122.5994 & 2.17053 \\
\hline 2271 & 41.5903 & 289.5151 & 309.743 & 300 & 122.5994 & 2.16969 \\
\hline 2272 & 41.6073 & 278.3675 & 310.3101 & 300 & 122.5994 & 2.16884 \\
\hline 2273 & 41.6243 & 290.4446 & 310.9659 & 300 & 122.5994 & 2.16799 \\
\hline 2274 & 41.6413 & 280.192 & 311.6058 & 300 & 122.5994 & 2.16715 \\
\hline 2275 & 41.6583 & 301.0146 & 311.9969 & 300 & 122.5994 & 2.1663 \\
\hline 2276 & 41.6753 & 306.1146 & 312.6128 & 300 & 122.5994 & 2.16546 \\
\hline 2277 & 41.6923 & 337.5959 & 314.5308 & 300 & 122.5994 & 2.16461 \\
\hline 2278 & 41.7093 & 345.8719 & 319.7916 & 301 & 122.5994 & 2.16377 \\
\hline 2279 & 41.7263 & 353.0943 & 326.9479 & 301 & 122.5994 & 2.16293 \\
\hline 2280 & 41.7433 & 332.0765 & 336.4568 & 301 & 122.5994 & 2.16209 \\
\hline 2281 & 41.7603 & 353.4061 & 345.2525 & 301 & 122.5994 & 2.16124 \\
\hline 2282 & 41.7773 & 352.5273 & 348.3943 & 301 & 122.5994 & 2.1604 \\
\hline 2283 & 41.7943 & 345.3251 & 344.3055 & 301 & 122.5994 & 2.15957 \\
\hline 2284 & 41.8113 & 371.5204 & 337.7451 & 301 & 122.5994 & 2.15873 \\
\hline 2285 & 41.8283 & 365.9588 & 334.0665 & 302 & 122.5994 & 2.15789 \\
\hline 2286 & 41.8453 & 372.4543 & 332.2445 & 302 & 122.5994 & 2.15705 \\
\hline 2287 & 41.8623 & 367.5965 & 334.267 & 302 & 122.5994 & 2.15621 \\
\hline 2288 & 41.8793 & 341.3648 & 334.0643 & 302 & 122.5994 & 2.15538 \\
\hline 2289 & 41.8963 & 318.3063 & 331.237 & 302 & 122.5994 & 2.15454 \\
\hline 2290 & 41.9133 & 305.5275 & 326.7048 & 302 & 122.5994 & 2.15371 \\
\hline 2291 & 41.9303 & 327.9326 & 322.6879 & 302 & 122.5994 & 2.15287 \\
\hline 2292 & 41.9473 & 332.222 & 320.2397 & 302 & 122.5994 & 2.15204 \\
\hline 2293 & 41.9643 & 313.4705 & 319.4087 & 302 & 122.5994 & 2.15121 \\
\hline 2294 & 41.9813 & 302.7153 & 322.4818 & 303 & 122.5994 & 2.15038 \\
\hline 2295 & 41.9983 & 299.6101 & 325.1039 & 303 & 122.5994 & 2.14955 \\
\hline 2296 & 42.0153 & 311.5985 & 328.243 & 303 & 122.5994 & 2.14872 \\
\hline 2297 & 42.0323 & 319.0196 & 330.1494 & 303 & 122.5994 & 2.14789 \\
\hline 2298 & 42.0493 & 306.4791 & 329.6817 & 303 & 122.5994 & 2.14706 \\
\hline 2299 & 42.0663 & 293.9737 & 327.9011 & 303 & 122.5994 & 2.14623 \\
\hline 2300 & 42.0833 & 291.0451 & 326.5836 & 303 & 122.5994 & 2.1454 \\
\hline 2301 & 42.1003 & 309.5994 & 327.6341 & 304 & 122.5994 & 2.14457 \\
\hline
\end{tabular}




\begin{tabular}{|c|c|c|c|c|c|c|}
\hline 2302 & 42.1173 & 304.7355 & 329.0282 & 304 & 122.5994 & 2.14375 \\
\hline 2303 & 42.1343 & 284.4712 & 331.1299 & 304 & 122.5994 & 2.14292 \\
\hline 2304 & 42.1513 & 302.0274 & 333.1072 & 304 & 122.5994 & 2.1421 \\
\hline 2305 & 42.1683 & 301.7473 & 335.0364 & 304 & 122.5994 & 2.14127 \\
\hline 2306 & 42.1853 & 302.0047 & 337.8371 & 304 & 122.5994 & 2.14045 \\
\hline 2307 & 42.2023 & 335.3864 & 342.0816 & 304 & 122.5994 & 2.13963 \\
\hline 2308 & 42.2193 & 325.539 & 347.8311 & 304 & 122.5994 & 2.1388 \\
\hline 2309 & 42.2363 & 316.8264 & 354.7995 & 304 & 122.5994 & 2.13798 \\
\hline 2310 & 42.2533 & 322.8242 & 361.4406 & 304 & 122.5994 & 2.13716 \\
\hline 2311 & 42.2703 & 347.38 & 365.2392 & 304 & 122.5994 & 2.13634 \\
\hline 2312 & 42.2873 & 371.0427 & 365.2943 & 304 & 122.5994 & 2.13552 \\
\hline 2313 & 42.3043 & 399.9427 & 364.053 & 304 & 122.5994 & 2.1347 \\
\hline 2314 & 42.3213 & 398.8651 & 364.0442 & 303 & 122.5994 & 2.13388 \\
\hline 2315 & 42.3383 & 374.3877 & 369.4028 & 303 & 122.5994 & 2.13307 \\
\hline 2316 & 42.3553 & 413.837 & 380.0616 & 303 & 122.5994 & 2.13225 \\
\hline 2317 & 42.3723 & 470.2698 & 396.9969 & 303 & 122.5994 & 2.13143 \\
\hline 2318 & 42.3893 & 498.5753 & 423.2814 & 303 & 122.5994 & 2.13062 \\
\hline 2319 & 42.4063 & 545.8782 & 468.6361 & 303 & 122.5994 & 2.1298 \\
\hline 2320 & 42.4233 & 617.3937 & 544.9981 & 303 & 122.5994 & 2.12899 \\
\hline 2321 & 42.4403 & 765.8866 & 658.3182 & 303 & 122.5994 & 2.12818 \\
\hline 2322 & 42.4573 & 961.516 & 798.4451 & 303 & 122.5994 & 2.12736 \\
\hline 2323 & 42.4743 & 1222.028 & 926.9009 & 303 & 122.5994 & 2.12655 \\
\hline 2324 & 42.4913 & 1337.54 & 978.4485 & 303 & 122.5994 & 2.12574 \\
\hline 2325 & 42.5083 & 1450.582 & 926.5495 & 303 & 122.5994 & 2.12493 \\
\hline 2326 & 42.5253 & 1128.869 & 826.5106 & 303 & 122.5994 & 2.12412 \\
\hline 2327 & 42.5423 & 861.7385 & 742.7164 & 303 & 122.5994 & 2.12331 \\
\hline 2328 & 42.5593 & 690.397 & 703.5242 & 303 & 122.5994 & 2.1225 \\
\hline 2329 & 42.5763 & 749.8852 & 702.064 & 302 & 122.5994 & 2.12169 \\
\hline 2330 & 42.5933 & 804.1804 & 705.4523 & 302 & 122.5994 & 2.12089 \\
\hline 2331 & 42.6103 & 863.8327 & 671.621 & 301 & 122.5994 & 2.12008 \\
\hline 2332 & 42.6273 & 758.7685 & 600.7916 & 300 & 122.5994 & 2.11927 \\
\hline 2333 & 42.6443 & 583.3737 & 521.4584 & 299 & 122.5994 & 2.11847 \\
\hline 2334 & 42.6613 & 408.9681 & 454.0059 & 298 & 122.5994 & 2.11766 \\
\hline 2335 & 42.6783 & 387.4753 & 405.3105 & 297 & 122.5994 & 2.11686 \\
\hline 2336 & 42.6953 & 354.4711 & 373.6349 & 296 & 122.5994 & 2.11605 \\
\hline 2337 & 42.7123 & 341.1974 & 354.4739 & 296 & 122.5994 & 2.11525 \\
\hline 2338 & 42.7293 & 320.5342 & 341.2514 & 295 & 122.5994 & 2.11445 \\
\hline 2339 & 42.7463 & 313.7239 & 332.4188 & 294 & 122.5994 & 2.11365 \\
\hline 2340 & 42.7633 & 336.1372 & 326.8834 & 293 & 122.5994 & 2.11285 \\
\hline 2341 & 42.7803 & 315.3959 & 324.1542 & 292 & 122.5994 & 2.11205 \\
\hline 2342 & 42.7973 & 297.1258 & 323.8993 & 291 & 122.5994 & 2.11125 \\
\hline 2343 & 42.8143 & 315.4214 & 325.5496 & 290 & 122.5994 & 2.11045 \\
\hline 2344 & 42.8313 & 333.0211 & 328.5702 & 290 & 122.5994 & 2.10965 \\
\hline 2345 & 42.8483 & 348.0463 & 328.4671 & 289 & 122.5994 & 2.10885 \\
\hline 2346 & 42.8653 & 327.9845 & 324.7414 & 288 & 122.5994 & 2.10805 \\
\hline 2347 & 42.8823 & 320.5523 & 318.8004 & 287 & 122.5994 & 2.10726 \\
\hline 2348 & 42.8993 & 322.1795 & 313.2393 & 286 & 122.5994 & 2.10646 \\
\hline 2349 & 42.9163 & 316.5412 & 309.3076 & 285 & 122.5994 & 2.10567 \\
\hline 2350 & 42.9333 & 291.4006 & 307.2908 & 284 & 122.5994 & 2.10487 \\
\hline
\end{tabular}




\begin{tabular}{|c|c|c|c|c|c|c|}
\hline 2351 & 42.9503 & 282.7127 & 306.0937 & 283 & 122.5994 & 2.10408 \\
\hline 2352 & 42.9673 & 291.5645 & 305.0168 & 283 & 122.5994 & 2.10329 \\
\hline 2353 & 42.9843 & 304.3204 & 301.5174 & 282 & 122.5994 & 2.10249 \\
\hline 2354 & 43.0013 & 318.6703 & 297.4428 & 281 & 122.5994 & 2.1017 \\
\hline 2355 & 43.0183 & 294.6822 & 293.6932 & 280 & 122.5994 & 2.10091 \\
\hline 2356 & 43.0353 & 263.5708 & 290.6037 & 279 & 122.5994 & 2.10012 \\
\hline 2357 & 43.0523 & 260.4338 & 285.7014 & 278 & 122.5994 & 2.09933 \\
\hline 2358 & 43.0693 & 271.842 & 283.8949 & 277 & 122.5994 & 2.09854 \\
\hline 2359 & 43.0863 & 282.924 & 283.3911 & 277 & 122.5994 & 2.09775 \\
\hline 2360 & 43.1033 & 275.5343 & 282.2121 & 276 & 122.5994 & 2.09696 \\
\hline 2361 & 43.1203 & 295.974 & 281.2607 & 275 & 122.5994 & 2.09618 \\
\hline 2362 & 43.1373 & 280.9748 & 280.3172 & 274 & 122.5994 & 2.09539 \\
\hline 2363 & 43.1543 & 283.8529 & 279.1299 & 273 & 122.5994 & 2.0946 \\
\hline 2364 & 43.1713 & 266.052 & 277.6261 & 272 & 122.5994 & 2.09382 \\
\hline 2365 & 43.1883 & 248.685 & 276.1183 & 271 & 122.5994 & 2.09303 \\
\hline 2366 & 43.2053 & 287.1514 & 275.7814 & 271 & 122.5994 & 2.09225 \\
\hline 2367 & 43.2223 & 286.2115 & 273.6915 & 270 & 122.5994 & 2.09147 \\
\hline 2368 & 43.2393 & 276.3109 & 272.9229 & 269 & 122.5994 & 2.09068 \\
\hline 2369 & 43.2563 & 269.2603 & 272.2412 & 268 & 122.5994 & 2.0899 \\
\hline 2370 & 43.2733 & 241.0858 & 271.4749 & 267 & 122.5994 & 2.08912 \\
\hline 2371 & 43.2903 & 272.0208 & 270.742 & 266 & 122.5994 & 2.08834 \\
\hline 2372 & 43.3073 & 250.1087 & 269.9405 & 265 & 122.5994 & 2.08756 \\
\hline 2373 & 43.3243 & 280.2318 & 269.0711 & 264 & 122.5994 & 2.08678 \\
\hline 2374 & 43.3413 & 229.1156 & 269.0753 & 264 & 122.5994 & 2.086 \\
\hline 2375 & 43.3583 & 247.2234 & 267.9976 & 263 & 122.5994 & 2.08522 \\
\hline 2376 & 43.3753 & 259.0322 & 266.8734 & 262 & 122.5994 & 2.08444 \\
\hline 2377 & 43.3923 & 281.9503 & 265.6226 & 261 & 122.5994 & 2.08367 \\
\hline 2378 & 43.4093 & 255.059 & 265.7241 & 261 & 122.5994 & 2.08289 \\
\hline 2379 & 43.4263 & 251.2594 & 266.024 & 261 & 122.5994 & 2.08211 \\
\hline 2380 & 43.4433 & 260.3979 & 265.5735 & 260 & 122.5994 & 2.08134 \\
\hline 2381 & 43.4603 & 267.0952 & 266.4067 & 260 & 122.5994 & 2.08056 \\
\hline 2382 & 43.4773 & 266.2931 & 267.5363 & 260 & 122.5994 & 2.07979 \\
\hline 2383 & 43.4943 & 253.8451 & 268.9891 & 260 & 122.5994 & 2.07902 \\
\hline 2384 & 43.5113 & 273.7298 & 270.8183 & 260 & 122.5994 & 2.07824 \\
\hline 2385 & 43.5283 & 267.0126 & 271.9419 & 259 & 122.5994 & 2.07747 \\
\hline 2386 & 43.5453 & 266.6258 & 274.342 & 259 & 122.5994 & 2.0767 \\
\hline 2387 & 43.5623 & 273.3041 & 276.6557 & 259 & 122.5994 & 2.07593 \\
\hline 2388 & 43.5793 & 272.5651 & 277.5917 & 259 & 122.5994 & 2.07516 \\
\hline 2389 & 43.5963 & 287.9544 & 275.7267 & 258 & 122.5994 & 2.07439 \\
\hline 2390 & 43.6133 & 280.0945 & 274.3973 & 258 & 122.5994 & 2.07362 \\
\hline 2391 & 43.6303 & 263.1071 & 273.4655 & 258 & 122.5994 & 2.07285 \\
\hline 2392 & 43.6473 & 268.1604 & 272.7173 & 258 & 122.5994 & 2.07208 \\
\hline 2393 & 43.6643 & 269.5694 & 272.0953 & 258 & 122.5994 & 2.07131 \\
\hline 2394 & 43.6813 & 284.2897 & 270.6403 & 257 & 122.5994 & 2.07055 \\
\hline 2395 & 43.6983 & 270.6082 & 269.8835 & 257 & 122.5994 & 2.06978 \\
\hline 2396 & 43.7153 & 263.7521 & 268.7555 & 257 & 122.5994 & 2.06902 \\
\hline 2397 & 43.7323 & 270.0665 & 267.5273 & 257 & 122.5994 & 2.06825 \\
\hline 2398 & 43.7493 & 263.9426 & 266.0054 & 257 & 122.5994 & 2.06749 \\
\hline 2399 & 43.7663 & 260.462 & 263.2537 & 256 & 122.5994 & 2.06672 \\
\hline
\end{tabular}




\begin{tabular}{|c|c|c|c|c|c|c|}
\hline 2400 & 43.7833 & 253.8669 & 261.659 & 256 & 122.5994 & 2.06596 \\
\hline 2401 & 43.8003 & 252.5437 & 260.551 & 256 & 122.5994 & 2.0652 \\
\hline 2402 & 43.8173 & 256.0341 & 259.9996 & 256 & 122.5994 & 2.06444 \\
\hline 2403 & 43.8343 & 269.3178 & 259.8506 & 256 & 122.5994 & 2.06367 \\
\hline 2404 & 43.8513 & 277.8764 & 258.7422 & 255 & 122.5994 & 2.06291 \\
\hline 2405 & 43.8683 & 274.1119 & 258.5803 & 255 & 122.5994 & 2.06215 \\
\hline 2406 & 43.8853 & 260.0914 & 258.4465 & 255 & 122.5994 & 2.06139 \\
\hline 2407 & 43.9023 & 263.4786 & 257.4552 & 254 & 122.5994 & 2.06064 \\
\hline 2408 & 43.9193 & 270.8448 & 257.6249 & 254 & 122.5994 & 2.05988 \\
\hline 2409 & 43.9363 & 251.3299 & 257.8751 & 254 & 122.5994 & 2.05912 \\
\hline 2410 & 43.9533 & 265.2348 & 258.0133 & 254 & 122.5994 & 2.05836 \\
\hline 2411 & 43.9703 & 274.5717 & 257.9268 & 254 & 122.5994 & 2.05761 \\
\hline 2412 & 43.9873 & 264.1059 & 256.8634 & 253 & 122.5994 & 2.05685 \\
\hline 2413 & 44.0043 & 256.6366 & 256.823 & 253 & 122.5994 & 2.0561 \\
\hline 2414 & 44.0213 & 253.1029 & 256.9924 & 253 & 122.5994 & 2.05534 \\
\hline 2415 & 44.0383 & 271.0654 & 256.462 & 252 & 122.5994 & 2.05459 \\
\hline 2416 & 44.0553 & 259.6829 & 257.3017 & 252 & 122.5994 & 2.05383 \\
\hline 2417 & 44.0723 & 275.3229 & 258.4757 & 252 & 122.5994 & 2.05308 \\
\hline 2418 & 44.0893 & 275.6799 & 259.7899 & 252 & 122.5994 & 2.05233 \\
\hline 2419 & 44.1063 & 291.8506 & 261.2002 & 252 & 122.5994 & 2.05158 \\
\hline 2420 & 44.1233 & 272.7012 & 260.9789 & 251 & 122.5994 & 2.05083 \\
\hline 2421 & 44.1403 & 279.7956 & 261.1804 & 251 & 122.5994 & 2.05008 \\
\hline 2422 & 44.1573 & 246.4664 & 261.4284 & 251 & 122.5994 & 2.04933 \\
\hline 2423 & 44.1743 & 248.6828 & 260.8338 & 250 & 122.5994 & 2.04858 \\
\hline 2424 & 44.1913 & 263.5151 & 260.8694 & 250 & 122.5994 & 2.04783 \\
\hline 2425 & 44.2083 & 248.6963 & 260.2171 & 250 & 122.5994 & 2.04708 \\
\hline 2426 & 44.2253 & 244.4481 & 259.162 & 250 & 122.5994 & 2.04633 \\
\hline 2427 & 44.2423 & 252.1557 & 256.9703 & 249 & 122.5994 & 2.04559 \\
\hline 2428 & 44.2593 & 266.0949 & 255.8894 & 249 & 122.5994 & 2.04484 \\
\hline 2429 & 44.2763 & 271.8 & 255.2466 & 249 & 122.5994 & 2.04409 \\
\hline 2430 & 44.2933 & 259.1379 & 253.857 & 248 & 122.5994 & 2.04335 \\
\hline 2431 & 44.3103 & 278.0706 & 253.3476 & 248 & 122.5994 & 2.0426 \\
\hline 2432 & 44.3273 & 259.1851 & 252.6141 & 248 & 122.5994 & 2.04186 \\
\hline 2433 & 44.3443 & 265.6921 & 250.9275 & 247 & 122.5994 & 2.04112 \\
\hline 2434 & 44.3613 & 289.638 & 250.7487 & 247 & 122.5994 & 2.04037 \\
\hline 2435 & 44.3783 & 293.4651 & 250.6919 & 247 & 122.5994 & 2.03963 \\
\hline 2436 & 44.3953 & 260.6142 & 249.9112 & 246 & 122.5994 & 2.03889 \\
\hline 2437 & 44.4123 & 258.1729 & 250.1163 & 246 & 122.5994 & 2.03815 \\
\hline 2438 & 44.4293 & 269.9597 & 250.2949 & 246 & 122.5994 & 2.03741 \\
\hline 2439 & 44.4463 & 268.0682 & 249.3051 & 245 & 122.5994 & 2.03667 \\
\hline 2440 & 44.4633 & 259.2446 & 249.6183 & 245 & 122.5994 & 2.03593 \\
\hline 2441 & 44.4803 & 248.1675 & 250.5475 & 245 & 122.5994 & 2.03519 \\
\hline 2442 & 44.4973 & 247.0132 & 251.364 & 244 & 122.5994 & 2.03445 \\
\hline 2443 & 44.5143 & 273.8858 & 254.1994 & 244 & 122.5994 & 2.03372 \\
\hline 2444 & 44.5313 & 272.2254 & 257.8572 & 244 & 122.5994 & 2.03298 \\
\hline 2445 & 44.5483 & 274.6504 & 260.7234 & 243 & 122.5994 & 2.03224 \\
\hline 2446 & 44.5653 & 273.4866 & 263.3295 & 243 & 122.5994 & 2.03151 \\
\hline 2447 & 44.5823 & 259.6316 & 263.5154 & 243 & 122.5994 & 2.03077 \\
\hline 2448 & 44.5993 & 239.744 & 261.0666 & 242 & 122.5994 & 2.03004 \\
\hline
\end{tabular}




\begin{tabular}{|c|c|c|c|c|c|c|}
\hline 2449 & 44.6163 & 219.0564 & 260.0122 & 242 & 122.5994 & 2.0293 \\
\hline 2450 & 44.6333 & 247.3087 & 261.7275 & 243 & 122.5994 & 2.02857 \\
\hline 2451 & 44.6503 & 291.2351 & 265.4303 & 244 & 122.5994 & 2.02784 \\
\hline 2452 & 44.6673 & 252.2292 & 270.1223 & 245 & 122.5994 & 2.0271 \\
\hline 2453 & 44.6843 & 283.4799 & 274.0193 & 246 & 122.5994 & 2.02637 \\
\hline 2454 & 44.7013 & 263.0984 & 276.9637 & 247 & 122.5994 & 2.02564 \\
\hline 2455 & 44.7183 & 242.7261 & 280.3573 & 248 & 122.5994 & 2.02491 \\
\hline 2456 & 44.7353 & 235.215 & 284.8076 & 249 & 122.5994 & 2.02418 \\
\hline 2457 & 44.7523 & 280.4114 & 288.6516 & 250 & 122.5994 & 2.02345 \\
\hline 2458 & 44.7693 & 263.1915 & 288.5144 & 250 & 122.5994 & 2.02272 \\
\hline 2459 & 44.7863 & 259.2446 & 286.3589 & 251 & 122.5994 & 2.02199 \\
\hline 2460 & 44.8033 & 265.0721 & 283.164 & 252 & 122.5994 & 2.02127 \\
\hline 2461 & 44.8203 & 274.9059 & 280.4987 & 253 & 122.5994 & 2.02054 \\
\hline 2462 & 44.8373 & 301.4159 & 279.2355 & 254 & 122.5994 & 2.01981 \\
\hline 2463 & 44.8543 & 292.6606 & 279.3805 & 255 & 122.5994 & 2.01909 \\
\hline 2464 & 44.8713 & 292.449 & 279.8392 & 256 & 122.5994 & 2.01836 \\
\hline 2465 & 44.8883 & 292.1228 & 279.4377 & 257 & 122.5994 & 2.01764 \\
\hline 2466 & 44.9053 & 272.1002 & 278.3696 & 258 & 122.5994 & 2.01691 \\
\hline 2467 & 44.9223 & 269.3869 & 277.5256 & 259 & 122.5994 & 2.01619 \\
\hline 2468 & 44.9393 & 275.8022 & 277.0366 & 260 & 122.5994 & 2.01547 \\
\hline 2469 & 44.9563 & 278.8632 & 276.5581 & 261 & 122.5994 & 2.01474 \\
\hline 2470 & 44.9733 & 281.0246 & 276.1471 & 262 & 122.5994 & 2.01402 \\
\hline 2471 & 44.9903 & 298.3447 & 276.4517 & 263 & 122.5994 & 2.0133 \\
\hline 2472 & 45.0073 & 312.1574 & 278.4029 & 264 & 122.5994 & 2.01258 \\
\hline 2473 & 45.0243 & 301.0706 & 281.9548 & 265 & 122.5994 & 2.01186 \\
\hline 2474 & 45.0413 & 278.1651 & 285.4244 & 265 & 122.5994 & 2.01114 \\
\hline 2475 & 45.0583 & 293.6105 & 288.1949 & 265 & 122.5994 & 2.01042 \\
\hline 2476 & 45.0753 & 349.6456 & 289.3917 & 266 & 122.5994 & 2.0097 \\
\hline 2477 & 45.0923 & 336.5967 & 286.6032 & 266 & 122.5994 & 2.00898 \\
\hline 2478 & 45.1093 & 328.0048 & 283.0213 & 266 & 122.5994 & 2.00826 \\
\hline 2479 & 45.1263 & 315.9577 & 281.6883 & 267 & 122.5994 & 2.00755 \\
\hline 2480 & 45.1433 & 299.2265 & 281.2264 & 267 & 122.5994 & 2.00683 \\
\hline 2481 & 45.1603 & 302.0223 & 283.1059 & 268 & 122.5994 & 2.00611 \\
\hline 2482 & 45.1773 & 320.4067 & 283.96 & 268 & 122.5994 & 2.0054 \\
\hline 2483 & 45.1943 & 309.8501 & 283.3344 & 268 & 122.5994 & 2.00468 \\
\hline 2484 & 45.2113 & 325.0555 & 282.0343 & 269 & 122.5994 & 2.00397 \\
\hline 2485 & 45.2283 & 324.7798 & 280.6798 & 269 & 122.5994 & 2.00326 \\
\hline 2486 & 45.2453 & 300.687 & 278.7826 & 269 & 122.5994 & 2.00254 \\
\hline 2487 & 45.2623 & 300.8968 & 278.8469 & 270 & 122.5994 & 2.00183 \\
\hline 2488 & 45.2793 & 316.7416 & 278.7614 & 270 & 122.5994 & 2.00112 \\
\hline 2489 & 45.2963 & 319.0355 & 279.8977 & 271 & 122.5994 & 2.00041 \\
\hline 2490 & 45.3133 & 334.0791 & 279.7719 & 271 & 122.5994 & 1.9997 \\
\hline 2491 & 45.3303 & 333.8684 & 279.3731 & 271 & 122.5994 & 1.99899 \\
\hline 2492 & 45.3473 & 359.0074 & 280.8009 & 272 & 122.5994 & 1.99828 \\
\hline 2493 & 45.3643 & 375.4142 & 280.9493 & 272 & 122.5994 & 1.99757 \\
\hline 2494 & 45.3813 & 362.7586 & 281.4918 & 272 & 122.5994 & 1.99686 \\
\hline 2495 & 45.3983 & 364.7372 & 283.9737 & 273 & 122.5994 & 1.99615 \\
\hline 2496 & 45.4153 & 360.8803 & 285.9743 & 273 & 122.5994 & 1.99544 \\
\hline 2497 & 45.4323 & 392.9912 & 289.1503 & 274 & 122.5994 & 1.99473 \\
\hline
\end{tabular}




\begin{tabular}{|c|c|c|c|c|c|c|}
\hline 2498 & 45.4493 & 393.6055 & 291.0691 & 274 & 122.5994 & 1.99403 \\
\hline 2499 & 45.4663 & 424.4798 & 292.3934 & 274 & 122.5994 & 1.99332 \\
\hline 2500 & 45.4833 & 424.0318 & 293.7565 & 275 & 122.5994 & 1.99262 \\
\hline 2501 & 45.5003 & 430.1034 & 293.2898 & 275 & 122.5994 & 1.99191 \\
\hline 2502 & 45.5173 & 435.9853 & 293.0151 & 275 & 122.5994 & 1.99121 \\
\hline 2503 & 45.5343 & 454.5036 & 294.6089 & 276 & 122.5994 & 1.9905 \\
\hline 2504 & 45.5513 & 443.4112 & 296.2119 & 276 & 122.5994 & 1.9898 \\
\hline 2505 & 45.5683 & 455.8259 & 299.576 & 277 & 122.5994 & 1.9891 \\
\hline 2506 & 45.5853 & 388.2132 & 302.1835 & 277 & 122.5994 & 1.98839 \\
\hline 2507 & 45.6023 & 451.6859 & 304.3369 & 277 & 122.5994 & 1.98769 \\
\hline 2508 & 45.6193 & 411.0273 & 306.4014 & 278 & 122.5994 & 1.98699 \\
\hline 2509 & 45.6363 & 400.2599 & 306.3065 & 278 & 122.5994 & 1.98629 \\
\hline 2510 & 45.6533 & 415.9621 & 305.8739 & 278 & 122.5994 & 1.98559 \\
\hline 2511 & 45.6703 & 364.7971 & 307.2354 & 279 & 122.5994 & 1.98489 \\
\hline 2512 & 45.6873 & 368.3757 & 309.1094 & 279 & 122.5994 & 1.98419 \\
\hline 2513 & 45.7043 & 359.8564 & 313.9525 & 280 & 122.5994 & 1.98349 \\
\hline 2514 & 45.7213 & 413.8876 & 320.6873 & 280 & 122.5994 & 1.9828 \\
\hline 2515 & 45.7383 & 438.7121 & 331.9248 & 280 & 122.5994 & 1.9821 \\
\hline 2516 & 45.7553 & 466.49 & 352.0847 & 281 & 122.5994 & 1.9814 \\
\hline 2517 & 45.7723 & 544.673 & 384.7222 & 281 & 122.5994 & 1.9807 \\
\hline 2518 & 45.7893 & 640.5331 & 436.5953 & 281 & 122.5994 & 1.98001 \\
\hline 2519 & 45.8063 & 801.2337 & 510.0922 & 282 & 122.5994 & 1.97931 \\
\hline 2520 & 45.8233 & 929.4593 & 595.6506 & 282 & 122.5994 & 1.97862 \\
\hline 2521 & 45.8403 & 1011.579 & 676.6935 & 283 & 122.5994 & 1.97792 \\
\hline 2522 & 45.8573 & 917.2861 & 715.8867 & 282 & 122.5994 & 1.97723 \\
\hline 2523 & 45.8743 & 710.129 & 692.1913 & 282 & 122.5994 & 1.97654 \\
\hline 2524 & 45.8913 & 562.4626 & 630.5975 & 282 & 122.5994 & 1.97585 \\
\hline 2525 & 45.9083 & 565.4334 & 573.6921 & 282 & 122.5994 & 1.97515 \\
\hline 2526 & 45.9253 & 600.2809 & 542.4053 & 282 & 122.5994 & 1.97446 \\
\hline 2527 & 45.9423 & 622.3097 & 534.6516 & 281 & 122.5994 & 1.97377 \\
\hline 2528 & 45.9593 & 660.5921 & 540.0103 & 281 & 122.5994 & 1.97308 \\
\hline 2529 & 45.9763 & 624.4725 & 537.6355 & 281 & 122.5994 & 1.97239 \\
\hline 2530 & 45.9933 & 494.2102 & 510.2037 & 281 & 122.5994 & 1.9717 \\
\hline 2531 & 46.0103 & 422.0741 & 464.6531 & 281 & 122.5994 & 1.97101 \\
\hline 2532 & 46.0273 & 369.2152 & 418.0695 & 280 & 122.5994 & 1.97032 \\
\hline 2533 & 46.0443 & 358.6451 & 381.7963 & 280 & 122.5994 & 1.96964 \\
\hline 2534 & 46.0613 & 334.2871 & 355.0334 & 280 & 122.5994 & 1.96895 \\
\hline 2535 & 46.0783 & 292.382 & 335.1618 & 280 & 122.5994 & 1.96826 \\
\hline 2536 & 46.0953 & 301.884 & 320.9072 & 280 & 122.5994 & 1.96757 \\
\hline 2537 & 46.1123 & 305.7737 & 311.3799 & 280 & 122.5994 & 1.96689 \\
\hline 2538 & 46.1293 & 292.7354 & 304.4021 & 279 & 122.5994 & 1.9662 \\
\hline 2539 & 46.1463 & 285.5826 & 300.8934 & 279 & 122.5994 & 1.96552 \\
\hline 2540 & 46.1633 & 277.232 & 298.818 & 279 & 122.5994 & 1.96483 \\
\hline 2541 & 46.1803 & 274.3799 & 297.1657 & 279 & 122.5994 & 1.96415 \\
\hline 2542 & 46.1973 & 268.8932 & 295.2281 & 279 & 122.5994 & 1.96347 \\
\hline 2543 & 46.2143 & 261.2255 & 292.1053 & 278 & 122.5994 & 1.96278 \\
\hline 2544 & 46.2313 & 282.9516 & 290.3836 & 278 & 122.5994 & 1.9621 \\
\hline 2545 & 46.2483 & 292.449 & 289.234 & 278 & 122.5994 & 1.96142 \\
\hline 2546 & 46.2653 & 262.3285 & 287.7096 & 277 & 122.5994 & 1.96074 \\
\hline
\end{tabular}




\begin{tabular}{|c|c|c|c|c|c|c|}
\hline 2547 & 46.2823 & 275.7514 & 286.6789 & 276 & 122.5994 & 1.96006 \\
\hline 2548 & 46.2993 & 258.6749 & 286.8711 & 276 & 122.5994 & 1.95938 \\
\hline 2549 & 46.3163 & 255.4125 & 285.8958 & 275 & 122.5994 & 1.9587 \\
\hline 2550 & 46.3333 & 264.2228 & 284.3657 & 274 & 122.5994 & 1.95802 \\
\hline 2551 & 46.3503 & 262.8484 & 283.3903 & 274 & 122.5994 & 1.95734 \\
\hline 2552 & 46.3673 & 274.1273 & 281.3638 & 273 & 122.5994 & 1.95666 \\
\hline 2553 & 46.3843 & 235.0401 & 279.6345 & 272 & 122.5994 & 1.95599 \\
\hline 2554 & 46.4013 & 254.255 & 278.3705 & 271 & 122.5994 & 1.95531 \\
\hline 2555 & 46.4183 & 265.2651 & 277.7463 & 270 & 122.5994 & 1.95463 \\
\hline 2556 & 46.4353 & 272.0543 & 276.6167 & 270 & 122.5994 & 1.95396 \\
\hline 2557 & 46.4523 & 252.93 & 276.1313 & 269 & 122.5994 & 1.95328 \\
\hline 2558 & 46.4693 & 253.0606 & 275.5364 & 268 & 122.5994 & 1.95261 \\
\hline 2559 & 46.4863 & 268.8694 & 275.8011 & 268 & 122.5994 & 1.95193 \\
\hline 2560 & 46.5033 & 265.0822 & 274.0212 & 267 & 122.5994 & 1.95126 \\
\hline 2561 & 46.5203 & 256.0341 & 273.478 & 266 & 122.5994 & 1.95058 \\
\hline 2562 & 46.5373 & 258.6824 & 272.9295 & 265 & 122.5994 & 1.94991 \\
\hline 2563 & 46.5543 & 280.212 & 271.8836 & 264 & 122.5994 & 1.94924 \\
\hline 2564 & 46.5713 & 268.0846 & 271.188 & 264 & 122.5994 & 1.94857 \\
\hline 2565 & 46.5883 & 277.8138 & 269.292 & 263 & 122.5994 & 1.9479 \\
\hline 2566 & 46.6053 & 255.4303 & 267.5764 & 262 & 122.5994 & 1.94722 \\
\hline 2567 & 46.6223 & 248.1984 & 267.0677 & 262 & 122.5994 & 1.94655 \\
\hline 2568 & 46.6393 & 238.3672 & 265.9722 & 261 & 122.5994 & 1.94588 \\
\hline 2569 & 46.6563 & 267.4399 & 265.0957 & 260 & 122.5994 & 1.94521 \\
\hline 2570 & 46.6733 & 276.7637 & 266.1439 & 261 & 122.5994 & 1.94455 \\
\hline 2571 & 46.6903 & 265.3872 & 265.9569 & 261 & 122.5994 & 1.94388 \\
\hline 2572 & 46.7073 & 271.1767 & 266.7745 & 262 & 122.5994 & 1.94321 \\
\hline 2573 & 46.7243 & 257.1425 & 267.9681 & 263 & 122.5994 & 1.94254 \\
\hline 2574 & 46.7413 & 241.9847 & 268.9481 & 263 & 122.5994 & 1.94188 \\
\hline 2575 & 46.7583 & 252.3918 & 271.998 & 264 & 122.5994 & 1.94121 \\
\hline 2576 & 46.7753 & 243.9428 & 276.6677 & 265 & 122.5994 & 1.94054 \\
\hline 2577 & 46.7923 & 255.2634 & 282.2075 & 265 & 122.5994 & 1.93988 \\
\hline 2578 & 46.8093 & 268.2803 & 290.2149 & 266 & 122.5994 & 1.93921 \\
\hline 2579 & 46.8263 & 256.1841 & 298.2527 & 267 & 122.5994 & 1.93855 \\
\hline 2580 & 46.8433 & 272.2699 & 302.5609 & 267 & 122.5994 & 1.93788 \\
\hline 2581 & 46.8603 & 273.7407 & 302.2726 & 268 & 122.5994 & 1.93722 \\
\hline 2582 & 46.8773 & 266.4719 & 297.4994 & 269 & 122.5994 & 1.93656 \\
\hline 2583 & 46.8943 & 279.2413 & 291.3874 & 269 & 122.5994 & 1.9359 \\
\hline 2584 & 46.9113 & 272.6723 & 288.6465 & 270 & 122.5994 & 1.93523 \\
\hline 2585 & 46.9283 & 254.2415 & 288.8228 & 271 & 122.5994 & 1.93457 \\
\hline 2586 & 46.9453 & 240.686 & 289.9856 & 271 & 122.5994 & 1.93391 \\
\hline 2587 & 46.9623 & 233.2675 & 292.3207 & 272 & 122.5994 & 1.93325 \\
\hline 2588 & 46.9793 & 267.1765 & 292.8747 & 273 & 122.5994 & 1.93259 \\
\hline 2589 & 46.9963 & 274.1935 & 289.7919 & 273 & 122.5994 & 1.93193 \\
\hline 2590 & 47.0133 & 280.7454 & 286.9241 & 274 & 122.5994 & 1.93127 \\
\hline 2591 & 47.0303 & 283.1323 & 284.7202 & 275 & 122.5994 & 1.93061 \\
\hline 2592 & 47.0473 & 279.7693 & 282.6348 & 275 & 122.5994 & 1.92996 \\
\hline 2593 & 47.0643 & 268.697 & 282.5282 & 276 & 122.5994 & 1.9293 \\
\hline 2594 & 47.0813 & 270.9198 & 282.0249 & 276 & 122.5994 & 1.92864 \\
\hline 2595 & 47.0983 & 274.9157 & 282.831 & 277 & 122.5994 & 1.92799 \\
\hline
\end{tabular}




\begin{tabular}{|c|c|c|c|c|c|c|}
\hline 2596 & 47.1153 & 272.7708 & 282.6885 & 277 & 122.5994 & 1.92733 \\
\hline 2597 & 47.1323 & 242.2383 & 282.5388 & 277 & 122.5994 & 1.92667 \\
\hline 2598 & 47.1493 & 259.3263 & 283.4294 & 278 & 122.5994 & 1.92602 \\
\hline 2599 & 47.1663 & 248.1299 & 283.4703 & 278 & 122.5994 & 1.92536 \\
\hline 2600 & 47.1833 & 261.1531 & 283.799 & 278 & 122.5994 & 1.92471 \\
\hline 2601 & 47.2003 & 253.3412 & 285.7044 & 279 & 122.5994 & 1.92406 \\
\hline 2602 & 47.2173 & 311.1386 & 287.3777 & 279 & 122.5994 & 1.9234 \\
\hline 2603 & 47.2343 & 269.582 & 290.0771 & 279 & 122.5994 & 1.92275 \\
\hline 2604 & 47.2513 & 259.3199 & 294.3325 & 280 & 122.5994 & 1.9221 \\
\hline 2605 & 47.2683 & 278.4584 & 297.4737 & 280 & 122.5994 & 1.92145 \\
\hline 2606 & 47.2853 & 261.3072 & 298.7202 & 280 & 122.5994 & 1.9208 \\
\hline 2607 & 47.3023 & 242.7458 & 298.9795 & 281 & 122.5994 & 1.92014 \\
\hline 2608 & 47.3193 & 274.0764 & 298.1292 & 281 & 122.5994 & 1.91949 \\
\hline 2609 & 47.3363 & 258.4529 & 298.5836 & 281 & 122.5994 & 1.91885 \\
\hline 2610 & 47.3533 & 254.5961 & 301.4981 & 282 & 122.5994 & 1.9182 \\
\hline 2611 & 47.3703 & 267.2188 & 303.7253 & 282 & 122.5994 & 1.91755 \\
\hline 2612 & 47.3873 & 276.1293 & 304.4759 & 282 & 122.5994 & 1.9169 \\
\hline 2613 & 47.4043 & 278.2594 & 304.2616 & 283 & 122.5994 & 1.91625 \\
\hline 2614 & 47.4213 & 273.3428 & 301.7616 & 283 & 122.5994 & 1.9156 \\
\hline 2615 & 47.4383 & 265.7254 & 299.2841 & 283 & 122.5994 & 1.91496 \\
\hline 2616 & 47.4553 & 274.5697 & 298.7287 & 284 & 122.5994 & 1.91431 \\
\hline 2617 & 47.4723 & 298.3408 & 298.2357 & 284 & 122.5994 & 1.91366 \\
\hline 2618 & 47.4893 & 286.5222 & 297.2095 & 283 & 122.5994 & 1.91302 \\
\hline 2619 & 47.5063 & 273.3458 & 296.6384 & 283 & 122.5994 & 1.91237 \\
\hline 2620 & 47.5233 & 283.1563 & 294.0689 & 282 & 122.5994 & 1.91173 \\
\hline 2621 & 47.5403 & 272.2239 & 291.9637 & 282 & 122.5994 & 1.91109 \\
\hline 2622 & 47.5573 & 265.8798 & 288.9507 & 281 & 122.5994 & 1.91044 \\
\hline 2623 & 47.5743 & 244.341 & 286.3978 & 280 & 122.5994 & 1.9098 \\
\hline 2624 & 47.5913 & 265.3346 & 285.3434 & 280 & 122.5994 & 1.90916 \\
\hline 2625 & 47.6083 & 294.4334 & 283.74 & 279 & 122.5994 & 1.90851 \\
\hline 2626 & 47.6253 & 273.8132 & 282.339 & 278 & 122.5994 & 1.90787 \\
\hline 2627 & 47.6423 & 284.0522 & 282.0239 & 278 & 122.5994 & 1.90723 \\
\hline 2628 & 47.6593 & 271.6622 & 280.7223 & 277 & 122.5994 & 1.90659 \\
\hline 2629 & 47.6763 & 267.0541 & 279.4515 & 276 & 122.5994 & 1.90595 \\
\hline 2630 & 47.6933 & 256.9816 & 279.4114 & 276 & 122.5994 & 1.90531 \\
\hline 2631 & 47.7103 & 247.6087 & 278.5184 & 275 & 122.5994 & 1.90467 \\
\hline 2632 & 47.7273 & 266.7068 & 278.7213 & 275 & 122.5994 & 1.90403 \\
\hline 2633 & 47.7443 & 284.0113 & 277.9506 & 274 & 122.5994 & 1.9034 \\
\hline 2634 & 47.7613 & 259.2614 & 277.1174 & 273 & 122.5994 & 1.90276 \\
\hline 2635 & 47.7783 & 262.8044 & 277.3881 & 273 & 122.5994 & 1.90212 \\
\hline 2636 & 47.7953 & 263.2169 & 276.8213 & 272 & 122.5994 & 1.90148 \\
\hline 2637 & 47.8123 & 273.2942 & 277.2208 & 272 & 122.5994 & 1.90085 \\
\hline 2638 & 47.8293 & 269.1171 & 276.3586 & 271 & 122.5994 & 1.90021 \\
\hline 2639 & 47.8463 & 265.7254 & 275.1681 & 270 & 122.5994 & 1.89958 \\
\hline 2640 & 47.8633 & 258.0926 & 274.9886 & 270 & 122.5994 & 1.89894 \\
\hline 2641 & 47.8803 & 266.4419 & 274.0707 & 269 & 122.5994 & 1.89831 \\
\hline 2642 & 47.8973 & 279.8834 & 274.4698 & 269 & 122.5994 & 1.89767 \\
\hline 2643 & 47.9143 & 276.5623 & 276.1003 & 270 & 122.5994 & 1.89704 \\
\hline 2644 & 47.9313 & 244.9633 & 276.4504 & 270 & 122.5994 & 1.89641 \\
\hline
\end{tabular}




\begin{tabular}{|c|c|c|c|c|c|c|}
\hline 2645 & 47.9483 & 274.577 & 276.1909 & 270 & 122.5994 & 1.89577 \\
\hline 2646 & 47.9653 & 249.4229 & 276.5903 & 271 & 122.5994 & 1.89514 \\
\hline 2647 & 47.9823 & 279.787 & 276.166 & 271 & 122.5994 & 1.89451 \\
\hline 2648 & 47.9993 & 298.5381 & 276.203 & 271 & 122.5994 & 1.89388 \\
\hline 2649 & 48.0163 & 289.2273 & 277.5021 & 272 & 122.5994 & 1.89325 \\
\hline 2650 & 48.0333 & 295.7886 & 277.9009 & 272 & 122.5994 & 1.89262 \\
\hline 2651 & 48.0503 & 246.9922 & 278.8611 & 273 & 122.5994 & 1.89199 \\
\hline 2652 & 48.0673 & 274.4415 & 278.2352 & 273 & 122.5994 & 1.89136 \\
\hline 2653 & 48.0843 & 247.048 & 277.4177 & 273 & 122.5994 & 1.89073 \\
\hline 2654 & 48.1013 & 256.2405 & 277.8141 & 274 & 122.5994 & 1.8901 \\
\hline 2655 & 48.1183 & 260.2608 & 277.5887 & 274 & 122.5994 & 1.88947 \\
\hline 2656 & 48.1353 & 272.3117 & 277.7318 & 274 & 122.5994 & 1.88884 \\
\hline 2657 & 48.1523 & 301.6315 & 279.1013 & 275 & 122.5994 & 1.88822 \\
\hline 2658 & 48.1693 & 333.0205 & 279.5375 & 275 & 122.5994 & 1.88759 \\
\hline 2659 & 48.1863 & 319.3392 & 280.0887 & 275 & 122.5994 & 1.88696 \\
\hline 2660 & 48.2033 & 307.5783 & 281.9613 & 276 & 122.5994 & 1.88634 \\
\hline 2661 & 48.2203 & 327.7649 & 283.3886 & 276 & 122.5994 & 1.88571 \\
\hline 2662 & 48.2373 & 298.6752 & 285.4433 & 276 & 122.5994 & 1.88509 \\
\hline 2663 & 48.2543 & 325.8854 & 288.9649 & 277 & 122.5994 & 1.88446 \\
\hline 2664 & 48.2713 & 347.52 & 291.7001 & 277 & 122.5994 & 1.88384 \\
\hline 2665 & 48.2883 & 335.2426 & 295.5445 & 278 & 122.5994 & 1.88322 \\
\hline 2666 & 48.3053 & 321.6769 & 298.3854 & 278 & 122.5994 & 1.88259 \\
\hline 2667 & 48.3223 & 322.6678 & 300.478 & 278 & 122.5994 & 1.88197 \\
\hline 2668 & 48.3393 & 338.8919 & 301.8799 & 279 & 122.5994 & 1.88135 \\
\hline 2669 & 48.3563 & 350.5881 & 300.7694 & 279 & 122.5994 & 1.88073 \\
\hline 2670 & 48.3733 & 334.9362 & 299.137 & 279 & 122.5994 & 1.8801 \\
\hline 2671 & 48.3903 & 339.8324 & 298.7388 & 280 & 122.5994 & 1.87948 \\
\hline 2672 & 48.4073 & 325.8819 & 297.7007 & 280 & 122.5994 & 1.87886 \\
\hline 2673 & 48.4243 & 338.4358 & 297.1156 & 280 & 122.5994 & 1.87824 \\
\hline 2674 & 48.4413 & 336.5411 & 297.5737 & 281 & 122.5994 & 1.87762 \\
\hline 2675 & 48.4583 & 336.0286 & 296.3541 & 281 & 122.5994 & 1.87701 \\
\hline 2676 & 48.4753 & 321.7082 & 294.5125 & 281 & 122.5994 & 1.87639 \\
\hline 2677 & 48.4923 & 309.3971 & 293.4552 & 282 & 122.5994 & 1.87577 \\
\hline 2678 & 48.5093 & 310.5368 & 291.6607 & 282 & 122.5994 & 1.87515 \\
\hline 2679 & 48.5263 & 291.3198 & 290.2307 & 282 & 122.5994 & 1.87453 \\
\hline 2680 & 48.5433 & 299.8816 & 290.1775 & 283 & 122.5994 & 1.87392 \\
\hline 2681 & 48.5603 & 300.5629 & 289.2391 & 283 & 122.5994 & 1.8733 \\
\hline 2682 & 48.5773 & 295.8116 & 289.3617 & 284 & 122.5994 & 1.87269 \\
\hline 2683 & 48.5943 & 310.7212 & 288.5522 & 284 & 122.5994 & 1.87207 \\
\hline 2684 & 48.6113 & 272.5077 & 288.1023 & 284 & 122.5994 & 1.87145 \\
\hline 2685 & 48.6283 & 258.3816 & 289.0549 & 285 & 122.5994 & 1.87084 \\
\hline 2686 & 48.6453 & 290.1559 & 289.3093 & 285 & 122.5994 & 1.87023 \\
\hline 2687 & 48.6623 & 310.3585 & 289.6246 & 285 & 122.5994 & 1.86961 \\
\hline 2688 & 48.6793 & 290.7917 & 290.6897 & 286 & 122.5994 & 1.869 \\
\hline 2689 & 48.6963 & 277.1835 & 290.4076 & 286 & 122.5994 & 1.86839 \\
\hline 2690 & 48.7133 & 272.7588 & 290.0036 & 286 & 122.5994 & 1.86777 \\
\hline 2691 & 48.7303 & 281.0031 & 289.7361 & 286 & 122.5994 & 1.86716 \\
\hline 2692 & 48.7473 & 294.4559 & 289.7455 & 286 & 122.5994 & 1.86655 \\
\hline 2693 & 48.7643 & 280.2537 & 290.1463 & 286 & 122.5994 & 1.86594 \\
\hline
\end{tabular}




\begin{tabular}{|c|c|c|c|c|c|c|}
\hline 2694 & 48.7813 & 236.6038 & 289.8653 & 285 & 122.5994 & 1.86533 \\
\hline 2695 & 48.7983 & 274.1373 & 290.9746 & 285 & 122.5994 & 1.86472 \\
\hline 2696 & 48.8153 & 278.8181 & 292.4412 & 285 & 122.5994 & 1.86411 \\
\hline 2697 & 48.8323 & 243.6473 & 294.2758 & 285 & 122.5994 & 1.8635 \\
\hline 2698 & 48.8493 & 276.5368 & 296.2343 & 285 & 122.5994 & 1.86289 \\
\hline 2699 & 48.8663 & 308.9167 & 297.6393 & 285 & 122.5994 & 1.86228 \\
\hline 2700 & 48.8833 & 293.1313 & 298.0266 & 285 & 122.5994 & 1.86168 \\
\hline 2701 & 48.9003 & 301.5062 & 297.139 & 284 & 122.5994 & 1.86107 \\
\hline 2702 & 48.9173 & 285.5323 & 298.1524 & 284 & 122.5994 & 1.86046 \\
\hline 2703 & 48.9343 & 258.7457 & 301.3199 & 284 & 122.5994 & 1.85985 \\
\hline 2704 & 48.9513 & 261.8067 & 307.9533 & 284 & 122.5994 & 1.85925 \\
\hline 2705 & 48.9683 & 289.9488 & 318.8315 & 284 & 122.5994 & 1.85864 \\
\hline 2706 & 48.9853 & 282.3445 & 333.2737 & 284 & 122.5994 & 1.85804 \\
\hline 2707 & 49.0023 & 298.9163 & 346.8676 & 284 & 122.5994 & 1.85743 \\
\hline 2708 & 49.0193 & 300.6232 & 351.7581 & 284 & 122.5994 & 1.85683 \\
\hline 2709 & 49.0363 & 275.779 & 344.3862 & 284 & 122.5994 & 1.85622 \\
\hline 2710 & 49.0533 & 282.693 & 330.4351 & 283 & 122.5994 & 1.85562 \\
\hline 2711 & 49.0703 & 277.662 & 319.5171 & 283 & 122.5994 & 1.85502 \\
\hline 2712 & 49.0873 & 280.0046 & 314.5067 & 283 & 122.5994 & 1.85441 \\
\hline 2713 & 49.1043 & 283.9555 & 316.05 & 283 & 122.5994 & 1.85381 \\
\hline 2714 & 49.1213 & 294.1496 & 321.6368 & 282 & 122.5994 & 1.85321 \\
\hline 2715 & 49.1383 & 271.3714 & 329.1385 & 282 & 122.5994 & 1.85261 \\
\hline 2716 & 49.1553 & 261.8628 & 330.8368 & 281 & 122.5994 & 1.85201 \\
\hline 2717 & 49.1723 & 284.7871 & 326.5817 & 281 & 122.5994 & 1.85141 \\
\hline 2718 & 49.1893 & 283.1793 & 317.1704 & 280 & 122.5994 & 1.85081 \\
\hline 2719 & 49.2063 & 264.404 & 308.2539 & 280 & 122.5994 & 1.85021 \\
\hline 2720 & 49.2233 & 272.629 & 300.293 & 279 & 122.5994 & 1.84961 \\
\hline 2721 & 49.2403 & 281.9258 & 296.1505 & 279 & 122.5994 & 1.84901 \\
\hline 2722 & 49.2573 & 284.7289 & 293.33 & 278 & 122.5994 & 1.84841 \\
\hline 2723 & 49.2743 & 304.2956 & 292.9964 & 278 & 122.5994 & 1.84781 \\
\hline 2724 & 49.2913 & 283.3202 & 292.0667 & 277 & 122.5994 & 1.84722 \\
\hline 2725 & 49.3083 & 262.475 & 291.5429 & 277 & 122.5994 & 1.84662 \\
\hline 2726 & 49.3253 & 277.0549 & 289.1949 & 276 & 122.5994 & 1.84602 \\
\hline 2727 & 49.3423 & 276.8548 & 286.6276 & 275 & 122.5994 & 1.84543 \\
\hline 2728 & 49.3593 & 265.6653 & 285.4927 & 275 & 122.5994 & 1.84483 \\
\hline 2729 & 49.3763 & 269.246 & 284.0569 & 274 & 122.5994 & 1.84424 \\
\hline 2730 & 49.3933 & 272.2863 & 284.1792 & 274 & 122.5994 & 1.84364 \\
\hline 2731 & 49.4103 & 279.6106 & 283.3652 & 273 & 122.5994 & 1.84305 \\
\hline 2732 & 49.4273 & 279.7281 & 283.9061 & 273 & 122.5994 & 1.84245 \\
\hline 2733 & 49.4443 & 258.456 & 283.6091 & 272 & 122.5994 & 1.84186 \\
\hline 2734 & 49.4613 & 268.4137 & 284.6859 & 272 & 122.5994 & 1.84126 \\
\hline 2735 & 49.4783 & 275.0189 & 284.9369 & 271 & 122.5994 & 1.84067 \\
\hline 2736 & 49.4953 & 256.2649 & 285.7613 & 271 & 122.5994 & 1.84008 \\
\hline 2737 & 49.5123 & 281.3346 & 284.8705 & 270 & 122.5994 & 1.83949 \\
\hline 2738 & 49.5293 & 264.9603 & 285.6228 & 271 & 122.5994 & 1.8389 \\
\hline 2739 & 49.5463 & 283.5335 & 285.534 & 271 & 122.5994 & 1.8383 \\
\hline 2740 & 49.5633 & 261.0138 & 286.7374 & 272 & 122.5994 & 1.83771 \\
\hline 2741 & 49.5803 & 307.4369 & 288.171 & 273 & 122.5994 & 1.83712 \\
\hline 2742 & 49.5973 & 257.3216 & 289.6944 & 274 & 122.5994 & 1.83653 \\
\hline
\end{tabular}




\begin{tabular}{|c|c|c|c|c|c|c|}
\hline 2743 & 49.6143 & 262.4 & 289.9679 & 274 & 122.5994 & 1.83594 \\
\hline 2744 & 49.6313 & 265.5404 & 290.9883 & 275 & 122.5994 & 1.83536 \\
\hline 2745 & 49.6483 & 259.8486 & 291.8531 & 276 & 122.5994 & 1.83477 \\
\hline 2746 & 49.6653 & 276.8587 & 291.8878 & 276 & 122.5994 & 1.83418 \\
\hline 2747 & 49.6823 & 294.1152 & 293.7333 & 277 & 122.5994 & 1.83359 \\
\hline 2748 & 49.6993 & 275.5967 & 296.5391 & 278 & 122.5994 & 1.833 \\
\hline 2749 & 49.7163 & 280.7645 & 300.3881 & 279 & 122.5994 & 1.83242 \\
\hline 2750 & 49.7333 & 314.7932 & 313.929 & 279 & 122.5994 & 1.83183 \\
\hline 2751 & 49.7503 & 304.5188 & 320.3344 & 280 & 122.5994 & 1.83124 \\
\hline 2752 & 49.7673 & 315.3078 & 327.6169 & 281 & 122.5994 & 1.83066 \\
\hline 2753 & 49.7843 & 318.7834 & 338.2032 & 281 & 122.5994 & 1.83007 \\
\hline 2754 & 49.8013 & 303.3729 & 346.0079 & 282 & 122.5994 & 1.82949 \\
\hline 2755 & 49.8183 & 332.1151 & 351.4226 & 283 & 122.5994 & 1.8289 \\
\hline 2756 & 49.8353 & 366.8635 & 352.6236 & 284 & 122.5994 & 1.82832 \\
\hline 2757 & 49.8523 & 347.8191 & 349.9503 & 284 & 122.5994 & 1.82774 \\
\hline 2758 & 49.8693 & 355.0403 & 348.152 & 285 & 122.5994 & 1.82715 \\
\hline 2759 & 49.8863 & 357.8871 & 347.8249 & 286 & 122.5994 & 1.82657 \\
\hline 2760 & 49.9033 & 386.7132 & 347.9408 & 286 & 122.5994 & 1.82599 \\
\hline 2761 & 49.9203 & 399.5965 & 350.0306 & 287 & 122.5994 & 1.82541 \\
\hline 2762 & 49.9373 & 427.7829 & 351.5305 & 287 & 122.5994 & 1.82482 \\
\hline 2763 & 49.9543 & 429.0548 & 352.5216 & 287 & 122.5994 & 1.82424 \\
\hline 2764 & 49.9713 & 422.4723 & 352.5233 & 287 & 122.5994 & 1.82366 \\
\hline 2765 & 49.9883 & 412.6562 & 352.5341 & 287 & 122.5994 & 1.82308 \\
\hline 2766 & 50.0053 & 426.3441 & 353.9567 & 287 & 122.5994 & 1.8225 \\
\hline 2767 & 50.0223 & 471.8127 & 358.5422 & 288 & 122.5994 & 1.82192 \\
\hline 2768 & 50.0393 & 522.0975 & 364.399 & 288 & 122.5994 & 1.82134 \\
\hline 2769 & 50.0563 & 568.7165 & 372.441 & 288 & 122.5994 & 1.82076 \\
\hline 2770 & 50.0733 & 611.391 & 382.6602 & 288 & 122.5994 & 1.82019 \\
\hline 2771 & 50.0903 & 763.5342 & 396.0677 & 288 & 122.5994 & 1.81961 \\
\hline 2772 & 50.1073 & 951.352 & 412.4893 & 288 & 122.5994 & 1.81903 \\
\hline 2773 & 50.1243 & 1255.553 & 435.9207 & 288 & 122.5994 & 1.81845 \\
\hline 2774 & 50.1413 & 1628.093 & 472.7988 & 288 & 122.5994 & 1.81788 \\
\hline 2775 & 50.1583 & 2096.34 & 535.6651 & 288 & 122.5994 & 1.8173 \\
\hline 2776 & 50.1753 & 2662.183 & 647.629 & 288 & 122.5994 & 1.81672 \\
\hline 2777 & 50.1923 & 2723.419 & 842.4924 & 288 & 122.5994 & 1.81615 \\
\hline 2778 & 50.2093 & 2188.868 & 1153.231 & 288 & 122.5994 & 1.81557 \\
\hline 2779 & 50.2263 & 1535.85 & 1587.501 & 288 & 122.5994 & 1.815 \\
\hline 2780 & 50.2433 & 1140.774 & 2091.591 & 289 & 122.5994 & 1.81442 \\
\hline 2781 & 50.2603 & 1043.946 & 2502.53 & 289 & 122.5994 & 1.81385 \\
\hline 2782 & 50.2773 & 1142.39 & 2587.316 & 289 & 122.5994 & 1.81328 \\
\hline 2783 & 50.2943 & 1371.624 & 2302.082 & 289 & 122.5994 & 1.8127 \\
\hline 2784 & 50.3113 & 1514.274 & 1872.289 & 289 & 122.5994 & 1.81213 \\
\hline 2785 & 50.3283 & 1500.615 & 1515.246 & 289 & 122.5994 & 1.81156 \\
\hline 2786 & 50.3453 & 1182.53 & 1330.482 & 289 & 122.5994 & 1.81099 \\
\hline 2787 & 50.3623 & 727.3858 & 1326.409 & 289 & 122.5994 & 1.81042 \\
\hline 2788 & 50.3793 & 539.3135 & 1441.57 & 289 & 122.5994 & 1.80985 \\
\hline 2789 & 50.3963 & 446.3923 & 1551.516 & 289 & 122.5994 & 1.80927 \\
\hline 2790 & 50.4133 & 380.4781 & 1514.336 & 289 & 122.5994 & 1.8087 \\
\hline 2791 & 50.4303 & 397.982 & 1311.689 & 289 & 122.5994 & 1.80813 \\
\hline
\end{tabular}




\begin{tabular}{|c|c|c|c|c|c|c|}
\hline 2792 & 50.4473 & 336.8919 & 1049.132 & 289 & 122.5994 & 1.80756 \\
\hline 2793 & 50.4643 & 368.1046 & 814.7497 & 289 & 122.5994 & 1.807 \\
\hline 2794 & 50.4813 & 311.604 & 643.2706 & 290 & 122.5994 & 1.80643 \\
\hline 2795 & 50.4983 & 299.582 & 532.3942 & 290 & 122.5994 & 1.80586 \\
\hline 2796 & 50.5153 & 325.1159 & 467.6173 & 290 & 122.5994 & 1.80529 \\
\hline 2797 & 50.5323 & 312.0742 & 431.9334 & 290 & 122.5994 & 1.80472 \\
\hline 2798 & 50.5493 & 325.2796 & 413.6783 & 290 & 122.5994 & 1.80416 \\
\hline 2799 & 50.5663 & 329.7425 & 407.6986 & 290 & 122.5994 & 1.80359 \\
\hline 2800 & 50.5833 & 327.3061 & 413.5076 & 290 & 122.5994 & 1.80302 \\
\hline 2801 & 50.6003 & 343.1066 & 432.1898 & 290 & 122.5994 & 1.80246 \\
\hline 2802 & 50.6173 & 367.624 & 462.3253 & 290 & 122.5994 & 1.80189 \\
\hline 2803 & 50.6343 & 394.8263 & 496.4348 & 290 & 122.5994 & 1.80133 \\
\hline 2804 & 50.6513 & 393.3239 & 517.5831 & 290 & 122.5994 & 1.80076 \\
\hline 2805 & 50.6683 & 410.8936 & 513.56 & 290 & 122.5994 & 1.8002 \\
\hline 2806 & 50.6853 & 427.8971 & 495.7403 & 290 & 122.5994 & 1.79963 \\
\hline 2807 & 50.7023 & 399.5783 & 479.8536 & 290 & 122.5994 & 1.79907 \\
\hline 2808 & 50.7193 & 387.3317 & 472.3905 & 291 & 122.5994 & 1.79851 \\
\hline 2809 & 50.7363 & 380.3007 & 469.8759 & 291 & 122.5994 & 1.79794 \\
\hline 2810 & 50.7533 & 395.9861 & 469.7833 & 290 & 122.5994 & 1.79738 \\
\hline 2811 & 50.7703 & 399.5783 & 471.5993 & 289 & 122.5994 & 1.79682 \\
\hline 2812 & 50.7873 & 371.0165 & 471.5999 & 289 & 122.5994 & 1.79626 \\
\hline 2813 & 50.8043 & 372.6314 & 463.726 & 288 & 122.5994 & 1.7957 \\
\hline 2814 & 50.8213 & 368.072 & 456.3166 & 288 & 122.5994 & 1.79514 \\
\hline 2815 & 50.8383 & 351.3557 & 451.1516 & 287 & 122.5994 & 1.79458 \\
\hline 2816 & 50.8553 & 356.1656 & 445.9908 & 287 & 122.5994 & 1.79402 \\
\hline 2817 & 50.8723 & 337.0232 & 431.9417 & 286 & 122.5994 & 1.79346 \\
\hline 2818 & 50.8893 & 326.9994 & 403.2979 & 286 & 122.5994 & 1.7929 \\
\hline 2819 & 50.9063 & 340.6008 & 380.1868 & 285 & 122.5994 & 1.79234 \\
\hline 2820 & 50.9233 & 304.232 & 361.4899 & 284 & 122.5994 & 1.79178 \\
\hline 2821 & 50.9403 & 303.2672 & 349.7491 & 284 & 122.5994 & 1.79122 \\
\hline 2822 & 50.9573 & 273.3051 & 343.0871 & 283 & 122.5994 & 1.79066 \\
\hline 2823 & 50.9743 & 284.6138 & 341.5666 & 283 & 122.5994 & 1.79011 \\
\hline 2824 & 50.9913 & 273.5538 & 339.6896 & 282 & 122.5994 & 1.78955 \\
\hline 2825 & 51.0083 & 260.7274 & 336.2687 & 282 & 122.5994 & 1.78899 \\
\hline 2826 & 51.0253 & 282.5866 & 325.6679 & 281 & 122.5994 & 1.78844 \\
\hline 2827 & 51.0423 & 283.5307 & 318.801 & 280 & 122.5994 & 1.78788 \\
\hline 2828 & 51.0593 & 245.0134 & 314.5474 & 280 & 122.5994 & 1.78733 \\
\hline 2829 & 51.0763 & 245.0495 & 311.2651 & 279 & 122.5994 & 1.78677 \\
\hline 2830 & 51.0933 & 283.4273 & 310.3991 & 279 & 122.5994 & 1.78622 \\
\hline 2831 & 51.1103 & 265.3705 & 308.6223 & 278 & 122.5994 & 1.78566 \\
\hline 2832 & 51.1273 & 302.393 & 307.3839 & 278 & 122.5994 & 1.78511 \\
\hline 2833 & 51.1443 & 274.5286 & 305.3593 & 277 & 122.5994 & 1.78456 \\
\hline 2834 & 51.1613 & 253.6469 & 303.9996 & 276 & 122.5994 & 1.784 \\
\hline 2835 & 51.1783 & 281.5396 & 302.7747 & 275 & 122.5994 & 1.78345 \\
\hline 2836 & 51.1953 & 320.397 & 299.355 & 273 & 122.5994 & 1.7829 \\
\hline 2837 & 51.2123 & 299.4707 & 297.2686 & 272 & 122.5994 & 1.78235 \\
\hline 2838 & 51.2293 & 305.7871 & 295.6868 & 271 & 122.5994 & 1.78179 \\
\hline 2839 & 51.2463 & 293.971 & 294.7112 & 270 & 122.5994 & 1.78124 \\
\hline 2840 & 51.2633 & 266.7828 & 294.6364 & 269 & 122.5994 & 1.78069 \\
\hline
\end{tabular}




\begin{tabular}{|c|c|c|c|c|c|c|}
\hline 2841 & 51.2803 & 282.5144 & 296.2212 & 268 & 122.5994 & 1.78014 \\
\hline 2842 & 51.2973 & 310.6635 & 299.0152 & 266 & 122.5994 & 1.77959 \\
\hline 2843 & 51.3143 & 295.4974 & 305.0355 & 265 & 122.5994 & 1.77904 \\
\hline 2844 & 51.3313 & 307.4542 & 312.5792 & 264 & 122.5994 & 1.77849 \\
\hline 2845 & 51.3483 & 280.8072 & 320.505 & 263 & 122.5994 & 1.77794 \\
\hline 2846 & 51.3653 & 291.2398 & 325.8037 & 262 & 122.5994 & 1.7774 \\
\hline 2847 & 51.3823 & 297.6421 & 326.7196 & 261 & 122.5994 & 1.77685 \\
\hline 2848 & 51.3993 & 292.382 & 322.2926 & 259 & 122.5994 & 1.7763 \\
\hline 2849 & 51.4163 & 288.6848 & 317.3182 & 258 & 122.5994 & 1.77575 \\
\hline 2850 & 51.4333 & 287.0668 & 311.9612 & 257 & 122.5994 & 1.77521 \\
\hline 2851 & 51.4503 & 294.3025 & 307.2798 & 256 & 122.5994 & 1.77466 \\
\hline 2852 & 51.4673 & 273.488 & 303.9891 & 255 & 122.5994 & 1.77411 \\
\hline 2853 & 51.4843 & 280.9924 & 301.9368 & 254 & 122.5994 & 1.77357 \\
\hline 2854 & 51.5013 & 278.9272 & 298.4743 & 252 & 122.5994 & 1.77302 \\
\hline 2855 & 51.5183 & 265.6635 & 294.9028 & 251 & 122.5994 & 1.77248 \\
\hline 2856 & 51.5353 & 243.8456 & 290.0409 & 250 & 122.5994 & 1.77193 \\
\hline 2857 & 51.5523 & 244.4569 & 284.9647 & 249 & 122.5994 & 1.77139 \\
\hline 2858 & 51.5693 & 251.1699 & 281.1247 & 249 & 122.5994 & 1.77084 \\
\hline 2859 & 51.5863 & 254.3601 & 276.3706 & 248 & 122.5994 & 1.7703 \\
\hline 2860 & 51.6033 & 252.7003 & 272.5568 & 248 & 122.5994 & 1.76976 \\
\hline 2861 & 51.6203 & 247.983 & 267.4015 & 247 & 122.5994 & 1.76921 \\
\hline 2862 & 51.6373 & 262.6883 & 263.4323 & 247 & 122.5994 & 1.76867 \\
\hline 2863 & 51.6543 & 242.8333 & 259.1219 & 246 & 122.5994 & 1.76813 \\
\hline 2864 & 51.6713 & 241.2356 & 256.7203 & 246 & 122.5994 & 1.76759 \\
\hline 2865 & 51.6883 & 244.2472 & 254.3437 & 245 & 122.5994 & 1.76705 \\
\hline 2866 & 51.7053 & 235.214 & 253.9167 & 245 & 122.5994 & 1.7665 \\
\hline 2867 & 51.7223 & 246.5274 & 253.2446 & 244 & 122.5994 & 1.76596 \\
\hline 2868 & 51.7393 & 266.2036 & 253.462 & 244 & 122.5994 & 1.76542 \\
\hline 2869 & 51.7563 & 256.2011 & 253.3855 & 244 & 122.5994 & 1.76488 \\
\hline 2870 & 51.7733 & 256.6623 & 252.0001 & 243 & 122.5994 & 1.76434 \\
\hline 2871 & 51.7903 & 231.2208 & 251.7336 & 243 & 122.5994 & 1.7638 \\
\hline 2872 & 51.8073 & 245.8017 & 250.801 & 242 & 122.5994 & 1.76327 \\
\hline 2873 & 51.8243 & 245.3329 & 250.9834 & 242 & 122.5994 & 1.76273 \\
\hline 2874 & 51.8413 & 240.7982 & 249.8202 & 241 & 122.5994 & 1.76219 \\
\hline 2875 & 51.8583 & 224.9993 & 249.2673 & 241 & 122.5994 & 1.76165 \\
\hline 2876 & 51.8753 & 227.1444 & 247.5308 & 240 & 122.5994 & 1.76111 \\
\hline 2877 & 51.8923 & 249.8297 & 246.9318 & 240 & 122.5994 & 1.76058 \\
\hline 2878 & 51.9093 & 219.7091 & 245.4555 & 239 & 122.5994 & 1.76004 \\
\hline 2879 & 51.9263 & 218.7944 & 245.1377 & 239 & 122.5994 & 1.7595 \\
\hline 2880 & 51.9433 & 269.3755 & 244.0546 & 238 & 122.5994 & 1.75897 \\
\hline 2881 & 51.9603 & 220.7778 & 243.9973 & 238 & 122.5994 & 1.75843 \\
\hline 2882 & 51.9773 & 249.3173 & 244.9576 & 239 & 122.5994 & 1.7579 \\
\hline 2883 & 51.9943 & 219.2273 & 244.6787 & 239 & 122.5994 & 1.75736 \\
\hline 2884 & 52.0113 & 240.7777 & 245.264 & 240 & 122.5994 & 1.75683 \\
\hline 2885 & 52.0283 & 256.3161 & 245.8669 & 241 & 122.5994 & 1.75629 \\
\hline 2886 & 52.0453 & 260.8209 & 246.4448 & 242 & 122.5994 & 1.75576 \\
\hline 2887 & 52.0623 & 241.7384 & 245.8392 & 242 & 122.5994 & 1.75523 \\
\hline 2888 & 52.0793 & 252.3881 & 246.6784 & 243 & 122.5994 & 1.75469 \\
\hline 2889 & 52.0963 & 225.8182 & 247.7969 & 244 & 122.5994 & 1.75416 \\
\hline
\end{tabular}




\begin{tabular}{|c|c|c|c|c|c|c|}
\hline 2890 & 52.1133 & 234.0023 & 248.4728 & 244 & 122.5994 & 1.75363 \\
\hline 2891 & 52.1303 & 248.5297 & 250.8103 & 245 & 122.5994 & 1.7531 \\
\hline 2892 & 52.1473 & 234.2697 & 253.8492 & 246 & 122.5994 & 1.75257 \\
\hline 2893 & 52.1643 & 214.6324 & 257.3355 & 247 & 122.5994 & 1.75204 \\
\hline 2894 & 52.1813 & 260.3355 & 259.425 & 247 & 122.5994 & 1.7515 \\
\hline 2895 & 52.1983 & 240.8 & 260.8271 & 248 & 122.5994 & 1.75097 \\
\hline 2896 & 52.2153 & 253.7525 & 260.8783 & 249 & 122.5994 & 1.75044 \\
\hline 2897 & 52.2323 & 242.1681 & 259.6848 & 249 & 122.5994 & 1.74991 \\
\hline 2898 & 52.2493 & 237.3893 & 260.1826 & 250 & 122.5994 & 1.74938 \\
\hline 2899 & 52.2663 & 232.1085 & 261.7207 & 251 & 122.5994 & 1.74886 \\
\hline 2900 & 52.2833 & 251.284 & 263.1381 & 251 & 122.5994 & 1.74833 \\
\hline 2901 & 52.3003 & 250.9157 & 266.0905 & 252 & 122.5994 & 1.7478 \\
\hline 2902 & 52.3173 & 262.4148 & 268.709 & 253 & 122.5994 & 1.74727 \\
\hline 2903 & 52.3343 & 256.8392 & 270.0681 & 254 & 122.5994 & 1.74674 \\
\hline 2904 & 52.3513 & 260.0554 & 269.2784 & 254 & 122.5994 & 1.74622 \\
\hline 2905 & 52.3683 & 259.8349 & 269.4441 & 255 & 122.5994 & 1.74569 \\
\hline 2906 & 52.3853 & 248.0564 & 269.2857 & 255 & 122.5994 & 1.74516 \\
\hline 2907 & 52.4023 & 247.9585 & 269.5257 & 255 & 122.5994 & 1.74464 \\
\hline 2908 & 52.4193 & 244.8081 & 269.3403 & 255 & 122.5994 & 1.74411 \\
\hline 2909 & 52.4363 & 257.9101 & 268.5721 & 255 & 122.5994 & 1.74359 \\
\hline 2910 & 52.4533 & 265.143 & 267.8523 & 255 & 122.5994 & 1.74306 \\
\hline 2911 & 52.4703 & 252.6479 & 268.5197 & 256 & 122.5994 & 1.74254 \\
\hline 2912 & 52.4873 & 256.6351 & 268.7962 & 256 & 122.5994 & 1.74201 \\
\hline 2913 & 52.5043 & 254.0255 & 269.679 & 256 & 122.5994 & 1.74149 \\
\hline 2914 & 52.5213 & 234.9761 & 270.7808 & 256 & 122.5994 & 1.74096 \\
\hline 2915 & 52.5383 & 238.7621 & 271.7009 & 256 & 122.5994 & 1.74044 \\
\hline 2916 & 52.5553 & 271.3265 & 272.001 & 256 & 122.5994 & 1.73992 \\
\hline 2917 & 52.5723 & 267.5949 & 271.365 & 256 & 122.5994 & 1.73939 \\
\hline 2918 & 52.5893 & 269.8996 & 269.7863 & 256 & 122.5994 & 1.73887 \\
\hline 2919 & 52.6063 & 252.0179 & 267.7243 & 256 & 122.5994 & 1.73835 \\
\hline 2920 & 52.6233 & 267.2865 & 266.0024 & 256 & 122.5994 & 1.73783 \\
\hline 2921 & 52.6403 & 261.4338 & 264.9745 & 256 & 122.5994 & 1.73731 \\
\hline 2922 & 52.6573 & 264.7468 & 264.5182 & 256 & 122.5994 & 1.73679 \\
\hline 2923 & 52.6743 & 235.5005 & 264.3504 & 256 & 122.5994 & 1.73627 \\
\hline 2924 & 52.6913 & 279.7573 & 265.2766 & 257 & 122.5994 & 1.73575 \\
\hline 2925 & 52.7083 & 241.3185 & 265.0936 & 257 & 122.5994 & 1.73523 \\
\hline 2926 & 52.7253 & 254.7531 & 264.6317 & 257 & 122.5994 & 1.73471 \\
\hline 2927 & 52.7423 & 235.0734 & 263.8878 & 257 & 122.5994 & 1.73419 \\
\hline 2928 & 52.7593 & 255.4976 & 263.2164 & 257 & 122.5994 & 1.73367 \\
\hline 2929 & 52.7763 & 262.4725 & 262.9192 & 257 & 122.5994 & 1.73315 \\
\hline 2930 & 52.7933 & 256.3802 & 263.0728 & 257 & 122.5994 & 1.73263 \\
\hline 2931 & 52.8103 & 272.0833 & 263.9586 & 257 & 122.5994 & 1.73212 \\
\hline 2932 & 52.8273 & 269.1452 & 265.4554 & 257 & 122.5994 & 1.7316 \\
\hline 2933 & 52.8443 & 272.5702 & 267.2964 & 257 & 122.5994 & 1.73108 \\
\hline 2934 & 52.8613 & 275.5336 & 268.7776 & 257 & 122.5994 & 1.73056 \\
\hline 2935 & 52.8783 & 271.4475 & 268.9963 & 257 & 122.5994 & 1.73005 \\
\hline 2936 & 52.8953 & 244.2804 & 268.094 & 257 & 122.5994 & 1.72953 \\
\hline 2937 & 52.9123 & 251.2592 & 267.0846 & 257 & 122.5994 & 1.72902 \\
\hline 2938 & 52.9293 & 259.8691 & 266.5891 & 257 & 122.5994 & 1.7285 \\
\hline
\end{tabular}




\begin{tabular}{|c|c|c|c|c|c|c|}
\hline 2939 & 52.9463 & 261.0317 & 266.6812 & 257 & 122.5994 & 1.72799 \\
\hline 2940 & 52.9633 & 257.5567 & 267.1955 & 257 & 122.5994 & 1.72747 \\
\hline 2941 & 52.9803 & 259.9318 & 268.0773 & 257 & 122.5994 & 1.72696 \\
\hline 2942 & 52.9973 & 249.3333 & 269.2374 & 257 & 122.5994 & 1.72644 \\
\hline 2943 & 53.0143 & 237.4556 & 270.5184 & 257 & 122.5994 & 1.72593 \\
\hline 2944 & 53.0313 & 246.4412 & 271.7284 & 257 & 122.5994 & 1.72542 \\
\hline 2945 & 53.0483 & 274.3867 & 273.064 & 257 & 122.5994 & 1.7249 \\
\hline 2946 & 53.0653 & 272.9432 & 274.3304 & 257 & 122.5994 & 1.72439 \\
\hline 2947 & 53.0823 & 275.43 & 275.0332 & 257 & 122.5994 & 1.72388 \\
\hline 2948 & 53.0993 & 257.874 & 275.3794 & 257 & 122.5994 & 1.72337 \\
\hline 2949 & 53.1163 & 255.9754 & 275.7698 & 257 & 122.5994 & 1.72286 \\
\hline 2950 & 53.1333 & 266.3698 & 276.3334 & 257 & 122.5994 & 1.72234 \\
\hline 2951 & 53.1503 & 295.133 & 277.1689 & 257 & 122.5994 & 1.72183 \\
\hline 2952 & 53.1673 & 284.9957 & 277.993 & 257 & 122.5994 & 1.72132 \\
\hline 2953 & 53.1843 & 266.069 & 277.967 & 257 & 122.5994 & 1.72081 \\
\hline 2954 & 53.2013 & 268.5424 & 276.7575 & 257 & 122.5994 & 1.7203 \\
\hline 2955 & 53.2183 & 284.9577 & 274.9291 & 257 & 122.5994 & 1.71979 \\
\hline 2956 & 53.2353 & 279.3245 & 273.2188 & 257 & 122.5994 & 1.71928 \\
\hline 2957 & 53.2523 & 280.522 & 272.0724 & 257 & 122.5994 & 1.71878 \\
\hline 2958 & 53.2693 & 292.382 & 271.2966 & 257 & 122.5994 & 1.71827 \\
\hline 2959 & 53.2863 & 277.3181 & 270.5461 & 257 & 122.5994 & 1.71776 \\
\hline 2960 & 53.3033 & 277.7874 & 269.7248 & 257 & 122.5994 & 1.71725 \\
\hline 2961 & 53.3203 & 264.1577 & 268.6528 & 257 & 122.5994 & 1.71674 \\
\hline 2962 & 53.3373 & 281.8753 & 267.1123 & 257 & 122.5994 & 1.71624 \\
\hline 2963 & 53.3543 & 289.8887 & 265.3887 & 257 & 122.5994 & 1.71573 \\
\hline 2964 & 53.3713 & 291.6056 & 263.9276 & 257 & 122.5994 & 1.71522 \\
\hline 2965 & 53.3883 & 279.3364 & 262.873 & 257 & 122.5994 & 1.71472 \\
\hline 2966 & 53.4053 & 280.9131 & 262.216 & 257 & 122.5994 & 1.71421 \\
\hline 2967 & 53.4223 & 284.9672 & 261.8304 & 257 & 122.5994 & 1.71371 \\
\hline 2968 & 53.4393 & 274.3775 & 261.6238 & 257 & 122.5994 & 1.7132 \\
\hline 2969 & 53.4563 & 275.1341 & 261.6163 & 257 & 122.5994 & 1.7127 \\
\hline 2970 & 53.4733 & 275.5336 & 261.6451 & 257 & 122.5994 & 1.71219 \\
\hline 2971 & 53.4903 & 229.9194 & 261.5098 & 257 & 122.5994 & 1.71169 \\
\hline 2972 & 53.5073 & 277.9167 & 261.0981 & 257 & 122.5994 & 1.71118 \\
\hline 2973 & 53.5243 & 263.537 & 260.5351 & 257 & 122.5994 & 1.71068 \\
\hline 2974 & 53.5413 & 275.1139 & 259.9761 & 257 & 122.5994 & 1.71018 \\
\hline 2975 & 53.5583 & 256.1821 & 259.6279 & 257 & 122.5994 & 1.70968 \\
\hline 2976 & 53.5753 & 256.2613 & 259.5328 & 257 & 122.5994 & 1.70917 \\
\hline 2977 & 53.5923 & 280.7107 & 259.7231 & 257 & 122.5994 & 1.70867 \\
\hline 2978 & 53.6093 & 234.4 & 259.1573 & 256 & 122.5994 & 1.70817 \\
\hline 2979 & 53.6263 & 240.6973 & 259.8091 & 256 & 122.5994 & 1.70767 \\
\hline 2980 & 53.6433 & 245.6175 & 259.6382 & 255 & 122.5994 & 1.70717 \\
\hline 2981 & 53.6603 & 237.5033 & 259.6572 & 254 & 122.5994 & 1.70667 \\
\hline 2982 & 53.6773 & 241.5976 & 260.6969 & 254 & 122.5994 & 1.70617 \\
\hline 2983 & 53.6943 & 248.6017 & 260.3098 & 253 & 122.5994 & 1.70567 \\
\hline 2984 & 53.7113 & 240.0294 & 260.1111 & 253 & 122.5994 & 1.70517 \\
\hline 2985 & 53.7283 & 231.9775 & 258.2516 & 252 & 122.5994 & 1.70467 \\
\hline 2986 & 53.7453 & 257.0222 & 256.398 & 251 & 122.5994 & 1.70417 \\
\hline 2987 & 53.7623 & 248.8931 & 255.9982 & 251 & 122.5994 & 1.70367 \\
\hline
\end{tabular}




\begin{tabular}{|c|c|c|c|c|c|c|}
\hline 2988 & 53.7793 & 243.9558 & 255.2657 & 250 & 122.5994 & 1.70317 \\
\hline 2989 & 53.7963 & 237.1916 & 256.1183 & 250 & 122.5994 & 1.70267 \\
\hline 2990 & 53.8133 & 238.1018 & 255.9947 & 249 & 122.5994 & 1.70217 \\
\hline 2991 & 53.8303 & 246.5515 & 255.3552 & 248 & 122.5994 & 1.70168 \\
\hline 2992 & 53.8473 & 261.5464 & 254.9887 & 248 & 122.5994 & 1.70118 \\
\hline 2993 & 53.8643 & 262.0148 & 253.0862 & 247 & 122.5994 & 1.70068 \\
\hline 2994 & 53.8813 & 247.3075 & 251.104 & 246 & 122.5994 & 1.70019 \\
\hline 2995 & 53.8983 & 239.272 & 250.3728 & 246 & 122.5994 & 1.69969 \\
\hline 2996 & 53.9153 & 250.7202 & 249.107 & 245 & 122.5994 & 1.69919 \\
\hline 2997 & 53.9323 & 243.0222 & 248.2807 & 244 & 122.5994 & 1.6987 \\
\hline 2998 & 53.9493 & 229.4553 & 248.8233 & 244 & 122.5994 & 1.6982 \\
\hline 2999 & 53.9663 & 243.6969 & 248.3972 & 243 & 122.5994 & 1.69771 \\
\hline 3000 & 53.9833 & 249.3107 & 248.9597 & 243 & 122.5994 & 1.69721 \\
\hline 3001 & 54.0003 & 236.6297 & 248.4605 & 242 & 122.5994 & 1.69672 \\
\hline 3002 & 54.0173 & 232.7574 & 250.0361 & 243 & 122.5994 & 1.69623 \\
\hline 3003 & 54.0343 & 260.5785 & 252.5932 & 245 & 122.5994 & 1.69573 \\
\hline 3004 & 54.0513 & 259.0526 & 253.804 & 246 & 122.5994 & 1.69524 \\
\hline 3005 & 54.0683 & 254.5414 & 254.64 & 247 & 122.5994 & 1.69475 \\
\hline 3006 & 54.0853 & 242.7537 & 255.4441 & 248 & 122.5994 & 1.69425 \\
\hline 3007 & 54.1023 & 244.6089 & 257.4539 & 250 & 122.5994 & 1.69376 \\
\hline 3008 & 54.1193 & 247.4697 & 258.7432 & 251 & 122.5994 & 1.69327 \\
\hline 3009 & 54.1363 & 255.6371 & 260.2818 & 252 & 122.5994 & 1.69278 \\
\hline 3010 & 54.1533 & 271.3233 & 262.877 & 254 & 122.5994 & 1.69229 \\
\hline 3011 & 54.1703 & 241.5815 & 264.4532 & 255 & 122.5994 & 1.6918 \\
\hline 3012 & 54.1873 & 240.6947 & 265.8561 & 256 & 122.5994 & 1.69131 \\
\hline 3013 & 54.2043 & 258.7922 & 267.8011 & 258 & 122.5994 & 1.69082 \\
\hline 3014 & 54.2213 & 257.0791 & 268.2426 & 259 & 122.5994 & 1.69033 \\
\hline 3015 & 54.2383 & 272.6637 & 268.4796 & 260 & 122.5994 & 1.68984 \\
\hline 3016 & 54.2553 & 266.9308 & 269.033 & 261 & 122.5994 & 1.68935 \\
\hline 3017 & 54.2723 & 277.4524 & 271.228 & 263 & 122.5994 & 1.68886 \\
\hline 3018 & 54.2893 & 255.0725 & 273.1784 & 264 & 122.5994 & 1.68837 \\
\hline 3019 & 54.3063 & 283.4896 & 275.857 & 265 & 122.5994 & 1.68788 \\
\hline 3020 & 54.3233 & 254.2341 & 280.0394 & 267 & 122.5994 & 1.68739 \\
\hline 3021 & 54.3403 & 294.9191 & 283.0262 & 268 & 122.5994 & 1.6869 \\
\hline 3022 & 54.3573 & 275.5967 & 285.317 & 269 & 122.5994 & 1.68642 \\
\hline 3023 & 54.3743 & 275.3875 & 287.1228 & 270 & 122.5994 & 1.68593 \\
\hline 3024 & 54.3913 & 268.8527 & 289.4167 & 272 & 122.5994 & 1.68544 \\
\hline 3025 & 54.4083 & 266.5252 & 291.8029 & 273 & 122.5994 & 1.68496 \\
\hline 3026 & 54.4253 & 279.8053 & 291.8488 & 274 & 122.5994 & 1.68447 \\
\hline 3027 & 54.4423 & 286.0964 & 290.8751 & 274 & 122.5994 & 1.68398 \\
\hline 3028 & 54.4593 & 263.6872 & 291.1098 & 275 & 122.5994 & 1.6835 \\
\hline 3029 & 54.4763 & 278.4976 & 291.5477 & 276 & 122.5994 & 1.68301 \\
\hline 3030 & 54.4933 & 306.1696 & 291.0869 & 276 & 122.5994 & 1.68253 \\
\hline 3031 & 54.5103 & 272.9298 & 291.8117 & 277 & 122.5994 & 1.68204 \\
\hline 3032 & 54.5273 & 272.7018 & 291.852 & 277 & 122.5994 & 1.68156 \\
\hline 3033 & 54.5443 & 260.13 & 293.9825 & 278 & 122.5994 & 1.68108 \\
\hline 3034 & 54.5613 & 250.397 & 295.0692 & 279 & 122.5994 & 1.68059 \\
\hline 3035 & 54.5783 & 252.3632 & 295.0297 & 279 & 122.5994 & 1.68011 \\
\hline 3036 & 54.5953 & 275.5967 & 295.9495 & 280 & 122.5994 & 1.67963 \\
\hline
\end{tabular}




\begin{tabular}{|c|c|c|c|c|c|c|}
\hline 3037 & 54.6123 & 272.3416 & 295.7478 & 280 & 122.5994 & 1.67914 \\
\hline 3038 & 54.6293 & 276.1286 & 296.4435 & 281 & 122.5994 & 1.67866 \\
\hline 3039 & 54.6463 & 289.6 & 297.1861 & 282 & 122.5994 & 1.67818 \\
\hline 3040 & 54.6633 & 277.5716 & 297.168 & 282 & 122.5994 & 1.6777 \\
\hline 3041 & 54.6803 & 274.2412 & 298.5772 & 283 & 122.5994 & 1.67722 \\
\hline 3042 & 54.6973 & 276.5292 & 300.4679 & 284 & 122.5994 & 1.67674 \\
\hline 3043 & 54.7143 & 302.8124 & 301.645 & 284 & 122.5994 & 1.67625 \\
\hline 3044 & 54.7313 & 312.5187 & 304.1408 & 285 & 122.5994 & 1.67577 \\
\hline 3045 & 54.7483 & 304.4572 & 307.2465 & 286 & 122.5994 & 1.67529 \\
\hline 3046 & 54.7653 & 322.6137 & 310.1371 & 286 & 122.5994 & 1.67481 \\
\hline 3047 & 54.7823 & 341.1439 & 314.6684 & 287 & 122.5994 & 1.67433 \\
\hline 3048 & 54.7993 & 356.0721 & 318.3609 & 287 & 122.5994 & 1.67386 \\
\hline 3049 & 54.8163 & 397.8148 & 322.8328 & 288 & 122.5994 & 1.67338 \\
\hline 3050 & 54.8333 & 471.1071 & 326.2734 & 288 & 122.5994 & 1.6729 \\
\hline 3051 & 54.8503 & 548.0864 & 330.5786 & 288 & 122.5994 & 1.67242 \\
\hline 3052 & 54.8673 & 630.5319 & 336.9688 & 288 & 122.5994 & 1.67194 \\
\hline 3053 & 54.8843 & 769.5512 & 347.6966 & 288 & 122.5994 & 1.67146 \\
\hline 3054 & 54.9013 & 867.9577 & 367.235 & 288 & 122.5994 & 1.67099 \\
\hline 3055 & 54.9183 & 953.303 & 402.6656 & 288 & 122.5994 & 1.67051 \\
\hline 3056 & 54.9353 & 938.936 & 462.4892 & 288 & 122.5994 & 1.67003 \\
\hline 3057 & 54.9523 & 745.0739 & 551.9085 & 288 & 122.5994 & 1.66956 \\
\hline 3058 & 54.9693 & 553.6837 & 665.8082 & 288 & 122.5994 & 1.66908 \\
\hline 3059 & 54.9863 & 486.814 & 778.7234 & 288 & 122.5994 & 1.6686 \\
\hline 3060 & 55.0033 & 476.0977 & 838.7358 & 288 & 122.5994 & 1.66813 \\
\hline 3061 & 55.0203 & 482.5509 & 807.0421 & 288 & 122.5994 & 1.66765 \\
\hline 3062 & 55.0373 & 555.0806 & 710.0632 & 287 & 122.5994 & 1.66718 \\
\hline 3063 & 55.0543 & 600.5695 & 608.3999 & 287 & 122.5994 & 1.6667 \\
\hline 3064 & 55.0713 & 650.4962 & 535.4069 & 287 & 122.5994 & 1.66623 \\
\hline 3065 & 55.0883 & 590.8924 & 503.6246 & 287 & 122.5994 & 1.66576 \\
\hline 3066 & 55.1053 & 480.9977 & 511.7094 & 287 & 122.5994 & 1.66528 \\
\hline 3067 & 55.1223 & 402.3967 & 546.9126 & 287 & 122.5994 & 1.66481 \\
\hline 3068 & 55.1393 & 342.7656 & 584.6031 & 287 & 122.5994 & 1.66434 \\
\hline 3069 & 55.1563 & 356.7785 & 591.9651 & 287 & 122.5994 & 1.66386 \\
\hline 3070 & 55.1733 & 323.4936 & 555.7507 & 287 & 122.5994 & 1.66339 \\
\hline 3071 & 55.1903 & 309.2251 & 495.9922 & 287 & 122.5994 & 1.66292 \\
\hline 3072 & 55.2073 & 299.7934 & 437.9842 & 287 & 122.5994 & 1.66245 \\
\hline 3073 & 55.2243 & 306.9815 & 392.9617 & 287 & 122.5994 & 1.66197 \\
\hline 3074 & 55.2413 & 312.0014 & 362.7973 & 287 & 122.5994 & 1.6615 \\
\hline 3075 & 55.2583 & 310.4053 & 344.7627 & 287 & 122.5994 & 1.66103 \\
\hline 3076 & 55.2753 & 359.116 & 334.3812 & 287 & 122.5994 & 1.66056 \\
\hline 3077 & 55.2923 & 366.5014 & 327.9816 & 287 & 122.5994 & 1.66009 \\
\hline 3078 & 55.3093 & 375.3558 & 323.7049 & 287 & 122.5994 & 1.65962 \\
\hline 3079 & 55.3263 & 444.2113 & 320.9143 & 287 & 122.5994 & 1.65915 \\
\hline 3080 & 55.3433 & 457.2885 & 319.218 & 287 & 122.5994 & 1.65868 \\
\hline 3081 & 55.3603 & 530.2747 & 318.0242 & 287 & 122.5994 & 1.65821 \\
\hline 3082 & 55.3773 & 566.7084 & 317.0322 & 287 & 122.5994 & 1.65774 \\
\hline 3083 & 55.3943 & 474.513 & 316.6117 & 287 & 122.5994 & 1.65728 \\
\hline 3084 & 55.4113 & 406.9614 & 317.357 & 287 & 122.5994 & 1.65681 \\
\hline 3085 & 55.4283 & 367.0955 & 319.2213 & 286 & 122.5994 & 1.65634 \\
\hline
\end{tabular}




\begin{tabular}{|c|c|c|c|c|c|c|}
\hline 3086 & 55.4453 & 361.493 & 325.6328 & 286 & 122.5994 & 1.65587 \\
\hline 3087 & 55.4623 & 367.076 & 338.2533 & 286 & 122.5994 & 1.65541 \\
\hline 3088 & 55.4793 & 383.049 & 360.6301 & 286 & 122.5994 & 1.65494 \\
\hline 3089 & 55.4963 & 409.7918 & 395.9596 & 286 & 122.5994 & 1.65447 \\
\hline 3090 & 55.5133 & 435.4117 & 444.5141 & 286 & 122.5994 & 1.65401 \\
\hline 3091 & 55.5303 & 451.8936 & 499.8358 & 286 & 122.5994 & 1.65354 \\
\hline 3092 & 55.5473 & 451.8309 & 544.3922 & 286 & 122.5994 & 1.65307 \\
\hline 3093 & 55.5643 & 490.2289 & 552.3777 & 286 & 122.5994 & 1.65261 \\
\hline 3094 & 55.5813 & 522.7778 & 518.2143 & 286 & 122.5994 & 1.65214 \\
\hline 3095 & 55.5983 & 542.3558 & 466.5419 & 286 & 122.5994 & 1.65168 \\
\hline 3096 & 55.6153 & 530.2859 & 421.6239 & 286 & 122.5994 & 1.65121 \\
\hline 3097 & 55.6323 & 449.4555 & 394.9616 & 286 & 122.5994 & 1.65075 \\
\hline 3098 & 55.6493 & 387.8555 & 386.325 & 285 & 122.5994 & 1.65028 \\
\hline 3099 & 55.6663 & 334.9384 & 397.2846 & 285 & 122.5994 & 1.64982 \\
\hline 3100 & 55.6833 & 337.9745 & 414.4733 & 284 & 122.5994 & 1.64936 \\
\hline 3101 & 55.7003 & 362.9289 & 428.28 & 283 & 122.5994 & 1.64889 \\
\hline 3102 & 55.7173 & 371.469 & 423.3357 & 282 & 122.5994 & 1.64843 \\
\hline 3103 & 55.7343 & 401.8954 & 400.1323 & 282 & 122.5994 & 1.64797 \\
\hline 3104 & 55.7513 & 415.2589 & 369.3832 & 281 & 122.5994 & 1.64751 \\
\hline 3105 & 55.7683 & 406.5637 & 342.2336 & 280 & 122.5994 & 1.64704 \\
\hline 3106 & 55.7853 & 363.1131 & 323.3024 & 280 & 122.5994 & 1.64658 \\
\hline 3107 & 55.8023 & 341.1426 & 310.2942 & 279 & 122.5994 & 1.64612 \\
\hline 3108 & 55.8193 & 323.0815 & 302.2709 & 278 & 122.5994 & 1.64566 \\
\hline 3109 & 55.8363 & 308.0957 & 297.0518 & 277 & 122.5994 & 1.6452 \\
\hline 3110 & 55.8533 & 296.9131 & 294.2723 & 277 & 122.5994 & 1.64474 \\
\hline 3111 & 55.8703 & 256.4751 & 291.2548 & 276 & 122.5994 & 1.64428 \\
\hline 3112 & 55.8873 & 280.3664 & 288.8741 & 275 & 122.5994 & 1.64382 \\
\hline 3113 & 55.9043 & 272.6367 & 288.1595 & 275 & 122.5994 & 1.64336 \\
\hline 3114 & 55.9213 & 275.5309 & 287.0732 & 274 & 122.5994 & 1.6429 \\
\hline 3115 & 55.9383 & 292.3955 & 286.4461 & 273 & 122.5994 & 1.64244 \\
\hline 3116 & 55.9553 & 262.1914 & 287.0573 & 273 & 122.5994 & 1.64198 \\
\hline 3117 & 55.9723 & 253.2155 & 286.6717 & 272 & 122.5994 & 1.64152 \\
\hline 3118 & 55.9893 & 264.2353 & 284.4278 & 271 & 122.5994 & 1.64107 \\
\hline 3119 & 56.0063 & 285.7985 & 284.2722 & 270 & 122.5994 & 1.64061 \\
\hline 3120 & 56.0233 & 253.5435 & 285.4431 & 270 & 122.5994 & 1.64015 \\
\hline 3121 & 56.0403 & 262.6374 & 285.2874 & 269 & 122.5994 & 1.63969 \\
\hline 3122 & 56.0573 & 282.1166 & 283.9876 & 268 & 122.5994 & 1.63924 \\
\hline 3123 & 56.0743 & 262.9798 & 282.9506 & 268 & 122.5994 & 1.63878 \\
\hline 3124 & 56.0913 & 261.9156 & 281.0295 & 267 & 122.5994 & 1.63832 \\
\hline 3125 & 56.1083 & 241.8615 & 280.4423 & 267 & 122.5994 & 1.63787 \\
\hline 3126 & 56.1253 & 230.8508 & 278.8117 & 266 & 122.5994 & 1.63741 \\
\hline 3127 & 56.1423 & 249.6733 & 277.0035 & 265 & 122.5994 & 1.63696 \\
\hline 3128 & 56.1593 & 258.7896 & 276.4132 & 265 & 122.5994 & 1.6365 \\
\hline 3129 & 56.1763 & 280.1901 & 275.1752 & 264 & 122.5994 & 1.63605 \\
\hline 3130 & 56.1933 & 271.3397 & 274.7305 & 264 & 122.5994 & 1.63559 \\
\hline 3131 & 56.2103 & 246.383 & 272.7399 & 263 & 122.5994 & 1.63514 \\
\hline 3132 & 56.2273 & 230.7872 & 271.476 & 263 & 122.5994 & 1.63468 \\
\hline 3133 & 56.2443 & 257.224 & 269.4018 & 262 & 122.5994 & 1.63423 \\
\hline 3134 & 56.2613 & 250.1907 & 267.6619 & 261 & 122.5994 & 1.63378 \\
\hline
\end{tabular}




\begin{tabular}{|c|c|c|c|c|c|c|}
\hline 3135 & 56.2783 & 246.6634 & 267.1258 & 261 & 122.5994 & 1.63332 \\
\hline 3136 & 56.2953 & 239.6325 & 265.6631 & 260 & 122.5994 & 1.63287 \\
\hline 3137 & 56.3123 & 221.782 & 265.3802 & 260 & 122.5994 & 1.63242 \\
\hline 3138 & 56.3293 & 241.8856 & 263.6268 & 259 & 122.5994 & 1.63196 \\
\hline 3139 & 56.3463 & 246.2719 & 262.1194 & 258 & 122.5994 & 1.63151 \\
\hline 3140 & 56.3633 & 222.617 & 261.4821 & 258 & 122.5994 & 1.63106 \\
\hline 3141 & 56.3803 & 216.8273 & 259.9589 & 257 & 122.5994 & 1.63061 \\
\hline 3142 & 56.3973 & 229.0781 & 259.7195 & 257 & 122.5994 & 1.63016 \\
\hline 3143 & 56.4143 & 235.9221 & 258.7359 & 256 & 122.5994 & 1.62971 \\
\hline 3144 & 56.4313 & 255.0436 & 259.0284 & 256 & 122.5994 & 1.62926 \\
\hline 3145 & 56.4483 & 261.471 & 258.5886 & 255 & 122.5994 & 1.62881 \\
\hline 3146 & 56.4653 & 244.4976 & 259.5314 & 255 & 122.5994 & 1.62836 \\
\hline 3147 & 56.4823 & 268.2522 & 259.9626 & 254 & 122.5994 & 1.62791 \\
\hline 3148 & 56.4993 & 263.5396 & 261.8411 & 254 & 122.5994 & 1.62746 \\
\hline 3149 & 56.5163 & 246.7654 & 262.9287 & 253 & 122.5994 & 1.62701 \\
\hline 3150 & 56.5333 & 236.3405 & 264.4225 & 253 & 122.5994 & 1.62656 \\
\hline 3151 & 56.5503 & 195.9788 & 263.148 & 252 & 122.5994 & 1.62611 \\
\hline 3152 & 56.5673 & 216.8853 & 261.2767 & 252 & 122.5994 & 1.62566 \\
\hline 3153 & 56.5843 & 261.052 & 259.1341 & 252 & 122.5994 & 1.62521 \\
\hline 3154 & 56.6013 & 226.9591 & 256.5531 & 251 & 122.5994 & 1.62477 \\
\hline 3155 & 56.6183 & 250.8884 & 255.9294 & 251 & 122.5994 & 1.62432 \\
\hline 3156 & 56.6353 & 228.6327 & 255.2349 & 250 & 122.5994 & 1.62387 \\
\hline 3157 & 56.6523 & 247.2859 & 256.4057 & 250 & 122.5994 & 1.62342 \\
\hline 3158 & 56.6693 & 243.8518 & 258.1993 & 250 & 122.5994 & 1.62298 \\
\hline 3159 & 56.6863 & 234.5449 & 258.6386 & 249 & 122.5994 & 1.62253 \\
\hline 3160 & 56.7033 & 231.1096 & 259.3381 & 249 & 122.5994 & 1.62209 \\
\hline 3161 & 56.7203 & 213.3432 & 258.4973 & 248 & 122.5994 & 1.62164 \\
\hline 3162 & 56.7373 & 261.9482 & 258.5858 & 248 & 122.5994 & 1.62119 \\
\hline 3163 & 56.7543 & 221.8864 & 257.4681 & 247 & 122.5994 & 1.62075 \\
\hline 3164 & 56.7713 & 220.2591 & 256.7589 & 247 & 122.5994 & 1.6203 \\
\hline 3165 & 56.7883 & 240.227 & 255.5864 & 247 & 122.5994 & 1.61986 \\
\hline 3166 & 56.8053 & 241.156 & 253.6162 & 246 & 122.5994 & 1.61941 \\
\hline 3167 & 56.8223 & 248.5409 & 253.2843 & 246 & 122.5994 & 1.61897 \\
\hline 3168 & 56.8393 & 241.1131 & 252.3597 & 245 & 122.5994 & 1.61853 \\
\hline 3169 & 56.8563 & 247.5572 & 252.6592 & 245 & 122.5994 & 1.61808 \\
\hline 3170 & 56.8733 & 248.7565 & 252.8932 & 245 & 122.5994 & 1.61764 \\
\hline 3171 & 56.8903 & 231.6477 & 253.1153 & 245 & 122.5994 & 1.6172 \\
\hline 3172 & 56.9073 & 241.3308 & 253.1372 & 245 & 122.5994 & 1.61675 \\
\hline 3173 & 56.9243 & 252.5545 & 252.8905 & 245 & 122.5994 & 1.61631 \\
\hline 3174 & 56.9413 & 218.0378 & 251.6142 & 244 & 122.5994 & 1.61587 \\
\hline 3175 & 56.9583 & 230.1311 & 251.6133 & 244 & 122.5994 & 1.61543 \\
\hline 3176 & 56.9753 & 231.8873 & 251.8008 & 244 & 122.5994 & 1.61499 \\
\hline 3177 & 56.9923 & 235.1074 & 251.7248 & 244 & 122.5994 & 1.61454 \\
\hline 3178 & 57.0093 & 233.7781 & 251.1527 & 244 & 122.5994 & 1.6141 \\
\hline 3179 & 57.0263 & 249.6733 & 250.279 & 244 & 122.5994 & 1.61366 \\
\hline 3180 & 57.0433 & 239.7551 & 249.5667 & 244 & 122.5994 & 1.61322 \\
\hline 3181 & 57.0603 & 231.4632 & 249.1777 & 244 & 122.5994 & 1.61278 \\
\hline 3182 & 57.0773 & 234.8013 & 249.2052 & 244 & 122.5994 & 1.61234 \\
\hline 3183 & 57.0943 & 235.1211 & 249.6799 & 244 & 122.5994 & 1.6119 \\
\hline
\end{tabular}




\begin{tabular}{|c|c|c|c|c|c|c|}
\hline 3184 & 57.1113 & 241.8799 & 249.5638 & 243 & 122.5994 & 1.61146 \\
\hline 3185 & 57.1283 & 241.8061 & 250.7639 & 243 & 122.5994 & 1.61102 \\
\hline 3186 & 57.1453 & 240.1101 & 252.1676 & 243 & 122.5994 & 1.61058 \\
\hline 3187 & 57.1623 & 240.5524 & 253.9052 & 243 & 122.5994 & 1.61015 \\
\hline 3188 & 57.1793 & 234.6049 & 256.7579 & 243 & 122.5994 & 1.60971 \\
\hline 3189 & 57.1963 & 233.4223 & 261.8676 & 243 & 122.5994 & 1.60927 \\
\hline 3190 & 57.2133 & 252.6926 & 270.1691 & 243 & 122.5994 & 1.60883 \\
\hline 3191 & 57.2303 & 252.3153 & 281.9837 & 243 & 122.5994 & 1.60839 \\
\hline 3192 & 57.2473 & 265.6058 & 296.4529 & 243 & 122.5994 & 1.60796 \\
\hline 3193 & 57.2643 & 266.6746 & 309.973 & 242 & 122.5994 & 1.60752 \\
\hline 3194 & 57.2813 & 278.6677 & 318.7292 & 242 & 122.5994 & 1.60708 \\
\hline 3195 & 57.2983 & 295.5402 & 316.2013 & 242 & 122.5994 & 1.60665 \\
\hline 3196 & 57.3153 & 259.95 & 305.1089 & 242 & 122.5994 & 1.60621 \\
\hline 3197 & 57.3323 & 241.6868 & 292.2304 & 242 & 122.5994 & 1.60578 \\
\hline 3198 & 57.3493 & 225.9162 & 282.0189 & 242 & 122.5994 & 1.60534 \\
\hline 3199 & 57.3663 & 219.5859 & 276.5602 & 242 & 122.5994 & 1.6049 \\
\hline 3200 & 57.3833 & 234.121 & 276.4144 & 242 & 122.5994 & 1.60447 \\
\hline 3201 & 57.4003 & 245.0873 & 280.8951 & 242 & 122.5994 & 1.60403 \\
\hline 3202 & 57.4173 & 249.2179 & 288.0951 & 242 & 122.5994 & 1.6036 \\
\hline 3203 & 57.4343 & 261.1313 & 293.3098 & 241 & 122.5994 & 1.60317 \\
\hline 3204 & 57.4513 & 250.6826 & 293.9456 & 241 & 122.5994 & 1.60273 \\
\hline 3205 & 57.4683 & 246.7178 & 287.9477 & 241 & 122.5994 & 1.6023 \\
\hline 3206 & 57.4853 & 243.4626 & 278.2098 & 241 & 122.5994 & 1.60186 \\
\hline 3207 & 57.5023 & 222.4417 & 268.329 & 241 & 122.5994 & 1.60143 \\
\hline 3208 & 57.5193 & 272.1728 & 260.4318 & 241 & 122.5994 & 1.601 \\
\hline 3209 & 57.5363 & 252.096 & 255.1799 & 241 & 122.5994 & 1.60057 \\
\hline 3210 & 57.5533 & 223.5419 & 252.4046 & 241 & 122.5994 & 1.60013 \\
\hline 3211 & 57.5703 & 253.3464 & 251.5407 & 241 & 122.5994 & 1.5997 \\
\hline 3212 & 57.5873 & 217.1274 & 251.8739 & 241 & 122.5994 & 1.59927 \\
\hline 3213 & 57.6043 & 229.3368 & 251.5293 & 240 & 122.5994 & 1.59884 \\
\hline 3214 & 57.6213 & 236.7106 & 251.5354 & 240 & 122.5994 & 1.59841 \\
\hline 3215 & 57.6383 & 218.7303 & 250.5774 & 240 & 122.5994 & 1.59798 \\
\hline 3216 & 57.6553 & 248.0309 & 249.1811 & 240 & 122.5994 & 1.59755 \\
\hline 3217 & 57.6723 & 240.227 & 247.9591 & 240 & 122.5994 & 1.59711 \\
\hline 3218 & 57.6893 & 240.9599 & 247.0623 & 240 & 122.5994 & 1.59668 \\
\hline 3219 & 57.7063 & 244.8401 & 245.5648 & 239 & 122.5994 & 1.59625 \\
\hline 3220 & 57.7233 & 253.8022 & 245.5619 & 239 & 122.5994 & 1.59582 \\
\hline 3221 & 57.7403 & 261.1918 & 245.1269 & 238 & 122.5994 & 1.5954 \\
\hline 3222 & 57.7573 & 252.5242 & 246.2995 & 238 & 122.5994 & 1.59497 \\
\hline 3223 & 57.7743 & 255.1037 & 248.1278 & 238 & 122.5994 & 1.59454 \\
\hline 3224 & 57.7913 & 249.7659 & 249.5899 & 237 & 122.5994 & 1.59411 \\
\hline 3225 & 57.8083 & 257.1579 & 252.3983 & 237 & 122.5994 & 1.59368 \\
\hline 3226 & 57.8253 & 255.512 & 254.7624 & 237 & 122.5994 & 1.59325 \\
\hline 3227 & 57.8423 & 247.0322 & 254.3707 & 236 & 122.5994 & 1.59282 \\
\hline 3228 & 57.8593 & 240.7072 & 252.6938 & 236 & 122.5994 & 1.5924 \\
\hline 3229 & 57.8763 & 245.7003 & 249.9079 & 236 & 122.5994 & 1.59197 \\
\hline 3230 & 57.8933 & 262.2052 & 246.2549 & 235 & 122.5994 & 1.59154 \\
\hline 3231 & 57.9103 & 270.5713 & 244.4024 & 235 & 122.5994 & 1.59112 \\
\hline 3232 & 57.9273 & 258.8543 & 242.3201 & 234 & 122.5994 & 1.59069 \\
\hline
\end{tabular}




\begin{tabular}{|c|c|c|c|c|c|c|}
\hline 3233 & 57.9443 & 239.8187 & 242.3435 & 234 & 122.5994 & 1.59026 \\
\hline 3234 & 57.9613 & 232.3532 & 243.0085 & 234 & 122.5994 & 1.58984 \\
\hline 3235 & 57.9783 & 232.8512 & 242.9339 & 233 & 122.5994 & 1.58941 \\
\hline 3236 & 57.9953 & 243.4776 & 243.4825 & 233 & 122.5994 & 1.58899 \\
\hline 3237 & 58.0123 & 239.9266 & 242.1078 & 232 & 122.5994 & 1.58856 \\
\hline 3238 & 58.0293 & 242.1054 & 241.2128 & 232 & 122.5994 & 1.58814 \\
\hline 3239 & 58.0463 & 228.0848 & 240.3332 & 232 & 122.5994 & 1.58771 \\
\hline 3240 & 58.0633 & 242.4139 & 238.8317 & 231 & 122.5994 & 1.58729 \\
\hline 3241 & 58.0803 & 232.9654 & 238.0119 & 231 & 122.5994 & 1.58686 \\
\hline 3242 & 58.0973 & 222.8343 & 237.1727 & 231 & 122.5994 & 1.58644 \\
\hline 3243 & 58.1143 & 226.5234 & 237.3036 & 232 & 122.5994 & 1.58602 \\
\hline 3244 & 58.1313 & 242.3507 & 236.6221 & 232 & 122.5994 & 1.58559 \\
\hline 3245 & 58.1483 & 244.0225 & 236.1542 & 232 & 122.5994 & 1.58517 \\
\hline 3246 & 58.1653 & 223.3878 & 237.0026 & 233 & 122.5994 & 1.58475 \\
\hline 3247 & 58.1823 & 234.3247 & 237.0155 & 233 & 122.5994 & 1.58432 \\
\hline 3248 & 58.1993 & 231.8536 & 238.2634 & 234 & 122.5994 & 1.5839 \\
\hline 3249 & 58.2163 & 247.5315 & 238.8999 & 234 & 122.5994 & 1.58348 \\
\hline 3250 & 58.2333 & 261.2181 & 239.4463 & 234 & 122.5994 & 1.58306 \\
\hline 3251 & 58.2503 & 238.9594 & 240.985 & 235 & 122.5994 & 1.58264 \\
\hline 3252 & 58.2673 & 225.646 & 241.5181 & 235 & 122.5994 & 1.58221 \\
\hline 3253 & 58.2843 & 227.9008 & 242.9391 & 236 & 122.5994 & 1.58179 \\
\hline 3254 & 58.3013 & 219.1373 & 243.0242 & 236 & 122.5994 & 1.58137 \\
\hline 3255 & 58.3183 & 231.1456 & 242.7669 & 236 & 122.5994 & 1.58095 \\
\hline 3256 & 58.3353 & 239.5286 & 243.4235 & 237 & 122.5994 & 1.58053 \\
\hline 3257 & 58.3523 & 219.1881 & 243.2656 & 237 & 122.5994 & 1.58011 \\
\hline 3258 & 58.3693 & 222.2746 & 243.39 & 237 & 122.5994 & 1.57969 \\
\hline 3259 & 58.3863 & 226.1945 & 244.7292 & 238 & 122.5994 & 1.57927 \\
\hline 3260 & 58.4033 & 236.4471 & 245.388 & 238 & 122.5994 & 1.57885 \\
\hline 3261 & 58.4203 & 234.5891 & 246.1256 & 238 & 122.5994 & 1.57843 \\
\hline 3262 & 58.4373 & 253.3846 & 248.0943 & 239 & 122.5994 & 1.57802 \\
\hline 3263 & 58.4543 & 242.03 & 249.1519 & 239 & 122.5994 & 1.5776 \\
\hline 3264 & 58.4713 & 244.5015 & 251.2247 & 240 & 122.5994 & 1.57718 \\
\hline 3265 & 58.4883 & 227.1136 & 252.7216 & 240 & 122.5994 & 1.57676 \\
\hline 3266 & 58.5053 & 238.7595 & 255.6635 & 241 & 122.5994 & 1.57634 \\
\hline 3267 & 58.5223 & 256.2899 & 257.6849 & 241 & 122.5994 & 1.57593 \\
\hline 3268 & 58.5393 & 259.1669 & 260.1909 & 242 & 122.5994 & 1.57551 \\
\hline 3269 & 58.5563 & 240.4154 & 261.9484 & 243 & 122.5994 & 1.57509 \\
\hline 3270 & 58.5733 & 239.3581 & 263.6408 & 244 & 122.5994 & 1.57468 \\
\hline 3271 & 58.5903 & 250.7516 & 265.3333 & 244 & 122.5994 & 1.57426 \\
\hline 3272 & 58.6073 & 250.443 & 269.6548 & 245 & 122.5994 & 1.57384 \\
\hline 3273 & 58.6243 & 246.4664 & 275.8272 & 246 & 122.5994 & 1.57343 \\
\hline 3274 & 58.6413 & 245.1433 & 282.9796 & 246 & 122.5994 & 1.57301 \\
\hline 3275 & 58.6583 & 257.7002 & 292.9369 & 247 & 122.5994 & 1.5726 \\
\hline 3276 & 58.6753 & 277.0846 & 303.9969 & 248 & 122.5994 & 1.57218 \\
\hline 3277 & 58.6923 & 269.3349 & 314.3051 & 249 & 122.5994 & 1.57177 \\
\hline 3278 & 58.7093 & 265.8203 & 320.6192 & 249 & 122.5994 & 1.57135 \\
\hline 3279 & 58.7263 & 279.3735 & 323.1136 & 250 & 122.5994 & 1.57094 \\
\hline 3280 & 58.7433 & 271.0124 & 320.358 & 251 & 122.5994 & 1.57052 \\
\hline 3281 & 58.7603 & 261.037 & 315.3516 & 251 & 122.5994 & 1.57011 \\
\hline
\end{tabular}




\begin{tabular}{|c|c|c|c|c|c|c|}
\hline 3282 & 58.7773 & 280.3062 & 314.43 & 252 & 122.5994 & 1.5697 \\
\hline 3283 & 58.7943 & 273.9087 & 316.6891 & 253 & 122.5994 & 1.56928 \\
\hline 3284 & 58.8113 & 254.3081 & 317.3615 & 253 & 122.5994 & 1.56887 \\
\hline 3285 & 58.8283 & 245.4746 & 315.3112 & 254 & 122.5994 & 1.56846 \\
\hline 3286 & 58.8453 & 272.1039 & 311.0753 & 255 & 122.5994 & 1.56804 \\
\hline 3287 & 58.8623 & 280.9271 & 307.0602 & 256 & 122.5994 & 1.56763 \\
\hline 3288 & 58.8793 & 246.4302 & 302.7874 & 256 & 122.5994 & 1.56722 \\
\hline 3289 & 58.8963 & 245.7153 & 299.4102 & 257 & 122.5994 & 1.56681 \\
\hline 3290 & 58.9133 & 259.4339 & 295.0577 & 257 & 122.5994 & 1.5664 \\
\hline 3291 & 58.9303 & 267.6171 & 292.3097 & 257 & 122.5994 & 1.56599 \\
\hline 3292 & 58.9473 & 243.1733 & 291.2866 & 257 & 122.5994 & 1.56557 \\
\hline 3293 & 58.9643 & 255.4721 & 290.0684 & 257 & 122.5994 & 1.56516 \\
\hline 3294 & 58.9813 & 237.8132 & 286.4543 & 257 & 122.5994 & 1.56475 \\
\hline 3295 & 58.9983 & 244.9124 & 281.8264 & 258 & 122.5994 & 1.56434 \\
\hline 3296 & 59.0153 & 249.346 & 276.3569 & 258 & 122.5994 & 1.56393 \\
\hline 3297 & 59.0323 & 249.6161 & 272.4872 & 258 & 122.5994 & 1.56352 \\
\hline 3298 & 59.0493 & 226.4773 & 270.614 & 258 & 122.5994 & 1.56311 \\
\hline 3299 & 59.0663 & 233.7629 & 270.4197 & 258 & 122.5994 & 1.5627 \\
\hline 3300 & 59.0833 & 225.1399 & 271.1066 & 258 & 122.5994 & 1.56229 \\
\hline 3301 & 59.1003 & 259.0699 & 271.7987 & 258 & 122.5994 & 1.56189 \\
\hline 3302 & 59.1173 & 228.4294 & 272.0634 & 258 & 122.5994 & 1.56148 \\
\hline 3303 & 59.1343 & 243.483 & 272.1705 & 258 & 122.5994 & 1.56107 \\
\hline 3304 & 59.1513 & 246.2529 & 272.4892 & 258 & 122.5994 & 1.56066 \\
\hline 3305 & 59.1683 & 241.7431 & 272.8655 & 258 & 122.5994 & 1.56025 \\
\hline 3306 & 59.1853 & 225.6504 & 272.4848 & 258 & 122.5994 & 1.55984 \\
\hline 3307 & 59.2023 & 234.3881 & 271.2505 & 258 & 122.5994 & 1.55944 \\
\hline 3308 & 59.2193 & 254.9939 & 270.8074 & 259 & 122.5994 & 1.55903 \\
\hline 3309 & 59.2363 & 246.8832 & 269.6582 & 259 & 122.5994 & 1.55862 \\
\hline 3310 & 59.2533 & 237.1907 & 268.9379 & 259 & 122.5994 & 1.55822 \\
\hline 3311 & 59.2703 & 255.2877 & 268.4545 & 259 & 122.5994 & 1.55781 \\
\hline 3312 & 59.2873 & 266.3629 & 268.2706 & 259 & 122.5994 & 1.5574 \\
\hline 3313 & 59.3043 & 244.6088 & 268.378 & 259 & 122.5994 & 1.557 \\
\hline 3314 & 59.3213 & 270.4179 & 268.6089 & 259 & 122.5994 & 1.55659 \\
\hline 3315 & 59.3383 & 265.6752 & 269.3962 & 260 & 122.5994 & 1.55619 \\
\hline 3316 & 59.3553 & 228.977 & 268.929 & 260 & 122.5994 & 1.55578 \\
\hline 3317 & 59.3723 & 243.1299 & 268.9724 & 261 & 122.5994 & 1.55538 \\
\hline 3318 & 59.3893 & 264.4638 & 267.9401 & 261 & 122.5994 & 1.55497 \\
\hline 3319 & 59.4063 & 267.6061 & 268.0789 & 262 & 122.5994 & 1.55457 \\
\hline 3320 & 59.4233 & 270.1214 & 267.5182 & 262 & 122.5994 & 1.55416 \\
\hline 3321 & 59.4403 & 259.679 & 268.0943 & 263 & 122.5994 & 1.55376 \\
\hline 3322 & 59.4573 & 274.126 & 267.907 & 263 & 122.5994 & 1.55336 \\
\hline 3323 & 59.4743 & 272.2714 & 267.6927 & 263 & 122.5994 & 1.55295 \\
\hline 3324 & 59.4913 & 246.5229 & 268.3113 & 264 & 122.5994 & 1.55255 \\
\hline 3325 & 59.5083 & 240.0174 & 274.8731 & 264 & 122.5994 & 1.55215 \\
\hline 3326 & 59.5253 & 244.2623 & 276.0602 & 265 & 122.5994 & 1.55174 \\
\hline 3327 & 59.5423 & 254.5822 & 276.364 & 265 & 122.5994 & 1.55134 \\
\hline 3328 & 59.5593 & 254.1107 & 277.8614 & 266 & 122.5994 & 1.55094 \\
\hline 3329 & 59.5763 & 245.2296 & 281.1431 & 266 & 122.5994 & 1.55054 \\
\hline 3330 & 59.5933 & 281.6509 & 283.4903 & 267 & 122.5994 & 1.55014 \\
\hline
\end{tabular}




\begin{tabular}{|c|c|c|c|c|c|c|}
\hline 3331 & 59.6103 & 306.2908 & 285.343 & 267 & 122.5994 & 1.54973 \\
\hline 3332 & 59.6273 & 283.4166 & 288.665 & 268 & 122.5994 & 1.54933 \\
\hline 3333 & 59.6443 & 270.0084 & 291.3036 & 268 & 122.5994 & 1.54893 \\
\hline 3334 & 59.6613 & 271.2825 & 293.9577 & 268 & 122.5994 & 1.54853 \\
\hline 3335 & 59.6783 & 279.5685 & 297.5467 & 269 & 122.5994 & 1.54813 \\
\hline 3336 & 59.6953 & 264.8475 & 300.292 & 269 & 122.5994 & 1.54773 \\
\hline 3337 & 59.7123 & 301.2737 & 304.0276 & 270 & 122.5994 & 1.54733 \\
\hline 3338 & 59.7293 & 313.3318 & 306.0359 & 270 & 122.5994 & 1.54693 \\
\hline 3339 & 59.7463 & 307.4777 & 307.1177 & 270 & 122.5994 & 1.54653 \\
\hline 3340 & 59.7633 & 324.2219 & 308.4215 & 270 & 122.5994 & 1.54613 \\
\hline 3341 & 59.7803 & 309.19 & 310.9689 & 270 & 122.5994 & 1.54573 \\
\hline 3342 & 59.7973 & 334.8417 & 315.1559 & 270 & 122.5994 & 1.54533 \\
\hline 3343 & 59.8143 & 345.3204 & 320.9988 & 270 & 122.5994 & 1.54494 \\
\hline 3344 & 59.8313 & 335.6439 & 327.436 & 269 & 122.5994 & 1.54454 \\
\hline 3345 & 59.8483 & 378.0514 & 336.5838 & 269 & 122.5994 & 1.54414 \\
\hline 3346 & 59.8653 & 407.2245 & 348.1319 & 269 & 122.5994 & 1.54374 \\
\hline 3347 & 59.8823 & 453.3489 & 363.4423 & 269 & 122.5994 & 1.54334 \\
\hline 3348 & 59.8993 & 540.2465 & 385.5888 & 269 & 122.5994 & 1.54295 \\
\hline 3349 & 59.9163 & 684.948 & 422.1979 & 269 & 122.5994 & 1.54255 \\
\hline 3350 & 59.9333 & 926.3942 & 487.9603 & 269 & 122.5994 & 1.54215 \\
\hline 3351 & 59.9503 & 1094.15 & 604.9734 & 269 & 122.5994 & 1.54176 \\
\hline 3352 & 59.9673 & 1485.181 & 797.2715 & 269 & 122.5994 & 1.54136 \\
\hline 3353 & 59.9843 & 1747.508 & 1076.146 & 269 & 122.5994 & 1.54096 \\
\hline 3354 & 60.0013 & 1970.462 & 1416.621 & 269 & 122.5994 & 1.54057 \\
\hline 3355 & 60.0183 & 1780.223 & 1726.874 & 269 & 122.5994 & 1.54017 \\
\hline 3356 & 60.0353 & 1332.417 & 1844.756 & 269 & 122.5994 & 1.53978 \\
\hline 3357 & 60.0523 & 1014.847 & 1687.754 & 269 & 122.5994 & 1.53938 \\
\hline 3358 & 60.0693 & 777.5406 & 1376.421 & 269 & 122.5994 & 1.53899 \\
\hline 3359 & 60.0863 & 752.2203 & 1069.098 & 269 & 122.5994 & 1.53859 \\
\hline 3360 & 60.1033 & 762.671 & 849.4275 & 269 & 122.5994 & 1.5382 \\
\hline 3361 & 60.1203 & 858.8196 & 744.3055 & 269 & 122.5994 & 1.5378 \\
\hline 3362 & 60.1373 & 968.173 & 749.2132 & 269 & 122.5994 & 1.53741 \\
\hline 3363 & 60.1543 & 1039.438 & 841.0934 & 269 & 122.5994 & 1.53701 \\
\hline 3364 & 60.1713 & 1039.249 & 978.5021 & 269 & 122.5994 & 1.53662 \\
\hline 3365 & 60.1883 & 928.4764 & 1089.697 & 269 & 122.5994 & 1.53623 \\
\hline 3366 & 60.2053 & 771.2648 & 1088.044 & 269 & 122.5994 & 1.53583 \\
\hline 3367 & 60.2223 & 605.9131 & 961.9511 & 269 & 122.5994 & 1.53544 \\
\hline 3368 & 60.2393 & 481.6315 & 786.308 & 269 & 122.5994 & 1.53505 \\
\hline 3369 & 60.2563 & 398.8344 & 625.8419 & 269 & 122.5994 & 1.53466 \\
\hline 3370 & 60.2733 & 358.8801 & 506.6027 & 269 & 122.5994 & 1.53426 \\
\hline 3371 & 60.2903 & 355.2388 & 429.6302 & 269 & 122.5994 & 1.53387 \\
\hline 3372 & 60.3073 & 328.8841 & 383.7971 & 268 & 122.5994 & 1.53348 \\
\hline 3373 & 60.3243 & 332.5296 & 358.7487 & 268 & 122.5994 & 1.53309 \\
\hline 3374 & 60.3413 & 313.5896 & 343.9845 & 268 & 122.5994 & 1.5327 \\
\hline 3375 & 60.3583 & 300.6982 & 333.6644 & 268 & 122.5994 & 1.53231 \\
\hline 3376 & 60.3753 & 289.3269 & 325.4067 & 268 & 122.5994 & 1.53192 \\
\hline 3377 & 60.3923 & 264.2393 & 318.747 & 268 & 122.5994 & 1.53153 \\
\hline 3378 & 60.4093 & 286.9205 & 313.4931 & 268 & 122.5994 & 1.53113 \\
\hline 3379 & 60.4263 & 288.7373 & 309.5466 & 268 & 122.5994 & 1.53074 \\
\hline
\end{tabular}




\begin{tabular}{|c|c|c|c|c|c|c|}
\hline 3380 & 60.4433 & 282.2776 & 295.4924 & 268 & 122.5994 & 1.53035 \\
\hline 3381 & 60.4603 & 272.5847 & 294.958 & 268 & 122.5994 & 1.52997 \\
\hline 3382 & 60.4773 & 254.513 & 295.4442 & 268 & 122.5994 & 1.52958 \\
\hline 3383 & 60.4943 & 269.5822 & 296.552 & 268 & 122.5994 & 1.52919 \\
\hline 3384 & 60.5113 & 250.1113 & 297.2765 & 268 & 122.5994 & 1.5288 \\
\hline 3385 & 60.5283 & 264.1581 & 296.7151 & 268 & 122.5994 & 1.52841 \\
\hline 3386 & 60.5453 & 277.2017 & 294.2886 & 267 & 122.5994 & 1.52802 \\
\hline 3387 & 60.5623 & 293.4064 & 292.4551 & 267 & 122.5994 & 1.52763 \\
\hline 3388 & 60.5793 & 289.8222 & 288.8866 & 266 & 122.5994 & 1.52724 \\
\hline 3389 & 60.5963 & 270.995 & 286.0283 & 266 & 122.5994 & 1.52686 \\
\hline 3390 & 60.6133 & 275.0594 & 282.7817 & 265 & 122.5994 & 1.52647 \\
\hline 3391 & 60.6303 & 245.261 & 281.6259 & 265 & 122.5994 & 1.52608 \\
\hline 3392 & 60.6473 & 255.5495 & 280.6301 & 264 & 122.5994 & 1.52569 \\
\hline 3393 & 60.6643 & 246.6251 & 281.4896 & 264 & 122.5994 & 1.52531 \\
\hline 3394 & 60.6813 & 292.4818 & 281.5165 & 263 & 122.5994 & 1.52492 \\
\hline 3395 & 60.6983 & 298.3815 & 282.0289 & 263 & 122.5994 & 1.52453 \\
\hline 3396 & 60.7153 & 264.807 & 280.9178 & 262 & 122.5994 & 1.52415 \\
\hline 3397 & 60.7323 & 247.796 & 280.3879 & 262 & 122.5994 & 1.52376 \\
\hline 3398 & 60.7493 & 270.0524 & 278.4993 & 261 & 122.5994 & 1.52338 \\
\hline 3399 & 60.7663 & 310.1508 & 276.6135 & 260 & 122.5994 & 1.52299 \\
\hline 3400 & 60.7833 & 282.9184 & 276.0185 & 260 & 122.5994 & 1.52261 \\
\hline 3401 & 60.8003 & 267.2592 & 272.0625 & 259 & 122.5994 & 1.52222 \\
\hline 3402 & 60.8173 & 271.511 & 272.0728 & 259 & 122.5994 & 1.52184 \\
\hline 3403 & 60.8343 & 254.1139 & 271.155 & 258 & 122.5994 & 1.52145 \\
\hline 3404 & 60.8513 & 261.329 & 271.2485 & 258 & 122.5994 & 1.52107 \\
\hline 3405 & 60.8683 & 270.59 & 270.2379 & 257 & 122.5994 & 1.52068 \\
\hline 3406 & 60.8853 & 272.0836 & 269.848 & 257 & 122.5994 & 1.5203 \\
\hline 3407 & 60.9023 & 257.8791 & 267.8174 & 256 & 122.5994 & 1.51992 \\
\hline 3408 & 60.9193 & 256.438 & 266.4235 & 256 & 122.5994 & 1.51953 \\
\hline 3409 & 60.9363 & 266.2108 & 264.0481 & 255 & 122.5994 & 1.51915 \\
\hline 3410 & 60.9533 & 243.56 & 262.9699 & 255 & 122.5994 & 1.51877 \\
\hline 3411 & 60.9703 & 250.7906 & 262.1978 & 255 & 122.5994 & 1.51838 \\
\hline 3412 & 60.9873 & 257.8339 & 261.6691 & 255 & 122.5994 & 1.518 \\
\hline 3413 & 61.0043 & 269.8075 & 261.2684 & 255 & 122.5994 & 1.51762 \\
\hline 3414 & 61.0213 & 281.38 & 260.9641 & 255 & 122.5994 & 1.51724 \\
\hline 3415 & 61.0383 & 275.4348 & 260.7595 & 255 & 122.5994 & 1.51685 \\
\hline 3416 & 61.0553 & 263.8464 & 260.5127 & 255 & 122.5994 & 1.51647 \\
\hline 3417 & 61.0723 & 262.5194 & 260.078 & 255 & 122.5994 & 1.51609 \\
\hline 3418 & 61.0893 & 246.1571 & 259.5733 & 255 & 122.5994 & 1.51571 \\
\hline 3419 & 61.1063 & 254.3908 & 259.1922 & 255 & 122.5994 & 1.51533 \\
\hline 3420 & 61.1233 & 256.318 & 259.1279 & 255 & 122.5994 & 1.51495 \\
\hline 3421 & 61.1403 & 253.9156 & 258.2886 & 254 & 122.5994 & 1.51457 \\
\hline 3422 & 61.1573 & 267.1635 & 258.5228 & 254 & 122.5994 & 1.51419 \\
\hline 3423 & 61.1743 & 276.3985 & 258.5627 & 254 & 122.5994 & 1.51381 \\
\hline 3424 & 61.1913 & 255.7643 & 258.5879 & 254 & 122.5994 & 1.51343 \\
\hline 3425 & 61.2083 & 247.6112 & 258.5091 & 254 & 122.5994 & 1.51305 \\
\hline 3426 & 61.2253 & 267.5837 & 258.4008 & 254 & 122.5994 & 1.51267 \\
\hline 3427 & 61.2423 & 266.218 & 258.2544 & 254 & 122.5994 & 1.51229 \\
\hline 3428 & 61.2593 & 262.4148 & 258.1216 & 254 & 122.5994 & 1.51191 \\
\hline
\end{tabular}




\begin{tabular}{|c|c|c|c|c|c|c|}
\hline 3429 & 61.2763 & 252.7737 & 258.0332 & 254 & 122.5994 & 1.51153 \\
\hline 3430 & 61.2933 & 242.2981 & 258.0567 & 254 & 122.5994 & 1.51115 \\
\hline 3431 & 61.3103 & 242.7173 & 258.0871 & 254 & 122.5994 & 1.51078 \\
\hline 3432 & 61.3273 & 248.5571 & 257.9824 & 254 & 122.5994 & 1.5104 \\
\hline 3433 & 61.3443 & 239.0851 & 257.8346 & 254 & 122.5994 & 1.51002 \\
\hline 3434 & 61.3613 & 282.0578 & 257.834 & 254 & 122.5994 & 1.50964 \\
\hline 3435 & 61.3783 & 262.5517 & 258.0604 & 254 & 122.5994 & 1.50926 \\
\hline 3436 & 61.3953 & 233.5979 & 257.6657 & 253 & 122.5994 & 1.50889 \\
\hline 3437 & 61.4123 & 254.2827 & 258.7776 & 253 & 122.5994 & 1.50851 \\
\hline 3438 & 61.4293 & 223.6379 & 260.4306 & 253 & 122.5994 & 1.50813 \\
\hline 3439 & 61.4463 & 261.3794 & 262.3237 & 253 & 122.5994 & 1.50776 \\
\hline 3440 & 61.4633 & 275.4621 & 263.7424 & 253 & 122.5994 & 1.50738 \\
\hline 3441 & 61.4803 & 295.487 & 262.5842 & 252 & 122.5994 & 1.50701 \\
\hline 3442 & 61.4973 & 274.9216 & 260.9707 & 252 & 122.5994 & 1.50663 \\
\hline 3443 & 61.5143 & 259.8206 & 259.0203 & 252 & 122.5994 & 1.50625 \\
\hline 3444 & 61.5313 & 270.8219 & 257.4463 & 252 & 122.5994 & 1.50588 \\
\hline 3445 & 61.5483 & 290.7278 & 255.5111 & 251 & 122.5994 & 1.5055 \\
\hline 3446 & 61.5653 & 275.9451 & 255.2443 & 251 & 122.5994 & 1.50513 \\
\hline 3447 & 61.5823 & 279.349 & 255.5501 & 251 & 122.5994 & 1.50475 \\
\hline 3448 & 61.5993 & 281.1645 & 256.3518 & 251 & 122.5994 & 1.50438 \\
\hline 3449 & 61.6163 & 273.6774 & 257.4353 & 251 & 122.5994 & 1.504 \\
\hline 3450 & 61.6333 & 272.0481 & 257.4737 & 250 & 122.5994 & 1.50363 \\
\hline 3451 & 61.6503 & 293.4472 & 258.1181 & 250 & 122.5994 & 1.50326 \\
\hline 3452 & 61.6673 & 281.4393 & 258.8049 & 250 & 122.5994 & 1.50288 \\
\hline 3453 & 61.6843 & 272.5756 & 260.4824 & 250 & 122.5994 & 1.50251 \\
\hline 3454 & 61.7013 & 287.2532 & 263.8593 & 250 & 122.5994 & 1.50214 \\
\hline 3455 & 61.7183 & 294.9897 & 267.6842 & 249 & 122.5994 & 1.50176 \\
\hline 3456 & 61.7353 & 282.1788 & 272.6086 & 249 & 122.5994 & 1.50139 \\
\hline 3457 & 61.7523 & 265.1217 & 274.8085 & 249 & 122.5994 & 1.50102 \\
\hline 3458 & 61.7693 & 260.1142 & 272.8708 & 249 & 122.5994 & 1.50065 \\
\hline 3459 & 61.7863 & 278.2476 & 268.858 & 249 & 122.5994 & 1.50027 \\
\hline 3460 & 61.8033 & 276.4679 & 263.952 & 248 & 122.5994 & 1.4999 \\
\hline 3461 & 61.8203 & 285.2296 & 260.845 & 248 & 122.5994 & 1.49953 \\
\hline 3462 & 61.8373 & 275.0998 & 258.6838 & 248 & 122.5994 & 1.49916 \\
\hline 3463 & 61.8543 & 257.6144 & 257.7262 & 248 & 122.5994 & 1.49879 \\
\hline 3464 & 61.8713 & 266.0287 & 258.2459 & 248 & 122.5994 & 1.49842 \\
\hline 3465 & 61.8883 & 282.6889 & 258.9032 & 247 & 122.5994 & 1.49805 \\
\hline 3466 & 61.9053 & 288.9723 & 260.9513 & 247 & 122.5994 & 1.49768 \\
\hline 3467 & 61.9223 & 281.3044 & 261.9183 & 247 & 122.5994 & 1.49731 \\
\hline 3468 & 61.9393 & 275.5336 & 260.9176 & 247 & 122.5994 & 1.49694 \\
\hline 3469 & 61.9563 & 257.6161 & 257.9118 & 246 & 122.5994 & 1.49657 \\
\hline 3470 & 61.9733 & 242.4731 & 255.9598 & 246 & 122.5994 & 1.4962 \\
\hline 3471 & 61.9903 & 261.4532 & 254.331 & 246 & 122.5994 & 1.49583 \\
\hline 3472 & 62.0073 & 272.2239 & 252.9659 & 246 & 122.5994 & 1.49546 \\
\hline 3473 & 62.0243 & 272.2239 & 251.8296 & 246 & 122.5994 & 1.49509 \\
\hline 3474 & 62.0413 & 267.5231 & 250.0877 & 245 & 122.5994 & 1.49472 \\
\hline 3475 & 62.0583 & 273.0424 & 249.7149 & 245 & 122.5994 & 1.49435 \\
\hline 3476 & 62.0753 & 258.0984 & 249.7374 & 245 & 122.5994 & 1.49398 \\
\hline 3477 & 62.0923 & 270.7714 & 250.2152 & 245 & 122.5994 & 1.49361 \\
\hline
\end{tabular}




\begin{tabular}{|c|c|c|c|c|c|c|}
\hline 3478 & 62.1093 & 239.3833 & 251.1902 & 245 & 122.5994 & 1.49325 \\
\hline 3479 & 62.1263 & 236.1727 & 251.7748 & 244 & 122.5994 & 1.49288 \\
\hline 3480 & 62.1433 & 258.7534 & 253.9745 & 244 & 122.5994 & 1.49251 \\
\hline 3481 & 62.1603 & 245.7472 & 256.4968 & 244 & 122.5994 & 1.49214 \\
\hline 3482 & 62.1773 & 237.7682 & 257.6461 & 243 & 122.5994 & 1.49178 \\
\hline 3483 & 62.1943 & 242.8943 & 258.8172 & 243 & 122.5994 & 1.49141 \\
\hline 3484 & 62.2113 & 240.4095 & 258.2525 & 242 & 122.5994 & 1.49104 \\
\hline 3485 & 62.2283 & 252.3176 & 259.0095 & 242 & 122.5994 & 1.49068 \\
\hline 3486 & 62.2453 & 275.2361 & 259.6752 & 241 & 122.5994 & 1.49031 \\
\hline 3487 & 62.2623 & 252.7345 & 262.6212 & 241 & 122.5994 & 1.48994 \\
\hline 3488 & 62.2793 & 234.4055 & 265.4139 & 240 & 122.5994 & 1.48958 \\
\hline 3489 & 62.2963 & 246.1626 & 268.4107 & 240 & 122.5994 & 1.48921 \\
\hline 3490 & 62.3133 & 241.1653 & 267.4294 & 239 & 122.5994 & 1.48885 \\
\hline 3491 & 62.3303 & 252.4949 & 264.5151 & 239 & 122.5994 & 1.48848 \\
\hline 3492 & 62.3473 & 247.2206 & 259.8739 & 238 & 122.5994 & 1.48812 \\
\hline 3493 & 62.3643 & 227.9881 & 257.0828 & 238 & 122.5994 & 1.48775 \\
\hline 3494 & 62.3813 & 224.8094 & 254.4185 & 237 & 122.5994 & 1.48739 \\
\hline 3495 & 62.3983 & 211.5524 & 252.7043 & 236 & 122.5994 & 1.48702 \\
\hline 3496 & 62.4153 & 230.4139 & 252.4831 & 236 & 122.5994 & 1.48666 \\
\hline 3497 & 62.4323 & 230.7449 & 251.7453 & 235 & 122.5994 & 1.48629 \\
\hline 3498 & 62.4493 & 244.8224 & 252.6179 & 235 & 122.5994 & 1.48593 \\
\hline 3499 & 62.4663 & 249.4229 & 252.443 & 234 & 122.5994 & 1.48557 \\
\hline 3500 & 62.4833 & 224.5663 & 252.4034 & 234 & 122.5994 & 1.4852 \\
\hline 3501 & 62.5003 & 234.8542 & 250.3303 & 233 & 122.5994 & 1.48484 \\
\hline 3502 & 62.5173 & 242.315 & 249.2104 & 233 & 122.5994 & 1.48448 \\
\hline 3503 & 62.5343 & 231.7726 & 247.4748 & 232 & 122.5994 & 1.48411 \\
\hline 3504 & 62.5513 & 216.1188 & 246.9235 & 232 & 122.5994 & 1.48375 \\
\hline 3505 & 62.5683 & 224.3499 & 245.5852 & 231 & 122.5994 & 1.48339 \\
\hline 3506 & 62.5853 & 245.0037 & 245.5662 & 231 & 122.5994 & 1.48303 \\
\hline 3507 & 62.6023 & 243.7697 & 244.7814 & 230 & 122.5994 & 1.48267 \\
\hline 3508 & 62.6193 & 223.8844 & 244.7339 & 230 & 122.5994 & 1.4823 \\
\hline 3509 & 62.6363 & 216.2518 & 244.3878 & 230 & 122.5994 & 1.48194 \\
\hline 3510 & 62.6533 & 214.6062 & 244.5517 & 230 & 122.5994 & 1.48158 \\
\hline 3511 & 62.6703 & 228.7355 & 244.7644 & 229 & 122.5994 & 1.48122 \\
\hline 3512 & 62.6873 & 241.9177 & 246.7971 & 229 & 122.5994 & 1.48086 \\
\hline 3513 & 62.7043 & 252.3522 & 248.9123 & 229 & 122.5994 & 1.4805 \\
\hline 3514 & 62.7213 & 233.8865 & 249.802 & 228 & 122.5994 & 1.48014 \\
\hline 3515 & 62.7383 & 225.1607 & 251.3707 & 228 & 122.5994 & 1.47978 \\
\hline 3516 & 62.7553 & 231.0708 & 251.9054 & 228 & 122.5994 & 1.47942 \\
\hline 3517 & 62.7723 & 247.2716 & 249.8262 & 227 & 122.5994 & 1.47906 \\
\hline 3518 & 62.7893 & 243.3578 & 247.4075 & 227 & 122.5994 & 1.4787 \\
\hline 3519 & 62.8063 & 254.6882 & 244.3219 & 227 & 122.5994 & 1.47834 \\
\hline 3520 & 62.8233 & 235.6196 & 241.4206 & 227 & 122.5994 & 1.47798 \\
\hline 3521 & 62.8403 & 227.2494 & 238.5667 & 226 & 122.5994 & 1.47762 \\
\hline 3522 & 62.8573 & 230.2872 & 237.8998 & 226 & 122.5994 & 1.47726 \\
\hline 3523 & 62.8743 & 226.3572 & 238.0271 & 226 & 122.5994 & 1.4769 \\
\hline 3524 & 62.8913 & 260.2266 & 237.6409 & 225 & 122.5994 & 1.47655 \\
\hline 3525 & 62.9083 & 232.7959 & 238.3549 & 225 & 122.5994 & 1.47619 \\
\hline 3526 & 62.9253 & 267.6963 & 238.549 & 225 & 122.5994 & 1.47583 \\
\hline
\end{tabular}




\begin{tabular}{|c|c|c|c|c|c|c|}
\hline 3527 & 62.9423 & 218.9546 & 237.8337 & 225 & 122.5994 & 1.47547 \\
\hline 3528 & 62.9593 & 228.7388 & 235.6836 & 224 & 122.5994 & 1.47512 \\
\hline 3529 & 62.9763 & 228.2624 & 234.5383 & 224 & 122.5994 & 1.47476 \\
\hline 3530 & 62.9933 & 216.9425 & 233.6586 & 224 & 122.5994 & 1.4744 \\
\hline 3531 & 63.0103 & 221.7078 & 234.1804 & 225 & 122.5994 & 1.47404 \\
\hline 3532 & 63.0273 & 239.7522 & 234.0417 & 225 & 122.5994 & 1.47369 \\
\hline 3533 & 63.0443 & 231.1132 & 235.5089 & 226 & 122.5994 & 1.47333 \\
\hline 3534 & 63.0613 & 234.2264 & 236.3295 & 226 & 122.5994 & 1.47297 \\
\hline 3535 & 63.0783 & 239.7557 & 236.912 & 226 & 122.5994 & 1.47262 \\
\hline 3536 & 63.0953 & 229.5321 & 237.7575 & 227 & 122.5994 & 1.47226 \\
\hline 3537 & 63.1123 & 251.01 & 237.1507 & 227 & 122.5994 & 1.47191 \\
\hline 3538 & 63.1293 & 234.5864 & 237.7863 & 228 & 122.5994 & 1.47155 \\
\hline 3539 & 63.1463 & 263.725 & 237.9858 & 228 & 122.5994 & 1.4712 \\
\hline 3540 & 63.1633 & 248.6988 & 238.8133 & 228 & 122.5994 & 1.47084 \\
\hline 3541 & 63.1803 & 239.8119 & 241.4347 & 229 & 122.5994 & 1.47049 \\
\hline 3542 & 63.1973 & 227.8139 & 243.6864 & 229 & 122.5994 & 1.47013 \\
\hline 3543 & 63.2143 & 254.8735 & 247.2125 & 230 & 122.5994 & 1.46978 \\
\hline 3544 & 63.2313 & 251.6524 & 248.803 & 230 & 122.5994 & 1.46942 \\
\hline 3545 & 63.2483 & 220.6344 & 248.5888 & 230 & 122.5994 & 1.46907 \\
\hline 3546 & 63.2653 & 238.4016 & 247.9932 & 231 & 122.5994 & 1.46872 \\
\hline 3547 & 63.2823 & 244.0977 & 245.9989 & 231 & 122.5994 & 1.46836 \\
\hline 3548 & 63.2993 & 231.0708 & 245.6178 & 232 & 122.5994 & 1.46801 \\
\hline 3549 & 63.3163 & 232.0541 & 245.3541 & 232 & 122.5994 & 1.46765 \\
\hline 3550 & 63.3333 & 240.8288 & 246.3104 & 232 & 122.5994 & 1.4673 \\
\hline 3551 & 63.3503 & 237.6646 & 249.4004 & 233 & 122.5994 & 1.46695 \\
\hline 3552 & 63.3673 & 239.7108 & 252.0701 & 233 & 122.5994 & 1.4666 \\
\hline 3553 & 63.3843 & 269.1784 & 254.9529 & 234 & 122.5994 & 1.46624 \\
\hline 3554 & 63.4013 & 261.7727 & 253.9092 & 233 & 122.5994 & 1.46589 \\
\hline 3555 & 63.4183 & 262.918 & 252.0654 & 233 & 122.5994 & 1.46554 \\
\hline 3556 & 63.4353 & 245.231 & 249.7282 & 233 & 122.5994 & 1.46519 \\
\hline 3557 & 63.4523 & 246.9242 & 247.9566 & 233 & 122.5994 & 1.46484 \\
\hline 3558 & 63.4693 & 245.8441 & 247.0857 & 233 & 122.5994 & 1.46449 \\
\hline 3559 & 63.4863 & 230.6939 & 246.5252 & 233 & 122.5994 & 1.46413 \\
\hline 3560 & 63.5033 & 222.8689 & 246.3609 & 233 & 122.5994 & 1.46378 \\
\hline 3561 & 63.5203 & 226.1944 & 246.9868 & 233 & 122.5994 & 1.46343 \\
\hline 3562 & 63.5373 & 238.6485 & 248.2589 & 233 & 122.5994 & 1.46308 \\
\hline 3563 & 63.5543 & 255.6231 & 249.3152 & 233 & 122.5994 & 1.46273 \\
\hline 3564 & 63.5713 & 243.6347 & 249.3408 & 233 & 122.5994 & 1.46238 \\
\hline 3565 & 63.5883 & 232.0347 & 246.8145 & 232 & 122.5994 & 1.46203 \\
\hline 3566 & 63.6053 & 248.6273 & 245.0529 & 232 & 122.5994 & 1.46168 \\
\hline 3567 & 63.6223 & 282.51 & 243.7283 & 232 & 122.5994 & 1.46133 \\
\hline 3568 & 63.6393 & 282.2612 & 243.9311 & 232 & 122.5994 & 1.46098 \\
\hline 3569 & 63.6563 & 271.2172 & 243.8695 & 232 & 122.5994 & 1.46063 \\
\hline 3570 & 63.6733 & 248.7297 & 244.2441 & 232 & 122.5994 & 1.46028 \\
\hline 3571 & 63.6903 & 243.3367 & 245.189 & 232 & 122.5994 & 1.45994 \\
\hline 3572 & 63.7073 & 230.5795 & 246.8044 & 232 & 122.5994 & 1.45959 \\
\hline 3573 & 63.7243 & 233.1935 & 248.8377 & 232 & 122.5994 & 1.45924 \\
\hline 3574 & 63.7413 & 253.2949 & 250.8085 & 232 & 122.5994 & 1.45889 \\
\hline 3575 & 63.7583 & 261.825 & 251.8493 & 232 & 122.5994 & 1.45854 \\
\hline
\end{tabular}




\begin{tabular}{|c|c|c|c|c|c|c|}
\hline 3576 & 63.7753 & 274.4935 & 251.2795 & 231 & 122.5994 & 1.45819 \\
\hline 3577 & 63.7923 & 272.4778 & 252.0271 & 231 & 122.5994 & 1.45785 \\
\hline 3578 & 63.8093 & 278.6228 & 253.8987 & 231 & 122.5994 & 1.4575 \\
\hline 3579 & 63.8263 & 282.2281 & 256.7394 & 231 & 122.5994 & 1.45715 \\
\hline 3580 & 63.8433 & 259.2722 & 259.3009 & 231 & 122.5994 & 1.4568 \\
\hline 3581 & 63.8603 & 272.8573 & 260.1447 & 231 & 122.5994 & 1.45646 \\
\hline 3582 & 63.8773 & 292.8968 & 258.9886 & 231 & 122.5994 & 1.45611 \\
\hline 3583 & 63.8943 & 299.9218 & 257.069 & 231 & 122.5994 & 1.45576 \\
\hline 3584 & 63.9113 & 264.5653 & 255.7621 & 231 & 122.5994 & 1.45542 \\
\hline 3585 & 63.9283 & 283.368 & 255.4085 & 231 & 122.5994 & 1.45507 \\
\hline 3586 & 63.9453 & 268.8319 & 255.6326 & 231 & 122.5994 & 1.45473 \\
\hline 3587 & 63.9623 & 294.4779 & 256.3775 & 231 & 122.5994 & 1.45438 \\
\hline 3588 & 63.9793 & 324.0432 & 258.2243 & 231 & 122.5994 & 1.45404 \\
\hline 3589 & 63.9963 & 337.6023 & 260.8512 & 231 & 122.5994 & 1.45369 \\
\hline 3590 & 64.0133 & 397.4474 & 264.7043 & 231 & 122.5994 & 1.45335 \\
\hline 3591 & 64.0303 & 428.4965 & 267.7186 & 231 & 122.5994 & 1.453 \\
\hline 3592 & 64.0473 & 469.8648 & 272.0078 & 231 & 122.5994 & 1.45266 \\
\hline 3593 & 64.0643 & 500.4862 & 278.9958 & 231 & 122.5994 & 1.45231 \\
\hline 3594 & 64.0813 & 549.4392 & 288.7014 & 231 & 122.5994 & 1.45197 \\
\hline 3595 & 64.0983 & 510.5422 & 297.8828 & 231 & 122.5994 & 1.45162 \\
\hline 3596 & 64.1153 & 429.4005 & 301.407 & 231 & 122.5994 & 1.45128 \\
\hline 3597 & 64.1323 & 378.5326 & 299.5054 & 231 & 122.5994 & 1.45094 \\
\hline 3598 & 64.1493 & 334.9511 & 298.292 & 231 & 122.5994 & 1.45059 \\
\hline 3599 & 64.1663 & 330.0928 & 303.8687 & 231 & 122.5994 & 1.45025 \\
\hline 3600 & 64.1833 & 333.8184 & 321.4822 & 231 & 122.5994 & 1.44991 \\
\hline 3601 & 64.2003 & 332.6774 & 355.5543 & 231 & 122.5994 & 1.44956 \\
\hline 3602 & 64.2173 & 347.4016 & 406.9326 & 231 & 122.5994 & 1.44922 \\
\hline 3603 & 64.2343 & 372.748 & 467.5518 & 230 & 122.5994 & 1.44888 \\
\hline 3604 & 64.2513 & 392.2861 & 518.567 & 230 & 122.5994 & 1.44854 \\
\hline 3605 & 64.2683 & 374.7557 & 528.9076 & 230 & 122.5994 & 1.44819 \\
\hline 3606 & 64.2853 & 353.0004 & 492.1115 & 230 & 122.5994 & 1.44785 \\
\hline 3607 & 64.3023 & 324.2038 & 433.0846 & 230 & 122.5994 & 1.44751 \\
\hline 3608 & 64.3193 & 293.7123 & 376.8703 & 230 & 122.5994 & 1.44717 \\
\hline 3609 & 64.3363 & 253.0279 & 336.1826 & 230 & 122.5994 & 1.44683 \\
\hline 3610 & 64.3533 & 246.3316 & 314.8159 & 230 & 122.5994 & 1.44649 \\
\hline 3611 & 64.3703 & 248.5846 & 312.0635 & 230 & 122.5994 & 1.44614 \\
\hline 3612 & 64.3873 & 257.4968 & 325.0533 & 230 & 122.5994 & 1.4458 \\
\hline 3613 & 64.4043 & 253.3005 & 348.6636 & 230 & 122.5994 & 1.44546 \\
\hline 3614 & 64.4213 & 245.0361 & 373.8868 & 230 & 122.5994 & 1.44512 \\
\hline 3615 & 64.4383 & 234.0286 & 386.2824 & 230 & 122.5994 & 1.44478 \\
\hline 3616 & 64.4553 & 250.4647 & 374.9984 & 230 & 122.5994 & 1.44444 \\
\hline 3617 & 64.4723 & 243.3886 & 347.4539 & 230 & 122.5994 & 1.4441 \\
\hline 3618 & 64.4893 & 232.688 & 318.1207 & 230 & 122.5994 & 1.44376 \\
\hline 3619 & 64.5063 & 220.8161 & 295.4872 & 230 & 122.5994 & 1.44342 \\
\hline 3620 & 64.5233 & 219.0692 & 282.2784 & 230 & 122.5994 & 1.44308 \\
\hline 3621 & 64.5403 & 227.0438 & 277.1438 & 230 & 122.5994 & 1.44275 \\
\hline 3622 & 64.5573 & 244.7568 & 275.7871 & 230 & 122.5994 & 1.44241 \\
\hline 3623 & 64.5743 & 199.4726 & 273.0067 & 230 & 122.5994 & 1.44207 \\
\hline 3624 & 64.5913 & 241.185 & 267.224 & 230 & 122.5994 & 1.44173 \\
\hline
\end{tabular}




$\begin{array}{rrrrrrr}3625 & 64.6083 & 237.3464 & 260.8151 & 230 & 122.5994 & 1.44139 \\ 3626 & 64.6253 & 234.0241 & 255.7663 & 230 & 122.5994 & 1.44105 \\ 3627 & 64.6423 & 233.9562 & 253.0908 & 230 & 122.5994 & 1.44071 \\ 3628 & 64.6593 & 224.9268 & 253.2819 & 230 & 122.5994 & 1.44038 \\ 3629 & 64.6763 & 225.9601 & 256.3821 & 230 & 122.5994 & 1.44004 \\ 3630 & 64.6933 & 241.058 & 261.7651 & 230 & 122.5994 & 1.4397 \\ 3631 & 64.7103 & 250.6713 & 267.4142 & 230 & 122.5994 & 1.43936 \\ 3632 & 64.7273 & 259.531 & 269.8828 & 230 & 122.5994 & 1.43903 \\ 3633 & 64.7443 & 261.0581 & 267.3787 & 230 & 122.5994 & 1.43869 \\ 3634 & 64.7613 & 254.469 & 261.8393 & 230 & 122.5994 & 1.43835 \\ 3635 & 64.7783 & 256.9691 & 255.1545 & 229 & 122.5994 & 1.43802 \\ 3636 & 64.7953 & 234.1212 & 251.6324 & 229 & 122.5994 & 1.43768 \\ 3637 & 64.8123 & 234.8373 & 250.9341 & 229 & 122.5994 & 1.43735 \\ 3638 & 64.8293 & 241.1811 & 252.618 & 229 & 122.5994 & 1.43701 \\ 3639 & 64.8463 & 250.1186 & 254.2668 & 229 & 122.5994 & 1.43667 \\ 3640 & 64.8633 & 239.7241 & 256.0832 & 229 & 122.5994 & 1.43634 \\ 3641 & 64.8803 & 237.0118 & 256.7134 & 229 & 122.5994 & 1.436 \\ 3642 & 64.8973 & 249.5588 & 256.4521 & 229 & 122.5994 & 1.43567 \\ 3643 & 64.9143 & 251.9772 & 254.8222 & 229 & 122.5994 & 1.43533 \\ 3644 & 64.9313 & 248.8015 & 252.0814 & 229 & 122.5994 & 1.435 \\ 3645 & 64.9483 & 234.7097 & 249.0306 & 229 & 122.5994 & 1.43466 \\ 3646 & 64.9653 & 228.3874 & 246.0337 & 229 & 122.5994 & 1.43433 \\ 3647 & 64.9823 & 229.1058 & 243.7207 & 229 & 122.5994 & 1.434\end{array}$


No. Pos. [ ${ }^{\circ} 2$ Th. lobs [cts] Icalc [cts] lback [cts] CT [s] ESD D spacings

\begin{tabular}{|c|c|c|c|c|c|c|}
\hline 1 & 3.0003 & 2088.484 & 2088.484 & 2088.484 & 122.5994 & 29.42347 \\
\hline 2 & 3.0173 & 2070.651 & 2062.922 & 2062.922 & 122.5994 & 29.25773 \\
\hline 3 & 3.0343 & 2080.429 & 2037.36 & 2037.36 & 122.5994 & 29.09385 \\
\hline 4 & 3.0513 & 1899.285 & 2011.798 & 2011.798 & 122.5994 & 28.9318 \\
\hline 5 & 3.0683 & 1874.127 & 1986.237 & 1986.237 & 122.5994 & 28.77154 \\
\hline 6 & 3.0853 & 1857.061 & 1960.675 & 1960.675 & 122.5994 & 28.61305 \\
\hline 7 & 3.1023 & 1766.901 & 1935.113 & 1935.113 & 122.5994 & 28.45629 \\
\hline 8 & 3.1193 & 1680.239 & 1909.551 & 1909.551 & 122.5994 & 28.30124 \\
\hline 9 & 3.1363 & 1716.57 & 1883.989 & 1883.989 & 122.5994 & 28.14788 \\
\hline 10 & 3.1533 & 1679.736 & 1858.427 & 1858.427 & 122.5994 & 27.99617 \\
\hline 11 & 3.1703 & 1645.481 & 1832.866 & 1832.866 & 122.5994 & 27.84608 \\
\hline 12 & 3.1873 & 1653.145 & 1807.304 & 1807.304 & 122.5994 & 27.6976 \\
\hline 13 & 3.2043 & 1601.642 & 1781.742 & 1781.742 & 122.5994 & 27.55069 \\
\hline 14 & 3.2213 & 1564.688 & 1756.18 & 1756.18 & 122.5994 & 27.40534 \\
\hline 15 & 3.2383 & 1561.832 & 1730.618 & 1730.618 & 122.5994 & 27.26151 \\
\hline 16 & 3.2553 & 1518.773 & 1705.057 & 1705.056 & 122.5994 & 27.11918 \\
\hline 17 & 3.2723 & 1516.518 & 1679.495 & 1679.495 & 122.5994 & 26.97833 \\
\hline 18 & 3.2893 & 1535.183 & 1653.933 & 1653.933 & 122.5994 & 26.83894 \\
\hline 19 & 3.3063 & 1532.809 & 1628.371 & 1628.371 & 122.5994 & 26.70098 \\
\hline 20 & 3.3233 & 1534.59 & 1602.809 & 1602.809 & 122.5994 & 26.56443 \\
\hline 21 & 3.3403 & 1481.172 & 1577.247 & 1577.247 & 122.5994 & 26.42928 \\
\hline 22 & 3.3573 & 1500.472 & 1551.686 & 1551.685 & 122.5994 & 26.29549 \\
\hline 23 & 3.3743 & 1524.307 & 1526.124 & 1526.124 & 122.5994 & 26.16305 \\
\hline 24 & 3.3913 & 1498.343 & 1500.562 & 1500.562 & 122.5994 & 26.03194 \\
\hline 25 & 3.4083 & 1487.095 & 1475 & 1475 & 122.5994 & 25.90213 \\
\hline 26 & 3.4253 & 1459.722 & 1461.625 & 1461.625 & 122.5994 & 25.77362 \\
\hline 27 & 3.4423 & 1364.089 & 1448.25 & 1448.25 & 122.5994 & 25.64637 \\
\hline 28 & 3.4593 & 1359.152 & 1434.875 & 1434.875 & 122.5994 & 25.52038 \\
\hline 29 & 3.4763 & 1357.169 & 1421.5 & 1421.5 & 122.5994 & 25.39561 \\
\hline 30 & 3.4933 & 1353.325 & 1408.125 & 1408.125 & 122.5994 & 25.27207 \\
\hline 31 & 3.5103 & 1385.576 & 1394.75 & 1394.75 & 122.5994 & 25.14971 \\
\hline 32 & 3.5273 & 1403.762 & 1381.375 & 1381.375 & 122.5994 & 25.02854 \\
\hline 33 & 3.5443 & 1350.286 & 1368 & 1368 & 122.5994 & 24.90853 \\
\hline 34 & 3.5613 & 1340.518 & 1354.625 & 1354.625 & 122.5994 & 24.78967 \\
\hline 35 & 3.5783 & 1325.535 & 1341.25 & 1341.25 & 122.5994 & 24.67194 \\
\hline 36 & 3.5953 & 1339.685 & 1327.875 & 1327.875 & 122.5994 & 24.55532 \\
\hline 37 & 3.6123 & 1352.589 & 1314.5 & 1314.5 & 122.5994 & 24.4398 \\
\hline 38 & 3.6293 & 1307.862 & 1301.125 & 1301.125 & 122.5994 & 24.32536 \\
\hline 39 & 3.6463 & 1243.686 & 1287.75 & 1287.75 & 122.5994 & 24.21198 \\
\hline 40 & 3.6633 & 1243.83 & 1274.375 & 1274.375 & 122.5994 & 24.09966 \\
\hline 41 & 3.6803 & 1276.674 & 1261 & 1261 & 122.5994 & 23.98838 \\
\hline 42 & 3.6973 & 1275.338 & 1247.625 & 1247.625 & 122.5994 & 23.87812 \\
\hline 43 & 3.7143 & 1210.876 & 1234.25 & 1234.25 & 122.5994 & 23.76887 \\
\hline 44 & 3.7313 & 1223.424 & 1220.875 & 1220.875 & 122.5994 & 23.66062 \\
\hline 45 & 3.7483 & 1265.108 & 1207.5 & 1207.5 & 122.5994 & 23.55335 \\
\hline 46 & 3.7653 & 1226.351 & 1194.125 & 1194.125 & 122.5994 & 23.44704 \\
\hline 47 & 3.7823 & 1143.291 & 1180.75 & 1180.75 & 122.5994 & 23.3417 \\
\hline
\end{tabular}




\begin{tabular}{|c|c|c|c|c|c|c|}
\hline 48 & 3.7993 & 1123.255 & 1167.375 & 1167.375 & 122.5994 & 23.23729 \\
\hline 49 & 3.8163 & 1208.134 & 1154 & 1154 & 122.5994 & 23.13382 \\
\hline 50 & 3.8333 & 1124.441 & 1148.635 & 1148.635 & 122.5994 & 23.03126 \\
\hline 51 & 3.8503 & 1161.271 & 1143.271 & 1143.271 & 122.5994 & 22.92961 \\
\hline 52 & 3.8673 & 1137.243 & 1137.906 & 1137.906 & 122.5994 & 22.82886 \\
\hline 53 & 3.8843 & 1094.538 & 1132.542 & 1132.542 & 122.5994 & 22.72898 \\
\hline 54 & 3.9013 & 1133.808 & 1127.177 & 1127.177 & 122.5994 & 22.62998 \\
\hline 55 & 3.9183 & 1110.52 & 1121.813 & 1121.813 & 122.5994 & 22.53184 \\
\hline 56 & 3.9353 & 1056.946 & 1116.448 & 1116.448 & 122.5994 & 22.43454 \\
\hline 57 & 3.9523 & 1086.308 & 1111.083 & 1111.083 & 122.5994 & 22.33808 \\
\hline 58 & 3.9693 & 1067.014 & 1105.719 & 1105.719 & 122.5994 & 22.24245 \\
\hline 59 & 3.9863 & 1058.022 & 1100.354 & 1100.354 & 122.5994 & 22.14763 \\
\hline 60 & 4.0033 & 1115.06 & 1094.99 & 1094.99 & 122.5994 & 22.05362 \\
\hline 61 & 4.0203 & 1093.676 & 1089.625 & 1089.625 & 122.5994 & 21.9604 \\
\hline 62 & 4.0373 & 1074.736 & 1084.26 & 1084.26 & 122.5994 & 21.86797 \\
\hline 63 & 4.0543 & 1043.28 & 1078.896 & 1078.896 & 122.5994 & 21.77632 \\
\hline 64 & 4.0713 & 1045.61 & 1073.531 & 1073.531 & 122.5994 & 21.68543 \\
\hline 65 & 4.0883 & 1067.037 & 1068.167 & 1068.167 & 122.5994 & 21.59529 \\
\hline 66 & 4.1053 & 1082.507 & 1062.802 & 1062.802 & 122.5994 & 21.50591 \\
\hline 67 & 4.1223 & 1054.601 & 1057.438 & 1057.438 & 122.5994 & 21.41726 \\
\hline 68 & 4.1393 & 1043.28 & 1052.073 & 1052.073 & 122.5994 & 21.32933 \\
\hline 69 & 4.1563 & 1034.346 & 1046.708 & 1046.708 & 122.5994 & 21.24213 \\
\hline 70 & 4.1733 & 1045.968 & 1041.344 & 1041.344 & 122.5994 & 21.15564 \\
\hline 71 & 4.1903 & 1065.974 & 1035.979 & 1035.979 & 122.5994 & 21.06985 \\
\hline 72 & 4.2073 & 1069.651 & 1030.615 & 1030.615 & 122.5994 & 20.98475 \\
\hline 73 & 4.2243 & 1029.954 & 1025.25 & 1025.25 & 122.5994 & 20.90034 \\
\hline 74 & 4.2413 & 1021.368 & 1019.885 & 1019.885 & 122.5994 & 20.81661 \\
\hline 75 & 4.2583 & 1028.072 & 1014.521 & 1014.521 & 122.5994 & 20.73354 \\
\hline 76 & 4.2753 & 1021.551 & 1009.156 & 1009.156 & 122.5994 & 20.65114 \\
\hline 77 & 4.2923 & 983.97 & 1003.792 & 1003.792 & 122.5994 & 20.56939 \\
\hline 78 & 4.3093 & 965.0688 & 998.4271 & 998.4271 & 122.5994 & 20.48828 \\
\hline 79 & 4.3263 & 958.8174 & 993.0625 & 993.0625 & 122.5994 & 20.40781 \\
\hline 80 & 4.3433 & 933.3521 & 987.6979 & 987.6979 & 122.5994 & 20.32797 \\
\hline 81 & 4.3603 & 1005.426 & 982.3333 & 982.3333 & 122.5994 & 20.24875 \\
\hline 82 & 4.3773 & 1003.737 & 976.9688 & 976.9688 & 122.5994 & 20.17015 \\
\hline 83 & 4.3943 & 954.207 & 971.6042 & 971.6042 & 122.5994 & 20.09216 \\
\hline 84 & 4.4113 & 942.4806 & 966.2396 & 966.2396 & 122.5994 & 20.01477 \\
\hline 85 & 4.4283 & 947.473 & 960.875 & 960.875 & 122.5994 & 19.93797 \\
\hline 86 & 4.4453 & 908.3139 & 955.5104 & 955.5104 & 122.5994 & 19.86176 \\
\hline 87 & 4.4623 & 910.2558 & 950.1458 & 950.1458 & 122.5994 & 19.78613 \\
\hline 88 & 4.4793 & 927.9086 & 944.7813 & 944.7813 & 122.5994 & 19.71108 \\
\hline 89 & 4.4963 & 946.6708 & 939.4167 & 939.4167 & 122.5994 & 19.63659 \\
\hline 90 & 4.5133 & 898.6734 & 934.0521 & 934.0521 & 122.5994 & 19.56267 \\
\hline 91 & 4.5303 & 910.8856 & 928.6875 & 928.6875 & 122.5994 & 19.48929 \\
\hline 92 & 4.5473 & 941.2012 & 923.3229 & 923.3229 & 122.5994 & 19.41647 \\
\hline 93 & 4.5643 & 942.5076 & 917.9583 & 917.9583 & 122.5994 & 19.34419 \\
\hline 94 & 4.5813 & 918.0992 & 912.5938 & 912.5938 & 122.5994 & 19.27245 \\
\hline 95 & 4.5983 & 935.8293 & 907.2292 & 907.2292 & 122.5994 & 19.20124 \\
\hline 96 & 4.6153 & 914.4609 & 901.8646 & 901.8646 & 122.5994 & 19.13055 \\
\hline
\end{tabular}




\begin{tabular}{|c|c|c|c|c|c|c|}
\hline 97 & 4.6323 & 960.8444 & 896.5 & 896.5 & 122.5994 & 19.06038 \\
\hline 98 & 4.6493 & 870.2663 & 890.7292 & 890.7292 & 122.5994 & 18.99073 \\
\hline 99 & 4.6663 & 873.472 & 884.9583 & 884.9583 & 122.5994 & 18.92158 \\
\hline 100 & 4.6833 & 894.5906 & 879.1875 & 879.1875 & 122.5994 & 18.85293 \\
\hline 101 & 4.7003 & 862.9869 & 873.4167 & 873.4167 & 122.5994 & 18.78479 \\
\hline 102 & 4.7173 & 877.8851 & 867.6458 & 867.6458 & 122.5994 & 18.71713 \\
\hline 103 & 4.7343 & 832.8128 & 861.875 & 861.875 & 122.5994 & 18.64996 \\
\hline 104 & 4.7513 & 851.8931 & 856.1042 & 856.1042 & 122.5994 & 18.58327 \\
\hline 105 & 4.7683 & 867.4461 & 850.3333 & 850.3333 & 122.5994 & 18.51705 \\
\hline 106 & 4.7853 & 861.5545 & 844.5625 & 844.5625 & 122.5994 & 18.45131 \\
\hline 107 & 4.8023 & 860.4046 & 838.7917 & 838.7917 & 122.5994 & 18.38603 \\
\hline 108 & 4.8193 & 836.9516 & 833.0208 & 833.0208 & 122.5994 & 18.32121 \\
\hline 109 & 4.8363 & 839.1411 & 827.25 & 827.25 & 122.5994 & 18.25685 \\
\hline 110 & 4.8533 & 845.2665 & 821.4792 & 821.4792 & 122.5994 & 18.19294 \\
\hline 111 & 4.8703 & 853.6596 & 815.7083 & 815.7083 & 122.5994 & 18.12947 \\
\hline 112 & 4.8873 & 851.1273 & 809.9375 & 809.9375 & 122.5994 & 18.06645 \\
\hline 113 & 4.9043 & 833.8081 & 804.1667 & 804.1667 & 122.5994 & 18.00386 \\
\hline 114 & 4.9213 & 809.7758 & 798.3958 & 798.3958 & 122.5994 & 17.94171 \\
\hline 115 & 4.9383 & 822.2405 & 792.625 & 792.625 & 122.5994 & 17.87998 \\
\hline 116 & 4.9553 & 786.3859 & 786.8542 & 786.8542 & 122.5994 & 17.81868 \\
\hline 117 & 4.9723 & 795.2321 & 781.0833 & 781.0833 & 122.5994 & 17.7578 \\
\hline 118 & 4.9893 & 789.4391 & 775.3125 & 775.3125 & 122.5994 & 17.69733 \\
\hline 119 & 5.0063 & 790.0497 & 769.5417 & 769.5417 & 122.5994 & 17.63727 \\
\hline 120 & 5.0233 & 785.8001 & 763.7708 & 763.7708 & 122.5994 & 17.57762 \\
\hline 121 & 5.0403 & 746.5331 & 758 & 758 & 122.5994 & 17.51837 \\
\hline 122 & 5.0573 & 749.9623 & 757.0833 & 757.0833 & 122.5994 & 17.45953 \\
\hline 123 & 5.0743 & 736.6274 & 756.1667 & 756.1667 & 122.5994 & 17.40107 \\
\hline 124 & 5.0913 & 749.9647 & 755.25 & 755.25 & 122.5994 & 17.34301 \\
\hline 125 & 5.1083 & 785.9534 & 754.3333 & 754.3333 & 122.5994 & 17.28533 \\
\hline 126 & 5.1253 & 791.3501 & 753.4167 & 753.4167 & 122.5994 & 17.22803 \\
\hline 127 & 5.1423 & 762.0002 & 752.5 & 752.5 & 122.5994 & 17.17112 \\
\hline 128 & 5.1593 & 787.4759 & 751.5833 & 751.5833 & 122.5994 & 17.11458 \\
\hline 129 & 5.1763 & 763.102 & 750.6667 & 750.6667 & 122.5994 & 17.05841 \\
\hline 130 & 5.1933 & 763.5733 & 749.75 & 749.75 & 122.5994 & 17.00261 \\
\hline 131 & 5.2103 & 715.4383 & 748.8333 & 748.8333 & 122.5994 & 16.94717 \\
\hline 132 & 5.2273 & 806.3866 & 747.9167 & 747.9167 & 122.5994 & 16.89209 \\
\hline 133 & 5.2443 & 786.5588 & 747 & 747 & 122.5994 & 16.83737 \\
\hline 134 & 5.2613 & 745.1681 & 746.0833 & 746.0833 & 122.5994 & 16.78301 \\
\hline 135 & 5.2783 & 773.9186 & 745.1667 & 745.1667 & 122.5994 & 16.72899 \\
\hline 136 & 5.2953 & 733.7011 & 744.25 & 744.25 & 122.5994 & 16.67532 \\
\hline 137 & 5.3123 & 724.0005 & 743.3333 & 743.3333 & 122.5994 & 16.622 \\
\hline 138 & 5.3293 & 733.8591 & 742.4167 & 742.4167 & 122.5994 & 16.56901 \\
\hline 139 & 5.3463 & 744.9481 & 741.5 & 741.5 & 122.5994 & 16.51637 \\
\hline 140 & 5.3633 & 718.4827 & 740.5833 & 740.5833 & 122.5994 & 16.46405 \\
\hline 141 & 5.3803 & 723.8021 & 739.6667 & 739.6667 & 122.5994 & 16.41207 \\
\hline 142 & 5.3973 & 740.387 & 738.75 & 738.75 & 122.5994 & 16.36041 \\
\hline 143 & 5.4143 & 756.2641 & 737.8333 & 737.8333 & 122.5994 & 16.30908 \\
\hline 144 & 5.4313 & 753.7349 & 736.9167 & 736.9167 & 122.5994 & 16.25807 \\
\hline 145 & 5.4483 & 720.9822 & 736 & 736 & 122.5994 & 16.20738 \\
\hline
\end{tabular}




\begin{tabular}{|c|c|c|c|c|c|c|}
\hline 146 & 5.4653 & 705.4033 & 735.2083 & 735.2083 & 122.5994 & 16.15701 \\
\hline 147 & 5.4823 & 767.2824 & 734.4167 & 734.4167 & 122.5994 & 16.10695 \\
\hline 148 & 5.4993 & 763.699 & 733.625 & 733.625 & 122.5994 & 16.05719 \\
\hline 149 & 5.5163 & 745.3039 & 732.8333 & 732.8333 & 122.5994 & 16.00775 \\
\hline 150 & 5.5333 & 741.9281 & 732.0417 & 732.0417 & 122.5994 & 15.9586 \\
\hline 151 & 5.5503 & 726.5662 & 731.25 & 731.25 & 122.5994 & 15.90976 \\
\hline 152 & 5.5673 & 719.5698 & 730.4583 & 730.4583 & 122.5994 & 15.86122 \\
\hline 153 & 5.5843 & 717.8256 & 729.6667 & 729.6667 & 122.5994 & 15.81297 \\
\hline 154 & 5.6013 & 718.0313 & 728.875 & 728.875 & 122.5994 & 15.76502 \\
\hline 155 & 5.6183 & 749.3954 & 728.0833 & 728.0833 & 122.5994 & 15.71735 \\
\hline 156 & 5.6353 & 723.6286 & 727.2917 & 727.2917 & 122.5994 & 15.66998 \\
\hline 157 & 5.6523 & 709.8791 & 726.5 & 726.5 & 122.5994 & 15.62289 \\
\hline 158 & 5.6693 & 754.9626 & 725.7083 & 725.7083 & 122.5994 & 15.57608 \\
\hline 159 & 5.6863 & 777.7235 & 724.9167 & 724.9167 & 122.5994 & 15.52955 \\
\hline 160 & 5.7033 & 729.8759 & 724.125 & 724.125 & 122.5994 & 15.4833 \\
\hline 161 & 5.7203 & 716.499 & 723.3333 & 723.3333 & 122.5994 & 15.43732 \\
\hline 162 & 5.7373 & 707.553 & 722.5417 & 722.5417 & 122.5994 & 15.39162 \\
\hline 163 & 5.7543 & 715.3678 & 721.75 & 721.75 & 122.5994 & 15.34618 \\
\hline 164 & 5.7713 & 715.463 & 720.9583 & 720.9583 & 122.5994 & 15.30102 \\
\hline 165 & 5.7883 & 742.0638 & 720.1667 & 720.1667 & 122.5994 & 15.25612 \\
\hline 166 & 5.8053 & 751.7105 & 719.375 & 719.375 & 122.5994 & 15.21148 \\
\hline 167 & 5.8223 & 725.1583 & 718.5833 & 718.5833 & 122.5994 & 15.16711 \\
\hline 168 & 5.8393 & 721.4184 & 717.7917 & 717.7917 & 122.5994 & 15.12299 \\
\hline 169 & 5.8563 & 726.937 & 717 & 717 & 122.5994 & 15.07913 \\
\hline 170 & 5.8733 & 708.6074 & 718.0684 & 716.5 & 122.5994 & 15.03552 \\
\hline 171 & 5.8903 & 703.9252 & 718.4914 & 716 & 122.5994 & 14.99216 \\
\hline 172 & 5.9073 & 725.8121 & 718.2168 & 715.5 & 122.5994 & 14.94906 \\
\hline 173 & 5.9243 & 745.8607 & 717.9741 & 715 & 122.5994 & 14.9062 \\
\hline 174 & 5.9413 & 762.7141 & 717.7694 & 714.5 & 122.5994 & 14.86358 \\
\hline 175 & 5.9583 & 779.7119 & 717.6107 & 714 & 122.5994 & 14.82121 \\
\hline 176 & 5.9753 & 729.5687 & 717.5078 & 713.5 & 122.5994 & 14.77909 \\
\hline 177 & 5.9923 & 768.8582 & 717.4736 & 713 & 122.5994 & 14.7372 \\
\hline 178 & 6.0093 & 774.9706 & 717.5246 & 712.5 & 122.5994 & 14.69554 \\
\hline 179 & 6.0263 & 745.4459 & 717.6827 & 712 & 122.5994 & 14.65413 \\
\hline 180 & 6.0433 & 796.6609 & 717.9771 & 711.5 & 122.5994 & 14.61294 \\
\hline 181 & 6.0603 & 738.8474 & 718.4476 & 711 & 122.5994 & 14.57199 \\
\hline 182 & 6.0773 & 798.3653 & 719.1501 & 710.5 & 122.5994 & 14.53126 \\
\hline 183 & 6.0943 & 765.5395 & 720.1667 & 710 & 122.5994 & 14.49077 \\
\hline 184 & 6.1113 & 814.4387 & 721.6318 & 709.5 & 122.5994 & 14.4505 \\
\hline 185 & 6.1283 & 758.7729 & 723.8015 & 709 & 122.5994 & 14.41045 \\
\hline 186 & 6.1453 & 805.5941 & 727.2279 & 708.5 & 122.5994 & 14.37062 \\
\hline 187 & 6.1623 & 823.5458 & 733.1192 & 708 & 122.5994 & 14.33102 \\
\hline 188 & 6.1793 & 758.1238 & 743.8615 & 707.5 & 122.5994 & 14.29163 \\
\hline 189 & 6.1963 & 834.1672 & 763.3489 & 707 & 122.5994 & 14.25246 \\
\hline 190 & 6.2133 & 815.7106 & 796.3358 & 706.5 & 122.5994 & 14.2135 \\
\hline 191 & 6.2303 & 870.181 & 846.0515 & 706 & 122.5994 & 14.17475 \\
\hline 192 & 6.2473 & 929.5732 & 910.038 & 705.5 & 122.5994 & 14.13622 \\
\hline 193 & 6.2643 & 922.5617 & 974.337 & 705 & 122.5994 & 14.0979 \\
\hline 194 & 6.2813 & 921.4295 & 1010.572 & 704.5 & 122.5994 & 14.05978 \\
\hline
\end{tabular}




\begin{tabular}{|c|c|c|c|c|c|c|}
\hline 195 & 6.2983 & 976.7061 & 995.4883 & 704 & 122.5994 & 14.02187 \\
\hline 196 & 6.3153 & 987.3685 & 939.4379 & 703.5 & 122.5994 & 13.98416 \\
\hline 197 & 6.3323 & 942.3164 & 871.9681 & 703 & 122.5994 & 13.94666 \\
\hline 198 & 6.3493 & 838.9301 & 813.877 & 702.5 & 122.5994 & 13.90935 \\
\hline 199 & 6.3663 & 790.8751 & 772.27 & 702 & 122.5994 & 13.87225 \\
\hline 200 & 6.3833 & 779.9772 & 746.0114 & 701.5 & 122.5994 & 13.83534 \\
\hline 201 & 6.4003 & 778.9249 & 730.6902 & 701 & 122.5994 & 13.79863 \\
\hline 202 & 6.4173 & 763.7187 & 721.8669 & 700.5 & 122.5994 & 13.76212 \\
\hline 203 & 6.4343 & 733.886 & 716.4648 & 700 & 122.5994 & 13.72579 \\
\hline 204 & 6.4513 & 697.52 & 712.7888 & 699.5 & 122.5994 & 13.68966 \\
\hline 205 & 6.4683 & 732.9887 & 710.0305 & 699 & 122.5994 & 13.65372 \\
\hline 206 & 6.4853 & 779.722 & 707.8222 & 698.5 & 122.5994 & 13.61797 \\
\hline 207 & 6.5023 & 758.7458 & 705.9832 & 698 & 122.5994 & 13.5824 \\
\hline 208 & 6.5193 & 738.0062 & 704.4111 & 697.5 & 122.5994 & 13.54702 \\
\hline 209 & 6.5363 & 759.133 & 703.039 & 697 & 122.5994 & 13.51183 \\
\hline 210 & 6.5533 & 708.6602 & 701.8207 & 696.5 & 122.5994 & 13.47682 \\
\hline 211 & 6.5703 & 710.1468 & 700.7221 & 696 & 122.5994 & 13.44198 \\
\hline 212 & 6.5873 & 747.3822 & 699.7184 & 695.5 & 122.5994 & 13.40733 \\
\hline 213 & 6.6043 & 733.7466 & 698.7906 & 695 & 122.5994 & 13.37286 \\
\hline 214 & 6.6213 & 704.2023 & 697.9243 & 694.5 & 122.5994 & 13.33856 \\
\hline 215 & 6.6383 & 695.99 & 697.1083 & 694 & 122.5994 & 13.30444 \\
\hline 216 & 6.6553 & 685.8302 & 696.334 & 693.5 & 122.5994 & 13.2705 \\
\hline 217 & 6.6723 & 689.5653 & 695.5942 & 693 & 122.5994 & 13.23672 \\
\hline 218 & 6.6893 & 714.7826 & 693.8329 & 693 & 122.5994 & 13.20312 \\
\hline 219 & 6.7063 & 687.1618 & 693 & 693 & 122.5994 & 13.16969 \\
\hline 220 & 6.7233 & 704.5767 & 693 & 693 & 122.5994 & 13.13643 \\
\hline 221 & 6.7403 & 722.1721 & 693 & 693 & 122.5994 & 13.10334 \\
\hline 222 & 6.7573 & 715.5288 & 693 & 693 & 122.5994 & 13.07041 \\
\hline 223 & 6.7743 & 742.2933 & 693 & 693 & 122.5994 & 13.03765 \\
\hline 224 & 6.7913 & 703.6074 & 693 & 693 & 122.5994 & 13.00505 \\
\hline 225 & 6.8083 & 717.8664 & 693 & 693 & 122.5994 & 12.97261 \\
\hline 226 & 6.8253 & 692.3367 & 693 & 693 & 122.5994 & 12.94034 \\
\hline 227 & 6.8423 & 677.4269 & 693 & 693 & 122.5994 & 12.90823 \\
\hline 228 & 6.8593 & 708.6309 & 693 & 693 & 122.5994 & 12.87627 \\
\hline 229 & 6.8763 & 656.1492 & 693 & 693 & 122.5994 & 12.84448 \\
\hline 230 & 6.8933 & 669.3071 & 693 & 693 & 122.5994 & 12.81284 \\
\hline 231 & 6.9103 & 656.1643 & 693 & 693 & 122.5994 & 12.78136 \\
\hline 232 & 6.9273 & 700.8489 & 693 & 693 & 122.5994 & 12.75003 \\
\hline 233 & 6.9443 & 696.9966 & 693 & 693 & 122.5994 & 12.71886 \\
\hline 234 & 6.9613 & 661.1798 & 693 & 693 & 122.5994 & 12.68783 \\
\hline 235 & 6.9783 & 711.9625 & 693 & 693 & 122.5994 & 12.65696 \\
\hline 236 & 6.9953 & 684.7021 & 693 & 693 & 122.5994 & 12.62624 \\
\hline 237 & 7.0123 & 662.3534 & 693 & 693 & 122.5994 & 12.59567 \\
\hline 238 & 7.0293 & 741.5546 & 693 & 693 & 122.5994 & 12.56525 \\
\hline 239 & 7.0463 & 703.1107 & 693 & 693 & 122.5994 & 12.53497 \\
\hline 240 & 7.0633 & 673.7763 & 693 & 693 & 122.5994 & 12.50484 \\
\hline 241 & 7.0803 & 694.3043 & 693 & 693 & 122.5994 & 12.47485 \\
\hline 242 & 7.0973 & 703.6367 & 692.8333 & 692.8333 & 122.5994 & 12.44501 \\
\hline 243 & 7.1143 & 683.1988 & 692.6667 & 692.6667 & 122.5994 & 12.41531 \\
\hline
\end{tabular}




\begin{tabular}{|c|c|c|c|c|c|c|}
\hline 244 & 7.1313 & 669.6414 & 692.5 & 692.5 & 122.5994 & 12.38575 \\
\hline 245 & 7.1483 & 674.7849 & 692.3333 & 692.3333 & 122.5994 & 12.35633 \\
\hline 246 & 7.1653 & 690.906 & 692.1667 & 692.1667 & 122.5994 & 12.32706 \\
\hline 247 & 7.1823 & 672.7387 & 692 & 692 & 122.5994 & 12.29792 \\
\hline 248 & 7.1993 & 698.9217 & 691.8333 & 691.8333 & 122.5994 & 12.26892 \\
\hline 249 & 7.2163 & 691.6831 & 691.6667 & 691.6667 & 122.5994 & 12.24005 \\
\hline 250 & 7.2333 & 701.1962 & 691.5 & 691.5 & 122.5994 & 12.21132 \\
\hline 251 & 7.2503 & 704.1959 & 691.3333 & 691.3333 & 122.5994 & 12.18273 \\
\hline 252 & 7.2673 & 681.2032 & 691.1667 & 691.1667 & 122.5994 & 12.15427 \\
\hline 253 & 7.2843 & 694.5952 & 691 & 691 & 122.5994 & 12.12594 \\
\hline 254 & 7.3013 & 708.1628 & 690.8333 & 690.8333 & 122.5994 & 12.09774 \\
\hline 255 & 7.3183 & 707.1919 & 690.6667 & 690.6667 & 122.5994 & 12.06968 \\
\hline 256 & 7.3353 & 718.1203 & 690.5 & 690.5 & 122.5994 & 12.04175 \\
\hline 257 & 7.3523 & 718.1553 & 690.3333 & 690.3333 & 122.5994 & 12.01394 \\
\hline 258 & 7.3693 & 701.6539 & 690.1667 & 690.1667 & 122.5994 & 11.98627 \\
\hline 259 & 7.3863 & 666.0553 & 690 & 690 & 122.5994 & 11.95872 \\
\hline 260 & 7.4033 & 662.816 & 689.8333 & 689.8333 & 122.5994 & 11.93129 \\
\hline 261 & 7.4203 & 685.3364 & 689.6667 & 689.6667 & 122.5994 & 11.904 \\
\hline 262 & 7.4373 & 714.6569 & 689.5 & 689.5 & 122.5994 & 11.87683 \\
\hline 263 & 7.4543 & 698.5381 & 689.3333 & 689.3333 & 122.5994 & 11.84978 \\
\hline 264 & 7.4713 & 702.0933 & 689.1667 & 689.1667 & 122.5994 & 11.82285 \\
\hline 265 & 7.4883 & 687.7866 & 689 & 689 & 122.5994 & 11.79605 \\
\hline 266 & 7.5053 & 657.9217 & 688.5 & 688.5 & 122.5994 & 11.76937 \\
\hline 267 & 7.5223 & 703.3265 & 688 & 688 & 122.5994 & 11.74281 \\
\hline 268 & 7.5393 & 699.8272 & 687.5 & 687.5 & 122.5994 & 11.71637 \\
\hline 269 & 7.5563 & 671.7196 & 687 & 687 & 122.5994 & 11.69005 \\
\hline 270 & 7.5733 & 690.3291 & 686.5 & 686.5 & 122.5994 & 11.66385 \\
\hline 271 & 7.5903 & 684.2051 & 686 & 686 & 122.5994 & 11.63776 \\
\hline 272 & 7.6073 & 689.9111 & 685.5 & 685.5 & 122.5994 & 11.61179 \\
\hline 273 & 7.6243 & 742.0564 & 685 & 685 & 122.5994 & 11.58594 \\
\hline 274 & 7.6413 & 708.3575 & 684.5 & 684.5 & 122.5994 & 11.5602 \\
\hline 275 & 7.6583 & 674.0901 & 684 & 684 & 122.5994 & 11.53458 \\
\hline 276 & 7.6753 & 685.155 & 683.5 & 683.5 & 122.5994 & 11.50907 \\
\hline 277 & 7.6923 & 686.4332 & 683 & 683 & 122.5994 & 11.48367 \\
\hline 278 & 7.7093 & 737.697 & 682.5 & 682.5 & 122.5994 & 11.45839 \\
\hline 279 & 7.7263 & 667.0242 & 682 & 682 & 122.5994 & 11.43321 \\
\hline 280 & 7.7433 & 734.2416 & 681.5 & 681.5 & 122.5994 & 11.40815 \\
\hline 281 & 7.7603 & 682.5295 & 681 & 681 & 122.5994 & 11.3832 \\
\hline 282 & 7.7773 & 732.9211 & 680.5 & 680.5 & 122.5994 & 11.35835 \\
\hline 283 & 7.7943 & 703.3441 & 680 & 680 & 122.5994 & 11.33362 \\
\hline 284 & 7.8113 & 721.1307 & 679.5 & 679.5 & 122.5994 & 11.30899 \\
\hline 285 & 7.8283 & 742.4828 & 679 & 679 & 122.5994 & 11.28447 \\
\hline 286 & 7.8453 & 678.7825 & 678.5 & 678.5 & 122.5994 & 11.26006 \\
\hline 287 & 7.8623 & 691.3491 & 678 & 678 & 122.5994 & 11.23575 \\
\hline 288 & 7.8793 & 660.4834 & 677.5 & 677.5 & 122.5994 & 11.21154 \\
\hline 289 & 7.8963 & 672.918 & 677 & 677 & 122.5994 & 11.18745 \\
\hline 290 & 7.9133 & 707.5952 & 676.7044 & 676.7044 & 122.5994 & 11.16345 \\
\hline 291 & 7.9303 & 663.9814 & 676.4089 & 676.4089 & 122.5994 & 11.13956 \\
\hline 292 & 7.9473 & 708.9657 & 676.1133 & 676.1133 & 122.5994 & 11.11577 \\
\hline
\end{tabular}




\begin{tabular}{|c|c|c|c|c|c|c|}
\hline 293 & 7.9643 & 706.8885 & 675.8177 & 675.8177 & 122.5994 & 11.09208 \\
\hline 294 & 7.9813 & 693.2866 & 675.5222 & 675.5221 & 122.5994 & 11.06849 \\
\hline 295 & 7.9983 & 678.4267 & 675.2266 & 675.2266 & 122.5994 & 11.045 \\
\hline 296 & 8.0153 & 654.383 & 674.931 & 674.931 & 122.5994 & 11.02162 \\
\hline 297 & 8.0323 & 696.8614 & 674.6354 & 674.6354 & 122.5994 & 10.99833 \\
\hline 298 & 8.0493 & 717.3175 & 674.3398 & 674.3398 & 122.5994 & 10.97514 \\
\hline 299 & 8.0663 & 720.8677 & 674.0443 & 674.0443 & 122.5994 & 10.95204 \\
\hline 300 & 8.0833 & 723.5629 & 679.7476 & 673.7487 & 122.5994 & 10.92905 \\
\hline 301 & 8.1003 & 683.2279 & 682.6638 & 673.4531 & 122.5994 & 10.90615 \\
\hline 302 & 8.1173 & 665.4188 & 682.7805 & 673.1576 & 122.5994 & 10.88335 \\
\hline 303 & 8.1343 & 689.1215 & 682.9255 & 672.862 & 122.5994 & 10.86064 \\
\hline 304 & 8.1513 & 707.5327 & 683.1013 & 672.5664 & 122.5994 & 10.83803 \\
\hline 305 & 8.1683 & 725.0349 & 683.3107 & 672.2708 & 122.5994 & 10.81551 \\
\hline 306 & 8.1853 & 739.8538 & 683.557 & 671.9753 & 122.5994 & 10.79309 \\
\hline 307 & 8.2023 & 714.7714 & 683.844 & 671.6797 & 122.5994 & 10.77075 \\
\hline 308 & 8.2193 & 703.3912 & 684.1757 & 671.3841 & 122.5994 & 10.74852 \\
\hline 309 & 8.2363 & 710.9153 & 684.5569 & 671.0885 & 122.5994 & 10.72637 \\
\hline 310 & 8.2533 & 709.4059 & 684.9927 & 670.793 & 122.5994 & 10.70431 \\
\hline 311 & 8.2703 & 725.5638 & 685.4892 & 670.4974 & 122.5994 & 10.68235 \\
\hline 312 & 8.2873 & 723.241 & 686.0533 & 670.2018 & 122.5994 & 10.66047 \\
\hline 313 & 8.3043 & 718.2123 & 686.6928 & 669.9063 & 122.5994 & 10.63869 \\
\hline 314 & 8.3213 & 766.5598 & 686.9238 & 669.1178 & 122.5994 & 10.61699 \\
\hline 315 & 8.3383 & 746.5186 & 687.2493 & 668.3294 & 122.5994 & 10.59538 \\
\hline 316 & 8.3553 & 764.3091 & 687.6816 & 667.541 & 122.5994 & 10.57386 \\
\hline 317 & 8.3723 & 750.794 & 688.2345 & 666.7526 & 122.5994 & 10.55243 \\
\hline 318 & 8.3893 & 795.5863 & 688.924 & 665.9642 & 122.5994 & 10.53109 \\
\hline 319 & 8.4063 & 773.5017 & 689.7691 & 665.1758 & 122.5994 & 10.50983 \\
\hline 320 & 8.4233 & 786.5377 & 690.7922 & 664.3874 & 122.5994 & 10.48865 \\
\hline 321 & 8.4403 & 779.7103 & 692.0198 & 663.599 & 122.5994 & 10.46757 \\
\hline 322 & 8.4573 & 773.3739 & 693.4839 & 662.8105 & 122.5994 & 10.44656 \\
\hline 323 & 8.4743 & 820.0106 & 695.2234 & 662.0221 & 122.5994 & 10.42565 \\
\hline 324 & 8.4913 & 823.7054 & 697.2866 & 661.2337 & 122.5994 & 10.40481 \\
\hline 325 & 8.5083 & 846.1618 & 699.7361 & 660.4453 & 122.5994 & 10.38406 \\
\hline 326 & 8.5253 & 864.2005 & 702.6563 & 659.6569 & 122.5994 & 10.36339 \\
\hline 327 & 8.5423 & 858.6746 & 706.1649 & 658.8685 & 122.5994 & 10.34281 \\
\hline 328 & 8.5593 & 868.9617 & 710.4346 & 658.0801 & 122.5994 & 10.3223 \\
\hline 329 & 8.5763 & 837.9138 & 715.7241 & 657.2917 & 122.5994 & 10.30188 \\
\hline 330 & 8.5933 & 884.4689 & 722.4248 & 656.5033 & 122.5994 & 10.28154 \\
\hline 331 & 8.6103 & 918.7938 & 731.1263 & 655.7148 & 122.5994 & 10.26128 \\
\hline 332 & 8.6273 & 975.174 & 742.6896 & 654.9264 & 122.5994 & 10.24109 \\
\hline 333 & 8.6443 & 950.5495 & 758.3284 & 654.138 & 122.5994 & 10.22099 \\
\hline 334 & 8.6613 & 999.5653 & 779.6681 & 653.3496 & 122.5994 & 10.20097 \\
\hline 335 & 8.6783 & 1013.274 & 808.7521 & 652.5612 & 122.5994 & 10.18102 \\
\hline 336 & 8.6953 & 1056.57 & 847.9681 & 651.7728 & 122.5994 & 10.16116 \\
\hline 337 & 8.7123 & 1100.816 & 899.8327 & 650.9844 & 122.5994 & 10.14137 \\
\hline 338 & 8.7293 & 1181.964 & 966.2544 & 649.7979 & 122.5994 & 10.12166 \\
\hline 339 & 8.7463 & 1248.724 & 1049.25 & 648.6113 & 122.5994 & 10.10202 \\
\hline 340 & 8.7633 & 1297.887 & 1149.16 & 647.4248 & 122.5994 & 10.08246 \\
\hline 341 & 8.7803 & 1387.396 & 1264.405 & 646.2383 & 122.5994 & 10.06298 \\
\hline
\end{tabular}




\begin{tabular}{|c|c|c|c|c|c|c|}
\hline 342 & 8.7973 & 1509.413 & 1390.65 & 645.0518 & 122.5994 & 10.04357 \\
\hline 343 & 8.8143 & 1599.354 & 1519.88 & 643.8652 & 122.5994 & 10.02424 \\
\hline 344 & 8.8313 & 1652.469 & 1639.542 & 642.6787 & 122.5994 & 10.00498 \\
\hline 345 & 8.8483 & 1808.568 & 1733.018 & 641.4922 & 122.5994 & 9.9858 \\
\hline 346 & 8.8653 & 1972.941 & 1783.152 & 640.3057 & 122.5994 & 9.96669 \\
\hline 347 & 8.8823 & 2211.671 & 1779.017 & 639.1191 & 122.5994 & 9.94765 \\
\hline 348 & 8.8993 & 2458.329 & 1721.445 & 637.9326 & 122.5994 & 9.92869 \\
\hline 349 & 8.9163 & 2733.53 & 1622.427 & 636.7461 & 122.5994 & 9.90979 \\
\hline 350 & 8.9333 & 2802.861 & 1499.103 & 635.5596 & 122.5994 & 9.89097 \\
\hline 351 & 8.9503 & 2516.879 & 1367.597 & 634.373 & 122.5994 & 9.87222 \\
\hline 352 & 8.9673 & 1932.53 & 1239.932 & 633.1865 & 122.5994 & 9.85355 \\
\hline 353 & 8.9843 & 1333.198 & 1123.742 & 632 & 122.5994 & 9.83494 \\
\hline 354 & 9.0013 & 1021.644 & 1023.086 & 630.8135 & 122.5994 & 9.8164 \\
\hline 355 & 9.0183 & 885.8011 & 939.3439 & 629.627 & 122.5994 & 9.79794 \\
\hline 356 & 9.0353 & 771.6311 & 872.0378 & 628.4404 & 122.5994 & 9.77954 \\
\hline 357 & 9.0523 & 721.9141 & 819.4688 & 627.2539 & 122.5994 & 9.76121 \\
\hline 358 & 9.0693 & 725.5429 & 779.3 & 626.0674 & 122.5994 & 9.74295 \\
\hline 359 & 9.0863 & 713.9452 & 749.0228 & 624.8809 & 122.5994 & 9.72476 \\
\hline 360 & 9.1033 & 688.4865 & 726.2816 & 623.6943 & 122.5994 & 9.70664 \\
\hline 361 & 9.1203 & 685.1237 & 709.0751 & 622.5078 & 122.5994 & 9.68859 \\
\hline 362 & 9.1373 & 666.5003 & 695.8202 & 621.3213 & 122.5994 & 9.6706 \\
\hline 363 & 9.1543 & 693.4469 & 685.3399 & 620.1348 & 122.5994 & 9.65268 \\
\hline 364 & 9.1713 & 685.2116 & 676.8012 & 618.9482 & 122.5994 & 9.63482 \\
\hline 365 & 9.1883 & 646.9754 & 669.635 & 617.7617 & 122.5994 & 9.61704 \\
\hline 366 & 9.2053 & 657.5123 & 663.4633 & 616.5752 & 122.5994 & 9.59931 \\
\hline 367 & 9.2223 & 647.0888 & 658.0359 & 615.3887 & 122.5994 & 9.58166 \\
\hline 368 & 9.2393 & 668.1293 & 653.1857 & 614.2021 & 122.5994 & 9.56407 \\
\hline 369 & 9.2563 & 633.3683 & 648.7982 & 613.0156 & 122.5994 & 9.54654 \\
\hline 370 & 9.2733 & 611.3063 & 644.7908 & 611.8291 & 122.5994 & 9.52908 \\
\hline 371 & 9.2903 & 605.3287 & 641.1028 & 610.6426 & 122.5994 & 9.51168 \\
\hline 372 & 9.3073 & 624.665 & 637.6863 & 609.4561 & 122.5994 & 9.49434 \\
\hline 373 & 9.3243 & 615.1585 & 634.5033 & 608.2695 & 122.5994 & 9.47707 \\
\hline 374 & 9.3413 & 650.9207 & 631.5222 & 607.083 & 122.5994 & 9.45986 \\
\hline 375 & 9.3583 & 671.5282 & 628.7169 & 605.8965 & 122.5994 & 9.44272 \\
\hline 376 & 9.3753 & 584.1621 & 626.0655 & 604.71 & 122.5994 & 9.42563 \\
\hline 377 & 9.3923 & 626.899 & 623.5492 & 603.5234 & 122.5994 & 9.40861 \\
\hline 378 & 9.4093 & 598.6807 & 621.1522 & 602.3369 & 122.5994 & 9.39165 \\
\hline 379 & 9.4263 & 622.3345 & 618.8605 & 601.1504 & 122.5994 & 9.37475 \\
\hline 380 & 9.4433 & 617.7429 & 616.6626 & 599.9639 & 122.5994 & 9.35791 \\
\hline 381 & 9.4603 & 670.4041 & 614.5482 & 598.7773 & 122.5994 & 9.34113 \\
\hline 382 & 9.4773 & 596.3037 & 612.5084 & 597.5908 & 122.5994 & 9.32441 \\
\hline 383 & 9.4943 & 603.5034 & 610.5355 & 596.4043 & 122.5994 & 9.30776 \\
\hline 384 & 9.5113 & 634.774 & 608.6227 & 595.2178 & 122.5994 & 9.29116 \\
\hline 385 & 9.5283 & 671.5728 & 606.7642 & 594.0313 & 122.5994 & 9.27462 \\
\hline 386 & 9.5453 & 594.7448 & 604.5564 & 592.4466 & 122.5994 & 9.25814 \\
\hline 387 & 9.5623 & 598.9191 & 602.3931 & 590.862 & 122.5994 & 9.24172 \\
\hline 388 & 9.5793 & 648.4145 & 600.27 & 589.2773 & 122.5994 & 9.22536 \\
\hline 389 & 9.5963 & 648.0278 & 598.1835 & 587.6927 & 122.5994 & 9.20905 \\
\hline 390 & 9.6133 & 607.7729 & 596.1305 & 586.1081 & 122.5994 & 9.19281 \\
\hline
\end{tabular}




\begin{tabular}{|c|c|c|c|c|c|c|}
\hline 391 & 9.6303 & 599.172 & 594.108 & 584.5234 & 122.5994 & 9.17662 \\
\hline 392 & 9.6473 & 601.1253 & 592.1134 & 582.9388 & 122.5994 & 9.16048 \\
\hline 393 & 9.6643 & 610.4229 & 584.3831 & 581.3542 & 122.5994 & 9.14441 \\
\hline 394 & 9.6813 & 614.1897 & 579.7695 & 579.7695 & 122.5994 & 9.12839 \\
\hline 395 & 9.6983 & 595.3711 & 578.1849 & 578.1849 & 122.5994 & 9.11243 \\
\hline 396 & 9.7153 & 590.5214 & 576.6003 & 576.6003 & 122.5994 & 9.09652 \\
\hline 397 & 9.7323 & 565.7998 & 575.0156 & 575.0156 & 122.5994 & 9.08067 \\
\hline 398 & 9.7493 & 562.9835 & 573.431 & 573.431 & 122.5994 & 9.06487 \\
\hline 399 & 9.7663 & 541.8013 & 571.8464 & 571.8464 & 122.5994 & 9.04913 \\
\hline 400 & 9.7833 & 556.4226 & 570.2617 & 570.2617 & 122.5994 & 9.03344 \\
\hline 401 & 9.8003 & 585.6509 & 568.6771 & 568.6771 & 122.5994 & 9.01781 \\
\hline 402 & 9.8173 & 579.5967 & 567.0925 & 567.0924 & 122.5994 & 9.00224 \\
\hline 403 & 9.8343 & 579.1352 & 565.5078 & 565.5078 & 122.5994 & 8.98671 \\
\hline 404 & 9.8513 & 603.7576 & 563.9232 & 563.9232 & 122.5994 & 8.97124 \\
\hline 405 & 9.8683 & 571.564 & 562.3386 & 562.3385 & 122.5994 & 8.95583 \\
\hline 406 & 9.8853 & 565.8 & 560.7539 & 560.7539 & 122.5994 & 8.94046 \\
\hline 407 & 9.9023 & 560.7057 & 559.1693 & 559.1693 & 122.5994 & 8.92515 \\
\hline 408 & 9.9193 & 564.148 & 557.5847 & 557.5846 & 122.5994 & 8.90989 \\
\hline 409 & 9.9363 & 544.4921 & 556 & 556 & 122.5994 & 8.89469 \\
\hline 410 & 9.9533 & 572.2814 & 555.8333 & 555.8333 & 122.5994 & 8.87953 \\
\hline 411 & 9.9703 & 542.0375 & 555.6667 & 555.6667 & 122.5994 & 8.86443 \\
\hline 412 & 9.9873 & 569.3639 & 555.5 & 555.5 & 122.5994 & 8.84938 \\
\hline 413 & 10.0043 & 547.3478 & 555.3333 & 555.3333 & 122.5994 & 8.83438 \\
\hline 414 & 10.0213 & 580.7864 & 555.1667 & 555.1667 & 122.5994 & 8.81943 \\
\hline 415 & 10.0383 & 563.91 & 555 & 555 & 122.5994 & 8.80454 \\
\hline 416 & 10.0553 & 583.9236 & 554.8333 & 554.8333 & 122.5994 & 8.78969 \\
\hline 417 & 10.0723 & 553.9045 & 554.6667 & 554.6667 & 122.5994 & 8.77489 \\
\hline 418 & 10.0893 & 598.9312 & 554.5 & 554.5 & 122.5994 & 8.76014 \\
\hline 419 & 10.1063 & 585.7076 & 554.3333 & 554.3333 & 122.5994 & 8.74545 \\
\hline 420 & 10.1233 & 571.2942 & 554.1667 & 554.1667 & 122.5994 & 8.7308 \\
\hline 421 & 10.1403 & 577.0514 & 554 & 554 & 122.5994 & 8.7162 \\
\hline 422 & 10.1573 & 600.4592 & 553.8333 & 553.8333 & 122.5994 & 8.70165 \\
\hline 423 & 10.1743 & 523.3293 & 553.6667 & 553.6667 & 122.5994 & 8.68715 \\
\hline 424 & 10.1913 & 514.946 & 553.5 & 553.5 & 122.5994 & 8.6727 \\
\hline 425 & 10.2083 & 548.2771 & 553.3333 & 553.3333 & 122.5994 & 8.65829 \\
\hline 426 & 10.2253 & 523.179 & 553.1667 & 553.1667 & 122.5994 & 8.64394 \\
\hline 427 & 10.2423 & 542.0975 & 553 & 553 & 122.5994 & 8.62963 \\
\hline 428 & 10.2593 & 541.7591 & 552.8333 & 552.8333 & 122.5994 & 8.61537 \\
\hline 429 & 10.2763 & 556.9704 & 552.6667 & 552.6667 & 122.5994 & 8.60115 \\
\hline 430 & 10.2933 & 552.6242 & 552.5 & 552.5 & 122.5994 & 8.58698 \\
\hline 431 & 10.3103 & 544.1594 & 552.3333 & 552.3333 & 122.5994 & 8.57286 \\
\hline 432 & 10.3273 & 561.7005 & 552.1667 & 552.1667 & 122.5994 & 8.55879 \\
\hline 433 & 10.3443 & 556.1221 & 552 & 552 & 122.5994 & 8.54476 \\
\hline 434 & 10.3613 & 545.863 & 551.7083 & 551.7083 & 122.5994 & 8.53078 \\
\hline 435 & 10.3783 & 545.9585 & 551.4167 & 551.4167 & 122.5994 & 8.51685 \\
\hline 436 & 10.3953 & 533.5894 & 551.125 & 551.125 & 122.5994 & 8.50296 \\
\hline 437 & 10.4123 & 540.7235 & 550.8333 & 550.8333 & 122.5994 & 8.48911 \\
\hline 438 & 10.4293 & 550.372 & 550.5417 & 550.5417 & 122.5994 & 8.47531 \\
\hline 439 & 10.4463 & 567.8111 & 550.25 & 550.25 & 122.5994 & 8.46156 \\
\hline
\end{tabular}




\begin{tabular}{|c|c|c|c|c|c|c|}
\hline 440 & 10.4633 & 599.7384 & 549.9583 & 549.9583 & 122.5994 & 8.44785 \\
\hline 441 & 10.4803 & 598.3179 & 549.6667 & 549.6667 & 122.5994 & 8.43418 \\
\hline 442 & 10.4973 & 578.7151 & 549.375 & 549.375 & 122.5994 & 8.42056 \\
\hline 443 & 10.5143 & 576.2462 & 549.0833 & 549.0833 & 122.5994 & 8.40699 \\
\hline 444 & 10.5313 & 601.2836 & 548.7917 & 548.7917 & 122.5994 & 8.39345 \\
\hline 445 & 10.5483 & 631.6786 & 548.5 & 548.5 & 122.5994 & 8.37996 \\
\hline 446 & 10.5653 & 633.5854 & 548.2083 & 548.2083 & 122.5994 & 8.36652 \\
\hline 447 & 10.5823 & 613.1817 & 547.9167 & 547.9167 & 122.5994 & 8.35312 \\
\hline 448 & 10.5993 & 590.1278 & 547.625 & 547.625 & 122.5994 & 8.33976 \\
\hline 449 & 10.6163 & 571.6011 & 547.3333 & 547.3333 & 122.5994 & 8.32644 \\
\hline 450 & 10.6333 & 532.6498 & 547.0417 & 547.0417 & 122.5994 & 8.31317 \\
\hline 451 & 10.6503 & 512.2086 & 546.75 & 546.75 & 122.5994 & 8.29994 \\
\hline 452 & 10.6673 & 510.7403 & 546.4583 & 546.4583 & 122.5994 & 8.28675 \\
\hline 453 & 10.6843 & 520.6708 & 546.1667 & 546.1667 & 122.5994 & 8.2736 \\
\hline 454 & 10.7013 & 548.7314 & 545.875 & 545.875 & 122.5994 & 8.26049 \\
\hline 455 & 10.7183 & 549.7157 & 545.5833 & 545.5833 & 122.5994 & 8.24743 \\
\hline 456 & 10.7353 & 547.5702 & 545.2917 & 545.2917 & 122.5994 & 8.23441 \\
\hline 457 & 10.7523 & 559.036 & 545 & 545 & 122.5994 & 8.22143 \\
\hline 458 & 10.7693 & 508.0279 & 544.75 & 544.75 & 122.5994 & 8.20849 \\
\hline 459 & 10.7863 & 556.3852 & 544.5 & 544.5 & 122.5994 & 8.19559 \\
\hline 460 & 10.8033 & 521.6434 & 544.25 & 544.25 & 122.5994 & 8.18273 \\
\hline 461 & 10.8203 & 519.3536 & 544 & 544 & 122.5994 & 8.16991 \\
\hline 462 & 10.8373 & 495.0365 & 543.75 & 543.75 & 122.5994 & 8.15713 \\
\hline 463 & 10.8543 & 484.5348 & 543.5 & 543.5 & 122.5994 & 8.1444 \\
\hline 464 & 10.8713 & 509.725 & 543.25 & 543.25 & 122.5994 & 8.1317 \\
\hline 465 & 10.8883 & 537.7977 & 543 & 543 & 122.5994 & 8.11904 \\
\hline 466 & 10.9053 & 510.7632 & 542.75 & 542.75 & 122.5994 & 8.10642 \\
\hline 467 & 10.9223 & 514.2348 & 542.5 & 542.5 & 122.5994 & 8.09384 \\
\hline 468 & 10.9393 & 477.615 & 542.25 & 542.25 & 122.5994 & 8.0813 \\
\hline 469 & 10.9563 & 525.6759 & 542 & 542 & 122.5994 & 8.0688 \\
\hline 470 & 10.9733 & 541.216 & 541.75 & 541.75 & 122.5994 & 8.05634 \\
\hline 471 & 10.9903 & 527.0281 & 541.5 & 541.5 & 122.5994 & 8.04392 \\
\hline 472 & 11.0073 & 545.6675 & 541.25 & 541.25 & 122.5994 & 8.03153 \\
\hline 473 & 11.0243 & 535.0647 & 541 & 541 & 122.5994 & 8.01919 \\
\hline 474 & 11.0413 & 542.0761 & 540.75 & 540.75 & 122.5994 & 8.00688 \\
\hline 475 & 11.0583 & 530.9708 & 540.5 & 540.5 & 122.5994 & 7.99461 \\
\hline 476 & 11.0753 & 500.785 & 540.25 & 540.25 & 122.5994 & 7.98237 \\
\hline 477 & 11.0923 & 502.7511 & 540 & 540 & 122.5994 & 7.97018 \\
\hline 478 & 11.1093 & 525.8877 & 539.75 & 539.75 & 122.5994 & 7.95802 \\
\hline 479 & 11.1263 & 545.5657 & 539.5 & 539.5 & 122.5994 & 7.9459 \\
\hline 480 & 11.1433 & 546.5507 & 539.25 & 539.25 & 122.5994 & 7.93381 \\
\hline 481 & 11.1603 & 535.1071 & 539 & 539 & 122.5994 & 7.92177 \\
\hline 482 & 11.1773 & 535.0223 & 538.375 & 538.375 & 122.5994 & 7.90976 \\
\hline 483 & 11.1943 & 522.2454 & 537.75 & 537.75 & 122.5994 & 7.89778 \\
\hline 484 & 11.2113 & 510.7403 & 537.125 & 537.125 & 122.5994 & 7.88585 \\
\hline 485 & 11.2283 & 526 & 536.5 & 536.5 & 122.5994 & 7.87394 \\
\hline 486 & 11.2453 & 535.5007 & 535.875 & 535.875 & 122.5994 & 7.86208 \\
\hline 487 & 11.2623 & 524.005 & 535.25 & 535.25 & 122.5994 & 7.85025 \\
\hline 488 & 11.2793 & 515.9777 & 534.625 & 534.625 & 122.5994 & 7.83846 \\
\hline
\end{tabular}




\begin{tabular}{|c|c|c|c|c|c|c|}
\hline 489 & 11.2963 & 513.0934 & 534 & 534 & 122.5994 & 7.8267 \\
\hline 490 & 11.3133 & 534.0569 & 533.375 & 533.375 & 122.5994 & 7.81498 \\
\hline 491 & 11.3303 & 535.3272 & 532.75 & 532.75 & 122.5994 & 7.80329 \\
\hline 492 & 11.3473 & 518.7134 & 532.125 & 532.125 & 122.5994 & 7.79164 \\
\hline 493 & 11.3643 & 508.1265 & 531.5 & 531.5 & 122.5994 & 7.78002 \\
\hline 494 & 11.3813 & 495.3924 & 530.875 & 530.875 & 122.5994 & 7.76844 \\
\hline 495 & 11.3983 & 477.3982 & 530.25 & 530.25 & 122.5994 & 7.75689 \\
\hline 496 & 11.4153 & 508.7043 & 529.625 & 529.625 & 122.5994 & 7.74537 \\
\hline 497 & 11.4323 & 544.605 & 529 & 529 & 122.5994 & 7.73389 \\
\hline 498 & 11.4493 & 523.483 & 528.375 & 528.375 & 122.5994 & 7.72245 \\
\hline 499 & 11.4663 & 520.5311 & 527.75 & 527.75 & 122.5994 & 7.71104 \\
\hline 500 & 11.4833 & 507.7055 & 527.125 & 527.125 & 122.5994 & 7.69966 \\
\hline 501 & 11.5003 & 532.9519 & 526.5 & 526.5 & 122.5994 & 7.68832 \\
\hline 502 & 11.5173 & 535.6647 & 525.875 & 525.875 & 122.5994 & 7.67701 \\
\hline 503 & 11.5343 & 522.5575 & 525.25 & 525.25 & 122.5994 & 7.66573 \\
\hline 504 & 11.5513 & 534.9873 & 524.625 & 524.625 & 122.5994 & 7.65449 \\
\hline 505 & 11.5683 & 507.4526 & 524 & 524 & 122.5994 & 7.64328 \\
\hline 506 & 11.5853 & 537.3471 & 523.5286 & 523.5286 & 122.5994 & 7.6321 \\
\hline 507 & 11.6023 & 522.4493 & 523.0573 & 523.0573 & 122.5994 & 7.62095 \\
\hline 508 & 11.6193 & 471.4302 & 522.5859 & 522.5859 & 122.5994 & 7.60984 \\
\hline 509 & 11.6363 & 495.1286 & 522.1146 & 522.1146 & 122.5994 & 7.59876 \\
\hline 510 & 11.6533 & 531.8682 & 521.6432 & 521.6432 & 122.5994 & 7.58772 \\
\hline 511 & 11.6703 & 529.133 & 521.1719 & 521.1719 & 122.5994 & 7.5767 \\
\hline 512 & 11.6873 & 515.3409 & 520.7005 & 520.7005 & 122.5994 & 7.56572 \\
\hline 513 & 11.7043 & 519.554 & 520.2292 & 520.2292 & 122.5994 & 7.55477 \\
\hline 514 & 11.7213 & 502.7712 & 519.7578 & 519.7578 & 122.5994 & 7.54385 \\
\hline 515 & 11.7383 & 526.0778 & 519.2864 & 519.2865 & 122.5994 & 7.53296 \\
\hline 516 & 11.7553 & 482.139 & 518.8151 & 518.8151 & 122.5994 & 7.52211 \\
\hline 517 & 11.7723 & 507.5512 & 518.3438 & 518.3438 & 122.5994 & 7.51128 \\
\hline 518 & 11.7893 & 481.8098 & 517.8724 & 517.8724 & 122.5994 & 7.50049 \\
\hline 519 & 11.8063 & 496.0077 & 517.4011 & 517.401 & 122.5994 & 7.48973 \\
\hline 520 & 11.8233 & 497.4853 & 516.9297 & 516.9297 & 122.5994 & 7.479 \\
\hline 521 & 11.8403 & 540.2824 & 516.4583 & 516.4583 & 122.5994 & 7.4683 \\
\hline 522 & 11.8573 & 526.1308 & 515.987 & 515.987 & 122.5994 & 7.45763 \\
\hline 523 & 11.8743 & 515.2701 & 515.5156 & 515.5156 & 122.5994 & 7.44699 \\
\hline 524 & 11.8913 & 522.2827 & 515.0443 & 515.0443 & 122.5994 & 7.43638 \\
\hline 525 & 11.9083 & 526.7322 & 514.5729 & 514.5729 & 122.5994 & 7.4258 \\
\hline 526 & 11.9253 & 495.9436 & 514.1016 & 514.1016 & 122.5994 & 7.41525 \\
\hline 527 & 11.9423 & 533.2606 & 513.6302 & 513.6302 & 122.5994 & 7.40474 \\
\hline 528 & 11.9593 & 541.2704 & 513.1589 & 513.1589 & 122.5994 & 7.39425 \\
\hline 529 & 11.9763 & 508.4872 & 512.6875 & 512.6875 & 122.5994 & 7.38379 \\
\hline 530 & 11.9933 & 500.4287 & 512.2161 & 512.2161 & 122.5994 & 7.37336 \\
\hline 531 & 12.0103 & 525.4078 & 511.7448 & 511.7448 & 122.5994 & 7.36297 \\
\hline 532 & 12.0273 & 541.5136 & 511.2734 & 511.2734 & 122.5994 & 7.3526 \\
\hline 533 & 12.0443 & 539.8255 & 510.8021 & 510.8021 & 122.5994 & 7.34226 \\
\hline 534 & 12.0613 & 526.5688 & 510.3307 & 510.3307 & 122.5994 & 7.33195 \\
\hline 535 & 12.0783 & 522.2958 & 509.8594 & 509.8594 & 122.5994 & 7.32166 \\
\hline 536 & 12.0953 & 522.2786 & 509.388 & 509.388 & 122.5994 & 7.31141 \\
\hline 537 & 12.1123 & 538.8974 & 508.9167 & 508.9167 & 122.5994 & 7.30119 \\
\hline
\end{tabular}




\begin{tabular}{|c|c|c|c|c|c|c|}
\hline 538 & 12.1293 & 522.1847 & 510.9279 & 508.4453 & 122.5994 & 7.29099 \\
\hline 539 & 12.1463 & 528.1098 & 510.6771 & 507.974 & 122.5994 & 7.28083 \\
\hline 540 & 12.1633 & 539.2382 & 511.7086 & 507.5026 & 122.5994 & 7.27069 \\
\hline 541 & 12.1803 & 542.2525 & 511.6591 & 507.0313 & 122.5994 & 7.26058 \\
\hline 542 & 12.1973 & 556.5862 & 511.6486 & 506.5599 & 122.5994 & 7.2505 \\
\hline 543 & 12.2143 & 571.1713 & 511.7212 & 506.0885 & 122.5994 & 7.24045 \\
\hline 544 & 12.2313 & 550.2069 & 511.8714 & 505.6172 & 122.5994 & 7.23042 \\
\hline 545 & 12.2483 & 558.0958 & 512.1295 & 505.1458 & 122.5994 & 7.22042 \\
\hline 546 & 12.2653 & 563.0003 & 512.5217 & 504.6745 & 122.5994 & 7.21045 \\
\hline 547 & 12.2823 & 597.8994 & 513.0827 & 504.2031 & 122.5994 & 7.20051 \\
\hline 548 & 12.2993 & 587.5387 & 513.8591 & 503.7318 & 122.5994 & 7.1906 \\
\hline 549 & 12.3163 & 600.0799 & 514.9145 & 503.2604 & 122.5994 & 7.18071 \\
\hline 550 & 12.3333 & 597.2092 & 516.3386 & 502.7891 & 122.5994 & 7.17085 \\
\hline 551 & 12.3503 & 603.5096 & 518.2668 & 502.3177 & 122.5994 & 7.16102 \\
\hline 552 & 12.3673 & 605.1713 & 520.9281 & 501.8464 & 122.5994 & 7.15121 \\
\hline 553 & 12.3843 & 600.8652 & 524.7758 & 501.375 & 122.5994 & 7.14144 \\
\hline 554 & 12.4013 & 654.3515 & 530.5287 & 500.6406 & 122.5994 & 7.13168 \\
\hline 555 & 12.4183 & 655.7826 & 540.5331 & 499.9063 & 122.5994 & 7.12196 \\
\hline 556 & 12.4353 & 719.6334 & 558.6821 & 499.1719 & 122.5994 & 7.11226 \\
\hline 557 & 12.4523 & 687.7861 & 590.8825 & 498.4375 & 122.5994 & 7.10259 \\
\hline 558 & 12.4693 & 777.0035 & 643.6766 & 497.7031 & 122.5994 & 7.09294 \\
\hline 559 & 12.4863 & 729.4767 & 720.4691 & 496.9688 & 122.5994 & 7.08333 \\
\hline 560 & 12.5033 & 812.4228 & 815.2217 & 496.2344 & 122.5994 & 7.07373 \\
\hline 561 & 12.5203 & 824.0939 & 903.9208 & 495.5 & 122.5994 & 7.06417 \\
\hline 562 & 12.5373 & 941.5893 & 947.2103 & 494.7656 & 122.5994 & 7.05463 \\
\hline 563 & 12.5543 & 937.7303 & 925.6588 & 494.0313 & 122.5994 & 7.04511 \\
\hline 564 & 12.5713 & 1014.169 & 854.0118 & 493.2969 & 122.5994 & 7.03562 \\
\hline 565 & 12.5883 & 1028.231 & 761.0631 & 492.5625 & 122.5994 & 7.02616 \\
\hline 566 & 12.6053 & 944.6532 & 675.117 & 491.8281 & 122.5994 & 7.01672 \\
\hline 567 & 12.6223 & 766.6119 & 610.0498 & 491.0938 & 122.5994 & 7.00731 \\
\hline 568 & 12.6393 & 650.3993 & 566.7374 & 490.3594 & 122.5994 & 6.99792 \\
\hline 569 & 12.6563 & 604.6921 & 540.238 & 489.625 & 122.5994 & 6.98856 \\
\hline 570 & 12.6733 & 579.8365 & 524.5762 & 488.8906 & 122.5994 & 6.97923 \\
\hline 571 & 12.6903 & 551.8169 & 515.0658 & 488.1563 & 122.5994 & 6.96991 \\
\hline 572 & 12.7073 & 519.2299 & 508.8105 & 487.4219 & 122.5994 & 6.96063 \\
\hline 573 & 12.7243 & 513.2511 & 504.2857 & 486.6875 & 122.5994 & 6.95137 \\
\hline 574 & 12.7413 & 518.5533 & 500.7542 & 485.9531 & 122.5994 & 6.94213 \\
\hline 575 & 12.7583 & 498.8653 & 497.8573 & 485.2188 & 122.5994 & 6.93292 \\
\hline 576 & 12.7753 & 508.4928 & 495.4037 & 484.4844 & 122.5994 & 6.92373 \\
\hline 577 & 12.7923 & 507.485 & 493.2767 & 483.75 & 122.5994 & 6.91457 \\
\hline 578 & 12.8093 & 490.2291 & 491.0181 & 482.6354 & 122.5994 & 6.90543 \\
\hline 579 & 12.8263 & 504.6325 & 488.9526 & 481.5208 & 122.5994 & 6.89631 \\
\hline 580 & 12.8433 & 503.4366 & 487.0391 & 480.4063 & 122.5994 & 6.88722 \\
\hline 581 & 12.8603 & 498.9827 & 485.2472 & 479.2917 & 122.5994 & 6.87816 \\
\hline 582 & 12.8773 & 501.8337 & 483.5535 & 478.1771 & 122.5994 & 6.86912 \\
\hline 583 & 12.8943 & 493.0589 & 481.9399 & 477.0625 & 122.5994 & 6.8601 \\
\hline 584 & 12.9113 & 514.5204 & 480.3924 & 475.9479 & 122.5994 & 6.8511 \\
\hline 585 & 12.9283 & 518.6949 & 478.8999 & 474.8333 & 122.5994 & 6.84213 \\
\hline 586 & 12.9453 & 477.2667 & 475.1172 & 473.7188 & 122.5994 & 6.83319 \\
\hline
\end{tabular}




\begin{tabular}{|c|c|c|c|c|c|c|}
\hline 587 & 12.9623 & 480.2042 & 473.8878 & 472.6042 & 122.5994 & 6.82426 \\
\hline 588 & 12.9793 & 494.4955 & 471.5247 & 471.4896 & 122.5994 & 6.81536 \\
\hline 589 & 12.9963 & 470.1215 & 470.3868 & 470.375 & 122.5994 & 6.80649 \\
\hline 590 & 13.0133 & 457.9423 & 469.2713 & 469.2604 & 122.5994 & 6.79763 \\
\hline 591 & 13.0303 & 473.0068 & 468.1458 & 468.1458 & 122.5994 & 6.7888 \\
\hline 592 & 13.0473 & 461.0565 & 467.0313 & 467.0313 & 122.5994 & 6.78 \\
\hline 593 & 13.0643 & 444.3121 & 465.9167 & 465.9167 & 122.5994 & 6.77121 \\
\hline 594 & 13.0813 & 483.7412 & 464.8021 & 464.8021 & 122.5994 & 6.76245 \\
\hline 595 & 13.0983 & 486.9239 & 463.6875 & 463.6875 & 122.5994 & 6.75371 \\
\hline 596 & 13.1153 & 483.9813 & 462.5729 & 462.5729 & 122.5994 & 6.74499 \\
\hline 597 & 13.1323 & 480.393 & 461.4583 & 461.4583 & 122.5994 & 6.7363 \\
\hline 598 & 13.1493 & 457.7104 & 460.3438 & 460.3438 & 122.5994 & 6.72763 \\
\hline 599 & 13.1663 & 457.3734 & 459.2292 & 459.2292 & 122.5994 & 6.71898 \\
\hline 600 & 13.1833 & 469.4537 & 458.1146 & 458.1146 & 122.5994 & 6.71036 \\
\hline 601 & 13.2003 & 443.7053 & 457 & 457 & 122.5994 & 6.70175 \\
\hline 602 & 13.2173 & 481.0273 & 456.7083 & 456.7083 & 122.5994 & 6.69317 \\
\hline 603 & 13.2343 & 427.1841 & 456.4167 & 456.4167 & 122.5994 & 6.68461 \\
\hline 604 & 13.2513 & 473.9421 & 456.125 & 456.125 & 122.5994 & 6.67607 \\
\hline 605 & 13.2683 & 453.7619 & 455.8333 & 455.8333 & 122.5994 & 6.66756 \\
\hline 606 & 13.2853 & 479.3175 & 455.5417 & 455.5417 & 122.5994 & 6.65907 \\
\hline 607 & 13.3023 & 462.5678 & 455.25 & 455.25 & 122.5994 & 6.65059 \\
\hline 608 & 13.3193 & 469.0941 & 454.9583 & 454.9583 & 122.5994 & 6.64214 \\
\hline 609 & 13.3363 & 442.3975 & 454.6667 & 454.6667 & 122.5994 & 6.63371 \\
\hline 610 & 13.3533 & 457.9423 & 454.375 & 454.375 & 122.5994 & 6.62531 \\
\hline 611 & 13.3703 & 463.4403 & 454.0833 & 454.0833 & 122.5994 & 6.61692 \\
\hline 612 & 13.3873 & 491.646 & 453.7917 & 453.7917 & 122.5994 & 6.60856 \\
\hline 613 & 13.4043 & 441.2486 & 453.5 & 453.5 & 122.5994 & 6.60021 \\
\hline 614 & 13.4213 & 484.8446 & 453.2083 & 453.2083 & 122.5994 & 6.59189 \\
\hline 615 & 13.4383 & 487.4886 & 452.9167 & 452.9167 & 122.5994 & 6.58359 \\
\hline 616 & 13.4553 & 479.9268 & 453.0266 & 452.625 & 122.5994 & 6.57531 \\
\hline 617 & 13.4723 & 465.4739 & 452.7711 & 452.3333 & 122.5994 & 6.56705 \\
\hline 618 & 13.4893 & 459.8999 & 452.7941 & 452.0417 & 122.5994 & 6.55881 \\
\hline 619 & 13.5063 & 467.9509 & 452.5744 & 451.75 & 122.5994 & 6.5506 \\
\hline 620 & 13.5233 & 489.1827 & 452.4022 & 451.4583 & 122.5994 & 6.5424 \\
\hline 621 & 13.5403 & 462.2586 & 452.2095 & 451.1667 & 122.5994 & 6.53423 \\
\hline 622 & 13.5573 & 460.7072 & 452.0657 & 450.875 & 122.5994 & 6.52607 \\
\hline 623 & 13.5743 & 464.5626 & 451.9124 & 450.5833 & 122.5994 & 6.51793 \\
\hline 624 & 13.5913 & 459.6981 & 451.8006 & 450.2917 & 122.5994 & 6.50982 \\
\hline 625 & 13.6083 & 436.8182 & 451.7061 & 450 & 122.5994 & 6.50173 \\
\hline 626 & 13.6253 & 452.0495 & 451.8402 & 449.8958 & 122.5994 & 6.49365 \\
\hline 627 & 13.6423 & 466.8449 & 452.0275 & 449.7917 & 122.5994 & 6.4856 \\
\hline 628 & 13.6593 & 466.4986 & 452.3338 & 449.6875 & 122.5994 & 6.47757 \\
\hline 629 & 13.6763 & 488.9836 & 452.6929 & 449.5833 & 122.5994 & 6.46955 \\
\hline 630 & 13.6933 & 503.8629 & 453.2197 & 449.4792 & 122.5994 & 6.46156 \\
\hline 631 & 13.7103 & 499.2873 & 453.9609 & 449.375 & 122.5994 & 6.45358 \\
\hline 632 & 13.7273 & 510.2767 & 455.1389 & 449.2708 & 122.5994 & 6.44563 \\
\hline 633 & 13.7443 & 509.0326 & 457.1662 & 449.1667 & 122.5994 & 6.4377 \\
\hline 634 & 13.7613 & 498.9987 & 460.7808 & 449.0625 & 122.5994 & 6.42978 \\
\hline 635 & 13.7783 & 480.3403 & 467.045 & 448.9583 & 122.5994 & 6.4218 \\
\hline
\end{tabular}




\begin{tabular}{|c|c|c|c|c|c|c|}
\hline 636 & 13.7953 & 453.877 & 477.049 & 448.8542 & 122.5994 & 6.41401 \\
\hline 637 & 13.8123 & 486.9926 & 491.2131 & 448.75 & 122.5994 & 6.40615 \\
\hline 638 & 13.8293 & 509.4139 & 508.1283 & 448.6458 & 122.5994 & 6.39832 \\
\hline 639 & 13.8463 & 504.223 & 523.1895 & 448.5417 & 122.5994 & 6.3905 \\
\hline 640 & 13.8633 & 508.5097 & 530.2104 & 448.4375 & 122.5994 & 6.3827 \\
\hline 641 & 13.8803 & 510.7403 & 527.1532 & 448.3333 & 122.5994 & 6.37492 \\
\hline 642 & 13.8973 & 510.7695 & 516.1632 & 448.2292 & 122.5994 & 6.36716 \\
\hline 643 & 13.9143 & 525.6073 & 501.554 & 448.125 & 122.5994 & 6.35942 \\
\hline 644 & 13.9313 & 588.4697 & 487.8376 & 448.0208 & 122.5994 & 6.3517 \\
\hline 645 & 13.9483 & 543.1851 & 477.2776 & 447.9167 & 122.5994 & 6.344 \\
\hline 646 & 13.9653 & 513.5572 & 469.998 & 447.8125 & 122.5994 & 6.33631 \\
\hline 647 & 13.9823 & 498.6241 & 465.2811 & 447.7083 & 122.5994 & 6.32865 \\
\hline 648 & 13.9993 & 493.2007 & 462.4573 & 447.6042 & 122.5994 & 6.321 \\
\hline 649 & 14.0163 & 501.2569 & 461.0137 & 447.5 & 122.5994 & 6.31337 \\
\hline 650 & 14.0333 & 471.9806 & 460.5007 & 447.3958 & 122.5994 & 6.30576 \\
\hline 651 & 14.0503 & 433.2004 & 460.1885 & 447.2917 & 122.5994 & 6.29817 \\
\hline 652 & 14.0673 & 470.5426 & 459.2958 & 447.1875 & 122.5994 & 6.2906 \\
\hline 653 & 14.0843 & 425.2071 & 457.6661 & 447.0833 & 122.5994 & 6.28304 \\
\hline 654 & 14.1013 & 454.1277 & 455.5614 & 446.9792 & 122.5994 & 6.27551 \\
\hline 655 & 14.1183 & 461.9249 & 453.3953 & 446.875 & 122.5994 & 6.26799 \\
\hline 656 & 14.1353 & 456.3886 & 451.5541 & 446.7708 & 122.5994 & 6.26049 \\
\hline 657 & 14.1523 & 441.0082 & 450.1761 & 446.6667 & 122.5994 & 6.25301 \\
\hline 658 & 14.1693 & 443.2924 & 449.2149 & 446.5625 & 122.5994 & 6.24554 \\
\hline 659 & 14.1863 & 456.6249 & 448.557 & 446.4583 & 122.5994 & 6.2381 \\
\hline 660 & 14.2033 & 452.517 & 448.0917 & 446.3542 & 122.5994 & 6.23067 \\
\hline 661 & 14.2203 & 459.7336 & 447.7387 & 446.25 & 122.5994 & 6.22326 \\
\hline 662 & 14.2373 & 434.5061 & 447.4501 & 446.1458 & 122.5994 & 6.21587 \\
\hline 663 & 14.2543 & 434.9525 & 447.2004 & 446.0417 & 122.5994 & 6.20849 \\
\hline 664 & 14.2713 & 426.0994 & 446.6158 & 445.9375 & 122.5994 & 6.20113 \\
\hline 665 & 14.2883 & 452.4796 & 446.4386 & 445.8333 & 122.5994 & 6.19379 \\
\hline 666 & 14.3053 & 449.4484 & 446.0296 & 445.7292 & 122.5994 & 6.18647 \\
\hline 667 & 14.3223 & 455.0958 & 445.8918 & 445.625 & 122.5994 & 6.17917 \\
\hline 668 & 14.3393 & 457.5615 & 445.7278 & 445.5208 & 122.5994 & 6.17188 \\
\hline 669 & 14.3563 & 465.3389 & 445.6021 & 445.4167 & 122.5994 & 6.16461 \\
\hline 670 & 14.3733 & 485.5288 & 445.4494 & 445.3125 & 122.5994 & 6.15736 \\
\hline 671 & 14.3903 & 460.8259 & 445.3318 & 445.2083 & 122.5994 & 6.15012 \\
\hline 672 & 14.4073 & 461.5159 & 445.201 & 445.1042 & 122.5994 & 6.1429 \\
\hline 673 & 14.4243 & 437.9375 & 445.088 & 445 & 122.5994 & 6.1357 \\
\hline 674 & 14.4413 & 420.404 & 444.4762 & 444.3958 & 122.5994 & 6.12851 \\
\hline 675 & 14.4583 & 456.8933 & 443.8653 & 443.7917 & 122.5994 & 6.12135 \\
\hline 676 & 14.4753 & 474.4741 & 443.2126 & 443.1875 & 122.5994 & 6.1142 \\
\hline 677 & 14.4923 & 435.448 & 442.6064 & 442.5833 & 122.5994 & 6.10706 \\
\hline 678 & 14.5093 & 432.0133 & 441.9792 & 441.9792 & 122.5994 & 6.09995 \\
\hline 679 & 14.5263 & 463.7133 & 441.375 & 441.375 & 122.5994 & 6.09284 \\
\hline 680 & 14.5433 & 433.5104 & 440.7708 & 440.7708 & 122.5994 & 6.08576 \\
\hline 681 & 14.5603 & 419.4662 & 440.1667 & 440.1667 & 122.5994 & 6.07869 \\
\hline 682 & 14.5773 & 439.4096 & 439.6971 & 439.5625 & 122.5994 & 6.07164 \\
\hline 683 & 14.5943 & 428.3676 & 439.1049 & 438.9583 & 122.5994 & 6.06461 \\
\hline 684 & 14.6113 & 455.4841 & 438.5803 & 438.3542 & 122.5994 & 6.05759 \\
\hline
\end{tabular}




\begin{tabular}{|c|c|c|c|c|c|c|}
\hline 685 & 14.6283 & 440.4914 & 437.9976 & 437.75 & 122.5994 & 6.05059 \\
\hline 686 & 14.6453 & 434.6189 & 437.4182 & 437.1458 & 122.5994 & 6.0436 \\
\hline 687 & 14.6623 & 414.9481 & 436.8426 & 436.5417 & 122.5994 & 6.03664 \\
\hline 688 & 14.6793 & 424.0335 & 436.2717 & 435.9375 & 122.5994 & 6.02968 \\
\hline 689 & 14.6963 & 431.8067 & 435.7067 & 435.3333 & 122.5994 & 6.02275 \\
\hline 690 & 14.7133 & 429.2255 & 435.1488 & 434.7292 & 122.5994 & 6.01583 \\
\hline 691 & 14.7303 & 449.8235 & 434.6001 & 434.125 & 122.5994 & 6.00892 \\
\hline 692 & 14.7473 & 435.4068 & 434.063 & 433.5208 & 122.5994 & 6.00203 \\
\hline 693 & 14.7643 & 407.1885 & 433.541 & 432.9167 & 122.5994 & 5.99516 \\
\hline 694 & 14.7813 & 437.708 & 433.0389 & 432.3125 & 122.5994 & 5.9883 \\
\hline 695 & 14.7983 & 425.4699 & 432.5644 & 431.7083 & 122.5994 & 5.98146 \\
\hline 696 & 14.8153 & 472.8978 & 432.1302 & 431.1042 & 122.5994 & 5.97464 \\
\hline 697 & 14.8323 & 429.6514 & 431.7624 & 430.5 & 122.5994 & 5.96783 \\
\hline 698 & 14.8493 & 436.767 & 431.5173 & 429.8958 & 122.5994 & 5.96103 \\
\hline 699 & 14.8663 & 436.2894 & 431.5129 & 429.2917 & 122.5994 & 5.95425 \\
\hline 700 & 14.8833 & 409.3379 & 431.964 & 428.6875 & 122.5994 & 5.94749 \\
\hline 701 & 14.9003 & 441.0082 & 433.1841 & 428.0833 & 122.5994 & 5.94074 \\
\hline 702 & 14.9173 & 440.6608 & 435.5 & 427.4792 & 122.5994 & 5.93401 \\
\hline 703 & 14.9343 & 435.0642 & 439.0453 & 426.875 & 122.5994 & 5.9273 \\
\hline 704 & 14.9513 & 423.934 & 443.4236 & 426.2708 & 122.5994 & 5.92059 \\
\hline 705 & 14.9683 & 451.9731 & 447.274 & 425.6667 & 122.5994 & 5.91391 \\
\hline 706 & 14.9853 & 460.8112 & 448.6151 & 425.0625 & 122.5994 & 5.90724 \\
\hline 707 & 15.0023 & 435.0425 & 446.8158 & 424.4583 & 122.5994 & 5.90058 \\
\hline 708 & 15.0193 & 444.3257 & 442.7292 & 423.8542 & 122.5994 & 5.89394 \\
\hline 709 & 15.0363 & 444.9693 & 437.5128 & 423.25 & 122.5994 & 5.88732 \\
\hline 710 & 15.0533 & 466.8379 & 432.5013 & 422.6458 & 122.5994 & 5.88071 \\
\hline 711 & 15.0703 & 482.0216 & 428.4916 & 422.0417 & 122.5994 & 5.87411 \\
\hline 712 & 15.0873 & 461.4253 & 425.5929 & 421.4375 & 122.5994 & 5.86753 \\
\hline 713 & 15.1043 & 471.308 & 423.5795 & 420.8333 & 122.5994 & 5.86096 \\
\hline 714 & 15.1213 & 442.2916 & 422.1527 & 420.2292 & 122.5994 & 5.85441 \\
\hline 715 & 15.1383 & 426.2633 & 421.0666 & 419.625 & 122.5994 & 5.84788 \\
\hline 716 & 15.1553 & 432.0107 & 420.1625 & 419.0208 & 122.5994 & 5.84136 \\
\hline 717 & 15.1723 & 421.0415 & 419.3546 & 418.4167 & 122.5994 & 5.83485 \\
\hline 718 & 15.1893 & 424.9894 & 418.6008 & 417.8125 & 122.5994 & 5.82836 \\
\hline 719 & 15.2063 & 423.8847 & 417.8813 & 417.2083 & 122.5994 & 5.82188 \\
\hline 720 & 15.2233 & 408.0476 & 417.1854 & 416.6042 & 122.5994 & 5.81542 \\
\hline 721 & 15.2403 & 410.0392 & 416.507 & 416 & 122.5994 & 5.80897 \\
\hline 722 & 15.2573 & 429.072 & 416.071 & 415.625 & 122.5994 & 5.80253 \\
\hline 723 & 15.2743 & 423.1863 & 415.6454 & 415.25 & 122.5994 & 5.79611 \\
\hline 724 & 15.2913 & 399.5672 & 415.2278 & 414.875 & 122.5994 & 5.78971 \\
\hline 725 & 15.3083 & 402.9453 & 414.8168 & 414.5 & 122.5994 & 5.78332 \\
\hline 726 & 15.3253 & 445.714 & 414.4109 & 414.125 & 122.5994 & 5.77694 \\
\hline 727 & 15.3423 & 447.1517 & 414.0094 & 413.75 & 122.5994 & 5.77058 \\
\hline 728 & 15.3593 & 443.4142 & 413.6114 & 413.375 & 122.5994 & 5.76423 \\
\hline 729 & 15.3763 & 427.3554 & 413.2162 & 413 & 122.5994 & 5.75789 \\
\hline 730 & 15.3933 & 444.817 & 412.6994 & 412.625 & 122.5994 & 5.75157 \\
\hline 731 & 15.4103 & 467.0821 & 412.3182 & 412.25 & 122.5994 & 5.74527 \\
\hline 732 & 15.4273 & 443.0375 & 411.875 & 411.875 & 122.5994 & 5.73897 \\
\hline 733 & 15.4443 & 387.5395 & 411.5 & 411.5 & 122.5994 & 5.73269 \\
\hline
\end{tabular}




\begin{tabular}{|c|c|c|c|c|c|c|}
\hline 734 & 15.4613 & 435.1144 & 411.1952 & 411.125 & 122.5994 & 5.72643 \\
\hline 735 & 15.4783 & 443.636 & 410.8265 & 410.75 & 122.5994 & 5.72018 \\
\hline 736 & 15.4953 & 424.1077 & 410.4927 & 410.375 & 122.5994 & 5.71394 \\
\hline 737 & 15.5123 & 448.1883 & 410.129 & 410 & 122.5994 & 5.70772 \\
\hline 738 & 15.5293 & 453.6985 & 409.767 & 409.625 & 122.5994 & 5.70151 \\
\hline 739 & 15.5463 & 438.8604 & 409.407 & 409.25 & 122.5994 & 5.69531 \\
\hline 740 & 15.5633 & 440.5663 & 409.0496 & 408.875 & 122.5994 & 5.68913 \\
\hline 741 & 15.5803 & 441.0082 & 408.6952 & 408.5 & 122.5994 & 5.68296 \\
\hline 742 & 15.5973 & 425.3071 & 408.3447 & 408.125 & 122.5994 & 5.6768 \\
\hline 743 & 15.6143 & 416.4302 & 407.9991 & 407.75 & 122.5994 & 5.67066 \\
\hline 744 & 15.6313 & 412.1524 & 407.6597 & 407.375 & 122.5994 & 5.66453 \\
\hline 745 & 15.6483 & 396.1577 & 407.3284 & 407 & 122.5994 & 5.65842 \\
\hline 746 & 15.6653 & 415.9272 & 407.6121 & 407.2292 & 122.5994 & 5.65231 \\
\hline 747 & 15.6823 & 407.2291 & 407.9109 & 407.4583 & 122.5994 & 5.64622 \\
\hline 748 & 15.6993 & 392.603 & 408.2321 & 407.6875 & 122.5994 & 5.64015 \\
\hline 749 & 15.7163 & 401.9144 & 408.5915 & 407.9167 & 122.5994 & 5.63409 \\
\hline 750 & 15.7333 & 422.1739 & 409.0225 & 408.1458 & 122.5994 & 5.62804 \\
\hline 751 & 15.7503 & 436.7931 & 409.594 & 408.375 & 122.5994 & 5.622 \\
\hline 752 & 15.7673 & 430.9758 & 410.4263 & 408.6042 & 122.5994 & 5.61598 \\
\hline 753 & 15.7843 & 404.3984 & 411.6847 & 408.8333 & 122.5994 & 5.60997 \\
\hline 754 & 15.8013 & 432.0137 & 413.524 & 409.0625 & 122.5994 & 5.60397 \\
\hline 755 & 15.8183 & 448.3339 & 415.9713 & 409.2917 & 122.5994 & 5.59798 \\
\hline 756 & 15.8353 & 409.0186 & 418.7423 & 409.5208 & 122.5994 & 5.59201 \\
\hline 757 & 15.8523 & 441.3139 & 421.0399 & 409.75 & 122.5994 & 5.58605 \\
\hline 758 & 15.8693 & 453.6725 & 421.9247 & 409.9792 & 122.5994 & 5.58011 \\
\hline 759 & 15.8863 & 456.3465 & 421.3171 & 410.2083 & 122.5994 & 5.57418 \\
\hline 760 & 15.9033 & 453.841 & 419.696 & 410.4375 & 122.5994 & 5.56826 \\
\hline 761 & 15.9203 & 425.2357 & 417.6017 & 410.6667 & 122.5994 & 5.56235 \\
\hline 762 & 15.9373 & 414.7222 & 415.6667 & 410.8958 & 122.5994 & 5.55645 \\
\hline 763 & 15.9543 & 421.3269 & 414.2471 & 411.125 & 122.5994 & 5.55057 \\
\hline 764 & 15.9713 & 412.4785 & 413.3732 & 411.3542 & 122.5994 & 5.5447 \\
\hline 765 & 15.9883 & 429.8265 & 412.9265 & 411.5833 & 122.5994 & 5.53884 \\
\hline 766 & 16.0053 & 419.8635 & 412.7603 & 411.8125 & 122.5994 & 5.533 \\
\hline 767 & 16.0223 & 429.9781 & 412.7564 & 412.0417 & 122.5994 & 5.52717 \\
\hline 768 & 16.0393 & 455.8153 & 412.8394 & 412.2708 & 122.5994 & 5.52135 \\
\hline 769 & 16.0563 & 429.8574 & 412.9685 & 412.5 & 122.5994 & 5.51554 \\
\hline 770 & 16.0733 & 407.6855 & 412.655 & 412.2604 & 122.5994 & 5.50974 \\
\hline 771 & 16.0903 & 403.9712 & 412.3582 & 412.0208 & 122.5994 & 5.50396 \\
\hline 772 & 16.1073 & 394.6404 & 412.0731 & 411.7813 & 122.5994 & 5.49819 \\
\hline 773 & 16.1243 & 425.5545 & 411.7965 & 411.5417 & 122.5994 & 5.49243 \\
\hline 774 & 16.1413 & 452.1348 & 411.5265 & 411.3021 & 122.5994 & 5.48668 \\
\hline 775 & 16.1583 & 410.821 & 411.2616 & 411.0625 & 122.5994 & 5.48095 \\
\hline 776 & 16.1753 & 415.3735 & 411.0008 & 410.8229 & 122.5994 & 5.47523 \\
\hline 777 & 16.1923 & 414.2507 & 410.7432 & 410.5833 & 122.5994 & 5.46952 \\
\hline 778 & 16.2093 & 385.2511 & 410.4881 & 410.3438 & 122.5994 & 5.46382 \\
\hline 779 & 16.2263 & 417.9943 & 410.2352 & 410.1042 & 122.5994 & 5.45813 \\
\hline 780 & 16.2433 & 435.8222 & 409.9841 & 409.8646 & 122.5994 & 5.45246 \\
\hline 781 & 16.2603 & 411.1451 & 409.7344 & 409.625 & 122.5994 & 5.4468 \\
\hline 782 & 16.2773 & 391.2139 & 409.4233 & 409.3854 & 122.5994 & 5.44115 \\
\hline
\end{tabular}




\begin{tabular}{|c|c|c|c|c|c|c|}
\hline 783 & 16.2943 & 391.3591 & 409.1806 & 409.1458 & 122.5994 & 5.43551 \\
\hline 784 & 16.3113 & 405.5577 & 408.9063 & 408.9063 & 122.5994 & 5.42988 \\
\hline 785 & 16.3283 & 422.2255 & 408.6667 & 408.6667 & 122.5994 & 5.42427 \\
\hline 786 & 16.3453 & 409.8386 & 408.4271 & 408.4271 & 122.5994 & 5.41866 \\
\hline 787 & 16.3623 & 400.7167 & 408.1875 & 408.1875 & 122.5994 & 5.41307 \\
\hline 788 & 16.3793 & 392.578 & 407.9479 & 407.9479 & 122.5994 & 5.40749 \\
\hline 789 & 16.3963 & 430.8155 & 407.7083 & 407.7083 & 122.5994 & 5.40192 \\
\hline 790 & 16.4133 & 385.2674 & 407.4688 & 407.4688 & 122.5994 & 5.39637 \\
\hline 791 & 16.4303 & 420.1791 & 407.2292 & 407.2292 & 122.5994 & 5.39082 \\
\hline 792 & 16.4473 & 440.6856 & 406.9896 & 406.9896 & 122.5994 & 5.38529 \\
\hline 793 & 16.4643 & 423.2077 & 406.75 & 406.75 & 122.5994 & 5.37977 \\
\hline 794 & 16.4813 & 397.0217 & 406.5104 & 406.5104 & 122.5994 & 5.37426 \\
\hline 795 & 16.4983 & 411.0364 & 406.2708 & 406.2708 & 122.5994 & 5.36876 \\
\hline 796 & 16.5153 & 404.7733 & 406.0313 & 406.0313 & 122.5994 & 5.36327 \\
\hline 797 & 16.5323 & 439.4789 & 405.7917 & 405.7917 & 122.5994 & 5.35779 \\
\hline 798 & 16.5493 & 404.2378 & 405.5521 & 405.5521 & 122.5994 & 5.35233 \\
\hline 799 & 16.5663 & 425.0211 & 405.3125 & 405.3125 & 122.5994 & 5.34687 \\
\hline 800 & 16.5833 & 411.9649 & 405.0729 & 405.0729 & 122.5994 & 5.34143 \\
\hline 801 & 16.6003 & 427.5693 & 404.8333 & 404.8333 & 122.5994 & 5.336 \\
\hline 802 & 16.6173 & 421.4905 & 404.5938 & 404.5938 & 122.5994 & 5.33058 \\
\hline 803 & 16.6343 & 413.1398 & 404.3542 & 404.3542 & 122.5994 & 5.32517 \\
\hline 804 & 16.6513 & 409.4726 & 404.1146 & 404.1146 & 122.5994 & 5.31977 \\
\hline 805 & 16.6683 & 389.6903 & 403.875 & 403.875 & 122.5994 & 5.31438 \\
\hline 806 & 16.6853 & 390.5321 & 403.6354 & 403.6354 & 122.5994 & 5.309 \\
\hline 807 & 16.7023 & 420.9653 & 403.3958 & 403.3958 & 122.5994 & 5.30364 \\
\hline 808 & 16.7193 & 438.5725 & 403.1563 & 403.1563 & 122.5994 & 5.29828 \\
\hline 809 & 16.7363 & 413.6652 & 402.9167 & 402.9167 & 122.5994 & 5.29294 \\
\hline 810 & 16.7533 & 426.2696 & 402.6771 & 402.6771 & 122.5994 & 5.28761 \\
\hline 811 & 16.7703 & 425.1688 & 402.4375 & 402.4375 & 122.5994 & 5.28229 \\
\hline 812 & 16.7873 & 402.2141 & 402.1979 & 402.1979 & 122.5994 & 5.27698 \\
\hline 813 & 16.8043 & 413.3851 & 401.9583 & 401.9583 & 122.5994 & 5.27168 \\
\hline 814 & 16.8213 & 428.498 & 401.7206 & 401.7188 & 122.5994 & 5.26639 \\
\hline 815 & 16.8383 & 415.9095 & 401.4812 & 401.4792 & 122.5994 & 5.26111 \\
\hline 816 & 16.8553 & 393.3825 & 401.2419 & 401.2396 & 122.5994 & 5.25584 \\
\hline 817 & 16.8723 & 399.7327 & 401.0034 & 401 & 122.5994 & 5.25058 \\
\hline 818 & 16.8893 & 400.492 & 401.3788 & 401.375 & 122.5994 & 5.24534 \\
\hline 819 & 16.9063 & 401.6533 & 401.7542 & 401.75 & 122.5994 & 5.2401 \\
\hline 820 & 16.9233 & 418.1486 & 402.1297 & 402.125 & 122.5994 & 5.23487 \\
\hline 821 & 16.9403 & 403.5755 & 402.5052 & 402.5 & 122.5994 & 5.22966 \\
\hline 822 & 16.9573 & 416.2817 & 402.8946 & 402.875 & 122.5994 & 5.22445 \\
\hline 823 & 16.9743 & 408.9425 & 403.2716 & 403.25 & 122.5994 & 5.21926 \\
\hline 824 & 16.9913 & 411.4477 & 403.6555 & 403.625 & 122.5994 & 5.21408 \\
\hline 825 & 17.0083 & 419.1513 & 406.4617 & 404 & 122.5994 & 5.2089 \\
\hline 826 & 17.0253 & 420.2097 & 406.9492 & 404.375 & 122.5994 & 5.20374 \\
\hline 827 & 17.0423 & 436.2857 & 408.6114 & 404.75 & 122.5994 & 5.19859 \\
\hline 828 & 17.0593 & 441.0082 & 409.1675 & 405.125 & 122.5994 & 5.19345 \\
\hline 829 & 17.0763 & 414.1295 & 409.7374 & 405.5 & 122.5994 & 5.18831 \\
\hline 830 & 17.0933 & 401.3179 & 410.3231 & 405.875 & 122.5994 & 5.18319 \\
\hline 831 & 17.1103 & 442.4549 & 410.9276 & 406.25 & 122.5994 & 5.17808 \\
\hline
\end{tabular}




\begin{tabular}{|c|c|c|c|c|c|c|}
\hline 832 & 17.1273 & 423.7101 & 411.5556 & 406.625 & 122.5994 & 5.17298 \\
\hline 833 & 17.1443 & 409.2503 & 412.2132 & 407 & 122.5994 & 5.16789 \\
\hline 834 & 17.1613 & 438.4715 & 412.9074 & 407.375 & 122.5994 & 5.16281 \\
\hline 835 & 17.1783 & 429.1914 & 413.6438 & 407.75 & 122.5994 & 5.15774 \\
\hline 836 & 17.1953 & 444.6516 & 414.4224 & 408.125 & 122.5994 & 5.15268 \\
\hline 837 & 17.2123 & 424.6256 & 415.233 & 408.5 & 122.5994 & 5.14762 \\
\hline 838 & 17.2293 & 388.5787 & 416.0592 & 408.875 & 122.5994 & 5.14258 \\
\hline 839 & 17.2463 & 439.0281 & 416.9082 & 409.25 & 122.5994 & 5.13755 \\
\hline 840 & 17.2633 & 379.0866 & 417.8213 & 409.625 & 122.5994 & 5.13253 \\
\hline 841 & 17.2803 & 427.8907 & 418.8471 & 410 & 122.5994 & 5.12752 \\
\hline 842 & 17.2973 & 417.9493 & 419.5739 & 409.9167 & 122.5994 & 5.12252 \\
\hline 843 & 17.3143 & 400.7317 & 420.4772 & 409.8333 & 122.5994 & 5.11753 \\
\hline 844 & 17.3313 & 435.6684 & 421.4987 & 409.75 & 122.5994 & 5.11255 \\
\hline 845 & 17.3483 & 429.827 & 422.479 & 409.6667 & 122.5994 & 5.10758 \\
\hline 846 & 17.3653 & 432.3373 & 423.2767 & 409.5833 & 122.5994 & 5.10261 \\
\hline 847 & 17.3823 & 412.9231 & 423.9409 & 409.5 & 122.5994 & 5.09766 \\
\hline 848 & 17.3993 & 423.6336 & 424.5929 & 409.4167 & 122.5994 & 5.09272 \\
\hline 849 & 17.4163 & 434.3199 & 425.3665 & 409.3333 & 122.5994 & 5.08779 \\
\hline 850 & 17.4333 & 395.6001 & 426.4436 & 409.25 & 122.5994 & 5.08286 \\
\hline 851 & 17.4503 & 412.6586 & 427.9717 & 409.1667 & 122.5994 & 5.07795 \\
\hline 852 & 17.4673 & 409.0578 & 430.0254 & 409.0833 & 122.5994 & 5.07305 \\
\hline 853 & 17.4843 & 424.37 & 432.5961 & 409 & 122.5994 & 5.06815 \\
\hline 854 & 17.5013 & 446.1667 & 435.5598 & 408.9167 & 122.5994 & 5.06327 \\
\hline 855 & 17.5183 & 464.3974 & 438.7549 & 408.8333 & 122.5994 & 5.05839 \\
\hline 856 & 17.5353 & 440.4434 & 442.3228 & 408.75 & 122.5994 & 5.05353 \\
\hline 857 & 17.5523 & 428.9297 & 446.7452 & 408.6667 & 122.5994 & 5.04867 \\
\hline 858 & 17.5693 & 446.5849 & 452.6226 & 408.5833 & 122.5994 & 5.04382 \\
\hline 859 & 17.5863 & 457.6445 & 460.748 & 408.5 & 122.5994 & 5.03899 \\
\hline 860 & 17.6033 & 456.0775 & 472.0556 & 408.4167 & 122.5994 & 5.03416 \\
\hline 861 & 17.6203 & 449.3969 & 487.4918 & 408.3333 & 122.5994 & 5.02934 \\
\hline 862 & 17.6373 & 479.3009 & 507.9709 & 408.25 & 122.5994 & 5.02453 \\
\hline 863 & 17.6543 & 501.3582 & 534.2985 & 408.1667 & 122.5994 & 5.01973 \\
\hline 864 & 17.6713 & 492.2506 & 567.0068 & 408.0833 & 122.5994 & 5.01494 \\
\hline 865 & 17.6883 & 517.823 & 606.151 & 408 & 122.5994 & 5.01016 \\
\hline 866 & 17.7053 & 521.4428 & 651.0398 & 407.9167 & 122.5994 & 5.00539 \\
\hline 867 & 17.7223 & 540.0213 & 699.8889 & 407.8333 & 122.5994 & 5.00062 \\
\hline 868 & 17.7393 & 591.6841 & 749.4249 & 407.75 & 122.5994 & 4.99587 \\
\hline 869 & 17.7563 & 637.254 & 794.6694 & 407.6667 & 122.5994 & 4.99112 \\
\hline 870 & 17.7733 & 657.406 & 829.4238 & 407.5833 & 122.5994 & 4.98639 \\
\hline 871 & 17.7903 & 705.897 & 848.1155 & 407.5 & 122.5994 & 4.98166 \\
\hline 872 & 17.8073 & 768.3553 & 847.7618 & 407.4167 & 122.5994 & 4.97694 \\
\hline 873 & 17.8243 & 836.3355 & 828.9261 & 407.3333 & 122.5994 & 4.97224 \\
\hline 874 & 17.8413 & 857.1427 & 795.0184 & 407.25 & 122.5994 & 4.96754 \\
\hline 875 & 17.8583 & 812.1263 & 751.1212 & 407.1667 & 122.5994 & 4.96285 \\
\hline 876 & 17.8753 & 800.905 & 702.6587 & 407.0833 & 122.5994 & 4.95816 \\
\hline 877 & 17.8923 & 699.3835 & 654.288 & 407 & 122.5994 & 4.95349 \\
\hline 878 & 17.9093 & 619.3619 & 609.3407 & 406.9167 & 122.5994 & 4.94883 \\
\hline 879 & 17.9263 & 566.9603 & 569.7283 & 406.8333 & 122.5994 & 4.94417 \\
\hline 880 & 17.9433 & 531.7978 & 536.3503 & 406.75 & 122.5994 & 4.93953 \\
\hline
\end{tabular}




\begin{tabular}{|c|c|c|c|c|c|c|}
\hline 881 & 17.9603 & 499.3832 & 509.1622 & 406.6667 & 122.5994 & 4.93489 \\
\hline 882 & 17.9773 & 455.6249 & 487.6835 & 406.5833 & 122.5994 & 4.93026 \\
\hline 883 & 17.9943 & 445.4104 & 471.1577 & 406.5 & 122.5994 & 4.92564 \\
\hline 884 & 18.0113 & 457.9544 & 458.6462 & 406.4167 & 122.5994 & 4.92103 \\
\hline 885 & 18.0283 & 469.4842 & 449.2781 & 406.3333 & 122.5994 & 4.91643 \\
\hline 886 & 18.0453 & 405.2165 & 442.272 & 406.25 & 122.5994 & 4.91184 \\
\hline 887 & 18.0623 & 429.823 & 436.989 & 406.1667 & 122.5994 & 4.90725 \\
\hline 888 & 18.0793 & 390.136 & 432.9395 & 406.0833 & 122.5994 & 4.90267 \\
\hline 889 & 18.0963 & 410.3854 & 429.7678 & 406 & 122.5994 & 4.89811 \\
\hline 890 & 18.1133 & 401.2406 & 428.472 & 407.1563 & 122.5994 & 4.89355 \\
\hline 891 & 18.1303 & 434.2934 & 427.66 & 408.3125 & 122.5994 & 4.889 \\
\hline 892 & 18.1473 & 422.3762 & 427.2453 & 409.4688 & 122.5994 & 4.88446 \\
\hline 893 & 18.1643 & 430.2629 & 427.1815 & 410.625 & 122.5994 & 4.87992 \\
\hline 894 & 18.1813 & 420.2579 & 427.4222 & 411.7813 & 122.5994 & 4.8754 \\
\hline 895 & 18.1983 & 417.3814 & 427.9233 & 412.9375 & 122.5994 & 4.87088 \\
\hline 896 & 18.2153 & 412.2437 & 428.5406 & 414.0938 & 122.5994 & 4.86638 \\
\hline 897 & 18.2323 & 430.1267 & 429.0935 & 415.25 & 122.5994 & 4.86188 \\
\hline 898 & 18.2493 & 438.9619 & 429.5425 & 416.4063 & 122.5994 & 4.85739 \\
\hline 899 & 18.2663 & 427.0308 & 430.0157 & 417.5625 & 122.5994 & 4.8529 \\
\hline 900 & 18.2833 & 435.5167 & 430.6337 & 418.7188 & 122.5994 & 4.84843 \\
\hline 901 & 18.3003 & 439.4803 & 431.5376 & 419.875 & 122.5994 & 4.84396 \\
\hline 902 & 18.3173 & 441.696 & 432.7323 & 421.0313 & 122.5994 & 4.83951 \\
\hline 903 & 18.3343 & 463.4846 & 434.0836 & 422.1875 & 122.5994 & 4.83506 \\
\hline 904 & 18.3513 & 451.4097 & 435.2271 & 423.3438 & 122.5994 & 4.83062 \\
\hline 905 & 18.3683 & 417.2644 & 435.8834 & 424.5 & 122.5994 & 4.82618 \\
\hline 906 & 18.3853 & 444.4406 & 436.1769 & 425.6563 & 122.5994 & 4.82176 \\
\hline 907 & 18.4023 & 433.5015 & 436.2491 & 426.8125 & 122.5994 & 4.81734 \\
\hline 908 & 18.4193 & 410.2785 & 436.2322 & 427.9688 & 122.5994 & 4.81294 \\
\hline 909 & 18.4363 & 407.4925 & 436.3221 & 429.125 & 122.5994 & 4.80854 \\
\hline 910 & 18.4533 & 432.3183 & 436.6263 & 430.2813 & 122.5994 & 4.80414 \\
\hline 911 & 18.4703 & 448.1353 & 437.1461 & 431.4375 & 122.5994 & 4.79976 \\
\hline 912 & 18.4873 & 435.9811 & 437.8369 & 432.5938 & 122.5994 & 4.79539 \\
\hline 913 & 18.5043 & 456.4726 & 438.6469 & 433.75 & 122.5994 & 4.79102 \\
\hline 914 & 18.5213 & 459.8151 & 439.0818 & 434.4427 & 122.5994 & 4.78666 \\
\hline 915 & 18.5383 & 479.7698 & 439.5582 & 435.1354 & 122.5994 & 4.78231 \\
\hline 916 & 18.5553 & 454.2803 & 440.0738 & 435.8281 & 122.5994 & 4.77797 \\
\hline 917 & 18.5723 & 442.7728 & 440.6333 & 436.5208 & 122.5994 & 4.77363 \\
\hline 918 & 18.5893 & 436.0369 & 438.9901 & 437.2135 & 122.5994 & 4.7693 \\
\hline 919 & 18.6063 & 445.281 & 439.7626 & 437.9063 & 122.5994 & 4.76498 \\
\hline 920 & 18.6233 & 426.8701 & 439.5155 & 438.599 & 122.5994 & 4.76067 \\
\hline 921 & 18.6403 & 457.0757 & 440.6223 & 439.2917 & 122.5994 & 4.75637 \\
\hline 922 & 18.6573 & 466.5152 & 441.9611 & 439.9844 & 122.5994 & 4.75207 \\
\hline 923 & 18.6743 & 462.957 & 443.5007 & 440.6771 & 122.5994 & 4.74779 \\
\hline 924 & 18.6913 & 469.6849 & 445.0691 & 441.3698 & 122.5994 & 4.74351 \\
\hline 925 & 18.7083 & 429.9033 & 446.3484 & 442.0625 & 122.5994 & 4.73923 \\
\hline 926 & 18.7253 & 439.387 & 447.0919 & 442.7552 & 122.5994 & 4.73497 \\
\hline 927 & 18.7423 & 452.7354 & 447.4167 & 443.4479 & 122.5994 & 4.73071 \\
\hline 928 & 18.7593 & 461.7403 & 447.4586 & 444.1406 & 122.5994 & 4.72646 \\
\hline 929 & 18.7763 & 474.821 & 447.3933 & 444.8333 & 122.5994 & 4.72222 \\
\hline
\end{tabular}




\begin{tabular}{|c|c|c|c|c|c|c|}
\hline 930 & 18.7933 & 449.7426 & 447.3857 & 445.526 & 122.5994 & 4.71799 \\
\hline 931 & 18.8103 & 492.9399 & 447.5549 & 446.2188 & 122.5994 & 4.71376 \\
\hline 932 & 18.8273 & 502.4875 & 447.9602 & 446.9115 & 122.5994 & 4.70955 \\
\hline 933 & 18.8443 & 525.3414 & 448.5774 & 447.6042 & 122.5994 & 4.70534 \\
\hline 934 & 18.8613 & 544.6584 & 449.382 & 448.2969 & 122.5994 & 4.70113 \\
\hline 935 & 18.8783 & 561.968 & 450.3375 & 448.9896 & 122.5994 & 4.69694 \\
\hline 936 & 18.8953 & 513.0697 & 451.3741 & 449.6823 & 122.5994 & 4.69275 \\
\hline 937 & 18.9123 & 468.3862 & 452.3615 & 450.375 & 122.5994 & 4.68857 \\
\hline 938 & 18.9293 & 496.4443 & 453.1717 & 451.0677 & 122.5994 & 4.6844 \\
\hline 939 & 18.9463 & 456.2899 & 453.9198 & 451.7604 & 122.5994 & 4.68023 \\
\hline 940 & 18.9633 & 494.6842 & 454.5187 & 452.4531 & 122.5994 & 4.67608 \\
\hline 941 & 18.9803 & 437.4112 & 455.1046 & 453.1458 & 122.5994 & 4.67193 \\
\hline 942 & 18.9973 & 406.0878 & 455.7648 & 453.8385 & 122.5994 & 4.66778 \\
\hline 943 & 19.0143 & 444.8018 & 456.4117 & 454.5313 & 122.5994 & 4.66365 \\
\hline 944 & 19.0313 & 459.159 & 457.1229 & 455.224 & 122.5994 & 4.65952 \\
\hline 945 & 19.0483 & 435.7774 & 457.6475 & 455.9167 & 122.5994 & 4.6554 \\
\hline 946 & 19.0653 & 417.5945 & 458.1322 & 456.6094 & 122.5994 & 4.65129 \\
\hline 947 & 19.0823 & 420.1172 & 458.6484 & 457.3021 & 122.5994 & 4.64718 \\
\hline 948 & 19.0993 & 460.7899 & 459.3951 & 457.9948 & 122.5994 & 4.64309 \\
\hline 949 & 19.1163 & 467.6278 & 459.8914 & 458.6875 & 122.5994 & 4.63899 \\
\hline 950 & 19.1333 & 449.4484 & 460.4735 & 459.3802 & 122.5994 & 4.63491 \\
\hline 951 & 19.1503 & 445.7304 & 461.2497 & 460.0729 & 122.5994 & 4.63084 \\
\hline 952 & 19.1673 & 444.8565 & 461.9193 & 460.7656 & 122.5994 & 4.62677 \\
\hline 953 & 19.1843 & 443.3421 & 462.6296 & 461.4583 & 122.5994 & 4.6227 \\
\hline 954 & 19.2013 & 465.1141 & 463.3583 & 462.151 & 122.5994 & 4.61865 \\
\hline 955 & 19.2183 & 479.0115 & 464.1001 & 462.8438 & 122.5994 & 4.6146 \\
\hline 956 & 19.2353 & 441.187 & 464.8527 & 463.5365 & 122.5994 & 4.61056 \\
\hline 957 & 19.2523 & 446.6369 & 465.6153 & 464.2292 & 122.5994 & 4.60653 \\
\hline 958 & 19.2693 & 476.823 & 466.3887 & 464.9219 & 122.5994 & 4.6025 \\
\hline 959 & 19.2863 & 457.6684 & 467.1743 & 465.6146 & 122.5994 & 4.59849 \\
\hline 960 & 19.3033 & 482.3838 & 467.9749 & 466.3073 & 122.5994 & 4.59447 \\
\hline 961 & 19.3203 & 460.7316 & 468.7967 & 467 & 122.5994 & 4.59047 \\
\hline 962 & 19.3373 & 451.1405 & 469.3798 & 467.4323 & 122.5994 & 4.58647 \\
\hline 963 & 19.3543 & 488.5722 & 470.0214 & 467.8646 & 122.5994 & 4.58248 \\
\hline 964 & 19.3713 & 504.9453 & 470.731 & 468.2969 & 122.5994 & 4.5785 \\
\hline 965 & 19.3883 & 502.9294 & 471.5197 & 468.7292 & 122.5994 & 4.57452 \\
\hline 966 & 19.4053 & 474.9046 & 472.3943 & 469.1615 & 122.5994 & 4.57055 \\
\hline 967 & 19.4223 & 497.715 & 473.307 & 469.5938 & 122.5994 & 4.56659 \\
\hline 968 & 19.4393 & 509.8958 & 474.9013 & 470.026 & 122.5994 & 4.56264 \\
\hline 969 & 19.4563 & 456.5067 & 475.6933 & 470.4583 & 122.5994 & 4.55869 \\
\hline 970 & 19.4733 & 481.854 & 476.423 & 470.8906 & 122.5994 & 4.55475 \\
\hline 971 & 19.4903 & 465.6399 & 477.1773 & 471.3229 & 122.5994 & 4.55081 \\
\hline 972 & 19.5073 & 458.4307 & 477.9706 & 471.7552 & 122.5994 & 4.54688 \\
\hline 973 & 19.5243 & 489.8431 & 478.9193 & 472.1875 & 122.5994 & 4.54296 \\
\hline 974 & 19.5413 & 501.1927 & 480.1482 & 472.6198 & 122.5994 & 4.53905 \\
\hline 975 & 19.5583 & 501.7694 & 481.8338 & 473.0521 & 122.5994 & 4.53514 \\
\hline 976 & 19.5753 & 514.9486 & 484.279 & 473.4844 & 122.5994 & 4.53124 \\
\hline 977 & 19.5923 & 524.4183 & 487.9547 & 473.9167 & 122.5994 & 4.52735 \\
\hline 978 & 19.6093 & 488.705 & 493.4273 & 474.349 & 122.5994 & 4.52346 \\
\hline
\end{tabular}




\begin{tabular}{|c|c|c|c|c|c|c|}
\hline 979 & 19.6263 & 503.1373 & 501.1076 & 474.7813 & 122.5994 & 4.51958 \\
\hline 980 & 19.6433 & 529.5756 & 510.8184 & 475.2135 & 122.5994 & 4.51571 \\
\hline 981 & 19.6603 & 477.0299 & 521.1501 & 475.6458 & 122.5994 & 4.51184 \\
\hline 982 & 19.6773 & 540.9147 & 529.2474 & 476.0781 & 122.5994 & 4.50798 \\
\hline 983 & 19.6943 & 532.3168 & 533.3448 & 476.5104 & 122.5994 & 4.50413 \\
\hline 984 & 19.7113 & 593.086 & 534.7901 & 476.9427 & 122.5994 & 4.50029 \\
\hline 985 & 19.7283 & 637.6686 & 534.9968 & 477.375 & 122.5994 & 4.49645 \\
\hline 986 & 19.7453 & 646.3441 & 534.4741 & 477.487 & 122.5994 & 4.49261 \\
\hline 987 & 19.7623 & 625.6668 & 534.9986 & 477.599 & 122.5994 & 4.48879 \\
\hline 988 & 19.7793 & 607.7739 & 537.5279 & 477.7109 & 122.5994 & 4.48497 \\
\hline 989 & 19.7963 & 609.2394 & 542.0573 & 477.8229 & 122.5994 & 4.48115 \\
\hline 990 & 19.8133 & 646.5044 & 548.2051 & 477.9349 & 122.5994 & 4.47735 \\
\hline 991 & 19.8303 & 688.7327 & 555.7721 & 478.0469 & 122.5994 & 4.47355 \\
\hline 992 & 19.8473 & 725.2232 & 565.0829 & 478.1589 & 122.5994 & 4.46975 \\
\hline 993 & 19.8643 & 725.0143 & 577.075 & 478.2708 & 122.5994 & 4.46597 \\
\hline 994 & 19.8813 & 698.9072 & 592.8828 & 478.3828 & 122.5994 & 4.46219 \\
\hline 995 & 19.8983 & 706.3825 & 612.9183 & 478.4948 & 122.5994 & 4.45841 \\
\hline 996 & 19.9153 & 715.0899 & 635.1309 & 478.6068 & 122.5994 & 4.45465 \\
\hline 997 & 19.9323 & 702.3219 & 652.6154 & 478.7188 & 122.5994 & 4.45088 \\
\hline 998 & 19.9493 & 676.5243 & 655.8035 & 478.8307 & 122.5994 & 4.44713 \\
\hline 999 & 19.9663 & 665.9381 & 643.5515 & 478.9427 & 122.5994 & 4.44338 \\
\hline 1000 & 19.9833 & 640.4002 & 622.505 & 479.0547 & 122.5994 & 4.43964 \\
\hline 1001 & 20.0003 & 612.3552 & 596.189 & 479.1667 & 122.5994 & 4.4359 \\
\hline 1002 & 20.0173 & 599.9009 & 568.2177 & 479.2786 & 122.5994 & 4.43218 \\
\hline 1003 & 20.0343 & 571.2585 & 543.8649 & 479.3906 & 122.5994 & 4.42845 \\
\hline 1004 & 20.0513 & 582.9938 & 525.6365 & 479.5026 & 122.5994 & 4.42474 \\
\hline 1005 & 20.0683 & 576.1357 & 513.3485 & 479.6146 & 122.5994 & 4.42103 \\
\hline 1006 & 20.0853 & 548.1294 & 505.5069 & 479.7266 & 122.5994 & 4.41732 \\
\hline 1007 & 20.1023 & 516.3568 & 501.0677 & 479.8385 & 122.5994 & 4.41363 \\
\hline 1008 & 20.1193 & 526.0975 & 498.5328 & 479.9505 & 122.5994 & 4.40994 \\
\hline 1009 & 20.1363 & 535.0627 & 500.4575 & 480.0625 & 122.5994 & 4.40625 \\
\hline 1010 & 20.1533 & 550.1975 & 499.8367 & 480.1745 & 122.5994 & 4.40257 \\
\hline 1011 & 20.1703 & 543.2769 & 499.6721 & 480.2865 & 122.5994 & 4.3989 \\
\hline 1012 & 20.1873 & 510.7221 & 499.7949 & 480.3984 & 122.5994 & 4.39523 \\
\hline 1013 & 20.2043 & 526.4356 & 502.1338 & 480.5104 & 122.5994 & 4.39157 \\
\hline 1014 & 20.2213 & 548.4571 & 502.509 & 480.6224 & 122.5994 & 4.38792 \\
\hline 1015 & 20.2383 & 500.0699 & 502.6967 & 480.7344 & 122.5994 & 4.38427 \\
\hline 1016 & 20.2553 & 518.6251 & 502.5642 & 480.8464 & 122.5994 & 4.38063 \\
\hline 1017 & 20.2723 & 501.0806 & 502.1254 & 480.9583 & 122.5994 & 4.377 \\
\hline 1018 & 20.2893 & 501.1849 & 501.4574 & 481.0703 & 122.5994 & 4.37337 \\
\hline 1019 & 20.3063 & 538.9321 & 500.6605 & 481.1823 & 122.5994 & 4.36974 \\
\hline 1020 & 20.3233 & 534.3154 & 499.8749 & 481.2943 & 122.5994 & 4.36613 \\
\hline 1021 & 20.3403 & 445.9541 & 499.2214 & 481.4063 & 122.5994 & 4.36252 \\
\hline 1022 & 20.3573 & 482.6386 & 498.7758 & 481.5182 & 122.5994 & 4.35891 \\
\hline 1023 & 20.3743 & 501.5858 & 498.5765 & 481.6302 & 122.5994 & 4.35531 \\
\hline 1024 & 20.3913 & 510.8705 & 498.632 & 481.7422 & 122.5994 & 4.35172 \\
\hline 1025 & 20.4083 & 518.3932 & 498.935 & 481.8542 & 122.5994 & 4.34813 \\
\hline 1026 & 20.4253 & 475.7236 & 499.4738 & 481.9661 & 122.5994 & 4.34455 \\
\hline 1027 & 20.4423 & 484.6785 & 500.2392 & 482.0781 & 122.5994 & 4.34098 \\
\hline
\end{tabular}




\begin{tabular}{|c|c|c|c|c|c|c|}
\hline 1028 & 20.4593 & 491.9894 & 501.2289 & 482.1901 & 122.5994 & 4.33741 \\
\hline 1029 & 20.4763 & 478.8502 & 502.4495 & 482.3021 & 122.5994 & 4.33385 \\
\hline 1030 & 20.4933 & 445.1994 & 503.9186 & 482.4141 & 122.5994 & 4.33029 \\
\hline 1031 & 20.5103 & 481.0439 & 505.6664 & 482.526 & 122.5994 & 4.32674 \\
\hline 1032 & 20.5273 & 527.7823 & 507.6961 & 482.638 & 122.5994 & 4.3232 \\
\hline 1033 & 20.5443 & 502.689 & 510.1519 & 482.75 & 122.5994 & 4.31966 \\
\hline 1034 & 20.5613 & 506.2595 & 512.7479 & 482.5417 & 122.5994 & 4.31612 \\
\hline 1035 & 20.5783 & 512.9806 & 515.8921 & 482.3333 & 122.5994 & 4.3126 \\
\hline 1036 & 20.5953 & 531.1423 & 519.713 & 482.125 & 122.5994 & 4.30907 \\
\hline 1037 & 20.6123 & 538.0315 & 524.7673 & 481.9167 & 122.5994 & 4.30556 \\
\hline 1038 & 20.6293 & 581.8261 & 530.4128 & 481.7083 & 122.5994 & 4.30205 \\
\hline 1039 & 20.6463 & 592.242 & 537.2287 & 481.5 & 122.5994 & 4.29855 \\
\hline 1040 & 20.6633 & 564.7622 & 545.4257 & 481.2917 & 122.5994 & 4.29505 \\
\hline 1041 & 20.6803 & 561.7005 & 555.1568 & 481.0833 & 122.5994 & 4.29156 \\
\hline 1042 & 20.6973 & 563.5286 & 567.4389 & 480.875 & 122.5994 & 4.28807 \\
\hline 1043 & 20.7143 & 591.0424 & 582.9956 & 480.6667 & 122.5994 & 4.28459 \\
\hline 1044 & 20.7313 & 640.6797 & 603.5652 & 480.4583 & 122.5994 & 4.28111 \\
\hline 1045 & 20.7483 & 677.3884 & 635.0965 & 480.25 & 122.5994 & 4.27764 \\
\hline 1046 & 20.7653 & 758.3798 & 687.5947 & 480.0417 & 122.5994 & 4.27418 \\
\hline 1047 & 20.7823 & 859.2931 & 779.4813 & 479.8333 & 122.5994 & 4.27072 \\
\hline 1048 & 20.7993 & 945.7462 & 936.204 & 479.625 & 122.5994 & 4.26727 \\
\hline 1049 & 20.8163 & 1041.77 & 1181.802 & 479.4167 & 122.5994 & 4.26382 \\
\hline 1050 & 20.8333 & 1200.197 & 1521.671 & 479.2083 & 122.5994 & 4.26038 \\
\hline 1051 & 20.8503 & 1426.316 & 1916.073 & 479 & 122.5994 & 4.25695 \\
\hline 1052 & 20.8673 & 1754.188 & 2248.956 & 478.7917 & 122.5994 & 4.25352 \\
\hline 1053 & 20.8843 & 2243.61 & 2378.678 & 478.5833 & 122.5994 & 4.25009 \\
\hline 1054 & 20.9013 & 2824 & 2307.337 & 478.375 & 122.5994 & 4.24667 \\
\hline 1055 & 20.9183 & 3262.817 & 2129.85 & 478.1667 & 122.5994 & 4.24326 \\
\hline 1056 & 20.9353 & 2994.095 & 1877.887 & 477.9583 & 122.5994 & 4.23985 \\
\hline 1057 & 20.9523 & 2308.553 & 1581.025 & 477.75 & 122.5994 & 4.23645 \\
\hline 1058 & 20.9693 & 1877.306 & 1298.66 & 477.2188 & 122.5994 & 4.23306 \\
\hline 1059 & 20.9863 & 1580.32 & 1069.966 & 476.6875 & 122.5994 & 4.22967 \\
\hline 1060 & 21.0033 & 1098.808 & 902.9521 & 476.1563 & 122.5994 & 4.22628 \\
\hline 1061 & 21.0203 & 774.2021 & 784.5475 & 475.625 & 122.5994 & 4.2229 \\
\hline 1062 & 21.0373 & 692.8184 & 700.3378 & 475.0938 & 122.5994 & 4.21953 \\
\hline 1063 & 21.0543 & 668.7666 & 641.4076 & 474.5625 & 122.5994 & 4.21616 \\
\hline 1064 & 21.0713 & 600.1342 & 600.712 & 474.0313 & 122.5994 & 4.2128 \\
\hline 1065 & 21.0883 & 630.3006 & 572.5881 & 473.5 & 122.5994 & 4.20944 \\
\hline 1066 & 21.1053 & 580.97 & 552.939 & 472.9688 & 122.5994 & 4.20609 \\
\hline 1067 & 21.1223 & 545.0091 & 538.8271 & 472.4375 & 122.5994 & 4.20274 \\
\hline 1068 & 21.1393 & 560.9079 & 528.219 & 471.9063 & 122.5994 & 4.1994 \\
\hline 1069 & 21.1563 & 516.2566 & 519.9028 & 471.375 & 122.5994 & 4.19606 \\
\hline 1070 & 21.1733 & 533.5323 & 513.1885 & 470.8438 & 122.5994 & 4.19273 \\
\hline 1071 & 21.1903 & 441.7236 & 507.6512 & 470.3125 & 122.5994 & 4.18941 \\
\hline 1072 & 21.2073 & 475.4407 & 503.0058 & 469.7813 & 122.5994 & 4.18609 \\
\hline 1073 & 21.2243 & 479.333 & 499.0509 & 469.25 & 122.5994 & 4.18277 \\
\hline 1074 & 21.2413 & 476.3943 & 495.6379 & 468.7188 & 122.5994 & 4.17946 \\
\hline 1075 & 21.2583 & 486.5181 & 492.6587 & 468.1875 & 122.5994 & 4.17616 \\
\hline 1076 & 21.2753 & 465.385 & 490.0369 & 467.6563 & 122.5994 & 4.17286 \\
\hline
\end{tabular}




\begin{tabular}{|c|c|c|c|c|c|c|}
\hline 1077 & 21.2923 & 438.1955 & 487.722 & 467.125 & 122.5994 & 4.16957 \\
\hline 1078 & 21.3093 & 490.5605 & 485.6822 & 466.5938 & 122.5994 & 4.16628 \\
\hline 1079 & 21.3263 & 480.473 & 483.8954 & 466.0625 & 122.5994 & 4.163 \\
\hline 1080 & 21.3433 & 480.2408 & 482.337 & 465.5313 & 122.5994 & 4.15972 \\
\hline 1081 & 21.3603 & 455.5654 & 480.9619 & 465 & 122.5994 & 4.15645 \\
\hline 1082 & 21.3773 & 436.7779 & 479.9247 & 464.7083 & 122.5994 & 4.15318 \\
\hline 1083 & 21.3943 & 484.009 & 478.8856 & 464.4167 & 122.5994 & 4.14992 \\
\hline 1084 & 21.4113 & 473.5133 & 477.8335 & 464.125 & 122.5994 & 4.14666 \\
\hline 1085 & 21.4283 & 453.5941 & 476.8265 & 463.8333 & 122.5994 & 4.14341 \\
\hline 1086 & 21.4453 & 446.3501 & 475.8921 & 463.5417 & 122.5994 & 4.14016 \\
\hline 1087 & 21.4623 & 434.6493 & 475.0594 & 463.25 & 122.5994 & 4.13692 \\
\hline 1088 & 21.4793 & 476.7715 & 474.3864 & 462.9583 & 122.5994 & 4.13369 \\
\hline 1089 & 21.4963 & 483.8548 & 473.9214 & 462.6667 & 122.5994 & 4.13046 \\
\hline 1090 & 21.5133 & 463.4284 & 473.6843 & 462.375 & 122.5994 & 4.12723 \\
\hline 1091 & 21.5303 & 463.4547 & 473.6239 & 462.0833 & 122.5994 & 4.12401 \\
\hline 1092 & 21.5473 & 473.1859 & 473.8713 & 461.7917 & 122.5994 & 4.12079 \\
\hline 1093 & 21.5643 & 470.8988 & 474.3494 & 461.5 & 122.5994 & 4.11758 \\
\hline 1094 & 21.5813 & 464.2098 & 471.3159 & 461.2083 & 122.5994 & 4.11438 \\
\hline 1095 & 21.5983 & 460.2803 & 472.3034 & 460.9167 & 122.5994 & 4.11118 \\
\hline 1096 & 21.6153 & 459.802 & 473.3197 & 460.625 & 122.5994 & 4.10798 \\
\hline 1097 & 21.6323 & 491.3242 & 474.2199 & 460.3333 & 122.5994 & 4.10479 \\
\hline 1098 & 21.6493 & 478.1331 & 474.8286 & 460.0417 & 122.5994 & 4.10161 \\
\hline 1099 & 21.6663 & 456.3668 & 476.3904 & 459.75 & 122.5994 & 4.09843 \\
\hline 1100 & 21.6833 & 480.5158 & 476.159 & 459.4583 & 122.5994 & 4.09525 \\
\hline 1101 & 21.7003 & 447.1075 & 475.4423 & 459.1667 & 122.5994 & 4.09209 \\
\hline 1102 & 21.7173 & 456.396 & 473.2446 & 458.875 & 122.5994 & 4.08892 \\
\hline 1103 & 21.7343 & 470.8988 & 472.0303 & 458.5833 & 122.5994 & 4.08576 \\
\hline 1104 & 21.7513 & 445.937 & 470.6526 & 458.2917 & 122.5994 & 4.08261 \\
\hline 1105 & 21.7683 & 479.0638 & 469.2939 & 458 & 122.5994 & 4.07946 \\
\hline 1106 & 21.7853 & 452.6464 & 468.4437 & 458.2292 & 122.5994 & 4.07631 \\
\hline 1107 & 21.8023 & 431.0371 & 467.9879 & 458.4583 & 122.5994 & 4.07317 \\
\hline 1108 & 21.8193 & 477.4918 & 467.8 & 458.6875 & 122.5994 & 4.07004 \\
\hline 1109 & 21.8363 & 481.0271 & 467.9318 & 458.9167 & 122.5994 & 4.06691 \\
\hline 1110 & 21.8533 & 464.6626 & 468.4105 & 459.1458 & 122.5994 & 4.06378 \\
\hline 1111 & 21.8703 & 447.211 & 469.2746 & 459.375 & 122.5994 & 4.06066 \\
\hline 1112 & 21.8873 & 456.7521 & 470.5704 & 459.6042 & 122.5994 & 4.05754 \\
\hline 1113 & 21.9043 & 494.394 & 472.4134 & 459.8333 & 122.5994 & 4.05443 \\
\hline 1114 & 21.9213 & 455.9283 & 475.1246 & 460.0625 & 122.5994 & 4.05133 \\
\hline 1115 & 21.9383 & 478.8176 & 479.4719 & 460.2917 & 122.5994 & 4.04823 \\
\hline 1116 & 21.9553 & 501.5616 & 486.9653 & 460.5208 & 122.5994 & 4.04513 \\
\hline 1117 & 21.9723 & 493.6309 & 500.0589 & 460.75 & 122.5994 & 4.04204 \\
\hline 1118 & 21.9893 & 556.0274 & 521.7899 & 460.9792 & 122.5994 & 4.03895 \\
\hline 1119 & 22.0063 & 531.5412 & 554.4022 & 461.2083 & 122.5994 & 4.03587 \\
\hline 1120 & 22.0233 & 580.5381 & 596.9614 & 461.4375 & 122.5994 & 4.03279 \\
\hline 1121 & 22.0403 & 641.653 & 642.1357 & 461.6667 & 122.5994 & 4.02972 \\
\hline 1122 & 22.0573 & 700.3698 & 672.8333 & 461.8958 & 122.5994 & 4.02665 \\
\hline 1123 & 22.0743 & 696.3161 & 676.581 & 462.125 & 122.5994 & 4.02359 \\
\hline 1124 & 22.0913 & 747.4718 & 660.562 & 462.3542 & 122.5994 & 4.02053 \\
\hline 1125 & 22.1083 & 685.2728 & 635.1519 & 462.5833 & 122.5994 & 4.01748 \\
\hline
\end{tabular}




\begin{tabular}{|c|c|c|c|c|c|c|}
\hline 1126 & 22.1253 & 622.5263 & 601.8465 & 462.8125 & 122.5994 & 4.01443 \\
\hline 1127 & 22.1423 & 666.149 & 565.5749 & 463.0417 & 122.5994 & 4.01139 \\
\hline 1128 & 22.1593 & 636.6029 & 534.1223 & 463.2708 & 122.5994 & 4.00835 \\
\hline 1129 & 22.1763 & 578.675 & 510.9055 & 463.5 & 122.5994 & 4.00532 \\
\hline 1130 & 22.1933 & 519.138 & 495.2451 & 463.4375 & 122.5994 & 4.00229 \\
\hline 1131 & 22.2103 & 484.009 & 485.5764 & 463.375 & 122.5994 & 3.99926 \\
\hline 1132 & 22.2273 & 478.1134 & 479.8319 & 463.3125 & 122.5994 & 3.99624 \\
\hline 1133 & 22.2443 & 458.9594 & 476.3683 & 463.25 & 122.5994 & 3.99322 \\
\hline 1134 & 22.2613 & 448.4233 & 474.144 & 463.1875 & 122.5994 & 3.99021 \\
\hline 1135 & 22.2783 & 472.9013 & 472.6032 & 463.125 & 122.5994 & 3.98721 \\
\hline 1136 & 22.2953 & 488.7727 & 471.4798 & 463.0625 & 122.5994 & 3.98421 \\
\hline 1137 & 22.3123 & 476.7006 & 470.649 & 463 & 122.5994 & 3.98121 \\
\hline 1138 & 22.3293 & 498.4711 & 470.0496 & 462.9375 & 122.5994 & 3.97822 \\
\hline 1139 & 22.3463 & 500.1083 & 469.6502 & 462.875 & 122.5994 & 3.97523 \\
\hline 1140 & 22.3633 & 488.4913 & 469.435 & 462.8125 & 122.5994 & 3.97224 \\
\hline 1141 & 22.3803 & 478.9583 & 469.3966 & 462.75 & 122.5994 & 3.96927 \\
\hline 1142 & 22.3973 & 483.4735 & 469.5308 & 462.6875 & 122.5994 & 3.96629 \\
\hline 1143 & 22.4143 & 472.3757 & 469.832 & 462.625 & 122.5994 & 3.96332 \\
\hline 1144 & 22.4313 & 446.1205 & 470.2878 & 462.5625 & 122.5994 & 3.96036 \\
\hline 1145 & 22.4483 & 492.2718 & 470.8742 & 462.5 & 122.5994 & 3.9574 \\
\hline 1146 & 22.4653 & 493.1412 & 471.4816 & 462.4375 & 122.5994 & 3.95444 \\
\hline 1147 & 22.4823 & 449.9747 & 472.1822 & 462.375 & 122.5994 & 3.95149 \\
\hline 1148 & 22.4993 & 428.3621 & 472.8176 & 462.3125 & 122.5994 & 3.94854 \\
\hline 1149 & 22.5163 & 433.0921 & 472.2332 & 462.25 & 122.5994 & 3.9456 \\
\hline 1150 & 22.5333 & 458.2523 & 471.9582 & 462.1875 & 122.5994 & 3.94266 \\
\hline 1151 & 22.5503 & 469.7889 & 472.1057 & 462.125 & 122.5994 & 3.93973 \\
\hline 1152 & 22.5673 & 451.2478 & 472.0664 & 462.0625 & 122.5994 & 3.9368 \\
\hline 1153 & 22.5843 & 479.2065 & 471.926 & 462 & 122.5994 & 3.93387 \\
\hline 1154 & 22.6013 & 463.8065 & 471.7985 & 461.9375 & 122.5994 & 3.93095 \\
\hline 1155 & 22.6183 & 479.5774 & 471.8253 & 461.875 & 122.5994 & 3.92803 \\
\hline 1156 & 22.6353 & 476.4966 & 472.1467 & 461.8125 & 122.5994 & 3.92512 \\
\hline 1157 & 22.6523 & 486.7435 & 472.9125 & 461.75 & 122.5994 & 3.92222 \\
\hline 1158 & 22.6693 & 484.4752 & 474.652 & 461.6875 & 122.5994 & 3.91931 \\
\hline 1159 & 22.6863 & 479.9884 & 476.7294 & 461.625 & 122.5994 & 3.91641 \\
\hline 1160 & 22.7033 & 435.5896 & 479.5892 & 461.5625 & 122.5994 & 3.91352 \\
\hline 1161 & 22.7203 & 477.6889 & 483.4608 & 461.5 & 122.5994 & 3.91063 \\
\hline 1162 & 22.7373 & 449.9869 & 488.5102 & 461.4375 & 122.5994 & 3.90774 \\
\hline 1163 & 22.7543 & 475.1918 & 493.7093 & 461.375 & 122.5994 & 3.90486 \\
\hline 1164 & 22.7713 & 446.2308 & 499.3052 & 461.3125 & 122.5994 & 3.90199 \\
\hline 1165 & 22.7883 & 487.6312 & 505.123 & 461.25 & 122.5994 & 3.89911 \\
\hline 1166 & 22.8053 & 470.8988 & 510.0329 & 461.1875 & 122.5994 & 3.89625 \\
\hline 1167 & 22.8223 & 470.4641 & 513.6111 & 461.125 & 122.5994 & 3.89338 \\
\hline 1168 & 22.8393 & 465.6274 & 515.3606 & 461.0625 & 122.5994 & 3.89052 \\
\hline 1169 & 22.8563 & 463.7062 & 515.1443 & 461 & 122.5994 & 3.88767 \\
\hline 1170 & 22.8733 & 501.0876 & 513.1582 & 460.9375 & 122.5994 & 3.88482 \\
\hline 1171 & 22.8903 & 502.8189 & 509.7931 & 460.875 & 122.5994 & 3.88197 \\
\hline 1172 & 22.9073 & 539.144 & 505.5627 & 460.8125 & 122.5994 & 3.87913 \\
\hline 1173 & 22.9243 & 574.6194 & 501.08 & 460.75 & 122.5994 & 3.87629 \\
\hline 1174 & 22.9413 & 543.38 & 497.02 & 460.6875 & 122.5994 & 3.87346 \\
\hline
\end{tabular}




\begin{tabular}{|c|c|c|c|c|c|c|}
\hline 1175 & 22.9583 & 528.6033 & 494.1209 & 460.625 & 122.5994 & 3.87063 \\
\hline 1176 & 22.9753 & 569.1429 & 493.26 & 460.5625 & 122.5994 & 3.8678 \\
\hline 1177 & 22.9923 & 577.469 & 495.504 & 460.5 & 122.5994 & 3.86498 \\
\hline 1178 & 23.0093 & 583.9639 & 501.6892 & 460.1458 & 122.5994 & 3.86216 \\
\hline 1179 & 23.0263 & 578.8919 & 512.8908 & 459.7917 & 122.5994 & 3.85935 \\
\hline 1180 & 23.0433 & 551.7337 & 528.8873 & 459.4375 & 122.5994 & 3.85654 \\
\hline 1181 & 23.0603 & 519.9809 & 547.2552 & 459.0833 & 122.5994 & 3.85374 \\
\hline 1182 & 23.0773 & 491.3035 & 563.6863 & 458.7292 & 122.5994 & 3.85093 \\
\hline 1183 & 23.0943 & 511.56 & 578.6391 & 458.375 & 122.5994 & 3.84814 \\
\hline 1184 & 23.1113 & 530.6957 & 589.3315 & 458.0208 & 122.5994 & 3.84535 \\
\hline 1185 & 23.1283 & 531.6763 & 592.5576 & 457.6667 & 122.5994 & 3.84256 \\
\hline 1186 & 23.1453 & 524.3597 & 583.4306 & 457.3125 & 122.5994 & 3.83977 \\
\hline 1187 & 23.1623 & 529.188 & 566.4332 & 456.9583 & 122.5994 & 3.83699 \\
\hline 1188 & 23.1793 & 521.2852 & 548.0703 & 456.6042 & 122.5994 & 3.83422 \\
\hline 1189 & 23.1963 & 500.7101 & 529.3618 & 456.25 & 122.5994 & 3.83145 \\
\hline 1190 & 23.2133 & 503.2945 & 511.0992 & 455.8958 & 122.5994 & 3.82868 \\
\hline 1191 & 23.2303 & 471.1497 & 495.7346 & 455.5417 & 122.5994 & 3.82592 \\
\hline 1192 & 23.2473 & 474.9762 & 484.4179 & 455.1875 & 122.5994 & 3.82316 \\
\hline 1193 & 23.2643 & 475.8361 & 476.8215 & 454.8333 & 122.5994 & 3.8204 \\
\hline 1194 & 23.2813 & 455.1169 & 472.0744 & 454.4792 & 122.5994 & 3.81765 \\
\hline 1195 & 23.2983 & 479.4138 & 469.2637 & 454.125 & 122.5994 & 3.8149 \\
\hline 1196 & 23.3153 & 461.9492 & 467.6331 & 453.7708 & 122.5994 & 3.81216 \\
\hline 1197 & 23.3323 & 443.2575 & 466.8141 & 453.4167 & 122.5994 & 3.80942 \\
\hline 1198 & 23.3493 & 440.9577 & 466.549 & 453.0625 & 122.5994 & 3.80669 \\
\hline 1199 & 23.3663 & 441.0082 & 466.7708 & 452.7083 & 122.5994 & 3.80396 \\
\hline 1200 & 23.3833 & 451.135 & 467.4941 & 452.3542 & 122.5994 & 3.80123 \\
\hline 1201 & 23.4003 & 450.2154 & 468.9042 & 452 & 122.5994 & 3.79851 \\
\hline 1202 & 23.4173 & 449.4484 & 472.0703 & 452.3477 & 122.5994 & 3.79579 \\
\hline 1203 & 23.4343 & 479.2603 & 477.217 & 452.6953 & 122.5994 & 3.79307 \\
\hline 1204 & 23.4513 & 483.9536 & 486.1078 & 453.043 & 122.5994 & 3.79036 \\
\hline 1205 & 23.4683 & 484.009 & 501.2213 & 453.3906 & 122.5994 & 3.78765 \\
\hline 1206 & 23.4853 & 471.8547 & 524.9441 & 453.7383 & 122.5994 & 3.78495 \\
\hline 1207 & 23.5023 & 534.7895 & 557.9683 & 454.0859 & 122.5994 & 3.78225 \\
\hline 1208 & 23.5193 & 489.9064 & 596.8326 & 454.4336 & 122.5994 & 3.77955 \\
\hline 1209 & 23.5363 & 542.4473 & 630.7791 & 454.7813 & 122.5994 & 3.77686 \\
\hline 1210 & 23.5533 & 543.0851 & 645.4447 & 455.1289 & 122.5994 & 3.77418 \\
\hline 1211 & 23.5703 & 559.5039 & 640.2926 & 455.4766 & 122.5994 & 3.77149 \\
\hline 1212 & 23.5873 & 630.5884 & 627.1356 & 455.8242 & 122.5994 & 3.76881 \\
\hline 1213 & 23.6043 & 638.5407 & 610.8782 & 456.1719 & 122.5994 & 3.76614 \\
\hline 1214 & 23.6213 & 620.517 & 590.8892 & 456.5195 & 122.5994 & 3.76346 \\
\hline 1215 & 23.6383 & 627.4474 & 571.3554 & 456.8672 & 122.5994 & 3.7608 \\
\hline 1216 & 23.6553 & 635.9449 & 554.5165 & 457.2148 & 122.5994 & 3.75813 \\
\hline 1217 & 23.6723 & 580.8209 & 539.5581 & 457.5625 & 122.5994 & 3.75547 \\
\hline 1218 & 23.6893 & 583.5171 & 526.3328 & 457.9102 & 122.5994 & 3.75281 \\
\hline 1219 & 23.7063 & 592.3615 & 517.4115 & 458.2578 & 122.5994 & 3.75016 \\
\hline 1220 & 23.7233 & 575.374 & 510.6593 & 458.6055 & 122.5994 & 3.74751 \\
\hline 1221 & 23.7403 & 551.1349 & 505.2306 & 458.9531 & 122.5994 & 3.74487 \\
\hline 1222 & 23.7573 & 527.3588 & 502.2501 & 459.3008 & 122.5994 & 3.74223 \\
\hline 1223 & 23.7743 & 522.9208 & 501.5433 & 459.6484 & 122.5994 & 3.73959 \\
\hline
\end{tabular}




\begin{tabular}{|c|c|c|c|c|c|c|}
\hline 1224 & 23.7913 & 543.5072 & 503.341 & 459.9961 & 122.5994 & 3.73696 \\
\hline 1225 & 23.8083 & 530.628 & 507.2636 & 460.3438 & 122.5994 & 3.73433 \\
\hline 1226 & 23.8253 & 544.5696 & 512.1288 & 460.6914 & 122.5994 & 3.7317 \\
\hline 1227 & 23.8423 & 560.1724 & 517.1524 & 461.0391 & 122.5994 & 3.72908 \\
\hline 1228 & 23.8593 & 569.318 & 521.5627 & 461.3867 & 122.5994 & 3.72646 \\
\hline 1229 & 23.8763 & 550.2813 & 524.1166 & 461.7344 & 122.5994 & 3.72384 \\
\hline 1230 & 23.8933 & 547.5702 & 524.6155 & 462.082 & 122.5994 & 3.72123 \\
\hline 1231 & 23.9103 & 541.083 & 523.104 & 462.4297 & 122.5994 & 3.71863 \\
\hline 1232 & 23.9273 & 513.7825 & 520.6024 & 462.7773 & 122.5994 & 3.71602 \\
\hline 1233 & 23.9443 & 523.0358 & 516.2026 & 463.125 & 122.5994 & 3.71342 \\
\hline 1234 & 23.9613 & 557.1958 & 510.0074 & 463.4727 & 122.5994 & 3.71083 \\
\hline 1235 & 23.9783 & 538.6386 & 505.0369 & 463.8203 & 122.5994 & 3.70823 \\
\hline 1236 & 23.9953 & 530.3855 & 500.0812 & 464.168 & 122.5994 & 3.70565 \\
\hline 1237 & 24.0123 & 538.2501 & 495.8427 & 464.5156 & 122.5994 & 3.70306 \\
\hline 1238 & 24.0293 & 546.8755 & 492.2479 & 464.8633 & 122.5994 & 3.70048 \\
\hline 1239 & 24.0463 & 534.5206 & 490.8275 & 465.2109 & 122.5994 & 3.6979 \\
\hline 1240 & 24.0633 & 571.0889 & 491.6788 & 465.5586 & 122.5994 & 3.69533 \\
\hline 1241 & 24.0803 & 578.9453 & 495.3768 & 465.9063 & 122.5994 & 3.69276 \\
\hline 1242 & 24.0973 & 548.0859 & 503.0238 & 466.2539 & 122.5994 & 3.69019 \\
\hline 1243 & 24.1143 & 576.0387 & 514.1801 & 466.6016 & 122.5994 & 3.68763 \\
\hline 1244 & 24.1313 & 558.5284 & 526.8511 & 466.9492 & 122.5994 & 3.68507 \\
\hline 1245 & 24.1483 & 512.4739 & 537.0062 & 467.2969 & 122.5994 & 3.68251 \\
\hline 1246 & 24.1653 & 548.9197 & 540.852 & 467.6445 & 122.5994 & 3.67996 \\
\hline 1247 & 24.1823 & 535.2461 & 540.671 & 467.9922 & 122.5994 & 3.67741 \\
\hline 1248 & 24.1993 & 536.6864 & 540.7856 & 468.3398 & 122.5994 & 3.67487 \\
\hline 1249 & 24.2163 & 599.4894 & 542.3809 & 468.6875 & 122.5994 & 3.67233 \\
\hline 1250 & 24.2333 & 543.2688 & 545.3219 & 468.6589 & 122.5994 & 3.66979 \\
\hline 1251 & 24.2503 & 563.0157 & 550.8353 & 468.6302 & 122.5994 & 3.66725 \\
\hline 1252 & 24.2673 & 566.3857 & 558.6646 & 468.6016 & 122.5994 & 3.66472 \\
\hline 1253 & 24.2843 & 544.3525 & 568.9158 & 468.5729 & 122.5994 & 3.6622 \\
\hline 1254 & 24.3013 & 607.4042 & 580.4745 & 468.5443 & 122.5994 & 3.65967 \\
\hline 1255 & 24.3183 & 629.6512 & 586.8218 & 468.5156 & 122.5994 & 3.65715 \\
\hline 1256 & 24.3353 & 625.0716 & 581.7455 & 468.487 & 122.5994 & 3.65464 \\
\hline 1257 & 24.3523 & 645.422 & 568.8805 & 468.4583 & 122.5994 & 3.65212 \\
\hline 1258 & 24.3693 & 656.4843 & 554.3047 & 468.4297 & 122.5994 & 3.64961 \\
\hline 1259 & 24.3863 & 674.1252 & 538.7763 & 468.401 & 122.5994 & 3.64711 \\
\hline 1260 & 24.4033 & 573.4797 & 522.1969 & 468.3724 & 122.5994 & 3.64461 \\
\hline 1261 & 24.4203 & 547.0541 & 507.0643 & 468.3438 & 122.5994 & 3.64211 \\
\hline 1262 & 24.4373 & 536.2903 & 495.2807 & 468.3151 & 122.5994 & 3.63961 \\
\hline 1263 & 24.4543 & 496.2975 & 487.0351 & 468.2865 & 122.5994 & 3.63712 \\
\hline 1264 & 24.4713 & 515.2406 & 481.6848 & 468.2578 & 122.5994 & 3.63463 \\
\hline 1265 & 24.4883 & 512.6402 & 478.3603 & 468.2292 & 122.5994 & 3.63215 \\
\hline 1266 & 24.5053 & 497.8961 & 476.2825 & 468.2005 & 122.5994 & 3.62967 \\
\hline 1267 & 24.5223 & 487.1937 & 474.908 & 468.1719 & 122.5994 & 3.62719 \\
\hline 1268 & 24.5393 & 488.1482 & 473.9223 & 468.1432 & 122.5994 & 3.62471 \\
\hline 1269 & 24.5563 & 469.654 & 473.1649 & 468.1146 & 122.5994 & 3.62224 \\
\hline 1270 & 24.5733 & 481.7182 & 472.5563 & 468.0859 & 122.5994 & 3.61978 \\
\hline 1271 & 24.5903 & 472.2481 & 472.0543 & 468.0573 & 122.5994 & 3.61731 \\
\hline 1272 & 24.6073 & 469.08 & 471.633 & 468.0286 & 122.5994 & 3.61485 \\
\hline
\end{tabular}




\begin{tabular}{|c|c|c|c|c|c|c|}
\hline 1273 & 24.6243 & 453.6332 & 472.8278 & 468 & 122.5994 & 3.6124 \\
\hline 1274 & 24.6413 & 478.6675 & 473.943 & 469.3125 & 122.5994 & 3.60994 \\
\hline 1275 & 24.6583 & 473.286 & 475.106 & 470.625 & 122.5994 & 3.60749 \\
\hline 1276 & 24.6753 & 454.2251 & 476.3141 & 471.9375 & 122.5994 & 3.60504 \\
\hline 1277 & 24.6923 & 439.9215 & 477.5811 & 473.25 & 122.5994 & 3.6026 \\
\hline 1278 & 24.7093 & 457.0368 & 478.8824 & 474.5625 & 122.5994 & 3.60016 \\
\hline 1279 & 24.7263 & 475.3963 & 479.5949 & 475.875 & 122.5994 & 3.59772 \\
\hline 1280 & 24.7433 & 462.2586 & 480.3882 & 477.1875 & 122.5994 & 3.59529 \\
\hline 1281 & 24.7603 & 471.6484 & 481.8668 & 478.5 & 122.5994 & 3.59286 \\
\hline 1282 & 24.7773 & 469.8024 & 483.3744 & 479.8125 & 122.5994 & 3.59043 \\
\hline 1283 & 24.7943 & 449.852 & 484.6502 & 481.125 & 122.5994 & 3.58801 \\
\hline 1284 & 24.8113 & 456.4817 & 486.2539 & 482.4375 & 122.5994 & 3.58559 \\
\hline 1285 & 24.8283 & 469.2832 & 487.9054 & 483.75 & 122.5994 & 3.58317 \\
\hline 1286 & 24.8453 & 493.8279 & 489.6152 & 485.0625 & 122.5994 & 3.58076 \\
\hline 1287 & 24.8623 & 503.2512 & 491.3949 & 486.375 & 122.5994 & 3.57835 \\
\hline 1288 & 24.8793 & 486.3973 & 493.2566 & 487.6875 & 122.5994 & 3.57594 \\
\hline 1289 & 24.8963 & 464.1839 & 495.2115 & 489 & 122.5994 & 3.57354 \\
\hline 1290 & 24.9133 & 510.1454 & 497.2696 & 490.3125 & 122.5994 & 3.57114 \\
\hline 1291 & 24.9303 & 485.0784 & 499.4375 & 491.625 & 122.5994 & 3.56874 \\
\hline 1292 & 24.9473 & 512.5128 & 501.7176 & 492.9375 & 122.5994 & 3.56635 \\
\hline 1293 & 24.9643 & 499.193 & 504.1059 & 494.25 & 122.5994 & 3.56396 \\
\hline 1294 & 24.9813 & 514.9461 & 506.5929 & 495.5625 & 122.5994 & 3.56157 \\
\hline 1295 & 24.9983 & 512.2041 & 509.171 & 496.875 & 122.5994 & 3.55919 \\
\hline 1296 & 25.0153 & 534.5004 & 511.8506 & 498.1875 & 122.5994 & 3.55681 \\
\hline 1297 & 25.0323 & 490.2422 & 514.6854 & 499.5 & 122.5994 & 3.55443 \\
\hline 1298 & 25.0493 & 508.2706 & 517.7975 & 500.8125 & 122.5994 & 3.55206 \\
\hline 1299 & 25.0663 & 510.7695 & 521.4299 & 502.125 & 122.5994 & 3.54969 \\
\hline 1300 & 25.0833 & 489.4876 & 526.0848 & 503.4375 & 122.5994 & 3.54732 \\
\hline 1301 & 25.1003 & 532.3141 & 532.7972 & 504.75 & 122.5994 & 3.54496 \\
\hline 1302 & 25.1173 & 556.7986 & 543.4696 & 506.0625 & 122.5994 & 3.5426 \\
\hline 1303 & 25.1343 & 581.6635 & 560.9438 & 507.375 & 122.5994 & 3.54024 \\
\hline 1304 & 25.1513 & 640.8313 & 588.3066 & 508.6875 & 122.5994 & 3.53789 \\
\hline 1305 & 25.1683 & 661.6893 & 627.2271 & 510 & 122.5994 & 3.53553 \\
\hline 1306 & 25.1853 & 683.638 & 674.8339 & 511.3125 & 122.5994 & 3.53319 \\
\hline 1307 & 25.2023 & 713.4396 & 720.566 & 512.625 & 122.5994 & 3.53084 \\
\hline 1308 & 25.2193 & 713.7441 & 746.5448 & 513.9375 & 122.5994 & 3.5285 \\
\hline 1309 & 25.2363 & 682.7246 & 746.6008 & 515.25 & 122.5994 & 3.52616 \\
\hline 1310 & 25.2533 & 687.4087 & 734.3015 & 516.5625 & 122.5994 & 3.52383 \\
\hline 1311 & 25.2703 & 660.8904 & 719.7982 & 517.875 & 122.5994 & 3.52149 \\
\hline 1312 & 25.2873 & 641.0822 & 702.0074 & 519.1875 & 122.5994 & 3.51917 \\
\hline 1313 & 25.3043 & 637.1293 & 682.5981 & 520.5 & 122.5994 & 3.51684 \\
\hline 1314 & 25.3213 & 607.6328 & 669.2344 & 521.8125 & 122.5994 & 3.51452 \\
\hline 1315 & 25.3383 & 600.2612 & 666.6066 & 523.125 & 122.5994 & 3.5122 \\
\hline 1316 & 25.3553 & 583.4008 & 674.3121 & 524.4375 & 122.5994 & 3.50988 \\
\hline 1317 & 25.3723 & 567.2296 & 687.3955 & 525.75 & 122.5994 & 3.50757 \\
\hline 1318 & 25.3893 & 598.7093 & 697.3311 & 527.0625 & 122.5994 & 3.50526 \\
\hline 1319 & 25.4063 & 546.9487 & 698.0814 & 528.375 & 122.5994 & 3.50295 \\
\hline 1320 & 25.4233 & 537.8441 & 691.5483 & 529.6875 & 122.5994 & 3.50065 \\
\hline 1321 & 25.4403 & 577.4705 & 681.2621 & 531 & 122.5994 & 3.49835 \\
\hline
\end{tabular}




\begin{tabular}{|c|c|c|c|c|c|c|}
\hline 1322 & 25.4573 & 617.154 & 667.157 & 531.9583 & 122.5994 & 3.49605 \\
\hline 1323 & 25.4743 & 613.4197 & 649.918 & 532.9167 & 122.5994 & 3.49375 \\
\hline 1324 & 25.4913 & 612.0219 & 632.4233 & 533.875 & 122.5994 & 3.49146 \\
\hline 1325 & 25.5083 & 644.7879 & 617.3743 & 534.8333 & 122.5994 & 3.48917 \\
\hline 1326 & 25.5253 & 657.7651 & 605.8847 & 535.7917 & 122.5994 & 3.48689 \\
\hline 1327 & 25.5423 & 647.8728 & 597.7469 & 536.75 & 122.5994 & 3.48461 \\
\hline 1328 & 25.5593 & 647.4518 & 591.5959 & 537.7083 & 122.5994 & 3.48233 \\
\hline 1329 & 25.5763 & 653.9664 & 585.7114 & 538.6667 & 122.5994 & 3.48005 \\
\hline 1330 & 25.5933 & 666.5679 & 579.7936 & 539.625 & 122.5994 & 3.47778 \\
\hline 1331 & 25.6103 & 664.459 & 574.6169 & 540.5833 & 122.5994 & 3.47551 \\
\hline 1332 & 25.6273 & 644.2613 & 570.3224 & 541.5417 & 122.5994 & 3.47324 \\
\hline 1333 & 25.6443 & 605.6626 & 566.4106 & 542.5 & 122.5994 & 3.47098 \\
\hline 1334 & 25.6613 & 617.4231 & 562.963 & 543.4583 & 122.5994 & 3.46872 \\
\hline 1335 & 25.6783 & 622.9942 & 560.3004 & 544.4167 & 122.5994 & 3.46646 \\
\hline 1336 & 25.6953 & 605.6407 & 558.4767 & 545.375 & 122.5994 & 3.4642 \\
\hline 1337 & 25.7123 & 637.547 & 557.329 & 546.3333 & 122.5994 & 3.46195 \\
\hline 1338 & 25.7293 & 638.5923 & 556.7359 & 547.2917 & 122.5994 & 3.4597 \\
\hline 1339 & 25.7463 & 592.276 & 556.5446 & 548.25 & 122.5994 & 3.45746 \\
\hline 1340 & 25.7633 & 604.5471 & 556.5998 & 549.2083 & 122.5994 & 3.45521 \\
\hline 1341 & 25.7803 & 686.7652 & 556.8079 & 550.1667 & 122.5994 & 3.45297 \\
\hline 1342 & 25.7973 & 594.4565 & 557.1426 & 551.125 & 122.5994 & 3.45074 \\
\hline 1343 & 25.8143 & 571.8204 & 557.5801 & 552.0833 & 122.5994 & 3.4485 \\
\hline 1344 & 25.8313 & 571.2207 & 558.1512 & 553.0417 & 122.5994 & 3.44627 \\
\hline 1345 & 25.8483 & 536.7083 & 558.8344 & 554 & 122.5994 & 3.44404 \\
\hline 1346 & 25.8653 & 568.942 & 557.9518 & 554.4884 & 122.5994 & 3.44182 \\
\hline 1347 & 25.8823 & 526.2482 & 558.4313 & 554.9769 & 122.5994 & 3.4396 \\
\hline 1348 & 25.8993 & 570.4001 & 577.0793 & 555.4653 & 122.5994 & 3.43738 \\
\hline 1349 & 25.9163 & 519.9402 & 578.4814 & 555.9538 & 122.5994 & 3.43516 \\
\hline 1350 & 25.9333 & 541.9673 & 579.983 & 556.4422 & 122.5994 & 3.43295 \\
\hline 1351 & 25.9503 & 509.3123 & 581.7015 & 556.9307 & 122.5994 & 3.43074 \\
\hline 1352 & 25.9673 & 569.2799 & 592.4249 & 557.4191 & 122.5994 & 3.42853 \\
\hline 1353 & 25.9843 & 538.7932 & 595.1741 & 557.9076 & 122.5994 & 3.42633 \\
\hline 1354 & 26.0013 & 504.9515 & 598.1181 & 558.396 & 122.5994 & 3.42413 \\
\hline 1355 & 26.0183 & 563.318 & 600.9334 & 558.8845 & 122.5994 & 3.42193 \\
\hline 1356 & 26.0353 & 545.0747 & 609.2777 & 559.3729 & 122.5994 & 3.41973 \\
\hline 1357 & 26.0523 & 554.4928 & 611.6274 & 559.8614 & 122.5994 & 3.41754 \\
\hline 1358 & 26.0693 & 547.5542 & 614.0635 & 560.3498 & 122.5994 & 3.41535 \\
\hline 1359 & 26.0863 & 570.6585 & 616.6754 & 560.8383 & 122.5994 & 3.41316 \\
\hline 1360 & 26.1033 & 584.4218 & 622.3793 & 561.3267 & 122.5994 & 3.41098 \\
\hline 1361 & 26.1203 & 577.0543 & 625.4844 & 561.8151 & 122.5994 & 3.40879 \\
\hline 1362 & 26.1373 & 567.8036 & 629.0277 & 562.3036 & 122.5994 & 3.40662 \\
\hline 1363 & 26.1543 & 581.5817 & 633.1166 & 562.792 & 122.5994 & 3.40444 \\
\hline 1364 & 26.1713 & 606.782 & 637.7998 & 563.2805 & 122.5994 & 3.40227 \\
\hline 1365 & 26.1883 & 610.1014 & 643.1122 & 563.7689 & 122.5994 & 3.4001 \\
\hline 1366 & 26.2053 & 611.8614 & 648.5725 & 564.2574 & 122.5994 & 3.39793 \\
\hline 1367 & 26.2223 & 605.4473 & 655.3471 & 564.7458 & 122.5994 & 3.39577 \\
\hline 1368 & 26.2393 & 608.8888 & 663.0057 & 565.2343 & 122.5994 & 3.3936 \\
\hline 1369 & 26.2563 & 624.2166 & 671.416 & 565.7227 & 122.5994 & 3.39145 \\
\hline 1370 & 26.2733 & 616.8976 & 681.0581 & 565.8342 & 122.5994 & 3.38929 \\
\hline
\end{tabular}




\begin{tabular}{|c|c|c|c|c|c|c|}
\hline 1371 & 26.2903 & 654.3401 & 692.3726 & 565.9457 & 122.5994 & 3.38714 \\
\hline 1372 & 26.3073 & 643.3851 & 706.0422 & 566.0572 & 122.5994 & 3.38499 \\
\hline 1373 & 26.3243 & 650.58 & 723.2626 & 566.1686 & 122.5994 & 3.38284 \\
\hline 1374 & 26.3413 & 702.7165 & 745.5833 & 566.2801 & 122.5994 & 3.38069 \\
\hline 1375 & 26.3583 & 698.6325 & 774.1674 & 566.3916 & 122.5994 & 3.37855 \\
\hline 1376 & 26.3753 & 697.8796 & 808.9496 & 566.5031 & 122.5994 & 3.37641 \\
\hline 1377 & 26.3923 & 758.6565 & 847.5447 & 566.6145 & 122.5994 & 3.37428 \\
\hline 1378 & 26.4093 & 813.0265 & 885.0353 & 566.726 & 122.5994 & 3.37214 \\
\hline 1379 & 26.4263 & 888.9507 & 919.7509 & 566.8375 & 122.5994 & 3.37001 \\
\hline 1380 & 26.4433 & 940.6018 & 957.8404 & 566.949 & 122.5994 & 3.36789 \\
\hline 1381 & 26.4603 & 961.5169 & 1005.257 & 567.0605 & 122.5994 & 3.36576 \\
\hline 1382 & 26.4773 & 1068.388 & 1062.125 & 567.1719 & 122.5994 & 3.36364 \\
\hline 1383 & 26.4943 & 1241.249 & 1127.821 & 567.2834 & 122.5994 & 3.36152 \\
\hline 1384 & 26.5113 & 1388.969 & 1211.275 & 567.3949 & 122.5994 & 3.3594 \\
\hline 1385 & 26.5283 & 1554.221 & 1336.854 & 567.5064 & 122.5994 & 3.35729 \\
\hline 1386 & 26.5453 & 1818.352 & 1548.327 & 567.6178 & 122.5994 & 3.35517 \\
\hline 1387 & 26.5623 & 2103.378 & 1921.212 & 567.7293 & 122.5994 & 3.35307 \\
\hline 1388 & 26.5793 & 2608.823 & 2571.54 & 567.8408 & 122.5994 & 3.35096 \\
\hline 1389 & 26.5963 & 3047.193 & 3631.781 & 567.9523 & 122.5994 & 3.34886 \\
\hline 1390 & 26.6133 & 3672.311 & 5176.205 & 568.0638 & 122.5994 & 3.34676 \\
\hline 1391 & 26.6303 & 4843.919 & 7106.875 & 568.1752 & 122.5994 & 3.34466 \\
\hline 1392 & 26.6473 & 6253.247 & 8994.598 & 568.2867 & 122.5994 & 3.34256 \\
\hline 1393 & 26.6643 & 8394.941 & 10065.51 & 568.3982 & 122.5994 & 3.34047 \\
\hline 1394 & 26.6813 & 10934.57 & 9953.54 & 568.5097 & 122.5994 & 3.33838 \\
\hline 1395 & 26.6983 & 13905.9 & 9220.236 & 568.6212 & 122.5994 & 3.33629 \\
\hline 1396 & 26.7153 & 13369.31 & 8391.433 & 568.7326 & 122.5994 & 3.33421 \\
\hline 1397 & 26.7323 & 9261.322 & 7420.719 & 568.8441 & 122.5994 & 3.33213 \\
\hline 1398 & 26.7493 & 7407.352 & 6194.811 & 568.9556 & 122.5994 & 3.33005 \\
\hline 1399 & 26.7663 & 7271.497 & 4950.542 & 569.0671 & 122.5994 & 3.32797 \\
\hline 1400 & 26.7833 & 6568.978 & 3933.799 & 569.1786 & 122.5994 & 3.3259 \\
\hline 1401 & 26.8003 & 4105.832 & 3207.976 & 569.29 & 122.5994 & 3.32383 \\
\hline 1402 & 26.8173 & 2544.199 & 2729.194 & 569.4015 & 122.5994 & 3.32176 \\
\hline 1403 & 26.8343 & 2160.866 & 2418.327 & 569.513 & 122.5994 & 3.31969 \\
\hline 1404 & 26.8513 & 1902.253 & 2199.719 & 569.6245 & 122.5994 & 3.31763 \\
\hline 1405 & 26.8683 & 1795.249 & 2022.01 & 569.736 & 122.5994 & 3.31557 \\
\hline 1406 & 26.8853 & 1650.915 & 1857.761 & 569.8474 & 122.5994 & 3.31351 \\
\hline 1407 & 26.9023 & 1598.312 & 1697.344 & 569.9589 & 122.5994 & 3.31146 \\
\hline 1408 & 26.9193 & 1438.487 & 1541.723 & 570.0704 & 122.5994 & 3.3094 \\
\hline 1409 & 26.9363 & 1269.101 & 1395.365 & 570.1819 & 122.5994 & 3.30735 \\
\hline 1410 & 26.9533 & 1204.231 & 1263.023 & 570.2934 & 122.5994 & 3.30531 \\
\hline 1411 & 26.9703 & 1078.533 & 1147.433 & 570.4048 & 122.5994 & 3.30326 \\
\hline 1412 & 26.9873 & 951.0598 & 1049.769 & 570.5163 & 122.5994 & 3.30122 \\
\hline 1413 & 27.0043 & 864.4129 & 969.1508 & 570.6278 & 122.5994 & 3.29918 \\
\hline 1414 & 27.0213 & 790.6782 & 903.9236 & 570.7393 & 122.5994 & 3.29714 \\
\hline 1415 & 27.0383 & 760.2958 & 851.941 & 570.8508 & 122.5994 & 3.29511 \\
\hline 1416 & 27.0553 & 738.678 & 810.9102 & 570.9622 & 122.5994 & 3.29308 \\
\hline 1417 & 27.0723 & 721.1154 & 720.5844 & 571.0737 & 122.5994 & 3.29105 \\
\hline 1418 & 27.0893 & 675.1447 & 699.1966 & 570.8082 & 122.5994 & 3.28902 \\
\hline 1419 & 27.1063 & 640.3468 & 682.4883 & 570.5428 & 122.5994 & 3.287 \\
\hline
\end{tabular}




\begin{tabular}{|c|c|c|c|c|c|c|}
\hline 1420 & 27.1233 & 694.9229 & 669.2332 & 570.2773 & 122.5994 & 3.28497 \\
\hline 1421 & 27.1403 & 734.4237 & 658.5084 & 570.0118 & 122.5994 & 3.28295 \\
\hline 1422 & 27.1573 & 703.8539 & 649.6406 & 569.7463 & 122.5994 & 3.28094 \\
\hline 1423 & 27.1743 & 684.3406 & 642.1531 & 569.4808 & 122.5994 & 3.27892 \\
\hline 1424 & 27.1913 & 670.4238 & 635.7137 & 569.2153 & 122.5994 & 3.27691 \\
\hline 1425 & 27.2083 & 625.134 & 630.0916 & 568.9498 & 122.5994 & 3.2749 \\
\hline 1426 & 27.2253 & 628.1783 & 625.1249 & 568.6843 & 122.5994 & 3.2729 \\
\hline 1427 & 27.2423 & 589.8594 & 624.9917 & 568.4188 & 122.5994 & 3.27089 \\
\hline 1428 & 27.2593 & 579.9619 & 621.2543 & 568.1533 & 122.5994 & 3.26889 \\
\hline 1429 & 27.2763 & 551.2741 & 617.9234 & 567.8878 & 122.5994 & 3.26689 \\
\hline 1430 & 27.2933 & 541.7226 & 614.9478 & 567.6224 & 122.5994 & 3.2649 \\
\hline 1431 & 27.3103 & 550.0916 & 612.7659 & 567.3569 & 122.5994 & 3.2629 \\
\hline 1432 & 27.3273 & 544.8051 & 610.4099 & 567.0914 & 122.5994 & 3.26091 \\
\hline 1433 & 27.3443 & 509.5324 & 608.3121 & 566.8259 & 122.5994 & 3.25892 \\
\hline 1434 & 27.3613 & 563.5972 & 606.4518 & 566.5604 & 122.5994 & 3.25694 \\
\hline 1435 & 27.3783 & 596.7286 & 604.8134 & 566.2949 & 122.5994 & 3.25495 \\
\hline 1436 & 27.3953 & 626.3274 & 603.3851 & 566.0294 & 122.5994 & 3.25297 \\
\hline 1437 & 27.4123 & 607.721 & 602.16 & 565.7639 & 122.5994 & 3.25099 \\
\hline 1438 & 27.4293 & 656.0168 & 601.1354 & 565.4984 & 122.5994 & 3.24902 \\
\hline 1439 & 27.4463 & 684.8293 & 600.3129 & 565.2329 & 122.5994 & 3.24704 \\
\hline 1440 & 27.4633 & 681.4221 & 599.7 & 564.9675 & 122.5994 & 3.24507 \\
\hline 1441 & 27.4803 & 722.9206 & 590.1765 & 564.702 & 122.5994 & 3.2431 \\
\hline 1442 & 27.4973 & 718.5539 & 590.048 & 564.0556 & 122.5994 & 3.24114 \\
\hline 1443 & 27.5143 & 779.9314 & 590.1721 & 563.4092 & 122.5994 & 3.23917 \\
\hline 1444 & 27.5313 & 834.029 & 590.5997 & 562.7628 & 122.5994 & 3.23721 \\
\hline 1445 & 27.5483 & 813.8605 & 591.1984 & 562.1164 & 122.5994 & 3.23525 \\
\hline 1446 & 27.5653 & 836.8121 & 592.5238 & 561.4701 & 122.5994 & 3.2333 \\
\hline 1447 & 27.5823 & 861.3913 & 594.6044 & 560.8237 & 122.5994 & 3.23134 \\
\hline 1448 & 27.5993 & 911.4091 & 597.9598 & 560.1773 & 122.5994 & 3.22939 \\
\hline 1449 & 27.6163 & 781.8793 & 598.0194 & 559.5309 & 122.5994 & 3.22744 \\
\hline 1450 & 27.6333 & 734.054 & 608.2565 & 558.8845 & 122.5994 & 3.22549 \\
\hline 1451 & 27.6503 & 718.3362 & 625.6364 & 558.2382 & 122.5994 & 3.22355 \\
\hline 1452 & 27.6673 & 756.9493 & 653.1054 & 557.5918 & 122.5994 & 3.22161 \\
\hline 1453 & 27.6843 & 729.4919 & 690.2281 & 556.9454 & 122.5994 & 3.21967 \\
\hline 1454 & 27.7013 & 686.0246 & 737.1104 & 556.299 & 122.5994 & 3.21773 \\
\hline 1455 & 27.7183 & 670.2346 & 780.366 & 555.6526 & 122.5994 & 3.2158 \\
\hline 1456 & 27.7353 & 717.3743 & 802.1827 & 555.0063 & 122.5994 & 3.21386 \\
\hline 1457 & 27.7523 & 800.2508 & 798.8553 & 554.3599 & 122.5994 & 3.21193 \\
\hline 1458 & 27.7693 & 947.955 & 786.7578 & 553.7135 & 122.5994 & 3.21001 \\
\hline 1459 & 27.7863 & 973.8203 & 778.3304 & 553.0671 & 122.5994 & 3.20808 \\
\hline 1460 & 27.8033 & 923.4899 & 774.6497 & 552.4207 & 122.5994 & 3.20616 \\
\hline 1461 & 27.8203 & 947.8185 & 778.8372 & 551.7744 & 122.5994 & 3.20424 \\
\hline 1462 & 27.8373 & 1026.692 & 805.0413 & 551.128 & 122.5994 & 3.20232 \\
\hline 1463 & 27.8543 & 1060.211 & 867.3872 & 550.4816 & 122.5994 & 3.2004 \\
\hline 1464 & 27.8713 & 1083.041 & 969.7292 & 549.8352 & 122.5994 & 3.19849 \\
\hline 1465 & 27.8883 & 1021.4 & 1098.667 & 549.1888 & 122.5994 & 3.19658 \\
\hline 1466 & 27.9053 & 1033.708 & 1214.447 & 548.0513 & 122.5994 & 3.19467 \\
\hline 1467 & 27.9223 & 1118.033 & 1269.998 & 546.9137 & 122.5994 & 3.19276 \\
\hline 1468 & 27.9393 & 1233.058 & 1269.004 & 545.7762 & 122.5994 & 3.19086 \\
\hline
\end{tabular}




\begin{tabular}{|c|c|c|c|c|c|c|}
\hline 1469 & 27.9563 & 1408.128 & 1250.39 & 544.6387 & 122.5994 & 3.18896 \\
\hline 1470 & 27.9733 & 1486.961 & 1221.055 & 543.5011 & 122.5994 & 3.18706 \\
\hline 1471 & 27.9903 & 1465.868 & 1161.732 & 542.3636 & 122.5994 & 3.18516 \\
\hline 1472 & 28.0073 & 1412.401 & 1076.136 & 541.226 & 122.5994 & 3.18327 \\
\hline 1473 & 28.0243 & 1401.369 & 989.9792 & 540.0885 & 122.5994 & 3.18137 \\
\hline 1474 & 28.0413 & 1329.665 & 917.023 & 538.9509 & 122.5994 & 3.17948 \\
\hline 1475 & 28.0583 & 1250.274 & 858.8027 & 537.8134 & 122.5994 & 3.1776 \\
\hline 1476 & 28.0753 & 1117.015 & 816.8357 & 536.6758 & 122.5994 & 3.17571 \\
\hline 1477 & 28.0923 & 956.2809 & 791.0204 & 535.5383 & 122.5994 & 3.17383 \\
\hline 1478 & 28.1093 & 876.2211 & 776.0548 & 534.4007 & 122.5994 & 3.17195 \\
\hline 1479 & 28.1263 & 777.7693 & 762.0883 & 533.2632 & 122.5994 & 3.17007 \\
\hline 1480 & 28.1433 & 729.4133 & 739.4188 & 532.1256 & 122.5994 & 3.16819 \\
\hline 1481 & 28.1603 & 728.09 & 713.0078 & 530.9881 & 122.5994 & 3.16632 \\
\hline 1482 & 28.1773 & 719.6999 & 688.1776 & 529.8505 & 122.5994 & 3.16445 \\
\hline 1483 & 28.1943 & 635.5379 & 666.564 & 528.713 & 122.5994 & 3.16258 \\
\hline 1484 & 28.2113 & 620.4775 & 645.213 & 527.5754 & 122.5994 & 3.16071 \\
\hline 1485 & 28.2283 & 579.0837 & 626.1893 & 526.4379 & 122.5994 & 3.15884 \\
\hline 1486 & 28.2453 & 608.4552 & 612.8248 & 525.3003 & 122.5994 & 3.15698 \\
\hline 1487 & 28.2623 & 595.697 & 608.1554 & 524.1628 & 122.5994 & 3.15512 \\
\hline 1488 & 28.2793 & 561.8801 & 612.869 & 523.0252 & 122.5994 & 3.15326 \\
\hline 1489 & 28.2963 & 561.7776 & 624.8614 & 521.8877 & 122.5994 & 3.15141 \\
\hline 1490 & 28.3133 & 565.9146 & 637.6779 & 520.309 & 122.5994 & 3.14955 \\
\hline 1491 & 28.3303 & 551.4294 & 639.4896 & 518.7304 & 122.5994 & 3.1477 \\
\hline 1492 & 28.3473 & 542.0149 & 635.1768 & 517.1517 & 122.5994 & 3.14585 \\
\hline 1493 & 28.3643 & 582.718 & 625.8776 & 515.5731 & 122.5994 & 3.14401 \\
\hline 1494 & 28.3813 & 555.2286 & 614.3269 & 513.9944 & 122.5994 & 3.14216 \\
\hline 1495 & 28.3983 & 546.3228 & 600.1962 & 512.4158 & 122.5994 & 3.14032 \\
\hline 1496 & 28.4153 & 557.1593 & 585.5466 & 510.8371 & 122.5994 & 3.13848 \\
\hline 1497 & 28.4323 & 504.9008 & 570.3295 & 509.2585 & 122.5994 & 3.13664 \\
\hline 1498 & 28.4493 & 485.1537 & 556.5504 & 507.6798 & 122.5994 & 3.13481 \\
\hline 1499 & 28.4663 & 516.902 & 545.1754 & 506.1012 & 122.5994 & 3.13297 \\
\hline 1500 & 28.4833 & 541.7677 & 536.3954 & 504.5225 & 122.5994 & 3.13114 \\
\hline 1501 & 28.5003 & 504.6361 & 529.742 & 502.9438 & 122.5994 & 3.12931 \\
\hline 1502 & 28.5173 & 489.7302 & 524.5236 & 501.3652 & 122.5994 & 3.12749 \\
\hline 1503 & 28.5343 & 497.1069 & 520.14 & 499.7865 & 122.5994 & 3.12566 \\
\hline 1504 & 28.5513 & 494.2173 & 516.1859 & 498.2079 & 122.5994 & 3.12384 \\
\hline 1505 & 28.5683 & 491.8448 & 512.4526 & 496.6292 & 122.5994 & 3.12202 \\
\hline 1506 & 28.5853 & 506.8125 & 508.8779 & 495.0506 & 122.5994 & 3.1202 \\
\hline 1507 & 28.6023 & 476.189 & 505.4732 & 493.4719 & 122.5994 & 3.11838 \\
\hline 1508 & 28.6193 & 489.6581 & 502.2669 & 491.8933 & 122.5994 & 3.11657 \\
\hline 1509 & 28.6363 & 499.2956 & 499.2754 & 490.3146 & 122.5994 & 3.11476 \\
\hline 1510 & 28.6533 & 510.1736 & 496.4983 & 488.736 & 122.5994 & 3.11295 \\
\hline 1511 & 28.6703 & 495.8863 & 493.9225 & 487.1573 & 122.5994 & 3.11114 \\
\hline 1512 & 28.6873 & 486.676 & 491.5283 & 485.5787 & 122.5994 & 3.10934 \\
\hline 1513 & 28.7043 & 478.4407 & 489.2939 & 484 & 122.5994 & 3.10753 \\
\hline 1514 & 28.7213 & 469.966 & 487.5687 & 482.7917 & 122.5994 & 3.10573 \\
\hline 1515 & 28.7383 & 500.1596 & 485.9633 & 481.5833 & 122.5994 & 3.10394 \\
\hline 1516 & 28.7553 & 517.9574 & 484.457 & 480.375 & 122.5994 & 3.10214 \\
\hline 1517 & 28.7723 & 497.6775 & 483.0234 & 479.1667 & 122.5994 & 3.10034 \\
\hline
\end{tabular}




\begin{tabular}{|c|c|c|c|c|c|c|}
\hline 1518 & 28.7893 & 464.6052 & 481.6229 & 477.9583 & 122.5994 & 3.09855 \\
\hline 1519 & 28.8063 & 450.8862 & 480.2117 & 476.75 & 122.5994 & 3.09676 \\
\hline 1520 & 28.8233 & 446.5583 & 478.0038 & 475.5417 & 122.5994 & 3.09497 \\
\hline 1521 & 28.8403 & 448.1996 & 476.6136 & 474.3333 & 122.5994 & 3.09319 \\
\hline 1522 & 28.8573 & 461.883 & 475.2424 & 473.125 & 122.5994 & 3.09141 \\
\hline 1523 & 28.8743 & 463.2925 & 473.8798 & 471.9167 & 122.5994 & 3.08962 \\
\hline 1524 & 28.8913 & 458.9585 & 471.2919 & 470.7083 & 122.5994 & 3.08784 \\
\hline 1525 & 28.9083 & 468.1937 & 469.9983 & 469.5 & 122.5994 & 3.08607 \\
\hline 1526 & 28.9253 & 453.474 & 468.7169 & 468.2917 & 122.5994 & 3.08429 \\
\hline 1527 & 28.9423 & 467.3351 & 467.451 & 467.0833 & 122.5994 & 3.08252 \\
\hline 1528 & 28.9593 & 452.5502 & 466.1992 & 465.875 & 122.5994 & 3.08075 \\
\hline 1529 & 28.9763 & 468.205 & 464.9583 & 464.6667 & 122.5994 & 3.07898 \\
\hline 1530 & 28.9933 & 459.2829 & 463.7248 & 463.4583 & 122.5994 & 3.07721 \\
\hline 1531 & 29.0103 & 490.0548 & 462.4959 & 462.25 & 122.5994 & 3.07545 \\
\hline 1532 & 29.0273 & 531.2317 & 461.2702 & 461.0417 & 122.5994 & 3.07369 \\
\hline 1533 & 29.0443 & 477.4111 & 460.0467 & 459.8333 & 122.5994 & 3.07193 \\
\hline 1534 & 29.0613 & 519.158 & 458.8248 & 458.625 & 122.5994 & 3.07017 \\
\hline 1535 & 29.0783 & 453.6985 & 457.6042 & 457.4167 & 122.5994 & 3.06841 \\
\hline 1536 & 29.0953 & 453.4654 & 456.3849 & 456.2083 & 122.5994 & 3.06666 \\
\hline 1537 & 29.1123 & 445.4257 & 455.1665 & 455 & 122.5994 & 3.0649 \\
\hline 1538 & 29.1293 & 453.1398 & 455.6782 & 455.5208 & 122.5994 & 3.06315 \\
\hline 1539 & 29.1463 & 497.7359 & 457.0395 & 456.0417 & 122.5994 & 3.06141 \\
\hline 1540 & 29.1633 & 461.3724 & 457.5903 & 456.5625 & 122.5994 & 3.05966 \\
\hline 1541 & 29.1803 & 483.7304 & 458.1442 & 457.0833 & 122.5994 & 3.05792 \\
\hline 1542 & 29.1973 & 457.757 & 458.7014 & 457.6042 & 122.5994 & 3.05618 \\
\hline 1543 & 29.2143 & 472.742 & 459.6757 & 458.125 & 122.5994 & 3.05444 \\
\hline 1544 & 29.2313 & 446.9757 & 460.2584 & 458.6458 & 122.5994 & 3.0527 \\
\hline 1545 & 29.2483 & 470.8988 & 460.8462 & 459.1667 & 122.5994 & 3.05096 \\
\hline 1546 & 29.2653 & 476.17 & 461.3759 & 459.6875 & 122.5994 & 3.04923 \\
\hline 1547 & 29.2823 & 478.0495 & 461.9783 & 460.2083 & 122.5994 & 3.0475 \\
\hline 1548 & 29.2993 & 450.2991 & 462.5873 & 460.7292 & 122.5994 & 3.04577 \\
\hline 1549 & 29.3163 & 484.009 & 463.2036 & 461.25 & 122.5994 & 3.04404 \\
\hline 1550 & 29.3333 & 474.0448 & 463.8281 & 461.7708 & 122.5994 & 3.04231 \\
\hline 1551 & 29.3503 & 456.6969 & 464.4282 & 462.2917 & 122.5994 & 3.04059 \\
\hline 1552 & 29.3673 & 475.23 & 465.0729 & 462.8125 & 122.5994 & 3.03887 \\
\hline 1553 & 29.3843 & 504.3775 & 465.7285 & 463.3333 & 122.5994 & 3.03715 \\
\hline 1554 & 29.4013 & 513.4207 & 466.3965 & 463.8542 & 122.5994 & 3.03543 \\
\hline 1555 & 29.4183 & 535.0629 & 467.0784 & 464.375 & 122.5994 & 3.03372 \\
\hline 1556 & 29.4353 & 602.4917 & 467.7759 & 464.8958 & 122.5994 & 3.032 \\
\hline 1557 & 29.4523 & 652.5209 & 468.4911 & 465.4167 & 122.5994 & 3.03029 \\
\hline 1558 & 29.4693 & 665.5948 & 469.2266 & 465.9375 & 122.5994 & 3.02858 \\
\hline 1559 & 29.4863 & 652.1388 & 469.985 & 466.4583 & 122.5994 & 3.02688 \\
\hline 1560 & 29.5033 & 648.8932 & 470.7698 & 466.9792 & 122.5994 & 3.02517 \\
\hline 1561 & 29.5203 & 675.1744 & 471.5851 & 467.5 & 122.5994 & 3.02347 \\
\hline 1562 & 29.5373 & 677.2791 & 472.0712 & 467.6563 & 122.5994 & 3.02177 \\
\hline 1563 & 29.5543 & 658.3124 & 472.599 & 467.8125 & 122.5994 & 3.02007 \\
\hline 1564 & 29.5713 & 628.0797 & 473.1764 & 467.9688 & 122.5994 & 3.01837 \\
\hline 1565 & 29.5883 & 595.8291 & 473.8142 & 468.125 & 122.5994 & 3.01667 \\
\hline 1566 & 29.6053 & 592.1921 & 474.5276 & 468.2813 & 122.5994 & 3.01498 \\
\hline
\end{tabular}




\begin{tabular}{|c|c|c|c|c|c|c|}
\hline 1567 & 29.6223 & 598.7672 & 475.3383 & 468.4375 & 122.5994 & 3.01329 \\
\hline 1568 & 29.6393 & 549.2441 & 476.2791 & 468.5938 & 122.5994 & 3.0116 \\
\hline 1569 & 29.6563 & 539.2547 & 477.3992 & 468.75 & 122.5994 & 3.00991 \\
\hline 1570 & 29.6733 & 525.4029 & 478.7714 & 468.9063 & 122.5994 & 3.00822 \\
\hline 1571 & 29.6903 & 563.6241 & 480.5011 & 469.0625 & 122.5994 & 3.00654 \\
\hline 1572 & 29.7073 & 549.4547 & 482.7348 & 469.2188 & 122.5994 & 3.00486 \\
\hline 1573 & 29.7243 & 535.2572 & 486.1517 & 469.375 & 122.5994 & 3.00318 \\
\hline 1574 & 29.7413 & 549.3731 & 490.0974 & 469.5313 & 122.5994 & 3.0015 \\
\hline 1575 & 29.7583 & 532.1105 & 495.2396 & 469.6875 & 122.5994 & 2.99983 \\
\hline 1576 & 29.7753 & 533.1045 & 501.9037 & 469.8438 & 122.5994 & 2.99815 \\
\hline 1577 & 29.7923 & 516.8947 & 510.3645 & 470 & 122.5994 & 2.99648 \\
\hline 1578 & 29.8093 & 511.0815 & 521.0913 & 470.1563 & 122.5994 & 2.99481 \\
\hline 1579 & 29.8263 & 523.7737 & 533.9131 & 470.3125 & 122.5994 & 2.99314 \\
\hline 1580 & 29.8433 & 584.0713 & 548.4133 & 470.4688 & 122.5994 & 2.99147 \\
\hline 1581 & 29.8603 & 655.0749 & 564.3896 & 470.625 & 122.5994 & 2.98981 \\
\hline 1582 & 29.8773 & 601.0521 & 580.944 & 470.7813 & 122.5994 & 2.98815 \\
\hline 1583 & 29.8943 & 650.2622 & 596.8011 & 470.9375 & 122.5994 & 2.98649 \\
\hline 1584 & 29.9113 & 650.2622 & 609.9073 & 471.0938 & 122.5994 & 2.98483 \\
\hline 1585 & 29.9283 & 648.6066 & 618.6758 & 471.25 & 122.5994 & 2.98317 \\
\hline 1586 & 29.9453 & 628.4749 & 622.2219 & 471.4063 & 122.5994 & 2.98152 \\
\hline 1587 & 29.9623 & 618.1794 & 620.7779 & 471.5625 & 122.5994 & 2.97986 \\
\hline 1588 & 29.9793 & 637.0911 & 615.2388 & 471.7188 & 122.5994 & 2.97821 \\
\hline 1589 & 29.9963 & 615.0674 & 606.6276 & 471.875 & 122.5994 & 2.97656 \\
\hline 1590 & 30.0133 & 584.1519 & 596.0966 & 472.0313 & 122.5994 & 2.97492 \\
\hline 1591 & 30.0303 & 560.9858 & 585.2792 & 472.1875 & 122.5994 & 2.97327 \\
\hline 1592 & 30.0473 & 572.6847 & 576.3505 & 472.3438 & 122.5994 & 2.97163 \\
\hline 1593 & 30.0643 & 549.9166 & 571.5233 & 472.5 & 122.5994 & 2.96999 \\
\hline 1594 & 30.0813 & 511.5146 & 572.5606 & 472.6563 & 122.5994 & 2.96835 \\
\hline 1595 & 30.0983 & 499.1209 & 578.2853 & 472.8125 & 122.5994 & 2.96671 \\
\hline 1596 & 30.1153 & 495.6898 & 585.9293 & 472.9688 & 122.5994 & 2.96507 \\
\hline 1597 & 30.1323 & 507.9164 & 588.5442 & 473.125 & 122.5994 & 2.96344 \\
\hline 1598 & 30.1493 & 497.4786 & 584.3449 & 473.2813 & 122.5994 & 2.96181 \\
\hline 1599 & 30.1663 & 478.194 & 578.528 & 473.4375 & 122.5994 & 2.96018 \\
\hline 1600 & 30.1833 & 518.0887 & 576.0125 & 473.5938 & 122.5994 & 2.95855 \\
\hline 1601 & 30.2003 & 511.718 & 575.2917 & 473.75 & 122.5994 & 2.95692 \\
\hline 1602 & 30.2173 & 498.4218 & 570.0079 & 473.9063 & 122.5994 & 2.9553 \\
\hline 1603 & 30.2343 & 498.2169 & 558.0809 & 474.0625 & 122.5994 & 2.95367 \\
\hline 1604 & 30.2513 & 492.7224 & 543.9308 & 474.2188 & 122.5994 & 2.95205 \\
\hline 1605 & 30.2683 & 494.9321 & 532.0892 & 474.375 & 122.5994 & 2.95043 \\
\hline 1606 & 30.2853 & 500.2885 & 523.2795 & 474.5313 & 122.5994 & 2.94881 \\
\hline 1607 & 30.3023 & 491.0521 & 515.9596 & 474.6875 & 122.5994 & 2.9472 \\
\hline 1608 & 30.3193 & 519.6562 & 509.1361 & 474.8438 & 122.5994 & 2.94558 \\
\hline 1609 & 30.3363 & 527.4419 & 503.4513 & 475 & 122.5994 & 2.94397 \\
\hline 1610 & 30.3533 & 519.2286 & 499.2953 & 474.7917 & 122.5994 & 2.94236 \\
\hline 1611 & 30.3703 & 498.5069 & 497.4187 & 474.5833 & 122.5994 & 2.94075 \\
\hline 1612 & 30.3873 & 484.009 & 498.3544 & 474.375 & 122.5994 & 2.93915 \\
\hline 1613 & 30.4043 & 502.2629 & 502.8078 & 474.1667 & 122.5994 & 2.93754 \\
\hline 1614 & 30.4213 & 510.7695 & 511.6088 & 473.9583 & 122.5994 & 2.93594 \\
\hline 1615 & 30.4383 & 510.7695 & 525.1691 & 473.75 & 122.5994 & 2.93434 \\
\hline
\end{tabular}




\begin{tabular}{|c|c|c|c|c|c|c|}
\hline 1616 & 30.4553 & 507.3924 & 542.4742 & 473.5417 & 122.5994 & 2.93274 \\
\hline 1617 & 30.4723 & 516.7284 & 559.7303 & 473.3333 & 122.5994 & 2.93114 \\
\hline 1618 & 30.4893 & 516.2467 & 571.8221 & 473.125 & 122.5994 & 2.92955 \\
\hline 1619 & 30.5063 & 549.1307 & 577.0831 & 472.9167 & 122.5994 & 2.92795 \\
\hline 1620 & 30.5233 & 560.9192 & 575.561 & 472.7083 & 122.5994 & 2.92636 \\
\hline 1621 & 30.5403 & 525.8615 & 568.1272 & 472.5 & 122.5994 & 2.92477 \\
\hline 1622 & 30.5573 & 511.8783 & 557.7052 & 472.2917 & 122.5994 & 2.92318 \\
\hline 1623 & 30.5743 & 486.7006 & 546.3687 & 472.0833 & 122.5994 & 2.9216 \\
\hline 1624 & 30.5913 & 517.0167 & 535.1497 & 471.875 & 122.5994 & 2.92001 \\
\hline 1625 & 30.6083 & 519.7901 & 523.9122 & 471.6667 & 122.5994 & 2.91843 \\
\hline 1626 & 30.6253 & 489.5534 & 513.4913 & 471.4583 & 122.5994 & 2.91685 \\
\hline 1627 & 30.6423 & 488.4191 & 506.1729 & 471.25 & 122.5994 & 2.91527 \\
\hline 1628 & 30.6593 & 505.8311 & 500.367 & 471.0417 & 122.5994 & 2.91369 \\
\hline 1629 & 30.6763 & 445.0981 & 495.7433 & 470.8333 & 122.5994 & 2.91211 \\
\hline 1630 & 30.6933 & 440.9577 & 491.7966 & 470.625 & 122.5994 & 2.91054 \\
\hline 1631 & 30.7103 & 442.5278 & 488.797 & 470.4167 & 122.5994 & 2.90897 \\
\hline 1632 & 30.7273 & 463.8739 & 486.2261 & 470.2083 & 122.5994 & 2.9074 \\
\hline 1633 & 30.7443 & 480.6272 & 484.5282 & 470 & 122.5994 & 2.90583 \\
\hline 1634 & 30.7613 & 484.8584 & 482.4197 & 469.5833 & 122.5994 & 2.90426 \\
\hline 1635 & 30.7783 & 464.9954 & 480.1752 & 469.1667 & 122.5994 & 2.9027 \\
\hline 1636 & 30.7953 & 481.9331 & 477.1538 & 468.75 & 122.5994 & 2.90113 \\
\hline 1637 & 30.8123 & 491.9605 & 475.4417 & 468.3333 & 122.5994 & 2.89957 \\
\hline 1638 & 30.8293 & 494.3902 & 474.0808 & 467.9167 & 122.5994 & 2.89801 \\
\hline 1639 & 30.8463 & 516.4083 & 473.0156 & 467.5 & 122.5994 & 2.89645 \\
\hline 1640 & 30.8633 & 523.5016 & 472.1545 & 467.0833 & 122.5994 & 2.89489 \\
\hline 1641 & 30.8803 & 538.9649 & 471.4237 & 466.6667 & 122.5994 & 2.89334 \\
\hline 1642 & 30.8973 & 563.9812 & 470.456 & 466.25 & 122.5994 & 2.89179 \\
\hline 1643 & 30.9143 & 618.6935 & 469.9506 & 465.8333 & 122.5994 & 2.89023 \\
\hline 1644 & 30.9313 & 654.6015 & 469.1816 & 465.4167 & 122.5994 & 2.88868 \\
\hline 1645 & 30.9483 & 639.7176 & 468.8581 & 465 & 122.5994 & 2.88714 \\
\hline 1646 & 30.9653 & 697.9412 & 468.418 & 464.5833 & 122.5994 & 2.88559 \\
\hline 1647 & 30.9823 & 734.9135 & 468.2257 & 464.1667 & 122.5994 & 2.88405 \\
\hline 1648 & 30.9993 & 743.5388 & 468.0895 & 463.75 & 122.5994 & 2.8825 \\
\hline 1649 & 31.0163 & 722.1051 & 467.8495 & 463.3333 & 122.5994 & 2.88096 \\
\hline 1650 & 31.0333 & 672.7398 & 467.8621 & 462.9167 & 122.5994 & 2.87942 \\
\hline 1651 & 31.0503 & 650.4089 & 467.965 & 462.5 & 122.5994 & 2.87788 \\
\hline 1652 & 31.0673 & 626.3191 & 468.1884 & 462.0833 & 122.5994 & 2.87635 \\
\hline 1653 & 31.0843 & 574.807 & 468.5234 & 461.6667 & 122.5994 & 2.87481 \\
\hline 1654 & 31.1013 & 564.3912 & 469.2273 & 461.25 & 122.5994 & 2.87328 \\
\hline 1655 & 31.1183 & 507.6674 & 470.4781 & 460.8333 & 122.5994 & 2.87175 \\
\hline 1656 & 31.1353 & 479.8846 & 473.3792 & 460.4167 & 122.5994 & 2.87022 \\
\hline 1657 & 31.1523 & 496.8424 & 477.3575 & 460 & 122.5994 & 2.86869 \\
\hline 1658 & 31.1693 & 487.3167 & 483.4646 & 459.2917 & 122.5994 & 2.86717 \\
\hline 1659 & 31.1863 & 482.5779 & 492.4265 & 458.5833 & 122.5994 & 2.86564 \\
\hline 1660 & 31.2033 & 502.6223 & 503.5475 & 457.875 & 122.5994 & 2.86412 \\
\hline 1661 & 31.2203 & 497.8627 & 514.5805 & 457.1667 & 122.5994 & 2.8626 \\
\hline 1662 & 31.2373 & 509.429 & 520.7308 & 456.4583 & 122.5994 & 2.86108 \\
\hline 1663 & 31.2543 & 522.2313 & 521.0656 & 455.75 & 122.5994 & 2.85956 \\
\hline 1664 & 31.2713 & 524.4198 & 519.6464 & 455.0417 & 122.5994 & 2.85805 \\
\hline
\end{tabular}




\begin{tabular}{|c|c|c|c|c|c|c|}
\hline 1665 & 31.2883 & 554.3602 & 520.1583 & 454.3333 & 122.5994 & 2.85653 \\
\hline 1666 & 31.3053 & 546.1969 & 522.8056 & 453.625 & 122.5994 & 2.85502 \\
\hline 1667 & 31.3223 & 579.0078 & 525.6686 & 452.9167 & 122.5994 & 2.85351 \\
\hline 1668 & 31.3393 & 556.6813 & 528.2582 & 452.2083 & 122.5994 & 2.852 \\
\hline 1669 & 31.3563 & 560.6539 & 531.9573 & 451.5 & 122.5994 & 2.85049 \\
\hline 1670 & 31.3733 & 548.8325 & 537.5815 & 450.7917 & 122.5994 & 2.84899 \\
\hline 1671 & 31.3903 & 530.2683 & 544.7462 & 450.0833 & 122.5994 & 2.84748 \\
\hline 1672 & 31.4073 & 546.7116 & 552.2297 & 449.375 & 122.5994 & 2.84598 \\
\hline 1673 & 31.4243 & 542.9534 & 558.5229 & 448.6667 & 122.5994 & 2.84448 \\
\hline 1674 & 31.4413 & 479.8284 & 562.4673 & 447.9583 & 122.5994 & 2.84298 \\
\hline 1675 & 31.4583 & 457.8906 & 563.4962 & 447.25 & 122.5994 & 2.84148 \\
\hline 1676 & 31.4753 & 453.6985 & 561.1913 & 446.5417 & 122.5994 & 2.83999 \\
\hline 1677 & 31.4923 & 454.4878 & 555.0052 & 445.8333 & 122.5994 & 2.83849 \\
\hline 1678 & 31.5093 & 467.9585 & 545.3752 & 445.125 & 122.5994 & 2.837 \\
\hline 1679 & 31.5263 & 484.009 & 533.9308 & 444.4167 & 122.5994 & 2.83551 \\
\hline 1680 & 31.5433 & 480.1954 & 522.0924 & 443.7083 & 122.5994 & 2.83402 \\
\hline 1681 & 31.5603 & 447.5684 & 510.4791 & 443 & 122.5994 & 2.83253 \\
\hline 1682 & 31.5773 & 415.5684 & 499.2227 & 442.2917 & 122.5994 & 2.83105 \\
\hline 1683 & 31.5943 & 442.4284 & 488.7542 & 441.5833 & 122.5994 & 2.82956 \\
\hline 1684 & 31.6113 & 486.769 & 479.6915 & 440.875 & 122.5994 & 2.82808 \\
\hline 1685 & 31.6283 & 499.3904 & 472.3343 & 440.1667 & 122.5994 & 2.8266 \\
\hline 1686 & 31.6453 & 498.3545 & 466.3336 & 439.4583 & 122.5994 & 2.82512 \\
\hline 1687 & 31.6623 & 485.0685 & 462.2112 & 438.75 & 122.5994 & 2.82364 \\
\hline 1688 & 31.6793 & 525.9461 & 459.4293 & 438.0417 & 122.5994 & 2.82216 \\
\hline 1689 & 31.6963 & 569.3531 & 457.8689 & 437.3333 & 122.5994 & 2.82069 \\
\hline 1690 & 31.7133 & 595.6075 & 457.4852 & 436.625 & 122.5994 & 2.81922 \\
\hline 1691 & 31.7303 & 640.9324 & 458.1736 & 435.9167 & 122.5994 & 2.81774 \\
\hline 1692 & 31.7473 & 684.4444 & 460.342 & 435.2083 & 122.5994 & 2.81627 \\
\hline 1693 & 31.7643 & 674.9084 & 463.8254 & 434.5 & 122.5994 & 2.81481 \\
\hline 1694 & 31.7813 & 652.5837 & 468.4665 & 433.7917 & 122.5994 & 2.81334 \\
\hline 1695 & 31.7983 & 621.6472 & 473.9778 & 433.0833 & 122.5994 & 2.81187 \\
\hline 1696 & 31.8153 & 584.7338 & 480.4256 & 432.375 & 122.5994 & 2.81041 \\
\hline 1697 & 31.8323 & 554.6902 & 488.1614 & 431.6667 & 122.5994 & 2.80895 \\
\hline 1698 & 31.8493 & 549.9767 & 497.2412 & 430.9583 & 122.5994 & 2.80749 \\
\hline 1699 & 31.8663 & 547.2561 & 507.1292 & 430.25 & 122.5994 & 2.80603 \\
\hline 1700 & 31.8833 & 525.0302 & 516.8169 & 429.5417 & 122.5994 & 2.80457 \\
\hline 1701 & 31.9003 & 487.7361 & 525.1351 & 428.8333 & 122.5994 & 2.80311 \\
\hline 1702 & 31.9173 & 476.1242 & 530.9539 & 428.125 & 122.5994 & 2.80166 \\
\hline 1703 & 31.9343 & 445.2721 & 533.4516 & 427.4167 & 122.5994 & 2.80021 \\
\hline 1704 & 31.9513 & 445.946 & 532.4958 & 426.7083 & 122.5994 & 2.79876 \\
\hline 1705 & 31.9683 & 475.2523 & 528.563 & 426 & 122.5994 & 2.79731 \\
\hline 1706 & 31.9853 & 463.2384 & 521.9784 & 425 & 122.5994 & 2.79586 \\
\hline 1707 & 32.0023 & 465.8016 & 513.497 & 424 & 122.5994 & 2.79441 \\
\hline 1708 & 32.0193 & 467.799 & 503.5311 & 423 & 122.5994 & 2.79297 \\
\hline 1709 & 32.0363 & 463.2838 & 492.6152 & 422 & 122.5994 & 2.79152 \\
\hline 1710 & 32.0533 & 479.0898 & 481.5375 & 421 & 122.5994 & 2.79008 \\
\hline 1711 & 32.0703 & 480.473 & 471.0741 & 420 & 122.5994 & 2.78864 \\
\hline 1712 & 32.0873 & 483.1386 & 461.7448 & 419 & 122.5994 & 2.7872 \\
\hline 1713 & 32.1043 & 487.7509 & 453.5603 & 418 & 122.5994 & 2.78577 \\
\hline
\end{tabular}




\begin{tabular}{|c|c|c|c|c|c|c|}
\hline 1714 & 32.1213 & 454.9533 & 446.4435 & 417 & 122.5994 & 2.78433 \\
\hline 1715 & 32.1383 & 468.3539 & 440.3053 & 416 & 122.5994 & 2.7829 \\
\hline 1716 & 32.1553 & 455.3581 & 435.0592 & 415 & 122.5994 & 2.78147 \\
\hline 1717 & 32.1723 & 426.707 & 430.8212 & 414 & 122.5994 & 2.78003 \\
\hline 1718 & 32.1893 & 472.3227 & 427.1495 & 413 & 122.5994 & 2.7786 \\
\hline 1719 & 32.2063 & 446.219 & 424.0623 & 412 & 122.5994 & 2.77718 \\
\hline 1720 & 32.2233 & 449.4424 & 420.8135 & 411 & 122.5994 & 2.77575 \\
\hline 1721 & 32.2403 & 429.1396 & 418.498 & 410 & 122.5994 & 2.77433 \\
\hline 1722 & 32.2573 & 500.4195 & 416.5164 & 409 & 122.5994 & 2.7729 \\
\hline 1723 & 32.2743 & 456.839 & 414.6959 & 408 & 122.5994 & 2.77148 \\
\hline 1724 & 32.2913 & 434.7802 & 413.0809 & 407 & 122.5994 & 2.77006 \\
\hline 1725 & 32.3083 & 406.4969 & 411.3152 & 406 & 122.5994 & 2.76864 \\
\hline 1726 & 32.3253 & 394.8817 & 410.048 & 405 & 122.5994 & 2.76722 \\
\hline 1727 & 32.3423 & 418.379 & 408.9343 & 404 & 122.5994 & 2.76581 \\
\hline 1728 & 32.3593 & 429.436 & 407.9854 & 403 & 122.5994 & 2.76439 \\
\hline 1729 & 32.3763 & 407.7272 & 407.2203 & 402 & 122.5994 & 2.76298 \\
\hline 1730 & 32.3933 & 382.5926 & 406.4875 & 400.8333 & 122.5994 & 2.76157 \\
\hline 1731 & 32.4103 & 366.2176 & 405.9478 & 399.6667 & 122.5994 & 2.76016 \\
\hline 1732 & 32.4273 & 389.9649 & 405.6049 & 398.5 & 122.5994 & 2.75875 \\
\hline 1733 & 32.4443 & 407.0213 & 405.5702 & 397.3333 & 122.5994 & 2.75735 \\
\hline 1734 & 32.4613 & 394.903 & 405.9688 & 396.1667 & 122.5994 & 2.75594 \\
\hline 1735 & 32.4783 & 383.4434 & 406.7227 & 395 & 122.5994 & 2.75454 \\
\hline 1736 & 32.4953 & 402.8021 & 407.5737 & 393.8333 & 122.5994 & 2.75313 \\
\hline 1737 & 32.5123 & 396.2181 & 408.643 & 392.6667 & 122.5994 & 2.75173 \\
\hline 1738 & 32.5293 & 387.9851 & 410.5779 & 391.5 & 122.5994 & 2.75033 \\
\hline 1739 & 32.5463 & 365.5069 & 413.5156 & 390.3333 & 122.5994 & 2.74894 \\
\hline 1740 & 32.5633 & 387.3607 & 416.26 & 389.1667 & 122.5994 & 2.74754 \\
\hline 1741 & 32.5803 & 394.1146 & 416.4757 & 388 & 122.5994 & 2.74615 \\
\hline 1742 & 32.5973 & 383.5327 & 413.3044 & 386.8333 & 122.5994 & 2.74475 \\
\hline 1743 & 32.6143 & 390.1907 & 408.7397 & 385.6667 & 122.5994 & 2.74336 \\
\hline 1744 & 32.6313 & 382.1547 & 404.7174 & 384.5 & 122.5994 & 2.74197 \\
\hline 1745 & 32.6483 & 362.4226 & 401.4956 & 383.3333 & 122.5994 & 2.74058 \\
\hline 1746 & 32.6653 & 355.8304 & 398.0805 & 382.1667 & 122.5994 & 2.73919 \\
\hline 1747 & 32.6823 & 356.7575 & 393.9056 & 381 & 122.5994 & 2.73781 \\
\hline 1748 & 32.6993 & 378.478 & 389.5608 & 379.8333 & 122.5994 & 2.73642 \\
\hline 1749 & 32.7163 & 379.266 & 385.1129 & 378.6667 & 122.5994 & 2.73504 \\
\hline 1750 & 32.7333 & 373.5684 & 381.9966 & 377.5 & 122.5994 & 2.73366 \\
\hline 1751 & 32.7503 & 361.3469 & 379.5323 & 376.3333 & 122.5994 & 2.73228 \\
\hline 1752 & 32.7673 & 366.3477 & 377.5664 & 375.1667 & 122.5994 & 2.7309 \\
\hline 1753 & 32.7843 & 386.0712 & 375.915 & 374 & 122.5994 & 2.72952 \\
\hline 1754 & 32.8013 & 375.2026 & 375.4518 & 374.1458 & 122.5994 & 2.72815 \\
\hline 1755 & 32.8183 & 368.6891 & 375.4029 & 374.2917 & 122.5994 & 2.72677 \\
\hline 1756 & 32.8353 & 357.0373 & 375.3959 & 374.4375 & 122.5994 & 2.7254 \\
\hline 1757 & 32.8523 & 350.5521 & 375.4337 & 374.5833 & 122.5994 & 2.72403 \\
\hline 1758 & 32.8693 & 361.8141 & 375.4992 & 374.7292 & 122.5994 & 2.72266 \\
\hline 1759 & 32.8863 & 368.0212 & 375.5895 & 374.875 & 122.5994 & 2.72129 \\
\hline 1760 & 32.9033 & 371.8662 & 375.6644 & 375.0208 & 122.5994 & 2.71992 \\
\hline 1761 & 32.9203 & 403.3319 & 375.8034 & 375.1667 & 122.5994 & 2.71856 \\
\hline 1762 & 32.9373 & 386.836 & 375.9727 & 375.3125 & 122.5994 & 2.71719 \\
\hline
\end{tabular}




\begin{tabular}{|c|c|c|c|c|c|c|}
\hline 1763 & 32.9543 & 435.9407 & 376.1679 & 375.4583 & 122.5994 & 2.71583 \\
\hline 1764 & 32.9713 & 373.5261 & 376.3896 & 375.6042 & 122.5994 & 2.71447 \\
\hline 1765 & 32.9883 & 383.4217 & 376.4778 & 375.75 & 122.5994 & 2.71311 \\
\hline 1766 & 33.0053 & 399.585 & 376.8166 & 375.8958 & 122.5994 & 2.71175 \\
\hline 1767 & 33.0223 & 384.2485 & 377.2154 & 376.0417 & 122.5994 & 2.71039 \\
\hline 1768 & 33.0393 & 424.7382 & 377.6124 & 376.1875 & 122.5994 & 2.70904 \\
\hline 1769 & 33.0563 & 438.8015 & 377.8833 & 376.3333 & 122.5994 & 2.70768 \\
\hline 1770 & 33.0733 & 389.6394 & 377.8846 & 376.4792 & 122.5994 & 2.70633 \\
\hline 1771 & 33.0903 & 413.773 & 377.8694 & 376.625 & 122.5994 & 2.70498 \\
\hline 1772 & 33.1073 & 426.6755 & 377.8729 & 376.7708 & 122.5994 & 2.70363 \\
\hline 1773 & 33.1243 & 377.3999 & 377.9243 & 376.9167 & 122.5994 & 2.70228 \\
\hline 1774 & 33.1413 & 388.2655 & 377.9744 & 377.0625 & 122.5994 & 2.70093 \\
\hline 1775 & 33.1583 & 402.1767 & 377.9771 & 377.2083 & 122.5994 & 2.69958 \\
\hline 1776 & 33.1753 & 363.8556 & 377.9574 & 377.3542 & 122.5994 & 2.69824 \\
\hline 1777 & 33.1923 & 408.0009 & 377.9597 & 377.5 & 122.5994 & 2.6969 \\
\hline 1778 & 33.2093 & 407.1496 & 378.0061 & 377.6458 & 122.5994 & 2.69556 \\
\hline 1779 & 33.2263 & 411.2969 & 378.1085 & 377.7917 & 122.5994 & 2.69422 \\
\hline 1780 & 33.2433 & 417.0787 & 378.2509 & 377.9375 & 122.5994 & 2.69288 \\
\hline 1781 & 33.2603 & 379.8305 & 378.436 & 378.0833 & 122.5994 & 2.69154 \\
\hline 1782 & 33.2773 & 389.4081 & 378.6578 & 378.2292 & 122.5994 & 2.6902 \\
\hline 1783 & 33.2943 & 385.3161 & 378.9033 & 378.375 & 122.5994 & 2.68887 \\
\hline 1784 & 33.3113 & 366.901 & 379.1482 & 378.5208 & 122.5994 & 2.68753 \\
\hline 1785 & 33.3283 & 361.6591 & 379.3506 & 378.6667 & 122.5994 & 2.6862 \\
\hline 1786 & 33.3453 & 370.1697 & 379.5138 & 378.8125 & 122.5994 & 2.68487 \\
\hline 1787 & 33.3623 & 370.8913 & 379.6782 & 378.9583 & 122.5994 & 2.68354 \\
\hline 1788 & 33.3793 & 379.1246 & 379.8723 & 379.1042 & 122.5994 & 2.68221 \\
\hline 1789 & 33.3963 & 388.379 & 380.0992 & 379.25 & 122.5994 & 2.68089 \\
\hline 1790 & 33.4133 & 380.2805 & 380.3404 & 379.3958 & 122.5994 & 2.67956 \\
\hline 1791 & 33.4303 & 364.5416 & 380.5893 & 379.5417 & 122.5994 & 2.67824 \\
\hline 1792 & 33.4473 & 378.4299 & 380.8584 & 379.6875 & 122.5994 & 2.67692 \\
\hline 1793 & 33.4643 & 397.7387 & 381.1461 & 379.8333 & 122.5994 & 2.6756 \\
\hline 1794 & 33.4813 & 407.7252 & 381.466 & 379.9792 & 122.5994 & 2.67428 \\
\hline 1795 & 33.4983 & 399.2724 & 381.7879 & 380.125 & 122.5994 & 2.67296 \\
\hline 1796 & 33.5153 & 371.213 & 382.0818 & 380.2708 & 122.5994 & 2.67164 \\
\hline 1797 & 33.5323 & 381.0153 & 382.3166 & 380.4167 & 122.5994 & 2.67033 \\
\hline 1798 & 33.5493 & 405.3295 & 382.7155 & 380.5625 & 122.5994 & 2.66901 \\
\hline 1799 & 33.5663 & 392.9754 & 382.8924 & 380.7083 & 122.5994 & 2.6677 \\
\hline 1800 & 33.5833 & 365.049 & 383.1013 & 380.8542 & 122.5994 & 2.66639 \\
\hline 1801 & 33.6003 & 368.403 & 383.3458 & 381 & 122.5994 & 2.66508 \\
\hline 1802 & 33.6173 & 396.1205 & 384.437 & 382.0133 & 122.5994 & 2.66377 \\
\hline 1803 & 33.6343 & 394.7274 & 385.5256 & 383.0267 & 122.5994 & 2.66246 \\
\hline 1804 & 33.6513 & 388.9563 & 386.3669 & 384.04 & 122.5994 & 2.66115 \\
\hline 1805 & 33.6683 & 394.5101 & 387.1614 & 385.0534 & 122.5994 & 2.65985 \\
\hline 1806 & 33.6853 & 376.3772 & 388.1022 & 386.0667 & 122.5994 & 2.65855 \\
\hline 1807 & 33.7023 & 392.6437 & 389.0328 & 387.0801 & 122.5994 & 2.65724 \\
\hline 1808 & 33.7193 & 385.5462 & 390.003 & 388.0934 & 122.5994 & 2.65594 \\
\hline 1809 & 33.7363 & 391.3147 & 390.9737 & 389.1068 & 122.5994 & 2.65464 \\
\hline 1810 & 33.7533 & 406.7685 & 391.9702 & 390.1201 & 122.5994 & 2.65335 \\
\hline 1811 & 33.7703 & 385.539 & 393.0773 & 391.1335 & 122.5994 & 2.65205 \\
\hline
\end{tabular}




\begin{tabular}{|c|c|c|c|c|c|c|}
\hline 1812 & 33.7873 & 422.9544 & 394.3188 & 392.1468 & 122.5994 & 2.65075 \\
\hline 1813 & 33.8043 & 420.3575 & 395.6098 & 393.1602 & 122.5994 & 2.64946 \\
\hline 1814 & 33.8213 & 423.9255 & 397.247 & 394.1735 & 122.5994 & 2.64817 \\
\hline 1815 & 33.8383 & 381.2742 & 399.071 & 395.1868 & 122.5994 & 2.64687 \\
\hline 1816 & 33.8553 & 412.2621 & 401.5118 & 396.2002 & 122.5994 & 2.64558 \\
\hline 1817 & 33.8723 & 416.5167 & 405.0135 & 397.2135 & 122.5994 & 2.6443 \\
\hline 1818 & 33.8893 & 417.9053 & 410.0512 & 398.2269 & 122.5994 & 2.64301 \\
\hline 1819 & 33.9063 & 396.4368 & 416.9475 & 399.2402 & 122.5994 & 2.64172 \\
\hline 1820 & 33.9233 & 401.7234 & 425.1849 & 400.2536 & 122.5994 & 2.64044 \\
\hline 1821 & 33.9403 & 420.0538 & 432.9588 & 401.2669 & 122.5994 & 2.63915 \\
\hline 1822 & 33.9573 & 461.5342 & 437.3576 & 402.2803 & 122.5994 & 2.63787 \\
\hline 1823 & 33.9743 & 450.275 & 437.1556 & 403.2936 & 122.5994 & 2.63659 \\
\hline 1824 & 33.9913 & 408.7311 & 435.0474 & 404.307 & 122.5994 & 2.63531 \\
\hline 1825 & 34.0083 & 388.4569 & 434.0213 & 405.3203 & 122.5994 & 2.63403 \\
\hline 1826 & 34.0253 & 450.5321 & 434.5626 & 405.9854 & 122.5994 & 2.63275 \\
\hline 1827 & 34.0423 & 431.4202 & 435.6847 & 406.6504 & 122.5994 & 2.63148 \\
\hline 1828 & 34.0593 & 393.7812 & 435.8455 & 407.3154 & 122.5994 & 2.6302 \\
\hline 1829 & 34.0763 & 434.0814 & 434.7348 & 407.9805 & 122.5994 & 2.62893 \\
\hline 1830 & 34.0933 & 439.7355 & 432.4978 & 408.6455 & 122.5994 & 2.62766 \\
\hline 1831 & 34.1103 & 442.1201 & 429.2344 & 409.3105 & 122.5994 & 2.62639 \\
\hline 1832 & 34.1273 & 438.4287 & 426.4256 & 409.9756 & 122.5994 & 2.62512 \\
\hline 1833 & 34.1443 & 442.7164 & 424.812 & 410.6406 & 122.5994 & 2.62385 \\
\hline 1834 & 34.1613 & 439.7461 & 424.1483 & 411.3057 & 122.5994 & 2.62258 \\
\hline 1835 & 34.1783 & 413.8893 & 423.7191 & 411.9707 & 122.5994 & 2.62132 \\
\hline 1836 & 34.1953 & 405.7026 & 422.7806 & 412.6357 & 122.5994 & 2.62005 \\
\hline 1837 & 34.2123 & 428.5541 & 421.7218 & 413.3008 & 122.5994 & 2.61879 \\
\hline 1838 & 34.2293 & 430.228 & 420.9291 & 413.9658 & 122.5994 & 2.61753 \\
\hline 1839 & 34.2463 & 428.9454 & 420.5844 & 414.6309 & 122.5994 & 2.61627 \\
\hline 1840 & 34.2633 & 454.3389 & 420.7174 & 415.2959 & 122.5994 & 2.61501 \\
\hline 1841 & 34.2803 & 448.3843 & 421.2939 & 415.9609 & 122.5994 & 2.61375 \\
\hline 1842 & 34.2973 & 450.7034 & 422.309 & 416.626 & 122.5994 & 2.61249 \\
\hline 1843 & 34.3143 & 451.5237 & 423.852 & 417.291 & 122.5994 & 2.61124 \\
\hline 1844 & 34.3313 & 470.556 & 426.6443 & 417.9561 & 122.5994 & 2.60998 \\
\hline 1845 & 34.3483 & 472.1652 & 430.0742 & 418.6211 & 122.5994 & 2.60873 \\
\hline 1846 & 34.3653 & 451.7287 & 434.2413 & 419.2861 & 122.5994 & 2.60748 \\
\hline 1847 & 34.3823 & 482.6132 & 438.831 & 419.9512 & 122.5994 & 2.60623 \\
\hline 1848 & 34.3993 & 487.513 & 442.4249 & 420.6162 & 122.5994 & 2.60498 \\
\hline 1849 & 34.4163 & 507.799 & 444.1803 & 421.2813 & 122.5994 & 2.60373 \\
\hline 1850 & 34.4333 & 501.1376 & 444.7325 & 421.9463 & 122.5994 & 2.60249 \\
\hline 1851 & 34.4503 & 465.6899 & 445.054 & 422.6113 & 122.5994 & 2.60124 \\
\hline 1852 & 34.4673 & 516.6354 & 446.2857 & 423.2764 & 122.5994 & 2.6 \\
\hline 1853 & 34.4843 & 505.9651 & 448.4564 & 423.9414 & 122.5994 & 2.59875 \\
\hline 1854 & 34.5013 & 517.4349 & 450.5703 & 424.6064 & 122.5994 & 2.59751 \\
\hline 1855 & 34.5183 & 544.6158 & 452.5494 & 425.2715 & 122.5994 & 2.59627 \\
\hline 1856 & 34.5353 & 509.8035 & 454.7563 & 425.9365 & 122.5994 & 2.59503 \\
\hline 1857 & 34.5523 & 493.6748 & 457.5435 & 426.6016 & 122.5994 & 2.5938 \\
\hline 1858 & 34.5693 & 518.7117 & 460.7401 & 427.2666 & 122.5994 & 2.59256 \\
\hline 1859 & 34.5863 & 542.3145 & 464.0127 & 427.9316 & 122.5994 & 2.59132 \\
\hline 1860 & 34.6033 & 492.9701 & 467.0632 & 428.5967 & 122.5994 & 2.59009 \\
\hline
\end{tabular}




\begin{tabular}{|c|c|c|c|c|c|c|}
\hline 1861 & 34.6203 & 528.8567 & 469.5887 & 429.2617 & 122.5994 & 2.58886 \\
\hline 1862 & 34.6373 & 524.8647 & 471.5599 & 429.9268 & 122.5994 & 2.58762 \\
\hline 1863 & 34.6543 & 534.441 & 473.0771 & 430.5918 & 122.5994 & 2.58639 \\
\hline 1864 & 34.6713 & 544.509 & 474.2204 & 431.2568 & 122.5994 & 2.58516 \\
\hline 1865 & 34.6883 & 516.1254 & 474.9911 & 431.9219 & 122.5994 & 2.58394 \\
\hline 1866 & 34.7053 & 554.0962 & 475.3805 & 432.5869 & 122.5994 & 2.58271 \\
\hline 1867 & 34.7223 & 505.781 & 475.4845 & 433.252 & 122.5994 & 2.58148 \\
\hline 1868 & 34.7393 & 535.3441 & 475.5446 & 433.917 & 122.5994 & 2.58026 \\
\hline 1869 & 34.7563 & 558.4268 & 475.8601 & 434.582 & 122.5994 & 2.57904 \\
\hline 1870 & 34.7733 & 525.3414 & 476.6703 & 435.2471 & 122.5994 & 2.57781 \\
\hline 1871 & 34.7903 & 535.6557 & 478.0898 & 435.9121 & 122.5994 & 2.57659 \\
\hline 1872 & 34.8073 & 563.0346 & 480.1228 & 436.5771 & 122.5994 & 2.57537 \\
\hline 1873 & 34.8243 & 569.0278 & 482.6011 & 437.2422 & 122.5994 & 2.57416 \\
\hline 1874 & 34.8413 & 538.1884 & 485.2971 & 437.5589 & 122.5994 & 2.57294 \\
\hline 1875 & 34.8583 & 535.2183 & 488.3361 & 437.8757 & 122.5994 & 2.57172 \\
\hline 1876 & 34.8753 & 538.6631 & 491.9919 & 438.1924 & 122.5994 & 2.57051 \\
\hline 1877 & 34.8923 & 560.3621 & 495.7966 & 438.5091 & 122.5994 & 2.56929 \\
\hline 1878 & 34.9093 & 581.4673 & 500.3256 & 438.8258 & 122.5994 & 2.56808 \\
\hline 1879 & 34.9263 & 595.1776 & 505.9774 & 439.1426 & 122.5994 & 2.56687 \\
\hline 1880 & 34.9433 & 582.8263 & 512.549 & 439.4593 & 122.5994 & 2.56566 \\
\hline 1881 & 34.9603 & 596.7362 & 519.9578 & 439.776 & 122.5994 & 2.56445 \\
\hline 1882 & 34.9773 & 636.2545 & 528.8854 & 440.0928 & 122.5994 & 2.56325 \\
\hline 1883 & 34.9943 & 670.8436 & 539.725 & 440.4095 & 122.5994 & 2.56204 \\
\hline 1884 & 35.0113 & 649.6635 & 550.0035 & 440.7262 & 122.5994 & 2.56083 \\
\hline 1885 & 35.0283 & 630.4726 & 555.7151 & 441.043 & 122.5994 & 2.55963 \\
\hline 1886 & 35.0453 & 621.052 & 556.8784 & 441.3597 & 122.5994 & 2.55843 \\
\hline 1887 & 35.0623 & 621.5775 & 557.6104 & 441.6764 & 122.5994 & 2.55723 \\
\hline 1888 & 35.0793 & 645.3362 & 560.695 & 441.9932 & 122.5994 & 2.55603 \\
\hline 1889 & 35.0963 & 624.038 & 565.528 & 442.3099 & 122.5994 & 2.55483 \\
\hline 1890 & 35.1133 & 595.7882 & 569.2394 & 442.6266 & 122.5994 & 2.55363 \\
\hline 1891 & 35.1303 & 606.7778 & 570.2855 & 442.9434 & 122.5994 & 2.55243 \\
\hline 1892 & 35.1473 & 571.8329 & 570.1987 & 443.2601 & 122.5994 & 2.55124 \\
\hline 1893 & 35.1643 & 559.015 & 570.8302 & 443.5768 & 122.5994 & 2.55004 \\
\hline 1894 & 35.1813 & 593.3802 & 572.2096 & 443.8936 & 122.5994 & 2.54885 \\
\hline 1895 & 35.1983 & 590.5636 & 571.7548 & 444.2103 & 122.5994 & 2.54766 \\
\hline 1896 & 35.2153 & 510.3937 & 565.6676 & 444.527 & 122.5994 & 2.54647 \\
\hline 1897 & 35.2323 & 513.4611 & 554.3973 & 444.8438 & 122.5994 & 2.54528 \\
\hline 1898 & 35.2493 & 505.0298 & 540.5046 & 444.7253 & 122.5994 & 2.54409 \\
\hline 1899 & 35.2663 & 443.8023 & 528.6149 & 444.6068 & 122.5994 & 2.5429 \\
\hline 1900 & 35.2833 & 484.4373 & 519.52 & 444.4883 & 122.5994 & 2.54171 \\
\hline 1901 & 35.3003 & 528.7063 & 511.7419 & 444.3698 & 122.5994 & 2.54053 \\
\hline 1902 & 35.3173 & 521.2533 & 504.0592 & 444.2513 & 122.5994 & 2.53934 \\
\hline 1903 & 35.3343 & 454.7439 & 497.5889 & 444.1328 & 122.5994 & 2.53816 \\
\hline 1904 & 35.3513 & 469.6751 & 492.8381 & 444.0143 & 122.5994 & 2.53698 \\
\hline 1905 & 35.3683 & 446.1197 & 490.3152 & 443.8958 & 122.5994 & 2.5358 \\
\hline 1906 & 35.3853 & 445.1673 & 489.9568 & 443.7773 & 122.5994 & 2.53462 \\
\hline 1907 & 35.4023 & 483.5238 & 490.4777 & 443.6589 & 122.5994 & 2.53344 \\
\hline 1908 & 35.4193 & 442.5591 & 489.7098 & 443.5404 & 122.5994 & 2.53226 \\
\hline 1909 & 35.4363 & 485.8473 & 486.3069 & 443.4219 & 122.5994 & 2.53109 \\
\hline
\end{tabular}




\begin{tabular}{|c|c|c|c|c|c|c|}
\hline 1910 & 35.4533 & 439.7183 & 481.7279 & 443.3034 & 122.5994 & 2.52991 \\
\hline 1911 & 35.4703 & 422.8476 & 477.833 & 443.1849 & 122.5994 & 2.52874 \\
\hline 1912 & 35.4873 & 415.9006 & 475.2174 & 443.0664 & 122.5994 & 2.52757 \\
\hline 1913 & 35.5043 & 474.6978 & 473.1977 & 442.9479 & 122.5994 & 2.5264 \\
\hline 1914 & 35.5213 & 468.7679 & 470.7949 & 442.8294 & 122.5994 & 2.52523 \\
\hline 1915 & 35.5383 & 463.5713 & 468.1512 & 442.7109 & 122.5994 & 2.52406 \\
\hline 1916 & 35.5553 & 482.1606 & 466.1046 & 442.5924 & 122.5994 & 2.52289 \\
\hline 1917 & 35.5723 & 459.8521 & 465.1087 & 442.474 & 122.5994 & 2.52172 \\
\hline 1918 & 35.5893 & 465.5586 & 465.21 & 442.3555 & 122.5994 & 2.52056 \\
\hline 1919 & 35.6063 & 449.845 & 466.158 & 442.237 & 122.5994 & 2.51939 \\
\hline 1920 & 35.6233 & 419.7891 & 467.7075 & 442.1185 & 122.5994 & 2.51823 \\
\hline 1921 & 35.6403 & 434.9697 & 469.503 & 442 & 122.5994 & 2.51707 \\
\hline 1922 & 35.6573 & 459.4337 & 471.701 & 442.2734 & 122.5994 & 2.51591 \\
\hline 1923 & 35.6743 & 465.9934 & 473.6631 & 442.5469 & 122.5994 & 2.51475 \\
\hline 1924 & 35.6913 & 448.9423 & 475.5774 & 442.8203 & 122.5994 & 2.51359 \\
\hline 1925 & 35.7083 & 448.8955 & 477.0736 & 443.0938 & 122.5994 & 2.51243 \\
\hline 1926 & 35.7253 & 440.7914 & 477.5796 & 443.3672 & 122.5994 & 2.51127 \\
\hline 1927 & 35.7423 & 456.1403 & 476.7179 & 443.6406 & 122.5994 & 2.51012 \\
\hline 1928 & 35.7593 & 449.7297 & 474.882 & 443.9141 & 122.5994 & 2.50896 \\
\hline 1929 & 35.7763 & 429.1602 & 473.4012 & 444.1875 & 122.5994 & 2.50781 \\
\hline 1930 & 35.7933 & 435.3073 & 472.574 & 444.4609 & 122.5994 & 2.50666 \\
\hline 1931 & 35.8103 & 469.3121 & 472.4621 & 444.7344 & 122.5994 & 2.50551 \\
\hline 1932 & 35.8273 & 453.9332 & 472.9441 & 445.0078 & 122.5994 & 2.50436 \\
\hline 1933 & 35.8443 & 423.214 & 474.2994 & 445.2813 & 122.5994 & 2.50321 \\
\hline 1934 & 35.8613 & 438.2953 & 477.2538 & 445.5547 & 122.5994 & 2.50206 \\
\hline 1935 & 35.8783 & 445.9776 & 482.5114 & 445.8281 & 122.5994 & 2.50091 \\
\hline 1936 & 35.8953 & 438.3015 & 490.5237 & 446.1016 & 122.5994 & 2.49977 \\
\hline 1937 & 35.9123 & 445.1483 & 500.9654 & 446.375 & 122.5994 & 2.49862 \\
\hline 1938 & 35.9293 & 424.1107 & 513.4681 & 446.6484 & 122.5994 & 2.49748 \\
\hline 1939 & 35.9463 & 456.2651 & 526.2626 & 446.9219 & 122.5994 & 2.49634 \\
\hline 1940 & 35.9633 & 453.5705 & 537.084 & 447.1953 & 122.5994 & 2.4952 \\
\hline 1941 & 35.9803 & 460.9982 & 545.2772 & 447.4688 & 122.5994 & 2.49406 \\
\hline 1942 & 35.9973 & 447.1162 & 551.1484 & 447.7422 & 122.5994 & 2.49292 \\
\hline 1943 & 36.0143 & 471.2498 & 554.7314 & 448.0156 & 122.5994 & 2.49178 \\
\hline 1944 & 36.0313 & 483.2132 & 556.2403 & 448.2891 & 122.5994 & 2.49064 \\
\hline 1945 & 36.0483 & 494.9529 & 554.6183 & 448.5625 & 122.5994 & 2.48951 \\
\hline 1946 & 36.0653 & 508.6791 & 549.5441 & 448.4375 & 122.5994 & 2.48837 \\
\hline 1947 & 36.0823 & 530.7237 & 542.1481 & 448.3125 & 122.5994 & 2.48724 \\
\hline 1948 & 36.0993 & 521.2742 & 538.8254 & 448.1875 & 122.5994 & 2.48611 \\
\hline 1949 & 36.1163 & 487.1596 & 529.8798 & 448.0625 & 122.5994 & 2.48498 \\
\hline 1950 & 36.1333 & 463.613 & 520.7897 & 447.9375 & 122.5994 & 2.48385 \\
\hline 1951 & 36.1503 & 470.5788 & 512.2726 & 447.8125 & 122.5994 & 2.48272 \\
\hline 1952 & 36.1673 & 470.8448 & 504.5575 & 447.6875 & 122.5994 & 2.48159 \\
\hline 1953 & 36.1843 & 408.0145 & 497.7563 & 447.5625 & 122.5994 & 2.48046 \\
\hline 1954 & 36.2013 & 412.1369 & 491.7834 & 447.4375 & 122.5994 & 2.47934 \\
\hline 1955 & 36.2183 & 415.8672 & 486.5523 & 447.3125 & 122.5994 & 2.47821 \\
\hline 1956 & 36.2353 & 407.4838 & 482.3574 & 447.1875 & 122.5994 & 2.47709 \\
\hline 1957 & 36.2523 & 401.6977 & 479.408 & 447.0625 & 122.5994 & 2.47597 \\
\hline 1958 & 36.2693 & 424.3553 & 477.7044 & 446.9375 & 122.5994 & 2.47485 \\
\hline
\end{tabular}




\begin{tabular}{|c|c|c|c|c|c|c|}
\hline 1959 & 36.2863 & 450.9673 & 477.0572 & 446.8125 & 122.5994 & 2.47372 \\
\hline 1960 & 36.3033 & 399.9039 & 477.2743 & 446.6875 & 122.5994 & 2.47261 \\
\hline 1961 & 36.3203 & 383.8673 & 478.3697 & 446.5625 & 122.5994 & 2.47149 \\
\hline 1962 & 36.3373 & 416.3872 & 480.4998 & 446.4375 & 122.5994 & 2.47037 \\
\hline 1963 & 36.3543 & 400.8235 & 483.8264 & 446.3125 & 122.5994 & 2.46925 \\
\hline 1964 & 36.3713 & 413.7093 & 488.4706 & 446.1875 & 122.5994 & 2.46814 \\
\hline 1965 & 36.3883 & 456.4154 & 494.5221 & 446.0625 & 122.5994 & 2.46702 \\
\hline 1966 & 36.4053 & 483.4675 & 502.1137 & 445.9375 & 122.5994 & 2.46591 \\
\hline 1967 & 36.4223 & 464.4946 & 511.8018 & 445.8125 & 122.5994 & 2.4648 \\
\hline 1968 & 36.4393 & 472.1009 & 525.1994 & 445.6875 & 122.5994 & 2.46369 \\
\hline 1969 & 36.4563 & 523.0617 & 545.459 & 445.5625 & 122.5994 & 2.46258 \\
\hline 1970 & 36.4733 & 546.1311 & 578.843 & 445.4375 & 122.5994 & 2.46147 \\
\hline 1971 & 36.4903 & 581.5522 & 634.7245 & 445.3125 & 122.5994 & 2.46036 \\
\hline 1972 & 36.5073 & 610.8373 & 724.429 & 445.1875 & 122.5994 & 2.45926 \\
\hline 1973 & 36.5243 & 669.0448 & 853.6522 & 445.0625 & 122.5994 & 2.45815 \\
\hline 1974 & 36.5413 & 808.2457 & 1011.356 & 444.9375 & 122.5994 & 2.45705 \\
\hline 1975 & 36.5583 & 974.5168 & 1156.918 & 444.8125 & 122.5994 & 2.45594 \\
\hline 1976 & 36.5753 & 1119.85 & 1219.992 & 444.6875 & 122.5994 & 2.45484 \\
\hline 1977 & 36.5923 & 1330.911 & 1171.845 & 444.5625 & 122.5994 & 2.45374 \\
\hline 1978 & 36.6093 & 1341.565 & 1073.931 & 444.4375 & 122.5994 & 2.45264 \\
\hline 1979 & 36.6263 & 1153.62 & 994.1528 & 444.3125 & 122.5994 & 2.45154 \\
\hline 1980 & 36.6433 & 970.8823 & 953.0325 & 444.1875 & 122.5994 & 2.45044 \\
\hline 1981 & 36.6603 & 875.1168 & 926.6044 & 444.0625 & 122.5994 & 2.44934 \\
\hline 1982 & 36.6773 & 899.37 & 873.7317 & 443.9375 & 122.5994 & 2.44825 \\
\hline 1983 & 36.6943 & 939.8654 & 789.2864 & 443.8125 & 122.5994 & 2.44715 \\
\hline 1984 & 36.7113 & 912.3375 & 701.4043 & 443.6875 & 122.5994 & 2.44606 \\
\hline 1985 & 36.7283 & 740.3066 & 630.7952 & 443.5625 & 122.5994 & 2.44496 \\
\hline 1986 & 36.7453 & 600.1933 & 583.1112 & 443.4375 & 122.5994 & 2.44387 \\
\hline 1987 & 36.7623 & 547.0436 & 554.1779 & 443.3125 & 122.5994 & 2.44278 \\
\hline 1988 & 36.7793 & 497.3597 & 535.35 & 443.1875 & 122.5994 & 2.44169 \\
\hline 1989 & 36.7963 & 497.8177 & 520.6406 & 443.0625 & 122.5994 & 2.4406 \\
\hline 1990 & 36.8133 & 477.1452 & 508.6455 & 442.9375 & 122.5994 & 2.43951 \\
\hline 1991 & 36.8303 & 485.2747 & 500.8877 & 442.8125 & 122.5994 & 2.43843 \\
\hline 1992 & 36.8473 & 473.666 & 496.2199 & 442.6875 & 122.5994 & 2.43734 \\
\hline 1993 & 36.8643 & 474.6961 & 492.842 & 442.5625 & 122.5994 & 2.43626 \\
\hline 1994 & 36.8813 & 467.5391 & 488.4768 & 442.0391 & 122.5994 & 2.43517 \\
\hline 1995 & 36.8983 & 458.7463 & 483.585 & 441.5156 & 122.5994 & 2.43409 \\
\hline 1996 & 36.9153 & 450.1399 & 479.1213 & 440.9922 & 122.5994 & 2.43301 \\
\hline 1997 & 36.9323 & 406.4001 & 476.2417 & 440.4688 & 122.5994 & 2.43193 \\
\hline 1998 & 36.9493 & 464.6179 & 474.3476 & 439.9453 & 122.5994 & 2.43085 \\
\hline 1999 & 36.9663 & 449.5748 & 472.2844 & 439.4219 & 122.5994 & 2.42977 \\
\hline 2000 & 36.9833 & 452.9811 & 469.0981 & 438.8984 & 122.5994 & 2.42869 \\
\hline 2001 & 37.0003 & 386.1064 & 465.2754 & 438.375 & 122.5994 & 2.42761 \\
\hline 2002 & 37.0173 & 444.5889 & 461.8281 & 437.8516 & 122.5994 & 2.42654 \\
\hline 2003 & 37.0343 & 431.9729 & 459.189 & 437.3281 & 122.5994 & 2.42546 \\
\hline 2004 & 37.0513 & 428.9395 & 457.1108 & 436.8047 & 122.5994 & 2.42439 \\
\hline 2005 & 37.0683 & 464.3525 & 455.3117 & 436.2813 & 122.5994 & 2.42331 \\
\hline 2006 & 37.0853 & 416.1201 & 452.769 & 435.7578 & 122.5994 & 2.42224 \\
\hline 2007 & 37.1023 & 417.5414 & 450.1912 & 435.2344 & 122.5994 & 2.42117 \\
\hline
\end{tabular}




\begin{tabular}{|c|c|c|c|c|c|c|}
\hline 2008 & 37.1193 & 424.3193 & 447.9942 & 434.7109 & 122.5994 & 2.4201 \\
\hline 2009 & 37.1363 & 424.4166 & 446.8049 & 434.1875 & 122.5994 & 2.41903 \\
\hline 2010 & 37.1533 & 417.9069 & 445.7976 & 433.6641 & 122.5994 & 2.41797 \\
\hline 2011 & 37.1703 & 405.141 & 445.3354 & 433.1406 & 122.5994 & 2.4169 \\
\hline 2012 & 37.1873 & 422.7357 & 445.3652 & 432.6172 & 122.5994 & 2.41583 \\
\hline 2013 & 37.2043 & 411.5028 & 445.8841 & 432.0938 & 122.5994 & 2.41477 \\
\hline 2014 & 37.2213 & 444.2529 & 446.9505 & 431.5703 & 122.5994 & 2.4137 \\
\hline 2015 & 37.2383 & 419.0484 & 448.756 & 431.0469 & 122.5994 & 2.41264 \\
\hline 2016 & 37.2553 & 405.0464 & 451.1618 & 430.5234 & 122.5994 & 2.41158 \\
\hline 2017 & 37.2723 & 437.9446 & 454.6078 & 430 & 122.5994 & 2.41052 \\
\hline 2018 & 37.2893 & 445.2636 & 458.6005 & 429.0833 & 122.5994 & 2.40946 \\
\hline 2019 & 37.3063 & 432.6977 & 463.2239 & 428.1667 & 122.5994 & 2.4084 \\
\hline 2020 & 37.3233 & 428.9445 & 467.6487 & 427.25 & 122.5994 & 2.40734 \\
\hline 2021 & 37.3403 & 424.4166 & 467.9843 & 426.3333 & 122.5994 & 2.40628 \\
\hline 2022 & 37.3573 & 440.5943 & 469.5518 & 425.4167 & 122.5994 & 2.40523 \\
\hline 2023 & 37.3743 & 426.6728 & 471.0657 & 424.5 & 122.5994 & 2.40417 \\
\hline 2024 & 37.3913 & 426.3951 & 474.4024 & 423.5833 & 122.5994 & 2.40312 \\
\hline 2025 & 37.4083 & 446.6814 & 480.493 & 422.6667 & 122.5994 & 2.40207 \\
\hline 2026 & 37.4253 & 458.1115 & 488.5743 & 421.75 & 122.5994 & 2.40101 \\
\hline 2027 & 37.4423 & 450.6717 & 495.7353 & 420.8333 & 122.5994 & 2.39996 \\
\hline 2028 & 37.4593 & 442.0223 & 497.5556 & 419.9167 & 122.5994 & 2.39891 \\
\hline 2029 & 37.4763 & 455.9878 & 491.4186 & 419 & 122.5994 & 2.39786 \\
\hline 2030 & 37.4933 & 453.7968 & 480.8485 & 418.0833 & 122.5994 & 2.39682 \\
\hline 2031 & 37.5103 & 449.4999 & 470.896 & 417.1667 & 122.5994 & 2.39577 \\
\hline 2032 & 37.5273 & 449.3969 & 464.1827 & 416.25 & 122.5994 & 2.39472 \\
\hline 2033 & 37.5443 & 440.3918 & 459.9436 & 415.3333 & 122.5994 & 2.39368 \\
\hline 2034 & 37.5613 & 430.59 & 455.454 & 414.4167 & 122.5994 & 2.39263 \\
\hline 2035 & 37.5783 & 470.9687 & 449.1252 & 413.5 & 122.5994 & 2.39159 \\
\hline 2036 & 37.5953 & 446.8794 & 442.3532 & 412.5833 & 122.5994 & 2.39055 \\
\hline 2037 & 37.6123 & 467.7833 & 436.9206 & 411.6667 & 122.5994 & 2.38951 \\
\hline 2038 & 37.6293 & 457.4447 & 433.3468 & 410.75 & 122.5994 & 2.38847 \\
\hline 2039 & 37.6463 & 468.2686 & 431.1101 & 409.8333 & 122.5994 & 2.38743 \\
\hline 2040 & 37.6633 & 459.6807 & 429.4472 & 408.9167 & 122.5994 & 2.38639 \\
\hline 2041 & 37.6803 & 395.5085 & 427.7052 & 408 & 122.5994 & 2.38535 \\
\hline 2042 & 37.6973 & 417.4668 & 425.871 & 407.0833 & 122.5994 & 2.38431 \\
\hline 2043 & 37.7143 & 484.1219 & 424.4744 & 406.1667 & 122.5994 & 2.38328 \\
\hline 2044 & 37.7313 & 434.953 & 424.0723 & 405.25 & 122.5994 & 2.38224 \\
\hline 2045 & 37.7483 & 449.1505 & 424.6555 & 404.3333 & 122.5994 & 2.38121 \\
\hline 2046 & 37.7653 & 497.3328 & 425.8343 & 403.4167 & 122.5994 & 2.38018 \\
\hline 2047 & 37.7823 & 470.7482 & 426.7135 & 402.5 & 122.5994 & 2.37914 \\
\hline 2048 & 37.7993 & 466.1392 & 426.725 & 401.5833 & 122.5994 & 2.37811 \\
\hline 2049 & 37.8163 & 455.8243 & 426.0247 & 400.6667 & 122.5994 & 2.37708 \\
\hline 2050 & 37.8333 & 422.5529 & 425.633 & 399.75 & 122.5994 & 2.37605 \\
\hline 2051 & 37.8503 & 406.7187 & 426.1549 & 398.8333 & 122.5994 & 2.37503 \\
\hline 2052 & 37.8673 & 427.9125 & 427.4789 & 397.9167 & 122.5994 & 2.374 \\
\hline 2053 & 37.8843 & 424.4166 & 428.9063 & 397 & 122.5994 & 2.37297 \\
\hline 2054 & 37.9013 & 429.3916 & 429.5516 & 396.0833 & 122.5994 & 2.37195 \\
\hline 2055 & 37.9183 & 447.1471 & 429.1634 & 395.1667 & 122.5994 & 2.37092 \\
\hline 2056 & 37.9353 & 422.9824 & 427.8635 & 394.25 & 122.5994 & 2.3699 \\
\hline
\end{tabular}




\begin{tabular}{|c|c|c|c|c|c|c|}
\hline 2057 & 37.9523 & 427.7193 & 425.748 & 393.3333 & 122.5994 & 2.36888 \\
\hline 2058 & 37.9693 & 412.1449 & 423.2652 & 392.4167 & 122.5994 & 2.36786 \\
\hline 2059 & 37.9863 & 414.3394 & 420.5827 & 391.5 & 122.5994 & 2.36684 \\
\hline 2060 & 38.0033 & 414.0958 & 417.7377 & 390.5833 & 122.5994 & 2.36582 \\
\hline 2061 & 38.0203 & 394.928 & 414.726 & 389.6667 & 122.5994 & 2.3648 \\
\hline 2062 & 38.0373 & 380.0971 & 411.6103 & 388.75 & 122.5994 & 2.36378 \\
\hline 2063 & 38.0543 & 375.3848 & 408.5473 & 387.8333 & 122.5994 & 2.36276 \\
\hline 2064 & 38.0713 & 381.7308 & 405.7431 & 386.9167 & 122.5994 & 2.36175 \\
\hline 2065 & 38.0883 & 381.0155 & 403.4358 & 386 & 122.5994 & 2.36073 \\
\hline 2066 & 38.1053 & 393.4829 & 402.3131 & 385.4167 & 122.5994 & 2.35972 \\
\hline 2067 & 38.1223 & 403.417 & 402.5961 & 384.8333 & 122.5994 & 2.3587 \\
\hline 2068 & 38.1393 & 373.4984 & 405.1612 & 384.25 & 122.5994 & 2.35769 \\
\hline 2069 & 38.1563 & 355.3072 & 411.1515 & 383.6667 & 122.5994 & 2.35668 \\
\hline 2070 & 38.1733 & 365.3429 & 421.5877 & 383.0833 & 122.5994 & 2.35567 \\
\hline 2071 & 38.1903 & 382.6427 & 436.0962 & 382.5 & 122.5994 & 2.35466 \\
\hline 2072 & 38.2073 & 381.4827 & 451.8862 & 381.9167 & 122.5994 & 2.35365 \\
\hline 2073 & 38.2243 & 360.8946 & 462.7783 & 381.3333 & 122.5994 & 2.35264 \\
\hline 2074 & 38.2413 & 361.0867 & 462.6257 & 380.75 & 122.5994 & 2.35164 \\
\hline 2075 & 38.2583 & 368.6891 & 452.8781 & 380.1667 & 122.5994 & 2.35063 \\
\hline 2076 & 38.2753 & 373.5937 & 442.0045 & 379.5833 & 122.5994 & 2.34963 \\
\hline 2077 & 38.2923 & 371.254 & 434.6234 & 379 & 122.5994 & 2.34862 \\
\hline 2078 & 38.3093 & 365.9645 & 431.1576 & 378.4167 & 122.5994 & 2.34762 \\
\hline 2079 & 38.3263 & 340.9154 & 428.0573 & 377.8333 & 122.5994 & 2.34662 \\
\hline 2080 & 38.3433 & 355.8705 & 421.466 & 377.25 & 122.5994 & 2.34562 \\
\hline 2081 & 38.3603 & 367.6621 & 411.7179 & 376.6667 & 122.5994 & 2.34462 \\
\hline 2082 & 38.3773 & 371.6747 & 401.7561 & 376.0833 & 122.5994 & 2.34362 \\
\hline 2083 & 38.3943 & 381.7539 & 393.5786 & 375.5 & 122.5994 & 2.34262 \\
\hline 2084 & 38.4113 & 362.0699 & 387.7594 & 374.9167 & 122.5994 & 2.34162 \\
\hline 2085 & 38.4283 & 360.441 & 384.0637 & 374.3333 & 122.5994 & 2.34062 \\
\hline 2086 & 38.4453 & 319.8694 & 381.9539 & 373.75 & 122.5994 & 2.33963 \\
\hline 2087 & 38.4623 & 407.7796 & 380.9272 & 373.1667 & 122.5994 & 2.33863 \\
\hline 2088 & 38.4793 & 384.0321 & 380.614 & 372.5833 & 122.5994 & 2.33764 \\
\hline 2089 & 38.4963 & 383.9217 & 380.7031 & 372 & 122.5994 & 2.33664 \\
\hline 2090 & 38.5133 & 347.9283 & 381.8307 & 372.3333 & 122.5994 & 2.33565 \\
\hline 2091 & 38.5303 & 335.2008 & 382.8208 & 372.6667 & 122.5994 & 2.33466 \\
\hline 2092 & 38.5473 & 364.5728 & 383.3082 & 373 & 122.5994 & 2.33367 \\
\hline 2093 & 38.5643 & 346.6134 & 383.2193 & 373.3333 & 122.5994 & 2.33268 \\
\hline 2094 & 38.5813 & 394.0443 & 382.9371 & 373.6667 & 122.5994 & 2.33169 \\
\hline 2095 & 38.5983 & 339.8439 & 382.6836 & 374 & 122.5994 & 2.3307 \\
\hline 2096 & 38.6153 & 358.4069 & 382.4738 & 374.3333 & 122.5994 & 2.32972 \\
\hline 2097 & 38.6323 & 333.123 & 382.3098 & 374.6667 & 122.5994 & 2.32873 \\
\hline 2098 & 38.6493 & 379.6389 & 382.0782 & 375 & 122.5994 & 2.32775 \\
\hline 2099 & 38.6663 & 351.872 & 381.7706 & 375.3333 & 122.5994 & 2.32676 \\
\hline 2100 & 38.6833 & 381.2845 & 381.5713 & 375.6667 & 122.5994 & 2.32578 \\
\hline 2101 & 38.7003 & 350.1041 & 381.6209 & 376 & 122.5994 & 2.3248 \\
\hline 2102 & 38.7173 & 368.6046 & 381.9106 & 376.3333 & 122.5994 & 2.32381 \\
\hline 2103 & 38.7343 & 366.9529 & 383.0569 & 376.6667 & 122.5994 & 2.32283 \\
\hline 2104 & 38.7513 & 359.0854 & 385.2367 & 377 & 122.5994 & 2.32185 \\
\hline 2105 & 38.7683 & 359.6829 & 388.7185 & 377.3333 & 122.5994 & 2.32087 \\
\hline
\end{tabular}




\begin{tabular}{|c|c|c|c|c|c|c|}
\hline 2106 & 38.7853 & 371.8427 & 393.4895 & 377.6667 & 122.5994 & 2.3199 \\
\hline 2107 & 38.8023 & 359.8604 & 398.9181 & 378 & 122.5994 & 2.31892 \\
\hline 2108 & 38.8193 & 346.0565 & 403.372 & 378.3333 & 122.5994 & 2.31794 \\
\hline 2109 & 38.8363 & 330.4702 & 408.1906 & 378.6667 & 122.5994 & 2.31697 \\
\hline 2110 & 38.8533 & 350.8207 & 408.7468 & 379 & 122.5994 & 2.31599 \\
\hline 2111 & 38.8703 & 372.5016 & 408.6244 & 379.3333 & 122.5994 & 2.31502 \\
\hline 2112 & 38.8873 & 371.2795 & 407.8847 & 379.6667 & 122.5994 & 2.31405 \\
\hline 2113 & 38.9043 & 382.9611 & 406.6352 & 380 & 122.5994 & 2.31307 \\
\hline 2114 & 38.9213 & 390.9721 & 405.008 & 380.25 & 122.5994 & 2.3121 \\
\hline 2115 & 38.9383 & 362.8062 & 403.0014 & 380.5 & 122.5994 & 2.31113 \\
\hline 2116 & 38.9553 & 378.4632 & 400.9232 & 380.75 & 122.5994 & 2.31016 \\
\hline 2117 & 38.9723 & 377.8621 & 399.0547 & 381 & 122.5994 & 2.30919 \\
\hline 2118 & 38.9893 & 358.1834 & 397.0047 & 381.25 & 122.5994 & 2.30823 \\
\hline 2119 & 39.0063 & 345.484 & 395.1741 & 381.5 & 122.5994 & 2.30726 \\
\hline 2120 & 39.0233 & 374.0971 & 393.7705 & 381.75 & 122.5994 & 2.30629 \\
\hline 2121 & 39.0403 & 376.802 & 392.9973 & 382 & 122.5994 & 2.30533 \\
\hline 2122 & 39.0573 & 369.7437 & 392.8498 & 382.25 & 122.5994 & 2.30436 \\
\hline 2123 & 39.0743 & 369.9229 & 393.1128 & 382.5 & 122.5994 & 2.3034 \\
\hline 2124 & 39.0913 & 360.9265 & 394.0506 & 382.75 & 122.5994 & 2.30244 \\
\hline 2125 & 39.1083 & 387.1529 & 395.6246 & 383 & 122.5994 & 2.30148 \\
\hline 2126 & 39.1253 & 358.267 & 398.2231 & 383.25 & 122.5994 & 2.30052 \\
\hline 2127 & 39.1423 & 391.2194 & 402.5415 & 383.5 & 122.5994 & 2.29956 \\
\hline 2128 & 39.1593 & 386.74 & 409.4918 & 383.75 & 122.5994 & 2.2986 \\
\hline 2129 & 39.1763 & 362.175 & 419.7538 & 384 & 122.5994 & 2.29764 \\
\hline 2130 & 39.1933 & 366.4126 & 433.028 & 384.25 & 122.5994 & 2.29668 \\
\hline 2131 & 39.2103 & 356.0766 & 446.9952 & 384.5 & 122.5994 & 2.29572 \\
\hline 2132 & 39.2273 & 389.6415 & 456.4883 & 384.75 & 122.5994 & 2.29477 \\
\hline 2133 & 39.2443 & 372.0885 & 456.8062 & 385 & 122.5994 & 2.29381 \\
\hline 2134 & 39.2613 & 372.1321 & 450.9802 & 385.25 & 122.5994 & 2.29286 \\
\hline 2135 & 39.2783 & 351.5766 & 445.1634 & 385.5 & 122.5994 & 2.29191 \\
\hline 2136 & 39.2953 & 393.154 & 442.9659 & 385.75 & 122.5994 & 2.29095 \\
\hline 2137 & 39.3123 & 388.3787 & 444.6273 & 386 & 122.5994 & 2.29 \\
\hline 2138 & 39.3293 & 388.1417 & 447.0369 & 386 & 122.5994 & 2.28905 \\
\hline 2139 & 39.3463 & 407.9664 & 447.5395 & 386 & 122.5994 & 2.2881 \\
\hline 2140 & 39.3633 & 388.4202 & 447.5407 & 386 & 122.5994 & 2.28715 \\
\hline 2141 & 39.3803 & 401.4239 & 451.2184 & 386 & 122.5994 & 2.2862 \\
\hline 2142 & 39.3973 & 434.3801 & 463.8625 & 386 & 122.5994 & 2.28526 \\
\hline 2143 & 39.4143 & 460.9809 & 492.3839 & 386 & 122.5994 & 2.28431 \\
\hline 2144 & 39.4313 & 497.2484 & 544.8015 & 386 & 122.5994 & 2.28336 \\
\hline 2145 & 39.4483 & 545.6476 & 627.0875 & 386 & 122.5994 & 2.28242 \\
\hline 2146 & 39.4653 & 649.1652 & 736.7948 & 386 & 122.5994 & 2.28148 \\
\hline 2147 & 39.4823 & 809.5533 & 853.9042 & 386 & 122.5994 & 2.28053 \\
\hline 2148 & 39.4993 & 959.8973 & 932.8437 & 386 & 122.5994 & 2.27959 \\
\hline 2149 & 39.5163 & 1086.859 & 929.4482 & 386 & 122.5994 & 2.27865 \\
\hline 2150 & 39.5333 & 1075.902 & 862.7847 & 386 & 122.5994 & 2.27771 \\
\hline 2151 & 39.5503 & 929.3869 & 790.1298 & 386 & 122.5994 & 2.27677 \\
\hline 2152 & 39.5673 & 738.6963 & 745.2396 & 386 & 122.5994 & 2.27583 \\
\hline 2153 & 39.5843 & 712.3551 & 730.9216 & 386 & 122.5994 & 2.27489 \\
\hline 2154 & 39.6013 & 727.2126 & 722.3637 & 386 & 122.5994 & 2.27395 \\
\hline
\end{tabular}




\begin{tabular}{|c|c|c|c|c|c|c|}
\hline 2155 & 39.6183 & 732.4674 & 688.3052 & 386 & 122.5994 & 2.27302 \\
\hline 2156 & 39.6353 & 717.156 & 628.1373 & 386 & 122.5994 & 2.27208 \\
\hline 2157 & 39.6523 & 637.0998 & 563.4604 & 386 & 122.5994 & 2.27115 \\
\hline 2158 & 39.6693 & 527.7954 & 508.9127 & 386 & 122.5994 & 2.27021 \\
\hline 2159 & 39.6863 & 481.7643 & 469.6759 & 386 & 122.5994 & 2.26928 \\
\hline 2160 & 39.7033 & 439.8936 & 444.4704 & 386 & 122.5994 & 2.26835 \\
\hline 2161 & 39.7203 & 438.504 & 429.3038 & 386 & 122.5994 & 2.26741 \\
\hline 2162 & 39.7373 & 415.031 & 420.2311 & 386 & 122.5994 & 2.26648 \\
\hline 2163 & 39.7543 & 388.3789 & 414.5164 & 386 & 122.5994 & 2.26555 \\
\hline 2164 & 39.7713 & 381.2896 & 410.6414 & 386 & 122.5994 & 2.26462 \\
\hline 2165 & 39.7883 & 386.2032 & 407.8728 & 386 & 122.5994 & 2.2637 \\
\hline 2166 & 39.8053 & 393.1118 & 405.8519 & 386 & 122.5994 & 2.26277 \\
\hline 2167 & 39.8223 & 369.3936 & 404.3738 & 386 & 122.5994 & 2.26184 \\
\hline 2168 & 39.8393 & 354.817 & 403.3401 & 386 & 122.5994 & 2.26092 \\
\hline 2169 & 39.8563 & 368.3237 & 402.8051 & 386 & 122.5994 & 2.25999 \\
\hline 2170 & 39.8733 & 371.1612 & 402.8816 & 386 & 122.5994 & 2.25907 \\
\hline 2171 & 39.8903 & 412.8741 & 403.5266 & 386 & 122.5994 & 2.25814 \\
\hline 2172 & 39.9073 & 376.2232 & 404.4316 & 386 & 122.5994 & 2.25722 \\
\hline 2173 & 39.9243 & 384.7105 & 405.0159 & 386 & 122.5994 & 2.2563 \\
\hline 2174 & 39.9413 & 352.6042 & 404.9495 & 386 & 122.5994 & 2.25538 \\
\hline 2175 & 39.9583 & 347.1677 & 401.2076 & 386 & 122.5994 & 2.25446 \\
\hline 2176 & 39.9753 & 361.8016 & 401.3293 & 386 & 122.5994 & 2.25354 \\
\hline 2177 & 39.9923 & 387.0864 & 401.8986 & 386 & 122.5994 & 2.25262 \\
\hline 2178 & 40.0093 & 365.987 & 402.8383 & 386 & 122.5994 & 2.2517 \\
\hline 2179 & 40.0263 & 370.8748 & 401.9971 & 386 & 122.5994 & 2.25078 \\
\hline 2180 & 40.0433 & 418.3974 & 402.8079 & 386 & 122.5994 & 2.24987 \\
\hline 2181 & 40.0603 & 384.3773 & 403.4559 & 386 & 122.5994 & 2.24895 \\
\hline 2182 & 40.0773 & 383.8378 & 404.2833 & 386 & 122.5994 & 2.24804 \\
\hline 2183 & 40.0943 & 384.2112 & 405.5668 & 386 & 122.5994 & 2.24712 \\
\hline 2184 & 40.1113 & 384.1232 & 407.4074 & 386 & 122.5994 & 2.24621 \\
\hline 2185 & 40.1283 & 392.0254 & 409.4926 & 386 & 122.5994 & 2.2453 \\
\hline 2186 & 40.1453 & 380.3665 & 411.2926 & 386.0833 & 122.5994 & 2.24439 \\
\hline 2187 & 40.1623 & 392.9274 & 412.482 & 386.1667 & 122.5994 & 2.24347 \\
\hline 2188 & 40.1793 & 414.3267 & 413.9234 & 386.25 & 122.5994 & 2.24256 \\
\hline 2189 & 40.1963 & 387.0152 & 416.8961 & 386.3333 & 122.5994 & 2.24166 \\
\hline 2190 & 40.2133 & 421.0264 & 423.128 & 386.4167 & 122.5994 & 2.24075 \\
\hline 2191 & 40.2303 & 428.5471 & 434.9091 & 386.5 & 122.5994 & 2.23984 \\
\hline 2192 & 40.2473 & 434.4775 & 455.0895 & 386.5833 & 122.5994 & 2.23893 \\
\hline 2193 & 40.2643 & 474.4359 & 486.2275 & 386.6667 & 122.5994 & 2.23803 \\
\hline 2194 & 40.2813 & 503.9116 & 528.5 & 386.75 & 122.5994 & 2.23712 \\
\hline 2195 & 40.2983 & 561.1067 & 575.8701 & 386.8333 & 122.5994 & 2.23622 \\
\hline 2196 & 40.3153 & 613.8514 & 612.0099 & 386.9167 & 122.5994 & 2.23531 \\
\hline 2197 & 40.3323 & 694.606 & 616.8444 & 387 & 122.5994 & 2.23441 \\
\hline 2198 & 40.3493 & 741.8697 & 592.4457 & 387.0833 & 122.5994 & 2.23351 \\
\hline 2199 & 40.3663 & 698.4273 & 561.7644 & 387.1667 & 122.5994 & 2.23261 \\
\hline 2200 & 40.3833 & 607.7786 & 541.622 & 387.25 & 122.5994 & 2.23171 \\
\hline 2201 & 40.4003 & 512.3223 & 535.3801 & 387.3333 & 122.5994 & 2.23081 \\
\hline 2202 & 40.4173 & 522.6374 & 534.8556 & 387.4167 & 122.5994 & 2.22991 \\
\hline 2203 & 40.4343 & 559.5086 & 526.0281 & 387.5 & 122.5994 & 2.22901 \\
\hline
\end{tabular}




\begin{tabular}{|c|c|c|c|c|c|c|}
\hline 2204 & 40.4513 & 576.9326 & 504.1107 & 387.5833 & 122.5994 & 2.22811 \\
\hline 2205 & 40.4683 & 537.1829 & 477.4382 & 387.6667 & 122.5994 & 2.22721 \\
\hline 2206 & 40.4853 & 457.6982 & 453.8322 & 387.75 & 122.5994 & 2.22632 \\
\hline 2207 & 40.5023 & 427.5185 & 436.1658 & 387.8333 & 122.5994 & 2.22542 \\
\hline 2208 & 40.5193 & 400.4686 & 423.9055 & 387.9167 & 122.5994 & 2.22453 \\
\hline 2209 & 40.5363 & 386.0566 & 415.5501 & 388 & 122.5994 & 2.22363 \\
\hline 2210 & 40.5533 & 392.5081 & 409.4456 & 387.75 & 122.5994 & 2.22274 \\
\hline 2211 & 40.5703 & 358.177 & 405.2 & 387.5 & 122.5994 & 2.22185 \\
\hline 2212 & 40.5873 & 372.0755 & 402.1489 & 387.25 & 122.5994 & 2.22096 \\
\hline 2213 & 40.6043 & 366.8181 & 399.8627 & 387 & 122.5994 & 2.22007 \\
\hline 2214 & 40.6213 & 364.1875 & 397.917 & 386.75 & 122.5994 & 2.21918 \\
\hline 2215 & 40.6383 & 358.453 & 396.1312 & 386.5 & 122.5994 & 2.21829 \\
\hline 2216 & 40.6553 & 353.3357 & 394.5361 & 386.25 & 122.5994 & 2.2174 \\
\hline 2217 & 40.6723 & 364.948 & 393.1897 & 386 & 122.5994 & 2.21651 \\
\hline 2218 & 40.6893 & 371.3904 & 392.0995 & 385.75 & 122.5994 & 2.21563 \\
\hline 2219 & 40.7063 & 350.5891 & 391.2367 & 385.5 & 122.5994 & 2.21474 \\
\hline 2220 & 40.7233 & 361.9484 & 390.576 & 385.25 & 122.5994 & 2.21385 \\
\hline 2221 & 40.7403 & 383.5058 & 390.0486 & 385 & 122.5994 & 2.21297 \\
\hline 2222 & 40.7573 & 378.9011 & 389.6537 & 384.75 & 122.5994 & 2.21209 \\
\hline 2223 & 40.7743 & 370.0769 & 389.3958 & 384.5 & 122.5994 & 2.2112 \\
\hline 2224 & 40.7913 & 375.017 & 389.2976 & 384.25 & 122.5994 & 2.21032 \\
\hline 2225 & 40.8083 & 410.3303 & 389.3885 & 384 & 122.5994 & 2.20944 \\
\hline 2226 & 40.8253 & 381.3474 & 389.6845 & 383.75 & 122.5994 & 2.20856 \\
\hline 2227 & 40.8423 & 372.9452 & 390.1201 & 383.5 & 122.5994 & 2.20768 \\
\hline 2228 & 40.8593 & 386.7556 & 390.6486 & 383.25 & 122.5994 & 2.2068 \\
\hline 2229 & 40.8763 & 395.4831 & 391.1042 & 383 & 122.5994 & 2.20592 \\
\hline 2230 & 40.8933 & 362.4593 & 391.3893 & 382.75 & 122.5994 & 2.20504 \\
\hline 2231 & 40.9103 & 403.1687 & 391.6279 & 382.5 & 122.5994 & 2.20417 \\
\hline 2232 & 40.9273 & 395.9499 & 391.9474 & 382.25 & 122.5994 & 2.20329 \\
\hline 2233 & 40.9443 & 387.855 & 392.3633 & 382 & 122.5994 & 2.20241 \\
\hline 2234 & 40.9613 & 374.5901 & 393.3085 & 382.1667 & 122.5994 & 2.20154 \\
\hline 2235 & 40.9783 & 341.071 & 394.3239 & 382.3333 & 122.5994 & 2.20066 \\
\hline 2236 & 40.9953 & 376.4102 & 395.4215 & 382.5 & 122.5994 & 2.19979 \\
\hline 2237 & 41.0123 & 376.7532 & 396.67 & 382.6667 & 122.5994 & 2.19892 \\
\hline 2238 & 41.0293 & 378.7681 & 397.5872 & 382.8333 & 122.5994 & 2.19805 \\
\hline 2239 & 41.0463 & 368.6046 & 398.7246 & 383 & 122.5994 & 2.19718 \\
\hline 2240 & 41.0633 & 377.6286 & 399.2551 & 383.1667 & 122.5994 & 2.19631 \\
\hline 2241 & 41.0803 & 413.2144 & 399.3063 & 383.3333 & 122.5994 & 2.19544 \\
\hline 2242 & 41.0973 & 381.58 & 399.33 & 383.5 & 122.5994 & 2.19457 \\
\hline 2243 & 41.1143 & 388.9525 & 399.657 & 383.6667 & 122.5994 & 2.1937 \\
\hline 2244 & 41.1313 & 427.1488 & 400.4742 & 383.8333 & 122.5994 & 2.19283 \\
\hline 2245 & 41.1483 & 388.1417 & 401.6377 & 384 & 122.5994 & 2.19196 \\
\hline 2246 & 41.1653 & 401.5986 & 403.1161 & 384.1667 & 122.5994 & 2.1911 \\
\hline 2247 & 41.1823 & 425.9099 & 404.7401 & 384.3333 & 122.5994 & 2.19023 \\
\hline 2248 & 41.1993 & 406.5631 & 407.2066 & 384.5 & 122.5994 & 2.18937 \\
\hline 2249 & 41.2163 & 404.9056 & 409.8944 & 384.6667 & 122.5994 & 2.18851 \\
\hline 2250 & 41.2333 & 409.2985 & 411.9552 & 384.8333 & 122.5994 & 2.18764 \\
\hline 2251 & 41.2503 & 408.7141 & 412.9928 & 385 & 122.5994 & 2.18678 \\
\hline 2252 & 41.2673 & 418.4641 & 413.4348 & 385.1667 & 122.5994 & 2.18592 \\
\hline
\end{tabular}




\begin{tabular}{|c|c|c|c|c|c|c|}
\hline 2253 & 41.2843 & 403.709 & 413.3385 & 385.3333 & 122.5994 & 2.18506 \\
\hline 2254 & 41.3013 & 425.6978 & 412.2419 & 385.5 & 122.5994 & 2.1842 \\
\hline 2255 & 41.3183 & 407.156 & 410.1821 & 385.6667 & 122.5994 & 2.18334 \\
\hline 2256 & 41.3353 & 372.6127 & 407.8179 & 385.8333 & 122.5994 & 2.18248 \\
\hline 2257 & 41.3523 & 384.8904 & 405.4979 & 386 & 122.5994 & 2.18162 \\
\hline 2258 & 41.3693 & 392.0024 & 403.046 & 385.7083 & 122.5994 & 2.18076 \\
\hline 2259 & 41.3863 & 387.4031 & 400.9791 & 385.4167 & 122.5994 & 2.17991 \\
\hline 2260 & 41.4033 & 367.8979 & 398.7819 & 385.125 & 122.5994 & 2.17905 \\
\hline 2261 & 41.4203 & 364.3394 & 396.2202 & 384.8333 & 122.5994 & 2.1782 \\
\hline 2262 & 41.4373 & 389.0226 & 393.6447 & 384.5417 & 122.5994 & 2.17734 \\
\hline 2263 & 41.4543 & 416.2782 & 391.4296 & 384.25 & 122.5994 & 2.17649 \\
\hline 2264 & 41.4713 & 380.523 & 389.6976 & 383.9583 & 122.5994 & 2.17564 \\
\hline 2265 & 41.4883 & 380.36 & 388.4122 & 383.6667 & 122.5994 & 2.17478 \\
\hline 2266 & 41.5053 & 373.7707 & 387.4807 & 383.375 & 122.5994 & 2.17393 \\
\hline 2267 & 41.5223 & 360.0486 & 386.776 & 383.0833 & 122.5994 & 2.17308 \\
\hline 2268 & 41.5393 & 363.0837 & 386.2328 & 382.7917 & 122.5994 & 2.17223 \\
\hline 2269 & 41.5563 & 367.9189 & 385.8126 & 382.5 & 122.5994 & 2.17138 \\
\hline 2270 & 41.5733 & 381.3379 & 385.5046 & 382.2083 & 122.5994 & 2.17053 \\
\hline 2271 & 41.5903 & 380.8315 & 385.3159 & 381.9167 & 122.5994 & 2.16969 \\
\hline 2272 & 41.6073 & 376.9416 & 385.2658 & 381.625 & 122.5994 & 2.16884 \\
\hline 2273 & 41.6243 & 333.1836 & 385.4114 & 381.3333 & 122.5994 & 2.16799 \\
\hline 2274 & 41.6413 & 364.8506 & 385.7301 & 381.0417 & 122.5994 & 2.16715 \\
\hline 2275 & 41.6583 & 372.1774 & 386.2825 & 380.75 & 122.5994 & 2.1663 \\
\hline 2276 & 41.6753 & 398.6645 & 387.0979 & 380.4583 & 122.5994 & 2.16546 \\
\hline 2277 & 41.6923 & 343.8824 & 388.1939 & 380.1667 & 122.5994 & 2.16461 \\
\hline 2278 & 41.7093 & 353.3231 & 389.5527 & 379.875 & 122.5994 & 2.16377 \\
\hline 2279 & 41.7263 & 372.5086 & 391.9938 & 379.5833 & 122.5994 & 2.16293 \\
\hline 2280 & 41.7433 & 376.4102 & 393.6606 & 379.2917 & 122.5994 & 2.16209 \\
\hline 2281 & 41.7603 & 376.3239 & 395.2814 & 379 & 122.5994 & 2.16124 \\
\hline 2282 & 41.7773 & 378.4953 & 397.1393 & 379 & 122.5994 & 2.1604 \\
\hline 2283 & 41.7943 & 403.6621 & 398.7474 & 379 & 122.5994 & 2.15957 \\
\hline 2284 & 41.8113 & 398.9937 & 400.0512 & 379 & 122.5994 & 2.15873 \\
\hline 2285 & 41.8283 & 394.3843 & 401.6044 & 379 & 122.5994 & 2.15789 \\
\hline 2286 & 41.8453 & 413.4285 & 402.3131 & 379 & 122.5994 & 2.15705 \\
\hline 2287 & 41.8623 & 429.7397 & 402.9473 & 379 & 122.5994 & 2.15621 \\
\hline 2288 & 41.8793 & 377.006 & 403.5065 & 379 & 122.5994 & 2.15538 \\
\hline 2289 & 41.8963 & 368.3133 & 403.5375 & 379 & 122.5994 & 2.15454 \\
\hline 2290 & 41.9133 & 376.4281 & 402.4742 & 379 & 122.5994 & 2.15371 \\
\hline 2291 & 41.9303 & 366.8658 & 400.6545 & 379 & 122.5994 & 2.15287 \\
\hline 2292 & 41.9473 & 380.6244 & 398.385 & 379 & 122.5994 & 2.15204 \\
\hline 2293 & 41.9643 & 405.0569 & 396.377 & 379 & 122.5994 & 2.15121 \\
\hline 2294 & 41.9813 & 407.9983 & 394.8651 & 379 & 122.5994 & 2.15038 \\
\hline 2295 & 41.9983 & 396.382 & 393.7679 & 379 & 122.5994 & 2.14955 \\
\hline 2296 & 42.0153 & 389.0833 & 392.7307 & 379 & 122.5994 & 2.14872 \\
\hline 2297 & 42.0323 & 360.2942 & 391.6038 & 379 & 122.5994 & 2.14789 \\
\hline 2298 & 42.0493 & 369.7286 & 390.4476 & 379 & 122.5994 & 2.14706 \\
\hline 2299 & 42.0663 & 392.0923 & 389.6193 & 379 & 122.5994 & 2.14623 \\
\hline 2300 & 42.0833 & 375.7739 & 389.135 & 379 & 122.5994 & 2.1454 \\
\hline 2301 & 42.1003 & 372.2252 & 389.0086 & 379 & 122.5994 & 2.14457 \\
\hline
\end{tabular}




\begin{tabular}{|c|c|c|c|c|c|c|}
\hline 2302 & 42.1173 & 378.2416 & 389.1974 & 379 & 122.5994 & 2.14375 \\
\hline 2303 & 42.1343 & 381.0165 & 389.6329 & 379 & 122.5994 & 2.14292 \\
\hline 2304 & 42.1513 & 383.4358 & 390.235 & 379 & 122.5994 & 2.1421 \\
\hline 2305 & 42.1683 & 376.3239 & 391.0004 & 379 & 122.5994 & 2.14127 \\
\hline 2306 & 42.1853 & 364.9313 & 391.5163 & 378.625 & 122.5994 & 2.14045 \\
\hline 2307 & 42.2023 & 403.765 & 392.2189 & 378.25 & 122.5994 & 2.13963 \\
\hline 2308 & 42.2193 & 411.7747 & 393.2028 & 377.875 & 122.5994 & 2.1388 \\
\hline 2309 & 42.2363 & 413.4109 & 394.3601 & 377.5 & 122.5994 & 2.13798 \\
\hline 2310 & 42.2533 & 406.4184 & 395.7704 & 377.125 & 122.5994 & 2.13716 \\
\hline 2311 & 42.2703 & 428.8641 & 397.4656 & 376.75 & 122.5994 & 2.13634 \\
\hline 2312 & 42.2873 & 447.0693 & 399.5743 & 376.375 & 122.5994 & 2.13552 \\
\hline 2313 & 42.3043 & 415.5268 & 402.3538 & 376 & 122.5994 & 2.1347 \\
\hline 2314 & 42.3213 & 422.8447 & 406.1595 & 375.625 & 122.5994 & 2.13388 \\
\hline 2315 & 42.3383 & 438.3145 & 411.4862 & 375.25 & 122.5994 & 2.13307 \\
\hline 2316 & 42.3553 & 441.0588 & 419.4335 & 374.875 & 122.5994 & 2.13225 \\
\hline 2317 & 42.3723 & 434.0048 & 432.3826 & 374.5 & 122.5994 & 2.13143 \\
\hline 2318 & 42.3893 & 511.0081 & 454.8453 & 374.125 & 122.5994 & 2.13062 \\
\hline 2319 & 42.4063 & 512.4193 & 493.2033 & 373.75 & 122.5994 & 2.1298 \\
\hline 2320 & 42.4233 & 573.488 & 553.4811 & 373.375 & 122.5994 & 2.12899 \\
\hline 2321 & 42.4403 & 654.0196 & 636.8126 & 373 & 122.5994 & 2.12818 \\
\hline 2322 & 42.4573 & 719.7117 & 732.7813 & 372.625 & 122.5994 & 2.12736 \\
\hline 2323 & 42.4743 & 904.7676 & 811.0092 & 372.25 & 122.5994 & 2.12655 \\
\hline 2324 & 42.4913 & 972.2666 & 830.088 & 371.875 & 122.5994 & 2.12574 \\
\hline 2325 & 42.5083 & 1101.865 & 786.747 & 371.5 & 122.5994 & 2.12493 \\
\hline 2326 & 42.5253 & 962.6261 & 722.8007 & 371.125 & 122.5994 & 2.12412 \\
\hline 2327 & 42.5423 & 786.3654 & 675.4491 & 370.75 & 122.5994 & 2.12331 \\
\hline 2328 & 42.5593 & 717.8054 & 658.435 & 370.375 & 122.5994 & 2.1225 \\
\hline 2329 & 42.5763 & 713.4666 & 663.4052 & 370 & 122.5994 & 2.12169 \\
\hline 2330 & 42.5933 & 718.1711 & 664.7411 & 369.625 & 122.5994 & 2.12089 \\
\hline 2331 & 42.6103 & 714.644 & 640.0009 & 369.25 & 122.5994 & 2.12008 \\
\hline 2332 & 42.6273 & 679.1752 & 594.7322 & 368.875 & 122.5994 & 2.11927 \\
\hline 2333 & 42.6443 & 599.8564 & 546.7142 & 368.5 & 122.5994 & 2.11847 \\
\hline 2334 & 42.6613 & 532.0117 & 507.1158 & 368.125 & 122.5994 & 2.11766 \\
\hline 2335 & 42.6783 & 438.5701 & 478.711 & 367.75 & 122.5994 & 2.11686 \\
\hline 2336 & 42.6953 & 399.0024 & 458.9988 & 367.375 & 122.5994 & 2.11605 \\
\hline 2337 & 42.7123 & 398.1592 & 444.1837 & 367 & 122.5994 & 2.11525 \\
\hline 2338 & 42.7293 & 405.1137 & 432.4742 & 366.625 & 122.5994 & 2.11445 \\
\hline 2339 & 42.7463 & 399.6149 & 423.1754 & 366.25 & 122.5994 & 2.11365 \\
\hline 2340 & 42.7633 & 390.6108 & 415.73 & 365.875 & 122.5994 & 2.11285 \\
\hline 2341 & 42.7803 & 403.7882 & 409.6425 & 365.5 & 122.5994 & 2.11205 \\
\hline 2342 & 42.7973 & 376.639 & 404.4248 & 365.125 & 122.5994 & 2.11125 \\
\hline 2343 & 42.8143 & 351.3258 & 399.5284 & 364.75 & 122.5994 & 2.11045 \\
\hline 2344 & 42.8313 & 353.1864 & 394.9407 & 364.375 & 122.5994 & 2.10965 \\
\hline 2345 & 42.8483 & 353.9535 & 391.1436 & 364 & 122.5994 & 2.10885 \\
\hline 2346 & 42.8653 & 352.0711 & 388.1846 & 363.625 & 122.5994 & 2.10805 \\
\hline 2347 & 42.8823 & 347.6028 & 385.4669 & 363.25 & 122.5994 & 2.10726 \\
\hline 2348 & 42.8993 & 334.9782 & 382.4073 & 362.875 & 122.5994 & 2.10646 \\
\hline 2349 & 42.9163 & 355.6206 & 379.1998 & 362.5 & 122.5994 & 2.10567 \\
\hline 2350 & 42.9333 & 377.7788 & 376.4304 & 362.125 & 122.5994 & 2.10487 \\
\hline
\end{tabular}




\begin{tabular}{|c|c|c|c|c|c|c|}
\hline 2351 & 42.9503 & 376.7531 & 374.4075 & 361.75 & 122.5994 & 2.10408 \\
\hline 2352 & 42.9673 & 367.0146 & 373.0833 & 361.375 & 122.5994 & 2.10329 \\
\hline 2353 & 42.9843 & 354.9363 & 372.2151 & 361 & 122.5994 & 2.10249 \\
\hline 2354 & 43.0013 & 350.6228 & 371.4023 & 360.625 & 122.5994 & 2.1017 \\
\hline 2355 & 43.0183 & 363.2744 & 370.1611 & 360.25 & 122.5994 & 2.10091 \\
\hline 2356 & 43.0353 & 358.4918 & 368.7732 & 359.875 & 122.5994 & 2.10012 \\
\hline 2357 & 43.0523 & 371.9666 & 367.449 & 359.5 & 122.5994 & 2.09933 \\
\hline 2358 & 43.0693 & 377.5234 & 366.2778 & 359.125 & 122.5994 & 2.09854 \\
\hline 2359 & 43.0863 & 373.478 & 365.173 & 358.75 & 122.5994 & 2.09775 \\
\hline 2360 & 43.1033 & 333.1142 & 364.2231 & 358.375 & 122.5994 & 2.09696 \\
\hline 2361 & 43.1203 & 337.3804 & 363.4188 & 358 & 122.5994 & 2.09618 \\
\hline 2362 & 43.1373 & 363.3254 & 362.7671 & 357.625 & 122.5994 & 2.09539 \\
\hline 2363 & 43.1543 & 343.9782 & 362.2663 & 357.25 & 122.5994 & 2.0946 \\
\hline 2364 & 43.1713 & 351.0703 & 361.9245 & 356.875 & 122.5994 & 2.09382 \\
\hline 2365 & 43.1883 & 376.6664 & 361.7998 & 356.5 & 122.5994 & 2.09303 \\
\hline 2366 & 43.2053 & 366.5337 & 360.9294 & 356.125 & 122.5994 & 2.09225 \\
\hline 2367 & 43.2223 & 371.744 & 361.4162 & 355.75 & 122.5994 & 2.09147 \\
\hline 2368 & 43.2393 & 390.7029 & 362.1084 & 355.375 & 122.5994 & 2.09068 \\
\hline 2369 & 43.2563 & 362.4992 & 362.6285 & 355 & 122.5994 & 2.0899 \\
\hline 2370 & 43.2733 & 402.8598 & 362.4163 & 354.625 & 122.5994 & 2.08912 \\
\hline 2371 & 43.2903 & 357.4727 & 361.3759 & 354.25 & 122.5994 & 2.08834 \\
\hline 2372 & 43.3073 & 349.885 & 360.0064 & 353.875 & 122.5994 & 2.08756 \\
\hline 2373 & 43.3243 & 364.3596 & 358.9413 & 353.5 & 122.5994 & 2.08678 \\
\hline 2374 & 43.3413 & 354.3715 & 358.3186 & 353.125 & 122.5994 & 2.086 \\
\hline 2375 & 43.3583 & 368.9111 & 358.0994 & 352.75 & 122.5994 & 2.08522 \\
\hline 2376 & 43.3753 & 369.4778 & 357.9028 & 352.375 & 122.5994 & 2.08444 \\
\hline 2377 & 43.3923 & 341.3143 & 357.0444 & 352 & 122.5994 & 2.08367 \\
\hline 2378 & 43.4093 & 335.9184 & 356.1362 & 351.7083 & 122.5994 & 2.08289 \\
\hline 2379 & 43.4263 & 349.2419 & 355.5196 & 351.4167 & 122.5994 & 2.08211 \\
\hline 2380 & 43.4433 & 372.1915 & 355.09 & 351.125 & 122.5994 & 2.08134 \\
\hline 2381 & 43.4603 & 321.9562 & 355.041 & 350.8333 & 122.5994 & 2.08056 \\
\hline 2382 & 43.4773 & 336.1432 & 355.6653 & 350.5417 & 122.5994 & 2.07979 \\
\hline 2383 & 43.4943 & 355.4886 & 357.2516 & 350.25 & 122.5994 & 2.07902 \\
\hline 2384 & 43.5113 & 333.6131 & 360.0648 & 349.9583 & 122.5994 & 2.07824 \\
\hline 2385 & 43.5283 & 351.8728 & 363.8849 & 349.6667 & 122.5994 & 2.07747 \\
\hline 2386 & 43.5453 & 346.7509 & 368.2992 & 349.375 & 122.5994 & 2.0767 \\
\hline 2387 & 43.5623 & 322.7562 & 371.3833 & 349.0833 & 122.5994 & 2.07593 \\
\hline 2388 & 43.5793 & 325.6574 & 371.169 & 348.7917 & 122.5994 & 2.07516 \\
\hline 2389 & 43.5963 & 340.4946 & 368.0708 & 348.5 & 122.5994 & 2.07439 \\
\hline 2390 & 43.6133 & 349.6565 & 364.4625 & 348.2083 & 122.5994 & 2.07362 \\
\hline 2391 & 43.6303 & 357.7701 & 362.0209 & 347.9167 & 122.5994 & 2.07285 \\
\hline 2392 & 43.6473 & 353.574 & 361.2361 & 347.625 & 122.5994 & 2.07208 \\
\hline 2393 & 43.6643 & 350.696 & 361.5787 & 347.3333 & 122.5994 & 2.07131 \\
\hline 2394 & 43.6813 & 348.5647 & 361.7421 & 347.0417 & 122.5994 & 2.07055 \\
\hline 2395 & 43.6983 & 348.5819 & 360.6327 & 346.75 & 122.5994 & 2.06978 \\
\hline 2396 & 43.7153 & 364.544 & 358.6054 & 346.4583 & 122.5994 & 2.06902 \\
\hline 2397 & 43.7323 & 348.558 & 356.6722 & 346.1667 & 122.5994 & 2.06825 \\
\hline 2398 & 43.7493 & 340.7253 & 355.3813 & 345.875 & 122.5994 & 2.06749 \\
\hline 2399 & 43.7663 & 335.6554 & 354.8748 & 345.5833 & 122.5994 & 2.06672 \\
\hline
\end{tabular}




\begin{tabular}{|c|c|c|c|c|c|c|}
\hline 2400 & 43.7833 & 347.3241 & 354.9729 & 345.2917 & 122.5994 & 2.06596 \\
\hline 2401 & 43.8003 & 347.6155 & 355.2914 & 345 & 122.5994 & 2.0652 \\
\hline 2402 & 43.8173 & 342.2956 & 355.6948 & 344.9583 & 122.5994 & 2.06444 \\
\hline 2403 & 43.8343 & 352.4217 & 355.7104 & 344.9167 & 122.5994 & 2.06367 \\
\hline 2404 & 43.8513 & 344.0547 & 355.4352 & 344.875 & 122.5994 & 2.06291 \\
\hline 2405 & 43.8683 & 346.6765 & 355.1256 & 344.8333 & 122.5994 & 2.06215 \\
\hline 2406 & 43.8853 & 349.6565 & 354.956 & 344.7917 & 122.5994 & 2.06139 \\
\hline 2407 & 43.9023 & 352.6176 & 354.9417 & 344.75 & 122.5994 & 2.06064 \\
\hline 2408 & 43.9193 & 356.3832 & 354.9365 & 344.7083 & 122.5994 & 2.05988 \\
\hline 2409 & 43.9363 & 363.3401 & 354.6698 & 344.6667 & 122.5994 & 2.05912 \\
\hline 2410 & 43.9533 & 355.3902 & 353.9915 & 344.625 & 122.5994 & 2.05836 \\
\hline 2411 & 43.9703 & 328.2592 & 353.0916 & 344.5833 & 122.5994 & 2.05761 \\
\hline 2412 & 43.9873 & 340.027 & 352.2598 & 344.5417 & 122.5994 & 2.05685 \\
\hline 2413 & 44.0043 & 329.0815 & 351.6754 & 344.5 & 122.5994 & 2.0561 \\
\hline 2414 & 44.0213 & 341.1063 & 351.3182 & 344.4583 & 122.5994 & 2.05534 \\
\hline 2415 & 44.0383 & 352.7393 & 351.1165 & 344.4167 & 122.5994 & 2.05459 \\
\hline 2416 & 44.0553 & 332.0556 & 350.998 & 344.375 & 122.5994 & 2.05383 \\
\hline 2417 & 44.0723 & 324.1645 & 350.927 & 344.3333 & 122.5994 & 2.05308 \\
\hline 2418 & 44.0893 & 342.407 & 350.9379 & 344.2917 & 122.5994 & 2.05233 \\
\hline 2419 & 44.1063 & 364.1804 & 350.8739 & 344.25 & 122.5994 & 2.05158 \\
\hline 2420 & 44.1233 & 339.9998 & 351.0193 & 344.2083 & 122.5994 & 2.05083 \\
\hline 2421 & 44.1403 & 298.8453 & 351.0829 & 344.1667 & 122.5994 & 2.05008 \\
\hline 2422 & 44.1573 & 339.8793 & 350.9955 & 344.125 & 122.5994 & 2.04933 \\
\hline 2423 & 44.1743 & 356.0655 & 350.7672 & 344.0833 & 122.5994 & 2.04858 \\
\hline 2424 & 44.1913 & 369.312 & 350.4511 & 344.0417 & 122.5994 & 2.04783 \\
\hline 2425 & 44.2083 & 325.1695 & 350.1022 & 344 & 122.5994 & 2.04708 \\
\hline 2426 & 44.2253 & 331.2082 & 349.9149 & 343.9583 & 122.5994 & 2.04633 \\
\hline 2427 & 44.2423 & 339.3925 & 349.6056 & 343.9167 & 122.5994 & 2.04559 \\
\hline 2428 & 44.2593 & 365.8851 & 349.2766 & 343.875 & 122.5994 & 2.04484 \\
\hline 2429 & 44.2763 & 334.3716 & 348.9409 & 343.8333 & 122.5994 & 2.04409 \\
\hline 2430 & 44.2933 & 321.2947 & 348.6125 & 343.7917 & 122.5994 & 2.04335 \\
\hline 2431 & 44.3103 & 354.4788 & 348.3186 & 343.75 & 122.5994 & 2.0426 \\
\hline 2432 & 44.3273 & 358.3354 & 348.0843 & 343.7083 & 122.5994 & 2.04186 \\
\hline 2433 & 44.3443 & 358.5713 & 348.0031 & 343.6667 & 122.5994 & 2.04112 \\
\hline 2434 & 44.3613 & 353.4061 & 347.9179 & 343.625 & 122.5994 & 2.04037 \\
\hline 2435 & 44.3783 & 354.8846 & 347.8917 & 343.5833 & 122.5994 & 2.03963 \\
\hline 2436 & 44.3953 & 367.743 & 347.9159 & 343.5417 & 122.5994 & 2.03889 \\
\hline 2437 & 44.4123 & 344.8381 & 348.0074 & 343.5 & 122.5994 & 2.03815 \\
\hline 2438 & 44.4293 & 299.5427 & 348.2273 & 343.4583 & 122.5994 & 2.03741 \\
\hline 2439 & 44.4463 & 316.5109 & 348.6709 & 343.4167 & 122.5994 & 2.03667 \\
\hline 2440 & 44.4633 & 336.2492 & 349.4204 & 343.375 & 122.5994 & 2.03593 \\
\hline 2441 & 44.4803 & 342.3135 & 350.476 & 343.3333 & 122.5994 & 2.03519 \\
\hline 2442 & 44.4973 & 331.2842 & 351.6782 & 343.2917 & 122.5994 & 2.03445 \\
\hline 2443 & 44.5143 & 321.4661 & 352.6824 & 343.25 & 122.5994 & 2.03372 \\
\hline 2444 & 44.5313 & 313.3318 & 353.2976 & 343.2083 & 122.5994 & 2.03298 \\
\hline 2445 & 44.5483 & 359.3923 & 354.0987 & 343.1667 & 122.5994 & 2.03224 \\
\hline 2446 & 44.5653 & 364.0214 & 355.9685 & 343.125 & 122.5994 & 2.03151 \\
\hline 2447 & 44.5823 & 353.081 & 359.319 & 343.0833 & 122.5994 & 2.03077 \\
\hline 2448 & 44.5993 & 352.2855 & 363.8084 & 343.0417 & 122.5994 & 2.03004 \\
\hline
\end{tabular}




\begin{tabular}{|c|c|c|c|c|c|c|}
\hline 2449 & 44.6163 & 334.9 & 367.731 & 343 & 122.5994 & 2.0293 \\
\hline 2450 & 44.6333 & 337.7295 & 369.2384 & 343.5833 & 122.5994 & 2.02857 \\
\hline 2451 & 44.6503 & 342.2172 & 366.9857 & 344.1667 & 122.5994 & 2.02784 \\
\hline 2452 & 44.6673 & 367.4155 & 363.2715 & 344.75 & 122.5994 & 2.0271 \\
\hline 2453 & 44.6843 & 373.391 & 363.7284 & 345.3333 & 122.5994 & 2.02637 \\
\hline 2454 & 44.7013 & 343.2302 & 362.7125 & 345.9167 & 122.5994 & 2.02564 \\
\hline 2455 & 44.7183 & 352.7346 & 363.2805 & 346.5 & 122.5994 & 2.02491 \\
\hline 2456 & 44.7353 & 369.8266 & 364.332 & 347.0833 & 122.5994 & 2.02418 \\
\hline 2457 & 44.7523 & 362.7567 & 364.4073 & 347.6667 & 122.5994 & 2.02345 \\
\hline 2458 & 44.7693 & 341.5454 & 363.0915 & 348.25 & 122.5994 & 2.02272 \\
\hline 2459 & 44.7863 & 351.8047 & 361.3093 & 348.8333 & 122.5994 & 2.02199 \\
\hline 2460 & 44.8033 & 374.2286 & 361.4977 & 349.4167 & 122.5994 & 2.02127 \\
\hline 2461 & 44.8203 & 365.6671 & 360.754 & 350 & 122.5994 & 2.02054 \\
\hline 2462 & 44.8373 & 386.9273 & 360.7152 & 350.5833 & 122.5994 & 2.01981 \\
\hline 2463 & 44.8543 & 388.0528 & 361.1928 & 351.1667 & 122.5994 & 2.01909 \\
\hline 2464 & 44.8713 & 376.4748 & 362.0655 & 351.75 & 122.5994 & 2.01836 \\
\hline 2465 & 44.8883 & 384.6631 & 363.206 & 352.3333 & 122.5994 & 2.01764 \\
\hline 2466 & 44.9053 & 410.707 & 364.5212 & 352.9167 & 122.5994 & 2.01691 \\
\hline 2467 & 44.9223 & 376.1809 & 365.9526 & 353.5 & 122.5994 & 2.01619 \\
\hline 2468 & 44.9393 & 373.0803 & 367.485 & 354.0833 & 122.5994 & 2.01547 \\
\hline 2469 & 44.9563 & 382.6392 & 369.318 & 354.6667 & 122.5994 & 2.01474 \\
\hline 2470 & 44.9733 & 401.6076 & 371.1956 & 355.25 & 122.5994 & 2.01402 \\
\hline 2471 & 44.9903 & 386.4225 & 372.8532 & 355.8333 & 122.5994 & 2.0133 \\
\hline 2472 & 45.0073 & 398.7255 & 374.0305 & 356.4167 & 122.5994 & 2.01258 \\
\hline 2473 & 45.0243 & 399.6446 & 374.943 & 357 & 122.5994 & 2.01186 \\
\hline 2474 & 45.0413 & 424.0866 & 375.5481 & 357.1563 & 122.5994 & 2.01114 \\
\hline 2475 & 45.0583 & 394.8609 & 376.4917 & 357.3125 & 122.5994 & 2.01042 \\
\hline 2476 & 45.0753 & 403.9712 & 377.8878 & 357.4688 & 122.5994 & 2.0097 \\
\hline 2477 & 45.0923 & 399.6959 & 379.6179 & 357.625 & 122.5994 & 2.00898 \\
\hline 2478 & 45.1093 & 392.7618 & 381.4656 & 357.7813 & 122.5994 & 2.00826 \\
\hline 2479 & 45.1263 & 402.7909 & 383.3512 & 357.9375 & 122.5994 & 2.00755 \\
\hline 2480 & 45.1433 & 416.7149 & 385.4597 & 358.0938 & 122.5994 & 2.00683 \\
\hline 2481 & 45.1603 & 438.8803 & 387.9826 & 358.25 & 122.5994 & 2.00611 \\
\hline 2482 & 45.1773 & 396.2291 & 392.0748 & 358.4063 & 122.5994 & 2.0054 \\
\hline 2483 & 45.1943 & 369.6312 & 395.5654 & 358.5625 & 122.5994 & 2.00468 \\
\hline 2484 & 45.2113 & 400.0715 & 399.9531 & 358.7188 & 122.5994 & 2.00397 \\
\hline 2485 & 45.2283 & 430.977 & 405.8478 & 358.875 & 122.5994 & 2.00326 \\
\hline 2486 & 45.2453 & 422.6326 & 413.9917 & 359.0313 & 122.5994 & 2.00254 \\
\hline 2487 & 45.2623 & 393.9457 & 425.1862 & 359.1875 & 122.5994 & 2.00183 \\
\hline 2488 & 45.2793 & 408.3565 & 440.1932 & 359.3438 & 122.5994 & 2.00112 \\
\hline 2489 & 45.2963 & 426.9045 & 459.6506 & 359.5 & 122.5994 & 2.00041 \\
\hline 2490 & 45.3133 & 434.9086 & 483.6304 & 359.6563 & 122.5994 & 1.9997 \\
\hline 2491 & 45.3303 & 451.5123 & 513.0259 & 359.8125 & 122.5994 & 1.99899 \\
\hline 2492 & 45.3473 & 459.6443 & 548.6788 & 359.9688 & 122.5994 & 1.99828 \\
\hline 2493 & 45.3643 & 474.0211 & 591.1051 & 360.125 & 122.5994 & 1.99757 \\
\hline 2494 & 45.3813 & 443.2568 & 640.3308 & 360.2813 & 122.5994 & 1.99686 \\
\hline 2495 & 45.3983 & 498.265 & 695.3226 & 360.4375 & 122.5994 & 1.99615 \\
\hline 2496 & 45.4153 & 538.3117 & 753.185 & 360.5938 & 122.5994 & 1.99544 \\
\hline 2497 & 45.4323 & 585.341 & 808.8232 & 360.75 & 122.5994 & 1.99473 \\
\hline
\end{tabular}




\begin{tabular}{|c|c|c|c|c|c|c|}
\hline 2498 & 45.4493 & 587.5562 & 854.8105 & 360.4792 & 122.5994 & 1.99403 \\
\hline 2499 & 45.4663 & 606.3474 & 884.7422 & 360.2083 & 122.5994 & 1.99332 \\
\hline 2500 & 45.4833 & 661.8655 & 895.046 & 359.9375 & 122.5994 & 1.99262 \\
\hline 2501 & 45.5003 & 709.4915 & 887.9259 & 359.6667 & 122.5994 & 1.99191 \\
\hline 2502 & 45.5173 & 744.5829 & 869.3726 & 359.3958 & 122.5994 & 1.99121 \\
\hline 2503 & 45.5343 & 671.3135 & 845.1544 & 359.125 & 122.5994 & 1.9905 \\
\hline 2504 & 45.5513 & 681.8781 & 818.4159 & 358.8542 & 122.5994 & 1.9898 \\
\hline 2505 & 45.5683 & 677.2218 & 789.4057 & 358.5833 & 122.5994 & 1.9891 \\
\hline 2506 & 45.5853 & 639.2953 & 756.7073 & 358.3125 & 122.5994 & 1.98839 \\
\hline 2507 & 45.6023 & 607.4975 & 719.3976 & 358.0417 & 122.5994 & 1.98769 \\
\hline 2508 & 45.6193 & 609.8817 & 678.8259 & 357.7708 & 122.5994 & 1.98699 \\
\hline 2509 & 45.6363 & 568.0052 & 638.1765 & 357.5 & 122.5994 & 1.98629 \\
\hline 2510 & 45.6533 & 552.5461 & 600.886 & 357.2292 & 122.5994 & 1.98559 \\
\hline 2511 & 45.6703 & 538.0296 & 569.3173 & 356.9583 & 122.5994 & 1.98489 \\
\hline 2512 & 45.6873 & 509.4977 & 544.4001 & 356.6875 & 122.5994 & 1.98419 \\
\hline 2513 & 45.7043 & 463.2681 & 525.6265 & 356.4167 & 122.5994 & 1.98349 \\
\hline 2514 & 45.7213 & 485.633 & 511.9711 & 356.1458 & 122.5994 & 1.9828 \\
\hline 2515 & 45.7383 & 502.1571 & 505.1385 & 355.875 & 122.5994 & 1.9821 \\
\hline 2516 & 45.7553 & 467.7036 & 510.158 & 355.6042 & 122.5994 & 1.9814 \\
\hline 2517 & 45.7723 & 516.4287 & 531.9046 & 355.3333 & 122.5994 & 1.9807 \\
\hline 2518 & 45.7893 & 565.1459 & 570.7587 & 355.0625 & 122.5994 & 1.98001 \\
\hline 2519 & 45.8063 & 636.9266 & 618.322 & 354.7917 & 122.5994 & 1.97931 \\
\hline 2520 & 45.8233 & 666.1628 & 653.6884 & 354.5208 & 122.5994 & 1.97862 \\
\hline 2521 & 45.8403 & 754.5153 & 652.1184 & 354.25 & 122.5994 & 1.97792 \\
\hline 2522 & 45.8573 & 741.3125 & 615.1454 & 353.9792 & 122.5994 & 1.97723 \\
\hline 2523 & 45.8743 & 625.7177 & 569.6108 & 353.7083 & 122.5994 & 1.97654 \\
\hline 2524 & 45.8913 & 521.2903 & 536.8448 & 353.4375 & 122.5994 & 1.97585 \\
\hline 2525 & 45.9083 & 507.2631 & 524.2944 & 353.1667 & 122.5994 & 1.97515 \\
\hline 2526 & 45.9253 & 527.4855 & 528.0237 & 352.8958 & 122.5994 & 1.97446 \\
\hline 2527 & 45.9423 & 537.9749 & 534.9747 & 352.625 & 122.5994 & 1.97377 \\
\hline 2528 & 45.9593 & 527.3642 & 529.0054 & 352.3542 & 122.5994 & 1.97308 \\
\hline 2529 & 45.9763 & 527.9769 & 506.3921 & 352.0833 & 122.5994 & 1.97239 \\
\hline 2530 & 45.9933 & 514.8191 & 477.1637 & 351.8125 & 122.5994 & 1.9717 \\
\hline 2531 & 46.0103 & 471.4746 & 450.1556 & 351.5417 & 122.5994 & 1.97101 \\
\hline 2532 & 46.0273 & 457.2321 & 428.7672 & 351.2708 & 122.5994 & 1.97032 \\
\hline 2533 & 46.0443 & 448.3746 & 413.2737 & 351 & 122.5994 & 1.96964 \\
\hline 2534 & 46.0613 & 398.4798 & 402.5381 & 350.7292 & 122.5994 & 1.96895 \\
\hline 2535 & 46.0783 & 391.2923 & 395.1197 & 350.4583 & 122.5994 & 1.96826 \\
\hline 2536 & 46.0953 & 382.3008 & 389.8554 & 350.1875 & 122.5994 & 1.96757 \\
\hline 2537 & 46.1123 & 357.1757 & 385.7478 & 349.9167 & 122.5994 & 1.96689 \\
\hline 2538 & 46.1293 & 363.9656 & 382.2563 & 349.6458 & 122.5994 & 1.9662 \\
\hline 2539 & 46.1463 & 366.2749 & 379.0323 & 349.375 & 122.5994 & 1.96552 \\
\hline 2540 & 46.1633 & 360.071 & 376.022 & 349.1042 & 122.5994 & 1.96483 \\
\hline 2541 & 46.1803 & 379.8628 & 373.2648 & 348.8333 & 122.5994 & 1.96415 \\
\hline 2542 & 46.1973 & 384.0702 & 370.6703 & 348.5625 & 122.5994 & 1.96347 \\
\hline 2543 & 46.2143 & 383.0159 & 368.1265 & 348.2917 & 122.5994 & 1.96278 \\
\hline 2544 & 46.2313 & 351.4801 & 365.736 & 348.0208 & 122.5994 & 1.9621 \\
\hline 2545 & 46.2483 & 352.3596 & 363.8762 & 347.75 & 122.5994 & 1.96142 \\
\hline 2546 & 46.2653 & 357.1757 & 359.1437 & 347.0521 & 122.5994 & 1.96074 \\
\hline
\end{tabular}




\begin{tabular}{|c|c|c|c|c|c|c|}
\hline 2547 & 46.2823 & 360.1333 & 358.5966 & 346.3542 & 122.5994 & 1.96006 \\
\hline 2548 & 46.2993 & 337.1807 & 358.7701 & 345.6563 & 122.5994 & 1.95938 \\
\hline 2549 & 46.3163 & 334.2137 & 359.257 & 344.9583 & 122.5994 & 1.9587 \\
\hline 2550 & 46.3333 & 334.9346 & 359.3134 & 344.2604 & 122.5994 & 1.95802 \\
\hline 2551 & 46.3503 & 325.4218 & 358.1938 & 343.5625 & 122.5994 & 1.95734 \\
\hline 2552 & 46.3673 & 320.8548 & 355.9132 & 342.8646 & 122.5994 & 1.95666 \\
\hline 2553 & 46.3843 & 343.6184 & 351.7208 & 342.1667 & 122.5994 & 1.95599 \\
\hline 2554 & 46.4013 & 342.4729 & 349.6035 & 341.4688 & 122.5994 & 1.95531 \\
\hline 2555 & 46.4183 & 314.7076 & 348.1463 & 340.7708 & 122.5994 & 1.95463 \\
\hline 2556 & 46.4353 & 356.0401 & 347.3376 & 340.0729 & 122.5994 & 1.95396 \\
\hline 2557 & 46.4523 & 299.5869 & 346.7461 & 339.375 & 122.5994 & 1.95328 \\
\hline 2558 & 46.4693 & 313.7529 & 345.9824 & 338.6771 & 122.5994 & 1.95261 \\
\hline 2559 & 46.4863 & 342.1529 & 344.7474 & 337.9792 & 122.5994 & 1.95193 \\
\hline 2560 & 46.5033 & 337.8695 & 343.3172 & 337.2813 & 122.5994 & 1.95126 \\
\hline 2561 & 46.5203 & 330.0732 & 342.0528 & 336.5833 & 122.5994 & 1.95058 \\
\hline 2562 & 46.5373 & 357.5667 & 340.0639 & 335.8854 & 122.5994 & 1.94991 \\
\hline 2563 & 46.5543 & 355.0934 & 339.6275 & 335.1875 & 122.5994 & 1.94924 \\
\hline 2564 & 46.5713 & 311.7201 & 339.5237 & 334.4896 & 122.5994 & 1.94857 \\
\hline 2565 & 46.5883 & 307.5057 & 339.5446 & 333.7917 & 122.5994 & 1.9479 \\
\hline 2566 & 46.6053 & 324.5829 & 339.441 & 333.0938 & 122.5994 & 1.94722 \\
\hline 2567 & 46.6223 & 302.5062 & 338.7405 & 332.3958 & 122.5994 & 1.94655 \\
\hline 2568 & 46.6393 & 325.9806 & 337.6755 & 331.6979 & 122.5994 & 1.94588 \\
\hline 2569 & 46.6563 & 330.6806 & 336.5959 & 331 & 122.5994 & 1.94521 \\
\hline 2570 & 46.6733 & 352.7751 & 336.8903 & 331.4583 & 122.5994 & 1.94455 \\
\hline 2571 & 46.6903 & 324.9076 & 337.4904 & 331.9167 & 122.5994 & 1.94388 \\
\hline 2572 & 46.7073 & 313.12 & 338.5413 & 332.375 & 122.5994 & 1.94321 \\
\hline 2573 & 46.7243 & 321.6731 & 339.522 & 332.8333 & 122.5994 & 1.94254 \\
\hline 2574 & 46.7413 & 318.8233 & 340.4291 & 333.2917 & 122.5994 & 1.94188 \\
\hline 2575 & 46.7583 & 327.9747 & 341.1402 & 333.75 & 122.5994 & 1.94121 \\
\hline 2576 & 46.7753 & 343.7449 & 342.0557 & 334.2083 & 122.5994 & 1.94054 \\
\hline 2577 & 46.7923 & 311.9772 & 343.1761 & 334.6667 & 122.5994 & 1.93988 \\
\hline 2578 & 46.8093 & 308.4808 & 344.4835 & 335.125 & 122.5994 & 1.93921 \\
\hline 2579 & 46.8263 & 337.7737 & 345.9189 & 335.5833 & 122.5994 & 1.93855 \\
\hline 2580 & 46.8433 & 337.2839 & 347.3668 & 336.0417 & 122.5994 & 1.93788 \\
\hline 2581 & 46.8603 & 311.3428 & 348.6855 & 336.5 & 122.5994 & 1.93722 \\
\hline 2582 & 46.8773 & 316.0611 & 349.6964 & 336.9583 & 122.5994 & 1.93656 \\
\hline 2583 & 46.8943 & 348.2265 & 350.5189 & 337.4167 & 122.5994 & 1.9359 \\
\hline 2584 & 46.9113 & 337.1217 & 351.3073 & 337.875 & 122.5994 & 1.93523 \\
\hline 2585 & 46.9283 & 325.4952 & 352.3721 & 338.3333 & 122.5994 & 1.93457 \\
\hline 2586 & 46.9453 & 313.5637 & 354.0471 & 338.7917 & 122.5994 & 1.93391 \\
\hline 2587 & 46.9623 & 305.0381 & 356.4756 & 339.25 & 122.5994 & 1.93325 \\
\hline 2588 & 46.9793 & 330.0746 & 359.4012 & 339.7083 & 122.5994 & 1.93259 \\
\hline 2589 & 46.9963 & 327.2779 & 361.9505 & 340.1667 & 122.5994 & 1.93193 \\
\hline 2590 & 47.0133 & 305.5487 & 362.6896 & 340.625 & 122.5994 & 1.93127 \\
\hline 2591 & 47.0303 & 322.4922 & 361.2532 & 341.0833 & 122.5994 & 1.93061 \\
\hline 2592 & 47.0473 & 339.5279 & 359.0512 & 341.5417 & 122.5994 & 1.92996 \\
\hline 2593 & 47.0643 & 362.542 & 357.6683 & 342 & 122.5994 & 1.9293 \\
\hline 2594 & 47.0813 & 330.4996 & 357.5105 & 342 & 122.5994 & 1.92864 \\
\hline 2595 & 47.0983 & 335.1396 & 359.1654 & 342 & 122.5994 & 1.92799 \\
\hline
\end{tabular}




\begin{tabular}{|c|c|c|c|c|c|c|}
\hline 2596 & 47.1153 & 360.4374 & 361.9542 & 342 & 122.5994 & 1.92733 \\
\hline 2597 & 47.1323 & 320.7415 & 364.3249 & 342 & 122.5994 & 1.92667 \\
\hline 2598 & 47.1493 & 332.2189 & 364.381 & 342 & 122.5994 & 1.92602 \\
\hline 2599 & 47.1663 & 334.9346 & 361.7939 & 342 & 122.5994 & 1.92536 \\
\hline 2600 & 47.1833 & 314.9686 & 358.2472 & 342 & 122.5994 & 1.92471 \\
\hline 2601 & 47.2003 & 326.7526 & 355.4802 & 342 & 122.5994 & 1.92406 \\
\hline 2602 & 47.2173 & 331.1525 & 354.3003 & 342 & 122.5994 & 1.9234 \\
\hline 2603 & 47.2343 & 303.1471 & 354.7057 & 342 & 122.5994 & 1.92275 \\
\hline 2604 & 47.2513 & 331.2836 & 356.075 & 342 & 122.5994 & 1.9221 \\
\hline 2605 & 47.2683 & 341.3698 & 357.0438 & 342 & 122.5994 & 1.92145 \\
\hline 2606 & 47.2853 & 310.8025 & 356.2825 & 342 & 122.5994 & 1.9208 \\
\hline 2607 & 47.3023 & 331.4686 & 353.9788 & 342 & 122.5994 & 1.92014 \\
\hline 2608 & 47.3193 & 335.619 & 351.4501 & 342 & 122.5994 & 1.91949 \\
\hline 2609 & 47.3363 & 342.2172 & 349.3638 & 342 & 122.5994 & 1.91885 \\
\hline 2610 & 47.3533 & 343.3079 & 348.1133 & 342 & 122.5994 & 1.9182 \\
\hline 2611 & 47.3703 & 342.2661 & 347.6102 & 342 & 122.5994 & 1.91755 \\
\hline 2612 & 47.3873 & 308.4789 & 347.4874 & 342 & 122.5994 & 1.9169 \\
\hline 2613 & 47.4043 & 332.7507 & 347.246 & 342 & 122.5994 & 1.91625 \\
\hline 2614 & 47.4213 & 329.6712 & 346.6274 & 342 & 122.5994 & 1.9156 \\
\hline 2615 & 47.4383 & 351.5686 & 345.9789 & 342 & 122.5994 & 1.91496 \\
\hline 2616 & 47.4553 & 358.1802 & 345.2528 & 342 & 122.5994 & 1.91431 \\
\hline 2617 & 47.4723 & 359.9616 & 344.6995 & 342 & 122.5994 & 1.91366 \\
\hline 2618 & 47.4893 & 363.076 & 344.3344 & 342 & 122.5994 & 1.91302 \\
\hline 2619 & 47.5063 & 362.6585 & 344.1209 & 342 & 122.5994 & 1.91237 \\
\hline 2620 & 47.5233 & 346.7868 & 344.0117 & 342 & 122.5994 & 1.91173 \\
\hline 2621 & 47.5403 & 356.0295 & 343.9667 & 342 & 122.5994 & 1.91109 \\
\hline 2622 & 47.5573 & 348.2404 & 343.9531 & 342 & 122.5994 & 1.91044 \\
\hline 2623 & 47.5743 & 359.1773 & 343.9722 & 342 & 122.5994 & 1.9098 \\
\hline 2624 & 47.5913 & 364.4302 & 344.0255 & 342 & 122.5994 & 1.90916 \\
\hline 2625 & 47.6083 & 388.0528 & 344.1251 & 342 & 122.5994 & 1.90851 \\
\hline 2626 & 47.6253 & 367.8238 & 344.2758 & 342 & 122.5994 & 1.90787 \\
\hline 2627 & 47.6423 & 361.261 & 344.4827 & 342 & 122.5994 & 1.90723 \\
\hline 2628 & 47.6593 & 364.3316 & 344.7621 & 342 & 122.5994 & 1.90659 \\
\hline 2629 & 47.6763 & 361.7692 & 345.0567 & 342 & 122.5994 & 1.90595 \\
\hline 2630 & 47.6933 & 366.4357 & 345.5664 & 342 & 122.5994 & 1.90531 \\
\hline 2631 & 47.7103 & 367.026 & 345.9797 & 342 & 122.5994 & 1.90467 \\
\hline 2632 & 47.7273 & 359.4268 & 346.3226 & 342 & 122.5994 & 1.90403 \\
\hline 2633 & 47.7443 & 355.7842 & 346.5903 & 342 & 122.5994 & 1.9034 \\
\hline 2634 & 47.7613 & 367.2268 & 346.8063 & 342 & 122.5994 & 1.90276 \\
\hline 2635 & 47.7783 & 349.5903 & 346.9862 & 342 & 122.5994 & 1.90212 \\
\hline 2636 & 47.7953 & 341.1715 & 346.9711 & 342 & 122.5994 & 1.90148 \\
\hline 2637 & 47.8123 & 350.2396 & 347.1422 & 342 & 122.5994 & 1.90085 \\
\hline 2638 & 47.8293 & 353.4061 & 347.3502 & 342 & 122.5994 & 1.90021 \\
\hline 2639 & 47.8463 & 333.8564 & 347.6015 & 342 & 122.5994 & 1.89958 \\
\hline 2640 & 47.8633 & 344.8322 & 347.9431 & 342 & 122.5994 & 1.89894 \\
\hline 2641 & 47.8803 & 358.8193 & 348.482 & 342 & 122.5994 & 1.89831 \\
\hline 2642 & 47.8973 & 315.8248 & 349.0882 & 341.6667 & 122.5994 & 1.89767 \\
\hline 2643 & 47.9143 & 355.7569 & 350.4694 & 341.3333 & 122.5994 & 1.89704 \\
\hline 2644 & 47.9313 & 326.3391 & 353.1478 & 341 & 122.5994 & 1.89641 \\
\hline
\end{tabular}




\begin{tabular}{|c|c|c|c|c|c|c|}
\hline 2645 & 47.9483 & 326.7799 & 357.5734 & 340.6667 & 122.5994 & 1.89577 \\
\hline 2646 & 47.9653 & 321.0972 & 363.7469 & 340.3333 & 122.5994 & 1.89514 \\
\hline 2647 & 47.9823 & 333.8753 & 370.692 & 340 & 122.5994 & 1.89451 \\
\hline 2648 & 47.9993 & 356.2211 & 375.7817 & 339.6667 & 122.5994 & 1.89388 \\
\hline 2649 & 48.0163 & 338.9898 & 375.9953 & 339.3333 & 122.5994 & 1.89325 \\
\hline 2650 & 48.0333 & 360.8898 & 371.8182 & 339 & 122.5994 & 1.89262 \\
\hline 2651 & 48.0503 & 320.7076 & 366.9468 & 338.6667 & 122.5994 & 1.89199 \\
\hline 2652 & 48.0673 & 339.0474 & 364.4601 & 338.3333 & 122.5994 & 1.89136 \\
\hline 2653 & 48.0843 & 347.8657 & 365.9534 & 338 & 122.5994 & 1.89073 \\
\hline 2654 & 48.1013 & 322.1828 & 371.5974 & 337.6667 & 122.5994 & 1.8901 \\
\hline 2655 & 48.1183 & 343.462 & 380.1756 & 337.3333 & 122.5994 & 1.88947 \\
\hline 2656 & 48.1353 & 353.4061 & 388.5938 & 337 & 122.5994 & 1.88884 \\
\hline 2657 & 48.1523 & 349.6824 & 392.9493 & 336.6667 & 122.5994 & 1.88822 \\
\hline 2658 & 48.1693 & 333.2121 & 390.4327 & 336.3333 & 122.5994 & 1.88759 \\
\hline 2659 & 48.1863 & 359.6089 & 381.8503 & 336 & 122.5994 & 1.88696 \\
\hline 2660 & 48.2033 & 353.4061 & 371.7258 & 335.6667 & 122.5994 & 1.88634 \\
\hline 2661 & 48.2203 & 360.5703 & 363.9218 & 335.3333 & 122.5994 & 1.88571 \\
\hline 2662 & 48.2373 & 367.054 & 359.854 & 335 & 122.5994 & 1.88509 \\
\hline 2663 & 48.2543 & 321.7965 & 359.2068 & 334.6667 & 122.5994 & 1.88446 \\
\hline 2664 & 48.2713 & 332.7657 & 360.2967 & 334.3333 & 122.5994 & 1.88384 \\
\hline 2665 & 48.2883 & 331.0755 & 360.5273 & 334 & 122.5994 & 1.88322 \\
\hline 2666 & 48.3053 & 343.6792 & 358.1238 & 333.7292 & 122.5994 & 1.88259 \\
\hline 2667 & 48.3223 & 345.3604 & 353.8528 & 333.4583 & 122.5994 & 1.88197 \\
\hline 2668 & 48.3393 & 341.1766 & 349.7354 & 333.1875 & 122.5994 & 1.88135 \\
\hline 2669 & 48.3563 & 357.2576 & 346.9653 & 332.9167 & 122.5994 & 1.88073 \\
\hline 2670 & 48.3733 & 349.2139 & 345.7189 & 332.6458 & 122.5994 & 1.8801 \\
\hline 2671 & 48.3903 & 352.0978 & 345.3722 & 332.375 & 122.5994 & 1.87948 \\
\hline 2672 & 48.4073 & 357.9455 & 344.802 & 332.1042 & 122.5994 & 1.87886 \\
\hline 2673 & 48.4243 & 374.4526 & 343.3574 & 331.8333 & 122.5994 & 1.87824 \\
\hline 2674 & 48.4413 & 391.8738 & 341.5616 & 331.5625 & 122.5994 & 1.87762 \\
\hline 2675 & 48.4583 & 367.8993 & 340.1003 & 331.2917 & 122.5994 & 1.87701 \\
\hline 2676 & 48.4753 & 361.4841 & 339.1407 & 331.0208 & 122.5994 & 1.87639 \\
\hline 2677 & 48.4923 & 351.7457 & 338.6054 & 330.75 & 122.5994 & 1.87577 \\
\hline 2678 & 48.5093 & 335.6985 & 338.2489 & 330.4792 & 122.5994 & 1.87515 \\
\hline 2679 & 48.5263 & 352.0421 & 337.8126 & 330.2083 & 122.5994 & 1.87453 \\
\hline 2680 & 48.5433 & 357.4751 & 336.9978 & 329.9375 & 122.5994 & 1.87392 \\
\hline 2681 & 48.5603 & 368.6987 & 335.853 & 329.6667 & 122.5994 & 1.8733 \\
\hline 2682 & 48.5773 & 373.6726 & 334.7531 & 329.3958 & 122.5994 & 1.87269 \\
\hline 2683 & 48.5943 & 351.1675 & 333.8812 & 329.125 & 122.5994 & 1.87207 \\
\hline 2684 & 48.6113 & 367.0551 & 333.2364 & 328.8542 & 122.5994 & 1.87145 \\
\hline 2685 & 48.6283 & 326.4011 & 332.7133 & 328.5833 & 122.5994 & 1.87084 \\
\hline 2686 & 48.6453 & 340.5969 & 332.2864 & 328.3125 & 122.5994 & 1.87023 \\
\hline 2687 & 48.6623 & 355.6173 & 331.993 & 328.0417 & 122.5994 & 1.86961 \\
\hline 2688 & 48.6793 & 363.4464 & 331.8579 & 327.7708 & 122.5994 & 1.869 \\
\hline 2689 & 48.6963 & 358.4235 & 331.7913 & 327.5 & 122.5994 & 1.86839 \\
\hline 2690 & 48.7133 & 357.2576 & 331.9721 & 327.2292 & 122.5994 & 1.86777 \\
\hline 2691 & 48.7303 & 340.7861 & 332.2892 & 326.9583 & 122.5994 & 1.86716 \\
\hline 2692 & 48.7473 & 328.6701 & 332.7486 & 326.6875 & 122.5994 & 1.86655 \\
\hline 2693 & 48.7643 & 314.4525 & 333.4004 & 326.4167 & 122.5994 & 1.86594 \\
\hline
\end{tabular}




\begin{tabular}{|c|c|c|c|c|c|c|}
\hline 2694 & 48.7813 & 365.4711 & 334.342 & 326.1458 & 122.5994 & 1.86533 \\
\hline 2695 & 48.7983 & 308.4865 & 335.6706 & 325.875 & 122.5994 & 1.86472 \\
\hline 2696 & 48.8153 & 334.47 & 337.3692 & 325.6042 & 122.5994 & 1.86411 \\
\hline 2697 & 48.8323 & 341.9283 & 339.1418 & 325.3333 & 122.5994 & 1.8635 \\
\hline 2698 & 48.8493 & 297.7231 & 340.2747 & 325.0625 & 122.5994 & 1.86289 \\
\hline 2699 & 48.8663 & 361.4376 & 340.0879 & 324.7917 & 122.5994 & 1.86228 \\
\hline 2700 & 48.8833 & 369.4453 & 338.8645 & 324.5208 & 122.5994 & 1.86168 \\
\hline 2701 & 48.9003 & 370.7674 & 337.3112 & 324.25 & 122.5994 & 1.86107 \\
\hline 2702 & 48.9173 & 286.1797 & 336.2858 & 323.9792 & 122.5994 & 1.86046 \\
\hline 2703 & 48.9343 & 338.2527 & 335.8076 & 323.7083 & 122.5994 & 1.85985 \\
\hline 2704 & 48.9513 & 314.4606 & 335.6718 & 323.4375 & 122.5994 & 1.85925 \\
\hline 2705 & 48.9683 & 324.3097 & 335.6021 & 323.1667 & 122.5994 & 1.85864 \\
\hline 2706 & 48.9853 & 339.344 & 335.4265 & 322.8958 & 122.5994 & 1.85804 \\
\hline 2707 & 49.0023 & 330.0208 & 335.2083 & 322.625 & 122.5994 & 1.85743 \\
\hline 2708 & 49.0193 & 343.1685 & 335.2532 & 322.3542 & 122.5994 & 1.85683 \\
\hline 2709 & 49.0363 & 354.1821 & 335.8759 & 322.0833 & 122.5994 & 1.85622 \\
\hline 2710 & 49.0533 & 345.9268 & 336.5403 & 321.8125 & 122.5994 & 1.85562 \\
\hline 2711 & 49.0703 & 346.0061 & 336.3492 & 321.5417 & 122.5994 & 1.85502 \\
\hline 2712 & 49.0873 & 342.2671 & 334.8224 & 321.2708 & 122.5994 & 1.85441 \\
\hline 2713 & 49.1043 & 321.1795 & 332.9025 & 321 & 122.5994 & 1.85381 \\
\hline 2714 & 49.1213 & 298.3974 & 332.2912 & 321.3333 & 122.5994 & 1.85321 \\
\hline 2715 & 49.1383 & 309.7042 & 333.1213 & 321.6667 & 122.5994 & 1.85261 \\
\hline 2716 & 49.1553 & 338.9557 & 335.8359 & 322 & 122.5994 & 1.85201 \\
\hline 2717 & 49.1723 & 354.8902 & 340.5187 & 322.3333 & 122.5994 & 1.85141 \\
\hline 2718 & 49.1893 & 332.2535 & 346.6498 & 322.6667 & 122.5994 & 1.85081 \\
\hline 2719 & 49.2063 & 330.8892 & 352.9161 & 323 & 122.5994 & 1.85021 \\
\hline 2720 & 49.2233 & 334.9335 & 357.4069 & 323.3333 & 122.5994 & 1.84961 \\
\hline 2721 & 49.2403 & 341.1211 & 357.2563 & 323.6667 & 122.5994 & 1.84901 \\
\hline 2722 & 49.2573 & 339.3103 & 353.015 & 324 & 122.5994 & 1.84841 \\
\hline 2723 & 49.2743 & 344.4477 & 347.7048 & 324.3333 & 122.5994 & 1.84781 \\
\hline 2724 & 49.2913 & 363.9056 & 343.8309 & 324.6667 & 122.5994 & 1.84722 \\
\hline 2725 & 49.3083 & 343.643 & 342.3834 & 325 & 122.5994 & 1.84662 \\
\hline 2726 & 49.3253 & 358.3815 & 343.2321 & 325.3333 & 122.5994 & 1.84602 \\
\hline 2727 & 49.3423 & 363.8137 & 345.3297 & 325.6667 & 122.5994 & 1.84543 \\
\hline 2728 & 49.3593 & 322.8634 & 346.9846 & 326 & 122.5994 & 1.84483 \\
\hline 2729 & 49.3763 & 331.2891 & 346.5677 & 326.3333 & 122.5994 & 1.84424 \\
\hline 2730 & 49.3933 & 326.6146 & 344.7777 & 326.6667 & 122.5994 & 1.84364 \\
\hline 2731 & 49.4103 & 320.3793 & 343.1255 & 327 & 122.5994 & 1.84305 \\
\hline 2732 & 49.4273 & 334.266 & 342.3846 & 327.3333 & 122.5994 & 1.84245 \\
\hline 2733 & 49.4443 & 324.3254 & 342.5078 & 327.6667 & 122.5994 & 1.84186 \\
\hline 2734 & 49.4613 & 323.2653 & 342.7644 & 328 & 122.5994 & 1.84126 \\
\hline 2735 & 49.4783 & 315.2015 & 342.1455 & 328.3333 & 122.5994 & 1.84067 \\
\hline 2736 & 49.4953 & 319.2904 & 340.5862 & 328.6667 & 122.5994 & 1.84008 \\
\hline 2737 & 49.5123 & 339.4235 & 338.926 & 329 & 122.5994 & 1.83949 \\
\hline 2738 & 49.5293 & 338.9329 & 337.7977 & 329.2839 & 122.5994 & 1.8389 \\
\hline 2739 & 49.5463 & 318.7305 & 337.4729 & 329.5677 & 122.5994 & 1.8383 \\
\hline 2740 & 49.5633 & 323.5633 & 337.7927 & 329.8516 & 122.5994 & 1.83771 \\
\hline 2741 & 49.5803 & 334.3638 & 338.5138 & 330.1354 & 122.5994 & 1.83712 \\
\hline 2742 & 49.5973 & 290.1375 & 339.109 & 330.4193 & 122.5994 & 1.83653 \\
\hline
\end{tabular}




\begin{tabular}{|c|c|c|c|c|c|c|}
\hline 2743 & 49.6143 & 364.6008 & 339.2397 & 330.7031 & 122.5994 & 1.83594 \\
\hline 2744 & 49.6313 & 321.0843 & 339.1861 & 330.987 & 122.5994 & 1.83536 \\
\hline 2745 & 49.6483 & 355.1078 & 339.4467 & 331.2708 & 122.5994 & 1.83477 \\
\hline 2746 & 49.6653 & 309.462 & 340.2266 & 331.5547 & 122.5994 & 1.83418 \\
\hline 2747 & 49.6823 & 354.0974 & 341.4696 & 331.8385 & 122.5994 & 1.83359 \\
\hline 2748 & 49.6993 & 323.5359 & 342.8297 & 332.1224 & 122.5994 & 1.833 \\
\hline 2749 & 49.7163 & 345.9461 & 344.3737 & 332.4063 & 122.5994 & 1.83242 \\
\hline 2750 & 49.7333 & 323.6931 & 346.7359 & 332.6901 & 122.5994 & 1.83183 \\
\hline 2751 & 49.7503 & 342.8471 & 350.5856 & 332.974 & 122.5994 & 1.83124 \\
\hline 2752 & 49.7673 & 352.4834 & 355.9497 & 333.2578 & 122.5994 & 1.83066 \\
\hline 2753 & 49.7843 & 372.212 & 370.9558 & 333.5417 & 122.5994 & 1.83007 \\
\hline 2754 & 49.8013 & 343.9602 & 376.3154 & 333.8255 & 122.5994 & 1.82949 \\
\hline 2755 & 49.8183 & 347.8325 & 379.2404 & 334.1094 & 122.5994 & 1.8289 \\
\hline 2756 & 49.8353 & 352.7564 & 379.5037 & 334.3932 & 122.5994 & 1.82832 \\
\hline 2757 & 49.8523 & 369.9687 & 378.3206 & 334.6771 & 122.5994 & 1.82774 \\
\hline 2758 & 49.8693 & 349.6108 & 378.0173 & 334.9609 & 122.5994 & 1.82715 \\
\hline 2759 & 49.8863 & 361.083 & 380.8123 & 335.2448 & 122.5994 & 1.82657 \\
\hline 2760 & 49.9033 & 371.4953 & 387.355 & 335.5286 & 122.5994 & 1.82599 \\
\hline 2761 & 49.9203 & 377.8212 & 401.1609 & 335.8125 & 122.5994 & 1.82541 \\
\hline 2762 & 49.9373 & 401.6003 & 410.544 & 335.6986 & 122.5994 & 1.82482 \\
\hline 2763 & 49.9543 & 411.8501 & 417.4272 & 335.5846 & 122.5994 & 1.82424 \\
\hline 2764 & 49.9713 & 389.4485 & 421.2242 & 335.4707 & 122.5994 & 1.82366 \\
\hline 2765 & 49.9883 & 386.8067 & 423.1617 & 335.3568 & 122.5994 & 1.82308 \\
\hline 2766 & 50.0053 & 408.1571 & 425.0226 & 335.2428 & 122.5994 & 1.8225 \\
\hline 2767 & 50.0223 & 432.5038 & 429.7063 & 335.1289 & 122.5994 & 1.82192 \\
\hline 2768 & 50.0393 & 442.3134 & 440.9843 & 335.015 & 122.5994 & 1.82134 \\
\hline 2769 & 50.0563 & 500.0501 & 462.9969 & 334.901 & 122.5994 & 1.82076 \\
\hline 2770 & 50.0733 & 536.3601 & 502.1799 & 334.7871 & 122.5994 & 1.82019 \\
\hline 2771 & 50.0903 & 599.3014 & 569.6161 & 334.6732 & 122.5994 & 1.81961 \\
\hline 2772 & 50.1073 & 692.098 & 681.3174 & 334.5592 & 122.5994 & 1.81903 \\
\hline 2773 & 50.1243 & 769.6058 & 851.3506 & 334.4453 & 122.5994 & 1.81845 \\
\hline 2774 & 50.1413 & 999.4241 & 1080.125 & 334.3314 & 122.5994 & 1.81788 \\
\hline 2775 & 50.1583 & 1270.006 & 1337.37 & 334.2174 & 122.5994 & 1.8173 \\
\hline 2776 & 50.1753 & 1560.078 & 1535.524 & 334.1035 & 122.5994 & 1.81672 \\
\hline 2777 & 50.1923 & 1776.146 & 1555.225 & 333.9896 & 122.5994 & 1.81615 \\
\hline 2778 & 50.2093 & 1643.52 & 1389.365 & 333.8757 & 122.5994 & 1.81557 \\
\hline 2779 & 50.2263 & 1237.986 & 1162.905 & 333.7617 & 122.5994 & 1.815 \\
\hline 2780 & 50.2433 & 962.8654 & 983.1779 & 333.6478 & 122.5994 & 1.81442 \\
\hline 2781 & 50.2603 & 827.0882 & 895.6603 & 333.5339 & 122.5994 & 1.81385 \\
\hline 2782 & 50.2773 & 825.8135 & 899.8943 & 333.4199 & 122.5994 & 1.81328 \\
\hline 2783 & 50.2943 & 864.3844 & 960.3998 & 333.306 & 122.5994 & 1.8127 \\
\hline 2784 & 50.3113 & 1005.646 & 1009.302 & 333.1921 & 122.5994 & 1.81213 \\
\hline 2785 & 50.3283 & 1052.968 & 975.6076 & 333.0781 & 122.5994 & 1.81156 \\
\hline 2786 & 50.3453 & 951.3972 & 859.4252 & 332.9642 & 122.5994 & 1.81099 \\
\hline 2787 & 50.3623 & 730.6269 & 718.9389 & 332.8503 & 122.5994 & 1.81042 \\
\hline 2788 & 50.3793 & 535.4068 & 597.4113 & 332.7363 & 122.5994 & 1.80985 \\
\hline 2789 & 50.3963 & 475.9863 & 509.7213 & 332.6224 & 122.5994 & 1.80927 \\
\hline 2790 & 50.4133 & 416.3262 & 454.1063 & 332.5085 & 122.5994 & 1.8087 \\
\hline 2791 & 50.4303 & 427.3407 & 421.7774 & 332.3945 & 122.5994 & 1.80813 \\
\hline
\end{tabular}




\begin{tabular}{|c|c|c|c|c|c|c|}
\hline 2792 & 50.4473 & 391.2761 & 403.3846 & 332.2806 & 122.5994 & 1.80756 \\
\hline 2793 & 50.4643 & 379.5487 & 392.3145 & 332.1667 & 122.5994 & 1.807 \\
\hline 2794 & 50.4813 & 414.2276 & 385.0573 & 332.0527 & 122.5994 & 1.80643 \\
\hline 2795 & 50.4983 & 351.3186 & 380.5463 & 331.9388 & 122.5994 & 1.80586 \\
\hline 2796 & 50.5153 & 313.7742 & 379.1461 & 331.8249 & 122.5994 & 1.80529 \\
\hline 2797 & 50.5323 & 362.2676 & 381.9059 & 331.7109 & 122.5994 & 1.80472 \\
\hline 2798 & 50.5493 & 375.0078 & 388.1527 & 331.597 & 122.5994 & 1.80416 \\
\hline 2799 & 50.5663 & 397.5899 & 397.0348 & 331.4831 & 122.5994 & 1.80359 \\
\hline 2800 & 50.5833 & 376.9209 & 405.0051 & 331.3691 & 122.5994 & 1.80302 \\
\hline 2801 & 50.6003 & 390.0677 & 406.7873 & 331.2552 & 122.5994 & 1.80246 \\
\hline 2802 & 50.6173 & 387.3772 & 401.1128 & 331.1413 & 122.5994 & 1.80189 \\
\hline 2803 & 50.6343 & 393.6863 & 392.7961 & 331.0273 & 122.5994 & 1.80133 \\
\hline 2804 & 50.6513 & 417.8972 & 386.2988 & 330.9134 & 122.5994 & 1.80076 \\
\hline 2805 & 50.6683 & 500.4074 & 382.7031 & 330.7995 & 122.5994 & 1.8002 \\
\hline 2806 & 50.6853 & 562.3105 & 380.8926 & 330.6855 & 122.5994 & 1.79963 \\
\hline 2807 & 50.7023 & 596.9099 & 379.9542 & 330.5716 & 122.5994 & 1.79907 \\
\hline 2808 & 50.7193 & 526.8178 & 378.8692 & 330.4577 & 122.5994 & 1.79851 \\
\hline 2809 & 50.7363 & 428.9363 & 375.5934 & 330.3438 & 122.5994 & 1.79794 \\
\hline 2810 & 50.7533 & 371.2509 & 369.4281 & 329.832 & 122.5994 & 1.79738 \\
\hline 2811 & 50.7703 & 379.1471 & 362.9324 & 329.3203 & 122.5994 & 1.79682 \\
\hline 2812 & 50.7873 & 401.8648 & 357.6799 & 328.8086 & 122.5994 & 1.79626 \\
\hline 2813 & 50.8043 & 418.3018 & 353.4817 & 328.2969 & 122.5994 & 1.7957 \\
\hline 2814 & 50.8213 & 450.8226 & 349.831 & 327.7852 & 122.5994 & 1.79514 \\
\hline 2815 & 50.8383 & 461.4401 & 346.3314 & 327.2734 & 122.5994 & 1.79458 \\
\hline 2816 & 50.8553 & 412.0047 & 343.2048 & 326.7617 & 122.5994 & 1.79402 \\
\hline 2817 & 50.8723 & 366.6233 & 340.5316 & 326.25 & 122.5994 & 1.79346 \\
\hline 2818 & 50.8893 & 343.5321 & 338.3325 & 325.7383 & 122.5994 & 1.7929 \\
\hline 2819 & 50.9063 & 358.7326 & 336.6165 & 325.2266 & 122.5994 & 1.79234 \\
\hline 2820 & 50.9233 & 364.9359 & 335.3529 & 324.7148 & 122.5994 & 1.79178 \\
\hline 2821 & 50.9403 & 331.1962 & 329.917 & 324.2031 & 122.5994 & 1.79122 \\
\hline 2822 & 50.9573 & 352.9357 & 329.6158 & 323.6914 & 122.5994 & 1.79066 \\
\hline 2823 & 50.9743 & 336.5159 & 329.6026 & 323.1797 & 122.5994 & 1.79011 \\
\hline 2824 & 50.9913 & 329.4043 & 329.8294 & 322.668 & 122.5994 & 1.78955 \\
\hline 2825 & 51.0083 & 331.2082 & 330.0308 & 322.1563 & 122.5994 & 1.78899 \\
\hline 2826 & 51.0253 & 328.4399 & 331.3249 & 321.6445 & 122.5994 & 1.78844 \\
\hline 2827 & 51.0423 & 321.7828 & 334.1292 & 321.1328 & 122.5994 & 1.78788 \\
\hline 2828 & 51.0593 & 320.4527 & 339.652 & 320.6211 & 122.5994 & 1.78733 \\
\hline 2829 & 51.0763 & 326.4852 & 349.3044 & 320.1094 & 122.5994 & 1.78677 \\
\hline 2830 & 51.0933 & 356.61 & 363.805 & 319.5977 & 122.5994 & 1.78622 \\
\hline 2831 & 51.1103 & 350.9696 & 382.4296 & 319.0859 & 122.5994 & 1.78566 \\
\hline 2832 & 51.1273 & 356.5187 & 400.5343 & 318.5742 & 122.5994 & 1.78511 \\
\hline 2833 & 51.1443 & 357.2576 & 409.2744 & 318.0625 & 122.5994 & 1.78456 \\
\hline 2834 & 51.1613 & 332.3587 & 402.2924 & 317.0599 & 122.5994 & 1.784 \\
\hline 2835 & 51.1783 & 379.0922 & 385.3218 & 316.0573 & 122.5994 & 1.78345 \\
\hline 2836 & 51.1953 & 342.3511 & 368.1555 & 315.0547 & 122.5994 & 1.7829 \\
\hline 2837 & 51.2123 & 369.3235 & 356.5846 & 314.0521 & 122.5994 & 1.78235 \\
\hline 2838 & 51.2293 & 396.6468 & 352.2888 & 313.0495 & 122.5994 & 1.78179 \\
\hline 2839 & 51.2463 & 321.3693 & 354.2137 & 312.0469 & 122.5994 & 1.78124 \\
\hline 2840 & 51.2633 & 335.7957 & 358.741 & 311.0443 & 122.5994 & 1.78069 \\
\hline
\end{tabular}




\begin{tabular}{|c|c|c|c|c|c|c|}
\hline 2841 & 51.2803 & 349.1534 & 360.2801 & 310.0417 & 122.5994 & 1.78014 \\
\hline 2842 & 51.2973 & 374.9342 & 354.7923 & 309.0391 & 122.5994 & 1.77959 \\
\hline 2843 & 51.3143 & 357.6175 & 344.2799 & 308.0365 & 122.5994 & 1.77904 \\
\hline 2844 & 51.3313 & 323.1356 & 333.1492 & 307.0339 & 122.5994 & 1.77849 \\
\hline 2845 & 51.3483 & 354.131 & 323.8854 & 306.0313 & 122.5994 & 1.77794 \\
\hline 2846 & 51.3653 & 355.6489 & 317.1382 & 305.0286 & 122.5994 & 1.7774 \\
\hline 2847 & 51.3823 & 341.9253 & 312.6264 & 304.026 & 122.5994 & 1.77685 \\
\hline 2848 & 51.3993 & 320.5071 & 309.5298 & 303.0234 & 122.5994 & 1.7763 \\
\hline 2849 & 51.4163 & 333.3222 & 307.2844 & 302.0208 & 122.5994 & 1.77575 \\
\hline 2850 & 51.4333 & 329.8922 & 305.4954 & 301.0182 & 122.5994 & 1.77521 \\
\hline 2851 & 51.4503 & 316.7069 & 303.9432 & 300.0156 & 122.5994 & 1.77466 \\
\hline 2852 & 51.4673 & 321.4422 & 302.5329 & 299.013 & 122.5994 & 1.77411 \\
\hline 2853 & 51.4843 & 317.1183 & 301.2255 & 298.0104 & 122.5994 & 1.77357 \\
\hline 2854 & 51.5013 & 325.714 & 300.017 & 297.0078 & 122.5994 & 1.77302 \\
\hline 2855 & 51.5183 & 292.3151 & 298.934 & 296.0052 & 122.5994 & 1.77248 \\
\hline 2856 & 51.5353 & 279.4116 & 297.9337 & 295.0026 & 122.5994 & 1.77193 \\
\hline 2857 & 51.5523 & 283.124 & 297.0275 & 294 & 122.5994 & 1.77139 \\
\hline 2858 & 51.5693 & 314.2372 & 297.1101 & 294.5417 & 122.5994 & 1.77084 \\
\hline 2859 & 51.5863 & 322.5813 & 297.805 & 295.0833 & 122.5994 & 1.7703 \\
\hline 2860 & 51.6033 & 315.3611 & 298.3772 & 295.625 & 122.5994 & 1.76976 \\
\hline 2861 & 51.6203 & 325.9803 & 298.8704 & 296.1667 & 122.5994 & 1.76921 \\
\hline 2862 & 51.6373 & 319.5876 & 299.4368 & 296.7083 & 122.5994 & 1.76867 \\
\hline 2863 & 51.6543 & 309.2508 & 300.1949 & 297.25 & 122.5994 & 1.76813 \\
\hline 2864 & 51.6713 & 306.2206 & 301.3102 & 297.7917 & 122.5994 & 1.76759 \\
\hline 2865 & 51.6883 & 301.8879 & 302.9489 & 298.3333 & 122.5994 & 1.76705 \\
\hline 2866 & 51.7053 & 312.9347 & 304.96 & 298.875 & 122.5994 & 1.7665 \\
\hline 2867 & 51.7223 & 290.5276 & 307.9875 & 299.4167 & 122.5994 & 1.76596 \\
\hline 2868 & 51.7393 & 299.1003 & 311.4294 & 299.9583 & 122.5994 & 1.76542 \\
\hline 2869 & 51.7563 & 302.3106 & 314.5314 & 300.5 & 122.5994 & 1.76488 \\
\hline 2870 & 51.7733 & 294.3054 & 316.1634 & 301.0417 & 122.5994 & 1.76434 \\
\hline 2871 & 51.7903 & 284.7369 & 315.9981 & 301.5833 & 122.5994 & 1.7638 \\
\hline 2872 & 51.8073 & 282.2129 & 314.596 & 302.125 & 122.5994 & 1.76327 \\
\hline 2873 & 51.8243 & 301.3607 & 312.9956 & 302.6667 & 122.5994 & 1.76273 \\
\hline 2874 & 51.8413 & 317.7783 & 311.9857 & 303.2083 & 122.5994 & 1.76219 \\
\hline 2875 & 51.8583 & 317.4615 & 311.9341 & 303.75 & 122.5994 & 1.76165 \\
\hline 2876 & 51.8753 & 301.7291 & 312.778 & 304.2917 & 122.5994 & 1.76111 \\
\hline 2877 & 51.8923 & 311.5912 & 314.0688 & 304.8333 & 122.5994 & 1.76058 \\
\hline 2878 & 51.9093 & 310.1464 & 315.0972 & 305.375 & 122.5994 & 1.76004 \\
\hline 2879 & 51.9263 & 300.0583 & 315.5027 & 305.9167 & 122.5994 & 1.7595 \\
\hline 2880 & 51.9433 & 280.0431 & 315.4373 & 306.4583 & 122.5994 & 1.75897 \\
\hline 2881 & 51.9603 & 308.7099 & 315.3156 & 307 & 122.5994 & 1.75843 \\
\hline 2882 & 51.9773 & 338.2701 & 315.2733 & 307.4375 & 122.5994 & 1.7579 \\
\hline 2883 & 51.9943 & 302.5625 & 315.3615 & 307.875 & 122.5994 & 1.75736 \\
\hline 2884 & 52.0113 & 286.2977 & 315.577 & 308.3125 & 122.5994 & 1.75683 \\
\hline 2885 & 52.0283 & 306.2206 & 315.8433 & 308.75 & 122.5994 & 1.75629 \\
\hline 2886 & 52.0453 & 308.1536 & 316.634 & 309.1875 & 122.5994 & 1.75576 \\
\hline 2887 & 52.0623 & 326.1846 & 318.241 & 309.625 & 122.5994 & 1.75523 \\
\hline 2888 & 52.0793 & 282.9731 & 320.8539 & 310.0625 & 122.5994 & 1.75469 \\
\hline 2889 & 52.0963 & 310.3569 & 324.5199 & 310.5 & 122.5994 & 1.75416 \\
\hline
\end{tabular}




\begin{tabular}{|c|c|c|c|c|c|c|}
\hline 2890 & 52.1133 & 298.0299 & 328.9289 & 310.9375 & 122.5994 & 1.75363 \\
\hline 2891 & 52.1303 & 321.8683 & 333.0565 & 311.375 & 122.5994 & 1.7531 \\
\hline 2892 & 52.1473 & 299.3631 & 335.199 & 311.8125 & 122.5994 & 1.75257 \\
\hline 2893 & 52.1643 & 313.3318 & 334.2902 & 312.25 & 122.5994 & 1.75204 \\
\hline 2894 & 52.1813 & 306.4342 & 331.6151 & 312.6875 & 122.5994 & 1.7515 \\
\hline 2895 & 52.1983 & 291.1144 & 328.9476 & 313.125 & 122.5994 & 1.75097 \\
\hline 2896 & 52.2153 & 313.5295 & 327.3047 & 313.5625 & 122.5994 & 1.75044 \\
\hline 2897 & 52.2323 & 325.6243 & 327.0168 & 314 & 122.5994 & 1.74991 \\
\hline 2898 & 52.2493 & 298.5817 & 327.9389 & 314.4375 & 122.5994 & 1.74938 \\
\hline 2899 & 52.2663 & 293.5833 & 329.5347 & 314.875 & 122.5994 & 1.74886 \\
\hline 2900 & 52.2833 & 321.2618 & 330.843 & 315.3125 & 122.5994 & 1.74833 \\
\hline 2901 & 52.3003 & 302.0861 & 331.0558 & 315.75 & 122.5994 & 1.7478 \\
\hline 2902 & 52.3173 & 318.3042 & 330.3004 & 316.1875 & 122.5994 & 1.74727 \\
\hline 2903 & 52.3343 & 328.444 & 329.1357 & 316.625 & 122.5994 & 1.74674 \\
\hline 2904 & 52.3513 & 345.3978 & 328.0025 & 317.0625 & 122.5994 & 1.74622 \\
\hline 2905 & 52.3683 & 349.8716 & 327.2169 & 317.5 & 122.5994 & 1.74569 \\
\hline 2906 & 52.3853 & 334.8418 & 326.521 & 317.6458 & 122.5994 & 1.74516 \\
\hline 2907 & 52.4023 & 323.9689 & 326.1183 & 317.7917 & 122.5994 & 1.74464 \\
\hline 2908 & 52.4193 & 318.1757 & 326.1244 & 317.9375 & 122.5994 & 1.74411 \\
\hline 2909 & 52.4363 & 331.8581 & 326.7726 & 318.0833 & 122.5994 & 1.74359 \\
\hline 2910 & 52.4533 & 343.8138 & 328.2337 & 318.2292 & 122.5994 & 1.74306 \\
\hline 2911 & 52.4703 & 339.9576 & 330.5447 & 318.375 & 122.5994 & 1.74254 \\
\hline 2912 & 52.4873 & 333.9643 & 333.5866 & 318.5208 & 122.5994 & 1.74201 \\
\hline 2913 & 52.5043 & 331.2082 & 336.973 & 318.6667 & 122.5994 & 1.74149 \\
\hline 2914 & 52.5213 & 343.6958 & 339.6818 & 318.8125 & 122.5994 & 1.74096 \\
\hline 2915 & 52.5383 & 331.7926 & 340.4005 & 318.9583 & 122.5994 & 1.74044 \\
\hline 2916 & 52.5553 & 334.4507 & 339.0809 & 319.1042 & 122.5994 & 1.73992 \\
\hline 2917 & 52.5723 & 349.6394 & 337.1326 & 319.25 & 122.5994 & 1.73939 \\
\hline 2918 & 52.5893 & 344.8684 & 335.6089 & 319.3958 & 122.5994 & 1.73887 \\
\hline 2919 & 52.6063 & 313.979 & 335.0361 & 319.5417 & 122.5994 & 1.73835 \\
\hline 2920 & 52.6233 & 344.328 & 335.5076 & 319.6875 & 122.5994 & 1.73783 \\
\hline 2921 & 52.6403 & 311.4702 & 336.839 & 319.8333 & 122.5994 & 1.73731 \\
\hline 2922 & 52.6573 & 323.9008 & 338.5158 & 319.9792 & 122.5994 & 1.73679 \\
\hline 2923 & 52.6743 & 356.6457 & 339.7979 & 320.125 & 122.5994 & 1.73627 \\
\hline 2924 & 52.6913 & 318.482 & 340.3694 & 320.2708 & 122.5994 & 1.73575 \\
\hline 2925 & 52.7083 & 323.0446 & 340.6822 & 320.4167 & 122.5994 & 1.73523 \\
\hline 2926 & 52.7253 & 304.109 & 341.1703 & 320.5625 & 122.5994 & 1.73471 \\
\hline 2927 & 52.7423 & 319.6857 & 341.9367 & 320.7083 & 122.5994 & 1.73419 \\
\hline 2928 & 52.7593 & 330.972 & 342.87 & 320.8542 & 122.5994 & 1.73367 \\
\hline 2929 & 52.7763 & 309.8097 & 343.7536 & 321 & 122.5994 & 1.73315 \\
\hline 2930 & 52.7933 & 349.2664 & 344.3676 & 321.1458 & 122.5994 & 1.73263 \\
\hline 2931 & 52.8103 & 327.7336 & 344.5316 & 321.2917 & 122.5994 & 1.73212 \\
\hline 2932 & 52.8273 & 330.057 & 344.477 & 321.4375 & 122.5994 & 1.7316 \\
\hline 2933 & 52.8443 & 312.7278 & 344.1839 & 321.5833 & 122.5994 & 1.73108 \\
\hline 2934 & 52.8613 & 310.8777 & 343.6649 & 321.7292 & 122.5994 & 1.73056 \\
\hline 2935 & 52.8783 & 348.3024 & 342.7456 & 321.875 & 122.5994 & 1.73005 \\
\hline 2936 & 52.8953 & 334.1995 & 341.7913 & 322.0208 & 122.5994 & 1.72953 \\
\hline 2937 & 52.9123 & 321.1654 & 340.7885 & 322.1667 & 122.5994 & 1.72902 \\
\hline 2938 & 52.9293 & 364.2157 & 339.8199 & 322.3125 & 122.5994 & 1.7285 \\
\hline
\end{tabular}




\begin{tabular}{|c|c|c|c|c|c|c|}
\hline 2939 & 52.9463 & 319.4796 & 338.7858 & 322.4583 & 122.5994 & 1.72799 \\
\hline 2940 & 52.9633 & 337.1323 & 337.4692 & 322.6042 & 122.5994 & 1.72747 \\
\hline 2941 & 52.9803 & 316.7283 & 336.1167 & 322.75 & 122.5994 & 1.72696 \\
\hline 2942 & 52.9973 & 306.2908 & 334.8413 & 322.8958 & 122.5994 & 1.72644 \\
\hline 2943 & 53.0143 & 310.4815 & 333.6322 & 323.0417 & 122.5994 & 1.72593 \\
\hline 2944 & 53.0313 & 321.5364 & 332.6027 & 323.1875 & 122.5994 & 1.72542 \\
\hline 2945 & 53.0483 & 321.5803 & 331.8072 & 323.3333 & 122.5994 & 1.7249 \\
\hline 2946 & 53.0653 & 335.7372 & 331.4109 & 323.4792 & 122.5994 & 1.72439 \\
\hline 2947 & 53.0823 & 362.806 & 331.5847 & 323.625 & 122.5994 & 1.72388 \\
\hline 2948 & 53.0993 & 332.7049 & 332.1717 & 323.7708 & 122.5994 & 1.72337 \\
\hline 2949 & 53.1163 & 310.3185 & 333.6408 & 323.9167 & 122.5994 & 1.72286 \\
\hline 2950 & 53.1333 & 328.6481 & 336.3397 & 324.0625 & 122.5994 & 1.72234 \\
\hline 2951 & 53.1503 & 345.5651 & 340.3672 & 324.2083 & 122.5994 & 1.72183 \\
\hline 2952 & 53.1673 & 355.3518 & 345.321 & 324.3542 & 122.5994 & 1.72132 \\
\hline 2953 & 53.1843 & 353.2952 & 349.9218 & 324.5 & 122.5994 & 1.72081 \\
\hline 2954 & 53.2013 & 351.7529 & 352.4585 & 324.3542 & 122.5994 & 1.7203 \\
\hline 2955 & 53.2183 & 351.3095 & 352.7131 & 324.2083 & 122.5994 & 1.71979 \\
\hline 2956 & 53.2353 & 326.4764 & 351.7974 & 324.0625 & 122.5994 & 1.71928 \\
\hline 2957 & 53.2523 & 338.3742 & 351.3069 & 323.9167 & 122.5994 & 1.71878 \\
\hline 2958 & 53.2693 & 345.5458 & 352.1407 & 323.7708 & 122.5994 & 1.71827 \\
\hline 2959 & 53.2863 & 331.5398 & 353.6759 & 323.625 & 122.5994 & 1.71776 \\
\hline 2960 & 53.3033 & 347.3117 & 354.4847 & 323.4792 & 122.5994 & 1.71725 \\
\hline 2961 & 53.3203 & 341.8178 & 354.0286 & 323.3333 & 122.5994 & 1.71674 \\
\hline 2962 & 53.3373 & 334.9346 & 352.3793 & 323.1875 & 122.5994 & 1.71624 \\
\hline 2963 & 53.3543 & 329.5351 & 349.5412 & 323.0417 & 122.5994 & 1.71573 \\
\hline 2964 & 53.3713 & 355.6578 & 345.9757 & 322.8958 & 122.5994 & 1.71522 \\
\hline 2965 & 53.3883 & 353.0688 & 342.825 & 322.75 & 122.5994 & 1.71472 \\
\hline 2966 & 53.4053 & 337.9578 & 340.6457 & 322.6042 & 122.5994 & 1.71421 \\
\hline 2967 & 53.4223 & 359.3111 & 339.3685 & 322.4583 & 122.5994 & 1.71371 \\
\hline 2968 & 53.4393 & 339.6808 & 338.363 & 322.3125 & 122.5994 & 1.7132 \\
\hline 2969 & 53.4563 & 328.6712 & 337.2595 & 322.1667 & 122.5994 & 1.7127 \\
\hline 2970 & 53.4733 & 367.1528 & 336.3387 & 322.0208 & 122.5994 & 1.71219 \\
\hline 2971 & 53.4903 & 338.5708 & 335.753 & 321.875 & 122.5994 & 1.71169 \\
\hline 2972 & 53.5073 & 348.5456 & 335.2393 & 321.7292 & 122.5994 & 1.71118 \\
\hline 2973 & 53.5243 & 342.6947 & 334.2433 & 321.5833 & 122.5994 & 1.71068 \\
\hline 2974 & 53.5413 & 353.0482 & 332.4205 & 321.4375 & 122.5994 & 1.71018 \\
\hline 2975 & 53.5583 & 338.6847 & 330.207 & 321.2917 & 122.5994 & 1.70968 \\
\hline 2976 & 53.5753 & 286.3214 & 328.2946 & 321.1458 & 122.5994 & 1.70917 \\
\hline 2977 & 53.5923 & 351.9515 & 326.8998 & 321 & 122.5994 & 1.70867 \\
\hline 2978 & 53.6093 & 306.9847 & 327.1774 & 321.6875 & 122.5994 & 1.70817 \\
\hline 2979 & 53.6263 & 322.0256 & 328.0948 & 322.375 & 122.5994 & 1.70767 \\
\hline 2980 & 53.6433 & 341.9376 & 329.3387 & 323.0625 & 122.5994 & 1.70717 \\
\hline 2981 & 53.6603 & 333.4672 & 330.605 & 323.75 & 122.5994 & 1.70667 \\
\hline 2982 & 53.6773 & 298.3242 & 331.653 & 324.4375 & 122.5994 & 1.70617 \\
\hline 2983 & 53.6943 & 316.1593 & 332.4534 & 325.125 & 122.5994 & 1.70567 \\
\hline 2984 & 53.7113 & 329.3004 & 333.4452 & 325.8125 & 122.5994 & 1.70517 \\
\hline 2985 & 53.7283 & 326.3697 & 334.6052 & 326.5 & 122.5994 & 1.70467 \\
\hline 2986 & 53.7453 & 347.9749 & 336.4495 & 327.1875 & 122.5994 & 1.70417 \\
\hline 2987 & 53.7623 & 338.6698 & 338.3391 & 327.875 & 122.5994 & 1.70367 \\
\hline
\end{tabular}




\begin{tabular}{|c|c|c|c|c|c|c|}
\hline 2988 & 53.7793 & 322.7754 & 340.0607 & 328.5625 & 122.5994 & 1.70317 \\
\hline 2989 & 53.7963 & 308.7808 & 341.7511 & 329.25 & 122.5994 & 1.70267 \\
\hline 2990 & 53.8133 & 308.4182 & 343.6603 & 329.9375 & 122.5994 & 1.70217 \\
\hline 2991 & 53.8303 & 310.9116 & 345.8044 & 330.625 & 122.5994 & 1.70168 \\
\hline 2992 & 53.8473 & 325.7393 & 348.1885 & 331.3125 & 122.5994 & 1.70118 \\
\hline 2993 & 53.8643 & 349.7565 & 350.69 & 332 & 122.5994 & 1.70068 \\
\hline 2994 & 53.8813 & 315.8185 & 353.2649 & 332.6875 & 122.5994 & 1.70019 \\
\hline 2995 & 53.8983 & 305.6407 & 356.0017 & 333.375 & 122.5994 & 1.69969 \\
\hline 2996 & 53.9153 & 328.8496 & 358.8386 & 334.0625 & 122.5994 & 1.69919 \\
\hline 2997 & 53.9323 & 343.4425 & 361.0406 & 334.75 & 122.5994 & 1.6987 \\
\hline 2998 & 53.9493 & 346.2012 & 361.6959 & 335.4375 & 122.5994 & 1.6982 \\
\hline 2999 & 53.9663 & 351.8838 & 360.647 & 336.125 & 122.5994 & 1.69771 \\
\hline 3000 & 53.9833 & 360.7677 & 359.0154 & 336.8125 & 122.5994 & 1.69721 \\
\hline 3001 & 54.0003 & 339.0279 & 357.7346 & 337.5 & 122.5994 & 1.69672 \\
\hline 3002 & 54.0173 & 326.0279 & 356.6607 & 337.8177 & 122.5994 & 1.69623 \\
\hline 3003 & 54.0343 & 351.8586 & 356.1511 & 338.1354 & 122.5994 & 1.69573 \\
\hline 3004 & 54.0513 & 361.6778 & 356.3156 & 338.4531 & 122.5994 & 1.69524 \\
\hline 3005 & 54.0683 & 338.3847 & 357.0927 & 338.7708 & 122.5994 & 1.69475 \\
\hline 3006 & 54.0853 & 344.6106 & 358.0599 & 339.0885 & 122.5994 & 1.69425 \\
\hline 3007 & 54.1023 & 335.8959 & 358.7607 & 339.4063 & 122.5994 & 1.69376 \\
\hline 3008 & 54.1193 & 303.0294 & 359.1307 & 339.724 & 122.5994 & 1.69327 \\
\hline 3009 & 54.1363 & 328.3152 & 359.2828 & 340.0417 & 122.5994 & 1.69278 \\
\hline 3010 & 54.1533 & 344.9715 & 358.4232 & 340.3594 & 122.5994 & 1.69229 \\
\hline 3011 & 54.1703 & 318.3478 & 357.7515 & 340.6771 & 122.5994 & 1.6918 \\
\hline 3012 & 54.1873 & 320.9153 & 356.8958 & 340.9948 & 122.5994 & 1.69131 \\
\hline 3013 & 54.2043 & 338.773 & 357.1866 & 341.3125 & 122.5994 & 1.69082 \\
\hline 3014 & 54.2213 & 342.2172 & 358.7234 & 341.6302 & 122.5994 & 1.69033 \\
\hline 3015 & 54.2383 & 314.6732 & 361.0955 & 341.9479 & 122.5994 & 1.68984 \\
\hline 3016 & 54.2553 & 325.2884 & 363.5504 & 342.2656 & 122.5994 & 1.68935 \\
\hline 3017 & 54.2723 & 324.3548 & 365.6869 & 342.5833 & 122.5994 & 1.68886 \\
\hline 3018 & 54.2893 & 334.004 & 367.3915 & 342.901 & 122.5994 & 1.68837 \\
\hline 3019 & 54.3063 & 348.7121 & 369.3286 & 343.2188 & 122.5994 & 1.68788 \\
\hline 3020 & 54.3233 & 360.5687 & 372.771 & 343.5365 & 122.5994 & 1.68739 \\
\hline 3021 & 54.3403 & 357.2543 & 377.7309 & 343.8542 & 122.5994 & 1.6869 \\
\hline 3022 & 54.3573 & 392.0539 & 383.8684 & 344.1719 & 122.5994 & 1.68642 \\
\hline 3023 & 54.3743 & 339.1086 & 389.2763 & 344.4896 & 122.5994 & 1.68593 \\
\hline 3024 & 54.3913 & 366.0509 & 392.5152 & 344.8073 & 122.5994 & 1.68544 \\
\hline 3025 & 54.4083 & 388.9977 & 392.6784 & 345.125 & 122.5994 & 1.68496 \\
\hline 3026 & 54.4253 & 344.7276 & 389.1862 & 345.4427 & 122.5994 & 1.68447 \\
\hline 3027 & 54.4423 & 335.6747 & 383.8061 & 345.7604 & 122.5994 & 1.68398 \\
\hline 3028 & 54.4593 & 370.964 & 379.1464 & 346.0781 & 122.5994 & 1.6835 \\
\hline 3029 & 54.4763 & 360.4745 & 376.6694 & 346.3958 & 122.5994 & 1.68301 \\
\hline 3030 & 54.4933 & 357.1757 & 376.3243 & 346.7135 & 122.5994 & 1.68253 \\
\hline 3031 & 54.5103 & 359.4798 & 377.2425 & 347.0313 & 122.5994 & 1.68204 \\
\hline 3032 & 54.5273 & 371.1105 & 378.1591 & 347.349 & 122.5994 & 1.68156 \\
\hline 3033 & 54.5443 & 381.1412 & 378.3712 & 347.6667 & 122.5994 & 1.68108 \\
\hline 3034 & 54.5613 & 376.2576 & 377.2574 & 347.9844 & 122.5994 & 1.68059 \\
\hline 3035 & 54.5783 & 357.6671 & 374.6747 & 348.3021 & 122.5994 & 1.68011 \\
\hline 3036 & 54.5953 & 364.0991 & 371.5551 & 348.6198 & 122.5994 & 1.67963 \\
\hline
\end{tabular}




\begin{tabular}{|c|c|c|c|c|c|c|}
\hline 3037 & 54.6123 & 354.6253 & 369.0771 & 348.9375 & 122.5994 & 1.67914 \\
\hline 3038 & 54.6293 & 352.0957 & 367.2816 & 349.2552 & 122.5994 & 1.67866 \\
\hline 3039 & 54.6463 & 339.52 & 366.4052 & 349.5729 & 122.5994 & 1.67818 \\
\hline 3040 & 54.6633 & 343.5155 & 366.3535 & 349.8906 & 122.5994 & 1.6777 \\
\hline 3041 & 54.6803 & 357.2576 & 367.0839 & 350.2083 & 122.5994 & 1.67722 \\
\hline 3042 & 54.6973 & 353.9349 & 368.4854 & 350.526 & 122.5994 & 1.67674 \\
\hline 3043 & 54.7143 & 348.0484 & 370.5515 & 350.8438 & 122.5994 & 1.67625 \\
\hline 3044 & 54.7313 & 377.2742 & 373.48 & 351.1615 & 122.5994 & 1.67577 \\
\hline 3045 & 54.7483 & 404.0837 & 377.5703 & 351.4792 & 122.5994 & 1.67529 \\
\hline 3046 & 54.7653 & 383.9007 & 382.9783 & 351.7969 & 122.5994 & 1.67481 \\
\hline 3047 & 54.7823 & 382.9823 & 390.2895 & 352.1146 & 122.5994 & 1.67433 \\
\hline 3048 & 54.7993 & 396.5512 & 400.2964 & 352.4323 & 122.5994 & 1.67386 \\
\hline 3049 & 54.8163 & 414.1291 & 414.42 & 352.75 & 122.5994 & 1.67338 \\
\hline 3050 & 54.8333 & 444.6853 & 435.428 & 352.6979 & 122.5994 & 1.6729 \\
\hline 3051 & 54.8503 & 468.7102 & 468.1815 & 352.6458 & 122.5994 & 1.67242 \\
\hline 3052 & 54.8673 & 543.4009 & 516.3861 & 352.5938 & 122.5994 & 1.67194 \\
\hline 3053 & 54.8843 & 574.4772 & 578.8629 & 352.5417 & 122.5994 & 1.67146 \\
\hline 3054 & 54.9013 & 639.7943 & 643.8632 & 352.4896 & 122.5994 & 1.67099 \\
\hline 3055 & 54.9183 & 763.3599 & 684.6174 & 352.4375 & 122.5994 & 1.67051 \\
\hline 3056 & 54.9353 & 748.1741 & 675.2095 & 352.3854 & 122.5994 & 1.67003 \\
\hline 3057 & 54.9523 & 669.7301 & 625.6946 & 352.3333 & 122.5994 & 1.66956 \\
\hline 3058 & 54.9693 & 545.5548 & 567.8552 & 352.2813 & 122.5994 & 1.66908 \\
\hline 3059 & 54.9863 & 508.0383 & 523.9586 & 352.2292 & 122.5994 & 1.6686 \\
\hline 3060 & 55.0033 & 480.9402 & 503.1652 & 352.1771 & 122.5994 & 1.66813 \\
\hline 3061 & 55.0203 & 464.2857 & 505.7054 & 352.125 & 122.5994 & 1.66765 \\
\hline 3062 & 55.0373 & 496.3466 & 524.7443 & 352.0729 & 122.5994 & 1.66718 \\
\hline 3063 & 55.0543 & 529.8306 & 546.6534 & 352.0208 & 122.5994 & 1.6667 \\
\hline 3064 & 55.0713 & 563.2459 & 552.6888 & 351.9688 & 122.5994 & 1.66623 \\
\hline 3065 & 55.0883 & 553.3767 & 532.7479 & 351.9167 & 122.5994 & 1.66576 \\
\hline 3066 & 55.1053 & 483.3824 & 497.1541 & 351.8646 & 122.5994 & 1.66528 \\
\hline 3067 & 55.1223 & 430.1393 & 461.0989 & 351.8125 & 122.5994 & 1.66481 \\
\hline 3068 & 55.1393 & 396.2805 & 432.5404 & 351.7604 & 122.5994 & 1.66434 \\
\hline 3069 & 55.1563 & 407.4838 & 413.5133 & 351.7083 & 122.5994 & 1.66386 \\
\hline 3070 & 55.1733 & 364.5213 & 402.649 & 351.6563 & 122.5994 & 1.66339 \\
\hline 3071 & 55.1903 & 357.8866 & 397.1416 & 351.6042 & 122.5994 & 1.66292 \\
\hline 3072 & 55.2073 & 368.6046 & 394.0867 & 351.5521 & 122.5994 & 1.66245 \\
\hline 3073 & 55.2243 & 366.8301 & 391.9245 & 351.5 & 122.5994 & 1.66197 \\
\hline 3074 & 55.2413 & 355.6696 & 389.8763 & 351.0208 & 122.5994 & 1.6615 \\
\hline 3075 & 55.2583 & 402.8157 & 388.7801 & 350.5417 & 122.5994 & 1.66103 \\
\hline 3076 & 55.2753 & 420.306 & 389.9155 & 350.0625 & 122.5994 & 1.66056 \\
\hline 3077 & 55.2923 & 431.1326 & 393.8748 & 349.5833 & 122.5994 & 1.66009 \\
\hline 3078 & 55.3093 & 476.99 & 402.9324 & 349.1042 & 122.5994 & 1.65962 \\
\hline 3079 & 55.3263 & 463.5927 & 418.6369 & 348.625 & 122.5994 & 1.65915 \\
\hline 3080 & 55.3433 & 455.8796 & 440.4782 & 348.1458 & 122.5994 & 1.65868 \\
\hline 3081 & 55.3603 & 482.7345 & 464.0209 & 347.6667 & 122.5994 & 1.65821 \\
\hline 3082 & 55.3773 & 540.9058 & 478.9861 & 347.1875 & 122.5994 & 1.65774 \\
\hline 3083 & 55.3943 & 509.0441 & 474.7573 & 346.7083 & 122.5994 & 1.65728 \\
\hline 3084 & 55.4113 & 422.9439 & 454.4141 & 346.2292 & 122.5994 & 1.65681 \\
\hline 3085 & 55.4283 & 407.4475 & 430.562 & 345.75 & 122.5994 & 1.65634 \\
\hline
\end{tabular}




\begin{tabular}{|c|c|c|c|c|c|c|}
\hline 3086 & 55.4453 & 404.0097 & 411.6887 & 345.2708 & 122.5994 & 1.65587 \\
\hline 3087 & 55.4623 & 409.5593 & 401.7023 & 344.7917 & 122.5994 & 1.65541 \\
\hline 3088 & 55.4793 & 376.8733 & 400.8594 & 344.3125 & 122.5994 & 1.65494 \\
\hline 3089 & 55.4963 & 393.3194 & 406.857 & 343.8333 & 122.5994 & 1.65447 \\
\hline 3090 & 55.5133 & 403.7827 & 415.193 & 343.3542 & 122.5994 & 1.65401 \\
\hline 3091 & 55.5303 & 432.2256 & 419.0471 & 342.875 & 122.5994 & 1.65354 \\
\hline 3092 & 55.5473 & 422.6024 & 413.3586 & 342.3958 & 122.5994 & 1.65307 \\
\hline 3093 & 55.5643 & 405.0443 & 400.8042 & 341.9167 & 122.5994 & 1.65261 \\
\hline 3094 & 55.5813 & 398.2192 & 387.29 & 341.4375 & 122.5994 & 1.65214 \\
\hline 3095 & 55.5983 & 350.2216 & 376.43 & 340.9583 & 122.5994 & 1.65168 \\
\hline 3096 & 55.6153 & 329.7306 & 369.3727 & 340.4792 & 122.5994 & 1.65121 \\
\hline 3097 & 55.6323 & 338.0496 & 365.9415 & 340 & 122.5994 & 1.65075 \\
\hline 3098 & 55.6493 & 354.1403 & 365.9601 & 339.8958 & 122.5994 & 1.65028 \\
\hline 3099 & 55.6663 & 356.077 & 368.3967 & 339.7917 & 122.5994 & 1.64982 \\
\hline 3100 & 55.6833 & 387.7417 & 372.2951 & 339.6875 & 122.5994 & 1.64936 \\
\hline 3101 & 55.7003 & 392.821 & 375.7898 & 339.5833 & 122.5994 & 1.64889 \\
\hline 3102 & 55.7173 & 388.1417 & 375.5532 & 339.4792 & 122.5994 & 1.64843 \\
\hline 3103 & 55.7343 & 379.332 & 372.3351 & 339.375 & 122.5994 & 1.64797 \\
\hline 3104 & 55.7513 & 385.3849 & 367.3411 & 339.2708 & 122.5994 & 1.64751 \\
\hline 3105 & 55.7683 & 366.7456 & 362.7534 & 339.1667 & 122.5994 & 1.64704 \\
\hline 3106 & 55.7853 & 367.1381 & 359.8028 & 339.0625 & 122.5994 & 1.64658 \\
\hline 3107 & 55.8023 & 375.0937 & 359.0339 & 338.9583 & 122.5994 & 1.64612 \\
\hline 3108 & 55.8193 & 360.0268 & 360.3488 & 338.8542 & 122.5994 & 1.64566 \\
\hline 3109 & 55.8363 & 401.6155 & 363.2306 & 338.75 & 122.5994 & 1.6452 \\
\hline 3110 & 55.8533 & 386.6499 & 366.2865 & 338.6458 & 122.5994 & 1.64474 \\
\hline 3111 & 55.8703 & 366.4916 & 369.1267 & 338.5417 & 122.5994 & 1.64428 \\
\hline 3112 & 55.8873 & 386.5921 & 371.1064 & 338.4375 & 122.5994 & 1.64382 \\
\hline 3113 & 55.9043 & 365.737 & 373.0085 & 338.3333 & 122.5994 & 1.64336 \\
\hline 3114 & 55.9213 & 339.1275 & 375.2449 & 338.2292 & 122.5994 & 1.6429 \\
\hline 3115 & 55.9383 & 346.0674 & 377.9481 & 338.125 & 122.5994 & 1.64244 \\
\hline 3116 & 55.9553 & 380.0334 & 381.2407 & 338.0208 & 122.5994 & 1.64198 \\
\hline 3117 & 55.9723 & 372.9751 & 385.5246 & 337.9167 & 122.5994 & 1.64152 \\
\hline 3118 & 55.9893 & 384.4717 & 390.9157 & 337.8125 & 122.5994 & 1.64107 \\
\hline 3119 & 56.0063 & 386.3471 & 397.0861 & 337.7083 & 122.5994 & 1.64061 \\
\hline 3120 & 56.0233 & 362.6368 & 403.2797 & 337.6042 & 122.5994 & 1.64015 \\
\hline 3121 & 56.0403 & 322.0161 & 408.4372 & 337.5 & 122.5994 & 1.63969 \\
\hline 3122 & 56.0573 & 373.3871 & 411.4035 & 337.3958 & 122.5994 & 1.63924 \\
\hline 3123 & 56.0743 & 340.0591 & 411.3718 & 337.2917 & 122.5994 & 1.63878 \\
\hline 3124 & 56.0913 & 365.5228 & 409.09 & 337.1875 & 122.5994 & 1.63832 \\
\hline 3125 & 56.1083 & 362.1646 & 405.178 & 337.0833 & 122.5994 & 1.63787 \\
\hline 3126 & 56.1253 & 345.6964 & 400.8392 & 336.9792 & 122.5994 & 1.63741 \\
\hline 3127 & 56.1423 & 366.7029 & 396.9248 & 336.875 & 122.5994 & 1.63696 \\
\hline 3128 & 56.1593 & 359.8989 & 393.7842 & 336.7708 & 122.5994 & 1.6365 \\
\hline 3129 & 56.1763 & 362.9747 & 391.3238 & 336.6667 & 122.5994 & 1.63605 \\
\hline 3130 & 56.1933 & 373.9053 & 389.1265 & 336.5625 & 122.5994 & 1.63559 \\
\hline 3131 & 56.2103 & 359.4767 & 386.5118 & 336.4583 & 122.5994 & 1.63514 \\
\hline 3132 & 56.2273 & 347.794 & 383.561 & 336.3542 & 122.5994 & 1.63468 \\
\hline 3133 & 56.2443 & 352.8 & 379.7726 & 336.25 & 122.5994 & 1.63423 \\
\hline 3134 & 56.2613 & 358.4885 & 374.9252 & 336.1458 & 122.5994 & 1.63378 \\
\hline
\end{tabular}




\begin{tabular}{|c|c|c|c|c|c|c|}
\hline 3135 & 56.2783 & 376.3239 & 369.4153 & 336.0417 & 122.5994 & 1.63332 \\
\hline 3136 & 56.2953 & 352.5814 & 364.0976 & 335.9375 & 122.5994 & 1.63287 \\
\hline 3137 & 56.3123 & 318.9549 & 359.5781 & 335.8333 & 122.5994 & 1.63242 \\
\hline 3138 & 56.3293 & 322.1724 & 356.1033 & 335.7292 & 122.5994 & 1.63196 \\
\hline 3139 & 56.3463 & 350.6636 & 353.7089 & 335.625 & 122.5994 & 1.63151 \\
\hline 3140 & 56.3633 & 342.5804 & 352.2923 & 335.5208 & 122.5994 & 1.63106 \\
\hline 3141 & 56.3803 & 338.9093 & 351.5552 & 335.4167 & 122.5994 & 1.63061 \\
\hline 3142 & 56.3973 & 355.6837 & 351.2599 & 335.3125 & 122.5994 & 1.63016 \\
\hline 3143 & 56.4143 & 352.6331 & 350.7094 & 335.2083 & 122.5994 & 1.62971 \\
\hline 3144 & 56.4313 & 345.0812 & 349.6869 & 335.1042 & 122.5994 & 1.62926 \\
\hline 3145 & 56.4483 & 351.8985 & 348.5471 & 335 & 122.5994 & 1.62881 \\
\hline 3146 & 56.4653 & 344.7846 & 347.3986 & 334.5833 & 122.5994 & 1.62836 \\
\hline 3147 & 56.4823 & 356.6457 & 346.7128 & 334.1667 & 122.5994 & 1.62791 \\
\hline 3148 & 56.4993 & 362.0637 & 346.4457 & 333.75 & 122.5994 & 1.62746 \\
\hline 3149 & 56.5163 & 336.5637 & 346.4412 & 333.3333 & 122.5994 & 1.62701 \\
\hline 3150 & 56.5333 & 355.6537 & 346.441 & 332.9167 & 122.5994 & 1.62656 \\
\hline 3151 & 56.5503 & 373.8255 & 346.3777 & 332.5 & 122.5994 & 1.62611 \\
\hline 3152 & 56.5673 & 367.1096 & 346.0339 & 332.0833 & 122.5994 & 1.62566 \\
\hline 3153 & 56.5843 & 334.376 & 345.5533 & 331.6667 & 122.5994 & 1.62521 \\
\hline 3154 & 56.6013 & 352.4676 & 344.729 & 331.25 & 122.5994 & 1.62477 \\
\hline 3155 & 56.6183 & 328.5398 & 343.9377 & 330.8333 & 122.5994 & 1.62432 \\
\hline 3156 & 56.6353 & 327.2341 & 343.3405 & 330.4167 & 122.5994 & 1.62387 \\
\hline 3157 & 56.6523 & 340.8327 & 342.8322 & 330 & 122.5994 & 1.62342 \\
\hline 3158 & 56.6693 & 342.2956 & 342.4074 & 329.5833 & 122.5994 & 1.62298 \\
\hline 3159 & 56.6863 & 349.2733 & 341.9525 & 329.1667 & 122.5994 & 1.62253 \\
\hline 3160 & 56.7033 & 324.7416 & 341.3452 & 328.75 & 122.5994 & 1.62209 \\
\hline 3161 & 56.7203 & 292.7224 & 340.51 & 328.3333 & 122.5994 & 1.62164 \\
\hline 3162 & 56.7373 & 331.476 & 339.4281 & 327.9167 & 122.5994 & 1.62119 \\
\hline 3163 & 56.7543 & 345.1703 & 338.2175 & 327.5 & 122.5994 & 1.62075 \\
\hline 3164 & 56.7713 & 323.4424 & 336.9586 & 327.0833 & 122.5994 & 1.6203 \\
\hline 3165 & 56.7883 & 312.7142 & 335.826 & 326.6667 & 122.5994 & 1.61986 \\
\hline 3166 & 56.8053 & 310.1496 & 334.7236 & 326.25 & 122.5994 & 1.61941 \\
\hline 3167 & 56.8223 & 343.7933 & 333.7511 & 325.8333 & 122.5994 & 1.61897 \\
\hline 3168 & 56.8393 & 327.5786 & 332.8938 & 325.4167 & 122.5994 & 1.61853 \\
\hline 3169 & 56.8563 & 325.891 & 331.6964 & 325 & 122.5994 & 1.61808 \\
\hline 3170 & 56.8733 & 316.1526 & 331.1268 & 324.75 & 122.5994 & 1.61764 \\
\hline 3171 & 56.8903 & 313.2599 & 330.6078 & 324.5 & 122.5994 & 1.6172 \\
\hline 3172 & 56.9073 & 311.7256 & 330.0612 & 324.25 & 122.5994 & 1.61675 \\
\hline 3173 & 56.9243 & 310.6505 & 329.5703 & 324 & 122.5994 & 1.61631 \\
\hline 3174 & 56.9413 & 323.0695 & 329.1172 & 323.75 & 122.5994 & 1.61587 \\
\hline 3175 & 56.9583 & 317.2626 & 328.6985 & 323.5 & 122.5994 & 1.61543 \\
\hline 3176 & 56.9753 & 314.2768 & 328.3064 & 323.25 & 122.5994 & 1.61499 \\
\hline 3177 & 56.9923 & 328.8569 & 327.9405 & 323 & 122.5994 & 1.61454 \\
\hline 3178 & 57.0093 & 333.3277 & 327.3986 & 322.75 & 122.5994 & 1.6141 \\
\hline 3179 & 57.0263 & 322.6834 & 327.1499 & 322.5 & 122.5994 & 1.61366 \\
\hline 3180 & 57.0433 & 329.1197 & 327.037 & 322.25 & 122.5994 & 1.61322 \\
\hline 3181 & 57.0603 & 340.739 & 327.0168 & 322 & 122.5994 & 1.61278 \\
\hline 3182 & 57.0773 & 318.0706 & 327.2002 & 321.75 & 122.5994 & 1.61234 \\
\hline 3183 & 57.0943 & 302.0847 & 327.678 & 321.5 & 122.5994 & 1.6119 \\
\hline
\end{tabular}




\begin{tabular}{|c|c|c|c|c|c|c|}
\hline 3184 & 57.1113 & 303.177 & 328.6466 & 321.25 & 122.5994 & 1.61146 \\
\hline 3185 & 57.1283 & 292.382 & 330.4331 & 321 & 122.5994 & 1.61102 \\
\hline 3186 & 57.1453 & 314.9384 & 333.4931 & 320.75 & 122.5994 & 1.61058 \\
\hline 3187 & 57.1623 & 307.7129 & 338.2381 & 320.5 & 122.5994 & 1.61015 \\
\hline 3188 & 57.1793 & 289.5409 & 344.8027 & 320.25 & 122.5994 & 1.60971 \\
\hline 3189 & 57.1963 & 309.0112 & 352.6903 & 320 & 122.5994 & 1.60927 \\
\hline 3190 & 57.2133 & 311.6663 & 360.3552 & 319.75 & 122.5994 & 1.60883 \\
\hline 3191 & 57.2303 & 308.5266 & 366.5628 & 319.5 & 122.5994 & 1.60839 \\
\hline 3192 & 57.2473 & 328.692 & 371.7856 & 319.25 & 122.5994 & 1.60796 \\
\hline 3193 & 57.2643 & 316.633 & 375.481 & 319 & 122.5994 & 1.60752 \\
\hline 3194 & 57.2813 & 298.9184 & 374.7483 & 318.9167 & 122.5994 & 1.60708 \\
\hline 3195 & 57.2983 & 351.3059 & 368.3671 & 318.8333 & 122.5994 & 1.60665 \\
\hline 3196 & 57.3153 & 328.378 & 360.0791 & 318.75 & 122.5994 & 1.60621 \\
\hline 3197 & 57.3323 & 310.5561 & 353.6611 & 318.6667 & 122.5994 & 1.60578 \\
\hline 3198 & 57.3493 & 335.3905 & 350.5298 & 318.5833 & 122.5994 & 1.60534 \\
\hline 3199 & 57.3663 & 333.3098 & 349.9931 & 318.5 & 122.5994 & 1.6049 \\
\hline 3200 & 57.3833 & 322.288 & 350.6666 & 318.4167 & 122.5994 & 1.60447 \\
\hline 3201 & 57.4003 & 308.3136 & 351.8904 & 318.3333 & 122.5994 & 1.60403 \\
\hline 3202 & 57.4173 & 318.5896 & 352.9779 & 318.25 & 122.5994 & 1.6036 \\
\hline 3203 & 57.4343 & 333.4147 & 351.9996 & 318.1667 & 122.5994 & 1.60317 \\
\hline 3204 & 57.4513 & 328.1852 & 347.8742 & 318.0833 & 122.5994 & 1.60273 \\
\hline 3205 & 57.4683 & 351.8882 & 342.0764 & 318 & 122.5994 & 1.6023 \\
\hline 3206 & 57.4853 & 321.7088 & 336.5525 & 317.9167 & 122.5994 & 1.60186 \\
\hline 3207 & 57.5023 & 323.9756 & 332.2515 & 317.8333 & 122.5994 & 1.60143 \\
\hline 3208 & 57.5193 & 331.1704 & 329.2805 & 317.75 & 122.5994 & 1.601 \\
\hline 3209 & 57.5363 & 318.2332 & 327.5 & 317.6667 & 122.5994 & 1.60057 \\
\hline 3210 & 57.5533 & 370.0744 & 326.5476 & 317.5833 & 122.5994 & 1.60013 \\
\hline 3211 & 57.5703 & 319.7355 & 326.192 & 317.5 & 122.5994 & 1.5997 \\
\hline 3212 & 57.5873 & 311.961 & 326.2448 & 317.4167 & 122.5994 & 1.59927 \\
\hline 3213 & 57.6043 & 329.8014 & 326.6768 & 317.3333 & 122.5994 & 1.59884 \\
\hline 3214 & 57.6213 & 293.6528 & 327.4209 & 317.25 & 122.5994 & 1.59841 \\
\hline 3215 & 57.6383 & 329.6394 & 328.3143 & 317.1667 & 122.5994 & 1.59798 \\
\hline 3216 & 57.6553 & 357.7686 & 329.0036 & 317.0833 & 122.5994 & 1.59755 \\
\hline 3217 & 57.6723 & 338.6342 & 329.1231 & 317 & 122.5994 & 1.59711 \\
\hline 3218 & 57.6893 & 327.2169 & 328.4847 & 316.9167 & 122.5994 & 1.59668 \\
\hline 3219 & 57.7063 & 339.4109 & 327.3247 & 316.8333 & 122.5994 & 1.59625 \\
\hline 3220 & 57.7233 & 337.4573 & 326.2961 & 316.75 & 122.5994 & 1.59582 \\
\hline 3221 & 57.7403 & 331.2997 & 325.896 & 316.6667 & 122.5994 & 1.5954 \\
\hline 3222 & 57.7573 & 346.2742 & 326.3367 & 316.5833 & 122.5994 & 1.59497 \\
\hline 3223 & 57.7743 & 329.2297 & 327.5454 & 316.5 & 122.5994 & 1.59454 \\
\hline 3224 & 57.7913 & 321.0029 & 329.1748 & 316.4167 & 122.5994 & 1.59411 \\
\hline 3225 & 57.8083 & 325.1208 & 330.468 & 316.3333 & 122.5994 & 1.59368 \\
\hline 3226 & 57.8253 & 304.07 & 330.8448 & 316.25 & 122.5994 & 1.59325 \\
\hline 3227 & 57.8423 & 321.2409 & 330.44 & 316.1667 & 122.5994 & 1.59282 \\
\hline 3228 & 57.8593 & 326.0902 & 329.7771 & 316.0833 & 122.5994 & 1.5924 \\
\hline 3229 & 57.8763 & 312.7378 & 329.3246 & 316 & 122.5994 & 1.59197 \\
\hline 3230 & 57.8933 & 300.9822 & 329.2002 & 315.9167 & 122.5994 & 1.59154 \\
\hline 3231 & 57.9103 & 315.096 & 329.2369 & 315.8333 & 122.5994 & 1.59112 \\
\hline 3232 & 57.9273 & 325.705 & 329.2838 & 315.75 & 122.5994 & 1.59069 \\
\hline
\end{tabular}




\begin{tabular}{|c|c|c|c|c|c|c|}
\hline 3233 & 57.9443 & 322.1022 & 329.351 & 315.6667 & 122.5994 & 1.59026 \\
\hline 3234 & 57.9613 & 332.655 & 329.1072 & 315.5833 & 122.5994 & 1.58984 \\
\hline 3235 & 57.9783 & 317.2188 & 328.5068 & 315.5 & 122.5994 & 1.58941 \\
\hline 3236 & 57.9953 & 309.8816 & 327.7698 & 315.4167 & 122.5994 & 1.58899 \\
\hline 3237 & 58.0123 & 303.7195 & 327.1871 & 315.3333 & 122.5994 & 1.58856 \\
\hline 3238 & 58.0293 & 309.4242 & 327.4073 & 315.25 & 122.5994 & 1.58814 \\
\hline 3239 & 58.0463 & 305.0575 & 328.4312 & 315.1667 & 122.5994 & 1.58771 \\
\hline 3240 & 58.0633 & 304.1412 & 330.2021 & 315.0833 & 122.5994 & 1.58729 \\
\hline 3241 & 58.0803 & 331.8781 & 332.4186 & 315 & 122.5994 & 1.58686 \\
\hline 3242 & 58.0973 & 315.8588 & 333.9096 & 314.4167 & 122.5994 & 1.58644 \\
\hline 3243 & 58.1143 & 303.583 & 334.4718 & 313.8333 & 122.5994 & 1.58602 \\
\hline 3244 & 58.1313 & 300.0341 & 333.8455 & 313.25 & 122.5994 & 1.58559 \\
\hline 3245 & 58.1483 & 307.6938 & 332.0461 & 312.6667 & 122.5994 & 1.58517 \\
\hline 3246 & 58.1653 & 324.372 & 329.0173 & 312.0833 & 122.5994 & 1.58475 \\
\hline 3247 & 58.1823 & 286.6355 & 325.7039 & 311.5 & 122.5994 & 1.58432 \\
\hline 3248 & 58.1993 & 349.7518 & 323.0398 & 310.9167 & 122.5994 & 1.5839 \\
\hline 3249 & 58.2163 & 300.3469 & 321.4012 & 310.3333 & 122.5994 & 1.58348 \\
\hline 3250 & 58.2333 & 317.7613 & 320.916 & 309.75 & 122.5994 & 1.58306 \\
\hline 3251 & 58.2503 & 291.2362 & 321.0966 & 309.1667 & 122.5994 & 1.58264 \\
\hline 3252 & 58.2673 & 276.2776 & 321.3344 & 308.5833 & 122.5994 & 1.58221 \\
\hline 3253 & 58.2843 & 322.5638 & 321.3282 & 308 & 122.5994 & 1.58179 \\
\hline 3254 & 58.3013 & 292.6887 & 321.1654 & 307.4167 & 122.5994 & 1.58137 \\
\hline 3255 & 58.3183 & 323.8204 & 320.8731 & 306.8333 & 122.5994 & 1.58095 \\
\hline 3256 & 58.3353 & 311.874 & 320.8614 & 306.25 & 122.5994 & 1.58053 \\
\hline 3257 & 58.3523 & 273.3718 & 321.5096 & 305.6667 & 122.5994 & 1.58011 \\
\hline 3258 & 58.3693 & 294.0899 & 322.6257 & 305.0833 & 122.5994 & 1.57969 \\
\hline 3259 & 58.3863 & 312.5559 & 323.7035 & 304.5 & 122.5994 & 1.57927 \\
\hline 3260 & 58.4033 & 304.6071 & 324.3869 & 303.9167 & 122.5994 & 1.57885 \\
\hline 3261 & 58.4203 & 294.8644 & 324.2138 & 303.3333 & 122.5994 & 1.57843 \\
\hline 3262 & 58.4373 & 312.0728 & 322.4177 & 302.75 & 122.5994 & 1.57802 \\
\hline 3263 & 58.4543 & 318.1627 & 319.3272 & 302.1667 & 122.5994 & 1.5776 \\
\hline 3264 & 58.4713 & 290.9705 & 316.315 & 301.5833 & 122.5994 & 1.57718 \\
\hline 3265 & 58.4883 & 294.4478 & 314.2933 & 301 & 122.5994 & 1.57676 \\
\hline 3266 & 58.5053 & 316.0512 & 315.1459 & 301.8958 & 122.5994 & 1.57634 \\
\hline 3267 & 58.5223 & 286.0021 & 317.2514 & 302.7917 & 122.5994 & 1.57593 \\
\hline 3268 & 58.5393 & 277.9041 & 320.2456 & 303.6875 & 122.5994 & 1.57551 \\
\hline 3269 & 58.5563 & 298.1741 & 323.7928 & 304.5833 & 122.5994 & 1.57509 \\
\hline 3270 & 58.5733 & 292.4342 & 327.6295 & 305.4792 & 122.5994 & 1.57468 \\
\hline 3271 & 58.5903 & 291.7257 & 331.4261 & 306.375 & 122.5994 & 1.57426 \\
\hline 3272 & 58.6073 & 304.126 & 335.3651 & 307.2708 & 122.5994 & 1.57384 \\
\hline 3273 & 58.6243 & 306.2206 & 339.9768 & 308.1667 & 122.5994 & 1.57343 \\
\hline 3274 & 58.6413 & 304.7454 & 344.8318 & 309.0625 & 122.5994 & 1.57301 \\
\hline 3275 & 58.6583 & 315.4552 & 347.646 & 309.9583 & 122.5994 & 1.5726 \\
\hline 3276 & 58.6753 & 319.5689 & 346.6095 & 310.8542 & 122.5994 & 1.57218 \\
\hline 3277 & 58.6923 & 331.1293 & 342.5818 & 311.75 & 122.5994 & 1.57177 \\
\hline 3278 & 58.7093 & 349.5636 & 338.0068 & 312.6458 & 122.5994 & 1.57135 \\
\hline 3279 & 58.7263 & 346.2424 & 334.8396 & 313.5417 & 122.5994 & 1.57094 \\
\hline 3280 & 58.7433 & 351.7261 & 333.8112 & 314.4375 & 122.5994 & 1.57052 \\
\hline 3281 & 58.7603 & 366.5466 & 334.7713 & 315.3333 & 122.5994 & 1.57011 \\
\hline
\end{tabular}




\begin{tabular}{|c|c|c|c|c|c|c|}
\hline 3282 & 58.7773 & 338.8854 & 336.9639 & 316.2292 & 122.5994 & 1.5697 \\
\hline 3283 & 58.7943 & 306.7694 & 339.4636 & 317.125 & 122.5994 & 1.56928 \\
\hline 3284 & 58.8113 & 305.0271 & 341.3607 & 318.0208 & 122.5994 & 1.56887 \\
\hline 3285 & 58.8283 & 310.8632 & 341.5269 & 318.9167 & 122.5994 & 1.56846 \\
\hline 3286 & 58.8453 & 313.2599 & 339.9702 & 319.8125 & 122.5994 & 1.56804 \\
\hline 3287 & 58.8623 & 318.3338 & 337.7069 & 320.7083 & 122.5994 & 1.56763 \\
\hline 3288 & 58.8793 & 360.8843 & 335.8256 & 321.6042 & 122.5994 & 1.56722 \\
\hline 3289 & 58.8963 & 342.7687 & 334.825 & 322.5 & 122.5994 & 1.56681 \\
\hline 3290 & 58.9133 & 325.655 & 334.2685 & 322.9063 & 122.5994 & 1.5664 \\
\hline 3291 & 58.9303 & 353.224 & 334.2475 & 323.3125 & 122.5994 & 1.56599 \\
\hline 3292 & 58.9473 & 319.4563 & 334.3706 & 323.7188 & 122.5994 & 1.56557 \\
\hline 3293 & 58.9643 & 312.6655 & 334.5693 & 324.125 & 122.5994 & 1.56516 \\
\hline 3294 & 58.9813 & 304.348 & 334.8668 & 324.5313 & 122.5994 & 1.56475 \\
\hline 3295 & 58.9983 & 343.775 & 335.2259 & 324.9375 & 122.5994 & 1.56434 \\
\hline 3296 & 59.0153 & 339.7616 & 335.5858 & 325.3438 & 122.5994 & 1.56393 \\
\hline 3297 & 59.0323 & 315.3385 & 335.9852 & 325.75 & 122.5994 & 1.56352 \\
\hline 3298 & 59.0493 & 326.7075 & 336.4691 & 326.1563 & 122.5994 & 1.56311 \\
\hline 3299 & 59.0663 & 320.7365 & 336.8422 & 326.5625 & 122.5994 & 1.5627 \\
\hline 3300 & 59.0833 & 306.4721 & 336.986 & 326.9688 & 122.5994 & 1.56229 \\
\hline 3301 & 59.1003 & 338.5005 & 337.2039 & 327.375 & 122.5994 & 1.56189 \\
\hline 3302 & 59.1173 & 331.5632 & 337.9468 & 327.7813 & 122.5994 & 1.56148 \\
\hline 3303 & 59.1343 & 341.0289 & 339.5575 & 328.1875 & 122.5994 & 1.56107 \\
\hline 3304 & 59.1513 & 319.0495 & 342.1124 & 328.5938 & 122.5994 & 1.56066 \\
\hline 3305 & 59.1683 & 347.7531 & 345.4882 & 329 & 122.5994 & 1.56025 \\
\hline 3306 & 59.1853 & 329.2073 & 349.1659 & 329.4063 & 122.5994 & 1.55984 \\
\hline 3307 & 59.2023 & 340.3499 & 352.1468 & 329.8125 & 122.5994 & 1.55944 \\
\hline 3308 & 59.2193 & 331.5648 & 353.2383 & 330.2188 & 122.5994 & 1.55903 \\
\hline 3309 & 59.2363 & 349.5046 & 352.4412 & 330.625 & 122.5994 & 1.55862 \\
\hline 3310 & 59.2533 & 332.0046 & 350.7805 & 331.0313 & 122.5994 & 1.55822 \\
\hline 3311 & 59.2703 & 331.6894 & 351.4229 & 331.4375 & 122.5994 & 1.55781 \\
\hline 3312 & 59.2873 & 328.3953 & 350.5721 & 331.8438 & 122.5994 & 1.5574 \\
\hline 3313 & 59.3043 & 353.4061 & 350.4931 & 332.25 & 122.5994 & 1.557 \\
\hline 3314 & 59.3213 & 353.4871 & 350.7862 & 332.2656 & 122.5994 & 1.55659 \\
\hline 3315 & 59.3383 & 345.5291 & 351.5841 & 332.2813 & 122.5994 & 1.55619 \\
\hline 3316 & 59.3553 & 337.2393 & 352.3005 & 332.2969 & 122.5994 & 1.55578 \\
\hline 3317 & 59.3723 & 343.2078 & 352.2071 & 332.3125 & 122.5994 & 1.55538 \\
\hline 3318 & 59.3893 & 330.3313 & 351.1042 & 332.3281 & 122.5994 & 1.55497 \\
\hline 3319 & 59.4063 & 347.6676 & 349.6771 & 332.3438 & 122.5994 & 1.55457 \\
\hline 3320 & 59.4233 & 331.0404 & 349.5367 & 332.3594 & 122.5994 & 1.55416 \\
\hline 3321 & 59.4403 & 308.2961 & 348.908 & 332.375 & 122.5994 & 1.55376 \\
\hline 3322 & 59.4573 & 316.7785 & 348.9767 & 332.3906 & 122.5994 & 1.55336 \\
\hline 3323 & 59.4743 & 342.397 & 349.8257 & 332.4063 & 122.5994 & 1.55295 \\
\hline 3324 & 59.4913 & 347.905 & 351.4012 & 332.4219 & 122.5994 & 1.55255 \\
\hline 3325 & 59.5083 & 334.334 & 353.3836 & 332.4375 & 122.5994 & 1.55215 \\
\hline 3326 & 59.5253 & 319.1336 & 355.026 & 332.4531 & 122.5994 & 1.55174 \\
\hline 3327 & 59.5423 & 326.0019 & 355.6467 & 332.4688 & 122.5994 & 1.55134 \\
\hline 3328 & 59.5593 & 329.7005 & 355.1909 & 332.4844 & 122.5994 & 1.55094 \\
\hline 3329 & 59.5763 & 316.1348 & 354.4329 & 332.5 & 122.5994 & 1.55054 \\
\hline 3330 & 59.5933 & 328.8155 & 353.9753 & 332.5156 & 122.5994 & 1.55014 \\
\hline
\end{tabular}




\begin{tabular}{|c|c|c|c|c|c|c|}
\hline 3331 & 59.6103 & 332.7901 & 353.8757 & 332.5313 & 122.5994 & 1.54973 \\
\hline 3332 & 59.6273 & 344.9029 & 354.0038 & 332.5469 & 122.5994 & 1.54933 \\
\hline 3333 & 59.6443 & 366.6765 & 354.3088 & 332.5625 & 122.5994 & 1.54893 \\
\hline 3334 & 59.6613 & 353.5178 & 354.9494 & 332.5781 & 122.5994 & 1.54853 \\
\hline 3335 & 59.6783 & 329.7819 & 355.9568 & 332.5938 & 122.5994 & 1.54813 \\
\hline 3336 & 59.6953 & 321.3261 & 357.0703 & 332.6094 & 122.5994 & 1.54773 \\
\hline 3337 & 59.7123 & 340.264 & 358.0439 & 332.625 & 122.5994 & 1.54733 \\
\hline 3338 & 59.7293 & 358.0368 & 358.9517 & 332.6406 & 122.5994 & 1.54693 \\
\hline 3339 & 59.7463 & 373.5811 & 360.288 & 332.6563 & 122.5994 & 1.54653 \\
\hline 3340 & 59.7633 & 341.3748 & 362.2184 & 332.6719 & 122.5994 & 1.54613 \\
\hline 3341 & 59.7803 & 360.7977 & 364.7918 & 332.6875 & 122.5994 & 1.54573 \\
\hline 3342 & 59.7973 & 316.0103 & 368.0441 & 332.7031 & 122.5994 & 1.54533 \\
\hline 3343 & 59.8143 & 356.3003 & 372.209 & 332.7188 & 122.5994 & 1.54494 \\
\hline 3344 & 59.8313 & 360.9654 & 377.7484 & 332.7344 & 122.5994 & 1.54454 \\
\hline 3345 & 59.8483 & 386.7984 & 385.704 & 332.75 & 122.5994 & 1.54414 \\
\hline 3346 & 59.8653 & 399.6289 & 396.825 & 332.7656 & 122.5994 & 1.54374 \\
\hline 3347 & 59.8823 & 461.7999 & 414.375 & 332.7813 & 122.5994 & 1.54334 \\
\hline 3348 & 59.8993 & 497.3762 & 444.7259 & 332.7969 & 122.5994 & 1.54295 \\
\hline 3349 & 59.9163 & 510.298 & 498.8372 & 332.8125 & 122.5994 & 1.54255 \\
\hline 3350 & 59.9333 & 621.5918 & 591.542 & 332.8281 & 122.5994 & 1.54215 \\
\hline 3351 & 59.9503 & 740.1495 & 735.5695 & 332.8438 & 122.5994 & 1.54176 \\
\hline 3352 & 59.9673 & 885.2611 & 929.5555 & 332.8594 & 122.5994 & 1.54136 \\
\hline 3353 & 59.9843 & 1027.88 & 1141.552 & 332.875 & 122.5994 & 1.54096 \\
\hline 3354 & 60.0013 & 1108.182 & 1290.791 & 332.8906 & 122.5994 & 1.54057 \\
\hline 3355 & 60.0183 & 1126.985 & 1282.828 & 332.9063 & 122.5994 & 1.54017 \\
\hline 3356 & 60.0353 & 1012.995 & 1127.766 & 332.9219 & 122.5994 & 1.53978 \\
\hline 3357 & 60.0523 & 821.1809 & 927.3355 & 332.9375 & 122.5994 & 1.53938 \\
\hline 3358 & 60.0693 & 678.7165 & 759.1168 & 332.9531 & 122.5994 & 1.53899 \\
\hline 3359 & 60.0863 & 610.3581 & 655.9984 & 332.9688 & 122.5994 & 1.53859 \\
\hline 3360 & 60.1033 & 626.4814 & 624.1492 & 332.9844 & 122.5994 & 1.5382 \\
\hline 3361 & 60.1203 & 614.2454 & 654.853 & 333 & 122.5994 & 1.5378 \\
\hline 3362 & 60.1373 & 685.743 & 728.6214 & 332.625 & 122.5994 & 1.53741 \\
\hline 3363 & 60.1543 & 734.9073 & 812.3473 & 332.25 & 122.5994 & 1.53701 \\
\hline 3364 & 60.1713 & 745.7572 & 853.1702 & 331.875 & 122.5994 & 1.53662 \\
\hline 3365 & 60.1883 & 707.4921 & 811.8796 & 331.5 & 122.5994 & 1.53623 \\
\hline 3366 & 60.2053 & 656.6578 & 712.7739 & 331.125 & 122.5994 & 1.53583 \\
\hline 3367 & 60.2223 & 530.5133 & 604.9725 & 330.75 & 122.5994 & 1.53544 \\
\hline 3368 & 60.2393 & 450.9804 & 516.6511 & 330.375 & 122.5994 & 1.53505 \\
\hline 3369 & 60.2563 & 412.1895 & 455.3904 & 330 & 122.5994 & 1.53466 \\
\hline 3370 & 60.2733 & 387.9706 & 417.8527 & 329.625 & 122.5994 & 1.53426 \\
\hline 3371 & 60.2903 & 386.0805 & 396.5954 & 329.25 & 122.5994 & 1.53387 \\
\hline 3372 & 60.3073 & 382.291 & 384.6423 & 328.875 & 122.5994 & 1.53348 \\
\hline 3373 & 60.3243 & 371.4044 & 377.0591 & 328.5 & 122.5994 & 1.53309 \\
\hline 3374 & 60.3413 & 360.9654 & 371.1283 & 328.125 & 122.5994 & 1.5327 \\
\hline 3375 & 60.3583 & 374.8524 & 366.1381 & 327.75 & 122.5994 & 1.53231 \\
\hline 3376 & 60.3753 & 340.3638 & 362.2945 & 327.375 & 122.5994 & 1.53192 \\
\hline 3377 & 60.3923 & 331.7619 & 359.7824 & 327 & 122.5994 & 1.53153 \\
\hline 3378 & 60.4093 & 380.4412 & 358.7941 & 326.625 & 122.5994 & 1.53113 \\
\hline 3379 & 60.4263 & 360.579 & 359.155 & 326.25 & 122.5994 & 1.53074 \\
\hline
\end{tabular}




\begin{tabular}{|c|c|c|c|c|c|c|}
\hline 3380 & 60.4433 & 321.9194 & 360.482 & 325.875 & 122.5994 & 1.53035 \\
\hline 3381 & 60.4603 & 328.9204 & 362.1796 & 325.5 & 122.5994 & 1.52997 \\
\hline 3382 & 60.4773 & 351.5184 & 363.4073 & 325.125 & 122.5994 & 1.52958 \\
\hline 3383 & 60.4943 & 371.9665 & 363.5728 & 324.75 & 122.5994 & 1.52919 \\
\hline 3384 & 60.5113 & 329.3322 & 363.3573 & 324.375 & 122.5994 & 1.5288 \\
\hline 3385 & 60.5283 & 299.8479 & 364.0587 & 324 & 122.5994 & 1.52841 \\
\hline 3386 & 60.5453 & 310.3401 & 367.379 & 324.4479 & 122.5994 & 1.52802 \\
\hline 3387 & 60.5623 & 347.6291 & 372.4654 & 324.8958 & 122.5994 & 1.52763 \\
\hline 3388 & 60.5793 & 363.3622 & 378.9472 & 325.3438 & 122.5994 & 1.52724 \\
\hline 3389 & 60.5963 & 331.404 & 385.9253 & 325.7917 & 122.5994 & 1.52686 \\
\hline 3390 & 60.6133 & 351.3842 & 392.1852 & 326.2396 & 122.5994 & 1.52647 \\
\hline 3391 & 60.6303 & 353.4061 & 396.977 & 326.6875 & 122.5994 & 1.52608 \\
\hline 3392 & 60.6473 & 332.1354 & 400.4058 & 327.1354 & 122.5994 & 1.52569 \\
\hline 3393 & 60.6643 & 313.5596 & 402.841 & 327.5833 & 122.5994 & 1.52531 \\
\hline 3394 & 60.6813 & 342.4767 & 404.0857 & 328.0313 & 122.5994 & 1.52492 \\
\hline 3395 & 60.6983 & 379.1219 & 402.6168 & 328.4792 & 122.5994 & 1.52453 \\
\hline 3396 & 60.7153 & 339.1375 & 395.325 & 328.9271 & 122.5994 & 1.52415 \\
\hline 3397 & 60.7323 & 349.9162 & 388.8483 & 329.375 & 122.5994 & 1.52376 \\
\hline 3398 & 60.7493 & 355.5706 & 383.0724 & 329.8229 & 122.5994 & 1.52338 \\
\hline 3399 & 60.7663 & 372.078 & 378.8022 & 330.2708 & 122.5994 & 1.52299 \\
\hline 3400 & 60.7833 & 345.6802 & 375.8546 & 330.7188 & 122.5994 & 1.52261 \\
\hline 3401 & 60.8003 & 392.0923 & 373.9496 & 331.1667 & 122.5994 & 1.52222 \\
\hline 3402 & 60.8173 & 382.5464 & 372.9609 & 331.6146 & 122.5994 & 1.52184 \\
\hline 3403 & 60.8343 & 351.5946 & 372.5785 & 332.0625 & 122.5994 & 1.52145 \\
\hline 3404 & 60.8513 & 396.1939 & 371.9571 & 332.5104 & 122.5994 & 1.52107 \\
\hline 3405 & 60.8683 & 364.1121 & 368.4689 & 332.9583 & 122.5994 & 1.52068 \\
\hline 3406 & 60.8853 & 351.6339 & 364.3412 & 333.4063 & 122.5994 & 1.5203 \\
\hline 3407 & 60.9023 & 363.7277 & 359.3716 & 333.8542 & 122.5994 & 1.51992 \\
\hline 3408 & 60.9193 & 375.5757 & 354.743 & 334.3021 & 122.5994 & 1.51953 \\
\hline 3409 & 60.9363 & 354.9779 & 350.9536 & 334.75 & 122.5994 & 1.51915 \\
\hline 3410 & 60.9533 & 338.7991 & 347.825 & 334.8542 & 122.5994 & 1.51877 \\
\hline 3411 & 60.9703 & 347.3088 & 345.6474 & 334.9583 & 122.5994 & 1.51838 \\
\hline 3412 & 60.9873 & 351.1262 & 344.23 & 335.0625 & 122.5994 & 1.518 \\
\hline 3413 & 61.0043 & 344.3055 & 343.3238 & 335.1667 & 122.5994 & 1.51762 \\
\hline 3414 & 61.0213 & 335.9976 & 342.7932 & 335.2708 & 122.5994 & 1.51724 \\
\hline 3415 & 61.0383 & 350.8579 & 342.5926 & 335.375 & 122.5994 & 1.51685 \\
\hline 3416 & 61.0553 & 352.0015 & 342.5987 & 335.4792 & 122.5994 & 1.51647 \\
\hline 3417 & 61.0723 & 346.0136 & 342.5637 & 335.5833 & 122.5994 & 1.51609 \\
\hline 3418 & 61.0893 & 373.7959 & 342.7408 & 335.6875 & 122.5994 & 1.51571 \\
\hline 3419 & 61.1063 & 383.2109 & 343.2179 & 335.7917 & 122.5994 & 1.51533 \\
\hline 3420 & 61.1233 & 347.2177 & 344.0559 & 335.8958 & 122.5994 & 1.51495 \\
\hline 3421 & 61.1403 & 353.8619 & 345.214 & 336 & 122.5994 & 1.51457 \\
\hline 3422 & 61.1573 & 370.2016 & 346.4348 & 336.1042 & 122.5994 & 1.51419 \\
\hline 3423 & 61.1743 & 363.8013 & 347.2502 & 336.2083 & 122.5994 & 1.51381 \\
\hline 3424 & 61.1913 & 348.7755 & 347.582 & 336.3125 & 122.5994 & 1.51343 \\
\hline 3425 & 61.2083 & 330.0357 & 347.817 & 336.4167 & 122.5994 & 1.51305 \\
\hline 3426 & 61.2253 & 311.6647 & 348.2504 & 336.5208 & 122.5994 & 1.51267 \\
\hline 3427 & 61.2423 & 340.0243 & 349.1206 & 336.625 & 122.5994 & 1.51229 \\
\hline 3428 & 61.2593 & 367.4319 & 350.6768 & 336.7292 & 122.5994 & 1.51191 \\
\hline
\end{tabular}




\begin{tabular}{|c|c|c|c|c|c|c|}
\hline 3429 & 61.2763 & 372.5397 & 353.0217 & 336.8333 & 122.5994 & 1.51153 \\
\hline 3430 & 61.2933 & 351.7785 & 356.1697 & 336.9375 & 122.5994 & 1.51115 \\
\hline 3431 & 61.3103 & 340.0012 & 360.1381 & 337.0417 & 122.5994 & 1.51078 \\
\hline 3432 & 61.3273 & 350.8425 & 364.6118 & 337.1458 & 122.5994 & 1.5104 \\
\hline 3433 & 61.3443 & 392.6946 & 368.9055 & 337.25 & 122.5994 & 1.51002 \\
\hline 3434 & 61.3613 & 340.5433 & 371.8771 & 337.0339 & 122.5994 & 1.50964 \\
\hline 3435 & 61.3783 & 371.2249 & 373.5373 & 336.8177 & 122.5994 & 1.50926 \\
\hline 3436 & 61.3953 & 358.9297 & 373.4196 & 336.6016 & 122.5994 & 1.50889 \\
\hline 3437 & 61.4123 & 343.3453 & 371.0423 & 336.3854 & 122.5994 & 1.50851 \\
\hline 3438 & 61.4293 & 345.7468 & 367.2375 & 336.1693 & 122.5994 & 1.50813 \\
\hline 3439 & 61.4463 & 353.2844 & 364.2993 & 335.9531 & 122.5994 & 1.50776 \\
\hline 3440 & 61.4633 & 353.4061 & 362.6584 & 335.737 & 122.5994 & 1.50738 \\
\hline 3441 & 61.4803 & 357.0554 & 361.775 & 335.5208 & 122.5994 & 1.50701 \\
\hline 3442 & 61.4973 & 350.6547 & 361.0533 & 335.3047 & 122.5994 & 1.50663 \\
\hline 3443 & 61.5143 & 377.8068 & 360.4732 & 335.0885 & 122.5994 & 1.50625 \\
\hline 3444 & 61.5313 & 397.4372 & 360.0522 & 334.8724 & 122.5994 & 1.50588 \\
\hline 3445 & 61.5483 & 371.4849 & 359.7609 & 334.6563 & 122.5994 & 1.5055 \\
\hline 3446 & 61.5653 & 396.9606 & 359.3691 & 334.4401 & 122.5994 & 1.50513 \\
\hline 3447 & 61.5823 & 403.9712 & 358.7188 & 334.224 & 122.5994 & 1.50475 \\
\hline 3448 & 61.5993 & 404.0639 & 358.4351 & 334.0078 & 122.5994 & 1.50438 \\
\hline 3449 & 61.6163 & 401.7202 & 359.8733 & 333.7917 & 122.5994 & 1.504 \\
\hline 3450 & 61.6333 & 392.9248 & 363.1743 & 333.5755 & 122.5994 & 1.50363 \\
\hline 3451 & 61.6503 & 403.4791 & 367.6032 & 333.3594 & 122.5994 & 1.50326 \\
\hline 3452 & 61.6673 & 427.5031 & 371.7411 & 333.1432 & 122.5994 & 1.50288 \\
\hline 3453 & 61.6843 & 417.6398 & 374.2564 & 332.9271 & 122.5994 & 1.50251 \\
\hline 3454 & 61.7013 & 382.8896 & 375.7032 & 332.7109 & 122.5994 & 1.50214 \\
\hline 3455 & 61.7183 & 358.0924 & 377.6234 & 332.4948 & 122.5994 & 1.50176 \\
\hline 3456 & 61.7353 & 385.381 & 380.3945 & 332.2786 & 122.5994 & 1.50139 \\
\hline 3457 & 61.7523 & 379.5843 & 383.2737 & 332.0625 & 122.5994 & 1.50102 \\
\hline 3458 & 61.7693 & 398.1105 & 386.0749 & 331.8464 & 122.5994 & 1.50065 \\
\hline 3459 & 61.7863 & 396.8837 & 389.3408 & 331.6302 & 122.5994 & 1.50027 \\
\hline 3460 & 61.8033 & 381.8746 & 393.0779 & 331.4141 & 122.5994 & 1.4999 \\
\hline 3461 & 61.8203 & 380.6424 & 396.5414 & 331.1979 & 122.5994 & 1.49953 \\
\hline 3462 & 61.8373 & 387.4713 & 398.6525 & 330.9818 & 122.5994 & 1.49916 \\
\hline 3463 & 61.8543 & 375.344 & 398.6143 & 330.7656 & 122.5994 & 1.49879 \\
\hline 3464 & 61.8713 & 357.4625 & 396.8642 & 330.5495 & 122.5994 & 1.49842 \\
\hline 3465 & 61.8883 & 370.9241 & 394.4756 & 330.3333 & 122.5994 & 1.49805 \\
\hline 3466 & 61.9053 & 391.6367 & 391.8731 & 330.1172 & 122.5994 & 1.49768 \\
\hline 3467 & 61.9223 & 390.3909 & 388.7517 & 329.901 & 122.5994 & 1.49731 \\
\hline 3468 & 61.9393 & 361.9729 & 385.0396 & 329.6849 & 122.5994 & 1.49694 \\
\hline 3469 & 61.9563 & 349.7366 & 381.1916 & 329.4688 & 122.5994 & 1.49657 \\
\hline 3470 & 61.9733 & 359.3325 & 377.3151 & 329.2526 & 122.5994 & 1.4962 \\
\hline 3471 & 61.9903 & 357.3363 & 373.4315 & 329.0365 & 122.5994 & 1.49583 \\
\hline 3472 & 62.0073 & 340.7361 & 369.6239 & 328.8203 & 122.5994 & 1.49546 \\
\hline 3473 & 62.0243 & 386.3876 & 365.9644 & 328.6042 & 122.5994 & 1.49509 \\
\hline 3474 & 62.0413 & 368.4499 & 362.4869 & 328.388 & 122.5994 & 1.49472 \\
\hline 3475 & 62.0583 & 359.0573 & 359.2654 & 328.1719 & 122.5994 & 1.49435 \\
\hline 3476 & 62.0753 & 363.9786 & 356.329 & 327.9557 & 122.5994 & 1.49398 \\
\hline 3477 & 62.0923 & 358.7666 & 353.6794 & 327.7396 & 122.5994 & 1.49361 \\
\hline
\end{tabular}




\begin{tabular}{|c|c|c|c|c|c|c|}
\hline 3478 & 62.1093 & 357.1757 & 351.2902 & 327.5234 & 122.5994 & 1.49325 \\
\hline 3479 & 62.1263 & 341.7101 & 349.0959 & 327.3073 & 122.5994 & 1.49288 \\
\hline 3480 & 62.1433 & 338.5275 & 347.3224 & 327.0911 & 122.5994 & 1.49251 \\
\hline 3481 & 62.1603 & 358.2787 & 345.7065 & 326.875 & 122.5994 & 1.49214 \\
\hline 3482 & 62.1773 & 318.1151 & 343.8778 & 326.3385 & 122.5994 & 1.49178 \\
\hline 3483 & 62.1943 & 294.1266 & 342.1142 & 325.8021 & 122.5994 & 1.49141 \\
\hline 3484 & 62.2113 & 353.3723 & 340.434 & 325.2656 & 122.5994 & 1.49104 \\
\hline 3485 & 62.2283 & 346.3462 & 338.9207 & 324.7292 & 122.5994 & 1.49068 \\
\hline 3486 & 62.2453 & 313.8497 & 337.5374 & 324.1927 & 122.5994 & 1.49031 \\
\hline 3487 & 62.2623 & 361.004 & 336.0332 & 323.6562 & 122.5994 & 1.48994 \\
\hline 3488 & 62.2793 & 352.8011 & 334.444 & 323.1198 & 122.5994 & 1.48958 \\
\hline 3489 & 62.2963 & 331.3859 & 332.9945 & 322.5833 & 122.5994 & 1.48921 \\
\hline 3490 & 62.3133 & 333.1674 & 331.8515 & 322.0469 & 122.5994 & 1.48885 \\
\hline 3491 & 62.3303 & 309.4083 & 330.9954 & 321.5104 & 122.5994 & 1.48848 \\
\hline 3492 & 62.3473 & 308.7129 & 330.2421 & 320.974 & 122.5994 & 1.48812 \\
\hline 3493 & 62.3643 & 327.0919 & 329.4401 & 320.4375 & 122.5994 & 1.48775 \\
\hline 3494 & 62.3813 & 325.176 & 328.4994 & 319.901 & 122.5994 & 1.48739 \\
\hline 3495 & 62.3983 & 331.8575 & 327.4706 & 319.3646 & 122.5994 & 1.48702 \\
\hline 3496 & 62.4153 & 335.5893 & 326.4155 & 318.8281 & 122.5994 & 1.48666 \\
\hline 3497 & 62.4323 & 332.2417 & 325.2258 & 318.2917 & 122.5994 & 1.48629 \\
\hline 3498 & 62.4493 & 304.6986 & 324.0102 & 317.7552 & 122.5994 & 1.48593 \\
\hline 3499 & 62.4663 & 301.1546 & 322.8349 & 317.2187 & 122.5994 & 1.48557 \\
\hline 3500 & 62.4833 & 320.7912 & 321.8951 & 316.6823 & 122.5994 & 1.4852 \\
\hline 3501 & 62.5003 & 307.6359 & 321.2237 & 316.1458 & 122.5994 & 1.48484 \\
\hline 3502 & 62.5173 & 345.0864 & 320.8428 & 315.6094 & 122.5994 & 1.48448 \\
\hline 3503 & 62.5343 & 317.8877 & 320.7646 & 315.0729 & 122.5994 & 1.48411 \\
\hline 3504 & 62.5513 & 315.9774 & 320.8744 & 314.5365 & 122.5994 & 1.48375 \\
\hline 3505 & 62.5683 & 312.079 & 321.0848 & 314 & 122.5994 & 1.48339 \\
\hline 3506 & 62.5853 & 308.0874 & 321.2198 & 313.5 & 122.5994 & 1.48303 \\
\hline 3507 & 62.6023 & 311.1694 & 321.0643 & 313 & 122.5994 & 1.48267 \\
\hline 3508 & 62.6193 & 297.6829 & 320.5298 & 312.5 & 122.5994 & 1.4823 \\
\hline 3509 & 62.6363 & 289.3631 & 319.7553 & 312 & 122.5994 & 1.48194 \\
\hline 3510 & 62.6533 & 283.8998 & 318.9806 & 311.5 & 122.5994 & 1.48158 \\
\hline 3511 & 62.6703 & 307.581 & 318.1169 & 311 & 122.5994 & 1.48122 \\
\hline 3512 & 62.6873 & 321.7236 & 317.3026 & 310.5 & 122.5994 & 1.48086 \\
\hline 3513 & 62.7043 & 316.8822 & 316.6578 & 310 & 122.5994 & 1.4805 \\
\hline 3514 & 62.7213 & 310.5964 & 316.2412 & 309.5 & 122.5994 & 1.48014 \\
\hline 3515 & 62.7383 & 321.4176 & 316.0003 & 309 & 122.5994 & 1.47978 \\
\hline 3516 & 62.7553 & 304.6825 & 315.9571 & 308.5 & 122.5994 & 1.47942 \\
\hline 3517 & 62.7723 & 284.5702 & 316.0562 & 308 & 122.5994 & 1.47906 \\
\hline 3518 & 62.7893 & 289.1204 & 316.2732 & 307.5 & 122.5994 & 1.4787 \\
\hline 3519 & 62.8063 & 304.4274 & 316.695 & 307 & 122.5994 & 1.47834 \\
\hline 3520 & 62.8233 & 302.2371 & 317.2841 & 306.5 & 122.5994 & 1.47798 \\
\hline 3521 & 62.8403 & 304.4617 & 317.6198 & 306 & 122.5994 & 1.47762 \\
\hline 3522 & 62.8573 & 303.5726 & 317.2286 & 305.5 & 122.5994 & 1.47726 \\
\hline 3523 & 62.8743 & 308.2632 & 315.9382 & 305 & 122.5994 & 1.4769 \\
\hline 3524 & 62.8913 & 298.5689 & 314.1251 & 304.5 & 122.5994 & 1.47655 \\
\hline 3525 & 62.9083 & 307.3422 & 312.3243 & 304 & 122.5994 & 1.47619 \\
\hline 3526 & 62.9253 & 330.9625 & 310.8489 & 303.5 & 122.5994 & 1.47583 \\
\hline
\end{tabular}




\begin{tabular}{|c|c|c|c|c|c|c|}
\hline 3527 & 62.9423 & 334.8579 & 309.8245 & 303 & 122.5994 & 1.47547 \\
\hline 3528 & 62.9593 & 297.2139 & 309.2808 & 302.5 & 122.5994 & 1.47512 \\
\hline 3529 & 62.9763 & 304.9678 & 309.0464 & 302 & 122.5994 & 1.47476 \\
\hline 3530 & 62.9933 & 283.9367 & 308.8369 & 301.2917 & 122.5994 & 1.4744 \\
\hline 3531 & 63.0103 & 305.0814 & 308.6125 & 300.5833 & 122.5994 & 1.47404 \\
\hline 3532 & 63.0273 & 313.0767 & 308.0261 & 299.875 & 122.5994 & 1.47369 \\
\hline 3533 & 63.0443 & 306.3233 & 306.9334 & 299.1667 & 122.5994 & 1.47333 \\
\hline 3534 & 63.0613 & 299.8755 & 305.4572 & 298.4583 & 122.5994 & 1.47297 \\
\hline 3535 & 63.0783 & 330.6539 & 303.8902 & 297.75 & 122.5994 & 1.47262 \\
\hline 3536 & 63.0953 & 315.4128 & 302.4873 & 297.0417 & 122.5994 & 1.47226 \\
\hline 3537 & 63.1123 & 294.9587 & 301.2718 & 296.3333 & 122.5994 & 1.47191 \\
\hline 3538 & 63.1293 & 322.5024 & 300.3319 & 295.625 & 122.5994 & 1.47155 \\
\hline 3539 & 63.1463 & 311.5761 & 299.6813 & 294.9167 & 122.5994 & 1.4712 \\
\hline 3540 & 63.1633 & 317.8695 & 299.3259 & 294.2083 & 122.5994 & 1.47084 \\
\hline 3541 & 63.1803 & 283.9832 & 299.2361 & 293.5 & 122.5994 & 1.47049 \\
\hline 3542 & 63.1973 & 290.4585 & 299.2839 & 292.7917 & 122.5994 & 1.47013 \\
\hline 3543 & 63.2143 & 286.6028 & 299.2652 & 292.0833 & 122.5994 & 1.46978 \\
\hline 3544 & 63.2313 & 306.0003 & 298.9349 & 291.375 & 122.5994 & 1.46942 \\
\hline 3545 & 63.2483 & 314.1238 & 298.2607 & 290.6667 & 122.5994 & 1.46907 \\
\hline 3546 & 63.2653 & 309.2059 & 297.2941 & 289.9583 & 122.5994 & 1.46872 \\
\hline 3547 & 63.2823 & 311.6027 & 296.2321 & 289.25 & 122.5994 & 1.46836 \\
\hline 3548 & 63.2993 & 308.3452 & 295.1128 & 288.5417 & 122.5994 & 1.46801 \\
\hline 3549 & 63.3163 & 320.3213 & 294.1678 & 287.8333 & 122.5994 & 1.46765 \\
\hline 3550 & 63.3333 & 301.2219 & 293.8681 & 287.125 & 122.5994 & 1.4673 \\
\hline 3551 & 63.3503 & 289.8457 & 293.4553 & 286.4167 & 122.5994 & 1.46695 \\
\hline 3552 & 63.3673 & 281.5199 & 293.3618 & 285.7083 & 122.5994 & 1.4666 \\
\hline 3553 & 63.3843 & 288.6675 & 293.6041 & 285 & 122.5994 & 1.46624 \\
\hline 3554 & 63.4013 & 291.0118 & 295.0522 & 285.1479 & 122.5994 & 1.46589 \\
\hline 3555 & 63.4183 & 281.7407 & 296.9537 & 285.2957 & 122.5994 & 1.46554 \\
\hline 3556 & 63.4353 & 304.3621 & 299.3854 & 285.4436 & 122.5994 & 1.46519 \\
\hline 3557 & 63.4523 & 327.9261 & 302.4411 & 285.5914 & 122.5994 & 1.46484 \\
\hline 3558 & 63.4693 & 327.0184 & 306.0282 & 285.7393 & 122.5994 & 1.46449 \\
\hline 3559 & 63.4863 & 308.9969 & 309.2467 & 285.8872 & 122.5994 & 1.46413 \\
\hline 3560 & 63.5033 & 308.8712 & 310.6754 & 286.035 & 122.5994 & 1.46378 \\
\hline 3561 & 63.5203 & 321.8226 & 309.586 & 286.1829 & 122.5994 & 1.46343 \\
\hline 3562 & 63.5373 & 320.908 & 307.3241 & 286.3308 & 122.5994 & 1.46308 \\
\hline 3563 & 63.5543 & 301.2222 & 305.2156 & 286.4786 & 122.5994 & 1.46273 \\
\hline 3564 & 63.5713 & 292.382 & 303.7492 & 286.6265 & 122.5994 & 1.46238 \\
\hline 3565 & 63.5883 & 287.7972 & 303.0332 & 286.7743 & 122.5994 & 1.46203 \\
\hline 3566 & 63.6053 & 292.8 & 303.0259 & 286.9222 & 122.5994 & 1.46168 \\
\hline 3567 & 63.6223 & 318.9813 & 303.6267 & 287.0701 & 122.5994 & 1.46133 \\
\hline 3568 & 63.6393 & 341.315 & 304.7837 & 287.2179 & 122.5994 & 1.46098 \\
\hline 3569 & 63.6563 & 318.0529 & 306.5322 & 287.3658 & 122.5994 & 1.46063 \\
\hline 3570 & 63.6733 & 309.8013 & 308.4984 & 287.5136 & 122.5994 & 1.46028 \\
\hline 3571 & 63.6903 & 327.195 & 310.3044 & 287.6615 & 122.5994 & 1.45994 \\
\hline 3572 & 63.7073 & 310.4245 & 311.9643 & 287.8094 & 122.5994 & 1.45959 \\
\hline 3573 & 63.7243 & 340.2179 & 313.4916 & 287.9572 & 122.5994 & 1.45924 \\
\hline 3574 & 63.7413 & 326.6556 & 314.2656 & 288.1051 & 122.5994 & 1.45889 \\
\hline 3575 & 63.7583 & 327.2893 & 313.2941 & 288.253 & 122.5994 & 1.45854 \\
\hline
\end{tabular}




\begin{tabular}{|c|c|c|c|c|c|c|}
\hline 3576 & 63.7753 & 314.3952 & 310.6774 & 288.4008 & 122.5994 & 1.45819 \\
\hline 3577 & 63.7923 & 337.1431 & 307.7886 & 288.5487 & 122.5994 & 1.45785 \\
\hline 3578 & 63.8093 & 327.975 & 305.7473 & 288.5994 & 122.5994 & 1.4575 \\
\hline 3579 & 63.8263 & 320.5592 & 305.3908 & 288.6501 & 122.5994 & 1.45715 \\
\hline 3580 & 63.8433 & 309.9471 & 307.188 & 288.7008 & 122.5994 & 1.4568 \\
\hline 3581 & 63.8603 & 342.2956 & 311.0395 & 288.7515 & 122.5994 & 1.45646 \\
\hline 3582 & 63.8773 & 341.3094 & 316.1305 & 288.8022 & 122.5994 & 1.45611 \\
\hline 3583 & 63.8943 & 324.5601 & 320.841 & 288.8528 & 122.5994 & 1.45576 \\
\hline 3584 & 63.9113 & 329.5422 & 323.2802 & 288.9035 & 122.5994 & 1.45542 \\
\hline 3585 & 63.9283 & 315.8041 & 322.6537 & 288.9542 & 122.5994 & 1.45507 \\
\hline 3586 & 63.9453 & 340.4929 & 320.1434 & 289.0049 & 122.5994 & 1.45473 \\
\hline 3587 & 63.9623 & 376.9358 & 317.946 & 289.0556 & 122.5994 & 1.45438 \\
\hline 3588 & 63.9793 & 378.223 & 318.0971 & 289.1063 & 122.5994 & 1.45404 \\
\hline 3589 & 63.9963 & 374.0888 & 322.3138 & 289.157 & 122.5994 & 1.45369 \\
\hline 3590 & 64.0133 & 396.0628 & 332.3799 & 289.2077 & 122.5994 & 1.45335 \\
\hline 3591 & 64.0303 & 402.9709 & 350.0594 & 289.2584 & 122.5994 & 1.453 \\
\hline 3592 & 64.0473 & 419.8995 & 376.0301 & 289.3091 & 122.5994 & 1.45266 \\
\hline 3593 & 64.0643 & 421.1737 & 407.9243 & 289.3598 & 122.5994 & 1.45231 \\
\hline 3594 & 64.0813 & 456.3883 & 437.6404 & 289.4105 & 122.5994 & 1.45197 \\
\hline 3595 & 64.0983 & 448.2669 & 450.8826 & 289.4612 & 122.5994 & 1.45162 \\
\hline 3596 & 64.1153 & 410.4042 & 438.8451 & 289.5119 & 122.5994 & 1.45128 \\
\hline 3597 & 64.1323 & 376.3104 & 411.0746 & 289.5626 & 122.5994 & 1.45094 \\
\hline 3598 & 64.1493 & 363.1521 & 381.866 & 289.6133 & 122.5994 & 1.45059 \\
\hline 3599 & 64.1663 & 360.7151 & 359.2561 & 289.664 & 122.5994 & 1.45025 \\
\hline 3600 & 64.1833 & 338.9451 & 345.9998 & 289.7147 & 122.5994 & 1.44991 \\
\hline 3601 & 64.2003 & 376.6882 & 342.2389 & 289.7654 & 122.5994 & 1.44956 \\
\hline 3602 & 64.2173 & 383.2345 & 346.7486 & 289.8161 & 122.5994 & 1.44922 \\
\hline 3603 & 64.2343 & 386.6766 & 357.5897 & 289.8668 & 122.5994 & 1.44888 \\
\hline 3604 & 64.2513 & 393.448 & 371.216 & 289.9174 & 122.5994 & 1.44854 \\
\hline 3605 & 64.2683 & 367.8745 & 381.0752 & 289.9681 & 122.5994 & 1.44819 \\
\hline 3606 & 64.2853 & 340.1016 & 379.858 & 290.0188 & 122.5994 & 1.44785 \\
\hline 3607 & 64.3023 & 334.77 & 367.5228 & 290.0695 & 122.5994 & 1.44751 \\
\hline 3608 & 64.3193 & 353.7223 & 351.0759 & 290.1202 & 122.5994 & 1.44717 \\
\hline 3609 & 64.3363 & 342.1156 & 335.9597 & 290.1709 & 122.5994 & 1.44683 \\
\hline 3610 & 64.3533 & 328.9164 & 324.3969 & 290.2216 & 122.5994 & 1.44649 \\
\hline 3611 & 64.3703 & 297.0783 & 316.4967 & 290.2723 & 122.5994 & 1.44614 \\
\hline 3612 & 64.3873 & 293.9998 & 311.5801 & 290.323 & 122.5994 & 1.4458 \\
\hline 3613 & 64.4043 & 319.6284 & 308.6587 & 290.3737 & 122.5994 & 1.44546 \\
\hline 3614 & 64.4213 & 311.8814 & 306.9734 & 290.4244 & 122.5994 & 1.44512 \\
\hline 3615 & 64.4383 & 298.3787 & 305.8953 & 290.4751 & 122.5994 & 1.44478 \\
\hline 3616 & 64.4553 & 303.8766 & 304.919 & 290.5258 & 122.5994 & 1.44444 \\
\hline 3617 & 64.4723 & 295.1788 & 303.7917 & 290.5765 & 122.5994 & 1.4441 \\
\hline 3618 & 64.4893 & 315.5362 & 302.5725 & 290.6272 & 122.5994 & 1.44376 \\
\hline 3619 & 64.5063 & 314.2842 & 301.2807 & 290.6779 & 122.5994 & 1.44342 \\
\hline 3620 & 64.5233 & 325.7527 & 300.1316 & 290.7286 & 122.5994 & 1.44308 \\
\hline 3621 & 64.5403 & 296.3597 & 299.1557 & 290.7793 & 122.5994 & 1.44275 \\
\hline 3622 & 64.5573 & 321.0689 & 298.4443 & 290.83 & 122.5994 & 1.44241 \\
\hline 3623 & 64.5743 & 282.0064 & 298.0578 & 290.8807 & 122.5994 & 1.44207 \\
\hline 3624 & 64.5913 & 295.0894 & 298.094 & 290.9314 & 122.5994 & 1.44173 \\
\hline
\end{tabular}




$\begin{array}{rrrrrrr}3625 & 64.6083 & 295.8794 & 298.5662 & 290.9821 & 122.5994 & 1.44139 \\ 3626 & 64.6253 & 313.1304 & 299.3218 & 291.0327 & 122.5994 & 1.44105 \\ 3627 & 64.6423 & 292.7638 & 299.9649 & 291.0834 & 122.5994 & 1.44071 \\ 3628 & 64.6593 & 309.2474 & 300.0139 & 291.1341 & 122.5994 & 1.44038 \\ 3629 & 64.6763 & 298.7023 & 299.4412 & 291.1848 & 122.5994 & 1.44004 \\ 3630 & 64.6933 & 292.5291 & 298.8886 & 291.2355 & 122.5994 & 1.4397 \\ 3631 & 64.7103 & 329.4122 & 298.6481 & 291.2862 & 122.5994 & 1.43936 \\ 3632 & 64.7273 & 308.4539 & 298.7271 & 291.3369 & 122.5994 & 1.43903 \\ 3633 & 64.7443 & 277.5741 & 298.8893 & 291.3876 & 122.5994 & 1.43869 \\ 3634 & 64.7613 & 294.3398 & 298.9689 & 291.4383 & 122.5994 & 1.43835 \\ 3635 & 64.7783 & 317.6159 & 299.1884 & 291.489 & 122.5994 & 1.43802 \\ 3636 & 64.7953 & 300.501 & 299.9461 & 291.5397 & 122.5994 & 1.43768 \\ 3637 & 64.8123 & 285.3094 & 301.4692 & 291.5904 & 122.5994 & 1.43735 \\ 3638 & 64.8293 & 302.7628 & 303.6681 & 291.6411 & 122.5994 & 1.43701 \\ 3639 & 64.8463 & 301.0372 & 306.1655 & 291.6918 & 122.5994 & 1.43667 \\ 3640 & 64.8633 & 309.6198 & 308.0178 & 291.7425 & 122.5994 & 1.43634 \\ 3641 & 64.8803 & 278.8068 & 308.7767 & 291.7932 & 122.5994 & 1.436 \\ 3642 & 64.8973 & 297.37 & 307.6082 & 291.8439 & 122.5994 & 1.43567 \\ 3643 & 64.9143 & 323.0609 & 305.2938 & 291.8946 & 122.5994 & 1.43533 \\ 3644 & 64.9313 & 322.1563 & 303.2684 & 291.9453 & 122.5994 & 1.435 \\ 3645 & 64.9483 & 311.9002 & 301.8213 & 291.996 & 122.5994 & 1.43466 \\ 3646 & 64.9653 & 306.6721 & 301.5107 & 292.0467 & 122.5994 & 1.43433 \\ 3647 & 64.9823 & 292.0973 & 302.4983 & 292.0467 & 122.5994 & 1.434\end{array}$


No. Pos. [ ${ }^{\circ} 2$ Th. lobs [cts] Icalc [cts] lback [cts] CT [s] ESD D spacings

\begin{tabular}{|c|c|c|c|c|c|c|}
\hline 1 & 3.0003 & 2237.283 & 2237.283 & 2237.283 & 122.5994 & 29.42347 \\
\hline 2 & 3.0173 & 2143.302 & 2210.23 & 2210.23 & 122.5994 & 29.25773 \\
\hline 3 & 3.0343 & 2121.295 & 2183.177 & 2183.177 & 122.5994 & 29.09385 \\
\hline 4 & 3.0513 & 2034.602 & 2156.123 & 2156.123 & 122.5994 & 28.9318 \\
\hline 5 & 3.0683 & 2044.579 & 2129.07 & 2129.07 & 122.5994 & 28.77154 \\
\hline 6 & 3.0853 & 2053.423 & 2102.016 & 2102.016 & 122.5994 & 28.61305 \\
\hline 7 & 3.1023 & 1969.965 & 2074.963 & 2074.963 & 122.5994 & 28.45629 \\
\hline 8 & 3.1193 & 1858.778 & 2047.909 & 2047.909 & 122.5994 & 28.30124 \\
\hline 9 & 3.1363 & 1945.931 & 2020.856 & 2020.856 & 122.5994 & 28.14788 \\
\hline 10 & 3.1533 & 1857.931 & 1993.802 & 1993.802 & 122.5994 & 27.99617 \\
\hline 11 & 3.1703 & 1808.71 & 1966.749 & 1966.749 & 122.5994 & 27.84608 \\
\hline 12 & 3.1873 & 1751.66 & 1939.695 & 1939.695 & 122.5994 & 27.6976 \\
\hline 13 & 3.2043 & 1747.552 & 1912.642 & 1912.642 & 122.5994 & 27.55069 \\
\hline 14 & 3.2213 & 1753.755 & 1885.588 & 1885.588 & 122.5994 & 27.40534 \\
\hline 15 & 3.2383 & 1730.855 & 1858.535 & 1858.535 & 122.5994 & 27.26151 \\
\hline 16 & 3.2553 & 1762.316 & 1831.481 & 1831.481 & 122.5994 & 27.11918 \\
\hline 17 & 3.2723 & 1688.76 & 1804.428 & 1804.428 & 122.5994 & 26.97833 \\
\hline 18 & 3.2893 & 1656.473 & 1777.374 & 1777.374 & 122.5994 & 26.83894 \\
\hline 19 & 3.3063 & 1643.949 & 1750.321 & 1750.321 & 122.5994 & 26.70098 \\
\hline 20 & 3.3233 & 1640.674 & 1723.267 & 1723.267 & 122.5994 & 26.56443 \\
\hline 21 & 3.3403 & 1626.519 & 1696.214 & 1696.214 & 122.5994 & 26.42928 \\
\hline 22 & 3.3573 & 1579.689 & 1669.16 & 1669.16 & 122.5994 & 26.29549 \\
\hline 23 & 3.3743 & 1584.047 & 1642.107 & 1642.107 & 122.5994 & 26.16305 \\
\hline 24 & 3.3913 & 1603.867 & 1615.053 & 1615.053 & 122.5994 & 26.03194 \\
\hline 25 & 3.4083 & 1611.634 & 1588 & 1588 & 122.5994 & 25.90213 \\
\hline 26 & 3.4253 & 1574.381 & 1576.667 & 1576.667 & 122.5994 & 25.77362 \\
\hline 27 & 3.4423 & 1534.823 & 1565.333 & 1565.333 & 122.5994 & 25.64637 \\
\hline 28 & 3.4593 & 1530.071 & 1554 & 1554 & 122.5994 & 25.52038 \\
\hline 29 & 3.4763 & 1550.871 & 1542.667 & 1542.667 & 122.5994 & 25.39561 \\
\hline 30 & 3.4933 & 1511.587 & 1531.333 & 1531.333 & 122.5994 & 25.27207 \\
\hline 31 & 3.5103 & 1447.76 & 1520 & 1520 & 122.5994 & 25.14971 \\
\hline 32 & 3.5273 & 1449.123 & 1508.667 & 1508.667 & 122.5994 & 25.02854 \\
\hline 33 & 3.5443 & 1464.514 & 1497.333 & 1497.333 & 122.5994 & 24.90853 \\
\hline 34 & 3.5613 & 1499.764 & 1486 & 1486 & 122.5994 & 24.78967 \\
\hline 35 & 3.5783 & 1418.576 & 1474.667 & 1474.667 & 122.5994 & 24.67194 \\
\hline 36 & 3.5953 & 1423.435 & 1463.333 & 1463.333 & 122.5994 & 24.55532 \\
\hline 37 & 3.6123 & 1413.055 & 1452 & 1452 & 122.5994 & 24.4398 \\
\hline 38 & 3.6293 & 1382.48 & 1440.667 & 1440.667 & 122.5994 & 24.32536 \\
\hline 39 & 3.6463 & 1346.287 & 1429.333 & 1429.333 & 122.5994 & 24.21198 \\
\hline 40 & 3.6633 & 1401.83 & 1418 & 1418 & 122.5994 & 24.09966 \\
\hline 41 & 3.6803 & 1331.064 & 1406.667 & 1406.667 & 122.5994 & 23.98838 \\
\hline 42 & 3.6973 & 1343.455 & 1395.333 & 1395.333 & 122.5994 & 23.87812 \\
\hline 43 & 3.7143 & 1340.272 & 1384 & 1384 & 122.5994 & 23.76887 \\
\hline 44 & 3.7313 & 1305.916 & 1372.667 & 1372.667 & 122.5994 & 23.66062 \\
\hline 45 & 3.7483 & 1351.527 & 1361.333 & 1361.333 & 122.5994 & 23.55335 \\
\hline 46 & 3.7653 & 1340.032 & 1350 & 1350 & 122.5994 & 23.44704 \\
\hline 47 & 3.7823 & 1398.159 & 1338.667 & 1338.667 & 122.5994 & 23.3417 \\
\hline
\end{tabular}




\begin{tabular}{|c|c|c|c|c|c|c|}
\hline 48 & 3.7993 & 1317.533 & 1327.333 & 1327.333 & 122.5994 & 23.23729 \\
\hline 49 & 3.8163 & 1303.216 & 1316 & 1316 & 122.5994 & 23.13382 \\
\hline 50 & 3.8333 & 1301.638 & 1309.563 & 1309.563 & 122.5994 & 23.03126 \\
\hline 51 & 3.8503 & 1279.984 & 1303.125 & 1303.125 & 122.5994 & 22.92961 \\
\hline 52 & 3.8673 & 1339.849 & 1296.688 & 1296.688 & 122.5994 & 22.82886 \\
\hline 53 & 3.8843 & 1276.179 & 1290.25 & 1290.25 & 122.5994 & 22.72898 \\
\hline 54 & 3.9013 & 1289.825 & 1283.813 & 1283.813 & 122.5994 & 22.62998 \\
\hline 55 & 3.9183 & 1294.842 & 1277.375 & 1277.375 & 122.5994 & 22.53184 \\
\hline 56 & 3.9353 & 1278.381 & 1270.938 & 1270.938 & 122.5994 & 22.43454 \\
\hline 57 & 3.9523 & 1234.9 & 1264.5 & 1264.5 & 122.5994 & 22.33808 \\
\hline 58 & 3.9693 & 1246.095 & 1258.063 & 1258.063 & 122.5994 & 22.24245 \\
\hline 59 & 3.9863 & 1246.095 & 1251.625 & 1251.625 & 122.5994 & 22.14763 \\
\hline 60 & 4.0033 & 1218.148 & 1245.188 & 1245.188 & 122.5994 & 22.05362 \\
\hline 61 & 4.0203 & 1158.35 & 1238.75 & 1238.75 & 122.5994 & 21.9604 \\
\hline 62 & 4.0373 & 1210.27 & 1232.313 & 1232.313 & 122.5994 & 21.86797 \\
\hline 63 & 4.0543 & 1232.004 & 1225.875 & 1225.875 & 122.5994 & 21.77632 \\
\hline 64 & 4.0713 & 1208.163 & 1219.438 & 1219.438 & 122.5994 & 21.68543 \\
\hline 65 & 4.0883 & 1222 & 1213 & 1213 & 122.5994 & 21.59529 \\
\hline 66 & 4.1053 & 1211.135 & 1206.563 & 1206.563 & 122.5994 & 21.50591 \\
\hline 67 & 4.1223 & 1149.86 & 1200.125 & 1200.125 & 122.5994 & 21.41726 \\
\hline 68 & 4.1393 & 1172.984 & 1193.688 & 1193.688 & 122.5994 & 21.32933 \\
\hline 69 & 4.1563 & 1164.569 & 1187.25 & 1187.25 & 122.5994 & 21.24213 \\
\hline 70 & 4.1733 & 1173.674 & 1180.813 & 1180.813 & 122.5994 & 21.15564 \\
\hline 71 & 4.1903 & 1176.821 & 1174.375 & 1174.375 & 122.5994 & 21.06985 \\
\hline 72 & 4.2073 & 1168.275 & 1167.938 & 1167.938 & 122.5994 & 20.98475 \\
\hline 73 & 4.2243 & 1183.963 & 1161.5 & 1161.5 & 122.5994 & 20.90034 \\
\hline 74 & 4.2413 & 1163.349 & 1155.063 & 1155.063 & 122.5994 & 20.81661 \\
\hline 75 & 4.2583 & 1126.212 & 1148.625 & 1148.625 & 122.5994 & 20.73354 \\
\hline 76 & 4.2753 & 1141.409 & 1142.188 & 1142.188 & 122.5994 & 20.65114 \\
\hline 77 & 4.2923 & 1110.951 & 1135.75 & 1135.75 & 122.5994 & 20.56939 \\
\hline 78 & 4.3093 & 1115.233 & 1129.313 & 1129.313 & 122.5994 & 20.48828 \\
\hline 79 & 4.3263 & 1128.981 & 1122.875 & 1122.875 & 122.5994 & 20.40781 \\
\hline 80 & 4.3433 & 1119.572 & 1116.438 & 1116.438 & 122.5994 & 20.32797 \\
\hline 81 & 4.3603 & 1091.638 & 1110 & 1110 & 122.5994 & 20.24875 \\
\hline 82 & 4.3773 & 1107.055 & 1103.563 & 1103.563 & 122.5994 & 20.17015 \\
\hline 83 & 4.3943 & 1110.451 & 1097.125 & 1097.125 & 122.5994 & 20.09216 \\
\hline 84 & 4.4113 & 1062.412 & 1090.688 & 1090.688 & 122.5994 & 20.01477 \\
\hline 85 & 4.4283 & 1119.157 & 1084.25 & 1084.25 & 122.5994 & 19.93797 \\
\hline 86 & 4.4453 & 1096.978 & 1077.813 & 1077.813 & 122.5994 & 19.86176 \\
\hline 87 & 4.4623 & 1050.148 & 1071.375 & 1071.375 & 122.5994 & 19.78613 \\
\hline 88 & 4.4793 & 1106.293 & 1064.938 & 1064.938 & 122.5994 & 19.71108 \\
\hline 89 & 4.4963 & 1080.062 & 1058.5 & 1058.5 & 122.5994 & 19.63659 \\
\hline 90 & 4.5133 & 1040.177 & 1052.063 & 1052.063 & 122.5994 & 19.56267 \\
\hline 91 & 4.5303 & 1061.103 & 1045.625 & 1045.625 & 122.5994 & 19.48929 \\
\hline 92 & 4.5473 & 1007.311 & 1039.188 & 1039.188 & 122.5994 & 19.41647 \\
\hline 93 & 4.5643 & 1067.989 & 1032.75 & 1032.75 & 122.5994 & 19.34419 \\
\hline 94 & 4.5813 & 1043.273 & 1026.313 & 1026.313 & 122.5994 & 19.27245 \\
\hline 95 & 4.5983 & 1035.713 & 1019.875 & 1019.875 & 122.5994 & 19.20124 \\
\hline 96 & 4.6153 & 987.3442 & 1013.438 & 1013.438 & 122.5994 & 19.13055 \\
\hline
\end{tabular}




\begin{tabular}{|c|c|c|c|c|c|c|}
\hline 97 & 4.6323 & 1016.766 & 1007 & 1007 & 122.5994 & 19.06038 \\
\hline 98 & 4.6493 & 1004.909 & 1003.708 & 1003.708 & 122.5994 & 18.99073 \\
\hline 99 & 4.6663 & 1003.507 & 1000.417 & 1000.417 & 122.5994 & 18.92158 \\
\hline 100 & 4.6833 & 992.2401 & 997.125 & 997.125 & 122.5994 & 18.85293 \\
\hline 101 & 4.7003 & 993.2254 & 993.8333 & 993.8333 & 122.5994 & 18.78479 \\
\hline 102 & 4.7173 & 995.2829 & 990.5417 & 990.5417 & 122.5994 & 18.71713 \\
\hline 103 & 4.7343 & 977.251 & 987.25 & 987.25 & 122.5994 & 18.64996 \\
\hline 104 & 4.7513 & 971.2815 & 983.9583 & 983.9583 & 122.5994 & 18.58327 \\
\hline 105 & 4.7683 & 994.9919 & 980.6667 & 980.6667 & 122.5994 & 18.51705 \\
\hline 106 & 4.7853 & 1007.688 & 977.375 & 977.375 & 122.5994 & 18.45131 \\
\hline 107 & 4.8023 & 966.2373 & 974.0833 & 974.0833 & 122.5994 & 18.38603 \\
\hline 108 & 4.8193 & 977.7913 & 970.7917 & 970.7917 & 122.5994 & 18.32121 \\
\hline 109 & 4.8363 & 981.5913 & 967.5 & 967.5 & 122.5994 & 18.25685 \\
\hline 110 & 4.8533 & 992.0347 & 964.2083 & 964.2083 & 122.5994 & 18.19294 \\
\hline 111 & 4.8703 & 972.8251 & 960.9167 & 960.9167 & 122.5994 & 18.12947 \\
\hline 112 & 4.8873 & 973.1529 & 957.625 & 957.625 & 122.5994 & 18.06645 \\
\hline 113 & 4.9043 & 974.3676 & 954.3333 & 954.3333 & 122.5994 & 18.00386 \\
\hline 114 & 4.9213 & 932.8438 & 951.0417 & 951.0417 & 122.5994 & 17.94171 \\
\hline 115 & 4.9383 & 923.0708 & 947.75 & 947.75 & 122.5994 & 17.87998 \\
\hline 116 & 4.9553 & 950.9851 & 944.4583 & 944.4583 & 122.5994 & 17.81868 \\
\hline 117 & 4.9723 & 957.9919 & 941.1667 & 941.1667 & 122.5994 & 17.7578 \\
\hline 118 & 4.9893 & 929.7204 & 937.875 & 937.875 & 122.5994 & 17.69733 \\
\hline 119 & 5.0063 & 921.8447 & 934.5833 & 934.5833 & 122.5994 & 17.63727 \\
\hline 120 & 5.0233 & 916.656 & 931.2917 & 931.2917 & 122.5994 & 17.57762 \\
\hline 121 & 5.0403 & 934.4267 & 928 & 928 & 122.5994 & 17.51837 \\
\hline 122 & 5.0573 & 947.3658 & 924.7083 & 924.7083 & 122.5994 & 17.45953 \\
\hline 123 & 5.0743 & 969.6482 & 921.4167 & 921.4167 & 122.5994 & 17.40107 \\
\hline 124 & 5.0913 & 969.8416 & 918.125 & 918.125 & 122.5994 & 17.34301 \\
\hline 125 & 5.1083 & 948.6255 & 914.8333 & 914.8333 & 122.5994 & 17.28533 \\
\hline 126 & 5.1253 & 929.8417 & 911.5417 & 911.5417 & 122.5994 & 17.22803 \\
\hline 127 & 5.1423 & 915.5593 & 908.25 & 908.25 & 122.5994 & 17.17112 \\
\hline 128 & 5.1593 & 903.2109 & 904.9583 & 904.9583 & 122.5994 & 17.11458 \\
\hline 129 & 5.1763 & 886.5109 & 901.6667 & 901.6667 & 122.5994 & 17.05841 \\
\hline 130 & 5.1933 & 863.777 & 898.375 & 898.375 & 122.5994 & 17.00261 \\
\hline 131 & 5.2103 & 877.2933 & 895.0833 & 895.0833 & 122.5994 & 16.94717 \\
\hline 132 & 5.2273 & 906.6249 & 891.7917 & 891.7917 & 122.5994 & 16.89209 \\
\hline 133 & 5.2443 & 896.883 & 888.5 & 888.5 & 122.5994 & 16.83737 \\
\hline 134 & 5.2613 & 888.857 & 885.2083 & 885.2083 & 122.5994 & 16.78301 \\
\hline 135 & 5.2783 & 909.2532 & 881.9167 & 881.9167 & 122.5994 & 16.72899 \\
\hline 136 & 5.2953 & 928.5055 & 878.625 & 878.625 & 122.5994 & 16.67532 \\
\hline 137 & 5.3123 & 852.9581 & 875.3333 & 875.3333 & 122.5994 & 16.622 \\
\hline 138 & 5.3293 & 949.1818 & 872.0417 & 872.0417 & 122.5994 & 16.56901 \\
\hline 139 & 5.3463 & 850.1218 & 868.75 & 868.75 & 122.5994 & 16.51637 \\
\hline 140 & 5.3633 & 875.8802 & 865.4583 & 865.4583 & 122.5994 & 16.46405 \\
\hline 141 & 5.3803 & 852.1356 & 862.1667 & 862.1667 & 122.5994 & 16.41207 \\
\hline 142 & 5.3973 & 812.6284 & 858.875 & 858.875 & 122.5994 & 16.36041 \\
\hline 143 & 5.4143 & 826.936 & 855.5833 & 855.5833 & 122.5994 & 16.30908 \\
\hline 144 & 5.4313 & 879.8187 & 852.2917 & 852.2917 & 122.5994 & 16.25807 \\
\hline 145 & 5.4483 & 850.9176 & 849 & 849 & 122.5994 & 16.20738 \\
\hline
\end{tabular}




\begin{tabular}{|c|c|c|c|c|c|c|}
\hline 146 & 5.4653 & 835.2256 & 848.6875 & 848.6875 & 122.5994 & 16.15701 \\
\hline 147 & 5.4823 & 836.0202 & 848.375 & 848.375 & 122.5994 & 16.10695 \\
\hline 148 & 5.4993 & 831.7075 & 848.0625 & 848.0625 & 122.5994 & 16.05719 \\
\hline 149 & 5.5163 & 795.5977 & 847.75 & 847.75 & 122.5994 & 16.00775 \\
\hline 150 & 5.5333 & 855.2917 & 847.4375 & 847.4375 & 122.5994 & 15.9586 \\
\hline 151 & 5.5503 & 892.144 & 849.2311 & 847.125 & 122.5994 & 15.90976 \\
\hline 152 & 5.5673 & 900.0458 & 850.0726 & 846.8125 & 122.5994 & 15.86122 \\
\hline 153 & 5.5843 & 861.6527 & 849.9207 & 846.5 & 122.5994 & 15.81297 \\
\hline 154 & 5.6013 & 813.2561 & 849.7809 & 846.1875 & 122.5994 & 15.76502 \\
\hline 155 & 5.6183 & 845.938 & 849.6545 & 845.875 & 122.5994 & 15.71735 \\
\hline 156 & 5.6353 & 888.0813 & 849.5428 & 845.5625 & 122.5994 & 15.66998 \\
\hline 157 & 5.6523 & 887.3093 & 849.4474 & 845.25 & 122.5994 & 15.62289 \\
\hline 158 & 5.6693 & 877.8253 & 849.3701 & 844.9375 & 122.5994 & 15.57608 \\
\hline 159 & 5.6863 & 897.5976 & 849.313 & 844.625 & 122.5994 & 15.52955 \\
\hline 160 & 5.7033 & 896.5491 & 849.2786 & 844.3125 & 122.5994 & 15.4833 \\
\hline 161 & 5.7203 & 892.064 & 849.2693 & 844 & 122.5994 & 15.43732 \\
\hline 162 & 5.7373 & 887.6134 & 849.2884 & 843.6875 & 122.5994 & 15.39162 \\
\hline 163 & 5.7543 & 888.2378 & 849.3394 & 843.375 & 122.5994 & 15.34618 \\
\hline 164 & 5.7713 & 855.3796 & 849.4267 & 843.0625 & 122.5994 & 15.30102 \\
\hline 165 & 5.7883 & 860.4111 & 849.5551 & 842.75 & 122.5994 & 15.25612 \\
\hline 166 & 5.8053 & 881.5203 & 849.7303 & 842.4375 & 122.5994 & 15.21148 \\
\hline 167 & 5.8223 & 849.4415 & 849.9591 & 842.125 & 122.5994 & 15.16711 \\
\hline 168 & 5.8393 & 829.4317 & 850.2496 & 841.8125 & 122.5994 & 15.12299 \\
\hline 169 & 5.8563 & 840.1336 & 850.6115 & 841.5 & 122.5994 & 15.07913 \\
\hline 170 & 5.8733 & 861.7152 & 851.0562 & 841.1875 & 122.5994 & 15.03552 \\
\hline 171 & 5.8903 & 897.7289 & 851.5977 & 840.875 & 122.5994 & 14.99216 \\
\hline 172 & 5.9073 & 871.7073 & 852.2537 & 840.5625 & 122.5994 & 14.94906 \\
\hline 173 & 5.9243 & 869.1142 & 853.0463 & 840.25 & 122.5994 & 14.9062 \\
\hline 174 & 5.9413 & 880.4598 & 854.005 & 839.9375 & 122.5994 & 14.86358 \\
\hline 175 & 5.9583 & 877.6703 & 855.1709 & 839.625 & 122.5994 & 14.82121 \\
\hline 176 & 5.9753 & 876.1513 & 856.604 & 839.3125 & 122.5994 & 14.77909 \\
\hline 177 & 5.9923 & 885.5245 & 858.3967 & 839 & 122.5994 & 14.7372 \\
\hline 178 & 6.0093 & 883.2213 & 860.6934 & 838.6875 & 122.5994 & 14.69554 \\
\hline 179 & 6.0263 & 916.9947 & 863.7204 & 838.375 & 122.5994 & 14.65413 \\
\hline 180 & 6.0433 & 945.0202 & 867.825 & 838.0625 & 122.5994 & 14.61294 \\
\hline 181 & 6.0603 & 943.262 & 873.5172 & 837.75 & 122.5994 & 14.57199 \\
\hline 182 & 6.0773 & 953.5001 & 881.5065 & 837.4375 & 122.5994 & 14.53126 \\
\hline 183 & 6.0943 & 932.3316 & 892.709 & 837.125 & 122.5994 & 14.49077 \\
\hline 184 & 6.1113 & 924.5315 & 908.2079 & 836.8125 & 122.5994 & 14.4505 \\
\hline 185 & 6.1283 & 971.4096 & 929.1378 & 836.5 & 122.5994 & 14.41045 \\
\hline 186 & 6.1453 & 1041.948 & 956.4885 & 836.1875 & 122.5994 & 14.37062 \\
\hline 187 & 6.1623 & 998.6272 & 990.8392 & 835.875 & 122.5994 & 14.33102 \\
\hline 188 & 6.1793 & 1037.933 & 1032.026 & 835.5625 & 122.5994 & 14.29163 \\
\hline 189 & 6.1963 & 1083.592 & 1078.771 & 835.25 & 122.5994 & 14.25246 \\
\hline 190 & 6.2133 & 1103.228 & 1128.208 & 834.9375 & 122.5994 & 14.2135 \\
\hline 191 & 6.2303 & 1036.248 & 1175.369 & 834.625 & 122.5994 & 14.17475 \\
\hline 192 & 6.2473 & 1165.051 & 1213.088 & 834.3125 & 122.5994 & 14.13622 \\
\hline 193 & 6.2643 & 1184.252 & 1233.401 & 834 & 122.5994 & 14.0979 \\
\hline 194 & 6.2813 & 1213.064 & 1230.67 & 833.3333 & 122.5994 & 14.05978 \\
\hline
\end{tabular}




\begin{tabular}{|c|c|c|c|c|c|c|}
\hline 195 & 6.2983 & 1340.823 & 1205.845 & 832.6667 & 122.5994 & 14.02187 \\
\hline 196 & 6.3153 & 1327.19 & 1164.978 & 832 & 122.5994 & 13.98416 \\
\hline 197 & 6.3323 & 1213.394 & 1116.045 & 831.3333 & 122.5994 & 13.94666 \\
\hline 198 & 6.3493 & 1070.927 & 1065.845 & 830.6667 & 122.5994 & 13.90935 \\
\hline 199 & 6.3663 & 987.3735 & 1018.946 & 830 & 122.5994 & 13.87225 \\
\hline 200 & 6.3833 & 961.2619 & 977.9091 & 829.3333 & 122.5994 & 13.83534 \\
\hline 201 & 6.4003 & 915.6363 & 943.7966 & 828.6667 & 122.5994 & 13.79863 \\
\hline 202 & 6.4173 & 868.7612 & 916.6215 & 828 & 122.5994 & 13.76212 \\
\hline 203 & 6.4343 & 849.0809 & 895.7156 & 827.3333 & 122.5994 & 13.72579 \\
\hline 204 & 6.4513 & 870.8724 & 880.0469 & 826.6667 & 122.5994 & 13.68966 \\
\hline 205 & 6.4683 & 872.9183 & 868.4803 & 826 & 122.5994 & 13.65372 \\
\hline 206 & 6.4853 & 841.8302 & 859.9567 & 825.3333 & 122.5994 & 13.61797 \\
\hline 207 & 6.5023 & 805.6949 & 853.5947 & 824.6667 & 122.5994 & 13.5824 \\
\hline 208 & 6.5193 & 831.5994 & 848.7215 & 824 & 122.5994 & 13.54702 \\
\hline 209 & 6.5363 & 857.0522 & 844.8572 & 823.3333 & 122.5994 & 13.51183 \\
\hline 210 & 6.5533 & 840.9596 & 841.6785 & 822.6667 & 122.5994 & 13.47682 \\
\hline 211 & 6.5703 & 840.6285 & 838.975 & 822 & 122.5994 & 13.44198 \\
\hline 212 & 6.5873 & 826.3271 & 836.6127 & 821.3333 & 122.5994 & 13.40733 \\
\hline 213 & 6.6043 & 779.665 & 834.506 & 820.6667 & 122.5994 & 13.37286 \\
\hline 214 & 6.6213 & 818.1523 & 832.5986 & 820 & 122.5994 & 13.33856 \\
\hline 215 & 6.6383 & 831.742 & 830.8517 & 819.3333 & 122.5994 & 13.30444 \\
\hline 216 & 6.6553 & 799.4886 & 829.2373 & 818.6667 & 122.5994 & 13.2705 \\
\hline 217 & 6.6723 & 821.1135 & 827.734 & 818 & 122.5994 & 13.23672 \\
\hline 218 & 6.6893 & 823.661 & 826.4293 & 817.4375 & 122.5994 & 13.20312 \\
\hline 219 & 6.7063 & 825.9029 & 825.2053 & 816.875 & 122.5994 & 13.16969 \\
\hline 220 & 6.7233 & 793.0949 & 824.0508 & 816.3125 & 122.5994 & 13.13643 \\
\hline 221 & 6.7403 & 807.4151 & 822.9565 & 815.75 & 122.5994 & 13.10334 \\
\hline 222 & 6.7573 & 831.0009 & 821.9147 & 815.1875 & 122.5994 & 13.07041 \\
\hline 223 & 6.7743 & 848.7381 & 820.9187 & 814.625 & 122.5994 & 13.03765 \\
\hline 224 & 6.7913 & 834.1151 & 819.9629 & 814.0625 & 122.5994 & 13.00505 \\
\hline 225 & 6.8083 & 824.7425 & 819.0425 & 813.5 & 122.5994 & 12.97261 \\
\hline 226 & 6.8253 & 804.5033 & 818.1534 & 812.9375 & 122.5994 & 12.94034 \\
\hline 227 & 6.8423 & 865.8152 & 817.2922 & 812.375 & 122.5994 & 12.90823 \\
\hline 228 & 6.8593 & 814.6357 & 816.4557 & 811.8125 & 122.5994 & 12.87627 \\
\hline 229 & 6.8763 & 791.2002 & 815.6414 & 811.25 & 122.5994 & 12.84448 \\
\hline 230 & 6.8933 & 768.9066 & 814.8469 & 810.6875 & 122.5994 & 12.81284 \\
\hline 231 & 6.9103 & 793.822 & 814.0701 & 810.125 & 122.5994 & 12.78136 \\
\hline 232 & 6.9273 & 856.6009 & 813.3094 & 809.5625 & 122.5994 & 12.75003 \\
\hline 233 & 6.9443 & 852.6796 & 812.5632 & 809 & 122.5994 & 12.71886 \\
\hline 234 & 6.9613 & 829.9741 & 811.8301 & 808.4375 & 122.5994 & 12.68783 \\
\hline 235 & 6.9783 & 867.2402 & 811.1089 & 807.875 & 122.5994 & 12.65696 \\
\hline 236 & 6.9953 & 786.2504 & 808.3671 & 807.3125 & 122.5994 & 12.62624 \\
\hline 237 & 7.0123 & 824.5785 & 806.75 & 806.75 & 122.5994 & 12.59567 \\
\hline 238 & 7.0293 & 832.944 & 806.1875 & 806.1875 & 122.5994 & 12.56525 \\
\hline 239 & 7.0463 & 810.1858 & 805.625 & 805.625 & 122.5994 & 12.53497 \\
\hline 240 & 7.0633 & 796.0366 & 805.0625 & 805.0625 & 122.5994 & 12.50484 \\
\hline 241 & 7.0803 & 805.5849 & 804.5 & 804.5 & 122.5994 & 12.47485 \\
\hline 242 & 7.0973 & 833.7336 & 803.9375 & 803.9375 & 122.5994 & 12.44501 \\
\hline 243 & 7.1143 & 851.4251 & 803.375 & 803.375 & 122.5994 & 12.41531 \\
\hline
\end{tabular}




\begin{tabular}{|c|c|c|c|c|c|c|}
\hline 244 & 7.1313 & 837.63 & 802.8125 & 802.8125 & 122.5994 & 12.38575 \\
\hline 245 & 7.1483 & 807.9743 & 802.25 & 802.25 & 122.5994 & 12.35633 \\
\hline 246 & 7.1653 & 809.1579 & 801.6875 & 801.6875 & 122.5994 & 12.32706 \\
\hline 247 & 7.1823 & 809.2706 & 801.125 & 801.125 & 122.5994 & 12.29792 \\
\hline 248 & 7.1993 & 831.2465 & 800.5625 & 800.5625 & 122.5994 & 12.26892 \\
\hline 249 & 7.2163 & 825.5745 & 800 & 800 & 122.5994 & 12.24005 \\
\hline 250 & 7.2333 & 792.5336 & 799.4375 & 799.4375 & 122.5994 & 12.21132 \\
\hline 251 & 7.2503 & 782.1684 & 798.875 & 798.875 & 122.5994 & 12.18273 \\
\hline 252 & 7.2673 & 799.7622 & 798.3125 & 798.3125 & 122.5994 & 12.15427 \\
\hline 253 & 7.2843 & 811.317 & 797.75 & 797.75 & 122.5994 & 12.12594 \\
\hline 254 & 7.3013 & 823.1982 & 797.1875 & 797.1875 & 122.5994 & 12.09774 \\
\hline 255 & 7.3183 & 808.8217 & 796.625 & 796.625 & 122.5994 & 12.06968 \\
\hline 256 & 7.3353 & 804.1785 & 796.0625 & 796.0625 & 122.5994 & 12.04175 \\
\hline 257 & 7.3523 & 789.6021 & 795.5 & 795.5 & 122.5994 & 12.01394 \\
\hline 258 & 7.3693 & 816.8219 & 794.9375 & 794.9375 & 122.5994 & 11.98627 \\
\hline 259 & 7.3863 & 831.4744 & 794.375 & 794.375 & 122.5994 & 11.95872 \\
\hline 260 & 7.4033 & 840.2618 & 793.8125 & 793.8125 & 122.5994 & 11.93129 \\
\hline 261 & 7.4203 & 818.5462 & 793.25 & 793.25 & 122.5994 & 11.904 \\
\hline 262 & 7.4373 & 819.8437 & 792.6875 & 792.6875 & 122.5994 & 11.87683 \\
\hline 263 & 7.4543 & 795.4558 & 792.125 & 792.125 & 122.5994 & 11.84978 \\
\hline 264 & 7.4713 & 795.069 & 791.5625 & 791.5625 & 122.5994 & 11.82285 \\
\hline 265 & 7.4883 & 783.6122 & 791 & 791 & 122.5994 & 11.79605 \\
\hline 266 & 7.5053 & 804.4978 & 789.9583 & 789.9583 & 122.5994 & 11.76937 \\
\hline 267 & 7.5223 & 781.3236 & 788.9167 & 788.9167 & 122.5994 & 11.74281 \\
\hline 268 & 7.5393 & 759.0592 & 787.875 & 787.875 & 122.5994 & 11.71637 \\
\hline 269 & 7.5563 & 781.808 & 786.8333 & 786.8333 & 122.5994 & 11.69005 \\
\hline 270 & 7.5733 & 794.0606 & 785.7917 & 785.7917 & 122.5994 & 11.66385 \\
\hline 271 & 7.5903 & 860.7284 & 784.75 & 784.75 & 122.5994 & 11.63776 \\
\hline 272 & 7.6073 & 771.9562 & 783.7083 & 783.7083 & 122.5994 & 11.61179 \\
\hline 273 & 7.6243 & 788.6904 & 782.6667 & 782.6667 & 122.5994 & 11.58594 \\
\hline 274 & 7.6413 & 800.1699 & 781.625 & 781.625 & 122.5994 & 11.5602 \\
\hline 275 & 7.6583 & 751.095 & 780.5833 & 780.5833 & 122.5994 & 11.53458 \\
\hline 276 & 7.6753 & 791.1073 & 779.5417 & 779.5417 & 122.5994 & 11.50907 \\
\hline 277 & 7.6923 & 784.6834 & 778.5 & 778.5 & 122.5994 & 11.48367 \\
\hline 278 & 7.7093 & 762.6419 & 777.4583 & 777.4583 & 122.5994 & 11.45839 \\
\hline 279 & 7.7263 & 778.1167 & 776.4167 & 776.4167 & 122.5994 & 11.43321 \\
\hline 280 & 7.7433 & 817.849 & 775.375 & 775.375 & 122.5994 & 11.40815 \\
\hline 281 & 7.7603 & 784.5688 & 774.3333 & 774.3333 & 122.5994 & 11.3832 \\
\hline 282 & 7.7773 & 805.7711 & 773.2917 & 773.2917 & 122.5994 & 11.35835 \\
\hline 283 & 7.7943 & 789.6248 & 772.25 & 772.25 & 122.5994 & 11.33362 \\
\hline 284 & 7.8113 & 788.6041 & 771.2083 & 771.2083 & 122.5994 & 11.30899 \\
\hline 285 & 7.8283 & 775.951 & 770.1667 & 770.1667 & 122.5994 & 11.28447 \\
\hline 286 & 7.8453 & 754.0955 & 769.125 & 769.125 & 122.5994 & 11.26006 \\
\hline 287 & 7.8623 & 743.2008 & 768.0833 & 768.0833 & 122.5994 & 11.23575 \\
\hline 288 & 7.8793 & 762.7211 & 767.0417 & 767.0417 & 122.5994 & 11.21154 \\
\hline 289 & 7.8963 & 766.2166 & 766 & 766 & 122.5994 & 11.18745 \\
\hline 290 & 7.9133 & 774.0156 & 765.2622 & 765.2622 & 122.5994 & 11.16345 \\
\hline 291 & 7.9303 & 801.8432 & 764.5244 & 764.5244 & 122.5994 & 11.13956 \\
\hline 292 & 7.9473 & 757.9975 & 763.7866 & 763.7866 & 122.5994 & 11.11577 \\
\hline
\end{tabular}




\begin{tabular}{|c|c|c|c|c|c|c|}
\hline 293 & 7.9643 & 797.7426 & 763.0488 & 763.0488 & 122.5994 & 11.09208 \\
\hline 294 & 7.9813 & 827.691 & 762.311 & 762.311 & 122.5994 & 11.06849 \\
\hline 295 & 7.9983 & 778.5486 & 761.5732 & 761.5732 & 122.5994 & 11.045 \\
\hline 296 & 8.0153 & 782.919 & 760.8354 & 760.8354 & 122.5994 & 11.02162 \\
\hline 297 & 8.0323 & 810.1665 & 760.0977 & 760.0977 & 122.5994 & 10.99833 \\
\hline 298 & 8.0493 & 797.7234 & 759.3599 & 759.3599 & 122.5994 & 10.97514 \\
\hline 299 & 8.0663 & 790.9708 & 758.6221 & 758.6221 & 122.5994 & 10.95204 \\
\hline 300 & 8.0833 & 828.4118 & 757.8843 & 757.8843 & 122.5994 & 10.92905 \\
\hline 301 & 8.1003 & 856.3438 & 757.1465 & 757.1465 & 122.5994 & 10.90615 \\
\hline 302 & 8.1173 & 819.576 & 756.4087 & 756.4087 & 122.5994 & 10.88335 \\
\hline 303 & 8.1343 & 803.7411 & 764.0435 & 755.6709 & 122.5994 & 10.86064 \\
\hline 304 & 8.1513 & 812.0072 & 763.6998 & 754.9331 & 122.5994 & 10.83803 \\
\hline 305 & 8.1683 & 785.3322 & 767.6859 & 754.1953 & 122.5994 & 10.81551 \\
\hline 306 & 8.1853 & 786.2036 & 767.6074 & 753.4575 & 122.5994 & 10.79309 \\
\hline 307 & 8.2023 & 814.1733 & 767.5782 & 752.7197 & 122.5994 & 10.77075 \\
\hline 308 & 8.2193 & 823.7054 & 767.6034 & 751.9819 & 122.5994 & 10.74852 \\
\hline 309 & 8.2363 & 798.8187 & 767.6884 & 751.2441 & 122.5994 & 10.72637 \\
\hline 310 & 8.2533 & 843.7358 & 767.8396 & 750.5063 & 122.5994 & 10.70431 \\
\hline 311 & 8.2703 & 833.3542 & 768.0644 & 749.7686 & 122.5994 & 10.68235 \\
\hline 312 & 8.2873 & 828.1437 & 768.371 & 749.0308 & 122.5994 & 10.66047 \\
\hline 313 & 8.3043 & 818.8061 & 768.7689 & 748.293 & 122.5994 & 10.63869 \\
\hline 314 & 8.3213 & 824.8927 & 768.8459 & 747.1322 & 122.5994 & 10.61699 \\
\hline 315 & 8.3383 & 816.4578 & 769.0376 & 745.9714 & 122.5994 & 10.59538 \\
\hline 316 & 8.3553 & 871.2038 & 769.3583 & 744.8105 & 122.5994 & 10.57386 \\
\hline 317 & 8.3723 & 832.0024 & 769.825 & 743.6497 & 122.5994 & 10.55243 \\
\hline 318 & 8.3893 & 856.0543 & 770.4571 & 742.4889 & 122.5994 & 10.53109 \\
\hline 319 & 8.4063 & 874.3142 & 771.2775 & 741.3281 & 122.5994 & 10.50983 \\
\hline 320 & 8.4233 & 850.7771 & 772.313 & 740.1673 & 122.5994 & 10.48865 \\
\hline 321 & 8.4403 & 878.0563 & 773.5961 & 739.0065 & 122.5994 & 10.46757 \\
\hline 322 & 8.4573 & 855.4027 & 775.1642 & 737.8457 & 122.5994 & 10.44656 \\
\hline 323 & 8.4743 & 924.6469 & 777.0634 & 736.6849 & 122.5994 & 10.42565 \\
\hline 324 & 8.4913 & 941.8925 & 779.3489 & 735.5241 & 122.5994 & 10.40481 \\
\hline 325 & 8.5083 & 901.2371 & 782.0895 & 734.3633 & 122.5994 & 10.38406 \\
\hline 326 & 8.5253 & 972.8748 & 785.373 & 733.2025 & 122.5994 & 10.36339 \\
\hline 327 & 8.5423 & 936.9984 & 789.3149 & 732.0417 & 122.5994 & 10.34281 \\
\hline 328 & 8.5593 & 982.3936 & 794.0759 & 730.8809 & 122.5994 & 10.3223 \\
\hline 329 & 8.5763 & 1054.42 & 799.8895 & 729.7201 & 122.5994 & 10.30188 \\
\hline 330 & 8.5933 & 1031.769 & 807.113 & 728.5592 & 122.5994 & 10.28154 \\
\hline 331 & 8.6103 & 1116.892 & 816.2997 & 727.3984 & 122.5994 & 10.26128 \\
\hline 332 & 8.6273 & 1075.765 & 828.3115 & 726.2376 & 122.5994 & 10.24109 \\
\hline 333 & 8.6443 & 1135.006 & 844.4608 & 725.0768 & 122.5994 & 10.22099 \\
\hline 334 & 8.6613 & 1213.295 & 866.6684 & 723.916 & 122.5994 & 10.20097 \\
\hline 335 & 8.6783 & 1228.955 & 897.6057 & 722.7552 & 122.5994 & 10.18102 \\
\hline 336 & 8.6953 & 1274.762 & 940.7313 & 721.5944 & 122.5994 & 10.16116 \\
\hline 337 & 8.7123 & 1397.59 & 1000.165 & 720.4336 & 122.5994 & 10.14137 \\
\hline 338 & 8.7293 & 1457.502 & 1080.299 & 719.2728 & 122.5994 & 10.12166 \\
\hline 339 & 8.7463 & 1523.422 & 1185.104 & 718.112 & 122.5994 & 10.10202 \\
\hline 340 & 8.7633 & 1625.37 & 1317.181 & 716.9512 & 122.5994 & 10.08246 \\
\hline 341 & 8.7803 & 1726.774 & 1476.541 & 715.7904 & 122.5994 & 10.06298 \\
\hline
\end{tabular}




\begin{tabular}{|c|c|c|c|c|c|c|}
\hline 342 & 8.7973 & 1792.475 & 1659.226 & 714.6296 & 122.5994 & 10.04357 \\
\hline 343 & 8.8143 & 1933.654 & 1855.607 & 713.4688 & 122.5994 & 10.02424 \\
\hline 344 & 8.8313 & 2220.642 & 2048.45 & 712.3079 & 122.5994 & 10.00498 \\
\hline 345 & 8.8483 & 2434.946 & 2212.064 & 711.1471 & 122.5994 & 9.9858 \\
\hline 346 & 8.8653 & 2675.91 & 2315.971 & 709.9863 & 122.5994 & 9.96669 \\
\hline 347 & 8.8823 & 3064.116 & 2336.021 & 708.8255 & 122.5994 & 9.94765 \\
\hline 348 & 8.8993 & 3423.891 & 2267.161 & 707.6647 & 122.5994 & 9.92869 \\
\hline 349 & 8.9163 & 3879.092 & 2126.169 & 706.5039 & 122.5994 & 9.90979 \\
\hline 350 & 8.9333 & 4043.81 & 1941.876 & 705.3431 & 122.5994 & 9.89097 \\
\hline 351 & 8.9503 & 3497.4 & 1742.708 & 704.1823 & 122.5994 & 9.87222 \\
\hline 352 & 8.9673 & 2562.727 & 1549.854 & 703.0215 & 122.5994 & 9.85355 \\
\hline 353 & 8.9843 & 1699.908 & 1376.375 & 701.8607 & 122.5994 & 9.83494 \\
\hline 354 & 9.0013 & 1177.311 & 1228.773 & 700.6999 & 122.5994 & 9.8164 \\
\hline 355 & 9.0183 & 971.0621 & 1108.765 & 699.5391 & 122.5994 & 9.79794 \\
\hline 356 & 9.0353 & 918.6445 & 1014.865 & 698.3783 & 122.5994 & 9.77954 \\
\hline 357 & 9.0523 & 908.0784 & 943.6432 & 697.2174 & 122.5994 & 9.76121 \\
\hline 358 & 9.0693 & 889.6787 & 890.8236 & 696.0566 & 122.5994 & 9.74295 \\
\hline 359 & 9.0863 & 861.4085 & 852.0994 & 694.8958 & 122.5994 & 9.72476 \\
\hline 360 & 9.1033 & 819.2777 & 823.6783 & 693.735 & 122.5994 & 9.70664 \\
\hline 361 & 9.1203 & 760.8745 & 802.5145 & 692.5742 & 122.5994 & 9.68859 \\
\hline 362 & 9.1373 & 766.4022 & 785.9188 & 690.9904 & 122.5994 & 9.6706 \\
\hline 363 & 9.1543 & 759.7666 & 772.7274 & 689.4066 & 122.5994 & 9.65268 \\
\hline 364 & 9.1713 & 734.1798 & 761.8774 & 687.8228 & 122.5994 & 9.63482 \\
\hline 365 & 9.1883 & 751.6415 & 752.6805 & 686.2389 & 122.5994 & 9.61704 \\
\hline 366 & 9.2053 & 714.7097 & 744.694 & 684.6551 & 122.5994 & 9.59931 \\
\hline 367 & 9.2223 & 721.6167 & 737.6307 & 683.0713 & 122.5994 & 9.58166 \\
\hline 368 & 9.2393 & 723.5821 & 731.2985 & 681.4875 & 122.5994 & 9.56407 \\
\hline 369 & 9.2563 & 713.7826 & 725.561 & 679.9036 & 122.5994 & 9.54654 \\
\hline 370 & 9.2733 & 688.0527 & 720.319 & 678.3198 & 122.5994 & 9.52908 \\
\hline 371 & 9.2903 & 637.0176 & 715.4951 & 676.736 & 122.5994 & 9.51168 \\
\hline 372 & 9.3073 & 695.891 & 711.0277 & 675.1522 & 122.5994 & 9.49434 \\
\hline 373 & 9.3243 & 630.1088 & 706.8669 & 673.5684 & 122.5994 & 9.47707 \\
\hline 374 & 9.3413 & 661.0323 & 702.9708 & 671.9845 & 122.5994 & 9.45986 \\
\hline 375 & 9.3583 & 682.4641 & 699.3048 & 670.4007 & 122.5994 & 9.44272 \\
\hline 376 & 9.3753 & 704.6575 & 695.8398 & 668.8169 & 122.5994 & 9.42563 \\
\hline 377 & 9.3923 & 667.0557 & 692.5508 & 667.2331 & 122.5994 & 9.40861 \\
\hline 378 & 9.4093 & 681.8067 & 689.4169 & 665.6493 & 122.5994 & 9.39165 \\
\hline 379 & 9.4263 & 687.1125 & 686.4199 & 664.0654 & 122.5994 & 9.37475 \\
\hline 380 & 9.4433 & 694.1575 & 683.5443 & 662.4816 & 122.5994 & 9.35791 \\
\hline 381 & 9.4603 & 704.7768 & 680.7767 & 660.8978 & 122.5994 & 9.34113 \\
\hline 382 & 9.4773 & 696.9118 & 678.1054 & 659.314 & 122.5994 & 9.32441 \\
\hline 383 & 9.4943 & 718.2534 & 675.5204 & 657.7301 & 122.5994 & 9.30776 \\
\hline 384 & 9.5113 & 718.2534 & 673.0128 & 656.1463 & 122.5994 & 9.29116 \\
\hline 385 & 9.5283 & 715.4116 & 670.575 & 654.5625 & 122.5994 & 9.27462 \\
\hline 386 & 9.5453 & 686.9946 & 667.677 & 652.4557 & 122.5994 & 9.25814 \\
\hline 387 & 9.5623 & 642.8218 & 664.8359 & 650.349 & 122.5994 & 9.24172 \\
\hline 388 & 9.5793 & 657.1183 & 662.0465 & 648.2422 & 122.5994 & 9.22536 \\
\hline 389 & 9.5963 & 646.7233 & 659.304 & 646.1354 & 122.5994 & 9.20905 \\
\hline 390 & 9.6133 & 661.1352 & 648.374 & 644.0286 & 122.5994 & 9.19281 \\
\hline
\end{tabular}




\begin{tabular}{|c|c|c|c|c|c|c|}
\hline 391 & 9.6303 & 670.7338 & 641.9219 & 641.9219 & 122.5994 & 9.17662 \\
\hline 392 & 9.6473 & 627.9533 & 639.8151 & 639.8151 & 122.5994 & 9.16048 \\
\hline 393 & 9.6643 & 640.4284 & 637.7083 & 637.7083 & 122.5994 & 9.14441 \\
\hline 394 & 9.6813 & 655.7964 & 635.6016 & 635.6016 & 122.5994 & 9.12839 \\
\hline 395 & 9.6983 & 645.4117 & 633.4948 & 633.4948 & 122.5994 & 9.11243 \\
\hline 396 & 9.7153 & 625.1337 & 631.388 & 631.388 & 122.5994 & 9.09652 \\
\hline 397 & 9.7323 & 633.6259 & 629.2813 & 629.2812 & 122.5994 & 9.08067 \\
\hline 398 & 9.7493 & 638.9185 & 627.1745 & 627.1745 & 122.5994 & 9.06487 \\
\hline 399 & 9.7663 & 627.8348 & 625.0677 & 625.0677 & 122.5994 & 9.04913 \\
\hline 400 & 9.7833 & 647.8155 & 622.9609 & 622.9609 & 122.5994 & 9.03344 \\
\hline 401 & 9.8003 & 629.9346 & 620.8542 & 620.8542 & 122.5994 & 9.01781 \\
\hline 402 & 9.8173 & 613.2102 & 618.7474 & 618.7474 & 122.5994 & 9.00224 \\
\hline 403 & 9.8343 & 583.4822 & 616.6406 & 616.6406 & 122.5994 & 8.98671 \\
\hline 404 & 9.8513 & 599.0312 & 614.5339 & 614.5339 & 122.5994 & 8.97124 \\
\hline 405 & 9.8683 & 621.9496 & 612.4271 & 612.4271 & 122.5994 & 8.95583 \\
\hline 406 & 9.8853 & 621.4482 & 610.3203 & 610.3203 & 122.5994 & 8.94046 \\
\hline 407 & 9.9023 & 587.6124 & 608.2136 & 608.2135 & 122.5994 & 8.92515 \\
\hline 408 & 9.9193 & 605.5692 & 606.1068 & 606.1068 & 122.5994 & 8.90989 \\
\hline 409 & 9.9363 & 599.7273 & 604 & 604 & 122.5994 & 8.89469 \\
\hline 410 & 9.9533 & 595.337 & 603.5 & 603.5 & 122.5994 & 8.87953 \\
\hline 411 & 9.9703 & 644.672 & 603 & 603 & 122.5994 & 8.86443 \\
\hline 412 & 9.9873 & 572.3998 & 602.5 & 602.5 & 122.5994 & 8.84938 \\
\hline 413 & 10.0043 & 594.3711 & 602 & 602 & 122.5994 & 8.83438 \\
\hline 414 & 10.0213 & 608.9817 & 601.5 & 601.5 & 122.5994 & 8.81943 \\
\hline 415 & 10.0383 & 623.6362 & 601 & 601 & 122.5994 & 8.80454 \\
\hline 416 & 10.0553 & 583.9127 & 600.5 & 600.5 & 122.5994 & 8.78969 \\
\hline 417 & 10.0723 & 613.3659 & 600 & 600 & 122.5994 & 8.77489 \\
\hline 418 & 10.0893 & 610.2386 & 599.5 & 599.5 & 122.5994 & 8.76014 \\
\hline 419 & 10.1063 & 590.5882 & 599 & 599 & 122.5994 & 8.74545 \\
\hline 420 & 10.1233 & 609.9504 & 598.5 & 598.5 & 122.5994 & 8.7308 \\
\hline 421 & 10.1403 & 599.511 & 598 & 598 & 122.5994 & 8.7162 \\
\hline 422 & 10.1573 & 581.3857 & 597.5 & 597.5 & 122.5994 & 8.70165 \\
\hline 423 & 10.1743 & 589.3434 & 597 & 597 & 122.5994 & 8.68715 \\
\hline 424 & 10.1913 & 574.5272 & 596.5 & 596.5 & 122.5994 & 8.6727 \\
\hline 425 & 10.2083 & 562.2843 & 596 & 596 & 122.5994 & 8.65829 \\
\hline 426 & 10.2253 & 565.7645 & 595.5 & 595.5 & 122.5994 & 8.64394 \\
\hline 427 & 10.2423 & 560.8835 & 595 & 595 & 122.5994 & 8.62963 \\
\hline 428 & 10.2593 & 567.8575 & 594.5 & 594.5 & 122.5994 & 8.61537 \\
\hline 429 & 10.2763 & 614.017 & 594 & 594 & 122.5994 & 8.60115 \\
\hline 430 & 10.2933 & 597.822 & 593.5 & 593.5 & 122.5994 & 8.58698 \\
\hline 431 & 10.3103 & 567.8794 & 593 & 593 & 122.5994 & 8.57286 \\
\hline 432 & 10.3273 & 602.9528 & 592.5 & 592.5 & 122.5994 & 8.55879 \\
\hline 433 & 10.3443 & 602.8534 & 592 & 592 & 122.5994 & 8.54476 \\
\hline 434 & 10.3613 & 581.0832 & 591.3333 & 591.3333 & 122.5994 & 8.53078 \\
\hline 435 & 10.3783 & 584.3652 & 590.6667 & 590.6667 & 122.5994 & 8.51685 \\
\hline 436 & 10.3953 & 599.5386 & 590 & 590 & 122.5994 & 8.50296 \\
\hline 437 & 10.4123 & 590.1005 & 589.3333 & 589.3333 & 122.5994 & 8.48911 \\
\hline 438 & 10.4293 & 587.6691 & 588.6667 & 588.6667 & 122.5994 & 8.47531 \\
\hline 439 & 10.4463 & 567.7373 & 588 & 588 & 122.5994 & 8.46156 \\
\hline
\end{tabular}




\begin{tabular}{|c|c|c|c|c|c|c|}
\hline 440 & 10.4633 & 580.3067 & 587.3333 & 587.3333 & 122.5994 & 8.44785 \\
\hline 441 & 10.4803 & 594.7702 & 586.6667 & 586.6667 & 122.5994 & 8.43418 \\
\hline 442 & 10.4973 & 637.2167 & 586 & 586 & 122.5994 & 8.42056 \\
\hline 443 & 10.5143 & 655.1496 & 585.3333 & 585.3333 & 122.5994 & 8.40699 \\
\hline 444 & 10.5313 & 648.2214 & 584.6667 & 584.6667 & 122.5994 & 8.39345 \\
\hline 445 & 10.5483 & 681.2743 & 584 & 584 & 122.5994 & 8.37996 \\
\hline 446 & 10.5653 & 711.4531 & 583.3333 & 583.3333 & 122.5994 & 8.36652 \\
\hline 447 & 10.5823 & 711.8853 & 582.6667 & 582.6667 & 122.5994 & 8.35312 \\
\hline 448 & 10.5993 & 701.0245 & 582 & 582 & 122.5994 & 8.33976 \\
\hline 449 & 10.6163 & 635.108 & 581.3333 & 581.3333 & 122.5994 & 8.32644 \\
\hline 450 & 10.6333 & 597.0298 & 580.6667 & 580.6667 & 122.5994 & 8.31317 \\
\hline 451 & 10.6503 & 569.1688 & 580 & 580 & 122.5994 & 8.29994 \\
\hline 452 & 10.6673 & 560.2676 & 579.3333 & 579.3333 & 122.5994 & 8.28675 \\
\hline 453 & 10.6843 & 561.7005 & 578.6667 & 578.6667 & 122.5994 & 8.2736 \\
\hline 454 & 10.7013 & 547.6588 & 578 & 578 & 122.5994 & 8.26049 \\
\hline 455 & 10.7183 & 560.9643 & 577.3333 & 577.3333 & 122.5994 & 8.24743 \\
\hline 456 & 10.7353 & 558.9622 & 576.6667 & 576.6667 & 122.5994 & 8.23441 \\
\hline 457 & 10.7523 & 592.098 & 576 & 576 & 122.5994 & 8.22143 \\
\hline 458 & 10.7693 & 588.0578 & 574.9167 & 574.9167 & 122.5994 & 8.20849 \\
\hline 459 & 10.7863 & 557.1205 & 573.8333 & 573.8333 & 122.5994 & 8.19559 \\
\hline 460 & 10.8033 & 564.6907 & 572.75 & 572.75 & 122.5994 & 8.18273 \\
\hline 461 & 10.8203 & 541.1094 & 571.6667 & 571.6667 & 122.5994 & 8.16991 \\
\hline 462 & 10.8373 & 572.9518 & 570.5833 & 570.5833 & 122.5994 & 8.15713 \\
\hline 463 & 10.8543 & 553.6729 & 569.5 & 569.5 & 122.5994 & 8.1444 \\
\hline 464 & 10.8713 & 521.0496 & 568.4167 & 568.4167 & 122.5994 & 8.1317 \\
\hline 465 & 10.8883 & 575.0862 & 567.3333 & 567.3333 & 122.5994 & 8.11904 \\
\hline 466 & 10.9053 & 529.0263 & 566.25 & 566.25 & 122.5994 & 8.10642 \\
\hline 467 & 10.9223 & 538.4978 & 565.1667 & 565.1667 & 122.5994 & 8.09384 \\
\hline 468 & 10.9393 & 548.2502 & 564.0833 & 564.0833 & 122.5994 & 8.0813 \\
\hline 469 & 10.9563 & 557.1861 & 563 & 563 & 122.5994 & 8.0688 \\
\hline 470 & 10.9733 & 500.1892 & 561.9167 & 561.9167 & 122.5994 & 8.05634 \\
\hline 471 & 10.9903 & 533.2282 & 560.8333 & 560.8333 & 122.5994 & 8.04392 \\
\hline 472 & 11.0073 & 567.7006 & 559.75 & 559.75 & 122.5994 & 8.03153 \\
\hline 473 & 11.0243 & 572.3133 & 558.6667 & 558.6667 & 122.5994 & 8.01919 \\
\hline 474 & 11.0413 & 553.9333 & 557.5833 & 557.5833 & 122.5994 & 8.00688 \\
\hline 475 & 11.0583 & 565.4674 & 556.5 & 556.5 & 122.5994 & 7.99461 \\
\hline 476 & 11.0753 & 579.7616 & 555.4167 & 555.4167 & 122.5994 & 7.98237 \\
\hline 477 & 11.0923 & 577.1737 & 554.3333 & 554.3333 & 122.5994 & 7.97018 \\
\hline 478 & 11.1093 & 573.2694 & 553.25 & 553.25 & 122.5994 & 7.95802 \\
\hline 479 & 11.1263 & 565.9296 & 552.1667 & 552.1667 & 122.5994 & 7.9459 \\
\hline 480 & 11.1433 & 584.1216 & 551.0833 & 551.0833 & 122.5994 & 7.93381 \\
\hline 481 & 11.1603 & 541.4551 & 550 & 550 & 122.5994 & 7.92177 \\
\hline 482 & 11.1773 & 529.0099 & 549.5833 & 549.5833 & 122.5994 & 7.90976 \\
\hline 483 & 11.1943 & 534.1611 & 549.1667 & 549.1667 & 122.5994 & 7.89778 \\
\hline 484 & 11.2113 & 546.5413 & 548.75 & 548.75 & 122.5994 & 7.88585 \\
\hline 485 & 11.2283 & 548.3865 & 548.3333 & 548.3333 & 122.5994 & 7.87394 \\
\hline 486 & 11.2453 & 546.9756 & 547.9167 & 547.9167 & 122.5994 & 7.86208 \\
\hline 487 & 11.2623 & 567.4841 & 547.5 & 547.5 & 122.5994 & 7.85025 \\
\hline 488 & 11.2793 & 578.7473 & 547.0833 & 547.0833 & 122.5994 & 7.83846 \\
\hline
\end{tabular}




\begin{tabular}{|c|c|c|c|c|c|c|}
\hline 489 & 11.2963 & 573.6155 & 546.6667 & 546.6667 & 122.5994 & 7.8267 \\
\hline 490 & 11.3133 & 551.5008 & 546.25 & 546.25 & 122.5994 & 7.81498 \\
\hline 491 & 11.3303 & 544.1609 & 545.8333 & 545.8333 & 122.5994 & 7.80329 \\
\hline 492 & 11.3473 & 559.6405 & 545.4167 & 545.4167 & 122.5994 & 7.79164 \\
\hline 493 & 11.3643 & 545.2446 & 545 & 545 & 122.5994 & 7.78002 \\
\hline 494 & 11.3813 & 542.0642 & 544.5833 & 544.5833 & 122.5994 & 7.76844 \\
\hline 495 & 11.3983 & 544.6326 & 544.1667 & 544.1667 & 122.5994 & 7.75689 \\
\hline 496 & 11.4153 & 561.3247 & 543.75 & 543.75 & 122.5994 & 7.74537 \\
\hline 497 & 11.4323 & 536.6641 & 543.3333 & 543.3333 & 122.5994 & 7.73389 \\
\hline 498 & 11.4493 & 555.4798 & 542.9167 & 542.9167 & 122.5994 & 7.72245 \\
\hline 499 & 11.4663 & 541.0349 & 542.5 & 542.5 & 122.5994 & 7.71104 \\
\hline 500 & 11.4833 & 556.5583 & 542.0833 & 542.0833 & 122.5994 & 7.69966 \\
\hline 501 & 11.5003 & 562.8494 & 541.6667 & 541.6667 & 122.5994 & 7.68832 \\
\hline 502 & 11.5173 & 572.8577 & 541.25 & 541.25 & 122.5994 & 7.67701 \\
\hline 503 & 11.5343 & 568.3187 & 540.8333 & 540.8333 & 122.5994 & 7.66573 \\
\hline 504 & 11.5513 & 535.649 & 540.4167 & 540.4167 & 122.5994 & 7.65449 \\
\hline 505 & 11.5683 & 544.6788 & 540 & 540 & 122.5994 & 7.64328 \\
\hline 506 & 11.5853 & 515.6809 & 539.7969 & 539.7969 & 122.5994 & 7.6321 \\
\hline 507 & 11.6023 & 547.5811 & 539.5938 & 539.5938 & 122.5994 & 7.62095 \\
\hline 508 & 11.6193 & 552.2603 & 539.3906 & 539.3906 & 122.5994 & 7.60984 \\
\hline 509 & 11.6363 & 543.5215 & 539.1875 & 539.1875 & 122.5994 & 7.59876 \\
\hline 510 & 11.6533 & 503.7531 & 538.9844 & 538.9844 & 122.5994 & 7.58772 \\
\hline 511 & 11.6703 & 519.3671 & 538.7813 & 538.7813 & 122.5994 & 7.5767 \\
\hline 512 & 11.6873 & 538.1243 & 538.5781 & 538.5781 & 122.5994 & 7.56572 \\
\hline 513 & 11.7043 & 556.6643 & 538.375 & 538.375 & 122.5994 & 7.55477 \\
\hline 514 & 11.7213 & 538.2192 & 538.1719 & 538.1719 & 122.5994 & 7.54385 \\
\hline 515 & 11.7383 & 538.8034 & 537.9688 & 537.9688 & 122.5994 & 7.53296 \\
\hline 516 & 11.7553 & 547.5702 & 537.7656 & 537.7656 & 122.5994 & 7.52211 \\
\hline 517 & 11.7723 & 544.7009 & 537.5625 & 537.5625 & 122.5994 & 7.51128 \\
\hline 518 & 11.7893 & 523.8658 & 537.3594 & 537.3594 & 122.5994 & 7.50049 \\
\hline 519 & 11.8063 & 548.8786 & 537.1563 & 537.1563 & 122.5994 & 7.48973 \\
\hline 520 & 11.8233 & 529.7491 & 536.9531 & 536.9531 & 122.5994 & 7.479 \\
\hline 521 & 11.8403 & 533.62 & 536.7501 & 536.75 & 122.5994 & 7.4683 \\
\hline 522 & 11.8573 & 534.5923 & 536.9052 & 536.5469 & 122.5994 & 7.45763 \\
\hline 523 & 11.8743 & 546.9597 & 536.7197 & 536.3438 & 122.5994 & 7.44699 \\
\hline 524 & 11.8913 & 572.3902 & 536.5356 & 536.1406 & 122.5994 & 7.43638 \\
\hline 525 & 11.9083 & 552.7257 & 536.3531 & 535.9375 & 122.5994 & 7.4258 \\
\hline 526 & 11.9253 & 531.7148 & 536.1721 & 535.7344 & 122.5994 & 7.41525 \\
\hline 527 & 11.9423 & 541.2097 & 535.993 & 535.5313 & 122.5994 & 7.40474 \\
\hline 528 & 11.9593 & 555.5898 & 535.8159 & 535.3281 & 122.5994 & 7.39425 \\
\hline 529 & 11.9763 & 562.6605 & 535.6411 & 535.125 & 122.5994 & 7.38379 \\
\hline 530 & 11.9933 & 551.162 & 535.1927 & 534.6458 & 122.5994 & 7.37336 \\
\hline 531 & 12.0103 & 568.5468 & 534.7473 & 534.1667 & 122.5994 & 7.36297 \\
\hline 532 & 12.0273 & 577.4551 & 534.3049 & 533.6875 & 122.5994 & 7.3526 \\
\hline 533 & 12.0443 & 562.2163 & 533.8662 & 533.2083 & 122.5994 & 7.34226 \\
\hline 534 & 12.0613 & 556.9704 & 533.4316 & 532.7292 & 122.5994 & 7.33195 \\
\hline 535 & 12.0783 & 556.9704 & 533.0017 & 532.25 & 122.5994 & 7.32166 \\
\hline 536 & 12.0953 & 554.5307 & 532.5771 & 531.7708 & 122.5994 & 7.31141 \\
\hline 537 & 12.1123 & 564.9128 & 532.1587 & 531.2917 & 122.5994 & 7.30119 \\
\hline
\end{tabular}




\begin{tabular}{|c|c|c|c|c|c|c|}
\hline 538 & 12.1293 & 592.1733 & 533.9222 & 530.8125 & 122.5994 & 7.29099 \\
\hline 539 & 12.1463 & 599.4943 & 533.7101 & 530.3333 & 122.5994 & 7.28083 \\
\hline 540 & 12.1633 & 607.1256 & 533.5339 & 529.8542 & 122.5994 & 7.27069 \\
\hline 541 & 12.1803 & 611.9884 & 533.4 & 529.375 & 122.5994 & 7.26058 \\
\hline 542 & 12.1973 & 629.2753 & 533.3168 & 528.8958 & 122.5994 & 7.2505 \\
\hline 543 & 12.2143 & 623.5231 & 533.2947 & 528.4167 & 122.5994 & 7.24045 \\
\hline 544 & 12.2313 & 635.6344 & 533.3467 & 527.9375 & 122.5994 & 7.23042 \\
\hline 545 & 12.2483 & 623.9052 & 533.4898 & 527.4583 & 122.5994 & 7.22042 \\
\hline 546 & 12.2653 & 629.579 & 533.7457 & 526.9792 & 122.5994 & 7.21045 \\
\hline 547 & 12.2823 & 657.938 & 534.1431 & 526.5 & 122.5994 & 7.20051 \\
\hline 548 & 12.2993 & 697.8671 & 534.7203 & 526.0208 & 122.5994 & 7.1906 \\
\hline 549 & 12.3163 & 690.8689 & 535.5297 & 525.5417 & 122.5994 & 7.18071 \\
\hline 550 & 12.3333 & 725.4265 & 536.6439 & 525.0625 & 122.5994 & 7.17085 \\
\hline 551 & 12.3503 & 748.0165 & 538.1708 & 524.5833 & 122.5994 & 7.16102 \\
\hline 552 & 12.3673 & 736.7871 & 540.2871 & 524.1042 & 122.5994 & 7.15121 \\
\hline 553 & 12.3843 & 739.1859 & 543.3299 & 523.625 & 122.5994 & 7.14144 \\
\hline 554 & 12.4013 & 841.1605 & 548.0204 & 523.1458 & 122.5994 & 7.13168 \\
\hline 555 & 12.4183 & 841.9469 & 555.9108 & 522.6667 & 122.5994 & 7.12196 \\
\hline 556 & 12.4353 & 824.7174 & 570.0104 & 522.1875 & 122.5994 & 7.11226 \\
\hline 557 & 12.4523 & 874.1885 & 595.15 & 521.7083 & 122.5994 & 7.10259 \\
\hline 558 & 12.4693 & 876.1764 & 637.192 & 521.2292 & 122.5994 & 7.09294 \\
\hline 559 & 12.4863 & 900.5629 & 700.2366 & 520.75 & 122.5994 & 7.08333 \\
\hline 560 & 12.5033 & 999.8132 & 781.6426 & 520.2708 & 122.5994 & 7.07373 \\
\hline 561 & 12.5203 & 1051.552 & 864.7332 & 519.7917 & 122.5994 & 7.06417 \\
\hline 562 & 12.5373 & 1220.068 & 916.1717 & 519.3125 & 122.5994 & 7.05463 \\
\hline 563 & 12.5543 & 1350.613 & 910.9423 & 518.8333 & 122.5994 & 7.04511 \\
\hline 564 & 12.5713 & 1449.851 & 856.0668 & 518.3542 & 122.5994 & 7.03562 \\
\hline 565 & 12.5883 & 1349.336 & 775.4028 & 517.875 & 122.5994 & 7.02616 \\
\hline 566 & 12.6053 & 1147.643 & 695.8265 & 517.3958 & 122.5994 & 7.01672 \\
\hline 567 & 12.6223 & 950.2066 & 633.3137 & 516.9167 & 122.5994 & 7.00731 \\
\hline 568 & 12.6393 & 733.0229 & 590.8327 & 516.4375 & 122.5994 & 6.99792 \\
\hline 569 & 12.6563 & 634.5599 & 564.6324 & 515.9583 & 122.5994 & 6.98856 \\
\hline 570 & 12.6733 & 615.0515 & 549.2609 & 515.4792 & 122.5994 & 6.97923 \\
\hline 571 & 12.6903 & 603.434 & 540.1464 & 515 & 122.5994 & 6.96991 \\
\hline 572 & 12.7073 & 576.7307 & 534.3455 & 514.5208 & 122.5994 & 6.96063 \\
\hline 573 & 12.7243 & 560.4909 & 530.2759 & 514.0417 & 122.5994 & 6.95137 \\
\hline 574 & 12.7413 & 498.7131 & 527.1741 & 513.5625 & 122.5994 & 6.94213 \\
\hline 575 & 12.7583 & 523.6619 & 524.678 & 513.0833 & 122.5994 & 6.93292 \\
\hline 576 & 12.7753 & 530.5385 & 522.6005 & 512.6042 & 122.5994 & 6.92373 \\
\hline 577 & 12.7923 & 516.1208 & 520.83 & 512.125 & 122.5994 & 6.91457 \\
\hline 578 & 12.8093 & 549.9189 & 519.0165 & 511.3698 & 122.5994 & 6.90543 \\
\hline 579 & 12.8263 & 548.1079 & 517.3835 & 510.6146 & 122.5994 & 6.89631 \\
\hline 580 & 12.8433 & 505.9139 & 515.8925 & 509.8594 & 122.5994 & 6.88722 \\
\hline 581 & 12.8603 & 496.8109 & 514.5145 & 509.1042 & 122.5994 & 6.87816 \\
\hline 582 & 12.8773 & 499.5729 & 513.2277 & 508.349 & 122.5994 & 6.86912 \\
\hline 583 & 12.8943 & 488.4191 & 512.0151 & 507.5938 & 122.5994 & 6.8601 \\
\hline 584 & 12.9113 & 500.5491 & 510.8637 & 506.8385 & 122.5994 & 6.8511 \\
\hline 585 & 12.9283 & 515.9044 & 509.7631 & 506.0833 & 122.5994 & 6.84213 \\
\hline 586 & 12.9453 & 496.2327 & 506.5699 & 505.3281 & 122.5994 & 6.83319 \\
\hline
\end{tabular}




\begin{tabular}{|c|c|c|c|c|c|c|}
\hline 587 & 12.9623 & 484.9465 & 505.712 & 504.5729 & 122.5994 & 6.82426 \\
\hline 588 & 12.9793 & 496.8305 & 504.8662 & 503.8177 & 122.5994 & 6.81536 \\
\hline 589 & 12.9963 & 528.5695 & 504.0307 & 503.0625 & 122.5994 & 6.80649 \\
\hline 590 & 13.0133 & 536.6229 & 503.2041 & 502.3073 & 122.5994 & 6.79763 \\
\hline 591 & 13.0303 & 549.8391 & 502.3851 & 501.5521 & 122.5994 & 6.7888 \\
\hline 592 & 13.0473 & 514.2381 & 501.5726 & 500.7969 & 122.5994 & 6.78 \\
\hline 593 & 13.0643 & 497.2708 & 500.7658 & 500.0417 & 122.5994 & 6.77121 \\
\hline 594 & 13.0813 & 500.6927 & 499.9641 & 499.2865 & 122.5994 & 6.76245 \\
\hline 595 & 13.0983 & 494.8056 & 499.1666 & 498.5313 & 122.5994 & 6.75371 \\
\hline 596 & 13.1153 & 514.4795 & 498.3729 & 497.776 & 122.5994 & 6.74499 \\
\hline 597 & 13.1323 & 490.3282 & 497.5826 & 497.0208 & 122.5994 & 6.7363 \\
\hline 598 & 13.1493 & 469.2681 & 496.7953 & 496.2656 & 122.5994 & 6.72763 \\
\hline 599 & 13.1663 & 512.7207 & 496.0107 & 495.5104 & 122.5994 & 6.71898 \\
\hline 600 & 13.1833 & 503.7901 & 495.2285 & 494.7552 & 122.5994 & 6.71036 \\
\hline 601 & 13.2003 & 481.6176 & 494.4484 & 494 & 122.5994 & 6.70175 \\
\hline 602 & 13.2173 & 500.219 & 493.9463 & 493.5208 & 122.5994 & 6.69317 \\
\hline 603 & 13.2343 & 480.6649 & 493.4458 & 493.0417 & 122.5994 & 6.68461 \\
\hline 604 & 13.2513 & 462.7045 & 492.9469 & 492.5625 & 122.5994 & 6.67607 \\
\hline 605 & 13.2683 & 501.5767 & 492.4495 & 492.0833 & 122.5994 & 6.66756 \\
\hline 606 & 13.2853 & 484.009 & 491.6042 & 491.6042 & 122.5994 & 6.65907 \\
\hline 607 & 13.3023 & 484.4975 & 491.125 & 491.125 & 122.5994 & 6.65059 \\
\hline 608 & 13.3193 & 495.7053 & 490.6458 & 490.6458 & 122.5994 & 6.64214 \\
\hline 609 & 13.3363 & 469.4899 & 490.1667 & 490.1667 & 122.5994 & 6.63371 \\
\hline 610 & 13.3533 & 457.2622 & 490.3606 & 489.6875 & 122.5994 & 6.62531 \\
\hline 611 & 13.3703 & 482.4964 & 489.926 & 489.2083 & 122.5994 & 6.61692 \\
\hline 612 & 13.3873 & 449.2525 & 489.83 & 488.7292 & 122.5994 & 6.60856 \\
\hline 613 & 13.4043 & 497.2708 & 489.4272 & 488.25 & 122.5994 & 6.60021 \\
\hline 614 & 13.4213 & 499.8515 & 489.0325 & 487.7708 & 122.5994 & 6.59189 \\
\hline 615 & 13.4383 & 505.123 & 488.6615 & 487.2917 & 122.5994 & 6.58359 \\
\hline 616 & 13.4553 & 473.9844 & 488.2881 & 486.8125 & 122.5994 & 6.57531 \\
\hline 617 & 13.4723 & 463.3621 & 487.9343 & 486.3333 & 122.5994 & 6.56705 \\
\hline 618 & 13.4893 & 520.272 & 487.609 & 485.8542 & 122.5994 & 6.55881 \\
\hline 619 & 13.5063 & 513.5958 & 487.282 & 485.375 & 122.5994 & 6.5506 \\
\hline 620 & 13.5233 & 530.518 & 486.9855 & 484.8958 & 122.5994 & 6.5424 \\
\hline 621 & 13.5403 & 462.3274 & 486.7039 & 484.4167 & 122.5994 & 6.53423 \\
\hline 622 & 13.5573 & 440.1444 & 486.4514 & 483.9375 & 122.5994 & 6.52607 \\
\hline 623 & 13.5743 & 492.3086 & 486.2339 & 483.4583 & 122.5994 & 6.51793 \\
\hline 624 & 13.5913 & 500.042 & 486.059 & 482.9792 & 122.5994 & 6.50982 \\
\hline 625 & 13.6083 & 499.6024 & 485.9365 & 482.5 & 122.5994 & 6.50173 \\
\hline 626 & 13.6253 & 515.9121 & 485.5146 & 481.6563 & 122.5994 & 6.49365 \\
\hline 627 & 13.6423 & 492.5856 & 485.1759 & 480.8125 & 122.5994 & 6.4856 \\
\hline 628 & 13.6593 & 506.1325 & 484.9478 & 479.9688 & 122.5994 & 6.47757 \\
\hline 629 & 13.6763 & 527.0254 & 484.8752 & 479.125 & 122.5994 & 6.46955 \\
\hline 630 & 13.6933 & 535.7788 & 485.0377 & 478.2813 & 122.5994 & 6.46156 \\
\hline 631 & 13.7103 & 554.4985 & 485.5791 & 477.4375 & 122.5994 & 6.45358 \\
\hline 632 & 13.7273 & 531.132 & 486.7526 & 476.5938 & 122.5994 & 6.44563 \\
\hline 633 & 13.7443 & 499.095 & 488.9707 & 475.75 & 122.5994 & 6.4377 \\
\hline 634 & 13.7613 & 505.6484 & 492.8366 & 474.9063 & 122.5994 & 6.42978 \\
\hline 635 & 13.7783 & 504.9685 & 499.1134 & 474.0625 & 122.5994 & 6.42189 \\
\hline
\end{tabular}




\begin{tabular}{|c|c|c|c|c|c|c|}
\hline 636 & 13.7953 & 504.7455 & 508.5816 & 473.2188 & 122.5994 & 6.41401 \\
\hline 637 & 13.8123 & 528.1527 & 521.7715 & 472.375 & 122.5994 & 6.40615 \\
\hline 638 & 13.8293 & 512.4697 & 538.5723 & 471.5313 & 122.5994 & 6.39832 \\
\hline 639 & 13.8463 & 485.6577 & 557.707 & 470.6875 & 122.5994 & 6.3905 \\
\hline 640 & 13.8633 & 551.8077 & 576.1211 & 469.8438 & 122.5994 & 6.3827 \\
\hline 641 & 13.8803 & 539.7689 & 589.0298 & 469 & 122.5994 & 6.37492 \\
\hline 642 & 13.8973 & 597.1481 & 592.1949 & 468.1563 & 122.5994 & 6.36716 \\
\hline 643 & 13.9143 & 599.0424 & 584.87 & 467.3125 & 122.5994 & 6.35942 \\
\hline 644 & 13.9313 & 615.9185 & 569.6132 & 466.4688 & 122.5994 & 6.3517 \\
\hline 645 & 13.9483 & 574.1397 & 550.4922 & 465.625 & 122.5994 & 6.344 \\
\hline 646 & 13.9653 & 541.6905 & 531.392 & 464.7813 & 122.5994 & 6.33631 \\
\hline 647 & 13.9823 & 542.4019 & 514.7366 & 463.9375 & 122.5994 & 6.32865 \\
\hline 648 & 13.9993 & 500.9011 & 501.407 & 463.0938 & 122.5994 & 6.321 \\
\hline 649 & 14.0163 & 501.5126 & 491.2568 & 462.25 & 122.5994 & 6.31337 \\
\hline 650 & 14.0333 & 484.6192 & 483.6531 & 461.4063 & 122.5994 & 6.30576 \\
\hline 651 & 14.0503 & 488.3555 & 477.897 & 460.5625 & 122.5994 & 6.29817 \\
\hline 652 & 14.0673 & 441.2872 & 473.421 & 459.7188 & 122.5994 & 6.2906 \\
\hline 653 & 14.0843 & 484.009 & 469.8333 & 458.875 & 122.5994 & 6.28304 \\
\hline 654 & 14.1013 & 483.5698 & 466.8911 & 458.0313 & 122.5994 & 6.27551 \\
\hline 655 & 14.1183 & 473.6782 & 464.4391 & 457.1875 & 122.5994 & 6.26799 \\
\hline 656 & 14.1353 & 461.575 & 462.3646 & 456.3438 & 122.5994 & 6.26049 \\
\hline 657 & 14.1523 & 532.5349 & 460.5793 & 455.5 & 122.5994 & 6.25301 \\
\hline 658 & 14.1693 & 486.0054 & 459.0117 & 454.6563 & 122.5994 & 6.24554 \\
\hline 659 & 14.1863 & 474.3604 & 457.6045 & 453.8125 & 122.5994 & 6.2381 \\
\hline 660 & 14.2033 & 494.448 & 456.3136 & 452.9688 & 122.5994 & 6.23067 \\
\hline 661 & 14.2203 & 487.867 & 455.1068 & 452.125 & 122.5994 & 6.22326 \\
\hline 662 & 14.2373 & 441.1493 & 453.9615 & 451.2813 & 122.5994 & 6.21587 \\
\hline 663 & 14.2543 & 464.6383 & 452.8626 & 450.4375 & 122.5994 & 6.20849 \\
\hline 664 & 14.2713 & 453.1587 & 451.7999 & 449.5938 & 122.5994 & 6.20113 \\
\hline 665 & 14.2883 & 465.4288 & 450.7662 & 448.75 & 122.5994 & 6.19379 \\
\hline 666 & 14.3053 & 451.5657 & 449.7563 & 447.9063 & 122.5994 & 6.18647 \\
\hline 667 & 14.3223 & 469.5487 & 448.7662 & 447.0625 & 122.5994 & 6.17917 \\
\hline 668 & 14.3393 & 460.9639 & 447.7928 & 446.2188 & 122.5994 & 6.17188 \\
\hline 669 & 14.3563 & 468.1833 & 446.8338 & 445.375 & 122.5994 & 6.16461 \\
\hline 670 & 14.3733 & 469.191 & 445.887 & 444.5313 & 122.5994 & 6.15736 \\
\hline 671 & 14.3903 & 455.9117 & 444.9508 & 443.6875 & 122.5994 & 6.15012 \\
\hline 672 & 14.4073 & 452.189 & 444.0237 & 442.8438 & 122.5994 & 6.1429 \\
\hline 673 & 14.4243 & 445.314 & 443.0769 & 442 & 122.5994 & 6.1357 \\
\hline 674 & 14.4413 & 426.1865 & 442.363 & 441.9375 & 122.5994 & 6.12851 \\
\hline 675 & 14.4583 & 443.2147 & 442.3232 & 441.875 & 122.5994 & 6.12135 \\
\hline 676 & 14.4753 & 464.9802 & 441.9496 & 441.8125 & 122.5994 & 6.1142 \\
\hline 677 & 14.4923 & 458.8556 & 441.9181 & 441.75 & 122.5994 & 6.10706 \\
\hline 678 & 14.5093 & 482.3541 & 441.8585 & 441.6875 & 122.5994 & 6.09995 \\
\hline 679 & 14.5263 & 495.033 & 441.7866 & 441.625 & 122.5994 & 6.09284 \\
\hline 680 & 14.5433 & 475.9903 & 441.7302 & 441.5625 & 122.5994 & 6.08576 \\
\hline 681 & 14.5603 & 465.4463 & 441.6683 & 441.5 & 122.5994 & 6.07869 \\
\hline 682 & 14.5773 & 490.9734 & 441.5962 & 441.4375 & 122.5994 & 6.07164 \\
\hline 683 & 14.5943 & 470.8005 & 441.5453 & 441.375 & 122.5994 & 6.06461 \\
\hline 684 & 14.6113 & 450.7421 & 441.4868 & 441.3125 & 122.5994 & 6.05759 \\
\hline
\end{tabular}




\begin{tabular}{|c|c|c|c|c|c|c|}
\hline 685 & 14.6283 & 431.4621 & 441.4401 & 441.25 & 122.5994 & 6.05059 \\
\hline 686 & 14.6453 & 436.6345 & 441.3956 & 441.1875 & 122.5994 & 6.0436 \\
\hline 687 & 14.6623 & 450.6548 & 441.3538 & 441.125 & 122.5994 & 6.03664 \\
\hline 688 & 14.6793 & 437.2436 & 441.3151 & 441.0625 & 122.5994 & 6.02968 \\
\hline 689 & 14.6963 & 452.9598 & 441.2803 & 441 & 122.5994 & 6.02275 \\
\hline 690 & 14.7133 & 479.4442 & 441.2503 & 440.9375 & 122.5994 & 6.01583 \\
\hline 691 & 14.7303 & 484.009 & 441.2262 & 440.875 & 122.5994 & 6.00892 \\
\hline 692 & 14.7473 & 476.38 & 441.2097 & 440.8125 & 122.5994 & 6.00203 \\
\hline 693 & 14.7643 & 471.3806 & 441.2031 & 440.75 & 122.5994 & 5.99516 \\
\hline 694 & 14.7813 & 474.8608 & 441.2106 & 440.6875 & 122.5994 & 5.9883 \\
\hline 695 & 14.7983 & 471.1662 & 441.2391 & 440.625 & 122.5994 & 5.98146 \\
\hline 696 & 14.8153 & 483.4033 & 441.3015 & 440.5625 & 122.5994 & 5.97464 \\
\hline 697 & 14.8323 & 488.3103 & 441.4201 & 440.5 & 122.5994 & 5.96783 \\
\hline 698 & 14.8493 & 479.6191 & 441.6323 & 440.4375 & 122.5994 & 5.96103 \\
\hline 699 & 14.8663 & 470.3495 & 441.9925 & 440.375 & 122.5994 & 5.95425 \\
\hline 700 & 14.8833 & 440.6498 & 442.571 & 440.3125 & 122.5994 & 5.94749 \\
\hline 701 & 14.9003 & 433.1613 & 443.4411 & 440.25 & 122.5994 & 5.94074 \\
\hline 702 & 14.9173 & 442.4147 & 444.6557 & 440.1875 & 122.5994 & 5.93401 \\
\hline 703 & 14.9343 & 461.1214 & 446.2114 & 440.125 & 122.5994 & 5.9273 \\
\hline 704 & 14.9513 & 477.79 & 448.0009 & 440.0625 & 122.5994 & 5.92059 \\
\hline 705 & 14.9683 & 411.344 & 449.7548 & 440 & 122.5994 & 5.91391 \\
\hline 706 & 14.9853 & 468.7428 & 451.0344 & 439.9375 & 122.5994 & 5.90724 \\
\hline 707 & 15.0023 & 527.5394 & 451.4288 & 439.875 & 122.5994 & 5.90058 \\
\hline 708 & 15.0193 & 459.5427 & 450.8375 & 439.8125 & 122.5994 & 5.89394 \\
\hline 709 & 15.0363 & 451.4383 & 449.4636 & 439.75 & 122.5994 & 5.88732 \\
\hline 710 & 15.0533 & 467.9728 & 447.6554 & 439.6875 & 122.5994 & 5.88071 \\
\hline 711 & 15.0703 & 501.0247 & 445.7851 & 439.625 & 122.5994 & 5.87411 \\
\hline 712 & 15.0873 & 500.1809 & 444.1189 & 439.5625 & 122.5994 & 5.86753 \\
\hline 713 & 15.1043 & 473.8315 & 442.7763 & 439.5 & 122.5994 & 5.86096 \\
\hline 714 & 15.1213 & 481.429 & 441.7686 & 439.4375 & 122.5994 & 5.85441 \\
\hline 715 & 15.1383 & 492.1519 & 441.0485 & 439.375 & 122.5994 & 5.84788 \\
\hline 716 & 15.1553 & 473.5507 & 440.547 & 439.3125 & 122.5994 & 5.84136 \\
\hline 717 & 15.1723 & 472.6634 & 440.1966 & 439.25 & 122.5994 & 5.83485 \\
\hline 718 & 15.1893 & 460.6216 & 439.9435 & 439.1875 & 122.5994 & 5.82836 \\
\hline 719 & 15.2063 & 428.0118 & 439.7501 & 439.125 & 122.5994 & 5.82188 \\
\hline 720 & 15.2233 & 419.8092 & 439.593 & 439.0625 & 122.5994 & 5.81542 \\
\hline 721 & 15.2403 & 442.9897 & 439.4584 & 439 & 122.5994 & 5.80897 \\
\hline 722 & 15.2573 & 444.9475 & 439.0262 & 438.625 & 122.5994 & 5.80253 \\
\hline 723 & 15.2743 & 467.2202 & 438.6044 & 438.25 & 122.5994 & 5.79611 \\
\hline 724 & 15.2913 & 470.6807 & 438.1905 & 437.875 & 122.5994 & 5.78971 \\
\hline 725 & 15.3083 & 449.4226 & 437.7826 & 437.5 & 122.5994 & 5.78332 \\
\hline 726 & 15.3253 & 427.0643 & 437.3795 & 437.125 & 122.5994 & 5.77694 \\
\hline 727 & 15.3423 & 430.0462 & 437.0146 & 436.75 & 122.5994 & 5.77058 \\
\hline 728 & 15.3593 & 438.2917 & 436.621 & 436.375 & 122.5994 & 5.76423 \\
\hline 729 & 15.3763 & 468.1454 & 436.247 & 436 & 122.5994 & 5.75789 \\
\hline 730 & 15.3933 & 440.3156 & 435.86 & 435.625 & 122.5994 & 5.75157 \\
\hline 731 & 15.4103 & 440.8672 & 435.4752 & 435.25 & 122.5994 & 5.74527 \\
\hline 732 & 15.4273 & 425.2072 & 435.0926 & 434.875 & 122.5994 & 5.73897 \\
\hline 733 & 15.4443 & 416.1678 & 434.7119 & 434.5 & 122.5994 & 5.73269 \\
\hline
\end{tabular}




\begin{tabular}{|c|c|c|c|c|c|c|}
\hline 734 & 15.4613 & 425.6555 & 434.333 & 434.125 & 122.5994 & 5.72643 \\
\hline 735 & 15.4783 & 479.5242 & 433.9559 & 433.75 & 122.5994 & 5.72018 \\
\hline 736 & 15.4953 & 482.0736 & 433.5806 & 433.375 & 122.5994 & 5.71394 \\
\hline 737 & 15.5123 & 454.3705 & 433.207 & 433 & 122.5994 & 5.70772 \\
\hline 738 & 15.5293 & 475.319 & 432.8354 & 432.625 & 122.5994 & 5.70151 \\
\hline 739 & 15.5463 & 483.4779 & 432.4076 & 432.25 & 122.5994 & 5.69531 \\
\hline 740 & 15.5633 & 445.2595 & 432.0437 & 431.875 & 122.5994 & 5.68913 \\
\hline 741 & 15.5803 & 456.6461 & 431.6529 & 431.5 & 122.5994 & 5.68296 \\
\hline 742 & 15.5973 & 426.2643 & 431.296 & 431.125 & 122.5994 & 5.6768 \\
\hline 743 & 15.6143 & 424.3436 & 430.9424 & 430.75 & 122.5994 & 5.67066 \\
\hline 744 & 15.6313 & 424.3679 & 430.5932 & 430.375 & 122.5994 & 5.66453 \\
\hline 745 & 15.6483 & 416.5515 & 430.2499 & 430 & 122.5994 & 5.65842 \\
\hline 746 & 15.6653 & 465.0758 & 430.165 & 429.875 & 122.5994 & 5.65231 \\
\hline 747 & 15.6823 & 416.5873 & 430.0932 & 429.75 & 122.5994 & 5.64622 \\
\hline 748 & 15.6993 & 424.945 & 430.0427 & 429.625 & 122.5994 & 5.64015 \\
\hline 749 & 15.7163 & 431.0962 & 430.0278 & 429.5 & 122.5994 & 5.63409 \\
\hline 750 & 15.7333 & 416.1678 & 430.071 & 429.375 & 122.5994 & 5.62804 \\
\hline 751 & 15.7503 & 423.0579 & 430.2043 & 429.25 & 122.5994 & 5.622 \\
\hline 752 & 15.7673 & 463.8515 & 430.4658 & 429.125 & 122.5994 & 5.61598 \\
\hline 753 & 15.7843 & 448.6859 & 430.8913 & 429 & 122.5994 & 5.60997 \\
\hline 754 & 15.8013 & 439.3564 & 431.4999 & 428.875 & 122.5994 & 5.60397 \\
\hline 755 & 15.8183 & 421.6898 & 432.2734 & 428.75 & 122.5994 & 5.59798 \\
\hline 756 & 15.8353 & 445.16 & 433.1286 & 428.625 & 122.5994 & 5.59201 \\
\hline 757 & 15.8523 & 462.5708 & 433.8894 & 428.5 & 122.5994 & 5.58605 \\
\hline 758 & 15.8693 & 462.9608 & 434.3159 & 428.375 & 122.5994 & 5.58011 \\
\hline 759 & 15.8863 & 437.8451 & 434.2462 & 428.25 & 122.5994 & 5.57418 \\
\hline 760 & 15.9033 & 448.0488 & 433.6977 & 428.125 & 122.5994 & 5.56826 \\
\hline 761 & 15.9203 & 452.7157 & 432.8035 & 428 & 122.5994 & 5.56235 \\
\hline 762 & 15.9373 & 460.1676 & 431.7458 & 427.875 & 122.5994 & 5.55645 \\
\hline 763 & 15.9543 & 444.4541 & 430.7033 & 427.75 & 122.5994 & 5.55057 \\
\hline 764 & 15.9713 & 424.7987 & 429.7912 & 427.625 & 122.5994 & 5.5447 \\
\hline 765 & 15.9883 & 426.1991 & 429.0524 & 427.5 & 122.5994 & 5.53884 \\
\hline 766 & 16.0053 & 459.2879 & 428.4819 & 427.375 & 122.5994 & 5.533 \\
\hline 767 & 16.0223 & 466.739 & 428.0505 & 427.25 & 122.5994 & 5.52717 \\
\hline 768 & 16.0393 & 432.9645 & 427.722 & 427.125 & 122.5994 & 5.52135 \\
\hline 769 & 16.0563 & 421.222 & 427.4633 & 427 & 122.5994 & 5.51554 \\
\hline 770 & 16.0733 & 412.3711 & 427.5406 & 427.1667 & 122.5994 & 5.50974 \\
\hline 771 & 16.0903 & 415.0421 & 427.6451 & 427.3333 & 122.5994 & 5.50396 \\
\hline 772 & 16.1073 & 427.771 & 427.7661 & 427.5 & 122.5994 & 5.49819 \\
\hline 773 & 16.1243 & 431.9693 & 427.8975 & 427.6667 & 122.5994 & 5.49243 \\
\hline 774 & 16.1413 & 447.4878 & 428.036 & 427.8333 & 122.5994 & 5.48668 \\
\hline 775 & 16.1583 & 439.9398 & 428.1794 & 428 & 122.5994 & 5.48095 \\
\hline 776 & 16.1753 & 464.3601 & 428.3267 & 428.1667 & 122.5994 & 5.47523 \\
\hline 777 & 16.1923 & 464.5356 & 428.4769 & 428.3333 & 122.5994 & 5.46952 \\
\hline 778 & 16.2093 & 419.174 & 428.6295 & 428.5 & 122.5994 & 5.46382 \\
\hline 779 & 16.2263 & 406.9983 & 428.7841 & 428.6667 & 122.5994 & 5.45813 \\
\hline 780 & 16.2433 & 423.6138 & 428.9403 & 428.8333 & 122.5994 & 5.45246 \\
\hline 781 & 16.2603 & 418.8471 & 429.0978 & 429 & 122.5994 & 5.4468 \\
\hline 782 & 16.2773 & 449.7854 & 429.2564 & 429.1667 & 122.5994 & 5.44115 \\
\hline
\end{tabular}




\begin{tabular}{|c|c|c|c|c|c|c|}
\hline 783 & 16.2943 & 416.7454 & 429.416 & 429.3333 & 122.5994 & 5.43551 \\
\hline 784 & 16.3113 & 443.0752 & 429.5764 & 429.5 & 122.5994 & 5.42988 \\
\hline 785 & 16.3283 & 456.8501 & 429.7374 & 429.6667 & 122.5994 & 5.42427 \\
\hline 786 & 16.3453 & 465.6249 & 429.8991 & 429.8333 & 122.5994 & 5.41866 \\
\hline 787 & 16.3623 & 443.2628 & 430.0612 & 430 & 122.5994 & 5.41307 \\
\hline 788 & 16.3793 & 485.2581 & 430.2238 & 430.1667 & 122.5994 & 5.40749 \\
\hline 789 & 16.3963 & 467.555 & 430.3869 & 430.3333 & 122.5994 & 5.40192 \\
\hline 790 & 16.4133 & 409.3821 & 430.5184 & 430.5 & 122.5994 & 5.39637 \\
\hline 791 & 16.4303 & 436.7554 & 430.6839 & 430.6667 & 122.5994 & 5.39082 \\
\hline 792 & 16.4473 & 453.6161 & 430.8495 & 430.8333 & 122.5994 & 5.38529 \\
\hline 793 & 16.4643 & 449.9726 & 431 & 431 & 122.5994 & 5.37977 \\
\hline 794 & 16.4813 & 461.9907 & 431.1667 & 431.1667 & 122.5994 & 5.37426 \\
\hline 795 & 16.4983 & 456.4935 & 431.3333 & 431.3333 & 122.5994 & 5.36876 \\
\hline 796 & 16.5153 & 441.0082 & 431.5 & 431.5 & 122.5994 & 5.36327 \\
\hline 797 & 16.5323 & 439.9469 & 431.6667 & 431.6667 & 122.5994 & 5.35779 \\
\hline 798 & 16.5493 & 429.5967 & 431.8333 & 431.8333 & 122.5994 & 5.35233 \\
\hline 799 & 16.5663 & 419.8965 & 432 & 432 & 122.5994 & 5.34687 \\
\hline 800 & 16.5833 & 451.5323 & 432.1667 & 432.1667 & 122.5994 & 5.34143 \\
\hline 801 & 16.6003 & 426.0827 & 432.3333 & 432.3333 & 122.5994 & 5.336 \\
\hline 802 & 16.6173 & 434.6086 & 432.5 & 432.5 & 122.5994 & 5.33058 \\
\hline 803 & 16.6343 & 438.8677 & 432.6667 & 432.6667 & 122.5994 & 5.32517 \\
\hline 804 & 16.6513 & 437.3114 & 432.8333 & 432.8333 & 122.5994 & 5.31977 \\
\hline 805 & 16.6683 & 446.9239 & 433 & 433 & 122.5994 & 5.31438 \\
\hline 806 & 16.6853 & 441.0082 & 433.1667 & 433.1667 & 122.5994 & 5.309 \\
\hline 807 & 16.7023 & 435.2657 & 433.3333 & 433.3333 & 122.5994 & 5.30364 \\
\hline 808 & 16.7193 & 433.4871 & 433.5 & 433.5 & 122.5994 & 5.29828 \\
\hline 809 & 16.7363 & 444.5841 & 433.6667 & 433.6667 & 122.5994 & 5.29294 \\
\hline 810 & 16.7533 & 433.4346 & 433.8333 & 433.8333 & 122.5994 & 5.28761 \\
\hline 811 & 16.7703 & 412.8898 & 434 & 434 & 122.5994 & 5.28229 \\
\hline 812 & 16.7873 & 397.4308 & 434.1667 & 434.1667 & 122.5994 & 5.27698 \\
\hline 813 & 16.8043 & 419.4255 & 434.3333 & 434.3333 & 122.5994 & 5.27168 \\
\hline 814 & 16.8213 & 434.7636 & 434.5 & 434.5 & 122.5994 & 5.26639 \\
\hline 815 & 16.8383 & 436.9471 & 434.6667 & 434.6667 & 122.5994 & 5.26111 \\
\hline 816 & 16.8553 & 429.891 & 434.8333 & 434.8333 & 122.5994 & 5.25584 \\
\hline 817 & 16.8723 & 422.5484 & 435 & 435 & 122.5994 & 5.25058 \\
\hline 818 & 16.8893 & 429.1607 & 434.8333 & 434.8333 & 122.5994 & 5.24534 \\
\hline 819 & 16.9063 & 466.131 & 434.6667 & 434.6667 & 122.5994 & 5.2401 \\
\hline 820 & 16.9233 & 491.1177 & 434.5 & 434.5 & 122.5994 & 5.23487 \\
\hline 821 & 16.9403 & 435.7022 & 434.3333 & 434.3333 & 122.5994 & 5.22966 \\
\hline 822 & 16.9573 & 420.2934 & 434.1667 & 434.1667 & 122.5994 & 5.22445 \\
\hline 823 & 16.9743 & 422.8421 & 434 & 434 & 122.5994 & 5.21926 \\
\hline 824 & 16.9913 & 453.0423 & 433.8333 & 433.8333 & 122.5994 & 5.21408 \\
\hline 825 & 17.0083 & 466.5687 & 433.6667 & 433.6667 & 122.5994 & 5.2089 \\
\hline 826 & 17.0253 & 441.0451 & 433.5 & 433.5 & 122.5994 & 5.20374 \\
\hline 827 & 17.0423 & 462.1933 & 433.3333 & 433.3333 & 122.5994 & 5.19859 \\
\hline 828 & 17.0593 & 447.1673 & 436.4812 & 433.1667 & 122.5994 & 5.19345 \\
\hline 829 & 17.0763 & 417.4323 & 436.4707 & 433 & 122.5994 & 5.18831 \\
\hline 830 & 17.0933 & 382.1906 & 436.4714 & 432.8333 & 122.5994 & 5.18319 \\
\hline 831 & 17.1103 & 442.7599 & 438.162 & 432.6667 & 122.5994 & 5.17808 \\
\hline
\end{tabular}




\begin{tabular}{|c|c|c|c|c|c|c|}
\hline 832 & 17.1273 & 461.1792 & 438.2684 & 432.5 & 122.5994 & 5.17298 \\
\hline 833 & 17.1443 & 431.8233 & 438.3955 & 432.3333 & 122.5994 & 5.16789 \\
\hline 834 & 17.1613 & 463.6387 & 438.5455 & 432.1667 & 122.5994 & 5.16281 \\
\hline 835 & 17.1783 & 434.5282 & 438.7209 & 432 & 122.5994 & 5.15774 \\
\hline 836 & 17.1953 & 448.879 & 438.9243 & 431.8333 & 122.5994 & 5.15268 \\
\hline 837 & 17.2123 & 466.3571 & 439.159 & 431.6667 & 122.5994 & 5.14762 \\
\hline 838 & 17.2293 & 416.4321 & 439.4284 & 431.5 & 122.5994 & 5.14258 \\
\hline 839 & 17.2463 & 444.7061 & 439.7367 & 431.3333 & 122.5994 & 5.13755 \\
\hline 840 & 17.2633 & 429.1506 & 440.0887 & 431.1667 & 122.5994 & 5.13253 \\
\hline 841 & 17.2803 & 438.8775 & 440.4897 & 431 & 122.5994 & 5.12752 \\
\hline 842 & 17.2973 & 415.6549 & 441.5504 & 431.4375 & 122.5994 & 5.12252 \\
\hline 843 & 17.3143 & 448.9034 & 442.6738 & 431.875 & 122.5994 & 5.11753 \\
\hline 844 & 17.3313 & 446.9803 & 443.8687 & 432.3125 & 122.5994 & 5.11255 \\
\hline 845 & 17.3483 & 454.5903 & 445.1451 & 432.75 & 122.5994 & 5.10758 \\
\hline 846 & 17.3653 & 433.8164 & 446.5151 & 433.1875 & 122.5994 & 5.10261 \\
\hline 847 & 17.3823 & 429.6495 & 447.993 & 433.625 & 122.5994 & 5.09766 \\
\hline 848 & 17.3993 & 467.5401 & 449.5958 & 434.0625 & 122.5994 & 5.09272 \\
\hline 849 & 17.4163 & 462.4695 & 451.3443 & 434.5 & 122.5994 & 5.08779 \\
\hline 850 & 17.4333 & 443.618 & 453.2644 & 434.9375 & 122.5994 & 5.08286 \\
\hline 851 & 17.4503 & 468.5406 & 455.3887 & 435.375 & 122.5994 & 5.07795 \\
\hline 852 & 17.4673 & 477.2587 & 457.7603 & 435.8125 & 122.5994 & 5.07305 \\
\hline 853 & 17.4843 & 469.4773 & 460.4388 & 436.25 & 122.5994 & 5.06815 \\
\hline 854 & 17.5013 & 479.3756 & 463.5107 & 436.6875 & 122.5994 & 5.06327 \\
\hline 855 & 17.5183 & 475.7591 & 467.1068 & 437.125 & 122.5994 & 5.05839 \\
\hline 856 & 17.5353 & 470.31 & 471.4293 & 437.5625 & 122.5994 & 5.05353 \\
\hline 857 & 17.5523 & 467.5861 & 476.7912 & 438 & 122.5994 & 5.04867 \\
\hline 858 & 17.5693 & 479.4063 & 483.6658 & 438.4375 & 122.5994 & 5.04382 \\
\hline 859 & 17.5863 & 501.5856 & 492.7473 & 438.875 & 122.5994 & 5.03899 \\
\hline 860 & 17.6033 & 508.4858 & 504.9956 & 439.3125 & 122.5994 & 5.03416 \\
\hline 861 & 17.6203 & 479.7589 & 521.658 & 439.75 & 122.5994 & 5.02934 \\
\hline 862 & 17.6373 & 495.1876 & 544.2289 & 440.1875 & 122.5994 & 5.02453 \\
\hline 863 & 17.6543 & 524.7977 & 574.3223 & 440.625 & 122.5994 & 5.01973 \\
\hline 864 & 17.6713 & 561.0164 & 613.4408 & 441.0625 & 122.5994 & 5.01494 \\
\hline 865 & 17.6883 & 579.7356 & 662.6429 & 441.5 & 122.5994 & 5.01016 \\
\hline 866 & 17.7053 & 582.6968 & 722.1191 & 441.9375 & 122.5994 & 5.00539 \\
\hline 867 & 17.7223 & 647.7009 & 790.675 & 442.375 & 122.5994 & 5.00062 \\
\hline 868 & 17.7393 & 642.8908 & 865.0787 & 442.8125 & 122.5994 & 4.99587 \\
\hline 869 & 17.7563 & 690.0665 & 939.3838 & 443.25 & 122.5994 & 4.99112 \\
\hline 870 & 17.7733 & 789.4463 & 1004.628 & 443.6875 & 122.5994 & 4.98639 \\
\hline 871 & 17.7903 & 872.6689 & 1050.331 & 444.125 & 122.5994 & 4.98166 \\
\hline 872 & 17.8073 & 962.1354 & 1068.21 & 444.5625 & 122.5994 & 4.97694 \\
\hline 873 & 17.8243 & 1131.21 & 1055.689 & 445 & 122.5994 & 4.97224 \\
\hline 874 & 17.8413 & 1181.761 & 1016.105 & 445.4375 & 122.5994 & 4.96754 \\
\hline 875 & 17.8583 & 1107.403 & 956.6522 & 445.875 & 122.5994 & 4.96285 \\
\hline 876 & 17.8753 & 1029.999 & 886.2589 & 446.3125 & 122.5994 & 4.95816 \\
\hline 877 & 17.8923 & 877.6892 & 813.4811 & 446.75 & 122.5994 & 4.95349 \\
\hline 878 & 17.9093 & 754.5414 & 744.8397 & 447.1875 & 122.5994 & 4.94883 \\
\hline 879 & 17.9263 & 664.553 & 684.2723 & 447.625 & 122.5994 & 4.94417 \\
\hline 880 & 17.9433 & 574.1341 & 633.5449 & 448.0625 & 122.5994 & 4.93953 \\
\hline
\end{tabular}




\begin{tabular}{|c|c|c|c|c|c|c|}
\hline 881 & 17.9603 & 544.4478 & 592.8391 & 448.5 & 122.5994 & 4.93489 \\
\hline 882 & 17.9773 & 474.08 & 561.3311 & 448.9375 & 122.5994 & 4.93026 \\
\hline 883 & 17.9943 & 466.6668 & 537.653 & 449.375 & 122.5994 & 4.92564 \\
\hline 884 & 18.0113 & 479.6044 & 520.2793 & 449.8125 & 122.5994 & 4.92103 \\
\hline 885 & 18.0283 & 483.9536 & 507.6557 & 450.25 & 122.5994 & 4.91643 \\
\hline 886 & 18.0453 & 481.5114 & 498.5382 & 450.6875 & 122.5994 & 4.91184 \\
\hline 887 & 18.0623 & 426.2279 & 491.8783 & 451.125 & 122.5994 & 4.90725 \\
\hline 888 & 18.0793 & 454.7918 & 486.9315 & 451.5625 & 122.5994 & 4.90267 \\
\hline 889 & 18.0963 & 465.653 & 483.1673 & 452 & 122.5994 & 4.89811 \\
\hline 890 & 18.1133 & 455.7974 & 480.3207 & 452.5313 & 122.5994 & 4.89355 \\
\hline 891 & 18.1303 & 443.4922 & 478.0626 & 453.0625 & 122.5994 & 4.889 \\
\hline 892 & 18.1473 & 460.1793 & 476.2399 & 453.5938 & 122.5994 & 4.88446 \\
\hline 893 & 18.1643 & 473.4226 & 474.7621 & 454.125 & 122.5994 & 4.87992 \\
\hline 894 & 18.1813 & 473.9083 & 473.5403 & 454.6563 & 122.5994 & 4.8754 \\
\hline 895 & 18.1983 & 442.8113 & 472.5412 & 455.1875 & 122.5994 & 4.87088 \\
\hline 896 & 18.2153 & 466.7221 & 471.7189 & 455.7188 & 122.5994 & 4.86638 \\
\hline 897 & 18.2323 & 472.3444 & 471.0504 & 456.25 & 122.5994 & 4.86188 \\
\hline 898 & 18.2493 & 452.4232 & 470.5133 & 456.7813 & 122.5994 & 4.85739 \\
\hline 899 & 18.2663 & 466.2747 & 470.0897 & 457.3125 & 122.5994 & 4.8529 \\
\hline 900 & 18.2833 & 495.6752 & 469.7644 & 457.8438 & 122.5994 & 4.84843 \\
\hline 901 & 18.3003 & 479.2282 & 469.5251 & 458.375 & 122.5994 & 4.84396 \\
\hline 902 & 18.3173 & 464.256 & 469.3611 & 458.9063 & 122.5994 & 4.83951 \\
\hline 903 & 18.3343 & 457.1358 & 469.2635 & 459.4375 & 122.5994 & 4.83506 \\
\hline 904 & 18.3513 & 473.4856 & 469.2249 & 459.9688 & 122.5994 & 4.83062 \\
\hline 905 & 18.3683 & 474.6953 & 469.2389 & 460.5 & 122.5994 & 4.82618 \\
\hline 906 & 18.3853 & 470.2655 & 469.3001 & 461.0313 & 122.5994 & 4.82176 \\
\hline 907 & 18.4023 & 462.777 & 469.404 & 461.5625 & 122.5994 & 4.81734 \\
\hline 908 & 18.4193 & 456.4107 & 469.5468 & 462.0938 & 122.5994 & 4.81294 \\
\hline 909 & 18.4363 & 459.3836 & 469.7257 & 462.625 & 122.5994 & 4.80854 \\
\hline 910 & 18.4533 & 456.5482 & 469.9388 & 463.1563 & 122.5994 & 4.80414 \\
\hline 911 & 18.4703 & 472.3239 & 470.189 & 463.6875 & 122.5994 & 4.79976 \\
\hline 912 & 18.4873 & 469.1799 & 470.4695 & 464.2188 & 122.5994 & 4.79539 \\
\hline 913 & 18.5043 & 478.5528 & 470.7862 & 464.75 & 122.5994 & 4.79102 \\
\hline 914 & 18.5213 & 494.1414 & 470.8699 & 465.0052 & 122.5994 & 4.78666 \\
\hline 915 & 18.5383 & 457.0236 & 467.7541 & 465.2604 & 122.5994 & 4.78231 \\
\hline 916 & 18.5553 & 457.2169 & 468.0853 & 465.5156 & 122.5994 & 4.77797 \\
\hline 917 & 18.5723 & 445.4793 & 468.4794 & 465.7708 & 122.5994 & 4.77363 \\
\hline 918 & 18.5893 & 452.2666 & 467.3631 & 466.026 & 122.5994 & 4.7693 \\
\hline 919 & 18.6063 & 470.9624 & 467.9937 & 466.2813 & 122.5994 & 4.76498 \\
\hline 920 & 18.6233 & 458.5801 & 468.719 & 466.5365 & 122.5994 & 4.76067 \\
\hline 921 & 18.6403 & 513.5721 & 469.539 & 466.7917 & 122.5994 & 4.75637 \\
\hline 922 & 18.6573 & 485.0569 & 470.4405 & 467.0469 & 122.5994 & 4.75207 \\
\hline 923 & 18.6743 & 486.2851 & 471.39 & 467.3021 & 122.5994 & 4.74779 \\
\hline 924 & 18.6913 & 444.0679 & 472.3282 & 467.5573 & 122.5994 & 4.74351 \\
\hline 925 & 18.7083 & 494.0426 & 473.1713 & 467.8125 & 122.5994 & 4.73923 \\
\hline 926 & 18.7253 & 526.3559 & 473.8318 & 468.0677 & 122.5994 & 4.73497 \\
\hline 927 & 18.7423 & 479.329 & 474.2565 & 468.3229 & 122.5994 & 4.73071 \\
\hline 928 & 18.7593 & 491.836 & 474.4475 & 468.5781 & 122.5994 & 4.72646 \\
\hline 929 & 18.7763 & 527.8188 & 474.4505 & 468.8333 & 122.5994 & 4.72222 \\
\hline
\end{tabular}




\begin{tabular}{|c|c|c|c|c|c|c|}
\hline 930 & 18.7933 & 561.3277 & 474.3339 & 469.0885 & 122.5994 & 4.71799 \\
\hline 931 & 18.8103 & 529.3423 & 474.1711 & 469.3438 & 122.5994 & 4.71376 \\
\hline 932 & 18.8273 & 564.0259 & 474.021 & 469.599 & 122.5994 & 4.70955 \\
\hline 933 & 18.8443 & 638.8316 & 473.913 & 469.8542 & 122.5994 & 4.70534 \\
\hline 934 & 18.8613 & 704.8295 & 473.8464 & 470.1094 & 122.5994 & 4.70113 \\
\hline 935 & 18.8783 & 669.8298 & 473.8032 & 470.3646 & 122.5994 & 4.69694 \\
\hline 936 & 18.8953 & 651.6904 & 473.7673 & 470.6198 & 122.5994 & 4.69275 \\
\hline 937 & 18.9123 & 588.3694 & 473.735 & 470.875 & 122.5994 & 4.68857 \\
\hline 938 & 18.9293 & 590.7204 & 473.7135 & 471.1302 & 122.5994 & 4.6844 \\
\hline 939 & 18.9463 & 514.4151 & 473.7149 & 471.3854 & 122.5994 & 4.68023 \\
\hline 940 & 18.9633 & 502.6459 & 473.7509 & 471.6406 & 122.5994 & 4.67608 \\
\hline 941 & 18.9803 & 469.9233 & 473.8376 & 471.8958 & 122.5994 & 4.67193 \\
\hline 942 & 18.9973 & 470.8733 & 473.9367 & 472.151 & 122.5994 & 4.66778 \\
\hline 943 & 19.0143 & 473.5994 & 474.0371 & 472.4063 & 122.5994 & 4.66365 \\
\hline 944 & 19.0313 & 442.7832 & 474.4996 & 472.6615 & 122.5994 & 4.65952 \\
\hline 945 & 19.0483 & 439.3831 & 474.7346 & 472.9167 & 122.5994 & 4.6554 \\
\hline 946 & 19.0653 & 458.1295 & 474.828 & 473.1719 & 122.5994 & 4.65129 \\
\hline 947 & 19.0823 & 459.9677 & 475.0477 & 473.4271 & 122.5994 & 4.64718 \\
\hline 948 & 19.0993 & 423.9305 & 475.2155 & 473.6823 & 122.5994 & 4.64309 \\
\hline 949 & 19.1163 & 460.8562 & 475.3727 & 473.9375 & 122.5994 & 4.63899 \\
\hline 950 & 19.1333 & 475.4784 & 475.603 & 474.1927 & 122.5994 & 4.63491 \\
\hline 951 & 19.1503 & 445.2183 & 476.0717 & 474.4479 & 122.5994 & 4.63084 \\
\hline 952 & 19.1673 & 463.0861 & 476.3513 & 474.7031 & 122.5994 & 4.62677 \\
\hline 953 & 19.1843 & 463.2195 & 476.7033 & 474.9583 & 122.5994 & 4.6227 \\
\hline 954 & 19.2013 & 441.0082 & 477.1311 & 475.2135 & 122.5994 & 4.61865 \\
\hline 955 & 19.2183 & 445.4523 & 477.4572 & 475.4688 & 122.5994 & 4.6146 \\
\hline 956 & 19.2353 & 493.0539 & 477.8318 & 475.724 & 122.5994 & 4.61056 \\
\hline 957 & 19.2523 & 474.1214 & 478.1995 & 475.9792 & 122.5994 & 4.60653 \\
\hline 958 & 19.2693 & 478.7705 & 478.5695 & 476.2344 & 122.5994 & 4.6025 \\
\hline 959 & 19.2863 & 497.0785 & 478.9543 & 476.4896 & 122.5994 & 4.59849 \\
\hline 960 & 19.3033 & 488.4191 & 479.3611 & 476.7448 & 122.5994 & 4.59447 \\
\hline 961 & 19.3203 & 465.6552 & 479.7802 & 477 & 122.5994 & 4.59047 \\
\hline 962 & 19.3373 & 465.346 & 480.787 & 477.8229 & 122.5994 & 4.58647 \\
\hline 963 & 19.3543 & 489.3448 & 481.8169 & 478.6458 & 122.5994 & 4.58248 \\
\hline 964 & 19.3713 & 465.8445 & 482.873 & 479.4688 & 122.5994 & 4.5785 \\
\hline 965 & 19.3883 & 479.4492 & 483.9597 & 480.2917 & 122.5994 & 4.57452 \\
\hline 966 & 19.4053 & 485.0534 & 485.0821 & 481.1146 & 122.5994 & 4.57055 \\
\hline 967 & 19.4223 & 477.1347 & 486.247 & 481.9375 & 122.5994 & 4.56659 \\
\hline 968 & 19.4393 & 507.5401 & 487.463 & 482.7604 & 122.5994 & 4.56264 \\
\hline 969 & 19.4563 & 500.3991 & 488.713 & 483.5833 & 122.5994 & 4.55869 \\
\hline 970 & 19.4733 & 489.7011 & 490.0718 & 484.4063 & 122.5994 & 4.55475 \\
\hline 971 & 19.4903 & 500.0438 & 492.2562 & 485.2292 & 122.5994 & 4.55081 \\
\hline 972 & 19.5073 & 482.005 & 493.8758 & 486.0521 & 122.5994 & 4.54688 \\
\hline 973 & 19.5243 & 508.0849 & 495.6987 & 486.875 & 122.5994 & 4.54296 \\
\hline 974 & 19.5413 & 477.5452 & 497.8087 & 487.6979 & 122.5994 & 4.53905 \\
\hline 975 & 19.5583 & 557.9732 & 500.2701 & 488.5208 & 122.5994 & 4.53514 \\
\hline 976 & 19.5753 & 511.8101 & 503.2765 & 489.3438 & 122.5994 & 4.53124 \\
\hline 977 & 19.5923 & 547.5644 & 507.1024 & 490.1667 & 122.5994 & 4.52735 \\
\hline 978 & 19.6093 & 511.1601 & 512.1906 & 490.9896 & 122.5994 & 4.52346 \\
\hline
\end{tabular}




\begin{tabular}{|c|c|c|c|c|c|c|}
\hline 979 & 19.6263 & 528.6165 & 519.2755 & 491.8125 & 122.5994 & 4.51958 \\
\hline 980 & 19.6433 & 523.4711 & 529.3272 & 492.6354 & 122.5994 & 4.51571 \\
\hline 981 & 19.6603 & 573.9046 & 543.3625 & 493.4583 & 122.5994 & 4.51184 \\
\hline 982 & 19.6773 & 555.3288 & 561.9409 & 494.2813 & 122.5994 & 4.50798 \\
\hline 983 & 19.6943 & 583.9613 & 584.2623 & 495.1042 & 122.5994 & 4.50413 \\
\hline 984 & 19.7113 & 607.4064 & 607.0098 & 495.9271 & 122.5994 & 4.50029 \\
\hline 985 & 19.7283 & 620.0216 & 624.4991 & 496.75 & 122.5994 & 4.49645 \\
\hline 986 & 19.7453 & 620.0216 & 633.3128 & 497.0677 & 122.5994 & 4.49261 \\
\hline 987 & 19.7623 & 623.182 & 635.8062 & 497.3854 & 122.5994 & 4.48879 \\
\hline 988 & 19.7793 & 631.4968 & 633.3174 & 497.7031 & 122.5994 & 4.48497 \\
\hline 989 & 19.7963 & 631.7972 & 627.2072 & 498.0208 & 122.5994 & 4.48115 \\
\hline 990 & 19.8133 & 662.7781 & 620.267 & 498.3385 & 122.5994 & 4.47735 \\
\hline 991 & 19.8303 & 677.5481 & 614.3442 & 498.6563 & 122.5994 & 4.47355 \\
\hline 992 & 19.8473 & 718.6752 & 609.7211 & 498.974 & 122.5994 & 4.46975 \\
\hline 993 & 19.8643 & 693.2733 & 605.8165 & 499.2917 & 122.5994 & 4.46597 \\
\hline 994 & 19.8813 & 709.97 & 601.7345 & 499.6094 & 122.5994 & 4.46219 \\
\hline 995 & 19.8983 & 727.9043 & 596.717 & 499.9271 & 122.5994 & 4.45841 \\
\hline 996 & 19.9153 & 664.4468 & 590.5103 & 500.2448 & 122.5994 & 4.45465 \\
\hline 997 & 19.9323 & 644.7207 & 583.1453 & 500.5625 & 122.5994 & 4.45088 \\
\hline 998 & 19.9493 & 650.127 & 575.1364 & 500.8802 & 122.5994 & 4.44713 \\
\hline 999 & 19.9663 & 617.9547 & 567.129 & 501.1979 & 122.5994 & 4.44338 \\
\hline 1000 & 19.9833 & 622.3748 & 559.7128 & 501.5156 & 122.5994 & 4.43964 \\
\hline 1001 & 20.0003 & 640.2764 & 553.5279 & 501.8333 & 122.5994 & 4.4359 \\
\hline 1002 & 20.0173 & 592.6834 & 549.2074 & 502.151 & 122.5994 & 4.43218 \\
\hline 1003 & 20.0343 & 555.4735 & 547.6259 & 502.4688 & 122.5994 & 4.42845 \\
\hline 1004 & 20.0513 & 550.7803 & 550.254 & 502.7865 & 122.5994 & 4.42474 \\
\hline 1005 & 20.0683 & 531.7678 & 559.4174 & 503.1042 & 122.5994 & 4.42103 \\
\hline 1006 & 20.0853 & 532.9871 & 577.9902 & 503.4219 & 122.5994 & 4.41732 \\
\hline 1007 & 20.1023 & 538.2501 & 608.124 & 503.7396 & 122.5994 & 4.41363 \\
\hline 1008 & 20.1193 & 535.1624 & 648.9659 & 504.0573 & 122.5994 & 4.40994 \\
\hline 1009 & 20.1363 & 517.8895 & 693.1957 & 504.375 & 122.5994 & 4.40625 \\
\hline 1010 & 20.1533 & 533.5805 & 724.2071 & 504.263 & 122.5994 & 4.40257 \\
\hline 1011 & 20.1703 & 549.7469 & 729.1402 & 504.151 & 122.5994 & 4.3989 \\
\hline 1012 & 20.1873 & 527.7951 & 713.2549 & 504.0391 & 122.5994 & 4.39523 \\
\hline 1013 & 20.2043 & 563.3428 & 685.3165 & 503.9271 & 122.5994 & 4.39157 \\
\hline 1014 & 20.2213 & 524.0808 & 648.8102 & 503.8151 & 122.5994 & 4.38792 \\
\hline 1015 & 20.2383 & 533.235 & 617.5045 & 503.7031 & 122.5994 & 4.38427 \\
\hline 1016 & 20.2553 & 515.1929 & 586.4785 & 503.5911 & 122.5994 & 4.38063 \\
\hline 1017 & 20.2723 & 518.5837 & 563.9852 & 503.4792 & 122.5994 & 4.377 \\
\hline 1018 & 20.2893 & 499.9008 & 549.1935 & 503.3672 & 122.5994 & 4.37337 \\
\hline 1019 & 20.3063 & 509.47 & 539.7283 & 503.2552 & 122.5994 & 4.36974 \\
\hline 1020 & 20.3233 & 510.7695 & 533.8384 & 503.1432 & 122.5994 & 4.36613 \\
\hline 1021 & 20.3403 & 545.6049 & 530.0866 & 503.0313 & 122.5994 & 4.36252 \\
\hline 1022 & 20.3573 & 498.9014 & 527.5996 & 502.9193 & 122.5994 & 4.35891 \\
\hline 1023 & 20.3743 & 506.1669 & 525.9163 & 502.8073 & 122.5994 & 4.35531 \\
\hline 1024 & 20.3913 & 497.6009 & 524.8049 & 502.6953 & 122.5994 & 4.35172 \\
\hline 1025 & 20.4083 & 524.122 & 524.146 & 502.5833 & 122.5994 & 4.34813 \\
\hline 1026 & 20.4253 & 515.2996 & 523.8743 & 502.4714 & 122.5994 & 4.34455 \\
\hline 1027 & 20.4423 & 513.5995 & 523.9544 & 502.3594 & 122.5994 & 4.34098 \\
\hline
\end{tabular}




\begin{tabular}{|c|c|c|c|c|c|c|}
\hline 1028 & 20.4593 & 496.3662 & 524.2429 & 502.2474 & 122.5994 & 4.33741 \\
\hline 1029 & 20.4763 & 525.7163 & 525.0059 & 502.1354 & 122.5994 & 4.33385 \\
\hline 1030 & 20.4933 & 499.2791 & 525.991 & 502.0234 & 122.5994 & 4.33029 \\
\hline 1031 & 20.5103 & 492.0336 & 527.2677 & 501.9115 & 122.5994 & 4.32674 \\
\hline 1032 & 20.5273 & 529.4319 & 529.2354 & 501.7995 & 122.5994 & 4.3232 \\
\hline 1033 & 20.5443 & 520.2324 & 531.6195 & 501.6875 & 122.5994 & 4.31966 \\
\hline 1034 & 20.5613 & 553.7856 & 534.5173 & 501.5755 & 122.5994 & 4.31612 \\
\hline 1035 & 20.5783 & 545.1571 & 538.0443 & 501.4635 & 122.5994 & 4.3126 \\
\hline 1036 & 20.5953 & 557.3665 & 542.2122 & 501.3516 & 122.5994 & 4.30907 \\
\hline 1037 & 20.6123 & 567.0761 & 546.989 & 501.2396 & 122.5994 & 4.30556 \\
\hline 1038 & 20.6293 & 555.4991 & 552.1158 & 501.1276 & 122.5994 & 4.30205 \\
\hline 1039 & 20.6463 & 555.4125 & 558.0643 & 501.0156 & 122.5994 & 4.29855 \\
\hline 1040 & 20.6633 & 566.787 & 564.8427 & 500.9036 & 122.5994 & 4.29505 \\
\hline 1041 & 20.6803 & 579.6449 & 572.6194 & 500.7917 & 122.5994 & 4.29156 \\
\hline 1042 & 20.6973 & 585.5838 & 582.2566 & 500.6797 & 122.5994 & 4.28807 \\
\hline 1043 & 20.7143 & 625.7849 & 594.8635 & 500.5677 & 122.5994 & 4.28459 \\
\hline 1044 & 20.7313 & 697.5707 & 612.7353 & 500.4557 & 122.5994 & 4.28111 \\
\hline 1045 & 20.7483 & 764.4251 & 640.7098 & 500.3438 & 122.5994 & 4.27764 \\
\hline 1046 & 20.7653 & 815.2085 & 687.6433 & 500.2318 & 122.5994 & 4.27418 \\
\hline 1047 & 20.7823 & 890.6878 & 772.3879 & 500.1198 & 122.5994 & 4.27072 \\
\hline 1048 & 20.7993 & 960.5762 & 917.4573 & 500.0078 & 122.5994 & 4.26727 \\
\hline 1049 & 20.8163 & 1084.093 & 1145.2 & 499.8958 & 122.5994 & 4.26382 \\
\hline 1050 & 20.8333 & 1313.516 & 1461.772 & 499.7839 & 122.5994 & 4.26038 \\
\hline 1051 & 20.8503 & 1554.445 & 1830.542 & 499.6719 & 122.5994 & 4.25695 \\
\hline 1052 & 20.8673 & 1882.655 & 2146.419 & 499.5599 & 122.5994 & 4.25352 \\
\hline 1053 & 20.8843 & 2423.453 & 2279.531 & 499.4479 & 122.5994 & 4.25009 \\
\hline 1054 & 20.9013 & 3157.576 & 2229.224 & 499.3359 & 122.5994 & 4.24667 \\
\hline 1055 & 20.9183 & 3438.209 & 2077.165 & 499.224 & 122.5994 & 4.24326 \\
\hline 1056 & 20.9353 & 2826.317 & 1843.021 & 499.112 & 122.5994 & 4.23985 \\
\hline 1057 & 20.9523 & 2280.004 & 1551.59 & 499 & 122.5994 & 4.23645 \\
\hline 1058 & 20.9693 & 1919.278 & 1272.224 & 498.4583 & 122.5994 & 4.23306 \\
\hline 1059 & 20.9863 & 1466.857 & 1048.687 & 497.9167 & 122.5994 & 4.22967 \\
\hline 1060 & 21.0033 & 987.8199 & 883.2898 & 497.375 & 122.5994 & 4.22628 \\
\hline 1061 & 21.0203 & 725.3194 & 766.5205 & 496.8333 & 122.5994 & 4.2229 \\
\hline 1062 & 21.0373 & 686.4528 & 688.3247 & 496.2917 & 122.5994 & 4.21953 \\
\hline 1063 & 21.0543 & 690.9169 & 637.6871 & 495.75 & 122.5994 & 4.21616 \\
\hline 1064 & 21.0713 & 633.5604 & 605.0176 & 495.2083 & 122.5994 & 4.2128 \\
\hline 1065 & 21.0883 & 576.9574 & 583.7624 & 494.6667 & 122.5994 & 4.20944 \\
\hline 1066 & 21.1053 & 588.7923 & 569.882 & 494.125 & 122.5994 & 4.20609 \\
\hline 1067 & 21.1223 & 581.4374 & 561.2261 & 493.5833 & 122.5994 & 4.20274 \\
\hline 1068 & 21.1393 & 540.0448 & 557.139 & 493.0417 & 122.5994 & 4.1994 \\
\hline 1069 & 21.1563 & 511.3363 & 558.0292 & 492.5 & 122.5994 & 4.19606 \\
\hline 1070 & 21.1733 & 492.8791 & 564.5948 & 491.9583 & 122.5994 & 4.19273 \\
\hline 1071 & 21.1903 & 523.5545 & 576.8072 & 491.4167 & 122.5994 & 4.18941 \\
\hline 1072 & 21.2073 & 489.9996 & 592.4568 & 490.875 & 122.5994 & 4.18609 \\
\hline 1073 & 21.2243 & 524.7202 & 605.5663 & 490.3333 & 122.5994 & 4.18277 \\
\hline 1074 & 21.2413 & 526.252 & 608.718 & 489.7917 & 122.5994 & 4.17946 \\
\hline 1075 & 21.2583 & 502.2472 & 601.4778 & 489.25 & 122.5994 & 4.17616 \\
\hline 1076 & 21.2753 & 538.7219 & 588.7543 & 488.7083 & 122.5994 & 4.17286 \\
\hline
\end{tabular}




\begin{tabular}{|c|c|c|c|c|c|c|}
\hline 1077 & 21.2923 & 514.8732 & 572.4131 & 488.1667 & 122.5994 & 4.16957 \\
\hline 1078 & 21.3093 & 529.6296 & 554.1022 & 487.625 & 122.5994 & 4.16628 \\
\hline 1079 & 21.3263 & 485.0109 & 537.4176 & 487.0833 & 122.5994 & 4.163 \\
\hline 1080 & 21.3433 & 477.5688 & 524.4173 & 486.5417 & 122.5994 & 4.15972 \\
\hline 1081 & 21.3603 & 494.4346 & 515.1945 & 486 & 122.5994 & 4.15645 \\
\hline 1082 & 21.3773 & 474.2961 & 509.3535 & 485.8333 & 122.5994 & 4.15318 \\
\hline 1083 & 21.3943 & 500.5378 & 505.5132 & 485.6667 & 122.5994 & 4.14992 \\
\hline 1084 & 21.4113 & 508.2785 & 502.9949 & 485.5 & 122.5994 & 4.14666 \\
\hline 1085 & 21.4283 & 440.8185 & 501.2122 & 485.3333 & 122.5994 & 4.14341 \\
\hline 1086 & 21.4453 & 472.5884 & 490.8423 & 485.1667 & 122.5994 & 4.14016 \\
\hline 1087 & 21.4623 & 471.336 & 490.3561 & 485 & 122.5994 & 4.13692 \\
\hline 1088 & 21.4793 & 468.0253 & 490.0879 & 484.8333 & 122.5994 & 4.13369 \\
\hline 1089 & 21.4963 & 484.7071 & 490.0304 & 484.6667 & 122.5994 & 4.13046 \\
\hline 1090 & 21.5133 & 468.3823 & 490.7479 & 484.5 & 122.5994 & 4.12723 \\
\hline 1091 & 21.5303 & 455.47 & 491.1876 & 484.3333 & 122.5994 & 4.12401 \\
\hline 1092 & 21.5473 & 459.1984 & 491.8864 & 484.1667 & 122.5994 & 4.12079 \\
\hline 1093 & 21.5643 & 481.8325 & 493.1222 & 484 & 122.5994 & 4.11758 \\
\hline 1094 & 21.5813 & 491.0778 & 494.3581 & 483.8333 & 122.5994 & 4.11438 \\
\hline 1095 & 21.5983 & 492.1992 & 495.8044 & 483.6667 & 122.5994 & 4.11118 \\
\hline 1096 & 21.6153 & 501.4848 & 497.3722 & 483.5 & 122.5994 & 4.10798 \\
\hline 1097 & 21.6323 & 494.5404 & 498.923 & 483.3333 & 122.5994 & 4.10479 \\
\hline 1098 & 21.6493 & 492.9832 & 500.291 & 483.1667 & 122.5994 & 4.10161 \\
\hline 1099 & 21.6663 & 482.4791 & 501.344 & 483 & 122.5994 & 4.09843 \\
\hline 1100 & 21.6833 & 490.8539 & 502.0773 & 482.8333 & 122.5994 & 4.09525 \\
\hline 1101 & 21.7003 & 489.9065 & 502.4662 & 482.6667 & 122.5994 & 4.09209 \\
\hline 1102 & 21.7173 & 502.4013 & 502.4404 & 482.5 & 122.5994 & 4.08892 \\
\hline 1103 & 21.7343 & 520.4735 & 502.4538 & 482.3333 & 122.5994 & 4.08576 \\
\hline 1104 & 21.7513 & 475.7486 & 502.3324 & 482.1667 & 122.5994 & 4.08261 \\
\hline 1105 & 21.7683 & 467.7176 & 501.5961 & 482 & 122.5994 & 4.07946 \\
\hline 1106 & 21.7853 & 498.3211 & 501.6563 & 482.125 & 122.5994 & 4.07631 \\
\hline 1107 & 21.8023 & 460.9789 & 501.7212 & 482.25 & 122.5994 & 4.07317 \\
\hline 1108 & 21.8193 & 466.9531 & 501.8621 & 482.375 & 122.5994 & 4.07004 \\
\hline 1109 & 21.8363 & 470.8988 & 502.1508 & 482.5 & 122.5994 & 4.06691 \\
\hline 1110 & 21.8533 & 482.1936 & 502.687 & 482.625 & 122.5994 & 4.06378 \\
\hline 1111 & 21.8703 & 472.4319 & 503.6763 & 482.75 & 122.5994 & 4.06066 \\
\hline 1112 & 21.8873 & 478.787 & 505.2713 & 482.875 & 122.5994 & 4.05754 \\
\hline 1113 & 21.9043 & 475.5717 & 507.8278 & 483 & 122.5994 & 4.05443 \\
\hline 1114 & 21.9213 & 496.0883 & 511.7566 & 483.125 & 122.5994 & 4.05133 \\
\hline 1115 & 21.9383 & 528.0296 & 517.4532 & 483.25 & 122.5994 & 4.04823 \\
\hline 1116 & 21.9553 & 488.7999 & 525.3425 & 483.375 & 122.5994 & 4.04513 \\
\hline 1117 & 21.9723 & 575.1057 & 535.6672 & 483.5 & 122.5994 & 4.04204 \\
\hline 1118 & 21.9893 & 504.5984 & 548.2845 & 483.625 & 122.5994 & 4.03895 \\
\hline 1119 & 22.0063 & 589.1469 & 562.2657 & 483.75 & 122.5994 & 4.03587 \\
\hline 1120 & 22.0233 & 647.5622 & 575.3967 & 483.875 & 122.5994 & 4.03279 \\
\hline 1121 & 22.0403 & 618.0098 & 584.2887 & 484 & 122.5994 & 4.02972 \\
\hline 1122 & 22.0573 & 653.2829 & 586.3494 & 484.125 & 122.5994 & 4.02665 \\
\hline 1123 & 22.0743 & 706.958 & 581.8276 & 484.25 & 122.5994 & 4.02359 \\
\hline 1124 & 22.0913 & 684.734 & 572.5062 & 484.375 & 122.5994 & 4.02053 \\
\hline 1125 & 22.1083 & 742.43 & 559.8719 & 484.5 & 122.5994 & 4.01748 \\
\hline
\end{tabular}




\begin{tabular}{|c|c|c|c|c|c|c|}
\hline 1126 & 22.1253 & 679.0046 & 545.6371 & 484.625 & 122.5994 & 4.01443 \\
\hline 1127 & 22.1423 & 655.8995 & 531.9086 & 484.75 & 122.5994 & 4.01139 \\
\hline 1128 & 22.1593 & 596.723 & 520.2026 & 484.875 & 122.5994 & 4.00835 \\
\hline 1129 & 22.1763 & 558.5668 & 511.3286 & 485 & 122.5994 & 4.00532 \\
\hline 1130 & 22.1933 & 508.7964 & 504.4221 & 484.8542 & 122.5994 & 4.00229 \\
\hline 1131 & 22.2103 & 479.1739 & 499.5872 & 484.7083 & 122.5994 & 3.99926 \\
\hline 1132 & 22.2273 & 494.2686 & 496.4521 & 484.5625 & 122.5994 & 3.99624 \\
\hline 1133 & 22.2443 & 499.621 & 494.3225 & 484.4167 & 122.5994 & 3.99322 \\
\hline 1134 & 22.2613 & 509.6453 & 492.962 & 484.2708 & 122.5994 & 3.99021 \\
\hline 1135 & 22.2783 & 480.6292 & 492.1279 & 484.125 & 122.5994 & 3.98721 \\
\hline 1136 & 22.2953 & 460.4525 & 491.6697 & 483.9792 & 122.5994 & 3.98421 \\
\hline 1137 & 22.3123 & 483.3398 & 491.4997 & 483.8333 & 122.5994 & 3.98121 \\
\hline 1138 & 22.3293 & 504.8245 & 491.5632 & 483.6875 & 122.5994 & 3.97822 \\
\hline 1139 & 22.3463 & 488.5574 & 491.8159 & 483.5417 & 122.5994 & 3.97523 \\
\hline 1140 & 22.3633 & 518.6143 & 492.2036 & 483.3958 & 122.5994 & 3.97224 \\
\hline 1141 & 22.3803 & 516.1853 & 492.6617 & 483.25 & 122.5994 & 3.96927 \\
\hline 1142 & 22.3973 & 496.2489 & 493.0334 & 483.1042 & 122.5994 & 3.96629 \\
\hline 1143 & 22.4143 & 478.6549 & 493.3508 & 482.9583 & 122.5994 & 3.96332 \\
\hline 1144 & 22.4313 & 475.9748 & 493.4991 & 482.8125 & 122.5994 & 3.96036 \\
\hline 1145 & 22.4483 & 496.624 & 493.4861 & 482.6667 & 122.5994 & 3.9574 \\
\hline 1146 & 22.4653 & 487.679 & 493.3365 & 482.5208 & 122.5994 & 3.95444 \\
\hline 1147 & 22.4823 & 454.2264 & 493.1799 & 482.375 & 122.5994 & 3.95149 \\
\hline 1148 & 22.4993 & 467.2322 & 493.0157 & 482.2292 & 122.5994 & 3.94854 \\
\hline 1149 & 22.5163 & 504.6213 & 492.9866 & 482.0833 & 122.5994 & 3.9456 \\
\hline 1150 & 22.5333 & 515.2406 & 493.3531 & 481.9375 & 122.5994 & 3.94266 \\
\hline 1151 & 22.5503 & 505.2375 & 493.5099 & 481.7917 & 122.5994 & 3.93973 \\
\hline 1152 & 22.5673 & 494.9435 & 493.6698 & 481.6458 & 122.5994 & 3.9368 \\
\hline 1153 & 22.5843 & 482.4403 & 494.2845 & 481.5 & 122.5994 & 3.93387 \\
\hline 1154 & 22.6013 & 483.1674 & 494.1305 & 481.3542 & 122.5994 & 3.93095 \\
\hline 1155 & 22.6183 & 473.1299 & 493.7531 & 481.2083 & 122.5994 & 3.92803 \\
\hline 1156 & 22.6353 & 493.3179 & 493.1888 & 481.0625 & 122.5994 & 3.92512 \\
\hline 1157 & 22.6523 & 451.8445 & 492.5334 & 480.9167 & 122.5994 & 3.92222 \\
\hline 1158 & 22.6693 & 516.0487 & 491.8796 & 480.7708 & 122.5994 & 3.91931 \\
\hline 1159 & 22.6863 & 471.4374 & 491.2941 & 480.625 & 122.5994 & 3.91641 \\
\hline 1160 & 22.7033 & 491.2034 & 491.0267 & 480.4792 & 122.5994 & 3.91352 \\
\hline 1161 & 22.7203 & 443.1384 & 491.1414 & 480.3333 & 122.5994 & 3.91063 \\
\hline 1162 & 22.7373 & 478.8089 & 491.7576 & 480.1875 & 122.5994 & 3.90774 \\
\hline 1163 & 22.7543 & 449.5394 & 492.4726 & 480.0417 & 122.5994 & 3.90486 \\
\hline 1164 & 22.7713 & 496.2775 & 494.8647 & 479.8958 & 122.5994 & 3.90199 \\
\hline 1165 & 22.7883 & 453.6985 & 497.6967 & 479.75 & 122.5994 & 3.89911 \\
\hline 1166 & 22.8053 & 454.8424 & 501.3951 & 479.6042 & 122.5994 & 3.89625 \\
\hline 1167 & 22.8223 & 475.1488 & 505.9426 & 479.4583 & 122.5994 & 3.89338 \\
\hline 1168 & 22.8393 & 518.8558 & 511.2183 & 479.3125 & 122.5994 & 3.89052 \\
\hline 1169 & 22.8563 & 513.7628 & 516.9556 & 479.1667 & 122.5994 & 3.88767 \\
\hline 1170 & 22.8733 & 529.4467 & 522.6997 & 479.0208 & 122.5994 & 3.88482 \\
\hline 1171 & 22.8903 & 514.2665 & 527.8381 & 478.875 & 122.5994 & 3.88197 \\
\hline 1172 & 22.9073 & 552.2603 & 531.7928 & 478.7292 & 122.5994 & 3.87913 \\
\hline 1173 & 22.9243 & 554.3101 & 534.3375 & 478.5833 & 122.5994 & 3.87629 \\
\hline 1174 & 22.9413 & 567.3496 & 535.7598 & 478.4375 & 122.5994 & 3.87346 \\
\hline
\end{tabular}




\begin{tabular}{|c|c|c|c|c|c|c|}
\hline 1175 & 22.9583 & 579.4218 & 536.7305 & 478.2917 & 122.5994 & 3.87063 \\
\hline 1176 & 22.9753 & 565.7306 & 538.1343 & 478.1458 & 122.5994 & 3.8678 \\
\hline 1177 & 22.9923 & 573.1125 & 541.0549 & 478 & 122.5994 & 3.86498 \\
\hline 1178 & 23.0093 & 558.804 & 546.5065 & 477.5833 & 122.5994 & 3.86216 \\
\hline 1179 & 23.0263 & 539.6871 & 556.0718 & 477.1667 & 122.5994 & 3.85935 \\
\hline 1180 & 23.0433 & 528.4963 & 570.6119 & 476.75 & 122.5994 & 3.85654 \\
\hline 1181 & 23.0603 & 519.8497 & 590.8063 & 476.3333 & 122.5994 & 3.85374 \\
\hline 1182 & 23.0773 & 529.3169 & 613.6271 & 475.9167 & 122.5994 & 3.85093 \\
\hline 1183 & 23.0943 & 548.982 & 636.1584 & 475.5 & 122.5994 & 3.84814 \\
\hline 1184 & 23.1113 & 561.2724 & 650.1613 & 475.0833 & 122.5994 & 3.84535 \\
\hline 1185 & 23.1283 & 542.1376 & 646.6307 & 474.6667 & 122.5994 & 3.84256 \\
\hline 1186 & 23.1453 & 512.9903 & 630.4139 & 474.25 & 122.5994 & 3.83977 \\
\hline 1187 & 23.1623 & 520.223 & 608.6528 & 473.8333 & 122.5994 & 3.83699 \\
\hline 1188 & 23.1793 & 502.3483 & 583.825 & 473.4167 & 122.5994 & 3.83422 \\
\hline 1189 & 23.1963 & 497.1051 & 557.458 & 473 & 122.5994 & 3.83145 \\
\hline 1190 & 23.2133 & 505.5236 & 533.7159 & 472.5833 & 122.5994 & 3.82868 \\
\hline 1191 & 23.2303 & 488.3814 & 515.512 & 472.1667 & 122.5994 & 3.82592 \\
\hline 1192 & 23.2473 & 482.3114 & 502.9227 & 471.75 & 122.5994 & 3.82316 \\
\hline 1193 & 23.2643 & 489.7319 & 494.8371 & 471.3333 & 122.5994 & 3.8204 \\
\hline 1194 & 23.2813 & 498.8159 & 489.8622 & 470.9167 & 122.5994 & 3.81765 \\
\hline 1195 & 23.2983 & 507.9834 & 486.804 & 470.5 & 122.5994 & 3.8149 \\
\hline 1196 & 23.3153 & 482.3828 & 484.8149 & 470.0833 & 122.5994 & 3.81216 \\
\hline 1197 & 23.3323 & 467.7227 & 483.5289 & 469.6667 & 122.5994 & 3.80942 \\
\hline 1198 & 23.3493 & 460.0228 & 482.6873 & 469.25 & 122.5994 & 3.80669 \\
\hline 1199 & 23.3663 & 464.6462 & 482.2039 & 468.8333 & 122.5994 & 3.80396 \\
\hline 1200 & 23.3833 & 439.5003 & 482.0482 & 468.4167 & 122.5994 & 3.80123 \\
\hline 1201 & 23.4003 & 471.142 & 482.3127 & 468 & 122.5994 & 3.79851 \\
\hline 1202 & 23.4173 & 483.3113 & 484.0312 & 468.5013 & 122.5994 & 3.79579 \\
\hline 1203 & 23.4343 & 499.329 & 486.5518 & 469.0026 & 122.5994 & 3.79307 \\
\hline 1204 & 23.4513 & 486.0115 & 490.3222 & 469.5039 & 122.5994 & 3.79036 \\
\hline 1205 & 23.4683 & 472.1371 & 496.0038 & 470.0052 & 122.5994 & 3.78765 \\
\hline 1206 & 23.4853 & 499.5184 & 504.4335 & 470.5065 & 122.5994 & 3.78495 \\
\hline 1207 & 23.5023 & 510.3071 & 516.4759 & 471.0078 & 122.5994 & 3.78225 \\
\hline 1208 & 23.5193 & 510.7695 & 532.7354 & 471.5091 & 122.5994 & 3.77955 \\
\hline 1209 & 23.5363 & 614.1848 & 553.1563 & 472.0104 & 122.5994 & 3.77686 \\
\hline 1210 & 23.5533 & 591.3538 & 576.4655 & 472.5117 & 122.5994 & 3.77418 \\
\hline 1211 & 23.5703 & 656.0881 & 599.4622 & 473.013 & 122.5994 & 3.77149 \\
\hline 1212 & 23.5873 & 674.8523 & 617.0086 & 473.5143 & 122.5994 & 3.76881 \\
\hline 1213 & 23.6043 & 658.6067 & 624.7393 & 474.0156 & 122.5994 & 3.76614 \\
\hline 1214 & 23.6213 & 693.3521 & 622.6755 & 474.5169 & 122.5994 & 3.76346 \\
\hline 1215 & 23.6383 & 656.2144 & 613.8467 & 475.0182 & 122.5994 & 3.7608 \\
\hline 1216 & 23.6553 & 624.2303 & 600.7863 & 475.5195 & 122.5994 & 3.75813 \\
\hline 1217 & 23.6723 & 623.3102 & 586.3267 & 476.0208 & 122.5994 & 3.75547 \\
\hline 1218 & 23.6893 & 633.629 & 574.1768 & 476.5221 & 122.5994 & 3.75281 \\
\hline 1219 & 23.7063 & 596.1797 & 566.6218 & 477.0234 & 122.5994 & 3.75016 \\
\hline 1220 & 23.7233 & 567.1702 & 566.8802 & 477.5247 & 122.5994 & 3.74751 \\
\hline 1221 & 23.7403 & 577.3062 & 571.8896 & 478.026 & 122.5994 & 3.74487 \\
\hline 1222 & 23.7573 & 559.9464 & 575.9318 & 478.5273 & 122.5994 & 3.74223 \\
\hline 1223 & 23.7743 & 572.4564 & 575.6369 & 479.0286 & 122.5994 & 3.73959 \\
\hline
\end{tabular}




\begin{tabular}{|c|c|c|c|c|c|c|}
\hline 1224 & 23.7913 & 559.7275 & 573.02 & 479.5299 & 122.5994 & 3.73696 \\
\hline 1225 & 23.8083 & 571.2034 & 570.3505 & 480.0313 & 122.5994 & 3.73433 \\
\hline 1226 & 23.8253 & 548.3738 & 566.7161 & 480.5326 & 122.5994 & 3.7317 \\
\hline 1227 & 23.8423 & 553.164 & 561.979 & 481.0339 & 122.5994 & 3.72908 \\
\hline 1228 & 23.8593 & 606.1148 & 557.252 & 481.5352 & 122.5994 & 3.72646 \\
\hline 1229 & 23.8763 & 584.6066 & 552.9048 & 482.0365 & 122.5994 & 3.72384 \\
\hline 1230 & 23.8933 & 538.7845 & 548.6218 & 482.5378 & 122.5994 & 3.72123 \\
\hline 1231 & 23.9103 & 535.7731 & 544.0695 & 483.0391 & 122.5994 & 3.71863 \\
\hline 1232 & 23.9273 & 540.5126 & 539.0454 & 483.5404 & 122.5994 & 3.71602 \\
\hline 1233 & 23.9443 & 538.1849 & 533.5224 & 484.0417 & 122.5994 & 3.71342 \\
\hline 1234 & 23.9613 & 538.4728 & 527.7234 & 484.543 & 122.5994 & 3.71083 \\
\hline 1235 & 23.9783 & 522.7092 & 522.0552 & 485.0443 & 122.5994 & 3.70823 \\
\hline 1236 & 23.9953 & 519.286 & 516.9259 & 485.5456 & 122.5994 & 3.70565 \\
\hline 1237 & 24.0123 & 523.5676 & 512.631 & 486.0469 & 122.5994 & 3.70306 \\
\hline 1238 & 24.0293 & 534.0408 & 509.3411 & 486.5482 & 122.5994 & 3.70048 \\
\hline 1239 & 24.0463 & 563.9979 & 507.1502 & 487.0495 & 122.5994 & 3.6979 \\
\hline 1240 & 24.0633 & 595.1502 & 506.1282 & 487.5508 & 122.5994 & 3.69533 \\
\hline 1241 & 24.0803 & 578.8356 & 506.3568 & 488.0521 & 122.5994 & 3.69276 \\
\hline 1242 & 24.0973 & 559.7986 & 507.9407 & 488.5534 & 122.5994 & 3.69019 \\
\hline 1243 & 24.1143 & 546.7946 & 510.9936 & 489.0547 & 122.5994 & 3.68763 \\
\hline 1244 & 24.1313 & 542.9001 & 515.5939 & 489.556 & 122.5994 & 3.68507 \\
\hline 1245 & 24.1483 & 532.2563 & 520.9361 & 490.0573 & 122.5994 & 3.68251 \\
\hline 1246 & 24.1653 & 550.9738 & 528.2743 & 490.5586 & 122.5994 & 3.67996 \\
\hline 1247 & 24.1823 & 572.3786 & 536.1204 & 491.0599 & 122.5994 & 3.67741 \\
\hline 1248 & 24.1993 & 556.2796 & 543.0796 & 491.5612 & 122.5994 & 3.67487 \\
\hline 1249 & 24.2163 & 564.3548 & 550.4949 & 492.0625 & 122.5994 & 3.67233 \\
\hline 1250 & 24.2333 & 587.4668 & 557.0333 & 492.2266 & 122.5994 & 3.66979 \\
\hline 1251 & 24.2503 & 603.6364 & 562.4921 & 492.3906 & 122.5994 & 3.66725 \\
\hline 1252 & 24.2673 & 618.7166 & 564.9631 & 492.5547 & 122.5994 & 3.66472 \\
\hline 1253 & 24.2843 & 610.7132 & 563.4055 & 492.7188 & 122.5994 & 3.6622 \\
\hline 1254 & 24.3013 & 629.2223 & 558.3857 & 492.8828 & 122.5994 & 3.65967 \\
\hline 1255 & 24.3183 & 644.8902 & 551.3312 & 493.0469 & 122.5994 & 3.65715 \\
\hline 1256 & 24.3353 & 610.0868 & 543.0555 & 493.2109 & 122.5994 & 3.65464 \\
\hline 1257 & 24.3523 & 605.6575 & 534.1545 & 493.375 & 122.5994 & 3.65212 \\
\hline 1258 & 24.3693 & 625.6331 & 525.5123 & 493.5391 & 122.5994 & 3.64961 \\
\hline 1259 & 24.3863 & 634.813 & 518.0211 & 493.7031 & 122.5994 & 3.64711 \\
\hline 1260 & 24.4033 & 559.2015 & 512.0715 & 493.8672 & 122.5994 & 3.64461 \\
\hline 1261 & 24.4203 & 535.1619 & 507.6445 & 494.0313 & 122.5994 & 3.64211 \\
\hline 1262 & 24.4373 & 548.1896 & 504.519 & 494.1953 & 122.5994 & 3.63961 \\
\hline 1263 & 24.4543 & 543.8184 & 502.3957 & 494.3594 & 122.5994 & 3.63712 \\
\hline 1264 & 24.4713 & 503.5991 & 500.9951 & 494.5234 & 122.5994 & 3.63463 \\
\hline 1265 & 24.4883 & 528.2422 & 500.0842 & 494.6875 & 122.5994 & 3.63215 \\
\hline 1266 & 24.5053 & 509.2233 & 499.4869 & 494.8516 & 122.5994 & 3.62967 \\
\hline 1267 & 24.5223 & 511.2615 & 499.09 & 495.0156 & 122.5994 & 3.62719 \\
\hline 1268 & 24.5393 & 481.7213 & 499.2881 & 495.1797 & 122.5994 & 3.62471 \\
\hline 1269 & 24.5563 & 472.7951 & 499.1322 & 495.3438 & 122.5994 & 3.62224 \\
\hline 1270 & 24.5733 & 473.1602 & 499.0419 & 495.5078 & 122.5994 & 3.61978 \\
\hline 1271 & 24.5903 & 486.983 & 499.0029 & 495.6719 & 122.5994 & 3.61731 \\
\hline 1272 & 24.6073 & 502.8404 & 499.2408 & 495.8359 & 122.5994 & 3.61485 \\
\hline
\end{tabular}




\begin{tabular}{|c|c|c|c|c|c|c|}
\hline 1273 & 24.6243 & 515.0206 & 499.069 & 496 & 122.5994 & 3.6124 \\
\hline 1274 & 24.6413 & 480.3886 & 500.3132 & 497.3073 & 122.5994 & 3.60994 \\
\hline 1275 & 24.6583 & 474.1217 & 501.5906 & 498.6146 & 122.5994 & 3.60749 \\
\hline 1276 & 24.6753 & 486.2716 & 502.7868 & 499.9219 & 122.5994 & 3.60504 \\
\hline 1277 & 24.6923 & 510.5517 & 504.143 & 501.2292 & 122.5994 & 3.6026 \\
\hline 1278 & 24.7093 & 494.7775 & 505.5476 & 502.5365 & 122.5994 & 3.60016 \\
\hline 1279 & 24.7263 & 482.1007 & 506.9963 & 503.8438 & 122.5994 & 3.59772 \\
\hline 1280 & 24.7433 & 475.8449 & 508.3559 & 505.151 & 122.5994 & 3.59529 \\
\hline 1281 & 24.7603 & 498.0685 & 509.9403 & 506.4583 & 122.5994 & 3.59286 \\
\hline 1282 & 24.7773 & 486.3183 & 512.3831 & 507.7656 & 122.5994 & 3.59043 \\
\hline 1283 & 24.7943 & 504.8258 & 514.0933 & 509.0729 & 122.5994 & 3.58801 \\
\hline 1284 & 24.8113 & 509.8134 & 515.8887 & 510.3802 & 122.5994 & 3.58559 \\
\hline 1285 & 24.8283 & 511.4361 & 517.6597 & 511.6875 & 122.5994 & 3.58317 \\
\hline 1286 & 24.8453 & 565.6624 & 519.9725 & 512.9948 & 122.5994 & 3.58076 \\
\hline 1287 & 24.8623 & 537.0834 & 522.1359 & 514.3021 & 122.5994 & 3.57835 \\
\hline 1288 & 24.8793 & 539.3973 & 524.1906 & 515.6094 & 122.5994 & 3.57594 \\
\hline 1289 & 24.8963 & 532.4122 & 526.4441 & 516.9167 & 122.5994 & 3.57354 \\
\hline 1290 & 24.9133 & 531.1769 & 528.7422 & 518.224 & 122.5994 & 3.57114 \\
\hline 1291 & 24.9303 & 526.6647 & 531.1069 & 519.5313 & 122.5994 & 3.56874 \\
\hline 1292 & 24.9473 & 538.7888 & 533.5505 & 520.8385 & 122.5994 & 3.56635 \\
\hline 1293 & 24.9643 & 569.3576 & 536.0631 & 522.1458 & 122.5994 & 3.56396 \\
\hline 1294 & 24.9813 & 548.7781 & 538.6084 & 523.4531 & 122.5994 & 3.56157 \\
\hline 1295 & 24.9983 & 612.0394 & 541.1259 & 524.7604 & 122.5994 & 3.55919 \\
\hline 1296 & 25.0153 & 499.4577 & 543.5633 & 526.0677 & 122.5994 & 3.55681 \\
\hline 1297 & 25.0323 & 569.1912 & 545.9326 & 527.375 & 122.5994 & 3.55443 \\
\hline 1298 & 25.0493 & 616.361 & 548.3819 & 528.6823 & 122.5994 & 3.55206 \\
\hline 1299 & 25.0663 & 597.1422 & 551.0301 & 529.9896 & 122.5994 & 3.54969 \\
\hline 1300 & 25.0833 & 604.6089 & 554.2694 & 531.2969 & 122.5994 & 3.54732 \\
\hline 1301 & 25.1003 & 562.9564 & 558.7893 & 532.6042 & 122.5994 & 3.54496 \\
\hline 1302 & 25.1173 & 580.6802 & 565.9238 & 533.9115 & 122.5994 & 3.5426 \\
\hline 1303 & 25.1343 & 692.0043 & 577.7802 & 535.2188 & 122.5994 & 3.54024 \\
\hline 1304 & 25.1513 & 712.1454 & 597.1384 & 536.526 & 122.5994 & 3.53789 \\
\hline 1305 & 25.1683 & 696.3516 & 626.2881 & 537.8333 & 122.5994 & 3.53553 \\
\hline 1306 & 25.1853 & 779.0466 & 665.0865 & 539.1406 & 122.5994 & 3.53319 \\
\hline 1307 & 25.2023 & 859.8251 & 708.0803 & 540.4479 & 122.5994 & 3.53084 \\
\hline 1308 & 25.2193 & 921.2352 & 741.6736 & 541.7552 & 122.5994 & 3.5285 \\
\hline 1309 & 25.2363 & 900.0168 & 751.9412 & 543.0625 & 122.5994 & 3.52616 \\
\hline 1310 & 25.2533 & 887.4375 & 742.9431 & 544.3698 & 122.5994 & 3.52383 \\
\hline 1311 & 25.2703 & 824.7771 & 727.6028 & 545.6771 & 122.5994 & 3.52149 \\
\hline 1312 & 25.2873 & 818.7564 & 709.0701 & 546.9844 & 122.5994 & 3.51917 \\
\hline 1313 & 25.3043 & 762.1761 & 685.7382 & 548.2917 & 122.5994 & 3.51684 \\
\hline 1314 & 25.3213 & 698.3537 & 662.3653 & 549.599 & 122.5994 & 3.51452 \\
\hline 1315 & 25.3383 & 666.6139 & 645.2775 & 550.9063 & 122.5994 & 3.5122 \\
\hline 1316 & 25.3553 & 623.6855 & 637.0027 & 552.2135 & 122.5994 & 3.50988 \\
\hline 1317 & 25.3723 & 556.5424 & 637.3239 & 553.5208 & 122.5994 & 3.50757 \\
\hline 1318 & 25.3893 & 592.4526 & 644.5721 & 554.8281 & 122.5994 & 3.50526 \\
\hline 1319 & 25.4063 & 628.6103 & 656.149 & 556.1354 & 122.5994 & 3.50295 \\
\hline 1320 & 25.4233 & 640.8311 & 668.862 & 557.4427 & 122.5994 & 3.50065 \\
\hline 1321 & 25.4403 & 654.3735 & 679.817 & 558.75 & 122.5994 & 3.49835 \\
\hline
\end{tabular}




\begin{tabular}{|c|c|c|c|c|c|c|}
\hline 1322 & 25.4573 & 660.5023 & 687.4365 & 559.6771 & 122.5994 & 3.49605 \\
\hline 1323 & 25.4743 & 658.2217 & 692.2412 & 560.6042 & 122.5994 & 3.49375 \\
\hline 1324 & 25.4913 & 684.5986 & 693.9643 & 561.5313 & 122.5994 & 3.49146 \\
\hline 1325 & 25.5083 & 718.2534 & 691.8651 & 562.4583 & 122.5994 & 3.48917 \\
\hline 1326 & 25.5253 & 663.1985 & 686.056 & 563.3854 & 122.5994 & 3.48689 \\
\hline 1327 & 25.5423 & 645.073 & 677.6554 & 564.3125 & 122.5994 & 3.48461 \\
\hline 1328 & 25.5593 & 681.7769 & 667.7713 & 565.2396 & 122.5994 & 3.48233 \\
\hline 1329 & 25.5763 & 665.6919 & 656.9982 & 566.1667 & 122.5994 & 3.48005 \\
\hline 1330 & 25.5933 & 690.6475 & 645.7408 & 567.0938 & 122.5994 & 3.47778 \\
\hline 1331 & 25.6103 & 698.6449 & 634.6052 & 568.0208 & 122.5994 & 3.47551 \\
\hline 1332 & 25.6273 & 663.358 & 624.2495 & 568.9479 & 122.5994 & 3.47324 \\
\hline 1333 & 25.6443 & 646.9043 & 615.1223 & 569.875 & 122.5994 & 3.47098 \\
\hline 1334 & 25.6613 & 673.2931 & 607.3613 & 570.8021 & 122.5994 & 3.46872 \\
\hline 1335 & 25.6783 & 675.4687 & 601.0131 & 571.7292 & 122.5994 & 3.46646 \\
\hline 1336 & 25.6953 & 682.0646 & 596.0482 & 572.6563 & 122.5994 & 3.4642 \\
\hline 1337 & 25.7123 & 655.6697 & 592.3657 & 573.5833 & 122.5994 & 3.46195 \\
\hline 1338 & 25.7293 & 697.138 & 589.8116 & 574.5104 & 122.5994 & 3.4597 \\
\hline 1339 & 25.7463 & 696.1811 & 588.0522 & 575.4375 & 122.5994 & 3.45746 \\
\hline 1340 & 25.7633 & 670.5941 & 587.0071 & 576.3646 & 122.5994 & 3.45521 \\
\hline 1341 & 25.7803 & 644.3985 & 586.4587 & 577.2917 & 122.5994 & 3.45297 \\
\hline 1342 & 25.7973 & 644.3547 & 586.2887 & 578.2188 & 122.5994 & 3.45074 \\
\hline 1343 & 25.8143 & 588.878 & 586.3345 & 579.1458 & 122.5994 & 3.4485 \\
\hline 1344 & 25.8313 & 563.6827 & 586.5856 & 580.0729 & 122.5994 & 3.44627 \\
\hline 1345 & 25.8483 & 579.2504 & 586.9724 & 581 & 122.5994 & 3.44404 \\
\hline 1346 & 25.8653 & 594.4894 & 587.1551 & 581.6199 & 122.5994 & 3.44182 \\
\hline 1347 & 25.8823 & 614.2826 & 587.4123 & 582.2397 & 122.5994 & 3.4396 \\
\hline 1348 & 25.8993 & 576.6952 & 587.7285 & 582.8596 & 122.5994 & 3.43738 \\
\hline 1349 & 25.9163 & 605.152 & 588.0771 & 583.4794 & 122.5994 & 3.43516 \\
\hline 1350 & 25.9333 & 601.2755 & 588.8358 & 584.0993 & 122.5994 & 3.43295 \\
\hline 1351 & 25.9503 & 639.3928 & 611.0674 & 584.7191 & 122.5994 & 3.43074 \\
\hline 1352 & 25.9673 & 624.6697 & 612.6167 & 585.339 & 122.5994 & 3.42853 \\
\hline 1353 & 25.9843 & 617.8015 & 614.0392 & 585.9588 & 122.5994 & 3.42633 \\
\hline 1354 & 26.0013 & 602.5639 & 615.8465 & 586.5787 & 122.5994 & 3.42413 \\
\hline 1355 & 26.0183 & 653.7981 & 628.6553 & 587.1985 & 122.5994 & 3.42193 \\
\hline 1356 & 26.0353 & 602.6209 & 631.4047 & 587.8184 & 122.5994 & 3.41973 \\
\hline 1357 & 26.0523 & 565.0673 & 634.5085 & 588.4382 & 122.5994 & 3.41754 \\
\hline 1358 & 26.0693 & 582.7339 & 638.0719 & 589.0581 & 122.5994 & 3.41535 \\
\hline 1359 & 26.0863 & 590.501 & 642.2667 & 589.6779 & 122.5994 & 3.41316 \\
\hline 1360 & 26.1033 & 591.605 & 655.4822 & 590.2978 & 122.5994 & 3.41098 \\
\hline 1361 & 26.1203 & 603.1402 & 660.9481 & 590.9176 & 122.5994 & 3.40879 \\
\hline 1362 & 26.1373 & 638.0057 & 666.2677 & 591.5375 & 122.5994 & 3.40662 \\
\hline 1363 & 26.1543 & 678.8031 & 670.9502 & 592.1573 & 122.5994 & 3.40444 \\
\hline 1364 & 26.1713 & 690.125 & 679.9391 & 592.7772 & 122.5994 & 3.40227 \\
\hline 1365 & 26.1883 & 681.4768 & 685.2727 & 593.397 & 122.5994 & 3.4001 \\
\hline 1366 & 26.2053 & 683.9048 & 691.089 & 594.0169 & 122.5994 & 3.39793 \\
\hline 1367 & 26.2223 & 672.8529 & 697.2996 & 594.6367 & 122.5994 & 3.39577 \\
\hline 1368 & 26.2393 & 652.903 & 704.3672 & 595.2566 & 122.5994 & 3.3936 \\
\hline 1369 & 26.2563 & 626.5488 & 712.0961 & 595.8764 & 122.5994 & 3.39145 \\
\hline 1370 & 26.2733 & 623.6793 & 721.2001 & 596.0937 & 122.5994 & 3.38929 \\
\hline
\end{tabular}




\begin{tabular}{|c|c|c|c|c|c|c|}
\hline 1371 & 26.2903 & 631.711 & 731.8571 & 596.3111 & 122.5994 & 3.38714 \\
\hline 1372 & 26.3073 & 663.0031 & 744.3484 & 596.5284 & 122.5994 & 3.38499 \\
\hline 1373 & 26.3243 & 676.2337 & 758.8547 & 596.7458 & 122.5994 & 3.38284 \\
\hline 1374 & 26.3413 & 730.8716 & 776.542 & 596.9631 & 122.5994 & 3.38069 \\
\hline 1375 & 26.3583 & 788.6571 & 798.159 & 597.1805 & 122.5994 & 3.37855 \\
\hline 1376 & 26.3753 & 814.2409 & 825.1406 & 597.3978 & 122.5994 & 3.37641 \\
\hline 1377 & 26.3923 & 820.2305 & 859.3285 & 597.6152 & 122.5994 & 3.37428 \\
\hline 1378 & 26.4093 & 858.1466 & 902.6837 & 597.8325 & 122.5994 & 3.37214 \\
\hline 1379 & 26.4263 & 933.3507 & 956.6206 & 598.0498 & 122.5994 & 3.37001 \\
\hline 1380 & 26.4433 & 994.7258 & 1020.969 & 598.2672 & 122.5994 & 3.36789 \\
\hline 1381 & 26.4603 & 1102.111 & 1092.769 & 598.4845 & 122.5994 & 3.36576 \\
\hline 1382 & 26.4773 & 1272.782 & 1166.822 & 598.7019 & 122.5994 & 3.36364 \\
\hline 1383 & 26.4943 & 1346.521 & 1243.73 & 598.9192 & 122.5994 & 3.36152 \\
\hline 1384 & 26.5113 & 1510.052 & 1338.086 & 599.1366 & 122.5994 & 3.3594 \\
\hline 1385 & 26.5283 & 1725.445 & 1476.251 & 599.3539 & 122.5994 & 3.35729 \\
\hline 1386 & 26.5453 & 1967.331 & 1702.554 & 599.5712 & 122.5994 & 3.35517 \\
\hline 1387 & 26.5623 & 2372.804 & 2099.448 & 599.7886 & 122.5994 & 3.35307 \\
\hline 1388 & 26.5793 & 2815.729 & 2797.554 & 600.0059 & 122.5994 & 3.35096 \\
\hline 1389 & 26.5963 & 3420.639 & 3943.404 & 600.2233 & 122.5994 & 3.34886 \\
\hline 1390 & 26.6133 & 4388.9 & 5618.091 & 600.4406 & 122.5994 & 3.34676 \\
\hline 1391 & 26.6303 & 5810.724 & 7714.635 & 600.658 & 122.5994 & 3.34466 \\
\hline 1392 & 26.6473 & 7405.553 & 9765.08 & 600.8753 & 122.5994 & 3.34256 \\
\hline 1393 & 26.6643 & 9763.889 & 10925.97 & 601.0927 & 122.5994 & 3.34047 \\
\hline 1394 & 26.6813 & 12953.93 & 10799.17 & 601.31 & 122.5994 & 3.33838 \\
\hline 1395 & 26.6983 & 14727.57 & 9998.743 & 601.5273 & 122.5994 & 3.33629 \\
\hline 1396 & 26.7153 & 11890.29 & 9100.528 & 601.7447 & 122.5994 & 3.33421 \\
\hline 1397 & 26.7323 & 8292.685 & 8056.757 & 601.962 & 122.5994 & 3.33213 \\
\hline 1398 & 26.7493 & 7648.112 & 6746.737 & 602.1794 & 122.5994 & 3.33005 \\
\hline 1399 & 26.7663 & 7654.188 & 5431.071 & 602.3967 & 122.5994 & 3.32797 \\
\hline 1400 & 26.7833 & 5765.022 & 4378.318 & 602.6141 & 122.5994 & 3.3259 \\
\hline 1401 & 26.8003 & 3509.81 & 3655.365 & 602.8314 & 122.5994 & 3.32383 \\
\hline 1402 & 26.8173 & 2652.554 & 3205.867 & 603.0488 & 122.5994 & 3.32176 \\
\hline 1403 & 26.8343 & 2425.643 & 2928.782 & 603.2661 & 122.5994 & 3.31969 \\
\hline 1404 & 26.8513 & 2511.354 & 2731.583 & 603.4835 & 122.5994 & 3.31763 \\
\hline 1405 & 26.8683 & 2579.601 & 2556.328 & 603.7008 & 122.5994 & 3.31557 \\
\hline 1406 & 26.8853 & 2592.155 & 2376.451 & 603.9182 & 122.5994 & 3.31351 \\
\hline 1407 & 26.9023 & 2303.537 & 2183.736 & 604.1355 & 122.5994 & 3.31146 \\
\hline 1408 & 26.9193 & 1994.514 & 1980.943 & 604.3528 & 122.5994 & 3.3094 \\
\hline 1409 & 26.9363 & 1799.632 & 1777.962 & 604.5702 & 122.5994 & 3.30735 \\
\hline 1410 & 26.9533 & 1575.78 & 1586.437 & 604.7875 & 122.5994 & 3.30531 \\
\hline 1411 & 26.9703 & 1369.733 & 1415.213 & 605.0049 & 122.5994 & 3.30326 \\
\hline 1412 & 26.9873 & 1171.017 & 1268.703 & 605.2222 & 122.5994 & 3.30122 \\
\hline 1413 & 27.0043 & 1006.57 & 1147.632 & 605.4396 & 122.5994 & 3.29918 \\
\hline 1414 & 27.0213 & 890.9256 & 1049.676 & 605.6569 & 122.5994 & 3.29714 \\
\hline 1415 & 27.0383 & 831.0447 & 973.1511 & 605.8743 & 122.5994 & 3.29511 \\
\hline 1416 & 27.0553 & 830.7508 & 913.8381 & 606.0916 & 122.5994 & 3.29308 \\
\hline 1417 & 27.0723 & 791.7265 & 868.2023 & 606.309 & 122.5994 & 3.29105 \\
\hline 1418 & 27.0893 & 745.7679 & 773.9 & 606.1238 & 122.5994 & 3.28902 \\
\hline 1419 & 27.1063 & 726.264 & 750.5147 & 605.9386 & 122.5994 & 3.287 \\
\hline
\end{tabular}




\begin{tabular}{|c|c|c|c|c|c|c|}
\hline 1420 & 27.1233 & 709.3748 & 732.3804 & 605.7535 & 122.5994 & 3.28497 \\
\hline 1421 & 27.1403 & 679.6504 & 717.9977 & 605.5683 & 122.5994 & 3.28295 \\
\hline 1422 & 27.1573 & 665.6524 & 707.7324 & 605.3832 & 122.5994 & 3.28094 \\
\hline 1423 & 27.1743 & 678.0525 & 698.0575 & 605.198 & 122.5994 & 3.27892 \\
\hline 1424 & 27.1913 & 651.5966 & 689.8412 & 605.0129 & 122.5994 & 3.27691 \\
\hline 1425 & 27.2083 & 617.2884 & 682.7493 & 604.8277 & 122.5994 & 3.2749 \\
\hline 1426 & 27.2253 & 616.5851 & 676.8699 & 604.6425 & 122.5994 & 3.2729 \\
\hline 1427 & 27.2423 & 596.5751 & 671.4232 & 604.4574 & 122.5994 & 3.27089 \\
\hline 1428 & 27.2593 & 620.4145 & 666.5936 & 604.2722 & 122.5994 & 3.26889 \\
\hline 1429 & 27.2763 & 578.4243 & 662.2894 & 604.0871 & 122.5994 & 3.26689 \\
\hline 1430 & 27.2933 & 622.8369 & 658.4398 & 603.9019 & 122.5994 & 3.2649 \\
\hline 1431 & 27.3103 & 592.035 & 654.9868 & 603.7168 & 122.5994 & 3.2629 \\
\hline 1432 & 27.3273 & 572.1619 & 651.8831 & 603.5316 & 122.5994 & 3.26091 \\
\hline 1433 & 27.3443 & 559.66 & 649.0896 & 603.3464 & 122.5994 & 3.25892 \\
\hline 1434 & 27.3613 & 556.9704 & 646.5742 & 603.1613 & 122.5994 & 3.25694 \\
\hline 1435 & 27.3783 & 598.1499 & 644.3105 & 602.9761 & 122.5994 & 3.25495 \\
\hline 1436 & 27.3953 & 635.7658 & 642.2768 & 602.791 & 122.5994 & 3.25297 \\
\hline 1437 & 27.4123 & 649.2197 & 640.4563 & 602.6058 & 122.5994 & 3.25099 \\
\hline 1438 & 27.4293 & 669.1558 & 627.5236 & 602.4206 & 122.5994 & 3.24902 \\
\hline 1439 & 27.4463 & 725.4658 & 633.4124 & 602.2355 & 122.5994 & 3.24704 \\
\hline 1440 & 27.4633 & 755.2256 & 633.0743 & 602.0503 & 122.5994 & 3.24507 \\
\hline 1441 & 27.4803 & 816.9522 & 632.9714 & 601.8652 & 122.5994 & 3.2431 \\
\hline 1442 & 27.4973 & 835.9785 & 632.4844 & 601.2743 & 122.5994 & 3.24114 \\
\hline 1443 & 27.5143 & 984.1779 & 635.7717 & 600.6834 & 122.5994 & 3.23917 \\
\hline 1444 & 27.5313 & 918.585 & 636.1871 & 600.0925 & 122.5994 & 3.23721 \\
\hline 1445 & 27.5483 & 933.2684 & 636.9643 & 599.5016 & 122.5994 & 3.23525 \\
\hline 1446 & 27.5653 & 980.4048 & 638.1802 & 598.9107 & 122.5994 & 3.2333 \\
\hline 1447 & 27.5823 & 1045.444 & 632.0854 & 598.3198 & 122.5994 & 3.23134 \\
\hline 1448 & 27.5993 & 998.7685 & 635.1207 & 597.7289 & 122.5994 & 3.22939 \\
\hline 1449 & 27.6163 & 911.5818 & 639.4489 & 597.138 & 122.5994 & 3.22744 \\
\hline 1450 & 27.6333 & 861.7495 & 646.0336 & 596.5471 & 122.5994 & 3.22549 \\
\hline 1451 & 27.6503 & 777.8434 & 651.0972 & 595.9562 & 122.5994 & 3.22355 \\
\hline 1452 & 27.6673 & 723.1683 & 663.8356 & 595.3653 & 122.5994 & 3.22161 \\
\hline 1453 & 27.6843 & 773.0696 & 680.8041 & 594.7744 & 122.5994 & 3.21967 \\
\hline 1454 & 27.7013 & 737.8912 & 702.345 & 594.1836 & 122.5994 & 3.21773 \\
\hline 1455 & 27.7183 & 701.6568 & 727.6461 & 593.5927 & 122.5994 & 3.2158 \\
\hline 1456 & 27.7353 & 729.8868 & 754.1275 & 593.0018 & 122.5994 & 3.21386 \\
\hline 1457 & 27.7523 & 736.3037 & 778.3257 & 592.4109 & 122.5994 & 3.21193 \\
\hline 1458 & 27.7693 & 817.3424 & 797.454 & 591.82 & 122.5994 & 3.21001 \\
\hline 1459 & 27.7863 & 880.6024 & 812.7568 & 591.2291 & 122.5994 & 3.20808 \\
\hline 1460 & 27.8033 & 894.7273 & 828.4928 & 590.6382 & 122.5994 & 3.20616 \\
\hline 1461 & 27.8203 & 875.6835 & 848.1449 & 590.0473 & 122.5994 & 3.20424 \\
\hline 1462 & 27.8373 & 905.1539 & 875.1848 & 589.4564 & 122.5994 & 3.20232 \\
\hline 1463 & 27.8543 & 940.1027 & 914.7003 & 588.8655 & 122.5994 & 3.2004 \\
\hline 1464 & 27.8713 & 1028.731 & 973.5986 & 588.2746 & 122.5994 & 3.19849 \\
\hline 1465 & 27.8883 & 1095.638 & 1058.214 & 587.6837 & 122.5994 & 3.19658 \\
\hline 1466 & 27.9053 & 1144.555 & 1172.032 & 586.5701 & 122.5994 & 3.19467 \\
\hline 1467 & 27.9223 & 1236.739 & 1314.324 & 585.4565 & 122.5994 & 3.19276 \\
\hline 1468 & 27.9393 & 1416.595 & 1474.276 & 584.3429 & 122.5994 & 3.19086 \\
\hline
\end{tabular}




\begin{tabular}{|c|c|c|c|c|c|c|}
\hline 1469 & 27.9563 & 1609.969 & 1625.581 & 583.2293 & 122.5994 & 3.18896 \\
\hline 1470 & 27.9733 & 1748.736 & 1728.609 & 582.1157 & 122.5994 & 3.18706 \\
\hline 1471 & 27.9903 & 1734.699 & 1753.936 & 581.002 & 122.5994 & 3.18516 \\
\hline 1472 & 28.0073 & 1613.379 & 1709.118 & 579.8884 & 122.5994 & 3.18327 \\
\hline 1473 & 28.0243 & 1638.515 & 1622.516 & 578.7748 & 122.5994 & 3.18137 \\
\hline 1474 & 28.0413 & 1771.081 & 1510.646 & 577.6612 & 122.5994 & 3.17948 \\
\hline 1475 & 28.0583 & 1588.102 & 1377.542 & 576.5476 & 122.5994 & 3.1776 \\
\hline 1476 & 28.0753 & 1211.369 & 1233.873 & 575.434 & 122.5994 & 3.17571 \\
\hline 1477 & 28.0923 & 1015.726 & 1098.578 & 574.3204 & 122.5994 & 3.17383 \\
\hline 1478 & 28.1093 & 1005.686 & 985.4089 & 573.2068 & 122.5994 & 3.17195 \\
\hline 1479 & 28.1263 & 898.3118 & 897.9254 & 572.0931 & 122.5994 & 3.17007 \\
\hline 1480 & 28.1433 & 785.5678 & 833.0267 & 570.9795 & 122.5994 & 3.16819 \\
\hline 1481 & 28.1603 & 707.854 & 786.0616 & 569.8659 & 122.5994 & 3.16632 \\
\hline 1482 & 28.1773 & 701.552 & 752.8941 & 568.7523 & 122.5994 & 3.16445 \\
\hline 1483 & 28.1943 & 740.445 & 729.9062 & 567.6387 & 122.5994 & 3.16258 \\
\hline 1484 & 28.2113 & 677.6269 & 713.6528 & 566.5251 & 122.5994 & 3.16071 \\
\hline 1485 & 28.2283 & 680.4966 & 701.061 & 565.4115 & 122.5994 & 3.15884 \\
\hline 1486 & 28.2453 & 599.5167 & 689.6393 & 564.2979 & 122.5994 & 3.15698 \\
\hline 1487 & 28.2623 & 633.9175 & 677.6444 & 563.1843 & 122.5994 & 3.15512 \\
\hline 1488 & 28.2793 & 645.1181 & 664.7986 & 562.0706 & 122.5994 & 3.15326 \\
\hline 1489 & 28.2963 & 665.0739 & 652.0676 & 560.957 & 122.5994 & 3.15141 \\
\hline 1490 & 28.3133 & 629.644 & 638.8882 & 559.3755 & 122.5994 & 3.14955 \\
\hline 1491 & 28.3303 & 618.2708 & 627.0692 & 557.7939 & 122.5994 & 3.1477 \\
\hline 1492 & 28.3473 & 591.6177 & 615.7394 & 556.2124 & 122.5994 & 3.14585 \\
\hline 1493 & 28.3643 & 605.1713 & 605.2046 & 554.6309 & 122.5994 & 3.14401 \\
\hline 1494 & 28.3813 & 597.4623 & 595.5872 & 553.0493 & 122.5994 & 3.14216 \\
\hline 1495 & 28.3983 & 549.818 & 587.7659 & 551.4678 & 122.5994 & 3.14032 \\
\hline 1496 & 28.4153 & 584.9059 & 581.3448 & 549.8862 & 122.5994 & 3.13848 \\
\hline 1497 & 28.4323 & 551.7026 & 576.1604 & 548.3047 & 122.5994 & 3.13664 \\
\hline 1498 & 28.4493 & 523.2336 & 572.0181 & 546.7231 & 122.5994 & 3.13481 \\
\hline 1499 & 28.4663 & 512.3656 & 568.7374 & 545.1416 & 122.5994 & 3.13297 \\
\hline 1500 & 28.4833 & 549.9474 & 566.1853 & 543.5601 & 122.5994 & 3.13114 \\
\hline 1501 & 28.5003 & 559.572 & 564.3049 & 541.9785 & 122.5994 & 3.12931 \\
\hline 1502 & 28.5173 & 567.8623 & 563.139 & 540.397 & 122.5994 & 3.12749 \\
\hline 1503 & 28.5343 & 560.7527 & 562.8332 & 538.8154 & 122.5994 & 3.12566 \\
\hline 1504 & 28.5513 & 541.3471 & 563.5743 & 537.2339 & 122.5994 & 3.12384 \\
\hline 1505 & 28.5683 & 540.8802 & 565.4318 & 535.6523 & 122.5994 & 3.12202 \\
\hline 1506 & 28.5853 & 543.3116 & 568.121 & 534.0708 & 122.5994 & 3.1202 \\
\hline 1507 & 28.6023 & 558.8321 & 570.7162 & 532.4893 & 122.5994 & 3.11838 \\
\hline 1508 & 28.6193 & 539.6811 & 571.5059 & 530.9077 & 122.5994 & 3.11657 \\
\hline 1509 & 28.6363 & 552.1479 & 568.6583 & 529.3262 & 122.5994 & 3.11476 \\
\hline 1510 & 28.6533 & 560.8309 & 564.3729 & 527.7446 & 122.5994 & 3.11295 \\
\hline 1511 & 28.6703 & 547.4144 & 559.6232 & 526.1631 & 122.5994 & 3.11114 \\
\hline 1512 & 28.6873 & 537.999 & 554.7592 & 524.5815 & 122.5994 & 3.10934 \\
\hline 1513 & 28.7043 & 512.6114 & 542.8085 & 523 & 122.5994 & 3.10753 \\
\hline 1514 & 28.7213 & 501.2942 & 536.8961 & 521.125 & 122.5994 & 3.10573 \\
\hline 1515 & 28.7383 & 523.5507 & 531.2216 & 519.25 & 122.5994 & 3.10394 \\
\hline 1516 & 28.7553 & 534.5441 & 526.2493 & 517.375 & 122.5994 & 3.10214 \\
\hline 1517 & 28.7723 & 501.1709 & 522.0709 & 515.5 & 122.5994 & 3.10034 \\
\hline
\end{tabular}




\begin{tabular}{|c|c|c|c|c|c|c|}
\hline 1518 & 28.7893 & 484.009 & 518.4708 & 513.625 & 122.5994 & 3.09855 \\
\hline 1519 & 28.8063 & 475.334 & 515.2595 & 511.75 & 122.5994 & 3.09676 \\
\hline 1520 & 28.8233 & 467.9403 & 512.6488 & 509.875 & 122.5994 & 3.09497 \\
\hline 1521 & 28.8403 & 481.9683 & 510.2939 & 508 & 122.5994 & 3.09319 \\
\hline 1522 & 28.8573 & 475.694 & 508.0447 & 506.125 & 122.5994 & 3.09141 \\
\hline 1523 & 28.8743 & 464.3844 & 505.7676 & 504.25 & 122.5994 & 3.08962 \\
\hline 1524 & 28.8913 & 452.4042 & 503.6859 & 502.375 & 122.5994 & 3.08784 \\
\hline 1525 & 28.9083 & 473.3071 & 501.6504 & 500.5 & 122.5994 & 3.08607 \\
\hline 1526 & 28.9253 & 486.7676 & 499.6671 & 498.625 & 122.5994 & 3.08429 \\
\hline 1527 & 28.9423 & 488.4191 & 497.6903 & 496.75 & 122.5994 & 3.08252 \\
\hline 1528 & 28.9593 & 473.4323 & 495.7318 & 494.875 & 122.5994 & 3.08075 \\
\hline 1529 & 28.9763 & 501.8497 & 493.7874 & 493 & 122.5994 & 3.07898 \\
\hline 1530 & 28.9933 & 510.3111 & 491.8541 & 491.125 & 122.5994 & 3.07721 \\
\hline 1531 & 29.0103 & 494.2849 & 489.93 & 489.25 & 122.5994 & 3.07545 \\
\hline 1532 & 29.0273 & 526.8917 & 488.0135 & 487.375 & 122.5994 & 3.07369 \\
\hline 1533 & 29.0443 & 494.1887 & 486.1035 & 485.5 & 122.5994 & 3.07193 \\
\hline 1534 & 29.0613 & 475.5218 & 484.1992 & 483.625 & 122.5994 & 3.07017 \\
\hline 1535 & 29.0783 & 479.5413 & 482.2999 & 481.75 & 122.5994 & 3.06841 \\
\hline 1536 & 29.0953 & 467.6595 & 480.4052 & 479.875 & 122.5994 & 3.06666 \\
\hline 1537 & 29.1123 & 503.683 & 478.515 & 478 & 122.5994 & 3.0649 \\
\hline 1538 & 29.1293 & 455.837 & 478.7144 & 478.2109 & 122.5994 & 3.06315 \\
\hline 1539 & 29.1463 & 482.0061 & 478.9178 & 478.4219 & 122.5994 & 3.06141 \\
\hline 1540 & 29.1633 & 459.7936 & 479.125 & 478.6328 & 122.5994 & 3.05966 \\
\hline 1541 & 29.1803 & 445.0447 & 479.362 & 478.8438 & 122.5994 & 3.05792 \\
\hline 1542 & 29.1973 & 470.2208 & 480.3542 & 479.0547 & 122.5994 & 3.05618 \\
\hline 1543 & 29.2143 & 467.34 & 480.6134 & 479.2656 & 122.5994 & 3.05444 \\
\hline 1544 & 29.2313 & 473.5162 & 480.8818 & 479.4766 & 122.5994 & 3.0527 \\
\hline 1545 & 29.2483 & 493.9623 & 481.1613 & 479.6875 & 122.5994 & 3.05096 \\
\hline 1546 & 29.2653 & 528.9493 & 481.4675 & 479.8984 & 122.5994 & 3.04923 \\
\hline 1547 & 29.2823 & 511.3786 & 482.173 & 480.1094 & 122.5994 & 3.0475 \\
\hline 1548 & 29.2993 & 470.9974 & 482.524 & 480.3203 & 122.5994 & 3.04577 \\
\hline 1549 & 29.3163 & 490.1335 & 482.9028 & 480.5313 & 122.5994 & 3.04404 \\
\hline 1550 & 29.3333 & 479.6992 & 483.3153 & 480.7422 & 122.5994 & 3.04231 \\
\hline 1551 & 29.3503 & 470.8988 & 483.6696 & 480.9531 & 122.5994 & 3.04059 \\
\hline 1552 & 29.3673 & 489.0395 & 484.1761 & 481.1641 & 122.5994 & 3.03887 \\
\hline 1553 & 29.3843 & 516.2792 & 484.7396 & 481.375 & 122.5994 & 3.03715 \\
\hline 1554 & 29.4013 & 522.1674 & 485.3674 & 481.5859 & 122.5994 & 3.03543 \\
\hline 1555 & 29.4183 & 550.5538 & 486.0152 & 481.7969 & 122.5994 & 3.03372 \\
\hline 1556 & 29.4353 & 584.1781 & 486.7888 & 482.0078 & 122.5994 & 3.032 \\
\hline 1557 & 29.4523 & 619.495 & 487.6411 & 482.2188 & 122.5994 & 3.03029 \\
\hline 1558 & 29.4693 & 652.8925 & 488.5799 & 482.4297 & 122.5994 & 3.02858 \\
\hline 1559 & 29.4863 & 670.8225 & 489.6094 & 482.6406 & 122.5994 & 3.02688 \\
\hline 1560 & 29.5033 & 685.0446 & 490.7186 & 482.8516 & 122.5994 & 3.02517 \\
\hline 1561 & 29.5203 & 721.2173 & 491.8702 & 483.0625 & 122.5994 & 3.02347 \\
\hline 1562 & 29.5373 & 702.384 & 492.8732 & 482.9063 & 122.5994 & 3.02177 \\
\hline 1563 & 29.5543 & 632.9087 & 493.534 & 482.75 & 122.5994 & 3.02007 \\
\hline 1564 & 29.5713 & 643.2739 & 494.0021 & 482.5938 & 122.5994 & 3.01837 \\
\hline 1565 & 29.5883 & 672.3249 & 494.2535 & 482.4375 & 122.5994 & 3.01667 \\
\hline 1566 & 29.6053 & 679.5188 & 494.3286 & 482.2813 & 122.5994 & 3.01498 \\
\hline
\end{tabular}




\begin{tabular}{|c|c|c|c|c|c|c|}
\hline 1567 & 29.6223 & 624.9965 & 494.42 & 482.125 & 122.5994 & 3.01329 \\
\hline 1568 & 29.6393 & 576.0523 & 494.3492 & 481.9688 & 122.5994 & 3.0116 \\
\hline 1569 & 29.6563 & 552.5254 & 494.3023 & 481.8125 & 122.5994 & 3.00991 \\
\hline 1570 & 29.6733 & 569.159 & 494.3784 & 481.6563 & 122.5994 & 3.00822 \\
\hline 1571 & 29.6903 & 561.0837 & 494.7124 & 481.5 & 122.5994 & 3.00654 \\
\hline 1572 & 29.7073 & 572.2415 & 495.5865 & 481.3438 & 122.5994 & 3.00486 \\
\hline 1573 & 29.7243 & 556.4505 & 496.9586 & 481.1875 & 122.5994 & 3.00318 \\
\hline 1574 & 29.7413 & 576.3971 & 499.1709 & 481.0313 & 122.5994 & 3.0015 \\
\hline 1575 & 29.7583 & 543.9272 & 502.4987 & 480.875 & 122.5994 & 2.99983 \\
\hline 1576 & 29.7753 & 571.7883 & 507.2573 & 480.7188 & 122.5994 & 2.99815 \\
\hline 1577 & 29.7923 & 584.7134 & 513.837 & 480.5625 & 122.5994 & 2.99648 \\
\hline 1578 & 29.8093 & 594.7017 & 522.4139 & 480.4063 & 122.5994 & 2.99481 \\
\hline 1579 & 29.8263 & 540.9538 & 533.2388 & 480.25 & 122.5994 & 2.99314 \\
\hline 1580 & 29.8433 & 589.153 & 546.3176 & 480.0938 & 122.5994 & 2.99147 \\
\hline 1581 & 29.8603 & 600.2196 & 561.3615 & 479.9375 & 122.5994 & 2.98981 \\
\hline 1582 & 29.8773 & 670.3922 & 577.6509 & 479.7813 & 122.5994 & 2.98815 \\
\hline 1583 & 29.8943 & 652.0712 & 593.8962 & 479.625 & 122.5994 & 2.98649 \\
\hline 1584 & 29.9113 & 691.4409 & 608.2533 & 479.4688 & 122.5994 & 2.98483 \\
\hline 1585 & 29.9283 & 610.9659 & 618.7479 & 479.3125 & 122.5994 & 2.98317 \\
\hline 1586 & 29.9453 & 677.0914 & 624.1792 & 479.1563 & 122.5994 & 2.98152 \\
\hline 1587 & 29.9623 & 689.09 & 625.2279 & 479 & 122.5994 & 2.97986 \\
\hline 1588 & 29.9793 & 696.8833 & 622.1327 & 478.8438 & 122.5994 & 2.97821 \\
\hline 1589 & 29.9963 & 626.7614 & 616.5665 & 478.6875 & 122.5994 & 2.97656 \\
\hline 1590 & 30.0133 & 583.8255 & 609.383 & 478.5313 & 122.5994 & 2.97492 \\
\hline 1591 & 30.0303 & 573.9946 & 601.3438 & 478.375 & 122.5994 & 2.97327 \\
\hline 1592 & 30.0473 & 565.3466 & 592.9847 & 478.2188 & 122.5994 & 2.97163 \\
\hline 1593 & 30.0643 & 560.4993 & 584.0266 & 478.0625 & 122.5994 & 2.96999 \\
\hline 1594 & 30.0813 & 566.1402 & 574.1069 & 477.9063 & 122.5994 & 2.96835 \\
\hline 1595 & 30.0983 & 577.7543 & 563.3148 & 477.75 & 122.5994 & 2.96671 \\
\hline 1596 & 30.1153 & 553.5826 & 552.7374 & 477.5938 & 122.5994 & 2.96507 \\
\hline 1597 & 30.1323 & 553.7214 & 543.3671 & 477.4375 & 122.5994 & 2.96344 \\
\hline 1598 & 30.1493 & 532.3504 & 535.3661 & 477.2813 & 122.5994 & 2.96181 \\
\hline 1599 & 30.1663 & 538.2501 & 528.5151 & 477.125 & 122.5994 & 2.96018 \\
\hline 1600 & 30.1833 & 530.746 & 522.91 & 476.9688 & 122.5994 & 2.95855 \\
\hline 1601 & 30.2003 & 515.9815 & 518.838 & 476.8125 & 122.5994 & 2.95692 \\
\hline 1602 & 30.2173 & 527.2393 & 516.1903 & 476.6563 & 122.5994 & 2.9553 \\
\hline 1603 & 30.2343 & 543.1984 & 514.3181 & 476.5 & 122.5994 & 2.95367 \\
\hline 1604 & 30.2513 & 545.1236 & 512.3754 & 476.3438 & 122.5994 & 2.95205 \\
\hline 1605 & 30.2683 & 564.424 & 510.0049 & 476.1875 & 122.5994 & 2.95043 \\
\hline 1606 & 30.2853 & 548.4177 & 507.4764 & 476.0313 & 122.5994 & 2.94881 \\
\hline 1607 & 30.3023 & 529.5268 & 505.0852 & 475.875 & 122.5994 & 2.9472 \\
\hline 1608 & 30.3193 & 525.6796 & 502.96 & 475.7188 & 122.5994 & 2.94558 \\
\hline 1609 & 30.3363 & 506.3608 & 501.0969 & 475.5625 & 122.5994 & 2.94397 \\
\hline 1610 & 30.3533 & 505.6468 & 499.5024 & 475.0391 & 122.5994 & 2.94236 \\
\hline 1611 & 30.3703 & 499.2784 & 499.1741 & 474.5156 & 122.5994 & 2.94075 \\
\hline 1612 & 30.3873 & 478.3011 & 500.7958 & 473.9922 & 122.5994 & 2.93915 \\
\hline 1613 & 30.4043 & 529.7343 & 504.8429 & 473.4688 & 122.5994 & 2.93754 \\
\hline 1614 & 30.4213 & 518.0136 & 511.5447 & 472.9453 & 122.5994 & 2.93594 \\
\hline 1615 & 30.4383 & 524.933 & 520.6921 & 472.4219 & 122.5994 & 2.93434 \\
\hline
\end{tabular}




\begin{tabular}{|c|c|c|c|c|c|c|}
\hline 1616 & 30.4553 & 530.168 & 531.368 & 471.8984 & 122.5994 & 2.93274 \\
\hline 1617 & 30.4723 & 550.5487 & 541.605 & 471.375 & 122.5994 & 2.93114 \\
\hline 1618 & 30.4893 & 534.8295 & 548.528 & 470.8516 & 122.5994 & 2.92955 \\
\hline 1619 & 30.5063 & 571.0863 & 550.0615 & 470.3281 & 122.5994 & 2.92795 \\
\hline 1620 & 30.5233 & 597.05 & 546.8203 & 469.8047 & 122.5994 & 2.92636 \\
\hline 1621 & 30.5403 & 571.3074 & 541.499 & 469.2813 & 122.5994 & 2.92477 \\
\hline 1622 & 30.5573 & 497.9477 & 534.2924 & 468.7578 & 122.5994 & 2.92318 \\
\hline 1623 & 30.5743 & 484.4526 & 525.7161 & 468.2344 & 122.5994 & 2.9216 \\
\hline 1624 & 30.5913 & 508.6543 & 515.8924 & 467.7109 & 122.5994 & 2.92001 \\
\hline 1625 & 30.6083 & 519.2679 & 505.8426 & 467.1875 & 122.5994 & 2.91843 \\
\hline 1626 & 30.6253 & 489.5534 & 497.1037 & 466.6641 & 122.5994 & 2.91685 \\
\hline 1627 & 30.6423 & 484.0732 & 489.5023 & 466.1406 & 122.5994 & 2.91527 \\
\hline 1628 & 30.6593 & 474.9792 & 483.5628 & 465.6172 & 122.5994 & 2.91369 \\
\hline 1629 & 30.6763 & 437.7912 & 478.3417 & 465.0938 & 122.5994 & 2.91211 \\
\hline 1630 & 30.6933 & 472.1641 & 475.1008 & 464.5703 & 122.5994 & 2.91054 \\
\hline 1631 & 30.7103 & 492.0842 & 472.7311 & 464.0469 & 122.5994 & 2.90897 \\
\hline 1632 & 30.7273 & 424.3679 & 470.9835 & 463.5234 & 122.5994 & 2.9074 \\
\hline 1633 & 30.7443 & 435.3078 & 469.6341 & 463 & 122.5994 & 2.90583 \\
\hline 1634 & 30.7613 & 515.5717 & 468.14 & 462.6914 & 122.5994 & 2.90426 \\
\hline 1635 & 30.7783 & 491.9426 & 467.4523 & 462.3828 & 122.5994 & 2.9027 \\
\hline 1636 & 30.7953 & 516.4645 & 466.7604 & 462.0742 & 122.5994 & 2.90113 \\
\hline 1637 & 30.8123 & 540.1138 & 466.2722 & 461.7656 & 122.5994 & 2.89957 \\
\hline 1638 & 30.8293 & 539.4504 & 465.8487 & 461.457 & 122.5994 & 2.89801 \\
\hline 1639 & 30.8463 & 573.6813 & 465.4807 & 461.1484 & 122.5994 & 2.89645 \\
\hline 1640 & 30.8633 & 572.8288 & 465.2544 & 460.8398 & 122.5994 & 2.89489 \\
\hline 1641 & 30.8803 & 589.1103 & 465.0042 & 460.5313 & 122.5994 & 2.89334 \\
\hline 1642 & 30.8973 & 607.069 & 464.7924 & 460.2227 & 122.5994 & 2.89179 \\
\hline 1643 & 30.9143 & 650.3378 & 464.628 & 459.9141 & 122.5994 & 2.89023 \\
\hline 1644 & 30.9313 & 643.6705 & 464.5164 & 459.6055 & 122.5994 & 2.88868 \\
\hline 1645 & 30.9483 & 683.6584 & 464.4572 & 459.2969 & 122.5994 & 2.88714 \\
\hline 1646 & 30.9653 & 772.9161 & 464.4714 & 458.9883 & 122.5994 & 2.88559 \\
\hline 1647 & 30.9823 & 857.9372 & 464.5513 & 458.6797 & 122.5994 & 2.88405 \\
\hline 1648 & 30.9993 & 919.1939 & 464.8999 & 458.3711 & 122.5994 & 2.8825 \\
\hline 1649 & 31.0163 & 871.6326 & 465.2103 & 458.0625 & 122.5994 & 2.88096 \\
\hline 1650 & 31.0333 & 789.856 & 465.5378 & 457.7539 & 122.5994 & 2.87942 \\
\hline 1651 & 31.0503 & 770.0879 & 465.981 & 457.4453 & 122.5994 & 2.87788 \\
\hline 1652 & 31.0673 & 742.0015 & 467.0841 & 457.1367 & 122.5994 & 2.87635 \\
\hline 1653 & 31.0843 & 708.6822 & 468.7732 & 456.8281 & 122.5994 & 2.87481 \\
\hline 1654 & 31.1013 & 665.3452 & 471.0554 & 456.5195 & 122.5994 & 2.87328 \\
\hline 1655 & 31.1183 & 590.4774 & 474.0254 & 456.2109 & 122.5994 & 2.87175 \\
\hline 1656 & 31.1353 & 549.4021 & 478.4115 & 455.9023 & 122.5994 & 2.87022 \\
\hline 1657 & 31.1523 & 518.6723 & 484.2436 & 455.5938 & 122.5994 & 2.86869 \\
\hline 1658 & 31.1693 & 513.5665 & 491.4171 & 454.9342 & 122.5994 & 2.86717 \\
\hline 1659 & 31.1863 & 526.0238 & 500.455 & 454.2747 & 122.5994 & 2.86564 \\
\hline 1660 & 31.2033 & 513.1858 & 511.3784 & 453.6152 & 122.5994 & 2.86412 \\
\hline 1661 & 31.2203 & 573.5979 & 523.9417 & 452.9557 & 122.5994 & 2.8626 \\
\hline 1662 & 31.2373 & 582.3521 & 537.4603 & 452.2962 & 122.5994 & 2.86108 \\
\hline 1663 & 31.2543 & 561.5393 & 550.692 & 451.6367 & 122.5994 & 2.85956 \\
\hline 1664 & 31.2713 & 571.8519 & 561.9537 & 450.9772 & 122.5994 & 2.85805 \\
\hline
\end{tabular}




\begin{tabular}{|c|c|c|c|c|c|c|}
\hline 1665 & 31.2883 & 652.1549 & 569.5808 & 450.3177 & 122.5994 & 2.85653 \\
\hline 1666 & 31.3053 & 633.0449 & 572.4222 & 449.6582 & 122.5994 & 2.85502 \\
\hline 1667 & 31.3223 & 608.1538 & 570.1545 & 448.9987 & 122.5994 & 2.85351 \\
\hline 1668 & 31.3393 & 584.0449 & 564.0586 & 448.3392 & 122.5994 & 2.852 \\
\hline 1669 & 31.3563 & 602.4716 & 554.3602 & 447.6797 & 122.5994 & 2.85049 \\
\hline 1670 & 31.3733 & 618.695 & 542.7797 & 447.0202 & 122.5994 & 2.84899 \\
\hline 1671 & 31.3903 & 574.5293 & 530.1525 & 446.3607 & 122.5994 & 2.84748 \\
\hline 1672 & 31.4073 & 571.2207 & 517.2714 & 445.7012 & 122.5994 & 2.84598 \\
\hline 1673 & 31.4243 & 543.5383 & 505.3862 & 445.0417 & 122.5994 & 2.84448 \\
\hline 1674 & 31.4413 & 529.0559 & 494.8276 & 444.3822 & 122.5994 & 2.84298 \\
\hline 1675 & 31.4583 & 497.0566 & 486.2954 & 443.7227 & 122.5994 & 2.84148 \\
\hline 1676 & 31.4753 & 484.8945 & 479.7386 & 443.0632 & 122.5994 & 2.83999 \\
\hline 1677 & 31.4923 & 506.8139 & 474.7681 & 442.4036 & 122.5994 & 2.83849 \\
\hline 1678 & 31.5093 & 514.8657 & 470.9061 & 441.7441 & 122.5994 & 2.837 \\
\hline 1679 & 31.5263 & 508.4387 & 467.8884 & 441.0846 & 122.5994 & 2.83551 \\
\hline 1680 & 31.5433 & 486.762 & 465.6825 & 440.4251 & 122.5994 & 2.83402 \\
\hline 1681 & 31.5603 & 477.8424 & 464.2543 & 439.7656 & 122.5994 & 2.83253 \\
\hline 1682 & 31.5773 & 481.2835 & 463.4626 & 439.1061 & 122.5994 & 2.83105 \\
\hline 1683 & 31.5943 & 436.5648 & 463.1631 & 438.4466 & 122.5994 & 2.82956 \\
\hline 1684 & 31.6113 & 488.3632 & 463.3461 & 437.7871 & 122.5994 & 2.82808 \\
\hline 1685 & 31.6283 & 482.3138 & 464.099 & 437.1276 & 122.5994 & 2.8266 \\
\hline 1686 & 31.6453 & 468.9273 & 465.4681 & 436.4681 & 122.5994 & 2.82512 \\
\hline 1687 & 31.6623 & 490.874 & 467.3891 & 435.8086 & 122.5994 & 2.82364 \\
\hline 1688 & 31.6793 & 493.339 & 469.6026 & 435.1491 & 122.5994 & 2.82216 \\
\hline 1689 & 31.6963 & 470.2696 & 471.7136 & 434.4896 & 122.5994 & 2.82069 \\
\hline 1690 & 31.7133 & 443.8353 & 473.1996 & 433.8301 & 122.5994 & 2.81922 \\
\hline 1691 & 31.7303 & 447.9151 & 473.5775 & 433.1706 & 122.5994 & 2.81774 \\
\hline 1692 & 31.7473 & 463.5089 & 472.6205 & 432.5111 & 122.5994 & 2.81627 \\
\hline 1693 & 31.7643 & 491.0526 & 470.52 & 431.8516 & 122.5994 & 2.81481 \\
\hline 1694 & 31.7813 & 480.9366 & 467.7379 & 431.1921 & 122.5994 & 2.81334 \\
\hline 1695 & 31.7983 & 468.9563 & 464.5889 & 430.5326 & 122.5994 & 2.81187 \\
\hline 1696 & 31.8153 & 466.5687 & 461.2162 & 429.873 & 122.5994 & 2.81041 \\
\hline 1697 & 31.8323 & 453.9506 & 457.776 & 429.2135 & 122.5994 & 2.80895 \\
\hline 1698 & 31.8493 & 438.8583 & 454.5906 & 428.554 & 122.5994 & 2.80749 \\
\hline 1699 & 31.8663 & 445.8429 & 452.0633 & 427.8945 & 122.5994 & 2.80603 \\
\hline 1700 & 31.8833 & 463.2647 & 450.5232 & 427.235 & 122.5994 & 2.80457 \\
\hline 1701 & 31.9003 & 488.7328 & 450.2183 & 426.5755 & 122.5994 & 2.80311 \\
\hline 1702 & 31.9173 & 470.4167 & 451.3504 & 425.916 & 122.5994 & 2.80166 \\
\hline 1703 & 31.9343 & 479.0959 & 454.0897 & 425.2565 & 122.5994 & 2.80021 \\
\hline 1704 & 31.9513 & 453.1157 & 458.5638 & 424.597 & 122.5994 & 2.79876 \\
\hline 1705 & 31.9683 & 471.2096 & 464.8228 & 423.9375 & 122.5994 & 2.79731 \\
\hline 1706 & 31.9853 & 505.1293 & 472.4333 & 422.9271 & 122.5994 & 2.79586 \\
\hline 1707 & 32.0023 & 501.1918 & 481.4548 & 421.9167 & 122.5994 & 2.79441 \\
\hline 1708 & 32.0193 & 497.3563 & 490.6393 & 420.9062 & 122.5994 & 2.79297 \\
\hline 1709 & 32.0363 & 552.6648 & 500.2868 & 419.8958 & 122.5994 & 2.79152 \\
\hline 1710 & 32.0533 & 493.898 & 508.3091 & 418.8854 & 122.5994 & 2.79008 \\
\hline 1711 & 32.0703 & 491.7756 & 513.2943 & 417.875 & 122.5994 & 2.78864 \\
\hline 1712 & 32.0873 & 511.8989 & 514.4886 & 416.8646 & 122.5994 & 2.7872 \\
\hline 1713 & 32.1043 & 526.8189 & 511.7766 & 415.8542 & 122.5994 & 2.78577 \\
\hline
\end{tabular}




\begin{tabular}{|c|c|c|c|c|c|c|}
\hline 1714 & 32.1213 & 497.9484 & 506.6637 & 414.8437 & 122.5994 & 2.78433 \\
\hline 1715 & 32.1383 & 512.6386 & 499.4337 & 413.8333 & 122.5994 & 2.7829 \\
\hline 1716 & 32.1553 & 471.3644 & 490.5365 & 412.8229 & 122.5994 & 2.78147 \\
\hline 1717 & 32.1723 & 503.2181 & 480.3862 & 411.8125 & 122.5994 & 2.78003 \\
\hline 1718 & 32.1893 & 476.9446 & 469.6338 & 410.8021 & 122.5994 & 2.7786 \\
\hline 1719 & 32.2063 & 434.0904 & 459.0182 & 409.7917 & 122.5994 & 2.77718 \\
\hline 1720 & 32.2233 & 457.6767 & 449.1412 & 408.7812 & 122.5994 & 2.77575 \\
\hline 1721 & 32.2403 & 432.9024 & 440.3919 & 407.7708 & 122.5994 & 2.77433 \\
\hline 1722 & 32.2573 & 457.4156 & 432.9153 & 406.7604 & 122.5994 & 2.7729 \\
\hline 1723 & 32.2743 & 443.8244 & 426.6703 & 405.75 & 122.5994 & 2.77148 \\
\hline 1724 & 32.2913 & 491.4681 & 421.6913 & 404.7396 & 122.5994 & 2.77006 \\
\hline 1725 & 32.3083 & 418.455 & 417.447 & 403.7292 & 122.5994 & 2.76864 \\
\hline 1726 & 32.3253 & 441.0588 & 413.9341 & 402.7187 & 122.5994 & 2.76722 \\
\hline 1727 & 32.3423 & 435.8876 & 411.0447 & 401.7083 & 122.5994 & 2.76581 \\
\hline 1728 & 32.3593 & 407.9492 & 408.6226 & 400.6979 & 122.5994 & 2.76439 \\
\hline 1729 & 32.3763 & 424.0391 & 406.6439 & 399.6875 & 122.5994 & 2.76298 \\
\hline 1730 & 32.3933 & 413.128 & 404.3991 & 398.2422 & 122.5994 & 2.76157 \\
\hline 1731 & 32.4103 & 438.4646 & 402.3349 & 396.7969 & 122.5994 & 2.76016 \\
\hline 1732 & 32.4273 & 399.9616 & 400.4185 & 395.3516 & 122.5994 & 2.75875 \\
\hline 1733 & 32.4443 & 398.9909 & 398.6055 & 393.9063 & 122.5994 & 2.75735 \\
\hline 1734 & 32.4613 & 392.5628 & 396.7111 & 392.4609 & 122.5994 & 2.75594 \\
\hline 1735 & 32.4783 & 389.0196 & 395.0501 & 391.0156 & 122.5994 & 2.75454 \\
\hline 1736 & 32.4953 & 410.8496 & 393.4585 & 389.5703 & 122.5994 & 2.75313 \\
\hline 1737 & 32.5123 & 425.361 & 391.9302 & 388.125 & 122.5994 & 2.75173 \\
\hline 1738 & 32.5293 & 406.1336 & 390.386 & 386.6797 & 122.5994 & 2.75033 \\
\hline 1739 & 32.5463 & 399.1341 & 389.062 & 385.2344 & 122.5994 & 2.74894 \\
\hline 1740 & 32.5633 & 404.0639 & 387.8436 & 383.7891 & 122.5994 & 2.74754 \\
\hline 1741 & 32.5803 & 403.9712 & 386.8802 & 382.3438 & 122.5994 & 2.74615 \\
\hline 1742 & 32.5973 & 380.7218 & 386.3893 & 380.8984 & 122.5994 & 2.74475 \\
\hline 1743 & 32.6143 & 364.5207 & 386.7661 & 379.4531 & 122.5994 & 2.74336 \\
\hline 1744 & 32.6313 & 372.4421 & 388.3466 & 378.0078 & 122.5994 & 2.74197 \\
\hline 1745 & 32.6483 & 385.1364 & 391.3601 & 376.5625 & 122.5994 & 2.74058 \\
\hline 1746 & 32.6653 & 420.0558 & 395.534 & 375.1172 & 122.5994 & 2.73919 \\
\hline 1747 & 32.6823 & 411.4596 & 399.5916 & 373.6719 & 122.5994 & 2.73781 \\
\hline 1748 & 32.6993 & 385.0586 & 401.151 & 372.2266 & 122.5994 & 2.73642 \\
\hline 1749 & 32.7163 & 392.2685 & 398.8145 & 370.7813 & 122.5994 & 2.73504 \\
\hline 1750 & 32.7333 & 385.1122 & 394.411 & 369.3359 & 122.5994 & 2.73366 \\
\hline 1751 & 32.7503 & 373.7951 & 390.2613 & 367.8906 & 122.5994 & 2.73228 \\
\hline 1752 & 32.7673 & 375.1523 & 386.9267 & 366.4453 & 122.5994 & 2.7309 \\
\hline 1753 & 32.7843 & 360.3597 & 383.4125 & 365 & 122.5994 & 2.72952 \\
\hline 1754 & 32.8013 & 358.8337 & 381.2371 & 365.9583 & 122.5994 & 2.72815 \\
\hline 1755 & 32.8183 & 375.2852 & 378.0806 & 366.9167 & 122.5994 & 2.72677 \\
\hline 1756 & 32.8353 & 389.1858 & 375.8494 & 367.875 & 122.5994 & 2.7254 \\
\hline 1757 & 32.8523 & 398.3222 & 374.4424 & 368.8333 & 122.5994 & 2.72403 \\
\hline 1758 & 32.8693 & 399.9616 & 373.8012 & 369.7917 & 122.5994 & 2.72266 \\
\hline 1759 & 32.8863 & 367.85 & 373.7804 & 370.75 & 122.5994 & 2.72129 \\
\hline 1760 & 32.9033 & 400.8152 & 373.9106 & 371.7083 & 122.5994 & 2.71992 \\
\hline 1761 & 32.9203 & 408.0009 & 374.5421 & 372.6667 & 122.5994 & 2.71856 \\
\hline 1762 & 32.9373 & 400.937 & 375.3178 & 373.625 & 122.5994 & 2.71719 \\
\hline
\end{tabular}




\begin{tabular}{|c|c|c|c|c|c|c|}
\hline 1763 & 32.9543 & 389.104 & 376.148 & 374.5833 & 122.5994 & 2.71583 \\
\hline 1764 & 32.9713 & 388.1417 & 377.0251 & 375.5417 & 122.5994 & 2.71447 \\
\hline 1765 & 32.9883 & 410.9658 & 377.9377 & 376.5 & 122.5994 & 2.71311 \\
\hline 1766 & 33.0053 & 436.1804 & 378.881 & 377.4583 & 122.5994 & 2.71175 \\
\hline 1767 & 33.0223 & 466.5521 & 379.8539 & 378.4167 & 122.5994 & 2.71039 \\
\hline 1768 & 33.0393 & 392.3237 & 380.8588 & 379.375 & 122.5994 & 2.70904 \\
\hline 1769 & 33.0563 & 406.3446 & 381.9018 & 380.3333 & 122.5994 & 2.70768 \\
\hline 1770 & 33.0733 & 460.4639 & 382.9929 & 381.2917 & 122.5994 & 2.70633 \\
\hline 1771 & 33.0903 & 434.0067 & 384.147 & 382.25 & 122.5994 & 2.70498 \\
\hline 1772 & 33.1073 & 445.0399 & 385.3841 & 383.2083 & 122.5994 & 2.70363 \\
\hline 1773 & 33.1243 & 408.6083 & 386.5563 & 384.1667 & 122.5994 & 2.70228 \\
\hline 1774 & 33.1413 & 415.0983 & 388.0468 & 385.125 & 122.5994 & 2.70093 \\
\hline 1775 & 33.1583 & 430.5918 & 389.6954 & 386.0833 & 122.5994 & 2.69958 \\
\hline 1776 & 33.1753 & 424.1809 & 391.5248 & 387.0417 & 122.5994 & 2.69824 \\
\hline 1777 & 33.1923 & 428.03 & 393.449 & 388 & 122.5994 & 2.6969 \\
\hline 1778 & 33.2093 & 383.0649 & 395.6487 & 388.9583 & 122.5994 & 2.69556 \\
\hline 1779 & 33.2263 & 406.6895 & 397.979 & 389.9167 & 122.5994 & 2.69422 \\
\hline 1780 & 33.2433 & 439.1345 & 400.3543 & 390.875 & 122.5994 & 2.69288 \\
\hline 1781 & 33.2603 & 406.0567 & 402.6546 & 391.8333 & 122.5994 & 2.69154 \\
\hline 1782 & 33.2773 & 374.947 & 404.7623 & 392.7917 & 122.5994 & 2.6902 \\
\hline 1783 & 33.2943 & 348.8152 & 406.5953 & 393.75 & 122.5994 & 2.68887 \\
\hline 1784 & 33.3113 & 377.109 & 408.1011 & 394.7083 & 122.5994 & 2.68753 \\
\hline 1785 & 33.3283 & 377.2281 & 409.2437 & 395.6667 & 122.5994 & 2.6862 \\
\hline 1786 & 33.3453 & 365.8938 & 410.0286 & 396.625 & 122.5994 & 2.68487 \\
\hline 1787 & 33.3623 & 403.1912 & 410.5353 & 397.5833 & 122.5994 & 2.68354 \\
\hline 1788 & 33.3793 & 437.8674 & 410.8955 & 398.5417 & 122.5994 & 2.68221 \\
\hline 1789 & 33.3963 & 431.8998 & 411.2401 & 399.5 & 122.5994 & 2.68089 \\
\hline 1790 & 33.4133 & 402.173 & 411.6718 & 400.4583 & 122.5994 & 2.67956 \\
\hline 1791 & 33.4303 & 394.0432 & 412.2762 & 401.4167 & 122.5994 & 2.67824 \\
\hline 1792 & 33.4473 & 411.2502 & 413.1331 & 402.375 & 122.5994 & 2.67692 \\
\hline 1793 & 33.4643 & 401.9226 & 414.2978 & 403.3333 & 122.5994 & 2.6756 \\
\hline 1794 & 33.4813 & 400.3766 & 415.7726 & 404.2917 & 122.5994 & 2.67428 \\
\hline 1795 & 33.4983 & 416.9481 & 417.6264 & 405.25 & 122.5994 & 2.67296 \\
\hline 1796 & 33.5153 & 410.1145 & 419.4635 & 406.2083 & 122.5994 & 2.67164 \\
\hline 1797 & 33.5323 & 404.0639 & 421.235 & 407.1667 & 122.5994 & 2.67033 \\
\hline 1798 & 33.5493 & 385.5925 & 422.7721 & 408.125 & 122.5994 & 2.66901 \\
\hline 1799 & 33.5663 & 392.6353 & 423.9602 & 409.0833 & 122.5994 & 2.6677 \\
\hline 1800 & 33.5833 & 423.063 & 424.8266 & 410.0417 & 122.5994 & 2.66639 \\
\hline 1801 & 33.6003 & 414.7541 & 425.263 & 411 & 122.5994 & 2.66508 \\
\hline 1802 & 33.6173 & 404.975 & 425.4259 & 412 & 122.5994 & 2.66377 \\
\hline 1803 & 33.6343 & 413.3541 & 425.3351 & 413 & 122.5994 & 2.66246 \\
\hline 1804 & 33.6513 & 422.7255 & 425.0653 & 414 & 122.5994 & 2.66115 \\
\hline 1805 & 33.6683 & 417.6337 & 424.7036 & 415 & 122.5994 & 2.65985 \\
\hline 1806 & 33.6853 & 409.4193 & 424.3403 & 416 & 122.5994 & 2.65855 \\
\hline 1807 & 33.7023 & 397.6744 & 424.0546 & 417 & 122.5994 & 2.65724 \\
\hline 1808 & 33.7193 & 389.0039 & 424.0362 & 418 & 122.5994 & 2.65594 \\
\hline 1809 & 33.7363 & 388.1417 & 424.0651 & 419 & 122.5994 & 2.65464 \\
\hline 1810 & 33.7533 & 406.4643 & 424.28 & 420 & 122.5994 & 2.65335 \\
\hline 1811 & 33.7703 & 427.4101 & 424.6768 & 421 & 122.5994 & 2.65205 \\
\hline
\end{tabular}




\begin{tabular}{|c|c|c|c|c|c|c|}
\hline 1812 & 33.7873 & 432.4576 & 425.2398 & 422 & 122.5994 & 2.65075 \\
\hline 1813 & 33.8043 & 380.9572 & 425.9774 & 423 & 122.5994 & 2.64946 \\
\hline 1814 & 33.8213 & 369.0653 & 426.8202 & 424 & 122.5994 & 2.64817 \\
\hline 1815 & 33.8383 & 419.2025 & 427.7949 & 425 & 122.5994 & 2.64687 \\
\hline 1816 & 33.8553 & 384.1232 & 428.8948 & 426 & 122.5994 & 2.64558 \\
\hline 1817 & 33.8723 & 387.4759 & 430.3005 & 427 & 122.5994 & 2.6443 \\
\hline 1818 & 33.8893 & 425.7405 & 431.8995 & 428 & 122.5994 & 2.64301 \\
\hline 1819 & 33.9063 & 402.3205 & 433.7441 & 429 & 122.5994 & 2.64172 \\
\hline 1820 & 33.9233 & 415.3207 & 436.046 & 430 & 122.5994 & 2.64044 \\
\hline 1821 & 33.9403 & 393.8641 & 438.9062 & 431 & 122.5994 & 2.63915 \\
\hline 1822 & 33.9573 & 446.0359 & 442.4436 & 432 & 122.5994 & 2.63787 \\
\hline 1823 & 33.9743 & 447.6867 & 446.4575 & 433 & 122.5994 & 2.63659 \\
\hline 1824 & 33.9913 & 441.0588 & 450.7481 & 434 & 122.5994 & 2.63531 \\
\hline 1825 & 34.0083 & 444.0756 & 454.6694 & 435 & 122.5994 & 2.63403 \\
\hline 1826 & 34.0253 & 445.8119 & 457.4844 & 436 & 122.5994 & 2.63275 \\
\hline 1827 & 34.0423 & 410.5558 & 458.8455 & 437 & 122.5994 & 2.63148 \\
\hline 1828 & 34.0593 & 401.6351 & 458.9525 & 438 & 122.5994 & 2.6302 \\
\hline 1829 & 34.0763 & 436.1464 & 458.6278 & 439 & 122.5994 & 2.62893 \\
\hline 1830 & 34.0933 & 418.3271 & 458.1809 & 440 & 122.5994 & 2.62766 \\
\hline 1831 & 34.1103 & 460.2038 & 457.5969 & 441 & 122.5994 & 2.62639 \\
\hline 1832 & 34.1273 & 442.7386 & 456.6791 & 442 & 122.5994 & 2.62512 \\
\hline 1833 & 34.1443 & 439.6167 & 455.5193 & 443 & 122.5994 & 2.62385 \\
\hline 1834 & 34.1613 & 445.7914 & 454.7428 & 444 & 122.5994 & 2.62258 \\
\hline 1835 & 34.1783 & 450.7501 & 454.1232 & 445 & 122.5994 & 2.62132 \\
\hline 1836 & 34.1953 & 454.0904 & 454.0099 & 446 & 122.5994 & 2.62005 \\
\hline 1837 & 34.2123 & 424.4072 & 454.4826 & 447 & 122.5994 & 2.61879 \\
\hline 1838 & 34.2293 & 436.8292 & 455.6222 & 448 & 122.5994 & 2.61753 \\
\hline 1839 & 34.2463 & 452.642 & 457.9419 & 449 & 122.5994 & 2.61627 \\
\hline 1840 & 34.2633 & 443.3506 & 461.147 & 450 & 122.5994 & 2.61501 \\
\hline 1841 & 34.2803 & 455.3234 & 465.731 & 451 & 122.5994 & 2.61375 \\
\hline 1842 & 34.2973 & 462.2057 & 471.6486 & 452 & 122.5994 & 2.61249 \\
\hline 1843 & 34.3143 & 467.7816 & 478.048 & 453 & 122.5994 & 2.61124 \\
\hline 1844 & 34.3313 & 459.5871 & 483.6248 & 454 & 122.5994 & 2.60998 \\
\hline 1845 & 34.3483 & 486.1388 & 485.6508 & 455 & 122.5994 & 2.60873 \\
\hline 1846 & 34.3653 & 465.3291 & 485.4113 & 456 & 122.5994 & 2.60748 \\
\hline 1847 & 34.3823 & 449.3969 & 485.0405 & 457 & 122.5994 & 2.60623 \\
\hline 1848 & 34.3993 & 440.1454 & 485.7526 & 458 & 122.5994 & 2.60498 \\
\hline 1849 & 34.4163 & 462.7397 & 487.1917 & 459 & 122.5994 & 2.60373 \\
\hline 1850 & 34.4333 & 474.253 & 488.2296 & 459.875 & 122.5994 & 2.60249 \\
\hline 1851 & 34.4503 & 485.9071 & 488.3796 & 460.75 & 122.5994 & 2.60124 \\
\hline 1852 & 34.4673 & 514.3422 & 488.6874 & 461.625 & 122.5994 & 2.6 \\
\hline 1853 & 34.4843 & 531.5892 & 489.9009 & 462.5 & 122.5994 & 2.59875 \\
\hline 1854 & 34.5013 & 549.7689 & 492.3033 & 463.375 & 122.5994 & 2.59751 \\
\hline 1855 & 34.5183 & 527.5102 & 495.834 & 464.25 & 122.5994 & 2.59627 \\
\hline 1856 & 34.5353 & 545.4889 & 500.2845 & 465.125 & 122.5994 & 2.59503 \\
\hline 1857 & 34.5523 & 525.8824 & 505.1928 & 466 & 122.5994 & 2.5938 \\
\hline 1858 & 34.5693 & 511.4335 & 510.08 & 466.875 & 122.5994 & 2.59256 \\
\hline 1859 & 34.5863 & 515.1377 & 514.7653 & 467.75 & 122.5994 & 2.59132 \\
\hline 1860 & 34.6033 & 506.3291 & 518.7251 & 468.625 & 122.5994 & 2.59009 \\
\hline
\end{tabular}




\begin{tabular}{|c|c|c|c|c|c|c|}
\hline 1861 & 34.6203 & 565.9106 & 521.8521 & 469.5 & 122.5994 & 2.58886 \\
\hline 1862 & 34.6373 & 541.9984 & 524.5126 & 470.375 & 122.5994 & 2.58762 \\
\hline 1863 & 34.6543 & 520.3694 & 527.2399 & 471.25 & 122.5994 & 2.58639 \\
\hline 1864 & 34.6713 & 527.5221 & 530.8885 & 472.125 & 122.5994 & 2.58516 \\
\hline 1865 & 34.6883 & 508.335 & 535.6879 & 473 & 122.5994 & 2.58394 \\
\hline 1866 & 34.7053 & 490.1834 & 541.1838 & 473.875 & 122.5994 & 2.58271 \\
\hline 1867 & 34.7223 & 509.2541 & 545.855 & 474.75 & 122.5994 & 2.58148 \\
\hline 1868 & 34.7393 & 550.5983 & 547.5588 & 475.625 & 122.5994 & 2.58026 \\
\hline 1869 & 34.7563 & 534.1866 & 546.6105 & 476.5 & 122.5994 & 2.57904 \\
\hline 1870 & 34.7733 & 506.9948 & 546.5941 & 477.375 & 122.5994 & 2.57781 \\
\hline 1871 & 34.7903 & 539.691 & 549.8412 & 478.25 & 122.5994 & 2.57659 \\
\hline 1872 & 34.8073 & 530.1338 & 556.541 & 479.125 & 122.5994 & 2.57537 \\
\hline 1873 & 34.8243 & 536.2665 & 564.5577 & 480 & 122.5994 & 2.57416 \\
\hline 1874 & 34.8413 & 554.2144 & 570.6223 & 480.526 & 122.5994 & 2.57294 \\
\hline 1875 & 34.8583 & 576.1353 & 573.4476 & 481.0521 & 122.5994 & 2.57172 \\
\hline 1876 & 34.8753 & 586.6063 & 573.7661 & 481.5781 & 122.5994 & 2.57051 \\
\hline 1877 & 34.8923 & 632.2884 & 574.1462 & 482.1042 & 122.5994 & 2.56929 \\
\hline 1878 & 34.9093 & 622.2623 & 576.3841 & 482.6302 & 122.5994 & 2.56808 \\
\hline 1879 & 34.9263 & 611.917 & 580.0283 & 483.1563 & 122.5994 & 2.56687 \\
\hline 1880 & 34.9433 & 650.1876 & 584.098 & 483.6823 & 122.5994 & 2.56566 \\
\hline 1881 & 34.9603 & 643.4824 & 587.8853 & 484.2083 & 122.5994 & 2.56445 \\
\hline 1882 & 34.9773 & 646.9931 & 592.1492 & 484.7344 & 122.5994 & 2.56325 \\
\hline 1883 & 34.9943 & 665.6731 & 598.0401 & 485.2604 & 122.5994 & 2.56204 \\
\hline 1884 & 35.0113 & 639.4669 & 606.1979 & 485.7865 & 122.5994 & 2.56083 \\
\hline 1885 & 35.0283 & 634.4714 & 616.107 & 486.3125 & 122.5994 & 2.55963 \\
\hline 1886 & 35.0453 & 652.4447 & 625.3959 & 486.8385 & 122.5994 & 2.55843 \\
\hline 1887 & 35.0623 & 635.5522 & 629.917 & 487.3646 & 122.5994 & 2.55723 \\
\hline 1888 & 35.0793 & 643.3379 & 626.3482 & 487.8906 & 122.5994 & 2.55603 \\
\hline 1889 & 35.0963 & 645.7886 & 616.7245 & 488.4167 & 122.5994 & 2.55483 \\
\hline 1890 & 35.1133 & 650.0144 & 605.1905 & 488.9427 & 122.5994 & 2.55363 \\
\hline 1891 & 35.1303 & 602.4623 & 594.6597 & 489.4688 & 122.5994 & 2.55243 \\
\hline 1892 & 35.1473 & 579.2501 & 584.8083 & 489.9948 & 122.5994 & 2.55124 \\
\hline 1893 & 35.1643 & 554.0085 & 573.8335 & 490.5208 & 122.5994 & 2.55004 \\
\hline 1894 & 35.1813 & 563.5187 & 561.349 & 491.0469 & 122.5994 & 2.54885 \\
\hline 1895 & 35.1983 & 539.5484 & 548.9489 & 491.5729 & 122.5994 & 2.54766 \\
\hline 1896 & 35.2153 & 494.1498 & 538.2534 & 492.099 & 122.5994 & 2.54647 \\
\hline 1897 & 35.2323 & 534.5929 & 529.9375 & 492.625 & 122.5994 & 2.54528 \\
\hline 1898 & 35.2493 & 528.608 & 523.6177 & 492.7526 & 122.5994 & 2.54409 \\
\hline 1899 & 35.2663 & 501.6382 & 519.3492 & 492.8802 & 122.5994 & 2.5429 \\
\hline 1900 & 35.2833 & 505.0571 & 516.7184 & 493.0078 & 122.5994 & 2.54171 \\
\hline 1901 & 35.3003 & 518.1726 & 515.3033 & 493.1354 & 122.5994 & 2.54053 \\
\hline 1902 & 35.3173 & 495.5409 & 515.597 & 493.263 & 122.5994 & 2.53934 \\
\hline 1903 & 35.3343 & 488.6996 & 515.5456 & 493.3906 & 122.5994 & 2.53816 \\
\hline 1904 & 35.3513 & 526.7358 & 515.4691 & 493.5182 & 122.5994 & 2.53698 \\
\hline 1905 & 35.3683 & 520.2126 & 515.3201 & 493.6458 & 122.5994 & 2.5358 \\
\hline 1906 & 35.3853 & 501.9191 & 515.0549 & 493.7734 & 122.5994 & 2.53462 \\
\hline 1907 & 35.4023 & 467.2782 & 515.1557 & 493.901 & 122.5994 & 2.53344 \\
\hline 1908 & 35.4193 & 541.9456 & 514.9893 & 494.0286 & 122.5994 & 2.53226 \\
\hline 1909 & 35.4363 & 508.5953 & 514.9293 & 494.1563 & 122.5994 & 2.53109 \\
\hline
\end{tabular}




\begin{tabular}{|c|c|c|c|c|c|c|}
\hline 1910 & 35.4533 & 470.2013 & 515.0015 & 494.2839 & 122.5994 & 2.52991 \\
\hline 1911 & 35.4703 & 465.2524 & 515.4788 & 494.4115 & 122.5994 & 2.52874 \\
\hline 1912 & 35.4873 & 490.7237 & 516.4958 & 494.5391 & 122.5994 & 2.52757 \\
\hline 1913 & 35.5043 & 474.6978 & 518.5721 & 494.6667 & 122.5994 & 2.5264 \\
\hline 1914 & 35.5213 & 479.4415 & 522.5474 & 494.7943 & 122.5994 & 2.52523 \\
\hline 1915 & 35.5383 & 523.6469 & 529.5547 & 494.9219 & 122.5994 & 2.52406 \\
\hline 1916 & 35.5553 & 517.9406 & 540.5519 & 495.0495 & 122.5994 & 2.52289 \\
\hline 1917 & 35.5723 & 524.3193 & 555.7572 & 495.1771 & 122.5994 & 2.52172 \\
\hline 1918 & 35.5893 & 568.9369 & 572.8859 & 495.3047 & 122.5994 & 2.52056 \\
\hline 1919 & 35.6063 & 547.9774 & 586.4131 & 495.4323 & 122.5994 & 2.51939 \\
\hline 1920 & 35.6233 & 499.2723 & 589.2718 & 495.5599 & 122.5994 & 2.51823 \\
\hline 1921 & 35.6403 & 483.2546 & 582.168 & 495.6875 & 122.5994 & 2.51707 \\
\hline 1922 & 35.6573 & 526.0807 & 573.0383 & 495.4167 & 122.5994 & 2.51591 \\
\hline 1923 & 35.6743 & 542.2695 & 567.512 & 495.1458 & 122.5994 & 2.51475 \\
\hline 1924 & 35.6913 & 525.6112 & 565.7153 & 494.875 & 122.5994 & 2.51359 \\
\hline 1925 & 35.7083 & 517.8152 & 563.6681 & 494.6042 & 122.5994 & 2.51243 \\
\hline 1926 & 35.7253 & 494.5074 & 558.1252 & 494.3333 & 122.5994 & 2.51127 \\
\hline 1927 & 35.7423 & 501.5765 & 551.1152 & 494.0625 & 122.5994 & 2.51012 \\
\hline 1928 & 35.7593 & 471.9261 & 544.9055 & 493.7917 & 122.5994 & 2.50896 \\
\hline 1929 & 35.7763 & 474.7738 & 540.3599 & 493.5208 & 122.5994 & 2.50781 \\
\hline 1930 & 35.7933 & 492.9057 & 537.9525 & 493.25 & 122.5994 & 2.50666 \\
\hline 1931 & 35.8103 & 476.6909 & 536.7996 & 492.9792 & 122.5994 & 2.50551 \\
\hline 1932 & 35.8273 & 496.3769 & 537.2027 & 492.7083 & 122.5994 & 2.50436 \\
\hline 1933 & 35.8443 & 484.0885 & 538.2821 & 492.4375 & 122.5994 & 2.50321 \\
\hline 1934 & 35.8613 & 456.1695 & 541.6818 & 492.1667 & 122.5994 & 2.50206 \\
\hline 1935 & 35.8783 & 484.8493 & 544.5481 & 491.8958 & 122.5994 & 2.50091 \\
\hline 1936 & 35.8953 & 463.1939 & 548.8477 & 491.625 & 122.5994 & 2.49977 \\
\hline 1937 & 35.9123 & 438.3119 & 555.3355 & 491.3542 & 122.5994 & 2.49862 \\
\hline 1938 & 35.9293 & 471.7016 & 564.1639 & 491.0833 & 122.5994 & 2.49748 \\
\hline 1939 & 35.9463 & 467.201 & 575.6321 & 490.8125 & 122.5994 & 2.49634 \\
\hline 1940 & 35.9633 & 489.4554 & 589.6708 & 490.5417 & 122.5994 & 2.4952 \\
\hline 1941 & 35.9803 & 498.3974 & 606.1241 & 490.2708 & 122.5994 & 2.49406 \\
\hline 1942 & 35.9973 & 521.5557 & 620.7034 & 490 & 122.5994 & 2.49292 \\
\hline 1943 & 36.0143 & 536.1987 & 631.5956 & 489.7292 & 122.5994 & 2.49178 \\
\hline 1944 & 36.0313 & 516.1569 & 637.4975 & 489.4583 & 122.5994 & 2.49064 \\
\hline 1945 & 36.0483 & 584.7762 & 638.9165 & 489.1875 & 122.5994 & 2.48951 \\
\hline 1946 & 36.0653 & 587.7877 & 636.5656 & 488.9167 & 122.5994 & 2.48837 \\
\hline 1947 & 36.0823 & 524.4639 & 631.1592 & 488.6458 & 122.5994 & 2.48724 \\
\hline 1948 & 36.0993 & 565.1283 & 622.8395 & 488.375 & 122.5994 & 2.48611 \\
\hline 1949 & 36.1163 & 516.0484 & 612.1323 & 488.1042 & 122.5994 & 2.48498 \\
\hline 1950 & 36.1333 & 532.0802 & 599.6059 & 487.8333 & 122.5994 & 2.48385 \\
\hline 1951 & 36.1503 & 486.212 & 586.1552 & 487.5625 & 122.5994 & 2.48272 \\
\hline 1952 & 36.1673 & 510.132 & 572.647 & 487.2917 & 122.5994 & 2.48159 \\
\hline 1953 & 36.1843 & 453.6589 & 559.9149 & 487.0208 & 122.5994 & 2.48046 \\
\hline 1954 & 36.2013 & 491.9612 & 548.623 & 486.75 & 122.5994 & 2.47934 \\
\hline 1955 & 36.2183 & 453.0265 & 539.0813 & 486.4792 & 122.5994 & 2.47821 \\
\hline 1956 & 36.2353 & 438.1283 & 531.3792 & 486.2083 & 122.5994 & 2.47709 \\
\hline 1957 & 36.2523 & 454.3325 & 525.4445 & 485.9375 & 122.5994 & 2.47597 \\
\hline 1958 & 36.2693 & 416.2155 & 520.8687 & 485.6667 & 122.5994 & 2.47485 \\
\hline
\end{tabular}




\begin{tabular}{|c|c|c|c|c|c|c|}
\hline 1959 & 36.2863 & 419.8617 & 518.0068 & 485.3958 & 122.5994 & 2.47372 \\
\hline 1960 & 36.3033 & 449.1329 & 516.4616 & 485.125 & 122.5994 & 2.47261 \\
\hline 1961 & 36.3203 & 467.1582 & 515.9946 & 484.8542 & 122.5994 & 2.47149 \\
\hline 1962 & 36.3373 & 451.1487 & 516.6495 & 484.5833 & 122.5994 & 2.47037 \\
\hline 1963 & 36.3543 & 449.8107 & 518.297 & 484.3125 & 122.5994 & 2.46925 \\
\hline 1964 & 36.3713 & 439.4755 & 521.4565 & 484.0417 & 122.5994 & 2.46814 \\
\hline 1965 & 36.3883 & 505.3178 & 526.223 & 483.7708 & 122.5994 & 2.46702 \\
\hline 1966 & 36.4053 & 508.9674 & 533.083 & 483.5 & 122.5994 & 2.46591 \\
\hline 1967 & 36.4223 & 481.1184 & 542.628 & 483.2292 & 122.5994 & 2.4648 \\
\hline 1968 & 36.4393 & 472.0516 & 556.1399 & 482.9583 & 122.5994 & 2.46369 \\
\hline 1969 & 36.4563 & 523.4928 & 576.1101 & 482.6875 & 122.5994 & 2.46258 \\
\hline 1970 & 36.4733 & 540.7225 & 607.1265 & 482.0182 & 122.5994 & 2.46147 \\
\hline 1971 & 36.4903 & 558.4225 & 658.3502 & 481.349 & 122.5994 & 2.46036 \\
\hline 1972 & 36.5073 & 634.1888 & 740.6064 & 480.6797 & 122.5994 & 2.45926 \\
\hline 1973 & 36.5243 & 727.2156 & 859.469 & 480.0104 & 122.5994 & 2.45815 \\
\hline 1974 & 36.5413 & 856.3314 & 1005.169 & 479.3411 & 122.5994 & 2.45705 \\
\hline 1975 & 36.5583 & 1017.837 & 1139.814 & 478.6719 & 122.5994 & 2.45594 \\
\hline 1976 & 36.5753 & 1255.51 & 1198.858 & 478.0026 & 122.5994 & 2.45484 \\
\hline 1977 & 36.5923 & 1486.391 & 1155.723 & 477.3333 & 122.5994 & 2.45374 \\
\hline 1978 & 36.6093 & 1437.26 & 1066.904 & 476.6641 & 122.5994 & 2.45264 \\
\hline 1979 & 36.6263 & 1174.855 & 995.2483 & 475.9948 & 122.5994 & 2.45154 \\
\hline 1980 & 36.6433 & 972.4073 & 959.5873 & 475.3255 & 122.5994 & 2.45044 \\
\hline 1981 & 36.6603 & 912.1616 & 937.7343 & 474.6563 & 122.5994 & 2.44934 \\
\hline 1982 & 36.6773 & 954.1499 & 891.6421 & 473.987 & 122.5994 & 2.44825 \\
\hline 1983 & 36.6943 & 991.7706 & 815.48 & 473.3177 & 122.5994 & 2.44715 \\
\hline 1984 & 36.7113 & 905.2901 & 733.8103 & 472.6484 & 122.5994 & 2.44606 \\
\hline 1985 & 36.7283 & 701.1561 & 664.8329 & 471.9792 & 122.5994 & 2.44496 \\
\hline 1986 & 36.7453 & 591.7523 & 613.9678 & 471.3099 & 122.5994 & 2.44387 \\
\hline 1987 & 36.7623 & 534.8738 & 579.7557 & 470.6406 & 122.5994 & 2.44278 \\
\hline 1988 & 36.7793 & 523.2302 & 557.011 & 469.9714 & 122.5994 & 2.44169 \\
\hline 1989 & 36.7963 & 486.4031 & 542.5771 & 469.3021 & 122.5994 & 2.4406 \\
\hline 1990 & 36.8133 & 468.2868 & 532.1003 & 468.6328 & 122.5994 & 2.43951 \\
\hline 1991 & 36.8303 & 463.0989 & 523.5579 & 467.9635 & 122.5994 & 2.43843 \\
\hline 1992 & 36.8473 & 469.4536 & 516.0201 & 467.2943 & 122.5994 & 2.43734 \\
\hline 1993 & 36.8643 & 478.4566 & 509.475 & 466.625 & 122.5994 & 2.43626 \\
\hline 1994 & 36.8813 & 475.7099 & 503.3234 & 465.5573 & 122.5994 & 2.43517 \\
\hline 1995 & 36.8983 & 491.0938 & 498.8139 & 464.4896 & 122.5994 & 2.43409 \\
\hline 1996 & 36.9153 & 464.6093 & 495.5735 & 463.4219 & 122.5994 & 2.43301 \\
\hline 1997 & 36.9323 & 442.0938 & 493.4944 & 462.3542 & 122.5994 & 2.43193 \\
\hline 1998 & 36.9493 & 453.3439 & 492.3217 & 461.2865 & 122.5994 & 2.43085 \\
\hline 1999 & 36.9663 & 432.8173 & 491.6718 & 460.2188 & 122.5994 & 2.42977 \\
\hline 2000 & 36.9833 & 441.1992 & 491.1129 & 459.151 & 122.5994 & 2.42869 \\
\hline 2001 & 37.0003 & 452.6937 & 490.3312 & 458.0833 & 122.5994 & 2.42761 \\
\hline 2002 & 37.0173 & 426.9355 & 489.1297 & 457.0156 & 122.5994 & 2.42654 \\
\hline 2003 & 37.0343 & 469.5453 & 487.4935 & 455.9479 & 122.5994 & 2.42546 \\
\hline 2004 & 37.0513 & 455.0015 & 485.4176 & 454.8802 & 122.5994 & 2.42439 \\
\hline 2005 & 37.0683 & 467.5449 & 482.9454 & 453.8125 & 122.5994 & 2.42331 \\
\hline 2006 & 37.0853 & 476.4212 & 480.5877 & 452.7448 & 122.5994 & 2.42224 \\
\hline 2007 & 37.1023 & 479.1635 & 477.6344 & 451.6771 & 122.5994 & 2.42117 \\
\hline
\end{tabular}




\begin{tabular}{|c|c|c|c|c|c|c|}
\hline 2008 & 37.1193 & 452.8682 & 474.5858 & 450.6094 & 122.5994 & 2.4201 \\
\hline 2009 & 37.1363 & 444.3644 & 471.5432 & 449.5417 & 122.5994 & 2.41903 \\
\hline 2010 & 37.1533 & 426.1921 & 468.6445 & 448.474 & 122.5994 & 2.41797 \\
\hline 2011 & 37.1703 & 429.627 & 466.0577 & 447.4063 & 122.5994 & 2.4169 \\
\hline 2012 & 37.1873 & 433.8448 & 464.1287 & 446.3385 & 122.5994 & 2.41583 \\
\hline 2013 & 37.2043 & 470.8448 & 462.5895 & 445.2708 & 122.5994 & 2.41477 \\
\hline 2014 & 37.2213 & 452.5881 & 461.7021 & 444.2031 & 122.5994 & 2.4137 \\
\hline 2015 & 37.2383 & 420.3007 & 459.6112 & 443.1354 & 122.5994 & 2.41264 \\
\hline 2016 & 37.2553 & 435.3862 & 460.2474 & 442.0677 & 122.5994 & 2.41158 \\
\hline 2017 & 37.2723 & 434.1698 & 461.5963 & 441 & 122.5994 & 2.41052 \\
\hline 2018 & 37.2893 & 455.5608 & 463.4927 & 439.8333 & 122.5994 & 2.40946 \\
\hline 2019 & 37.3063 & 467.7772 & 465.8834 & 438.6667 & 122.5994 & 2.4084 \\
\hline 2020 & 37.3233 & 454.5892 & 468.4903 & 437.5 & 122.5994 & 2.40734 \\
\hline 2021 & 37.3403 & 458.6964 & 469.9905 & 436.3333 & 122.5994 & 2.40628 \\
\hline 2022 & 37.3573 & 449.3969 & 471.8787 & 435.1667 & 122.5994 & 2.40523 \\
\hline 2023 & 37.3743 & 466.9881 & 472.898 & 434 & 122.5994 & 2.40417 \\
\hline 2024 & 37.3913 & 472.4816 & 472.9507 & 432.8333 & 122.5994 & 2.40312 \\
\hline 2025 & 37.4083 & 452.853 & 472.093 & 431.6667 & 122.5994 & 2.40207 \\
\hline 2026 & 37.4253 & 447.6826 & 470.5078 & 430.5 & 122.5994 & 2.40101 \\
\hline 2027 & 37.4423 & 444.401 & 468.3751 & 429.3333 & 122.5994 & 2.39996 \\
\hline 2028 & 37.4593 & 454.0975 & 465.6687 & 428.1667 & 122.5994 & 2.39891 \\
\hline 2029 & 37.4763 & 476.017 & 462.4847 & 427 & 122.5994 & 2.39786 \\
\hline 2030 & 37.4933 & 442.1064 & 459.0084 & 425.8333 & 122.5994 & 2.39682 \\
\hline 2031 & 37.5103 & 457.5934 & 455.338 & 424.6667 & 122.5994 & 2.39577 \\
\hline 2032 & 37.5273 & 469.1228 & 451.7938 & 423.5 & 122.5994 & 2.39472 \\
\hline 2033 & 37.5443 & 476.0331 & 448.4171 & 422.3333 & 122.5994 & 2.39368 \\
\hline 2034 & 37.5613 & 486.0986 & 445.2701 & 421.1667 & 122.5994 & 2.39263 \\
\hline 2035 & 37.5783 & 455.461 & 442.7002 & 420 & 122.5994 & 2.39159 \\
\hline 2036 & 37.5953 & 451.8295 & 440.8641 & 418.8333 & 122.5994 & 2.39055 \\
\hline 2037 & 37.6123 & 492.4417 & 440.1682 & 417.6667 & 122.5994 & 2.38951 \\
\hline 2038 & 37.6293 & 458.469 & 441.2607 & 416.5 & 122.5994 & 2.38847 \\
\hline 2039 & 37.6463 & 438.6598 & 444.9646 & 415.3333 & 122.5994 & 2.38743 \\
\hline 2040 & 37.6633 & 474.1802 & 452.3854 & 414.1667 & 122.5994 & 2.38639 \\
\hline 2041 & 37.6803 & 460.2263 & 464.2678 & 413 & 122.5994 & 2.38535 \\
\hline 2042 & 37.6973 & 446.2547 & 480.1881 & 411.8333 & 122.5994 & 2.38431 \\
\hline 2043 & 37.7143 & 441.3277 & 497.1987 & 410.6667 & 122.5994 & 2.38328 \\
\hline 2044 & 37.7313 & 425.0218 & 508.6625 & 409.5 & 122.5994 & 2.38224 \\
\hline 2045 & 37.7483 & 495.8395 & 508.5157 & 408.3333 & 122.5994 & 2.38121 \\
\hline 2046 & 37.7653 & 466.6615 & 499.9095 & 407.1667 & 122.5994 & 2.38018 \\
\hline 2047 & 37.7823 & 510.4105 & 490.9678 & 406 & 122.5994 & 2.37914 \\
\hline 2048 & 37.7993 & 496.2781 & 485.9605 & 404.8333 & 122.5994 & 2.37811 \\
\hline 2049 & 37.8163 & 473.8944 & 483.6079 & 403.6667 & 122.5994 & 2.37708 \\
\hline 2050 & 37.8333 & 456.2774 & 479.6526 & 402.5 & 122.5994 & 2.37605 \\
\hline 2051 & 37.8503 & 480.7774 & 470.7036 & 401.3333 & 122.5994 & 2.37503 \\
\hline 2052 & 37.8673 & 448.7725 & 458.5999 & 400.1667 & 122.5994 & 2.374 \\
\hline 2053 & 37.8843 & 418.0837 & 446.5821 & 399 & 122.5994 & 2.37297 \\
\hline 2054 & 37.9013 & 429.1666 & 436.47 & 397.8333 & 122.5994 & 2.37195 \\
\hline 2055 & 37.9183 & 435.8637 & 428.2851 & 396.6667 & 122.5994 & 2.37092 \\
\hline 2056 & 37.9353 & 444.0824 & 421.7534 & 395.5 & 122.5994 & 2.3699 \\
\hline
\end{tabular}




\begin{tabular}{|c|c|c|c|c|c|c|}
\hline 2057 & 37.9523 & 416.6456 & 416.3495 & 394.3333 & 122.5994 & 2.36888 \\
\hline 2058 & 37.9693 & 389.0377 & 411.7166 & 393.1667 & 122.5994 & 2.36786 \\
\hline 2059 & 37.9863 & 381.3103 & 407.6712 & 392 & 122.5994 & 2.36684 \\
\hline 2060 & 38.0033 & 395.0194 & 404.1339 & 390.8333 & 122.5994 & 2.36582 \\
\hline 2061 & 38.0203 & 408.6284 & 401.0588 & 389.6667 & 122.5994 & 2.3648 \\
\hline 2062 & 38.0373 & 384.1232 & 398.4097 & 388.5 & 122.5994 & 2.36378 \\
\hline 2063 & 38.0543 & 384.2112 & 396.149 & 387.3333 & 122.5994 & 2.36276 \\
\hline 2064 & 38.0713 & 384.1232 & 394.244 & 386.1667 & 122.5994 & 2.36175 \\
\hline 2065 & 38.0883 & 387.4397 & 392.6081 & 385 & 122.5994 & 2.36073 \\
\hline 2066 & 38.1053 & 381.914 & 391.3986 & 383.9167 & 122.5994 & 2.35972 \\
\hline 2067 & 38.1223 & 393.8207 & 390.783 & 382.8333 & 122.5994 & 2.3587 \\
\hline 2068 & 38.1393 & 409.0904 & 390.9306 & 381.75 & 122.5994 & 2.35769 \\
\hline 2069 & 38.1563 & 392.0024 & 392.1895 & 380.6667 & 122.5994 & 2.35668 \\
\hline 2070 & 38.1733 & 406.6844 & 395.0024 & 379.5833 & 122.5994 & 2.35567 \\
\hline 2071 & 38.1903 & 415.814 & 399.4857 & 378.5 & 122.5994 & 2.35466 \\
\hline 2072 & 38.2073 & 405.2787 & 404.9573 & 377.4167 & 122.5994 & 2.35365 \\
\hline 2073 & 38.2243 & 375.1598 & 409.8596 & 376.3333 & 122.5994 & 2.35264 \\
\hline 2074 & 38.2413 & 366.3923 & 411.2595 & 375.25 & 122.5994 & 2.35164 \\
\hline 2075 & 38.2583 & 379.8314 & 408.412 & 374.1667 & 122.5994 & 2.35063 \\
\hline 2076 & 38.2753 & 364.2703 & 403.9763 & 373.0833 & 122.5994 & 2.34963 \\
\hline 2077 & 38.2923 & 336.2353 & 400.4731 & 372 & 122.5994 & 2.34862 \\
\hline 2078 & 38.3093 & 337.6702 & 398.3672 & 370.9167 & 122.5994 & 2.34762 \\
\hline 2079 & 38.3263 & 347.5051 & 397.6223 & 369.8333 & 122.5994 & 2.34662 \\
\hline 2080 & 38.3433 & 374.9821 & 396.4899 & 368.75 & 122.5994 & 2.34562 \\
\hline 2081 & 38.3603 & 390.0632 & 395.0739 & 367.6667 & 122.5994 & 2.34462 \\
\hline 2082 & 38.3773 & 356.3591 & 395.3918 & 366.5833 & 122.5994 & 2.34362 \\
\hline 2083 & 38.3943 & 364.0001 & 399.5117 & 365.5 & 122.5994 & 2.34262 \\
\hline 2084 & 38.4113 & 359.2515 & 408.4595 & 364.4167 & 122.5994 & 2.34162 \\
\hline 2085 & 38.4283 & 393.1991 & 421.4435 & 363.3333 & 122.5994 & 2.34062 \\
\hline 2086 & 38.4453 & 406.8871 & 434.7562 & 362.25 & 122.5994 & 2.33963 \\
\hline 2087 & 38.4623 & 400.9949 & 441.436 & 361.1667 & 122.5994 & 2.33863 \\
\hline 2088 & 38.4793 & 357.8509 & 437.2361 & 360.0833 & 122.5994 & 2.33764 \\
\hline 2089 & 38.4963 & 346.56 & 427.192 & 359 & 122.5994 & 2.33664 \\
\hline 2090 & 38.5133 & 349.461 & 420.2293 & 359.5417 & 122.5994 & 2.33565 \\
\hline 2091 & 38.5303 & 371.7481 & 417.58 & 360.0833 & 122.5994 & 2.33466 \\
\hline 2092 & 38.5473 & 387.9927 & 417.3853 & 360.625 & 122.5994 & 2.33367 \\
\hline 2093 & 38.5643 & 372.7289 & 414.7472 & 361.1667 & 122.5994 & 2.33268 \\
\hline 2094 & 38.5813 & 388.7222 & 407.722 & 361.7083 & 122.5994 & 2.33169 \\
\hline 2095 & 38.5983 & 406.5603 & 398.4575 & 362.25 & 122.5994 & 2.3307 \\
\hline 2096 & 38.6153 & 379.6189 & 390.0671 & 362.7917 & 122.5994 & 2.32972 \\
\hline 2097 & 38.6323 & 373.2151 & 383.8386 & 363.3333 & 122.5994 & 2.32873 \\
\hline 2098 & 38.6493 & 382.6646 & 379.6267 & 363.875 & 122.5994 & 2.32775 \\
\hline 2099 & 38.6663 & 395.5363 & 376.7198 & 364.4167 & 122.5994 & 2.32676 \\
\hline 2100 & 38.6833 & 371.2152 & 374.8264 & 364.9583 & 122.5994 & 2.32578 \\
\hline 2101 & 38.7003 & 384.1232 & 373.635 & 365.5 & 122.5994 & 2.3248 \\
\hline 2102 & 38.7173 & 380.9322 & 373.091 & 366.0417 & 122.5994 & 2.32381 \\
\hline 2103 & 38.7343 & 378.5633 & 373.0311 & 366.5833 & 122.5994 & 2.32283 \\
\hline 2104 & 38.7513 & 402.203 & 373.4886 & 367.125 & 122.5994 & 2.32185 \\
\hline 2105 & 38.7683 & 386.5614 & 374.4428 & 367.6667 & 122.5994 & 2.32087 \\
\hline
\end{tabular}




\begin{tabular}{|c|c|c|c|c|c|c|}
\hline 2106 & 38.7853 & 410.3156 & 376.5744 & 368.2083 & 122.5994 & 2.3199 \\
\hline 2107 & 38.8023 & 377.6293 & 378.3087 & 368.75 & 122.5994 & 2.31892 \\
\hline 2108 & 38.8193 & 380.2135 & 380.3756 & 369.2917 & 122.5994 & 2.31794 \\
\hline 2109 & 38.8363 & 381.6064 & 382.2749 & 369.8333 & 122.5994 & 2.31697 \\
\hline 2110 & 38.8533 & 387.1889 & 383.6476 & 370.375 & 122.5994 & 2.31599 \\
\hline 2111 & 38.8703 & 392.0024 & 384.3153 & 370.9167 & 122.5994 & 2.31502 \\
\hline 2112 & 38.8873 & 385.4917 & 384.8152 & 371.4583 & 122.5994 & 2.31405 \\
\hline 2113 & 38.9043 & 376.3239 & 384.7935 & 372 & 122.5994 & 2.31307 \\
\hline 2114 & 38.9213 & 355.3975 & 384.793 & 372.5208 & 122.5994 & 2.3121 \\
\hline 2115 & 38.9383 & 353.1874 & 384.8679 & 373.0417 & 122.5994 & 2.31113 \\
\hline 2116 & 38.9553 & 396.4971 & 388.5425 & 373.5625 & 122.5994 & 2.31016 \\
\hline 2117 & 38.9723 & 395.1806 & 388.7427 & 374.0833 & 122.5994 & 2.30919 \\
\hline 2118 & 38.9893 & 382.7746 & 388.9973 & 374.6042 & 122.5994 & 2.30823 \\
\hline 2119 & 39.0063 & 381.437 & 389.5193 & 375.125 & 122.5994 & 2.30726 \\
\hline 2120 & 39.0233 & 369.4747 & 390.5158 & 375.6458 & 122.5994 & 2.30629 \\
\hline 2121 & 39.0403 & 364.775 & 392.4269 & 376.1667 & 122.5994 & 2.30533 \\
\hline 2122 & 39.0573 & 364.8586 & 395.6727 & 376.6875 & 122.5994 & 2.30436 \\
\hline 2123 & 39.0743 & 369.8246 & 401.0308 & 377.2083 & 122.5994 & 2.3034 \\
\hline 2124 & 39.0913 & 359.602 & 409.3387 & 377.7292 & 122.5994 & 2.30244 \\
\hline 2125 & 39.1083 & 366.7519 & 421.5517 & 378.25 & 122.5994 & 2.30148 \\
\hline 2126 & 39.1253 & 383.7172 & 437.6971 & 378.7708 & 122.5994 & 2.30052 \\
\hline 2127 & 39.1423 & 388.1417 & 457.4635 & 379.2917 & 122.5994 & 2.29956 \\
\hline 2128 & 39.1593 & 397.1646 & 477.7432 & 379.8125 & 122.5994 & 2.2986 \\
\hline 2129 & 39.1763 & 393.7912 & 491.207 & 380.3333 & 122.5994 & 2.29764 \\
\hline 2130 & 39.1933 & 398.4351 & 490.7397 & 380.8542 & 122.5994 & 2.29668 \\
\hline 2131 & 39.2103 & 396.7425 & 480.1714 & 381.375 & 122.5994 & 2.29572 \\
\hline 2132 & 39.2273 & 375.9519 & 469.0914 & 381.8958 & 122.5994 & 2.29477 \\
\hline 2133 & 39.2443 & 414.1304 & 462.9986 & 382.4167 & 122.5994 & 2.29381 \\
\hline 2134 & 39.2613 & 357.2067 & 462.1179 & 382.9375 & 122.5994 & 2.29286 \\
\hline 2135 & 39.2783 & 370.5341 & 462.0783 & 383.4583 & 122.5994 & 2.29191 \\
\hline 2136 & 39.2953 & 409.6581 & 458.0574 & 383.9792 & 122.5994 & 2.29095 \\
\hline 2137 & 39.3123 & 374.0838 & 450.7611 & 384.5 & 122.5994 & 2.29 \\
\hline 2138 & 39.3293 & 399.5106 & 443.8919 & 384.6667 & 122.5994 & 2.28905 \\
\hline 2139 & 39.3463 & 424.3336 & 440.6766 & 384.8333 & 122.5994 & 2.2881 \\
\hline 2140 & 39.3633 & 432.0283 & 442.7683 & 385 & 122.5994 & 2.28715 \\
\hline 2141 & 39.3803 & 404.3332 & 452.1326 & 385.1667 & 122.5994 & 2.2862 \\
\hline 2142 & 39.3973 & 416.0521 & 472.5788 & 385.3333 & 122.5994 & 2.28526 \\
\hline 2143 & 39.4143 & 474.3482 & 511.4696 & 385.5 & 122.5994 & 2.28431 \\
\hline 2144 & 39.4313 & 517.841 & 578.498 & 385.6667 & 122.5994 & 2.28336 \\
\hline 2145 & 39.4483 & 591.2251 & 681.2646 & 385.8333 & 122.5994 & 2.28242 \\
\hline 2146 & 39.4653 & 705.6879 & 816.9432 & 386 & 122.5994 & 2.28148 \\
\hline 2147 & 39.4823 & 825.8666 & 960.8713 & 386.1667 & 122.5994 & 2.28053 \\
\hline 2148 & 39.4993 & 1057.742 & 1056.665 & 386.3333 & 122.5994 & 2.27959 \\
\hline 2149 & 39.5163 & 1173.84 & 1050.044 & 386.5 & 122.5994 & 2.27865 \\
\hline 2150 & 39.5333 & 1040.967 & 965.9058 & 386.6667 & 122.5994 & 2.27771 \\
\hline 2151 & 39.5503 & 853.7001 & 876.0388 & 386.8333 & 122.5994 & 2.27677 \\
\hline 2152 & 39.5673 & 722.2218 & 823.1717 & 387 & 122.5994 & 2.27583 \\
\hline 2153 & 39.5843 & 665.0871 & 808.8563 & 387.1667 & 122.5994 & 2.27489 \\
\hline 2154 & 39.6013 & 752.428 & 801.2827 & 387.3333 & 122.5994 & 2.27395 \\
\hline
\end{tabular}




\begin{tabular}{|c|c|c|c|c|c|c|}
\hline 2155 & 39.6183 & 783.7308 & 762.0209 & 387.5 & 122.5994 & 2.27302 \\
\hline 2156 & 39.6353 & 731.4795 & 689.4734 & 387.6667 & 122.5994 & 2.27208 \\
\hline 2157 & 39.6523 & 618.4587 & 611.009 & 387.8333 & 122.5994 & 2.27115 \\
\hline 2158 & 39.6693 & 506.5234 & 545.3909 & 388 & 122.5994 & 2.27021 \\
\hline 2159 & 39.6863 & 433.867 & 498.6207 & 388.1667 & 122.5994 & 2.26928 \\
\hline 2160 & 39.7033 & 424.3193 & 469.2891 & 388.3333 & 122.5994 & 2.26835 \\
\hline 2161 & 39.7203 & 420.5194 & 452.9444 & 388.5 & 122.5994 & 2.26741 \\
\hline 2162 & 39.7373 & 414.0986 & 444.0643 & 388.6667 & 122.5994 & 2.26648 \\
\hline 2163 & 39.7543 & 403.7193 & 438.2234 & 388.8333 & 122.5994 & 2.26555 \\
\hline 2164 & 39.7713 & 402.5561 & 434.0207 & 389 & 122.5994 & 2.26462 \\
\hline 2165 & 39.7883 & 399.022 & 429.4579 & 389.1667 & 122.5994 & 2.2637 \\
\hline 2166 & 39.8053 & 380.698 & 424.9299 & 389.3333 & 122.5994 & 2.26277 \\
\hline 2167 & 39.8223 & 393.9353 & 421.2421 & 389.5 & 122.5994 & 2.26184 \\
\hline 2168 & 39.8393 & 402.9705 & 418.754 & 389.6667 & 122.5994 & 2.26092 \\
\hline 2169 & 39.8563 & 365.0722 & 417.3391 & 389.8333 & 122.5994 & 2.25999 \\
\hline 2170 & 39.8733 & 387.4422 & 416.4333 & 390 & 122.5994 & 2.25907 \\
\hline 2171 & 39.8903 & 397.0482 & 415.5657 & 390.1667 & 122.5994 & 2.25814 \\
\hline 2172 & 39.9073 & 394.8283 & 414.8088 & 390.3333 & 122.5994 & 2.25722 \\
\hline 2173 & 39.9243 & 398.9893 & 410.0341 & 390.5 & 122.5994 & 2.2563 \\
\hline 2174 & 39.9413 & 390.971 & 410.7333 & 390.6667 & 122.5994 & 2.25538 \\
\hline 2175 & 39.9583 & 397.2985 & 412.094 & 390.8333 & 122.5994 & 2.25446 \\
\hline 2176 & 39.9753 & 396.8695 & 414.02 & 391 & 122.5994 & 2.25354 \\
\hline 2177 & 39.9923 & 412.4823 & 416.3019 & 391.1667 & 122.5994 & 2.25262 \\
\hline 2178 & 40.0093 & 389.3029 & 418.6583 & 391.3333 & 122.5994 & 2.2517 \\
\hline 2179 & 40.0263 & 411.7212 & 418.5046 & 391.5 & 122.5994 & 2.25078 \\
\hline 2180 & 40.0433 & 412.6172 & 420.0562 & 391.6667 & 122.5994 & 2.24987 \\
\hline 2181 & 40.0603 & 397.4899 & 420.7942 & 391.8333 & 122.5994 & 2.24895 \\
\hline 2182 & 40.0773 & 418.4408 & 421.0883 & 392 & 122.5994 & 2.24804 \\
\hline 2183 & 40.0943 & 401.0973 & 421.4234 & 392.1667 & 122.5994 & 2.24712 \\
\hline 2184 & 40.1113 & 419.6041 & 422.1325 & 392.3333 & 122.5994 & 2.24621 \\
\hline 2185 & 40.1283 & 388.4146 & 423.1776 & 392.5 & 122.5994 & 2.2453 \\
\hline 2186 & 40.1453 & 419.441 & 424.0171 & 392.3125 & 122.5994 & 2.24439 \\
\hline 2187 & 40.1623 & 362.2603 & 424.9338 & 392.125 & 122.5994 & 2.24347 \\
\hline 2188 & 40.1793 & 397.1013 & 426.2836 & 391.9375 & 122.5994 & 2.24256 \\
\hline 2189 & 40.1963 & 416.2155 & 428.8781 & 391.75 & 122.5994 & 2.24166 \\
\hline 2190 & 40.2133 & 417.7535 & 434.4977 & 391.5625 & 122.5994 & 2.24075 \\
\hline 2191 & 40.2303 & 434.7265 & 445.4563 & 391.375 & 122.5994 & 2.23984 \\
\hline 2192 & 40.2473 & 461.3645 & 464.9564 & 391.1875 & 122.5994 & 2.23893 \\
\hline 2193 & 40.2643 & 530.5616 & 495.7385 & 391 & 122.5994 & 2.23803 \\
\hline 2194 & 40.2813 & 523.0782 & 537.6775 & 390.8125 & 122.5994 & 2.23712 \\
\hline 2195 & 40.2983 & 579.3679 & 584.4413 & 390.625 & 122.5994 & 2.23622 \\
\hline 2196 & 40.3153 & 688.9378 & 619.6038 & 390.4375 & 122.5994 & 2.23531 \\
\hline 2197 & 40.3323 & 784.179 & 623.2499 & 390.25 & 122.5994 & 2.23441 \\
\hline 2198 & 40.3493 & 794.7888 & 597.5835 & 390.0625 & 122.5994 & 2.23351 \\
\hline 2199 & 40.3663 & 667.0799 & 565.5678 & 389.875 & 122.5994 & 2.23261 \\
\hline 2200 & 40.3833 & 591.3521 & 544.0033 & 389.6875 & 122.5994 & 2.23171 \\
\hline 2201 & 40.4003 & 532.1741 & 536.5231 & 389.5 & 122.5994 & 2.23081 \\
\hline 2202 & 40.4173 & 555.1246 & 535.3919 & 389.3125 & 122.5994 & 2.22991 \\
\hline 2203 & 40.4343 & 582.065 & 526.7686 & 389.125 & 122.5994 & 2.22901 \\
\hline
\end{tabular}




\begin{tabular}{|c|c|c|c|c|c|c|}
\hline 2204 & 40.4513 & 557.1272 & 505.5422 & 388.9375 & 122.5994 & 2.22811 \\
\hline 2205 & 40.4683 & 504.4388 & 479.1787 & 388.75 & 122.5994 & 2.22721 \\
\hline 2206 & 40.4853 & 470.9528 & 455.3302 & 388.5625 & 122.5994 & 2.22632 \\
\hline 2207 & 40.5023 & 425.1022 & 437.0659 & 388.375 & 122.5994 & 2.22542 \\
\hline 2208 & 40.5193 & 387.8746 & 424.5717 & 388.1875 & 122.5994 & 2.22453 \\
\hline 2209 & 40.5363 & 393.8888 & 416.515 & 388 & 122.5994 & 2.22363 \\
\hline 2210 & 40.5533 & 388.2945 & 411.7816 & 388.2396 & 122.5994 & 2.22274 \\
\hline 2211 & 40.5703 & 374.9891 & 408.7074 & 388.4792 & 122.5994 & 2.22185 \\
\hline 2212 & 40.5873 & 374.2227 & 406.3702 & 388.7188 & 122.5994 & 2.22096 \\
\hline 2213 & 40.6043 & 391.251 & 404.4518 & 388.9583 & 122.5994 & 2.22007 \\
\hline 2214 & 40.6213 & 365.1665 & 402.8259 & 389.1979 & 122.5994 & 2.21918 \\
\hline 2215 & 40.6383 & 336.6274 & 401.4691 & 389.4375 & 122.5994 & 2.21829 \\
\hline 2216 & 40.6553 & 358.374 & 400.4089 & 389.6771 & 122.5994 & 2.2174 \\
\hline 2217 & 40.6723 & 402.1492 & 399.7098 & 389.9167 & 122.5994 & 2.21651 \\
\hline 2218 & 40.6893 & 380.1866 & 399.2477 & 390.1563 & 122.5994 & 2.21563 \\
\hline 2219 & 40.7063 & 370.3281 & 399.0884 & 390.3958 & 122.5994 & 2.21474 \\
\hline 2220 & 40.7233 & 369.6725 & 399.2009 & 390.6354 & 122.5994 & 2.21385 \\
\hline 2221 & 40.7403 & 386.5319 & 399.6793 & 390.875 & 122.5994 & 2.21297 \\
\hline 2222 & 40.7573 & 404.6966 & 400.4897 & 391.1146 & 122.5994 & 2.21209 \\
\hline 2223 & 40.7743 & 395.0538 & 401.782 & 391.3542 & 122.5994 & 2.2112 \\
\hline 2224 & 40.7913 & 388.7215 & 403.5379 & 391.5938 & 122.5994 & 2.21032 \\
\hline 2225 & 40.8083 & 384.7923 & 405.6956 & 391.8333 & 122.5994 & 2.20944 \\
\hline 2226 & 40.8253 & 433.7834 & 407.9474 & 392.0729 & 122.5994 & 2.20856 \\
\hline 2227 & 40.8423 & 422.4806 & 409.6599 & 392.3125 & 122.5994 & 2.20768 \\
\hline 2228 & 40.8593 & 409.0161 & 410.122 & 392.5521 & 122.5994 & 2.2068 \\
\hline 2229 & 40.8763 & 419.456 & 409.4105 & 392.7917 & 122.5994 & 2.20592 \\
\hline 2230 & 40.8933 & 393.2294 & 408.3583 & 393.0313 & 122.5994 & 2.20504 \\
\hline 2231 & 40.9103 & 403.7479 & 407.6411 & 393.2708 & 122.5994 & 2.20417 \\
\hline 2232 & 40.9273 & 408.035 & 407.5421 & 393.5104 & 122.5994 & 2.20329 \\
\hline 2233 & 40.9443 & 419.909 & 407.7439 & 393.75 & 122.5994 & 2.20241 \\
\hline 2234 & 40.9613 & 403.241 & 407.368 & 393.7135 & 122.5994 & 2.20154 \\
\hline 2235 & 40.9783 & 388.0528 & 406.302 & 393.6771 & 122.5994 & 2.20066 \\
\hline 2236 & 40.9953 & 386.0713 & 404.847 & 393.6406 & 122.5994 & 2.19979 \\
\hline 2237 & 41.0123 & 369.1011 & 403.5022 & 393.6042 & 122.5994 & 2.19892 \\
\hline 2238 & 41.0293 & 401.9623 & 402.7025 & 393.5677 & 122.5994 & 2.19805 \\
\hline 2239 & 41.0463 & 388.0528 & 402.4647 & 393.5313 & 122.5994 & 2.19718 \\
\hline 2240 & 41.0633 & 388.7434 & 402.1956 & 393.4948 & 122.5994 & 2.19631 \\
\hline 2241 & 41.0803 & 391.3161 & 402.682 & 393.4583 & 122.5994 & 2.19544 \\
\hline 2242 & 41.0973 & 393.2828 & 403.2874 & 393.4219 & 122.5994 & 2.19457 \\
\hline 2243 & 41.1143 & 410.2092 & 404.028 & 393.3854 & 122.5994 & 2.1937 \\
\hline 2244 & 41.1313 & 408.2097 & 405.1004 & 393.349 & 122.5994 & 2.19283 \\
\hline 2245 & 41.1483 & 427.3607 & 406.7783 & 393.3125 & 122.5994 & 2.19196 \\
\hline 2246 & 41.1653 & 433.8654 & 408.7693 & 393.276 & 122.5994 & 2.1911 \\
\hline 2247 & 41.1823 & 427.0479 & 411.0794 & 393.2396 & 122.5994 & 2.19023 \\
\hline 2248 & 41.1993 & 447.7141 & 412.6296 & 393.2031 & 122.5994 & 2.18937 \\
\hline 2249 & 41.2163 & 424.2568 & 412.9987 & 393.1667 & 122.5994 & 2.18851 \\
\hline 2250 & 41.2333 & 448.5782 & 412.6694 & 393.1302 & 122.5994 & 2.18764 \\
\hline 2251 & 41.2503 & 434.8561 & 412.2597 & 393.0938 & 122.5994 & 2.18678 \\
\hline 2252 & 41.2673 & 422.2738 & 412.2279 & 393.0573 & 122.5994 & 2.18592 \\
\hline
\end{tabular}




\begin{tabular}{|c|c|c|c|c|c|c|}
\hline 2253 & 41.2843 & 442.973 & 412.3292 & 393.0208 & 122.5994 & 2.18506 \\
\hline 2254 & 41.3013 & 427.4703 & 412.2594 & 392.9844 & 122.5994 & 2.1842 \\
\hline 2255 & 41.3183 & 416.2178 & 411.6137 & 392.9479 & 122.5994 & 2.18334 \\
\hline 2256 & 41.3353 & 411.6721 & 410.3798 & 392.9115 & 122.5994 & 2.18248 \\
\hline 2257 & 41.3523 & 419.9612 & 408.7264 & 392.875 & 122.5994 & 2.18162 \\
\hline 2258 & 41.3693 & 436.7681 & 406.9537 & 392.8385 & 122.5994 & 2.18076 \\
\hline 2259 & 41.3863 & 419.9759 & 405.2 & 392.8021 & 122.5994 & 2.17991 \\
\hline 2260 & 41.4033 & 403.2276 & 403.6327 & 392.7656 & 122.5994 & 2.17905 \\
\hline 2261 & 41.4203 & 399.9616 & 402.2712 & 392.7292 & 122.5994 & 2.1782 \\
\hline 2262 & 41.4373 & 382.2162 & 401.0543 & 392.6927 & 122.5994 & 2.17734 \\
\hline 2263 & 41.4543 & 375.0464 & 399.9128 & 392.6563 & 122.5994 & 2.17649 \\
\hline 2264 & 41.4713 & 368.3402 & 398.8304 & 392.6198 & 122.5994 & 2.17564 \\
\hline 2265 & 41.4883 & 381.4544 & 397.8472 & 392.5833 & 122.5994 & 2.17478 \\
\hline 2266 & 41.5053 & 393.8684 & 397.0067 & 392.5469 & 122.5994 & 2.17393 \\
\hline 2267 & 41.5223 & 393.0617 & 396.3146 & 392.5104 & 122.5994 & 2.17308 \\
\hline 2268 & 41.5393 & 410.7525 & 395.7695 & 392.474 & 122.5994 & 2.17223 \\
\hline 2269 & 41.5563 & 390.6469 & 395.3568 & 392.4375 & 122.5994 & 2.17138 \\
\hline 2270 & 41.5733 & 397.1189 & 395.0255 & 392.401 & 122.5994 & 2.17053 \\
\hline 2271 & 41.5903 & 367.1291 & 394.7866 & 392.3646 & 122.5994 & 2.16969 \\
\hline 2272 & 41.6073 & 374.3116 & 394.646 & 392.3281 & 122.5994 & 2.16884 \\
\hline 2273 & 41.6243 & 342.8736 & 394.5429 & 392.2917 & 122.5994 & 2.16799 \\
\hline 2274 & 41.6413 & 390.6881 & 394.4702 & 392.2552 & 122.5994 & 2.16715 \\
\hline 2275 & 41.6583 & 381.3468 & 394.45 & 392.2188 & 122.5994 & 2.1663 \\
\hline 2276 & 41.6753 & 376.6066 & 394.4548 & 392.1823 & 122.5994 & 2.16546 \\
\hline 2277 & 41.6923 & 403.174 & 394.5083 & 392.1458 & 122.5994 & 2.16461 \\
\hline 2278 & 41.7093 & 407.971 & 394.6313 & 392.1094 & 122.5994 & 2.16377 \\
\hline 2279 & 41.7263 & 407.6106 & 394.8409 & 392.0729 & 122.5994 & 2.16293 \\
\hline 2280 & 41.7433 & 380.8632 & 395.0717 & 392.0365 & 122.5994 & 2.16209 \\
\hline 2281 & 41.7603 & 395.7453 & 396.3403 & 392 & 122.5994 & 2.16124 \\
\hline 2282 & 41.7773 & 391.6922 & 396.2867 & 391.7604 & 122.5994 & 2.1604 \\
\hline 2283 & 41.7943 & 390.1404 & 396.2416 & 391.5208 & 122.5994 & 2.15957 \\
\hline 2284 & 41.8113 & 407.0232 & 396.3519 & 391.2813 & 122.5994 & 2.15873 \\
\hline 2285 & 41.8283 & 401.2051 & 396.7136 & 391.0417 & 122.5994 & 2.15789 \\
\hline 2286 & 41.8453 & 406.0198 & 397.3396 & 390.8021 & 122.5994 & 2.15705 \\
\hline 2287 & 41.8623 & 397.5534 & 398.9334 & 390.5625 & 122.5994 & 2.15621 \\
\hline 2288 & 41.8793 & 408.1988 & 400.2786 & 390.3229 & 122.5994 & 2.15538 \\
\hline 2289 & 41.8963 & 420.5794 & 401.9887 & 390.0833 & 122.5994 & 2.15454 \\
\hline 2290 & 41.9133 & 399.178 & 404.0977 & 389.8438 & 122.5994 & 2.15371 \\
\hline 2291 & 41.9303 & 383.2347 & 406.5585 & 389.6042 & 122.5994 & 2.15287 \\
\hline 2292 & 41.9473 & 413.341 & 409.1583 & 389.3646 & 122.5994 & 2.15204 \\
\hline 2293 & 41.9643 & 404.7975 & 411.7063 & 389.125 & 122.5994 & 2.15121 \\
\hline 2294 & 41.9813 & 380.2135 & 413.6577 & 388.8854 & 122.5994 & 2.15038 \\
\hline 2295 & 41.9983 & 384.5259 & 414.6807 & 388.6458 & 122.5994 & 2.14955 \\
\hline 2296 & 42.0153 & 386.0472 & 414.7233 & 388.4063 & 122.5994 & 2.14872 \\
\hline 2297 & 42.0323 & 382.7048 & 413.9905 & 388.1667 & 122.5994 & 2.14789 \\
\hline 2298 & 42.0493 & 392.0024 & 412.9888 & 387.9271 & 122.5994 & 2.14706 \\
\hline 2299 & 42.0663 & 385.1223 & 411.9025 & 387.6875 & 122.5994 & 2.14623 \\
\hline 2300 & 42.0833 & 396.7737 & 410.73 & 387.4479 & 122.5994 & 2.1454 \\
\hline 2301 & 42.1003 & 395.0574 & 409.4088 & 387.2083 & 122.5994 & 2.14457 \\
\hline
\end{tabular}




\begin{tabular}{|c|c|c|c|c|c|c|}
\hline 2302 & 42.1173 & 376.5889 & 407.8931 & 386.9688 & 122.5994 & 2.14375 \\
\hline 2303 & 42.1343 & 382.0492 & 406.2765 & 386.7292 & 122.5994 & 2.14292 \\
\hline 2304 & 42.1513 & 400.1256 & 404.7139 & 386.4896 & 122.5994 & 2.1421 \\
\hline 2305 & 42.1683 & 395.5259 & 403.4519 & 386.25 & 122.5994 & 2.14127 \\
\hline 2306 & 42.1853 & 391.9631 & 402.5522 & 386.0104 & 122.5994 & 2.14045 \\
\hline 2307 & 42.2023 & 400.0533 & 402.0549 & 385.7708 & 122.5994 & 2.13963 \\
\hline 2308 & 42.2193 & 379.9545 & 401.9908 & 385.5313 & 122.5994 & 2.1388 \\
\hline 2309 & 42.2363 & 402.7328 & 402.3665 & 385.2917 & 122.5994 & 2.13798 \\
\hline 2310 & 42.2533 & 437.2088 & 403.2156 & 385.0521 & 122.5994 & 2.13716 \\
\hline 2311 & 42.2703 & 399.6218 & 404.632 & 384.8125 & 122.5994 & 2.13634 \\
\hline 2312 & 42.2873 & 397.4187 & 406.716 & 384.5729 & 122.5994 & 2.13552 \\
\hline 2313 & 42.3043 & 460.3344 & 409.6654 & 384.3333 & 122.5994 & 2.1347 \\
\hline 2314 & 42.3213 & 406.4453 & 413.7702 & 384.0938 & 122.5994 & 2.13388 \\
\hline 2315 & 42.3383 & 406.1491 & 419.572 & 383.8542 & 122.5994 & 2.13307 \\
\hline 2316 & 42.3553 & 452.7877 & 428.2014 & 383.6146 & 122.5994 & 2.13225 \\
\hline 2317 & 42.3723 & 502.5506 & 441.9601 & 383.375 & 122.5994 & 2.13143 \\
\hline 2318 & 42.3893 & 476.8057 & 464.9445 & 383.1354 & 122.5994 & 2.13062 \\
\hline 2319 & 42.4063 & 509.6839 & 502.8698 & 382.8958 & 122.5994 & 2.1298 \\
\hline 2320 & 42.4233 & 609.0879 & 561.0665 & 382.6563 & 122.5994 & 2.12899 \\
\hline 2321 & 42.4403 & 685.8822 & 640.0751 & 382.4167 & 122.5994 & 2.12818 \\
\hline 2322 & 42.4573 & 804.8589 & 729.5223 & 382.1771 & 122.5994 & 2.12736 \\
\hline 2323 & 42.4743 & 937.505 & 800.8647 & 381.9375 & 122.5994 & 2.12655 \\
\hline 2324 & 42.4913 & 1047.772 & 816.1084 & 381.6979 & 122.5994 & 2.12574 \\
\hline 2325 & 42.5083 & 1169.183 & 773.3646 & 381.4583 & 122.5994 & 2.12493 \\
\hline 2326 & 42.5253 & 1006.692 & 712.1292 & 381.2188 & 122.5994 & 2.12412 \\
\hline 2327 & 42.5423 & 827.1205 & 666.0178 & 380.9792 & 122.5994 & 2.12331 \\
\hline 2328 & 42.5593 & 659.0322 & 646.6273 & 380.7396 & 122.5994 & 2.1225 \\
\hline 2329 & 42.5763 & 648.6648 & 646.0297 & 380.5 & 122.5994 & 2.12169 \\
\hline 2330 & 42.5933 & 735.5519 & 640.7889 & 379.9792 & 122.5994 & 2.12089 \\
\hline 2331 & 42.6103 & 738.798 & 611.551 & 379.4583 & 122.5994 & 2.12008 \\
\hline 2332 & 42.6273 & 685.1643 & 564.2709 & 378.9375 & 122.5994 & 2.11927 \\
\hline 2333 & 42.6443 & 603.1328 & 515.6132 & 378.4167 & 122.5994 & 2.11847 \\
\hline 2334 & 42.6613 & 507.173 & 475.5399 & 377.8958 & 122.5994 & 2.11766 \\
\hline 2335 & 42.6783 & 432.246 & 446.8635 & 377.375 & 122.5994 & 2.11686 \\
\hline 2336 & 42.6953 & 419.0843 & 428.2528 & 376.8542 & 122.5994 & 2.11605 \\
\hline 2337 & 42.7123 & 421.0811 & 416.9333 & 376.3333 & 122.5994 & 2.11525 \\
\hline 2338 & 42.7293 & 404.5426 & 410.2746 & 375.8125 & 122.5994 & 2.11445 \\
\hline 2339 & 42.7463 & 407.6505 & 406.4043 & 375.2917 & 122.5994 & 2.11365 \\
\hline 2340 & 42.7633 & 375.5133 & 404.4026 & 374.7708 & 122.5994 & 2.11285 \\
\hline 2341 & 42.7803 & 359.023 & 403.3592 & 374.25 & 122.5994 & 2.11205 \\
\hline 2342 & 42.7973 & 388.0334 & 402.4493 & 373.7292 & 122.5994 & 2.11125 \\
\hline 2343 & 42.8143 & 406.425 & 400.9205 & 373.2083 & 122.5994 & 2.11045 \\
\hline 2344 & 42.8313 & 391.0559 & 398.369 & 372.6875 & 122.5994 & 2.10965 \\
\hline 2345 & 42.8483 & 369.1203 & 395.1208 & 372.1667 & 122.5994 & 2.10885 \\
\hline 2346 & 42.8653 & 379.0783 & 392.101 & 371.6458 & 122.5994 & 2.10805 \\
\hline 2347 & 42.8823 & 386.5955 & 389.7464 & 371.125 & 122.5994 & 2.10726 \\
\hline 2348 & 42.8993 & 376.3239 & 387.9495 & 370.6042 & 122.5994 & 2.10646 \\
\hline 2349 & 42.9163 & 372.3939 & 386.3687 & 370.0833 & 122.5994 & 2.10567 \\
\hline 2350 & 42.9333 & 360.3713 & 384.6936 & 369.5625 & 122.5994 & 2.10487 \\
\hline
\end{tabular}




\begin{tabular}{|c|c|c|c|c|c|c|}
\hline 2351 & 42.9503 & 373.0061 & 382.4824 & 369.0417 & 122.5994 & 2.10408 \\
\hline 2352 & 42.9673 & 383.4684 & 380.0921 & 368.5208 & 122.5994 & 2.10329 \\
\hline 2353 & 42.9843 & 368.6277 & 377.8756 & 368 & 122.5994 & 2.10249 \\
\hline 2354 & 43.0013 & 353.8649 & 375.9345 & 367.4167 & 122.5994 & 2.1017 \\
\hline 2355 & 43.0183 & 356.9992 & 374.3599 & 366.8333 & 122.5994 & 2.10091 \\
\hline 2356 & 43.0353 & 376.5857 & 373.0998 & 366.25 & 122.5994 & 2.10012 \\
\hline 2357 & 43.0523 & 350.0136 & 372.0889 & 365.6667 & 122.5994 & 2.09933 \\
\hline 2358 & 43.0693 & 353.5758 & 371.2264 & 365.0833 & 122.5994 & 2.09854 \\
\hline 2359 & 43.0863 & 375.1212 & 370.4837 & 364.5 & 122.5994 & 2.09775 \\
\hline 2360 & 43.1033 & 351.534 & 369.8404 & 363.9167 & 122.5994 & 2.09696 \\
\hline 2361 & 43.1203 & 350.9279 & 369.3058 & 363.3333 & 122.5994 & 2.09618 \\
\hline 2362 & 43.1373 & 374.8503 & 367.8219 & 362.75 & 122.5994 & 2.09539 \\
\hline 2363 & 43.1543 & 383.5128 & 367.7175 & 362.1667 & 122.5994 & 2.0946 \\
\hline 2364 & 43.1713 & 364.6009 & 367.9058 & 361.5833 & 122.5994 & 2.09382 \\
\hline 2365 & 43.1883 & 364.3422 & 368.4196 & 361 & 122.5994 & 2.09303 \\
\hline 2366 & 43.2053 & 358.0414 & 369.1812 & 360.4167 & 122.5994 & 2.09225 \\
\hline 2367 & 43.2223 & 346.9673 & 369.9409 & 359.8333 & 122.5994 & 2.09147 \\
\hline 2368 & 43.2393 & 370.6543 & 369.3692 & 359.25 & 122.5994 & 2.09068 \\
\hline 2369 & 43.2563 & 372.4543 & 368.8827 & 358.6667 & 122.5994 & 2.0899 \\
\hline 2370 & 43.2733 & 391.545 & 367.9501 & 358.0833 & 122.5994 & 2.08912 \\
\hline 2371 & 43.2903 & 342.5331 & 367.0256 & 357.5 & 122.5994 & 2.08834 \\
\hline 2372 & 43.3073 & 387.6434 & 366.2757 & 356.9167 & 122.5994 & 2.08756 \\
\hline 2373 & 43.3243 & 360.4159 & 365.6817 & 356.3333 & 122.5994 & 2.08678 \\
\hline 2374 & 43.3413 & 347.1028 & 364.992 & 355.75 & 122.5994 & 2.086 \\
\hline 2375 & 43.3583 & 366.3153 & 364.3557 & 355.1667 & 122.5994 & 2.08522 \\
\hline 2376 & 43.3753 & 379.5941 & 363.7028 & 354.5833 & 122.5994 & 2.08444 \\
\hline 2377 & 43.3923 & 338.9802 & 363.3068 & 354 & 122.5994 & 2.08367 \\
\hline 2378 & 43.4093 & 343.2892 & 363.8091 & 353.8542 & 122.5994 & 2.08289 \\
\hline 2379 & 43.4263 & 350.3581 & 364.6477 & 353.7083 & 122.5994 & 2.08211 \\
\hline 2380 & 43.4433 & 352.7042 & 365.6385 & 353.5625 & 122.5994 & 2.08134 \\
\hline 2381 & 43.4603 & 343.6226 & 366.4095 & 353.4167 & 122.5994 & 2.08056 \\
\hline 2382 & 43.4773 & 326.2617 & 366.5402 & 353.2708 & 122.5994 & 2.07979 \\
\hline 2383 & 43.4943 & 348.8398 & 365.8988 & 353.125 & 122.5994 & 2.07902 \\
\hline 2384 & 43.5113 & 353.0871 & 364.7836 & 352.9792 & 122.5994 & 2.07824 \\
\hline 2385 & 43.5283 & 344.4099 & 363.7409 & 352.8333 & 122.5994 & 2.07747 \\
\hline 2386 & 43.5453 & 347.3828 & 362.9645 & 352.6875 & 122.5994 & 2.0767 \\
\hline 2387 & 43.5623 & 348.5508 & 362.5334 & 352.5417 & 122.5994 & 2.07593 \\
\hline 2388 & 43.5793 & 366.5692 & 362.2401 & 352.3958 & 122.5994 & 2.07516 \\
\hline 2389 & 43.5963 & 367.5945 & 361.8331 & 352.25 & 122.5994 & 2.07439 \\
\hline 2390 & 43.6133 & 364.1913 & 361.1962 & 352.1042 & 122.5994 & 2.07362 \\
\hline 2391 & 43.6303 & 365.764 & 360.4514 & 351.9583 & 122.5994 & 2.07285 \\
\hline 2392 & 43.6473 & 349.2581 & 359.6773 & 351.8125 & 122.5994 & 2.07208 \\
\hline 2393 & 43.6643 & 334.737 & 358.9318 & 351.6667 & 122.5994 & 2.07131 \\
\hline 2394 & 43.6813 & 331.0385 & 358.2092 & 351.5208 & 122.5994 & 2.07055 \\
\hline 2395 & 43.6983 & 361.3663 & 357.5246 & 351.375 & 122.5994 & 2.06978 \\
\hline 2396 & 43.7153 & 372.4543 & 356.9028 & 351.2292 & 122.5994 & 2.06902 \\
\hline 2397 & 43.7323 & 374.594 & 356.3719 & 351.0833 & 122.5994 & 2.06825 \\
\hline 2398 & 43.7493 & 367.5849 & 355.9023 & 350.9375 & 122.5994 & 2.06749 \\
\hline 2399 & 43.7663 & 365.5187 & 355.4794 & 350.7917 & 122.5994 & 2.06672 \\
\hline
\end{tabular}




\begin{tabular}{|c|c|c|c|c|c|c|}
\hline 2400 & 43.7833 & 359.3011 & 355.0772 & 350.6458 & 122.5994 & 2.06596 \\
\hline 2401 & 43.8003 & 378.0735 & 354.7006 & 350.5 & 122.5994 & 2.0652 \\
\hline 2402 & 43.8173 & 381.7324 & 354.3505 & 350.3542 & 122.5994 & 2.06444 \\
\hline 2403 & 43.8343 & 376.3239 & 354.0761 & 350.2083 & 122.5994 & 2.06367 \\
\hline 2404 & 43.8513 & 365.5175 & 353.9503 & 350.0625 & 122.5994 & 2.06291 \\
\hline 2405 & 43.8683 & 377.8436 & 353.9967 & 349.9167 & 122.5994 & 2.06215 \\
\hline 2406 & 43.8853 & 358.3874 & 354.262 & 349.7708 & 122.5994 & 2.06139 \\
\hline 2407 & 43.9023 & 370.2262 & 354.7371 & 349.625 & 122.5994 & 2.06064 \\
\hline 2408 & 43.9193 & 370.2275 & 355.3523 & 349.4792 & 122.5994 & 2.05988 \\
\hline 2409 & 43.9363 & 397.2934 & 355.8948 & 349.3333 & 122.5994 & 2.05912 \\
\hline 2410 & 43.9533 & 377.8155 & 356.1739 & 349.1875 & 122.5994 & 2.05836 \\
\hline 2411 & 43.9703 & 343.4722 & 356.2921 & 349.0417 & 122.5994 & 2.05761 \\
\hline 2412 & 43.9873 & 351.6374 & 356.2642 & 348.8958 & 122.5994 & 2.05685 \\
\hline 2413 & 44.0043 & 363.6077 & 356.16 & 348.75 & 122.5994 & 2.0561 \\
\hline 2414 & 44.0213 & 382.6392 & 356.0663 & 348.6042 & 122.5994 & 2.05534 \\
\hline 2415 & 44.0383 & 384.1232 & 356.0388 & 348.4583 & 122.5994 & 2.05459 \\
\hline 2416 & 44.0553 & 347.3849 & 356.0514 & 348.3125 & 122.5994 & 2.05383 \\
\hline 2417 & 44.0723 & 368.3092 & 355.9604 & 348.1667 & 122.5994 & 2.05308 \\
\hline 2418 & 44.0893 & 357.3521 & 355.9258 & 348.0208 & 122.5994 & 2.05233 \\
\hline 2419 & 44.1063 & 375.8192 & 355.8273 & 347.875 & 122.5994 & 2.05158 \\
\hline 2420 & 44.1233 & 355.4175 & 355.6074 & 347.7292 & 122.5994 & 2.05083 \\
\hline 2421 & 44.1403 & 322.2266 & 355.1974 & 347.5833 & 122.5994 & 2.05008 \\
\hline 2422 & 44.1573 & 344.9211 & 354.6807 & 347.4375 & 122.5994 & 2.04933 \\
\hline 2423 & 44.1743 & 334.524 & 354.0714 & 347.2917 & 122.5994 & 2.04858 \\
\hline 2424 & 44.1913 & 333.1795 & 353.4412 & 347.1458 & 122.5994 & 2.04783 \\
\hline 2425 & 44.2083 & 347.7562 & 352.8455 & 347 & 122.5994 & 2.04708 \\
\hline 2426 & 44.2253 & 359.1987 & 353.2036 & 347.7534 & 122.5994 & 2.04633 \\
\hline 2427 & 44.2423 & 362.6366 & 353.6042 & 348.5068 & 122.5994 & 2.04559 \\
\hline 2428 & 44.2593 & 345.2244 & 354.0245 & 349.2603 & 122.5994 & 2.04484 \\
\hline 2429 & 44.2763 & 364.0287 & 354.5575 & 350.0137 & 122.5994 & 2.04409 \\
\hline 2430 & 44.2933 & 349.7366 & 355.0197 & 350.7671 & 122.5994 & 2.04335 \\
\hline 2431 & 44.3103 & 352.8747 & 355.5327 & 351.5205 & 122.5994 & 2.0426 \\
\hline 2432 & 44.3273 & 357.5049 & 356.1168 & 352.2739 & 122.5994 & 2.04186 \\
\hline 2433 & 44.3443 & 357.2063 & 356.7826 & 353.0273 & 122.5994 & 2.04112 \\
\hline 2434 & 44.3613 & 356.7895 & 357.5334 & 353.7808 & 122.5994 & 2.04037 \\
\hline 2435 & 44.3783 & 350.0728 & 358.3679 & 354.5342 & 122.5994 & 2.03963 \\
\hline 2436 & 44.3953 & 375.9445 & 359.3341 & 355.2876 & 122.5994 & 2.03889 \\
\hline 2437 & 44.4123 & 361.9869 & 360.3018 & 356.041 & 122.5994 & 2.03815 \\
\hline 2438 & 44.4293 & 357.1203 & 361.3581 & 356.7944 & 122.5994 & 2.03741 \\
\hline 2439 & 44.4463 & 345.6143 & 362.4566 & 357.5479 & 122.5994 & 2.03667 \\
\hline 2440 & 44.4633 & 360.6406 & 363.5829 & 358.3013 & 122.5994 & 2.03593 \\
\hline 2441 & 44.4803 & 362.9356 & 364.7371 & 359.0547 & 122.5994 & 2.03519 \\
\hline 2442 & 44.4973 & 361.4111 & 365.9261 & 359.8081 & 122.5994 & 2.03445 \\
\hline 2443 & 44.5143 & 346.3361 & 367.1478 & 360.5615 & 122.5994 & 2.03372 \\
\hline 2444 & 44.5313 & 352.9833 & 368.3672 & 361.3149 & 122.5994 & 2.03298 \\
\hline 2445 & 44.5483 & 363.5031 & 369.5726 & 362.0684 & 122.5994 & 2.03224 \\
\hline 2446 & 44.5653 & 350.3444 & 370.7049 & 362.8218 & 122.5994 & 2.03151 \\
\hline 2447 & 44.5823 & 353.081 & 371.7384 & 363.5752 & 122.5994 & 2.03077 \\
\hline 2448 & 44.5993 & 349.8599 & 372.621 & 364.3286 & 122.5994 & 2.03004 \\
\hline
\end{tabular}




\begin{tabular}{|c|c|c|c|c|c|c|}
\hline 2449 & 44.6163 & 385.0126 & 373.2918 & 365.082 & 122.5994 & 2.0293 \\
\hline 2450 & 44.6333 & 390.2079 & 373.3462 & 365.4615 & 122.5994 & 2.02857 \\
\hline 2451 & 44.6503 & 367.5687 & 373.2232 & 365.8411 & 122.5994 & 2.02784 \\
\hline 2452 & 44.6673 & 355.4807 & 373.0858 & 366.2206 & 122.5994 & 2.0271 \\
\hline 2453 & 44.6843 & 367.8433 & 372.9532 & 366.6001 & 122.5994 & 2.02637 \\
\hline 2454 & 44.7013 & 348.9674 & 372.885 & 366.9796 & 122.5994 & 2.02564 \\
\hline 2455 & 44.7183 & 372.6524 & 372.9269 & 367.3591 & 122.5994 & 2.02491 \\
\hline 2456 & 44.7353 & 377.1009 & 373.0268 & 367.7386 & 122.5994 & 2.02418 \\
\hline 2457 & 44.7523 & 392.9084 & 373.181 & 368.1182 & 122.5994 & 2.02345 \\
\hline 2458 & 44.7693 & 385.7168 & 378.2394 & 368.4977 & 122.5994 & 2.02272 \\
\hline 2459 & 44.7863 & 370.4439 & 379.0721 & 368.8772 & 122.5994 & 2.02199 \\
\hline 2460 & 44.8033 & 368.6046 & 380.5157 & 369.2567 & 122.5994 & 2.02127 \\
\hline 2461 & 44.8203 & 379.488 & 382.9851 & 369.6362 & 122.5994 & 2.02054 \\
\hline 2462 & 44.8373 & 376.512 & 386.9665 & 370.0157 & 122.5994 & 2.01981 \\
\hline 2463 & 44.8543 & 395.4429 & 392.7036 & 370.3953 & 122.5994 & 2.01909 \\
\hline 2464 & 44.8713 & 365.0304 & 399.8236 & 370.7748 & 122.5994 & 2.01836 \\
\hline 2465 & 44.8883 & 392.4167 & 409.1342 & 371.1543 & 122.5994 & 2.01764 \\
\hline 2466 & 44.9053 & 411.0003 & 413.1527 & 371.5338 & 122.5994 & 2.01691 \\
\hline 2467 & 44.9223 & 386.8378 & 412.3094 & 371.9133 & 122.5994 & 2.01619 \\
\hline 2468 & 44.9393 & 427.7842 & 408.7057 & 372.2928 & 122.5994 & 2.01547 \\
\hline 2469 & 44.9563 & 417.3297 & 405.4373 & 372.6724 & 122.5994 & 2.01474 \\
\hline 2470 & 44.9733 & 396.0916 & 404.1086 & 373.0519 & 122.5994 & 2.01402 \\
\hline 2471 & 44.9903 & 424.3193 & 404.8893 & 373.4314 & 122.5994 & 2.0133 \\
\hline 2472 & 45.0073 & 417.7108 & 406.7523 & 373.8109 & 122.5994 & 2.01258 \\
\hline 2473 & 45.0243 & 393.2486 & 407.8805 & 374.1904 & 122.5994 & 2.01186 \\
\hline 2474 & 45.0413 & 428.2902 & 406.6788 & 374.197 & 122.5994 & 2.01114 \\
\hline 2475 & 45.0583 & 404.7803 & 404.3772 & 374.2037 & 122.5994 & 2.01042 \\
\hline 2476 & 45.0753 & 405.324 & 402.2658 & 374.2103 & 122.5994 & 2.0097 \\
\hline 2477 & 45.0923 & 477.2814 & 400.9931 & 374.2169 & 122.5994 & 2.00898 \\
\hline 2478 & 45.1093 & 462.0036 & 400.7144 & 374.2235 & 122.5994 & 2.00826 \\
\hline 2479 & 45.1263 & 442.3952 & 401.3572 & 374.2301 & 122.5994 & 2.00755 \\
\hline 2480 & 45.1433 & 450.9415 & 403.4604 & 374.2367 & 122.5994 & 2.00683 \\
\hline 2481 & 45.1603 & 457.5904 & 405.5624 & 374.2433 & 122.5994 & 2.00611 \\
\hline 2482 & 45.1773 & 441.4984 & 408.2781 & 374.2499 & 122.5994 & 2.0054 \\
\hline 2483 & 45.1943 & 433.4948 & 411.663 & 374.2566 & 122.5994 & 2.00468 \\
\hline 2484 & 45.2113 & 441.0588 & 415.7828 & 374.2632 & 122.5994 & 2.00397 \\
\hline 2485 & 45.2283 & 429.8686 & 420.7627 & 374.2698 & 122.5994 & 2.00326 \\
\hline 2486 & 45.2453 & 427.8424 & 426.8061 & 374.2764 & 122.5994 & 2.00254 \\
\hline 2487 & 45.2623 & 432.8126 & 434.2968 & 374.283 & 122.5994 & 2.00183 \\
\hline 2488 & 45.2793 & 430.6802 & 443.9662 & 374.2896 & 122.5994 & 2.00112 \\
\hline 2489 & 45.2963 & 464.3484 & 457.0129 & 374.2962 & 122.5994 & 2.00041 \\
\hline 2490 & 45.3133 & 490.8173 & 474.9289 & 374.3028 & 122.5994 & 1.9997 \\
\hline 2491 & 45.3303 & 477.7781 & 499.461 & 374.3094 & 122.5994 & 1.99899 \\
\hline 2492 & 45.3473 & 495.6096 & 532.9835 & 374.3161 & 122.5994 & 1.99828 \\
\hline 2493 & 45.3643 & 523.7198 & 575.7852 & 374.3227 & 122.5994 & 1.99757 \\
\hline 2494 & 45.3813 & 546.9683 & 629.5086 & 374.3293 & 122.5994 & 1.99686 \\
\hline 2495 & 45.3983 & 566.3336 & 694.3152 & 374.3359 & 122.5994 & 1.99615 \\
\hline 2496 & 45.4153 & 635.7999 & 769.2192 & 374.3425 & 122.5994 & 1.99544 \\
\hline 2497 & 45.4323 & 661.9474 & 851.4308 & 374.3491 & 122.5994 & 1.99473 \\
\hline
\end{tabular}




\begin{tabular}{|c|c|c|c|c|c|c|}
\hline 2498 & 45.4493 & 690.9067 & 935.4127 & 374.3557 & 122.5994 & 1.99403 \\
\hline 2499 & 45.4663 & 667.1238 & 1012.277 & 374.3623 & 122.5994 & 1.99332 \\
\hline 2500 & 45.4833 & 720.2709 & 1070.848 & 374.369 & 122.5994 & 1.99262 \\
\hline 2501 & 45.5003 & 792.6976 & 1101.863 & 374.3756 & 122.5994 & 1.99191 \\
\hline 2502 & 45.5173 & 843.9075 & 1103.789 & 374.3822 & 122.5994 & 1.99121 \\
\hline 2503 & 45.5343 & 823.8367 & 1083.47 & 374.3888 & 122.5994 & 1.9905 \\
\hline 2504 & 45.5513 & 842.5553 & 1051.052 & 374.3954 & 122.5994 & 1.9898 \\
\hline 2505 & 45.5683 & 762.5253 & 1014.11 & 374.402 & 122.5994 & 1.9891 \\
\hline 2506 & 45.5853 & 741.0505 & 975.2086 & 374.4086 & 122.5994 & 1.98839 \\
\hline 2507 & 45.6023 & 662.07 & 932.6318 & 374.4152 & 122.5994 & 1.98769 \\
\hline 2508 & 45.6193 & 694.6088 & 883.6113 & 374.4219 & 122.5994 & 1.98699 \\
\hline 2509 & 45.6363 & 608.5079 & 827.7896 & 374.4285 & 122.5994 & 1.98629 \\
\hline 2510 & 45.6533 & 624.7722 & 768.5008 & 374.4351 & 122.5994 & 1.98559 \\
\hline 2511 & 45.6703 & 614.7003 & 710.4856 & 374.4417 & 122.5994 & 1.98489 \\
\hline 2512 & 45.6873 & 565.9977 & 657.8703 & 374.4483 & 122.5994 & 1.98419 \\
\hline 2513 & 45.7043 & 547.2794 & 613.6015 & 374.4549 & 122.5994 & 1.98349 \\
\hline 2514 & 45.7213 & 540.6973 & 579.9319 & 374.4615 & 122.5994 & 1.9828 \\
\hline 2515 & 45.7383 & 511.5456 & 559.7154 & 374.4681 & 122.5994 & 1.9821 \\
\hline 2516 & 45.7553 & 519.2745 & 556.2712 & 374.4748 & 122.5994 & 1.9814 \\
\hline 2517 & 45.7723 & 522.2632 & 572.4076 & 374.4814 & 122.5994 & 1.9807 \\
\hline 2518 & 45.7893 & 580.2933 & 607.5637 & 374.488 & 122.5994 & 1.98001 \\
\hline 2519 & 45.8063 & 664.7419 & 653.707 & 374.4946 & 122.5994 & 1.97931 \\
\hline 2520 & 45.8233 & 721.625 & 690.5353 & 374.5012 & 122.5994 & 1.97862 \\
\hline 2521 & 45.8403 & 774.832 & 692.2675 & 374.5078 & 122.5994 & 1.97792 \\
\hline 2522 & 45.8573 & 739.6274 & 656.8055 & 374.1415 & 122.5994 & 1.97723 \\
\hline 2523 & 45.8743 & 646.9887 & 608.9121 & 373.7752 & 122.5994 & 1.97654 \\
\hline 2524 & 45.8913 & 560.88 & 571.2463 & 373.4089 & 122.5994 & 1.97585 \\
\hline 2525 & 45.9083 & 507.9477 & 553.4764 & 373.0426 & 122.5994 & 1.97515 \\
\hline 2526 & 45.9253 & 495.9894 & 553.4944 & 372.6764 & 122.5994 & 1.97446 \\
\hline 2527 & 45.9423 & 512.8163 & 559.0839 & 372.3101 & 122.5994 & 1.97377 \\
\hline 2528 & 45.9593 & 592.018 & 553.3904 & 371.9438 & 122.5994 & 1.97308 \\
\hline 2529 & 45.9763 & 602.428 & 530.7488 & 371.5775 & 122.5994 & 1.97239 \\
\hline 2530 & 45.9933 & 509.5724 & 500.2619 & 371.2112 & 122.5994 & 1.9717 \\
\hline 2531 & 46.0103 & 440.3606 & 471.9613 & 370.8449 & 122.5994 & 1.97101 \\
\hline 2532 & 46.0273 & 413.5839 & 450.5021 & 370.4786 & 122.5994 & 1.97032 \\
\hline 2533 & 46.0443 & 413.6201 & 436.3327 & 370.1123 & 122.5994 & 1.96964 \\
\hline 2534 & 46.0613 & 404.4171 & 427.6675 & 369.746 & 122.5994 & 1.96895 \\
\hline 2535 & 46.0783 & 394.9806 & 422.1165 & 369.3797 & 122.5994 & 1.96826 \\
\hline 2536 & 46.0953 & 394.8301 & 417.8637 & 369.0134 & 122.5994 & 1.96757 \\
\hline 2537 & 46.1123 & 373.6512 & 414.0218 & 368.6471 & 122.5994 & 1.96689 \\
\hline 2538 & 46.1293 & 411.1555 & 410.4221 & 368.2808 & 122.5994 & 1.9662 \\
\hline 2539 & 46.1463 & 425.8476 & 407.1637 & 367.9146 & 122.5994 & 1.96552 \\
\hline 2540 & 46.1633 & 388.7057 & 404.3271 & 367.5483 & 122.5994 & 1.96483 \\
\hline 2541 & 46.1803 & 377.5089 & 401.8772 & 367.182 & 122.5994 & 1.96415 \\
\hline 2542 & 46.1973 & 372.4025 & 399.7106 & 366.8157 & 122.5994 & 1.96347 \\
\hline 2543 & 46.2143 & 363.3836 & 397.7479 & 366.4494 & 122.5994 & 1.96278 \\
\hline 2544 & 46.2313 & 374.7456 & 396.0779 & 366.0831 & 122.5994 & 1.9621 \\
\hline 2545 & 46.2483 & 386.0827 & 390.4799 & 365.7168 & 122.5994 & 1.96142 \\
\hline 2546 & 46.2653 & 394.0635 & 389.745 & 364.9786 & 122.5994 & 1.96074 \\
\hline
\end{tabular}




\begin{tabular}{|c|c|c|c|c|c|c|}
\hline 2547 & 46.2823 & 380.6382 & 389.3838 & 364.2404 & 122.5994 & 1.96006 \\
\hline 2548 & 46.2993 & 379.5299 & 388.4626 & 363.5022 & 122.5994 & 1.95938 \\
\hline 2549 & 46.3163 & 339.8579 & 385.9925 & 362.764 & 122.5994 & 1.9587 \\
\hline 2550 & 46.3333 & 350.0446 & 382.3049 & 362.0258 & 122.5994 & 1.95802 \\
\hline 2551 & 46.3503 & 353.4061 & 378.6146 & 361.2876 & 122.5994 & 1.95734 \\
\hline 2552 & 46.3673 & 370.4061 & 373.54 & 360.5494 & 122.5994 & 1.95666 \\
\hline 2553 & 46.3843 & 368.9525 & 371.7939 & 359.8112 & 122.5994 & 1.95599 \\
\hline 2554 & 46.4013 & 347.4901 & 370.9477 & 359.073 & 122.5994 & 1.95531 \\
\hline 2555 & 46.4183 & 342.4719 & 370.3523 & 358.3348 & 122.5994 & 1.95463 \\
\hline 2556 & 46.4353 & 342.2172 & 369.2892 & 357.5966 & 122.5994 & 1.95396 \\
\hline 2557 & 46.4523 & 327.7186 & 367.6761 & 356.8584 & 122.5994 & 1.95328 \\
\hline 2558 & 46.4693 & 357.1757 & 366.0325 & 356.1202 & 122.5994 & 1.95261 \\
\hline 2559 & 46.4863 & 357.1118 & 364.7721 & 355.382 & 122.5994 & 1.95193 \\
\hline 2560 & 46.5033 & 355.2521 & 363.2782 & 354.6438 & 122.5994 & 1.95126 \\
\hline 2561 & 46.5203 & 380.7855 & 363.0525 & 353.9056 & 122.5994 & 1.95058 \\
\hline 2562 & 46.5373 & 346.9461 & 363.1461 & 353.1674 & 122.5994 & 1.94991 \\
\hline 2563 & 46.5543 & 385.2361 & 363.3495 & 352.4292 & 122.5994 & 1.94924 \\
\hline 2564 & 46.5713 & 362.0186 & 363.434 & 351.691 & 122.5994 & 1.94857 \\
\hline 2565 & 46.5883 & 344.6862 & 363.4875 & 350.9528 & 122.5994 & 1.9479 \\
\hline 2566 & 46.6053 & 345.7607 & 362.8521 & 350.2146 & 122.5994 & 1.94722 \\
\hline 2567 & 46.6223 & 368.3586 & 361.4106 & 349.4764 & 122.5994 & 1.94655 \\
\hline 2568 & 46.6393 & 339.4626 & 360.1744 & 348.7382 & 122.5994 & 1.94588 \\
\hline 2569 & 46.6563 & 342.2172 & 358.8678 & 348 & 122.5994 & 1.94521 \\
\hline 2570 & 46.6733 & 348.4259 & 358.3279 & 348 & 122.5994 & 1.94455 \\
\hline 2571 & 46.6903 & 360.3701 & 357.8286 & 348 & 122.5994 & 1.94388 \\
\hline 2572 & 46.7073 & 348.4693 & 357.3249 & 348 & 122.5994 & 1.94321 \\
\hline 2573 & 46.7243 & 349.8303 & 356.7812 & 348 & 122.5994 & 1.94254 \\
\hline 2574 & 46.7413 & 357.1757 & 356.1561 & 348 & 122.5994 & 1.94188 \\
\hline 2575 & 46.7583 & 358.7092 & 355.507 & 348 & 122.5994 & 1.94121 \\
\hline 2576 & 46.7753 & 353.3825 & 354.8585 & 348 & 122.5994 & 1.94054 \\
\hline 2577 & 46.7923 & 348.5878 & 354.2586 & 348 & 122.5994 & 1.93988 \\
\hline 2578 & 46.8093 & 337.6038 & 353.727 & 348 & 122.5994 & 1.93921 \\
\hline 2579 & 46.8263 & 308.3223 & 353.1591 & 348 & 122.5994 & 1.93855 \\
\hline 2580 & 46.8433 & 309.7075 & 352.8415 & 348 & 122.5994 & 1.93788 \\
\hline 2581 & 46.8603 & 335.5481 & 352.6835 & 348 & 122.5994 & 1.93722 \\
\hline 2582 & 46.8773 & 378.333 & 352.7575 & 348 & 122.5994 & 1.93656 \\
\hline 2583 & 46.8943 & 347.9256 & 353.1906 & 348 & 122.5994 & 1.9359 \\
\hline 2584 & 46.9113 & 308.244 & 354.1785 & 348 & 122.5994 & 1.93523 \\
\hline 2585 & 46.9283 & 333.6843 & 355.9992 & 348 & 122.5994 & 1.93457 \\
\hline 2586 & 46.9453 & 340.4872 & 358.7935 & 348 & 122.5994 & 1.93391 \\
\hline 2587 & 46.9623 & 325.6502 & 362.6017 & 348 & 122.5994 & 1.93325 \\
\hline 2588 & 46.9793 & 322.758 & 366.6053 & 348 & 122.5994 & 1.93259 \\
\hline 2589 & 46.9963 & 323.9689 & 369.2403 & 348 & 122.5994 & 1.93193 \\
\hline 2590 & 47.0133 & 336.9219 & 369.0048 & 348 & 122.5994 & 1.93127 \\
\hline 2591 & 47.0303 & 326.3373 & 366.7284 & 348 & 122.5994 & 1.93061 \\
\hline 2592 & 47.0473 & 339.5833 & 364.5163 & 348 & 122.5994 & 1.92996 \\
\hline 2593 & 47.0643 & 357.5102 & 363.6971 & 348 & 122.5994 & 1.9293 \\
\hline 2594 & 47.0813 & 352.0926 & 364.4278 & 347.8542 & 122.5994 & 1.92864 \\
\hline 2595 & 47.0983 & 346.6231 & 366.3455 & 347.7083 & 122.5994 & 1.92799 \\
\hline
\end{tabular}




\begin{tabular}{|c|c|c|c|c|c|c|}
\hline 2596 & 47.1153 & 349.0401 & 368.1284 & 347.5625 & 122.5994 & 1.92733 \\
\hline 2597 & 47.1323 & 356.1761 & 368.2741 & 347.4167 & 122.5994 & 1.92667 \\
\hline 2598 & 47.1493 & 344.0449 & 366.5968 & 347.2708 & 122.5994 & 1.92602 \\
\hline 2599 & 47.1663 & 352.3597 & 364.3699 & 347.125 & 122.5994 & 1.92536 \\
\hline 2600 & 47.1833 & 339.7046 & 362.825 & 346.9792 & 122.5994 & 1.92471 \\
\hline 2601 & 47.2003 & 338.5275 & 362.4819 & 346.8333 & 122.5994 & 1.92406 \\
\hline 2602 & 47.2173 & 371.3093 & 363.1569 & 346.6875 & 122.5994 & 1.9234 \\
\hline 2603 & 47.2343 & 353.6845 & 363.815 & 346.5417 & 122.5994 & 1.92275 \\
\hline 2604 & 47.2513 & 353.5365 & 363.2283 & 346.3958 & 122.5994 & 1.9221 \\
\hline 2605 & 47.2683 & 372.8722 & 361.1585 & 346.25 & 122.5994 & 1.92145 \\
\hline 2606 & 47.2853 & 382.9671 & 358.5615 & 346.1042 & 122.5994 & 1.9208 \\
\hline 2607 & 47.3023 & 359.6278 & 356.4437 & 345.9583 & 122.5994 & 1.92014 \\
\hline 2608 & 47.3193 & 343.6867 & 355.0561 & 345.8125 & 122.5994 & 1.91949 \\
\hline 2609 & 47.3363 & 354.6222 & 354.3871 & 345.6667 & 122.5994 & 1.91885 \\
\hline 2610 & 47.3533 & 339.0054 & 354.1806 & 345.5208 & 122.5994 & 1.9182 \\
\hline 2611 & 47.3703 & 366.5938 & 353.7574 & 345.375 & 122.5994 & 1.91755 \\
\hline 2612 & 47.3873 & 369.5616 & 352.7944 & 345.2292 & 122.5994 & 1.9169 \\
\hline 2613 & 47.4043 & 346.1857 & 351.5769 & 345.0833 & 122.5994 & 1.91625 \\
\hline 2614 & 47.4213 & 375.4649 & 350.5127 & 344.9375 & 122.5994 & 1.9156 \\
\hline 2615 & 47.4383 & 381.2002 & 349.6871 & 344.7917 & 122.5994 & 1.91496 \\
\hline 2616 & 47.4553 & 392.536 & 349.1045 & 344.6458 & 122.5994 & 1.91431 \\
\hline 2617 & 47.4723 & 349.7366 & 348.7016 & 344.5 & 122.5994 & 1.91366 \\
\hline 2618 & 47.4893 & 350.8119 & 348.3639 & 344.3542 & 122.5994 & 1.91302 \\
\hline 2619 & 47.5063 & 359.6836 & 348.0157 & 344.2083 & 122.5994 & 1.91237 \\
\hline 2620 & 47.5233 & 367.1737 & 347.6545 & 344.0625 & 122.5994 & 1.91173 \\
\hline 2621 & 47.5403 & 358.5879 & 347.2871 & 343.9167 & 122.5994 & 1.91109 \\
\hline 2622 & 47.5573 & 361.0481 & 347.0835 & 343.7708 & 122.5994 & 1.91044 \\
\hline 2623 & 47.5743 & 370.5922 & 346.7854 & 343.625 & 122.5994 & 1.9098 \\
\hline 2624 & 47.5913 & 380.8002 & 346.5199 & 343.4792 & 122.5994 & 1.90916 \\
\hline 2625 & 47.6083 & 374.5486 & 346.2598 & 343.3333 & 122.5994 & 1.90851 \\
\hline 2626 & 47.6253 & 363.3073 & 346.0127 & 343.1875 & 122.5994 & 1.90787 \\
\hline 2627 & 47.6423 & 347.8719 & 345.7834 & 343.0417 & 122.5994 & 1.90723 \\
\hline 2628 & 47.6593 & 323.381 & 345.502 & 342.8958 & 122.5994 & 1.90659 \\
\hline 2629 & 47.6763 & 350.8834 & 345.3283 & 342.75 & 122.5994 & 1.90595 \\
\hline 2630 & 47.6933 & 377.0383 & 345.3688 & 342.6042 & 122.5994 & 1.90531 \\
\hline 2631 & 47.7103 & 353.0948 & 345.4293 & 342.4583 & 122.5994 & 1.90467 \\
\hline 2632 & 47.7273 & 363.0811 & 345.5691 & 342.3125 & 122.5994 & 1.90403 \\
\hline 2633 & 47.7443 & 357.148 & 345.8448 & 342.1667 & 122.5994 & 1.9034 \\
\hline 2634 & 47.7613 & 338.7837 & 346.4043 & 342.0208 & 122.5994 & 1.90276 \\
\hline 2635 & 47.7783 & 337.3309 & 347.6024 & 341.875 & 122.5994 & 1.90212 \\
\hline 2636 & 47.7953 & 325.9817 & 349.5622 & 341.7292 & 122.5994 & 1.90148 \\
\hline 2637 & 47.8123 & 333.3915 & 352.3314 & 341.5833 & 122.5994 & 1.90085 \\
\hline 2638 & 47.8293 & 366.437 & 355.4231 & 341.4375 & 122.5994 & 1.90021 \\
\hline 2639 & 47.8463 & 361.8563 & 357.7712 & 341.2917 & 122.5994 & 1.89958 \\
\hline 2640 & 47.8633 & 336.8824 & 358.3851 & 341.1458 & 122.5994 & 1.89894 \\
\hline 2641 & 47.8803 & 346.1007 & 357.7777 & 341 & 122.5994 & 1.89831 \\
\hline 2642 & 47.8973 & 331.4518 & 357.4927 & 341.0469 & 122.5994 & 1.89767 \\
\hline 2643 & 47.9143 & 327.5786 & 357.9502 & 341.0938 & 122.5994 & 1.89704 \\
\hline 2644 & 47.9313 & 321.4073 & 359.0997 & 341.1406 & 122.5994 & 1.89641 \\
\hline
\end{tabular}




\begin{tabular}{|c|c|c|c|c|c|c|}
\hline 2645 & 47.9483 & 343.0926 & 360.3258 & 341.1875 & 122.5994 & 1.89577 \\
\hline 2646 & 47.9653 & 335.9255 & 360.9107 & 341.2344 & 122.5994 & 1.89514 \\
\hline 2647 & 47.9823 & 369.9028 & 360.3771 & 341.2813 & 122.5994 & 1.89451 \\
\hline 2648 & 47.9993 & 372.5397 & 358.9235 & 341.3281 & 122.5994 & 1.89388 \\
\hline 2649 & 48.0163 & 364.9654 & 357.2888 & 341.375 & 122.5994 & 1.89325 \\
\hline 2650 & 48.0333 & 338.6159 & 356.0133 & 341.4219 & 122.5994 & 1.89262 \\
\hline 2651 & 48.0503 & 387.8406 & 355.2343 & 341.4688 & 122.5994 & 1.89199 \\
\hline 2652 & 48.0673 & 375.0547 & 354.7602 & 341.5156 & 122.5994 & 1.89136 \\
\hline 2653 & 48.0843 & 343.3639 & 354.4135 & 341.5625 & 122.5994 & 1.89073 \\
\hline 2654 & 48.1013 & 358.5078 & 354.1694 & 341.6094 & 122.5994 & 1.8901 \\
\hline 2655 & 48.1183 & 333.5981 & 354.211 & 341.6563 & 122.5994 & 1.88947 \\
\hline 2656 & 48.1353 & 353.8798 & 354.7834 & 341.7031 & 122.5994 & 1.88884 \\
\hline 2657 & 48.1523 & 360.6514 & 356.006 & 341.75 & 122.5994 & 1.88822 \\
\hline 2658 & 48.1693 & 375.6901 & 358.0377 & 341.7969 & 122.5994 & 1.88759 \\
\hline 2659 & 48.1863 & 360.0834 & 360.9387 & 341.8438 & 122.5994 & 1.88696 \\
\hline 2660 & 48.2033 & 378.3443 & 364.6477 & 341.8906 & 122.5994 & 1.88634 \\
\hline 2661 & 48.2203 & 345.706 & 368.9081 & 341.9375 & 122.5994 & 1.88571 \\
\hline 2662 & 48.2373 & 357.2576 & 373.2002 & 341.9844 & 122.5994 & 1.88509 \\
\hline 2663 & 48.2543 & 372.338 & 376.5017 & 342.0313 & 122.5994 & 1.88446 \\
\hline 2664 & 48.2713 & 406.1714 & 377.6329 & 342.0781 & 122.5994 & 1.88384 \\
\hline 2665 & 48.2883 & 384.5296 & 376.2437 & 342.125 & 122.5994 & 1.88322 \\
\hline 2666 & 48.3053 & 364.7915 & 372.972 & 341.8516 & 122.5994 & 1.88259 \\
\hline 2667 & 48.3223 & 372.6837 & 369.5023 & 341.5781 & 122.5994 & 1.88197 \\
\hline 2668 & 48.3393 & 390.8876 & 366.6768 & 341.3047 & 122.5994 & 1.88135 \\
\hline 2669 & 48.3563 & 376.4102 & 364.7581 & 341.0313 & 122.5994 & 1.88073 \\
\hline 2670 & 48.3733 & 353.9709 & 363.4901 & 340.7578 & 122.5994 & 1.8801 \\
\hline 2671 & 48.3903 & 333.8936 & 362.1066 & 340.4844 & 122.5994 & 1.87948 \\
\hline 2672 & 48.4073 & 362.0424 & 359.9957 & 340.2109 & 122.5994 & 1.87886 \\
\hline 2673 & 48.4243 & 384.0661 & 357.1118 & 339.9375 & 122.5994 & 1.87824 \\
\hline 2674 & 48.4413 & 380.1382 & 353.9633 & 339.6641 & 122.5994 & 1.87762 \\
\hline 2675 & 48.4583 & 380.792 & 351.0418 & 339.3906 & 122.5994 & 1.87701 \\
\hline 2676 & 48.4753 & 379.4663 & 348.6505 & 339.1172 & 122.5994 & 1.87639 \\
\hline 2677 & 48.4923 & 354.9894 & 346.8434 & 338.8438 & 122.5994 & 1.87577 \\
\hline 2678 & 48.5093 & 358.1082 & 345.7657 & 338.5703 & 122.5994 & 1.87515 \\
\hline 2679 & 48.5263 & 380.6755 & 345.4195 & 338.2969 & 122.5994 & 1.87453 \\
\hline 2680 & 48.5433 & 385.5296 & 345.8727 & 338.0234 & 122.5994 & 1.87392 \\
\hline 2681 & 48.5603 & 366.325 & 347.1757 & 337.75 & 122.5994 & 1.8733 \\
\hline 2682 & 48.5773 & 367.6441 & 349.3254 & 337.4766 & 122.5994 & 1.87269 \\
\hline 2683 & 48.5943 & 383.565 & 351.7386 & 337.2031 & 122.5994 & 1.87207 \\
\hline 2684 & 48.6113 & 396.7253 & 353.5435 & 336.9297 & 122.5994 & 1.87145 \\
\hline 2685 & 48.6283 & 356.6728 & 353.6172 & 336.6563 & 122.5994 & 1.87084 \\
\hline 2686 & 48.6453 & 393.0355 & 352.074 & 336.3828 & 122.5994 & 1.87023 \\
\hline 2687 & 48.6623 & 376.77 & 350.0441 & 336.1094 & 122.5994 & 1.86961 \\
\hline 2688 & 48.6793 & 381.3272 & 348.4674 & 335.8359 & 122.5994 & 1.869 \\
\hline 2689 & 48.6963 & 384.1232 & 347.8337 & 335.5625 & 122.5994 & 1.86839 \\
\hline 2690 & 48.7133 & 357.8725 & 347.9536 & 335.2891 & 122.5994 & 1.86777 \\
\hline 2691 & 48.7303 & 363.3981 & 348.3654 & 335.0156 & 122.5994 & 1.86716 \\
\hline 2692 & 48.7473 & 337.8404 & 348.3369 & 334.7422 & 122.5994 & 1.86655 \\
\hline 2693 & 48.7643 & 338.3179 & 347.3822 & 334.4688 & 122.5994 & 1.86594 \\
\hline
\end{tabular}




\begin{tabular}{|c|c|c|c|c|c|c|}
\hline 2694 & 48.7813 & 374.1839 & 345.8639 & 334.1953 & 122.5994 & 1.86533 \\
\hline 2695 & 48.7983 & 332.8721 & 344.3094 & 333.9219 & 122.5994 & 1.86472 \\
\hline 2696 & 48.8153 & 345.7275 & 343.0761 & 333.6484 & 122.5994 & 1.86411 \\
\hline 2697 & 48.8323 & 338.9351 & 342.2132 & 333.375 & 122.5994 & 1.8635 \\
\hline 2698 & 48.8493 & 379.2076 & 341.596 & 333.1016 & 122.5994 & 1.86289 \\
\hline 2699 & 48.8663 & 345.2556 & 341.1124 & 332.8281 & 122.5994 & 1.86228 \\
\hline 2700 & 48.8833 & 330.4241 & 340.6845 & 332.5547 & 122.5994 & 1.86168 \\
\hline 2701 & 48.9003 & 321.1299 & 340.3167 & 332.2813 & 122.5994 & 1.86107 \\
\hline 2702 & 48.9173 & 332.7611 & 340.0695 & 332.0078 & 122.5994 & 1.86046 \\
\hline 2703 & 48.9343 & 369.8464 & 340.0954 & 331.7344 & 122.5994 & 1.85985 \\
\hline 2704 & 48.9513 & 359.6069 & 340.5332 & 331.4609 & 122.5994 & 1.85925 \\
\hline 2705 & 48.9683 & 383.7096 & 341.4502 & 331.1875 & 122.5994 & 1.85864 \\
\hline 2706 & 48.9853 & 347.5142 & 343.1964 & 330.9141 & 122.5994 & 1.85804 \\
\hline 2707 & 49.0023 & 343.6639 & 345.534 & 330.6406 & 122.5994 & 1.85743 \\
\hline 2708 & 49.0193 & 313.2856 & 347.927 & 330.3672 & 122.5994 & 1.85683 \\
\hline 2709 & 49.0363 & 327.8434 & 349.2065 & 330.0938 & 122.5994 & 1.85622 \\
\hline 2710 & 49.0533 & 334.3412 & 348.3289 & 329.8203 & 122.5994 & 1.85562 \\
\hline 2711 & 49.0703 & 329.6856 & 345.774 & 329.5469 & 122.5994 & 1.85502 \\
\hline 2712 & 49.0873 & 337.3773 & 343.065 & 329.2734 & 122.5994 & 1.85441 \\
\hline 2713 & 49.1043 & 332.8105 & 341.2053 & 329 & 122.5994 & 1.85381 \\
\hline 2714 & 49.1213 & 323.5937 & 341.0352 & 329.0417 & 122.5994 & 1.85321 \\
\hline 2715 & 49.1383 & 319.4808 & 342.1198 & 329.0833 & 122.5994 & 1.85261 \\
\hline 2716 & 49.1553 & 354.7804 & 343.9516 & 329.125 & 122.5994 & 1.85201 \\
\hline 2717 & 49.1723 & 349.2042 & 345.7034 & 329.1667 & 122.5994 & 1.85141 \\
\hline 2718 & 49.1893 & 369.4193 & 347.0612 & 329.2083 & 122.5994 & 1.85081 \\
\hline 2719 & 49.2063 & 376.8499 & 348.1912 & 329.25 & 122.5994 & 1.85021 \\
\hline 2720 & 49.2233 & 349.8126 & 349.5093 & 329.2917 & 122.5994 & 1.84961 \\
\hline 2721 & 49.2403 & 377.4673 & 350.8947 & 329.3333 & 122.5994 & 1.84901 \\
\hline 2722 & 49.2573 & 398.6572 & 352.0542 & 329.375 & 122.5994 & 1.84841 \\
\hline 2723 & 49.2743 & 356.2571 & 352.7768 & 329.4167 & 122.5994 & 1.84781 \\
\hline 2724 & 49.2913 & 354.7294 & 352.603 & 329.4583 & 122.5994 & 1.84722 \\
\hline 2725 & 49.3083 & 364.0254 & 351.4402 & 329.5 & 122.5994 & 1.84662 \\
\hline 2726 & 49.3253 & 349.1181 & 349.2773 & 329.5417 & 122.5994 & 1.84602 \\
\hline 2727 & 49.3423 & 344.6408 & 347.4204 & 329.5833 & 122.5994 & 1.84543 \\
\hline 2728 & 49.3593 & 346.0061 & 346.2401 & 329.625 & 122.5994 & 1.84483 \\
\hline 2729 & 49.3763 & 351.1355 & 345.5529 & 329.6667 & 122.5994 & 1.84424 \\
\hline 2730 & 49.3933 & 367.1649 & 345.0974 & 329.7083 & 122.5994 & 1.84364 \\
\hline 2731 & 49.4103 & 352.8376 & 344.5753 & 329.75 & 122.5994 & 1.84305 \\
\hline 2732 & 49.4273 & 315.7842 & 343.6086 & 329.7917 & 122.5994 & 1.84245 \\
\hline 2733 & 49.4443 & 352.043 & 342.2192 & 329.8333 & 122.5994 & 1.84186 \\
\hline 2734 & 49.4613 & 312.1031 & 340.5581 & 329.875 & 122.5994 & 1.84126 \\
\hline 2735 & 49.4783 & 307.9735 & 339.2995 & 329.9167 & 122.5994 & 1.84067 \\
\hline 2736 & 49.4953 & 324.8488 & 338.5605 & 329.9583 & 122.5994 & 1.84008 \\
\hline 2737 & 49.5123 & 318.2986 & 341.9861 & 330 & 122.5994 & 1.83949 \\
\hline 2738 & 49.5293 & 356.114 & 342.7306 & 330.6302 & 122.5994 & 1.8389 \\
\hline 2739 & 49.5463 & 347.2569 & 343.4725 & 331.2604 & 122.5994 & 1.8383 \\
\hline 2740 & 49.5633 & 356.5037 & 344.0614 & 331.8906 & 122.5994 & 1.83771 \\
\hline 2741 & 49.5803 & 353.5771 & 344.5672 & 332.5208 & 122.5994 & 1.83712 \\
\hline 2742 & 49.5973 & 357.1673 & 345.1824 & 333.151 & 122.5994 & 1.83653 \\
\hline
\end{tabular}




\begin{tabular}{|c|c|c|c|c|c|c|}
\hline 2743 & 49.6143 & 338.5704 & 347.7638 & 333.7813 & 122.5994 & 1.83594 \\
\hline 2744 & 49.6313 & 310.3528 & 349.1989 & 334.4115 & 122.5994 & 1.83536 \\
\hline 2745 & 49.6483 & 342.978 & 351.0862 & 335.0417 & 122.5994 & 1.83477 \\
\hline 2746 & 49.6653 & 359.8813 & 353.3427 & 335.6719 & 122.5994 & 1.83418 \\
\hline 2747 & 49.6823 & 342.8395 & 355.8019 & 336.3021 & 122.5994 & 1.83359 \\
\hline 2748 & 49.6993 & 348.1782 & 358.2487 & 336.9323 & 122.5994 & 1.833 \\
\hline 2749 & 49.7163 & 330.3467 & 360.4605 & 337.5625 & 122.5994 & 1.83242 \\
\hline 2750 & 49.7333 & 300.8833 & 362.2373 & 338.1927 & 122.5994 & 1.83183 \\
\hline 2751 & 49.7503 & 317.1467 & 363.8707 & 338.8229 & 122.5994 & 1.83124 \\
\hline 2752 & 49.7673 & 356.3125 & 365.7907 & 339.4531 & 122.5994 & 1.83066 \\
\hline 2753 & 49.7843 & 365.4537 & 368.5695 & 340.0833 & 122.5994 & 1.83007 \\
\hline 2754 & 49.8013 & 363.3794 & 372.6501 & 340.7135 & 122.5994 & 1.82949 \\
\hline 2755 & 49.8183 & 384.2082 & 378.2639 & 341.3438 & 122.5994 & 1.8289 \\
\hline 2756 & 49.8353 & 349.6565 & 385.2983 & 341.974 & 122.5994 & 1.82832 \\
\hline 2757 & 49.8523 & 362.3094 & 393.1356 & 342.6042 & 122.5994 & 1.82774 \\
\hline 2758 & 49.8693 & 378.5225 & 400.6361 & 343.2344 & 122.5994 & 1.82715 \\
\hline 2759 & 49.8863 & 354.8251 & 406.719 & 343.8646 & 122.5994 & 1.82657 \\
\hline 2760 & 49.9033 & 395.6207 & 411.3882 & 344.4948 & 122.5994 & 1.82599 \\
\hline 2761 & 49.9203 & 427.3031 & 415.6464 & 345.125 & 122.5994 & 1.82541 \\
\hline 2762 & 49.9373 & 405.2323 & 419.428 & 345.2357 & 122.5994 & 1.82482 \\
\hline 2763 & 49.9543 & 405.4411 & 423.2188 & 345.3464 & 122.5994 & 1.82424 \\
\hline 2764 & 49.9713 & 389.0892 & 427.651 & 345.457 & 122.5994 & 1.82366 \\
\hline 2765 & 49.9883 & 415.4284 & 433.5569 & 345.5677 & 122.5994 & 1.82308 \\
\hline 2766 & 50.0053 & 442.965 & 441.5944 & 345.6784 & 122.5994 & 1.8225 \\
\hline 2767 & 50.0223 & 442.9283 & 452.2924 & 345.7891 & 122.5994 & 1.82192 \\
\hline 2768 & 50.0393 & 472.0196 & 467.2751 & 345.8997 & 122.5994 & 1.82134 \\
\hline 2769 & 50.0563 & 496.2653 & 490.1771 & 346.0104 & 122.5994 & 1.82076 \\
\hline 2770 & 50.0733 & 506.6608 & 528.4479 & 346.1211 & 122.5994 & 1.82019 \\
\hline 2771 & 50.0903 & 594.9859 & 595.9775 & 346.2318 & 122.5994 & 1.81961 \\
\hline 2772 & 50.1073 & 697.3676 & 713.4323 & 346.3424 & 122.5994 & 1.81903 \\
\hline 2773 & 50.1243 & 823.5686 & 900.6591 & 346.4531 & 122.5994 & 1.81845 \\
\hline 2774 & 50.1413 & 1066.827 & 1160.441 & 346.5638 & 122.5994 & 1.81788 \\
\hline 2775 & 50.1583 & 1376.612 & 1456.167 & 346.6745 & 122.5994 & 1.8173 \\
\hline 2776 & 50.1753 & 1643.816 & 1685.383 & 346.7852 & 122.5994 & 1.81672 \\
\hline 2777 & 50.1923 & 1750.68 & 1712.012 & 346.8958 & 122.5994 & 1.81615 \\
\hline 2778 & 50.2093 & 1478.46 & 1526.997 & 347.0065 & 122.5994 & 1.81557 \\
\hline 2779 & 50.2263 & 1057.461 & 1271.183 & 347.1172 & 122.5994 & 1.815 \\
\hline 2780 & 50.2433 & 842.8405 & 1066.751 & 347.2279 & 122.5994 & 1.81442 \\
\hline 2781 & 50.2603 & 739.9172 & 966.8801 & 347.3385 & 122.5994 & 1.81385 \\
\hline 2782 & 50.2773 & 817.0933 & 972.1636 & 347.4492 & 122.5994 & 1.81328 \\
\hline 2783 & 50.2943 & 911.4686 & 1042.165 & 347.5599 & 122.5994 & 1.8127 \\
\hline 2784 & 50.3113 & 1075.137 & 1099.837 & 347.6706 & 122.5994 & 1.81213 \\
\hline 2785 & 50.3283 & 996.6785 & 1064.323 & 347.7813 & 122.5994 & 1.81156 \\
\hline 2786 & 50.3453 & 884.7527 & 935.4694 & 347.4674 & 122.5994 & 1.81099 \\
\hline 2787 & 50.3623 & 643.6218 & 778.7183 & 347.1536 & 122.5994 & 1.81042 \\
\hline 2788 & 50.3793 & 532.3961 & 642.4471 & 346.8398 & 122.5994 & 1.80985 \\
\hline 2789 & 50.3963 & 438.0394 & 543.5245 & 346.526 & 122.5994 & 1.80927 \\
\hline 2790 & 50.4133 & 392.0923 & 480.0804 & 346.2122 & 122.5994 & 1.8087 \\
\hline 2791 & 50.4303 & 392.612 & 442.6341 & 345.8984 & 122.5994 & 1.80813 \\
\hline
\end{tabular}




\begin{tabular}{|c|c|c|c|c|c|c|}
\hline 2792 & 50.4473 & 410.8957 & 420.8311 & 345.5846 & 122.5994 & 1.80756 \\
\hline 2793 & 50.4643 & 385.7839 & 407.4548 & 345.2708 & 122.5994 & 1.807 \\
\hline 2794 & 50.4813 & 361.6168 & 398.4676 & 344.957 & 122.5994 & 1.80643 \\
\hline 2795 & 50.4983 & 404.0639 & 391.79 & 344.6432 & 122.5994 & 1.80586 \\
\hline 2796 & 50.5153 & 403.418 & 386.8777 & 344.3294 & 122.5994 & 1.80529 \\
\hline 2797 & 50.5323 & 395.0478 & 383.3153 & 344.0156 & 122.5994 & 1.80472 \\
\hline 2798 & 50.5493 & 374.2819 & 381.0583 & 343.7018 & 122.5994 & 1.80416 \\
\hline 2799 & 50.5663 & 399.9616 & 380.395 & 343.388 & 122.5994 & 1.80359 \\
\hline 2800 & 50.5833 & 401.8551 & 381.8689 & 343.0742 & 122.5994 & 1.80302 \\
\hline 2801 & 50.6003 & 412.0078 & 384.9727 & 342.7604 & 122.5994 & 1.80246 \\
\hline 2802 & 50.6173 & 429.9871 & 390.276 & 342.4466 & 122.5994 & 1.80189 \\
\hline 2803 & 50.6343 & 443.9523 & 398.0946 & 342.1328 & 122.5994 & 1.80133 \\
\hline 2804 & 50.6513 & 433.3523 & 408.1328 & 341.819 & 122.5994 & 1.80076 \\
\hline 2805 & 50.6683 & 418.8397 & 418.4723 & 341.5052 & 122.5994 & 1.8002 \\
\hline 2806 & 50.6853 & 422.8681 & 426.1772 & 341.1914 & 122.5994 & 1.79963 \\
\hline 2807 & 50.7023 & 406.7819 & 429.6342 & 340.8776 & 122.5994 & 1.79907 \\
\hline 2808 & 50.7193 & 395.1877 & 428.5785 & 340.5638 & 122.5994 & 1.79851 \\
\hline 2809 & 50.7363 & 403.768 & 422.9413 & 340.25 & 122.5994 & 1.79794 \\
\hline 2810 & 50.7533 & 388.5413 & 415.0208 & 339.9362 & 122.5994 & 1.79738 \\
\hline 2811 & 50.7703 & 375.2097 & 407.5205 & 339.6224 & 122.5994 & 1.79682 \\
\hline 2812 & 50.7873 & 363.0211 & 402.3052 & 339.3086 & 122.5994 & 1.79626 \\
\hline 2813 & 50.8043 & 384.6601 & 398.9552 & 338.9948 & 122.5994 & 1.7957 \\
\hline 2814 & 50.8213 & 412.3603 & 396.0993 & 338.681 & 122.5994 & 1.79514 \\
\hline 2815 & 50.8383 & 407.284 & 392.7094 & 338.3672 & 122.5994 & 1.79458 \\
\hline 2816 & 50.8553 & 391.0493 & 388.1106 & 338.0534 & 122.5994 & 1.79402 \\
\hline 2817 & 50.8723 & 368.2266 & 382.0042 & 337.7396 & 122.5994 & 1.79346 \\
\hline 2818 & 50.8893 & 359.4925 & 375.0607 & 337.4258 & 122.5994 & 1.7929 \\
\hline 2819 & 50.9063 & 353.8897 & 365.0846 & 337.112 & 122.5994 & 1.79234 \\
\hline 2820 & 50.9233 & 357.0571 & 359.3654 & 336.7982 & 122.5994 & 1.79178 \\
\hline 2821 & 50.9403 & 353.4026 & 354.7408 & 336.4844 & 122.5994 & 1.79122 \\
\hline 2822 & 50.9573 & 365.5575 & 351.2027 & 336.1706 & 122.5994 & 1.79066 \\
\hline 2823 & 50.9743 & 389.3613 & 346.7143 & 335.8568 & 122.5994 & 1.79011 \\
\hline 2824 & 50.9913 & 332.196 & 345.0306 & 335.543 & 122.5994 & 1.78955 \\
\hline 2825 & 51.0083 & 331.6731 & 343.9446 & 335.2292 & 122.5994 & 1.78899 \\
\hline 2826 & 51.0253 & 353.2184 & 343.3058 & 334.9154 & 122.5994 & 1.78844 \\
\hline 2827 & 51.0423 & 345.1333 & 342.9678 & 334.6016 & 122.5994 & 1.78788 \\
\hline 2828 & 51.0593 & 386.7349 & 342.8689 & 334.2878 & 122.5994 & 1.78733 \\
\hline 2829 & 51.0763 & 376.026 & 342.998 & 333.974 & 122.5994 & 1.78677 \\
\hline 2830 & 51.0933 & 362.4641 & 343.44 & 333.6602 & 122.5994 & 1.78622 \\
\hline 2831 & 51.1103 & 361.0481 & 344.3733 & 333.3464 & 122.5994 & 1.78566 \\
\hline 2832 & 51.1273 & 407.5868 & 346.1166 & 333.0326 & 122.5994 & 1.78511 \\
\hline 2833 & 51.1443 & 357.355 & 349.1574 & 332.7188 & 122.5994 & 1.78456 \\
\hline 2834 & 51.1613 & 386.5178 & 353.5626 & 331.9805 & 122.5994 & 1.784 \\
\hline 2835 & 51.1783 & 384.2131 & 360.2767 & 331.2422 & 122.5994 & 1.78345 \\
\hline 2836 & 51.1953 & 384.2112 & 369.5427 & 330.5039 & 122.5994 & 1.7829 \\
\hline 2837 & 51.2123 & 371.7052 & 380.9414 & 329.7656 & 122.5994 & 1.78235 \\
\hline 2838 & 51.2293 & 334.239 & 393.5249 & 329.0273 & 122.5994 & 1.78179 \\
\hline 2839 & 51.2463 & 402.8074 & 404.6898 & 328.2891 & 122.5994 & 1.78124 \\
\hline 2840 & 51.2633 & 380.8869 & 410.3872 & 327.5508 & 122.5994 & 1.78069 \\
\hline
\end{tabular}




\begin{tabular}{|c|c|c|c|c|c|c|}
\hline 2841 & 51.2803 & 346.7207 & 409.1902 & 326.8125 & 122.5994 & 1.78014 \\
\hline 2842 & 51.2973 & 353.0079 & 401.8243 & 326.0742 & 122.5994 & 1.77959 \\
\hline 2843 & 51.3143 & 353.0584 & 392.1515 & 325.3359 & 122.5994 & 1.77904 \\
\hline 2844 & 51.3313 & 368.0068 & 383.525 & 324.5977 & 122.5994 & 1.77849 \\
\hline 2845 & 51.3483 & 344.504 & 377.6423 & 323.8594 & 122.5994 & 1.77794 \\
\hline 2846 & 51.3653 & 343.4057 & 374.6389 & 323.1211 & 122.5994 & 1.7774 \\
\hline 2847 & 51.3823 & 357.4257 & 373.2802 & 322.3828 & 122.5994 & 1.77685 \\
\hline 2848 & 51.3993 & 347.8474 & 371.1435 & 321.6445 & 122.5994 & 1.7763 \\
\hline 2849 & 51.4163 & 362.0401 & 366.7476 & 320.9063 & 122.5994 & 1.77575 \\
\hline 2850 & 51.4333 & 374.9121 & 359.8105 & 320.168 & 122.5994 & 1.77521 \\
\hline 2851 & 51.4503 & 346.3501 & 351.7397 & 319.4297 & 122.5994 & 1.77466 \\
\hline 2852 & 51.4673 & 332.4598 & 343.8935 & 318.6914 & 122.5994 & 1.77411 \\
\hline 2853 & 51.4843 & 326.9884 & 337.0802 & 317.9531 & 122.5994 & 1.77357 \\
\hline 2854 & 51.5013 & 320.2601 & 331.6102 & 317.2148 & 122.5994 & 1.77302 \\
\hline 2855 & 51.5183 & 328.2587 & 327.4958 & 316.4766 & 122.5994 & 1.77248 \\
\hline 2856 & 51.5353 & 330.2901 & 324.455 & 315.7383 & 122.5994 & 1.77193 \\
\hline 2857 & 51.5523 & 301.3368 & 322.2468 & 315 & 122.5994 & 1.77139 \\
\hline 2858 & 51.5693 & 302.0373 & 321.2272 & 314.875 & 122.5994 & 1.77084 \\
\hline 2859 & 51.5863 & 330.116 & 320.5824 & 314.75 & 122.5994 & 1.7703 \\
\hline 2860 & 51.6033 & 308.0281 & 320.1713 & 314.625 & 122.5994 & 1.76976 \\
\hline 2861 & 51.6203 & 300.4911 & 319.922 & 314.5 & 122.5994 & 1.76921 \\
\hline 2862 & 51.6373 & 292.382 & 319.7501 & 314.375 & 122.5994 & 1.76867 \\
\hline 2863 & 51.6543 & 302.5064 & 319.6722 & 314.25 & 122.5994 & 1.76813 \\
\hline 2864 & 51.6713 & 309.7303 & 319.7938 & 314.125 & 122.5994 & 1.76759 \\
\hline 2865 & 51.6883 & 325.3742 & 320.1269 & 314 & 122.5994 & 1.76705 \\
\hline 2866 & 51.7053 & 325.6071 & 320.8487 & 313.875 & 122.5994 & 1.7665 \\
\hline 2867 & 51.7223 & 297.2168 & 322.0532 & 313.75 & 122.5994 & 1.76596 \\
\hline 2868 & 51.7393 & 290.3213 & 323.7144 & 313.625 & 122.5994 & 1.76542 \\
\hline 2869 & 51.7563 & 314.7685 & 325.5655 & 313.5 & 122.5994 & 1.76488 \\
\hline 2870 & 51.7733 & 311.0802 & 326.9987 & 313.375 & 122.5994 & 1.76434 \\
\hline 2871 & 51.7903 & 308.8592 & 327.1005 & 313.25 & 122.5994 & 1.7638 \\
\hline 2872 & 51.8073 & 317.9643 & 325.6391 & 313.125 & 122.5994 & 1.76327 \\
\hline 2873 & 51.8243 & 334.8783 & 323.4719 & 313 & 122.5994 & 1.76273 \\
\hline 2874 & 51.8413 & 335.532 & 321.5649 & 312.875 & 122.5994 & 1.76219 \\
\hline 2875 & 51.8583 & 293.6445 & 320.3725 & 312.75 & 122.5994 & 1.76165 \\
\hline 2876 & 51.8753 & 294.3731 & 319.9758 & 312.625 & 122.5994 & 1.76111 \\
\hline 2877 & 51.8923 & 301.9061 & 320.1145 & 312.5 & 122.5994 & 1.76058 \\
\hline 2878 & 51.9093 & 318.727 & 320.3919 & 312.375 & 122.5994 & 1.76004 \\
\hline 2879 & 51.9263 & 307.2985 & 319.845 & 312.25 & 122.5994 & 1.7595 \\
\hline 2880 & 51.9433 & 296.4489 & 318.9994 & 312.125 & 122.5994 & 1.75897 \\
\hline 2881 & 51.9603 & 319.5671 & 317.768 & 312 & 122.5994 & 1.75843 \\
\hline 2882 & 51.9773 & 303.0057 & 317.1437 & 312.4167 & 122.5994 & 1.7579 \\
\hline 2883 & 51.9943 & 342.0379 & 316.8073 & 312.8333 & 122.5994 & 1.75736 \\
\hline 2884 & 52.0113 & 324.6176 & 316.8171 & 313.25 & 122.5994 & 1.75683 \\
\hline 2885 & 52.0283 & 340.6854 & 317.1013 & 313.6667 & 122.5994 & 1.75629 \\
\hline 2886 & 52.0453 & 312.5468 & 317.6636 & 314.0833 & 122.5994 & 1.75576 \\
\hline 2887 & 52.0623 & 306.5205 & 318.3918 & 314.5 & 122.5994 & 1.75523 \\
\hline 2888 & 52.0793 & 340.1492 & 319.1534 & 314.9167 & 122.5994 & 1.75469 \\
\hline 2889 & 52.0963 & 323.4698 & 320.3483 & 315.3333 & 122.5994 & 1.75416 \\
\hline
\end{tabular}




\begin{tabular}{|c|c|c|c|c|c|c|}
\hline 2890 & 52.1133 & 315.8866 & 321.7809 & 315.75 & 122.5994 & 1.75363 \\
\hline 2891 & 52.1303 & 297.5479 & 323.4131 & 316.1667 & 122.5994 & 1.7531 \\
\hline 2892 & 52.1473 & 325.5793 & 325.1624 & 316.5833 & 122.5994 & 1.75257 \\
\hline 2893 & 52.1643 & 344.3398 & 326.9387 & 317 & 122.5994 & 1.75204 \\
\hline 2894 & 52.1813 & 332.2961 & 328.7158 & 317.4167 & 122.5994 & 1.7515 \\
\hline 2895 & 52.1983 & 327.068 & 330.5286 & 317.8333 & 122.5994 & 1.75097 \\
\hline 2896 & 52.2153 & 336.1472 & 332.364 & 318.25 & 122.5994 & 1.75044 \\
\hline 2897 & 52.2323 & 288.3332 & 333.7345 & 318.6667 & 122.5994 & 1.74991 \\
\hline 2898 & 52.2493 & 342.3085 & 334.4773 & 319.0833 & 122.5994 & 1.74938 \\
\hline 2899 & 52.2663 & 330.5853 & 334.6351 & 319.5 & 122.5994 & 1.74886 \\
\hline 2900 & 52.2833 & 298.48 & 334.5069 & 319.9167 & 122.5994 & 1.74833 \\
\hline 2901 & 52.3003 & 318.4173 & 334.3568 & 320.3333 & 122.5994 & 1.7478 \\
\hline 2902 & 52.3173 & 325.6849 & 334.3312 & 320.75 & 122.5994 & 1.74727 \\
\hline 2903 & 52.3343 & 312.1522 & 334.4955 & 321.1667 & 122.5994 & 1.74674 \\
\hline 2904 & 52.3513 & 320.6631 & 334.9001 & 321.5833 & 122.5994 & 1.74622 \\
\hline 2905 & 52.3683 & 320.3431 & 335.2218 & 322 & 122.5994 & 1.74569 \\
\hline 2906 & 52.3853 & 322.1567 & 335.4475 & 322.5417 & 122.5994 & 1.74516 \\
\hline 2907 & 52.4023 & 333.8238 & 335.3437 & 323.0833 & 122.5994 & 1.74464 \\
\hline 2908 & 52.4193 & 337.9417 & 335.0118 & 323.625 & 122.5994 & 1.74411 \\
\hline 2909 & 52.4363 & 320.9898 & 334.6493 & 324.1667 & 122.5994 & 1.74359 \\
\hline 2910 & 52.4533 & 326.0957 & 334.4267 & 324.7083 & 122.5994 & 1.74306 \\
\hline 2911 & 52.4703 & 357.882 & 334.4607 & 325.25 & 122.5994 & 1.74254 \\
\hline 2912 & 52.4873 & 348.7015 & 334.8545 & 325.7917 & 122.5994 & 1.74201 \\
\hline 2913 & 52.5043 & 339.6817 & 335.519 & 326.3333 & 122.5994 & 1.74149 \\
\hline 2914 & 52.5213 & 339.7197 & 336.3932 & 326.875 & 122.5994 & 1.74096 \\
\hline 2915 & 52.5383 & 333.4922 & 337.3391 & 327.4167 & 122.5994 & 1.74044 \\
\hline 2916 & 52.5553 & 291.124 & 338.076 & 327.9583 & 122.5994 & 1.73992 \\
\hline 2917 & 52.5723 & 327.8021 & 338.4156 & 328.5 & 122.5994 & 1.73939 \\
\hline 2918 & 52.5893 & 375.0724 & 338.4028 & 329.0417 & 122.5994 & 1.73887 \\
\hline 2919 & 52.6063 & 351.2492 & 338.3017 & 329.5833 & 122.5994 & 1.73835 \\
\hline 2920 & 52.6233 & 327.5853 & 338.3647 & 330.125 & 122.5994 & 1.73783 \\
\hline 2921 & 52.6403 & 326.9561 & 338.8469 & 330.6667 & 122.5994 & 1.73731 \\
\hline 2922 & 52.6573 & 321.5401 & 339.5967 & 331.2083 & 122.5994 & 1.73679 \\
\hline 2923 & 52.6743 & 308.0525 & 340.6864 & 331.75 & 122.5994 & 1.73627 \\
\hline 2924 & 52.6913 & 341.7194 & 341.9715 & 332.2917 & 122.5994 & 1.73575 \\
\hline 2925 & 52.7083 & 319.3227 & 343.2821 & 332.8333 & 122.5994 & 1.73523 \\
\hline 2926 & 52.7253 & 323.5044 & 344.5103 & 333.375 & 122.5994 & 1.73471 \\
\hline 2927 & 52.7423 & 324.0432 & 345.6557 & 333.9167 & 122.5994 & 1.73419 \\
\hline 2928 & 52.7593 & 338.21 & 346.7376 & 334.4583 & 122.5994 & 1.73367 \\
\hline 2929 & 52.7763 & 342.2942 & 347.7617 & 335 & 122.5994 & 1.73315 \\
\hline 2930 & 52.7933 & 320.9969 & 348.4086 & 335.1875 & 122.5994 & 1.73263 \\
\hline 2931 & 52.8103 & 346.8957 & 349.2503 & 335.375 & 122.5994 & 1.73212 \\
\hline 2932 & 52.8273 & 365.7415 & 350.1927 & 335.5625 & 122.5994 & 1.7316 \\
\hline 2933 & 52.8443 & 326.2471 & 351.4731 & 335.75 & 122.5994 & 1.73108 \\
\hline 2934 & 52.8613 & 348.8588 & 353.1482 & 335.9375 & 122.5994 & 1.73056 \\
\hline 2935 & 52.8783 & 338.5639 & 355.1711 & 336.125 & 122.5994 & 1.73005 \\
\hline 2936 & 52.8953 & 322.1564 & 357.4457 & 336.3125 & 122.5994 & 1.72953 \\
\hline 2937 & 52.9123 & 369.8307 & 359.7065 & 336.5 & 122.5994 & 1.72902 \\
\hline 2938 & 52.9293 & 362.4683 & 361.6241 & 336.6875 & 122.5994 & 1.7285 \\
\hline
\end{tabular}




\begin{tabular}{|c|c|c|c|c|c|c|}
\hline 2939 & 52.9463 & 383.2834 & 362.8783 & 336.875 & 122.5994 & 1.72799 \\
\hline 2940 & 52.9633 & 377.5559 & 363.3097 & 337.0625 & 122.5994 & 1.72747 \\
\hline 2941 & 52.9803 & 357.0948 & 362.9183 & 337.25 & 122.5994 & 1.72696 \\
\hline 2942 & 52.9973 & 329.1438 & 361.9544 & 337.4375 & 122.5994 & 1.72644 \\
\hline 2943 & 53.0143 & 344.4559 & 360.8088 & 337.625 & 122.5994 & 1.72593 \\
\hline 2944 & 53.0313 & 347.2583 & 359.9165 & 337.8125 & 122.5994 & 1.72542 \\
\hline 2945 & 53.0483 & 354.0287 & 359.5139 & 338 & 122.5994 & 1.7249 \\
\hline 2946 & 53.0653 & 366.5787 & 359.5353 & 338.1875 & 122.5994 & 1.72439 \\
\hline 2947 & 53.0823 & 354.1141 & 359.8064 & 338.375 & 122.5994 & 1.72388 \\
\hline 2948 & 53.0993 & 364.0861 & 359.9667 & 338.5625 & 122.5994 & 1.72337 \\
\hline 2949 & 53.1163 & 373.5956 & 359.6802 & 338.75 & 122.5994 & 1.72286 \\
\hline 2950 & 53.1333 & 350.0657 & 358.6971 & 338.9375 & 122.5994 & 1.72234 \\
\hline 2951 & 53.1503 & 334.0323 & 357.1521 & 339.125 & 122.5994 & 1.72183 \\
\hline 2952 & 53.1673 & 361.0166 & 355.5753 & 339.3125 & 122.5994 & 1.72132 \\
\hline 2953 & 53.1843 & 355.0479 & 354.3828 & 339.5 & 122.5994 & 1.72081 \\
\hline 2954 & 53.2013 & 342.0273 & 353.9143 & 339.6875 & 122.5994 & 1.7203 \\
\hline 2955 & 53.2183 & 340.5906 & 354.2978 & 339.875 & 122.5994 & 1.71979 \\
\hline 2956 & 53.2353 & 340.0751 & 355.5968 & 340.0625 & 122.5994 & 1.71928 \\
\hline 2957 & 53.2523 & 352.1178 & 357.6053 & 340.25 & 122.5994 & 1.71878 \\
\hline 2958 & 53.2693 & 358.602 & 359.9695 & 340.4375 & 122.5994 & 1.71827 \\
\hline 2959 & 53.2863 & 352.4054 & 362.1675 & 340.625 & 122.5994 & 1.71776 \\
\hline 2960 & 53.3033 & 364.8563 & 363.7629 & 340.8125 & 122.5994 & 1.71725 \\
\hline 2961 & 53.3203 & 346.5825 & 364.4055 & 341 & 122.5994 & 1.71674 \\
\hline 2962 & 53.3373 & 334.9346 & 364.1552 & 341.1875 & 122.5994 & 1.71624 \\
\hline 2963 & 53.3543 & 348.4207 & 363.2982 & 341.375 & 122.5994 & 1.71573 \\
\hline 2964 & 53.3713 & 328.6222 & 362.2977 & 341.5625 & 122.5994 & 1.71522 \\
\hline 2965 & 53.3883 & 340.1605 & 361.5288 & 341.75 & 122.5994 & 1.71472 \\
\hline 2966 & 53.4053 & 340.1167 & 361.2381 & 341.9375 & 122.5994 & 1.71421 \\
\hline 2967 & 53.4223 & 359.9813 & 361.4816 & 342.125 & 122.5994 & 1.71371 \\
\hline 2968 & 53.4393 & 348.9653 & 362.1901 & 342.3125 & 122.5994 & 1.7132 \\
\hline 2969 & 53.4563 & 355.6851 & 363.1311 & 342.5 & 122.5994 & 1.7127 \\
\hline 2970 & 53.4733 & 353.8514 & 364.0152 & 342.6875 & 122.5994 & 1.71219 \\
\hline 2971 & 53.4903 & 320.4216 & 364.549 & 342.875 & 122.5994 & 1.71169 \\
\hline 2972 & 53.5073 & 310.2617 & 364.7392 & 343.0625 & 122.5994 & 1.71118 \\
\hline 2973 & 53.5243 & 309.8013 & 364.8208 & 343.25 & 122.5994 & 1.71068 \\
\hline 2974 & 53.5413 & 381.7434 & 364.8606 & 343.4375 & 122.5994 & 1.71018 \\
\hline 2975 & 53.5583 & 357.4605 & 364.327 & 343.625 & 122.5994 & 1.70968 \\
\hline 2976 & 53.5753 & 364.4891 & 362.8306 & 343.8125 & 122.5994 & 1.70917 \\
\hline 2977 & 53.5923 & 337.8983 & 360.8135 & 344 & 122.5994 & 1.70867 \\
\hline 2978 & 53.6093 & 317.9817 & 358.8651 & 344.0417 & 122.5994 & 1.70817 \\
\hline 2979 & 53.6263 & 338.2509 & 357.388 & 344.0833 & 122.5994 & 1.70767 \\
\hline 2980 & 53.6433 & 331.4803 & 356.4287 & 344.125 & 122.5994 & 1.70717 \\
\hline 2981 & 53.6603 & 306.571 & 355.9921 & 344.1667 & 122.5994 & 1.70667 \\
\hline 2982 & 53.6773 & 360.5171 & 356.1098 & 344.2083 & 122.5994 & 1.70617 \\
\hline 2983 & 53.6943 & 353.5706 & 356.4423 & 344.25 & 122.5994 & 1.70567 \\
\hline 2984 & 53.7113 & 336.9624 & 356.9979 & 344.2917 & 122.5994 & 1.70517 \\
\hline 2985 & 53.7283 & 342.9152 & 357.6281 & 344.3333 & 122.5994 & 1.70467 \\
\hline 2986 & 53.7453 & 332.8626 & 358.4948 & 344.375 & 122.5994 & 1.70417 \\
\hline 2987 & 53.7623 & 337.6962 & 359.3457 & 344.4167 & 122.5994 & 1.70367 \\
\hline
\end{tabular}




\begin{tabular}{|c|c|c|c|c|c|c|}
\hline 2988 & 53.7793 & 346.0167 & 359.3576 & 344.4583 & 122.5994 & 1.70317 \\
\hline 2989 & 53.7963 & 367.5719 & 358.1603 & 344.5 & 122.5994 & 1.70267 \\
\hline 2990 & 53.8133 & 345.9268 & 356.5484 & 344.5417 & 122.5994 & 1.70217 \\
\hline 2991 & 53.8303 & 352.2147 & 355.1623 & 344.5833 & 122.5994 & 1.70168 \\
\hline 2992 & 53.8473 & 360.8197 & 354.6259 & 344.625 & 122.5994 & 1.70118 \\
\hline 2993 & 53.8643 & 351.9937 & 354.9662 & 344.6667 & 122.5994 & 1.70068 \\
\hline 2994 & 53.8813 & 349.7366 & 356.018 & 344.7083 & 122.5994 & 1.70019 \\
\hline 2995 & 53.8983 & 338.874 & 357.4109 & 344.75 & 122.5994 & 1.69969 \\
\hline 2996 & 53.9153 & 327.2387 & 358.5611 & 344.7917 & 122.5994 & 1.69919 \\
\hline 2997 & 53.9323 & 334.5958 & 358.9637 & 344.8333 & 122.5994 & 1.6987 \\
\hline 2998 & 53.9493 & 338.5275 & 358.6664 & 344.875 & 122.5994 & 1.6982 \\
\hline 2999 & 53.9663 & 346.1322 & 358.0732 & 344.9167 & 122.5994 & 1.69771 \\
\hline 3000 & 53.9833 & 339.7866 & 357.3947 & 344.9583 & 122.5994 & 1.69721 \\
\hline 3001 & 54.0003 & 337.7264 & 356.6977 & 345 & 122.5994 & 1.69672 \\
\hline 3002 & 54.0173 & 361.058 & 356.9779 & 345.5553 & 122.5994 & 1.69623 \\
\hline 3003 & 54.0343 & 337.4219 & 357.8132 & 346.1107 & 122.5994 & 1.69573 \\
\hline 3004 & 54.0513 & 328.8327 & 359.1721 & 346.666 & 122.5994 & 1.69524 \\
\hline 3005 & 54.0683 & 345.5932 & 361.0866 & 347.2214 & 122.5994 & 1.69475 \\
\hline 3006 & 54.0853 & 357.1614 & 363.411 & 347.7767 & 122.5994 & 1.69425 \\
\hline 3007 & 54.1023 & 355.8404 & 366.1134 & 348.332 & 122.5994 & 1.69376 \\
\hline 3008 & 54.1193 & 345.6591 & 368.79 & 348.8874 & 122.5994 & 1.69327 \\
\hline 3009 & 54.1363 & 336.9108 & 371.0706 & 349.4427 & 122.5994 & 1.69278 \\
\hline 3010 & 54.1533 & 371.4356 & 372.071 & 349.998 & 122.5994 & 1.69229 \\
\hline 3011 & 54.1703 & 369.427 & 371.3687 & 350.5534 & 122.5994 & 1.6918 \\
\hline 3012 & 54.1873 & 347.055 & 369.6901 & 351.1087 & 122.5994 & 1.69131 \\
\hline 3013 & 54.2043 & 324.6683 & 368.107 & 351.6641 & 122.5994 & 1.69082 \\
\hline 3014 & 54.2213 & 357.1661 & 367.225 & 352.2194 & 122.5994 & 1.69033 \\
\hline 3015 & 54.2383 & 355.1921 & 368.0001 & 352.7747 & 122.5994 & 1.68984 \\
\hline 3016 & 54.2553 & 350.1376 & 368.5951 & 353.3301 & 122.5994 & 1.68935 \\
\hline 3017 & 54.2723 & 356.372 & 369.522 & 353.8854 & 122.5994 & 1.68886 \\
\hline 3018 & 54.2893 & 357.2576 & 370.3059 & 354.4408 & 122.5994 & 1.68837 \\
\hline 3019 & 54.3063 & 346.6447 & 370.5462 & 354.9961 & 122.5994 & 1.68788 \\
\hline 3020 & 54.3233 & 371.415 & 370.1021 & 355.5514 & 122.5994 & 1.68739 \\
\hline 3021 & 54.3403 & 361.2037 & 369.4861 & 356.1068 & 122.5994 & 1.6869 \\
\hline 3022 & 54.3573 & 331.3082 & 369.5691 & 356.6621 & 122.5994 & 1.68642 \\
\hline 3023 & 54.3743 & 365.4657 & 369.8693 & 357.2174 & 122.5994 & 1.68593 \\
\hline 3024 & 54.3913 & 395.716 & 370.8862 & 357.7728 & 122.5994 & 1.68544 \\
\hline 3025 & 54.4083 & 387.7965 & 372.725 & 358.3281 & 122.5994 & 1.68496 \\
\hline 3026 & 54.4253 & 379.2995 & 375.0975 & 358.5332 & 122.5994 & 1.68447 \\
\hline 3027 & 54.4423 & 328.7642 & 378.5758 & 358.7383 & 122.5994 & 1.68398 \\
\hline 3028 & 54.4593 & 339.9711 & 383.0999 & 358.9434 & 122.5994 & 1.6835 \\
\hline 3029 & 54.4763 & 354.9535 & 388.3873 & 359.1484 & 122.5994 & 1.68301 \\
\hline 3030 & 54.4933 & 385.4846 & 393.7707 & 359.3535 & 122.5994 & 1.68253 \\
\hline 3031 & 54.5103 & 391.3243 & 397.6981 & 359.5586 & 122.5994 & 1.68204 \\
\hline 3032 & 54.5273 & 385.521 & 399.8397 & 359.7637 & 122.5994 & 1.68156 \\
\hline 3033 & 54.5443 & 378.1745 & 400.5755 & 359.9688 & 122.5994 & 1.68108 \\
\hline 3034 & 54.5613 & 382.1917 & 400.0303 & 360.1738 & 122.5994 & 1.68059 \\
\hline 3035 & 54.5783 & 361.646 & 398.2077 & 360.3789 & 122.5994 & 1.68011 \\
\hline 3036 & 54.5953 & 341.0294 & 396.8734 & 360.584 & 122.5994 & 1.67963 \\
\hline
\end{tabular}




\begin{tabular}{|c|c|c|c|c|c|c|}
\hline 3037 & 54.6123 & 381.478 & 397.8757 & 360.7891 & 122.5994 & 1.67914 \\
\hline 3038 & 54.6293 & 380.1845 & 402.1817 & 360.9941 & 122.5994 & 1.67866 \\
\hline 3039 & 54.6463 & 361.3723 & 409.2079 & 361.1992 & 122.5994 & 1.67818 \\
\hline 3040 & 54.6633 & 345.1031 & 417.6346 & 361.4043 & 122.5994 & 1.6777 \\
\hline 3041 & 54.6803 & 375.2917 & 424.9884 & 361.6094 & 122.5994 & 1.67722 \\
\hline 3042 & 54.6973 & 377.2076 & 428.4501 & 361.8145 & 122.5994 & 1.67674 \\
\hline 3043 & 54.7143 & 360.5995 & 428.019 & 362.0195 & 122.5994 & 1.67625 \\
\hline 3044 & 54.7313 & 381.1824 & 424.6738 & 362.2246 & 122.5994 & 1.67577 \\
\hline 3045 & 54.7483 & 368.58 & 420.8372 & 362.4297 & 122.5994 & 1.67529 \\
\hline 3046 & 54.7653 & 386.765 & 418.5222 & 362.6348 & 122.5994 & 1.67481 \\
\hline 3047 & 54.7823 & 408.6641 & 419.3425 & 362.8398 & 122.5994 & 1.67433 \\
\hline 3048 & 54.7993 & 404.9281 & 424.301 & 363.0449 & 122.5994 & 1.67386 \\
\hline 3049 & 54.8163 & 447.1459 & 434.956 & 363.25 & 122.5994 & 1.67338 \\
\hline 3050 & 54.8333 & 465.7804 & 454.0367 & 363.4551 & 122.5994 & 1.6729 \\
\hline 3051 & 54.8503 & 503.5691 & 485.5167 & 363.6602 & 122.5994 & 1.67242 \\
\hline 3052 & 54.8673 & 536.5502 & 533.631 & 363.8652 & 122.5994 & 1.67194 \\
\hline 3053 & 54.8843 & 630.2979 & 596.8097 & 364.0703 & 122.5994 & 1.67146 \\
\hline 3054 & 54.9013 & 708.7455 & 662.7902 & 364.2754 & 122.5994 & 1.67099 \\
\hline 3055 & 54.9183 & 807.147 & 702.9797 & 364.4805 & 122.5994 & 1.67051 \\
\hline 3056 & 54.9353 & 735.4507 & 689.5993 & 364.6855 & 122.5994 & 1.67003 \\
\hline 3057 & 54.9523 & 646.5815 & 633.5616 & 364.8906 & 122.5994 & 1.66956 \\
\hline 3058 & 54.9693 & 506.5858 & 569.6135 & 365.0957 & 122.5994 & 1.66908 \\
\hline 3059 & 54.9863 & 470.8448 & 521.3303 & 365.3008 & 122.5994 & 1.6686 \\
\hline 3060 & 55.0033 & 456.877 & 498.0121 & 365.5059 & 122.5994 & 1.66813 \\
\hline 3061 & 55.0203 & 494.0146 & 499.4675 & 365.7109 & 122.5994 & 1.66765 \\
\hline 3062 & 55.0373 & 549.3777 & 518.9637 & 365.916 & 122.5994 & 1.66718 \\
\hline 3063 & 55.0543 & 599.4598 & 543.3781 & 366.1211 & 122.5994 & 1.6667 \\
\hline 3064 & 55.0713 & 583.083 & 553.5806 & 366.3262 & 122.5994 & 1.66623 \\
\hline 3065 & 55.0883 & 554.4432 & 538.3009 & 366.5313 & 122.5994 & 1.66576 \\
\hline 3066 & 55.1053 & 547.754 & 507.4338 & 366.7363 & 122.5994 & 1.66528 \\
\hline 3067 & 55.1223 & 469.145 & 476.6021 & 366.9414 & 122.5994 & 1.66481 \\
\hline 3068 & 55.1393 & 408.5566 & 453.7662 & 367.1465 & 122.5994 & 1.66434 \\
\hline 3069 & 55.1563 & 396.262 & 440.5036 & 367.3516 & 122.5994 & 1.66386 \\
\hline 3070 & 55.1733 & 420.7625 & 435.275 & 367.5566 & 122.5994 & 1.66339 \\
\hline 3071 & 55.1903 & 437.0988 & 434.1714 & 367.7617 & 122.5994 & 1.66292 \\
\hline 3072 & 55.2073 & 440.6374 & 434.7915 & 367.9668 & 122.5994 & 1.66245 \\
\hline 3073 & 55.2243 & 436.4591 & 435.4651 & 368.1719 & 122.5994 & 1.66197 \\
\hline 3074 & 55.2413 & 429.6583 & 435.6187 & 368.0267 & 122.5994 & 1.6615 \\
\hline 3075 & 55.2583 & 412.0558 & 436.5199 & 367.8815 & 122.5994 & 1.66103 \\
\hline 3076 & 55.2753 & 431.5352 & 439.5344 & 367.7363 & 122.5994 & 1.66056 \\
\hline 3077 & 55.2923 & 413.113 & 446.5611 & 367.5911 & 122.5994 & 1.66009 \\
\hline 3078 & 55.3093 & 454.6287 & 460.007 & 367.446 & 122.5994 & 1.65962 \\
\hline 3079 & 55.3263 & 465.8367 & 481.6378 & 367.3008 & 122.5994 & 1.65915 \\
\hline 3080 & 55.3433 & 512.3988 & 510.1374 & 367.1556 & 122.5994 & 1.65868 \\
\hline 3081 & 55.3603 & 569.8666 & 539.8172 & 367.0104 & 122.5994 & 1.65821 \\
\hline 3082 & 55.3773 & 556.8134 & 557.4146 & 366.8652 & 122.5994 & 1.65774 \\
\hline 3083 & 55.3943 & 504.6737 & 549.5881 & 366.7201 & 122.5994 & 1.65728 \\
\hline 3084 & 55.4113 & 445.2043 & 520.961 & 366.5749 & 122.5994 & 1.65681 \\
\hline 3085 & 55.4283 & 434.6212 & 488.0518 & 366.4297 & 122.5994 & 1.65634 \\
\hline
\end{tabular}




\begin{tabular}{|c|c|c|c|c|c|c|}
\hline 3086 & 55.4453 & 410.7226 & 462.0988 & 366.2845 & 122.5994 & 1.65587 \\
\hline 3087 & 55.4623 & 433.442 & 447.7011 & 366.1393 & 122.5994 & 1.65541 \\
\hline 3088 & 55.4793 & 450.0004 & 444.9535 & 365.9941 & 122.5994 & 1.65494 \\
\hline 3089 & 55.4963 & 449.1121 & 451.1159 & 365.849 & 122.5994 & 1.65447 \\
\hline 3090 & 55.5133 & 428.857 & 460.5229 & 365.7038 & 122.5994 & 1.65401 \\
\hline 3091 & 55.5303 & 428.3373 & 464.7437 & 365.5586 & 122.5994 & 1.65354 \\
\hline 3092 & 55.5473 & 412.1167 & 457.3816 & 365.4134 & 122.5994 & 1.65307 \\
\hline 3093 & 55.5643 & 409.0784 & 442.0215 & 365.2682 & 122.5994 & 1.65261 \\
\hline 3094 & 55.5813 & 408.7335 & 425.8961 & 365.123 & 122.5994 & 1.65214 \\
\hline 3095 & 55.5983 & 389.2916 & 412.8276 & 364.9779 & 122.5994 & 1.65168 \\
\hline 3096 & 55.6153 & 372.6518 & 403.8244 & 364.8327 & 122.5994 & 1.65121 \\
\hline 3097 & 55.6323 & 376.7489 & 398.7501 & 364.6875 & 122.5994 & 1.65075 \\
\hline 3098 & 55.6493 & 384.1232 & 396.1673 & 364.1172 & 122.5994 & 1.65028 \\
\hline 3099 & 55.6663 & 397.2636 & 394.5265 & 363.5469 & 122.5994 & 1.64982 \\
\hline 3100 & 55.6833 & 369.6356 & 394.6698 & 362.9766 & 122.5994 & 1.64936 \\
\hline 3101 & 55.7003 & 394.4841 & 395.9165 & 362.4063 & 122.5994 & 1.64889 \\
\hline 3102 & 55.7173 & 386.3464 & 398.7401 & 361.8359 & 122.5994 & 1.64843 \\
\hline 3103 & 55.7343 & 393.8994 & 403.528 & 361.2656 & 122.5994 & 1.64797 \\
\hline 3104 & 55.7513 & 393.963 & 409.9525 & 360.6953 & 122.5994 & 1.64751 \\
\hline 3105 & 55.7683 & 410.9736 & 416.4377 & 360.125 & 122.5994 & 1.64704 \\
\hline 3106 & 55.7853 & 422.7453 & 420.5166 & 359.5547 & 122.5994 & 1.64658 \\
\hline 3107 & 55.8023 & 404.2075 & 421.1855 & 358.9844 & 122.5994 & 1.64612 \\
\hline 3108 & 55.8193 & 389.826 & 421.3391 & 358.4141 & 122.5994 & 1.64566 \\
\hline 3109 & 55.8363 & 391.5888 & 422.5847 & 357.8438 & 122.5994 & 1.6452 \\
\hline 3110 & 55.8533 & 402.7047 & 425.5856 & 357.2734 & 122.5994 & 1.64474 \\
\hline 3111 & 55.8703 & 426.4812 & 429.7431 & 356.7031 & 122.5994 & 1.64428 \\
\hline 3112 & 55.8873 & 424.5784 & 433.9813 & 356.1328 & 122.5994 & 1.64382 \\
\hline 3113 & 55.9043 & 404.8121 & 436.867 & 355.5625 & 122.5994 & 1.64336 \\
\hline 3114 & 55.9213 & 404.0639 & 436.9666 & 354.9922 & 122.5994 & 1.6429 \\
\hline 3115 & 55.9383 & 412.1966 & 433.4794 & 354.4219 & 122.5994 & 1.64244 \\
\hline 3116 & 55.9553 & 439.5479 & 426.9998 & 353.8516 & 122.5994 & 1.64198 \\
\hline 3117 & 55.9723 & 380.388 & 419.9599 & 353.2813 & 122.5994 & 1.64152 \\
\hline 3118 & 55.9893 & 384.2112 & 413.5788 & 352.7109 & 122.5994 & 1.64107 \\
\hline 3119 & 56.0063 & 383.0997 & 408.3169 & 352.1406 & 122.5994 & 1.64061 \\
\hline 3120 & 56.0233 & 365.6804 & 403.8816 & 351.5703 & 122.5994 & 1.64015 \\
\hline 3121 & 56.0403 & 315.4422 & 399.685 & 351 & 122.5994 & 1.63969 \\
\hline 3122 & 56.0573 & 356.3906 & 395.6835 & 350.7708 & 122.5994 & 1.63924 \\
\hline 3123 & 56.0743 & 372.4543 & 391.2368 & 350.5417 & 122.5994 & 1.63878 \\
\hline 3124 & 56.0913 & 369.4707 & 386.4405 & 350.3125 & 122.5994 & 1.63832 \\
\hline 3125 & 56.1083 & 348.5011 & 381.2006 & 350.0833 & 122.5994 & 1.63787 \\
\hline 3126 & 56.1253 & 330.0725 & 376.63 & 349.8542 & 122.5994 & 1.63741 \\
\hline 3127 & 56.1423 & 370.543 & 372.4533 & 349.625 & 122.5994 & 1.63696 \\
\hline 3128 & 56.1593 & 365.8528 & 368.7509 & 349.3958 & 122.5994 & 1.6365 \\
\hline 3129 & 56.1763 & 369.7705 & 365.5958 & 349.1667 & 122.5994 & 1.63605 \\
\hline 3130 & 56.1933 & 368.8115 & 362.9489 & 348.9375 & 122.5994 & 1.63559 \\
\hline 3131 & 56.2103 & 366.2782 & 360.922 & 348.7083 & 122.5994 & 1.63514 \\
\hline 3132 & 56.2273 & 376.4102 & 359.1945 & 348.4792 & 122.5994 & 1.63468 \\
\hline 3133 & 56.2443 & 376.3239 & 358.0318 & 348.25 & 122.5994 & 1.63423 \\
\hline 3134 & 56.2613 & 384.4786 & 357.1366 & 348.0208 & 122.5994 & 1.63378 \\
\hline
\end{tabular}




\begin{tabular}{|c|c|c|c|c|c|c|}
\hline 3135 & 56.2783 & 387.4827 & 356.3856 & 347.7917 & 122.5994 & 1.63332 \\
\hline 3136 & 56.2953 & 371.0866 & 355.7405 & 347.5625 & 122.5994 & 1.63287 \\
\hline 3137 & 56.3123 & 368.5074 & 355.1707 & 347.3333 & 122.5994 & 1.63242 \\
\hline 3138 & 56.3293 & 357.4396 & 354.7012 & 347.1042 & 122.5994 & 1.63196 \\
\hline 3139 & 56.3463 & 327.2895 & 354.3098 & 346.875 & 122.5994 & 1.63151 \\
\hline 3140 & 56.3633 & 336.9586 & 353.9864 & 346.6458 & 122.5994 & 1.63106 \\
\hline 3141 & 56.3803 & 346.713 & 353.7175 & 346.4167 & 122.5994 & 1.63061 \\
\hline 3142 & 56.3973 & 358.3747 & 353.4761 & 346.1875 & 122.5994 & 1.63016 \\
\hline 3143 & 56.4143 & 363.224 & 353.2468 & 345.9583 & 122.5994 & 1.62971 \\
\hline 3144 & 56.4313 & 357.2576 & 353.1373 & 345.7292 & 122.5994 & 1.62926 \\
\hline 3145 & 56.4483 & 367.0641 & 353.2072 & 345.5 & 122.5994 & 1.62881 \\
\hline 3146 & 56.4653 & 335.3211 & 353.5178 & 345.2708 & 122.5994 & 1.62836 \\
\hline 3147 & 56.4823 & 344.6737 & 354.4283 & 345.0417 & 122.5994 & 1.62791 \\
\hline 3148 & 56.4993 & 357.1757 & 356.0465 & 344.8125 & 122.5994 & 1.62746 \\
\hline 3149 & 56.5163 & 343.5818 & 358.4323 & 344.5833 & 122.5994 & 1.62701 \\
\hline 3150 & 56.5333 & 308.8902 & 361.3522 & 344.3542 & 122.5994 & 1.62656 \\
\hline 3151 & 56.5503 & 332.6822 & 364.031 & 344.125 & 122.5994 & 1.62611 \\
\hline 3152 & 56.5673 & 348.1526 & 365.1196 & 343.8958 & 122.5994 & 1.62566 \\
\hline 3153 & 56.5843 & 371.5907 & 363.894 & 343.6667 & 122.5994 & 1.62521 \\
\hline 3154 & 56.6013 & 350.7622 & 361.2671 & 343.4375 & 122.5994 & 1.62477 \\
\hline 3155 & 56.6183 & 378.8654 & 358.6446 & 343.2083 & 122.5994 & 1.62432 \\
\hline 3156 & 56.6353 & 360.3897 & 356.9014 & 342.9792 & 122.5994 & 1.62387 \\
\hline 3157 & 56.6523 & 327.1095 & 356.2966 & 342.75 & 122.5994 & 1.62342 \\
\hline 3158 & 56.6693 & 307.5892 & 357.2051 & 342.5208 & 122.5994 & 1.62298 \\
\hline 3159 & 56.6863 & 329.9213 & 359.0811 & 342.2917 & 122.5994 & 1.62253 \\
\hline 3160 & 56.7033 & 356.3946 & 360.9642 & 342.0625 & 122.5994 & 1.62209 \\
\hline 3161 & 56.7203 & 353.5197 & 361.5902 & 341.8333 & 122.5994 & 1.62164 \\
\hline 3162 & 56.7373 & 356.9192 & 360.4008 & 341.6042 & 122.5994 & 1.62119 \\
\hline 3163 & 56.7543 & 332.1029 & 358.1977 & 341.375 & 122.5994 & 1.62075 \\
\hline 3164 & 56.7713 & 354.3987 & 356.0203 & 341.1458 & 122.5994 & 1.6203 \\
\hline 3165 & 56.7883 & 309.299 & 354.4769 & 340.9167 & 122.5994 & 1.61986 \\
\hline 3166 & 56.8053 & 347.0399 & 353.2669 & 340.6875 & 122.5994 & 1.61941 \\
\hline 3167 & 56.8223 & 356.3508 & 352.7006 & 340.4583 & 122.5994 & 1.61897 \\
\hline 3168 & 56.8393 & 344.4873 & 352.3521 & 340.2292 & 122.5994 & 1.61853 \\
\hline 3169 & 56.8563 & 319.289 & 351.9244 & 340 & 122.5994 & 1.61808 \\
\hline 3170 & 56.8733 & 348.2795 & 351.0317 & 339.6667 & 122.5994 & 1.61764 \\
\hline 3171 & 56.8903 & 342.2172 & 349.7812 & 339.3333 & 122.5994 & 1.6172 \\
\hline 3172 & 56.9073 & 342.2956 & 348.3938 & 339 & 122.5994 & 1.61675 \\
\hline 3173 & 56.9243 & 339.4694 & 347.127 & 338.6667 & 122.5994 & 1.61631 \\
\hline 3174 & 56.9413 & 330.2996 & 346.0663 & 338.3333 & 122.5994 & 1.61587 \\
\hline 3175 & 56.9583 & 323.388 & 345.2101 & 338 & 122.5994 & 1.61543 \\
\hline 3176 & 56.9753 & 321.2739 & 344.4619 & 337.6667 & 122.5994 & 1.61499 \\
\hline 3177 & 56.9923 & 338.5275 & 343.7631 & 337.3333 & 122.5994 & 1.61454 \\
\hline 3178 & 57.0093 & 333.3277 & 343.081 & 337 & 122.5994 & 1.6141 \\
\hline 3179 & 57.0263 & 335.1945 & 342.4348 & 336.6667 & 122.5994 & 1.61366 \\
\hline 3180 & 57.0433 & 351.9053 & 341.8641 & 336.3333 & 122.5994 & 1.61322 \\
\hline 3181 & 57.0603 & 329.9391 & 341.3837 & 336 & 122.5994 & 1.61278 \\
\hline 3182 & 57.0773 & 302.731 & 341.049 & 335.6667 & 122.5994 & 1.61234 \\
\hline 3183 & 57.0943 & 310.9328 & 340.9229 & 335.3333 & 122.5994 & 1.6119 \\
\hline
\end{tabular}




\begin{tabular}{|c|c|c|c|c|c|c|}
\hline 3184 & 57.1113 & 316.99 & 341.1091 & 335 & 122.5994 & 1.61146 \\
\hline 3185 & 57.1283 & 315.0625 & 341.8725 & 334.6667 & 122.5994 & 1.61102 \\
\hline 3186 & 57.1453 & 313.134 & 343.4857 & 334.3333 & 122.5994 & 1.61058 \\
\hline 3187 & 57.1623 & 305.8745 & 346.5887 & 334 & 122.5994 & 1.61015 \\
\hline 3188 & 57.1793 & 306.8077 & 351.8963 & 333.6667 & 122.5994 & 1.60971 \\
\hline 3189 & 57.1963 & 318.1874 & 359.9311 & 333.3333 & 122.5994 & 1.60927 \\
\hline 3190 & 57.2133 & 363.4049 & 370.4499 & 333 & 122.5994 & 1.60883 \\
\hline 3191 & 57.2303 & 354.2549 & 381.5498 & 332.6667 & 122.5994 & 1.60839 \\
\hline 3192 & 57.2473 & 332.4547 & 389.9508 & 332.3333 & 122.5994 & 1.60796 \\
\hline 3193 & 57.2643 & 341.2944 & 393.3427 & 332 & 122.5994 & 1.60752 \\
\hline 3194 & 57.2813 & 330.5227 & 390.3387 & 331.6667 & 122.5994 & 1.60708 \\
\hline 3195 & 57.2983 & 348.0577 & 381.445 & 331.3333 & 122.5994 & 1.60665 \\
\hline 3196 & 57.3153 & 359.9975 & 371.1218 & 331 & 122.5994 & 1.60621 \\
\hline 3197 & 57.3323 & 378.7048 & 363.3466 & 330.6667 & 122.5994 & 1.60578 \\
\hline 3198 & 57.3493 & 339.6724 & 359.8521 & 330.3333 & 122.5994 & 1.60534 \\
\hline 3199 & 57.3663 & 321.7536 & 360.4583 & 330 & 122.5994 & 1.6049 \\
\hline 3200 & 57.3833 & 338.5946 & 363.672 & 329.6667 & 122.5994 & 1.60447 \\
\hline 3201 & 57.4003 & 326.6665 & 367.1523 & 329.3333 & 122.5994 & 1.60403 \\
\hline 3202 & 57.4173 & 333.4815 & 369.0708 & 329 & 122.5994 & 1.6036 \\
\hline 3203 & 57.4343 & 325.1863 & 368.2293 & 328.6667 & 122.5994 & 1.60317 \\
\hline 3204 & 57.4513 & 357.8825 & 364.1057 & 328.3333 & 122.5994 & 1.60273 \\
\hline 3205 & 57.4683 & 329.7206 & 358.2291 & 328 & 122.5994 & 1.6023 \\
\hline 3206 & 57.4853 & 348.7677 & 352.4708 & 327.6667 & 122.5994 & 1.60186 \\
\hline 3207 & 57.5023 & 349.7366 & 347.7862 & 327.3333 & 122.5994 & 1.60143 \\
\hline 3208 & 57.5193 & 338.4893 & 344.4121 & 327 & 122.5994 & 1.601 \\
\hline 3209 & 57.5363 & 325.599 & 342.1171 & 326.6667 & 122.5994 & 1.60057 \\
\hline 3210 & 57.5533 & 385.1241 & 340.6768 & 326.3333 & 122.5994 & 1.60013 \\
\hline 3211 & 57.5703 & 325.0237 & 339.8329 & 326 & 122.5994 & 1.5997 \\
\hline 3212 & 57.5873 & 336.5995 & 339.2837 & 325.6667 & 122.5994 & 1.59927 \\
\hline 3213 & 57.6043 & 316.8096 & 338.8209 & 325.3333 & 122.5994 & 1.59884 \\
\hline 3214 & 57.6213 & 316.4142 & 338.1467 & 325 & 122.5994 & 1.59841 \\
\hline 3215 & 57.6383 & 319.4132 & 337.1966 & 324.6667 & 122.5994 & 1.59798 \\
\hline 3216 & 57.6553 & 349.6042 & 336.0707 & 324.3333 & 122.5994 & 1.59755 \\
\hline 3217 & 57.6723 & 337.0509 & 335.0045 & 324 & 122.5994 & 1.59711 \\
\hline 3218 & 57.6893 & 356.9611 & 333.7324 & 323.3333 & 122.5994 & 1.59668 \\
\hline 3219 & 57.7063 & 341.3338 & 332.8646 & 322.6667 & 122.5994 & 1.59625 \\
\hline 3220 & 57.7233 & 331.9367 & 332.4207 & 322 & 122.5994 & 1.59582 \\
\hline 3221 & 57.7403 & 317.3767 & 332.4249 & 321.3333 & 122.5994 & 1.5954 \\
\hline 3222 & 57.7573 & 326.4815 & 332.8753 & 320.6667 & 122.5994 & 1.59497 \\
\hline 3223 & 57.7743 & 326.4001 & 333.6039 & 320 & 122.5994 & 1.59454 \\
\hline 3224 & 57.7913 & 340.2187 & 334.3316 & 319.3333 & 122.5994 & 1.59411 \\
\hline 3225 & 57.8083 & 338.4033 & 334.6406 & 318.6667 & 122.5994 & 1.59368 \\
\hline 3226 & 57.8253 & 323.2335 & 334.1081 & 318 & 122.5994 & 1.59325 \\
\hline 3227 & 57.8423 & 337.6093 & 332.6383 & 317.3333 & 122.5994 & 1.59282 \\
\hline 3228 & 57.8593 & 314.3201 & 330.5832 & 316.6667 & 122.5994 & 1.5924 \\
\hline 3229 & 57.8763 & 312.6566 & 328.3476 & 316 & 122.5994 & 1.59197 \\
\hline 3230 & 57.8933 & 313.7102 & 326.3459 & 315.3333 & 122.5994 & 1.59154 \\
\hline 3231 & 57.9103 & 309.7236 & 324.7761 & 314.6667 & 122.5994 & 1.59112 \\
\hline 3232 & 57.9273 & 305.9939 & 323.6814 & 314 & 122.5994 & 1.59069 \\
\hline
\end{tabular}




\begin{tabular}{|c|c|c|c|c|c|c|}
\hline 3233 & 57.9443 & 320.5615 & 322.9551 & 313.3333 & 122.5994 & 1.59026 \\
\hline 3234 & 57.9613 & 330.3437 & 322.1933 & 312.6667 & 122.5994 & 1.58984 \\
\hline 3235 & 57.9783 & 313.6821 & 321.4595 & 312 & 122.5994 & 1.58941 \\
\hline 3236 & 57.9953 & 332.5082 & 320.4095 & 311.3333 & 122.5994 & 1.58899 \\
\hline 3237 & 58.0123 & 318.1461 & 319.114 & 310.6667 & 122.5994 & 1.58856 \\
\hline 3238 & 58.0293 & 322.3378 & 317.9254 & 310 & 122.5994 & 1.58814 \\
\hline 3239 & 58.0463 & 308.7086 & 316.7901 & 309.3333 & 122.5994 & 1.58771 \\
\hline 3240 & 58.0633 & 288.8076 & 315.9633 & 308.6667 & 122.5994 & 1.58729 \\
\hline 3241 & 58.0803 & 300.2929 & 315.5128 & 308 & 122.5994 & 1.58686 \\
\hline 3242 & 58.0973 & 330.0179 & 316.6928 & 308.625 & 122.5994 & 1.58644 \\
\hline 3243 & 58.1143 & 322.1121 & 318.127 & 309.25 & 122.5994 & 1.58602 \\
\hline 3244 & 58.1313 & 316.8096 & 319.6051 & 309.875 & 122.5994 & 1.58559 \\
\hline 3245 & 58.1483 & 294.6424 & 321.159 & 310.5 & 122.5994 & 1.58517 \\
\hline 3246 & 58.1653 & 320.6428 & 322.765 & 311.125 & 122.5994 & 1.58475 \\
\hline 3247 & 58.1823 & 327.6537 & 324.7342 & 311.75 & 122.5994 & 1.58432 \\
\hline 3248 & 58.1993 & 327.5786 & 327.2411 & 312.375 & 122.5994 & 1.5839 \\
\hline 3249 & 58.2163 & 327.6537 & 330.1644 & 313 & 122.5994 & 1.58348 \\
\hline 3250 & 58.2333 & 311.3992 & 333.4709 & 313.625 & 122.5994 & 1.58306 \\
\hline 3251 & 58.2503 & 316.2993 & 336.7804 & 314.25 & 122.5994 & 1.58264 \\
\hline 3252 & 58.2673 & 316.8822 & 339.0675 & 314.875 & 122.5994 & 1.58221 \\
\hline 3253 & 58.2843 & 334.3343 & 339.2303 & 315.5 & 122.5994 & 1.58179 \\
\hline 3254 & 58.3013 & 317.0192 & 337.5702 & 316.125 & 122.5994 & 1.58137 \\
\hline 3255 & 58.3183 & 313.0658 & 335.6277 & 316.75 & 122.5994 & 1.58095 \\
\hline 3256 & 58.3353 & 300.2076 & 334.5682 & 317.375 & 122.5994 & 1.58053 \\
\hline 3257 & 58.3523 & 322.9582 & 334.7824 & 318 & 122.5994 & 1.58011 \\
\hline 3258 & 58.3693 & 307.9695 & 336.2434 & 318.625 & 122.5994 & 1.57969 \\
\hline 3259 & 58.3863 & 325.4424 & 338.4762 & 319.25 & 122.5994 & 1.57927 \\
\hline 3260 & 58.4033 & 309.3311 & 340.7851 & 319.875 & 122.5994 & 1.57885 \\
\hline 3261 & 58.4203 & 328.1349 & 342.2723 & 320.5 & 122.5994 & 1.57843 \\
\hline 3262 & 58.4373 & 312.6666 & 342.2592 & 321.125 & 122.5994 & 1.57802 \\
\hline 3263 & 58.4543 & 327.6537 & 340.8666 & 321.75 & 122.5994 & 1.5776 \\
\hline 3264 & 58.4713 & 327.5786 & 339.0452 & 322.375 & 122.5994 & 1.57718 \\
\hline 3265 & 58.4883 & 331.8599 & 337.688 & 323 & 122.5994 & 1.57676 \\
\hline 3266 & 58.5053 & 345.9268 & 337.0898 & 323.625 & 122.5994 & 1.57634 \\
\hline 3267 & 58.5223 & 340.9345 & 337.2472 & 324.25 & 122.5994 & 1.57593 \\
\hline 3268 & 58.5393 & 323.4477 & 338.1457 & 324.875 & 122.5994 & 1.57551 \\
\hline 3269 & 58.5563 & 312.1475 & 339.3153 & 325.5 & 122.5994 & 1.57509 \\
\hline 3270 & 58.5733 & 317.0353 & 340.3859 & 326.125 & 122.5994 & 1.57468 \\
\hline 3271 & 58.5903 & 327.3289 & 341.2226 & 326.75 & 122.5994 & 1.57426 \\
\hline 3272 & 58.6073 & 338.7729 & 342.0131 & 327.375 & 122.5994 & 1.57384 \\
\hline 3273 & 58.6243 & 349.2514 & 343.0983 & 328 & 122.5994 & 1.57343 \\
\hline 3274 & 58.6413 & 329.6108 & 344.8622 & 328.625 & 122.5994 & 1.57301 \\
\hline 3275 & 58.6583 & 332.5912 & 347.5236 & 329.25 & 122.5994 & 1.5726 \\
\hline 3276 & 58.6753 & 318.7165 & 351.2651 & 329.875 & 122.5994 & 1.57218 \\
\hline 3277 & 58.6923 & 331.0756 & 356.041 & 330.5 & 122.5994 & 1.57177 \\
\hline 3278 & 58.7093 & 344.0391 & 361.2922 & 331.125 & 122.5994 & 1.57135 \\
\hline 3279 & 58.7263 & 344.2637 & 366.0487 & 331.75 & 122.5994 & 1.57094 \\
\hline 3280 & 58.7433 & 350.055 & 369.025 & 332.375 & 122.5994 & 1.57052 \\
\hline 3281 & 58.7603 & 349.1177 & 369.4035 & 333 & 122.5994 & 1.57011 \\
\hline
\end{tabular}




\begin{tabular}{|c|c|c|c|c|c|c|}
\hline 3282 & 58.7773 & 361.7907 & 367.9399 & 333.625 & 122.5994 & 1.5697 \\
\hline 3283 & 58.7943 & 340.3526 & 365.9238 & 334.25 & 122.5994 & 1.56928 \\
\hline 3284 & 58.8113 & 327.3627 & 364.3578 & 334.875 & 122.5994 & 1.56887 \\
\hline 3285 & 58.8283 & 338.4689 & 363.3217 & 335.5 & 122.5994 & 1.56846 \\
\hline 3286 & 58.8453 & 349.8362 & 362.5429 & 336.125 & 122.5994 & 1.56804 \\
\hline 3287 & 58.8623 & 335.3407 & 362.0727 & 336.75 & 122.5994 & 1.56763 \\
\hline 3288 & 58.8793 & 322.3661 & 362.0383 & 337.375 & 122.5994 & 1.56722 \\
\hline 3289 & 58.8963 & 345.0799 & 362.1189 & 338 & 122.5994 & 1.56681 \\
\hline 3290 & 58.9133 & 344.8231 & 361.6161 & 338.5 & 122.5994 & 1.5664 \\
\hline 3291 & 58.9303 & 339.3854 & 360.4438 & 339 & 122.5994 & 1.56599 \\
\hline 3292 & 58.9473 & 323.8244 & 358.9601 & 339.5 & 122.5994 & 1.56557 \\
\hline 3293 & 58.9643 & 344.7259 & 357.528 & 340 & 122.5994 & 1.56516 \\
\hline 3294 & 58.9813 & 349.7366 & 356.208 & 340.5 & 122.5994 & 1.56475 \\
\hline 3295 & 58.9983 & 336.7127 & 354.7788 & 341 & 122.5994 & 1.56434 \\
\hline 3296 & 59.0153 & 361.7499 & 353.3687 & 341.5 & 122.5994 & 1.56393 \\
\hline 3297 & 59.0323 & 375.3739 & 352.2025 & 342 & 122.5994 & 1.56352 \\
\hline 3298 & 59.0493 & 320.4304 & 351.5445 & 342.5 & 122.5994 & 1.56311 \\
\hline 3299 & 59.0663 & 366.6476 & 351.4901 & 343 & 122.5994 & 1.5627 \\
\hline 3300 & 59.0833 & 339.0618 & 352.0559 & 343.5 & 122.5994 & 1.56229 \\
\hline 3301 & 59.1003 & 368.4665 & 353.1676 & 344 & 122.5994 & 1.56189 \\
\hline 3302 & 59.1173 & 330.8292 & 354.8301 & 344.5 & 122.5994 & 1.56148 \\
\hline 3303 & 59.1343 & 315.3139 & 357.0751 & 345 & 122.5994 & 1.56107 \\
\hline 3304 & 59.1513 & 356.4087 & 359.9202 & 345.5 & 122.5994 & 1.56066 \\
\hline 3305 & 59.1683 & 346.3412 & 363.6009 & 346 & 122.5994 & 1.56025 \\
\hline 3306 & 59.1853 & 347.6038 & 368.0049 & 346.5 & 122.5994 & 1.55984 \\
\hline 3307 & 59.2023 & 337.7978 & 372.823 & 347 & 122.5994 & 1.55944 \\
\hline 3308 & 59.2193 & 376.0761 & 377.5011 & 347.5 & 122.5994 & 1.55903 \\
\hline 3309 & 59.2363 & 355.4043 & 381.4167 & 348 & 122.5994 & 1.55862 \\
\hline 3310 & 59.2533 & 362.6143 & 383.7801 & 348.5 & 122.5994 & 1.55822 \\
\hline 3311 & 59.2703 & 355.894 & 384.0629 & 349 & 122.5994 & 1.55781 \\
\hline 3312 & 59.2873 & 361.2228 & 382.8861 & 349.5 & 122.5994 & 1.5574 \\
\hline 3313 & 59.3043 & 349.6565 & 381.3864 & 350 & 122.5994 & 1.557 \\
\hline 3314 & 59.3213 & 346.5809 & 382.3981 & 350.3958 & 122.5994 & 1.55659 \\
\hline 3315 & 59.3383 & 331.9039 & 381.5453 & 350.7917 & 122.5994 & 1.55619 \\
\hline 3316 & 59.3553 & 323.8985 & 381.1414 & 351.1875 & 122.5994 & 1.55578 \\
\hline 3317 & 59.3723 & 341.1805 & 381.0886 & 351.5833 & 122.5994 & 1.55538 \\
\hline 3318 & 59.3893 & 351.4833 & 381.1729 & 351.9792 & 122.5994 & 1.55497 \\
\hline 3319 & 59.4063 & 346.5776 & 381.0703 & 352.375 & 122.5994 & 1.55457 \\
\hline 3320 & 59.4233 & 337.6686 & 380.3174 & 352.7708 & 122.5994 & 1.55416 \\
\hline 3321 & 59.4403 & 350.7625 & 378.8434 & 353.1667 & 122.5994 & 1.55376 \\
\hline 3322 & 59.4573 & 337.2012 & 376.9502 & 353.5625 & 122.5994 & 1.55336 \\
\hline 3323 & 59.4743 & 334.6999 & 376.455 & 353.9583 & 122.5994 & 1.55295 \\
\hline 3324 & 59.4913 & 353.6047 & 375.0782 & 354.3542 & 122.5994 & 1.55255 \\
\hline 3325 & 59.5083 & 347.2659 & 373.983 & 354.75 & 122.5994 & 1.55215 \\
\hline 3326 & 59.5253 & 314.1918 & 373.2096 & 355.1458 & 122.5994 & 1.55174 \\
\hline 3327 & 59.5423 & 331.2712 & 372.7725 & 355.5417 & 122.5994 & 1.55134 \\
\hline 3328 & 59.5593 & 344.7078 & 372.7091 & 355.9375 & 122.5994 & 1.55094 \\
\hline 3329 & 59.5763 & 347.6256 & 372.7995 & 356.3333 & 122.5994 & 1.55054 \\
\hline 3330 & 59.5933 & 351.0525 & 373.1542 & 356.7292 & 122.5994 & 1.55014 \\
\hline
\end{tabular}




\begin{tabular}{|c|c|c|c|c|c|c|}
\hline 3331 & 59.6103 & 369.5788 & 373.6304 & 357.125 & 122.5994 & 1.54973 \\
\hline 3332 & 59.6273 & 344.9076 & 374.2272 & 357.5208 & 122.5994 & 1.54933 \\
\hline 3333 & 59.6443 & 350.7714 & 374.9688 & 357.9167 & 122.5994 & 1.54893 \\
\hline 3334 & 59.6613 & 388.8026 & 375.8748 & 358.3125 & 122.5994 & 1.54853 \\
\hline 3335 & 59.6783 & 385.4415 & 377.0255 & 358.7083 & 122.5994 & 1.54813 \\
\hline 3336 & 59.6953 & 377.3566 & 378.4406 & 359.1042 & 122.5994 & 1.54773 \\
\hline 3337 & 59.7123 & 385.453 & 380.2017 & 359.5 & 122.5994 & 1.54733 \\
\hline 3338 & 59.7293 & 381.8704 & 381.8919 & 359.4479 & 122.5994 & 1.54693 \\
\hline 3339 & 59.7463 & 361.29 & 383.9813 & 359.3958 & 122.5994 & 1.54653 \\
\hline 3340 & 59.7633 & 373.3145 & 386.5176 & 359.3438 & 122.5994 & 1.54613 \\
\hline 3341 & 59.7803 & 373.0649 & 389.5834 & 359.2917 & 122.5994 & 1.54573 \\
\hline 3342 & 59.7973 & 411.1383 & 393.3489 & 359.2396 & 122.5994 & 1.54533 \\
\hline 3343 & 59.8143 & 390.7426 & 397.9417 & 359.1875 & 122.5994 & 1.54494 \\
\hline 3344 & 59.8313 & 406.5842 & 403.7581 & 359.1354 & 122.5994 & 1.54454 \\
\hline 3345 & 59.8483 & 443.4262 & 411.4602 & 359.0833 & 122.5994 & 1.54414 \\
\hline 3346 & 59.8653 & 441.0753 & 422.4236 & 359.0313 & 122.5994 & 1.54374 \\
\hline 3347 & 59.8823 & 479.3123 & 439.6455 & 358.9792 & 122.5994 & 1.54334 \\
\hline 3348 & 59.8993 & 488.8327 & 469.2332 & 358.9271 & 122.5994 & 1.54295 \\
\hline 3349 & 59.9163 & 525.4332 & 521.8507 & 358.875 & 122.5994 & 1.54255 \\
\hline 3350 & 59.9333 & 673.8086 & 611.694 & 358.8229 & 122.5994 & 1.54215 \\
\hline 3351 & 59.9503 & 739.954 & 750.76 & 358.7708 & 122.5994 & 1.54176 \\
\hline 3352 & 59.9673 & 948.4308 & 937.1376 & 358.7188 & 122.5994 & 1.54136 \\
\hline 3353 & 59.9843 & 1120.446 & 1139.582 & 358.6667 & 122.5994 & 1.54096 \\
\hline 3354 & 60.0013 & 1202.255 & 1280.544 & 358.6146 & 122.5994 & 1.54057 \\
\hline 3355 & 60.0183 & 1154.239 & 1270.45 & 358.5625 & 122.5994 & 1.54017 \\
\hline 3356 & 60.0353 & 942.3266 & 1120.318 & 358.5104 & 122.5994 & 1.53978 \\
\hline 3357 & 60.0523 & 777.0811 & 927.6473 & 358.4583 & 122.5994 & 1.53938 \\
\hline 3358 & 60.0693 & 646.0602 & 766.4674 & 358.4063 & 122.5994 & 1.53899 \\
\hline 3359 & 60.0863 & 583.324 & 668.3339 & 358.3542 & 122.5994 & 1.53859 \\
\hline 3360 & 60.1033 & 594.4402 & 638.3143 & 358.3021 & 122.5994 & 1.5382 \\
\hline 3361 & 60.1203 & 624.5929 & 668.1116 & 358.25 & 122.5994 & 1.5378 \\
\hline 3362 & 60.1373 & 690.856 & 739.1639 & 358.1979 & 122.5994 & 1.53741 \\
\hline 3363 & 60.1543 & 740.0317 & 819.0466 & 358.1458 & 122.5994 & 1.53701 \\
\hline 3364 & 60.1713 & 748.7228 & 857.2979 & 358.0938 & 122.5994 & 1.53662 \\
\hline 3365 & 60.1883 & 703.2474 & 817.0475 & 358.0417 & 122.5994 & 1.53623 \\
\hline 3366 & 60.2053 & 619.1598 & 721.9629 & 357.9896 & 122.5994 & 1.53583 \\
\hline 3367 & 60.2223 & 535.2688 & 619.0616 & 357.9375 & 122.5994 & 1.53544 \\
\hline 3368 & 60.2393 & 437.0343 & 535.0122 & 357.8854 & 122.5994 & 1.53505 \\
\hline 3369 & 60.2563 & 435.6336 & 476.9613 & 357.8333 & 122.5994 & 1.53466 \\
\hline 3370 & 60.2733 & 437.5072 & 441.0731 & 357.7813 & 122.5994 & 1.53426 \\
\hline 3371 & 60.2903 & 403.9167 & 420.3329 & 357.7292 & 122.5994 & 1.53387 \\
\hline 3372 & 60.3073 & 373.4965 & 408.2952 & 357.6771 & 122.5994 & 1.53348 \\
\hline 3373 & 60.3243 & 410.6081 & 400.8137 & 357.625 & 122.5994 & 1.53309 \\
\hline 3374 & 60.3413 & 418.2353 & 395.7472 & 357.5729 & 122.5994 & 1.5327 \\
\hline 3375 & 60.3583 & 399.2269 & 392.0665 & 357.5208 & 122.5994 & 1.53231 \\
\hline 3376 & 60.3753 & 384.5089 & 389.2542 & 357.4688 & 122.5994 & 1.53192 \\
\hline 3377 & 60.3923 & 362.6922 & 387.0214 & 357.4167 & 122.5994 & 1.53153 \\
\hline 3378 & 60.4093 & 372.4762 & 385.2838 & 357.3646 & 122.5994 & 1.53113 \\
\hline 3379 & 60.4263 & 382.88 & 384.16 & 357.3125 & 122.5994 & 1.53074 \\
\hline
\end{tabular}




\begin{tabular}{|c|c|c|c|c|c|c|}
\hline 3380 & 60.4433 & 362.5966 & 383.475 & 357.2604 & 122.5994 & 1.53035 \\
\hline 3381 & 60.4603 & 364.4264 & 383.1827 & 357.2083 & 122.5994 & 1.52997 \\
\hline 3382 & 60.4773 & 354.4596 & 383 & 357.1563 & 122.5994 & 1.52958 \\
\hline 3383 & 60.4943 & 355.5127 & 382.5398 & 357.1042 & 122.5994 & 1.52919 \\
\hline 3384 & 60.5113 & 363.176 & 381.536 & 357.0521 & 122.5994 & 1.5288 \\
\hline 3385 & 60.5283 & 367.9654 & 380.097 & 357 & 122.5994 & 1.52841 \\
\hline 3386 & 60.5453 & 346.6328 & 378.6005 & 357 & 122.5994 & 1.52802 \\
\hline 3387 & 60.5623 & 342.2956 & 377.3854 & 357 & 122.5994 & 1.52763 \\
\hline 3388 & 60.5793 & 319.9669 & 376.5867 & 357 & 122.5994 & 1.52724 \\
\hline 3389 & 60.5963 & 346.2784 & 376.4506 & 357 & 122.5994 & 1.52686 \\
\hline 3390 & 60.6133 & 326.1347 & 376.956 & 357 & 122.5994 & 1.52647 \\
\hline 3391 & 60.6303 & 365.9368 & 378.1108 & 357 & 122.5994 & 1.52608 \\
\hline 3392 & 60.6473 & 354.0699 & 379.91 & 357 & 122.5994 & 1.52569 \\
\hline 3393 & 60.6643 & 357.1128 & 382.2144 & 357 & 122.5994 & 1.52531 \\
\hline 3394 & 60.6813 & 353.5413 & 385.2689 & 357 & 122.5994 & 1.52492 \\
\hline 3395 & 60.6983 & 364.2814 & 389.4541 & 357 & 122.5994 & 1.52453 \\
\hline 3396 & 60.7153 & 347.6463 & 394.9899 & 357 & 122.5994 & 1.52415 \\
\hline 3397 & 60.7323 & 378.6179 & 399.5021 & 357 & 122.5994 & 1.52376 \\
\hline 3398 & 60.7493 & 363.3618 & 406.2599 & 357 & 122.5994 & 1.52338 \\
\hline 3399 & 60.7663 & 413.4519 & 411.7625 & 357 & 122.5994 & 1.52299 \\
\hline 3400 & 60.7833 & 334.6189 & 415.2836 & 357 & 122.5994 & 1.52261 \\
\hline 3401 & 60.8003 & 379.0948 & 417.0911 & 357 & 122.5994 & 1.52222 \\
\hline 3402 & 60.8173 & 375.911 & 416.9979 & 357 & 122.5994 & 1.52184 \\
\hline 3403 & 60.8343 & 386.6881 & 414.7355 & 357 & 122.5994 & 1.52145 \\
\hline 3404 & 60.8513 & 364.775 & 411.0556 & 357 & 122.5994 & 1.52107 \\
\hline 3405 & 60.8683 & 358.5184 & 407.2329 & 357 & 122.5994 & 1.52068 \\
\hline 3406 & 60.8853 & 347.4496 & 404.2867 & 357 & 122.5994 & 1.5203 \\
\hline 3407 & 60.9023 & 364.9098 & 401.4909 & 357 & 122.5994 & 1.51992 \\
\hline 3408 & 60.9193 & 344.8708 & 400.621 & 357 & 122.5994 & 1.51953 \\
\hline 3409 & 60.9363 & 345.8813 & 399.9503 & 357 & 122.5994 & 1.51915 \\
\hline 3410 & 60.9533 & 364.2113 & 399.292 & 357.2813 & 122.5994 & 1.51877 \\
\hline 3411 & 60.9703 & 389.9921 & 398.154 & 357.5625 & 122.5994 & 1.51838 \\
\hline 3412 & 60.9873 & 387.6706 & 396.3272 & 357.8438 & 122.5994 & 1.518 \\
\hline 3413 & 61.0043 & 367.1051 & 394.0953 & 358.125 & 122.5994 & 1.51762 \\
\hline 3414 & 61.0213 & 368.1158 & 391.4673 & 358.4063 & 122.5994 & 1.51724 \\
\hline 3415 & 61.0383 & 372.5397 & 389.0529 & 358.6875 & 122.5994 & 1.51685 \\
\hline 3416 & 61.0553 & 350.9983 & 387.1095 & 358.9688 & 122.5994 & 1.51647 \\
\hline 3417 & 61.0723 & 342.4455 & 385.7267 & 359.25 & 122.5994 & 1.51609 \\
\hline 3418 & 61.0893 & 363.1324 & 384.8575 & 359.5313 & 122.5994 & 1.51571 \\
\hline 3419 & 61.1063 & 362.4031 & 384.4558 & 359.8125 & 122.5994 & 1.51533 \\
\hline 3420 & 61.1233 & 348.817 & 384.4555 & 360.0938 & 122.5994 & 1.51495 \\
\hline 3421 & 61.1403 & 357.6721 & 384.4654 & 360.375 & 122.5994 & 1.51457 \\
\hline 3422 & 61.1573 & 366.3632 & 384.426 & 360.6563 & 122.5994 & 1.51419 \\
\hline 3423 & 61.1743 & 374.4822 & 384.5126 & 360.9375 & 122.5994 & 1.51381 \\
\hline 3424 & 61.1913 & 375.1686 & 384.7453 & 361.2188 & 122.5994 & 1.51343 \\
\hline 3425 & 61.2083 & 372.5397 & 385.5365 & 361.5 & 122.5994 & 1.51305 \\
\hline 3426 & 61.2253 & 348.9342 & 387.1049 & 361.7813 & 122.5994 & 1.51267 \\
\hline 3427 & 61.2423 & 362.8598 & 389.8528 & 362.0625 & 122.5994 & 1.51229 \\
\hline 3428 & 61.2593 & 363.9946 & 394.1529 & 362.3438 & 122.5994 & 1.51191 \\
\hline
\end{tabular}




\begin{tabular}{|c|c|c|c|c|c|c|}
\hline 3429 & 61.2763 & 378.6014 & 400.1147 & 362.625 & 122.5994 & 1.51153 \\
\hline 3430 & 61.2933 & 363.1201 & 407.2274 & 362.9063 & 122.5994 & 1.51115 \\
\hline 3431 & 61.3103 & 369.4113 & 413.6399 & 363.1875 & 122.5994 & 1.51078 \\
\hline 3432 & 61.3273 & 369.3734 & 416.3651 & 363.4688 & 122.5994 & 1.5104 \\
\hline 3433 & 61.3443 & 381.7008 & 413.969 & 363.75 & 122.5994 & 1.51002 \\
\hline 3434 & 61.3613 & 394.4671 & 408.2489 & 363.5313 & 122.5994 & 1.50964 \\
\hline 3435 & 61.3783 & 378.5445 & 402.6677 & 363.3125 & 122.5994 & 1.50926 \\
\hline 3436 & 61.3953 & 348.7111 & 399.1232 & 363.0938 & 122.5994 & 1.50889 \\
\hline 3437 & 61.4123 & 377.8946 & 398.4656 & 362.875 & 122.5994 & 1.50851 \\
\hline 3438 & 61.4293 & 358.2936 & 400.9154 & 362.6563 & 122.5994 & 1.50813 \\
\hline 3439 & 61.4463 & 368.3794 & 406.2451 & 362.4375 & 122.5994 & 1.50776 \\
\hline 3440 & 61.4633 & 407.7862 & 414.0376 & 362.2188 & 122.5994 & 1.50738 \\
\hline 3441 & 61.4803 & 372.8947 & 422.8396 & 362 & 122.5994 & 1.50701 \\
\hline 3442 & 61.4973 & 399.9616 & 430.2956 & 361.7813 & 122.5994 & 1.50663 \\
\hline 3443 & 61.5143 & 400.0533 & 434.0083 & 361.5625 & 122.5994 & 1.50625 \\
\hline 3444 & 61.5313 & 397.1289 & 432.4377 & 361.3438 & 122.5994 & 1.50588 \\
\hline 3445 & 61.5483 & 367.2366 & 425.2643 & 361.125 & 122.5994 & 1.5055 \\
\hline 3446 & 61.5653 & 388.5409 & 415.1333 & 360.9063 & 122.5994 & 1.50513 \\
\hline 3447 & 61.5823 & 386.9524 & 406.3112 & 360.6875 & 122.5994 & 1.50475 \\
\hline 3448 & 61.5993 & 368.4474 & 400.4906 & 360.4688 & 122.5994 & 1.50438 \\
\hline 3449 & 61.6163 & 418.9878 & 398.1521 & 360.25 & 122.5994 & 1.504 \\
\hline 3450 & 61.6333 & 394.4019 & 399.1334 & 360.0313 & 122.5994 & 1.50363 \\
\hline 3451 & 61.6503 & 398.3496 & 402.7001 & 359.8125 & 122.5994 & 1.50326 \\
\hline 3452 & 61.6673 & 426.4793 & 407.907 & 359.5938 & 122.5994 & 1.50288 \\
\hline 3453 & 61.6843 & 410.2274 & 413.4172 & 359.375 & 122.5994 & 1.50251 \\
\hline 3454 & 61.7013 & 408.9897 & 417.4577 & 359.1563 & 122.5994 & 1.50214 \\
\hline 3455 & 61.7183 & 401.0424 & 418.8149 & 358.9375 & 122.5994 & 1.50176 \\
\hline 3456 & 61.7353 & 413.3512 & 418.1106 & 358.7188 & 122.5994 & 1.50139 \\
\hline 3457 & 61.7523 & 402.9504 & 417.2411 & 358.5 & 122.5994 & 1.50102 \\
\hline 3458 & 61.7693 & 404.9315 & 417.1566 & 358.2813 & 122.5994 & 1.50065 \\
\hline 3459 & 61.7863 & 400.0533 & 418.4051 & 358.0625 & 122.5994 & 1.50027 \\
\hline 3460 & 61.8033 & 405.0978 & 420.8235 & 357.8438 & 122.5994 & 1.4999 \\
\hline 3461 & 61.8203 & 397.8419 & 423.0708 & 357.625 & 122.5994 & 1.49953 \\
\hline 3462 & 61.8373 & 382.1302 & 422.4679 & 357.4063 & 122.5994 & 1.49916 \\
\hline 3463 & 61.8543 & 399.7814 & 417.4917 & 357.1875 & 122.5994 & 1.49879 \\
\hline 3464 & 61.8713 & 403.8909 & 410.1072 & 356.9688 & 122.5994 & 1.49842 \\
\hline 3465 & 61.8883 & 407.0211 & 402.9646 & 356.75 & 122.5994 & 1.49805 \\
\hline 3466 & 61.9053 & 417.9446 & 397.5755 & 356.5313 & 122.5994 & 1.49768 \\
\hline 3467 & 61.9223 & 397.8008 & 394.0772 & 356.3125 & 122.5994 & 1.49731 \\
\hline 3468 & 61.9393 & 372.5735 & 392.2315 & 356.0938 & 122.5994 & 1.49694 \\
\hline 3469 & 61.9563 & 376.8272 & 391.6139 & 355.875 & 122.5994 & 1.49657 \\
\hline 3470 & 61.9733 & 394.2599 & 391.7368 & 355.6563 & 122.5994 & 1.4962 \\
\hline 3471 & 61.9903 & 366.7683 & 391.7854 & 355.4375 & 122.5994 & 1.49583 \\
\hline 3472 & 62.0073 & 384.0192 & 390.457 & 355.2188 & 122.5994 & 1.49546 \\
\hline 3473 & 62.0243 & 391.5718 & 386.9459 & 355 & 122.5994 & 1.49509 \\
\hline 3474 & 62.0413 & 339 & 382.3578 & 354.7813 & 122.5994 & 1.49472 \\
\hline 3475 & 62.0583 & 362.2024 & 377.971 & 354.5625 & 122.5994 & 1.49435 \\
\hline 3476 & 62.0753 & 355.8967 & 374.7259 & 354.3438 & 122.5994 & 1.49398 \\
\hline 3477 & 62.0923 & 367.6817 & 372.6051 & 354.125 & 122.5994 & 1.49361 \\
\hline
\end{tabular}




\begin{tabular}{|c|c|c|c|c|c|c|}
\hline 3478 & 62.1093 & 363.1244 & 371.5011 & 353.9063 & 122.5994 & 1.49325 \\
\hline 3479 & 62.1263 & 373.853 & 371.1992 & 353.6875 & 122.5994 & 1.49288 \\
\hline 3480 & 62.1433 & 353.5192 & 371.5089 & 353.4688 & 122.5994 & 1.49251 \\
\hline 3481 & 62.1603 & 379.9571 & 372.0627 & 353.25 & 122.5994 & 1.49214 \\
\hline 3482 & 62.1773 & 349.8142 & 371.7505 & 352.5313 & 122.5994 & 1.49178 \\
\hline 3483 & 62.1943 & 339.1997 & 370.4134 & 351.8125 & 122.5994 & 1.49141 \\
\hline 3484 & 62.2113 & 359.6483 & 368.1637 & 351.0938 & 122.5994 & 1.49104 \\
\hline 3485 & 62.2283 & 339.364 & 365.5917 & 350.375 & 122.5994 & 1.49068 \\
\hline 3486 & 62.2453 & 360.6924 & 362.9544 & 349.6563 & 122.5994 & 1.49031 \\
\hline 3487 & 62.2623 & 357.0639 & 360.947 & 348.9375 & 122.5994 & 1.48994 \\
\hline 3488 & 62.2793 & 324.3657 & 359.3735 & 348.2188 & 122.5994 & 1.48958 \\
\hline 3489 & 62.2963 & 338.5275 & 358.259 & 347.5 & 122.5994 & 1.48921 \\
\hline 3490 & 62.3133 & 338.6051 & 357.5085 & 346.7813 & 122.5994 & 1.48885 \\
\hline 3491 & 62.3303 & 339.5486 & 356.981 & 346.0625 & 122.5994 & 1.48848 \\
\hline 3492 & 62.3473 & 348.4745 & 356.3917 & 345.3438 & 122.5994 & 1.48812 \\
\hline 3493 & 62.3643 & 340.028 & 355.3585 & 344.625 & 122.5994 & 1.48775 \\
\hline 3494 & 62.3813 & 350.9132 & 353.8738 & 343.9063 & 122.5994 & 1.48739 \\
\hline 3495 & 62.3983 & 349.3631 & 352.2587 & 343.1875 & 122.5994 & 1.48702 \\
\hline 3496 & 62.4153 & 310.4697 & 350.7122 & 342.4688 & 122.5994 & 1.48666 \\
\hline 3497 & 62.4323 & 303.9964 & 349.6436 & 341.75 & 122.5994 & 1.48629 \\
\hline 3498 & 62.4493 & 318.6536 & 348.9532 & 341.0313 & 122.5994 & 1.48593 \\
\hline 3499 & 62.4663 & 322.9422 & 348.5829 & 340.3125 & 122.5994 & 1.48557 \\
\hline 3500 & 62.4833 & 342.3234 & 348.1371 & 339.5938 & 122.5994 & 1.4852 \\
\hline 3501 & 62.5003 & 319.6702 & 347.5478 & 338.875 & 122.5994 & 1.48484 \\
\hline 3502 & 62.5173 & 308.5772 & 346.4164 & 338.1563 & 122.5994 & 1.48448 \\
\hline 3503 & 62.5343 & 321.4218 & 345.0189 & 337.4375 & 122.5994 & 1.48411 \\
\hline 3504 & 62.5513 & 338.8566 & 343.486 & 336.7188 & 122.5994 & 1.48375 \\
\hline 3505 & 62.5683 & 331.8864 & 342.2632 & 336 & 122.5994 & 1.48339 \\
\hline 3506 & 62.5853 & 349.7366 & 341.4975 & 335.5 & 122.5994 & 1.48303 \\
\hline 3507 & 62.6023 & 332.244 & 340.9553 & 335 & 122.5994 & 1.48267 \\
\hline 3508 & 62.6193 & 309.7303 & 340.619 & 334.5 & 122.5994 & 1.4823 \\
\hline 3509 & 62.6363 & 327.1463 & 340.4098 & 334 & 122.5994 & 1.48194 \\
\hline 3510 & 62.6533 & 333.1374 & 340.1302 & 333.5 & 122.5994 & 1.48158 \\
\hline 3511 & 62.6703 & 326.1083 & 339.7511 & 333 & 122.5994 & 1.48122 \\
\hline 3512 & 62.6873 & 343.2769 & 339.1 & 332.5 & 122.5994 & 1.48086 \\
\hline 3513 & 62.7043 & 357.7607 & 338.3258 & 332 & 122.5994 & 1.4805 \\
\hline 3514 & 62.7213 & 347.7997 & 337.5762 & 331.5 & 122.5994 & 1.48014 \\
\hline 3515 & 62.7383 & 321.0135 & 337.1017 & 331 & 122.5994 & 1.47978 \\
\hline 3516 & 62.7553 & 318.7693 & 336.9963 & 330.5 & 122.5994 & 1.47942 \\
\hline 3517 & 62.7723 & 328.6836 & 337.4233 & 330 & 122.5994 & 1.47906 \\
\hline 3518 & 62.7893 & 312.1216 & 338.269 & 329.5 & 122.5994 & 1.4787 \\
\hline 3519 & 62.8063 & 314.9847 & 339.1569 & 329 & 122.5994 & 1.47834 \\
\hline 3520 & 62.8233 & 330.7424 & 339.3814 & 328.5 & 122.5994 & 1.47798 \\
\hline 3521 & 62.8403 & 332.2434 & 338.4192 & 328 & 122.5994 & 1.47762 \\
\hline 3522 & 62.8573 & 328.4539 & 336.7729 & 327.5 & 122.5994 & 1.47726 \\
\hline 3523 & 62.8743 & 324.8364 & 335.3214 & 327 & 122.5994 & 1.4769 \\
\hline 3524 & 62.8913 & 344.5678 & 334.4081 & 326.5 & 122.5994 & 1.47655 \\
\hline 3525 & 62.9083 & 313.9649 & 334.13 & 326 & 122.5994 & 1.47619 \\
\hline 3526 & 62.9253 & 327.3345 & 334.3044 & 325.5 & 122.5994 & 1.47583 \\
\hline
\end{tabular}




\begin{tabular}{|c|c|c|c|c|c|c|}
\hline 3527 & 62.9423 & 334.3703 & 334.5493 & 325 & 122.5994 & 1.47547 \\
\hline 3528 & 62.9593 & 306.4044 & 334.5315 & 324.5 & 122.5994 & 1.47512 \\
\hline 3529 & 62.9763 & 325.3292 & 334.2159 & 324 & 122.5994 & 1.47476 \\
\hline 3530 & 62.9933 & 320.9502 & 334.2303 & 324.0833 & 122.5994 & 1.4744 \\
\hline 3531 & 63.0103 & 355.4296 & 333.8536 & 324.1667 & 122.5994 & 1.47404 \\
\hline 3532 & 63.0273 & 310.9654 & 333.271 & 324.25 & 122.5994 & 1.47369 \\
\hline 3533 & 63.0443 & 338.4724 & 332.8054 & 324.3333 & 122.5994 & 1.47333 \\
\hline 3534 & 63.0613 & 338.4801 & 332.6388 & 324.4167 & 122.5994 & 1.47297 \\
\hline 3535 & 63.0783 & 331.49 & 332.7252 & 324.5 & 122.5994 & 1.47262 \\
\hline 3536 & 63.0953 & 338.6051 & 332.928 & 324.5833 & 122.5994 & 1.47226 \\
\hline 3537 & 63.1123 & 338.8285 & 333.0581 & 324.6667 & 122.5994 & 1.47191 \\
\hline 3538 & 63.1293 & 339.9679 & 332.8146 & 324.75 & 122.5994 & 1.47155 \\
\hline 3539 & 63.1463 & 319.1253 & 332.3042 & 324.8333 & 122.5994 & 1.4712 \\
\hline 3540 & 63.1633 & 311.8102 & 331.762 & 324.9167 & 122.5994 & 1.47084 \\
\hline 3541 & 63.1803 & 327.1039 & 331.3554 & 325 & 122.5994 & 1.47049 \\
\hline 3542 & 63.1973 & 336.0724 & 331.1494 & 325.0833 & 122.5994 & 1.47013 \\
\hline 3543 & 63.2143 & 319.8027 & 331.1535 & 325.1667 & 122.5994 & 1.46978 \\
\hline 3544 & 63.2313 & 351.9931 & 331.3691 & 325.25 & 122.5994 & 1.46942 \\
\hline 3545 & 63.2483 & 331.1855 & 331.7857 & 325.3333 & 122.5994 & 1.46907 \\
\hline 3546 & 63.2653 & 325.4488 & 332.4214 & 325.4167 & 122.5994 & 1.46872 \\
\hline 3547 & 63.2823 & 329.9829 & 333.2807 & 325.5 & 122.5994 & 1.46836 \\
\hline 3548 & 63.2993 & 316.6392 & 334.2718 & 325.5833 & 122.5994 & 1.46801 \\
\hline 3549 & 63.3163 & 337.4768 & 335.1292 & 325.6667 & 122.5994 & 1.46765 \\
\hline 3550 & 63.3333 & 327.7365 & 335.5417 & 325.75 & 122.5994 & 1.4673 \\
\hline 3551 & 63.3503 & 301.0632 & 335.3001 & 325.8333 & 122.5994 & 1.46695 \\
\hline 3552 & 63.3673 & 318.9361 & 334.8471 & 325.9167 & 122.5994 & 1.4666 \\
\hline 3553 & 63.3843 & 324.5697 & 334.898 & 326 & 122.5994 & 1.46624 \\
\hline 3554 & 63.4013 & 343.1556 & 334.797 & 325.9551 & 122.5994 & 1.46589 \\
\hline 3555 & 63.4183 & 333.0026 & 335.0772 & 325.9101 & 122.5994 & 1.46554 \\
\hline 3556 & 63.4353 & 316.7134 & 335.5534 & 325.8652 & 122.5994 & 1.46519 \\
\hline 3557 & 63.4523 & 322.1315 & 336.2763 & 325.8203 & 122.5994 & 1.46484 \\
\hline 3558 & 63.4693 & 320.4527 & 337.2013 & 325.7753 & 122.5994 & 1.46449 \\
\hline 3559 & 63.4863 & 338.7069 & 338.3043 & 325.7304 & 122.5994 & 1.46413 \\
\hline 3560 & 63.5033 & 344.9084 & 339.5388 & 325.6855 & 122.5994 & 1.46378 \\
\hline 3561 & 63.5203 & 327.6748 & 340.8428 & 325.6405 & 122.5994 & 1.46343 \\
\hline 3562 & 63.5373 & 292.6006 & 342.0429 & 325.5956 & 122.5994 & 1.46308 \\
\hline 3563 & 63.5543 & 320.7244 & 342.7454 & 325.5507 & 122.5994 & 1.46273 \\
\hline 3564 & 63.5713 & 329.0015 & 342.9096 & 325.5057 & 122.5994 & 1.46238 \\
\hline 3565 & 63.5883 & 315.582 & 342.1972 & 325.4608 & 122.5994 & 1.46203 \\
\hline 3566 & 63.6053 & 320.8159 & 341.1458 & 325.4159 & 122.5994 & 1.46168 \\
\hline 3567 & 63.6223 & 311.1361 & 339.9709 & 325.3709 & 122.5994 & 1.46133 \\
\hline 3568 & 63.6393 & 301.0105 & 339.1313 & 325.326 & 122.5994 & 1.46098 \\
\hline 3569 & 63.6563 & 307.3232 & 338.9625 & 325.2811 & 122.5994 & 1.46063 \\
\hline 3570 & 63.6733 & 332.6008 & 339.7358 & 325.2361 & 122.5994 & 1.46028 \\
\hline 3571 & 63.6903 & 341.5277 & 341.5554 & 325.1912 & 122.5994 & 1.45994 \\
\hline 3572 & 63.7073 & 339.2155 & 344.2083 & 325.1463 & 122.5994 & 1.45959 \\
\hline 3573 & 63.7243 & 313.8707 & 346.9613 & 325.1013 & 122.5994 & 1.45924 \\
\hline 3574 & 63.7413 & 297.5147 & 348.9233 & 325.0564 & 122.5994 & 1.45889 \\
\hline 3575 & 63.7583 & 340.9478 & 349.8614 & 325.0115 & 122.5994 & 1.45854 \\
\hline
\end{tabular}




\begin{tabular}{|c|c|c|c|c|c|c|}
\hline 3576 & 63.7753 & 325.7098 & 350.1765 & 324.9665 & 122.5994 & 1.45819 \\
\hline 3577 & 63.7923 & 341.2398 & 349.8936 & 324.9216 & 122.5994 & 1.45785 \\
\hline 3578 & 63.8093 & 338.6611 & 348.6976 & 324.9062 & 122.5994 & 1.4575 \\
\hline 3579 & 63.8263 & 342.2411 & 346.7957 & 324.8908 & 122.5994 & 1.45715 \\
\hline 3580 & 63.8433 & 334.7613 & 345.1985 & 324.8754 & 122.5994 & 1.4568 \\
\hline 3581 & 63.8603 & 321.0713 & 344.6882 & 324.86 & 122.5994 & 1.45646 \\
\hline 3582 & 63.8773 & 340.7766 & 345.5109 & 324.8446 & 122.5994 & 1.45611 \\
\hline 3583 & 63.8943 & 312.8292 & 347.4611 & 324.8292 & 122.5994 & 1.45576 \\
\hline 3584 & 63.9113 & 308.8788 & 349.964 & 324.8138 & 122.5994 & 1.45542 \\
\hline 3585 & 63.9283 & 334.9346 & 352.4218 & 324.7984 & 122.5994 & 1.45507 \\
\hline 3586 & 63.9453 & 333.8687 & 354.6398 & 324.7829 & 122.5994 & 1.45473 \\
\hline 3587 & 63.9623 & 332.2347 & 356.9962 & 324.7675 & 122.5994 & 1.45438 \\
\hline 3588 & 63.9793 & 359.992 & 360.3421 & 324.7521 & 122.5994 & 1.45404 \\
\hline 3589 & 63.9963 & 373.2297 & 366.3441 & 324.7367 & 122.5994 & 1.45369 \\
\hline 3590 & 64.0133 & 389.8314 & 377.8071 & 324.7213 & 122.5994 & 1.45335 \\
\hline 3591 & 64.0303 & 450.5212 & 397.8259 & 324.7059 & 122.5994 & 1.453 \\
\hline 3592 & 64.0473 & 485.7192 & 427.7895 & 324.6905 & 122.5994 & 1.45266 \\
\hline 3593 & 64.0643 & 474.1517 & 465.1551 & 324.6751 & 122.5994 & 1.45231 \\
\hline 3594 & 64.0813 & 498.428 & 500.5809 & 324.6597 & 122.5994 & 1.45197 \\
\hline 3595 & 64.0983 & 484.8648 & 517.3257 & 324.6443 & 122.5994 & 1.45162 \\
\hline 3596 & 64.1153 & 419.8404 & 504.5984 & 324.6289 & 122.5994 & 1.45128 \\
\hline 3597 & 64.1323 & 397.5497 & 472.9731 & 324.6135 & 122.5994 & 1.45094 \\
\hline 3598 & 64.1493 & 381.5326 & 439.1139 & 324.5981 & 122.5994 & 1.45059 \\
\hline 3599 & 64.1663 & 344.2632 & 412.3736 & 324.5827 & 122.5994 & 1.45025 \\
\hline 3600 & 64.1833 & 344.2935 & 395.9949 & 324.5673 & 122.5994 & 1.44991 \\
\hline 3601 & 64.2003 & 382.6297 & 390.1627 & 324.5519 & 122.5994 & 1.44956 \\
\hline 3602 & 64.2173 & 398.3317 & 393.6723 & 324.5365 & 122.5994 & 1.44922 \\
\hline 3603 & 64.2343 & 396.1277 & 404.4047 & 324.521 & 122.5994 & 1.44888 \\
\hline 3604 & 64.2513 & 408.292 & 418.4055 & 324.5056 & 122.5994 & 1.44854 \\
\hline 3605 & 64.2683 & 403.2009 & 428.4252 & 324.4902 & 122.5994 & 1.44819 \\
\hline 3606 & 64.2853 & 384.9385 & 426.0105 & 324.4748 & 122.5994 & 1.44785 \\
\hline 3607 & 64.3023 & 360.7343 & 411.2043 & 324.4594 & 122.5994 & 1.44751 \\
\hline 3608 & 64.3193 & 319.0217 & 391.9786 & 324.444 & 122.5994 & 1.44717 \\
\hline 3609 & 64.3363 & 317.0517 & 374.505 & 324.4286 & 122.5994 & 1.44683 \\
\hline 3610 & 64.3533 & 302.4698 & 361.1838 & 324.4132 & 122.5994 & 1.44649 \\
\hline 3611 & 64.3703 & 295.9731 & 351.9807 & 324.3978 & 122.5994 & 1.44614 \\
\hline 3612 & 64.3873 & 287.3412 & 345.8618 & 324.3824 & 122.5994 & 1.4458 \\
\hline 3613 & 64.4043 & 320.0147 & 341.6856 & 324.367 & 122.5994 & 1.44546 \\
\hline 3614 & 64.4213 & 303.5673 & 338.6588 & 324.3516 & 122.5994 & 1.44512 \\
\hline 3615 & 64.4383 & 300.3155 & 336.3415 & 324.3362 & 122.5994 & 1.44478 \\
\hline 3616 & 64.4553 & 319.2552 & 334.5258 & 324.3208 & 122.5994 & 1.44444 \\
\hline 3617 & 64.4723 & 326.9416 & 333.08 & 324.3054 & 122.5994 & 1.4441 \\
\hline 3618 & 64.4893 & 342.784 & 331.9044 & 324.29 & 122.5994 & 1.44376 \\
\hline 3619 & 64.5063 & 342.7098 & 331.0914 & 324.2746 & 122.5994 & 1.44342 \\
\hline 3620 & 64.5233 & 329.5971 & 330.4686 & 324.2591 & 122.5994 & 1.44308 \\
\hline 3621 & 64.5403 & 305.5612 & 329.9921 & 324.2437 & 122.5994 & 1.44275 \\
\hline 3622 & 64.5573 & 341.9832 & 329.7054 & 324.2283 & 122.5994 & 1.44241 \\
\hline 3623 & 64.5743 & 349.482 & 329.6107 & 324.2129 & 122.5994 & 1.44207 \\
\hline 3624 & 64.5913 & 295.1188 & 329.6994 & 324.1975 & 122.5994 & 1.44173 \\
\hline
\end{tabular}




$\begin{array}{lrrrrrr}3625 & 64.6083 & 316.2725 & 329.9468 & 324.1821 & 122.5994 & 1.44139 \\ 3626 & 64.6253 & 353.2039 & 330.3345 & 324.1667 & 122.5994 & 1.44105 \\ 3627 & 64.6423 & 327.523 & 330.9278 & 324.1513 & 122.5994 & 1.44071 \\ 3628 & 64.6593 & 322.0524 & 331.6752 & 324.1359 & 122.5994 & 1.44038 \\ 3629 & 64.6763 & 363.4828 & 332.6187 & 324.1205 & 122.5994 & 1.44004 \\ 3630 & 64.6933 & 340.1958 & 333.4953 & 324.1051 & 122.5994 & 1.4397 \\ 3631 & 64.7103 & 316.512 & 334.0195 & 324.0897 & 122.5994 & 1.43936 \\ 3632 & 64.7273 & 314.985 & 333.9739 & 324.0743 & 122.5994 & 1.43903 \\ 3633 & 64.7443 & 328.7248 & 333.3941 & 324.0589 & 122.5994 & 1.43869 \\ 3634 & 64.7613 & 328.9262 & 332.5248 & 324.0435 & 122.5994 & 1.43835 \\ 3635 & 64.7783 & 307.3537 & 331.6115 & 324.0281 & 122.5994 & 1.43802 \\ 3636 & 64.7953 & 334.6774 & 330.8596 & 324.0127 & 122.5994 & 1.43768 \\ 3637 & 64.8123 & 308.7611 & 329.8899 & 323.9972 & 122.5994 & 1.43735 \\ 3638 & 64.8293 & 313.293 & 329.6332 & 323.9818 & 122.5994 & 1.43701 \\ 3639 & 64.8463 & 307.5349 & 329.6548 & 323.9664 & 122.5994 & 1.43667 \\ 3640 & 64.8633 & 330.2876 & 329.9136 & 323.951 & 122.5994 & 1.43634 \\ 3641 & 64.8803 & 354.6913 & 330.219 & 323.9356 & 122.5994 & 1.436 \\ 3642 & 64.8973 & 345.7826 & 330.4071 & 323.9202 & 122.5994 & 1.43567 \\ 3643 & 64.9143 & 315.092 & 330.7097 & 323.9048 & 122.5994 & 1.43533 \\ 3644 & 64.9313 & 312.9966 & 331.21 & 323.8894 & 122.5994 & 1.435 \\ 3645 & 64.9483 & 319.098 & 332.1754 & 323.874 & 122.5994 & 1.43466 \\ 3646 & 64.9653 & 298.6968 & 333.7737 & 323.8586 & 122.5994 & 1.43433 \\ 3647 & 64.9823 & 323.8432 & 335.8554 & 323.8586 & 122.5994 & 1.434\end{array}$


No. Pos. [ ${ }^{\circ} 2$ Th. lobs [cts] Icalc [cts] lback [cts] CT [s] ESD D spacings

\begin{tabular}{|c|c|c|c|c|c|c|}
\hline 1 & 3.0003 & 2256.243 & 2256.243 & 2256.243 & 122.5994 & 29.42347 \\
\hline 2 & 3.0173 & 2105.642 & 2225.358 & 2225.358 & 122.5994 & 29.25773 \\
\hline 3 & 3.0343 & 2051.709 & 2194.473 & 2194.473 & 122.5994 & 29.09385 \\
\hline 4 & 3.0513 & 2037.849 & 2163.588 & 2163.588 & 122.5994 & 28.9318 \\
\hline 5 & 3.0683 & 1954.187 & 2132.703 & 2132.703 & 122.5994 & 28.77154 \\
\hline 6 & 3.0853 & 1861.628 & 2101.818 & 2101.818 & 122.5994 & 28.61305 \\
\hline 7 & 3.1023 & 1832.201 & 2070.933 & 2070.933 & 122.5994 & 28.45629 \\
\hline 8 & 3.1193 & 1872.916 & 2040.047 & 2040.047 & 122.5994 & 28.30124 \\
\hline 9 & 3.1363 & 1774.213 & 2009.162 & 2009.162 & 122.5994 & 28.14788 \\
\hline 10 & 3.1533 & 1816.714 & 1978.277 & 1978.277 & 122.5994 & 27.99617 \\
\hline 11 & 3.1703 & 1769.51 & 1947.392 & 1947.392 & 122.5994 & 27.84608 \\
\hline 12 & 3.1873 & 1695.5 & 1916.507 & 1916.507 & 122.5994 & 27.6976 \\
\hline 13 & 3.2043 & 1695.608 & 1885.622 & 1885.622 & 122.5994 & 27.55069 \\
\hline 14 & 3.2213 & 1704.748 & 1854.737 & 1854.737 & 122.5994 & 27.40534 \\
\hline 15 & 3.2383 & 1680.983 & 1823.851 & 1823.851 & 122.5994 & 27.26151 \\
\hline 16 & 3.2553 & 1660.042 & 1792.966 & 1792.966 & 122.5994 & 27.11918 \\
\hline 17 & 3.2723 & 1624.382 & 1762.081 & 1762.081 & 122.5994 & 26.97833 \\
\hline 18 & 3.2893 & 1628.441 & 1731.196 & 1731.196 & 122.5994 & 26.83894 \\
\hline 19 & 3.3063 & 1601.82 & 1700.311 & 1700.311 & 122.5994 & 26.70098 \\
\hline 20 & 3.3233 & 1575.808 & 1669.426 & 1669.426 & 122.5994 & 26.56443 \\
\hline 21 & 3.3403 & 1548.958 & 1638.541 & 1638.541 & 122.5994 & 26.42928 \\
\hline 22 & 3.3573 & 1512.9 & 1607.655 & 1607.655 & 122.5994 & 26.29549 \\
\hline 23 & 3.3743 & 1489.184 & 1576.77 & 1576.77 & 122.5994 & 26.16305 \\
\hline 24 & 3.3913 & 1497.786 & 1545.885 & 1545.885 & 122.5994 & 26.03194 \\
\hline 25 & 3.4083 & 1501.951 & 1515 & 1515 & 122.5994 & 25.90213 \\
\hline 26 & 3.4253 & 1538.52 & 1505.333 & 1505.333 & 122.5994 & 25.77362 \\
\hline 27 & 3.4423 & 1555.843 & 1495.667 & 1495.667 & 122.5994 & 25.64637 \\
\hline 28 & 3.4593 & 1498.248 & 1486 & 1486 & 122.5994 & 25.52038 \\
\hline 29 & 3.4763 & 1436.383 & 1476.333 & 1476.333 & 122.5994 & 25.39561 \\
\hline 30 & 3.4933 & 1444.976 & 1466.667 & 1466.667 & 122.5994 & 25.27207 \\
\hline 31 & 3.5103 & 1474.078 & 1457 & 1457 & 122.5994 & 25.14971 \\
\hline 32 & 3.5273 & 1436.573 & 1447.333 & 1447.333 & 122.5994 & 25.02854 \\
\hline 33 & 3.5443 & 1439.827 & 1437.667 & 1437.667 & 122.5994 & 24.90853 \\
\hline 34 & 3.5613 & 1435.428 & 1428 & 1428 & 122.5994 & 24.78967 \\
\hline 35 & 3.5783 & 1385.07 & 1418.333 & 1418.333 & 122.5994 & 24.67194 \\
\hline 36 & 3.5953 & 1335.841 & 1408.667 & 1408.667 & 122.5994 & 24.55532 \\
\hline 37 & 3.6123 & 1353.355 & 1399 & 1399 & 122.5994 & 24.4398 \\
\hline 38 & 3.6293 & 1379.294 & 1389.333 & 1389.333 & 122.5994 & 24.32536 \\
\hline 39 & 3.6463 & 1377.771 & 1379.667 & 1379.667 & 122.5994 & 24.21198 \\
\hline 40 & 3.6633 & 1426.764 & 1370 & 1370 & 122.5994 & 24.09966 \\
\hline 41 & 3.6803 & 1391.152 & 1360.333 & 1360.333 & 122.5994 & 23.98838 \\
\hline 42 & 3.6973 & 1351.25 & 1350.667 & 1350.667 & 122.5994 & 23.87812 \\
\hline 43 & 3.7143 & 1287.93 & 1341 & 1341 & 122.5994 & 23.76887 \\
\hline 44 & 3.7313 & 1248.737 & 1331.333 & 1331.333 & 122.5994 & 23.66062 \\
\hline 45 & 3.7483 & 1293.346 & 1321.667 & 1321.667 & 122.5994 & 23.55335 \\
\hline 46 & 3.7653 & 1275.179 & 1312 & 1312 & 122.5994 & 23.44704 \\
\hline 47 & 3.7823 & 1260.401 & 1302.333 & 1302.333 & 122.5994 & 23.3417 \\
\hline
\end{tabular}




\begin{tabular}{|c|c|c|c|c|c|c|}
\hline 48 & 3.7993 & 1239.768 & 1292.667 & 1292.667 & 122.5994 & 23.23729 \\
\hline 49 & 3.8163 & 1304.407 & 1283 & 1283 & 122.5994 & 23.13382 \\
\hline 50 & 3.8333 & 1332.569 & 1274.958 & 1274.958 & 122.5994 & 23.03126 \\
\hline 51 & 3.8503 & 1215.856 & 1266.917 & 1266.917 & 122.5994 & 22.92961 \\
\hline 52 & 3.8673 & 1267.643 & 1258.875 & 1258.875 & 122.5994 & 22.82886 \\
\hline 53 & 3.8843 & 1209.069 & 1250.833 & 1250.833 & 122.5994 & 22.72898 \\
\hline 54 & 3.9013 & 1234.406 & 1242.792 & 1242.792 & 122.5994 & 22.62998 \\
\hline 55 & 3.9183 & 1167.257 & 1234.75 & 1234.75 & 122.5994 & 22.53184 \\
\hline 56 & 3.9353 & 1191.505 & 1226.708 & 1226.708 & 122.5994 & 22.43454 \\
\hline 57 & 3.9523 & 1211.55 & 1218.667 & 1218.667 & 122.5994 & 22.33808 \\
\hline 58 & 3.9693 & 1256.191 & 1210.625 & 1210.625 & 122.5994 & 22.24245 \\
\hline 59 & 3.9863 & 1204.328 & 1202.583 & 1202.583 & 122.5994 & 22.14763 \\
\hline 60 & 4.0033 & 1142.91 & 1194.542 & 1194.542 & 122.5994 & 22.05362 \\
\hline 61 & 4.0203 & 1108.484 & 1186.5 & 1186.5 & 122.5994 & 21.9604 \\
\hline 62 & 4.0373 & 1156.022 & 1178.458 & 1178.458 & 122.5994 & 21.86797 \\
\hline 63 & 4.0543 & 1158.263 & 1170.417 & 1170.417 & 122.5994 & 21.77632 \\
\hline 64 & 4.0713 & 1141.297 & 1162.375 & 1162.375 & 122.5994 & 21.68543 \\
\hline 65 & 4.0883 & 1109.774 & 1154.333 & 1154.333 & 122.5994 & 21.59529 \\
\hline 66 & 4.1053 & 1132.984 & 1146.292 & 1146.292 & 122.5994 & 21.50591 \\
\hline 67 & 4.1223 & 1143.552 & 1138.25 & 1138.25 & 122.5994 & 21.41726 \\
\hline 68 & 4.1393 & 1129.744 & 1130.208 & 1130.208 & 122.5994 & 21.32933 \\
\hline 69 & 4.1563 & 1119.141 & 1122.167 & 1122.167 & 122.5994 & 21.24213 \\
\hline 70 & 4.1733 & 1096.331 & 1114.125 & 1114.125 & 122.5994 & 21.15564 \\
\hline 71 & 4.1903 & 1089.191 & 1106.083 & 1106.083 & 122.5994 & 21.06985 \\
\hline 72 & 4.2073 & 1088.299 & 1098.042 & 1098.042 & 122.5994 & 20.98475 \\
\hline 73 & 4.2243 & 1086.717 & 1090 & 1090 & 122.5994 & 20.90034 \\
\hline 74 & 4.2413 & 1099.383 & 1085.25 & 1085.25 & 122.5994 & 20.81661 \\
\hline 75 & 4.2583 & 1082.716 & 1080.5 & 1080.5 & 122.5994 & 20.73354 \\
\hline 76 & 4.2753 & 1061.307 & 1075.75 & 1075.75 & 122.5994 & 20.65114 \\
\hline 77 & 4.2923 & 1043.91 & 1071 & 1071 & 122.5994 & 20.56939 \\
\hline 78 & 4.3093 & 1049.577 & 1066.25 & 1066.25 & 122.5994 & 20.48828 \\
\hline 79 & 4.3263 & 1087.464 & 1061.5 & 1061.5 & 122.5994 & 20.40781 \\
\hline 80 & 4.3433 & 1139.849 & 1056.75 & 1056.75 & 122.5994 & 20.32797 \\
\hline 81 & 4.3603 & 1074.692 & 1052 & 1052 & 122.5994 & 20.24875 \\
\hline 82 & 4.3773 & 1048.116 & 1047.25 & 1047.25 & 122.5994 & 20.17015 \\
\hline 83 & 4.3943 & 1064.677 & 1042.5 & 1042.5 & 122.5994 & 20.09216 \\
\hline 84 & 4.4113 & 1064.148 & 1037.75 & 1037.75 & 122.5994 & 20.01477 \\
\hline 85 & 4.4283 & 1041.853 & 1033 & 1033 & 122.5994 & 19.93797 \\
\hline 86 & 4.4453 & 1026.187 & 1028.25 & 1028.25 & 122.5994 & 19.86176 \\
\hline 87 & 4.4623 & 1079.296 & 1023.5 & 1023.5 & 122.5994 & 19.78613 \\
\hline 88 & 4.4793 & 1015.652 & 1018.75 & 1018.75 & 122.5994 & 19.71108 \\
\hline 89 & 4.4963 & 1074.138 & 1014 & 1014 & 122.5994 & 19.63659 \\
\hline 90 & 4.5133 & 994.1823 & 1009.25 & 1009.25 & 122.5994 & 19.56267 \\
\hline 91 & 4.5303 & 956.7792 & 1004.5 & 1004.5 & 122.5994 & 19.48929 \\
\hline 92 & 4.5473 & 1058.214 & 999.75 & 999.75 & 122.5994 & 19.41647 \\
\hline 93 & 4.5643 & 1050.046 & 995 & 995 & 122.5994 & 19.34419 \\
\hline 94 & 4.5813 & 973.4429 & 990.25 & 990.25 & 122.5994 & 19.27245 \\
\hline 95 & 4.5983 & 986.4051 & 985.5 & 985.5 & 122.5994 & 19.20124 \\
\hline 96 & 4.6153 & 1004.601 & 980.75 & 980.75 & 122.5994 & 19.13055 \\
\hline
\end{tabular}




\begin{tabular}{|c|c|c|c|c|c|c|}
\hline 97 & 4.6323 & 995.2777 & 976 & 976 & 122.5994 & 19.06038 \\
\hline 98 & 4.6493 & 947.5883 & 973.4167 & 973.4167 & 122.5994 & 18.99073 \\
\hline 99 & 4.6663 & 935.6872 & 970.8333 & 970.8333 & 122.5994 & 18.92158 \\
\hline 100 & 4.6833 & 931.8332 & 968.25 & 968.25 & 122.5994 & 18.85293 \\
\hline 101 & 4.7003 & 942.5076 & 965.6667 & 965.6667 & 122.5994 & 18.78479 \\
\hline 102 & 4.7173 & 946.7607 & 963.0833 & 963.0833 & 122.5994 & 18.71713 \\
\hline 103 & 4.7343 & 964.7867 & 960.5 & 960.5 & 122.5994 & 18.64996 \\
\hline 104 & 4.7513 & 957.5085 & 957.9167 & 957.9167 & 122.5994 & 18.58327 \\
\hline 105 & 4.7683 & 951.2253 & 955.3333 & 955.3333 & 122.5994 & 18.51705 \\
\hline 106 & 4.7853 & 925.5659 & 952.75 & 952.75 & 122.5994 & 18.45131 \\
\hline 107 & 4.8023 & 982.8095 & 950.1667 & 950.1667 & 122.5994 & 18.38603 \\
\hline 108 & 4.8193 & 933.2775 & 947.5833 & 947.5833 & 122.5994 & 18.32121 \\
\hline 109 & 4.8363 & 927.9089 & 945 & 945 & 122.5994 & 18.25685 \\
\hline 110 & 4.8533 & 944.0856 & 942.4167 & 942.4167 & 122.5994 & 18.19294 \\
\hline 111 & 4.8703 & 909.8192 & 939.8333 & 939.8333 & 122.5994 & 18.12947 \\
\hline 112 & 4.8873 & 899.0488 & 937.25 & 937.25 & 122.5994 & 18.06645 \\
\hline 113 & 4.9043 & 890.4778 & 934.6667 & 934.6667 & 122.5994 & 18.00386 \\
\hline 114 & 4.9213 & 922.896 & 932.0833 & 932.0833 & 122.5994 & 17.94171 \\
\hline 115 & 4.9383 & 936.1148 & 929.5 & 929.5 & 122.5994 & 17.87998 \\
\hline 116 & 4.9553 & 903.15 & 926.9167 & 926.9167 & 122.5994 & 17.81868 \\
\hline 117 & 4.9723 & 888.1693 & 924.3333 & 924.3333 & 122.5994 & 17.7578 \\
\hline 118 & 4.9893 & 882.3233 & 921.75 & 921.75 & 122.5994 & 17.69733 \\
\hline 119 & 5.0063 & 891.2485 & 919.1667 & 919.1667 & 122.5994 & 17.63727 \\
\hline 120 & 5.0233 & 897.358 & 916.5833 & 916.5833 & 122.5994 & 17.57762 \\
\hline 121 & 5.0403 & 917.6212 & 914 & 914 & 122.5994 & 17.51837 \\
\hline 122 & 5.0573 & 922.9026 & 911.125 & 911.125 & 122.5994 & 17.45953 \\
\hline 123 & 5.0743 & 937.1738 & 908.25 & 908.25 & 122.5994 & 17.40107 \\
\hline 124 & 5.0913 & 853.136 & 905.375 & 905.375 & 122.5994 & 17.34301 \\
\hline 125 & 5.1083 & 871.1038 & 902.5 & 902.5 & 122.5994 & 17.28533 \\
\hline 126 & 5.1253 & 869.2012 & 899.625 & 899.625 & 122.5994 & 17.22803 \\
\hline 127 & 5.1423 & 886.3316 & 896.75 & 896.75 & 122.5994 & 17.17112 \\
\hline 128 & 5.1593 & 882.5267 & 893.875 & 893.875 & 122.5994 & 17.11458 \\
\hline 129 & 5.1763 & 862.8515 & 891 & 891 & 122.5994 & 17.05841 \\
\hline 130 & 5.1933 & 872.1864 & 888.125 & 888.125 & 122.5994 & 17.00261 \\
\hline 131 & 5.2103 & 862.0663 & 885.25 & 885.25 & 122.5994 & 16.94717 \\
\hline 132 & 5.2273 & 839.448 & 882.375 & 882.375 & 122.5994 & 16.89209 \\
\hline 133 & 5.2443 & 869.6059 & 879.5 & 879.5 & 122.5994 & 16.83737 \\
\hline 134 & 5.2613 & 901.8718 & 876.625 & 876.625 & 122.5994 & 16.78301 \\
\hline 135 & 5.2783 & 911.3526 & 873.75 & 873.75 & 122.5994 & 16.72899 \\
\hline 136 & 5.2953 & 863.5843 & 870.875 & 870.875 & 122.5994 & 16.67532 \\
\hline 137 & 5.3123 & 902.4947 & 868 & 868 & 122.5994 & 16.622 \\
\hline 138 & 5.3293 & 860.9903 & 865.125 & 865.125 & 122.5994 & 16.56901 \\
\hline 139 & 5.3463 & 864.1709 & 862.25 & 862.25 & 122.5994 & 16.51637 \\
\hline 140 & 5.3633 & 876.0423 & 859.375 & 859.375 & 122.5994 & 16.46405 \\
\hline 141 & 5.3803 & 886.8917 & 856.5 & 856.5 & 122.5994 & 16.41207 \\
\hline 142 & 5.3973 & 795.8068 & 853.625 & 853.625 & 122.5994 & 16.36041 \\
\hline 143 & 5.4143 & 812.5811 & 850.75 & 850.75 & 122.5994 & 16.30908 \\
\hline 144 & 5.4313 & 819.7219 & 847.875 & 847.875 & 122.5994 & 16.25807 \\
\hline 145 & 5.4483 & 845.0321 & 845 & 845 & 122.5994 & 16.20738 \\
\hline
\end{tabular}




\begin{tabular}{|c|c|c|c|c|c|c|}
\hline 146 & 5.4653 & 880.6873 & 845.0417 & 845.0417 & 122.5994 & 16.15701 \\
\hline 147 & 5.4823 & 866.9275 & 845.0833 & 845.0833 & 122.5994 & 16.10695 \\
\hline 148 & 5.4993 & 844.9122 & 845.125 & 845.125 & 122.5994 & 16.05719 \\
\hline 149 & 5.5163 & 826.8163 & 845.1667 & 845.1667 & 122.5994 & 16.00775 \\
\hline 150 & 5.5333 & 797.804 & 845.2083 & 845.2083 & 122.5994 & 15.9586 \\
\hline 151 & 5.5503 & 842.7844 & 845.25 & 845.25 & 122.5994 & 15.90976 \\
\hline 152 & 5.5673 & 873.442 & 845.2917 & 845.2917 & 122.5994 & 15.86122 \\
\hline 153 & 5.5843 & 824.7824 & 845.3333 & 845.3333 & 122.5994 & 15.81297 \\
\hline 154 & 5.6013 & 805.6542 & 845.375 & 845.375 & 122.5994 & 15.76502 \\
\hline 155 & 5.6183 & 814.8836 & 845.4167 & 845.4167 & 122.5994 & 15.71735 \\
\hline 156 & 5.6353 & 852.7233 & 845.4583 & 845.4583 & 122.5994 & 15.66998 \\
\hline 157 & 5.6523 & 842.9624 & 845.5 & 845.5 & 122.5994 & 15.62289 \\
\hline 158 & 5.6693 & 863.7032 & 845.5417 & 845.5417 & 122.5994 & 15.57608 \\
\hline 159 & 5.6863 & 830.5982 & 845.5833 & 845.5833 & 122.5994 & 15.52955 \\
\hline 160 & 5.7033 & 826.5131 & 845.625 & 845.625 & 122.5994 & 15.4833 \\
\hline 161 & 5.7203 & 824.3668 & 845.6667 & 845.6667 & 122.5994 & 15.43732 \\
\hline 162 & 5.7373 & 860.4232 & 845.7083 & 845.7083 & 122.5994 & 15.39162 \\
\hline 163 & 5.7543 & 839.6218 & 845.75 & 845.75 & 122.5994 & 15.34618 \\
\hline 164 & 5.7713 & 815.3612 & 845.7917 & 845.7917 & 122.5994 & 15.30102 \\
\hline 165 & 5.7883 & 823.3627 & 845.8333 & 845.8333 & 122.5994 & 15.25612 \\
\hline 166 & 5.8053 & 808.5835 & 845.875 & 845.875 & 122.5994 & 15.21148 \\
\hline 167 & 5.8223 & 807.3765 & 847.4838 & 845.9167 & 122.5994 & 15.16711 \\
\hline 168 & 5.8393 & 812.2419 & 848.4339 & 845.9583 & 122.5994 & 15.12299 \\
\hline 169 & 5.8563 & 844.2993 & 848.6771 & 846 & 122.5994 & 15.07913 \\
\hline 170 & 5.8733 & 875.6321 & 849.175 & 846.2708 & 122.5994 & 15.03552 \\
\hline 171 & 5.8903 & 889.9216 & 849.7029 & 846.5417 & 122.5994 & 14.99216 \\
\hline 172 & 5.9073 & 861.9643 & 850.2661 & 846.8125 & 122.5994 & 14.94906 \\
\hline 173 & 5.9243 & 867.3699 & 850.8715 & 847.0833 & 122.5994 & 14.9062 \\
\hline 174 & 5.9413 & 830.0123 & 851.5276 & 847.3542 & 122.5994 & 14.86358 \\
\hline 175 & 5.9583 & 829.3204 & 852.2449 & 847.625 & 122.5994 & 14.82121 \\
\hline 176 & 5.9753 & 857.5114 & 853.0369 & 847.8958 & 122.5994 & 14.77909 \\
\hline 177 & 5.9923 & 864.3762 & 853.9213 & 848.1667 & 122.5994 & 14.7372 \\
\hline 178 & 6.0093 & 869.1128 & 854.9207 & 848.4375 & 122.5994 & 14.69554 \\
\hline 179 & 6.0263 & 865.3533 & 856.0652 & 848.7083 & 122.5994 & 14.65413 \\
\hline 180 & 6.0433 & 889.6209 & 857.3962 & 848.9792 & 122.5994 & 14.61294 \\
\hline 181 & 6.0603 & 878.4193 & 858.9717 & 849.25 & 122.5994 & 14.57199 \\
\hline 182 & 6.0773 & 919.0843 & 860.8813 & 849.5208 & 122.5994 & 14.53126 \\
\hline 183 & 6.0943 & 902.0474 & 863.2813 & 849.7917 & 122.5994 & 14.49077 \\
\hline 184 & 6.1113 & 855.592 & 866.476 & 850.0625 & 122.5994 & 14.4505 \\
\hline 185 & 6.1283 & 892.3047 & 871.0899 & 850.3333 & 122.5994 & 14.41045 \\
\hline 186 & 6.1453 & 905.793 & 878.3455 & 850.6042 & 122.5994 & 14.37062 \\
\hline 187 & 6.1623 & 942.4436 & 890.367 & 850.875 & 122.5994 & 14.33102 \\
\hline 188 & 6.1793 & 925.6328 & 910.2366 & 851.1458 & 122.5994 & 14.29163 \\
\hline 189 & 6.1963 & 986.8031 & 941.3645 & 851.4167 & 122.5994 & 14.25246 \\
\hline 190 & 6.2133 & 1005.303 & 985.8988 & 851.6875 & 122.5994 & 14.2135 \\
\hline 191 & 6.2303 & 1016.373 & 1042.221 & 851.9583 & 122.5994 & 14.17475 \\
\hline 192 & 6.2473 & 1071.44 & 1101.584 & 852.2292 & 122.5994 & 14.13622 \\
\hline 193 & 6.2643 & 1101.361 & 1145.243 & 852.5 & 122.5994 & 14.0979 \\
\hline 194 & 6.2813 & 1177.136 & 1151.693 & 852.7708 & 122.5994 & 14.05978 \\
\hline
\end{tabular}




\begin{tabular}{|c|c|c|c|c|c|c|}
\hline 195 & 6.2983 & 1138.912 & 1117.568 & 853.0417 & 122.5994 & 14.02187 \\
\hline 196 & 6.3153 & 1109.356 & 1061.243 & 853.3125 & 122.5994 & 13.98416 \\
\hline 197 & 6.3323 & 1038.254 & 1003.411 & 853.5833 & 122.5994 & 13.94666 \\
\hline 198 & 6.3493 & 960.2182 & 955.5952 & 853.8542 & 122.5994 & 13.90935 \\
\hline 199 & 6.3663 & 905.6575 & 921.1723 & 854.125 & 122.5994 & 13.87225 \\
\hline 200 & 6.3833 & 866.928 & 898.8332 & 854.3958 & 122.5994 & 13.83534 \\
\hline 201 & 6.4003 & 865.6381 & 885.3528 & 854.6667 & 122.5994 & 13.79863 \\
\hline 202 & 6.4173 & 880.1805 & 877.4633 & 854.9375 & 122.5994 & 13.76212 \\
\hline 203 & 6.4343 & 876.1513 & 872.7489 & 855.2083 & 122.5994 & 13.72579 \\
\hline 204 & 6.4513 & 898.2926 & 869.7483 & 855.4792 & 122.5994 & 13.68966 \\
\hline 205 & 6.4683 & 915.2485 & 867.6899 & 855.75 & 122.5994 & 13.65372 \\
\hline 206 & 6.4853 & 848.7213 & 866.1942 & 856.0208 & 122.5994 & 13.61797 \\
\hline 207 & 6.5023 & 808.2621 & 865.071 & 856.2917 & 122.5994 & 13.5824 \\
\hline 208 & 6.5193 & 839.0352 & 864.2154 & 856.5625 & 122.5994 & 13.54702 \\
\hline 209 & 6.5363 & 839.5791 & 863.5614 & 856.8333 & 122.5994 & 13.51183 \\
\hline 210 & 6.5533 & 823.6505 & 863.0637 & 857.1042 & 122.5994 & 13.47682 \\
\hline 211 & 6.5703 & 875.0563 & 862.6892 & 857.375 & 122.5994 & 13.44198 \\
\hline 212 & 6.5873 & 861.2341 & 862.4131 & 857.6458 & 122.5994 & 13.40733 \\
\hline 213 & 6.6043 & 844.0026 & 862.2167 & 857.9167 & 122.5994 & 13.37286 \\
\hline 214 & 6.6213 & 867.8099 & 862.0853 & 858.1875 & 122.5994 & 13.33856 \\
\hline 215 & 6.6383 & 873.8138 & 862.0073 & 858.4583 & 122.5994 & 13.30444 \\
\hline 216 & 6.6553 & 883.6134 & 861.9739 & 858.7292 & 122.5994 & 13.2705 \\
\hline 217 & 6.6723 & 846.3632 & 861.9777 & 859 & 122.5994 & 13.23672 \\
\hline 218 & 6.6893 & 846.9639 & 859.8672 & 857.125 & 122.5994 & 13.20312 \\
\hline 219 & 6.7063 & 838.4452 & 857.7834 & 855.25 & 122.5994 & 13.16969 \\
\hline 220 & 6.7233 & 801.0701 & 855.7225 & 853.375 & 122.5994 & 13.13643 \\
\hline 221 & 6.7403 & 785.4868 & 851.5 & 851.5 & 122.5994 & 13.10334 \\
\hline 222 & 6.7573 & 831.4431 & 849.625 & 849.625 & 122.5994 & 13.07041 \\
\hline 223 & 6.7743 & 828.8462 & 847.75 & 847.75 & 122.5994 & 13.03765 \\
\hline 224 & 6.7913 & 819.1086 & 845.875 & 845.875 & 122.5994 & 13.00505 \\
\hline 225 & 6.8083 & 817.9753 & 844 & 844 & 122.5994 & 12.97261 \\
\hline 226 & 6.8253 & 832.4619 & 842.125 & 842.125 & 122.5994 & 12.94034 \\
\hline 227 & 6.8423 & 815.3948 & 840.25 & 840.25 & 122.5994 & 12.90823 \\
\hline 228 & 6.8593 & 842.8233 & 838.375 & 838.375 & 122.5994 & 12.87627 \\
\hline 229 & 6.8763 & 868.0314 & 836.5 & 836.5 & 122.5994 & 12.84448 \\
\hline 230 & 6.8933 & 769.6475 & 834.625 & 834.625 & 122.5994 & 12.81284 \\
\hline 231 & 6.9103 & 809.6767 & 832.75 & 832.75 & 122.5994 & 12.78136 \\
\hline 232 & 6.9273 & 774.004 & 830.875 & 830.875 & 122.5994 & 12.75003 \\
\hline 233 & 6.9443 & 795.0478 & 829 & 829 & 122.5994 & 12.71886 \\
\hline 234 & 6.9613 & 801.185 & 827.125 & 827.125 & 122.5994 & 12.68783 \\
\hline 235 & 6.9783 & 824.0826 & 825.25 & 825.25 & 122.5994 & 12.65696 \\
\hline 236 & 6.9953 & 834.2539 & 823.375 & 823.375 & 122.5994 & 12.62624 \\
\hline 237 & 7.0123 & 817.5074 & 821.5 & 821.5 & 122.5994 & 12.59567 \\
\hline 238 & 7.0293 & 809.4579 & 819.625 & 819.625 & 122.5994 & 12.56525 \\
\hline 239 & 7.0463 & 792.2893 & 817.75 & 817.75 & 122.5994 & 12.53497 \\
\hline 240 & 7.0633 & 842.8582 & 815.875 & 815.875 & 122.5994 & 12.50484 \\
\hline 241 & 7.0803 & 787.7127 & 814 & 814 & 122.5994 & 12.47485 \\
\hline 242 & 7.0973 & 815.0964 & 813.125 & 813.125 & 122.5994 & 12.44501 \\
\hline 243 & 7.1143 & 838.4277 & 812.25 & 812.25 & 122.5994 & 12.41531 \\
\hline
\end{tabular}




\begin{tabular}{|c|c|c|c|c|c|c|}
\hline 244 & 7.1313 & 786.5826 & 811.375 & 811.375 & 122.5994 & 12.38575 \\
\hline 245 & 7.1483 & 795.2321 & 810.5 & 810.5 & 122.5994 & 12.35633 \\
\hline 246 & 7.1653 & 789.1509 & 809.625 & 809.625 & 122.5994 & 12.32706 \\
\hline 247 & 7.1823 & 777.7683 & 808.75 & 808.75 & 122.5994 & 12.29792 \\
\hline 248 & 7.1993 & 793.1499 & 807.875 & 807.875 & 122.5994 & 12.26892 \\
\hline 249 & 7.2163 & 818.2715 & 807 & 807 & 122.5994 & 12.24005 \\
\hline 250 & 7.2333 & 863.1862 & 806.125 & 806.125 & 122.5994 & 12.21132 \\
\hline 251 & 7.2503 & 836.4678 & 805.25 & 805.25 & 122.5994 & 12.18273 \\
\hline 252 & 7.2673 & 793.494 & 804.375 & 804.375 & 122.5994 & 12.15427 \\
\hline 253 & 7.2843 & 831.2903 & 803.5 & 803.5 & 122.5994 & 12.12594 \\
\hline 254 & 7.3013 & 838.5357 & 802.625 & 802.625 & 122.5994 & 12.09774 \\
\hline 255 & 7.3183 & 850.7931 & 801.75 & 801.75 & 122.5994 & 12.06968 \\
\hline 256 & 7.3353 & 810.3039 & 800.875 & 800.875 & 122.5994 & 12.04175 \\
\hline 257 & 7.3523 & 810.0153 & 800 & 800 & 122.5994 & 12.01394 \\
\hline 258 & 7.3693 & 829.0999 & 799.125 & 799.125 & 122.5994 & 11.98627 \\
\hline 259 & 7.3863 & 842.8873 & 798.25 & 798.25 & 122.5994 & 11.95872 \\
\hline 260 & 7.4033 & 825.4933 & 797.375 & 797.375 & 122.5994 & 11.93129 \\
\hline 261 & 7.4203 & 838.6218 & 796.5 & 796.5 & 122.5994 & 11.904 \\
\hline 262 & 7.4373 & 839.3087 & 795.625 & 795.625 & 122.5994 & 11.87683 \\
\hline 263 & 7.4543 & 812.7655 & 794.75 & 794.75 & 122.5994 & 11.84978 \\
\hline 264 & 7.4713 & 791.7127 & 793.875 & 793.875 & 122.5994 & 11.82285 \\
\hline 265 & 7.4883 & 787.8087 & 793 & 793 & 122.5994 & 11.79605 \\
\hline 266 & 7.5053 & 801.4978 & 793.5417 & 793.5417 & 122.5994 & 11.76937 \\
\hline 267 & 7.5223 & 778.1694 & 794.0833 & 794.0833 & 122.5994 & 11.74281 \\
\hline 268 & 7.5393 & 796.5325 & 794.625 & 794.625 & 122.5994 & 11.71637 \\
\hline 269 & 7.5563 & 802.2067 & 795.1667 & 795.1667 & 122.5994 & 11.69005 \\
\hline 270 & 7.5733 & 765.7336 & 795.7083 & 795.7083 & 122.5994 & 11.66385 \\
\hline 271 & 7.5903 & 801.5686 & 796.25 & 796.25 & 122.5994 & 11.63776 \\
\hline 272 & 7.6073 & 774.8846 & 796.7917 & 796.7917 & 122.5994 & 11.61179 \\
\hline 273 & 7.6243 & 810.4973 & 797.3333 & 797.3333 & 122.5994 & 11.58594 \\
\hline 274 & 7.6413 & 853.3728 & 797.875 & 797.875 & 122.5994 & 11.5602 \\
\hline 275 & 7.6583 & 806.9036 & 798.4167 & 798.4167 & 122.5994 & 11.53458 \\
\hline 276 & 7.6753 & 800.905 & 798.9583 & 798.9583 & 122.5994 & 11.50907 \\
\hline 277 & 7.6923 & 790.2958 & 799.5 & 799.5 & 122.5994 & 11.48367 \\
\hline 278 & 7.7093 & 811.3365 & 800.0417 & 800.0417 & 122.5994 & 11.45839 \\
\hline 279 & 7.7263 & 795.5693 & 800.5833 & 800.5833 & 122.5994 & 11.43321 \\
\hline 280 & 7.7433 & 800.7136 & 801.125 & 801.125 & 122.5994 & 11.40815 \\
\hline 281 & 7.7603 & 746.3926 & 801.6667 & 801.6667 & 122.5994 & 11.3832 \\
\hline 282 & 7.7773 & 787.8123 & 802.2083 & 802.2083 & 122.5994 & 11.35835 \\
\hline 283 & 7.7943 & 753.4005 & 802.75 & 802.75 & 122.5994 & 11.33362 \\
\hline 284 & 7.8113 & 789.6021 & 803.2917 & 803.2917 & 122.5994 & 11.30899 \\
\hline 285 & 7.8283 & 794.6463 & 803.8333 & 803.8333 & 122.5994 & 11.28447 \\
\hline 286 & 7.8453 & 827.7194 & 804.375 & 804.375 & 122.5994 & 11.26006 \\
\hline 287 & 7.8623 & 780.9883 & 804.9167 & 804.9167 & 122.5994 & 11.23575 \\
\hline 288 & 7.8793 & 800.2155 & 805.4583 & 805.4583 & 122.5994 & 11.21154 \\
\hline 289 & 7.8963 & 818.1093 & 806 & 806 & 122.5994 & 11.18745 \\
\hline 290 & 7.9133 & 800.1897 & 806.75 & 806.75 & 122.5994 & 11.16345 \\
\hline 291 & 7.9303 & 815.2191 & 807.5 & 807.5 & 122.5994 & 11.13956 \\
\hline 292 & 7.9473 & 809.5533 & 808.25 & 808.25 & 122.5994 & 11.11577 \\
\hline
\end{tabular}




\begin{tabular}{|c|c|c|c|c|c|c|}
\hline 293 & 7.9643 & 806.769 & 809 & 809 & 122.5994 & 11.09208 \\
\hline 294 & 7.9813 & 780.1031 & 809.75 & 809.75 & 122.5994 & 11.06849 \\
\hline 295 & 7.9983 & 798.795 & 810.5 & 810.5 & 122.5994 & 11.045 \\
\hline 296 & 8.0153 & 815.6225 & 811.25 & 811.25 & 122.5994 & 11.02162 \\
\hline 297 & 8.0323 & 783.4394 & 812 & 812 & 122.5994 & 10.99833 \\
\hline 298 & 8.0493 & 768.4855 & 812.75 & 812.75 & 122.5994 & 10.97514 \\
\hline 299 & 8.0663 & 782.5685 & 813.5 & 813.5 & 122.5994 & 10.95204 \\
\hline 300 & 8.0833 & 812.2652 & 814.25 & 814.25 & 122.5994 & 10.92905 \\
\hline 301 & 8.1003 & 791.7681 & 815 & 815 & 122.5994 & 10.90615 \\
\hline 302 & 8.1173 & 780.6196 & 815.75 & 815.75 & 122.5994 & 10.88335 \\
\hline 303 & 8.1343 & 798.0682 & 816.5 & 816.5 & 122.5994 & 10.86064 \\
\hline 304 & 8.1513 & 809.7254 & 817.25 & 817.25 & 122.5994 & 10.83803 \\
\hline 305 & 8.1683 & 811.8085 & 822.9 & 818 & 122.5994 & 10.81551 \\
\hline 306 & 8.1853 & 829.2148 & 823.8954 & 818.75 & 122.5994 & 10.79309 \\
\hline 307 & 8.2023 & 870.8538 & 827.4323 & 819.5 & 122.5994 & 10.77075 \\
\hline 308 & 8.2193 & 828.4581 & 828.5959 & 820.25 & 122.5994 & 10.74852 \\
\hline 309 & 8.2363 & 810.9938 & 829.7925 & 821 & 122.5994 & 10.72637 \\
\hline 310 & 8.2533 & 850.0328 & 831.0258 & 821.75 & 122.5994 & 10.70431 \\
\hline 311 & 8.2703 & 806.7863 & 832.2997 & 822.5 & 122.5994 & 10.68235 \\
\hline 312 & 8.2873 & 801.8274 & 833.619 & 823.25 & 122.5994 & 10.66047 \\
\hline 313 & 8.3043 & 836.8617 & 834.9889 & 824 & 122.5994 & 10.63869 \\
\hline 314 & 8.3213 & 851.1085 & 834.1865 & 822.5208 & 122.5994 & 10.61699 \\
\hline 315 & 8.3383 & 809.2546 & 833.4482 & 821.0417 & 122.5994 & 10.59538 \\
\hline 316 & 8.3553 & 839.8923 & 832.7819 & 819.5625 & 122.5994 & 10.57386 \\
\hline 317 & 8.3723 & 866.6798 & 832.1974 & 818.0833 & 122.5994 & 10.55243 \\
\hline 318 & 8.3893 & 889.7654 & 831.7061 & 816.6042 & 122.5994 & 10.53109 \\
\hline 319 & 8.4063 & 874.0159 & 831.3209 & 815.125 & 122.5994 & 10.50983 \\
\hline 320 & 8.4233 & 855.007 & 831.0575 & 813.6458 & 122.5994 & 10.48865 \\
\hline 321 & 8.4403 & 863.0307 & 830.9344 & 812.1667 & 122.5994 & 10.46757 \\
\hline 322 & 8.4573 & 924.122 & 830.9739 & 810.6875 & 122.5994 & 10.44656 \\
\hline 323 & 8.4743 & 918.9268 & 831.2025 & 809.2083 & 122.5994 & 10.42565 \\
\hline 324 & 8.4913 & 929.6563 & 831.6526 & 807.7292 & 122.5994 & 10.40481 \\
\hline 325 & 8.5083 & 930.2674 & 832.3643 & 806.25 & 122.5994 & 10.38406 \\
\hline 326 & 8.5253 & 991.7662 & 833.3875 & 804.7708 & 122.5994 & 10.36339 \\
\hline 327 & 8.5423 & 936.1157 & 834.7882 & 803.2917 & 122.5994 & 10.34281 \\
\hline 328 & 8.5593 & 920.0365 & 836.6553 & 801.8125 & 122.5994 & 10.3223 \\
\hline 329 & 8.5763 & 973.4582 & 839.1172 & 800.3333 & 122.5994 & 10.30188 \\
\hline 330 & 8.5933 & 972.477 & 842.3694 & 798.8542 & 122.5994 & 10.28154 \\
\hline 331 & 8.6103 & 971.1751 & 846.7217 & 797.375 & 122.5994 & 10.26128 \\
\hline 332 & 8.6273 & 1061.195 & 852.6702 & 795.8958 & 122.5994 & 10.24109 \\
\hline 333 & 8.6443 & 1065.017 & 861 & 794.4167 & 122.5994 & 10.22099 \\
\hline 334 & 8.6613 & 1147.09 & 872.9096 & 792.9375 & 122.5994 & 10.20097 \\
\hline 335 & 8.6783 & 1122.545 & 890.1282 & 791.4583 & 122.5994 & 10.18102 \\
\hline 336 & 8.6953 & 1213.966 & 914.9814 & 789.9792 & 122.5994 & 10.16116 \\
\hline 337 & 8.7123 & 1247.889 & 950.3152 & 788.5 & 122.5994 & 10.14137 \\
\hline 338 & 8.7293 & 1328.649 & 999.2244 & 787.0208 & 122.5994 & 10.12166 \\
\hline 339 & 8.7463 & 1361.858 & 1064.524 & 785.5417 & 122.5994 & 10.10202 \\
\hline 340 & 8.7633 & 1436.896 & 1147.978 & 784.0625 & 122.5994 & 10.08246 \\
\hline 341 & 8.7803 & 1481.886 & 1249.359 & 782.5833 & 122.5994 & 10.06298 \\
\hline
\end{tabular}




\begin{tabular}{|c|c|c|c|c|c|c|}
\hline 342 & 8.7973 & 1537.61 & 1365.276 & 781.1042 & 122.5994 & 10.04357 \\
\hline 343 & 8.8143 & 1663.019 & 1487.79 & 779.625 & 122.5994 & 10.02424 \\
\hline 344 & 8.8313 & 1815.676 & 1602.946 & 778.1458 & 122.5994 & 10.00498 \\
\hline 345 & 8.8483 & 1973.765 & 1690.923 & 776.6667 & 122.5994 & 9.9858 \\
\hline 346 & 8.8653 & 2085.545 & 1731.163 & 775.1875 & 122.5994 & 9.96669 \\
\hline 347 & 8.8823 & 2288.857 & 1712.472 & 773.7083 & 122.5994 & 9.94765 \\
\hline 348 & 8.8993 & 2588.891 & 1639.943 & 772.2292 & 122.5994 & 9.92869 \\
\hline 349 & 8.9163 & 2704.449 & 1531.344 & 770.75 & 122.5994 & 9.90979 \\
\hline 350 & 8.9333 & 2451.411 & 1407.405 & 769.2708 & 122.5994 & 9.89097 \\
\hline 351 & 8.9503 & 1959.56 & 1284.636 & 767.7917 & 122.5994 & 9.87222 \\
\hline 352 & 8.9673 & 1455.361 & 1173.455 & 766.3125 & 122.5994 & 9.85355 \\
\hline 353 & 8.9843 & 1093.865 & 1079.091 & 764.8333 & 122.5994 & 9.83494 \\
\hline 354 & 9.0013 & 941.9769 & 1003.032 & 763.3542 & 122.5994 & 9.8164 \\
\hline 355 & 9.0183 & 893.5335 & 944.2781 & 761.875 & 122.5994 & 9.79794 \\
\hline 356 & 9.0353 & 867.0433 & 900.3735 & 760.3958 & 122.5994 & 9.77954 \\
\hline 357 & 9.0523 & 820.5012 & 868.2848 & 758.9167 & 122.5994 & 9.76121 \\
\hline 358 & 9.0693 & 797.0098 & 845.0214 & 757.4375 & 122.5994 & 9.74295 \\
\hline 359 & 9.0863 & 779.5117 & 828.0211 & 755.9583 & 122.5994 & 9.72476 \\
\hline 360 & 9.1033 & 776.8891 & 815.2982 & 754.4792 & 122.5994 & 9.70664 \\
\hline 361 & 9.1203 & 778.4245 & 805.4349 & 753 & 122.5994 & 9.68859 \\
\hline 362 & 9.1373 & 707.8053 & 795.2502 & 749.2917 & 122.5994 & 9.6706 \\
\hline 363 & 9.1543 & 724.2964 & 786.3618 & 745.5833 & 122.5994 & 9.65268 \\
\hline 364 & 9.1713 & 738.8244 & 778.387 & 741.875 & 122.5994 & 9.63482 \\
\hline 365 & 9.1883 & 712.9573 & 771.0851 & 738.1667 & 122.5994 & 9.61704 \\
\hline 366 & 9.2053 & 712.1221 & 764.3016 & 734.4583 & 122.5994 & 9.59931 \\
\hline 367 & 9.2223 & 708.2397 & 757.932 & 730.75 & 122.5994 & 9.58166 \\
\hline 368 & 9.2393 & 683.9109 & 751.9012 & 727.0417 & 122.5994 & 9.56407 \\
\hline 369 & 9.2563 & 695.6812 & 746.153 & 723.3333 & 122.5994 & 9.54654 \\
\hline 370 & 9.2733 & 653.0875 & 740.643 & 719.625 & 122.5994 & 9.52908 \\
\hline 371 & 9.2903 & 746.8159 & 735.3358 & 715.9167 & 122.5994 & 9.51168 \\
\hline 372 & 9.3073 & 718.8217 & 730.2021 & 712.2083 & 122.5994 & 9.49434 \\
\hline 373 & 9.3243 & 697.0408 & 725.2183 & 708.5 & 122.5994 & 9.47707 \\
\hline 374 & 9.3413 & 721.7473 & 720.3641 & 704.7917 & 122.5994 & 9.45986 \\
\hline 375 & 9.3583 & 649.7835 & 715.6227 & 701.0833 & 122.5994 & 9.44272 \\
\hline 376 & 9.3753 & 641.8177 & 710.9799 & 697.375 & 122.5994 & 9.42563 \\
\hline 377 & 9.3923 & 667.527 & 706.4236 & 693.6667 & 122.5994 & 9.40861 \\
\hline 378 & 9.4093 & 683.5553 & 701.9436 & 689.9583 & 122.5994 & 9.39165 \\
\hline 379 & 9.4263 & 659.7815 & 697.5311 & 686.25 & 122.5994 & 9.37475 \\
\hline 380 & 9.4433 & 656.1657 & 693.1785 & 682.5417 & 122.5994 & 9.35791 \\
\hline 381 & 9.4603 & 662.3208 & 688.879 & 678.8333 & 122.5994 & 9.34113 \\
\hline 382 & 9.4773 & 667.7299 & 684.6273 & 675.125 & 122.5994 & 9.32441 \\
\hline 383 & 9.4943 & 657.6258 & 680.4183 & 671.4167 & 122.5994 & 9.30776 \\
\hline 384 & 9.5113 & 671.924 & 676.2476 & 667.7083 & 122.5994 & 9.29116 \\
\hline 385 & 9.5283 & 672.8666 & 672.1115 & 664 & 122.5994 & 9.27462 \\
\hline 386 & 9.5453 & 637.388 & 670.0482 & 662.3333 & 122.5994 & 9.25814 \\
\hline 387 & 9.5623 & 684.4223 & 663.2111 & 660.6667 & 122.5994 & 9.24172 \\
\hline 388 & 9.5793 & 691.6633 & 659 & 659 & 122.5994 & 9.22536 \\
\hline 389 & 9.5963 & 677.2688 & 657.3333 & 657.3333 & 122.5994 & 9.20905 \\
\hline 390 & 9.6133 & 654.0494 & 655.6667 & 655.6667 & 122.5994 & 9.19281 \\
\hline
\end{tabular}




\begin{tabular}{|c|c|c|c|c|c|c|}
\hline 391 & 9.6303 & 666.534 & 654 & 654 & 122.5994 & 9.17662 \\
\hline 392 & 9.6473 & 661.1868 & 652.3333 & 652.3333 & 122.5994 & 9.16048 \\
\hline 393 & 9.6643 & 633.5469 & 650.6667 & 650.6667 & 122.5994 & 9.14441 \\
\hline 394 & 9.6813 & 631.855 & 649 & 649 & 122.5994 & 9.12839 \\
\hline 395 & 9.6983 & 604.9409 & 647.3333 & 647.3333 & 122.5994 & 9.11243 \\
\hline 396 & 9.7153 & 593.179 & 645.6667 & 645.6667 & 122.5994 & 9.09652 \\
\hline 397 & 9.7323 & 614.8235 & 644 & 644 & 122.5994 & 9.08067 \\
\hline 398 & 9.7493 & 607.4296 & 642.3333 & 642.3333 & 122.5994 & 9.06487 \\
\hline 399 & 9.7663 & 630.5791 & 640.6667 & 640.6667 & 122.5994 & 9.04913 \\
\hline 400 & 9.7833 & 618.116 & 639 & 639 & 122.5994 & 9.03344 \\
\hline 401 & 9.8003 & 583.6292 & 637.3333 & 637.3333 & 122.5994 & 9.01781 \\
\hline 402 & 9.8173 & 609.9311 & 635.6667 & 635.6667 & 122.5994 & 9.00224 \\
\hline 403 & 9.8343 & 623.526 & 634 & 634 & 122.5994 & 8.98671 \\
\hline 404 & 9.8513 & 633.4608 & 632.3333 & 632.3333 & 122.5994 & 8.97124 \\
\hline 405 & 9.8683 & 633.1207 & 630.6667 & 630.6667 & 122.5994 & 8.95583 \\
\hline 406 & 9.8853 & 626.444 & 629 & 629 & 122.5994 & 8.94046 \\
\hline 407 & 9.9023 & 614.0081 & 627.3333 & 627.3333 & 122.5994 & 8.92515 \\
\hline 408 & 9.9193 & 613.8492 & 625.6667 & 625.6667 & 122.5994 & 8.90989 \\
\hline 409 & 9.9363 & 650.442 & 624 & 624 & 122.5994 & 8.89469 \\
\hline 410 & 9.9533 & 624.0979 & 622.8333 & 622.8333 & 122.5994 & 8.87953 \\
\hline 411 & 9.9703 & 594.8792 & 621.6667 & 621.6667 & 122.5994 & 8.86443 \\
\hline 412 & 9.9873 & 598.7308 & 620.5 & 620.5 & 122.5994 & 8.84938 \\
\hline 413 & 10.0043 & 600.2268 & 619.3333 & 619.3333 & 122.5994 & 8.83438 \\
\hline 414 & 10.0213 & 622.1954 & 618.1667 & 618.1667 & 122.5994 & 8.81943 \\
\hline 415 & 10.0383 & 629.5596 & 617 & 617 & 122.5994 & 8.80454 \\
\hline 416 & 10.0553 & 584.267 & 615.8333 & 615.8333 & 122.5994 & 8.78969 \\
\hline 417 & 10.0723 & 618.0913 & 614.6667 & 614.6667 & 122.5994 & 8.77489 \\
\hline 418 & 10.0893 & 558.7174 & 613.5 & 613.5 & 122.5994 & 8.76014 \\
\hline 419 & 10.1063 & 634.5335 & 612.3333 & 612.3333 & 122.5994 & 8.74545 \\
\hline 420 & 10.1233 & 605.4757 & 611.1667 & 611.1667 & 122.5994 & 8.7308 \\
\hline 421 & 10.1403 & 624.7921 & 610 & 610 & 122.5994 & 8.7162 \\
\hline 422 & 10.1573 & 616.6183 & 608.8333 & 608.8333 & 122.5994 & 8.70165 \\
\hline 423 & 10.1743 & 564.781 & 607.6667 & 607.6667 & 122.5994 & 8.68715 \\
\hline 424 & 10.1913 & 603.2435 & 606.5 & 606.5 & 122.5994 & 8.6727 \\
\hline 425 & 10.2083 & 623.975 & 605.3333 & 605.3333 & 122.5994 & 8.65829 \\
\hline 426 & 10.2253 & 582.9224 & 604.1667 & 604.1667 & 122.5994 & 8.64394 \\
\hline 427 & 10.2423 & 600.2547 & 603 & 603 & 122.5994 & 8.62963 \\
\hline 428 & 10.2593 & 618.5428 & 601.8333 & 601.8333 & 122.5994 & 8.61537 \\
\hline 429 & 10.2763 & 593.5668 & 600.6667 & 600.6667 & 122.5994 & 8.60115 \\
\hline 430 & 10.2933 & 607.4136 & 599.5 & 599.5 & 122.5994 & 8.58698 \\
\hline 431 & 10.3103 & 598.0467 & 598.3333 & 598.3333 & 122.5994 & 8.57286 \\
\hline 432 & 10.3273 & 557.9863 & 597.1667 & 597.1667 & 122.5994 & 8.55879 \\
\hline 433 & 10.3443 & 589.9275 & 596 & 596 & 122.5994 & 8.54476 \\
\hline 434 & 10.3613 & 616.0885 & 594.75 & 594.75 & 122.5994 & 8.53078 \\
\hline 435 & 10.3783 & 641.7256 & 593.5 & 593.5 & 122.5994 & 8.51685 \\
\hline 436 & 10.3953 & 621.3947 & 592.25 & 592.25 & 122.5994 & 8.50296 \\
\hline 437 & 10.4123 & 602.0067 & 591 & 591 & 122.5994 & 8.48911 \\
\hline 438 & 10.4293 & 576.9556 & 589.75 & 589.75 & 122.5994 & 8.47531 \\
\hline 439 & 10.4463 & 587.6772 & 588.5 & 588.5 & 122.5994 & 8.46156 \\
\hline
\end{tabular}




\begin{tabular}{|c|c|c|c|c|c|c|}
\hline 440 & 10.4633 & 594.9095 & 587.25 & 587.25 & 122.5994 & 8.44785 \\
\hline 441 & 10.4803 & 601.2429 & 586 & 586 & 122.5994 & 8.43418 \\
\hline 442 & 10.4973 & 632.4436 & 584.75 & 584.75 & 122.5994 & 8.42056 \\
\hline 443 & 10.5143 & 627.3837 & 583.5 & 583.5 & 122.5994 & 8.40699 \\
\hline 444 & 10.5313 & 633.3944 & 582.25 & 582.25 & 122.5994 & 8.39345 \\
\hline 445 & 10.5483 & 628.4727 & 581 & 581 & 122.5994 & 8.37996 \\
\hline 446 & 10.5653 & 612.2066 & 579.75 & 579.75 & 122.5994 & 8.36652 \\
\hline 447 & 10.5823 & 592.6426 & 578.5 & 578.5 & 122.5994 & 8.35312 \\
\hline 448 & 10.5993 & 583.8049 & 577.25 & 577.25 & 122.5994 & 8.33976 \\
\hline 449 & 10.6163 & 594.9573 & 576 & 576 & 122.5994 & 8.32644 \\
\hline 450 & 10.6333 & 600.2612 & 574.75 & 574.75 & 122.5994 & 8.31317 \\
\hline 451 & 10.6503 & 583.7142 & 573.5 & 573.5 & 122.5994 & 8.29994 \\
\hline 452 & 10.6673 & 603.4553 & 572.25 & 572.25 & 122.5994 & 8.28675 \\
\hline 453 & 10.6843 & 576.3533 & 571 & 571 & 122.5994 & 8.2736 \\
\hline 454 & 10.7013 & 590.501 & 569.75 & 569.75 & 122.5994 & 8.26049 \\
\hline 455 & 10.7183 & 549.0221 & 568.5 & 568.5 & 122.5994 & 8.24743 \\
\hline 456 & 10.7353 & 563.3207 & 567.25 & 567.25 & 122.5994 & 8.23441 \\
\hline 457 & 10.7523 & 571.2207 & 566 & 566 & 122.5994 & 8.22143 \\
\hline 458 & 10.7693 & 567.2149 & 566.7083 & 566.7083 & 122.5994 & 8.20849 \\
\hline 459 & 10.7863 & 578.7292 & 567.4167 & 567.4167 & 122.5994 & 8.19559 \\
\hline 460 & 10.8033 & 576.6073 & 568.125 & 568.125 & 122.5994 & 8.18273 \\
\hline 461 & 10.8203 & 606.5726 & 568.8333 & 568.8333 & 122.5994 & 8.16991 \\
\hline 462 & 10.8373 & 565.6214 & 569.5417 & 569.5417 & 122.5994 & 8.15713 \\
\hline 463 & 10.8543 & 535.2901 & 570.25 & 570.25 & 122.5994 & 8.1444 \\
\hline 464 & 10.8713 & 565.1725 & 570.9583 & 570.9583 & 122.5994 & 8.1317 \\
\hline 465 & 10.8883 & 571.1421 & 571.6667 & 571.6667 & 122.5994 & 8.11904 \\
\hline 466 & 10.9053 & 552.2287 & 572.375 & 572.375 & 122.5994 & 8.10642 \\
\hline 467 & 10.9223 & 553.5365 & 573.0833 & 573.0833 & 122.5994 & 8.09384 \\
\hline 468 & 10.9393 & 599.0943 & 573.7917 & 573.7917 & 122.5994 & 8.0813 \\
\hline 469 & 10.9563 & 580.0869 & 574.5 & 574.5 & 122.5994 & 8.0688 \\
\hline 470 & 10.9733 & 628.8 & 575.2083 & 575.2083 & 122.5994 & 8.05634 \\
\hline 471 & 10.9903 & 562.7517 & 575.9167 & 575.9167 & 122.5994 & 8.04392 \\
\hline 472 & 11.0073 & 538.6333 & 576.625 & 576.625 & 122.5994 & 8.03153 \\
\hline 473 & 11.0243 & 571.9664 & 577.3333 & 577.3333 & 122.5994 & 8.01919 \\
\hline 474 & 11.0413 & 567.6816 & 578.0417 & 578.0417 & 122.5994 & 8.00688 \\
\hline 475 & 11.0583 & 533.5992 & 578.75 & 578.75 & 122.5994 & 7.99461 \\
\hline 476 & 11.0753 & 561.7544 & 579.4583 & 579.4583 & 122.5994 & 7.98237 \\
\hline 477 & 11.0923 & 593.0212 & 580.1667 & 580.1667 & 122.5994 & 7.97018 \\
\hline 478 & 11.1093 & 580.5631 & 580.875 & 580.875 & 122.5994 & 7.95802 \\
\hline 479 & 11.1263 & 569.1731 & 581.5833 & 581.5833 & 122.5994 & 7.9459 \\
\hline 480 & 11.1433 & 581.941 & 582.2917 & 582.2917 & 122.5994 & 7.93381 \\
\hline 481 & 11.1603 & 593.7407 & 583 & 583 & 122.5994 & 7.92177 \\
\hline 482 & 11.1773 & 582.0998 & 583.3333 & 583.3333 & 122.5994 & 7.90976 \\
\hline 483 & 11.1943 & 569.996 & 583.6667 & 583.6667 & 122.5994 & 7.89778 \\
\hline 484 & 11.2113 & 566.6597 & 584 & 584 & 122.5994 & 7.88585 \\
\hline 485 & 11.2283 & 558.1332 & 584.3333 & 584.3333 & 122.5994 & 7.87394 \\
\hline 486 & 11.2453 & 583.3096 & 584.6667 & 584.6667 & 122.5994 & 7.86208 \\
\hline 487 & 11.2623 & 570.3403 & 585 & 585 & 122.5994 & 7.85025 \\
\hline 488 & 11.2793 & 551.4956 & 585.3333 & 585.3333 & 122.5994 & 7.83846 \\
\hline
\end{tabular}




\begin{tabular}{|c|c|c|c|c|c|c|}
\hline 489 & 11.2963 & 571.2312 & 585.6667 & 585.6667 & 122.5994 & 7.8267 \\
\hline 490 & 11.3133 & 561.5069 & 586 & 586 & 122.5994 & 7.81498 \\
\hline 491 & 11.3303 & 554.3811 & 586.3333 & 586.3333 & 122.5994 & 7.80329 \\
\hline 492 & 11.3473 & 573.8469 & 586.6667 & 586.6667 & 122.5994 & 7.79164 \\
\hline 493 & 11.3643 & 582.0094 & 587 & 587 & 122.5994 & 7.78002 \\
\hline 494 & 11.3813 & 581.8342 & 587.3333 & 587.3333 & 122.5994 & 7.76844 \\
\hline 495 & 11.3983 & 570.5852 & 587.6667 & 587.6667 & 122.5994 & 7.75689 \\
\hline 496 & 11.4153 & 558.6232 & 588 & 588 & 122.5994 & 7.74537 \\
\hline 497 & 11.4323 & 582.7457 & 588.3333 & 588.3333 & 122.5994 & 7.73389 \\
\hline 498 & 11.4493 & 572.0177 & 588.6667 & 588.6667 & 122.5994 & 7.72245 \\
\hline 499 & 11.4663 & 518.8426 & 589 & 589 & 122.5994 & 7.71104 \\
\hline 500 & 11.4833 & 563.5275 & 589.3333 & 589.3333 & 122.5994 & 7.69966 \\
\hline 501 & 11.5003 & 563.9073 & 589.6667 & 589.6667 & 122.5994 & 7.68832 \\
\hline 502 & 11.5173 & 553.2981 & 590 & 590 & 122.5994 & 7.67701 \\
\hline 503 & 11.5343 & 571.2562 & 590.3333 & 590.3333 & 122.5994 & 7.66573 \\
\hline 504 & 11.5513 & 560.3467 & 590.6667 & 590.6667 & 122.5994 & 7.65449 \\
\hline 505 & 11.5683 & 606.0031 & 591 & 591 & 122.5994 & 7.64328 \\
\hline 506 & 11.5853 & 615.0163 & 589.875 & 589.875 & 122.5994 & 7.6321 \\
\hline 507 & 11.6023 & 588.9666 & 588.75 & 588.75 & 122.5994 & 7.62095 \\
\hline 508 & 11.6193 & 630.5444 & 587.625 & 587.625 & 122.5994 & 7.60984 \\
\hline 509 & 11.6363 & 630.3363 & 586.5 & 586.5 & 122.5994 & 7.59876 \\
\hline 510 & 11.6533 & 606.3699 & 585.375 & 585.375 & 122.5994 & 7.58772 \\
\hline 511 & 11.6703 & 629.3584 & 584.25 & 584.25 & 122.5994 & 7.5767 \\
\hline 512 & 11.6873 & 665.4304 & 583.125 & 583.125 & 122.5994 & 7.56572 \\
\hline 513 & 11.7043 & 572.43 & 582 & 582 & 122.5994 & 7.55477 \\
\hline 514 & 11.7213 & 643.4361 & 580.875 & 580.875 & 122.5994 & 7.54385 \\
\hline 515 & 11.7383 & 597.972 & 579.75 & 579.75 & 122.5994 & 7.53296 \\
\hline 516 & 11.7553 & 560.5574 & 578.625 & 578.625 & 122.5994 & 7.52211 \\
\hline 517 & 11.7723 & 549.4718 & 577.5 & 577.5 & 122.5994 & 7.51128 \\
\hline 518 & 11.7893 & 565.2759 & 576.375 & 576.375 & 122.5994 & 7.50049 \\
\hline 519 & 11.8063 & 554.2476 & 575.25 & 575.25 & 122.5994 & 7.48973 \\
\hline 520 & 11.8233 & 538.2192 & 574.125 & 574.125 & 122.5994 & 7.479 \\
\hline 521 & 11.8403 & 545.3664 & 573 & 573 & 122.5994 & 7.4683 \\
\hline 522 & 11.8573 & 577.0208 & 571.875 & 571.875 & 122.5994 & 7.45763 \\
\hline 523 & 11.8743 & 581.9056 & 570.75 & 570.75 & 122.5994 & 7.44699 \\
\hline 524 & 11.8913 & 576.0164 & 569.625 & 569.625 & 122.5994 & 7.43638 \\
\hline 525 & 11.9083 & 559.3564 & 568.5 & 568.5 & 122.5994 & 7.4258 \\
\hline 526 & 11.9253 & 575.0001 & 567.375 & 567.375 & 122.5994 & 7.41525 \\
\hline 527 & 11.9423 & 550.241 & 566.25 & 566.25 & 122.5994 & 7.40474 \\
\hline 528 & 11.9593 & 567.9573 & 567.5945 & 565.125 & 122.5994 & 7.39425 \\
\hline 529 & 11.9763 & 566.0168 & 566.6219 & 564 & 122.5994 & 7.38379 \\
\hline 530 & 11.9933 & 575.8894 & 567.3416 & 563.3125 & 122.5994 & 7.37336 \\
\hline 531 & 12.0103 & 550.3673 & 566.9142 & 562.625 & 122.5994 & 7.36297 \\
\hline 532 & 12.0273 & 559.6292 & 566.5128 & 561.9375 & 122.5994 & 7.3526 \\
\hline 533 & 12.0443 & 573.8713 & 566.1407 & 561.25 & 122.5994 & 7.34226 \\
\hline 534 & 12.0613 & 587.1156 & 565.8022 & 560.5625 & 122.5994 & 7.33195 \\
\hline 535 & 12.0783 & 595.6119 & 565.5021 & 559.875 & 122.5994 & 7.32166 \\
\hline 536 & 12.0953 & 585.6174 & 565.2463 & 559.1875 & 122.5994 & 7.31141 \\
\hline 537 & 12.1123 & 590.8291 & 565.0417 & 558.5 & 122.5994 & 7.30119 \\
\hline
\end{tabular}




\begin{tabular}{|c|c|c|c|c|c|c|}
\hline 538 & 12.1293 & 595.3711 & 564.8965 & 557.8125 & 122.5994 & 7.29099 \\
\hline 539 & 12.1463 & 603.8178 & 564.821 & 557.125 & 122.5994 & 7.28083 \\
\hline 540 & 12.1633 & 589.7401 & 564.8274 & 556.4375 & 122.5994 & 7.27069 \\
\hline 541 & 12.1803 & 578.9873 & 564.9308 & 555.75 & 122.5994 & 7.26058 \\
\hline 542 & 12.1973 & 599.5354 & 565.1499 & 555.0625 & 122.5994 & 7.2505 \\
\hline 543 & 12.2143 & 613.3775 & 565.5081 & 554.375 & 122.5994 & 7.24045 \\
\hline 544 & 12.2313 & 642.6048 & 566.0355 & 553.6875 & 122.5994 & 7.23042 \\
\hline 545 & 12.2483 & 658.9505 & 566.7709 & 553 & 122.5994 & 7.22042 \\
\hline 546 & 12.2653 & 660.5023 & 567.7681 & 552.3125 & 122.5994 & 7.21045 \\
\hline 547 & 12.2823 & 637.706 & 569.1058 & 551.625 & 122.5994 & 7.20051 \\
\hline 548 & 12.2993 & 702.0005 & 570.9081 & 550.9375 & 122.5994 & 7.1906 \\
\hline 549 & 12.3163 & 698.3772 & 573.3867 & 550.25 & 122.5994 & 7.18071 \\
\hline 550 & 12.3333 & 670.5384 & 576.9096 & 549.5625 & 122.5994 & 7.17085 \\
\hline 551 & 12.3503 & 669.9258 & 582.1104 & 548.875 & 122.5994 & 7.16102 \\
\hline 552 & 12.3673 & 670.8225 & 590.0219 & 548.1875 & 122.5994 & 7.15121 \\
\hline 553 & 12.3843 & 740.9393 & 602.1927 & 547.5 & 122.5994 & 7.14144 \\
\hline 554 & 12.4013 & 688.2414 & 620.7017 & 546.8125 & 122.5994 & 7.13168 \\
\hline 555 & 12.4183 & 745.2101 & 647.9463 & 546.125 & 122.5994 & 7.12196 \\
\hline 556 & 12.4353 & 792.6544 & 686.1317 & 545.4375 & 122.5994 & 7.11226 \\
\hline 557 & 12.4523 & 773.6374 & 736.4385 & 544.75 & 122.5994 & 7.10259 \\
\hline 558 & 12.4693 & 817.2781 & 797.9035 & 544.0625 & 122.5994 & 7.09294 \\
\hline 559 & 12.4863 & 823.9048 & 865.9961 & 543.375 & 122.5994 & 7.08333 \\
\hline 560 & 12.5033 & 866.8725 & 930.9785 & 542.6875 & 122.5994 & 7.07373 \\
\hline 561 & 12.5203 & 955.7432 & 978.0574 & 542 & 122.5994 & 7.06417 \\
\hline 562 & 12.5373 & 984.3839 & 993.1959 & 541.3125 & 122.5994 & 7.05463 \\
\hline 563 & 12.5543 & 1125.908 & 972.0268 & 540.625 & 122.5994 & 7.04511 \\
\hline 564 & 12.5713 & 1079.624 & 921.7174 & 539.9375 & 122.5994 & 7.03562 \\
\hline 565 & 12.5883 & 948.1774 & 855.7156 & 539.25 & 122.5994 & 7.02616 \\
\hline 566 & 12.6053 & 871.6217 & 787.3667 & 538.5625 & 122.5994 & 7.01672 \\
\hline 567 & 12.6223 & 687.3885 & 725.7738 & 537.875 & 122.5994 & 7.00731 \\
\hline 568 & 12.6393 & 679.3454 & 675.2494 & 537.1875 & 122.5994 & 6.99792 \\
\hline 569 & 12.6563 & 620.2053 & 636.6314 & 536.5 & 122.5994 & 6.98856 \\
\hline 570 & 12.6733 & 576.406 & 608.6835 & 535.8125 & 122.5994 & 6.97923 \\
\hline 571 & 12.6903 & 547.5389 & 589.2192 & 535.125 & 122.5994 & 6.96991 \\
\hline 572 & 12.7073 & 539.6482 & 575.9058 & 534.4375 & 122.5994 & 6.96063 \\
\hline 573 & 12.7243 & 525.5045 & 566.7368 & 533.75 & 122.5994 & 6.95137 \\
\hline 574 & 12.7413 & 557.0004 & 560.2194 & 533.0625 & 122.5994 & 6.94213 \\
\hline 575 & 12.7583 & 573.7379 & 555.3539 & 532.375 & 122.5994 & 6.93292 \\
\hline 576 & 12.7753 & 521.7759 & 551.523 & 531.6875 & 122.5994 & 6.92373 \\
\hline 577 & 12.7923 & 517.9502 & 548.3642 & 531 & 122.5994 & 6.91457 \\
\hline 578 & 12.8093 & 533.306 & 545.1052 & 529.75 & 122.5994 & 6.90543 \\
\hline 579 & 12.8263 & 536.0496 & 542.1844 & 528.5 & 122.5994 & 6.89631 \\
\hline 580 & 12.8433 & 544.166 & 539.5233 & 527.25 & 122.5994 & 6.88722 \\
\hline 581 & 12.8603 & 544.6522 & 537.0685 & 526 & 122.5994 & 6.87816 \\
\hline 582 & 12.8773 & 556.9714 & 534.7811 & 524.75 & 122.5994 & 6.86912 \\
\hline 583 & 12.8943 & 542.5209 & 532.6315 & 523.5 & 122.5994 & 6.8601 \\
\hline 584 & 12.9113 & 526.0988 & 530.5966 & 522.25 & 122.5994 & 6.8511 \\
\hline 585 & 12.9283 & 509.6252 & 528.6577 & 521 & 122.5994 & 6.84213 \\
\hline 586 & 12.9453 & 520.4669 & 526.8 & 519.75 & 122.5994 & 6.83319 \\
\hline
\end{tabular}




\begin{tabular}{|c|c|c|c|c|c|c|}
\hline 587 & 12.9623 & 522.4412 & 525.0114 & 518.5 & 122.5994 & 6.82426 \\
\hline 588 & 12.9793 & 506.7749 & 523.2817 & 517.25 & 122.5994 & 6.81536 \\
\hline 589 & 12.9963 & 528.5695 & 521.6028 & 516 & 122.5994 & 6.80649 \\
\hline 590 & 13.0133 & 515.1855 & 519.9678 & 514.75 & 122.5994 & 6.79763 \\
\hline 591 & 13.0303 & 523.8927 & 518.3709 & 513.5 & 122.5994 & 6.7888 \\
\hline 592 & 13.0473 & 543.6102 & 516.8073 & 512.25 & 122.5994 & 6.78 \\
\hline 593 & 13.0643 & 527.092 & 515.2728 & 511 & 122.5994 & 6.77121 \\
\hline 594 & 13.0813 & 544.454 & 513.7641 & 509.75 & 122.5994 & 6.76245 \\
\hline 595 & 13.0983 & 541.3228 & 512.2781 & 508.5 & 122.5994 & 6.75371 \\
\hline 596 & 13.1153 & 538.2192 & 508.5197 & 507.25 & 122.5994 & 6.74499 \\
\hline 597 & 13.1323 & 500.8539 & 507.1968 & 506 & 122.5994 & 6.7363 \\
\hline 598 & 13.1493 & 507.9959 & 504.75 & 504.75 & 122.5994 & 6.72763 \\
\hline 599 & 13.1663 & 530.5448 & 503.5 & 503.5 & 122.5994 & 6.71898 \\
\hline 600 & 13.1833 & 505.3292 & 502.25 & 502.25 & 122.5994 & 6.71036 \\
\hline 601 & 13.2003 & 489.6237 & 501 & 501 & 122.5994 & 6.70175 \\
\hline 602 & 13.2173 & 480.196 & 501.5 & 501.5 & 122.5994 & 6.69317 \\
\hline 603 & 13.2343 & 535.4816 & 502 & 502 & 122.5994 & 6.68461 \\
\hline 604 & 13.2513 & 515.8891 & 502.5 & 502.5 & 122.5994 & 6.67607 \\
\hline 605 & 13.2683 & 501.7968 & 503 & 503 & 122.5994 & 6.66756 \\
\hline 606 & 13.2853 & 511.3275 & 503.5 & 503.5 & 122.5994 & 6.65907 \\
\hline 607 & 13.3023 & 540.411 & 504 & 504 & 122.5994 & 6.65059 \\
\hline 608 & 13.3193 & 480.6865 & 504.5 & 504.5 & 122.5994 & 6.64214 \\
\hline 609 & 13.3363 & 498.4027 & 505 & 505 & 122.5994 & 6.63371 \\
\hline 610 & 13.3533 & 510.274 & 505.5 & 505.5 & 122.5994 & 6.62531 \\
\hline 611 & 13.3703 & 506.8229 & 506 & 506 & 122.5994 & 6.61692 \\
\hline 612 & 13.3873 & 508.145 & 506.5 & 506.5 & 122.5994 & 6.60856 \\
\hline 613 & 13.4043 & 494.319 & 507 & 507 & 122.5994 & 6.60021 \\
\hline 614 & 13.4213 & 509.5624 & 507.5 & 507.5 & 122.5994 & 6.59189 \\
\hline 615 & 13.4383 & 531.4044 & 508 & 508 & 122.5994 & 6.58359 \\
\hline 616 & 13.4553 & 492.5387 & 508.5 & 508.5 & 122.5994 & 6.57531 \\
\hline 617 & 13.4723 & 508.5558 & 509 & 509 & 122.5994 & 6.56705 \\
\hline 618 & 13.4893 & 524.1918 & 509.5 & 509.5 & 122.5994 & 6.55881 \\
\hline 619 & 13.5063 & 528.6594 & 510 & 510 & 122.5994 & 6.5506 \\
\hline 620 & 13.5233 & 501.2882 & 510.5 & 510.5 & 122.5994 & 6.5424 \\
\hline 621 & 13.5403 & 535.1075 & 511 & 511 & 122.5994 & 6.53423 \\
\hline 622 & 13.5573 & 519.1589 & 511.5 & 511.5 & 122.5994 & 6.52607 \\
\hline 623 & 13.5743 & 517.4982 & 512.0888 & 512 & 122.5994 & 6.51793 \\
\hline 624 & 13.5913 & 529.8987 & 512.7789 & 512.5 & 122.5994 & 6.50982 \\
\hline 625 & 13.6083 & 512.7002 & 513.3593 & 513 & 122.5994 & 6.50173 \\
\hline 626 & 13.6253 & 505.2201 & 513.2892 & 512.7917 & 122.5994 & 6.49365 \\
\hline 627 & 13.6423 & 501.2573 & 513.1756 & 512.5833 & 122.5994 & 6.4856 \\
\hline 628 & 13.6593 & 524.2549 & 513.0588 & 512.375 & 122.5994 & 6.47757 \\
\hline 629 & 13.6763 & 552.6636 & 512.9762 & 512.1667 & 122.5994 & 6.46955 \\
\hline 630 & 13.6933 & 534.3244 & 512.9148 & 511.9583 & 122.5994 & 6.46156 \\
\hline 631 & 13.7103 & 518.8921 & 512.8973 & 511.75 & 122.5994 & 6.45358 \\
\hline 632 & 13.7273 & 515.8583 & 512.943 & 511.5417 & 122.5994 & 6.44563 \\
\hline 633 & 13.7443 & 517.1245 & 513.0854 & 511.3333 & 122.5994 & 6.4377 \\
\hline 634 & 13.7613 & 524.4198 & 513.3958 & 511.125 & 122.5994 & 6.42978 \\
\hline 635 & 13.7783 & 506.9975 & 514.1182 & 510.9167 & 122.5994 & 6.42189 \\
\hline
\end{tabular}




\begin{tabular}{|c|c|c|c|c|c|c|}
\hline 636 & 13.7953 & 503.2384 & 515.6674 & 510.7083 & 122.5994 & 6.41401 \\
\hline 637 & 13.8123 & 521.829 & 519.2134 & 510.5 & 122.5994 & 6.40615 \\
\hline 638 & 13.8293 & 538.7839 & 526.2122 & 510.2917 & 122.5994 & 6.39832 \\
\hline 639 & 13.8463 & 519.5291 & 537.2291 & 510.0833 & 122.5994 & 6.3905 \\
\hline 640 & 13.8633 & 545.3698 & 549.0923 & 509.875 & 122.5994 & 6.3827 \\
\hline 641 & 13.8803 & 560.5825 & 555.0016 & 509.6667 & 122.5994 & 6.37492 \\
\hline 642 & 13.8973 & 543.1296 & 551.9866 & 509.4583 & 122.5994 & 6.36716 \\
\hline 643 & 13.9143 & 542.0327 & 543.7147 & 509.25 & 122.5994 & 6.35942 \\
\hline 644 & 13.9313 & 546.7682 & 533.5259 & 509.0417 & 122.5994 & 6.3517 \\
\hline 645 & 13.9483 & 500.7034 & 524.6757 & 508.8333 & 122.5994 & 6.344 \\
\hline 646 & 13.9653 & 561.6596 & 518.7294 & 508.625 & 122.5994 & 6.33631 \\
\hline 647 & 13.9823 & 520.8881 & 515.1633 & 508.4167 & 122.5994 & 6.32865 \\
\hline 648 & 13.9993 & 498.7211 & 513.4039 & 508.2083 & 122.5994 & 6.321 \\
\hline 649 & 14.0163 & 514.2655 & 513.205 & 508 & 122.5994 & 6.31337 \\
\hline 650 & 14.0333 & 502.2266 & 513.7706 & 507.1667 & 122.5994 & 6.30576 \\
\hline 651 & 14.0503 & 501.7694 & 515.1016 & 506.3333 & 122.5994 & 6.29817 \\
\hline 652 & 14.0673 & 528.7237 & 515.4801 & 505.5 & 122.5994 & 6.2906 \\
\hline 653 & 14.0843 & 493.4915 & 513.8925 & 504.6667 & 122.5994 & 6.28304 \\
\hline 654 & 14.1013 & 514.8463 & 511.1933 & 503.8333 & 122.5994 & 6.27551 \\
\hline 655 & 14.1183 & 504.3224 & 508.0199 & 503 & 122.5994 & 6.26799 \\
\hline 656 & 14.1353 & 481.6824 & 505.2636 & 502.1667 & 122.5994 & 6.26049 \\
\hline 657 & 14.1523 & 500.2382 & 503.2402 & 501.3333 & 122.5994 & 6.25301 \\
\hline 658 & 14.1693 & 493.9183 & 501.6963 & 500.5 & 122.5994 & 6.24554 \\
\hline 659 & 14.1863 & 524.714 & 500.3884 & 499.6667 & 122.5994 & 6.2381 \\
\hline 660 & 14.2033 & 498.5861 & 499.353 & 498.8333 & 122.5994 & 6.23067 \\
\hline 661 & 14.2203 & 484.8665 & 498.3408 & 498 & 122.5994 & 6.22326 \\
\hline 662 & 14.2373 & 491.3862 & 497.4238 & 497.1667 & 122.5994 & 6.21587 \\
\hline 663 & 14.2543 & 498.5148 & 496.5456 & 496.3333 & 122.5994 & 6.20849 \\
\hline 664 & 14.2713 & 487.2213 & 495.6681 & 495.5 & 122.5994 & 6.20113 \\
\hline 665 & 14.2883 & 480.2491 & 494.8093 & 494.6667 & 122.5994 & 6.19379 \\
\hline 666 & 14.3053 & 466.912 & 493.9559 & 493.8333 & 122.5994 & 6.18647 \\
\hline 667 & 14.3223 & 476.88 & 493.1064 & 493 & 122.5994 & 6.17917 \\
\hline 668 & 14.3393 & 502.734 & 492.2599 & 492.1667 & 122.5994 & 6.17188 \\
\hline 669 & 14.3563 & 487.8783 & 491.3655 & 491.3333 & 122.5994 & 6.16461 \\
\hline 670 & 14.3733 & 494.7645 & 490.5284 & 490.5 & 122.5994 & 6.15736 \\
\hline 671 & 14.3903 & 476.7281 & 489.6919 & 489.6667 & 122.5994 & 6.15012 \\
\hline 672 & 14.4073 & 476.1266 & 488.8333 & 488.8333 & 122.5994 & 6.1429 \\
\hline 673 & 14.4243 & 500.7561 & 488 & 488 & 122.5994 & 6.1357 \\
\hline 674 & 14.4413 & 493.9384 & 487.5 & 487.5 & 122.5994 & 6.12851 \\
\hline 675 & 14.4583 & 479.6935 & 487 & 487 & 122.5994 & 6.12135 \\
\hline 676 & 14.4753 & 483.8122 & 486.5 & 486.5 & 122.5994 & 6.1142 \\
\hline 677 & 14.4923 & 489.2269 & 486 & 486 & 122.5994 & 6.10706 \\
\hline 678 & 14.5093 & 497.2993 & 485.5 & 485.5 & 122.5994 & 6.09995 \\
\hline 679 & 14.5263 & 479.2282 & 485 & 485 & 122.5994 & 6.09284 \\
\hline 680 & 14.5433 & 471.8328 & 484.5 & 484.5 & 122.5994 & 6.08576 \\
\hline 681 & 14.5603 & 500.5234 & 484 & 484 & 122.5994 & 6.07869 \\
\hline 682 & 14.5773 & 475.099 & 483.5 & 483.5 & 122.5994 & 6.07164 \\
\hline 683 & 14.5943 & 483.0626 & 483 & 483 & 122.5994 & 6.06461 \\
\hline 684 & 14.6113 & 485.0007 & 482.5 & 482.5 & 122.5994 & 6.05759 \\
\hline
\end{tabular}




\begin{tabular}{|c|c|c|c|c|c|c|}
\hline 685 & 14.6283 & 495.504 & 482 & 482 & 122.5994 & 6.05059 \\
\hline 686 & 14.6453 & 508.3883 & 481.5 & 481.5 & 122.5994 & 6.0436 \\
\hline 687 & 14.6623 & 535.1938 & 481 & 481 & 122.5994 & 6.03664 \\
\hline 688 & 14.6793 & 514.2585 & 480.5 & 480.5 & 122.5994 & 6.02968 \\
\hline 689 & 14.6963 & 509.8528 & 480.1279 & 480 & 122.5994 & 6.02275 \\
\hline 690 & 14.7133 & 488.7001 & 479.6443 & 479.5 & 122.5994 & 6.01583 \\
\hline 691 & 14.7303 & 454.7626 & 479.2261 & 479 & 122.5994 & 6.00892 \\
\hline 692 & 14.7473 & 490.9435 & 478.7578 & 478.5 & 122.5994 & 6.00203 \\
\hline 693 & 14.7643 & 485.481 & 478.2968 & 478 & 122.5994 & 5.99516 \\
\hline 694 & 14.7813 & 492.0605 & 477.8452 & 477.5 & 122.5994 & 5.9883 \\
\hline 695 & 14.7983 & 501.1369 & 477.4063 & 477 & 122.5994 & 5.98146 \\
\hline 696 & 14.8153 & 484.8297 & 476.9852 & 476.5 & 122.5994 & 5.97464 \\
\hline 697 & 14.8323 & 492.6311 & 476.5893 & 476 & 122.5994 & 5.96783 \\
\hline 698 & 14.8493 & 436.9689 & 477.189 & 476.4583 & 122.5994 & 5.96103 \\
\hline 699 & 14.8663 & 480.026 & 477.8486 & 476.9167 & 122.5994 & 5.95425 \\
\hline 700 & 14.8833 & 501.0328 & 478.6234 & 477.375 & 122.5994 & 5.94749 \\
\hline 701 & 14.9003 & 486.7716 & 479.6743 & 477.8333 & 122.5994 & 5.94074 \\
\hline 702 & 14.9173 & 527.4973 & 481.4142 & 478.2917 & 122.5994 & 5.93401 \\
\hline 703 & 14.9343 & 507.9465 & 484.577 & 478.75 & 122.5994 & 5.9273 \\
\hline 704 & 14.9513 & 479.1239 & 489.8027 & 479.2083 & 122.5994 & 5.92059 \\
\hline 705 & 14.9683 & 450.2914 & 496.4627 & 479.6667 & 122.5994 & 5.91391 \\
\hline 706 & 14.9853 & 475.2123 & 501.0793 & 480.125 & 122.5994 & 5.90724 \\
\hline 707 & 15.0023 & 456.9748 & 500.771 & 480.5833 & 122.5994 & 5.90058 \\
\hline 708 & 15.0193 & 472.7476 & 497.6678 & 481.0417 & 122.5994 & 5.89394 \\
\hline 709 & 15.0363 & 476.5711 & 493.0827 & 481.5 & 122.5994 & 5.88732 \\
\hline 710 & 15.0533 & 473.2442 & 488.8724 & 481.9583 & 122.5994 & 5.88071 \\
\hline 711 & 15.0703 & 489.1902 & 486.2691 & 482.4167 & 122.5994 & 5.87411 \\
\hline 712 & 15.0873 & 478.3163 & 485.0833 & 482.875 & 122.5994 & 5.86753 \\
\hline 713 & 15.1043 & 468.3347 & 484.7447 & 483.3333 & 122.5994 & 5.86096 \\
\hline 714 & 15.1213 & 459.5657 & 484.8031 & 483.7917 & 122.5994 & 5.85441 \\
\hline 715 & 15.1383 & 480.4728 & 485.0283 & 484.25 & 122.5994 & 5.84788 \\
\hline 716 & 15.1553 & 487.1298 & 485.3303 & 484.7083 & 122.5994 & 5.84136 \\
\hline 717 & 15.1723 & 483.4216 & 485.6757 & 485.1667 & 122.5994 & 5.83485 \\
\hline 718 & 15.1893 & 488.5818 & 486.0493 & 485.625 & 122.5994 & 5.82836 \\
\hline 719 & 15.2063 & 503.1765 & 486.4423 & 486.0833 & 122.5994 & 5.82188 \\
\hline 720 & 15.2233 & 511.0904 & 486.8493 & 486.5417 & 122.5994 & 5.81542 \\
\hline 721 & 15.2403 & 456.3511 & 487.2666 & 487 & 122.5994 & 5.80897 \\
\hline 722 & 15.2573 & 477.0709 & 486.4416 & 486.2083 & 122.5994 & 5.80253 \\
\hline 723 & 15.2743 & 503.2943 & 485.6224 & 485.4167 & 122.5994 & 5.79611 \\
\hline 724 & 15.2913 & 485.4895 & 484.6965 & 484.625 & 122.5994 & 5.78971 \\
\hline 725 & 15.3083 & 447.6697 & 483.8968 & 483.8333 & 122.5994 & 5.78332 \\
\hline 726 & 15.3253 & 462.7535 & 483.0417 & 483.0417 & 122.5994 & 5.77694 \\
\hline 727 & 15.3423 & 477.542 & 482.25 & 482.25 & 122.5994 & 5.77058 \\
\hline 728 & 15.3593 & 476.4728 & 481.4583 & 481.4583 & 122.5994 & 5.76423 \\
\hline 729 & 15.3763 & 470.9557 & 480.6667 & 480.6667 & 122.5994 & 5.75789 \\
\hline 730 & 15.3933 & 432.0252 & 479.875 & 479.875 & 122.5994 & 5.75157 \\
\hline 731 & 15.4103 & 439.6684 & 479.0833 & 479.0833 & 122.5994 & 5.74527 \\
\hline 732 & 15.4273 & 490.8288 & 478.2917 & 478.2917 & 122.5994 & 5.73897 \\
\hline 733 & 15.4443 & 466.8428 & 477.5 & 477.5 & 122.5994 & 5.73269 \\
\hline
\end{tabular}




\begin{tabular}{|c|c|c|c|c|c|c|}
\hline 734 & 15.4613 & 440.651 & 476.7083 & 476.7083 & 122.5994 & 5.72643 \\
\hline 735 & 15.4783 & 460.4084 & 475.9167 & 475.9167 & 122.5994 & 5.72018 \\
\hline 736 & 15.4953 & 495.2385 & 475.125 & 475.125 & 122.5994 & 5.71394 \\
\hline 737 & 15.5123 & 486.894 & 474.3333 & 474.3333 & 122.5994 & 5.70772 \\
\hline 738 & 15.5293 & 484.009 & 473.5417 & 473.5417 & 122.5994 & 5.70151 \\
\hline 739 & 15.5463 & 499.6206 & 472.75 & 472.75 & 122.5994 & 5.69531 \\
\hline 740 & 15.5633 & 447.0257 & 471.9583 & 471.9583 & 122.5994 & 5.68913 \\
\hline 741 & 15.5803 & 441.0082 & 471.1667 & 471.1667 & 122.5994 & 5.68296 \\
\hline 742 & 15.5973 & 469.2118 & 470.375 & 470.375 & 122.5994 & 5.6768 \\
\hline 743 & 15.6143 & 442.0263 & 469.6632 & 469.5833 & 122.5994 & 5.67066 \\
\hline 744 & 15.6313 & 470.4974 & 468.8819 & 468.7917 & 122.5994 & 5.66453 \\
\hline 745 & 15.6483 & 483.7707 & 468.141 & 468 & 122.5994 & 5.65842 \\
\hline 746 & 15.6653 & 458.3516 & 467.8278 & 467.6667 & 122.5994 & 5.65231 \\
\hline 747 & 15.6823 & 470.2373 & 467.5193 & 467.3333 & 122.5994 & 5.64622 \\
\hline 748 & 15.6993 & 457.0162 & 467.2169 & 467 & 122.5994 & 5.64015 \\
\hline 749 & 15.7163 & 450.9664 & 466.9228 & 466.6667 & 122.5994 & 5.63409 \\
\hline 750 & 15.7333 & 506.2595 & 466.6405 & 466.3333 & 122.5994 & 5.62804 \\
\hline 751 & 15.7503 & 497.8568 & 466.3748 & 466 & 122.5994 & 5.622 \\
\hline 752 & 15.7673 & 448.6069 & 466.1343 & 465.6667 & 122.5994 & 5.61598 \\
\hline 753 & 15.7843 & 468.921 & 465.9359 & 465.3333 & 122.5994 & 5.60997 \\
\hline 754 & 15.8013 & 483.1866 & 465.8235 & 465 & 122.5994 & 5.60397 \\
\hline 755 & 15.8183 & 484.7032 & 465.9256 & 464.6667 & 122.5994 & 5.59798 \\
\hline 756 & 15.8353 & 454.788 & 466.5451 & 464.3333 & 122.5994 & 5.59201 \\
\hline 757 & 15.8523 & 496.1141 & 468.1469 & 464 & 122.5994 & 5.58605 \\
\hline 758 & 15.8693 & 491.546 & 470.9884 & 463.6667 & 122.5994 & 5.58011 \\
\hline 759 & 15.8863 & 485.6969 & 474.2874 & 463.3333 & 122.5994 & 5.57418 \\
\hline 760 & 15.9033 & 479.619 & 475.6111 & 463 & 122.5994 & 5.56826 \\
\hline 761 & 15.9203 & 473.4745 & 474.2227 & 462.6667 & 122.5994 & 5.56235 \\
\hline 762 & 15.9373 & 462.2586 & 471.6513 & 462.3333 & 122.5994 & 5.55645 \\
\hline 763 & 15.9543 & 477.8821 & 468.3704 & 462 & 122.5994 & 5.55057 \\
\hline 764 & 15.9713 & 481.6999 & 465.4396 & 461.6667 & 122.5994 & 5.5447 \\
\hline 765 & 15.9883 & 465.0629 & 463.4426 & 461.3333 & 122.5994 & 5.53884 \\
\hline 766 & 16.0053 & 477.5816 & 462.2258 & 461 & 122.5994 & 5.533 \\
\hline 767 & 16.0223 & 475.2489 & 461.4642 & 460.6667 & 122.5994 & 5.52717 \\
\hline 768 & 16.0393 & 462.2717 & 460.9125 & 460.3333 & 122.5994 & 5.52135 \\
\hline 769 & 16.0563 & 453.9174 & 460.4491 & 460 & 122.5994 & 5.51554 \\
\hline 770 & 16.0733 & 453.3161 & 461.3606 & 461 & 122.5994 & 5.50974 \\
\hline 771 & 16.0903 & 466.5178 & 462.2962 & 462 & 122.5994 & 5.50396 \\
\hline 772 & 16.1073 & 474.4828 & 463.2476 & 463 & 122.5994 & 5.49819 \\
\hline 773 & 16.1243 & 460.3973 & 464.2101 & 464 & 122.5994 & 5.49243 \\
\hline 774 & 16.1413 & 470.0821 & 465.1804 & 465 & 122.5994 & 5.48668 \\
\hline 775 & 16.1583 & 446.2059 & 466.1566 & 466 & 122.5994 & 5.48095 \\
\hline 776 & 16.1753 & 460.0615 & 467.1373 & 467 & 122.5994 & 5.47523 \\
\hline 777 & 16.1923 & 463.2101 & 468.0482 & 468 & 122.5994 & 5.46952 \\
\hline 778 & 16.2093 & 479.7825 & 469.0425 & 469 & 122.5994 & 5.46382 \\
\hline 779 & 16.2263 & 475.4605 & 470.0378 & 470 & 122.5994 & 5.45813 \\
\hline 780 & 16.2433 & 494.3964 & 471 & 471 & 122.5994 & 5.45246 \\
\hline 781 & 16.2603 & 474.2178 & 472 & 472 & 122.5994 & 5.4468 \\
\hline 782 & 16.2773 & 429.3239 & 473 & 473 & 122.5994 & 5.44115 \\
\hline
\end{tabular}




\begin{tabular}{|c|c|c|c|c|c|c|}
\hline 783 & 16.2943 & 458.5 & 474 & 474 & 122.5994 & 5.43551 \\
\hline 784 & 16.3113 & 459.2293 & 475 & 475 & 122.5994 & 5.42988 \\
\hline 785 & 16.3283 & 484.4217 & 476 & 476 & 122.5994 & 5.42427 \\
\hline 786 & 16.3453 & 446.2112 & 477 & 477 & 122.5994 & 5.41866 \\
\hline 787 & 16.3623 & 480.2161 & 478 & 478 & 122.5994 & 5.41307 \\
\hline 788 & 16.3793 & 475.8329 & 479 & 479 & 122.5994 & 5.40749 \\
\hline 789 & 16.3963 & 434.571 & 480 & 480 & 122.5994 & 5.40192 \\
\hline 790 & 16.4133 & 482.7413 & 481 & 481 & 122.5994 & 5.39637 \\
\hline 791 & 16.4303 & 470.9274 & 482 & 482 & 122.5994 & 5.39082 \\
\hline 792 & 16.4473 & 484.6157 & 483 & 483 & 122.5994 & 5.38529 \\
\hline 793 & 16.4643 & 513.6606 & 484 & 484 & 122.5994 & 5.37977 \\
\hline 794 & 16.4813 & 472.5881 & 483 & 483 & 122.5994 & 5.37426 \\
\hline 795 & 16.4983 & 435.4437 & 482 & 482 & 122.5994 & 5.36876 \\
\hline 796 & 16.5153 & 467.0252 & 481 & 481 & 122.5994 & 5.36327 \\
\hline 797 & 16.5323 & 470.8988 & 480 & 480 & 122.5994 & 5.35779 \\
\hline 798 & 16.5493 & 467.7138 & 479 & 479 & 122.5994 & 5.35233 \\
\hline 799 & 16.5663 & 449.4484 & 478 & 478 & 122.5994 & 5.34687 \\
\hline 800 & 16.5833 & 456.0685 & 477 & 477 & 122.5994 & 5.34143 \\
\hline 801 & 16.6003 & 488.6923 & 476 & 476 & 122.5994 & 5.336 \\
\hline 802 & 16.6173 & 504.1583 & 475 & 475 & 122.5994 & 5.33058 \\
\hline 803 & 16.6343 & 486.1358 & 474 & 474 & 122.5994 & 5.32517 \\
\hline 804 & 16.6513 & 454.8837 & 473 & 473 & 122.5994 & 5.31977 \\
\hline 805 & 16.6683 & 455.4201 & 472 & 472 & 122.5994 & 5.31438 \\
\hline 806 & 16.6853 & 456.3263 & 471 & 471 & 122.5994 & 5.309 \\
\hline 807 & 16.7023 & 472.3328 & 470 & 470 & 122.5994 & 5.30364 \\
\hline 808 & 16.7193 & 472.0928 & 469 & 469 & 122.5994 & 5.29828 \\
\hline 809 & 16.7363 & 468.2364 & 468 & 468 & 122.5994 & 5.29294 \\
\hline 810 & 16.7533 & 474.445 & 467 & 467 & 122.5994 & 5.28761 \\
\hline 811 & 16.7703 & 464.8886 & 466 & 466 & 122.5994 & 5.28229 \\
\hline 812 & 16.7873 & 464.6473 & 465 & 465 & 122.5994 & 5.27698 \\
\hline 813 & 16.8043 & 494.6532 & 464 & 464 & 122.5994 & 5.27168 \\
\hline 814 & 16.8213 & 488.703 & 463 & 463 & 122.5994 & 5.26639 \\
\hline 815 & 16.8383 & 453.3425 & 462 & 462 & 122.5994 & 5.26111 \\
\hline 816 & 16.8553 & 443.926 & 461 & 461 & 122.5994 & 5.25584 \\
\hline 817 & 16.8723 & 475.517 & 460 & 460 & 122.5994 & 5.25058 \\
\hline 818 & 16.8893 & 460.4168 & 459.9583 & 459.9583 & 122.5994 & 5.24534 \\
\hline 819 & 16.9063 & 457.2789 & 459.9167 & 459.9167 & 122.5994 & 5.2401 \\
\hline 820 & 16.9233 & 452.2594 & 459.875 & 459.875 & 122.5994 & 5.23487 \\
\hline 821 & 16.9403 & 438.3127 & 459.8333 & 459.8333 & 122.5994 & 5.22966 \\
\hline 822 & 16.9573 & 442.403 & 459.7917 & 459.7917 & 122.5994 & 5.22445 \\
\hline 823 & 16.9743 & 451.039 & 459.75 & 459.75 & 122.5994 & 5.21926 \\
\hline 824 & 16.9913 & 462.8096 & 459.7083 & 459.7083 & 122.5994 & 5.21408 \\
\hline 825 & 17.0083 & 472.9005 & 459.6667 & 459.6667 & 122.5994 & 5.2089 \\
\hline 826 & 17.0253 & 485.0836 & 459.625 & 459.625 & 122.5994 & 5.20374 \\
\hline 827 & 17.0423 & 458.3177 & 459.5833 & 459.5833 & 122.5994 & 5.19859 \\
\hline 828 & 17.0593 & 469.9326 & 459.5417 & 459.5417 & 122.5994 & 5.19345 \\
\hline 829 & 17.0763 & 478.8126 & 459.5 & 459.5 & 122.5994 & 5.18831 \\
\hline 830 & 17.0933 & 447.3012 & 461.45 & 459.4583 & 122.5994 & 5.18319 \\
\hline 831 & 17.1103 & 474.1574 & 461.5096 & 459.4167 & 122.5994 & 5.17808 \\
\hline
\end{tabular}




\begin{tabular}{|c|c|c|c|c|c|c|}
\hline 832 & 17.1273 & 456.7548 & 461.5769 & 459.375 & 122.5994 & 5.17298 \\
\hline 833 & 17.1443 & 501.0824 & 462.6625 & 459.3333 & 122.5994 & 5.16789 \\
\hline 834 & 17.1613 & 485.7215 & 462.8 & 459.2917 & 122.5994 & 5.16281 \\
\hline 835 & 17.1783 & 471.6255 & 462.9523 & 459.25 & 122.5994 & 5.15774 \\
\hline 836 & 17.1953 & 475.1014 & 463.121 & 459.2083 & 122.5994 & 5.15268 \\
\hline 837 & 17.2123 & 497.0268 & 463.3081 & 459.1667 & 122.5994 & 5.14762 \\
\hline 838 & 17.2293 & 501.4491 & 463.5157 & 459.125 & 122.5994 & 5.14258 \\
\hline 839 & 17.2463 & 465.9147 & 463.7463 & 459.0833 & 122.5994 & 5.13755 \\
\hline 840 & 17.2633 & 446.785 & 464.0028 & 459.0417 & 122.5994 & 5.13253 \\
\hline 841 & 17.2803 & 471.4988 & 464.2886 & 459 & 122.5994 & 5.12752 \\
\hline 842 & 17.2973 & 432.5966 & 464.9619 & 459.3125 & 122.5994 & 5.12252 \\
\hline 843 & 17.3143 & 504.0533 & 465.673 & 459.625 & 122.5994 & 5.11753 \\
\hline 844 & 17.3313 & 456.6766 & 466.4273 & 459.9375 & 122.5994 & 5.11255 \\
\hline 845 & 17.3483 & 473.174 & 467.2313 & 460.25 & 122.5994 & 5.10758 \\
\hline 846 & 17.3653 & 458.4068 & 468.0927 & 460.5625 & 122.5994 & 5.10261 \\
\hline 847 & 17.3823 & 451.5213 & 469.0205 & 460.875 & 122.5994 & 5.09766 \\
\hline 848 & 17.3993 & 491.8554 & 470.0258 & 461.1875 & 122.5994 & 5.09272 \\
\hline 849 & 17.4163 & 488.3632 & 471.1223 & 461.5 & 122.5994 & 5.08779 \\
\hline 850 & 17.4333 & 487.2404 & 472.3265 & 461.8125 & 122.5994 & 5.08286 \\
\hline 851 & 17.4503 & 477.7271 & 473.6602 & 462.125 & 122.5994 & 5.07795 \\
\hline 852 & 17.4673 & 478.7201 & 475.1517 & 462.4375 & 122.5994 & 5.07305 \\
\hline 853 & 17.4843 & 509.3255 & 476.8411 & 462.75 & 122.5994 & 5.06815 \\
\hline 854 & 17.5013 & 480.3877 & 478.7873 & 463.0625 & 122.5994 & 5.06327 \\
\hline 855 & 17.5183 & 449.3969 & 481.0816 & 463.375 & 122.5994 & 5.05839 \\
\hline 856 & 17.5353 & 470.1872 & 483.8693 & 463.6875 & 122.5994 & 5.05353 \\
\hline 857 & 17.5523 & 478.136 & 487.3808 & 464 & 122.5994 & 5.04867 \\
\hline 858 & 17.5693 & 470.0695 & 491.9728 & 464.3125 & 122.5994 & 5.04382 \\
\hline 859 & 17.5863 & 510.1041 & 498.1723 & 464.625 & 122.5994 & 5.03899 \\
\hline 860 & 17.6033 & 535.4883 & 506.7141 & 464.9375 & 122.5994 & 5.03416 \\
\hline 861 & 17.6203 & 531.2884 & 518.5399 & 465.25 & 122.5994 & 5.02934 \\
\hline 862 & 17.6373 & 522.7768 & 534.7463 & 465.5625 & 122.5994 & 5.02453 \\
\hline 863 & 17.6543 & 516.1976 & 556.4493 & 465.875 & 122.5994 & 5.01973 \\
\hline 864 & 17.6713 & 533.1533 & 584.5624 & 466.1875 & 122.5994 & 5.01494 \\
\hline 865 & 17.6883 & 559.7615 & 619.4934 & 466.5 & 122.5994 & 5.01016 \\
\hline 866 & 17.7053 & 570.4528 & 660.7638 & 466.8125 & 122.5994 & 5.00539 \\
\hline 867 & 17.7223 & 610.2074 & 706.5303 & 467.125 & 122.5994 & 5.00062 \\
\hline 868 & 17.7393 & 613.6834 & 753.0185 & 467.4375 & 122.5994 & 4.99587 \\
\hline 869 & 17.7563 & 635.6571 & 794.2177 & 467.75 & 122.5994 & 4.99112 \\
\hline 870 & 17.7733 & 682.8491 & 822.8767 & 468.0625 & 122.5994 & 4.98639 \\
\hline 871 & 17.7903 & 767.654 & 833.3176 & 468.375 & 122.5994 & 4.98166 \\
\hline 872 & 17.8073 & 837.7719 & 824.0831 & 468.6875 & 122.5994 & 4.97694 \\
\hline 873 & 17.8243 & 839.3699 & 797.7905 & 469 & 122.5994 & 4.97224 \\
\hline 874 & 17.8413 & 867.2212 & 759.4809 & 469.3125 & 122.5994 & 4.96754 \\
\hline 875 & 17.8583 & 788.5137 & 715.2178 & 469.625 & 122.5994 & 4.96285 \\
\hline 876 & 17.8753 & 731.1924 & 670.6262 & 469.9375 & 122.5994 & 4.95816 \\
\hline 877 & 17.8923 & 635.7423 & 629.7324 & 470.25 & 122.5994 & 4.95349 \\
\hline 878 & 17.9093 & 598.5808 & 594.7275 & 470.5625 & 122.5994 & 4.94883 \\
\hline 879 & 17.9263 & 595.3711 & 566.3409 & 470.875 & 122.5994 & 4.94417 \\
\hline 880 & 17.9433 & 573.2596 & 544.317 & 471.1875 & 122.5994 & 4.93953 \\
\hline
\end{tabular}




\begin{tabular}{|c|c|c|c|c|c|c|}
\hline 881 & 17.9603 & 531.7839 & 527.8481 & 471.5 & 122.5994 & 4.93489 \\
\hline 882 & 17.9773 & 511.5755 & 515.8806 & 471.8125 & 122.5994 & 4.93026 \\
\hline 883 & 17.9943 & 501.9732 & 507.3467 & 472.125 & 122.5994 & 4.92564 \\
\hline 884 & 18.0113 & 501.7694 & 501.3044 & 472.4375 & 122.5994 & 4.92103 \\
\hline 885 & 18.0283 & 471.0222 & 497.0015 & 472.75 & 122.5994 & 4.91643 \\
\hline 886 & 18.0453 & 480.3594 & 493.883 & 473.0625 & 122.5994 & 4.91184 \\
\hline 887 & 18.0623 & 497.2993 & 491.5635 & 473.375 & 122.5994 & 4.90725 \\
\hline 888 & 18.0793 & 495.4262 & 489.7888 & 473.6875 & 122.5994 & 4.90267 \\
\hline 889 & 18.0963 & 471.6029 & 488.3952 & 474 & 122.5994 & 4.89811 \\
\hline 890 & 18.1133 & 447.0776 & 487.2168 & 474.25 & 122.5994 & 4.89355 \\
\hline 891 & 18.1303 & 483.1746 & 486.2494 & 474.5 & 122.5994 & 4.889 \\
\hline 892 & 18.1473 & 467.3627 & 485.4485 & 474.75 & 122.5994 & 4.88446 \\
\hline 893 & 18.1643 & 524.0696 & 484.7827 & 475 & 122.5994 & 4.87992 \\
\hline 894 & 18.1813 & 477.5214 & 484.229 & 475.25 & 122.5994 & 4.8754 \\
\hline 895 & 18.1983 & 461.8778 & 483.7696 & 475.5 & 122.5994 & 4.87088 \\
\hline 896 & 18.2153 & 491.8516 & 483.3902 & 475.75 & 122.5994 & 4.86638 \\
\hline 897 & 18.2323 & 490.707 & 483.0794 & 476 & 122.5994 & 4.86188 \\
\hline 898 & 18.2493 & 463.5519 & 482.8276 & 476.25 & 122.5994 & 4.85739 \\
\hline 899 & 18.2663 & 472.1775 & 482.6268 & 476.5 & 122.5994 & 4.8529 \\
\hline 900 & 18.2833 & 485.5223 & 482.4705 & 476.75 & 122.5994 & 4.84843 \\
\hline 901 & 18.3003 & 493.2875 & 482.3751 & 477 & 122.5994 & 4.84396 \\
\hline 902 & 18.3173 & 503.5035 & 482.2934 & 477.25 & 122.5994 & 4.83951 \\
\hline 903 & 18.3343 & 484.7765 & 482.2419 & 477.5 & 122.5994 & 4.83506 \\
\hline 904 & 18.3513 & 478.1909 & 482.2284 & 477.75 & 122.5994 & 4.83062 \\
\hline 905 & 18.3683 & 496.7303 & 482.2284 & 478 & 122.5994 & 4.82618 \\
\hline 906 & 18.3853 & 483.7746 & 482.2498 & 478.25 & 122.5994 & 4.82176 \\
\hline 907 & 18.4023 & 481.9162 & 482.2906 & 478.5 & 122.5994 & 4.81734 \\
\hline 908 & 18.4193 & 468.5551 & 482.3487 & 478.75 & 122.5994 & 4.81294 \\
\hline 909 & 18.4363 & 469.2322 & 482.4304 & 479 & 122.5994 & 4.80854 \\
\hline 910 & 18.4533 & 482.5467 & 482.5195 & 479.25 & 122.5994 & 4.80414 \\
\hline 911 & 18.4703 & 490.9876 & 482.6222 & 479.5 & 122.5994 & 4.79976 \\
\hline 912 & 18.4873 & 490.1853 & 480.8935 & 479.75 & 122.5994 & 4.79539 \\
\hline 913 & 18.5043 & 469.4529 & 481.1053 & 480 & 122.5994 & 4.79102 \\
\hline 914 & 18.5213 & 466.3006 & 481.1891 & 481.0625 & 122.5994 & 4.78666 \\
\hline 915 & 18.5383 & 500.4663 & 482.2697 & 482.125 & 122.5994 & 4.78231 \\
\hline 916 & 18.5553 & 518.368 & 483.3546 & 483.1875 & 122.5994 & 4.77797 \\
\hline 917 & 18.5723 & 513.413 & 484.4458 & 484.25 & 122.5994 & 4.77363 \\
\hline 918 & 18.5893 & 500.9307 & 485.5467 & 485.3125 & 122.5994 & 4.7693 \\
\hline 919 & 18.6063 & 487.3058 & 486.6645 & 486.375 & 122.5994 & 4.76498 \\
\hline 920 & 18.6233 & 467.1855 & 487.8132 & 487.4375 & 122.5994 & 4.76067 \\
\hline 921 & 18.6403 & 469.1277 & 489.0168 & 488.5 & 122.5994 & 4.75637 \\
\hline 922 & 18.6573 & 510.81 & 490.3105 & 489.5625 & 122.5994 & 4.75207 \\
\hline 923 & 18.6743 & 464.417 & 491.735 & 490.625 & 122.5994 & 4.74779 \\
\hline 924 & 18.6913 & 502.7512 & 493.3204 & 491.6875 & 122.5994 & 4.74351 \\
\hline 925 & 18.7083 & 518.7695 & 495.0603 & 492.75 & 122.5994 & 4.73923 \\
\hline 926 & 18.7253 & 503.5309 & 496.8737 & 493.8125 & 122.5994 & 4.73497 \\
\hline 927 & 18.7423 & 518.4775 & 498.5751 & 494.875 & 122.5994 & 4.73071 \\
\hline 928 & 18.7593 & 541.5849 & 499.9264 & 495.9375 & 122.5994 & 4.72646 \\
\hline 929 & 18.7763 & 525.0285 & 500.8976 & 497 & 122.5994 & 4.72222 \\
\hline
\end{tabular}




\begin{tabular}{|c|c|c|c|c|c|c|}
\hline 930 & 18.7933 & 556.5992 & 501.6166 & 498.0625 & 122.5994 & 4.71799 \\
\hline 931 & 18.8103 & 581.0185 & 502.1921 & 499.125 & 122.5994 & 4.71376 \\
\hline 932 & 18.8273 & 599.6449 & 502.8232 & 500.1875 & 122.5994 & 4.70955 \\
\hline 933 & 18.8443 & 584.1728 & 503.532 & 501.25 & 122.5994 & 4.70534 \\
\hline 934 & 18.8613 & 642.9233 & 504.4063 & 502.3125 & 122.5994 & 4.70113 \\
\hline 935 & 18.8783 & 606.775 & 505.4095 & 503.375 & 122.5994 & 4.69694 \\
\hline 936 & 18.8953 & 530.0977 & 506.3799 & 504.4375 & 122.5994 & 4.69275 \\
\hline 937 & 18.9123 & 540.2011 & 507.3665 & 505.5 & 122.5994 & 4.68857 \\
\hline 938 & 18.9293 & 542.7073 & 508.2432 & 506.5625 & 122.5994 & 4.6844 \\
\hline 939 & 18.9463 & 505.3385 & 509.1027 & 507.625 & 122.5994 & 4.68023 \\
\hline 940 & 18.9633 & 544.9764 & 510.0962 & 508.6875 & 122.5994 & 4.67608 \\
\hline 941 & 18.9803 & 485.6206 & 511.1208 & 509.75 & 122.5994 & 4.67193 \\
\hline 942 & 18.9973 & 497.5318 & 512.3114 & 510.8125 & 122.5994 & 4.66778 \\
\hline 943 & 19.0143 & 473.2389 & 513.6172 & 511.875 & 122.5994 & 4.66365 \\
\hline 944 & 19.0313 & 480.8916 & 514.8056 & 512.9375 & 122.5994 & 4.65952 \\
\hline 945 & 19.0483 & 482.5869 & 515.7871 & 514 & 122.5994 & 4.6554 \\
\hline 946 & 19.0653 & 482.548 & 516.7331 & 515.0625 & 122.5994 & 4.65129 \\
\hline 947 & 19.0823 & 480.6758 & 517.6656 & 516.125 & 122.5994 & 4.64718 \\
\hline 948 & 19.0993 & 483.1843 & 518.5901 & 517.1875 & 122.5994 & 4.64309 \\
\hline 949 & 19.1163 & 500.1337 & 519.7622 & 518.25 & 122.5994 & 4.63899 \\
\hline 950 & 19.1333 & 482.1697 & 520.9061 & 519.3125 & 122.5994 & 4.63491 \\
\hline 951 & 19.1503 & 493.3413 & 522.199 & 520.375 & 122.5994 & 4.63084 \\
\hline 952 & 19.1673 & 498.3494 & 523.8399 & 521.4375 & 122.5994 & 4.62677 \\
\hline 953 & 19.1843 & 512.4811 & 525.6064 & 522.5 & 122.5994 & 4.6227 \\
\hline 954 & 19.2013 & 526.6741 & 527.7576 & 523.5625 & 122.5994 & 4.61865 \\
\hline 955 & 19.2183 & 515.5506 & 530.6885 & 524.625 & 122.5994 & 4.6146 \\
\hline 956 & 19.2353 & 527.3968 & 533.8549 & 525.6875 & 122.5994 & 4.61056 \\
\hline 957 & 19.2523 & 560.506 & 537.3921 & 526.75 & 122.5994 & 4.60653 \\
\hline 958 & 19.2693 & 563.8742 & 541.3624 & 527.8125 & 122.5994 & 4.6025 \\
\hline 959 & 19.2863 & 535.2063 & 544.4775 & 528.875 & 122.5994 & 4.59849 \\
\hline 960 & 19.3033 & 518.6367 & 547.1432 & 529.9375 & 122.5994 & 4.59447 \\
\hline 961 & 19.3203 & 533.3954 & 549.5593 & 531 & 122.5994 & 4.59047 \\
\hline 962 & 19.3373 & 542.9001 & 552.3829 & 532.875 & 122.5994 & 4.58647 \\
\hline 963 & 19.3543 & 520.4505 & 554.3948 & 534.75 & 122.5994 & 4.58248 \\
\hline 964 & 19.3713 & 547.3874 & 555.4489 & 536.625 & 122.5994 & 4.5785 \\
\hline 965 & 19.3883 & 537.4553 & 556.0663 & 538.5 & 122.5994 & 4.57452 \\
\hline 966 & 19.4053 & 522.0189 & 556.2804 & 540.375 & 122.5994 & 4.57055 \\
\hline 967 & 19.4223 & 552.691 & 556.4407 & 542.25 & 122.5994 & 4.56659 \\
\hline 968 & 19.4393 & 531.6426 & 556.8515 & 544.125 & 122.5994 & 4.56264 \\
\hline 969 & 19.4563 & 539.7177 & 557.8582 & 546 & 122.5994 & 4.55869 \\
\hline 970 & 19.4733 & 554.7866 & 559.4551 & 547.875 & 122.5994 & 4.55475 \\
\hline 971 & 19.4903 & 556.9704 & 561.5059 & 549.75 & 122.5994 & 4.55081 \\
\hline 972 & 19.5073 & 569.6857 & 563.6777 & 551.625 & 122.5994 & 4.54688 \\
\hline 973 & 19.5243 & 553.8944 & 565.6293 & 553.5 & 122.5994 & 4.54296 \\
\hline 974 & 19.5413 & 588.0288 & 567.3875 & 555.375 & 122.5994 & 4.53905 \\
\hline 975 & 19.5583 & 618.7488 & 569.0796 & 557.25 & 122.5994 & 4.53514 \\
\hline 976 & 19.5753 & 591.0704 & 570.8035 & 559.125 & 122.5994 & 4.53124 \\
\hline 977 & 19.5923 & 580.8224 & 572.7243 & 561 & 122.5994 & 4.52735 \\
\hline 978 & 19.6093 & 590.9207 & 575.0048 & 562.875 & 122.5994 & 4.52346 \\
\hline
\end{tabular}




\begin{tabular}{|c|c|c|c|c|c|c|}
\hline 979 & 19.6263 & 612.9321 & 577.7262 & 564.75 & 122.5994 & 4.51958 \\
\hline 980 & 19.6433 & 671.7626 & 580.9387 & 566.625 & 122.5994 & 4.51571 \\
\hline 981 & 19.6603 & 618.8623 & 584.7463 & 568.5 & 122.5994 & 4.51184 \\
\hline 982 & 19.6773 & 705.0818 & 589.3643 & 570.375 & 122.5994 & 4.50798 \\
\hline 983 & 19.6943 & 646.757 & 595.1268 & 572.25 & 122.5994 & 4.50413 \\
\hline 984 & 19.7113 & 692.4961 & 602.4502 & 574.125 & 122.5994 & 4.50029 \\
\hline 985 & 19.7283 & 692.4741 & 611.7214 & 576 & 122.5994 & 4.49645 \\
\hline 986 & 19.7453 & 681.0435 & 621.2125 & 575.9792 & 122.5994 & 4.49261 \\
\hline 987 & 19.7623 & 737.7305 & 632.723 & 575.9583 & 122.5994 & 4.48879 \\
\hline 988 & 19.7793 & 786.9756 & 645.2839 & 575.9375 & 122.5994 & 4.48497 \\
\hline 989 & 19.7963 & 778.4245 & 657.6102 & 575.9167 & 122.5994 & 4.48115 \\
\hline 990 & 19.8133 & 786.2954 & 668.8513 & 575.8958 & 122.5994 & 4.47735 \\
\hline 991 & 19.8303 & 820.4515 & 679.2861 & 575.875 & 122.5994 & 4.47355 \\
\hline 992 & 19.8473 & 834.1345 & 689.1168 & 575.8542 & 122.5994 & 4.46975 \\
\hline 993 & 19.8643 & 795.1637 & 698.4217 & 575.8333 & 122.5994 & 4.46597 \\
\hline 994 & 19.8813 & 807.7594 & 706.7682 & 575.8125 & 122.5994 & 4.46219 \\
\hline 995 & 19.8983 & 803.2523 & 712.4415 & 575.7917 & 122.5994 & 4.45841 \\
\hline 996 & 19.9153 & 803.7067 & 713.2557 & 575.7708 & 122.5994 & 4.45465 \\
\hline 997 & 19.9323 & 803.8208 & 708.0984 & 575.75 & 122.5994 & 4.45088 \\
\hline 998 & 19.9493 & 726.0586 & 697.6108 & 575.7292 & 122.5994 & 4.44713 \\
\hline 999 & 19.9663 & 716.3528 & 683.1802 & 575.7083 & 122.5994 & 4.44338 \\
\hline 1000 & 19.9833 & 712.7499 & 666.6117 & 575.6875 & 122.5994 & 4.43964 \\
\hline 1001 & 20.0003 & 693.696 & 649.9617 & 575.6667 & 122.5994 & 4.4359 \\
\hline 1002 & 20.0173 & 660.1239 & 634.8051 & 575.6458 & 122.5994 & 4.43218 \\
\hline 1003 & 20.0343 & 646.8313 & 622.2111 & 575.625 & 122.5994 & 4.42845 \\
\hline 1004 & 20.0513 & 634.2469 & 612.6131 & 575.6042 & 122.5994 & 4.42474 \\
\hline 1005 & 20.0683 & 612.0944 & 605.5034 & 575.5833 & 122.5994 & 4.42103 \\
\hline 1006 & 20.0853 & 609.4187 & 600.6262 & 575.5625 & 122.5994 & 4.41732 \\
\hline 1007 & 20.1023 & 634.4895 & 597.4543 & 575.5417 & 122.5994 & 4.41363 \\
\hline 1008 & 20.1193 & 618.4965 & 595.4354 & 575.5208 & 122.5994 & 4.40994 \\
\hline 1009 & 20.1363 & 618.8296 & 594.0516 & 575.5 & 122.5994 & 4.40625 \\
\hline 1010 & 20.1533 & 614.4262 & 592.8887 & 575.4792 & 122.5994 & 4.40257 \\
\hline 1011 & 20.1703 & 624.6728 & 591.6973 & 575.4583 & 122.5994 & 4.3989 \\
\hline 1012 & 20.1873 & 611.2397 & 590.4755 & 575.4375 & 122.5994 & 4.39523 \\
\hline 1013 & 20.2043 & 586.3135 & 589.062 & 575.4167 & 122.5994 & 4.39157 \\
\hline 1014 & 20.2213 & 580.8209 & 587.6099 & 575.3958 & 122.5994 & 4.38792 \\
\hline 1015 & 20.2383 & 592.7158 & 586.2175 & 575.375 & 122.5994 & 4.38427 \\
\hline 1016 & 20.2553 & 624.4443 & 584.9661 & 575.3542 & 122.5994 & 4.38063 \\
\hline 1017 & 20.2723 & 567.1558 & 583.8787 & 575.3333 & 122.5994 & 4.377 \\
\hline 1018 & 20.2893 & 599.4194 & 582.9157 & 575.3125 & 122.5994 & 4.37337 \\
\hline 1019 & 20.3063 & 574.496 & 587.3068 & 575.2917 & 122.5994 & 4.36974 \\
\hline 1020 & 20.3233 & 571.2207 & 586.907 & 575.2708 & 122.5994 & 4.36613 \\
\hline 1021 & 20.3403 & 557.7314 & 586.6401 & 575.25 & 122.5994 & 4.36252 \\
\hline 1022 & 20.3573 & 575.404 & 586.6483 & 575.2292 & 122.5994 & 4.35891 \\
\hline 1023 & 20.3743 & 561.8484 & 586.6802 & 575.2083 & 122.5994 & 4.35531 \\
\hline 1024 & 20.3913 & 543.4296 & 586.7913 & 575.1875 & 122.5994 & 4.35172 \\
\hline 1025 & 20.4083 & 590.3426 & 587.1139 & 575.1667 & 122.5994 & 4.34813 \\
\hline 1026 & 20.4253 & 584.6105 & 587.5746 & 575.1458 & 122.5994 & 4.34455 \\
\hline 1027 & 20.4423 & 566.8118 & 588.1775 & 575.125 & 122.5994 & 4.34098 \\
\hline
\end{tabular}




\begin{tabular}{|c|c|c|c|c|c|c|}
\hline 1028 & 20.4593 & 570.7567 & 588.9355 & 575.1042 & 122.5994 & 4.33741 \\
\hline 1029 & 20.4763 & 567.5203 & 589.8731 & 575.0833 & 122.5994 & 4.33385 \\
\hline 1030 & 20.4933 & 574.6703 & 590.8209 & 575.0625 & 122.5994 & 4.33029 \\
\hline 1031 & 20.5103 & 562.6413 & 592.2662 & 575.0417 & 122.5994 & 4.32674 \\
\hline 1032 & 20.5273 & 548.0909 & 594.0752 & 575.0208 & 122.5994 & 4.3232 \\
\hline 1033 & 20.5443 & 573.187 & 596.2764 & 575 & 122.5994 & 4.31966 \\
\hline 1034 & 20.5613 & 589.6941 & 598.2996 & 574.0625 & 122.5994 & 4.31612 \\
\hline 1035 & 20.5783 & 617.4926 & 601.1624 & 573.125 & 122.5994 & 4.3126 \\
\hline 1036 & 20.5953 & 608.7737 & 605.0872 & 572.1875 & 122.5994 & 4.30907 \\
\hline 1037 & 20.6123 & 605.1713 & 609.7787 & 571.25 & 122.5994 & 4.30556 \\
\hline 1038 & 20.6293 & 616.0828 & 616.4368 & 570.3125 & 122.5994 & 4.30205 \\
\hline 1039 & 20.6463 & 630.0844 & 624.549 & 569.375 & 122.5994 & 4.29855 \\
\hline 1040 & 20.6633 & 628.0162 & 633.7125 & 568.4375 & 122.5994 & 4.29505 \\
\hline 1041 & 20.6803 & 662.4438 & 643.9459 & 567.5 & 122.5994 & 4.29156 \\
\hline 1042 & 20.6973 & 686.5324 & 654.402 & 566.5625 & 122.5994 & 4.28807 \\
\hline 1043 & 20.7143 & 720.5085 & 664.685 & 565.625 & 122.5994 & 4.28459 \\
\hline 1044 & 20.7313 & 752.7611 & 675.5172 & 564.6875 & 122.5994 & 4.28111 \\
\hline 1045 & 20.7483 & 818.8085 & 689.7141 & 563.75 & 122.5994 & 4.27764 \\
\hline 1046 & 20.7653 & 872.7017 & 713.842 & 562.8125 & 122.5994 & 4.27418 \\
\hline 1047 & 20.7823 & 918.5616 & 760.9951 & 561.875 & 122.5994 & 4.27072 \\
\hline 1048 & 20.7993 & 978.6654 & 852.7882 & 560.9375 & 122.5994 & 4.26727 \\
\hline 1049 & 20.8163 & 1109.542 & 1014.636 & 560 & 122.5994 & 4.26382 \\
\hline 1050 & 20.8333 & 1230.212 & 1260.285 & 559.0625 & 122.5994 & 4.26038 \\
\hline 1051 & 20.8503 & 1456.711 & 1565.563 & 558.125 & 122.5994 & 4.25695 \\
\hline 1052 & 20.8673 & 1857.741 & 1833.022 & 557.1875 & 122.5994 & 4.25352 \\
\hline 1053 & 20.8843 & 2320.402 & 1925.163 & 556.25 & 122.5994 & 4.25009 \\
\hline 1054 & 20.9013 & 2650.822 & 1845.565 & 555.3125 & 122.5994 & 4.24667 \\
\hline 1055 & 20.9183 & 2482.174 & 1691.372 & 554.375 & 122.5994 & 4.24326 \\
\hline 1056 & 20.9353 & 2033.363 & 1483.769 & 553.4375 & 122.5994 & 4.23985 \\
\hline 1057 & 20.9523 & 1793.74 & 1245.693 & 552.5 & 122.5994 & 4.23645 \\
\hline 1058 & 20.9693 & 1435.783 & 1036.248 & 551.5625 & 122.5994 & 4.23306 \\
\hline 1059 & 20.9863 & 1018.664 & 882.7147 & 550.625 & 122.5994 & 4.22967 \\
\hline 1060 & 21.0033 & 758.0779 & 781.5458 & 549.6875 & 122.5994 & 4.22628 \\
\hline 1061 & 21.0203 & 725.9078 & 718.2666 & 548.75 & 122.5994 & 4.2229 \\
\hline 1062 & 21.0373 & 689.407 & 677.9619 & 547.8125 & 122.5994 & 4.21953 \\
\hline 1063 & 21.0543 & 654.8975 & 650.3511 & 546.875 & 122.5994 & 4.21616 \\
\hline 1064 & 21.0713 & 733.1212 & 630.0977 & 545.9375 & 122.5994 & 4.2128 \\
\hline 1065 & 21.0883 & 589.7864 & 614.7106 & 545 & 122.5994 & 4.20944 \\
\hline 1066 & 21.1053 & 606.7222 & 602.7598 & 544.0625 & 122.5994 & 4.20609 \\
\hline 1067 & 21.1223 & 564.7833 & 593.1323 & 543.125 & 122.5994 & 4.20274 \\
\hline 1068 & 21.1393 & 566.2503 & 585.105 & 542.1875 & 122.5994 & 4.1994 \\
\hline 1069 & 21.1563 & 575.8136 & 578.3293 & 541.25 & 122.5994 & 4.19606 \\
\hline 1070 & 21.1733 & 561.6437 & 572.4943 & 540.3125 & 122.5994 & 4.19273 \\
\hline 1071 & 21.1903 & 502.6318 & 567.3503 & 539.375 & 122.5994 & 4.18941 \\
\hline 1072 & 21.2073 & 543.1052 & 562.8331 & 538.4375 & 122.5994 & 4.18609 \\
\hline 1073 & 21.2243 & 545.7563 & 558.9354 & 537.5 & 122.5994 & 4.18277 \\
\hline 1074 & 21.2413 & 519.06 & 555.5743 & 536.5625 & 122.5994 & 4.17946 \\
\hline 1075 & 21.2583 & 510.7695 & 552.6638 & 535.625 & 122.5994 & 4.17616 \\
\hline 1076 & 21.2753 & 516.7775 & 550.1185 & 534.6875 & 122.5994 & 4.17286 \\
\hline
\end{tabular}




\begin{tabular}{|c|c|c|c|c|c|c|}
\hline 1077 & 21.2923 & 551.2843 & 547.8654 & 533.75 & 122.5994 & 4.16957 \\
\hline 1078 & 21.3093 & 528.7366 & 545.8445 & 532.8125 & 122.5994 & 4.16628 \\
\hline 1079 & 21.3263 & 566.62 & 544.0121 & 531.875 & 122.5994 & 4.163 \\
\hline 1080 & 21.3433 & 533.837 & 542.3405 & 530.9375 & 122.5994 & 4.15972 \\
\hline 1081 & 21.3603 & 505.8685 & 540.817 & 530 & 122.5994 & 4.15645 \\
\hline 1082 & 21.3773 & 555.6141 & 540.5995 & 530.4167 & 122.5994 & 4.15318 \\
\hline 1083 & 21.3943 & 502.3514 & 540.7675 & 530.8333 & 122.5994 & 4.14992 \\
\hline 1084 & 21.4113 & 530.6926 & 541.163 & 531.25 & 122.5994 & 4.14666 \\
\hline 1085 & 21.4283 & 561.8583 & 535.8279 & 531.6667 & 122.5994 & 4.14341 \\
\hline 1086 & 21.4453 & 553.886 & 537.3242 & 532.0833 & 122.5994 & 4.14016 \\
\hline 1087 & 21.4623 & 553.5235 & 539.3286 & 532.5 & 122.5994 & 4.13692 \\
\hline 1088 & 21.4793 & 553.056 & 541.9164 & 532.9167 & 122.5994 & 4.13369 \\
\hline 1089 & 21.4963 & 555.9105 & 545.0481 & 533.3333 & 122.5994 & 4.13046 \\
\hline 1090 & 21.5133 & 551.5606 & 548.471 & 533.75 & 122.5994 & 4.12723 \\
\hline 1091 & 21.5303 & 561.2622 & 551.6782 & 534.1667 & 122.5994 & 4.12401 \\
\hline 1092 & 21.5473 & 573.2657 & 554.2206 & 534.5833 & 122.5994 & 4.12079 \\
\hline 1093 & 21.5643 & 550.5924 & 555.7205 & 535 & 122.5994 & 4.11758 \\
\hline 1094 & 21.5813 & 510.4964 & 556.7737 & 535.4167 & 122.5994 & 4.11438 \\
\hline 1095 & 21.5983 & 539.1934 & 556.8602 & 535.8333 & 122.5994 & 4.11118 \\
\hline 1096 & 21.6153 & 563.1712 & 555.972 & 536.25 & 122.5994 & 4.10798 \\
\hline 1097 & 21.6323 & 562.486 & 554.3846 & 536.6667 & 122.5994 & 4.10479 \\
\hline 1098 & 21.6493 & 527.4257 & 552.4393 & 537.0833 & 122.5994 & 4.10161 \\
\hline 1099 & 21.6663 & 504.5751 & 550.3719 & 537.5 & 122.5994 & 4.09843 \\
\hline 1100 & 21.6833 & 545.1676 & 548.3614 & 537.9167 & 122.5994 & 4.09525 \\
\hline 1101 & 21.7003 & 508.4518 & 546.4658 & 538.3333 & 122.5994 & 4.09209 \\
\hline 1102 & 21.7173 & 549.329 & 545.0418 & 538.75 & 122.5994 & 4.08892 \\
\hline 1103 & 21.7343 & 537.4765 & 543.9894 & 539.1667 & 122.5994 & 4.08576 \\
\hline 1104 & 21.7513 & 529.9355 & 544.8082 & 539.5833 & 122.5994 & 4.08261 \\
\hline 1105 & 21.7683 & 530.1441 & 544.5532 & 540 & 122.5994 & 4.07946 \\
\hline 1106 & 21.7853 & 569.1895 & 543.9697 & 539.8333 & 122.5994 & 4.07631 \\
\hline 1107 & 21.8023 & 517.8138 & 543.5911 & 539.6667 & 122.5994 & 4.07317 \\
\hline 1108 & 21.8193 & 539.1979 & 543.3987 & 539.5 & 122.5994 & 4.07004 \\
\hline 1109 & 21.8363 & 543.6474 & 543.3287 & 539.3333 & 122.5994 & 4.06691 \\
\hline 1110 & 21.8533 & 523.0416 & 543.3901 & 539.1667 & 122.5994 & 4.06378 \\
\hline 1111 & 21.8703 & 536.0983 & 543.5874 & 539 & 122.5994 & 4.06066 \\
\hline 1112 & 21.8873 & 546.6811 & 543.9412 & 538.8333 & 122.5994 & 4.05754 \\
\hline 1113 & 21.9043 & 538.9385 & 544.4915 & 538.6667 & 122.5994 & 4.05443 \\
\hline 1114 & 21.9213 & 538.1884 & 545.3062 & 538.5 & 122.5994 & 4.05133 \\
\hline 1115 & 21.9383 & 565.5788 & 546.4988 & 538.3333 & 122.5994 & 4.04823 \\
\hline 1116 & 21.9553 & 566.4506 & 548.2809 & 538.1667 & 122.5994 & 4.04513 \\
\hline 1117 & 21.9723 & 575.8942 & 551.1581 & 538 & 122.5994 & 4.04204 \\
\hline 1118 & 21.9893 & 567.7565 & 556.5617 & 537.8333 & 122.5994 & 4.03895 \\
\hline 1119 & 22.0063 & 606.7497 & 568.5061 & 537.6667 & 122.5994 & 4.03587 \\
\hline 1120 & 22.0233 & 639.3959 & 594.2299 & 537.5 & 122.5994 & 4.03279 \\
\hline 1121 & 22.0403 & 692.2205 & 640.4711 & 537.3333 & 122.5994 & 4.02972 \\
\hline 1122 & 22.0573 & 706.7268 & 701.8583 & 537.1667 & 122.5994 & 4.02665 \\
\hline 1123 & 22.0743 & 788.0886 & 744.4081 & 537 & 122.5994 & 4.02359 \\
\hline 1124 & 22.0913 & 841.1987 & 734.4225 & 536.8333 & 122.5994 & 4.02053 \\
\hline 1125 & 22.1083 & 797.9149 & 705.0382 & 536.6667 & 122.5994 & 4.01748 \\
\hline
\end{tabular}




\begin{tabular}{|c|c|c|c|c|c|c|}
\hline 1126 & 22.1253 & 726.6332 & 681.4127 & 536.5 & 122.5994 & 4.01443 \\
\hline 1127 & 22.1423 & 692.2086 & 648.8281 & 536.3333 & 122.5994 & 4.01139 \\
\hline 1128 & 22.1593 & 655.9456 & 609.4523 & 536.1667 & 122.5994 & 4.00835 \\
\hline 1129 & 22.1763 & 584.9885 & 578.8901 & 536 & 122.5994 & 4.00532 \\
\hline 1130 & 22.1933 & 547.4685 & 560.6323 & 535.8333 & 122.5994 & 4.00229 \\
\hline 1131 & 22.2103 & 522.9873 & 551.3698 & 535.6667 & 122.5994 & 3.99926 \\
\hline 1132 & 22.2273 & 524.4013 & 547.0796 & 535.5 & 122.5994 & 3.99624 \\
\hline 1133 & 22.2443 & 512.8165 & 544.5286 & 535.3333 & 122.5994 & 3.99322 \\
\hline 1134 & 22.2613 & 499.0592 & 542.8853 & 535.1667 & 122.5994 & 3.99021 \\
\hline 1135 & 22.2783 & 534.5589 & 541.7603 & 535 & 122.5994 & 3.98721 \\
\hline 1136 & 22.2953 & 530.9508 & 541.0714 & 534.8333 & 122.5994 & 3.98421 \\
\hline 1137 & 22.3123 & 533.62 & 540.6265 & 534.6667 & 122.5994 & 3.98121 \\
\hline 1138 & 22.3293 & 533.5588 & 540.4449 & 534.5 & 122.5994 & 3.97822 \\
\hline 1139 & 22.3463 & 542.6697 & 540.4651 & 534.3333 & 122.5994 & 3.97523 \\
\hline 1140 & 22.3633 & 538.1444 & 540.7384 & 534.1667 & 122.5994 & 3.97224 \\
\hline 1141 & 22.3803 & 522.0042 & 539.9377 & 534 & 122.5994 & 3.96927 \\
\hline 1142 & 22.3973 & 509.9038 & 540.6225 & 533.8333 & 122.5994 & 3.96629 \\
\hline 1143 & 22.4143 & 522.6229 & 541.2957 & 533.6667 & 122.5994 & 3.96332 \\
\hline 1144 & 22.4313 & 529.9863 & 541.184 & 533.5 & 122.5994 & 3.96036 \\
\hline 1145 & 22.4483 & 524.4198 & 541.507 & 533.3333 & 122.5994 & 3.9574 \\
\hline 1146 & 22.4653 & 510.0114 & 541.4932 & 533.1667 & 122.5994 & 3.95444 \\
\hline 1147 & 22.4823 & 507.6884 & 541.1761 & 533 & 122.5994 & 3.95149 \\
\hline 1148 & 22.4993 & 516.9367 & 540.6415 & 532.8333 & 122.5994 & 3.94854 \\
\hline 1149 & 22.5163 & 526.2685 & 540.0061 & 532.6667 & 122.5994 & 3.9456 \\
\hline 1150 & 22.5333 & 519.0485 & 539.4164 & 532.5 & 122.5994 & 3.94266 \\
\hline 1151 & 22.5503 & 508.5767 & 539.0244 & 532.3333 & 122.5994 & 3.93973 \\
\hline 1152 & 22.5673 & 552.3185 & 538.9393 & 532.1667 & 122.5994 & 3.9368 \\
\hline 1153 & 22.5843 & 529.7875 & 539.1815 & 532 & 122.5994 & 3.93387 \\
\hline 1154 & 22.6013 & 523.5436 & 540.2593 & 532.4583 & 122.5994 & 3.93095 \\
\hline 1155 & 22.6183 & 528.2287 & 541.261 & 532.9167 & 122.5994 & 3.92803 \\
\hline 1156 & 22.6353 & 532.8735 & 541.8859 & 533.375 & 122.5994 & 3.92512 \\
\hline 1157 & 22.6523 & 521.6017 & 542.1385 & 533.8333 & 122.5994 & 3.92222 \\
\hline 1158 & 22.6693 & 476.0726 & 542.217 & 534.2917 & 122.5994 & 3.91931 \\
\hline 1159 & 22.6863 & 507.414 & 542.2228 & 534.75 & 122.5994 & 3.91641 \\
\hline 1160 & 22.7033 & 527.8676 & 542.2563 & 535.2083 & 122.5994 & 3.91352 \\
\hline 1161 & 22.7203 & 511.5191 & 542.5235 & 535.6667 & 122.5994 & 3.91063 \\
\hline 1162 & 22.7373 & 542.2096 & 543.2209 & 536.125 & 122.5994 & 3.90774 \\
\hline 1163 & 22.7543 & 552.2783 & 544.4883 & 536.5833 & 122.5994 & 3.90486 \\
\hline 1164 & 22.7713 & 560.613 & 546.441 & 537.0417 & 122.5994 & 3.90199 \\
\hline 1165 & 22.7883 & 516.1235 & 549.1897 & 537.5 & 122.5994 & 3.89911 \\
\hline 1166 & 22.8053 & 530.0286 & 553.0952 & 537.9583 & 122.5994 & 3.89625 \\
\hline 1167 & 22.8223 & 483.9067 & 557.7319 & 538.4167 & 122.5994 & 3.89338 \\
\hline 1168 & 22.8393 & 530.5303 & 563.3164 & 538.875 & 122.5994 & 3.89052 \\
\hline 1169 & 22.8563 & 547.8675 & 570.0953 & 539.3333 & 122.5994 & 3.88767 \\
\hline 1170 & 22.8733 & 590.6363 & 576.9326 & 539.7917 & 122.5994 & 3.88482 \\
\hline 1171 & 22.8903 & 640.7943 & 583.5452 & 540.25 & 122.5994 & 3.88197 \\
\hline 1172 & 22.9073 & 613.2988 & 589.1572 & 540.7083 & 122.5994 & 3.87913 \\
\hline 1173 & 22.9243 & 586.7041 & 592.6313 & 541.1667 & 122.5994 & 3.87629 \\
\hline 1174 & 22.9413 & 591.5951 & 593.6847 & 541.625 & 122.5994 & 3.87346 \\
\hline
\end{tabular}




\begin{tabular}{|c|c|c|c|c|c|c|}
\hline 1175 & 22.9583 & 596.643 & 592.4937 & 542.0833 & 122.5994 & 3.87063 \\
\hline 1176 & 22.9753 & 604.4265 & 589.5542 & 542.5417 & 122.5994 & 3.8678 \\
\hline 1177 & 22.9923 & 598.8778 & 585.5525 & 543 & 122.5994 & 3.86498 \\
\hline 1178 & 23.0093 & 566.3469 & 581.5632 & 543.4583 & 122.5994 & 3.86216 \\
\hline 1179 & 23.0263 & 584.4069 & 579.2451 & 543.9167 & 122.5994 & 3.85935 \\
\hline 1180 & 23.0433 & 575.6921 & 580.9326 & 544.375 & 122.5994 & 3.85654 \\
\hline 1181 & 23.0603 & 544.5483 & 586.6212 & 544.8333 & 122.5994 & 3.85374 \\
\hline 1182 & 23.0773 & 571.5373 & 593.637 & 545.2917 & 122.5994 & 3.85093 \\
\hline 1183 & 23.0943 & 557.2085 & 594.1591 & 545.75 & 122.5994 & 3.84814 \\
\hline 1184 & 23.1113 & 513.6827 & 588.0349 & 546.2083 & 122.5994 & 3.84535 \\
\hline 1185 & 23.1283 & 543.9956 & 582.3904 & 546.6667 & 122.5994 & 3.84256 \\
\hline 1186 & 23.1453 & 548.3874 & 578.0139 & 547.125 & 122.5994 & 3.83977 \\
\hline 1187 & 23.1623 & 506.105 & 571.7393 & 547.5833 & 122.5994 & 3.83699 \\
\hline 1188 & 23.1793 & 501.7694 & 565.5266 & 548.0417 & 122.5994 & 3.83422 \\
\hline 1189 & 23.1963 & 489.3354 & 561.2072 & 548.5 & 122.5994 & 3.83145 \\
\hline 1190 & 23.2133 & 511.0949 & 559.1666 & 548.9583 & 122.5994 & 3.82868 \\
\hline 1191 & 23.2303 & 525.2971 & 558.6481 & 549.4167 & 122.5994 & 3.82592 \\
\hline 1192 & 23.2473 & 519.7901 & 559.0127 & 549.875 & 122.5994 & 3.82316 \\
\hline 1193 & 23.2643 & 502.3705 & 559.9905 & 550.3333 & 122.5994 & 3.8204 \\
\hline 1194 & 23.2813 & 481.0764 & 561.5938 & 550.7917 & 122.5994 & 3.81765 \\
\hline 1195 & 23.2983 & 528.4202 & 563.9781 & 551.25 & 122.5994 & 3.8149 \\
\hline 1196 & 23.3153 & 535.7067 & 567.3576 & 551.7083 & 122.5994 & 3.81216 \\
\hline 1197 & 23.3323 & 502.4859 & 571.9343 & 552.1667 & 122.5994 & 3.80942 \\
\hline 1198 & 23.3493 & 527.0669 & 577.8168 & 552.625 & 122.5994 & 3.80669 \\
\hline 1199 & 23.3663 & 541.8607 & 584.9125 & 553.0833 & 122.5994 & 3.80396 \\
\hline 1200 & 23.3833 & 569.3222 & 592.7871 & 553.5417 & 122.5994 & 3.80123 \\
\hline 1201 & 23.4003 & 541.2704 & 600.5115 & 554 & 122.5994 & 3.79851 \\
\hline 1202 & 23.4173 & 561.2325 & 608.3464 & 556.0417 & 122.5994 & 3.79579 \\
\hline 1203 & 23.4343 & 546.1361 & 613.7859 & 558.0833 & 122.5994 & 3.79307 \\
\hline 1204 & 23.4513 & 518.4386 & 617.3484 & 560.125 & 122.5994 & 3.79036 \\
\hline 1205 & 23.4683 & 548.7694 & 621.2031 & 562.1667 & 122.5994 & 3.78765 \\
\hline 1206 & 23.4853 & 522.2129 & 628.8427 & 564.2083 & 122.5994 & 3.78495 \\
\hline 1207 & 23.5023 & 511.2361 & 643.8192 & 566.25 & 122.5994 & 3.78225 \\
\hline 1208 & 23.5193 & 597.5903 & 665.5832 & 568.2917 & 122.5994 & 3.77955 \\
\hline 1209 & 23.5363 & 690.9429 & 682.5645 & 570.3333 & 122.5994 & 3.77686 \\
\hline 1210 & 23.5533 & 655.4397 & 680.2292 & 572.375 & 122.5994 & 3.77418 \\
\hline 1211 & 23.5703 & 659.6197 & 670.2167 & 574.4167 & 122.5994 & 3.77149 \\
\hline 1212 & 23.5873 & 637.9414 & 665.1292 & 576.4583 & 122.5994 & 3.76881 \\
\hline 1213 & 23.6043 & 684.0363 & 659.0981 & 578.5 & 122.5994 & 3.76614 \\
\hline 1214 & 23.6213 & 658.9793 & 647.2393 & 580.5417 & 122.5994 & 3.76346 \\
\hline 1215 & 23.6383 & 682.442 & 636.4044 & 582.5833 & 122.5994 & 3.7608 \\
\hline 1216 & 23.6553 & 619.2653 & 629.8925 & 584.625 & 122.5994 & 3.75813 \\
\hline 1217 & 23.6723 & 645.1721 & 627.2611 & 586.6667 & 122.5994 & 3.75547 \\
\hline 1218 & 23.6893 & 642.3646 & 627.6701 & 588.7083 & 122.5994 & 3.75281 \\
\hline 1219 & 23.7063 & 633.1555 & 630.0552 & 590.75 & 122.5994 & 3.75016 \\
\hline 1220 & 23.7233 & 633.7987 & 634.1802 & 592.7917 & 122.5994 & 3.74751 \\
\hline 1221 & 23.7403 & 605.2878 & 639.8587 & 594.8333 & 122.5994 & 3.74487 \\
\hline 1222 & 23.7573 & 624.2125 & 646.6216 & 596.875 & 122.5994 & 3.74223 \\
\hline 1223 & 23.7743 & 582.7454 & 653.6116 & 598.9167 & 122.5994 & 3.73959 \\
\hline
\end{tabular}




\begin{tabular}{|c|c|c|c|c|c|c|}
\hline 1224 & 23.7913 & 568.8956 & 659.6694 & 600.9583 & 122.5994 & 3.73696 \\
\hline 1225 & 23.8083 & 611.8364 & 664.0663 & 603 & 122.5994 & 3.73433 \\
\hline 1226 & 23.8253 & 626.8026 & 663.2344 & 602.625 & 122.5994 & 3.7317 \\
\hline 1227 & 23.8423 & 626.2317 & 660.0542 & 602.25 & 122.5994 & 3.72908 \\
\hline 1228 & 23.8593 & 612.038 & 655.1332 & 601.875 & 122.5994 & 3.72646 \\
\hline 1229 & 23.8763 & 606.5021 & 648.7032 & 601.5 & 122.5994 & 3.72384 \\
\hline 1230 & 23.8933 & 621.6907 & 641.577 & 601.125 & 122.5994 & 3.72123 \\
\hline 1231 & 23.9103 & 611.6435 & 634.4768 & 600.75 & 122.5994 & 3.71863 \\
\hline 1232 & 23.9273 & 614.635 & 628.2188 & 600.375 & 122.5994 & 3.71602 \\
\hline 1233 & 23.9443 & 612.4356 & 622.7035 & 600 & 122.5994 & 3.71342 \\
\hline 1234 & 23.9613 & 585.5838 & 618.2861 & 599.625 & 122.5994 & 3.71083 \\
\hline 1235 & 23.9783 & 577.6991 & 615.0069 & 599.25 & 122.5994 & 3.70823 \\
\hline 1236 & 23.9953 & 602.0206 & 613.0785 & 598.875 & 122.5994 & 3.70565 \\
\hline 1237 & 24.0123 & 607.4016 & 612.4561 & 598.5 & 122.5994 & 3.70306 \\
\hline 1238 & 24.0293 & 583.4518 & 614.9657 & 598.125 & 122.5994 & 3.70048 \\
\hline 1239 & 24.0463 & 557.8888 & 622.9477 & 597.75 & 122.5994 & 3.6979 \\
\hline 1240 & 24.0633 & 595.2246 & 634.8185 & 597.375 & 122.5994 & 3.69533 \\
\hline 1241 & 24.0803 & 590.0879 & 648.0607 & 597 & 122.5994 & 3.69276 \\
\hline 1242 & 24.0973 & 559.3456 & 651.007 & 596.625 & 122.5994 & 3.69019 \\
\hline 1243 & 24.1143 & 528.8205 & 644.2757 & 596.25 & 122.5994 & 3.68763 \\
\hline 1244 & 24.1313 & 516.4611 & 638.8889 & 595.875 & 122.5994 & 3.68507 \\
\hline 1245 & 24.1483 & 525.8056 & 636.6431 & 595.5 & 122.5994 & 3.68251 \\
\hline 1246 & 24.1653 & 596.7244 & 633.8578 & 595.125 & 122.5994 & 3.67996 \\
\hline 1247 & 24.1823 & 627.166 & 633.385 & 594.75 & 122.5994 & 3.67741 \\
\hline 1248 & 24.1993 & 585.151 & 637.6062 & 594.375 & 122.5994 & 3.67487 \\
\hline 1249 & 24.2163 & 584.2271 & 641.5481 & 594 & 122.5994 & 3.67233 \\
\hline 1250 & 24.2333 & 585.6509 & 637.564 & 591.8333 & 122.5994 & 3.66979 \\
\hline 1251 & 24.2503 & 594.3542 & 634.4069 & 589.6667 & 122.5994 & 3.66725 \\
\hline 1252 & 24.2673 & 631.682 & 639.9844 & 587.5 & 122.5994 & 3.66472 \\
\hline 1253 & 24.2843 & 611.6401 & 656.0156 & 585.3333 & 122.5994 & 3.6622 \\
\hline 1254 & 24.3013 & 610.1014 & 680.8281 & 583.1667 & 122.5994 & 3.65967 \\
\hline 1255 & 24.3183 & 705.7601 & 703.7036 & 581 & 122.5994 & 3.65715 \\
\hline 1256 & 24.3353 & 660.4568 & 700.5675 & 578.8333 & 122.5994 & 3.65464 \\
\hline 1257 & 24.3523 & 646.0555 & 678.4702 & 576.6667 & 122.5994 & 3.65212 \\
\hline 1258 & 24.3693 & 679.7228 & 661.7236 & 574.5 & 122.5994 & 3.64961 \\
\hline 1259 & 24.3863 & 647.8869 & 648.9633 & 572.3333 & 122.5994 & 3.64711 \\
\hline 1260 & 24.4033 & 611.5771 & 628.2086 & 570.1667 & 122.5994 & 3.64461 \\
\hline 1261 & 24.4203 & 570.3085 & 605.3394 & 568 & 122.5994 & 3.64211 \\
\hline 1262 & 24.4373 & 527.4771 & 588.1901 & 565.8333 & 122.5994 & 3.63961 \\
\hline 1263 & 24.4543 & 550.4702 & 577.6235 & 563.6667 & 122.5994 & 3.63712 \\
\hline 1264 & 24.4713 & 558.2297 & 571.4806 & 561.5 & 122.5994 & 3.63463 \\
\hline 1265 & 24.4883 & 525.2982 & 567.5814 & 559.3333 & 122.5994 & 3.63215 \\
\hline 1266 & 24.5053 & 536.6068 & 564.4513 & 557.1667 & 122.5994 & 3.62967 \\
\hline 1267 & 24.5223 & 506.0025 & 562.2419 & 555 & 122.5994 & 3.62719 \\
\hline 1268 & 24.5393 & 531.2173 & 560.4113 & 552.8333 & 122.5994 & 3.62471 \\
\hline 1269 & 24.5563 & 523.1279 & 558.2031 & 550.6667 & 122.5994 & 3.62224 \\
\hline 1270 & 24.5733 & 521.1799 & 556.2805 & 548.5 & 122.5994 & 3.61978 \\
\hline 1271 & 24.5903 & 522.9316 & 553.9965 & 546.3333 & 122.5994 & 3.61731 \\
\hline 1272 & 24.6073 & 521.4364 & 551.28 & 544.1667 & 122.5994 & 3.61485 \\
\hline
\end{tabular}




\begin{tabular}{|c|c|c|c|c|c|c|}
\hline 1273 & 24.6243 & 543.4421 & 547.6138 & 542 & 122.5994 & 3.6124 \\
\hline 1274 & 24.6413 & 563.0894 & 549.7496 & 543.5 & 122.5994 & 3.60994 \\
\hline 1275 & 24.6583 & 548.694 & 550.4427 & 545 & 122.5994 & 3.60749 \\
\hline 1276 & 24.6753 & 552.8344 & 551.1655 & 546.5 & 122.5994 & 3.60504 \\
\hline 1277 & 24.6923 & 549.703 & 552.3658 & 548 & 122.5994 & 3.6026 \\
\hline 1278 & 24.7093 & 539.0007 & 553.5339 & 549.5 & 122.5994 & 3.60016 \\
\hline 1279 & 24.7263 & 524.4198 & 554.9011 & 551 & 122.5994 & 3.59772 \\
\hline 1280 & 24.7433 & 556.2197 & 556.4413 & 552.5 & 122.5994 & 3.59529 \\
\hline 1281 & 24.7603 & 555.0152 & 558.1262 & 554 & 122.5994 & 3.59286 \\
\hline 1282 & 24.7773 & 521.24 & 559.9352 & 555.5 & 122.5994 & 3.59043 \\
\hline 1283 & 24.7943 & 534.1402 & 561.8574 & 557 & 122.5994 & 3.58801 \\
\hline 1284 & 24.8113 & 544.7431 & 563.8889 & 558.5 & 122.5994 & 3.58559 \\
\hline 1285 & 24.8283 & 537.0824 & 566.659 & 560 & 122.5994 & 3.58317 \\
\hline 1286 & 24.8453 & 542.6658 & 568.9302 & 561.5 & 122.5994 & 3.58076 \\
\hline 1287 & 24.8623 & 532.0602 & 571.2848 & 563 & 122.5994 & 3.57835 \\
\hline 1288 & 24.8793 & 543.6083 & 573.6884 & 564.5 & 122.5994 & 3.57594 \\
\hline 1289 & 24.8963 & 555.6183 & 576.3655 & 566 & 122.5994 & 3.57354 \\
\hline 1290 & 24.9133 & 560.4445 & 578.7505 & 567.5 & 122.5994 & 3.57114 \\
\hline 1291 & 24.9303 & 583.4735 & 581.1022 & 569 & 122.5994 & 3.56874 \\
\hline 1292 & 24.9473 & 617.334 & 583.4659 & 570.5 & 122.5994 & 3.56635 \\
\hline 1293 & 24.9643 & 593.56 & 585.915 & 572 & 122.5994 & 3.56396 \\
\hline 1294 & 24.9813 & 593.5591 & 588.5492 & 573.5 & 122.5994 & 3.56157 \\
\hline 1295 & 24.9983 & 587.1866 & 591.5338 & 575 & 122.5994 & 3.55919 \\
\hline 1296 & 25.0153 & 585.6509 & 595.1198 & 576.5 & 122.5994 & 3.55681 \\
\hline 1297 & 25.0323 & 585.6509 & 599.6692 & 578 & 122.5994 & 3.55443 \\
\hline 1298 & 25.0493 & 585.6509 & 605.0287 & 578.6875 & 122.5994 & 3.55206 \\
\hline 1299 & 25.0663 & 550.3062 & 612.8163 & 579.375 & 122.5994 & 3.54969 \\
\hline 1300 & 25.0833 & 611.5859 & 624.1017 & 580.0625 & 122.5994 & 3.54732 \\
\hline 1301 & 25.1003 & 664.1905 & 640.0432 & 580.75 & 122.5994 & 3.54496 \\
\hline 1302 & 25.1173 & 640.2899 & 661.6765 & 581.4375 & 122.5994 & 3.5426 \\
\hline 1303 & 25.1343 & 671.7266 & 689.4283 & 582.125 & 122.5994 & 3.54024 \\
\hline 1304 & 25.1513 & 734.6179 & 722.6652 & 582.8125 & 122.5994 & 3.53789 \\
\hline 1305 & 25.1683 & 742.0633 & 758.8969 & 583.5 & 122.5994 & 3.53553 \\
\hline 1306 & 25.1853 & 775.2997 & 792.8928 & 584.1875 & 122.5994 & 3.53319 \\
\hline 1307 & 25.2023 & 738.7672 & 817.3939 & 584.875 & 122.5994 & 3.53084 \\
\hline 1308 & 25.2193 & 743.36 & 827.2443 & 585.5625 & 122.5994 & 3.5285 \\
\hline 1309 & 25.2363 & 837.0234 & 823.0328 & 586.25 & 122.5994 & 3.52616 \\
\hline 1310 & 25.2533 & 775.6799 & 808.4424 & 586.9375 & 122.5994 & 3.52383 \\
\hline 1311 & 25.2703 & 758.7046 & 786.1063 & 587.625 & 122.5994 & 3.52149 \\
\hline 1312 & 25.2873 & 739.5975 & 758.3187 & 588.3125 & 122.5994 & 3.51917 \\
\hline 1313 & 25.3043 & 710.9606 & 729.0206 & 589 & 122.5994 & 3.51684 \\
\hline 1314 & 25.3213 & 676.0969 & 702.2996 & 589.6875 & 122.5994 & 3.51452 \\
\hline 1315 & 25.3383 & 642.4153 & 680.8356 & 590.375 & 122.5994 & 3.5122 \\
\hline 1316 & 25.3553 & 638.0265 & 665.8351 & 591.0625 & 122.5994 & 3.50988 \\
\hline 1317 & 25.3723 & 638.8121 & 658.1257 & 591.75 & 122.5994 & 3.50757 \\
\hline 1318 & 25.3893 & 647.4076 & 658.8159 & 592.4375 & 122.5994 & 3.50526 \\
\hline 1319 & 25.4063 & 678.6811 & 668.7116 & 593.125 & 122.5994 & 3.50295 \\
\hline 1320 & 25.4233 & 663.2147 & 685.9366 & 593.8125 & 122.5994 & 3.50065 \\
\hline 1321 & 25.4403 & 667.7257 & 702.3473 & 594.5 & 122.5994 & 3.49835 \\
\hline
\end{tabular}




\begin{tabular}{|c|c|c|c|c|c|c|}
\hline 1322 & 25.4573 & 670.7411 & 709.1921 & 595.1875 & 122.5994 & 3.49605 \\
\hline 1323 & 25.4743 & 713.109 & 713.0894 & 595.875 & 122.5994 & 3.49375 \\
\hline 1324 & 25.4913 & 734.7177 & 720.6896 & 596.5625 & 122.5994 & 3.49146 \\
\hline 1325 & 25.5083 & 750.3868 & 727.4453 & 597.25 & 122.5994 & 3.48917 \\
\hline 1326 & 25.5253 & 764.4091 & 725.7653 & 597.9375 & 122.5994 & 3.48689 \\
\hline 1327 & 25.5423 & 756.2641 & 715.9781 & 598.625 & 122.5994 & 3.48461 \\
\hline 1328 & 25.5593 & 759.3064 & 703.579 & 599.3125 & 122.5994 & 3.48233 \\
\hline 1329 & 25.5763 & 764.9466 & 691.9907 & 600 & 122.5994 & 3.48005 \\
\hline 1330 & 25.5933 & 725.364 & 680.522 & 600.6875 & 122.5994 & 3.47778 \\
\hline 1331 & 25.6103 & 680.8484 & 667.8865 & 601.375 & 122.5994 & 3.47551 \\
\hline 1332 & 25.6273 & 725.3466 & 655.5627 & 602.0625 & 122.5994 & 3.47324 \\
\hline 1333 & 25.6443 & 713.6745 & 645.0277 & 602.75 & 122.5994 & 3.47098 \\
\hline 1334 & 25.6613 & 688.1265 & 636.6992 & 603.4375 & 122.5994 & 3.46872 \\
\hline 1335 & 25.6783 & 668.2162 & 630.3287 & 604.125 & 122.5994 & 3.46646 \\
\hline 1336 & 25.6953 & 667.9002 & 625.6479 & 604.8125 & 122.5994 & 3.4642 \\
\hline 1337 & 25.7123 & 698.2276 & 622.2802 & 605.5 & 122.5994 & 3.46195 \\
\hline 1338 & 25.7293 & 659.6968 & 619.941 & 606.1875 & 122.5994 & 3.4597 \\
\hline 1339 & 25.7463 & 605.8905 & 618.3839 & 606.875 & 122.5994 & 3.45746 \\
\hline 1340 & 25.7633 & 635.7251 & 617.4018 & 607.5625 & 122.5994 & 3.45521 \\
\hline 1341 & 25.7803 & 670.8265 & 616.8306 & 608.25 & 122.5994 & 3.45297 \\
\hline 1342 & 25.7973 & 653.9633 & 615.2638 & 608.9375 & 122.5994 & 3.45074 \\
\hline 1343 & 25.8143 & 623.8079 & 615.2541 & 609.625 & 122.5994 & 3.4485 \\
\hline 1344 & 25.8313 & 633.4937 & 615.385 & 610.3125 & 122.5994 & 3.44627 \\
\hline 1345 & 25.8483 & 580.8602 & 615.6235 & 611 & 122.5994 & 3.44404 \\
\hline 1346 & 25.8653 & 617.3545 & 615.9216 & 612.5417 & 122.5994 & 3.44182 \\
\hline 1347 & 25.8823 & 620.0216 & 617.277 & 614.0833 & 122.5994 & 3.4396 \\
\hline 1348 & 25.8993 & 600.6077 & 618.7609 & 615.625 & 122.5994 & 3.43738 \\
\hline 1349 & 25.9163 & 619.8677 & 620.3575 & 617.1667 & 122.5994 & 3.43516 \\
\hline 1350 & 25.9333 & 579.9743 & 622.695 & 618.7083 & 122.5994 & 3.43295 \\
\hline 1351 & 25.9503 & 550.2771 & 624.1039 & 620.25 & 122.5994 & 3.43074 \\
\hline 1352 & 25.9673 & 605.1713 & 625.4063 & 621.7917 & 122.5994 & 3.42853 \\
\hline 1353 & 25.9843 & 606.9582 & 626.7944 & 623.3333 & 122.5994 & 3.42633 \\
\hline 1354 & 26.0013 & 622.7282 & 645.2455 & 624.875 & 122.5994 & 3.42413 \\
\hline 1355 & 26.0183 & 600.6553 & 647.5847 & 626.4167 & 122.5994 & 3.42193 \\
\hline 1356 & 26.0353 & 573.4509 & 650.0098 & 627.9583 & 122.5994 & 3.41973 \\
\hline 1357 & 26.0523 & 587.3625 & 652.614 & 629.5 & 122.5994 & 3.41754 \\
\hline 1358 & 26.0693 & 599.2604 & 655.4359 & 631.0417 & 122.5994 & 3.41535 \\
\hline 1359 & 26.0863 & 605.4457 & 658.4847 & 632.5833 & 122.5994 & 3.41316 \\
\hline 1360 & 26.1033 & 578.1417 & 661.7607 & 634.125 & 122.5994 & 3.41098 \\
\hline 1361 & 26.1203 & 604.1379 & 680.0308 & 635.6667 & 122.5994 & 3.40879 \\
\hline 1362 & 26.1373 & 623.595 & 684.5546 & 637.2083 & 122.5994 & 3.40662 \\
\hline 1363 & 26.1543 & 624.0655 & 689.3047 & 638.75 & 122.5994 & 3.40444 \\
\hline 1364 & 26.1713 & 587.1487 & 694.2209 & 640.2917 & 122.5994 & 3.40227 \\
\hline 1365 & 26.1883 & 625.8669 & 701.7733 & 641.8333 & 122.5994 & 3.4001 \\
\hline 1366 & 26.2053 & 629.5234 & 706.655 & 643.375 & 122.5994 & 3.39793 \\
\hline 1367 & 26.2223 & 599.0968 & 712.3743 & 644.9167 & 122.5994 & 3.39577 \\
\hline 1368 & 26.2393 & 638.8552 & 718.5121 & 646.4583 & 122.5994 & 3.3936 \\
\hline 1369 & 26.2563 & 661.188 & 725.1772 & 648 & 122.5994 & 3.39145 \\
\hline 1370 & 26.2733 & 648.412 & 732.2641 & 649.5417 & 122.5994 & 3.38929 \\
\hline
\end{tabular}




\begin{tabular}{|c|c|c|c|c|c|c|}
\hline 1371 & 26.2903 & 682.5432 & 740.4602 & 651.0833 & 122.5994 & 3.38714 \\
\hline 1372 & 26.3073 & 712.9495 & 749.6921 & 652.625 & 122.5994 & 3.38499 \\
\hline 1373 & 26.3243 & 719.2837 & 760.1793 & 654.1667 & 122.5994 & 3.38284 \\
\hline 1374 & 26.3413 & 716.6197 & 772.1828 & 655.7083 & 122.5994 & 3.38069 \\
\hline 1375 & 26.3583 & 718.0714 & 786.0583 & 657.25 & 122.5994 & 3.37855 \\
\hline 1376 & 26.3753 & 779.9532 & 802.3329 & 658.7917 & 122.5994 & 3.37641 \\
\hline 1377 & 26.3923 & 801.9495 & 821.9067 & 660.3333 & 122.5994 & 3.37428 \\
\hline 1378 & 26.4093 & 842.2599 & 846.4647 & 661.875 & 122.5994 & 3.37214 \\
\hline 1379 & 26.4263 & 929.1994 & 878.807 & 663.4167 & 122.5994 & 3.37001 \\
\hline 1380 & 26.4433 & 1020.57 & 922.0349 & 664.9583 & 122.5994 & 3.36789 \\
\hline 1381 & 26.4603 & 1072.389 & 976.7686 & 666.5 & 122.5994 & 3.36576 \\
\hline 1382 & 26.4773 & 1130.126 & 1037.103 & 668.0417 & 122.5994 & 3.36364 \\
\hline 1383 & 26.4943 & 1305.46 & 1096.834 & 669.5833 & 122.5994 & 3.36152 \\
\hline 1384 & 26.5113 & 1446.978 & 1169.535 & 671.125 & 122.5994 & 3.3594 \\
\hline 1385 & 26.5283 & 1667.413 & 1275.926 & 672.6667 & 122.5994 & 3.35729 \\
\hline 1386 & 26.5453 & 1907.401 & 1440.179 & 674.2083 & 122.5994 & 3.35517 \\
\hline 1387 & 26.5623 & 2217.933 & 1715.388 & 675.75 & 122.5994 & 3.35307 \\
\hline 1388 & 26.5793 & 2515.675 & 2213.838 & 677.2917 & 122.5994 & 3.35096 \\
\hline 1389 & 26.5963 & 3136.151 & 3088.108 & 678.8333 & 122.5994 & 3.34886 \\
\hline 1390 & 26.6133 & 3884.512 & 4451.212 & 680.375 & 122.5994 & 3.34676 \\
\hline 1391 & 26.6303 & 5058.282 & 6237.703 & 681.9167 & 122.5994 & 3.34466 \\
\hline 1392 & 26.6473 & 6860.112 & 8003.912 & 683.4583 & 122.5994 & 3.34256 \\
\hline 1393 & 26.6643 & 9161.094 & 8883.729 & 685 & 122.5994 & 3.34047 \\
\hline 1394 & 26.6813 & 11119.87 & 8552.105 & 686.5417 & 122.5994 & 3.33838 \\
\hline 1395 & 26.6983 & 10409.03 & 7765.046 & 688.0833 & 122.5994 & 3.33629 \\
\hline 1396 & 26.7153 & 7223.749 & 7060.491 & 689.625 & 122.5994 & 3.33421 \\
\hline 1397 & 26.7323 & 6090.457 & 6263.47 & 691.1667 & 122.5994 & 3.33213 \\
\hline 1398 & 26.7493 & 6204.595 & 5191.185 & 692.7083 & 122.5994 & 3.33005 \\
\hline 1399 & 26.7663 & 5215.213 & 4112.567 & 694.25 & 122.5994 & 3.32797 \\
\hline 1400 & 26.7833 & 3439.424 & 3267.38 & 695.7917 & 122.5994 & 3.3259 \\
\hline 1401 & 26.8003 & 2276.671 & 2694.46 & 697.3333 & 122.5994 & 3.32383 \\
\hline 1402 & 26.8173 & 1866.036 & 2333.201 & 698.875 & 122.5994 & 3.32176 \\
\hline 1403 & 26.8343 & 1802.215 & 2098.93 & 700.4167 & 122.5994 & 3.31969 \\
\hline 1404 & 26.8513 & 1721.963 & 1925.266 & 701.9583 & 122.5994 & 3.31763 \\
\hline 1405 & 26.8683 & 1625.056 & 1773.805 & 703.5 & 122.5994 & 3.31557 \\
\hline 1406 & 26.8853 & 1522.365 & 1628.741 & 705.0417 & 122.5994 & 3.31351 \\
\hline 1407 & 26.9023 & 1433.239 & 1488.735 & 706.5833 & 122.5994 & 3.31146 \\
\hline 1408 & 26.9193 & 1268.097 & 1358.79 & 708.125 & 122.5994 & 3.3094 \\
\hline 1409 & 26.9363 & 1140.168 & 1244.03 & 709.6667 & 122.5994 & 3.30735 \\
\hline 1410 & 26.9533 & 1065.466 & 1147.067 & 711.2083 & 122.5994 & 3.30531 \\
\hline 1411 & 26.9703 & 963.0408 & 1068.003 & 712.75 & 122.5994 & 3.30326 \\
\hline 1412 & 26.9873 & 886.2375 & 1005.364 & 714.2917 & 122.5994 & 3.30122 \\
\hline 1413 & 27.0043 & 810.0113 & 956.8269 & 715.8333 & 122.5994 & 3.29918 \\
\hline 1414 & 27.0213 & 780.5851 & 919.791 & 717.375 & 122.5994 & 3.29714 \\
\hline 1415 & 27.0383 & 762.989 & 891.7395 & 718.9167 & 122.5994 & 3.29511 \\
\hline 1416 & 27.0553 & 708.4006 & 870.4634 & 720.4583 & 122.5994 & 3.29308 \\
\hline 1417 & 27.0723 & 713.4942 & 854.165 & 722 & 122.5994 & 3.29105 \\
\hline 1418 & 27.0893 & 748.2463 & 839.4182 & 721.4896 & 122.5994 & 3.28902 \\
\hline 1419 & 27.1063 & 707.2871 & 827.2774 & 720.9792 & 122.5994 & 3.287 \\
\hline
\end{tabular}




\begin{tabular}{|c|c|c|c|c|c|c|}
\hline 1420 & 27.1233 & 664.964 & 817.0472 & 720.4688 & 122.5994 & 3.28497 \\
\hline 1421 & 27.1403 & 663.9174 & 808.0668 & 719.9583 & 122.5994 & 3.28295 \\
\hline 1422 & 27.1573 & 682.8206 & 800.3915 & 719.4479 & 122.5994 & 3.28094 \\
\hline 1423 & 27.1743 & 701.1639 & 793.6041 & 718.9375 & 122.5994 & 3.27892 \\
\hline 1424 & 27.1913 & 665.8977 & 787.5459 & 718.4271 & 122.5994 & 3.27691 \\
\hline 1425 & 27.2083 & 617.7738 & 783.2406 & 717.9167 & 122.5994 & 3.2749 \\
\hline 1426 & 27.2253 & 618.2092 & 778.3751 & 717.4063 & 122.5994 & 3.2729 \\
\hline 1427 & 27.2423 & 614.8404 & 773.962 & 716.8958 & 122.5994 & 3.27089 \\
\hline 1428 & 27.2593 & 617.0823 & 769.9423 & 716.3854 & 122.5994 & 3.26889 \\
\hline 1429 & 27.2763 & 595.0947 & 766.2674 & 715.875 & 122.5994 & 3.26689 \\
\hline 1430 & 27.2933 & 574.8748 & 763.8063 & 715.3646 & 122.5994 & 3.2649 \\
\hline 1431 & 27.3103 & 616.3979 & 743.0862 & 714.8542 & 122.5994 & 3.2629 \\
\hline 1432 & 27.3273 & 641.9141 & 741.1586 & 714.3438 & 122.5994 & 3.26091 \\
\hline 1433 & 27.3443 & 596.8849 & 739.3858 & 713.8333 & 122.5994 & 3.25892 \\
\hline 1434 & 27.3613 & 610.1356 & 737.7537 & 713.3229 & 122.5994 & 3.25694 \\
\hline 1435 & 27.3783 & 606.6106 & 727.4929 & 712.8125 & 122.5994 & 3.25495 \\
\hline 1436 & 27.3953 & 631.3426 & 726.5469 & 712.3021 & 122.5994 & 3.25297 \\
\hline 1437 & 27.4123 & 695.3671 & 725.6812 & 711.7917 & 122.5994 & 3.25099 \\
\hline 1438 & 27.4293 & 702.2631 & 725.1047 & 711.2813 & 122.5994 & 3.24902 \\
\hline 1439 & 27.4463 & 737.5312 & 724.4072 & 710.7708 & 122.5994 & 3.24704 \\
\hline 1440 & 27.4633 & 755.6387 & 723.7892 & 710.2604 & 122.5994 & 3.24507 \\
\hline 1441 & 27.4803 & 811.3368 & 723.2535 & 709.75 & 122.5994 & 3.2431 \\
\hline 1442 & 27.4973 & 834.8313 & 716.673 & 707.5469 & 122.5994 & 3.24114 \\
\hline 1443 & 27.5143 & 756.2641 & 714.8767 & 705.3438 & 122.5994 & 3.23917 \\
\hline 1444 & 27.5313 & 756.7068 & 713.1735 & 703.1406 & 122.5994 & 3.23721 \\
\hline 1445 & 27.5483 & 771.6228 & 711.6319 & 700.9375 & 122.5994 & 3.23525 \\
\hline 1446 & 27.5653 & 824.3038 & 707.7777 & 698.7344 & 122.5994 & 3.2333 \\
\hline 1447 & 27.5823 & 770.9872 & 706.5938 & 696.5313 & 122.5994 & 3.23134 \\
\hline 1448 & 27.5993 & 746.9034 & 705.6076 & 694.3281 & 122.5994 & 3.22939 \\
\hline 1449 & 27.6163 & 687.2699 & 704.9664 & 692.125 & 122.5994 & 3.22744 \\
\hline 1450 & 27.6333 & 699.187 & 704.5953 & 689.9219 & 122.5994 & 3.22549 \\
\hline 1451 & 27.6503 & 715.6937 & 704.7156 & 687.7188 & 122.5994 & 3.22355 \\
\hline 1452 & 27.6673 & 654.9531 & 705.5596 & 685.5156 & 122.5994 & 3.22161 \\
\hline 1453 & 27.6843 & 718.0125 & 707.5811 & 683.3125 & 122.5994 & 3.21967 \\
\hline 1454 & 27.7013 & 750.774 & 711.9072 & 681.1094 & 122.5994 & 3.21773 \\
\hline 1455 & 27.7183 & 743.0439 & 721.5235 & 678.9063 & 122.5994 & 3.2158 \\
\hline 1456 & 27.7353 & 772.0576 & 742.7227 & 676.7031 & 122.5994 & 3.21386 \\
\hline 1457 & 27.7523 & 891.9203 & 783.4448 & 674.5 & 122.5994 & 3.21193 \\
\hline 1458 & 27.7693 & 922.6905 & 844.3286 & 672.2969 & 122.5994 & 3.21001 \\
\hline 1459 & 27.7863 & 911.3651 & 902.6691 & 670.0938 & 122.5994 & 3.20808 \\
\hline 1460 & 27.8033 & 896.2904 & 914.6028 & 667.8906 & 122.5994 & 3.20616 \\
\hline 1461 & 27.8203 & 975.5517 & 892.4445 & 665.6875 & 122.5994 & 3.20424 \\
\hline 1462 & 27.8373 & 1024.323 & 884.2841 & 663.4844 & 122.5994 & 3.20232 \\
\hline 1463 & 27.8543 & 1040.941 & 899.8768 & 661.2813 & 122.5994 & 3.2004 \\
\hline 1464 & 27.8713 & 1018.223 & 917.5282 & 659.0781 & 122.5994 & 3.19849 \\
\hline 1465 & 27.8883 & 1001.745 & 935.1853 & 656.875 & 122.5994 & 3.19658 \\
\hline 1466 & 27.9053 & 1073.594 & 977.2314 & 654.6719 & 122.5994 & 3.19467 \\
\hline 1467 & 27.9223 & 1138.797 & 1045.399 & 652.4688 & 122.5994 & 3.19276 \\
\hline 1468 & 27.9393 & 1234.198 & 1104.842 & 650.2656 & 122.5994 & 3.19086 \\
\hline
\end{tabular}




\begin{tabular}{|c|c|c|c|c|c|c|}
\hline 1469 & 27.9563 & 1344.773 & 1134.942 & 648.0625 & 122.5994 & 3.18896 \\
\hline 1470 & 27.9733 & 1320.318 & 1116.871 & 645.8594 & 122.5994 & 3.18706 \\
\hline 1471 & 27.9903 & 1213.884 & 1055.833 & 643.6563 & 122.5994 & 3.18516 \\
\hline 1472 & 28.0073 & 1174.118 & 996.6732 & 641.4531 & 122.5994 & 3.18327 \\
\hline 1473 & 28.0243 & 1108.875 & 953.8969 & 639.25 & 122.5994 & 3.18137 \\
\hline 1474 & 28.0413 & 1022.149 & 916.3118 & 637.0469 & 122.5994 & 3.17948 \\
\hline 1475 & 28.0583 & 915.3073 & 866.7778 & 634.8438 & 122.5994 & 3.1776 \\
\hline 1476 & 28.0753 & 843.33 & 813.5508 & 632.6406 & 122.5994 & 3.17571 \\
\hline 1477 & 28.0923 & 799.2632 & 762.6862 & 630.4375 & 122.5994 & 3.17383 \\
\hline 1478 & 28.1093 & 755.0744 & 728.2614 & 628.2344 & 122.5994 & 3.17195 \\
\hline 1479 & 28.1263 & 681.4963 & 709.6616 & 626.0313 & 122.5994 & 3.17007 \\
\hline 1480 & 28.1433 & 687.0821 & 697.0458 & 623.8281 & 122.5994 & 3.16819 \\
\hline 1481 & 28.1603 & 673.9998 & 680.7116 & 621.625 & 122.5994 & 3.16632 \\
\hline 1482 & 28.1773 & 675.315 & 664.6973 & 619.4219 & 122.5994 & 3.16445 \\
\hline 1483 & 28.1943 & 657.7024 & 652.0964 & 617.2188 & 122.5994 & 3.16258 \\
\hline 1484 & 28.2113 & 618.7351 & 643.3058 & 615.0156 & 122.5994 & 3.16071 \\
\hline 1485 & 28.2283 & 587.7038 & 637.3376 & 612.8125 & 122.5994 & 3.15884 \\
\hline 1486 & 28.2453 & 632.6664 & 633.4136 & 610.6094 & 122.5994 & 3.15698 \\
\hline 1487 & 28.2623 & 630.0841 & 631.8281 & 608.4063 & 122.5994 & 3.15512 \\
\hline 1488 & 28.2793 & 615.1313 & 634.5381 & 606.2031 & 122.5994 & 3.15326 \\
\hline 1489 & 28.2963 & 581.2163 & 645.0175 & 604 & 122.5994 & 3.15141 \\
\hline 1490 & 28.3133 & 605.5445 & 664.134 & 600.5833 & 122.5994 & 3.14955 \\
\hline 1491 & 28.3303 & 616.5372 & 687.216 & 597.1667 & 122.5994 & 3.1477 \\
\hline 1492 & 28.3473 & 639.2149 & 694.2999 & 593.75 & 122.5994 & 3.14585 \\
\hline 1493 & 28.3643 & 633.1388 & 676.6992 & 590.3333 & 122.5994 & 3.14401 \\
\hline 1494 & 28.3813 & 664.3649 & 656.7808 & 586.9167 & 122.5994 & 3.14216 \\
\hline 1495 & 28.3983 & 684.2037 & 647.2786 & 583.5 & 122.5994 & 3.14032 \\
\hline 1496 & 28.4153 & 638.4199 & 640.8246 & 580.0833 & 122.5994 & 3.13848 \\
\hline 1497 & 28.4323 & 631.9209 & 625.6273 & 576.6667 & 122.5994 & 3.13664 \\
\hline 1498 & 28.4493 & 627.5902 & 606.8457 & 573.25 & 122.5994 & 3.13481 \\
\hline 1499 & 28.4663 & 592.2037 & 591.9648 & 569.8333 & 122.5994 & 3.13297 \\
\hline 1500 & 28.4833 & 632.5709 & 582.2987 & 566.4167 & 122.5994 & 3.13114 \\
\hline 1501 & 28.5003 & 644.2925 & 576.2791 & 563 & 122.5994 & 3.12931 \\
\hline 1502 & 28.5173 & 641.974 & 572.1384 & 559.5833 & 122.5994 & 3.12749 \\
\hline 1503 & 28.5343 & 605.4246 & 568.9019 & 556.1667 & 122.5994 & 3.12566 \\
\hline 1504 & 28.5513 & 613.9062 & 566.0562 & 552.75 & 122.5994 & 3.12384 \\
\hline 1505 & 28.5683 & 632.2394 & 563.3564 & 549.3333 & 122.5994 & 3.12202 \\
\hline 1506 & 28.5853 & 607.8093 & 560.5522 & 545.9167 & 122.5994 & 3.1202 \\
\hline 1507 & 28.6023 & 602.2625 & 556.1938 & 542.5 & 122.5994 & 3.11838 \\
\hline 1508 & 28.6193 & 554.082 & 552.6676 & 539.0833 & 122.5994 & 3.11657 \\
\hline 1509 & 28.6363 & 551.7648 & 548.7265 & 535.6667 & 122.5994 & 3.11476 \\
\hline 1510 & 28.6533 & 567.7618 & 544.4653 & 532.25 & 122.5994 & 3.11295 \\
\hline 1511 & 28.6703 & 540.741 & 539.1639 & 528.8333 & 122.5994 & 3.11114 \\
\hline 1512 & 28.6873 & 521.9417 & 534.5121 & 525.4167 & 122.5994 & 3.10934 \\
\hline 1513 & 28.7043 & 527.8826 & 529.6741 & 522 & 122.5994 & 3.10753 \\
\hline 1514 & 28.7213 & 507.8333 & 528.5165 & 522.125 & 122.5994 & 3.10573 \\
\hline 1515 & 28.7383 & 516.0486 & 527.4822 & 522.25 & 122.5994 & 3.10394 \\
\hline 1516 & 28.7553 & 540.0614 & 526.621 & 522.375 & 122.5994 & 3.10214 \\
\hline 1517 & 28.7723 & 526.867 & 525.4072 & 522.5 & 122.5994 & 3.10034 \\
\hline
\end{tabular}




\begin{tabular}{|c|c|c|c|c|c|c|}
\hline 1518 & 28.7893 & 514.0369 & 524.9537 & 522.625 & 122.5994 & 3.09855 \\
\hline 1519 & 28.8063 & 510.7695 & 524.6541 & 522.75 & 122.5994 & 3.09676 \\
\hline 1520 & 28.8233 & 513.8647 & 524.4877 & 522.875 & 122.5994 & 3.09497 \\
\hline 1521 & 28.8403 & 493.2469 & 524.4391 & 523 & 122.5994 & 3.09319 \\
\hline 1522 & 28.8573 & 500.1697 & 524.5058 & 523.125 & 122.5994 & 3.09141 \\
\hline 1523 & 28.8743 & 502.8441 & 524.7034 & 523.25 & 122.5994 & 3.08962 \\
\hline 1524 & 28.8913 & 498.3307 & 525.0653 & 523.375 & 122.5994 & 3.08784 \\
\hline 1525 & 28.9083 & 469.5893 & 525.6304 & 523.5 & 122.5994 & 3.08607 \\
\hline 1526 & 28.9253 & 501.6655 & 526.4181 & 523.625 & 122.5994 & 3.08429 \\
\hline 1527 & 28.9423 & 549.2948 & 527.3927 & 523.75 & 122.5994 & 3.08252 \\
\hline 1528 & 28.9593 & 536.9965 & 528.4118 & 523.875 & 122.5994 & 3.08075 \\
\hline 1529 & 28.9763 & 513.5231 & 529.199 & 524 & 122.5994 & 3.07898 \\
\hline 1530 & 28.9933 & 527.1558 & 529.5212 & 524.125 & 122.5994 & 3.07721 \\
\hline 1531 & 29.0103 & 541.7874 & 529.4554 & 524.25 & 122.5994 & 3.07545 \\
\hline 1532 & 29.0273 & 556.1462 & 529.2148 & 524.375 & 122.5994 & 3.07369 \\
\hline 1533 & 29.0443 & 516.8433 & 528.8739 & 524.5 & 122.5994 & 3.07193 \\
\hline 1534 & 29.0613 & 515.2996 & 528.4015 & 524.625 & 122.5994 & 3.07017 \\
\hline 1535 & 29.0783 & 515.5195 & 527.8287 & 524.75 & 122.5994 & 3.06841 \\
\hline 1536 & 29.0953 & 550.5058 & 527.2673 & 524.875 & 122.5994 & 3.06666 \\
\hline 1537 & 29.1123 & 491.3186 & 526.796 & 525 & 122.5994 & 3.0649 \\
\hline 1538 & 29.1293 & 546.2608 & 525.4879 & 524.1667 & 122.5994 & 3.06315 \\
\hline 1539 & 29.1463 & 528.1664 & 524.306 & 523.3333 & 122.5994 & 3.06141 \\
\hline 1540 & 29.1633 & 518.2476 & 523.2326 & 522.5 & 122.5994 & 3.05966 \\
\hline 1541 & 29.1803 & 511.2075 & 523.1026 & 521.6667 & 122.5994 & 3.05792 \\
\hline 1542 & 29.1973 & 532.3076 & 522.2075 & 520.8333 & 122.5994 & 3.05618 \\
\hline 1543 & 29.2143 & 479.1763 & 521.3497 & 520 & 122.5994 & 3.05444 \\
\hline 1544 & 29.2313 & 506.4033 & 520.516 & 519.1667 & 122.5994 & 3.0527 \\
\hline 1545 & 29.2483 & 539.8279 & 520.1085 & 518.3333 & 122.5994 & 3.05096 \\
\hline 1546 & 29.2653 & 519.102 & 519.3248 & 517.5 & 122.5994 & 3.04923 \\
\hline 1547 & 29.2823 & 482.6477 & 518.5535 & 516.6667 & 122.5994 & 3.0475 \\
\hline 1548 & 29.2993 & 476.6244 & 517.732 & 515.8333 & 122.5994 & 3.04577 \\
\hline 1549 & 29.3163 & 497.6681 & 516.9868 & 515 & 122.5994 & 3.04404 \\
\hline 1550 & 29.3333 & 481.2361 & 516.2536 & 514.1667 & 122.5994 & 3.04231 \\
\hline 1551 & 29.3503 & 468.0854 & 515.533 & 513.3333 & 122.5994 & 3.04059 \\
\hline 1552 & 29.3673 & 479.0036 & 514.7951 & 512.5 & 122.5994 & 3.03887 \\
\hline 1553 & 29.3843 & 477.4541 & 514.1049 & 511.6667 & 122.5994 & 3.03715 \\
\hline 1554 & 29.4013 & 479.2482 & 513.4315 & 510.8333 & 122.5994 & 3.03543 \\
\hline 1555 & 29.4183 & 507.4462 & 512.7771 & 510 & 122.5994 & 3.03372 \\
\hline 1556 & 29.4353 & 481.2566 & 512.1152 & 509.1667 & 122.5994 & 3.032 \\
\hline 1557 & 29.4523 & 503.154 & 511.5093 & 508.3333 & 122.5994 & 3.03029 \\
\hline 1558 & 29.4693 & 540.5214 & 510.9319 & 507.5 & 122.5994 & 3.02858 \\
\hline 1559 & 29.4863 & 500.8595 & 510.3842 & 506.6667 & 122.5994 & 3.02688 \\
\hline 1560 & 29.5033 & 502.5027 & 509.88 & 505.8333 & 122.5994 & 3.02517 \\
\hline 1561 & 29.5203 & 508.5651 & 509.4097 & 505 & 122.5994 & 3.02347 \\
\hline 1562 & 29.5373 & 506.0259 & 510.5546 & 505.7083 & 122.5994 & 3.02177 \\
\hline 1563 & 29.5543 & 496.2606 & 511.7731 & 506.4167 & 122.5994 & 3.02007 \\
\hline 1564 & 29.5713 & 473.0218 & 513.0962 & 507.125 & 122.5994 & 3.01837 \\
\hline 1565 & 29.5883 & 504.2001 & 514.564 & 507.8333 & 122.5994 & 3.01667 \\
\hline 1566 & 29.6053 & 500.1933 & 516.2403 & 508.5417 & 122.5994 & 3.01498 \\
\hline
\end{tabular}




\begin{tabular}{|c|c|c|c|c|c|c|}
\hline 1567 & 29.6223 & 500.8089 & 518.2126 & 509.25 & 122.5994 & 3.01329 \\
\hline 1568 & 29.6393 & 506.2595 & 520.5908 & 509.9583 & 122.5994 & 3.0116 \\
\hline 1569 & 29.6563 & 506.2595 & 523.5012 & 510.6667 & 122.5994 & 3.00991 \\
\hline 1570 & 29.6733 & 530.543 & 527.0754 & 511.375 & 122.5994 & 3.00822 \\
\hline 1571 & 29.6903 & 509.715 & 531.4333 & 512.0833 & 122.5994 & 3.00654 \\
\hline 1572 & 29.7073 & 557.472 & 536.7495 & 512.7917 & 122.5994 & 3.00486 \\
\hline 1573 & 29.7243 & 540.2537 & 543.431 & 513.5 & 122.5994 & 3.00318 \\
\hline 1574 & 29.7413 & 529.724 & 552.0558 & 514.2083 & 122.5994 & 3.0015 \\
\hline 1575 & 29.7583 & 545.0951 & 563.0698 & 514.9167 & 122.5994 & 2.99983 \\
\hline 1576 & 29.7753 & 581.3927 & 576.6099 & 515.625 & 122.5994 & 2.99815 \\
\hline 1577 & 29.7923 & 598.8733 & 592.4884 & 516.3333 & 122.5994 & 2.99648 \\
\hline 1578 & 29.8093 & 609.4237 & 610.1155 & 517.0417 & 122.5994 & 2.99481 \\
\hline 1579 & 29.8263 & 638.6819 & 628.2156 & 517.75 & 122.5994 & 2.99314 \\
\hline 1580 & 29.8433 & 685.257 & 644.6396 & 518.4583 & 122.5994 & 2.99147 \\
\hline 1581 & 29.8603 & 660.613 & 657.3068 & 519.1667 & 122.5994 & 2.98981 \\
\hline 1582 & 29.8773 & 655.9218 & 663.2996 & 519.875 & 122.5994 & 2.98815 \\
\hline 1583 & 29.8943 & 691.2971 & 662.9918 & 520.5833 & 122.5994 & 2.98649 \\
\hline 1584 & 29.9113 & 679.0502 & 657.6216 & 521.2917 & 122.5994 & 2.98483 \\
\hline 1585 & 29.9283 & 647.2702 & 648.7136 & 522 & 122.5994 & 2.98317 \\
\hline 1586 & 29.9453 & 668.172 & 636.8626 & 522.7083 & 122.5994 & 2.98152 \\
\hline 1587 & 29.9623 & 638.9536 & 622.7399 & 523.4167 & 122.5994 & 2.97986 \\
\hline 1588 & 29.9793 & 598.1818 & 607.9393 & 524.125 & 122.5994 & 2.97821 \\
\hline 1589 & 29.9963 & 615.8856 & 593.9214 & 524.8333 & 122.5994 & 2.97656 \\
\hline 1590 & 30.0133 & 608.9782 & 581.7523 & 525.5417 & 122.5994 & 2.97492 \\
\hline 1591 & 30.0303 & 560.703 & 572.1511 & 526.25 & 122.5994 & 2.97327 \\
\hline 1592 & 30.0473 & 561.3958 & 565.2572 & 526.9583 & 122.5994 & 2.97163 \\
\hline 1593 & 30.0643 & 572.3766 & 561.9355 & 527.6667 & 122.5994 & 2.96999 \\
\hline 1594 & 30.0813 & 559.0365 & 563.9877 & 528.375 & 122.5994 & 2.96835 \\
\hline 1595 & 30.0983 & 553.6588 & 573.9717 & 529.0833 & 122.5994 & 2.96671 \\
\hline 1596 & 30.1153 & 563.0338 & 592.2628 & 529.7917 & 122.5994 & 2.96507 \\
\hline 1597 & 30.1323 & 566.3415 & 611.2989 & 530.5 & 122.5994 & 2.96344 \\
\hline 1598 & 30.1493 & 535.9039 & 614.1662 & 531.2083 & 122.5994 & 2.96181 \\
\hline 1599 & 30.1663 & 525.872 & 602.1634 & 531.9167 & 122.5994 & 2.96018 \\
\hline 1600 & 30.1833 & 546.6238 & 595.4413 & 532.625 & 122.5994 & 2.95855 \\
\hline 1601 & 30.2003 & 538.4532 & 601.5974 & 533.3333 & 122.5994 & 2.95692 \\
\hline 1602 & 30.2173 & 530.1657 & 611.7485 & 534.0417 & 122.5994 & 2.9553 \\
\hline 1603 & 30.2343 & 534.2315 & 608.0734 & 534.75 & 122.5994 & 2.95367 \\
\hline 1604 & 30.2513 & 526.6717 & 591.2036 & 535.4583 & 122.5994 & 2.95205 \\
\hline 1605 & 30.2683 & 533.7422 & 575.9704 & 536.1667 & 122.5994 & 2.95043 \\
\hline 1606 & 30.2853 & 521.1193 & 569.1516 & 536.875 & 122.5994 & 2.94881 \\
\hline 1607 & 30.3023 & 518.8882 & 567.3374 & 537.5833 & 122.5994 & 2.9472 \\
\hline 1608 & 30.3193 & 529.0099 & 564.1922 & 538.2917 & 122.5994 & 2.94558 \\
\hline 1609 & 30.3363 & 560.0015 & 558.8292 & 539 & 122.5994 & 2.94397 \\
\hline 1610 & 30.3533 & 543.0369 & 551.9675 & 537.125 & 122.5994 & 2.94236 \\
\hline 1611 & 30.3703 & 528.6797 & 547.3207 & 535.25 & 122.5994 & 2.94075 \\
\hline 1612 & 30.3873 & 521.4778 & 544.623 & 533.375 & 122.5994 & 2.93915 \\
\hline 1613 & 30.4043 & 546.9876 & 543.5649 & 531.5 & 122.5994 & 2.93754 \\
\hline 1614 & 30.4213 & 548.4326 & 544.831 & 529.625 & 122.5994 & 2.93594 \\
\hline 1615 & 30.4383 & 536.151 & 550.6973 & 527.75 & 122.5994 & 2.93434 \\
\hline
\end{tabular}




\begin{tabular}{|c|c|c|c|c|c|c|}
\hline 1616 & 30.4553 & 547.5778 & 564.3345 & 525.875 & 122.5994 & 2.93274 \\
\hline 1617 & 30.4723 & 537.9011 & 586.3566 & 524 & 122.5994 & 2.93114 \\
\hline 1618 & 30.4893 & 605.9898 & 608.1449 & 522.125 & 122.5994 & 2.92955 \\
\hline 1619 & 30.5063 & 596.8731 & 613.1676 & 520.25 & 122.5994 & 2.92795 \\
\hline 1620 & 30.5233 & 594.5667 & 601.0775 & 518.375 & 122.5994 & 2.92636 \\
\hline 1621 & 30.5403 & 603.7635 & 584.722 & 516.5 & 122.5994 & 2.92477 \\
\hline 1622 & 30.5573 & 583.7944 & 575.5551 & 514.625 & 122.5994 & 2.92318 \\
\hline 1623 & 30.5743 & 580.8209 & 569.3895 & 512.75 & 122.5994 & 2.9216 \\
\hline 1624 & 30.5913 & 520.4787 & 559.2082 & 510.875 & 122.5994 & 2.92001 \\
\hline 1625 & 30.6083 & 519.5285 & 544.8914 & 509 & 122.5994 & 2.91843 \\
\hline 1626 & 30.6253 & 524.2549 & 530.0861 & 507.125 & 122.5994 & 2.91685 \\
\hline 1627 & 30.6423 & 497.694 & 518.4233 & 505.25 & 122.5994 & 2.91527 \\
\hline 1628 & 30.6593 & 528.7561 & 511.6902 & 503.375 & 122.5994 & 2.91369 \\
\hline 1629 & 30.6763 & 492.5968 & 507.6838 & 501.5 & 122.5994 & 2.91211 \\
\hline 1630 & 30.6933 & 483.9536 & 504.5789 & 499.625 & 122.5994 & 2.91054 \\
\hline 1631 & 30.7103 & 481.8416 & 501.9849 & 497.75 & 122.5994 & 2.90897 \\
\hline 1632 & 30.7273 & 457.3411 & 499.6389 & 495.875 & 122.5994 & 2.9074 \\
\hline 1633 & 30.7443 & 496.9923 & 497.4401 & 494 & 122.5994 & 2.90583 \\
\hline 1634 & 30.7613 & 527.7631 & 498.0924 & 494.875 & 122.5994 & 2.90426 \\
\hline 1635 & 30.7783 & 522.7504 & 498.8193 & 495.75 & 122.5994 & 2.9027 \\
\hline 1636 & 30.7953 & 537.3593 & 499.1906 & 496.625 & 122.5994 & 2.90113 \\
\hline 1637 & 30.8123 & 535.4816 & 499.8248 & 497.5 & 122.5994 & 2.89957 \\
\hline 1638 & 30.8293 & 547.0763 & 500.7585 & 498.375 & 122.5994 & 2.89801 \\
\hline 1639 & 30.8463 & 557.1194 & 501.7194 & 499.25 & 122.5994 & 2.89645 \\
\hline 1640 & 30.8633 & 542.9001 & 502.7068 & 500.125 & 122.5994 & 2.89489 \\
\hline 1641 & 30.8803 & 551.0207 & 503.5252 & 501 & 122.5994 & 2.89334 \\
\hline 1642 & 30.8973 & 578.5974 & 504.2045 & 501.875 & 122.5994 & 2.89179 \\
\hline 1643 & 30.9143 & 593.7655 & 505.5778 & 502.75 & 122.5994 & 2.89023 \\
\hline 1644 & 30.9313 & 602.6221 & 506.7131 & 503.625 & 122.5994 & 2.88868 \\
\hline 1645 & 30.9483 & 621.2989 & 507.8865 & 504.5 & 122.5994 & 2.88714 \\
\hline 1646 & 30.9653 & 628.9349 & 509.1064 & 505.375 & 122.5994 & 2.88559 \\
\hline 1647 & 30.9823 & 639.7622 & 510.3841 & 506.25 & 122.5994 & 2.88405 \\
\hline 1648 & 30.9993 & 631.5383 & 511.7355 & 507.125 & 122.5994 & 2.8825 \\
\hline 1649 & 31.0163 & 628.6092 & 513.1846 & 508 & 122.5994 & 2.88096 \\
\hline 1650 & 31.0333 & 600.9298 & 514.7678 & 508.875 & 122.5994 & 2.87942 \\
\hline 1651 & 31.0503 & 578.4091 & 516.5433 & 509.75 & 122.5994 & 2.87788 \\
\hline 1652 & 31.0673 & 583.8607 & 518.6044 & 510.625 & 122.5994 & 2.87635 \\
\hline 1653 & 31.0843 & 587.4424 & 521.1101 & 511.5 & 122.5994 & 2.87481 \\
\hline 1654 & 31.1013 & 567.3106 & 524.3644 & 512.375 & 122.5994 & 2.87328 \\
\hline 1655 & 31.1183 & 570.9277 & 529.0094 & 513.25 & 122.5994 & 2.87175 \\
\hline 1656 & 31.1353 & 560.5575 & 536.2441 & 514.125 & 122.5994 & 2.87022 \\
\hline 1657 & 31.1523 & 562.4924 & 547.5266 & 515 & 122.5994 & 2.86869 \\
\hline 1658 & 31.1693 & 577.4846 & 563.1001 & 515.875 & 122.5994 & 2.86717 \\
\hline 1659 & 31.1863 & 577.5788 & 579.3739 & 516.75 & 122.5994 & 2.86564 \\
\hline 1660 & 31.2033 & 582.716 & 589.4709 & 517.625 & 122.5994 & 2.86412 \\
\hline 1661 & 31.2203 & 606.6631 & 594.9963 & 518.5 & 122.5994 & 2.8626 \\
\hline 1662 & 31.2373 & 589.772 & 603.2788 & 519.375 & 122.5994 & 2.86108 \\
\hline 1663 & 31.2543 & 577.165 & 615.4949 & 520.25 & 122.5994 & 2.85956 \\
\hline 1664 & 31.2713 & 602.6551 & 626.8188 & 521.125 & 122.5994 & 2.85805 \\
\hline
\end{tabular}




\begin{tabular}{|c|c|c|c|c|c|c|}
\hline 1665 & 31.2883 & 634.1951 & 631.4894 & 522 & 122.5994 & 2.85653 \\
\hline 1666 & 31.3053 & 644.283 & 629.9576 & 522.875 & 122.5994 & 2.85502 \\
\hline 1667 & 31.3223 & 566.4889 & 625.3817 & 523.75 & 122.5994 & 2.85351 \\
\hline 1668 & 31.3393 & 577.0394 & 619.3486 & 524.625 & 122.5994 & 2.852 \\
\hline 1669 & 31.3563 & 624.567 & 612.1958 & 525.5 & 122.5994 & 2.85049 \\
\hline 1670 & 31.3733 & 598.4666 & 604.4433 & 526.375 & 122.5994 & 2.84899 \\
\hline 1671 & 31.3903 & 581.8073 & 595.0626 & 527.25 & 122.5994 & 2.84748 \\
\hline 1672 & 31.4073 & 553.5817 & 585.4974 & 528.125 & 122.5994 & 2.84598 \\
\hline 1673 & 31.4243 & 529.5242 & 576.8687 & 529 & 122.5994 & 2.84448 \\
\hline 1674 & 31.4413 & 510.8299 & 570.2405 & 529.875 & 122.5994 & 2.84298 \\
\hline 1675 & 31.4583 & 551.9199 & 566.5802 & 530.75 & 122.5994 & 2.84148 \\
\hline 1676 & 31.4753 & 531.8211 & 564.922 & 531.625 & 122.5994 & 2.83999 \\
\hline 1677 & 31.4923 & 491.1914 & 563.0513 & 532.5 & 122.5994 & 2.83849 \\
\hline 1678 & 31.5093 & 538.2658 & 559.1315 & 533.375 & 122.5994 & 2.837 \\
\hline 1679 & 31.5263 & 582.6586 & 555.0241 & 534.25 & 122.5994 & 2.83551 \\
\hline 1680 & 31.5433 & 523.5435 & 552.9885 & 535.125 & 122.5994 & 2.83402 \\
\hline 1681 & 31.5603 & 569.8339 & 552.9436 & 536 & 122.5994 & 2.83253 \\
\hline 1682 & 31.5773 & 504.7462 & 551.1993 & 535.0833 & 122.5994 & 2.83105 \\
\hline 1683 & 31.5943 & 499.8644 & 548.6678 & 534.1667 & 122.5994 & 2.82956 \\
\hline 1684 & 31.6113 & 523.5172 & 546.3199 & 533.25 & 122.5994 & 2.82808 \\
\hline 1685 & 31.6283 & 565.7012 & 544.9308 & 532.3333 & 122.5994 & 2.8266 \\
\hline 1686 & 31.6453 & 548.7658 & 544.5492 & 531.4167 & 122.5994 & 2.82512 \\
\hline 1687 & 31.6623 & 564.1782 & 545.0158 & 530.5 & 122.5994 & 2.82364 \\
\hline 1688 & 31.6793 & 596.8283 & 546.3394 & 529.5833 & 122.5994 & 2.82216 \\
\hline 1689 & 31.6963 & 604.987 & 548.4908 & 528.6667 & 122.5994 & 2.82069 \\
\hline 1690 & 31.7133 & 633.9954 & 551.0375 & 527.75 & 122.5994 & 2.81922 \\
\hline 1691 & 31.7303 & 674.5923 & 552.9086 & 526.8333 & 122.5994 & 2.81774 \\
\hline 1692 & 31.7473 & 693.3883 & 552.8757 & 525.9167 & 122.5994 & 2.81627 \\
\hline 1693 & 31.7643 & 689.5722 & 550.9487 & 525 & 122.5994 & 2.81481 \\
\hline 1694 & 31.7813 & 625.596 & 548.3442 & 524.0833 & 122.5994 & 2.81334 \\
\hline 1695 & 31.7983 & 592.2053 & 545.9523 & 523.1667 & 122.5994 & 2.81187 \\
\hline 1696 & 31.8153 & 588.86 & 543.7593 & 522.25 & 122.5994 & 2.81041 \\
\hline 1697 & 31.8323 & 573.5901 & 541.489 & 521.3333 & 122.5994 & 2.80895 \\
\hline 1698 & 31.8493 & 551.9278 & 539.0762 & 520.4167 & 122.5994 & 2.80749 \\
\hline 1699 & 31.8663 & 528.7013 & 537.2142 & 519.5 & 122.5994 & 2.80603 \\
\hline 1700 & 31.8833 & 529.5366 & 536.4086 & 518.5833 & 122.5994 & 2.80457 \\
\hline 1701 & 31.9003 & 528.305 & 536.9844 & 517.6667 & 122.5994 & 2.80311 \\
\hline 1702 & 31.9173 & 502.8469 & 539.2223 & 516.75 & 122.5994 & 2.80166 \\
\hline 1703 & 31.9343 & 499.4359 & 543.195 & 515.8333 & 122.5994 & 2.80021 \\
\hline 1704 & 31.9513 & 506.2595 & 549.0634 & 514.9167 & 122.5994 & 2.79876 \\
\hline 1705 & 31.9683 & 512.2597 & 556.8398 & 514 & 122.5994 & 2.79731 \\
\hline 1706 & 31.9853 & 531.0266 & 564.9209 & 512.2083 & 122.5994 & 2.79586 \\
\hline 1707 & 32.0023 & 528.4148 & 574.8685 & 510.4167 & 122.5994 & 2.79441 \\
\hline 1708 & 32.0193 & 524.4799 & 585.1713 & 508.625 & 122.5994 & 2.79297 \\
\hline 1709 & 32.0363 & 527.8011 & 594.376 & 506.8333 & 122.5994 & 2.79152 \\
\hline 1710 & 32.0533 & 554.288 & 600.7636 & 505.0417 & 122.5994 & 2.79008 \\
\hline 1711 & 32.0703 & 539.3734 & 602.8635 & 503.25 & 122.5994 & 2.78864 \\
\hline 1712 & 32.0873 & 544.9177 & 601.3434 & 501.4583 & 122.5994 & 2.7872 \\
\hline 1713 & 32.1043 & 532.1186 & 596.5845 & 499.6667 & 122.5994 & 2.78577 \\
\hline
\end{tabular}




\begin{tabular}{|c|c|c|c|c|c|c|}
\hline 1714 & 32.1213 & 536.8709 & 588.9737 & 497.875 & 122.5994 & 2.78433 \\
\hline 1715 & 32.1383 & 514.1576 & 578.8116 & 496.0833 & 122.5994 & 2.7829 \\
\hline 1716 & 32.1553 & 552.3092 & 566.9195 & 494.2917 & 122.5994 & 2.78147 \\
\hline 1717 & 32.1723 & 518.6127 & 554.4219 & 492.5 & 122.5994 & 2.78003 \\
\hline 1718 & 32.1893 & 550.111 & 542.2802 & 490.7083 & 122.5994 & 2.7786 \\
\hline 1719 & 32.2063 & 473.6941 & 530.8453 & 488.9167 & 122.5994 & 2.77718 \\
\hline 1720 & 32.2233 & 470.9528 & 520.6866 & 487.125 & 122.5994 & 2.77575 \\
\hline 1721 & 32.2403 & 479.4989 & 511.9839 & 485.3333 & 122.5994 & 2.77433 \\
\hline 1722 & 32.2573 & 458.9453 & 504.8721 & 483.5417 & 122.5994 & 2.7729 \\
\hline 1723 & 32.2743 & 492.7927 & 499.2416 & 481.75 & 122.5994 & 2.77148 \\
\hline 1724 & 32.2913 & 491.6283 & 495.0587 & 479.9583 & 122.5994 & 2.77006 \\
\hline 1725 & 32.3083 & 473.9734 & 492.0763 & 478.1667 & 122.5994 & 2.76864 \\
\hline 1726 & 32.3253 & 463.8139 & 490.1313 & 476.375 & 122.5994 & 2.76722 \\
\hline 1727 & 32.3423 & 474.5982 & 489.0581 & 474.5833 & 122.5994 & 2.76581 \\
\hline 1728 & 32.3593 & 470.9528 & 488.6487 & 472.7917 & 122.5994 & 2.76439 \\
\hline 1729 & 32.3763 & 473.2064 & 488.5945 & 471 & 122.5994 & 2.76298 \\
\hline 1730 & 32.3933 & 476.9877 & 488.3442 & 469.125 & 122.5994 & 2.76157 \\
\hline 1731 & 32.4103 & 449.4999 & 487.4276 & 467.25 & 122.5994 & 2.76016 \\
\hline 1732 & 32.4273 & 445.2871 & 485.5237 & 465.375 & 122.5994 & 2.75875 \\
\hline 1733 & 32.4443 & 434.9237 & 482.7879 & 463.5 & 122.5994 & 2.75735 \\
\hline 1734 & 32.4613 & 440.9577 & 479.598 & 461.625 & 122.5994 & 2.75594 \\
\hline 1735 & 32.4783 & 448.8587 & 476.1708 & 459.75 & 122.5994 & 2.75454 \\
\hline 1736 & 32.4953 & 465.1966 & 472.5066 & 457.875 & 122.5994 & 2.75313 \\
\hline 1737 & 32.5123 & 447.4138 & 468.6067 & 456 & 122.5994 & 2.75173 \\
\hline 1738 & 32.5293 & 444.1381 & 464.6276 & 454.125 & 122.5994 & 2.75033 \\
\hline 1739 & 32.5463 & 477.0603 & 460.7869 & 452.25 & 122.5994 & 2.74894 \\
\hline 1740 & 32.5633 & 464.8253 & 457.2234 & 450.375 & 122.5994 & 2.74754 \\
\hline 1741 & 32.5803 & 462.2057 & 453.983 & 448.5 & 122.5994 & 2.74615 \\
\hline 1742 & 32.5973 & 452.436 & 451.0808 & 446.625 & 122.5994 & 2.74475 \\
\hline 1743 & 32.6143 & 433.0483 & 448.4175 & 444.75 & 122.5994 & 2.74336 \\
\hline 1744 & 32.6313 & 454.3073 & 445.9658 & 442.875 & 122.5994 & 2.74197 \\
\hline 1745 & 32.6483 & 498.0993 & 443.671 & 441 & 122.5994 & 2.74058 \\
\hline 1746 & 32.6653 & 505.798 & 441.4866 & 439.125 & 122.5994 & 2.73919 \\
\hline 1747 & 32.6823 & 469.7327 & 439.4198 & 437.25 & 122.5994 & 2.73781 \\
\hline 1748 & 32.6993 & 452.6765 & 437.3634 & 435.375 & 122.5994 & 2.73642 \\
\hline 1749 & 32.7163 & 465.8083 & 435.3416 & 433.5 & 122.5994 & 2.73504 \\
\hline 1750 & 32.7333 & 476.4641 & 433.373 & 431.625 & 122.5994 & 2.73366 \\
\hline 1751 & 32.7503 & 471.0365 & 431.3974 & 429.75 & 122.5994 & 2.73228 \\
\hline 1752 & 32.7673 & 413.6473 & 428.9471 & 427.875 & 122.5994 & 2.7309 \\
\hline 1753 & 32.7843 & 407.604 & 427.0312 & 426 & 122.5994 & 2.72952 \\
\hline 1754 & 32.8013 & 422.0072 & 427.5017 & 426.5 & 122.5994 & 2.72815 \\
\hline 1755 & 32.8183 & 452.4395 & 427.9833 & 427 & 122.5994 & 2.72677 \\
\hline 1756 & 32.8353 & 439.6722 & 428.1878 & 427.5 & 122.5994 & 2.7254 \\
\hline 1757 & 32.8523 & 410.2902 & 428.7106 & 428 & 122.5994 & 2.72403 \\
\hline 1758 & 32.8693 & 413.7408 & 429.2436 & 428.5 & 122.5994 & 2.72266 \\
\hline 1759 & 32.8863 & 426.3967 & 429.7958 & 429 & 122.5994 & 2.72129 \\
\hline 1760 & 32.9033 & 439.0467 & 430.3779 & 429.5 & 122.5994 & 2.71992 \\
\hline 1761 & 32.9203 & 466.9192 & 431.01 & 430 & 122.5994 & 2.71856 \\
\hline 1762 & 32.9373 & 459.4423 & 431.7258 & 430.5 & 122.5994 & 2.71719 \\
\hline
\end{tabular}




\begin{tabular}{|c|c|c|c|c|c|c|}
\hline 1763 & 32.9543 & 442.4547 & 432.5714 & 431 & 122.5994 & 2.71583 \\
\hline 1764 & 32.9713 & 448.9357 & 433.5958 & 431.5 & 122.5994 & 2.71447 \\
\hline 1765 & 32.9883 & 478.2004 & 434.8297 & 432 & 122.5994 & 2.71311 \\
\hline 1766 & 33.0053 & 471.0522 & 436.1581 & 432.5 & 122.5994 & 2.71175 \\
\hline 1767 & 33.0223 & 533.5334 & 437.6774 & 433 & 122.5994 & 2.71039 \\
\hline 1768 & 33.0393 & 554.0217 & 439.0709 & 433.5 & 122.5994 & 2.70904 \\
\hline 1769 & 33.0563 & 639.0078 & 440.1764 & 434 & 122.5994 & 2.70768 \\
\hline 1770 & 33.0733 & 615.122 & 441.1481 & 434.5 & 122.5994 & 2.70633 \\
\hline 1771 & 33.0903 & 607.3842 & 442.243 & 435 & 122.5994 & 2.70498 \\
\hline 1772 & 33.1073 & 524.8428 & 443.6529 & 435.5 & 122.5994 & 2.70363 \\
\hline 1773 & 33.1243 & 525.0468 & 445.0898 & 436 & 122.5994 & 2.70228 \\
\hline 1774 & 33.1413 & 496.5823 & 446.1842 & 436.5 & 122.5994 & 2.70093 \\
\hline 1775 & 33.1583 & 504.6076 & 446.8784 & 437 & 122.5994 & 2.69958 \\
\hline 1776 & 33.1753 & 553.6268 & 447.355 & 437.5 & 122.5994 & 2.69824 \\
\hline 1777 & 33.1923 & 514.0445 & 447.655 & 438 & 122.5994 & 2.6969 \\
\hline 1778 & 33.2093 & 482.7305 & 447.81 & 438.5 & 122.5994 & 2.69556 \\
\hline 1779 & 33.2263 & 447.2373 & 447.9789 & 439 & 122.5994 & 2.69422 \\
\hline 1780 & 33.2433 & 434.1651 & 448.3391 & 439.5 & 122.5994 & 2.69288 \\
\hline 1781 & 33.2603 & 414.9034 & 448.7854 & 440 & 122.5994 & 2.69154 \\
\hline 1782 & 33.2773 & 421.4132 & 449.0624 & 440.5 & 122.5994 & 2.6902 \\
\hline 1783 & 33.2943 & 431.0701 & 448.9724 & 441 & 122.5994 & 2.68887 \\
\hline 1784 & 33.3113 & 427.1693 & 448.5244 & 441.5 & 122.5994 & 2.68753 \\
\hline 1785 & 33.3283 & 462.049 & 448.0753 & 442 & 122.5994 & 2.6862 \\
\hline 1786 & 33.3453 & 476.9708 & 447.82 & 442.5 & 122.5994 & 2.68487 \\
\hline 1787 & 33.3623 & 457.7868 & 447.7609 & 443 & 122.5994 & 2.68354 \\
\hline 1788 & 33.3793 & 443.6265 & 447.7895 & 443.5 & 122.5994 & 2.68221 \\
\hline 1789 & 33.3963 & 472.5607 & 447.8031 & 444 & 122.5994 & 2.68089 \\
\hline 1790 & 33.4133 & 446.5325 & 447.8503 & 444.5 & 122.5994 & 2.67956 \\
\hline 1791 & 33.4303 & 440.3097 & 447.9703 & 445 & 122.5994 & 2.67824 \\
\hline 1792 & 33.4473 & 448.2386 & 448.1806 & 445.5 & 122.5994 & 2.67692 \\
\hline 1793 & 33.4643 & 454.4948 & 448.465 & 446 & 122.5994 & 2.6756 \\
\hline 1794 & 33.4813 & 460.3708 & 448.8011 & 446.5 & 122.5994 & 2.67428 \\
\hline 1795 & 33.4983 & 436.8227 & 449.1461 & 447 & 122.5994 & 2.67296 \\
\hline 1796 & 33.5153 & 436.2254 & 449.5527 & 447.5 & 122.5994 & 2.67164 \\
\hline 1797 & 33.5323 & 438.3635 & 449.9971 & 448 & 122.5994 & 2.67033 \\
\hline 1798 & 33.5493 & 436.7681 & 450.5131 & 448.5 & 122.5994 & 2.66901 \\
\hline 1799 & 33.5663 & 457.2869 & 451.1675 & 449 & 122.5994 & 2.6677 \\
\hline 1800 & 33.5833 & 454.3984 & 452.0128 & 449.5 & 122.5994 & 2.66639 \\
\hline 1801 & 33.6003 & 437.2458 & 453.037 & 450 & 122.5994 & 2.66508 \\
\hline 1802 & 33.6173 & 448.4035 & 454.9093 & 451.2917 & 122.5994 & 2.66377 \\
\hline 1803 & 33.6343 & 456.944 & 456.7696 & 452.5833 & 122.5994 & 2.66246 \\
\hline 1804 & 33.6513 & 431.3459 & 458.7417 & 453.875 & 122.5994 & 2.66115 \\
\hline 1805 & 33.6683 & 424.3193 & 461.0193 & 455.1667 & 122.5994 & 2.65985 \\
\hline 1806 & 33.6853 & 410.7572 & 462.7934 & 456.4583 & 122.5994 & 2.65855 \\
\hline 1807 & 33.7023 & 450.8518 & 464.0695 & 457.75 & 122.5994 & 2.65724 \\
\hline 1808 & 33.7193 & 450.4201 & 465.0527 & 459.0417 & 122.5994 & 2.65594 \\
\hline 1809 & 33.7363 & 404.9316 & 466.1168 & 460.3333 & 122.5994 & 2.65464 \\
\hline 1810 & 33.7533 & 430.0845 & 467.4706 & 461.625 & 122.5994 & 2.65335 \\
\hline 1811 & 33.7703 & 405.6557 & 469.0433 & 462.9167 & 122.5994 & 2.65205 \\
\hline
\end{tabular}




\begin{tabular}{|c|c|c|c|c|c|c|}
\hline 1812 & 33.7873 & 427.6178 & 470.6465 & 464.2083 & 122.5994 & 2.65075 \\
\hline 1813 & 33.8043 & 470.4215 & 472.3669 & 465.5 & 122.5994 & 2.64946 \\
\hline 1814 & 33.8213 & 429.0631 & 474.2384 & 466.7917 & 122.5994 & 2.64817 \\
\hline 1815 & 33.8383 & 495.507 & 476.3607 & 468.0833 & 122.5994 & 2.64687 \\
\hline 1816 & 33.8553 & 436.7681 & 478.1622 & 469.375 & 122.5994 & 2.64558 \\
\hline 1817 & 33.8723 & 436.8682 & 479.6874 & 470.6667 & 122.5994 & 2.6443 \\
\hline 1818 & 33.8893 & 435.9771 & 480.9678 & 471.9583 & 122.5994 & 2.64301 \\
\hline 1819 & 33.9063 & 434.4037 & 482.2211 & 473.25 & 122.5994 & 2.64172 \\
\hline 1820 & 33.9233 & 484.6026 & 483.8416 & 474.5417 & 122.5994 & 2.64044 \\
\hline 1821 & 33.9403 & 502.7186 & 486.1288 & 475.8333 & 122.5994 & 2.63915 \\
\hline 1822 & 33.9573 & 451.9138 & 490.1562 & 477.125 & 122.5994 & 2.63787 \\
\hline 1823 & 33.9743 & 522.5803 & 496.9966 & 478.4167 & 122.5994 & 2.63659 \\
\hline 1824 & 33.9913 & 516.7146 & 506.1955 & 479.7083 & 122.5994 & 2.63531 \\
\hline 1825 & 34.0083 & 457.9161 & 513.1893 & 481 & 122.5994 & 2.63403 \\
\hline 1826 & 34.0253 & 459.062 & 513.6054 & 483.9583 & 122.5994 & 2.63275 \\
\hline 1827 & 34.0423 & 473.7208 & 510.0053 & 486.9167 & 122.5994 & 2.63148 \\
\hline 1828 & 34.0593 & 501.7119 & 509.3856 & 489.875 & 122.5994 & 2.6302 \\
\hline 1829 & 34.0763 & 487.2925 & 513.1589 & 492.8333 & 122.5994 & 2.62893 \\
\hline 1830 & 34.0933 & 473.3887 & 518.5031 & 495.7917 & 122.5994 & 2.62766 \\
\hline 1831 & 34.1103 & 501.8269 & 520.6475 & 498.75 & 122.5994 & 2.62639 \\
\hline 1832 & 34.1273 & 496.4209 & 519.8896 & 501.7083 & 122.5994 & 2.62512 \\
\hline 1833 & 34.1443 & 488.3632 & 519.45 & 504.6667 & 122.5994 & 2.62385 \\
\hline 1834 & 34.1613 & 480.7769 & 520.4731 & 507.625 & 122.5994 & 2.62258 \\
\hline 1835 & 34.1783 & 483.0104 & 522.6362 & 510.5833 & 122.5994 & 2.62132 \\
\hline 1836 & 34.1953 & 480.4507 & 525.1066 & 513.5417 & 122.5994 & 2.62005 \\
\hline 1837 & 34.2123 & 486.6492 & 527.3627 & 516.5 & 122.5994 & 2.61879 \\
\hline 1838 & 34.2293 & 510.711 & 529.4634 & 519.4583 & 122.5994 & 2.61753 \\
\hline 1839 & 34.2463 & 508.3613 & 531.8273 & 522.4167 & 122.5994 & 2.61627 \\
\hline 1840 & 34.2633 & 503.6494 & 534.5526 & 525.375 & 122.5994 & 2.61501 \\
\hline 1841 & 34.2803 & 509.8099 & 537.5857 & 528.3333 & 122.5994 & 2.61375 \\
\hline 1842 & 34.2973 & 504.2345 & 541.0387 & 531.2917 & 122.5994 & 2.61249 \\
\hline 1843 & 34.3143 & 535.1332 & 544.8408 & 534.25 & 122.5994 & 2.61124 \\
\hline 1844 & 34.3313 & 529.6329 & 548.087 & 537.2083 & 122.5994 & 2.60998 \\
\hline 1845 & 34.3483 & 506.0951 & 551.4015 & 540.1667 & 122.5994 & 2.60873 \\
\hline 1846 & 34.3653 & 498.7087 & 555.1371 & 543.125 & 122.5994 & 2.60748 \\
\hline 1847 & 34.3823 & 530.029 & 559.739 & 546.0833 & 122.5994 & 2.60623 \\
\hline 1848 & 34.3993 & 539.5183 & 565.4361 & 549.0417 & 122.5994 & 2.60498 \\
\hline 1849 & 34.4163 & 552.4545 & 571.6876 & 552 & 122.5994 & 2.60373 \\
\hline 1850 & 34.4333 & 564.3341 & 575.9282 & 552.2083 & 122.5994 & 2.60249 \\
\hline 1851 & 34.4503 & 574.2127 & 580.659 & 552.4167 & 122.5994 & 2.60124 \\
\hline 1852 & 34.4673 & 529.5247 & 585.5742 & 552.625 & 122.5994 & 2.6 \\
\hline 1853 & 34.4843 & 500.782 & 590.1533 & 552.8333 & 122.5994 & 2.59875 \\
\hline 1854 & 34.5013 & 599.6421 & 594.0298 & 553.0417 & 122.5994 & 2.59751 \\
\hline 1855 & 34.5183 & 588.9182 & 596.7689 & 553.25 & 122.5994 & 2.59627 \\
\hline 1856 & 34.5353 & 630.6716 & 597.9671 & 553.4583 & 122.5994 & 2.59503 \\
\hline 1857 & 34.5523 & 620.9392 & 597.9153 & 553.6667 & 122.5994 & 2.5938 \\
\hline 1858 & 34.5693 & 587.2426 & 596.7853 & 553.875 & 122.5994 & 2.59256 \\
\hline 1859 & 34.5863 & 628.9421 & 595.0255 & 554.0833 & 122.5994 & 2.59132 \\
\hline 1860 & 34.6033 & 595.4839 & 592.8467 & 554.2917 & 122.5994 & 2.59009 \\
\hline
\end{tabular}




\begin{tabular}{|c|c|c|c|c|c|c|}
\hline 1861 & 34.6203 & 629.6476 & 590.254 & 554.5 & 122.5994 & 2.58886 \\
\hline 1862 & 34.6373 & 617.0227 & 587.5799 & 554.7083 & 122.5994 & 2.58762 \\
\hline 1863 & 34.6543 & 654.1784 & 585.2201 & 554.9167 & 122.5994 & 2.58639 \\
\hline 1864 & 34.6713 & 569.2828 & 583.5344 & 555.125 & 122.5994 & 2.58516 \\
\hline 1865 & 34.6883 & 649.1075 & 582.6077 & 555.3333 & 122.5994 & 2.58394 \\
\hline 1866 & 34.7053 & 633.8285 & 582.3192 & 555.5417 & 122.5994 & 2.58271 \\
\hline 1867 & 34.7223 & 599.3604 & 582.4167 & 555.75 & 122.5994 & 2.58148 \\
\hline 1868 & 34.7393 & 647.5897 & 582.6841 & 555.9583 & 122.5994 & 2.58026 \\
\hline 1869 & 34.7563 & 631.2756 & 583.0204 & 556.1667 & 122.5994 & 2.57904 \\
\hline 1870 & 34.7733 & 618.1827 & 583.5646 & 556.375 & 122.5994 & 2.57781 \\
\hline 1871 & 34.7903 & 627.7388 & 584.3682 & 556.5833 & 122.5994 & 2.57659 \\
\hline 1872 & 34.8073 & 623.8124 & 585.8509 & 556.7917 & 122.5994 & 2.57537 \\
\hline 1873 & 34.8243 & 637.944 & 588.3149 & 557 & 122.5994 & 2.57416 \\
\hline 1874 & 34.8413 & 651.2423 & 590.7019 & 555.6458 & 122.5994 & 2.57294 \\
\hline 1875 & 34.8583 & 684.2095 & 595.5614 & 554.2917 & 122.5994 & 2.57172 \\
\hline 1876 & 34.8753 & 696.7917 & 604.6977 & 552.9375 & 122.5994 & 2.57051 \\
\hline 1877 & 34.8923 & 667.843 & 620.0176 & 551.5833 & 122.5994 & 2.56929 \\
\hline 1878 & 34.9093 & 699.4988 & 641.6511 & 550.2292 & 122.5994 & 2.56808 \\
\hline 1879 & 34.9263 & 719.3263 & 663.9706 & 548.875 & 122.5994 & 2.56687 \\
\hline 1880 & 34.9433 & 771.9868 & 675.8818 & 547.5208 & 122.5994 & 2.56566 \\
\hline 1881 & 34.9603 & 751.3987 & 678.4882 & 546.1667 & 122.5994 & 2.56445 \\
\hline 1882 & 34.9773 & 741.3257 & 681.1436 & 544.8125 & 122.5994 & 2.56325 \\
\hline 1883 & 34.9943 & 753.8399 & 686.488 & 543.4583 & 122.5994 & 2.56204 \\
\hline 1884 & 35.0113 & 742.5765 & 691.265 & 542.1042 & 122.5994 & 2.56083 \\
\hline 1885 & 35.0283 & 730.8694 & 689.0624 & 540.75 & 122.5994 & 2.55963 \\
\hline 1886 & 35.0453 & 724.1774 & 678.2189 & 539.3958 & 122.5994 & 2.55843 \\
\hline 1887 & 35.0623 & 722.6933 & 664.1089 & 538.0417 & 122.5994 & 2.55723 \\
\hline 1888 & 35.0793 & 728.0583 & 649.6441 & 536.6875 & 122.5994 & 2.55603 \\
\hline 1889 & 35.0963 & 734.3396 & 635.2552 & 535.3333 & 122.5994 & 2.55483 \\
\hline 1890 & 35.1133 & 700.2289 & 621.1443 & 533.9792 & 122.5994 & 2.55363 \\
\hline 1891 & 35.1303 & 650.4064 & 607.5512 & 532.625 & 122.5994 & 2.55243 \\
\hline 1892 & 35.1473 & 614.4461 & 595.1263 & 531.2708 & 122.5994 & 2.55124 \\
\hline 1893 & 35.1643 & 644.4603 & 584.0345 & 529.9167 & 122.5994 & 2.55004 \\
\hline 1894 & 35.1813 & 588.2778 & 574.3613 & 528.5625 & 122.5994 & 2.54885 \\
\hline 1895 & 35.1983 & 601.8788 & 565.955 & 527.2083 & 122.5994 & 2.54766 \\
\hline 1896 & 35.2153 & 562.7973 & 558.8834 & 525.8542 & 122.5994 & 2.54647 \\
\hline 1897 & 35.2323 & 585.6326 & 552.9511 & 524.5 & 122.5994 & 2.54528 \\
\hline 1898 & 35.2493 & 548.6706 & 548.5072 & 523.1458 & 122.5994 & 2.54409 \\
\hline 1899 & 35.2663 & 515.1653 & 545.3419 & 521.7917 & 122.5994 & 2.5429 \\
\hline 1900 & 35.2833 & 550.5352 & 543.1933 & 520.4375 & 122.5994 & 2.54171 \\
\hline 1901 & 35.3003 & 569.4591 & 541.8765 & 519.0833 & 122.5994 & 2.54053 \\
\hline 1902 & 35.3173 & 562.694 & 541.7126 & 517.7292 & 122.5994 & 2.53934 \\
\hline 1903 & 35.3343 & 561.6361 & 541.9102 & 516.375 & 122.5994 & 2.53816 \\
\hline 1904 & 35.3513 & 522.0723 & 542.4824 & 515.0208 & 122.5994 & 2.53698 \\
\hline 1905 & 35.3683 & 524.3286 & 542.4336 & 513.6667 & 122.5994 & 2.5358 \\
\hline 1906 & 35.3853 & 519.8425 & 540.0884 & 512.3125 & 122.5994 & 2.53462 \\
\hline 1907 & 35.4023 & 533.107 & 536.0648 & 510.9583 & 122.5994 & 2.53344 \\
\hline 1908 & 35.4193 & 489.1824 & 532.1906 & 509.6042 & 122.5994 & 2.53226 \\
\hline 1909 & 35.4363 & 509.291 & 529.5632 & 508.25 & 122.5994 & 2.53109 \\
\hline
\end{tabular}




\begin{tabular}{|c|c|c|c|c|c|c|}
\hline 1910 & 35.4533 & 483.9536 & 528.0302 & 506.8958 & 122.5994 & 2.52991 \\
\hline 1911 & 35.4703 & 485.3422 & 526.4127 & 505.5417 & 122.5994 & 2.52874 \\
\hline 1912 & 35.4873 & 496.7192 & 524.0505 & 504.1875 & 122.5994 & 2.52757 \\
\hline 1913 & 35.5043 & 496.6642 & 521.6818 & 502.8333 & 122.5994 & 2.5264 \\
\hline 1914 & 35.5213 & 513.3282 & 519.8063 & 501.4792 & 122.5994 & 2.52523 \\
\hline 1915 & 35.5383 & 483.7113 & 517.971 & 500.125 & 122.5994 & 2.52406 \\
\hline 1916 & 35.5553 & 504.4679 & 516.1061 & 498.7708 & 122.5994 & 2.52289 \\
\hline 1917 & 35.5723 & 508.1967 & 514.038 & 497.4167 & 122.5994 & 2.52172 \\
\hline 1918 & 35.5893 & 492.9086 & 511.9478 & 496.0625 & 122.5994 & 2.52056 \\
\hline 1919 & 35.6063 & 518.5699 & 509.9908 & 494.7083 & 122.5994 & 2.51939 \\
\hline 1920 & 35.6233 & 507.5679 & 508.1935 & 493.3542 & 122.5994 & 2.51823 \\
\hline 1921 & 35.6403 & 482.9099 & 506.5359 & 492 & 122.5994 & 2.51707 \\
\hline 1922 & 35.6573 & 476.6733 & 507.1155 & 492.6667 & 122.5994 & 2.51591 \\
\hline 1923 & 35.6743 & 491.6215 & 507.9268 & 493.3333 & 122.5994 & 2.51475 \\
\hline 1924 & 35.6913 & 497.2423 & 509.5349 & 494 & 122.5994 & 2.51359 \\
\hline 1925 & 35.7083 & 495.5879 & 511.442 & 494.6667 & 122.5994 & 2.51243 \\
\hline 1926 & 35.7253 & 492.7927 & 514.7054 & 495.3333 & 122.5994 & 2.51127 \\
\hline 1927 & 35.7423 & 508.8329 & 518.7437 & 496 & 122.5994 & 2.51012 \\
\hline 1928 & 35.7593 & 510.2013 & 522.7467 & 496.6667 & 122.5994 & 2.50896 \\
\hline 1929 & 35.7763 & 481.998 & 525.5314 & 497.3333 & 122.5994 & 2.50781 \\
\hline 1930 & 35.7933 & 484.1906 & 527.0735 & 498 & 122.5994 & 2.50666 \\
\hline 1931 & 35.8103 & 490.4609 & 528.7172 & 498.6667 & 122.5994 & 2.50551 \\
\hline 1932 & 35.8273 & 462.4932 & 530.7859 & 499.3333 & 122.5994 & 2.50436 \\
\hline 1933 & 35.8443 & 483.2619 & 533.2231 & 500 & 122.5994 & 2.50321 \\
\hline 1934 & 35.8613 & 507.2461 & 535.9782 & 500.6667 & 122.5994 & 2.50206 \\
\hline 1935 & 35.8783 & 480.2663 & 539.0374 & 501.3333 & 122.5994 & 2.50091 \\
\hline 1936 & 35.8953 & 485.9961 & 542.9972 & 502 & 122.5994 & 2.49977 \\
\hline 1937 & 35.9123 & 465.9865 & 548.4034 & 502.6667 & 122.5994 & 2.49862 \\
\hline 1938 & 35.9293 & 509.5151 & 555.43 & 503.3333 & 122.5994 & 2.49748 \\
\hline 1939 & 35.9463 & 500.4167 & 564.0565 & 504 & 122.5994 & 2.49634 \\
\hline 1940 & 35.9633 & 509.7611 & 573.8209 & 504.6667 & 122.5994 & 2.4952 \\
\hline 1941 & 35.9803 & 533.4337 & 582.9557 & 505.3333 & 122.5994 & 2.49406 \\
\hline 1942 & 35.9973 & 513.8987 & 588.8747 & 506 & 122.5994 & 2.49292 \\
\hline 1943 & 36.0143 & 502.6787 & 589.8365 & 506.6667 & 122.5994 & 2.49178 \\
\hline 1944 & 36.0313 & 501.8269 & 587.6284 & 507.3333 & 122.5994 & 2.49064 \\
\hline 1945 & 36.0483 & 505.428 & 586.5476 & 508 & 122.5994 & 2.48951 \\
\hline 1946 & 36.0653 & 521.6203 & 583.0505 & 508.2292 & 122.5994 & 2.48837 \\
\hline 1947 & 36.0823 & 504.7584 & 579.087 & 508.4583 & 122.5994 & 2.48724 \\
\hline 1948 & 36.0993 & 546.7553 & 573.6773 & 508.6875 & 122.5994 & 2.48611 \\
\hline 1949 & 36.1163 & 510.2899 & 567.2473 & 508.9167 & 122.5994 & 2.48498 \\
\hline 1950 & 36.1333 & 523.1846 & 560.952 & 509.1458 & 122.5994 & 2.48385 \\
\hline 1951 & 36.1503 & 528.8718 & 556.7578 & 509.375 & 122.5994 & 2.48272 \\
\hline 1952 & 36.1673 & 537.9884 & 552.4905 & 509.6042 & 122.5994 & 2.48159 \\
\hline 1953 & 36.1843 & 519.7941 & 548.9607 & 509.8333 & 122.5994 & 2.48046 \\
\hline 1954 & 36.2013 & 479.7509 & 545.9764 & 510.0625 & 122.5994 & 2.47934 \\
\hline 1955 & 36.2183 & 488.4751 & 543.645 & 510.2917 & 122.5994 & 2.47821 \\
\hline 1956 & 36.2353 & 489.8005 & 542.4144 & 510.5208 & 122.5994 & 2.47709 \\
\hline 1957 & 36.2523 & 508.7949 & 541.9227 & 510.75 & 122.5994 & 2.47597 \\
\hline 1958 & 36.2693 & 487.5302 & 541.3845 & 510.9792 & 122.5994 & 2.47485 \\
\hline
\end{tabular}




\begin{tabular}{|c|c|c|c|c|c|c|}
\hline 1959 & 36.2863 & 480.1294 & 540.529 & 511.2083 & 122.5994 & 2.47372 \\
\hline 1960 & 36.3033 & 484.728 & 540.2405 & 511.4375 & 122.5994 & 2.47261 \\
\hline 1961 & 36.3203 & 484.6078 & 541.2086 & 511.6667 & 122.5994 & 2.47149 \\
\hline 1962 & 36.3373 & 466.6222 & 543.6654 & 511.8958 & 122.5994 & 2.47037 \\
\hline 1963 & 36.3543 & 478.9496 & 547.4269 & 512.125 & 122.5994 & 2.46925 \\
\hline 1964 & 36.3713 & 527.6286 & 552.2011 & 512.3542 & 122.5994 & 2.46814 \\
\hline 1965 & 36.3883 & 528.8995 & 558.2167 & 512.5833 & 122.5994 & 2.46702 \\
\hline 1966 & 36.4053 & 500.4628 & 565.5741 & 512.8125 & 122.5994 & 2.46591 \\
\hline 1967 & 36.4223 & 504.1457 & 573.8369 & 513.0417 & 122.5994 & 2.4648 \\
\hline 1968 & 36.4393 & 539.453 & 582.9421 & 513.2708 & 122.5994 & 2.46369 \\
\hline 1969 & 36.4563 & 579.2321 & 594.8696 & 513.5 & 122.5994 & 2.46258 \\
\hline 1970 & 36.4733 & 592.0335 & 614.0682 & 513.7292 & 122.5994 & 2.46147 \\
\hline 1971 & 36.4903 & 623.8204 & 647.7733 & 513.9583 & 122.5994 & 2.46036 \\
\hline 1972 & 36.5073 & 681.6808 & 706.2736 & 514.1875 & 122.5994 & 2.45926 \\
\hline 1973 & 36.5243 & 768.1116 & 799.0591 & 514.4167 & 122.5994 & 2.45815 \\
\hline 1974 & 36.5413 & 883.8828 & 922.1915 & 514.6458 & 122.5994 & 2.45705 \\
\hline 1975 & 36.5583 & 1066.679 & 1044.637 & 514.875 & 122.5994 & 2.45594 \\
\hline 1976 & 36.5753 & 1179.749 & 1100.506 & 515.1042 & 122.5994 & 2.45484 \\
\hline 1977 & 36.5923 & 1179.061 & 1054.623 & 515.3333 & 122.5994 & 2.45374 \\
\hline 1978 & 36.6093 & 1008.869 & 965.4512 & 515.5625 & 122.5994 & 2.45264 \\
\hline 1979 & 36.6263 & 854.2374 & 898.4993 & 515.7917 & 122.5994 & 2.45154 \\
\hline 1980 & 36.6433 & 831.2607 & 873.1705 & 516.0208 & 122.5994 & 2.45044 \\
\hline 1981 & 36.6603 & 855.1828 & 865.66 & 516.25 & 122.5994 & 2.44934 \\
\hline 1982 & 36.6773 & 879.0477 & 832.5121 & 516.4792 & 122.5994 & 2.44825 \\
\hline 1983 & 36.6943 & 839.0565 & 766.3081 & 516.7083 & 122.5994 & 2.44715 \\
\hline 1984 & 36.7113 & 697.5567 & 694.6185 & 516.9375 & 122.5994 & 2.44606 \\
\hline 1985 & 36.7283 & 612.6087 & 638.2835 & 517.1667 & 122.5994 & 2.44496 \\
\hline 1986 & 36.7453 & 576.3283 & 602.671 & 517.3958 & 122.5994 & 2.44387 \\
\hline 1987 & 36.7623 & 535.6482 & 586.3477 & 517.625 & 122.5994 & 2.44278 \\
\hline 1988 & 36.7793 & 527.8148 & 585.067 & 517.8542 & 122.5994 & 2.44169 \\
\hline 1989 & 36.7963 & 536.2259 & 592.0102 & 518.0833 & 122.5994 & 2.4406 \\
\hline 1990 & 36.8133 & 530.8351 & 595.6789 & 518.3125 & 122.5994 & 2.43951 \\
\hline 1991 & 36.8303 & 521.5826 & 587.0787 & 518.5417 & 122.5994 & 2.43843 \\
\hline 1992 & 36.8473 & 500.8734 & 575.4664 & 518.7708 & 122.5994 & 2.43734 \\
\hline 1993 & 36.8643 & 512.8458 & 570.5281 & 519 & 122.5994 & 2.43626 \\
\hline 1994 & 36.8813 & 531.3979 & 571.7839 & 517.9583 & 122.5994 & 2.43517 \\
\hline 1995 & 36.8983 & 546.1608 & 573.355 & 516.9167 & 122.5994 & 2.43409 \\
\hline 1996 & 36.9153 & 534.7289 & 567.2969 & 515.875 & 122.5994 & 2.43301 \\
\hline 1997 & 36.9323 & 555.6582 & 555.6176 & 514.8333 & 122.5994 & 2.43193 \\
\hline 1998 & 36.9493 & 516.6939 & 545.7443 & 513.7917 & 122.5994 & 2.43085 \\
\hline 1999 & 36.9663 & 528.8068 & 540.4133 & 512.75 & 122.5994 & 2.42977 \\
\hline 2000 & 36.9833 & 511.2353 & 538.0914 & 511.7083 & 122.5994 & 2.42869 \\
\hline 2001 & 37.0003 & 547.0543 & 536.114 & 510.6667 & 122.5994 & 2.42761 \\
\hline 2002 & 37.0173 & 532.9357 & 534.3494 & 509.625 & 122.5994 & 2.42654 \\
\hline 2003 & 37.0343 & 521.8845 & 533.9131 & 508.5833 & 122.5994 & 2.42546 \\
\hline 2004 & 37.0513 & 550.3975 & 534.967 & 507.5417 & 122.5994 & 2.42439 \\
\hline 2005 & 37.0683 & 579.4039 & 537.0983 & 506.5 & 122.5994 & 2.42331 \\
\hline 2006 & 37.0853 & 599.0142 & 538.9442 & 505.4583 & 122.5994 & 2.42224 \\
\hline 2007 & 37.1023 & 564.8565 & 539.663 & 504.4167 & 122.5994 & 2.42117 \\
\hline
\end{tabular}




\begin{tabular}{|c|c|c|c|c|c|c|}
\hline 2008 & 37.1193 & 575.5245 & 538.6733 & 503.375 & 122.5994 & 2.4201 \\
\hline 2009 & 37.1363 & 540.1585 & 536.7236 & 502.3333 & 122.5994 & 2.41903 \\
\hline 2010 & 37.1533 & 492.7927 & 534.4802 & 501.2917 & 122.5994 & 2.41797 \\
\hline 2011 & 37.1703 & 495.1164 & 532.6763 & 500.25 & 122.5994 & 2.4169 \\
\hline 2012 & 37.1873 & 501.7119 & 531.4946 & 499.2083 & 122.5994 & 2.41583 \\
\hline 2013 & 37.2043 & 516.3881 & 531.1562 & 498.1667 & 122.5994 & 2.41477 \\
\hline 2014 & 37.2213 & 531.0074 & 528.7676 & 497.125 & 122.5994 & 2.4137 \\
\hline 2015 & 37.2383 & 486.9039 & 527.9624 & 496.0833 & 122.5994 & 2.41264 \\
\hline 2016 & 37.2553 & 494.4872 & 524.5934 & 495.0417 & 122.5994 & 2.41158 \\
\hline 2017 & 37.2723 & 492.2344 & 520.3585 & 494 & 122.5994 & 2.41052 \\
\hline 2018 & 37.2893 & 498.2263 & 516.8945 & 492.7917 & 122.5994 & 2.40946 \\
\hline 2019 & 37.3063 & 499.3559 & 514.6841 & 491.5833 & 122.5994 & 2.4084 \\
\hline 2020 & 37.3233 & 490.4388 & 513.0929 & 490.375 & 122.5994 & 2.40734 \\
\hline 2021 & 37.3403 & 513.2615 & 510.8552 & 489.1667 & 122.5994 & 2.40628 \\
\hline 2022 & 37.3573 & 508.5265 & 507.6765 & 487.9583 & 122.5994 & 2.40523 \\
\hline 2023 & 37.3743 & 490.6256 & 504.7292 & 486.75 & 122.5994 & 2.40417 \\
\hline 2024 & 37.3913 & 498.7185 & 502.6677 & 485.5417 & 122.5994 & 2.40312 \\
\hline 2025 & 37.4083 & 500.1188 & 501.2694 & 484.3333 & 122.5994 & 2.40207 \\
\hline 2026 & 37.4253 & 517.6622 & 499.5303 & 483.125 & 122.5994 & 2.40101 \\
\hline 2027 & 37.4423 & 518.4916 & 496.9264 & 481.9167 & 122.5994 & 2.39996 \\
\hline 2028 & 37.4593 & 512.9205 & 494.4852 & 480.7083 & 122.5994 & 2.39891 \\
\hline 2029 & 37.4763 & 525.6068 & 493.0002 & 479.5 & 122.5994 & 2.39786 \\
\hline 2030 & 37.4933 & 508.134 & 492.557 & 478.2917 & 122.5994 & 2.39682 \\
\hline 2031 & 37.5103 & 497.3563 & 492.6645 & 477.0833 & 122.5994 & 2.39577 \\
\hline 2032 & 37.5273 & 525.8867 & 492.4382 & 475.875 & 122.5994 & 2.39472 \\
\hline 2033 & 37.5443 & 525.0427 & 491.838 & 474.6667 & 122.5994 & 2.39368 \\
\hline 2034 & 37.5613 & 533.4533 & 491.145 & 473.4583 & 122.5994 & 2.39263 \\
\hline 2035 & 37.5783 & 524.2502 & 490.7287 & 472.25 & 122.5994 & 2.39159 \\
\hline 2036 & 37.5953 & 516.2155 & 491.26 & 471.0417 & 122.5994 & 2.39055 \\
\hline 2037 & 37.6123 & 500.7136 & 492.7903 & 469.8333 & 122.5994 & 2.38951 \\
\hline 2038 & 37.6293 & 508.519 & 495.2714 & 468.625 & 122.5994 & 2.38847 \\
\hline 2039 & 37.6463 & 514.5621 & 497.2341 & 467.4167 & 122.5994 & 2.38743 \\
\hline 2040 & 37.6633 & 547.5795 & 497.2042 & 466.2083 & 122.5994 & 2.38639 \\
\hline 2041 & 37.6803 & 543.8367 & 495.5663 & 465 & 122.5994 & 2.38535 \\
\hline 2042 & 37.6973 & 521.756 & 494.3051 & 463.7917 & 122.5994 & 2.38431 \\
\hline 2043 & 37.7143 & 528.4855 & 494.9803 & 462.5833 & 122.5994 & 2.38328 \\
\hline 2044 & 37.7313 & 546.7239 & 497.0168 & 461.375 & 122.5994 & 2.38224 \\
\hline 2045 & 37.7483 & 524.9614 & 498.707 & 460.1667 & 122.5994 & 2.38121 \\
\hline 2046 & 37.7653 & 561.7691 & 499.0886 & 458.9583 & 122.5994 & 2.38018 \\
\hline 2047 & 37.7823 & 565.8603 & 497.8541 & 457.75 & 122.5994 & 2.37914 \\
\hline 2048 & 37.7993 & 541.83 & 494.8507 & 456.5417 & 122.5994 & 2.37811 \\
\hline 2049 & 37.8163 & 520.5082 & 489.717 & 455.3333 & 122.5994 & 2.37708 \\
\hline 2050 & 37.8333 & 528.9493 & 484.2354 & 454.125 & 122.5994 & 2.37605 \\
\hline 2051 & 37.8503 & 530.0726 & 479.6068 & 452.9167 & 122.5994 & 2.37503 \\
\hline 2052 & 37.8673 & 531.1442 & 475.8941 & 451.7083 & 122.5994 & 2.374 \\
\hline 2053 & 37.8843 & 486.7555 & 472.4959 & 450.5 & 122.5994 & 2.37297 \\
\hline 2054 & 37.9013 & 494.1371 & 468.5131 & 449.2917 & 122.5994 & 2.37195 \\
\hline 2055 & 37.9183 & 466.429 & 463.7898 & 448.0833 & 122.5994 & 2.37092 \\
\hline 2056 & 37.9353 & 496.0983 & 459.3723 & 446.875 & 122.5994 & 2.3699 \\
\hline
\end{tabular}




\begin{tabular}{|c|c|c|c|c|c|c|}
\hline 2057 & 37.9523 & 472.1102 & 455.6882 & 445.6667 & 122.5994 & 2.36888 \\
\hline 2058 & 37.9693 & 464.5501 & 452.7565 & 444.4583 & 122.5994 & 2.36786 \\
\hline 2059 & 37.9863 & 466.0202 & 450.321 & 443.25 & 122.5994 & 2.36684 \\
\hline 2060 & 38.0033 & 458.8395 & 448.6093 & 442.0417 & 122.5994 & 2.36582 \\
\hline 2061 & 38.0203 & 458.5762 & 447.0816 & 440.8333 & 122.5994 & 2.3648 \\
\hline 2062 & 38.0373 & 445.7944 & 445.9287 & 439.625 & 122.5994 & 2.36378 \\
\hline 2063 & 38.0543 & 458.4577 & 445.1628 & 438.4167 & 122.5994 & 2.36276 \\
\hline 2064 & 38.0713 & 431.9368 & 444.8975 & 437.2083 & 122.5994 & 2.36175 \\
\hline 2065 & 38.0883 & 419.3894 & 445.3107 & 436 & 122.5994 & 2.36073 \\
\hline 2066 & 38.1053 & 441.1604 & 447.4899 & 435.7917 & 122.5994 & 2.35972 \\
\hline 2067 & 38.1223 & 454.5606 & 450.5805 & 435.5833 & 122.5994 & 2.3587 \\
\hline 2068 & 38.1393 & 422.4676 & 454.5936 & 435.375 & 122.5994 & 2.35769 \\
\hline 2069 & 38.1563 & 412.1608 & 459.3474 & 435.1667 & 122.5994 & 2.35668 \\
\hline 2070 & 38.1733 & 418.8733 & 464.3657 & 434.9583 & 122.5994 & 2.35567 \\
\hline 2071 & 38.1903 & 446.2381 & 468.7723 & 434.75 & 122.5994 & 2.35466 \\
\hline 2072 & 38.2073 & 450.7747 & 471.5428 & 434.5417 & 122.5994 & 2.35365 \\
\hline 2073 & 38.2243 & 440.3826 & 471.9825 & 434.3333 & 122.5994 & 2.35264 \\
\hline 2074 & 38.2413 & 407.3812 & 470.655 & 434.125 & 122.5994 & 2.35164 \\
\hline 2075 & 38.2583 & 439.8776 & 468.5189 & 433.9167 & 122.5994 & 2.35063 \\
\hline 2076 & 38.2753 & 438.1187 & 466.4183 & 433.7083 & 122.5994 & 2.34963 \\
\hline 2077 & 38.2923 & 409.4593 & 464.2952 & 433.5 & 122.5994 & 2.34862 \\
\hline 2078 & 38.3093 & 415.1658 & 461.747 & 433.2917 & 122.5994 & 2.34762 \\
\hline 2079 & 38.3263 & 405.8172 & 458.5221 & 433.0833 & 122.5994 & 2.34662 \\
\hline 2080 & 38.3433 & 430.0813 & 454.9805 & 432.875 & 122.5994 & 2.34562 \\
\hline 2081 & 38.3603 & 437.7992 & 451.6778 & 432.6667 & 122.5994 & 2.34462 \\
\hline 2082 & 38.3773 & 436.8682 & 449.1566 & 432.4583 & 122.5994 & 2.34362 \\
\hline 2083 & 38.3943 & 423.5718 & 447.6031 & 432.25 & 122.5994 & 2.34262 \\
\hline 2084 & 38.4113 & 416.8628 & 447.0542 & 432.0417 & 122.5994 & 2.34162 \\
\hline 2085 & 38.4283 & 409.3951 & 447.4521 & 431.8333 & 122.5994 & 2.34062 \\
\hline 2086 & 38.4453 & 414.9952 & 448.7198 & 431.625 & 122.5994 & 2.33963 \\
\hline 2087 & 38.4623 & 434.4498 & 450.5227 & 431.4167 & 122.5994 & 2.33863 \\
\hline 2088 & 38.4793 & 459.7771 & 452.9915 & 431.2083 & 122.5994 & 2.33764 \\
\hline 2089 & 38.4963 & 427.2221 & 455.7333 & 431 & 122.5994 & 2.33664 \\
\hline 2090 & 38.5133 & 401.2379 & 457.4857 & 429.625 & 122.5994 & 2.33565 \\
\hline 2091 & 38.5303 & 431.5875 & 459.3325 & 428.25 & 122.5994 & 2.33466 \\
\hline 2092 & 38.5473 & 404.3374 & 460.9652 & 426.875 & 122.5994 & 2.33367 \\
\hline 2093 & 38.5643 & 407.9484 & 461.6257 & 425.5 & 122.5994 & 2.33268 \\
\hline 2094 & 38.5813 & 395.8388 & 460.753 & 424.125 & 122.5994 & 2.33169 \\
\hline 2095 & 38.5983 & 395.0274 & 457.9755 & 422.75 & 122.5994 & 2.3307 \\
\hline 2096 & 38.6153 & 443.2548 & 453.7611 & 421.375 & 122.5994 & 2.32972 \\
\hline 2097 & 38.6323 & 418.6174 & 449.1507 & 420 & 122.5994 & 2.32873 \\
\hline 2098 & 38.6493 & 408.4913 & 444.973 & 418.625 & 122.5994 & 2.32775 \\
\hline 2099 & 38.6663 & 439.778 & 441.4203 & 417.25 & 122.5994 & 2.32676 \\
\hline 2100 & 38.6833 & 432.0222 & 438.607 & 415.875 & 122.5994 & 2.32578 \\
\hline 2101 & 38.7003 & 424.6823 & 436.9684 & 414.5 & 122.5994 & 2.3248 \\
\hline 2102 & 38.7173 & 405.5274 & 436.8741 & 413.125 & 122.5994 & 2.32381 \\
\hline 2103 & 38.7343 & 399.717 & 437.2628 & 411.75 & 122.5994 & 2.32283 \\
\hline 2104 & 38.7513 & 413.0626 & 434.4771 & 410.375 & 122.5994 & 2.32185 \\
\hline 2105 & 38.7683 & 418.4321 & 427.7296 & 409 & 122.5994 & 2.32087 \\
\hline
\end{tabular}




\begin{tabular}{|c|c|c|c|c|c|c|}
\hline 2106 & 38.7853 & 426.7349 & 421.4594 & 407.625 & 122.5994 & 2.3199 \\
\hline 2107 & 38.8023 & 430.1028 & 417.7697 & 406.25 & 122.5994 & 2.31892 \\
\hline 2108 & 38.8193 & 421.5738 & 417.0844 & 404.875 & 122.5994 & 2.31794 \\
\hline 2109 & 38.8363 & 410.3633 & 418.0055 & 403.5 & 122.5994 & 2.31697 \\
\hline 2110 & 38.8533 & 407.7055 & 417.7816 & 402.125 & 122.5994 & 2.31599 \\
\hline 2111 & 38.8703 & 407.3681 & 414.8912 & 400.75 & 122.5994 & 2.31502 \\
\hline 2112 & 38.8873 & 389.8045 & 410.5486 & 399.375 & 122.5994 & 2.31405 \\
\hline 2113 & 38.9043 & 392.0024 & 406.0373 & 398 & 122.5994 & 2.31307 \\
\hline 2114 & 38.9213 & 405.0861 & 404.6846 & 398.5833 & 122.5994 & 2.3121 \\
\hline 2115 & 38.9383 & 408.5388 & 404.6094 & 399.1667 & 122.5994 & 2.31113 \\
\hline 2116 & 38.9553 & 408.2584 & 405.3388 & 399.75 & 122.5994 & 2.31016 \\
\hline 2117 & 38.9723 & 405.3966 & 405.937 & 400.3333 & 122.5994 & 2.30919 \\
\hline 2118 & 38.9893 & 407.5161 & 405.8938 & 400.9167 & 122.5994 & 2.30823 \\
\hline 2119 & 39.0063 & 422.6552 & 405.507 & 401.5 & 122.5994 & 2.30726 \\
\hline 2120 & 39.0233 & 402.811 & 405.3201 & 402.0833 & 122.5994 & 2.30629 \\
\hline 2121 & 39.0403 & 412.8755 & 405.4704 & 402.6667 & 122.5994 & 2.30533 \\
\hline 2122 & 39.0573 & 433.8393 & 405.8584 & 403.25 & 122.5994 & 2.30436 \\
\hline 2123 & 39.0743 & 415.2023 & 406.364 & 403.8333 & 122.5994 & 2.3034 \\
\hline 2124 & 39.0913 & 409.5513 & 411.2547 & 404.4167 & 122.5994 & 2.30244 \\
\hline 2125 & 39.1083 & 412.0505 & 412.3493 & 405 & 122.5994 & 2.30148 \\
\hline 2126 & 39.1253 & 397.5747 & 413.5731 & 405.5833 & 122.5994 & 2.30052 \\
\hline 2127 & 39.1423 & 422.2838 & 414.9339 & 406.1667 & 122.5994 & 2.29956 \\
\hline 2128 & 39.1593 & 423.1195 & 416.2654 & 406.75 & 122.5994 & 2.2986 \\
\hline 2129 & 39.1763 & 413.8865 & 417.9648 & 407.3333 & 122.5994 & 2.29764 \\
\hline 2130 & 39.1933 & 408.7776 & 419.8972 & 407.9167 & 122.5994 & 2.29668 \\
\hline 2131 & 39.2103 & 435.4438 & 422.1381 & 408.5 & 122.5994 & 2.29572 \\
\hline 2132 & 39.2273 & 406.0587 & 424.8611 & 409.0833 & 122.5994 & 2.29477 \\
\hline 2133 & 39.2443 & 432.0826 & 428.3164 & 409.6667 & 122.5994 & 2.29381 \\
\hline 2134 & 39.2613 & 448.1628 & 432.636 & 410.25 & 122.5994 & 2.29286 \\
\hline 2135 & 39.2783 & 415.196 & 438.276 & 410.8333 & 122.5994 & 2.29191 \\
\hline 2136 & 39.2953 & 415.9599 & 445.1783 & 411.4167 & 122.5994 & 2.29095 \\
\hline 2137 & 39.3123 & 389.2078 & 453.3705 & 412 & 122.5994 & 2.29 \\
\hline 2138 & 39.3293 & 407.7008 & 462.9489 & 413.0417 & 122.5994 & 2.28905 \\
\hline 2139 & 39.3463 & 483.9012 & 472.8623 & 414.0833 & 122.5994 & 2.2881 \\
\hline 2140 & 39.3633 & 453.6511 & 482.6483 & 415.125 & 122.5994 & 2.28715 \\
\hline 2141 & 39.3803 & 452.5507 & 493.345 & 416.1667 & 122.5994 & 2.2862 \\
\hline 2142 & 39.3973 & 522.0629 & 509.0211 & 417.2083 & 122.5994 & 2.28526 \\
\hline 2143 & 39.4143 & 553.0265 & 538.0085 & 418.25 & 122.5994 & 2.28431 \\
\hline 2144 & 39.4313 & 569.1028 & 591.5664 & 419.2917 & 122.5994 & 2.28336 \\
\hline 2145 & 39.4483 & 646.6237 & 678.8835 & 420.3333 & 122.5994 & 2.28242 \\
\hline 2146 & 39.4653 & 763.8427 & 797.5302 & 421.375 & 122.5994 & 2.28148 \\
\hline 2147 & 39.4823 & 826.0747 & 919.4674 & 422.4167 & 122.5994 & 2.28053 \\
\hline 2148 & 39.4993 & 938.6998 & 981.5979 & 423.4583 & 122.5994 & 2.27959 \\
\hline 2149 & 39.5163 & 981.9017 & 940.3725 & 424.5 & 122.5994 & 2.27865 \\
\hline 2150 & 39.5333 & 796.5072 & 846.0867 & 425.5417 & 122.5994 & 2.27771 \\
\hline 2151 & 39.5503 & 692.9934 & 769.3258 & 426.5833 & 122.5994 & 2.27677 \\
\hline 2152 & 39.5673 & 685.3396 & 738.9224 & 427.625 & 122.5994 & 2.27583 \\
\hline 2153 & 39.5843 & 666.3047 & 744.2171 & 428.6667 & 122.5994 & 2.27489 \\
\hline 2154 & 39.6013 & 705.6508 & 744.5082 & 429.7083 & 122.5994 & 2.27395 \\
\hline
\end{tabular}




\begin{tabular}{|c|c|c|c|c|c|c|}
\hline 2155 & 39.6183 & 701.6993 & 705.2711 & 430.75 & 122.5994 & 2.27302 \\
\hline 2156 & 39.6353 & 600.8994 & 640.5249 & 431.7917 & 122.5994 & 2.27208 \\
\hline 2157 & 39.6523 & 504.2435 & 577.1537 & 432.8333 & 122.5994 & 2.27115 \\
\hline 2158 & 39.6693 & 454.4182 & 529.3038 & 433.875 & 122.5994 & 2.27021 \\
\hline 2159 & 39.6863 & 436.884 & 499.5421 & 434.9167 & 122.5994 & 2.26928 \\
\hline 2160 & 39.7033 & 437.3157 & 483.9083 & 435.9583 & 122.5994 & 2.26835 \\
\hline 2161 & 39.7203 & 462.1084 & 476.7133 & 437 & 122.5994 & 2.26741 \\
\hline 2162 & 39.7373 & 443.5136 & 473.0099 & 437.7083 & 122.5994 & 2.26648 \\
\hline 2163 & 39.7543 & 415.9819 & 470.1285 & 438.4167 & 122.5994 & 2.26555 \\
\hline 2164 & 39.7713 & 427.7494 & 467.6217 & 439.125 & 122.5994 & 2.26462 \\
\hline 2165 & 39.7883 & 462.6248 & 465.6484 & 439.8333 & 122.5994 & 2.2637 \\
\hline 2166 & 39.8053 & 462.09 & 464.6075 & 440.5417 & 122.5994 & 2.26277 \\
\hline 2167 & 39.8223 & 414.713 & 464.1889 & 441.25 & 122.5994 & 2.26184 \\
\hline 2168 & 39.8393 & 449.539 & 463.7877 & 441.9583 & 122.5994 & 2.26092 \\
\hline 2169 & 39.8563 & 437.5158 & 463.136 & 442.6667 & 122.5994 & 2.25999 \\
\hline 2170 & 39.8733 & 437.0279 & 462.4234 & 443.375 & 122.5994 & 2.25907 \\
\hline 2171 & 39.8903 & 429.3475 & 461.8232 & 444.0833 & 122.5994 & 2.25814 \\
\hline 2172 & 39.9073 & 444.0126 & 461.358 & 444.7917 & 122.5994 & 2.25722 \\
\hline 2173 & 39.9243 & 438.3112 & 461.1339 & 445.5 & 122.5994 & 2.2563 \\
\hline 2174 & 39.9413 & 451.712 & 461.2207 & 446.2083 & 122.5994 & 2.25538 \\
\hline 2175 & 39.9583 & 431.8324 & 461.3958 & 446.9167 & 122.5994 & 2.25446 \\
\hline 2176 & 39.9753 & 444.4291 & 462.1511 & 447.625 & 122.5994 & 2.25354 \\
\hline 2177 & 39.9923 & 439.0483 & 463.2512 & 448.3333 & 122.5994 & 2.25262 \\
\hline 2178 & 40.0093 & 447.4903 & 464.5982 & 449.0417 & 122.5994 & 2.2517 \\
\hline 2179 & 40.0263 & 442.1179 & 466.1734 & 449.75 & 122.5994 & 2.25078 \\
\hline 2180 & 40.0433 & 452.2779 & 467.9199 & 450.4583 & 122.5994 & 2.24987 \\
\hline 2181 & 40.0603 & 449.7981 & 466.5843 & 451.1667 & 122.5994 & 2.24895 \\
\hline 2182 & 40.0773 & 445.476 & 468.3369 & 451.875 & 122.5994 & 2.24804 \\
\hline 2183 & 40.0943 & 437.3013 & 469.952 & 452.5833 & 122.5994 & 2.24712 \\
\hline 2184 & 40.1113 & 432.7232 & 471.6243 & 453.2917 & 122.5994 & 2.24621 \\
\hline 2185 & 40.1283 & 449.3574 & 473.7109 & 454 & 122.5994 & 2.2453 \\
\hline 2186 & 40.1453 & 475.3081 & 474.433 & 452.5 & 122.5994 & 2.24439 \\
\hline 2187 & 40.1623 & 482.4306 & 476.3181 & 451 & 122.5994 & 2.24347 \\
\hline 2188 & 40.1793 & 435.84 & 479.1144 & 449.5 & 122.5994 & 2.24256 \\
\hline 2189 & 40.1963 & 422.9041 & 481.3858 & 448 & 122.5994 & 2.24166 \\
\hline 2190 & 40.2133 & 455.3512 & 482.4704 & 446.5 & 122.5994 & 2.24075 \\
\hline 2191 & 40.2303 & 476.8641 & 485.8755 & 445 & 122.5994 & 2.23984 \\
\hline 2192 & 40.2473 & 519.1432 & 496.6398 & 443.5 & 122.5994 & 2.23893 \\
\hline 2193 & 40.2643 & 528.3403 & 518.6974 & 442 & 122.5994 & 2.23803 \\
\hline 2194 & 40.2813 & 600.9303 & 552.8763 & 440.5 & 122.5994 & 2.23712 \\
\hline 2195 & 40.2983 & 634.4078 & 593.4117 & 439 & 122.5994 & 2.23622 \\
\hline 2196 & 40.3153 & 674.4681 & 623.3907 & 437.5 & 122.5994 & 2.23531 \\
\hline 2197 & 40.3323 & 690.3226 & 622.0928 & 436 & 122.5994 & 2.23441 \\
\hline 2198 & 40.3493 & 674.5239 & 593.3628 & 434.5 & 122.5994 & 2.23351 \\
\hline 2199 & 40.3663 & 589.0391 & 561.741 & 433 & 122.5994 & 2.23261 \\
\hline 2200 & 40.3833 & 560.1498 & 542.9009 & 431.5 & 122.5994 & 2.23171 \\
\hline 2201 & 40.4003 & 549.1914 & 538.8198 & 430 & 122.5994 & 2.23081 \\
\hline 2202 & 40.4173 & 552.1493 & 540.5694 & 428.5 & 122.5994 & 2.22991 \\
\hline 2203 & 40.4343 & 566.0011 & 533.4044 & 427 & 122.5994 & 2.22901 \\
\hline
\end{tabular}




\begin{tabular}{|c|c|c|c|c|c|c|}
\hline 2204 & 40.4513 & 524.4103 & 512.8798 & 425.5 & 122.5994 & 2.22811 \\
\hline 2205 & 40.4683 & 508.8087 & 488.2256 & 424 & 122.5994 & 2.22721 \\
\hline 2206 & 40.4853 & 488.7172 & 467.3015 & 422.5 & 122.5994 & 2.22632 \\
\hline 2207 & 40.5023 & 456.5904 & 452.5013 & 421 & 122.5994 & 2.22542 \\
\hline 2208 & 40.5193 & 431.1361 & 443.0437 & 419.5 & 122.5994 & 2.22453 \\
\hline 2209 & 40.5363 & 403.8687 & 436.7913 & 418 & 122.5994 & 2.22363 \\
\hline 2210 & 40.5533 & 411.2974 & 434.5714 & 419.2917 & 122.5994 & 2.22274 \\
\hline 2211 & 40.5703 & 419.5107 & 432.9774 & 420.5833 & 122.5994 & 2.22185 \\
\hline 2212 & 40.5873 & 409.7322 & 432.1186 & 421.875 & 122.5994 & 2.22096 \\
\hline 2213 & 40.6043 & 403.318 & 431.9521 & 423.1667 & 122.5994 & 2.22007 \\
\hline 2214 & 40.6213 & 407.403 & 432.3526 & 424.4583 & 122.5994 & 2.21918 \\
\hline 2215 & 40.6383 & 419.9627 & 433.0182 & 425.75 & 122.5994 & 2.21829 \\
\hline 2216 & 40.6553 & 438.0504 & 433.6928 & 427.0417 & 122.5994 & 2.2174 \\
\hline 2217 & 40.6723 & 450.9336 & 434.2135 & 428.3333 & 122.5994 & 2.21651 \\
\hline 2218 & 40.6893 & 444.8467 & 434.8967 & 429.625 & 122.5994 & 2.21563 \\
\hline 2219 & 40.7063 & 431.9123 & 435.7876 & 430.9167 & 122.5994 & 2.21474 \\
\hline 2220 & 40.7233 & 428.5471 & 436.878 & 432.2083 & 122.5994 & 2.21385 \\
\hline 2221 & 40.7403 & 447.7552 & 438.093 & 433.5 & 122.5994 & 2.21297 \\
\hline 2222 & 40.7573 & 474.1696 & 439.4475 & 434.7917 & 122.5994 & 2.21209 \\
\hline 2223 & 40.7743 & 472.5078 & 440.9196 & 436.0833 & 122.5994 & 2.2112 \\
\hline 2224 & 40.7913 & 507.7241 & 442.6065 & 437.375 & 122.5994 & 2.21032 \\
\hline 2225 & 40.8083 & 481.2255 & 444.5048 & 438.6667 & 122.5994 & 2.20944 \\
\hline 2226 & 40.8253 & 463.8453 & 446.6099 & 439.9583 & 122.5994 & 2.20856 \\
\hline 2227 & 40.8423 & 428.3874 & 448.8899 & 441.25 & 122.5994 & 2.20768 \\
\hline 2228 & 40.8593 & 474.97 & 451.2632 & 442.5417 & 122.5994 & 2.2068 \\
\hline 2229 & 40.8763 & 455.2465 & 453.6615 & 443.8333 & 122.5994 & 2.20592 \\
\hline 2230 & 40.8933 & 499.8892 & 456.001 & 445.125 & 122.5994 & 2.20504 \\
\hline 2231 & 40.9103 & 484.285 & 458.1259 & 446.4167 & 122.5994 & 2.20417 \\
\hline 2232 & 40.9273 & 436.8034 & 459.9471 & 447.7083 & 122.5994 & 2.20329 \\
\hline 2233 & 40.9443 & 449.0892 & 461.4634 & 449 & 122.5994 & 2.20241 \\
\hline 2234 & 40.9613 & 433.564 & 460.7885 & 448.875 & 122.5994 & 2.20154 \\
\hline 2235 & 40.9783 & 452.7926 & 460.3463 & 448.75 & 122.5994 & 2.20066 \\
\hline 2236 & 40.9953 & 439.5789 & 459.7319 & 448.625 & 122.5994 & 2.19979 \\
\hline 2237 & 41.0123 & 420.7525 & 459.0271 & 448.5 & 122.5994 & 2.19892 \\
\hline 2238 & 41.0293 & 398.0023 & 458.2845 & 448.375 & 122.5994 & 2.19805 \\
\hline 2239 & 41.0463 & 444.0084 & 457.5683 & 448.25 & 122.5994 & 2.19718 \\
\hline 2240 & 41.0633 & 478.6539 & 456.8947 & 448.125 & 122.5994 & 2.19631 \\
\hline 2241 & 41.0803 & 449.6142 & 456.2844 & 448 & 122.5994 & 2.19544 \\
\hline 2242 & 41.0973 & 430.7941 & 455.5146 & 447.875 & 122.5994 & 2.19457 \\
\hline 2243 & 41.1143 & 418.5807 & 455.0646 & 447.75 & 122.5994 & 2.1937 \\
\hline 2244 & 41.1313 & 416.2082 & 454.6669 & 447.625 & 122.5994 & 2.19283 \\
\hline 2245 & 41.1483 & 465.9831 & 454.3602 & 447.5 & 122.5994 & 2.19196 \\
\hline 2246 & 41.1653 & 462.2415 & 454.3079 & 447.375 & 122.5994 & 2.1911 \\
\hline 2247 & 41.1823 & 436.6898 & 454.7476 & 447.25 & 122.5994 & 2.19023 \\
\hline 2248 & 41.1993 & 442.3535 & 455.932 & 447.125 & 122.5994 & 2.18937 \\
\hline 2249 & 41.2163 & 452.542 & 458.1012 & 447 & 122.5994 & 2.18851 \\
\hline 2250 & 41.2333 & 454.9159 & 461.0674 & 446.875 & 122.5994 & 2.18764 \\
\hline 2251 & 41.2503 & 433.3257 & 464.5695 & 446.75 & 122.5994 & 2.18678 \\
\hline 2252 & 41.2673 & 461.6402 & 469.1009 & 446.625 & 122.5994 & 2.18592 \\
\hline
\end{tabular}




\begin{tabular}{|c|c|c|c|c|c|c|}
\hline 2253 & 41.2843 & 484.6688 & 472.9543 & 446.5 & 122.5994 & 2.18506 \\
\hline 2254 & 41.3013 & 481.1772 & 472.6246 & 446.375 & 122.5994 & 2.1842 \\
\hline 2255 & 41.3183 & 441.207 & 469.0318 & 446.25 & 122.5994 & 2.18334 \\
\hline 2256 & 41.3353 & 445.9764 & 465.3344 & 446.125 & 122.5994 & 2.18248 \\
\hline 2257 & 41.3523 & 441.8196 & 463.2426 & 446 & 122.5994 & 2.18162 \\
\hline 2258 & 41.3693 & 455.3835 & 462.3647 & 445.2917 & 122.5994 & 2.18076 \\
\hline 2259 & 41.3863 & 442.5302 & 462.4897 & 444.5833 & 122.5994 & 2.17991 \\
\hline 2260 & 41.4033 & 421.1791 & 461.0384 & 443.875 & 122.5994 & 2.17905 \\
\hline 2261 & 41.4203 & 421.7434 & 457.5663 & 443.1667 & 122.5994 & 2.1782 \\
\hline 2262 & 41.4373 & 425.6487 & 453.4929 & 442.4583 & 122.5994 & 2.17734 \\
\hline 2263 & 41.4543 & 436.8282 & 450.054 & 441.75 & 122.5994 & 2.17649 \\
\hline 2264 & 41.4713 & 440.9577 & 447.4828 & 441.0417 & 122.5994 & 2.17564 \\
\hline 2265 & 41.4883 & 417.5623 & 445.58 & 440.3333 & 122.5994 & 2.17478 \\
\hline 2266 & 41.5053 & 396.2521 & 443.9509 & 439.625 & 122.5994 & 2.17393 \\
\hline 2267 & 41.5223 & 407.249 & 442.5063 & 438.9167 & 122.5994 & 2.17308 \\
\hline 2268 & 41.5393 & 390.3386 & 441.2018 & 438.2083 & 122.5994 & 2.17223 \\
\hline 2269 & 41.5563 & 416.3318 & 440.041 & 437.5 & 122.5994 & 2.17138 \\
\hline 2270 & 41.5733 & 424.3193 & 438.9611 & 436.7917 & 122.5994 & 2.17053 \\
\hline 2271 & 41.5903 & 390.4766 & 437.9706 & 436.0833 & 122.5994 & 2.16969 \\
\hline 2272 & 41.6073 & 404.8318 & 437.0206 & 435.375 & 122.5994 & 2.16884 \\
\hline 2273 & 41.6243 & 411.5848 & 436.1655 & 434.6667 & 122.5994 & 2.16799 \\
\hline 2274 & 41.6413 & 419.5426 & 435.3571 & 433.9583 & 122.5994 & 2.16715 \\
\hline 2275 & 41.6583 & 394.0308 & 434.5677 & 433.25 & 122.5994 & 2.1663 \\
\hline 2276 & 41.6753 & 388.3411 & 433.8258 & 432.5417 & 122.5994 & 2.16546 \\
\hline 2277 & 41.6923 & 407.4256 & 433.1066 & 431.8333 & 122.5994 & 2.16461 \\
\hline 2278 & 41.7093 & 428.2973 & 432.3895 & 431.125 & 122.5994 & 2.16377 \\
\hline 2279 & 41.7263 & 424.6121 & 431.7079 & 430.4167 & 122.5994 & 2.16293 \\
\hline 2280 & 41.7433 & 444.6726 & 431.2853 & 429.7083 & 122.5994 & 2.16209 \\
\hline 2281 & 41.7603 & 427.7447 & 430.6189 & 429 & 122.5994 & 2.16124 \\
\hline 2282 & 41.7773 & 416.4413 & 431.1773 & 429.4583 & 122.5994 & 2.1604 \\
\hline 2283 & 41.7943 & 421.5473 & 431.7641 & 429.9167 & 122.5994 & 2.15957 \\
\hline 2284 & 41.8113 & 430.5945 & 432.3896 & 430.375 & 122.5994 & 2.15873 \\
\hline 2285 & 41.8283 & 417.3901 & 433.0113 & 430.8333 & 122.5994 & 2.15789 \\
\hline 2286 & 41.8453 & 429.1492 & 434.7267 & 431.2917 & 122.5994 & 2.15705 \\
\hline 2287 & 41.8623 & 453.7505 & 435.718 & 431.75 & 122.5994 & 2.15621 \\
\hline 2288 & 41.8793 & 458.1233 & 436.9449 & 432.2083 & 122.5994 & 2.15538 \\
\hline 2289 & 41.8963 & 468.3126 & 438.4546 & 432.6667 & 122.5994 & 2.15454 \\
\hline 2290 & 41.9133 & 444.2134 & 440.2042 & 433.125 & 122.5994 & 2.15371 \\
\hline 2291 & 41.9303 & 440.9577 & 442.1453 & 433.5833 & 122.5994 & 2.15287 \\
\hline 2292 & 41.9473 & 441.0588 & 444.4506 & 434.0417 & 122.5994 & 2.15204 \\
\hline 2293 & 41.9643 & 431.8423 & 447.3428 & 434.5 & 122.5994 & 2.15121 \\
\hline 2294 & 41.9813 & 420.3743 & 451.0217 & 434.9583 & 122.5994 & 2.15038 \\
\hline 2295 & 41.9983 & 445.9852 & 455.4062 & 435.4167 & 122.5994 & 2.14955 \\
\hline 2296 & 42.0153 & 458.9546 & 459.7172 & 435.875 & 122.5994 & 2.14872 \\
\hline 2297 & 42.0323 & 448.6562 & 462.3983 & 436.3333 & 122.5994 & 2.14789 \\
\hline 2298 & 42.0493 & 440.9577 & 464.0428 & 436.7917 & 122.5994 & 2.14706 \\
\hline 2299 & 42.0663 & 448.5977 & 464.8042 & 437.25 & 122.5994 & 2.14623 \\
\hline 2300 & 42.0833 & 453.9463 & 465.5398 & 437.7083 & 122.5994 & 2.1454 \\
\hline 2301 & 42.1003 & 443.2416 & 466.3509 & 438.1667 & 122.5994 & 2.14457 \\
\hline
\end{tabular}




\begin{tabular}{|c|c|c|c|c|c|c|}
\hline 2302 & 42.1173 & 434.7457 & 467.1464 & 438.625 & 122.5994 & 2.14375 \\
\hline 2303 & 42.1343 & 426.0266 & 467.3948 & 439.0833 & 122.5994 & 2.14292 \\
\hline 2304 & 42.1513 & 429.4483 & 466.8123 & 439.5417 & 122.5994 & 2.1421 \\
\hline 2305 & 42.1683 & 448.8975 & 466.0035 & 440 & 122.5994 & 2.14127 \\
\hline 2306 & 42.1853 & 447.8263 & 464.3688 & 439.4583 & 122.5994 & 2.14045 \\
\hline 2307 & 42.2023 & 441.0588 & 463.0108 & 438.9167 & 122.5994 & 2.13963 \\
\hline 2308 & 42.2193 & 443.6436 & 461.9544 & 438.375 & 122.5994 & 2.1388 \\
\hline 2309 & 42.2363 & 453.6797 & 461.2613 & 437.8333 & 122.5994 & 2.13798 \\
\hline 2310 & 42.2533 & 443.5118 & 461.0526 & 437.2917 & 122.5994 & 2.13716 \\
\hline 2311 & 42.2703 & 454.745 & 461.5463 & 436.75 & 122.5994 & 2.13634 \\
\hline 2312 & 42.2873 & 456.0072 & 463.0155 & 436.2083 & 122.5994 & 2.13552 \\
\hline 2313 & 42.3043 & 492.8323 & 465.6941 & 435.6667 & 122.5994 & 2.1347 \\
\hline 2314 & 42.3213 & 472.561 & 469.7465 & 435.125 & 122.5994 & 2.13388 \\
\hline 2315 & 42.3383 & 472.4498 & 475.4283 & 434.5833 & 122.5994 & 2.13307 \\
\hline 2316 & 42.3553 & 493.1697 & 483.1195 & 434.0417 & 122.5994 & 2.13225 \\
\hline 2317 & 42.3723 & 497.2423 & 494.1912 & 433.5 & 122.5994 & 2.13143 \\
\hline 2318 & 42.3893 & 536.2683 & 512.2856 & 432.9583 & 122.5994 & 2.13062 \\
\hline 2319 & 42.4063 & 555.1457 & 543.0889 & 432.4167 & 122.5994 & 2.1298 \\
\hline 2320 & 42.4233 & 658.936 & 591.9911 & 431.875 & 122.5994 & 2.12899 \\
\hline 2321 & 42.4403 & 714.4318 & 658.2339 & 431.3333 & 122.5994 & 2.12818 \\
\hline 2322 & 42.4573 & 822.615 & 728.9049 & 430.7917 & 122.5994 & 2.12736 \\
\hline 2323 & 42.4743 & 903.1262 & 781.6884 & 430.25 & 122.5994 & 2.12655 \\
\hline 2324 & 42.4913 & 996.8362 & 784.6705 & 429.7083 & 122.5994 & 2.12574 \\
\hline 2325 & 42.5083 & 893.554 & 735.7527 & 429.1667 & 122.5994 & 2.12493 \\
\hline 2326 & 42.5253 & 743.3351 & 676.0267 & 428.625 & 122.5994 & 2.12412 \\
\hline 2327 & 42.5423 & 669.9344 & 637.0837 & 428.0833 & 122.5994 & 2.12331 \\
\hline 2328 & 42.5593 & 652.6615 & 625.2312 & 427.5417 & 122.5994 & 2.1225 \\
\hline 2329 & 42.5763 & 685.1247 & 629.9288 & 427 & 122.5994 & 2.12169 \\
\hline 2330 & 42.5933 & 671.324 & 631.0027 & 426.4583 & 122.5994 & 2.12089 \\
\hline 2331 & 42.6103 & 665.8719 & 608.9054 & 425.9167 & 122.5994 & 2.12008 \\
\hline 2332 & 42.6273 & 583.7355 & 568.1536 & 425.375 & 122.5994 & 2.11927 \\
\hline 2333 & 42.6443 & 495.1518 & 525.2828 & 424.8333 & 122.5994 & 2.11847 \\
\hline 2334 & 42.6613 & 500.1909 & 491.5795 & 424.2917 & 122.5994 & 2.11766 \\
\hline 2335 & 42.6783 & 474.2418 & 469.4597 & 423.75 & 122.5994 & 2.11686 \\
\hline 2336 & 42.6953 & 461.6492 & 456.7256 & 423.2083 & 122.5994 & 2.11605 \\
\hline 2337 & 42.7123 & 437.1002 & 450.0409 & 422.6667 & 122.5994 & 2.11525 \\
\hline 2338 & 42.7293 & 445.8166 & 446.156 & 422.125 & 122.5994 & 2.11445 \\
\hline 2339 & 42.7463 & 425.6365 & 442.3148 & 421.5833 & 122.5994 & 2.11365 \\
\hline 2340 & 42.7633 & 409.265 & 438.1422 & 421.0417 & 122.5994 & 2.11285 \\
\hline 2341 & 42.7803 & 434.369 & 434.6312 & 420.5 & 122.5994 & 2.11205 \\
\hline 2342 & 42.7973 & 429.939 & 431.7514 & 419.9583 & 122.5994 & 2.11125 \\
\hline 2343 & 42.8143 & 415.1667 & 429.3762 & 419.4167 & 122.5994 & 2.11045 \\
\hline 2344 & 42.8313 & 441.3847 & 427.4137 & 418.875 & 122.5994 & 2.10965 \\
\hline 2345 & 42.8483 & 448.1377 & 425.8088 & 418.3333 & 122.5994 & 2.10885 \\
\hline 2346 & 42.8653 & 429.7378 & 424.4883 & 417.7917 & 122.5994 & 2.10805 \\
\hline 2347 & 42.8823 & 422.0037 & 423.3206 & 417.25 & 122.5994 & 2.10726 \\
\hline 2348 & 42.8993 & 414.2438 & 422.3751 & 416.7083 & 122.5994 & 2.10646 \\
\hline 2349 & 42.9163 & 406.3431 & 421.551 & 416.1667 & 122.5994 & 2.10567 \\
\hline 2350 & 42.9333 & 396.9217 & 420.7976 & 415.625 & 122.5994 & 2.10487 \\
\hline
\end{tabular}




\begin{tabular}{|c|c|c|c|c|c|c|}
\hline 2351 & 42.9503 & 413.8699 & 420.1518 & 415.0833 & 122.5994 & 2.10408 \\
\hline 2352 & 42.9673 & 419.9452 & 419.5437 & 414.5417 & 122.5994 & 2.10329 \\
\hline 2353 & 42.9843 & 425.5402 & 418.9134 & 414 & 122.5994 & 2.10249 \\
\hline 2354 & 43.0013 & 411.1535 & 418.1317 & 413.3333 & 122.5994 & 2.1017 \\
\hline 2355 & 43.0183 & 383.2147 & 417.2639 & 412.6667 & 122.5994 & 2.10091 \\
\hline 2356 & 43.0353 & 394.9599 & 416.4203 & 412 & 122.5994 & 2.10012 \\
\hline 2357 & 43.0523 & 412.3827 & 415.7623 & 411.3333 & 122.5994 & 2.09933 \\
\hline 2358 & 43.0693 & 387.8004 & 415.1423 & 410.6667 & 122.5994 & 2.09854 \\
\hline 2359 & 43.0863 & 376.3239 & 414.5596 & 410 & 122.5994 & 2.09775 \\
\hline 2360 & 43.1033 & 385.29 & 413.8643 & 409.3333 & 122.5994 & 2.09696 \\
\hline 2361 & 43.1203 & 391.1276 & 412.062 & 408.6667 & 122.5994 & 2.09618 \\
\hline 2362 & 43.1373 & 373.2857 & 411.4651 & 408 & 122.5994 & 2.09539 \\
\hline 2363 & 43.1543 & 397.7459 & 411.0804 & 407.3333 & 122.5994 & 2.0946 \\
\hline 2364 & 43.1713 & 393.8814 & 410.8847 & 406.6667 & 122.5994 & 2.09382 \\
\hline 2365 & 43.1883 & 385.2794 & 410.8339 & 406 & 122.5994 & 2.09303 \\
\hline 2366 & 43.2053 & 419.7403 & 410.7935 & 405.3333 & 122.5994 & 2.09225 \\
\hline 2367 & 43.2223 & 413.1853 & 410.5967 & 404.6667 & 122.5994 & 2.09147 \\
\hline 2368 & 43.2393 & 400.9125 & 409.638 & 404 & 122.5994 & 2.09068 \\
\hline 2369 & 43.2563 & 407.6036 & 408.9446 & 403.3333 & 122.5994 & 2.0899 \\
\hline 2370 & 43.2733 & 419.9642 & 408.0603 & 402.6667 & 122.5994 & 2.08912 \\
\hline 2371 & 43.2903 & 380.4674 & 407.1588 & 402 & 122.5994 & 2.08834 \\
\hline 2372 & 43.3073 & 392.1227 & 406.4644 & 401.3333 & 122.5994 & 2.08756 \\
\hline 2373 & 43.3243 & 398.2607 & 406.1185 & 400.6667 & 122.5994 & 2.08678 \\
\hline 2374 & 43.3413 & 357.3742 & 406.3516 & 400 & 122.5994 & 2.086 \\
\hline 2375 & 43.3583 & 416.7159 & 407.1045 & 399.3333 & 122.5994 & 2.08522 \\
\hline 2376 & 43.3753 & 380.8541 & 407.5919 & 398.6667 & 122.5994 & 2.08444 \\
\hline 2377 & 43.3923 & 416.7491 & 406.4148 & 398 & 122.5994 & 2.08367 \\
\hline 2378 & 43.4093 & 390.8669 & 404.8868 & 398.3333 & 122.5994 & 2.08289 \\
\hline 2379 & 43.4263 & 387.5012 & 403.568 & 398.6667 & 122.5994 & 2.08211 \\
\hline 2380 & 43.4433 & 400.2561 & 403.1466 & 399 & 122.5994 & 2.08134 \\
\hline 2381 & 43.4603 & 386.6856 & 403.6774 & 399.3333 & 122.5994 & 2.08056 \\
\hline 2382 & 43.4773 & 393.2438 & 404.8643 & 399.6667 & 122.5994 & 2.07979 \\
\hline 2383 & 43.4943 & 382.2465 & 406.0779 & 400 & 122.5994 & 2.07902 \\
\hline 2384 & 43.5113 & 380.6093 & 406.8872 & 400.3333 & 122.5994 & 2.07824 \\
\hline 2385 & 43.5283 & 395.5302 & 408.3307 & 400.6667 & 122.5994 & 2.07747 \\
\hline 2386 & 43.5453 & 401.5329 & 411.916 & 401 & 122.5994 & 2.0767 \\
\hline 2387 & 43.5623 & 400.0357 & 418.2338 & 401.3333 & 122.5994 & 2.07593 \\
\hline 2388 & 43.5793 & 402.3433 & 425.3831 & 401.6667 & 122.5994 & 2.07516 \\
\hline 2389 & 43.5963 & 396.5613 & 427.7249 & 402 & 122.5994 & 2.07439 \\
\hline 2390 & 43.6133 & 386.6691 & 423.2302 & 402.3333 & 122.5994 & 2.07362 \\
\hline 2391 & 43.6303 & 380.9899 & 417.5181 & 402.6667 & 122.5994 & 2.07285 \\
\hline 2392 & 43.6473 & 385.3636 & 414.5695 & 403 & 122.5994 & 2.07208 \\
\hline 2393 & 43.6643 & 390.5455 & 415.0852 & 403.3333 & 122.5994 & 2.07131 \\
\hline 2394 & 43.6813 & 410.6459 & 418.136 & 403.6667 & 122.5994 & 2.07055 \\
\hline 2395 & 43.6983 & 388.0729 & 421.4088 & 404 & 122.5994 & 2.06978 \\
\hline 2396 & 43.7153 & 394.6733 & 421.5315 & 404.3333 & 122.5994 & 2.06902 \\
\hline 2397 & 43.7323 & 395.1689 & 418.765 & 404.6667 & 122.5994 & 2.06825 \\
\hline 2398 & 43.7493 & 416.1344 & 415.8494 & 405 & 122.5994 & 2.06749 \\
\hline 2399 & 43.7663 & 412.452 & 414.0139 & 405.3333 & 122.5994 & 2.06672 \\
\hline
\end{tabular}




\begin{tabular}{|c|c|c|c|c|c|c|}
\hline 2400 & 43.7833 & 421.2622 & 413.1895 & 405.6667 & 122.5994 & 2.06596 \\
\hline 2401 & 43.8003 & 405.6335 & 412.9756 & 406 & 122.5994 & 2.0652 \\
\hline 2402 & 43.8173 & 393.3745 & 412.4633 & 405.7083 & 122.5994 & 2.06444 \\
\hline 2403 & 43.8343 & 390.5696 & 412.1098 & 405.4167 & 122.5994 & 2.06367 \\
\hline 2404 & 43.8513 & 393.6248 & 411.9174 & 405.125 & 122.5994 & 2.06291 \\
\hline 2405 & 43.8683 & 401.8643 & 411.8155 & 404.8333 & 122.5994 & 2.06215 \\
\hline 2406 & 43.8853 & 406.9801 & 411.8385 & 404.5417 & 122.5994 & 2.06139 \\
\hline 2407 & 43.9023 & 363.2663 & 411.9431 & 404.25 & 122.5994 & 2.06064 \\
\hline 2408 & 43.9193 & 389.3854 & 412.1907 & 403.9583 & 122.5994 & 2.05988 \\
\hline 2409 & 43.9363 & 387.1138 & 412.5444 & 403.6667 & 122.5994 & 2.05912 \\
\hline 2410 & 43.9533 & 397.402 & 413.0026 & 403.375 & 122.5994 & 2.05836 \\
\hline 2411 & 43.9703 & 396.5535 & 413.3198 & 403.0833 & 122.5994 & 2.05761 \\
\hline 2412 & 43.9873 & 385.6843 & 413.3705 & 402.7917 & 122.5994 & 2.05685 \\
\hline 2413 & 44.0043 & 384.1232 & 413.2184 & 402.5 & 122.5994 & 2.0561 \\
\hline 2414 & 44.0213 & 406.1544 & 412.7715 & 402.2083 & 122.5994 & 2.05534 \\
\hline 2415 & 44.0383 & 427.2303 & 412.151 & 401.9167 & 122.5994 & 2.05459 \\
\hline 2416 & 44.0553 & 408.7815 & 411.7099 & 401.625 & 122.5994 & 2.05383 \\
\hline 2417 & 44.0723 & 392.3603 & 411.6359 & 401.3333 & 122.5994 & 2.05308 \\
\hline 2418 & 44.0893 & 380.418 & 411.9235 & 401.0417 & 122.5994 & 2.05233 \\
\hline 2419 & 44.1063 & 404.3993 & 412.552 & 400.75 & 122.5994 & 2.05158 \\
\hline 2420 & 44.1233 & 418.2986 & 413.4684 & 400.4583 & 122.5994 & 2.05083 \\
\hline 2421 & 44.1403 & 381.3949 & 414.4498 & 400.1667 & 122.5994 & 2.05008 \\
\hline 2422 & 44.1573 & 398.1741 & 415.2083 & 399.875 & 122.5994 & 2.04933 \\
\hline 2423 & 44.1743 & 411.5313 & 415.5492 & 399.5833 & 122.5994 & 2.04858 \\
\hline 2424 & 44.1913 & 351.5699 & 415.1076 & 399.2917 & 122.5994 & 2.04783 \\
\hline 2425 & 44.2083 & 415.3418 & 413.9161 & 399 & 122.5994 & 2.04708 \\
\hline 2426 & 44.2253 & 424.0182 & 412.3192 & 398.6667 & 122.5994 & 2.04633 \\
\hline 2427 & 44.2423 & 387.2659 & 410.763 & 398.3333 & 122.5994 & 2.04559 \\
\hline 2428 & 44.2593 & 398.3017 & 409.4496 & 398 & 122.5994 & 2.04484 \\
\hline 2429 & 44.2763 & 393.9246 & 408.3239 & 397.6667 & 122.5994 & 2.04409 \\
\hline 2430 & 44.2933 & 397.9588 & 407.2459 & 397.3333 & 122.5994 & 2.04335 \\
\hline 2431 & 44.3103 & 390.8784 & 406.0053 & 397 & 122.5994 & 2.0426 \\
\hline 2432 & 44.3273 & 385.654 & 404.6104 & 396.6667 & 122.5994 & 2.04186 \\
\hline 2433 & 44.3443 & 373.9941 & 403.2392 & 396.3333 & 122.5994 & 2.04112 \\
\hline 2434 & 44.3613 & 390.3578 & 402.096 & 396 & 122.5994 & 2.04037 \\
\hline 2435 & 44.3783 & 422.6389 & 401.3547 & 395.6667 & 122.5994 & 2.03963 \\
\hline 2436 & 44.3953 & 387.213 & 401.118 & 395.3333 & 122.5994 & 2.03889 \\
\hline 2437 & 44.4123 & 397.0449 & 401.283 & 395 & 122.5994 & 2.03815 \\
\hline 2438 & 44.4293 & 398.7622 & 401.3212 & 394.6667 & 122.5994 & 2.03741 \\
\hline 2439 & 44.4463 & 397.4679 & 400.5352 & 394.3333 & 122.5994 & 2.03667 \\
\hline 2440 & 44.4633 & 372.7626 & 399.1698 & 394 & 122.5994 & 2.03593 \\
\hline 2441 & 44.4803 & 369.2271 & 398.0958 & 393.6667 & 122.5994 & 2.03519 \\
\hline 2442 & 44.4973 & 402.1438 & 397.6486 & 393.3333 & 122.5994 & 2.03445 \\
\hline 2443 & 44.5143 & 380.6281 & 397.761 & 393 & 122.5994 & 2.03372 \\
\hline 2444 & 44.5313 & 371.9126 & 398.1369 & 392.6667 & 122.5994 & 2.03298 \\
\hline 2445 & 44.5483 & 391.7832 & 398.262 & 392.3333 & 122.5994 & 2.03224 \\
\hline 2446 & 44.5653 & 395.1281 & 397.6842 & 392 & 122.5994 & 2.03151 \\
\hline 2447 & 44.5823 & 384.6778 & 396.6123 & 391.6667 & 122.5994 & 2.03077 \\
\hline 2448 & 44.5993 & 377.6839 & 395.529 & 391.3333 & 122.5994 & 2.03004 \\
\hline
\end{tabular}




\begin{tabular}{|c|c|c|c|c|c|c|}
\hline 2449 & 44.6163 & 386.9463 & 394.6344 & 391 & 122.5994 & 2.0293 \\
\hline 2450 & 44.6333 & 392.0024 & 396.504 & 393.25 & 122.5994 & 2.02857 \\
\hline 2451 & 44.6503 & 439.1212 & 398.5463 & 395.5 & 122.5994 & 2.02784 \\
\hline 2452 & 44.6673 & 424.2407 & 400.5796 & 397.75 & 122.5994 & 2.0271 \\
\hline 2453 & 44.6843 & 430.7847 & 402.5321 & 400 & 122.5994 & 2.02637 \\
\hline 2454 & 44.7013 & 430.3827 & 404.4751 & 402.25 & 122.5994 & 2.02564 \\
\hline 2455 & 44.7183 & 399.2551 & 406.4012 & 404.5 & 122.5994 & 2.02491 \\
\hline 2456 & 44.7353 & 405.2535 & 408.3369 & 406.75 & 122.5994 & 2.02418 \\
\hline 2457 & 44.7523 & 391.1631 & 410.3185 & 409 & 122.5994 & 2.02345 \\
\hline 2458 & 44.7693 & 388.0528 & 415.2009 & 411.25 & 122.5994 & 2.02272 \\
\hline 2459 & 44.7863 & 442.5633 & 417.4215 & 413.5 & 122.5994 & 2.02199 \\
\hline 2460 & 44.8033 & 393.6612 & 419.5959 & 415.75 & 122.5994 & 2.02127 \\
\hline 2461 & 44.8203 & 418.0549 & 421.9206 & 418 & 122.5994 & 2.02054 \\
\hline 2462 & 44.8373 & 408.5912 & 424.2878 & 420.25 & 122.5994 & 2.01981 \\
\hline 2463 & 44.8543 & 411.9414 & 426.695 & 422.5 & 122.5994 & 2.01909 \\
\hline 2464 & 44.8713 & 400.1199 & 429.1411 & 424.75 & 122.5994 & 2.01836 \\
\hline 2465 & 44.8883 & 425.2399 & 433.0149 & 427 & 122.5994 & 2.01764 \\
\hline 2466 & 44.9053 & 473.7494 & 435.6251 & 429.25 & 122.5994 & 2.01691 \\
\hline 2467 & 44.9223 & 435.1636 & 438.2991 & 431.5 & 122.5994 & 2.01619 \\
\hline 2468 & 44.9393 & 411.3407 & 441.0696 & 433.75 & 122.5994 & 2.01547 \\
\hline 2469 & 44.9563 & 444.3495 & 444.0177 & 436 & 122.5994 & 2.01474 \\
\hline 2470 & 44.9733 & 407.2254 & 447.295 & 438.25 & 122.5994 & 2.01402 \\
\hline 2471 & 44.9903 & 460.4245 & 451.0445 & 440.5 & 122.5994 & 2.0133 \\
\hline 2472 & 45.0073 & 470.9996 & 455.1814 & 442.75 & 122.5994 & 2.01258 \\
\hline 2473 & 45.0243 & 446.2346 & 458.9578 & 445 & 122.5994 & 2.01186 \\
\hline 2474 & 45.0413 & 427.4411 & 459.9774 & 445.7552 & 122.5994 & 2.01114 \\
\hline 2475 & 45.0583 & 417.6703 & 460.3589 & 446.5104 & 122.5994 & 2.01042 \\
\hline 2476 & 45.0753 & 449.3969 & 461.1031 & 447.2656 & 122.5994 & 2.0097 \\
\hline 2477 & 45.0923 & 458.9822 & 462.5606 & 448.0208 & 122.5994 & 2.00898 \\
\hline 2478 & 45.1093 & 473.731 & 464.7233 & 448.776 & 122.5994 & 2.00826 \\
\hline 2479 & 45.1263 & 453.5663 & 467.4326 & 449.5313 & 122.5994 & 2.00755 \\
\hline 2480 & 45.1433 & 460.7519 & 470.2443 & 450.2865 & 122.5994 & 2.00683 \\
\hline 2481 & 45.1603 & 462.5745 & 472.8483 & 451.0417 & 122.5994 & 2.00611 \\
\hline 2482 & 45.1773 & 469.299 & 475.7158 & 451.7969 & 122.5994 & 2.0054 \\
\hline 2483 & 45.1943 & 449.4938 & 479.3271 & 452.5521 & 122.5994 & 2.00468 \\
\hline 2484 & 45.2113 & 442.3516 & 483.5701 & 453.3073 & 122.5994 & 2.00397 \\
\hline 2485 & 45.2283 & 482.2293 & 488.4669 & 454.0625 & 122.5994 & 2.00326 \\
\hline 2486 & 45.2453 & 495.5781 & 495.6343 & 454.8177 & 122.5994 & 2.00254 \\
\hline 2487 & 45.2623 & 508.2316 & 505.287 & 455.5729 & 122.5994 & 2.00183 \\
\hline 2488 & 45.2793 & 505.9582 & 519.1059 & 456.3281 & 122.5994 & 2.00112 \\
\hline 2489 & 45.2963 & 498.3159 & 537.7952 & 457.0833 & 122.5994 & 2.00041 \\
\hline 2490 & 45.3133 & 531.0975 & 561.5811 & 457.8385 & 122.5994 & 1.9997 \\
\hline 2491 & 45.3303 & 529.0574 & 590.3106 & 458.5938 & 122.5994 & 1.99899 \\
\hline 2492 & 45.3473 & 501.8368 & 626.1786 & 459.349 & 122.5994 & 1.99828 \\
\hline 2493 & 45.3643 & 541.2624 & 669.7645 & 460.1042 & 122.5994 & 1.99757 \\
\hline 2494 & 45.3813 & 565.1922 & 719.5361 & 460.8594 & 122.5994 & 1.99686 \\
\hline 2495 & 45.3983 & 583.9102 & 772.3581 & 461.6146 & 122.5994 & 1.99615 \\
\hline 2496 & 45.4153 & 650.5319 & 822.8459 & 462.3698 & 122.5994 & 1.99544 \\
\hline 2497 & 45.4323 & 632.982 & 862.8605 & 463.125 & 122.5994 & 1.99473 \\
\hline
\end{tabular}




\begin{tabular}{|c|c|c|c|c|c|c|}
\hline 2498 & 45.4493 & 648.1306 & 883.1102 & 462.0807 & 122.5994 & 1.99403 \\
\hline 2499 & 45.4663 & 664.2772 & 883.8112 & 461.0365 & 122.5994 & 1.99332 \\
\hline 2500 & 45.4833 & 701.9162 & 869.5466 & 459.9922 & 122.5994 & 1.99262 \\
\hline 2501 & 45.5003 & 712.985 & 847.6558 & 458.9479 & 122.5994 & 1.99191 \\
\hline 2502 & 45.5173 & 757.0038 & 824.1471 & 457.9036 & 122.5994 & 1.99121 \\
\hline 2503 & 45.5343 & 758.1869 & 801.3723 & 456.8594 & 122.5994 & 1.9905 \\
\hline 2504 & 45.5513 & 723.6697 & 777.6607 & 455.8151 & 122.5994 & 1.9898 \\
\hline 2505 & 45.5683 & 649.6599 & 749.3642 & 454.7708 & 122.5994 & 1.9891 \\
\hline 2506 & 45.5853 & 640.1753 & 715.4706 & 453.7266 & 122.5994 & 1.98839 \\
\hline 2507 & 45.6023 & 607.9024 & 678.5583 & 452.6823 & 122.5994 & 1.98769 \\
\hline 2508 & 45.6193 & 591.4306 & 642.0774 & 451.638 & 122.5994 & 1.98699 \\
\hline 2509 & 45.6363 & 595.1222 & 608.8134 & 450.5938 & 122.5994 & 1.98629 \\
\hline 2510 & 45.6533 & 571.6649 & 580.2395 & 449.5495 & 122.5994 & 1.98559 \\
\hline 2511 & 45.6703 & 566.0902 & 556.7494 & 448.5052 & 122.5994 & 1.98489 \\
\hline 2512 & 45.6873 & 515.8611 & 538.1051 & 447.4609 & 122.5994 & 1.98419 \\
\hline 2513 & 45.7043 & 532.8862 & 524.6772 & 446.4167 & 122.5994 & 1.98349 \\
\hline 2514 & 45.7213 & 517.3827 & 517.5782 & 445.3724 & 122.5994 & 1.9828 \\
\hline 2515 & 45.7383 & 487.6679 & 518.1757 & 444.3281 & 122.5994 & 1.9821 \\
\hline 2516 & 45.7553 & 526.9834 & 527.8622 & 443.2839 & 122.5994 & 1.9814 \\
\hline 2517 & 45.7723 & 555.4082 & 547.7404 & 442.2396 & 122.5994 & 1.9807 \\
\hline 2518 & 45.7893 & 607.4246 & 579.6852 & 441.1953 & 122.5994 & 1.98001 \\
\hline 2519 & 45.8063 & 628.2173 & 619.9067 & 440.151 & 122.5994 & 1.97931 \\
\hline 2520 & 45.8233 & 668.682 & 651.1437 & 439.1068 & 122.5994 & 1.97862 \\
\hline 2521 & 45.8403 & 676.7191 & 649.49 & 438.0625 & 122.5994 & 1.97792 \\
\hline 2522 & 45.8573 & 641.1006 & 616.7023 & 437.0182 & 122.5994 & 1.97723 \\
\hline 2523 & 45.8743 & 567.0782 & 578.5415 & 435.974 & 122.5994 & 1.97654 \\
\hline 2524 & 45.8913 & 533.0377 & 552.7463 & 434.9297 & 122.5994 & 1.97585 \\
\hline 2525 & 45.9083 & 537.1639 & 545.0522 & 433.8854 & 122.5994 & 1.97515 \\
\hline 2526 & 45.9253 & 538.2946 & 552.3079 & 432.8411 & 122.5994 & 1.97446 \\
\hline 2527 & 45.9423 & 563.0667 & 562.6325 & 431.7969 & 122.5994 & 1.97377 \\
\hline 2528 & 45.9593 & 548.3537 & 560.384 & 430.7526 & 122.5994 & 1.97308 \\
\hline 2529 & 45.9763 & 521.0362 & 542.4615 & 429.7083 & 122.5994 & 1.97239 \\
\hline 2530 & 45.9933 & 487.1181 & 519.6471 & 428.6641 & 122.5994 & 1.9717 \\
\hline 2531 & 46.0103 & 457.3083 & 499.5081 & 427.6198 & 122.5994 & 1.97101 \\
\hline 2532 & 46.0273 & 439.4144 & 484.202 & 426.5755 & 122.5994 & 1.97032 \\
\hline 2533 & 46.0443 & 413.2374 & 473.6375 & 425.5313 & 122.5994 & 1.96964 \\
\hline 2534 & 46.0613 & 402.0422 & 466.2149 & 424.487 & 122.5994 & 1.96895 \\
\hline 2535 & 46.0783 & 418.4573 & 460.7822 & 423.4427 & 122.5994 & 1.96826 \\
\hline 2536 & 46.0953 & 408.9997 & 457.0983 & 422.3984 & 122.5994 & 1.96757 \\
\hline 2537 & 46.1123 & 414.3306 & 454.856 & 421.3542 & 122.5994 & 1.96689 \\
\hline 2538 & 46.1293 & 414.1804 & 453.3823 & 420.3099 & 122.5994 & 1.9662 \\
\hline 2539 & 46.1463 & 426.4721 & 449.4438 & 419.2656 & 122.5994 & 1.96552 \\
\hline 2540 & 46.1633 & 443.7109 & 448.2238 & 418.2214 & 122.5994 & 1.96483 \\
\hline 2541 & 46.1803 & 450.6909 & 446.2957 & 417.1771 & 122.5994 & 1.96415 \\
\hline 2542 & 46.1973 & 414.8401 & 443.494 & 416.1328 & 122.5994 & 1.96347 \\
\hline 2543 & 46.2143 & 421.5 & 440.1891 & 415.0885 & 122.5994 & 1.96278 \\
\hline 2544 & 46.2313 & 423.729 & 436.746 & 414.0443 & 122.5994 & 1.9621 \\
\hline 2545 & 46.2483 & 399.501 & 433.5422 & 413 & 122.5994 & 1.96142 \\
\hline 2546 & 46.2653 & 407.2189 & 429.1751 & 411.75 & 122.5994 & 1.96074 \\
\hline
\end{tabular}




\begin{tabular}{|c|c|c|c|c|c|c|}
\hline 2547 & 46.2823 & 434.6103 & 426.6013 & 410.5 & 122.5994 & 1.96006 \\
\hline 2548 & 46.2993 & 389.2177 & 424.1872 & 409.25 & 122.5994 & 1.95938 \\
\hline 2549 & 46.3163 & 369.9671 & 421.8014 & 408 & 122.5994 & 1.9587 \\
\hline 2550 & 46.3333 & 415.7762 & 419.4156 & 406.75 & 122.5994 & 1.95802 \\
\hline 2551 & 46.3503 & 356.3867 & 417.0481 & 405.5 & 122.5994 & 1.95734 \\
\hline 2552 & 46.3673 & 373.0055 & 414.8104 & 404.25 & 122.5994 & 1.95666 \\
\hline 2553 & 46.3843 & 397.8258 & 412.7242 & 403 & 122.5994 & 1.95599 \\
\hline 2554 & 46.4013 & 381.5748 & 410.869 & 401.75 & 122.5994 & 1.95531 \\
\hline 2555 & 46.4183 & 369.2407 & 409.2141 & 400.5 & 122.5994 & 1.95463 \\
\hline 2556 & 46.4353 & 406.9821 & 407.7178 & 399.25 & 122.5994 & 1.95396 \\
\hline 2557 & 46.4523 & 361.2568 & 406.2714 & 398 & 122.5994 & 1.95328 \\
\hline 2558 & 46.4693 & 399.9616 & 404.8286 & 396.75 & 122.5994 & 1.95261 \\
\hline 2559 & 46.4863 & 399.899 & 403.292 & 395.5 & 122.5994 & 1.95193 \\
\hline 2560 & 46.5033 & 395.2599 & 401.8522 & 394.25 & 122.5994 & 1.95126 \\
\hline 2561 & 46.5203 & 383.8916 & 400.2702 & 393 & 122.5994 & 1.95058 \\
\hline 2562 & 46.5373 & 377.8367 & 399.5852 & 391.75 & 122.5994 & 1.94991 \\
\hline 2563 & 46.5543 & 363.0987 & 399.4529 & 390.5 & 122.5994 & 1.94924 \\
\hline 2564 & 46.5713 & 399.928 & 399.6682 & 389.25 & 122.5994 & 1.94857 \\
\hline 2565 & 46.5883 & 380.2872 & 399.539 & 388 & 122.5994 & 1.9479 \\
\hline 2566 & 46.6053 & 394.1169 & 398.5303 & 386.75 & 122.5994 & 1.94722 \\
\hline 2567 & 46.6223 & 374.3721 & 397.3096 & 385.5 & 122.5994 & 1.94655 \\
\hline 2568 & 46.6393 & 397.9218 & 396.1048 & 384.25 & 122.5994 & 1.94588 \\
\hline 2569 & 46.6563 & 393.0557 & 395.3311 & 383 & 122.5994 & 1.94521 \\
\hline 2570 & 46.6733 & 364.1275 & 396.1032 & 383.2917 & 122.5994 & 1.94455 \\
\hline 2571 & 46.6903 & 377.1707 & 396.675 & 383.5833 & 122.5994 & 1.94388 \\
\hline 2572 & 46.7073 & 395.6915 & 396.6797 & 383.875 & 122.5994 & 1.94321 \\
\hline 2573 & 46.7243 & 403.9866 & 396.2531 & 384.1667 & 122.5994 & 1.94254 \\
\hline 2574 & 46.7413 & 386.6327 & 395.6394 & 384.4583 & 122.5994 & 1.94188 \\
\hline 2575 & 46.7583 & 384.2112 & 395.1793 & 384.75 & 122.5994 & 1.94121 \\
\hline 2576 & 46.7753 & 393.7807 & 394.9129 & 385.0417 & 122.5994 & 1.94054 \\
\hline 2577 & 46.7923 & 391.2227 & 394.7026 & 385.3333 & 122.5994 & 1.93988 \\
\hline 2578 & 46.8093 & 350.3286 & 394.4098 & 385.625 & 122.5994 & 1.93921 \\
\hline 2579 & 46.8263 & 336.1831 & 393.922 & 385.9167 & 122.5994 & 1.93855 \\
\hline 2580 & 46.8433 & 374.4832 & 393.4233 & 386.2083 & 122.5994 & 1.93788 \\
\hline 2581 & 46.8603 & 380.0105 & 392.8609 & 386.5 & 122.5994 & 1.93722 \\
\hline 2582 & 46.8773 & 380.0917 & 392.4846 & 386.7917 & 122.5994 & 1.93656 \\
\hline 2583 & 46.8943 & 391.1696 & 392.2906 & 387.0833 & 122.5994 & 1.9359 \\
\hline 2584 & 46.9113 & 372.9684 & 392.3328 & 387.375 & 122.5994 & 1.93523 \\
\hline 2585 & 46.9283 & 378.7829 & 392.6439 & 387.6667 & 122.5994 & 1.93457 \\
\hline 2586 & 46.9453 & 388.0528 & 393.2368 & 387.9583 & 122.5994 & 1.93391 \\
\hline 2587 & 46.9623 & 385.6219 & 394.1032 & 388.25 & 122.5994 & 1.93325 \\
\hline 2588 & 46.9793 & 389.3445 & 395.1704 & 388.5417 & 122.5994 & 1.93259 \\
\hline 2589 & 46.9963 & 394.718 & 396.4329 & 388.8333 & 122.5994 & 1.93193 \\
\hline 2590 & 47.0133 & 382.1962 & 397.6824 & 389.125 & 122.5994 & 1.93127 \\
\hline 2591 & 47.0303 & 376.3239 & 398.6601 & 389.4167 & 122.5994 & 1.93061 \\
\hline 2592 & 47.0473 & 385.193 & 399.1041 & 389.7083 & 122.5994 & 1.92996 \\
\hline 2593 & 47.0643 & 391.095 & 399.0683 & 390 & 122.5994 & 1.9293 \\
\hline 2594 & 47.0813 & 401.5704 & 398.562 & 390.0625 & 122.5994 & 1.92864 \\
\hline 2595 & 47.0983 & 375.2069 & 398.167 & 390.125 & 122.5994 & 1.92799 \\
\hline
\end{tabular}




\begin{tabular}{|c|c|c|c|c|c|c|}
\hline 2596 & 47.1153 & 391.4493 & 398.2247 & 390.1875 & 122.5994 & 1.92733 \\
\hline 2597 & 47.1323 & 359.6096 & 399.0479 & 390.25 & 122.5994 & 1.92667 \\
\hline 2598 & 47.1493 & 356.7151 & 400.7331 & 390.3125 & 122.5994 & 1.92602 \\
\hline 2599 & 47.1663 & 360.6663 & 402.7551 & 390.375 & 122.5994 & 1.92536 \\
\hline 2600 & 47.1833 & 354.0041 & 403.5475 & 390.4375 & 122.5994 & 1.92471 \\
\hline 2601 & 47.2003 & 386.0541 & 401.6497 & 390.5 & 122.5994 & 1.92406 \\
\hline 2602 & 47.2173 & 391.949 & 398.6504 & 390.5625 & 122.5994 & 1.9234 \\
\hline 2603 & 47.2343 & 403.7963 & 396.3467 & 390.625 & 122.5994 & 1.92275 \\
\hline 2604 & 47.2513 & 372.5074 & 395.2393 & 390.6875 & 122.5994 & 1.9221 \\
\hline 2605 & 47.2683 & 379.5367 & 395.2209 & 390.75 & 122.5994 & 1.92145 \\
\hline 2606 & 47.2853 & 352.4802 & 395.9169 & 390.8125 & 122.5994 & 1.9208 \\
\hline 2607 & 47.3023 & 338.0937 & 396.5897 & 390.875 & 122.5994 & 1.92014 \\
\hline 2608 & 47.3193 & 384.0201 & 396.1915 & 390.9375 & 122.5994 & 1.91949 \\
\hline 2609 & 47.3363 & 416.9823 & 394.9921 & 391 & 122.5994 & 1.91885 \\
\hline 2610 & 47.3533 & 393.1753 & 393.8773 & 391.0625 & 122.5994 & 1.9182 \\
\hline 2611 & 47.3703 & 401.2277 & 393.1133 & 391.125 & 122.5994 & 1.91755 \\
\hline 2612 & 47.3873 & 405.1385 & 392.7834 & 391.1875 & 122.5994 & 1.9169 \\
\hline 2613 & 47.4043 & 400.2344 & 392.5948 & 391.25 & 122.5994 & 1.91625 \\
\hline 2614 & 47.4213 & 433.5241 & 392.4929 & 391.3125 & 122.5994 & 1.9156 \\
\hline 2615 & 47.4383 & 444.1158 & 392.4435 & 391.375 & 122.5994 & 1.91496 \\
\hline 2616 & 47.4553 & 465.3729 & 392.4343 & 391.4375 & 122.5994 & 1.91431 \\
\hline 2617 & 47.4723 & 445.6151 & 392.4563 & 391.5 & 122.5994 & 1.91366 \\
\hline 2618 & 47.4893 & 402.7357 & 392.4648 & 391.5625 & 122.5994 & 1.91302 \\
\hline 2619 & 47.5063 & 409.3498 & 392.6055 & 391.625 & 122.5994 & 1.91237 \\
\hline 2620 & 47.5233 & 415.7468 & 392.6542 & 391.6875 & 122.5994 & 1.91173 \\
\hline 2621 & 47.5403 & 387.6095 & 392.7358 & 391.75 & 122.5994 & 1.91109 \\
\hline 2622 & 47.5573 & 397.6908 & 392.8994 & 391.8125 & 122.5994 & 1.91044 \\
\hline 2623 & 47.5743 & 412.5352 & 393.109 & 391.875 & 122.5994 & 1.9098 \\
\hline 2624 & 47.5913 & 396.0628 & 393.425 & 391.9375 & 122.5994 & 1.90916 \\
\hline 2625 & 47.6083 & 390.536 & 393.8111 & 392 & 122.5994 & 1.90851 \\
\hline 2626 & 47.6253 & 373.0027 & 394.2173 & 392.0625 & 122.5994 & 1.90787 \\
\hline 2627 & 47.6423 & 370.7079 & 394.5219 & 392.125 & 122.5994 & 1.90723 \\
\hline 2628 & 47.6593 & 384.3156 & 394.5501 & 392.1875 & 122.5994 & 1.90659 \\
\hline 2629 & 47.6763 & 373.3811 & 394.4414 & 392.25 & 122.5994 & 1.90595 \\
\hline 2630 & 47.6933 & 342.0568 & 394.3487 & 392.3125 & 122.5994 & 1.90531 \\
\hline 2631 & 47.7103 & 349.4416 & 394.2634 & 392.375 & 122.5994 & 1.90467 \\
\hline 2632 & 47.7273 & 362.5369 & 394.303 & 392.4375 & 122.5994 & 1.90403 \\
\hline 2633 & 47.7443 & 380.545 & 394.4661 & 392.5 & 122.5994 & 1.9034 \\
\hline 2634 & 47.7613 & 376.8253 & 394.6633 & 392.5625 & 122.5994 & 1.90276 \\
\hline 2635 & 47.7783 & 343.4024 & 394.9107 & 392.625 & 122.5994 & 1.90212 \\
\hline 2636 & 47.7953 & 379.1071 & 395.1411 & 392.6875 & 122.5994 & 1.90148 \\
\hline 2637 & 47.8123 & 399.9616 & 395.4093 & 392.75 & 122.5994 & 1.90085 \\
\hline 2638 & 47.8293 & 379.6497 & 395.815 & 392.8125 & 122.5994 & 1.90021 \\
\hline 2639 & 47.8463 & 384.3956 & 396.3072 & 392.875 & 122.5994 & 1.89958 \\
\hline 2640 & 47.8633 & 391.1394 & 396.9089 & 392.9375 & 122.5994 & 1.89894 \\
\hline 2641 & 47.8803 & 408.1937 & 397.5872 & 393 & 122.5994 & 1.89831 \\
\hline 2642 & 47.8973 & 366.8027 & 397.7209 & 392.6667 & 122.5994 & 1.89767 \\
\hline 2643 & 47.9143 & 420.9824 & 397.7193 & 392.3333 & 122.5994 & 1.89704 \\
\hline 2644 & 47.9313 & 390.6386 & 397.6541 & 392 & 122.5994 & 1.89641 \\
\hline
\end{tabular}




\begin{tabular}{|c|c|c|c|c|c|c|}
\hline 2645 & 47.9483 & 384.1232 & 397.7101 & 391.6667 & 122.5994 & 1.89577 \\
\hline 2646 & 47.9653 & 391.3869 & 398.132 & 391.3333 & 122.5994 & 1.89514 \\
\hline 2647 & 47.9823 & 388.3208 & 399.1851 & 391 & 122.5994 & 1.89451 \\
\hline 2648 & 47.9993 & 388.1417 & 401.1129 & 390.6667 & 122.5994 & 1.89388 \\
\hline 2649 & 48.0163 & 376.6147 & 403.9629 & 390.3333 & 122.5994 & 1.89325 \\
\hline 2650 & 48.0333 & 399.882 & 407.8913 & 390 & 122.5994 & 1.89262 \\
\hline 2651 & 48.0503 & 383.8424 & 413.814 & 389.6667 & 122.5994 & 1.89199 \\
\hline 2652 & 48.0673 & 371.2325 & 421.5988 & 389.3333 & 122.5994 & 1.89136 \\
\hline 2653 & 48.0843 & 429.6335 & 426.2685 & 389 & 122.5994 & 1.89073 \\
\hline 2654 & 48.1013 & 384.7739 & 423.488 & 388.6667 & 122.5994 & 1.8901 \\
\hline 2655 & 48.1183 & 391.6806 & 419.3522 & 388.3333 & 122.5994 & 1.88947 \\
\hline 2656 & 48.1353 & 387.559 & 418.6435 & 388 & 122.5994 & 1.88884 \\
\hline 2657 & 48.1523 & 381.361 & 418.8119 & 387.6667 & 122.5994 & 1.88822 \\
\hline 2658 & 48.1693 & 376.2193 & 416.2682 & 387.3333 & 122.5994 & 1.88759 \\
\hline 2659 & 48.1863 & 424.2167 & 414.0139 & 387 & 122.5994 & 1.88696 \\
\hline 2660 & 48.2033 & 396.6091 & 413.5057 & 386.6667 & 122.5994 & 1.88634 \\
\hline 2661 & 48.2203 & 417.1988 & 411.8611 & 386.3333 & 122.5994 & 1.88571 \\
\hline 2662 & 48.2373 & 384.3173 & 409.0216 & 386 & 122.5994 & 1.88509 \\
\hline 2663 & 48.2543 & 391.6178 & 407.8099 & 385.6667 & 122.5994 & 1.88446 \\
\hline 2664 & 48.2713 & 384.9961 & 408.1566 & 385.3333 & 122.5994 & 1.88384 \\
\hline 2665 & 48.2883 & 389.6968 & 407.4949 & 385 & 122.5994 & 1.88322 \\
\hline 2666 & 48.3053 & 381.757 & 405.0212 & 384.3333 & 122.5994 & 1.88259 \\
\hline 2667 & 48.3223 & 383.5218 & 402.6422 & 383.6667 & 122.5994 & 1.88197 \\
\hline 2668 & 48.3393 & 376.0703 & 400.9362 & 383 & 122.5994 & 1.88135 \\
\hline 2669 & 48.3563 & 378.7019 & 399.7469 & 382.3333 & 122.5994 & 1.88073 \\
\hline 2670 & 48.3733 & 368.0537 & 398.8095 & 381.6667 & 122.5994 & 1.8801 \\
\hline 2671 & 48.3903 & 372.5397 & 397.9806 & 381 & 122.5994 & 1.87948 \\
\hline 2672 & 48.4073 & 359.9857 & 397.1531 & 380.3333 & 122.5994 & 1.87886 \\
\hline 2673 & 48.4243 & 353.3175 & 396.23 & 379.6667 & 122.5994 & 1.87824 \\
\hline 2674 & 48.4413 & 359.1037 & 395.1848 & 379 & 122.5994 & 1.87762 \\
\hline 2675 & 48.4583 & 365.3424 & 394.0182 & 378.3333 & 122.5994 & 1.87701 \\
\hline 2676 & 48.4753 & 381.2151 & 392.7574 & 377.6667 & 122.5994 & 1.87639 \\
\hline 2677 & 48.4923 & 385.783 & 391.3968 & 377 & 122.5994 & 1.87577 \\
\hline 2678 & 48.5093 & 377.2187 & 390.1604 & 376.3333 & 122.5994 & 1.87515 \\
\hline 2679 & 48.5263 & 404.6453 & 389.0144 & 375.6667 & 122.5994 & 1.87453 \\
\hline 2680 & 48.5433 & 373.9841 & 387.757 & 375 & 122.5994 & 1.87392 \\
\hline 2681 & 48.5603 & 371.3587 & 386.244 & 374.3333 & 122.5994 & 1.8733 \\
\hline 2682 & 48.5773 & 376.128 & 384.5803 & 373.6667 & 122.5994 & 1.87269 \\
\hline 2683 & 48.5943 & 372.5397 & 382.9445 & 373 & 122.5994 & 1.87207 \\
\hline 2684 & 48.6113 & 345.0751 & 381.5106 & 372.3333 & 122.5994 & 1.87145 \\
\hline 2685 & 48.6283 & 365.9434 & 380.328 & 371.6667 & 122.5994 & 1.87084 \\
\hline 2686 & 48.6453 & 361.6409 & 379.4166 & 371 & 122.5994 & 1.87023 \\
\hline 2687 & 48.6623 & 387.931 & 378.6478 & 370.3333 & 122.5994 & 1.86961 \\
\hline 2688 & 48.6793 & 367.5538 & 377.8263 & 369.6667 & 122.5994 & 1.869 \\
\hline 2689 & 48.6963 & 370.5679 & 376.8376 & 369 & 122.5994 & 1.86839 \\
\hline 2690 & 48.7133 & 362.6884 & 376.8567 & 369.5 & 122.5994 & 1.86777 \\
\hline 2691 & 48.7303 & 364.3136 & 376.8501 & 370 & 122.5994 & 1.86716 \\
\hline 2692 & 48.7473 & 400.0637 & 376.7597 & 370.5 & 122.5994 & 1.86655 \\
\hline 2693 & 48.7643 & 419.035 & 376.6671 & 371 & 122.5994 & 1.86594 \\
\hline
\end{tabular}




\begin{tabular}{|c|c|c|c|c|c|c|}
\hline 2694 & 48.7813 & 364.3197 & 376.7356 & 371.5 & 122.5994 & 1.86533 \\
\hline 2695 & 48.7983 & 364.7245 & 376.8459 & 372 & 122.5994 & 1.86472 \\
\hline 2696 & 48.8153 & 353.5601 & 376.9388 & 372.5 & 122.5994 & 1.86411 \\
\hline 2697 & 48.8323 & 368.781 & 377.0395 & 373 & 122.5994 & 1.8635 \\
\hline 2698 & 48.8493 & 380.6758 & 377.1731 & 373.5 & 122.5994 & 1.86289 \\
\hline 2699 & 48.8663 & 393.1337 & 377.3469 & 374 & 122.5994 & 1.86228 \\
\hline 2700 & 48.8833 & 337.7347 & 377.5784 & 374.5 & 122.5994 & 1.86168 \\
\hline 2701 & 48.9003 & 329.3796 & 377.8781 & 375 & 122.5994 & 1.86107 \\
\hline 2702 & 48.9173 & 348.1035 & 378.3018 & 375.5 & 122.5994 & 1.86046 \\
\hline 2703 & 48.9343 & 364.2534 & 378.835 & 376 & 122.5994 & 1.85985 \\
\hline 2704 & 48.9513 & 359.255 & 379.4876 & 376.5 & 122.5994 & 1.85925 \\
\hline 2705 & 48.9683 & 348.3175 & 380.3717 & 377 & 122.5994 & 1.85864 \\
\hline 2706 & 48.9853 & 341.5517 & 381.4248 & 377.5 & 122.5994 & 1.85804 \\
\hline 2707 & 49.0023 & 338.5275 & 382.7021 & 378 & 122.5994 & 1.85743 \\
\hline 2708 & 49.0193 & 347.8542 & 383.9679 & 378.5 & 122.5994 & 1.85683 \\
\hline 2709 & 49.0363 & 380.4964 & 384.998 & 379 & 122.5994 & 1.85622 \\
\hline 2710 & 49.0533 & 386.3813 & 385.7001 & 379.5 & 122.5994 & 1.85562 \\
\hline 2711 & 49.0703 & 374.9388 & 386.0816 & 380 & 122.5994 & 1.85502 \\
\hline 2712 & 49.0873 & 386.7428 & 386.2608 & 380.5 & 122.5994 & 1.85441 \\
\hline 2713 & 49.1043 & 390.3631 & 386.3765 & 381 & 122.5994 & 1.85381 \\
\hline 2714 & 49.1213 & 369.5845 & 385.9078 & 380.75 & 122.5994 & 1.85321 \\
\hline 2715 & 49.1383 & 369.3382 & 385.6384 & 380.5 & 122.5994 & 1.85261 \\
\hline 2716 & 49.1553 & 374.7974 & 385.4723 & 380.25 & 122.5994 & 1.85201 \\
\hline 2717 & 49.1723 & 375.8544 & 385.3265 & 380 & 122.5994 & 1.85141 \\
\hline 2718 & 49.1893 & 389.3818 & 385.1766 & 379.75 & 122.5994 & 1.85081 \\
\hline 2719 & 49.2063 & 388.5855 & 385.0672 & 379.5 & 122.5994 & 1.85021 \\
\hline 2720 & 49.2233 & 380.3326 & 385.184 & 379.25 & 122.5994 & 1.84961 \\
\hline 2721 & 49.2403 & 388.2546 & 385.9802 & 379 & 122.5994 & 1.84901 \\
\hline 2722 & 49.2573 & 392.0024 & 388.3932 & 378.75 & 122.5994 & 1.84841 \\
\hline 2723 & 49.2743 & 378.8159 & 394.0292 & 378.5 & 122.5994 & 1.84781 \\
\hline 2724 & 49.2913 & 408.7421 & 403.3157 & 378.25 & 122.5994 & 1.84722 \\
\hline 2725 & 49.3083 & 421.7783 & 413.4932 & 378 & 122.5994 & 1.84662 \\
\hline 2726 & 49.3253 & 404.4113 & 416.3001 & 377.75 & 122.5994 & 1.84602 \\
\hline 2727 & 49.3423 & 355.0424 & 408.6758 & 377.5 & 122.5994 & 1.84543 \\
\hline 2728 & 49.3593 & 353.9414 & 398.1043 & 377.25 & 122.5994 & 1.84483 \\
\hline 2729 & 49.3763 & 349.0655 & 390.7063 & 377 & 122.5994 & 1.84424 \\
\hline 2730 & 49.3933 & 361.2435 & 388.0711 & 376.75 & 122.5994 & 1.84364 \\
\hline 2731 & 49.4103 & 365.7727 & 389.7513 & 376.5 & 122.5994 & 1.84305 \\
\hline 2732 & 49.4273 & 367.7735 & 394.2316 & 376.25 & 122.5994 & 1.84245 \\
\hline 2733 & 49.4443 & 380.7488 & 398.2299 & 376 & 122.5994 & 1.84186 \\
\hline 2734 & 49.4613 & 426.4488 & 397.009 & 375.75 & 122.5994 & 1.84126 \\
\hline 2735 & 49.4783 & 399.9081 & 391.2944 & 375.5 & 122.5994 & 1.84067 \\
\hline 2736 & 49.4953 & 356.133 & 385.3904 & 375.25 & 122.5994 & 1.84008 \\
\hline 2737 & 49.5123 & 356.2687 & 381.4578 & 375 & 122.5994 & 1.83949 \\
\hline 2738 & 49.5293 & 398.8299 & 381.6071 & 376.8333 & 122.5994 & 1.8389 \\
\hline 2739 & 49.5463 & 375.2418 & 383.1026 & 378.6667 & 122.5994 & 1.8383 \\
\hline 2740 & 49.5633 & 376.1508 & 385.5185 & 380.5 & 122.5994 & 1.83771 \\
\hline 2741 & 49.5803 & 365.299 & 388.4336 & 382.3333 & 122.5994 & 1.83712 \\
\hline 2742 & 49.5973 & 364.8586 & 391.2612 & 384.1667 & 122.5994 & 1.83653 \\
\hline
\end{tabular}




\begin{tabular}{|c|c|c|c|c|c|c|}
\hline 2743 & 49.6143 & 357.1932 & 392.9704 & 386 & 122.5994 & 1.83594 \\
\hline 2744 & 49.6313 & 369.053 & 393.3835 & 387.8333 & 122.5994 & 1.83536 \\
\hline 2745 & 49.6483 & 390.589 & 393.6983 & 389.6667 & 122.5994 & 1.83477 \\
\hline 2746 & 49.6653 & 357.4935 & 394.6555 & 391.5 & 122.5994 & 1.83418 \\
\hline 2747 & 49.6823 & 364.9002 & 396.2916 & 393.3333 & 122.5994 & 1.83359 \\
\hline 2748 & 49.6993 & 402.742 & 398.5067 & 395.1667 & 122.5994 & 1.833 \\
\hline 2749 & 49.7163 & 363.6169 & 401.152 & 397 & 122.5994 & 1.83242 \\
\hline 2750 & 49.7333 & 413.6093 & 403.8699 & 398.8333 & 122.5994 & 1.83183 \\
\hline 2751 & 49.7503 & 436.8137 & 406.1407 & 400.6667 & 122.5994 & 1.83124 \\
\hline 2752 & 49.7673 & 395.2129 & 408.3832 & 402.5 & 122.5994 & 1.83066 \\
\hline 2753 & 49.7843 & 400.7343 & 411.9275 & 404.3333 & 122.5994 & 1.83007 \\
\hline 2754 & 49.8013 & 432.5985 & 417.8904 & 406.1667 & 122.5994 & 1.82949 \\
\hline 2755 & 49.8183 & 416.3182 & 435.1055 & 408 & 122.5994 & 1.8289 \\
\hline 2756 & 49.8353 & 376.1957 & 444.4841 & 409.8333 & 122.5994 & 1.82832 \\
\hline 2757 & 49.8523 & 406.9159 & 448.8401 & 411.6667 & 122.5994 & 1.82774 \\
\hline 2758 & 49.8693 & 438.0807 & 448.4327 & 413.5 & 122.5994 & 1.82715 \\
\hline 2759 & 49.8863 & 432.4963 & 450.5562 & 415.3333 & 122.5994 & 1.82657 \\
\hline 2760 & 49.9033 & 407.414 & 452.8104 & 417.1667 & 122.5994 & 1.82599 \\
\hline 2761 & 49.9203 & 420.2097 & 460.0117 & 419 & 122.5994 & 1.82541 \\
\hline 2762 & 49.9373 & 421.977 & 470.5694 & 418.6458 & 122.5994 & 1.82482 \\
\hline 2763 & 49.9543 & 420.8099 & 484.9834 & 418.2917 & 122.5994 & 1.82424 \\
\hline 2764 & 49.9713 & 408.8578 & 496.9868 & 417.9375 & 122.5994 & 1.82366 \\
\hline 2765 & 49.9883 & 431.4064 & 500.4662 & 417.5833 & 122.5994 & 1.82308 \\
\hline 2766 & 50.0053 & 453.9277 & 497.5472 & 417.2292 & 122.5994 & 1.8225 \\
\hline 2767 & 50.0223 & 467.7107 & 494.8708 & 416.875 & 122.5994 & 1.82192 \\
\hline 2768 & 50.0393 & 502.3896 & 499.6329 & 416.5208 & 122.5994 & 1.82134 \\
\hline 2769 & 50.0563 & 505.6984 & 516.2589 & 416.1667 & 122.5994 & 1.82076 \\
\hline 2770 & 50.0733 & 531.0409 & 549.4753 & 415.8125 & 122.5994 & 1.82019 \\
\hline 2771 & 50.0903 & 604.9037 & 607.4396 & 415.4583 & 122.5994 & 1.81961 \\
\hline 2772 & 50.1073 & 698.8121 & 701.8929 & 415.1042 & 122.5994 & 1.81903 \\
\hline 2773 & 50.1243 & 888.0597 & 851.7595 & 414.75 & 122.5994 & 1.81845 \\
\hline 2774 & 50.1413 & 1020.003 & 1069.341 & 414.3958 & 122.5994 & 1.81788 \\
\hline 2775 & 50.1583 & 1206.738 & 1323.804 & 414.0417 & 122.5994 & 1.8173 \\
\hline 2776 & 50.1753 & 1256.362 & 1506.994 & 413.6875 & 122.5994 & 1.81672 \\
\hline 2777 & 50.1923 & 1103.084 & 1482.755 & 413.3333 & 122.5994 & 1.81615 \\
\hline 2778 & 50.2093 & 906.0806 & 1280.31 & 412.9792 & 122.5994 & 1.81557 \\
\hline 2779 & 50.2263 & 771.179 & 1050.733 & 412.625 & 122.5994 & 1.815 \\
\hline 2780 & 50.2433 & 690.221 & 890.7784 & 412.2708 & 122.5994 & 1.81442 \\
\hline 2781 & 50.2603 & 719.1381 & 832.8615 & 411.9167 & 122.5994 & 1.81385 \\
\hline 2782 & 50.2773 & 747.3059 & 867.9027 & 411.5625 & 122.5994 & 1.81328 \\
\hline 2783 & 50.2943 & 848.1818 & 952.3322 & 411.2083 & 122.5994 & 1.8127 \\
\hline 2784 & 50.3113 & 818.6252 & 1007.208 & 410.8542 & 122.5994 & 1.81213 \\
\hline 2785 & 50.3283 & 746.9097 & 960.0853 & 410.5 & 122.5994 & 1.81156 \\
\hline 2786 & 50.3453 & 629.3428 & 834.5077 & 410.1458 & 122.5994 & 1.81099 \\
\hline 2787 & 50.3623 & 508.28 & 700.6335 & 409.7917 & 122.5994 & 1.81042 \\
\hline 2788 & 50.3793 & 471.8982 & 596.3823 & 409.4375 & 122.5994 & 1.80985 \\
\hline 2789 & 50.3963 & 408.8928 & 528.7873 & 409.0833 & 122.5994 & 1.80927 \\
\hline 2790 & 50.4133 & 400.3093 & 490.1623 & 408.7292 & 122.5994 & 1.8087 \\
\hline 2791 & 50.4303 & 427.5827 & 469.1462 & 408.375 & 122.5994 & 1.80813 \\
\hline
\end{tabular}




\begin{tabular}{|c|c|c|c|c|c|c|}
\hline 2792 & 50.4473 & 399.0253 & 457.5166 & 408.0208 & 122.5994 & 1.80756 \\
\hline 2793 & 50.4643 & 379.6434 & 450.58 & 407.6667 & 122.5994 & 1.807 \\
\hline 2794 & 50.4813 & 375.0648 & 445.9022 & 407.3125 & 122.5994 & 1.80643 \\
\hline 2795 & 50.4983 & 398.2073 & 442.1933 & 406.9583 & 122.5994 & 1.80586 \\
\hline 2796 & 50.5153 & 386.8617 & 438.4461 & 406.6042 & 122.5994 & 1.80529 \\
\hline 2797 & 50.5323 & 401.5228 & 434.7108 & 406.25 & 122.5994 & 1.80472 \\
\hline 2798 & 50.5493 & 388.7679 & 431.6625 & 405.8958 & 122.5994 & 1.80416 \\
\hline 2799 & 50.5663 & 394.4258 & 429.7579 & 405.5417 & 122.5994 & 1.80359 \\
\hline 2800 & 50.5833 & 405.8746 & 429.6644 & 405.1875 & 122.5994 & 1.80302 \\
\hline 2801 & 50.6003 & 411.0561 & 432.455 & 404.8333 & 122.5994 & 1.80246 \\
\hline 2802 & 50.6173 & 408.0944 & 438.9598 & 404.4792 & 122.5994 & 1.80189 \\
\hline 2803 & 50.6343 & 416.3037 & 447.9353 & 404.125 & 122.5994 & 1.80133 \\
\hline 2804 & 50.6513 & 429.199 & 453.709 & 403.7708 & 122.5994 & 1.80076 \\
\hline 2805 & 50.6683 & 409.3706 & 450.2965 & 403.4167 & 122.5994 & 1.8002 \\
\hline 2806 & 50.6853 & 399.7448 & 440.4946 & 403.0625 & 122.5994 & 1.79963 \\
\hline 2807 & 50.7023 & 393.58 & 430.6752 & 402.7083 & 122.5994 & 1.79907 \\
\hline 2808 & 50.7193 & 395.972 & 424.1028 & 402.3542 & 122.5994 & 1.79851 \\
\hline 2809 & 50.7363 & 399.4052 & 421.4753 & 402 & 122.5994 & 1.79794 \\
\hline 2810 & 50.7533 & 412.9095 & 422.5242 & 401.75 & 122.5994 & 1.79738 \\
\hline 2811 & 50.7703 & 411.26 & 425.7308 & 401.5 & 122.5994 & 1.79682 \\
\hline 2812 & 50.7873 & 403.8181 & 422.0573 & 401.25 & 122.5994 & 1.79626 \\
\hline 2813 & 50.8043 & 401.951 & 419.8488 & 401 & 122.5994 & 1.7957 \\
\hline 2814 & 50.8213 & 383.8546 & 414.7402 & 400.75 & 122.5994 & 1.79514 \\
\hline 2815 & 50.8383 & 398.0859 & 409.7378 & 400.5 & 122.5994 & 1.79458 \\
\hline 2816 & 50.8553 & 427.9536 & 406.1332 & 400.25 & 122.5994 & 1.79402 \\
\hline 2817 & 50.8723 & 408.0304 & 403.9038 & 400 & 122.5994 & 1.79346 \\
\hline 2818 & 50.8893 & 397.7843 & 402.6158 & 399.75 & 122.5994 & 1.7929 \\
\hline 2819 & 50.9063 & 404.0639 & 402.0957 & 399.5 & 122.5994 & 1.79234 \\
\hline 2820 & 50.9233 & 385.8786 & 401.6067 & 399.25 & 122.5994 & 1.79178 \\
\hline 2821 & 50.9403 & 395.1014 & 401.1202 & 399 & 122.5994 & 1.79122 \\
\hline 2822 & 50.9573 & 358.3255 & 400.7711 & 398.75 & 122.5994 & 1.79066 \\
\hline 2823 & 50.9743 & 343.8469 & 400.6153 & 398.5 & 122.5994 & 1.79011 \\
\hline 2824 & 50.9913 & 365.6512 & 400.5628 & 398.25 & 122.5994 & 1.78955 \\
\hline 2825 & 51.0083 & 341.0257 & 400.3992 & 398 & 122.5994 & 1.78899 \\
\hline 2826 & 51.0253 & 345.7407 & 400.0465 & 397.75 & 122.5994 & 1.78844 \\
\hline 2827 & 51.0423 & 368.0099 & 399.6931 & 397.5 & 122.5994 & 1.78788 \\
\hline 2828 & 51.0593 & 344.4923 & 399.5101 & 397.25 & 122.5994 & 1.78733 \\
\hline 2829 & 51.0763 & 354.3436 & 399.3928 & 397 & 122.5994 & 1.78677 \\
\hline 2830 & 51.0933 & 394.3801 & 399.3707 & 396.75 & 122.5994 & 1.78622 \\
\hline 2831 & 51.1103 & 361.9225 & 399.4265 & 396.5 & 122.5994 & 1.78566 \\
\hline 2832 & 51.1273 & 367.606 & 399.5076 & 396.25 & 122.5994 & 1.78511 \\
\hline 2833 & 51.1443 & 432.3725 & 399.5917 & 396 & 122.5994 & 1.78456 \\
\hline 2834 & 51.1613 & 386.4555 & 397.7671 & 393.9583 & 122.5994 & 1.784 \\
\hline 2835 & 51.1783 & 399.5992 & 396.2788 & 391.9167 & 122.5994 & 1.78345 \\
\hline 2836 & 51.1953 & 404.058 & 395.2854 & 389.875 & 122.5994 & 1.7829 \\
\hline 2837 & 51.2123 & 391.6189 & 395.4982 & 387.8333 & 122.5994 & 1.78235 \\
\hline 2838 & 51.2293 & 369.4147 & 398.4454 & 385.7917 & 122.5994 & 1.78179 \\
\hline 2839 & 51.2463 & 386.9106 & 406.6118 & 383.75 & 122.5994 & 1.78124 \\
\hline 2840 & 51.2633 & 371.9425 & 421.0097 & 381.7083 & 122.5994 & 1.78069 \\
\hline
\end{tabular}




\begin{tabular}{|c|c|c|c|c|c|c|}
\hline 2841 & 51.2803 & 406.724 & 436.3776 & 379.6667 & 122.5994 & 1.78014 \\
\hline 2842 & 51.2973 & 392.0758 & 437.7563 & 377.625 & 122.5994 & 1.77959 \\
\hline 2843 & 51.3143 & 366.4984 & 421.2615 & 375.5833 & 122.5994 & 1.77904 \\
\hline 2844 & 51.3313 & 377.0748 & 402.0145 & 373.5417 & 122.5994 & 1.77849 \\
\hline 2845 & 51.3483 & 375.7834 & 388.8635 & 371.5 & 122.5994 & 1.77794 \\
\hline 2846 & 51.3653 & 360.5264 & 382.9114 & 369.4583 & 122.5994 & 1.7774 \\
\hline 2847 & 51.3823 & 378.9287 & 382.9915 & 367.4167 & 122.5994 & 1.77685 \\
\hline 2848 & 51.3993 & 391.9094 & 387.6719 & 365.375 & 122.5994 & 1.7763 \\
\hline 2849 & 51.4163 & 368.6891 & 393.6508 & 363.3333 & 122.5994 & 1.77575 \\
\hline 2850 & 51.4333 & 364.0471 & 393.37 & 361.2917 & 122.5994 & 1.77521 \\
\hline 2851 & 51.4503 & 360.8556 & 384.1908 & 359.25 & 122.5994 & 1.77466 \\
\hline 2852 & 51.4673 & 374.9968 & 373.3634 & 357.2083 & 122.5994 & 1.77411 \\
\hline 2853 & 51.4843 & 368.2681 & 365.0818 & 355.1667 & 122.5994 & 1.77357 \\
\hline 2854 & 51.5013 & 356.6827 & 359.5667 & 353.125 & 122.5994 & 1.77302 \\
\hline 2855 & 51.5183 & 358.9452 & 355.8898 & 351.0833 & 122.5994 & 1.77248 \\
\hline 2856 & 51.5353 & 353.284 & 353.127 & 349.0417 & 122.5994 & 1.77193 \\
\hline 2857 & 51.5523 & 335.8447 & 350.8024 & 347 & 122.5994 & 1.77139 \\
\hline 2858 & 51.5693 & 349.5376 & 351.3422 & 347.5833 & 122.5994 & 1.77084 \\
\hline 2859 & 51.5863 & 353.4061 & 352.0669 & 348.1667 & 122.5994 & 1.7703 \\
\hline 2860 & 51.6033 & 355.3475 & 352.9557 & 348.75 & 122.5994 & 1.76976 \\
\hline 2861 & 51.6203 & 361.3358 & 353.9381 & 349.3333 & 122.5994 & 1.76921 \\
\hline 2862 & 51.6373 & 373.391 & 355.036 & 349.9167 & 122.5994 & 1.76867 \\
\hline 2863 & 51.6543 & 378.0457 & 356.4218 & 350.5 & 122.5994 & 1.76813 \\
\hline 2864 & 51.6713 & 371.6834 & 358.2033 & 351.0833 & 122.5994 & 1.76759 \\
\hline 2865 & 51.6883 & 347.5946 & 360.404 & 351.6667 & 122.5994 & 1.76705 \\
\hline 2866 & 51.7053 & 342.0656 & 363.0007 & 352.25 & 122.5994 & 1.7665 \\
\hline 2867 & 51.7223 & 336.1293 & 365.9689 & 352.8333 & 122.5994 & 1.76596 \\
\hline 2868 & 51.7393 & 336.3147 & 368.8456 & 353.4167 & 122.5994 & 1.76542 \\
\hline 2869 & 51.7563 & 351.744 & 370.8555 & 354 & 122.5994 & 1.76488 \\
\hline 2870 & 51.7733 & 349.0416 & 370.8123 & 354.5833 & 122.5994 & 1.76434 \\
\hline 2871 & 51.7903 & 337.6052 & 369.0863 & 355.1667 & 122.5994 & 1.7638 \\
\hline 2872 & 51.8073 & 337.6953 & 367.3224 & 355.75 & 122.5994 & 1.76327 \\
\hline 2873 & 51.8243 & 350.4317 & 366.3497 & 356.3333 & 122.5994 & 1.76273 \\
\hline 2874 & 51.8413 & 359.5767 & 366.2459 & 356.9167 & 122.5994 & 1.76219 \\
\hline 2875 & 51.8583 & 377.1771 & 366.8633 & 357.5 & 122.5994 & 1.76165 \\
\hline 2876 & 51.8753 & 357.176 & 367.8201 & 358.0833 & 122.5994 & 1.76111 \\
\hline 2877 & 51.8923 & 350.1034 & 368.7003 & 358.6667 & 122.5994 & 1.76058 \\
\hline 2878 & 51.9093 & 343.0231 & 368.8871 & 359.25 & 122.5994 & 1.76004 \\
\hline 2879 & 51.9263 & 366.5957 & 368.3962 & 359.8333 & 122.5994 & 1.7595 \\
\hline 2880 & 51.9433 & 354.3186 & 367.6997 & 360.4167 & 122.5994 & 1.75897 \\
\hline 2881 & 51.9603 & 375.5663 & 367.1559 & 361 & 122.5994 & 1.75843 \\
\hline 2882 & 51.9773 & 357.4804 & 365.9372 & 361.0417 & 122.5994 & 1.7579 \\
\hline 2883 & 51.9943 & 345.7466 & 364.9657 & 361.0833 & 122.5994 & 1.75736 \\
\hline 2884 & 52.0113 & 328.5853 & 364.4996 & 361.125 & 122.5994 & 1.75683 \\
\hline 2885 & 52.0283 & 357.7803 & 364.6609 & 361.1667 & 122.5994 & 1.75629 \\
\hline 2886 & 52.0453 & 368.4036 & 365.4843 & 361.2083 & 122.5994 & 1.75576 \\
\hline 2887 & 52.0623 & 362.256 & 366.6898 & 361.25 & 122.5994 & 1.75523 \\
\hline 2888 & 52.0793 & 339.9142 & 367.5215 & 361.2917 & 122.5994 & 1.75469 \\
\hline 2889 & 52.0963 & 350.702 & 367.1344 & 361.3333 & 122.5994 & 1.75416 \\
\hline
\end{tabular}




\begin{tabular}{|c|c|c|c|c|c|c|}
\hline 2890 & 52.1133 & 349.5622 & 366.1012 & 361.375 & 122.5994 & 1.75363 \\
\hline 2891 & 52.1303 & 318.4421 & 365.3488 & 361.4167 & 122.5994 & 1.7531 \\
\hline 2892 & 52.1473 & 362.6607 & 365.1386 & 361.4583 & 122.5994 & 1.75257 \\
\hline 2893 & 52.1643 & 380.7165 & 365.394 & 361.5 & 122.5994 & 1.75204 \\
\hline 2894 & 52.1813 & 372.4318 & 365.8267 & 361.5417 & 122.5994 & 1.7515 \\
\hline 2895 & 52.1983 & 373.885 & 366.8269 & 361.5833 & 122.5994 & 1.75097 \\
\hline 2896 & 52.2153 & 346.0061 & 368.5368 & 361.625 & 122.5994 & 1.75044 \\
\hline 2897 & 52.2323 & 343.6248 & 370.6736 & 361.6667 & 122.5994 & 1.74991 \\
\hline 2898 & 52.2493 & 344.8106 & 372.6535 & 361.7083 & 122.5994 & 1.74938 \\
\hline 2899 & 52.2663 & 362.5872 & 372.8123 & 361.75 & 122.5994 & 1.74886 \\
\hline 2900 & 52.2833 & 371.0928 & 370.9524 & 361.7917 & 122.5994 & 1.74833 \\
\hline 2901 & 52.3003 & 370.134 & 369.0519 & 361.8333 & 122.5994 & 1.7478 \\
\hline 2902 & 52.3173 & 377.3398 & 368.1611 & 361.875 & 122.5994 & 1.74727 \\
\hline 2903 & 52.3343 & 375.6915 & 368.2869 & 361.9167 & 122.5994 & 1.74674 \\
\hline 2904 & 52.3513 & 352.655 & 369.6242 & 361.9583 & 122.5994 & 1.74622 \\
\hline 2905 & 52.3683 & 353.1447 & 372.1156 & 362 & 122.5994 & 1.74569 \\
\hline 2906 & 52.3853 & 368.6046 & 375.4815 & 361.9167 & 122.5994 & 1.74516 \\
\hline 2907 & 52.4023 & 370.6305 & 378.9844 & 361.8333 & 122.5994 & 1.74464 \\
\hline 2908 & 52.4193 & 372.5397 & 381.8213 & 361.75 & 122.5994 & 1.74411 \\
\hline 2909 & 52.4363 & 357.3514 & 383.8712 & 361.6667 & 122.5994 & 1.74359 \\
\hline 2910 & 52.4533 & 368.5794 & 382.7827 & 361.5833 & 122.5994 & 1.74306 \\
\hline 2911 & 52.4703 & 369.9413 & 378.197 & 361.5 & 122.5994 & 1.74254 \\
\hline 2912 & 52.4873 & 349.1607 & 373.6796 & 361.4167 & 122.5994 & 1.74201 \\
\hline 2913 & 52.5043 & 393.3394 & 371.0024 & 361.3333 & 122.5994 & 1.74149 \\
\hline 2914 & 52.5213 & 387.8841 & 370.2352 & 361.25 & 122.5994 & 1.74096 \\
\hline 2915 & 52.5383 & 372.5397 & 370.8746 & 361.1667 & 122.5994 & 1.74044 \\
\hline 2916 & 52.5553 & 378.0407 & 372.3579 & 361.0833 & 122.5994 & 1.73992 \\
\hline 2917 & 52.5723 & 380.3007 & 374.0792 & 361 & 122.5994 & 1.73939 \\
\hline 2918 & 52.5893 & 374.2929 & 374.6213 & 360.9167 & 122.5994 & 1.73887 \\
\hline 2919 & 52.6063 & 406.7088 & 373.0948 & 360.8333 & 122.5994 & 1.73835 \\
\hline 2920 & 52.6233 & 393.5898 & 371.0631 & 360.75 & 122.5994 & 1.73783 \\
\hline 2921 & 52.6403 & 397.9079 & 369.8069 & 360.6667 & 122.5994 & 1.73731 \\
\hline 2922 & 52.6573 & 373.4251 & 369.527 & 360.5833 & 122.5994 & 1.73679 \\
\hline 2923 & 52.6743 & 368.6046 & 369.9777 & 360.5 & 122.5994 & 1.73627 \\
\hline 2924 & 52.6913 & 375.5768 & 370.8561 & 360.4167 & 122.5994 & 1.73575 \\
\hline 2925 & 52.7083 & 358.8284 & 371.9386 & 360.3333 & 122.5994 & 1.73523 \\
\hline 2926 & 52.7253 & 400.9349 & 373.0887 & 360.25 & 122.5994 & 1.73471 \\
\hline 2927 & 52.7423 & 396.4105 & 374.2473 & 360.1667 & 122.5994 & 1.73419 \\
\hline 2928 & 52.7593 & 358.022 & 375.4683 & 360.0833 & 122.5994 & 1.73367 \\
\hline 2929 & 52.7763 & 342.3015 & 376.8261 & 360 & 122.5994 & 1.73315 \\
\hline 2930 & 52.7933 & 361.1293 & 379.3423 & 360.875 & 122.5994 & 1.73263 \\
\hline 2931 & 52.8103 & 364.9386 & 382.0256 & 361.75 & 122.5994 & 1.73212 \\
\hline 2932 & 52.8273 & 370.3601 & 384.5059 & 362.625 & 122.5994 & 1.7316 \\
\hline 2933 & 52.8443 & 393.0594 & 386.4392 & 363.5 & 122.5994 & 1.73108 \\
\hline 2934 & 52.8613 & 360.9654 & 387.4908 & 364.375 & 122.5994 & 1.73056 \\
\hline 2935 & 52.8783 & 365.0479 & 387.6226 & 365.25 & 122.5994 & 1.73005 \\
\hline 2936 & 52.8953 & 389.0645 & 387.119 & 366.125 & 122.5994 & 1.72953 \\
\hline 2937 & 52.9123 & 368.5396 & 386.4233 & 367 & 122.5994 & 1.72902 \\
\hline 2938 & 52.9293 & 361.8015 & 386.03 & 367.875 & 122.5994 & 1.7285 \\
\hline
\end{tabular}




\begin{tabular}{|c|c|c|c|c|c|c|}
\hline 2939 & 52.9463 & 400.264 & 385.9344 & 368.75 & 122.5994 & 1.72799 \\
\hline 2940 & 52.9633 & 361.785 & 386.2981 & 369.625 & 122.5994 & 1.72747 \\
\hline 2941 & 52.9803 & 378.335 & 386.8663 & 370.5 & 122.5994 & 1.72696 \\
\hline 2942 & 52.9973 & 386.4136 & 387.0425 & 371.375 & 122.5994 & 1.72644 \\
\hline 2943 & 53.0143 & 389.6312 & 386.4843 & 372.25 & 122.5994 & 1.72593 \\
\hline 2944 & 53.0313 & 386.6631 & 385.6118 & 373.125 & 122.5994 & 1.72542 \\
\hline 2945 & 53.0483 & 389.3817 & 384.7939 & 374 & 122.5994 & 1.7249 \\
\hline 2946 & 53.0653 & 377.0527 & 384.2531 & 374.875 & 122.5994 & 1.72439 \\
\hline 2947 & 53.0823 & 372.348 & 384.0415 & 375.75 & 122.5994 & 1.72388 \\
\hline 2948 & 53.0993 & 388.9635 & 384.3045 & 376.625 & 122.5994 & 1.72337 \\
\hline 2949 & 53.1163 & 384.206 & 385.1318 & 377.5 & 122.5994 & 1.72286 \\
\hline 2950 & 53.1333 & 370.4276 & 386.7081 & 378.375 & 122.5994 & 1.72234 \\
\hline 2951 & 53.1503 & 361.6708 & 389.0757 & 379.25 & 122.5994 & 1.72183 \\
\hline 2952 & 53.1673 & 372.6469 & 392.5233 & 380.125 & 122.5994 & 1.72132 \\
\hline 2953 & 53.1843 & 387.8394 & 397.3103 & 381 & 122.5994 & 1.72081 \\
\hline 2954 & 53.2013 & 388.7745 & 402.0719 & 381.5417 & 122.5994 & 1.7203 \\
\hline 2955 & 53.2183 & 389.3467 & 405.1453 & 382.0833 & 122.5994 & 1.71979 \\
\hline 2956 & 53.2353 & 379.5951 & 404.6264 & 382.625 & 122.5994 & 1.71928 \\
\hline 2957 & 53.2523 & 374.0798 & 400.5549 & 383.1667 & 122.5994 & 1.71878 \\
\hline 2958 & 53.2693 & 372.4543 & 396.6939 & 383.7083 & 122.5994 & 1.71827 \\
\hline 2959 & 53.2863 & 390.2902 & 395.636 & 384.25 & 122.5994 & 1.71776 \\
\hline 2960 & 53.3033 & 389.4018 & 397.9541 & 384.7917 & 122.5994 & 1.71725 \\
\hline 2961 & 53.3203 & 386.8273 & 403.0202 & 385.3333 & 122.5994 & 1.71674 \\
\hline 2962 & 53.3373 & 379.8166 & 408.571 & 385.875 & 122.5994 & 1.71624 \\
\hline 2963 & 53.3543 & 393.6083 & 410.9607 & 386.4167 & 122.5994 & 1.71573 \\
\hline 2964 & 53.3713 & 406.1058 & 409.1822 & 386.9583 & 122.5994 & 1.71522 \\
\hline 2965 & 53.3883 & 371.5816 & 404.2994 & 387.5 & 122.5994 & 1.71472 \\
\hline 2966 & 53.4053 & 395.2784 & 399.538 & 388.0417 & 122.5994 & 1.71421 \\
\hline 2967 & 53.4223 & 375.0638 & 396.9185 & 388.5833 & 122.5994 & 1.71371 \\
\hline 2968 & 53.4393 & 418.8014 & 396.598 & 389.125 & 122.5994 & 1.7132 \\
\hline 2969 & 53.4563 & 400.6027 & 398.1479 & 389.6667 & 122.5994 & 1.7127 \\
\hline 2970 & 53.4733 & 388.9883 & 400.6162 & 390.2083 & 122.5994 & 1.71219 \\
\hline 2971 & 53.4903 & 391.7103 & 402.5546 & 390.75 & 122.5994 & 1.71169 \\
\hline 2972 & 53.5073 & 315.9059 & 403.2445 & 391.2917 & 122.5994 & 1.71118 \\
\hline 2973 & 53.5243 & 344.0645 & 403.2418 & 391.8333 & 122.5994 & 1.71068 \\
\hline 2974 & 53.5413 & 386.7052 & 403.5927 & 392.375 & 122.5994 & 1.71018 \\
\hline 2975 & 53.5583 & 380.2964 & 404.4299 & 392.9167 & 122.5994 & 1.70968 \\
\hline 2976 & 53.5753 & 435.9838 & 405.7274 & 393.4583 & 122.5994 & 1.70917 \\
\hline 2977 & 53.5923 & 365.7447 & 406.9696 & 394 & 122.5994 & 1.70867 \\
\hline 2978 & 53.6093 & 391.1563 & 407.3437 & 394.0417 & 122.5994 & 1.70817 \\
\hline 2979 & 53.6263 & 377.8052 & 407.3383 & 394.0833 & 122.5994 & 1.70767 \\
\hline 2980 & 53.6433 & 395.6776 & 407.1348 & 394.125 & 122.5994 & 1.70717 \\
\hline 2981 & 53.6603 & 392.0024 & 406.9499 & 394.1667 & 122.5994 & 1.70667 \\
\hline 2982 & 53.6773 & 387.2125 & 406.9128 & 394.2083 & 122.5994 & 1.70617 \\
\hline 2983 & 53.6943 & 360.8972 & 406.9844 & 394.25 & 122.5994 & 1.70567 \\
\hline 2984 & 53.7113 & 382.4603 & 407.2364 & 394.2917 & 122.5994 & 1.70517 \\
\hline 2985 & 53.7283 & 388.7944 & 407.5831 & 394.3333 & 122.5994 & 1.70467 \\
\hline 2986 & 53.7453 & 371.1437 & 408.0123 & 394.375 & 122.5994 & 1.70417 \\
\hline 2987 & 53.7623 & 366.0114 & 408.6071 & 394.4167 & 122.5994 & 1.70367 \\
\hline
\end{tabular}




\begin{tabular}{|c|c|c|c|c|c|c|}
\hline 2988 & 53.7793 & 398.63 & 409.5268 & 394.4583 & 122.5994 & 1.70317 \\
\hline 2989 & 53.7963 & 384.2112 & 411.2129 & 394.5 & 122.5994 & 1.70267 \\
\hline 2990 & 53.8133 & 384.1232 & 413.4084 & 394.5417 & 122.5994 & 1.70217 \\
\hline 2991 & 53.8303 & 380.2304 & 415.3906 & 394.5833 & 122.5994 & 1.70168 \\
\hline 2992 & 53.8473 & 372.5397 & 416.7356 & 394.625 & 122.5994 & 1.70118 \\
\hline 2993 & 53.8643 & 393.5463 & 418.0406 & 394.6667 & 122.5994 & 1.70068 \\
\hline 2994 & 53.8813 & 409.3335 & 419.4963 & 394.7083 & 122.5994 & 1.70019 \\
\hline 2995 & 53.8983 & 393.5772 & 420.7175 & 394.75 & 122.5994 & 1.69969 \\
\hline 2996 & 53.9153 & 406.8089 & 421.317 & 394.7917 & 122.5994 & 1.69919 \\
\hline 2997 & 53.9323 & 392.6312 & 421.1723 & 394.8333 & 122.5994 & 1.6987 \\
\hline 2998 & 53.9493 & 387.9387 & 420.6797 & 394.875 & 122.5994 & 1.6982 \\
\hline 2999 & 53.9663 & 416.8588 & 420.0486 & 394.9167 & 122.5994 & 1.69771 \\
\hline 3000 & 53.9833 & 406.8748 & 419.0837 & 394.9583 & 122.5994 & 1.69721 \\
\hline 3001 & 54.0003 & 382.849 & 418.0108 & 395 & 122.5994 & 1.69672 \\
\hline 3002 & 54.0173 & 388.0528 & 417.5951 & 395.5 & 122.5994 & 1.69623 \\
\hline 3003 & 54.0343 & 390.3902 & 417.4228 & 396 & 122.5994 & 1.69573 \\
\hline 3004 & 54.0513 & 424.7074 & 417.3755 & 396.5 & 122.5994 & 1.69524 \\
\hline 3005 & 54.0683 & 416.4665 & 417.2102 & 397 & 122.5994 & 1.69475 \\
\hline 3006 & 54.0853 & 379.466 & 416.5496 & 397.5 & 122.5994 & 1.69425 \\
\hline 3007 & 54.1023 & 373.8381 & 415.2764 & 398 & 122.5994 & 1.69376 \\
\hline 3008 & 54.1193 & 418.6981 & 413.945 & 398.5 & 122.5994 & 1.69327 \\
\hline 3009 & 54.1363 & 390.3951 & 412.8737 & 399 & 122.5994 & 1.69278 \\
\hline 3010 & 54.1533 & 355.5277 & 412.0847 & 399.5 & 122.5994 & 1.69229 \\
\hline 3011 & 54.1703 & 376.0732 & 411.5091 & 400 & 122.5994 & 1.6918 \\
\hline 3012 & 54.1873 & 371.9328 & 411.2195 & 400.5 & 122.5994 & 1.69131 \\
\hline 3013 & 54.2043 & 394.0002 & 411.2157 & 401 & 122.5994 & 1.69082 \\
\hline 3014 & 54.2213 & 377.1687 & 411.3253 & 401.5 & 122.5994 & 1.69033 \\
\hline 3015 & 54.2383 & 372.5397 & 411.251 & 402 & 122.5994 & 1.68984 \\
\hline 3016 & 54.2553 & 359.1362 & 411.5782 & 402.5 & 122.5994 & 1.68935 \\
\hline 3017 & 54.2723 & 384.7523 & 411.3645 & 403 & 122.5994 & 1.68886 \\
\hline 3018 & 54.2893 & 380.9707 & 411.2897 & 403.5 & 122.5994 & 1.68837 \\
\hline 3019 & 54.3063 & 421.5046 & 411.2816 & 404 & 122.5994 & 1.68788 \\
\hline 3020 & 54.3233 & 389.6792 & 411.4049 & 404.5 & 122.5994 & 1.68739 \\
\hline 3021 & 54.3403 & 411.5527 & 411.5716 & 405 & 122.5994 & 1.6869 \\
\hline 3022 & 54.3573 & 441.0152 & 411.7929 & 405.5 & 122.5994 & 1.68642 \\
\hline 3023 & 54.3743 & 381.099 & 412.0317 & 406 & 122.5994 & 1.68593 \\
\hline 3024 & 54.3913 & 420.8485 & 412.3348 & 406.5 & 122.5994 & 1.68544 \\
\hline 3025 & 54.4083 & 428.9286 & 413.0557 & 407 & 122.5994 & 1.68496 \\
\hline 3026 & 54.4253 & 378.8118 & 414.3732 & 408.2083 & 122.5994 & 1.68447 \\
\hline 3027 & 54.4423 & 400.5584 & 416.0294 & 409.4167 & 122.5994 & 1.68398 \\
\hline 3028 & 54.4593 & 373.9678 & 418.2269 & 410.625 & 122.5994 & 1.6835 \\
\hline 3029 & 54.4763 & 381.8623 & 421.3914 & 411.8333 & 122.5994 & 1.68301 \\
\hline 3030 & 54.4933 & 376.1114 & 425.7772 & 413.0417 & 122.5994 & 1.68253 \\
\hline 3031 & 54.5103 & 412.1449 & 430.6621 & 414.25 & 122.5994 & 1.68204 \\
\hline 3032 & 54.5273 & 413.8117 & 433.2248 & 415.4583 & 122.5994 & 1.68156 \\
\hline 3033 & 54.5443 & 407.9648 & 432.4557 & 416.6667 & 122.5994 & 1.68108 \\
\hline 3034 & 54.5613 & 374.7476 & 430.9951 & 417.875 & 122.5994 & 1.68059 \\
\hline 3035 & 54.5783 & 399.9104 & 430.2516 & 419.0833 & 122.5994 & 1.68011 \\
\hline 3036 & 54.5953 & 414.191 & 430.7789 & 420.2917 & 122.5994 & 1.67963 \\
\hline
\end{tabular}




\begin{tabular}{|c|c|c|c|c|c|c|}
\hline 3037 & 54.6123 & 403.8715 & 432.5252 & 421.5 & 122.5994 & 1.67914 \\
\hline 3038 & 54.6293 & 405.2267 & 435.3406 & 422.7083 & 122.5994 & 1.67866 \\
\hline 3039 & 54.6463 & 394.9195 & 438.9854 & 423.9167 & 122.5994 & 1.67818 \\
\hline 3040 & 54.6633 & 415.865 & 442.548 & 425.125 & 122.5994 & 1.6777 \\
\hline 3041 & 54.6803 & 419.3902 & 444.8883 & 426.3333 & 122.5994 & 1.67722 \\
\hline 3042 & 54.6973 & 382.9976 & 446.8589 & 427.5417 & 122.5994 & 1.67674 \\
\hline 3043 & 54.7143 & 398.1353 & 448.9521 & 428.75 & 122.5994 & 1.67625 \\
\hline 3044 & 54.7313 & 412.772 & 451.3287 & 429.9583 & 122.5994 & 1.67577 \\
\hline 3045 & 54.7483 & 392.1137 & 452.8695 & 431.1667 & 122.5994 & 1.67529 \\
\hline 3046 & 54.7653 & 415.78 & 454.1809 & 432.375 & 122.5994 & 1.67481 \\
\hline 3047 & 54.7823 & 436.1633 & 456.7384 & 433.5833 & 122.5994 & 1.67433 \\
\hline 3048 & 54.7993 & 457.1829 & 461.5316 & 434.7917 & 122.5994 & 1.67386 \\
\hline 3049 & 54.8163 & 479.564 & 470.207 & 436 & 122.5994 & 1.67338 \\
\hline 3050 & 54.8333 & 509.4678 & 485.9808 & 437.2083 & 122.5994 & 1.6729 \\
\hline 3051 & 54.8503 & 567.3594 & 513.9458 & 438.4167 & 122.5994 & 1.67242 \\
\hline 3052 & 54.8673 & 587.2999 & 558.7166 & 439.625 & 122.5994 & 1.67194 \\
\hline 3053 & 54.8843 & 618.7038 & 619.2756 & 440.8333 & 122.5994 & 1.67146 \\
\hline 3054 & 54.9013 & 641.9533 & 681.3947 & 442.0417 & 122.5994 & 1.67099 \\
\hline 3055 & 54.9183 & 682.0653 & 714.1158 & 443.25 & 122.5994 & 1.67051 \\
\hline 3056 & 54.9353 & 601.3241 & 693.1797 & 444.4583 & 122.5994 & 1.67003 \\
\hline 3057 & 54.9523 & 531.3853 & 639.376 & 445.6667 & 122.5994 & 1.66956 \\
\hline 3058 & 54.9693 & 498.8574 & 587.3748 & 446.875 & 122.5994 & 1.66908 \\
\hline 3059 & 54.9863 & 507.0697 & 554.4277 & 448.0833 & 122.5994 & 1.6686 \\
\hline 3060 & 55.0033 & 510.8281 & 543.6204 & 449.2917 & 122.5994 & 1.66813 \\
\hline 3061 & 55.0203 & 518.3362 & 551.7181 & 450.5 & 122.5994 & 1.66765 \\
\hline 3062 & 55.0373 & 560.0518 & 573.4427 & 451.7083 & 122.5994 & 1.66718 \\
\hline 3063 & 55.0543 & 579.0722 & 597.1041 & 452.9167 & 122.5994 & 1.6667 \\
\hline 3064 & 55.0713 & 542.2596 & 603.8783 & 454.125 & 122.5994 & 1.66623 \\
\hline 3065 & 55.0883 & 521.2749 & 586.1731 & 455.3333 & 122.5994 & 1.66576 \\
\hline 3066 & 55.1053 & 511.1883 & 558.2974 & 456.5417 & 122.5994 & 1.66528 \\
\hline 3067 & 55.1223 & 472.1946 & 534.7185 & 457.75 & 122.5994 & 1.66481 \\
\hline 3068 & 55.1393 & 441.2415 & 521.1534 & 458.9583 & 122.5994 & 1.66434 \\
\hline 3069 & 55.1563 & 424.4682 & 515.5215 & 460.1667 & 122.5994 & 1.66386 \\
\hline 3070 & 55.1733 & 436.2153 & 514.595 & 461.375 & 122.5994 & 1.66339 \\
\hline 3071 & 55.1903 & 420.306 & 515.3462 & 462.5833 & 122.5994 & 1.66292 \\
\hline 3072 & 55.2073 & 426.7845 & 516.9223 & 463.7917 & 122.5994 & 1.66245 \\
\hline 3073 & 55.2243 & 502.8486 & 519.1157 & 465 & 122.5994 & 1.66197 \\
\hline 3074 & 55.2413 & 472.9389 & 518.3796 & 463.3333 & 122.5994 & 1.6615 \\
\hline 3075 & 55.2583 & 482.8706 & 517.4733 & 461.6667 & 122.5994 & 1.66103 \\
\hline 3076 & 55.2753 & 448.1276 & 517.5616 & 460 & 122.5994 & 1.66056 \\
\hline 3077 & 55.2923 & 452.9645 & 521.438 & 458.3333 & 122.5994 & 1.66009 \\
\hline 3078 & 55.3093 & 512.8966 & 531.6357 & 456.6667 & 122.5994 & 1.65962 \\
\hline 3079 & 55.3263 & 541.3698 & 549.9496 & 455 & 122.5994 & 1.65915 \\
\hline 3080 & 55.3433 & 547.6873 & 574.6565 & 453.3333 & 122.5994 & 1.65868 \\
\hline 3081 & 55.3603 & 541.1447 & 597.9798 & 451.6667 & 122.5994 & 1.65821 \\
\hline 3082 & 55.3773 & 546.7864 & 604.1425 & 450 & 122.5994 & 1.65774 \\
\hline 3083 & 55.3943 & 492.9525 & 585.2238 & 448.3333 & 122.5994 & 1.65728 \\
\hline 3084 & 55.4113 & 446.6023 & 554.3875 & 446.6667 & 122.5994 & 1.65681 \\
\hline 3085 & 55.4283 & 434.1834 & 526.168 & 445 & 122.5994 & 1.65634 \\
\hline
\end{tabular}




\begin{tabular}{|c|c|c|c|c|c|c|}
\hline 3086 & 55.4453 & 448.005 & 507.2254 & 443.3333 & 122.5994 & 1.65587 \\
\hline 3087 & 55.4623 & 473.4761 & 498.9926 & 441.6667 & 122.5994 & 1.65541 \\
\hline 3088 & 55.4793 & 478.5481 & 500.1212 & 440 & 122.5994 & 1.65494 \\
\hline 3089 & 55.4963 & 474.7008 & 507.2509 & 438.3333 & 122.5994 & 1.65447 \\
\hline 3090 & 55.5133 & 467.6509 & 514.0457 & 436.6667 & 122.5994 & 1.65401 \\
\hline 3091 & 55.5303 & 451.5841 & 511.81 & 435 & 122.5994 & 1.65354 \\
\hline 3092 & 55.5473 & 440.9836 & 498.6299 & 433.3333 & 122.5994 & 1.65307 \\
\hline 3093 & 55.5643 & 423.8914 & 481.8357 & 431.6667 & 122.5994 & 1.65261 \\
\hline 3094 & 55.5813 & 429.7762 & 467.1533 & 430 & 122.5994 & 1.65214 \\
\hline 3095 & 55.5983 & 438.6646 & 455.8323 & 428.3333 & 122.5994 & 1.65168 \\
\hline 3096 & 55.6153 & 424.5261 & 448.9158 & 426.6667 & 122.5994 & 1.65121 \\
\hline 3097 & 55.6323 & 421.2542 & 444.8104 & 425 & 122.5994 & 1.65075 \\
\hline 3098 & 55.6493 & 429.5846 & 443.9667 & 424.7083 & 122.5994 & 1.65028 \\
\hline 3099 & 55.6663 & 419.185 & 444.6021 & 424.4167 & 122.5994 & 1.64982 \\
\hline 3100 & 55.6833 & 395.7551 & 446.4017 & 424.125 & 122.5994 & 1.64936 \\
\hline 3101 & 55.7003 & 425.1105 & 449.3319 & 423.8333 & 122.5994 & 1.64889 \\
\hline 3102 & 55.7173 & 435.0225 & 453.2525 & 423.5417 & 122.5994 & 1.64843 \\
\hline 3103 & 55.7343 & 451.423 & 458.0888 & 423.25 & 122.5994 & 1.64797 \\
\hline 3104 & 55.7513 & 464.601 & 463.6653 & 422.9583 & 122.5994 & 1.64751 \\
\hline 3105 & 55.7683 & 404.7961 & 470.3695 & 422.6667 & 122.5994 & 1.64704 \\
\hline 3106 & 55.7853 & 434.203 & 477.2695 & 422.375 & 122.5994 & 1.64658 \\
\hline 3107 & 55.8023 & 441.7767 & 483.3144 & 422.0833 & 122.5994 & 1.64612 \\
\hline 3108 & 55.8193 & 412.908 & 487.2834 & 421.7917 & 122.5994 & 1.64566 \\
\hline 3109 & 55.8363 & 433.2012 & 488.2164 & 421.5 & 122.5994 & 1.6452 \\
\hline 3110 & 55.8533 & 414.6661 & 486.2249 & 421.2083 & 122.5994 & 1.64474 \\
\hline 3111 & 55.8703 & 454.15 & 482.3167 & 420.9167 & 122.5994 & 1.64428 \\
\hline 3112 & 55.8873 & 430.2979 & 477.8369 & 420.625 & 122.5994 & 1.64382 \\
\hline 3113 & 55.9043 & 401.1389 & 473.8677 & 420.3333 & 122.5994 & 1.64336 \\
\hline 3114 & 55.9213 & 403.9841 & 470.8664 & 420.0417 & 122.5994 & 1.6429 \\
\hline 3115 & 55.9383 & 441.0513 & 468.5388 & 419.75 & 122.5994 & 1.64244 \\
\hline 3116 & 55.9553 & 436.8653 & 466.9709 & 419.4583 & 122.5994 & 1.64198 \\
\hline 3117 & 55.9723 & 440.0317 & 464.9522 & 419.1667 & 122.5994 & 1.64152 \\
\hline 3118 & 55.9893 & 420.035 & 462.779 & 418.875 & 122.5994 & 1.64107 \\
\hline 3119 & 56.0063 & 418.2985 & 459.8799 & 418.5833 & 122.5994 & 1.64061 \\
\hline 3120 & 56.0233 & 440.1447 & 456.1437 & 418.2917 & 122.5994 & 1.64015 \\
\hline 3121 & 56.0403 & 426.2589 & 451.7371 & 418 & 122.5994 & 1.63969 \\
\hline 3122 & 56.0573 & 387.8509 & 446.7149 & 417.1667 & 122.5994 & 1.63924 \\
\hline 3123 & 56.0743 & 413.0021 & 442.1183 & 416.3333 & 122.5994 & 1.63878 \\
\hline 3124 & 56.0913 & 430.3637 & 437.9711 & 415.5 & 122.5994 & 1.63832 \\
\hline 3125 & 56.1083 & 404.0639 & 434.2159 & 414.6667 & 122.5994 & 1.63787 \\
\hline 3126 & 56.1253 & 405.8969 & 431.3385 & 413.8333 & 122.5994 & 1.63741 \\
\hline 3127 & 56.1423 & 415.3348 & 429.1639 & 413 & 122.5994 & 1.63696 \\
\hline 3128 & 56.1593 & 423.1628 & 427.567 & 412.1667 & 122.5994 & 1.6365 \\
\hline 3129 & 56.1763 & 429.0489 & 426.3504 & 411.3333 & 122.5994 & 1.63605 \\
\hline 3130 & 56.1933 & 448.1274 & 425.4499 & 410.5 & 122.5994 & 1.63559 \\
\hline 3131 & 56.2103 & 457.0658 & 425.0423 & 409.6667 & 122.5994 & 1.63514 \\
\hline 3132 & 56.2273 & 479.6739 & 425.2746 & 408.8333 & 122.5994 & 1.63468 \\
\hline 3133 & 56.2443 & 491.68 & 426.047 & 408 & 122.5994 & 1.63423 \\
\hline 3134 & 56.2613 & 507.1326 & 427.0951 & 407.1667 & 122.5994 & 1.63378 \\
\hline
\end{tabular}




\begin{tabular}{|c|c|c|c|c|c|c|}
\hline 3135 & 56.2783 & 503.6517 & 428.1169 & 406.3333 & 122.5994 & 1.63332 \\
\hline 3136 & 56.2953 & 500.1207 & 428.6175 & 405.5 & 122.5994 & 1.63287 \\
\hline 3137 & 56.3123 & 505.7333 & 428.1227 & 404.6667 & 122.5994 & 1.63242 \\
\hline 3138 & 56.3293 & 497.6455 & 426.7665 & 403.8333 & 122.5994 & 1.63196 \\
\hline 3139 & 56.3463 & 493.7086 & 424.9955 & 403 & 122.5994 & 1.63151 \\
\hline 3140 & 56.3633 & 475.3411 & 423.2308 & 402.1667 & 122.5994 & 1.63106 \\
\hline 3141 & 56.3803 & 452.3201 & 421.7289 & 401.3333 & 122.5994 & 1.63061 \\
\hline 3142 & 56.3973 & 440.8502 & 420.5043 & 400.5 & 122.5994 & 1.63016 \\
\hline 3143 & 56.4143 & 451.4461 & 419.4348 & 399.6667 & 122.5994 & 1.62971 \\
\hline 3144 & 56.4313 & 455.9098 & 418.2846 & 398.8333 & 122.5994 & 1.62926 \\
\hline 3145 & 56.4483 & 435.8392 & 416.8186 & 398 & 122.5994 & 1.62881 \\
\hline 3146 & 56.4653 & 439.7294 & 414.83 & 397.1667 & 122.5994 & 1.62836 \\
\hline 3147 & 56.4823 & 416.7304 & 412.4424 & 396.3333 & 122.5994 & 1.62791 \\
\hline 3148 & 56.4993 & 406.8391 & 409.8985 & 395.5 & 122.5994 & 1.62746 \\
\hline 3149 & 56.5163 & 399.273 & 407.4161 & 394.6667 & 122.5994 & 1.62701 \\
\hline 3150 & 56.5333 & 408.1077 & 404.7086 & 393.8333 & 122.5994 & 1.62656 \\
\hline 3151 & 56.5503 & 384.4057 & 402.6344 & 393 & 122.5994 & 1.62611 \\
\hline 3152 & 56.5673 & 424.5495 & 400.7354 & 392.1667 & 122.5994 & 1.62566 \\
\hline 3153 & 56.5843 & 393.7254 & 398.9713 & 391.3333 & 122.5994 & 1.62521 \\
\hline 3154 & 56.6013 & 390.749 & 397.4309 & 390.5 & 122.5994 & 1.62477 \\
\hline 3155 & 56.6183 & 388.5964 & 395.9113 & 389.6667 & 122.5994 & 1.62432 \\
\hline 3156 & 56.6353 & 402.2137 & 394.5294 & 388.8333 & 122.5994 & 1.62387 \\
\hline 3157 & 56.6523 & 386.0003 & 393.2907 & 388 & 122.5994 & 1.62342 \\
\hline 3158 & 56.6693 & 380.5867 & 392.2408 & 387.1667 & 122.5994 & 1.62298 \\
\hline 3159 & 56.6863 & 395.1604 & 391.3108 & 386.3333 & 122.5994 & 1.62253 \\
\hline 3160 & 56.7033 & 388.291 & 390.6447 & 385.5 & 122.5994 & 1.62209 \\
\hline 3161 & 56.7203 & 407.9217 & 390.1363 & 384.6667 & 122.5994 & 1.62164 \\
\hline 3162 & 56.7373 & 372.3405 & 389.7164 & 383.8333 & 122.5994 & 1.62119 \\
\hline 3163 & 56.7543 & 357.9695 & 389.2573 & 383 & 122.5994 & 1.62075 \\
\hline 3164 & 56.7713 & 379.8653 & 388.703 & 382.1667 & 122.5994 & 1.6203 \\
\hline 3165 & 56.7883 & 369.824 & 388.0099 & 381.3333 & 122.5994 & 1.61986 \\
\hline 3166 & 56.8053 & 342.2675 & 387.0269 & 380.5 & 122.5994 & 1.61941 \\
\hline 3167 & 56.8223 & 377.8247 & 385.9659 & 379.6667 & 122.5994 & 1.61897 \\
\hline 3168 & 56.8393 & 387.4947 & 384.9564 & 378.8333 & 122.5994 & 1.61853 \\
\hline 3169 & 56.8563 & 385.5009 & 384.1126 & 378 & 122.5994 & 1.61808 \\
\hline 3170 & 56.8733 & 380.7495 & 384.7266 & 378.375 & 122.5994 & 1.61764 \\
\hline 3171 & 56.8903 & 339.7264 & 385.5594 & 378.75 & 122.5994 & 1.6172 \\
\hline 3172 & 56.9073 & 377.9058 & 386.5666 & 379.125 & 122.5994 & 1.61675 \\
\hline 3173 & 56.9243 & 393.0146 & 387.6863 & 379.5 & 122.5994 & 1.61631 \\
\hline 3174 & 56.9413 & 380.0019 & 388.708 & 379.875 & 122.5994 & 1.61587 \\
\hline 3175 & 56.9583 & 365.2598 & 389.6838 & 380.25 & 122.5994 & 1.61543 \\
\hline 3176 & 56.9753 & 363.2763 & 390.4598 & 380.625 & 122.5994 & 1.61499 \\
\hline 3177 & 56.9923 & 373.7322 & 390.9624 & 381 & 122.5994 & 1.61454 \\
\hline 3178 & 57.0093 & 372.7509 & 391.2295 & 381.375 & 122.5994 & 1.6141 \\
\hline 3179 & 57.0263 & 374.4523 & 391.4642 & 381.75 & 122.5994 & 1.61366 \\
\hline 3180 & 57.0433 & 370.9541 & 391.8866 & 382.125 & 122.5994 & 1.61322 \\
\hline 3181 & 57.0603 & 368.9909 & 392.7128 & 382.5 & 122.5994 & 1.61278 \\
\hline 3182 & 57.0773 & 343.5655 & 394.0967 & 382.875 & 122.5994 & 1.61234 \\
\hline 3183 & 57.0943 & 353.9828 & 396.0514 & 383.25 & 122.5994 & 1.6119 \\
\hline
\end{tabular}




\begin{tabular}{|c|c|c|c|c|c|c|}
\hline 3184 & 57.1113 & 356.4651 & 398.3755 & 383.625 & 122.5994 & 1.61146 \\
\hline 3185 & 57.1283 & 342.4805 & 401.022 & 384 & 122.5994 & 1.61102 \\
\hline 3186 & 57.1453 & 351.5018 & 403.415 & 384.375 & 122.5994 & 1.61058 \\
\hline 3187 & 57.1623 & 357.985 & 405.1556 & 384.75 & 122.5994 & 1.61015 \\
\hline 3188 & 57.1793 & 369.2553 & 406.1437 & 385.125 & 122.5994 & 1.60971 \\
\hline 3189 & 57.1963 & 370.1678 & 407.0713 & 385.5 & 122.5994 & 1.60927 \\
\hline 3190 & 57.2133 & 373.3549 & 409.1721 & 385.875 & 122.5994 & 1.60883 \\
\hline 3191 & 57.2303 & 371.4928 & 413.5133 & 386.25 & 122.5994 & 1.60839 \\
\hline 3192 & 57.2473 & 391.892 & 420.1192 & 386.625 & 122.5994 & 1.60796 \\
\hline 3193 & 57.2643 & 393.2376 & 427.0148 & 387 & 122.5994 & 1.60752 \\
\hline 3194 & 57.2813 & 379.8246 & 429.4081 & 386.8333 & 122.5994 & 1.60708 \\
\hline 3195 & 57.2983 & 382.0504 & 425.3798 & 386.6667 & 122.5994 & 1.60665 \\
\hline 3196 & 57.3153 & 384.1232 & 418.1284 & 386.5 & 122.5994 & 1.60621 \\
\hline 3197 & 57.3323 & 369.302 & 411.2109 & 386.3333 & 122.5994 & 1.60578 \\
\hline 3198 & 57.3493 & 367.8751 & 406.3657 & 386.1667 & 122.5994 & 1.60534 \\
\hline 3199 & 57.3663 & 359.3575 & 404.0984 & 386 & 122.5994 & 1.6049 \\
\hline 3200 & 57.3833 & 360.4059 & 404.3481 & 385.8333 & 122.5994 & 1.60447 \\
\hline 3201 & 57.4003 & 348.1499 & 406.5197 & 385.6667 & 122.5994 & 1.60403 \\
\hline 3202 & 57.4173 & 375.8795 & 409.4114 & 385.5 & 122.5994 & 1.6036 \\
\hline 3203 & 57.4343 & 369.9079 & 410.7556 & 385.3333 & 122.5994 & 1.60317 \\
\hline 3204 & 57.4513 & 368.6046 & 408.8641 & 385.1667 & 122.5994 & 1.60273 \\
\hline 3205 & 57.4683 & 372.3013 & 404.9895 & 385 & 122.5994 & 1.6023 \\
\hline 3206 & 57.4853 & 395.0253 & 401.1842 & 384.8333 & 122.5994 & 1.60186 \\
\hline 3207 & 57.5023 & 369.2046 & 398.3506 & 384.6667 & 122.5994 & 1.60143 \\
\hline 3208 & 57.5193 & 357.1462 & 396.6228 & 384.5 & 122.5994 & 1.601 \\
\hline 3209 & 57.5363 & 346.6501 & 395.7751 & 384.3333 & 122.5994 & 1.60057 \\
\hline 3210 & 57.5533 & 372.6311 & 395.5324 & 384.1667 & 122.5994 & 1.60013 \\
\hline 3211 & 57.5703 & 376.1495 & 395.5887 & 384 & 122.5994 & 1.5997 \\
\hline 3212 & 57.5873 & 372.4543 & 395.8301 & 383.8333 & 122.5994 & 1.59927 \\
\hline 3213 & 57.6043 & 373.3093 & 396.1567 & 383.6667 & 122.5994 & 1.59884 \\
\hline 3214 & 57.6213 & 377.7629 & 396.5099 & 383.5 & 122.5994 & 1.59841 \\
\hline 3215 & 57.6383 & 362.7263 & 396.8533 & 383.3333 & 122.5994 & 1.59798 \\
\hline 3216 & 57.6553 & 373.2165 & 397.2507 & 383.1667 & 122.5994 & 1.59755 \\
\hline 3217 & 57.6723 & 378.6268 & 397.9118 & 383 & 122.5994 & 1.59711 \\
\hline 3218 & 57.6893 & 394.2603 & 398.0158 & 381.875 & 122.5994 & 1.59668 \\
\hline 3219 & 57.7063 & 407.5234 & 398.2465 & 380.75 & 122.5994 & 1.59625 \\
\hline 3220 & 57.7233 & 371.2701 & 397.1615 & 379.625 & 122.5994 & 1.59582 \\
\hline 3221 & 57.7403 & 351.3306 & 394.1732 & 378.5 & 122.5994 & 1.5954 \\
\hline 3222 & 57.7573 & 374.2562 & 391.0642 & 377.375 & 122.5994 & 1.59497 \\
\hline 3223 & 57.7743 & 387.1529 & 389.0333 & 376.25 & 122.5994 & 1.59454 \\
\hline 3224 & 57.7913 & 375.3529 & 387.9002 & 375.125 & 122.5994 & 1.59411 \\
\hline 3225 & 57.8083 & 382.3061 & 386.9093 & 374 & 122.5994 & 1.59368 \\
\hline 3226 & 57.8253 & 366.5801 & 385.8937 & 372.875 & 122.5994 & 1.59325 \\
\hline 3227 & 57.8423 & 365.0549 & 385.064 & 371.75 & 122.5994 & 1.59282 \\
\hline 3228 & 57.8593 & 376.4102 & 384.7263 & 370.625 & 122.5994 & 1.5924 \\
\hline 3229 & 57.8763 & 364.202 & 383.8081 & 369.5 & 122.5994 & 1.59197 \\
\hline 3230 & 57.8933 & 366.15 & 381.5039 & 368.375 & 122.5994 & 1.59154 \\
\hline 3231 & 57.9103 & 376.4006 & 378.4677 & 367.25 & 122.5994 & 1.59112 \\
\hline 3232 & 57.9273 & 354.9081 & 375.7291 & 366.125 & 122.5994 & 1.59069 \\
\hline
\end{tabular}




\begin{tabular}{|c|c|c|c|c|c|c|}
\hline 3233 & 57.9443 & 348.3456 & 373.5426 & 365 & 122.5994 & 1.59026 \\
\hline 3234 & 57.9613 & 379.5764 & 371.635 & 363.875 & 122.5994 & 1.58984 \\
\hline 3235 & 57.9783 & 381.7477 & 369.8648 & 362.75 & 122.5994 & 1.58941 \\
\hline 3236 & 57.9953 & 361.6878 & 368.365 & 361.625 & 122.5994 & 1.58899 \\
\hline 3237 & 58.0123 & 359.6332 & 367.1492 & 360.5 & 122.5994 & 1.58856 \\
\hline 3238 & 58.0293 & 358.5086 & 366.0143 & 359.375 & 122.5994 & 1.58814 \\
\hline 3239 & 58.0463 & 354.6512 & 364.7735 & 358.25 & 122.5994 & 1.58771 \\
\hline 3240 & 58.0633 & 361.347 & 363.2841 & 357.125 & 122.5994 & 1.58729 \\
\hline 3241 & 58.0803 & 348.6964 & 361.8001 & 356 & 122.5994 & 1.58686 \\
\hline 3242 & 58.0973 & 356.0648 & 360.4959 & 354.9583 & 122.5994 & 1.58644 \\
\hline 3243 & 58.1143 & 369.6492 & 359.3931 & 353.9167 & 122.5994 & 1.58602 \\
\hline 3244 & 58.1313 & 343.2317 & 358.5819 & 352.875 & 122.5994 & 1.58559 \\
\hline 3245 & 58.1483 & 355.7925 & 358.2285 & 351.8333 & 122.5994 & 1.58517 \\
\hline 3246 & 58.1653 & 376.7555 & 358.6853 & 350.7917 & 122.5994 & 1.58475 \\
\hline 3247 & 58.1823 & 377.0256 & 360.6184 & 349.75 & 122.5994 & 1.58432 \\
\hline 3248 & 58.1993 & 346.8755 & 364.3697 & 348.7083 & 122.5994 & 1.5839 \\
\hline 3249 & 58.2163 & 379.3854 & 368.4521 & 347.6667 & 122.5994 & 1.58348 \\
\hline 3250 & 58.2333 & 413.1282 & 369.0754 & 346.625 & 122.5994 & 1.58306 \\
\hline 3251 & 58.2503 & 368.4762 & 364.3473 & 345.5833 & 122.5994 & 1.58264 \\
\hline 3252 & 58.2673 & 329.5381 & 357.7992 & 344.5417 & 122.5994 & 1.58221 \\
\hline 3253 & 58.2843 & 359.9968 & 352.6649 & 343.5 & 122.5994 & 1.58179 \\
\hline 3254 & 58.3013 & 361.0481 & 349.5558 & 342.4583 & 122.5994 & 1.58137 \\
\hline 3255 & 58.3183 & 356.8155 & 348.0445 & 341.4167 & 122.5994 & 1.58095 \\
\hline 3256 & 58.3353 & 331.807 & 347.9207 & 340.375 & 122.5994 & 1.58053 \\
\hline 3257 & 58.3523 & 347.6578 & 349.1133 & 339.3333 & 122.5994 & 1.58011 \\
\hline 3258 & 58.3693 & 373.7295 & 351.5013 & 338.2917 & 122.5994 & 1.57969 \\
\hline 3259 & 58.3863 & 344.0924 & 353.4576 & 337.25 & 122.5994 & 1.57927 \\
\hline 3260 & 58.4033 & 360.587 & 352.7651 & 336.2083 & 122.5994 & 1.57885 \\
\hline 3261 & 58.4203 & 354.5073 & 349.1736 & 335.1667 & 122.5994 & 1.57843 \\
\hline 3262 & 58.4373 & 337.9238 & 344.6783 & 334.125 & 122.5994 & 1.57802 \\
\hline 3263 & 58.4543 & 341.2172 & 341.3128 & 333.0833 & 122.5994 & 1.5776 \\
\hline 3264 & 58.4713 & 360.088 & 339.4315 & 332.0417 & 122.5994 & 1.57718 \\
\hline 3265 & 58.4883 & 322.7575 & 338.4811 & 331 & 122.5994 & 1.57676 \\
\hline 3266 & 58.5053 & 314.3188 & 340.4772 & 332.625 & 122.5994 & 1.57634 \\
\hline 3267 & 58.5223 & 334.5663 & 342.2481 & 334.25 & 122.5994 & 1.57593 \\
\hline 3268 & 58.5393 & 344.0272 & 343.8921 & 335.875 & 122.5994 & 1.57551 \\
\hline 3269 & 58.5563 & 376.312 & 345.7635 & 337.5 & 122.5994 & 1.57509 \\
\hline 3270 & 58.5733 & 349.8934 & 347.4997 & 339.125 & 122.5994 & 1.57468 \\
\hline 3271 & 58.5903 & 367.526 & 348.7679 & 340.75 & 122.5994 & 1.57426 \\
\hline 3272 & 58.6073 & 374.0097 & 350.1695 & 342.375 & 122.5994 & 1.57384 \\
\hline 3273 & 58.6243 & 379.4538 & 352.3203 & 344 & 122.5994 & 1.57343 \\
\hline 3274 & 58.6413 & 382.4704 & 355.1407 & 345.625 & 122.5994 & 1.57301 \\
\hline 3275 & 58.6583 & 375.1419 & 358.1841 & 347.25 & 122.5994 & 1.5726 \\
\hline 3276 & 58.6753 & 377.6637 & 361.4987 & 348.875 & 122.5994 & 1.57218 \\
\hline 3277 & 58.6923 & 374.9378 & 364.4544 & 350.5 & 122.5994 & 1.57177 \\
\hline 3278 & 58.7093 & 366.8118 & 366.4305 & 352.125 & 122.5994 & 1.57135 \\
\hline 3279 & 58.7263 & 389.2604 & 368.7822 & 353.75 & 122.5994 & 1.57094 \\
\hline 3280 & 58.7433 & 406.1954 & 372.8214 & 355.375 & 122.5994 & 1.57052 \\
\hline 3281 & 58.7603 & 394.31 & 377.5543 & 357 & 122.5994 & 1.57011 \\
\hline
\end{tabular}




\begin{tabular}{|c|c|c|c|c|c|c|}
\hline 3282 & 58.7773 & 388.8996 & 379.9203 & 358.625 & 122.5994 & 1.5697 \\
\hline 3283 & 58.7943 & 370.5335 & 379.8659 & 360.25 & 122.5994 & 1.56928 \\
\hline 3284 & 58.8113 & 382.0136 & 379.945 & 361.875 & 122.5994 & 1.56887 \\
\hline 3285 & 58.8283 & 350.8649 & 381.218 & 363.5 & 122.5994 & 1.56846 \\
\hline 3286 & 58.8453 & 366.0824 & 383.3581 & 365.125 & 122.5994 & 1.56804 \\
\hline 3287 & 58.8623 & 349.5975 & 385.314 & 366.75 & 122.5994 & 1.56763 \\
\hline 3288 & 58.8793 & 356.5185 & 386.9756 & 368.375 & 122.5994 & 1.56722 \\
\hline 3289 & 58.8963 & 379.286 & 389.051 & 370 & 122.5994 & 1.56681 \\
\hline 3290 & 58.9133 & 369.2813 & 389.9283 & 370.125 & 122.5994 & 1.5664 \\
\hline 3291 & 58.9303 & 395.7745 & 389.7624 & 370.25 & 122.5994 & 1.56599 \\
\hline 3292 & 58.9473 & 394.2745 & 387.7579 & 370.375 & 122.5994 & 1.56557 \\
\hline 3293 & 58.9643 & 408.6742 & 385.3004 & 370.5 & 122.5994 & 1.56516 \\
\hline 3294 & 58.9813 & 365.3235 & 383.5206 & 370.625 & 122.5994 & 1.56475 \\
\hline 3295 & 58.9983 & 373.924 & 382.6273 & 370.75 & 122.5994 & 1.56434 \\
\hline 3296 & 59.0153 & 404.8028 & 382.6742 & 370.875 & 122.5994 & 1.56393 \\
\hline 3297 & 59.0323 & 386.0874 & 383.7786 & 371 & 122.5994 & 1.56352 \\
\hline 3298 & 59.0493 & 387.8137 & 386.0529 & 371.125 & 122.5994 & 1.56311 \\
\hline 3299 & 59.0663 & 395.7506 & 388.8146 & 371.25 & 122.5994 & 1.5627 \\
\hline 3300 & 59.0833 & 392.0729 & 390.0124 & 371.375 & 122.5994 & 1.56229 \\
\hline 3301 & 59.1003 & 368.6332 & 388.6003 & 371.5 & 122.5994 & 1.56189 \\
\hline 3302 & 59.1173 & 376.4102 & 386.6859 & 371.625 & 122.5994 & 1.56148 \\
\hline 3303 & 59.1343 & 378.5686 & 385.4439 & 371.75 & 122.5994 & 1.56107 \\
\hline 3304 & 59.1513 & 420.2589 & 384.8889 & 371.875 & 122.5994 & 1.56066 \\
\hline 3305 & 59.1683 & 365.8962 & 384.9978 & 372 & 122.5994 & 1.56025 \\
\hline 3306 & 59.1853 & 378.0684 & 385.6758 & 372.125 & 122.5994 & 1.55984 \\
\hline 3307 & 59.2023 & 393.6831 & 386.7803 & 372.25 & 122.5994 & 1.55944 \\
\hline 3308 & 59.2193 & 402.124 & 388.1951 & 372.375 & 122.5994 & 1.55903 \\
\hline 3309 & 59.2363 & 394.1061 & 389.3301 & 372.5 & 122.5994 & 1.55862 \\
\hline 3310 & 59.2533 & 396.326 & 389.2356 & 372.625 & 122.5994 & 1.55822 \\
\hline 3311 & 59.2703 & 364.775 & 388.1244 & 372.75 & 122.5994 & 1.55781 \\
\hline 3312 & 59.2873 & 369.5116 & 386.897 & 372.875 & 122.5994 & 1.5574 \\
\hline 3313 & 59.3043 & 380.0587 & 386.1666 & 373 & 122.5994 & 1.557 \\
\hline 3314 & 59.3213 & 369.7807 & 386.7046 & 373.7917 & 122.5994 & 1.55659 \\
\hline 3315 & 59.3383 & 377.2116 & 387.8092 & 374.5833 & 122.5994 & 1.55619 \\
\hline 3316 & 59.3553 & 382.9613 & 389.217 & 375.375 & 122.5994 & 1.55578 \\
\hline 3317 & 59.3723 & 373.8865 & 390.6665 & 376.1667 & 122.5994 & 1.55538 \\
\hline 3318 & 59.3893 & 372.0568 & 392.0373 & 376.9583 & 122.5994 & 1.55497 \\
\hline 3319 & 59.4063 & 366.3565 & 393.203 & 377.75 & 122.5994 & 1.55457 \\
\hline 3320 & 59.4233 & 375.0069 & 396.0788 & 378.5417 & 122.5994 & 1.55416 \\
\hline 3321 & 59.4403 & 379.1445 & 396.2095 & 379.3333 & 122.5994 & 1.55376 \\
\hline 3322 & 59.4573 & 375.9896 & 395.8126 & 380.125 & 122.5994 & 1.55336 \\
\hline 3323 & 59.4743 & 387.7311 & 395.3296 & 380.9167 & 122.5994 & 1.55295 \\
\hline 3324 & 59.4913 & 398.0524 & 395.1137 & 381.7083 & 122.5994 & 1.55255 \\
\hline 3325 & 59.5083 & 385.6544 & 395.3077 & 382.5 & 122.5994 & 1.55215 \\
\hline 3326 & 59.5253 & 383.1304 & 395.919 & 383.2917 & 122.5994 & 1.55174 \\
\hline 3327 & 59.5423 & 374.5224 & 396.8996 & 384.0833 & 122.5994 & 1.55134 \\
\hline 3328 & 59.5593 & 395.6009 & 398.1723 & 384.875 & 122.5994 & 1.55094 \\
\hline 3329 & 59.5763 & 384.3878 & 399.6263 & 385.6667 & 122.5994 & 1.55054 \\
\hline 3330 & 59.5933 & 365.7605 & 402.2065 & 386.4583 & 122.5994 & 1.55014 \\
\hline
\end{tabular}




\begin{tabular}{|c|c|c|c|c|c|c|}
\hline 3331 & 59.6103 & 394.271 & 403.7666 & 387.25 & 122.5994 & 1.54973 \\
\hline 3332 & 59.6273 & 397.2696 & 405.3783 & 388.0417 & 122.5994 & 1.54933 \\
\hline 3333 & 59.6443 & 400.3797 & 407.1428 & 388.8333 & 122.5994 & 1.54893 \\
\hline 3334 & 59.6613 & 418.4966 & 409.0428 & 389.625 & 122.5994 & 1.54853 \\
\hline 3335 & 59.6783 & 394.7644 & 410.9908 & 390.4167 & 122.5994 & 1.54813 \\
\hline 3336 & 59.6953 & 396.1996 & 412.6938 & 391.2083 & 122.5994 & 1.54773 \\
\hline 3337 & 59.7123 & 375.5552 & 413.9593 & 392 & 122.5994 & 1.54733 \\
\hline 3338 & 59.7293 & 406.7011 & 413.8694 & 392.125 & 122.5994 & 1.54693 \\
\hline 3339 & 59.7463 & 389.8373 & 413.6909 & 392.25 & 122.5994 & 1.54653 \\
\hline 3340 & 59.7633 & 421.4825 & 414.204 & 392.375 & 122.5994 & 1.54613 \\
\hline 3341 & 59.7803 & 400.4712 & 415.8019 & 392.5 & 122.5994 & 1.54573 \\
\hline 3342 & 59.7973 & 417.4444 & 418.4677 & 392.625 & 122.5994 & 1.54533 \\
\hline 3343 & 59.8143 & 450.652 & 422.1116 & 392.75 & 122.5994 & 1.54494 \\
\hline 3344 & 59.8313 & 469.6235 & 426.7217 & 392.875 & 122.5994 & 1.54454 \\
\hline 3345 & 59.8483 & 470.9528 & 432.4378 & 393 & 122.5994 & 1.54414 \\
\hline 3346 & 59.8653 & 487.8737 & 439.8246 & 393.125 & 122.5994 & 1.54374 \\
\hline 3347 & 59.8823 & 479.6214 & 450.3816 & 393.25 & 122.5994 & 1.54334 \\
\hline 3348 & 59.8993 & 552.6237 & 467.76 & 393.375 & 122.5994 & 1.54295 \\
\hline 3349 & 59.9163 & 584.9444 & 500.4446 & 393.5 & 122.5994 & 1.54255 \\
\hline 3350 & 59.9333 & 696.6542 & 561.4286 & 393.625 & 122.5994 & 1.54215 \\
\hline 3351 & 59.9503 & 787.5174 & 665.4285 & 393.75 & 122.5994 & 1.54176 \\
\hline 3352 & 59.9673 & 902.1579 & 817.6516 & 393.875 & 122.5994 & 1.54136 \\
\hline 3353 & 59.9843 & 981.1131 & 996.6157 & 394 & 122.5994 & 1.54096 \\
\hline 3354 & 60.0013 & 1023.378 & 1131.677 & 394.125 & 122.5994 & 1.54057 \\
\hline 3355 & 60.0183 & 919.8937 & 1126.426 & 394.25 & 122.5994 & 1.54017 \\
\hline 3356 & 60.0353 & 778.36 & 988.2143 & 394.375 & 122.5994 & 1.53978 \\
\hline 3357 & 60.0523 & 642.7788 & 816.7711 & 394.5 & 122.5994 & 1.53938 \\
\hline 3358 & 60.0693 & 599.8305 & 680.7869 & 394.625 & 122.5994 & 1.53899 \\
\hline 3359 & 60.0863 & 617.629 & 603.116 & 394.75 & 122.5994 & 1.53859 \\
\hline 3360 & 60.1033 & 619.5212 & 583.2892 & 394.875 & 122.5994 & 1.5382 \\
\hline 3361 & 60.1203 & 624.5929 & 612.6188 & 395 & 122.5994 & 1.5378 \\
\hline 3362 & 60.1373 & 684.0513 & 677.8379 & 395.125 & 122.5994 & 1.53741 \\
\hline 3363 & 60.1543 & 727.6342 & 754.2805 & 395.25 & 122.5994 & 1.53701 \\
\hline 3364 & 60.1713 & 714.4447 & 795.2507 & 395.375 & 122.5994 & 1.53662 \\
\hline 3365 & 60.1883 & 615.6302 & 760.9221 & 395.5 & 122.5994 & 1.53623 \\
\hline 3366 & 60.2053 & 549.8281 & 675.7192 & 395.625 & 122.5994 & 1.53583 \\
\hline 3367 & 60.2223 & 497.5109 & 587.9046 & 395.75 & 122.5994 & 1.53544 \\
\hline 3368 & 60.2393 & 483.5935 & 521.287 & 395.875 & 122.5994 & 1.53505 \\
\hline 3369 & 60.2563 & 454.6347 & 479.3409 & 396 & 122.5994 & 1.53466 \\
\hline 3370 & 60.2733 & 428.2482 & 456.2181 & 396.125 & 122.5994 & 1.53426 \\
\hline 3371 & 60.2903 & 441.0588 & 444.1939 & 396.25 & 122.5994 & 1.53387 \\
\hline 3372 & 60.3073 & 429.9328 & 437.5025 & 396.375 & 122.5994 & 1.53348 \\
\hline 3373 & 60.3243 & 424.8625 & 433.0403 & 396.5 & 122.5994 & 1.53309 \\
\hline 3374 & 60.3413 & 445.3762 & 429.2278 & 396.625 & 122.5994 & 1.5327 \\
\hline 3375 & 60.3583 & 435.5444 & 425.8064 & 396.75 & 122.5994 & 1.53231 \\
\hline 3376 & 60.3753 & 405.2425 & 422.8441 & 396.875 & 122.5994 & 1.53192 \\
\hline 3377 & 60.3923 & 422.0383 & 420.5571 & 397 & 122.5994 & 1.53153 \\
\hline 3378 & 60.4093 & 412.5541 & 419.1436 & 397.125 & 122.5994 & 1.53113 \\
\hline 3379 & 60.4263 & 410.9819 & 418.8082 & 397.25 & 122.5994 & 1.53074 \\
\hline
\end{tabular}




\begin{tabular}{|c|c|c|c|c|c|c|}
\hline 3380 & 60.4433 & 413.3518 & 419.3975 & 397.375 & 122.5994 & 1.53035 \\
\hline 3381 & 60.4603 & 394.65 & 420.8714 & 397.5 & 122.5994 & 1.52997 \\
\hline 3382 & 60.4773 & 405.9673 & 422.9021 & 397.625 & 122.5994 & 1.52958 \\
\hline 3383 & 60.4943 & 421.5694 & 424.8168 & 397.75 & 122.5994 & 1.52919 \\
\hline 3384 & 60.5113 & 383.3674 & 425.3675 & 397.875 & 122.5994 & 1.5288 \\
\hline 3385 & 60.5283 & 401.3764 & 424.8903 & 398 & 122.5994 & 1.52841 \\
\hline 3386 & 60.5453 & 411.3728 & 423.7186 & 398 & 122.5994 & 1.52802 \\
\hline 3387 & 60.5623 & 375.0286 & 422.2994 & 398 & 122.5994 & 1.52763 \\
\hline 3388 & 60.5793 & 378.7709 & 421.9956 & 398 & 122.5994 & 1.52724 \\
\hline 3389 & 60.5963 & 390.79 & 423.4326 & 398 & 122.5994 & 1.52686 \\
\hline 3390 & 60.6133 & 418.0094 & 426.4071 & 398 & 122.5994 & 1.52647 \\
\hline 3391 & 60.6303 & 408.7306 & 430.6535 & 398 & 122.5994 & 1.52608 \\
\hline 3392 & 60.6473 & 431.7544 & 435.8901 & 398 & 122.5994 & 1.52569 \\
\hline 3393 & 60.6643 & 416.3952 & 441.4328 & 398 & 122.5994 & 1.52531 \\
\hline 3394 & 60.6813 & 400.1694 & 446.2704 & 398 & 122.5994 & 1.52492 \\
\hline 3395 & 60.6983 & 424.1046 & 448.2015 & 398 & 122.5994 & 1.52453 \\
\hline 3396 & 60.7153 & 415.4425 & 449.3867 & 398 & 122.5994 & 1.52415 \\
\hline 3397 & 60.7323 & 398.3245 & 447.4584 & 398 & 122.5994 & 1.52376 \\
\hline 3398 & 60.7493 & 381.5262 & 443.5409 & 398 & 122.5994 & 1.52338 \\
\hline 3399 & 60.7663 & 424.9796 & 439.2965 & 398 & 122.5994 & 1.52299 \\
\hline 3400 & 60.7833 & 366.3198 & 435.637 & 398 & 122.5994 & 1.52261 \\
\hline 3401 & 60.8003 & 378.8467 & 432.8067 & 398 & 122.5994 & 1.52222 \\
\hline 3402 & 60.8173 & 395.5475 & 429.9708 & 398 & 122.5994 & 1.52184 \\
\hline 3403 & 60.8343 & 404.2858 & 429.1452 & 398 & 122.5994 & 1.52145 \\
\hline 3404 & 60.8513 & 375.8845 & 429.1863 & 398 & 122.5994 & 1.52107 \\
\hline 3405 & 60.8683 & 385.3085 & 429.8554 & 398 & 122.5994 & 1.52068 \\
\hline 3406 & 60.8853 & 381.491 & 429.964 & 398 & 122.5994 & 1.5203 \\
\hline 3407 & 60.9023 & 395.972 & 429.1946 & 398 & 122.5994 & 1.51992 \\
\hline 3408 & 60.9193 & 398.511 & 427.7067 & 398 & 122.5994 & 1.51953 \\
\hline 3409 & 60.9363 & 392.3895 & 425.7994 & 398 & 122.5994 & 1.51915 \\
\hline 3410 & 60.9533 & 391.0312 & 423.8007 & 398.375 & 122.5994 & 1.51877 \\
\hline 3411 & 60.9703 & 420.5049 & 421.2813 & 398.75 & 122.5994 & 1.51838 \\
\hline 3412 & 60.9873 & 403.1128 & 418.9184 & 399.125 & 122.5994 & 1.518 \\
\hline 3413 & 61.0043 & 393.3915 & 417.1335 & 399.5 & 122.5994 & 1.51762 \\
\hline 3414 & 61.0213 & 398.6198 & 416.183 & 399.875 & 122.5994 & 1.51724 \\
\hline 3415 & 61.0383 & 427.0397 & 416.2497 & 400.25 & 122.5994 & 1.51685 \\
\hline 3416 & 61.0553 & 412.4458 & 416.9515 & 400.625 & 122.5994 & 1.51647 \\
\hline 3417 & 61.0723 & 396.1082 & 418.2259 & 401 & 122.5994 & 1.51609 \\
\hline 3418 & 61.0893 & 375.9205 & 419.7081 & 401.375 & 122.5994 & 1.51571 \\
\hline 3419 & 61.1063 & 371.0416 & 421.2019 & 401.75 & 122.5994 & 1.51533 \\
\hline 3420 & 61.1233 & 389.7482 & 422.3639 & 402.125 & 122.5994 & 1.51495 \\
\hline 3421 & 61.1403 & 411.8207 & 423.0753 & 402.5 & 122.5994 & 1.51457 \\
\hline 3422 & 61.1573 & 398.3891 & 423.1495 & 402.875 & 122.5994 & 1.51419 \\
\hline 3423 & 61.1743 & 369.5624 & 421.7038 & 403.25 & 122.5994 & 1.51381 \\
\hline 3424 & 61.1913 & 383.7262 & 419.7478 & 403.625 & 122.5994 & 1.51343 \\
\hline 3425 & 61.2083 & 417.882 & 418.4055 & 404 & 122.5994 & 1.51305 \\
\hline 3426 & 61.2253 & 403.1819 & 418.0084 & 404.375 & 122.5994 & 1.51267 \\
\hline 3427 & 61.2423 & 421.1149 & 418.5086 & 404.75 & 122.5994 & 1.51229 \\
\hline 3428 & 61.2593 & 420.4649 & 419.8314 & 405.125 & 122.5994 & 1.51191 \\
\hline
\end{tabular}




\begin{tabular}{|c|c|c|c|c|c|c|}
\hline 3429 & 61.2763 & 410.0612 & 421.9981 & 405.5 & 122.5994 & 1.51153 \\
\hline 3430 & 61.2933 & 404.8601 & 424.8179 & 405.875 & 122.5994 & 1.51115 \\
\hline 3431 & 61.3103 & 420.2666 & 427.2495 & 406.25 & 122.5994 & 1.51078 \\
\hline 3432 & 61.3273 & 414.3257 & 427.2895 & 406.625 & 122.5994 & 1.5104 \\
\hline 3433 & 61.3443 & 405.2324 & 425.0596 & 407 & 122.5994 & 1.51002 \\
\hline 3434 & 61.3613 & 400.5933 & 422.2545 & 406.6667 & 122.5994 & 1.50964 \\
\hline 3435 & 61.3783 & 403.5181 & 420.7641 & 406.3333 & 122.5994 & 1.50926 \\
\hline 3436 & 61.3953 & 378.8563 & 420.6794 & 406 & 122.5994 & 1.50889 \\
\hline 3437 & 61.4123 & 402.1237 & 421.5065 & 405.6667 & 122.5994 & 1.50851 \\
\hline 3438 & 61.4293 & 396.3602 & 423.8388 & 405.3333 & 122.5994 & 1.50813 \\
\hline 3439 & 61.4463 & 403.8471 & 427.5894 & 405 & 122.5994 & 1.50776 \\
\hline 3440 & 61.4633 & 453.3758 & 432.8007 & 404.6667 & 122.5994 & 1.50738 \\
\hline 3441 & 61.4803 & 457.6446 & 438.6727 & 404.3333 & 122.5994 & 1.50701 \\
\hline 3442 & 61.4973 & 440.028 & 443.1766 & 404 & 122.5994 & 1.50663 \\
\hline 3443 & 61.5143 & 418.4327 & 445.1615 & 403.6667 & 122.5994 & 1.50625 \\
\hline 3444 & 61.5313 & 452.625 & 445.4277 & 403.3333 & 122.5994 & 1.50588 \\
\hline 3445 & 61.5483 & 439.359 & 444.5367 & 403 & 122.5994 & 1.5055 \\
\hline 3446 & 61.5653 & 429.5339 & 442.6415 & 402.6667 & 122.5994 & 1.50513 \\
\hline 3447 & 61.5823 & 460.9955 & 439.7707 & 402.3333 & 122.5994 & 1.50475 \\
\hline 3448 & 61.5993 & 434.8911 & 436.8678 & 402 & 122.5994 & 1.50438 \\
\hline 3449 & 61.6163 & 464.0949 & 434.4173 & 401.6667 & 122.5994 & 1.504 \\
\hline 3450 & 61.6333 & 451.0893 & 432.9818 & 401.3333 & 122.5994 & 1.50363 \\
\hline 3451 & 61.6503 & 446.8654 & 432.8747 & 401 & 122.5994 & 1.50326 \\
\hline 3452 & 61.6673 & 471.0307 & 434.0034 & 400.6667 & 122.5994 & 1.50288 \\
\hline 3453 & 61.6843 & 486.3775 & 436.1344 & 400.3333 & 122.5994 & 1.50251 \\
\hline 3454 & 61.7013 & 482.4815 & 439.1413 & 400 & 122.5994 & 1.50214 \\
\hline 3455 & 61.7183 & 459.3821 & 441.6144 & 399.6667 & 122.5994 & 1.50176 \\
\hline 3456 & 61.7353 & 469.5722 & 440.6671 & 399.3333 & 122.5994 & 1.50139 \\
\hline 3457 & 61.7523 & 484.0645 & 437.0137 & 399 & 122.5994 & 1.50102 \\
\hline 3458 & 61.7693 & 482.2777 & 433.6536 & 398.6667 & 122.5994 & 1.50065 \\
\hline 3459 & 61.7863 & 490.4207 & 431.7061 & 398.3333 & 122.5994 & 1.50027 \\
\hline 3460 & 61.8033 & 476.7013 & 430.9389 & 398 & 122.5994 & 1.4999 \\
\hline 3461 & 61.8203 & 444.3008 & 430.5558 & 397.6667 & 122.5994 & 1.49953 \\
\hline 3462 & 61.8373 & 467.9325 & 430.1635 & 397.3333 & 122.5994 & 1.49916 \\
\hline 3463 & 61.8543 & 481.8055 & 429.6432 & 397 & 122.5994 & 1.49879 \\
\hline 3464 & 61.8713 & 462.0966 & 429.2628 & 396.6667 & 122.5994 & 1.49842 \\
\hline 3465 & 61.8883 & 454.2392 & 428.7026 & 396.3333 & 122.5994 & 1.49805 \\
\hline 3466 & 61.9053 & 450.4372 & 426.5884 & 396 & 122.5994 & 1.49768 \\
\hline 3467 & 61.9223 & 426.7644 & 423.0879 & 395.6667 & 122.5994 & 1.49731 \\
\hline 3468 & 61.9393 & 435.9842 & 419.9813 & 395.3333 & 122.5994 & 1.49694 \\
\hline 3469 & 61.9563 & 423.8928 & 418.0647 & 395 & 122.5994 & 1.49657 \\
\hline 3470 & 61.9733 & 426.4122 & 416.9865 & 394.6667 & 122.5994 & 1.4962 \\
\hline 3471 & 61.9903 & 445.2242 & 415.9649 & 394.3333 & 122.5994 & 1.49583 \\
\hline 3472 & 62.0073 & 437.9222 & 414.8423 & 394 & 122.5994 & 1.49546 \\
\hline 3473 & 62.0243 & 429.6751 & 413.6714 & 393.6667 & 122.5994 & 1.49509 \\
\hline 3474 & 62.0413 & 404.9637 & 412.5344 & 393.3333 & 122.5994 & 1.49472 \\
\hline 3475 & 62.0583 & 417.1639 & 411.4336 & 393 & 122.5994 & 1.49435 \\
\hline 3476 & 62.0753 & 411.8639 & 410.1139 & 392.6667 & 122.5994 & 1.49398 \\
\hline 3477 & 62.0923 & 392.2288 & 408.8622 & 392.3333 & 122.5994 & 1.49361 \\
\hline
\end{tabular}




\begin{tabular}{|c|c|c|c|c|c|c|}
\hline 3478 & 62.1093 & 410.8456 & 408.188 & 392 & 122.5994 & 1.49325 \\
\hline 3479 & 62.1263 & 383.0362 & 408.2935 & 391.6667 & 122.5994 & 1.49288 \\
\hline 3480 & 62.1433 & 403.4125 & 408.7859 & 391.3333 & 122.5994 & 1.49251 \\
\hline 3481 & 62.1603 & 401.0456 & 408.6927 & 391 & 122.5994 & 1.49214 \\
\hline 3482 & 62.1773 & 364.9342 & 406.9858 & 390.25 & 122.5994 & 1.49178 \\
\hline 3483 & 62.1943 & 400.6083 & 404.6229 & 389.5 & 122.5994 & 1.49141 \\
\hline 3484 & 62.2113 & 377.9468 & 402.183 & 388.75 & 122.5994 & 1.49104 \\
\hline 3485 & 62.2283 & 399.0805 & 399.9874 & 388 & 122.5994 & 1.49068 \\
\hline 3486 & 62.2453 & 372.9758 & 397.8751 & 387.25 & 122.5994 & 1.49031 \\
\hline 3487 & 62.2623 & 396.0166 & 396.2244 & 386.5 & 122.5994 & 1.48994 \\
\hline 3488 & 62.2793 & 388.1604 & 395.229 & 385.75 & 122.5994 & 1.48958 \\
\hline 3489 & 62.2963 & 391.8101 & 394.7984 & 385 & 122.5994 & 1.48921 \\
\hline 3490 & 62.3133 & 388.4195 & 394.7112 & 384.25 & 122.5994 & 1.48885 \\
\hline 3491 & 62.3303 & 393.1006 & 394.4791 & 383.5 & 122.5994 & 1.48848 \\
\hline 3492 & 62.3473 & 401.3696 & 393.6704 & 382.75 & 122.5994 & 1.48812 \\
\hline 3493 & 62.3643 & 378.6483 & 392.3377 & 382 & 122.5994 & 1.48775 \\
\hline 3494 & 62.3813 & 371.7323 & 390.8088 & 381.25 & 122.5994 & 1.48739 \\
\hline 3495 & 62.3983 & 385.2778 & 389.1328 & 380.5 & 122.5994 & 1.48702 \\
\hline 3496 & 62.4153 & 375.5634 & 387.3264 & 379.75 & 122.5994 & 1.48666 \\
\hline 3497 & 62.4323 & 387.2734 & 385.7297 & 379 & 122.5994 & 1.48629 \\
\hline 3498 & 62.4493 & 385.0757 & 384.434 & 378.25 & 122.5994 & 1.48593 \\
\hline 3499 & 62.4663 & 385.0447 & 383.3653 & 377.5 & 122.5994 & 1.48557 \\
\hline 3500 & 62.4833 & 375.2192 & 382.3978 & 376.75 & 122.5994 & 1.4852 \\
\hline 3501 & 62.5003 & 370.1812 & 381.4043 & 376 & 122.5994 & 1.48484 \\
\hline 3502 & 62.5173 & 369.8473 & 380.3581 & 375.25 & 122.5994 & 1.48448 \\
\hline 3503 & 62.5343 & 374.8105 & 379.1528 & 374.5 & 122.5994 & 1.48411 \\
\hline 3504 & 62.5513 & 367.7124 & 378.1389 & 373.75 & 122.5994 & 1.48375 \\
\hline 3505 & 62.5683 & 372.4543 & 377.1376 & 373 & 122.5994 & 1.48339 \\
\hline 3506 & 62.5853 & 377.3787 & 376.1411 & 372.2083 & 122.5994 & 1.48303 \\
\hline 3507 & 62.6023 & 372.371 & 375.3901 & 371.4167 & 122.5994 & 1.48267 \\
\hline 3508 & 62.6193 & 365.9367 & 375.1006 & 370.625 & 122.5994 & 1.4823 \\
\hline 3509 & 62.6363 & 369.0505 & 375.4234 & 369.8333 & 122.5994 & 1.48194 \\
\hline 3510 & 62.6533 & 370.6012 & 376.113 & 369.0417 & 122.5994 & 1.48158 \\
\hline 3511 & 62.6703 & 354.707 & 376.2555 & 368.25 & 122.5994 & 1.48122 \\
\hline 3512 & 62.6873 & 351.5401 & 374.9467 & 367.4583 & 122.5994 & 1.48086 \\
\hline 3513 & 62.7043 & 366.5241 & 372.7201 & 366.6667 & 122.5994 & 1.4805 \\
\hline 3514 & 62.7213 & 358.1042 & 370.8207 & 365.875 & 122.5994 & 1.48014 \\
\hline 3515 & 62.7383 & 367.3443 & 369.4261 & 365.0833 & 122.5994 & 1.47978 \\
\hline 3516 & 62.7553 & 361.9758 & 368.3371 & 364.2917 & 122.5994 & 1.47942 \\
\hline 3517 & 62.7723 & 368.3315 & 367.2687 & 363.5 & 122.5994 & 1.47906 \\
\hline 3518 & 62.7893 & 355.9557 & 366.3183 & 362.7083 & 122.5994 & 1.4787 \\
\hline 3519 & 62.8063 & 340.7893 & 365.7188 & 361.9167 & 122.5994 & 1.47834 \\
\hline 3520 & 62.8233 & 351.875 & 365.5063 & 361.125 & 122.5994 & 1.47798 \\
\hline 3521 & 62.8403 & 368.5241 & 365.3316 & 360.3333 & 122.5994 & 1.47762 \\
\hline 3522 & 62.8573 & 375.3906 & 364.5655 & 359.5417 & 122.5994 & 1.47726 \\
\hline 3523 & 62.8743 & 370.3855 & 363.2792 & 358.75 & 122.5994 & 1.4769 \\
\hline 3524 & 62.8913 & 377.9138 & 361.9382 & 357.9583 & 122.5994 & 1.47655 \\
\hline 3525 & 62.9083 & 370.7402 & 360.8896 & 357.1667 & 122.5994 & 1.47619 \\
\hline 3526 & 62.9253 & 340.0895 & 360.1302 & 356.375 & 122.5994 & 1.47583 \\
\hline
\end{tabular}




\begin{tabular}{|c|c|c|c|c|c|c|}
\hline 3527 & 62.9423 & 354.194 & 359.4948 & 355.5833 & 122.5994 & 1.47547 \\
\hline 3528 & 62.9593 & 343.9737 & 358.9029 & 354.7917 & 122.5994 & 1.47512 \\
\hline 3529 & 62.9763 & 366.2158 & 358.3716 & 354 & 122.5994 & 1.47476 \\
\hline 3530 & 62.9933 & 351.046 & 358.6409 & 353.9583 & 122.5994 & 1.4744 \\
\hline 3531 & 63.0103 & 356.8189 & 358.9261 & 353.9167 & 122.5994 & 1.47404 \\
\hline 3532 & 63.0273 & 372.0565 & 359.1755 & 353.875 & 122.5994 & 1.47369 \\
\hline 3533 & 63.0443 & 357.328 & 359.3398 & 353.8333 & 122.5994 & 1.47333 \\
\hline 3534 & 63.0613 & 365.1891 & 359.4588 & 353.7917 & 122.5994 & 1.47297 \\
\hline 3535 & 63.0783 & 381.9461 & 359.4594 & 353.75 & 122.5994 & 1.47262 \\
\hline 3536 & 63.0953 & 329.434 & 359.0483 & 353.7083 & 122.5994 & 1.47226 \\
\hline 3537 & 63.1123 & 357.4849 & 358.3464 & 353.6667 & 122.5994 & 1.47191 \\
\hline 3538 & 63.1293 & 358.6543 & 357.7621 & 353.625 & 122.5994 & 1.47155 \\
\hline 3539 & 63.1463 & 338.6051 & 357.4525 & 353.5833 & 122.5994 & 1.4712 \\
\hline 3540 & 63.1633 & 339.6084 & 357.3849 & 353.5417 & 122.5994 & 1.47084 \\
\hline 3541 & 63.1803 & 347.2606 & 357.4644 & 353.5 & 122.5994 & 1.47049 \\
\hline 3542 & 63.1973 & 355.5565 & 357.6646 & 353.4583 & 122.5994 & 1.47013 \\
\hline 3543 & 63.2143 & 360.1035 & 357.9923 & 353.4167 & 122.5994 & 1.46978 \\
\hline 3544 & 63.2313 & 345.6909 & 358.5362 & 353.375 & 122.5994 & 1.46942 \\
\hline 3545 & 63.2483 & 359.1032 & 359.4918 & 353.3333 & 122.5994 & 1.46907 \\
\hline 3546 & 63.2653 & 347.7782 & 361.2821 & 353.2917 & 122.5994 & 1.46872 \\
\hline 3547 & 63.2823 & 312.2636 & 364.365 & 353.25 & 122.5994 & 1.46836 \\
\hline 3548 & 63.2993 & 336.1029 & 369.006 & 353.2083 & 122.5994 & 1.46801 \\
\hline 3549 & 63.3163 & 338.5275 & 373.29 & 353.1667 & 122.5994 & 1.46765 \\
\hline 3550 & 63.3333 & 328.1256 & 373.1908 & 353.125 & 122.5994 & 1.4673 \\
\hline 3551 & 63.3503 & 326.5232 & 368.805 & 353.0833 & 122.5994 & 1.46695 \\
\hline 3552 & 63.3673 & 356.4622 & 364.4463 & 353.0417 & 122.5994 & 1.4666 \\
\hline 3553 & 63.3843 & 357.8283 & 361.7483 & 353 & 122.5994 & 1.46624 \\
\hline 3554 & 63.4013 & 351.7287 & 361.356 & 353.5 & 122.5994 & 1.46589 \\
\hline 3555 & 63.4183 & 356.8274 & 362.0501 & 354 & 122.5994 & 1.46554 \\
\hline 3556 & 63.4353 & 366.6532 & 363.4174 & 354.5 & 122.5994 & 1.46519 \\
\hline 3557 & 63.4523 & 355.098 & 365.6044 & 355 & 122.5994 & 1.46484 \\
\hline 3558 & 63.4693 & 346.2646 & 368.2276 & 355.5 & 122.5994 & 1.46449 \\
\hline 3559 & 63.4863 & 341.4443 & 370.3712 & 356 & 122.5994 & 1.46413 \\
\hline 3560 & 63.5033 & 347.8028 & 371.3667 & 356.5 & 122.5994 & 1.46378 \\
\hline 3561 & 63.5203 & 343.8295 & 369.6572 & 357 & 122.5994 & 1.46343 \\
\hline 3562 & 63.5373 & 351.0296 & 367.2344 & 357.5 & 122.5994 & 1.46308 \\
\hline 3563 & 63.5543 & 346.6637 & 365.597 & 358 & 122.5994 & 1.46273 \\
\hline 3564 & 63.5713 & 366.1446 & 364.9936 & 358.5 & 122.5994 & 1.46238 \\
\hline 3565 & 63.5883 & 361.9603 & 365.1341 & 359 & 122.5994 & 1.46203 \\
\hline 3566 & 63.6053 & 350.7604 & 365.5836 & 359.5 & 122.5994 & 1.46168 \\
\hline 3567 & 63.6223 & 369.1299 & 366.1906 & 360 & 122.5994 & 1.46133 \\
\hline 3568 & 63.6393 & 361.9886 & 366.8004 & 360.5 & 122.5994 & 1.46098 \\
\hline 3569 & 63.6563 & 360.094 & 367.1249 & 361 & 122.5994 & 1.46063 \\
\hline 3570 & 63.6733 & 331.7571 & 367.6942 & 361.5 & 122.5994 & 1.46028 \\
\hline 3571 & 63.6903 & 350.9709 & 368.5354 & 362 & 122.5994 & 1.45994 \\
\hline 3572 & 63.7073 & 350.9805 & 370.0463 & 362.5 & 122.5994 & 1.45959 \\
\hline 3573 & 63.7243 & 356.0946 & 372.614 & 363 & 122.5994 & 1.45924 \\
\hline 3574 & 63.7413 & 353.8673 & 376.3643 & 363.5 & 122.5994 & 1.45889 \\
\hline 3575 & 63.7583 & 377.5949 & 380.2964 & 364 & 122.5994 & 1.45854 \\
\hline
\end{tabular}




\begin{tabular}{|c|c|c|c|c|c|c|}
\hline 3576 & 63.7753 & 369.525 & 381.8802 & 364.5 & 122.5994 & 1.45819 \\
\hline 3577 & 63.7923 & 361.4901 & 380.9263 & 365 & 122.5994 & 1.45785 \\
\hline 3578 & 63.8093 & 372.0383 & 379.429 & 365.0969 & 122.5994 & 1.4575 \\
\hline 3579 & 63.8263 & 331.894 & 379.1855 & 365.1938 & 122.5994 & 1.45715 \\
\hline 3580 & 63.8433 & 380.0851 & 380.869 & 365.2906 & 122.5994 & 1.4568 \\
\hline 3581 & 63.8603 & 361.2645 & 385.0488 & 365.3875 & 122.5994 & 1.45646 \\
\hline 3582 & 63.8773 & 370.364 & 391.6518 & 365.4844 & 122.5994 & 1.45611 \\
\hline 3583 & 63.8943 & 401.8136 & 398.1704 & 365.5813 & 122.5994 & 1.45576 \\
\hline 3584 & 63.9113 & 373.5374 & 399.7378 & 365.6782 & 122.5994 & 1.45542 \\
\hline 3585 & 63.9283 & 381.5603 & 397.3589 & 365.7751 & 122.5994 & 1.45507 \\
\hline 3586 & 63.9453 & 369.0164 & 395.3023 & 365.8719 & 122.5994 & 1.45473 \\
\hline 3587 & 63.9623 & 415.7722 & 394.86 & 365.9688 & 122.5994 & 1.45438 \\
\hline 3588 & 63.9793 & 390.6821 & 397.169 & 366.0657 & 122.5994 & 1.45404 \\
\hline 3589 & 63.9963 & 390.5351 & 403.4268 & 366.1626 & 122.5994 & 1.45369 \\
\hline 3590 & 64.0133 & 421.7305 & 415.4066 & 366.2595 & 122.5994 & 1.45335 \\
\hline 3591 & 64.0303 & 455.8643 & 435.4443 & 366.3563 & 122.5994 & 1.453 \\
\hline 3592 & 64.0473 & 501.5969 & 464.6096 & 366.4532 & 122.5994 & 1.45266 \\
\hline 3593 & 64.0643 & 504.4055 & 499.0176 & 366.5501 & 122.5994 & 1.45231 \\
\hline 3594 & 64.0813 & 489.3307 & 525.1895 & 366.647 & 122.5994 & 1.45197 \\
\hline 3595 & 64.0983 & 435.3762 & 524.3793 & 366.7439 & 122.5994 & 1.45162 \\
\hline 3596 & 64.1153 & 413.0722 & 497.1587 & 366.8408 & 122.5994 & 1.45128 \\
\hline 3597 & 64.1323 & 376.3104 & 462.7744 & 366.9376 & 122.5994 & 1.45094 \\
\hline 3598 & 64.1493 & 375.7551 & 434.9035 & 367.0345 & 122.5994 & 1.45059 \\
\hline 3599 & 64.1663 & 407.8433 & 417.4816 & 367.1314 & 122.5994 & 1.45025 \\
\hline 3600 & 64.1833 & 394.3273 & 409.6244 & 367.2283 & 122.5994 & 1.44991 \\
\hline 3601 & 64.2003 & 395.7442 & 409.7693 & 367.3252 & 122.5994 & 1.44956 \\
\hline 3602 & 64.2173 & 413.5447 & 416.8777 & 367.422 & 122.5994 & 1.44922 \\
\hline 3603 & 64.2343 & 434.8225 & 429.2189 & 367.5189 & 122.5994 & 1.44888 \\
\hline 3604 & 64.2513 & 428.0604 & 442.6096 & 367.6158 & 122.5994 & 1.44854 \\
\hline 3605 & 64.2683 & 413.8087 & 448.8488 & 367.7127 & 122.5994 & 1.44819 \\
\hline 3606 & 64.2853 & 407.5707 & 441.3983 & 367.8096 & 122.5994 & 1.44785 \\
\hline 3607 & 64.3023 & 383.2743 & 425.3759 & 367.9065 & 122.5994 & 1.44751 \\
\hline 3608 & 64.3193 & 382.9496 & 409.356 & 368.0033 & 122.5994 & 1.44717 \\
\hline 3609 & 64.3363 & 372.3383 & 397.4856 & 368.1002 & 122.5994 & 1.44683 \\
\hline 3610 & 64.3533 & 358.576 & 390.1958 & 368.1971 & 122.5994 & 1.44649 \\
\hline 3611 & 64.3703 & 386.0943 & 386.348 & 368.294 & 122.5994 & 1.44614 \\
\hline 3612 & 64.3873 & 380.7476 & 384.3381 & 368.3909 & 122.5994 & 1.4458 \\
\hline 3613 & 64.4043 & 378.1017 & 382.2826 & 368.4877 & 122.5994 & 1.44546 \\
\hline 3614 & 64.4213 & 349.5167 & 379.7458 & 368.5846 & 122.5994 & 1.44512 \\
\hline 3615 & 64.4383 & 333.0415 & 377.6585 & 368.6815 & 122.5994 & 1.44478 \\
\hline 3616 & 64.4553 & 363.3586 & 376.3963 & 368.7784 & 122.5994 & 1.44444 \\
\hline 3617 & 64.4723 & 366.3734 & 375.7648 & 368.8753 & 122.5994 & 1.4441 \\
\hline 3618 & 64.4893 & 384.054 & 375.7039 & 368.9722 & 122.5994 & 1.44376 \\
\hline 3619 & 64.5063 & 365.0529 & 376.3934 & 369.069 & 122.5994 & 1.44342 \\
\hline 3620 & 64.5233 & 401.8498 & 377.8998 & 369.1659 & 122.5994 & 1.44308 \\
\hline 3621 & 64.5403 & 369.6615 & 379.9467 & 369.2628 & 122.5994 & 1.44275 \\
\hline 3622 & 64.5573 & 357.7737 & 381.2376 & 369.3597 & 122.5994 & 1.44241 \\
\hline 3623 & 64.5743 & 350.2499 & 380.5551 & 369.4566 & 122.5994 & 1.44207 \\
\hline 3624 & 64.5913 & 325.2406 & 378.6288 & 369.5534 & 122.5994 & 1.44173 \\
\hline
\end{tabular}




$\begin{array}{rrrrrrr}3625 & 64.6083 & 382.7096 & 376.812 & 369.6503 & 122.5994 & 1.44139 \\ 3626 & 64.6253 & 380.1393 & 375.817 & 369.7472 & 122.5994 & 1.44105 \\ 3627 & 64.6423 & 368.2102 & 375.3248 & 369.8441 & 122.5994 & 1.44071 \\ 3628 & 64.6593 & 342.1387 & 374.8766 & 369.941 & 122.5994 & 1.44038 \\ 3629 & 64.6763 & 344.1399 & 374.5861 & 370.0379 & 122.5994 & 1.44004 \\ 3630 & 64.6933 & 370.9654 & 374.7463 & 370.1347 & 122.5994 & 1.4397 \\ 3631 & 64.7103 & 348.5658 & 375.3888 & 370.2316 & 122.5994 & 1.43936 \\ 3632 & 64.7273 & 309.7441 & 376.1275 & 370.3285 & 122.5994 & 1.43903 \\ 3633 & 64.7443 & 337.6639 & 376.1568 & 370.4254 & 122.5994 & 1.43869 \\ 3634 & 64.7613 & 349.1276 & 375.1339 & 370.5223 & 122.5994 & 1.43835 \\ 3635 & 64.7783 & 326.1357 & 374.5165 & 370.6192 & 122.5994 & 1.43802 \\ 3636 & 64.7953 & 326.6489 & 374.2224 & 370.716 & 122.5994 & 1.43768 \\ 3637 & 64.8123 & 343.744 & 374.2301 & 370.8129 & 122.5994 & 1.43735 \\ 3638 & 64.8293 & 345.4664 & 374.3246 & 370.9098 & 122.5994 & 1.43701 \\ 3639 & 64.8463 & 404.5858 & 374.451 & 371.0067 & 122.5994 & 1.43667 \\ 3640 & 64.8633 & 385.6383 & 374.8617 & 371.1036 & 122.5994 & 1.43634 \\ 3641 & 64.8803 & 353.8806 & 375.9717 & 371.2004 & 122.5994 & 1.436 \\ 3642 & 64.8973 & 351.3245 & 378.4086 & 371.2973 & 122.5994 & 1.43567 \\ 3643 & 64.9143 & 365.3978 & 382.6899 & 371.3942 & 122.5994 & 1.43533 \\ 3644 & 64.9313 & 350.325 & 387.9257 & 371.4911 & 122.5994 & 1.435 \\ 3645 & 64.9483 & 354.2079 & 391.3068 & 371.588 & 122.5994 & 1.43466 \\ 3646 & 64.9653 & 356.0286 & 389.2458 & 371.6849 & 122.5994 & 1.43433 \\ 3647 & 64.9823 & 371.7817 & 384.7421 & 371.6849 & 122.5994 & 1.434\end{array}$




\begin{tabular}{|c|c|c|}
\hline International geochemical reference standard & $\begin{array}{l}\text { Alternative } \\
\text { Reference }\end{array}$ & Origin \\
\hline$G S D-1$ & GBW 07310 & China IGGE IRMA \\
\hline$G S D-2$ & GBW 07302 & China \\
\hline GSD-3 & GBW 07303 & China \\
\hline$G S D-4$ & GBW 07304 & China \\
\hline$G S D-5$ & GBW 07305 & China \\
\hline GSD-6 & GBW 07306 & China \\
\hline GSD-7 & GBW 07307 & China \\
\hline$G S D-8$ & GBW 07308 & China IGGE IRMA \\
\hline GSD-9 & GBW 01309 & China IGGE IRMA \\
\hline$G S D-10$ & GBW 07310 & China \\
\hline GSD-11 & GBW 07311 & China \\
\hline$G S D-12$ & GBW 07312 & China \\
\hline GSS-1 & GBW 07401 & China \\
\hline GSS-2 & GBW 07402 & China \\
\hline GSS-3 & GBW 07403 & China \\
\hline GSS-4 & GBW 07404 & China \\
\hline GSS-5 & GBW 07405 & China \\
\hline GSS-6 & GBW 07406 & China \\
\hline GSS-7 & GBW 07407 & China \\
\hline GSS-8 & GBW 07408 & China \\
\hline$J S D-1$ & & Geological Survey of Japan \\
\hline$M A G-1$ & & U.S. Dept. of the Int. Geo. Survey \\
\hline
\end{tabular}

IGGE: Institute of Geophysical and Geochemical Exploration

IRMA: Institute of Rock and Mineral Analysis

GSJ: Geological Survey of Japan

USGS: United States Geological Survey

ND: No data

BDL: Below detection limit 
Accepted values (\% concentration) for international geocher calibration software

\begin{tabular}{llrrrrr} 
Issuing Body & Description & SiO2 & TiO2 & Al2O3 & Fe2O3 & MnO \\
\hline IGGE & Stream Sediment & 58.43 & 0.98 & 14.83 & 4.83 & 0.12 \\
IGGE & Stream Sediment & 69.90 & 0.23 & 15.72 & 1.27 & 0.03 \\
IGGE & Stream Sediment & 71.28 & 1.06 & 12.04 & 5.72 & 0.05 \\
IGGE & Pond Sediment & 52.51 & 0.89 & 15.67 & 4.89 & 0.11 \\
IGGE & Pond Sediment & 56.36 & 0.9 & 15.37 & 4.80 & 0.15 \\
IGGE & Stream Sediment & 61.23 & 0.78 & 14.16 & 4.14 & 0.13 \\
IGGE & Stream Sediment & 64.63 & 0.75 & 13.44 & 4.87 & 0.09 \\
IGGE & Steam Sediment 142308 & 82.9 & 0.31 & 7.71 & 1.59 & 0.04 \\
IGGE & Sediment 300603 & 64.89 & 0.92 & 10.58 & 3.17 & 0.08 \\
IGGE & Stream Sediment & 88.89 & 0.21 & 2.84 & 3.59 & 0.13 \\
IGGE & Stream Sediment & 76.25 & 0.35 & 10.37 & 4.01 & 0.32 \\
IGGE & Stream Sediment & 77.29 & 0.25 & 9.3 & 3.56 & 0.18 \\
IGGE & Dark Brown Soil & 62.6 & 0.81 & 14.18 & 3.78 & 0.23 \\
IGGE & Chestnut Soil & 73.35 & 0.45 & 10.31 & 2.90 & 0.07 \\
IGGE & Yellow Brown Soil & 74.72 & 0.37 & 12.24 & 0.47 & 0.04 \\
IGGE & Limy Soil & 50.95 & 1.8 & 23.45 & 9.84 & 0.18 \\
IGGE & Yellow Red Soil & 52.57 & 1.05 & 21.58 & 1.38 & 0.18 \\
IGGE & Yellow Red Soil & 53.93 & 0.73 & 21.23 & 7.46 & 0.19 \\
IGGE & Laterite Soil & 32.69 & 3.36 & 29.26 & 17.59 & 0.23 \\
IGGE & Loess & 58.61 & 0.63 & 11.92 & 3.15 & 0.08 \\
GSJ & Stream Sediment & 66.42 & 0.65 & 14.66 & 3.55 & 0.093 \\
USGS-AEG & Marine mud (Gulf of Maine) & 50.36 & 0.751 & 16.37 & 3.49 & 0.098 \\
\hline & & & & & \\
\end{tabular}


mical reference standards used in the Bruker AXS S1CalProcess

Accepted $v$

: and subsequent LRCE.

\begin{tabular}{rrrrrrrr} 
MgO & \multicolumn{1}{r}{ CaO } & Na2O & K2O & P2O5 & LOI & Sum & Ba \\
\hline 4.14 & 4.6 & 3.5 & 2.77 & 0.34 & 2.29 & 96.83 & 950 \\
0.21 & 0.25 & 3.04 & 5.19 & 0.05 & 3.13 & 99.02 & 185 \\
0.7 & 0.22 & 0.32 & 2.46 & 0.14 & 4.99 & 98.98 & 615 \\
1.04 & 7.52 & 0.3 & 2.23 & 0.11 & 13.60 & 98.87 & 470 \\
0.98 & 5.34 & 0.4 & 2.1 & 0.14 & 12.20 & 98.74 & 440 \\
3 & 3.87 & 2.31 & 2.44 & 0.23 & 5.83 & 98.12 & 330 \\
3.06 & 1.66 & 1.21 & 3.55 & 0.19 & 4.60 & 98.05 & 720 \\
0.25 & 0.25 & 0.47 & 2.83 & 0.03 & 2.73 & 99.11 & 480 \\
2.39 & 5.35 & 1.44 & 1.99 & 0.15 & 7.42 & 98.38 & 430 \\
0.12 & 0.7 & 0.04 & 0.125 & 0.062 & 2.91 & 99.62 & 42 \\
0.62 & 0.47 & 0.46 & 3.28 & 0.059 & 2.95 & 99.14 & 260 \\
0.47 & 1.16 & 0.44 & 2.91 & 0.055 & 2.50 & 98.11 & 206 \\
1.81 & 1.72 & 1.66 & 2.59 & 0.17 & 8.59 & 98.14 & 590 \\
1.04 & 2.36 & 1.62 & 2.54 & 0.1 & 4.41 & 99.15 & 930 \\
0.58 & 1.27 & 2.71 & 3.04 & 0.073 & 2.65 & 98.16 & 1210 \\
0.49 & 0.26 & 0.112 & 1.03 & 0.16 & 10.88 & 99.15 & 213 \\
0.61 & 0.10 & 0.122 & 1.5 & 0.09 & 9.14 & 88.32 & 296 \\
0.34 & 0.22 & 0.19 & 1.7 & 0.07 & 10.00 & 96.06 & 118 \\
0.26 & 0.16 & 0.07 & 0.2 & 0.26 & 14.30 & 98.38 & 180 \\
2.38 & 8.27 & 1.72 & 2.24 & 0.18 & 9.15 & 98.33 & 480 \\
1.85 & 3.07 & 2.73 & 2.19 & 0.12 & 3.14 & 98.47 & ND \\
3 & 1.37 & 3.83 & 3.55 & 0.163 & & 82.98 & 479 \\
\hline
\end{tabular}


ralues (ppm concentration) for international geochemical reference standards used in the Bruker AXS S1CalProcess calibration software and subsequent LRCE.

\begin{tabular}{rrrrrrrr}
$\mathbf{V}$ & $\mathbf{C r}$ & $\mathbf{N i}$ & $\mathbf{C u}$ & $\mathbf{Z n}$ & $\mathbf{R b}$ & $\mathbf{Z r}$ & $\mathbf{S i O 2}$ \\
\hline 121 & 194 & 76 & 21.8 & 79 & 116 & 310 & 54.47 \\
16.5 & 12.20 & 5.50 & 4.9 & 44 & 470 & 460 & 80.92 \\
120 & 87 & 25.6 & 177 & 52 & 79 & 220 & 70.47 \\
118 & 81 & 40 & 37.3 & 101 & 130 & 188 & 69.74 \\
109 & 70 & 34 & 137 & 243 & 118 & 220 & 64.71 \\
142 & 190 & 78 & 383 & 144 & 107 & 170 & 65.63 \\
96 & 122 & 53 & 38 & 238 & 147 & 162 & 49.45 \\
26 & 7.60 & 2.70 & 4.1 & 43 & 132 & 490 & 52.10 \\
97 & 85 & 32.3 & 32.1 & 78 & 80 & 370 & 55.92 \\
107 & 136 & 30.2 & 22.6 & 46 & 9.2 & 70 & 59.98 \\
46.8 & 40 & 14.4 & 78.6 & 373 & 408 & 153 & 77.13 \\
46.6 & 35 & 12.8 & 1230 & 498 & 270 & 234 & 58.35 \\
86 & 62 & 20.4 & 21 & 680 & 140 & 245 & 58.73 \\
62 & 47 & 19.4 & 16.3 & 42.3 & 88 & 219 & 65.50 \\
36.5 & 32 & 12.2 & 11.4 & 31.4 & 85 & 246 & 69.29 \\
247 & 370 & 64.2 & 40.5 & 210 & 75 & 500 & 43.45 \\
166 & 118 & 40 & 144 & 494 & 117 & 272 & 45.16 \\
130 & 75 & 53 & 390 & 96.6 & 237 & 220 & 46.22 \\
245 & 410 & 276 & 97 & 142 & 15.8 & 318 & 33.06 \\
81.4 & 68 & 31.5 & 24.3 & 68 & 96 & 229 & 52.37 \\
81 & 22 & 6.9 & 22.2 & 99 & 65 & $\mathrm{ND}$ & 59.74 \\
140 & 97 & 53 & 30 & 130 & 149 & 126 & 48.20 \\
\hline & & & & & &
\end{tabular}


Minimum values (following Mudrock calibration of PXRF) (\%)

\begin{tabular}{|c|c|c|c|c|c|c|c|}
\hline TiO2 & Al2O3 & $\mathrm{Fe} 2 \mathrm{O3}$ & MnO & MgO & $\mathrm{CaO}$ & $\mathrm{Na} 2 \mathrm{O}$ & $\mathrm{K} 2 \mathrm{O}$ \\
\hline 0.57 & 14.18 & 6.40 & 0.08 & 2.23 & 4.17 & 0.91 & 0.51 \\
\hline 0.08 & 2.84 & 3.06 & 0.15 & 0.09 & 0.72 & 0.86 & 0.22 \\
\hline 0.19 & 10.51 & 3.50 & 0.29 & 0.71 & 0.53 & 0.30 & 1.35 \\
\hline 0.07 & 9.51 & 4.09 & 0.18 & 0.77 & 1.06 & 1.15 & 1.15 \\
\hline 0.13 & 14.89 & 3.05 & 0.05 & 0.08 & 0.40 & 1.98 & 3.06 \\
\hline 0.84 & 12.03 & 6.19 & 0.06 & 1.24 & 0.28 & 0.90 & 0.69 \\
\hline 0.52 & 14.92 & 5.58 & 0.09 & 1.36 & 6.79 & 0.87 & 0.56 \\
\hline 0.57 & 15.13 & 5.48 & 0.13 & 1.38 & 4.94 & 0.73 & 0.74 \\
\hline 0.43 & 13.51 & 5.59 & 0.10 & 2.09 & 3.63 & 1.09 & 0.75 \\
\hline 0.48 & 13.56 & 6.36 & 0.08 & 1.78 & 1.59 & 0.97 & 0.74 \\
\hline 0.51 & 6.98 & 2.75 & 0.06 & $\mathrm{BDL}$ & 0.27 & 0.41 & 1.58 \\
\hline 0.56 & 11.46 & 4.92 & 0.07 & 2.35 & 5.46 & 1.04 & 0.67 \\
\hline 0.62 & 13.40 & 4.99 & 0.21 & 1.53 & 1.59 & 0.98 & 1.17 \\
\hline 0.30 & 9.79 & 3.77 & 0.06 & 1.30 & 1.99 & 1.45 & 1.59 \\
\hline 0.32 & 10.88 & 2.91 & 0.05 & 0.06 & 1.21 & 1.64 & 2.13 \\
\hline 1.57 & 23.46 & 10.85 & 0.18 & 0.97 & 0.26 & 0.62 & 0.09 \\
\hline 0.80 & 22.23 & 12.24 & 0.17 & 0.84 & 0.16 & 0.41 & -0.06 \\
\hline 0.59 & 22.55 & 8.94 & 0.20 & 0.94 & 0.27 & 0.70 & 0.52 \\
\hline 2.85 & 26.77 & 17.13 & 0.21 & 14.93 & 0.12 & BDL & -0.22 \\
\hline 0.27 & 12.48 & 4.43 & 0.08 & 2.61 & 7.92 & 1.02 & 0.79 \\
\hline 0.47 & 13.56 & 5.27 & 0.10 & 1.31 & 2.77 & 1.22 & 1.00 \\
\hline 0.27 & 16.08 & 3.96 & 0.08 & 2.95 & 0.37 & BDL & 1.41 \\
\hline
\end{tabular}


Minimum values (following Mudrock calibration of PXRF) (ppm)

\begin{tabular}{rrrrrrrr} 
P2O5 & $\mathbf{B a}$ & $\mathbf{V}$ & $\mathbf{C r}$ & $\mathbf{N i}$ & $\mathbf{C u}$ & $\mathbf{Z n}$ & \multicolumn{2}{c}{$\mathbf{R b}$} \\
\hline 0.20 & 0.47 & 0.10 & 0.10 & 74.35 & 21.41 & 90.70 & 114.28 \\
0.04 & 0.17 & 0.06 & 0.11 & 28.32 & 21.55 & 45.95 & 9.69 \\
0.08 & 0.38 & 0.07 & 0.07 & 9.48 & 68.19 & 337.14 & 349.96 \\
0.09 & 0.32 & 0.06 & 0.07 & 14.99 & 1223.19 & 491.99 & 250.04 \\
0.08 & 0.27 & 0.06 & 0.07 & 6.59 & 11.23 & 42.94 & 476.78 \\
0.11 & 0.48 & 0.12 & 0.07 & 34.69 & 199.55 & 50.60 & 85.20 \\
0.17 & 0.47 & 0.10 & 0.04 & 39.81 & 35.45 & 105.80 & 129.96 \\
0.16 & 0.38 & 0.10 & 0.04 & 34.15 & 134.45 & 259.30 & 114.93 \\
0.18 & 0.36 & 0.09 & 0.10 & 78.04 & 428.92 & 163.79 & 116.16 \\
0.15 & 0.47 & 0.10 & 0.10 & 48.74 & 35.90 & 249.25 & 142.29 \\
0.04 & 0.46 & 0.09 & 0.03 & 2.59 & 5.10 & 47.16 & 121.66 \\
0.15 & 0.40 & 0.10 & 0.05 & 33.17 & 36.24 & 81.62 & 78.73 \\
0.15 & 0.45 & 0.10 & 0.06 & 21.43 & 26.58 & 721.80 & 144.98 \\
0.10 & 0.44 & 0.07 & 0.05 & 18.66 & 21.23 & 41.17 & 100.91 \\
0.08 & 0.44 & 0.07 & 0.05 & 11.63 & 14.08 & 27.03 & 96.44 \\
0.13 & 0.20 & 0.17 & 0.31 & 69.27 & 38.60 & 226.01 & 54.72 \\
0.12 & 0.27 & 0.12 & 0.15 & 34.09 & 99.09 & 384.63 & 62.75 \\
0.10 & 0.23 & 0.10 & 0.10 & 41.45 & 350.19 & 78.63 & 184.45 \\
0.15 & 0.12 & 0.27 & 0.44 & 275.54 & 86.03 & 151.62 & 8.18 \\
0.18 & 0.45 & 0.08 & 0.05 & 30.29 & 26.93 & 66.98 & 102.16 \\
0.14 & 0.46 & 0.09 & 0.04 & 11.95 & 31.22 & 103.51 & 77.37 \\
0.00 & -0.04 & 0.04 & 0.04 & 41.93 & 17.39 & 117.69 & 149.04 \\
\hline
\end{tabular}


Maximum values (following Mudrock calibration of PX

\begin{tabular}{|c|c|c|c|c|c|c|c|}
\hline $\mathrm{Zr}$ & $\mathrm{SiO2}$ & TiO2 & $\mathrm{Al2O3}$ & $\mathrm{Fe} 2 \mathrm{O3}$ & MnO & MgO & $\mathrm{CaO}$ \\
\hline 323.67 & 59.31 & 0.87 & 15.89 & 6.68 & 0.09 & 12.09 & 4.52 \\
\hline 66.23 & 89.81 & 0.29 & 5.37 & 4.35 & 0.16 & 8.19 & 0.92 \\
\hline 138.15 & 77.03 & 0.40 & 12.07 & 3.69 & 0.31 & 9.84 & 0.58 \\
\hline 207.25 & 76.93 & 0.29 & 11.79 & 4.46 & 0.19 & 10.26 & 1.22 \\
\hline 433.82 & 70.53 & 0.28 & 16.17 & 3.12 & 0.05 & 9.08 & 0.44 \\
\hline 204.60 & 72.06 & 1.09 & 14.43 & 6.88 & 0.06 & 10.92 & 0.35 \\
\hline 178.22 & 54.39 & 0.83 & 16.48 & 6.13 & 0.10 & 11.09 & 7.26 \\
\hline 198.49 & 57.22 & 0.83 & 16.48 & 6.13 & 0.14 & 10.93 & 5.39 \\
\hline 192.21 & 61.45 & 0.70 & 15.81 & 5.91 & 0.11 & 11.80 & 4.01 \\
\hline 156.85 & 66.21 & 0.76 & 15.74 & 6.64 & 0.08 & 12.24 & 1.74 \\
\hline 454.09 & 83.73 & 0.69 & 8.58 & 3.26 & 0.07 & 8.97 & 0.39 \\
\hline 359.61 & 64.19 & 0.85 & 12.77 & 4.94 & 0.08 & 10.68 & 6.00 \\
\hline 251.93 & 64.63 & 0.85 & 16.48 & 5.51 & 0.22 & 10.96 & 1.80 \\
\hline 209.28 & 73.45 & 0.50 & 13.11 & 3.89 & 0.07 & 10.02 & 2.50 \\
\hline 274.61 & 75.70 & 0.45 & 12.73 & 3.04 & 0.06 & 8.17 & 1.39 \\
\hline 418.32 & 49.25 & 1.87 & 24.67 & 11.19 & 0.19 & 13.21 & 0.35 \\
\hline 143.53 & 50.24 & 1.14 & 23.77 & 12.98 & 0.19 & 12.82 & 0.19 \\
\hline 161.07 & 53.57 & 0.84 & 23.91 & 9.24 & 0.21 & 11.64 & 0.32 \\
\hline 264.98 & 37.41 & 3.34 & 28.46 & 18.07 & 0.23 & 14.93 & 0.22 \\
\hline 217.08 & 56.96 & 0.56 & 13.60 & 4.83 & 0.08 & 10.73 & 8.41 \\
\hline 158.21 & 66.50 & 0.69 & 15.27 & 5.28 & 0.10 & 9.85 & 3.06 \\
\hline 113.97 & 50.43 & 0.63 & 16.83 & 4.48 & 0.09 & 5.26 & 0.53 \\
\hline
\end{tabular}




\begin{tabular}{rrrrrrrr} 
Na2O & \multicolumn{2}{c}{ K2O } & \multicolumn{2}{c}{ P2O5 } & $\mathbf{V}$ & $\mathbf{C r}$ & \multicolumn{2}{c}{$\mathbf{N i}$} \\
\hline 6.13 & 2.63 & 0.22 & 0.81 & 0.11 & 0.12 & 79.75 & 24.36 \\
2.48 & 0.55 & 0.11 & 0.42 & 0.08 & 0.13 & 32.54 & 24.83 \\
2.41 & 3.31 & 0.12 & 0.44 & 0.08 & 0.08 & 13.78 & 71.09 \\
2.81 & 2.91 & 0.12 & 0.42 & 0.07 & 0.09 & 21.24 & 1233.33 \\
2.79 & 5.47 & 0.10 & 0.42 & 0.07 & 0.08 & 10.30 & 13.08 \\
3.97 & 2.63 & 0.14 & 0.59 & 0.13 & 0.08 & 39.81 & 201.01 \\
6.32 & 2.26 & 0.18 & 0.58 & 0.11 & 0.05 & 47.16 & 37.47 \\
4.79 & 2.25 & 0.18 & 0.47 & 0.11 & 0.05 & 39.10 & 139.93 \\
4.30 & 2.42 & 0.18 & 0.46 & 0.10 & 0.12 & 86.23 & 435.24 \\
5.51 & 3.52 & 0.17 & 0.62 & 0.10 & 0.11 & 54.17 & 37.57 \\
2.34 & 3.24 & 0.11 & 0.55 & 0.09 & 0.04 & 6.50 & 6.91 \\
5.14 & 2.18 & 0.19 & 0.49 & 0.11 & 0.06 & 38.81 & 39.01 \\
2.40 & 2.60 & 0.15 & 0.68 & 0.11 & 0.07 & 26.15 & 30.00 \\
1.94 & 2.71 & 0.14 & 0.81 & 0.08 & 0.09 & 22.73 & 22.91 \\
1.99 & 3.43 & 0.12 & 1.09 & 0.08 & 0.06 & 15.47 & 14.55 \\
6.40 & 1.37 & 0.18 & 0.43 & 0.19 & 0.33 & 75.66 & 43.74 \\
8.03 & 1.77 & 0.17 & 0.47 & 0.14 & 0.17 & 43.22 & 99.64 \\
4.78 & 1.96 & 0.14 & 0.47 & 0.11 & 0.11 & 53.41 & 360.79 \\
15.37 & 0.59 & 0.25 & 0.22 & 0.29 & 0.46 & 284.48 & 90.04 \\
5.64 & 2.48 & 0.19 & 0.56 & 0.09 & 0.06 & 34.54 & 27.97 \\
2.55 & 2.28 & 0.16 & 0.60 & 0.10 & 0.05 & 16.80 & 31.62 \\
3.87 & 2.97 & 0.06 & 0.42 & 0.08 & 0.04 & 47.68 & 19.57 \\
\hline
\end{tabular}


ation of PXRF) (ppm)

\begin{tabular}{rrr}
$\mathbf{Z n} \mathbf{R b}$ & $\mathbf{Z r}$ \\
\hline 94.42 & 127.09 & 333.81 \\
48.95 & 15.53 & 96.01 \\
353.13 & 395.80 & 156.59 \\
499.31 & 265.75 & 234.65 \\
45.94 & 509.52 & 461.47 \\
55.58 & 86.52 & 244.99 \\
109.29 & 137.18 & 195.17 \\
268.04 & 119.88 & 210.73 \\
164.97 & 122.00 & 203.74 \\
254.76 & 155.32 & 177.86 \\
50.31 & 156.33 & 504.44 \\
84.73 & 93.86 & 375.25 \\
734.24 & 152.28 & 275.58 \\
43.96 & 103.53 & 239.67 \\
31.84 & 102.19 & 313.26 \\
239.81 & 74.78 & 462.92 \\
391.78 & 68.95 & 175.57 \\
80.54 & 205.10 & 185.50 \\
161.78 & 14.44 & 303.81 \\
70.06 & 106.02 & 240.80 \\
110.36 & 83.58 & 177.40 \\
125.66 & 173.19 & 135.70 \\
\hline & &
\end{tabular}

